Prepared for the U.S. Department of Energy

under Contract DE-AC05-76RL01830

\title{
Hanford Waste Physical and Rheological Properties: Data and Gaps
}

$\begin{array}{llll}\text { BE Wells } & \text { Y Onishi } & \text { CA Burns } & \text { RC Daniel } \\ \text { DE Kurath } & \text { JL Huckaby } & \text { EC Buck } & \text { KK Anderson } \\ \text { LA Mahoney } & \text { SK Cooley } & \text { JM Tingey } & \end{array}$

August 2011

Pacific Northwest NATIONAL LABORATORY

Proudly Operated by Battelle Since 1965 


\title{
DISCLAIMER
}

This report was prepared as an account of work sponsored by an agency of the United States Government. Neither the United States Government nor any agency thereof, nor Battelle Memorial Institute, nor any of their employees, makes any warranty, express or implied, or assumes any legal liability or responsibility for the accuracy, completeness, or usefulness of any information, apparatus, product, or process disclosed, or represents that its use would not infringe privately owned rights. Reference herein to any specific commercial product, process, or service by trade name, trademark, manufacturer, or otherwise does not necessarily constitute or imply its endorsement, recommendation, or favoring by the United States Government or any agency thereof, or Battelle Memorial Institute. The views and opinions of authors expressed herein do not necessarily state or reflect those of the United States Government or any agency thereof.

\author{
PACIFIC NORTHWEST NATIONAL LABORATORY \\ operated by \\ BATTELLE \\ for the \\ UNITED STATES DEPARTMENT OF ENERGY \\ under Contract DE-ACO5-76RL01830
}

Printed in the United States of America
Available to DOE and DOE contractors from the
Office of Scientific and Technical Information,
P.O. Box 62, Oak Ridge, TN 37831-0062;
ph: (865) 576-8401
fax: (865) 5765728
email: reports@adonis.osti.gov

\author{
Available to the public from the National Technical Information Service, \\ U.S. Department of Commerce, 5285 Port Royal Rd., Springfield, VA 22161 \\ ph: (800) 553-6847 \\ fax: (703) 605-6900 \\ email: orders@nits.fedworld.gov \\ online ordering: http://www.ntis.gov/ordering.htm
}


PNNL-20646

EMSP-RPT-006

\section{Hanford Waste Physical and Rheological Properties: Data and Gaps}

$\begin{array}{lll}\text { BE Wells } & \text { JL Huckaby } & \text { JM Tingey } \\ \text { DE Kurath } & \text { SK Cooley } & \text { RC Daniel } \\ \text { LA Mahoney } & \text { CA Burns } & \text { KK Anderson } \\ \text { Y Onishi } & \text { EC Buck } & \end{array}$

August 2011

Prepared for

the U.S. Department of Energy

under Contract DE-AC05-76RL01830

Pacific Northwest National Laboratory

Richland, Washington 99352 



\section{Executive Summary}

The Hanford Site in Washington State manages 177 underground storage tanks containing approximately $250,000 \mathrm{~m}^{3}$ of waste generated during past defense reprocessing and waste management operations. These tanks contain a mixture of sludge, saltcake and supernatant liquids. The insoluble sludge fraction of the waste consists of metal oxides and hydroxides and contains the bulk of many radionuclides such as the transuranic components and ${ }^{90} \mathrm{Sr}$. The saltcake, generated by extensive evaporation of aqueous solutions, consists primarily of dried sodium salts. The supernates consist of concentrated (5-15 $\underline{\mathrm{M}}$ ) aqueous solutions of sodium and potassium salts. The 177 storage tanks include 149 single-shell tanks (SSTs) and 28 double-shell tanks (DSTs).

Ultimately the wastes need to be retrieved from the tanks for treatment and disposal. The SSTs contain minimal amounts of liquid wastes, and the Tank Operations Contractor is continuing a program of moving solid wastes from SSTs to interim storage in the DSTs. The Hanford DST system provides the staging location for waste feed delivery to the Department of Energy (DOE) Office of River Protection's (ORP) Hanford Tank Waste Treatment and Immobilization Plant (WTP). The WTP is being designed and constructed to pretreat and then vitrify a large portion of the wastes in Hanford's 177 underground waste storage tanks.

The retrieval, transport, treatment and disposal operations involve the handling of a wide range of slurries. Solids in the slurry have a wide range of particle size, density and chemical characteristics. Depending on the solids concentration the slurries may exhibit a Newtonian or a non-Newtonian rheology.

The extent of knowledge of the physical and rheological properties is a key component to the success of the design and implementation of the waste processing facilities. These properties are used in engineering calculations in facility designs. Knowledge of the waste properties is also necessary for the development and fabrication of simulants that are used in testing at various scales. The expense and hazards associated with obtaining and using actual wastes dictates that simulants be used at many stages in the testing and scale-up of process equipment. The results presented in this report should be useful for estimating process and equipment performance and provide a technical basis for development of simulants for testing.

The purpose of this document is to provide an updated summary of the Hanford waste characterization data pertinent to safe storage, retrieval, transport and processing operations for both the tank farms and the WTP and thereby identify gaps in understanding and data. Important waste parameters for these operations are identified by examining examples of relevant mathematical models of selected phenomena including:

- Pipeline Critical Velocity

- Solid Settling Velocity

- Effective Cleaning Radius

- Vessel Wall/Bottom Erosion

- Critical Suspension Velocity 
- Suspended Solid Cloud Height

- Suspended Solid Concentration

- Solid Dissolution and Filtration

- Gas Generation, Retention, and Release

Typical engineering correlations are presented and discussed with the most important waste parameters identified. The identification is based on the functionality of the parameter in the correlations. The important parameters include physical and rheological properties of the waste. These properties of the as-stored Hanford waste, including both the liquid and solid phases, are presented by tank and waste type. In selected cases, composite results are presented that represent combinations of wastes.

The important parameters include:

- Liquid Density and pH

- Liquid Rheology (Viscosity)

- UDS Composition and Particle Density

- UDS Primary Particle Size and Shape

- UDS Particle Size Distribution

- UDS Particle Settling

- Slurry Rheology and Shear Strength

- Estimated Particle Size and Density Distributions

The data sets in Wells et al. (2007) (UDS composition and particle density, UDS primary particle size and shape, UDS particle size distributions [PSDs], and estimated particle size and density distributions [PSDDs]) and Poloski et al. (2007) (liquid and slurry rheology, and UDS particle settling) were updated with additional data. The primary source of additional data is from a recent series of tests sponsored by the WTP to support resolution of issue M12 raised by and External Flowsheet Review Team (EFRT). ${ }^{(a)}$ These tests involved an extensive suite of characterization and bench-scale process testing of 8 waste groups representing approximately $75 \%$ of the waste expected to be processed through the WTP. Additional information on the morphology of the waste solids was also included.

A summary of the updates from Wells et al. (2007) and Poloski et al. (2007) to the current work is provided in Table S.1. The data sets of the prior reports are expanded with additional waste data, and a more detailed list of UDS solid phase compounds is developed. The changes to the data set are generally thus expansion and refinement, not alteration of the prior work. The parameters are presented by 1) tank, 2) waste type (general, i.e., sludge or saltcake, and specific), and 3) composite. As in Wells et al. (2007), the composites are constructed using a volume weighted average of individual tanks or waste types. Data

(a) Barnes SM and R Voke. 2006. "Issue Response Plan for Implementation of External Flowsheet Review Team (EFRT) Recommendations - M12: Undemonstrated Leaching Process.” 24590-WTP-PL-ENG-06-0024 Rev 0. Bechtel National Inc., Richland, Washington. 
uncertainties are quantified where possible. Since this document contains the most extensive data and analyses it is recommended for use over the prior work.

An analysis of the effect of sample storage was conducted to make sure that the data sets represent equivalent or similar waste conditions. Further, the parameter values were evaluated with available in situ data pertaining to waste mobilization and mixing and undissolved solid (UDS) settling. The data therefore are as consistent and complete as possible.

Table S.1. Data Update Difference Summary

\begin{tabular}{|c|c|c|c|c|}
\hline \multicolumn{2}{|c|}{ Properties in Update } & $\begin{array}{l}\text { Wells et al. } \\
(2007)\end{array}$ & $\begin{array}{c}\text { Poloski et al. } \\
\text { (2007) }\end{array}$ & Update Difference \\
\hline \multirow{3}{*}{ Liquid } & Density & $\mathrm{n} / \mathrm{a}$ & $\mathrm{n} / \mathrm{a}$ & not included in prior work \\
\hline & $\mathrm{pH}$ & $\mathrm{n} / \mathrm{a}$ & $\mathrm{n} / \mathrm{a}$ & not included in prior work \\
\hline & Rheology & $\mathrm{n} / \mathrm{a}$ & included & additional data \\
\hline \multirow{7}{*}{ Solids } & Particle Density & included & $\mathrm{n} / \mathrm{a}$ & 52 solid phase compounds from 16 \\
\hline & $\begin{array}{l}\text { Primary Particle } \\
\text { Size and Shape }\end{array}$ & included & $\mathrm{n} / \mathrm{a}$ & 52 solid phase compounds from 16 \\
\hline & $\begin{array}{l}\text { Particle Size } \\
\text { Distribution }\end{array}$ & included & $\mathrm{n} / \mathrm{a}$ & $\begin{array}{c}\text { additional data, } \\
\text { alternate instrument configuration, } \\
\text { combined, maximum, and minimum } \\
\text { PSDs } \\
\text { by tank, waste type, and composites }\end{array}$ \\
\hline & $\begin{array}{c}\text { Particle Size and } \\
\text { Density } \\
\text { Distribution } \\
\end{array}$ & included & $\mathrm{n} / \mathrm{a}$ & by tank, waste type, and composites \\
\hline & Particle Settling & $\mathrm{n} / \mathrm{a}$ & included & $\begin{array}{c}\text { additional data, } \\
\text { comparison to PSDDs }\end{array}$ \\
\hline & Shear Strength & $\mathrm{n} / \mathrm{a}$ & included & additional data \\
\hline & Bingham Rheology & $\mathrm{n} / \mathrm{a}$ & included & additional data \\
\hline
\end{tabular}

The final step in this effort was to conduct a gap analysis to identify gaps in characterization data, analytical methods and data interpretation. The primary focus is on data gaps identified by considering the parameters by waste type, percent of waste type mass or volume represented and the uncertainty of the parameter. For the purpose of defining data gaps, a tank waste is treated as "represented" if the parameter of interest has been measured for at least one sample of that tank's waste. In this approach, a number of important factors are not considered beyond those used to select specific data for the overall data set. These factors include the extent to which a sample represents the tank contents, the number of samples, the number of measurements made on a sample or the measurement technique.

A summary of the number of tanks and waste types for which a given parameter is characterized is provided in Table S.2. The report sections in which each parameter is described are listed, as well as 
general categories of waste handling operations that are dependent on the parameter. The percentage of the Hanford UDS volume represented is determined from the primary waste types represented and their relative fractions to the waste inventory. In general, less than $50 \%$ of the Hanford UDS volume is represented by the waste parameter data. The primary waste type of a given tank is defined as the most prevalent waste type. The number of tanks and primary waste types are listed as well as the number of waste types that are considered to have more than $50 \%$ of the UDS volume represented by the data. Some of the information available from the WTP work to support resolution of the M12 issue is not reflected in the data gaps identified in Table S.2 as some of the composite samples used were composed on a number of different but similar waste types. The composite nature of these samples made it difficult to attribute the results to a particular waste type.

Specific gaps in the waste properties data base for which relatively little information is available include:

- Particle morphology: at least $50 \%$ of the solids are amorphous and are not well characterized

- $\quad$ Dry solids density

- Settled waste shear strength as a function of time

- Impact of sample storage on waste properties.

Specific gaps in analytical methods as applied to actual waste samples include:

- Methods to measure particle size and shape in a flowing fluid

- Routine methods for determining the volume fraction of solids in slurry and solids density

- Particle size and density distributions

- Routine methods for determining the fractal dimension of agglomerates

- Methods for determining the abrasive properties of tank waste slurries

- Solids settling rates in concentrated slurries.

Gaps in scale-up, data interpretation and analysis include:

- Interpretation of settling data

- Determination of technically defensible PSD uncertainties

- Critical shear stress for erosion. 
Table S.2. Summary of Waste Physical and Rheological Property Data Gaps

\begin{tabular}{|c|c|c|c|c|c|c|}
\hline \multicolumn{2}{|c|}{ Property } & $\begin{array}{l}\text { Report } \\
\text { Section }\end{array}$ & $\begin{array}{l}\text { Waste Handling } \\
\text { Operation } \\
\text { Importance } \\
\text { (see Section 2.0) }\end{array}$ & $\begin{array}{c}\text { Hanford UDS Volume } \\
\text { Represented }\end{array}$ & $\begin{array}{c}\text { Number of Individual Tanks (177 } \\
\text { total), Waste Types (44 total) } \\
\text { Represented }\end{array}$ & $\begin{array}{c}\text { Number of Waste } \\
\text { Types with }>50 \% \\
\text { UDS Volume } \\
\text { Representation } \\
\end{array}$ \\
\hline \multirow{3}{*}{ Liquid } & Density & 3.2 .1 & $\begin{array}{l}\text { storage, mixing, } \\
\text { transfer }\end{array}$ & not applicable & all & not applicable \\
\hline & $\mathrm{pH}$ & 3.2 .1 & storage, treatment & not applicable & all & not applicable \\
\hline & Rheology & 3.2 .2 & $\begin{array}{l}\text { storage, mixing, } \\
\text { transfer }\end{array}$ & not applicable & 11 tanks, 7 waste types & not applicable \\
\hline \multirow{12}{*}{ Solids } & $\begin{array}{l}\text { Particle } \\
\text { Density }\end{array}$ & 3.2 .3 & $\begin{array}{l}\text { storage, mixing, } \\
\text { transfer }\end{array}$ & See discussion in Sections 5.1.2 and & 1.3. & \\
\hline & $\begin{array}{l}\text { Primary } \\
\text { Particle Size } \\
\text { and Shape }\end{array}$ & 3.2 .4 & $\begin{array}{l}\text { storage, mixing, } \\
\text { transfer, treatment }\end{array}$ & $\begin{array}{l}\text { Images for limited solids phase comp } \\
\text { Finite set of images for specific solid } \\
\text { Images from a limited number of tanl }\end{array}$ & $\begin{array}{l}\text { ounds. } \\
\text { phase compounds. } \\
\text { ss. }\end{array}$ & \\
\hline & \multirow{3}{*}{$\begin{array}{l}\text { Particle Size } \\
\text { Distribution }\end{array}$} & \multirow{3}{*}{3.2 .5} & \multirow{3}{*}{$\begin{array}{l}\text { storage, mixing, } \\
\text { transfer, treatment }\end{array}$} & Flowing Sonicated, $40 \%$ & 22 tanks, 16 waste types & 5 waste types \\
\hline & & & & Flowing Unsonicated, 58\% & 22 tanks, 16 waste types & 5 waste types \\
\hline & & & & No-Flow Unsonicated, 41\% & 20 tanks, 11 waste types & 3 waste types \\
\hline & $\begin{array}{l}\text { Particle } \\
\text { Settling }\end{array}$ & 3.2.6 & $\begin{array}{l}\text { Not a specific input } \\
\text { parameter for listed } \\
\text { storage, mixing, } \\
\text { transfer, and } \\
\text { treatment models. }\end{array}$ & $23 \%$ & 20 tanks, 13 waste types & 3 waste types \\
\hline & \multirow{4}{*}{ Shear Strength } & \multirow{4}{*}{3.2 .7} & \multirow{4}{*}{ storage, mixing } & General, 52\% & 36 tanks, 15 waste types & 7 waste types \\
\hline & & & & Function of UDS Conc. $0.3 \%$ & 4 tanks, 2 waste types & 0 waste types \\
\hline & & & & Function of Time, $0.2 \%{ }^{(\mathrm{a})}$ & 1 tanks, 1 waste type & 0 waste types \\
\hline & & & & Function of Elevation, 39\% & 25 tanks, 12 waste types & 3 waste types \\
\hline & \multirow{2}{*}{$\begin{array}{l}\text { Bingham } \\
\text { Rheology }\end{array}$} & \multirow{2}{*}{3.2 .7} & \multirow{2}{*}{$\begin{array}{l}\text { mixing, transfer, } \\
\text { treatment. }\end{array}$} & General, 51\% & 29 tanks, 18 waste types & 5 waste types \\
\hline & & & & Function of UDS Conc. 26\% & 23 tanks, 13 waste types & 4 waste types \\
\hline
\end{tabular}





\section{Acknowledgments}

Staff from Bechtel National Inc., Washington River Protection Solutions, and Pacific Northwest National Laboratory provided critical expert comments and review to define pertinent parameters with the existing data. These staff include S Barnes, EC Buck, R Burk, WS Callaway III, G Cooke, PA Gauglitz, DA Greer, DL Herting, GJ Lumetta, RA Peterson, BM Rapko, JH Rasmussen, and JG Reynolds.

The authors thank EBK Baer, JA Bamberger, J Blanchard, MS Fountain, EC Golovich, JJ Jenks, DM Pfund, RJ Serne, and ST Yokuda for their contributions with data collection and review;

PP Schonewill for his independent technical review; WC Cosby, BK Wilson, and DR Payson for the technical editing; and MG Champion for administrative support. 



\section{Acronyms and Abbreviations}

\begin{tabular}{|c|c|}
\hline AISI & American Iron and Steel Institute \\
\hline $\mathrm{BBI}$ & Best Basis Inventory \\
\hline BBP & butylbenzyl phthalate (sludge) \\
\hline BDGRE & buoyant displacement gas release event \\
\hline BNFL & British Nuclear Fuels, Ltd. \\
\hline BNI & Bechtel National, Inc. \\
\hline $\mathrm{BP}$ & bismuth phosphate (sludge) \\
\hline $\mathrm{BR}$ & buoyancy ratio \\
\hline $\mathrm{CI}$ & confidence interval \\
\hline CUF & cells unit filter \\
\hline DI & deionized (water) \\
\hline DOE & U.S. Department of Energy \\
\hline DST & double-shell tank \\
\hline ECR & effective cleaning radius \\
\hline EDS & energy dispersive X-ray spectroscopy \\
\hline EFRT & External Flowsheet Review Team \\
\hline EM & Environmental Management \\
\hline EMSP & Environmental Management Science Program \\
\hline ER & energy ratio \\
\hline ESP & Environmental Simulation Program \\
\hline FTIR & Fourier transform infrared \\
\hline HAADF & high-angle annular dark field \\
\hline HGR & Hydrogen Generation Rate (model) \\
\hline HLW & high-level waste \\
\hline ITS & in-tank solidification \\
\hline LAW & low-activity waste \\
\hline LFL & lower flammability limit \\
\hline LLW & low-level waste \\
\hline LTL & lower tolerance level \\
\hline MW & mixed waste (sludge) \\
\hline NCAW & neutralized current acid waste \\
\hline NQA & Nuclear Quality Assurance \\
\hline ORP & Office of River Protection \\
\hline PJM & pulse jet mixing \\
\hline PNNL & Pacific Northwest National Laboratory \\
\hline PSD & particle size distribution \\
\hline
\end{tabular}


PSDD particle size density distribution

PUREX plutonium uranium extraction

QA quality assurance

REDOX reduction oxidation

RPL

Radiochemical Processing Laboratory

SEM

scanning electron microscopy

SRR

strontium recovery (waste)

SST

single-shell tank

STEM

scanning transmission electron microscopy

TBP

tributyl phosphate

TEM

transmission electron microscopy

TGA

thermogravimetric analysis

TI

tolerance interval

TWINS

Tank Waste Information Network System

UCL upper confidence limit

UDS

undissolved solids; those solids, whether soluble or insoluble, that are present as a solid phase and are not dissolved in the liquid phase of the waste

UL upper limit

UPA

ultrafine particle analyzer

UTL upper tolerance level

WRPS

Washington River Protection Solutions LLC

WTP

Hanford Tank Waste Treatment and Immobilization Plant

XRD

$\mathrm{X}$-ray diffraction 


\section{Symbols}

In a portion of this report, example models for phenomena of safe storage, retrieval, transport, and processing operations for Hanford waste are summarized. The model terms are defined on a model-by-model basis, and the symbols are typically preserved from the original reference. Given the breadth of phenomena and models as related to the waste parameters, there are instances of repeated terms and definitions as reflected in this list.

\begin{tabular}{|c|c|}
\hline$\% \mathrm{LFL}_{\mathrm{CH} 4}$ & methane concentration at $100 \% \mathrm{LFL}$ \\
\hline$\% \mathrm{LFL}_{\mathrm{H} 2}$ & hydrogen concentration at $100 \% \mathrm{LFL}$ \\
\hline$\% \mathrm{LFL}_{\mathrm{HS}}$ & headspace flammable gas concentration following gas release \\
\hline$\% \mathrm{LFL}_{\mathrm{NH} 3}$ & ammonia concentration at $100 \% \mathrm{LFL}$ \\
\hline$a$ & longest mutually perpendicular axis of particle, also, distance above the solid surface \\
\hline$A$ & constant, also, filtering surface area \\
\hline$A_{1}$ & area of sludge exposed to the bubble \\
\hline$A_{2}$ & area over which sludge strength applies \\
\hline$A_{R}$ & Archimedes Number \\
\hline$b$ & intermediate mutually perpendicular axis of particle, also, coefficient \\
\hline$c$ & shortest mutually perpendicular axis of particle \\
\hline$C$ & constant, also, solids concentration \\
\hline$C_{0}$ & solids volume percent at the tank bottom \\
\hline$C_{a}$ & solids concentration at elevation "a” above the tank bottom \\
\hline$C_{D}$ & drag coefficient \\
\hline $\mathrm{CF}$ & calibration factor \\
\hline$\left[\mathrm{CH}_{4}\right]_{\mathrm{RG}}$ & methane concentration in the released gas in nonconvective layer (vol\%) \\
\hline$C_{\max }$ & solids concentration (corresponding to $C_{V \max }$ ) \\
\hline$C_{r e f}$ & reference case solid concentration (wt\%) \\
\hline$C_{V}$ & solids volume fraction \\
\hline$C_{\operatorname{Vmax}}$ & maximum solids volume fraction (the packing factor) \\
\hline $\mathrm{C}_{\mathrm{W}}$ & maximum solid concentration in wt\% \\
\hline$D$ & tank or pipe diameter \\
\hline$d$ & total liquid depth \\
\hline$d_{50}$ & median diameter of the solids by volume \\
\hline
\end{tabular}




\begin{tabular}{|c|c|}
\hline$D C$ & PJM duty cycle \\
\hline$D_{F}$ & fractal dimension (between 0 and 3) \\
\hline$d_{f}$ & median floc size \\
\hline$d_{j}$ & nozzle diameter \\
\hline $\mathrm{D}_{\mathrm{L}}$ & design life (year) \\
\hline$d_{\max }$ & maximum stable channel depth \\
\hline$D_{P}$ & mean pore diameter \\
\hline d & particle diameter \\
\hline$d_{S}$ & spherical particle diameter \\
\hline$d_{S-r e f}$ & solids particle weighted-mean diameter from reference case \\
\hline E & modulus of elasticity \\
\hline$e$ & void ratio \\
\hline$E_{30^{\circ}}$ & erosion rate for with $30^{\circ}$ impingement angle \\
\hline$E_{90^{\circ}}$ & erosion rate for with $90^{\circ}$ impingement angle \\
\hline $\mathrm{E}_{\mathrm{d}}$ & PJM duty factor \\
\hline$E_{\text {pipe }}$ & erosion rate of horizontal mild steel straight pipe circumference (mm/year) \\
\hline$E_{\text {vessel }}$ & PJM wall erosion depth at end of design life \\
\hline$E_{\text {vessel-ref }}$ & PJM wall erosion depth rate of reference case \\
\hline$F$ & $\begin{array}{l}\text { buoyant force on a particle or vessel usage factor or cumulative percentile of the } \\
\text { distribution up to a specified value } x\end{array}$ \\
\hline $\mathrm{F}_{\mathrm{D}}$ & Densimetric Froude Number \\
\hline$F_{\text {gasRelease }}$ & fraction of gas released (assumed to be $100 \%$ ) \\
\hline$F_{L}$ & dimensionless factor (a densimetric Froude Number) \\
\hline$F_{S}$ & Solid Erosion by Jet shear \\
\hline$G$ & normal solids concentration (wt\%) or Gibbs free energy \\
\hline$g$ & gravitational acceleration \\
\hline $\mathrm{G}_{0}$ & initial shear strength \\
\hline $\mathrm{G}_{\mathrm{t}}$ & shear strength \\
\hline$g_{v}$ & volumetric gas generation rate per unit volume of gas-free slurry \\
\hline $\mathrm{G}_{\infty}$ & equilibrium shear strength \\
\hline
\end{tabular}




\begin{tabular}{|c|c|}
\hline $\mathrm{H}$ & waste thickness, height of saturated non-convective layer, or vessel operating height \\
\hline$h$ & hydraulic head \\
\hline$\left[\mathrm{H}_{2}\right]_{\mathrm{RG}}$ & hydrogen concentration in the released gas in nonconvective layer (vol\%) \\
\hline$H_{C}$ & cloud height \\
\hline$H_{S}$ & height of the lithostatic column above a bubble \\
\hline $\mathrm{HG}_{\mathrm{WNCL}}$ & hydrogen gas generation rate in saturated settled solid layer \\
\hline$I$ & fraction of time for maximum solids loading \\
\hline$J$ & constant \\
\hline $\mathrm{k}$ & gel time constant, also, erodibility coefficient or permeability \\
\hline K & $\begin{array}{l}\text { hydraulic conductivity, also, a constant that relates bond strength to material } \\
\text { properties and surface chemistry condition }\end{array}$ \\
\hline$K_{0}$ & ratio between horizontal and vertical effective stress \\
\hline $\mathrm{k}_{1}, \mathrm{k}_{2}$ & multiplying factors (for one-sided and two-sided TIs, respectively) \\
\hline $\mathrm{LL}_{\mathrm{i}}$ & lower prediction limit for the $\mathrm{i}^{\text {th }}$ data point \\
\hline$M$ & constant, function of scale factor \\
\hline$N$ & constant, function of scale factor \\
\hline$n$ & $\begin{array}{l}\text { sample size or number of operating jets/pulse tubes or the number of moles of } \\
\text { species } j\end{array}$ \\
\hline $\mathrm{N}_{\mathrm{H}}$ & Gas Holdup Number \\
\hline$\left[\mathrm{NH}_{3}\right]_{\mathrm{RG}}$ & ammonia concentration in the released gas in nonconvective layer (vol\%) \\
\hline$P$ & particle roundness, also, total pressure across a filter system \\
\hline$p$ & $\begin{array}{l}\text { pressure head or total pressure across a filter system or the number of linearly } \\
\text { independent mass-balance constrains }\end{array}$ \\
\hline $\mathrm{P}_{\mathrm{HS}}$ & tank headspace pressure \\
\hline $\mathrm{P}_{\mathrm{WNCL}}$ & representative retained gas pressure in the saturated settled solid layer \\
\hline$Q$ & transfer pump discharge \\
\hline$q$ & specific discharge \\
\hline$R$ & $\begin{array}{l}\text { Particle Reynolds Number or agglomerate size or radial distance from the pump } \\
\text { suction inlet }\end{array}$ \\
\hline$r$ & $\begin{array}{l}\text { primary (crystal) particle size or filter resistance including possible solids built-up on } \\
\text { a filter }\end{array}$ \\
\hline$R_{e J}$ & Jet Reynolds Number \\
\hline$r_{0}$ & undisturbed channel radius \\
\hline$r_{S}$ & solids particle radius \\
\hline
\end{tabular}


sample deviation

solid-to-liquid density ratio

scale factor

amount of solid eroded per unit bed surface area per unit time

Strouhal Number

time, also, gel time

liquid temperature in $(\mathrm{K})$

a student's $t$ statistic based on the prediction interval confidence level of $100(1-\alpha) \%$ and degrees of freedom equal to $n-p$, where $n$ is the number of data points, $p$ is the number of estimated model parameters, and for the $95 \%$ prediction limits calculated, $\alpha=0.05$

pulse jet mixing (PJM) cycle time, complete cycle includes discharge and re-fill

Non-dimensional Solids Settling Distance

temperature of the saturated settled solid layer

slurry or flow velocity

shear velocity

critical suspension velocity, all solids suspended at the end of the pulse

nozzle jet velocity

upper prediction limit for the $\mathrm{i}^{\text {th }}$ data point

gas bubble rise velocity at the waste surface

PJM jet velocity of reference case

hindered terminal settling velocity

accumulated solids volume

critical velocity

volume of headspace after gas release

slurry velocity

initial interface velocity

Poisson ratio

radial velocity toward the pump suction inlet

solids settling velocity

hindered settling velocity

settling velocity of a single particle

calculated void fraction in saturated settled solid layer 
weight of solids per unit volume of filtrate

mass fraction of UDS

$\mathrm{n} \times \mathrm{p}$ matrix of partial derivatives of the nonlinear model in Eq. (3.2.2.2) with respect to the model parameters

$\mathrm{i}^{\text {th }}$ row of the matrix $\mathbf{W}$

groundwater flow direction

sample mean

vertical distance above the solid surface

interface height

initial interface height

\section{Greek Letters}

constant, units of velocity

neutral buoyancy of saturated settled solid layer relative to the overlying convective layer constant, also, scale parameter

fraction of eddies having velocities equal to or greater than the settling velocity nonconvective layer strain at failure

constant, also, UDS concentration by volume

initial solids volume fraction in the well-mixed suspension

final solids volume fraction as time goes to infinity

jet density

volume fraction of non-salt solid

pulse volume fraction

ratio of pulse tube to vessel cross-section area

Solids volume fraction (a relative solids volume fraction to a reference solids volume fraction)

ratio of pressure head of convective layer to the headspace pressure

von Kármán constant

constant related to solids size

location parameter 


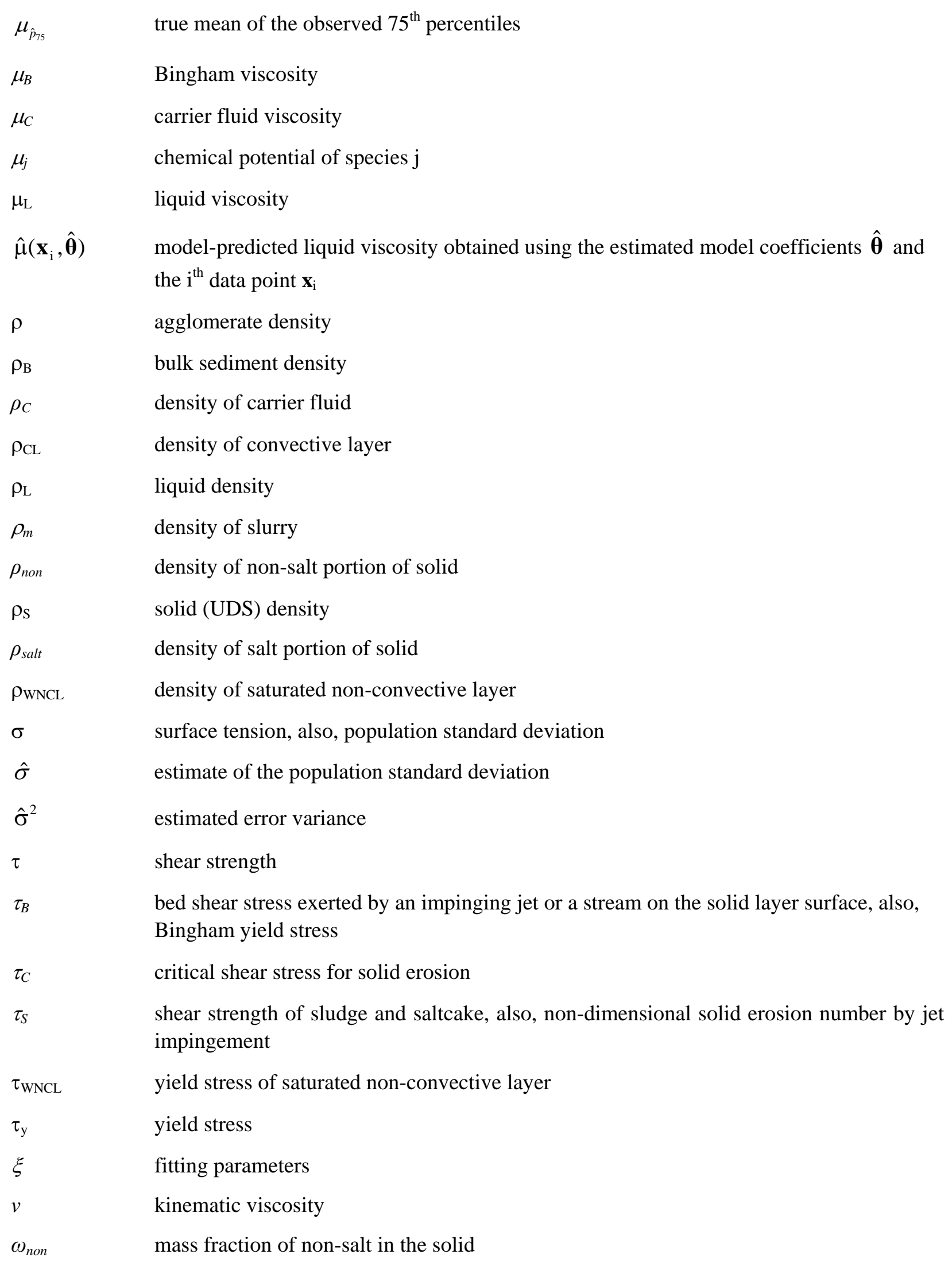




\section{Contents}

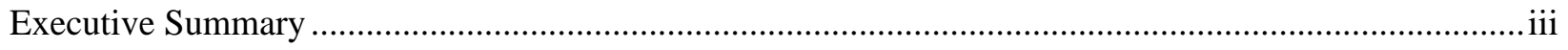

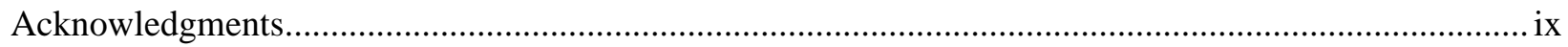

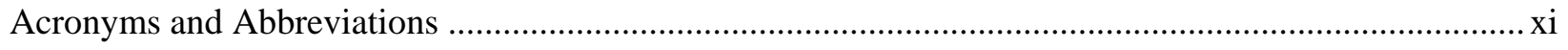

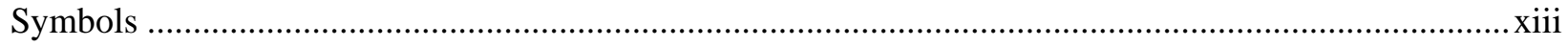

Greek Letters................................................................................................................................ Xvii

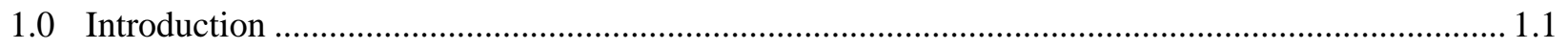

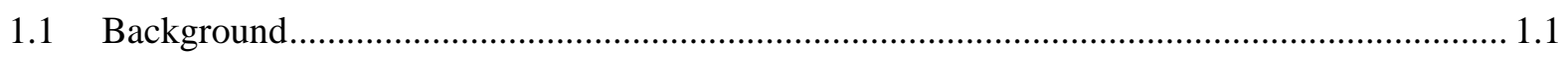

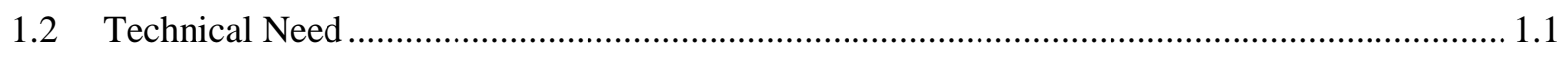

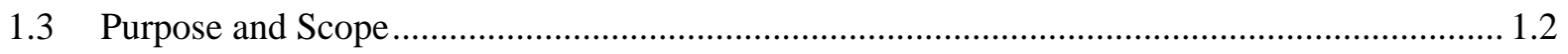

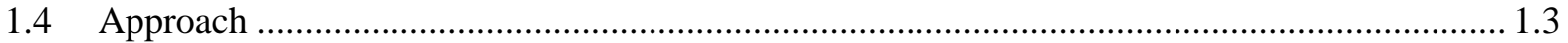

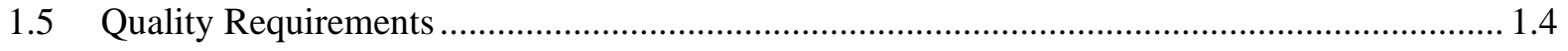

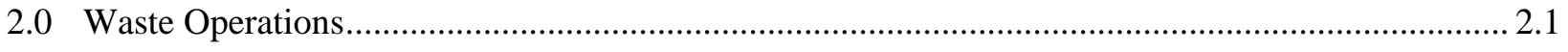

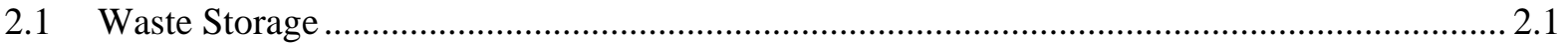

2.1.1 Solids Settling Velocity .............................................................................................. 2.1

2.1.2 Gas Generation, Retention, and Release ................................................................ 2.11

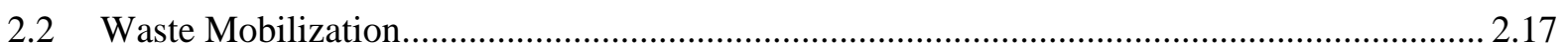

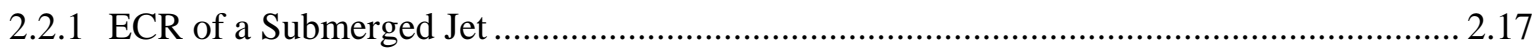

2.2.2 Pulsed Jet Mixer Resuspension of Settled Solids............................................................. 2.19

2.2.3 Jet Mixing Cloud Height ................................................................................................. 2.22

2.2.4 Suspended Solids Concentration.................................................................................. 2.24

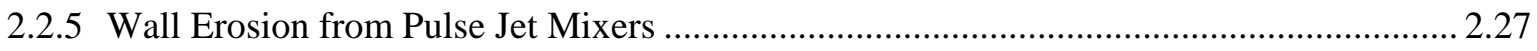

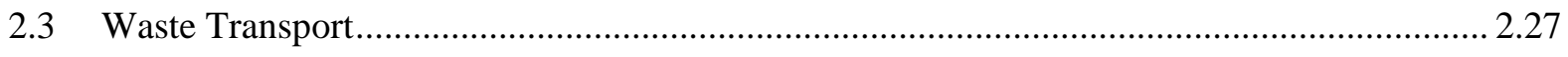

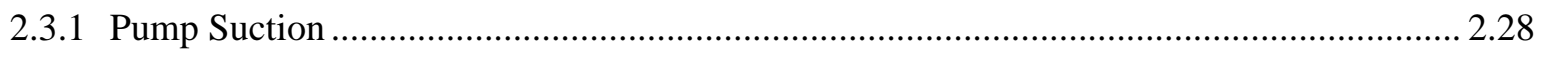

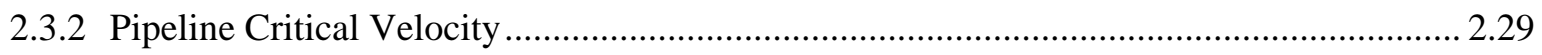

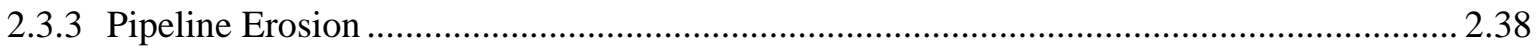

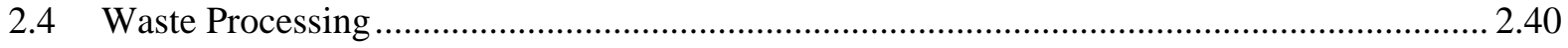

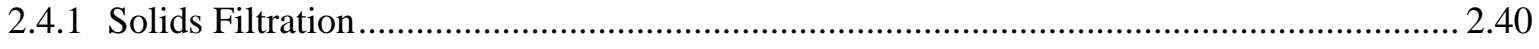

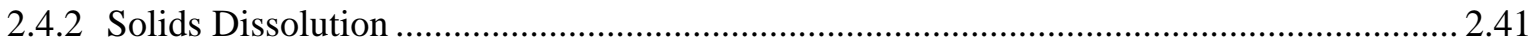

2.5 Summary of Physical and Rheological Parameters ........................................................... 2.41

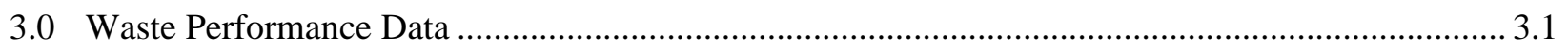

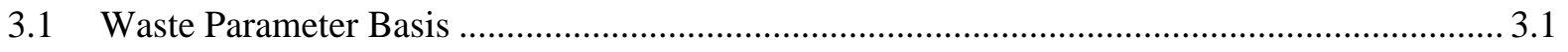

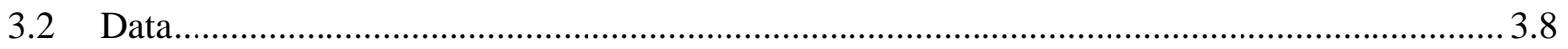

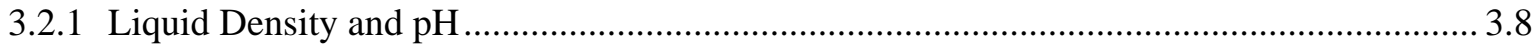




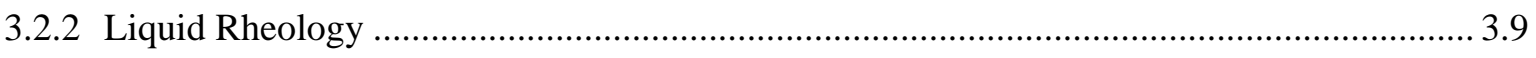

3.2.3 Undissolved Solid Composition and Particle Density ................................................... 3.19

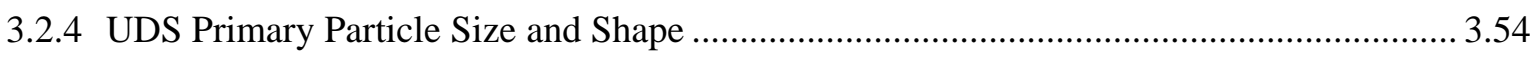

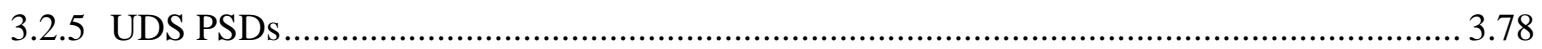

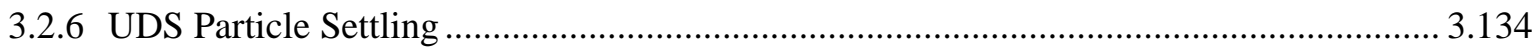

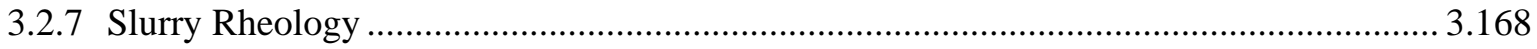

3.3 Estimated Particle Size and Density Distributions …........................................................... 3.228

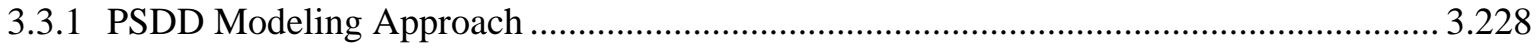

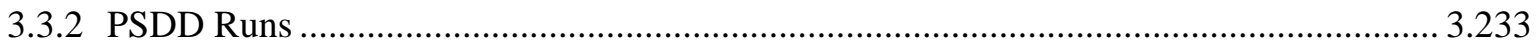

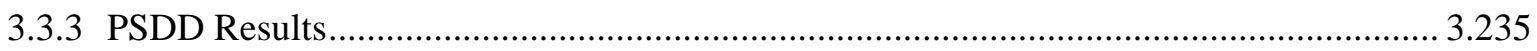

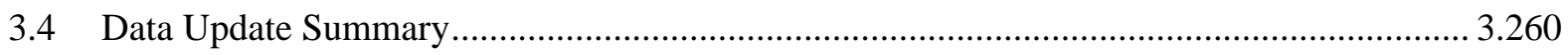

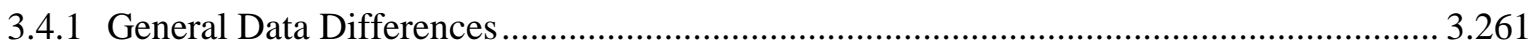

4.0 Effect of Pretreatment Processes on Waste Parameter Data............................................................ 4.1

4.1 Groups 1 and 2-Bismuth Phosphate Sludge and Saltcake Wastes ........................................ 4.1

4.2 Groups 3 and 4-PUREX Cladding and REDOX Cladding Wastes ...................................... 4.3

4.3 Groups 5 and 6-REDOX Sludge and S Saltcake Wastes ..................................................... 4.5

4.4 Group 7 with AY-102 Tank Waste-TBP Sludge and Tank AY-102 Wastes ......................... 4.6

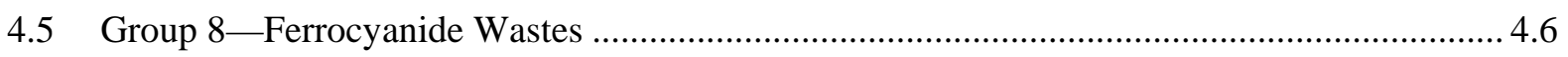

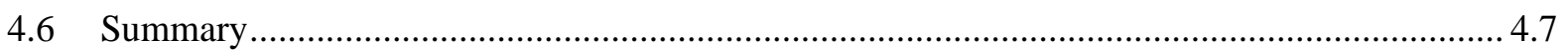

5.0 Summary of Hanford Waste Physical and Rheological Property Data Gaps .................................. 5.1

5.1 Data Gaps in Physical and Rheological Properties................................................................... 5.1

5.1.1 Variation of Parameters as a Function of Waste Type, UDS Concentration, and

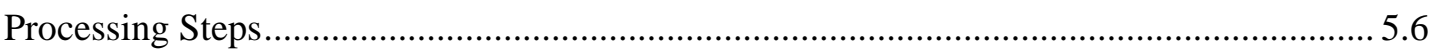

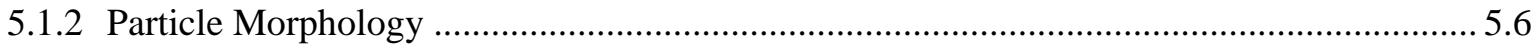

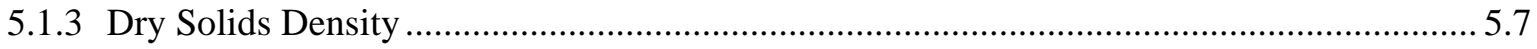

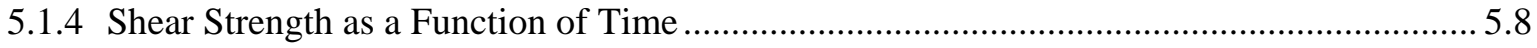

5.1 .5 Impact of Storage on Sample properties ........................................................................ 5.8

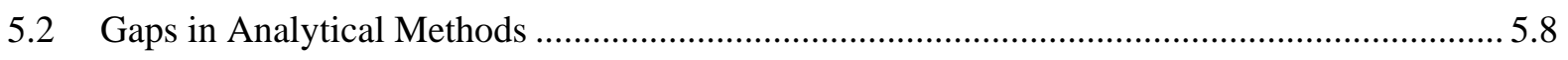

5.2.1 Particle Size and Shape .................................................................................................. 5.8

5.2.2 Volume Fraction of Solids in Slurry and Dry Solids Density ........................................... 5.8

5.2.3 Particle Size and Density Distributions............................................................................... 5.9

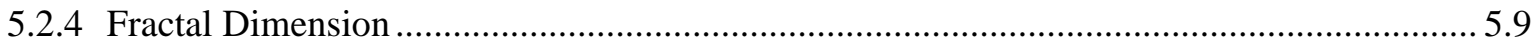

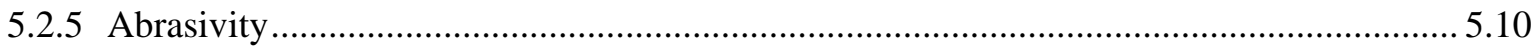

5.2.6 Solids Settling Rate in Concentrated Slurries .............................................................. 5.10

5.3 Gaps in Scale-Up, Data Interpretation and Analysis ............................................................ 5.10

5.3.1 Interpretation and Scale-up of Settling Data ................................................................... 5.10 


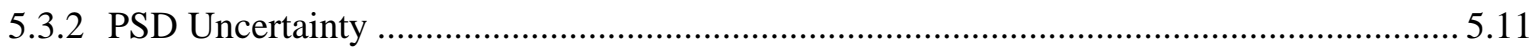

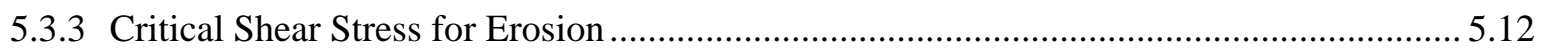

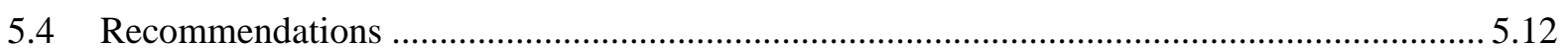

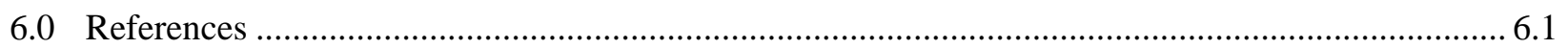

Appendix A: Density and pH of Liquid by Tank and by Waste Type ................................................. A.1

Appendix B: Expert Elicitation, Solid Phase Identification...............................................................1

Appendix C: Density and Composition of Existing and Fully-Washed Solid Phases by Tank,

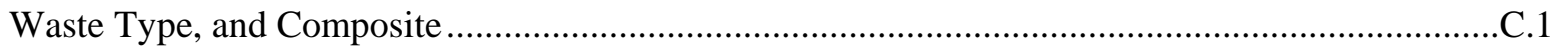

Appendix D: UDS Primary Particle Size and Shape ...................................................................... D.1

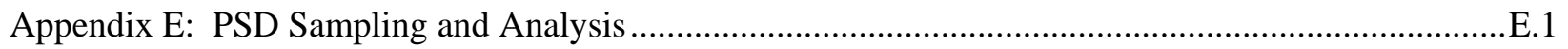

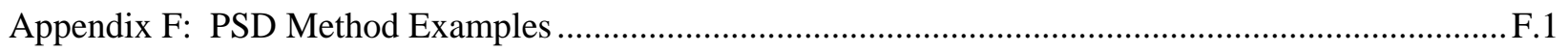

Appendix G: Particle Size Distribution Measurement Uncertainty - Additional Discussion ................. G.1

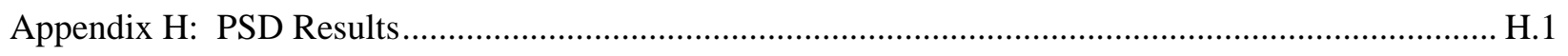

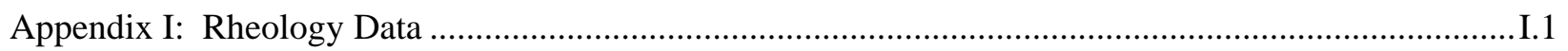




\section{Figures}

2.1. Variation of Drag Coefficient with Particle Reynolds Number ...................................................... 2.3

2.2. Measured Variation of Agglomerate Settling Velocity with Solids Concentrations ........................ 2.8

2.3. Measured Solids Settling Times of Hanford Waste .......................................................................... 2.9

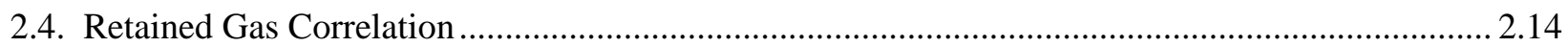

2.5. Vertical Distribution of Suspended Solids Concentrations for Various Rouse Number, z .............. 2.26

2.6. Pipeline Slurry Velocity and Pressure Gradient.............................................................................. 2.30

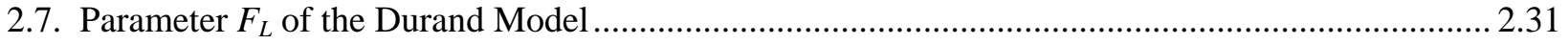

2.8. Comparison of Measured and Computed Critical Velocities Incorporated in the Value of $F_{L} \ldots . . . . .2 .32$

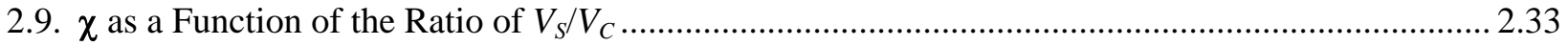

3.1. Comparison of Liquid Viscosity Measurements and Model Predictions by Tank.......................... 3.14

3.2. Comparison of Liquid Viscosity Measurements and Model Predictions for Entire Data Set.......... 3.17

3.3. Hanford Liquid Density and Mass Fraction of Water................................................................... 3.17

3.4. Comparison of Liquid Viscosity Measurements and Model Predictions for Entire Data Set

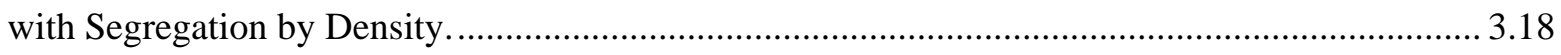

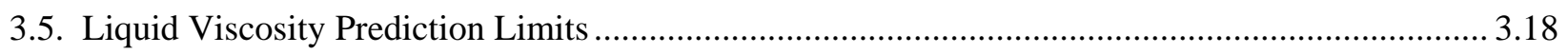

3.6. Measurement Techniques with SEM Example.......................................................................... 3.56

3.7. Euhedral Cubic $\mathrm{B}_{2} \mathrm{O}_{3}$ Particle Together with Botryoidal Bismuth Phosphate Agglomerates ......... 3.57

3.8. Different Forms of Bismuth in the Hanford Tank Waste.................................................................5. 3.57

3.9. TEM-EDS Analysis of Main Amorphous Bismuth Phase Found in the BP Sludge and Saltcake... 3.58

3.10.TEM Images of (a) BP Sludge and (b) Leached BP Sludge that Can Be Used for Estimating

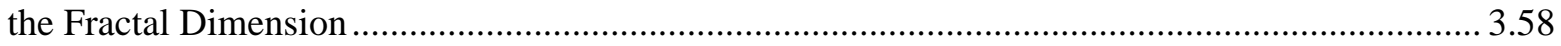

3.11. Cumulative Distribution of Particle Sizes for Bismuth-Iron Oxide Phase.................................... 3.59

3.12. TEM Images of Boehmite Particles from Unleached REDOX Sludge........................................ 3.60

3.13. Cumulative Distribution of Particle Sizes for Boehmite from REDOX Tank Sludge................... 3.61

3.14. Cumulative Distribution of Particle Sizes for $\mathrm{CrOOH}$ from REDOX Tank Sludge...................... 3.62

3.15. TEM Image of CrOOH Particles from Group 6 Tank Sludge ....................................................63

3.16. Particle Labeled (C) Consisted of a Fine Grained Aluminum and Chromium Bearing Particulate with Imbedded Uranium-Rich Particles..................................................................... 3.63

3.17. PSD for Hematite/ Ferrihydrite Phases. ......................................................................................63 3.63

3.18. Cumulative Distribution of Particle Sizes for Strontium Phosphate Phase from

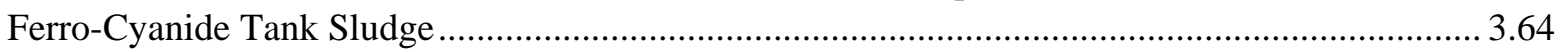

3.19. TEM Image of Strontium Phosphate Particles from Ferrocyanide Tank Sludge Group............... 3.65

3.20. Cumulative Distribution of Particle Sizes for Uranium Oxide Phase from Tank Sludge .............. 3.66

3.21. Cumulative Distribution of Particle Sizes for Actinide Phasesooke .............................................. 3.67 
3.22. Bright Field Image of Elongated Uranium Phases in REDOX Sludge Samples Associated with Boehmite Crystallites.

3.23. High-Resolution Images of Uranium Particles in M12 Group 3 ................................................68

3.24. STEM-HAADF Image (inverted contrast) and EDS Analyses of Regions of Interest .................. 3.68

3.25. TEM Image of Large Uranyl Phosphate in TBP Sludge.............................................................. 3.69

3.26. TEM-EDS Analysis of an Iron Phosphate Phase, Electron Diffraction of the Phase

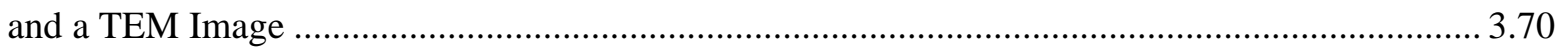

3.27. SEM Image of ZrOOH Particles from Hanford Tank Sludge Solids C-103............................... 3.71

3.28. Cumulative Distribution of Particle Sizes for Zirconium Oxide (ZrOOH) Phase from

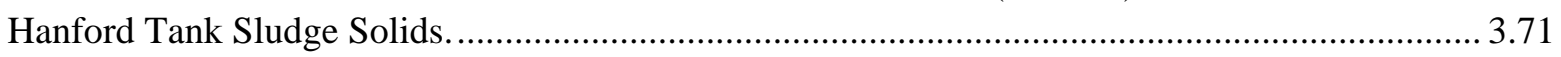

3.29. SEM Image of a Silver Particle from Hanford Tank C-103 ..................................................... 3.72

3.30. Ruthenium-Rich Iron Oxide Phase from Hanford Tank Sludge Solids C-103 ............................ 3.72

3.31. (a) Combined Elemental SEM Maps of Lead-Bearing Iron Oxide Phase from Hanford

Tank Sludge Solids C-103, (b) Backscattered Image of Particle, and (c) EDS Analysis

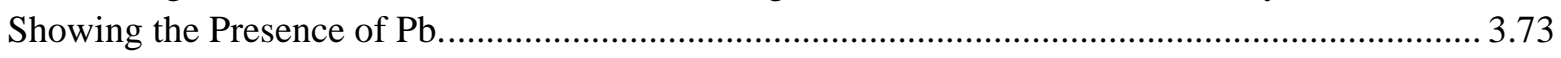

3.32. Cumulative Distribution of Particle Sizes for Iron Oxide (FeOOH) Phase from Hanford

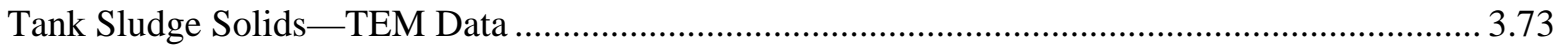

3.33. SEM Images from Hanford Tank Salts ................................................................................... 3.74

3.34. SEM Images of Oxalate and Carbonate Salts from Hanford Tank Salts .................................... 3.74

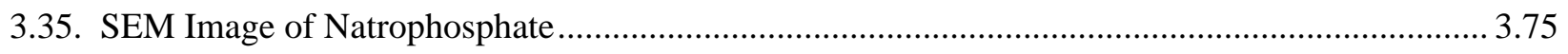

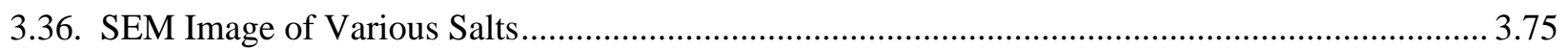

3.37. Waste Types Represented by Sludge, Flowing Sonicated PSDs ............................................... 3.93

3.38. Waste Types Represented by Sludge, Flowing Unsonicated PSDs ............................................. 3.94

3.39. Waste Types Represented by Sludge, No-Flow Unsonicated PSDs ............................................ 3.94

3.40. Waste Types Represented by Saltcake, Flowing Sonicated PSDs............................................. 3.95

3.41. Waste Types Represented by Saltcake, Flowing Unsonicated PSDs.......................................... 3.95

3.42. Waste Types Represented by Saltcake, No-Flow Unsonicated PSDs ........................................ 3.96

3.43. Graphical Representation of PSD Summary for AY-101 …................................................... 3.103

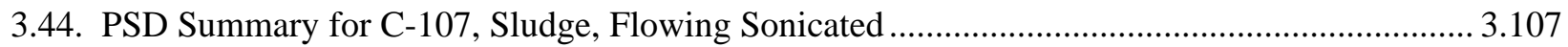

3.45. PSD Summary for C-107, Sludge, Flowing Unsonicated ........................................................ 3.108

3.46. PSD Summary for C-107, Sludge, No-Flow Unsonicated....................................................... 3.108

3.47. Tank Combined PSD Summary, Sludge, Flowing Sonicated.................................................. 3.110

3.48. Tank Combined PSD Summary, Sludge, Flowing Unsonicated............................................... 3.111

3.49. Tank Combined PSD Summary, Sludge, No-Flow Unsonicated............................................. 3.112

3.50. Tank Combined PSD Summary, Saltcake, Flowing Sonicated ................................................ 3.113

3.51. Tank Combined PSD Summary, Saltcake, Flowing Unsonicated .............................................. 3.114

3.52. Tank Combined PSD Summary, Saltcake, No-Flow Unsonicated ............................................. 3.115 
3.53. Waste Type Combined PSD Summary, Sludge, Flowing Sonicated.....

3.54. Waste Type Combined PSD Summary, Sludge, Flowing Unsonicated...................................... 3.118

3.55. Waste Type Combined PSD Summary, Sludge, No-Flow Unsonicated..................................... 3.119

3.56. Waste Type Combined PSD Summary, Saltcake, Flowing Sonicated ...................................... 3.120

3.57. Waste Type Combined PSD Summary, Saltcake, Flowing Unsonicated ................................... 3.121

3.58. Waste Type Combined PSD Summary, Saltcake, No-Flow Unsonicated .................................. 3.122

3.59. Composite Combined PSD Summary, Sludge............................................................................... 3.124

3.60. Composite Combined PSD Summary, Saltcake ....................................................................... 3.124

3.61. Sludge Composite Combined PSDs, Comparison to Wells et al. (2007) .................................... 3.125

3.62. UDS Waste Types Represented by Settling Rate Data Set..................................................... 3.137

3.63. Sedimentation Model and Fast and Slow Velocities Based on It ............................................. 3.140

3.64. Variation of the Fast Velocity with Initial Solids Fraction Concentration in 224

Post-1949 Sludges.

3.65. Variation of the Final Solids Concentration $\phi_{\text {final }}$ with Initial Solids Fraction Concentration in 224 Post-1949 Sludges

3.66. Variation of the Fast Velocity with Initial Solids Fraction Concentration in 1C/2C Wastes ....... 3.155

3.67. Variation of the Final Solids Concentration $\phi_{\text {final }}$ with Initial Solids Fraction Concentration in $1 \mathrm{C} / 2 \mathrm{C}$ Wastes

3.68. Overview of Interface Velocities

3.69. Interface Velocity for In-Tank Suspended AZ-101 Solids and Particle Velocity

Distributions for All AZ-101 Solids

3.70. Interface Velocity for In-Tank Suspended AZ-101 Solids and Particle Velocity

Distributions for All AZ-101 Solids

3.71. Interface Velocity and Particle Velocity Distribution for a Three-Component Simulant

3.72. Interface Velocity and Particle Velocity Distributions for AY-102 Solids

3.73. Interface Velocity and Particle Velocity Distributions for AY-102 Solids

3.74. Waste Types Represented by Shear-Strength Data Set.

3.75. Data Summary of Sludge Shear Vane Shear Strength, 132 Measurements. 3.174

3.76. Data Summary of Sludge Waste Extrusion Shear Strength, 124 Measurements 3.174

3.77. Data Summary of Saltcake Shear Vane Shear Strength, 87 Measurements 3.175

3.78. Data Summary of Saltcake Waste Extrusion Shear Strength, 147 Measurements ...................... 3.175

3.79. Data Summary of Saltcake Ball Rheometer Shear Strength, 170 Measurements....................... 3.176

3.80. Shear Strength as a Function of UDS Concentration, Shear Vane Data.....

3.81. Shear Strength as a Function of UDS Concentration; Shear Vane Data for Hanford Waste and Various Slurries.

3.82. AY-102 Sediment Shear Strength as a Function of Percent Water, Shear Vane Data. 3.181 
3.83. Shear Strength as a Function of Average UDS Concentration, Tanks with Primary

Waste Type Sludge

3.84. Shear Strength as a Function of Average UDS Concentration, Tanks with Primary

Waste Type Saltcake

3.85. Time to Reach Equilibrium Shear Strength, Comparison of Various Suspensions

3.184

3.86. Maximum Measured Shear Strength, Sludge: a) CWP1, b) CWZr2, c) Unidentified 3.185

3.87. Maximum Measured Shear Strength, Saltcake....

3.88. AY-102 Sediment Shear Strength for Waste Cores 270 Through 273 as a Function of Time. ... 3.187

3.89. AN-105 Sediment Shear Strength from Subsequent Ball Rheometer Passes

3.90. 224 Post-1949 Sludge Shear Strength as a Function of Elevation.

3.91. 224 Pre-1949 Sludge Shear Strength as a Function of Elevation

3.92. 2C Sludge Shear Strength as a Function of Elevation.

3.93. A1 Saltcake Shear Strength as a Function of Elevation.

3.94. A2 Saltcake Shear Strength as a Function of Elevation.

3.95. BL Sludge Shear Strength as a Function of Elevation.

3.96. CWP1 Sludge Shear Strength as a Function of Elevation. 3.195

3.97. CWZr2 Sludge Shear Strength as a Function of Elevation. 3.195

3.98. P3 Sludge Shear Strength as a Function of Elevation. 3.196

3.99. S1 Saltcake Shear Strength as a Function of Elevation. 3.196

3.100. S2 Saltcake Shear Strength as a Function of Elevation 3.197

3.101. Unidentified Sludge Shear Strength as a Function of Elevation 3.197

3.102. ECR, AZ-101 and SY-101 Results Compared to Scaled Tests

3.103. Bingham Parameters 3.202

3.104. Waste Types Represented by Bingham Rheological Model Data Set 3.203

3.105. Maximum Measured Bingham Yield Stress, Sludge 3.204

3.106. Maximum Measured Bingham Consistency, Sludge. 3.205

3.107. Maximum Measured Bingham Yield Stress, Saltcake. 3.206

3.108. Maximum Measured Bingham Consistency, Saltcake. 3.207

3.109. Primary Waste Types with Bingham Parameter Characterization as a Function of UDS Concentration....

3.110. Sludge and Saltcake Bingham Yield Stress as a Function of UDS Concentration, 20 to $95^{\circ} \mathrm{C}$

3.111. Sludge and Saltcake Bingham Viscosity as a Function of UDS Concentration, 20 to $95^{\circ} \mathrm{C}$ 3.210

3.112. 1C Sludge Bingham Parameters as Functions of UDS Concentration. 3.211

3.113. 224 Post-1949 Sludge Bingham Parameters as Functions of UDS Concentration 3.213

3.114. 224 Pre-1949 Sludge Bingham Parameters as Functions of UDS Concentration. 3.214 
3.115. 2C Sludge Bingham Parameters as Functions of UDS Concentration..................................... 3.215

3.116. BL Sludge Bingham Parameters as Functions of UDS Concentration ..................................... 3.216

3.117. CWP1 Sludge Bingham Parameters as Functions of UDS Concentration............................... 3.217

3.118. CWP2 Sludge Bingham Parameters as Functions of UDS Concentration................................. 3.218

3.119. P3 Sludge Bingham Parameters as Functions of UDS Concentration ..................................... 3.218

3.120. TFeCN Sludge Bingham Parameters as Functions of UDS Concentration .............................. 3.220

3.121. Unidentified Sludge Bingham Parameters as Functions of UDS Concentration ....................... 3.220

3.122. A2 Saltcake Bingham Parameters as Functions of UDS Concentration ................................... 3.221

3.123. R Saltcake Bingham Parameters as Functions of UDS Concentration ..................................... 3.223

3.124. S2 Saltcake Bingham Parameters as Functions of UDS Concentration.................................... 3.224

3.125. Bingham Yield Stress as a Function of Temperature............................................................. 3.226

3.126. Bingham Viscosity as a Function of Temperature ................................................................... 3.226

3.127. Solid Particles and Soft and Hard Agglomerates .................................................................... 3.230

3.128. Sludge, Flowing Unsonicated Composite PSDD Calculated Settling Velocity as a

Function of Fractal Dimension

3.129. Sludge Composite PSDD Calculated Settling Velocity as a Function of PSD Measurement, Fractal Dimension $=3$

3.130. Sludge Tank Flowing Unsonicated PSDDs, Calculated Settling Velocity Comparison,

Fractal Dimension $=3$

3.131. Sludge Tank No-Flow Unsonicated PSDDs, Calculated Settling Velocity Comparison, Fractal Dimension $=3$.

3.132. Saltcake Tank Flowing Unsonicated PSDDs, Calculated Settling Velocity Comparison, Fractal Dimension $=3$.

3.133. Saltcake Tank No-Flow Unsonicated PSDDs, Calculated Settling Velocity Comparison, Fractal Dimension $=3$.

3.134. $95^{\text {th }}$ Percentile Particle Identification, Example Applications, Sludge, Flowing Unsonicated PSDD with a Fractal Dimension of 3. 3.260

4.1. M-12 Waste Composite Groups 1 and 2 Leached and Washed Slurry PSDs ................................. 4.2

4.2. M-12 Waste Composite Groups 3 and 4 Leached and Washed Slurry PSDs ................................... 4.4

4.3. M-12 Waste Composite Groups 5 and 6 Leached and Washed Slurry PSDs .................................. 4.5

4.4. M-12 Waste Composite Group 8 Leached and Washed Slurry PSDs .............................................. 4.8 


\section{Tables}

S.1 Data Update Difference Summary …........................................................................ v

S.2 Summary of Waste Physical and Rheological Property Data Gaps...................................... vii

2.1 Summary of Some Examples of Critical Velocity Models ................................................. 2.36

2.2 Important Waste Properties.......................................................................................... 2.42

3.1 Primary and Secondary Waste Types by Tank, 2002 BBI, UDS Volume Basis ................... 3.2

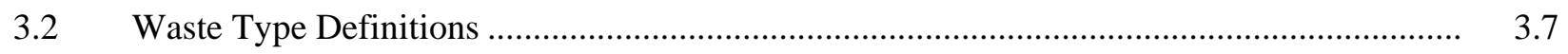

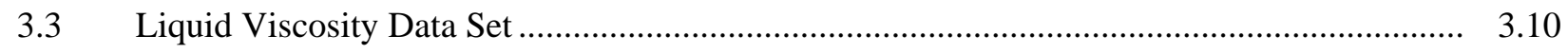

3.4 Liquid Viscosity Model Parameters............................................................................ 3.13

3.5 Linear Relationships that Approximate 95\% Prediction Limits .......................................... 3.14

3.6 Overview of Predicted and Observed Non-Salt Compounds and Their Densities ................. 3.24

3.7 Non-Salt Solid Phases to be Used in This Report............................................................. 3.35

3.8 Fully Washed ${ }^{(a)}$ Solid Phases for This Report..................................................................... 3.39

3.9 Tanks Identified to Represent Sludge Waste Types ........................................................ 3.42

3.10 Tanks Identified to Represent Saltcake Waste Types .......................................................... 3.49

3.11 UDS Phase and Density Summary ...........................................................................

3.12 Compositional Analysis of Bismuth Phosphate Sludge “As-Received”................................ 3.60

3.13 Particle Shape Factor Summary .................................................................................. 3.76

3.14 UDS Phase and Maximum Primary Particle Size Summary ….......................................... 3.77

3.15 PSD References Considered ................................................................................... 3.79

3.16 Instruments Used for PSD Analysis in Reports Listed in Table 3.15 ................................. 3.84

3.17 PSD Data Set, Composite, and Tank PSDs ............................................................ 3.89

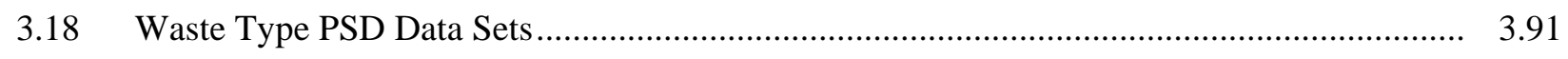

3.19 Initial Data Matrix, AZ-102 Example .......................................................................... 3.97

$3.20 \quad$ Final Data Matrix, AZ-102 Example .........................................................................

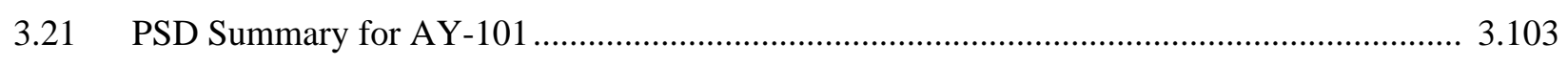

3.22 Example Interpolations to Form PSD Summary Based on Designated Size Bins

3.23 Example Designated Size Bins and Corresponding Cumulative Probabilities for

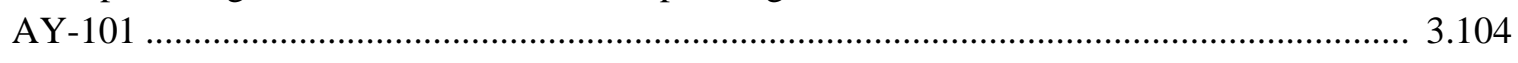

3.24 Example Designated Size Bins and Corresponding Probabilities for AY-101 ...................... 3.105

3.25 PSD Summary Based on Designated Size Bins for AY-101 ….......................................... 3.105

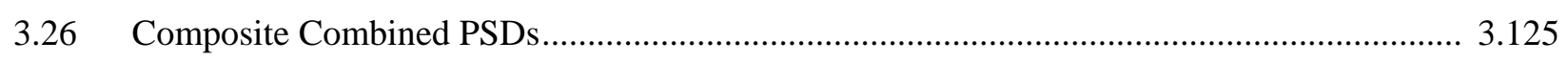

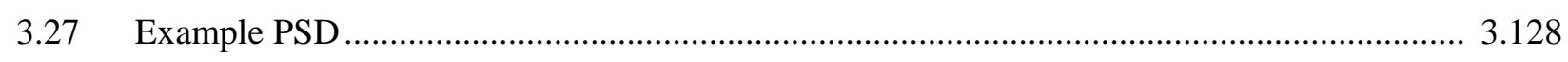

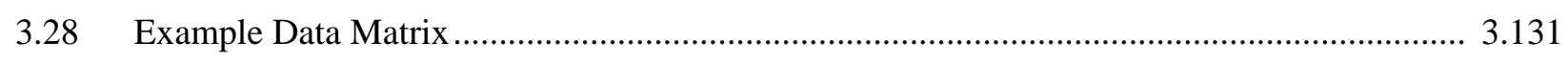




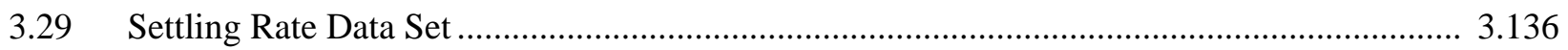

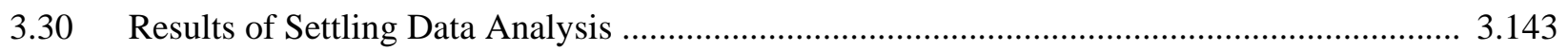

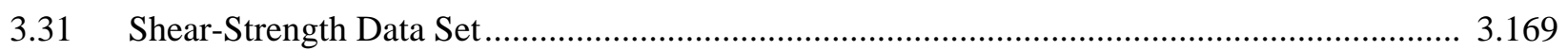

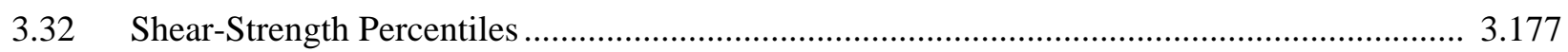

3.33 Power-Law Parameters for Shear Strength as a Function of UDS Concentration Available for Hanford Waste ............................................................................................ 3.179

3.34 Waste Type Representation of Shear Strength as a Function of Elevation............................. 3.198

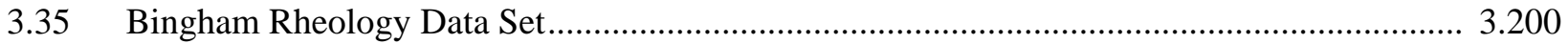

3.36 Bingham Yield Stress and Shear-Strength Comparison, Wells et al. (2010b)....................... 3.228

3.37 Measured Fractal Dimensions.............................................................................................. 3.232

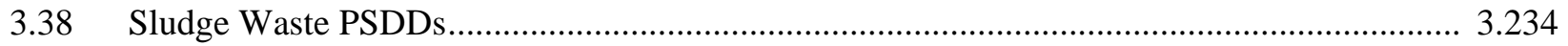

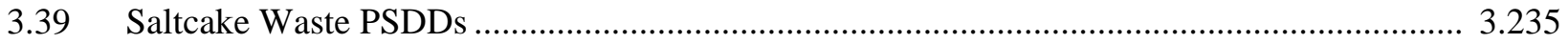

3.40 Sludge, Flowing Unsonicated PSDD, Fractal Dimension 3 ............................................. 3.237

3.41 Sludge, Flowing Unsonicated PSDD Volume Fraction, Fractal Dimension 2.25 ................. 3.240

3.42 Sludge, Flowing Unsonicated PSDD Density, Fractal Dimension 2.25 .............................. 3.243

3.43 Sludge, Flowing Unsonicated PSDD Volume Fraction, Fractal Dimension 1.6 ................... 3.246

3.44 Sludge, Flowing Unsonicated PSDD Density, Fractal Dimension 1.6 ….............................. 3.249

4.1 M-12 Waste Composite Groups 1 and 2 Leached and Washed Slurry Rheology Values ....... 4.2

4.2 M-12 Waste Composite Groups 3 and 4 Leached and Washed Slurry Rheology Values ....... 4.4

4.3 M-12 Waste Composite Groups 5 and 6 Leached and Washed Slurry Rheology Values ....... 4.6

4.4 M-12 Waste Composite Group 7 and Tank AY-102 Leached and Washed Slurry

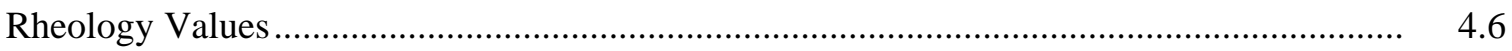

4.5 M-12 Waste Composite Group 8 Leached and Washed Slurry Rheology Values................... 4.8

5.1 Summary of Waste Physical and Rheological Property Data Gaps...................................... 5.3

$5.2 \quad$ Waste Type and Parameter Data ........................................................................................ 


\subsection{Introduction}

This document will identify data gaps in the Hanford waste physical and rheological properties characterization data by understanding the applications of the information and evaluating the currently available information.

\subsection{Background}

The Hanford Site in Washington State manages 177 underground storage tanks containing approximately $250,000 \mathrm{~m}^{3}$ of waste generated during past defense reprocessing and waste management operations. These tanks contain a mixture of sludge, saltcake and supernatant liquids. The insoluble sludge fraction of the waste consists of metal oxides and hydroxides and contains the bulk of many radionuclides such as the transuranic components and ${ }^{90} \mathrm{Sr}$. The saltcake, generated by extensive evaporation of aqueous solutions, consists primarily of dried sodium salts. The supernates consist of concentrated (5-15 $\underline{\mathrm{M}}$ ) aqueous solutions of sodium and potassium salts. The 177 storage tanks include 149 single-shell tanks (SSTs) and 28 double-shell tanks (DSTs).

Ultimately the wastes need to be retrieved from the tanks for treatment and disposal. The SSTs contain minimal amounts of liquid wastes, and the Tank Operations Contractor is continuing a program of moving solid wastes from SSTs to interim storage in the DSTs. The Hanford DST system provides the staging location for waste feed delivery to the Department of Energy (DOE) Office of River Protection's (ORP) Hanford Tank Waste Treatment and Immobilization Plant (WTP). The WTP is being designed and constructed to pretreat and then vitrify a large portion of the wastes in Hanford's 177 underground waste storage tanks.

\subsection{Technical Need}

The treatment and disposal operations involve the handling of a wide range of slurries. Solids in the slurry have a wide range of particle size, density and chemical characteristics. Depending on the solids concentration the slurries may exhibit a Newtonian or a non-Newtonian rheology.

Multi-phase mixing, suspension, and transport behavior is difficult to predict, in part, because slurry chemical and physical properties are not fully understood. In addition, simulation tools for slurry systems are not available or are inadequate. The difficulty of developing these simulation tools hinges on the lack of accurate physical and chemical property information (e.g., thermodynamics, kinetics, viscosity, particle size, and density, etc.). The lack of adequate slurry behavior predictive tools has resulted in an inability to scale-up waste processing systems with confidence, which increases process risk.

A workshop on Slurry Retrieval, Pipeline Transport \& Plugging and Mixing (Smith et. al. 2009) indicated that a key lesson from industry-academia experts is that solids handling is many times more difficult than handling gases or liquids. Difficulties handling slurries can severely reduce the expected throughput and yield and increase startup time and cost. The workshop also highlighted the importance of good chemical and physical characterization of the actual waste slurries for all unit operations to underpin the design basis. The workshop noted that process design cannot be based on average slurry properties but must cover a robust range of variable waste properties. 
To increase confidence for process scale-up, it is necessary to make sure that simulants are designed to emulate the specific chemical or physical behaviors of actual radioactive wastes. Simulated wastes can be developed to exhibit only a limited set of important properties for a specific application, or may be tailored to exhibit a broader range of chemical, physical, and rheological properties for a wide range of tests.

\subsection{Purpose and Scope}

The purpose of this document is to provide an updated summary of the Hanford waste characterization data pertinent to safe storage, retrieval, transport and processing operations for both the tank farms and the WTP and thereby identify gaps in understanding and data. Specific waste parameter characterization needed to estimate waste operations are identified by examining examples of relevant mathematical models of selected phenomena. The required waste characterization includes physical and rheological properties of the waste. These properties of the as-stored Hanford waste, including both the liquid and solid phases, are presented by tank and waste type. In selected cases composite results are presented that represent and overall blend of the wastes.

Many of the properties of the as-stored waste will be significantly affected by the retrieval and transport of the wastes. If low-salt solutions are used, soluble salts will be partially or completely dissolved resulting in changes to the bulk slurry properties. Properties such as the shear strength of settled solids will be altered as the solids structure is disrupted during the retrieval process. The particle size and density of the insoluble solids may be unaffected by some retrieval and staging activities. Data allowing the evaluation of potential property changes due to retrieval and staging activities are provided, and the effect of pretreatment processes are considered.

Characterizations include density, $\mathrm{pH}$, and rheology of the liquid, and composition, primary particle size and shape, particle size distribution (PSD), settling behavior, and slurry rheology for the solid fraction of the waste. The parameter data sets from Poloski et al. (2007) and Wells et al. (2007) are evaluated and expanded with additional data sets, primarily the extensive laboratory testing conducted on eight waste group composites in support of the WTP effort to resolve the External Flowsheet Review Team (EFRT) issue M12. ${ }^{\text {(a) }}$

The measured PSDs are related to solid composition via particle size and density distributions (PSDDs), the functionality of rheology with waste conditions is considered, and, where possible, these data are compared to process phenomena. Examples of the PSD and rheological changes due to the pretreatment processes of the WTP are also presented. Data gaps with respect to unquantified information as well as the waste volume represented for characterization of a specific parameter are discussed and a summary is provided.

(a) CCN 132846. 2006. Comprehensive Review of the Hanford Waste Treatment Plant Flowsheet and Throughput - Assessment Conducted by an Independent Team of External Experts. Chartered by the Hanford Waste Treatment and Immobilization Plant Project at the Direction of the U.S. Department of Energy, Office of Environmental Management, Washington, DC. 


\subsection{Approach}

The initial step was to examine various engineering applications addressing waste storage, mobilization, suspension, and transfer. These include:

- Pipeline Critical Velocity

- Solid Settling Velocity

- Effective Cleaning Radius

- Vessel Wall/Bottom Erosion

- Critical Suspension Velocity

- Suspended Solid Cloud Height

- Suspended Solid Concentration

- Solid Dissolution and Filtration

- Gas Generation, Retention, and Release

A summary of the available approaches for each application is presented. The salient waste parameters are identified and provide the basis for the waste parameters of interest. These parameters include:

- Liquid Density and pH

- Liquid Rheology (Viscosity)

- Undissolved Solid (UDS) ${ }^{(a)}$ Composition and Particle Density

- UDS Primary Particle Size and Shape

- UDS Particle Size Distribution

- UDS Particle Settling

- Slurry Rheology and Shear Strength

- Estimates of Particle Size and Density Distributions

For the available data, the parameters are presented by 1) tank, 2) waste type (general, i.e., sludge or saltcake, and specific), and 3) composite.

The data sets previously presented in Wells et al. (2007) and Poloski et al. (2007) were updated with the data from the additional waste types that have recently been characterized. An analysis of the effect of sample storage was conducted to make sure that the data sets represent equivalent or similar waste conditions. The parameter values were evaluated with in situ data pertaining to waste mobilization and UDS settling. The data set is therefore as consistent as possible both internally and with actual waste

(a) UDS; undissolved solids. Those solids, whether soluble or insoluble, that are present as a solid phase and are not dissolved in the liquid phase of the waste. 
behavior. Data uncertainties were quantified to the extent possible. Since this document contains the most extensive data and analyses, it is recommended for use over the prior work.

The final step in this effort was to conduct a gap analysis to identify gaps in characterization data, analytical methods, and data interpretation. The primary focus is on data gaps identified by considering the parameters by waste type, percent of waste type mass or volume represented and the uncertainty of the parameter. Gaps in analytical methods and data interpretation are also noted but they do not represent a comprehensive list. This gap analysis will help focus future efforts for waste characterization, method development, and data interpretation.

\subsection{Quality Requirements}

In accordance with the Project Quality Assurance Plan for the Environmental Management (EM)-31 Support Project, this work was designated as Quality Level 2. This designation is based on the fact that the work is eventually intended to support and could affect the quality of nuclear material applications, structures, systems and components of nuclear facilities (i.e., waste management, nuclear material processing, other related facilities). As such, the work was performed in accordance with applicable requirements of PNNL-NQA-EQAM-1 (Nuclear Quality Assurance [NQA]-1 based). The data are from numerous and varied sources as referenced. It is assumed that the data from these references have been fully reviewed and documented in accordance with the analysts' quality assurance (QA) programs.

Due to the large number of references and the length of elapsed time over which results were acquired and reported, no attempt was made to qualify or otherwise validate the referenced data as NQA-1.

However, based on a survey of the major programs under which the data were acquired it is likely that a large majority of the results were collected and reported under NQA-1 or equivalent quality programs. 


\subsection{Waste Operations}

Hanford waste characterization data are used to engineer safe storage, retrieval, transport, and processing operations. Whenever waste is stored or handled, it is important to anticipate its behavior and understand the physical phenomena behind its behavior. Several phenomena are particularly important to waste operations, such as the tendency of the solids to settle from waste slurries and the rheological behavior of settled layers of waste.

This section discusses selected phenomena known to be important to waste operations and identifies specific waste parameters that are needed to estimate the phenomena by examining relevant mathematical models of the phenomena. Both the phenomena and the models were selected as examples; the list of phenomena considered is not complete nor are all the available models presented. Given the breadth of phenomena and models as related to the waste parameters, there are instances of repeated terms in the models, e.g., solid volume fraction. The terms are defined on a model-by-model basis, and the symbols are typically preserved from the original reference.

Based on the sensitivity of the models to the input parameters, the order of significance of the waste parameters for specific phenomena can be established. However, the waste parameters can, in some instances, vary by several orders of magnitude, while others vary much less. Thus, the order of significance of the waste properties as indicated by the models can be affected by possible variations of these waste properties themselves.

Phenomena of importance in waste storage, including solids settling and the generation, retention, and release of flammable gases, are presented in Section 2.1. Section 2.2 discusses several phenomena of importance to the mobilization of settled waste solids; Section 2.3 examines phenomena of interest to the transport of waste; and Section 2.4 discusses phenomena of importance to waste processing operations. In Section 2.5, a summary is provided of the order of significance of the waste parameters as indicated by the sensitivity of the models to the input parameters.

\subsection{Waste Storage}

The Hanford waste consists of insoluble sludge solids, water-soluble salts, aqueous liquids, and possibly some gas. Slurries of solid waste transferred into storage and process vessels will settle, if not actively mixed, creating potential safety and solids accumulation problems. Section 2.1.1 discusses solids settling, presents models for various scenarios, and identifies waste properties that are needed by the models. Section 2.1.2 discusses the generation, retention, and release of flammable gas by Hanford waste.

\subsubsection{Solids Settling Velocity}

The majority of the Hanford waste undissolved solid particles are more dense than waste liquids, and thus suspended solid waste particles tend to settle. ${ }^{(a)}$ At low solids concentrations, individual particles can settle without interacting with other particles (individual particles undergoing unhindered settling). At

(a) Some tanks have floating crust layers comprised of solids, liquids, and gas. The buoyancy of these crusts is due to the retained gas. 
higher solids concentrations, the upward flow of fluid between the particles can reduce settling rates (hindering settling), and the interactions between particles may cause them to flocculate and settle as flocs or agglomerates (floc and agglomerate settling). In general, solid settling velocity is a function of

- solids particle size, shape, and density

- liquid density and viscosity

- waste chemistry affecting solids agglomeration

- suspended solids concentration.

\subsubsection{Examples of Solids Settling Velocity Models}

Example models for solids settling velocity under different scenarios are presented below.

\section{Individual Particle (Unhindered) Settling}

The settling velocity of an individual spherical particle is described by Vanoni (1975) as

$$
V_{S}^{2}=\frac{4}{3} \frac{g d_{S}}{C_{D}}\left(\frac{\rho_{S}-\rho_{L}}{\rho_{L}}\right)
$$

where $C_{D}=$ drag coefficient

$d_{S}=$ spherical particle diameter

$g=$ gravitational acceleration

$V_{S}=$ solids settling velocity

$\rho_{\mathrm{L}}=$ liquid density

$\rho_{\mathrm{S}}=$ solid density.

Figure 2.1 shows the drag coefficient, $C_{D}$, as a function of the particle Reynolds Number, $R$, defined by Equation (2.2).

$$
R=\frac{V_{S} \rho_{L} d_{S}}{\mu_{L}}
$$

where $\mu_{\mathrm{L}}$ is the liquid viscosity.

Equation (2.1) is applicable to spherical particles and all particle Reynolds numbers. As will be discussed later, Equation (2.1) can also be used for non-spherical particles by calculating equivalent spherical particle diameters for the non-spherical particles. 


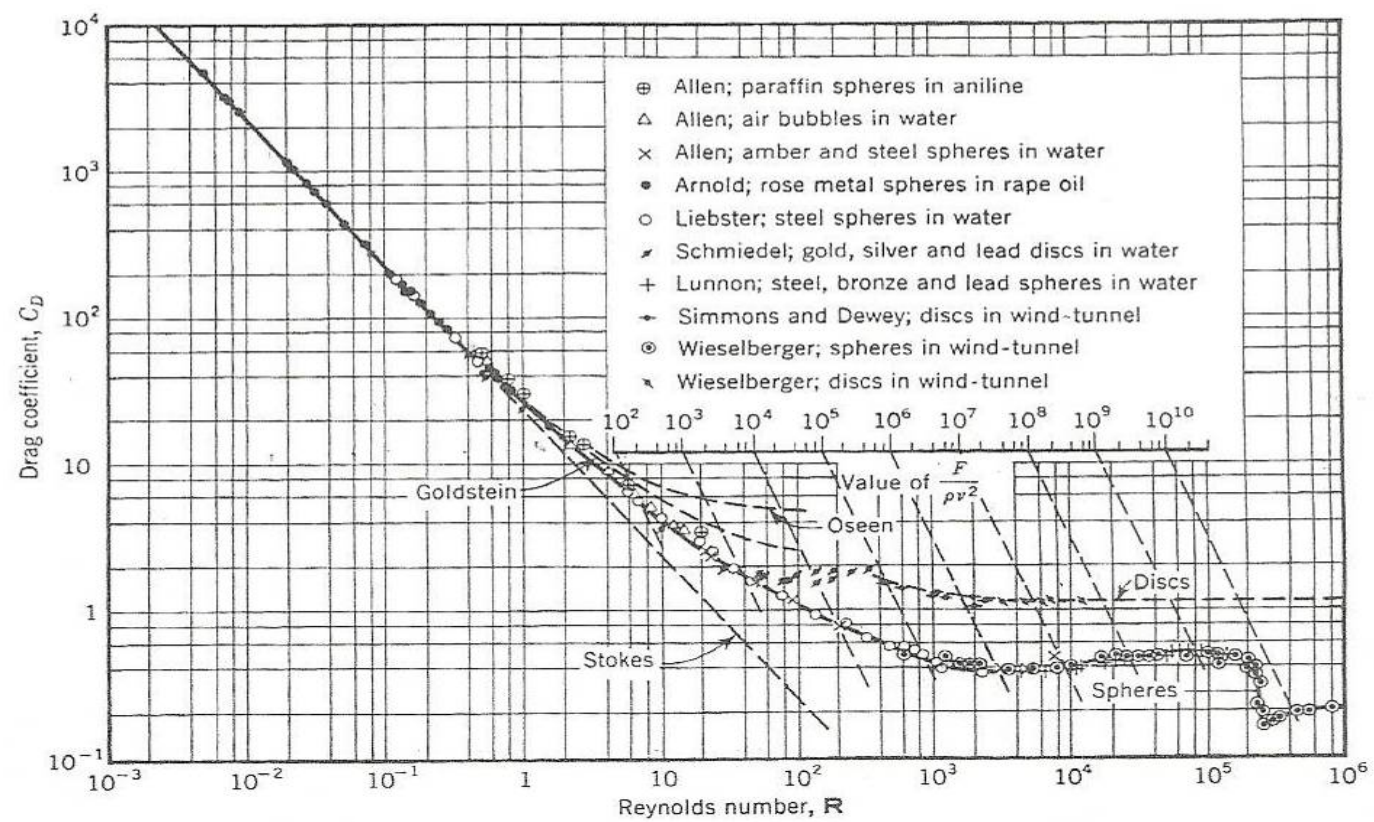

Figure 2.1. Variation of Drag Coefficient with Particle Reynolds Number (Vanoni 1975)

Because $R$ (Equation 2.2) contains the settling velocity in it, an iterative procedure is generally required to calculate the settling velocity with Equation (2.1). There are several ways to avoid iterations to estimate the solid settling velocity. One way is to use the third parameter, $F / \rho v^{2}$ (where $\rho$ is $\rho_{L}$ in this case, and $v=\mu_{L} / \rho_{L}$ is the kinematic viscosity of the liquid) to obtain the drag coefficient from Figure 2.1. $F$ is defined as

$$
F=\frac{\pi d_{S}^{3} g}{6}\left(\rho_{S}-\rho_{L}\right)
$$

An alternative way to estimate the solid settling velocity is to divide Figure 2.1 into the following three Reynolds number ranges:

1. The Stokes Law range, where $\mathrm{R}<0.3$

In the Stokes Law range is variably defined as $\mathrm{R}<0.1, \mathrm{R}<0.3$ or $\mathrm{R}<1$. In this report, we selected the Stokes Law range to be $\mathrm{R}<0.3$. As can be seen in Figure 2.1, in the Stokes Range, the drag coefficient, $C_{D}$, is

$$
C_{D}=\frac{24}{R}
$$

Substituting Equation (2.4) into Equation (2.1) yields the following well-known Stokes settling velocity formula (Wasp et al. 1977).

$$
V_{S}=\frac{g d_{S}{ }^{2}}{18 \mu_{L}}\left(\rho_{S}-\rho_{L}\right)
$$


2. The intermediate range, where $0.3 \leq \mathrm{R}<1000$

In the intermediate range, the drag coefficient is (Perry and Chilton 1973)

$$
C_{D} \approx \frac{18.5}{R^{0.6}}
$$

Substituting Equation (2.6) into Equation (2.1), the settling velocity can be estimated as

$$
V_{S} \approx \frac{g^{0.714} d_{S}^{1.14}\left(\rho_{S}-\rho_{L}\right)^{0.714}}{6.54 \mu_{L}^{0.428} \rho_{L}^{0.286}}
$$

3. The Newtonian Law range, where $1000 \leq \mathrm{R}<2 \times 10^{5}$

In the Newtonian Law range, the drag coefficient is (Perry and Chilton 1973)

$$
C_{D} \approx 0.44
$$

and the settling velocity is given by

$$
\mathrm{V}_{\mathrm{S}} \approx 1.74\left[\operatorname{gd}_{\mathrm{S}}\left(\frac{\rho_{\mathrm{S}}-\rho_{\mathrm{L}}}{\rho_{\mathrm{L}}}\right)\right]^{\frac{1}{2}}
$$

\section{Non-Spherical Particle Shape Factors}

The particle shape affects its settling velocity. Equations (2.2) through (2.9) are for a spherical particle or an equivalent spherical particle of a non-spherical solid. There are several approaches to express the shape factor of an arbitrarily shaped solid for the settling velocity, including the following (McNown et al. 1951, Vanoni 1975, Lansen et al. 1979, Simons and Senturk 1977):

- Corey’s shape factor,

$$
S_{F}=\frac{c}{\sqrt{a b}}
$$

where $a, b$, and $c$ are the longest, intermediate and shortest mutually perpendicular axes of the particle, respectively. 
- The sphericity is the ratio of the surface area of a sphere with equal volume as the particle to the surface area of the particle considered (or the ratio of the diameter of a sphere with equivalent volume to the diameter of a circumscribed sphere). ${ }^{\text {(a) }}$

- The roundness, $P$, is the ratio of the average radius of curvature to a radius of circle inscribed in the maximum projected area of a particle.

Corey's shape factor, calculated by Equation (2.10), is commonly used for an equivalent spherical diameter of a non-spherical particle. Equations (2.2) through (2.9) can then be used to obtain the settling velocity with Equation (2.1).

Another approach is to use the following to estimate the settling velocity of a non-spherical particle (Wu and Wang 2006).

$$
V_{S}=\frac{M v}{N d_{S}}\left[\sqrt{\frac{1}{4}+\left(\frac{4 N}{3 M^{2}} D_{*}^{3}\right)^{\frac{1}{n}}}-\frac{1}{2}\right]^{n}
$$

where

$$
\begin{aligned}
& D_{*}=d_{S}\left[\frac{\left(\frac{\rho_{S}}{\rho_{L}}-1\right) g}{v^{2}}\right]^{\frac{1}{3}} \\
& d_{S}=\sqrt[3]{a b c} \\
& M=53.5 e^{-0.65 S_{F}} \\
& N=5.65 e^{-2.5 S_{F}} \\
& n=0.7+0.9 S_{F} \\
& v=\mu_{L} / \rho_{L}=\text { kinematic viscosity. }
\end{aligned}
$$

Note that the parameter $\mathrm{D}_{*}^{3}$ is the Archimedes number, Equation (2.47).

The equivalent sphere is defined by Wu and Wang (2006) as a sphere having a settling velocity the same as the solid. The diameter of the equivalent sphere, $d_{E}$, is calculated by solving the following equation with the above calculated $V_{S}$ and known $\rho_{L}, \rho_{S}$ and $v$ :

$$
\left[\left(\frac{V_{S} d_{E}}{60.22 v}\right)^{0.625}+0.5\right]^{2}=0.25+\frac{1}{86.69} d_{E}^{1.875}\left\{\left(\frac{\rho_{S}}{\rho_{L}}-1\right) \frac{g}{v^{2}}\right\}^{0.625}
$$

(a) A circumscribed sphere of a polyhedron is a sphere that touches each vertex of the polyhedron and contains the polyhedron. 
These models would determine the solids settling velocity for Reynolds numbers up to $2 \times 10^{5}$ shown in Figure 2.1.

An alternative to Wu and Wang's model (Equation 2.11) to cover the Stokes Law, the intermediate, and the Newtonian Law ranges, is the following from Camenen (2007):

$$
V_{S}=\frac{\mu_{L}}{\rho_{L} d_{S}}\left[\sqrt{\frac{1}{4}\left(\frac{A}{B}\right)^{\frac{2}{m}}+\left(\frac{4}{3} \frac{D_{*}^{3}}{B}\right)^{\frac{1}{m}}}-\frac{1}{2}\left(\frac{A}{B}\right)^{\frac{1}{m}}\right]^{m}
$$

where

$$
\begin{aligned}
A & =a_{1}+a_{2}\left[1-\sin \left(\frac{\pi}{2} S_{F}\right)\right]^{a_{3}} \\
B & =b_{1}+b_{2}\left[1-\sin \left(\frac{\pi}{2} S_{F}\right)\right]^{b_{3}} \\
m & =m_{1} \sin ^{m 2}\left(\frac{\pi}{2} S_{F}\right) \\
a_{1} & =24 \\
a_{2} & =100 \\
a_{3} & =2.1+0.06 P \\
b_{1} & =0.39+0.22(6-P) \\
b_{2} & =20 \\
b_{3} & =1.75+0.35 P \\
m_{1} & =1.2+0.12 P \\
m_{2} & =0.47 \\
P & =\text { particle roundness. }
\end{aligned}
$$

Camenen (2007) provides $A, B$ and $m$ values for a sphere as 24, 0.4 and 2, respectively, so that Equation (2.14) for a spherical particle is

$$
\mathrm{V}_{\mathrm{S}}=\frac{\mu_{\mathrm{L}}}{\rho_{\mathrm{L}} \mathrm{d}_{\mathrm{S}}}\left[\sqrt{15+\sqrt{\frac{\mathrm{D}_{*}^{3}}{0.3}}}-\sqrt{15}\right]^{2}
$$

\section{Solids Floc and Agglomerate Settling}

When primary particles are small, normally less than $20 \mu \mathrm{m}$ for Hanford waste, they tend to flocculate and form agglomerates (Rector and Bunker 1995a). Although the agglomerate density, which includes the interstitial liquid, is less than the primary particle density, agglomerates usually settle faster than primary particles (MacLean 1999). 
When cohesive solids concentrations exceed some level, typically around $0.3 \mathrm{~g} / \mathrm{L}$, the small primary particles start to form agglomerates (Mehta and Pertheniades 1973). Two possible approaches to estimate the settling velocities of agglomerates are discussed here, the first based on a fractal analysis and the second based on measurements of silt and clay in estuaries and coastal waters.

From Wells et al. (2007), and as applied in Section 3.3, a fractal analysis indicates that the density of an agglomerate can be estimated by

$$
\rho=\left(\frac{\mathrm{R}}{\mathrm{r}}\right)^{\mathrm{D}_{\mathrm{F}}-3}\left(\rho_{\mathrm{S}}-\rho_{\mathrm{L}}\right)+\rho_{\mathrm{L}}
$$

where $D_{F}$ is the fractal dimension (between 0 and 3), $R$ is the agglomerate size, and $r$ is the primary (crystal) particle size.

Equation (2.15) provides a relationship between the agglomerate size and its density. The agglomerate density decreases as the size increases for a constant value of $D_{F}<3$. A further discussion for the fractal dimension is provided in Section 3.3. Given an appropriate fractal dimension value and agglomerate size, the density of the agglomerate can be estimated. With known size and density, the settling velocity equations presented above can be used to estimate the agglomerate settling velocity.

An alternative to the fractal dimension approach is an empirical approach developed with silt and clay particles. Krone (1962) conducted a series of experiments with San Francisco Bay sediments with densities of 2,650 kg/m $\mathrm{m}^{3}$ and solids sizes varying from 1 to $50 \mu \mathrm{m}$. The solids settling equation, Equation (2.16), accounts for flocculation and estimates the settling velocity of agglomerates as a function of solids concentration (see Figure 2.2).

$$
V_{S}=A C^{\frac{4}{3}}
$$

where $A$ is the constant, $C$ is the solids concentration, and $V_{S}$ is the solids settling velocity. 


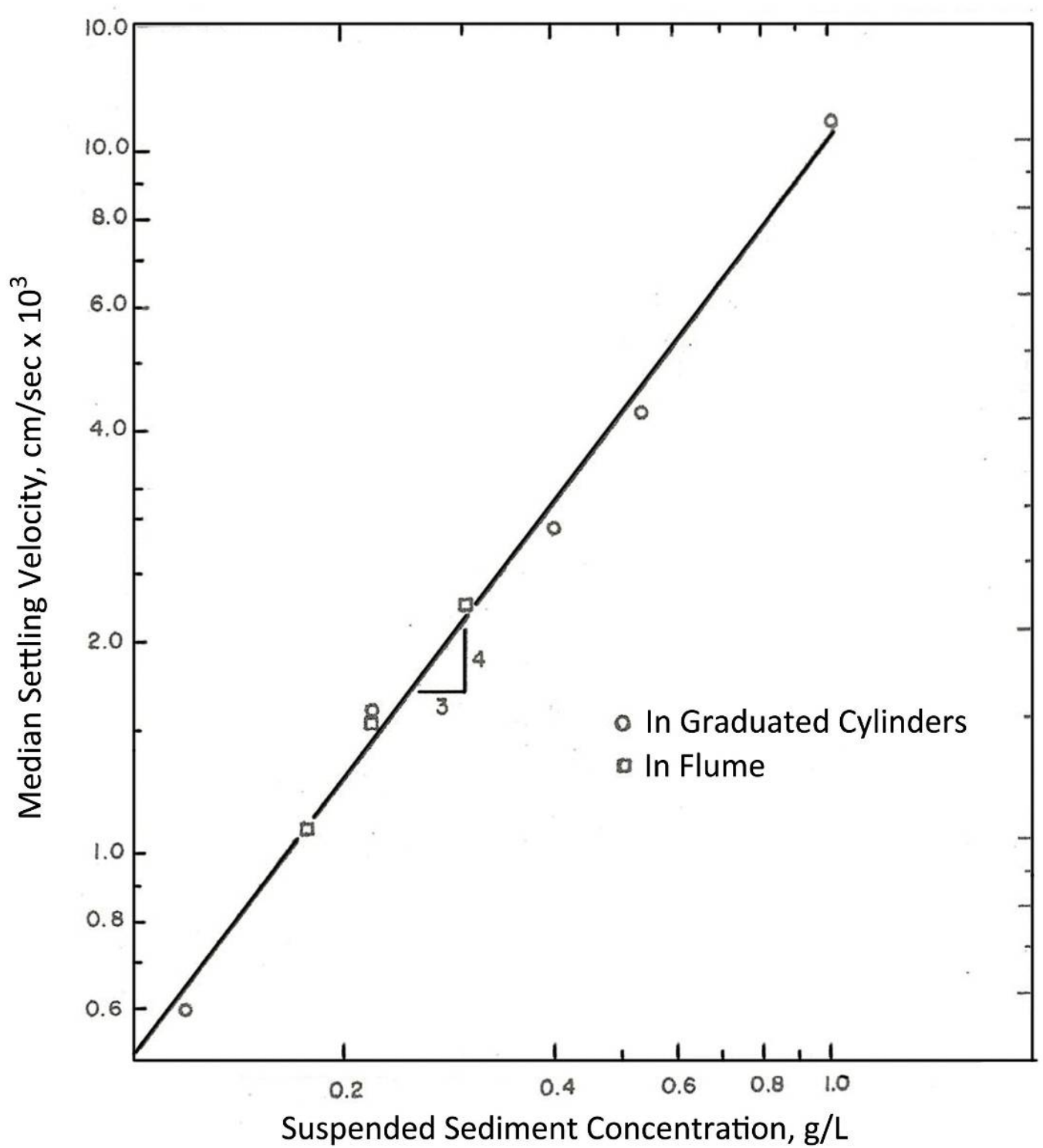

Figure 2.2. Measured Variation of Agglomerate Settling Velocity with Solids Concentrations (Krone 1962)

Because Equation (2.16) was derived with silt and clay in salt water, the use of an equation in the form of Equation (2.16) for waste solids settling tests would need to be conducted to determine the value of the constant, $A$, possibly for each solids mixture of interest.

\section{Hindered Solids Settling}

When the solids concentration exceeds a certain level, typically around $10 \mathrm{~g} / \mathrm{L}$, hindered settling occurs (Krone 1962, 1993; Mehta and Pertheniades 1973). Perry and Chilton (1973) suggest the following equation for hindered settling: 


$$
V_{S}=V_{S 0}\left(1-\frac{C_{V}}{C_{V \max }}\right)^{b}
$$

where $\quad b=$ coefficient (= 4.65 for the Stokes Law range)

$$
\begin{aligned}
C_{V}= & \text { solids volume fraction (volume of UDS per volume of suspension } \\
& \text { during settling) } \\
C_{V \max }= & \text { maximum solids volume fraction (volume of UDS per volume of sediment) } \\
V_{s 0}= & \text { settling velocity of a single particle. }
\end{aligned}
$$

Equation (2.17) was used in the computational chemical-fluid dynamic code ARIAL to assess waste pump jet mixing and retrieval in DSTs (Onishi 1999, Onishi et al. 2000, 2003). MacLean (1999) reported that a form of Equation (2.17) matched reasonably well with settling velocities of Hanford wastes (Tanks C-106, C-107, S-107, AZ-101, AZ-102, BY-104, BY-110 and SX-108) and simulants. Figure 2.3 shows three different modes of solids settling, possibly due to solids floc or agglomerate settling, hindered settling, and solids consolidation in that sequence over 50 days (MacLean 1999).

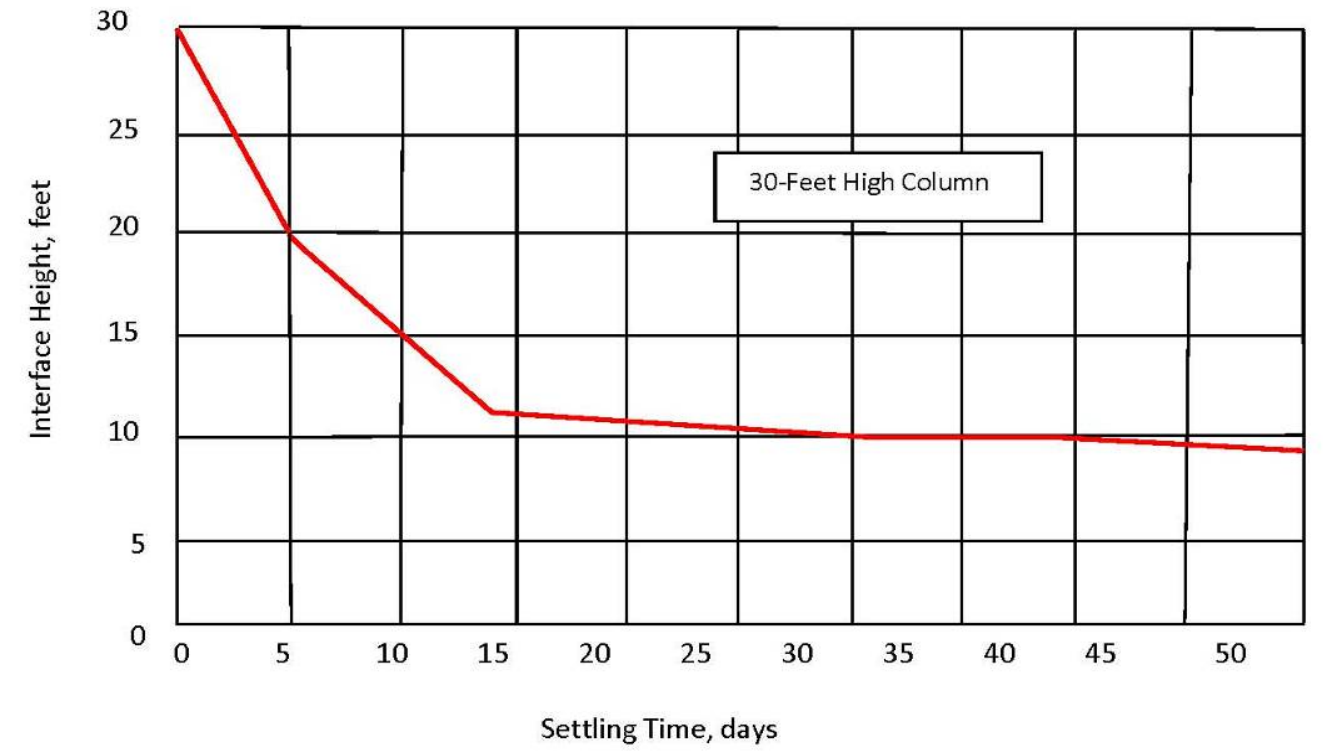

Figure 2.3. Measured Solids Settling Times of Hanford Waste (adopted from MacLean 1999)

Poloski et al. (2007) reported the following equation to represent sedimentation. This equation can also be used to estimate solids settling as done in Section 3.2.6.

$$
\frac{z}{z_{0}}=\frac{C \phi_{0}}{\alpha}+\left(1-\frac{C \phi_{0}}{\alpha}\right) e^{-\frac{\alpha}{\phi_{0} z_{0}} t}
$$


where $\quad z=$ interface height $(\mathrm{m})$ between the slurry layer and clarified liquid above at time $t$

$z_{0}=$ initial interface height $(\mathrm{m})$

$\phi_{0}=$ initial solids volume fraction in the well-mixed suspension (average volume fraction of UDS in initial well-mixed sample)

$C=$ adjustable constant found by fitting the model, units of velocity

$\alpha=$ adjustable constant found by fitting the model, units of velocity

$t=$ time

\section{Unified Solids Settling Velocity Methodology}

Onishi et al. (2009) developed a unified settling velocity calculation method to seamlessly cover the solids settling through a range of an individual particle settling, solids flocculation, and hindered settling, as discussed below.

The following three equations provide an approach to calculate the settling velocities of Hanford waste fine particles, whose single particle fall velocity is in the Stokes Law range.

$$
\begin{aligned}
& V_{S}=\frac{g d_{S}^{2}}{18 \mu_{L}}\left(\rho_{S}-\rho_{L}\right) \text { For } \mathrm{C}<0.3 \mathrm{~g} / \mathrm{L} \\
& V_{S}=A C^{\frac{4}{3}} \text { For } 0.3 \mathrm{~g} / \mathrm{L} \leq \mathrm{C}<10 \mathrm{~g} / \mathrm{L} \\
& V_{S}=B\left(1-\frac{C_{V}}{C_{V \max }}\right)^{4.65} \text { For } \mathrm{C} \geq 10 \mathrm{~g} / \mathrm{L}
\end{aligned}
$$

where $A$ and $B$ are constants.

Note that

$$
C_{V}=\frac{C}{\rho_{S}} \quad \text { and } \quad C_{V \max }=\frac{C_{\max }}{\rho_{S}}
$$

where $C$ is the solids concentration $(\mathrm{g} / \mathrm{L})$, and $C_{\max }$ is the solids concentration $(\mathrm{g} / \mathrm{L})$, corresponding to $C_{V \max }$.

Substituting Equation (2.22) to Equation (2.21) yields

$$
V_{S}=B\left(1-\frac{C}{C_{\max }}\right)^{4.65} \quad \text { For } \mathrm{C} \geq 10 \mathrm{~g} / \mathrm{L}
$$

The coefficients $A$ and $B$ must be determined for each given waste and set of conditions. Equations (2.19), (2.20), and (2.23) are unified to seamlessly cover all the solids concentration ranges. 


\subsubsection{Waste Properties That Affect Solids Settling}

Relevant waste properties and their degree of importance to solids settling velocities may be identified by examining Equations (2.2) through (2.23). For $R<0.3$ (the Stokes Law range), Equation (2.5) reveals that

$$
V_{S} \propto d_{S}^{2}\left(\rho_{S}-\rho_{L}\right) \mu_{L}^{-1}
$$

Thus, considering the sensitivity of the solids settling velocity to waste properties, the most important waste property is solids size (reflecting a shape factor), followed by the difference between the solids and liquid densities and the liquid viscosity. Note that the difference between solids and liquid densities is the solid's submerged weight in liquid divided by the gravitational acceleration constant; thus, both the solids and liquid densities are needed in Equation (2.24).

Note that the solids size can vary several orders of magnitude (Section 3.2.5), while both the liquid viscosity (Section 3.2.2) and solid density (Sections 3.2.3 and 3.3) can vary one order of magnitude, and the liquid density can increase by 50\% from water to salt-saturated liquid (Section 3.2.1). Thus, the order of importance of waste properties must also consider possible variations of these waste properties themselves. This is true throughout Section 2.0.

For $0.3 \leq R<1,000$ (the intermediate range), Equation (2.7) yields

$$
V_{S} \propto d_{S}^{1.14}\left(\rho_{S}-\rho_{L}\right)^{0.714} \mu_{L}^{-0.428} \rho_{L}^{-0.286}
$$

This equation indicates that the main waste properties controlling the solids settling velocity are solids size (and shape factor), the difference between solids and liquid densities, the liquid viscosity, and the liquid density, in that order of importance.

For $1,000 \leq R<2 \times 10^{5}$ (the Newtonian Law range), Equation (2.9) yields

$$
V_{S} \propto d_{S}^{0.5}\left(\rho_{S}-\rho_{L}\right)^{0.5} \rho_{L}^{-0.5}
$$

Thus, for this range of $R$, waste properties affecting settling velocity are solids size (and shape factor), the difference between solids and liquid densities, and liquid density. According to Equation (2.26), these are of equal importance.

When agglomeration and hindered settling become important, waste chemistry, temperature, and solids concentrations can affect the agglomerate size and density of solids, which in turn can affect the solids settling velocity, as indicated by Equations (2.19) through (2.23). Required waste chemistry information includes the identities and concentrations of aqueous chemical species as well as the identities and concentrations of solids phases.

\subsubsection{Gas Generation, Retention, and Release}

Tank waste generates flammable gases through thermo-chemical reactions, radiolysis of water and organic materials, and corrosion processes (Meacham 2009). Flammable gas generation, retention, and 
release are safety issues, and improved predictions of these phenomena can impact waste processing and WTP design margins.

\subsubsection{Gas Generation, Retention, and Release Discussion}

The primary flammable gases generated by Hanford waste are hydrogen, methane, and ammonia (Weber 2009). Hydrogen has been identified as the principle flammability concern, with methane generation assumed to be less than $10 \%$ that of hydrogen, and ammonia contributing no more than $2 \%$ of the mixture's lower flammability limit (LFL) (Meacham 2009).

Some fraction of these gases may be retained in sediment and crust layers, and some is released into the headspaces of the tanks. Gas bubbles are retained in solids layers by

- capillary force

- waste strength

- attachment to solid particles (sometimes called an "armored bubble”).

Gas releases can be essentially continuous, as when the amount of retained gas approaches a maximum level, and the gas release rate approaches the gas generation rate. Gas releases can also be episodic, as when the amount of retained gas exceeds criteria for a buoyant displacement gas release event (BDGRE), and a significant amount of gas is released over a short time (Meacham 2010). Retained gases can also be released by agitating the waste (e.g., by waste pump jet and pulsed jet mixers [PJMs]) to alter any or all of the three factors listed above.

\subsubsection{Example Gas Generation, Retention, and Release Calculation Models}

Hanford waste gas generation, retention, and release models are discussed in the following subsections.

\section{Gas Generation Models}

The Hydrogen Generation Rate (HGR) model has been used to estimate Hanford waste hydrogen gas generation (Hu 2004, Meacham 2009). It considers the primary three hydrogen generation pathways (thermo-chemical reactions, radiolysis, and corrosion), and estimates the rates of each. The three pathways are found to depend on waste temperature and chemistry (e.g., concentrations of nitrate, nitrite, total organic carbon), mass of waste present, liquid fraction, radiolytic heat load (alpha, beta, and gamma levels), and surface area of steel that can be corroded. Methods to estimate other flammable gases are described by Weber (2009).

\section{Gas Retention and Release Models}

Gauglitz et al. (1995, 1996, 2009) developed the following Bond Number to address the gas retention by capillary force and waste strength: 


$$
\text { Bond Number }=\frac{\left(\rho_{S}-\rho_{L}\right) g H_{S} D_{P}}{4 \sigma}+\frac{\tau_{y} D_{P}}{4 \sigma} \frac{A_{2}}{A_{1}}
$$

where $A_{1}=$ area of sludge exposed to the bubble

$A_{2}=$ area over which sludge strength applies

$D_{P}=$ mean pore diameter

$H_{S}=$ height of the lithostatic column above a bubble (depth of the solids layer above the bubble in the solids layer)

$\sigma=$ surface tension

$\tau_{\mathrm{y}}=$ yield stress.

The value of $A_{2} / A_{1}$ in Equation (2.56) is 2.8 based on experiments (Gauglitz et al. 1996). The mean pore diameter, $D_{P}$, in this equation may be represented by the solids particle diameter, $d_{S}$, (Gauglitz et al. 2009).

When the Bond number is greater than unity, a bubble is retained by capillary force and is a dendritic-shaped bubble, fingering between particles. When the Bond number is less than 1 , bubbles displace some solid particles to form a rounder bubble.

When the surface tension and/or waste strength are not high enough, gases are released from a solids layer (also called a non-convective layer). Bubbles retained by these two forces are released when solids holding them are mobilized by mixing. However, bubbles attaching to the surface of a solid particle may still remain attached to the solid particles and may even be suspended in the supernatant liquid layer (a convective layer) (Gauglitz et al. 1995). Thus, gas release phenomena discussed here depend on capillary forces and/or waste strength (i.e., shear strength or yield stress).

Gas retention and release from the Hanford high-level waste (HLW) by PJMs and air spargers were evaluated experimentally with several scale models. The data obtained were evaluated by using similarity analysis to derive the following gas retention correlation (Stewart et al. 2006):

$$
N_{H}=8.32 R_{e J}^{-0.76} \tau_{S} *^{0.16} S_{t}^{0.52}
$$

where

$$
\begin{gathered}
N_{H}=\frac{g_{V} H}{U_{R}} \equiv \text { Gas Holdup Number } \\
R_{e J}=\frac{U_{j} d_{j} \rho_{L}}{\mu_{L}} \equiv \text { Jet Reynolds Number } \\
\tau_{S}{ }^{*}=\frac{\tau_{S}}{\rho_{L} U_{j}^{2}} \equiv \text { Solid Erosion by Jet Impingement }
\end{gathered}
$$




$$
S_{t}=\frac{T_{C}}{\left(\frac{d_{j}}{U_{j}}\right)} \equiv \text { Strouhal Number }
$$

where $\mathrm{d}_{\mathrm{j}}=$ nozzle diameter

$g_{v}=$ volumetric gas generation rate per unit volume of gas-free slurry

$\mathrm{H}=$ waste thickness (depth in vessel)

$\mathrm{T}_{\mathrm{C}}=$ pulse jet mixing (PJM) cycle time, complete cycle includes discharge and re-fill

$\mathrm{U}_{\mathrm{j}}=$ nozzle jet velocity

$U_{R}=$ gas bubble rise velocity at the waste surface

$\tau_{\mathrm{S}}=$ shear strength of sludge and saltcake.

and the gas holdup number, $\mathrm{N}_{\mathrm{H}}$, is the volume fraction of gas bubbles in the waste during mixing.

As shown in Figure 2.4, this similarity-based gas retention correlation is independent of the physical model scale and thus is directly applicable to the WTP waste processing vessels (Stewart et al. 2006).

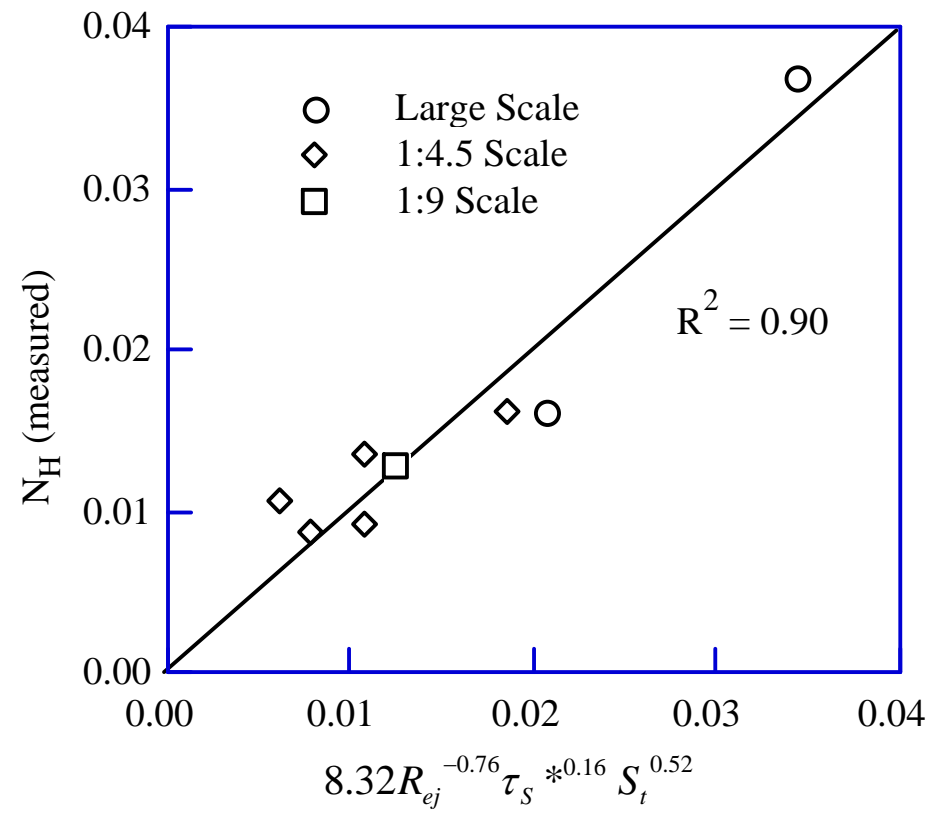

Figure 2.4. Retained Gas Correlation (Stewart et al. 1996b)

Based on the possible flammable gas concentration in the headspace of a tank and the possibility of a BDGRE for a safety analysis, a tank waste is assigned to a waste group (A, B, or C) (Weber 2009).

- Waste Group A: Tank waste has possibly enough flammable gas to exceed $100 \%$ of the LFL in the tank headspace, and can have BDGRE

- Waste Group B: Tank waste has possibly enough flammable gas to exceed $100 \%$ of the LFL level in the headspace, but does not have potential spontaneous BDGRE flammable gas hazard

- Waste Group C: A tank does not have a potential gas release event for flammable gas hazard. 
Weber (2009) provides equations to determine these waste groups, as described below.

Criterion 1: Retained gas flammability at headspace criterion; $\% \mathrm{LFL}_{\mathrm{HS}}<100 \%$

$$
\% L F L_{H S}=\left(\frac{\left[\mathrm{H}_{2}\right]_{R G}}{\% L F L_{H 2}}+\frac{\left[\mathrm{CH}_{4}\right]_{R G}}{\% L F L_{\mathrm{CH} 4}}+\frac{\left[\mathrm{NH}_{3}\right]_{R G}}{\% L F L_{N H 3}}\right) * \frac{V G_{W N C L} * F_{\text {Gas Release }}}{V_{H S}}
$$

where $\% \mathrm{LFL}_{\mathrm{CH} 4}=$ methane concentration at $100 \% \mathrm{LFL}$

$\% \mathrm{LFL}_{\mathrm{H} 2}=$ hydrogen concentration at $100 \% \mathrm{LFL}$

$\% \mathrm{LFL}_{\mathrm{HS}}=$ headspace flammable gas concentration following gas release

$\% \mathrm{LFL}_{\mathrm{NH} 3}=$ ammonia concentration at $100 \% \mathrm{LFL}$

$\left[\mathrm{CH}_{4}\right]_{\mathrm{RG}}=$ methane concentration in the released gas in nonconvective layer (vol\%)

$\left[\mathrm{H}_{2}\right]_{\mathrm{RG}}=$ hydrogen concentration in the released gas in nonconvective layer (vol\%)

$\left[\mathrm{NH}_{3}\right]_{\mathrm{RG}}=$ ammonia concentration in the released gas in nonconvective layer (vol\%)

$\mathrm{F}_{\text {gasRelease }}=$ fraction of gas released (assumed to be 100\%)

$\mathrm{V}_{\mathrm{HS}}=$ volume of headspace after gas release

$\mathrm{VG}_{\mathrm{WNCL}}=$ calculated void fraction in saturated settled solid layer.

If $\% \mathrm{LFL}_{\mathrm{HS}}$ is less than $100 \%$ of LFL, the waste group is Group C. Otherwise, the waste group is A or B.

Criterion 2: Energy Ratio (ER); ER < 3; The ER represents the buoyant potential energy of gas-bearing gobs to the energy required to yield the waste and release gas from these gobs, and is calculated by

$$
E R=\left\{\frac{\alpha_{N B} * \gamma * P_{H S}}{\left(1-\alpha_{N B}\right) * \tau_{W N C L} * \varepsilon_{y}}\right\} *\left\{\left(1+\frac{1}{\gamma}\right) * \operatorname{In}(1+\gamma)-1\right\}
$$

where $\quad \mathrm{P}_{\mathrm{HS}}=$ tank headspace pressure

$\alpha_{\mathrm{NB}}=$ neutral buoyancy of saturated settled solid layer relative to the overlying convective layer

$\varepsilon_{\mathrm{y}}=$ nonconvective layer strain at failure

$\gamma=$ ratio of pressure head of convective layer to the headspace pressure

$\tau_{\mathrm{WNCL}}=$ yield stress of saturated non-convective layer.

If the energy ratio is less than 3 and is not in Group C, the waste group is B.

Criterion 3: Buoyancy Ratio (BR); BR $<1$. The BR is the ratio of saturated settled solids layer gas fraction to the neutral buoyancy gas fraction. It is estimated by

$$
B R=\left(\frac{C F}{\rho_{W N C L}-\rho_{C L}}\right) *\left(\frac{\frac{H G_{W N C L}}{\left[H_{2}\right]_{R G}} T_{W N C L}}{P_{W N C L}}\right) * H_{W N C L}^{2}
$$


where

$$
\begin{aligned}
\mathrm{CF} & =\text { calibration factor } \\
\mathrm{H} & =\text { height of saturated non-convective layer } \\
\mathrm{HG}_{\mathrm{WNCL}} & =\text { hydrogen gas generation rate in saturated settled solid layer } \\
\mathrm{P}_{\mathrm{WNCL}} & =\text { representative retained gas pressure in the saturated settled solid layer } \\
\mathrm{T}_{\mathrm{WNCL}} & =\text { temperature of the saturated settled solid layer } \\
\rho_{\mathrm{CL}} & =\text { density of convective layer } \\
\rho_{\mathrm{WNCL}} & =\text { density of saturated non-convective layer. }
\end{aligned}
$$

If the BR is less than 1 and the waste type is not Group C, the waste group is Group B. If the BR is equal to or greater than 1 , the waste group is $\mathrm{A}$.

Meacham (2010) cites analogies between Hanford wastes and sludge deposits studied by van Kessel and van Kesteren (2002). Van Kessel and van Kesteren found that cracks and channels form and remain open in sludge with high shear strength as long as the depth of the sludge is less than a maximum value. The presence of stable cracks and channels in a layer of settled waste solids is desirable because they facilitate gas release and limit the amount of retained gas. The maximum sludge depth for stable channels is given by

$$
d_{\max }=\frac{\tau_{S}(1+e)}{K_{0}\left(\rho_{S}-\rho_{L}\right) g}\left[1+\ln \left(\frac{r_{0}^{2}-d_{f}^{2}}{d_{f}^{2}}\right)+\ln \left(\frac{E}{2 \tau_{S}\left(1+v_{R}\right)}\right)\right]
$$

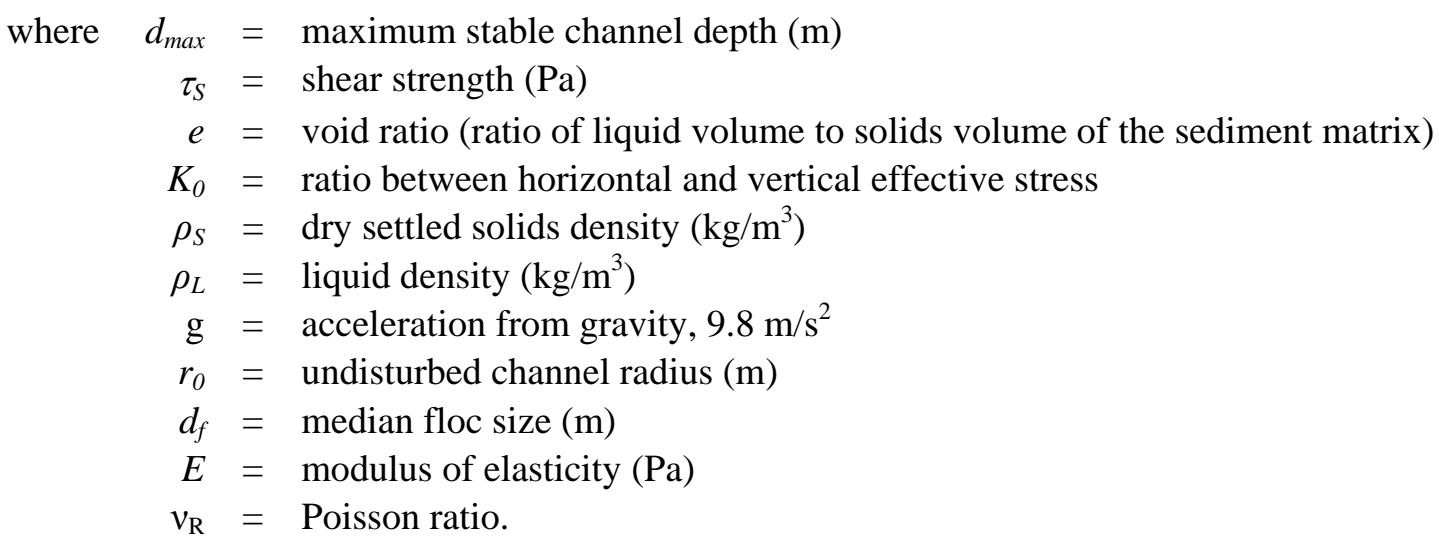

\subsubsection{Waste Properties That Affect Gas Generation, Retention and Release}

Gas generation rates are strongly affected by waste chemistry (e.g., concentrations of nitrate, nitrite, organic compounds), liquid weight fraction in the waste, waste temperature, alpha, beta, and gamma radiation levels and their heat load, waste volume and weight, and the tank carbon steel liner area exposed to wet waste.

Based on Equation (2.27), the waste properties affecting how gas is retained by slurry are solids density and particle size, solids layer thickness and weight, and surface tension. Waste properties affecting the amount of gas retained in the waste may be evaluated by examining Equation (2.28), as

$$
N_{H} \propto \rho_{L}{ }^{-0.92} \mu_{L}^{0.76} \tau_{S}^{0.16}
$$


Thus, the main waste properties affecting the volume fraction of gases retained in the waste during mixing operations are liquid density, liquid viscosity, and shear strength of the solids layer, in that order of importance. Shear strength depends not only on the waste itself but the history of that waste as well. The BDGRE models, Equation (2.34) and Equation (2.35), depend on these parameters as well as the sediment depth and density.

Equation (2.36) provides a means to estimate how deep a layer of waste could be before the beneficial cracks and channels are closed by lithostatic loads, given the waste parameters of shear strength, solids and liquid densities, mean floc size, and the modulus of elasticity.

\subsection{Waste Mobilization}

Wastes currently stored in the Hanford DSTs will be retrieved and transported to the WTP or a supplemental treatment facility for pretreatment and immobilization. The mobilization of settled solids in the DSTs using jet pumps, and specifically the effective cleaning radius (ECR) of a jet pump, is considered in Section 2.2.1. The jet mobilization of waste is also important to the WTP where arrays of stationary PJMs will be used to mix the contents of the process vessels. Correlations developed to characterize the effectiveness of PJMs to resuspend solids are discussed in Section 2.2.2. Models that predict the resulting cloud height and suspended solids concentration within the cloud are presented in Sections 2.2.3 and 2.2.4, respectively. Lastly, the erosion of vessel walls due to the abrasive nature of waste jets is discussed in Section 2.2.5.

\subsubsection{ECR of a Submerged Jet}

Hanford tank waste in DSTs and WTP waste process vessels will be mobilized by water and slurry jets. Thus, it is important to determine if these jets are capable of eroding the required amounts of settled sludge and saltcake in these tanks and vessels. The ECR is often used in the Hanford, the Savannah River, and the Oak Ridge Sites to express the jet eroding capacity. The ECR is the distance between the jet nozzle exit and the base of the non-mobilized sludge bank.

\subsubsection{Examples of ECR Calculation Models}

Various ECR models have been developed at the DOE sites (Powell et al. 1995a, b, Tedeschi 2000, Poirier 2004). Some examples of ECR models are presented here.

For pump jet mixing, Powell et al. (1997) and Gauglitz et al. (2009), respectively, derived the following ECR formulas for Hanford Site waste.

$$
\begin{gathered}
E C R=4.0 d_{j} U_{j} \tau_{S}^{-0.46} \\
E C R=5.78 d_{j}\left(\frac{\tau_{S}}{\rho_{L} U_{j}^{2}}\right)^{-0.5}
\end{gathered}
$$


Historically, the Hanford Site has used shear strength as a measure of the solids layer strength, while the Savannah River and the Oak Ridge Sites have used yield stress of a Bingham fluid to represent the solids layer strength. Poirier (2004) and Reshma et al. (2007) developed the following formula for the Savannah River and Oak Ridge Sites, respectively.

$$
\begin{aligned}
& E C R=0.97 d_{j} U_{j}\left(\frac{\tau_{y}}{\rho_{L}}\right)^{-0.5} \\
& E C R=4.4 d_{j} U_{j}\left(\frac{\tau_{y}}{\rho_{L}}\right)^{-0.5}
\end{aligned}
$$

where $\tau_{y}$ is the yield stress of the Bingham fluid.

There are many literature studies on solids erosion by water jets and streams (Pertheniades 1962, Onishi et al. 1993, Hanson and Simon 2001, Clark and Wynn 2007). These studies usually express the solids erosion model as

$$
S_{R}=k\left(\tau_{B}-\tau_{C}\right)
$$

where $\quad k=$ erodibility coefficient

$S_{R}=$ amount of solid eroded per unit bed surface area per unit time

$\tau_{B}=$ bed shear stress exerted by an impinging jet or a stream on the solid layer surface

$\tau_{C}=$ critical shear stress for solids erosion.

The solids erosion rate, $S_{R}$, can be determined by the solid layer's critical shear stress for erosion and the erodibility coefficient with the known bed shear stress of an imposing flow on the solids layer (e.g., Wells et al. 2009). The critical shear stress for erosion may be estimated from the shear strength and the plasticity index of the sludge (Dunn 1959). The erodibility coefficient is usually determined by an erosion experiment. Because necessary data have not been collected at the Hanford Site, Equation (2.42) has not been applied to Hanford tank waste to determine the solids erosion by a jet.

For the vertical jet representing the PJM, Gauglitz et al. (2009) derived the following non-dimensional ECR formula:

$$
E C R=1.16 d_{j}\left(\frac{\tau_{S}}{\rho_{L} U_{j}^{2}}\right)^{-0.5}
$$

\subsubsection{Waste Properties That Affect ECR}

Equation (2.38) indicates that

$$
E C R \propto d_{j} U_{j} \tau_{S}^{-0.46}
$$


while Equations (2.39) through (2.41) imply that

$$
E C R \propto d_{j} U_{j} \rho_{L}^{0.5} \tau_{S}{ }^{-0.5}\left(\text { or } \tau_{y}{ }^{-0.5}\right)
$$

These formulas indicate that key parameters for the ECR are the jet nozzle diameter, the jet exit velocity, the sludge shear strength (or yield stress), and possibly the liquid density, in that order of importance. Shear strength is expected to be the dominant property for ECR, and liquid density will have a smaller effect.

As stated above, the shear strength of a cohesive solid depends on both the waste itself and the history. For the solids erosion, the shear strength is the most critical parameter. For non-cohesive solids, the critical shear stress for erosion is the critical waste property and is inherent to a slurry containing a given specific solid size, density, and shape (Garcia 2008).

\subsubsection{Pulsed Jet Mixer Resuspension of Settled Solids}

The WTP design for mixing in storage and process vessels incorporates PJMs. These require no moving parts within the vessels, but their mixing performance differs from other industrial mixing systems (e.g., rotating impeller mixers), and much of the literature on other mixers is not directly applicable to PJMs. This section discusses some available models for describing solids resuspension by PJMs.

\subsubsection{Critical Resuspension Velocity}

The WTP defines critical suspension velocity as the lowest jet nozzle velocity that can suspend all solids in a process vessel. The critical suspension velocity depends on waste and jet properties as well as vessel and mixing equipment configuration, i.e., vessel dimensions and the positions, orientations, and number of jets.

\subsubsection{Examples of Critical Suspension Velocity Calculation Models}

The critical suspension velocity, $U_{C S}$, correlation derived by Meyer et al. (2010) is

$$
U_{C S}=2.302\left(U_{T H}+\frac{\phi_{J} D C \cdot U_{C S}}{1-\phi_{P T}}\right)^{0.478}\left\{D\left(\frac{\rho_{S}}{\rho_{L}}-1\right) g\right\}^{0.261} \phi_{J}^{-0.511} D C^{-0.261} \phi_{S}^{0.261} \phi_{P}{ }^{-0.234} A r^{-0.176}
$$

where the Archimedes Number, Ar, (referred to as the Galileo Number, Ga, in Meyer et al. (2010) is

$$
A r=\frac{\left(\frac{\rho_{S}}{\rho_{L}}-1\right) g d_{S}{ }^{3}}{\left(\frac{\mu_{L}}{\rho_{L}}\right)^{2}}=G a
$$


where

$$
\begin{aligned}
D & =\text { tank diameter } \\
D C & =\text { PJM duty cycle } \\
d_{j} & =\text { PJM nozzle diameter } \\
n & =\text { number of operating jets/pulse tubes } \\
\mathrm{U}_{\mathrm{TH}} & =\text { hindered terminal settling velocity } \\
\phi_{J} & =\text { jet density }\left(=\mathrm{nd}_{\mathrm{j}}^{2} / \mathrm{D}^{2}\right) \\
\phi_{P} & =\text { pulse volume fraction } \\
\phi_{P T}= & \text { ratio of pulse tube to vessel cross-section area } \\
\phi_{S}= & \text { Solids volume fraction (volume of UDS per a reference tank } \\
& \text { volume defined as } \left.\frac{\pi}{4} \mathrm{D}^{3}\right) .
\end{aligned}
$$

Onishi (2008) used the same scaled model data (Meyer et al. 2009) used to develop Equation (2.46) to derive the following correlation for the critical suspension velocity.

$$
\frac{U_{C S}^{2} \rho_{L}}{\tau_{S}}=304\left(\frac{d_{j}}{D}\right)^{-2.0}\left(F_{S}\right)^{0.483}\left(\phi_{S}\right)^{0.352}(D C)^{-0.526}(n)^{-0.803}\left(\phi_{P}\right)^{-0.775}\left(T_{S}\right)^{0.326}
$$

$$
\text { where } \begin{aligned}
F_{S}=\frac{\tau_{S}}{\left(\rho_{S}-\rho_{L}\right) g d_{S}} & \equiv \text { Solid Erosion by Jet shear } \\
T_{S}=\frac{V_{S} T_{C}}{H} & \equiv \text { Non-dimensional Solids Settling Distance } \\
H & =\text { vessel operating height } \\
V_{S} & =\text { solids settling velocity. }
\end{aligned}
$$

\subsubsection{Waste Properties That Affect Critical Suspension Velocity}

Equation (2.46) has the critical suspension velocity, $\mathrm{U}_{\mathrm{CS}}$, in both sides of the equation, and the right-hand side of the equation contains the power of a term consisting of the sum of $U_{\mathrm{CS}}$ and another variable, $\mathrm{U}_{\mathrm{TH}}$. Thus, Equation (2.46) does not allow a direct evaluation of the importance of various waste properties based on the degree of powers of waste properties. Equation (2.46) contains the solid size, solid density, liquid density, and liquid viscosity. Thus, these waste properties would influence the critical suspension velocity.

The critical suspension velocity expression, Equation (2.48), contains the solids settling velocity. Subsection 2.1.1 provides the relationships between the solids settling velocity and waste properties. Thus, these relationships were used to assess the importance of the waste properties included in Equation (2.48).

For the Stokes Law range (particle Reynolds Number, $R<0.3$ ), substituting Equation (2.24) into Equation (2.48) yields

$$
U_{C S} \propto \tau_{S}^{0.742} \rho_{L}{ }^{-0.500} \phi_{S}{ }^{0.176} \mu_{L}{ }^{-0.163} d_{S}{ }^{0.084}\left(\rho_{S}-\rho_{L}\right)^{-0.079}
$$


In this range, the most important waste property affecting the critical suspension velocity is the shear strength of the solids layer. This is consistent with models developed to describe the ECR of a waste pump jet on waste having a shear strength (or yield stress), as discussed below in Subsection 2.2.1. This is expected because both the ECR and the critical suspension velocity are addressing the capability of an impinging jet to mobilize the settled solids at a specific distance from a jet nozzle. Equation (2.49) indicates that $U_{C S}$ increases with increasing shear strength (as does critical shear stress for erosion).

The other waste properties affecting the critical suspension velocity in the Stokes Law range are liquid density, solids volume fraction, liquid viscosity, solids diameter, and difference between solids and liquid densities (i.e., the submerged weight of the solids divided by the gravitational acceleration constant). Note that the solids volume fraction is a process parameter, not a waste property, and that $\phi_{S}$ is a relative solids volume fraction selected by Meyer et al. (2009). Although the liquid density has an impact on the magnitude of the critical suspension velocity, the liquid density does not usually change significantly from one waste to another (compared to some other waste properties). The solids size and the submerged weight of the solids have relatively weak effects on the critical suspension velocity in the Stokes Law range.

It should be noted that the shear strength, solids density, and solids diameter are not independent of each other. For example, for a fine cohesive solid, the smaller the solids particle size, the greater the shear strength and the critical shear stress for erosion in general (Vanoni 1975). On the other hand, in general, for larger non-cohesive solids, the larger and/or the denser the solids, the greater the shear strength. Thus, Equation (2.49) and similar equations below should be carefully viewed with this variation in mind.

For Reynolds Numbers in the intermediate range $(0.3 \leq R<1,000)$, substituting Equation (2.25) into Equation (2.48) yields

$$
U_{C S} \propto \tau_{S}^{0.742} \rho_{L}^{-0.547} \phi_{S}^{0.176}\left(\rho_{S}-\rho_{L}\right)^{-0.126} \mu_{L}^{-0.070} d_{S}^{-0.056}
$$

Here the most important waste property affecting the critical suspension velocity is again shear strength, followed by liquid density, solids volume fraction, the difference between solids and liquid densities (thus, solids density), liquid viscosity, and solids diameter, in that order of importance. Liquid viscosity and solids size have relatively weak effects on the critical suspension velocity.

For the Newtonian Law range $\left(1,000 \leq R<2 \times 10^{5}\right)$, substituting Equation (2.26) into Equation (2.48) yields

$$
U_{C S} \propto \tau_{S}^{0.742} \rho_{L}^{-0.582} \phi_{S}^{0.176}\left(\rho_{S}-\rho_{L}\right)^{-0.160} d_{S}^{-0.160}
$$

In this range, Onishi's $U_{C S}$ model predicts that the key waste property affecting the critical suspension velocity is the shear strength again, followed by liquid density, solids volume fraction, difference between solids and liquid densities (thus, solids density), solids diameter, and liquid viscosity, in that order of importance. 


\subsubsection{Jet Mixing Cloud Height}

When a pulsed jet hits the sludge layer in a WTP waste process vessel, some eroded solids are lifted upward, often forming a distinct slurry layer above which a clear liquid exits. The cloud height expresses the height of this slurry layer above the sludge layer.

\subsubsection{Example Cloud Height Calculation Models}

Pacific Northwest National Laboratory (PNNL) conducted a series of experiments in scaled physical models to assess the PJM performance of WTP vessels (Meyer et al. 2009) and derived the following cloud height expression (Meyer et al. 2010).

$$
H_{C}=\frac{D C \cdot U_{T H}{ }^{2} \phi_{P}^{0.539} \phi_{J}^{1.662} n^{-0.658} R_{e J}^{0.143}}{\left(\frac{\rho_{S}}{\rho_{L}}-1\right) g \phi_{S}} \cdot \exp \left\{8.223\left(\frac{U_{j}}{U_{T H}}\right)^{0.1364}\right\}
$$

where the Jet Reynolds Number, $\mathrm{R}_{\mathrm{eJ}}$, is $R_{e J}=\frac{d_{j} U_{j} \rho_{L}}{\mu_{L}}$.

Onishi (2008) used the same scaled model data reported in Meyer et al. (2009) to derive the following correlation of the cloud height.

$\frac{H_{C}}{D}=2.39 \times 10^{-5}\left(\frac{d_{j}}{D}\right)^{1.10}\left(A_{r}\right)^{-0.20}\left(F_{S}\right)^{-6.89}\left(F_{D}\right)^{1.41}(z)^{-2.35}\left(R_{e^{*}}\right)^{-2.14}\left(\phi_{S}\right)^{-0.606}(D C)^{0.059}\left(S_{t}\right)^{-0.497}\left(\phi_{P}\right)^{0.645}$

where

$$
\begin{gathered}
F_{D}=\frac{U_{j}}{\sqrt{\left(\frac{\rho_{S}-\rho_{L}}{\rho_{L}}\right) g H}} \equiv \text { Densimetric Froude Number } \\
R_{e^{*}}=\frac{u_{*} d_{S} \rho_{L}}{\mu_{L}} \approx \frac{0.05 U_{j} d_{S} \rho_{L}}{\mu_{L}} \equiv \text { Particle Reynolds Number }
\end{gathered}
$$

$$
z=\frac{V_{S}}{\beta \kappa u_{*}}=\frac{V_{S}}{\beta \kappa \sqrt{\frac{\tau_{B}}{\rho_{L}}}} \equiv \text { Rouse Number }
$$

$u_{*}=$ Shear velocity

$\beta=$ constant $(\approx 1.0)$

$\kappa=$ von Kármán constant $(\approx 0.4)$. 


\subsubsection{Waste Properties That Affect Cloud Height}

Equation (2.52) contains both an exponential term and terms with powers in the right hand side of the equation. Thus, this equation cannot be used to obtain degrees of powers for waste proprieties to determine the order of importance of these waste properties. Because this equation contains solid size, solid density, liquid density, and liquid viscosity, these waste properties would affect the cloud height.

The alternative model for the suspension cloud height, Equation (2.53), was examined in the same manner that importance of waste properties to the critical suspension velocity was examined.

For Reynolds Numbers in the Stokes Law range (particle Reynolds Number, $R<0.3$ ), substituting Equation (2.24) into Equation (2.53) yields

$$
H_{C} \propto \tau_{S}^{-6.89} \mu_{L}^{4.89}\left(\rho_{S}-\rho_{L}\right)^{3.649} \rho_{L}^{-1.745} \phi_{S}^{-0.606} d_{S}^{-0.55}
$$

Thus, based on Equation (2.53), the most important waste property affecting the suspension cloud height model is the shear strength of the solids layer. This is again consistent with ECR models, Equations (2.38) through (2.45), which contain shear strength (or yield stress), as discussed in Section 2.2.1. Equation (2.57) indicates that the greater the shear strength, the smaller the cloud height.

The other waste properties affecting the suspension cloud height in the Stokes Law range are liquid viscosity, the difference between solids and liquid densities (i.e., submerged weight of solids divided by the gravitational acceleration constant), liquid density, solid volume fraction, and solid diameter, in that order of the importance. As indicated under the critical suspension velocity section above, the relationship between shear strength (and critical shear stress) and solid particle size/density are opposite for a fine cohesive solids and coarser non-cohesive solids. Liquid viscosity is important in the Stokes Law range, as expected.

For Reynolds Numbers in the intermediate range $(0.3 \leq R<1,000)$, substituting Equation (2.25) into Equation (2.53) yields

$$
H_{C} \propto \tau_{S}^{-6.89}\left(\rho_{S}-\rho_{L}\right)^{4.31} \mu_{L}^{3.55} d_{S}^{1,47} \rho_{L}^{-1.08} \phi_{S}^{-0.606}
$$

Here the most important waste property is again the shear strength, followed by the difference between the solids and liquid densities (thus, solid density), liquid viscosity, solids diameter, liquid density, and solids volume fraction, in that order of importance. The solids volume fraction has a relatively weak effect on the suspension cloud height in the intermediate range.

For Reynolds Numbers in the Newtonian Law range $\left(1,000 \leq R<2 \times 10^{5}\right)$, substituting Equation (2.26) into Equation (2.53) yields

$$
H_{C} \propto \tau_{S}^{-6.89}\left(\rho_{S}-\rho_{L}\right)^{4.81} d_{S}^{2.97} \mu_{L}^{2.54} \phi_{S}^{-0.606} \rho_{L}^{-0.57}
$$

In this range, the key waste property affecting cloud height is shear strength again, followed by liquid density, solid volume fraction, difference between solids and liquid densities (thus, solid density), solid 
diameter, and liquid viscosity, in that order of importance. The liquid viscosity has a relatively weak effect on the critical suspension velocity, as expected in the Newtonian Law range.

\subsubsection{Suspended Solids Concentration}

Transfer pumps will be used to retrieve waste slurries in the Hanford tanks and WTP waste processing vessels. The efficiency of solids retrieval depends on the degree to which solids are suspended, and thus, it is important to evaluate the suspended solids concentration distribution within a tank.

For many DSTs, the solids mobilized by mixer pumps have been predicted by PJM modeling (Onishi et al. 2000, Onishi and Wells 2004). The predicted vertical distributions of suspended solids concentrations are generally quite uniform due to fine solid particles having medium particle diameters equal to or less than $10 \mu \mathrm{m}$. AZ-101 PJM tests also imply that vertical distribution of the suspended solids was quite uniform during the PJM operation (Onishi and Recknagle 1997, Carlson et al. 2000, 2001). PNNL also conducted a series of scaled model experiments to obtain the solids concentration of suspended solids by PJMs.

\subsubsection{Examples of Suspended Solids Concentration Calculation Models}

Meyer et al. (2009) assumed that 1) the vertical distribution of suspended solids is linear and that 2) the radial concentration distribution is uniform within the suspended solid's cloud. With these assumptions, Meyer et al. (2009) derived the following suspended solids concentration correlation at the tank bottom, $C_{0}$ :

$$
C_{0}=\frac{2 \phi_{S}}{\left(\frac{H_{C}}{D}\right)}
$$

where $C_{0}$ is the solids vol\% at the tank bottom.

Equation (2.60) implies that the relative importance of these variables in the $C_{0}$ correlation is almost the same, but is inversely related to those in the cloud height correlation in Equation (2.52).

Substituting the $H_{C}$ correlation (Equation 2.52) into Equation (2.60), the $C_{0}$ correlation based on the suspension cloud height model of Meyer et al. is

$$
C_{0}=\frac{k \phi_{S} D\left(\frac{\rho_{S}}{\rho_{L}}-1\right) g \phi_{S}}{D C \cdot U_{T H}{ }^{2} \phi_{P}{ }^{0.539} \phi_{J}^{1.662} n^{-0.658} R_{e j}{ }^{0.143} \cdot \exp \left\{8.223\left(\frac{U}{U_{T H}}\right)^{0.1364}\right\}}
$$

With these two correlations, the vertical distribution of the suspended solids concentrations may be expressed as 


$$
C=C_{0}\left(1-\frac{y}{H_{C}}\right)
$$

where $y$ is the vertical distance above the solid surface.

The vertical distribution of the suspended solids is a result of the solids settling toward the tank bottom, counter-balanced by the upward component of the flow turbulence to lift solids upward. The turbulence intensity is usually related to the shear velocity, $u_{*}=\sqrt{\tau_{B} / \rho_{L}} \cdot \tau_{\mathrm{B}}$ is the bed shear stress. For a simple one-dimensional (vertical direction) steady state (longitudinally constant) uniform flow with the Prandtl-von Kármán velocity distribution, the vertical distribution of the solids is given by (Vanoni 1975)

$$
\frac{C}{C_{a}}=\left(\frac{d-y}{y} \frac{a}{H_{C}-a}\right)^{z}
$$

where $\quad a=$ distance above the solid surface

$C=$ solids concentration

$C_{a}=$ solids concentration at elevation "a" above the tank bottom

$d=$ total liquid depth.

The Rouse Number, Equation (2.63), is the ratio of the solids settling velocity and the shear velocity, as discussed above. Equation (2.62) indicates that the Rouse Number, $z$, uniquely determines the vertical distribution of the suspended solids concentrations. This is shown in Figure 2.5, indicating that the vertical solids concentration distribution is a function of the Rouse Number only. 


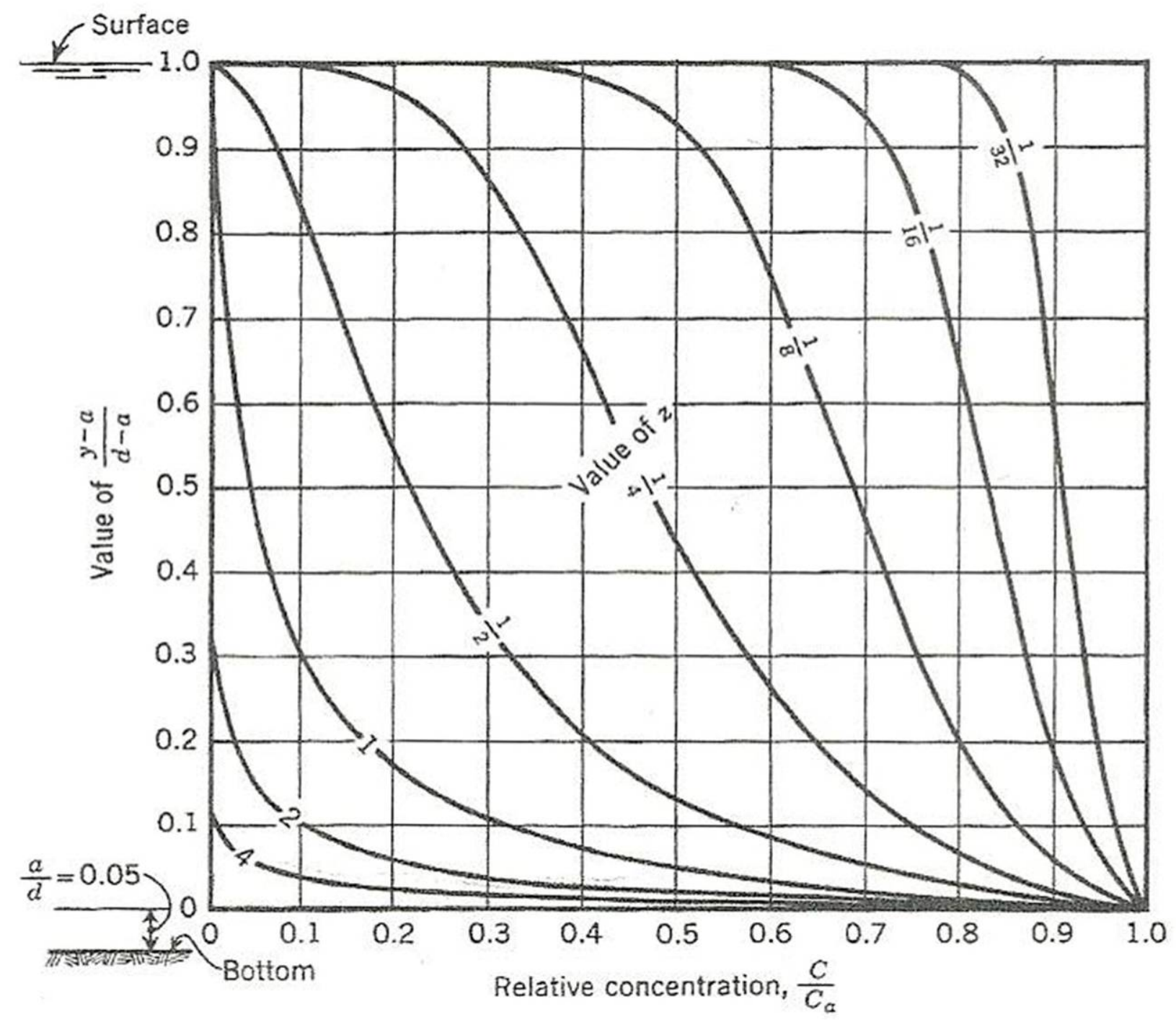

Figure 2.5. Vertical Distribution of Suspended Solids Concentrations for Various Rouse Number, z (Vanoni 1975)

Although Equation (2.63) provides a reasonably accurate picture of the vertical solids distribution in a vertically varying, horizontally constant (uniform), one-dimensional flow, a PJM-induced velocity field has a complex three-dimensional distribution, which is different from that expressed by the Prandtl-von Kármán velocity distribution (Vanoni 1975).

\subsubsection{Waste Properties That Affect Suspended Solids Concentration}

Relevant waste properties for the suspended solids concentration are basically the same as those associated with the suspension solids cloud height discussed in Section 2.2.3. 


\subsubsection{Wall Erosion from Pulse Jet Mixers}

In the WTP, radioactive waste will be mixed by PJMs in process vessels. A study on the 316-L stainless steel mixing vessel wall erosion by PJM operations has been conducted and reported by Papp (2008) and Papp and Duncan (2009). In their reports, the empirical relation to predict the extent of PJM vessel wall erosion is given as

$$
\begin{aligned}
& \frac{E_{\text {vessel }}}{E_{\text {vessel-ref }}}=\left(\frac{U_{j}}{U_{\text {ref }}}\right)^{3.08588}\left(\frac{d_{S}}{d_{S-r e f}}\right)^{1.982}\left[(1-I)\left(\frac{G}{C_{\text {ref }}}\right)^{0.8247}+I\left(\frac{C_{w}}{C_{r e f}}\right)^{0.8247}\right](F)\left(E_{d}\right)\left(D_{L}\right)(S C) \\
& \text { where } \quad E_{\text {vessel }}=\text { PJM wall erosion depth at end of design life (in.) } \\
& E_{\text {vessel-ref }}=\text { PJM wall erosion depth rate of reference case (in./year) } \\
& U_{\mathrm{j}}=\text { PJM jet velocity (m/s) } \\
& U_{r e f}=\text { PJM jet velocity of reference case (m/s) } \\
& d_{S}=\text { solids particle weighted-mean diameter (m) } \\
& d_{S-r e f}=\text { solids particle weighted-mean diameter from reference case (m) } \\
& I=\text { fraction of time for maximum solids loading } \\
& G=\text { normal solids concentration (wt\%) } \\
& C_{\mathrm{W}}=\text { maximum solids concentration (wt\%) } \\
& C_{r e f}=\text { reference case concentration (wt\%) } \\
& F=\text { vessel usage factor } \\
& E_{\mathrm{d}}=\text { PJM duty factor } \\
& \mathrm{D}_{\mathrm{L}}=\text { design life (year) } \\
& S c=\text { scale factor. }
\end{aligned}
$$

\subsubsection{Waste Properties That Affect Surface Erosion}

In Equation (2.64) the vessel wall erosion due to PJM operation is correlated with parameters of slurry concentration, particle diameter, and PJM jet velocity. Although particle hardness is likely a parameter affecting the wall erosion, no parameter for hardness is included in Equation (2.64). Based on this equation, PJM jet velocity is the dominant factor for the erosion of this system, having the largest exponent, 3.08588. As seen from Equation (2.64), the solids particle diameter is the only WTP waste property for the vessel wall erosion of the PJM system, so $E_{\text {vessel }} \propto d_{S}^{1.982}$.

\subsection{Waste Transport}

Once mobilized in the DSTs, waste solids will be transported by pumps and pipelines to other DSTs and eventually to WTP storage or process vessels. This section considers the effectiveness of the transfer pump suction to collect the solids (Section 2.3.1), the prevention of stationary beds of solids in the transfer pipes (Section 2.3.2), and pipe erosion (Section 2.3.3). 


\subsubsection{Pump Suction}

Waste sludge stored in tanks is usually removed as slurry by a waste transfer pump. The effectiveness of the pump suction is considered in this section.

\subsubsection{Examples of Solids Suction Calculation Models}

The flow induced by a waste transfer suction pump may be treated as an irrotational flow, and its velocity may be approximated by (Rouse 1961)

$$
V_{R}=\frac{Q}{2 \pi R^{2}}
$$

where $Q$ is the transfer pump discharge, $R$ is the radial distance from the pump suction inlet, and $V_{R}$ is the radial velocity toward the pump suction inlet.

The magnitude of the transfer pump-induced flow at any given location must overcome the critical shear stress for erosion to mobilize the settled solids at that location (Wells et al. 2009). The critical shear stress is usually expressed as a relationship between these two non-dimensional parameters:

$$
\begin{gathered}
F_{S, C}=\frac{\tau_{C}}{\left(\rho_{S}-\rho_{L}\right) g d_{S}} \equiv \text { Non-dimensional Critical Shear Stress for Erosion } \\
R_{e^{*}}=\frac{u_{*} d_{S}}{v}=\frac{\sqrt{\frac{\tau_{B}}{\rho_{L}}} d_{S} \rho_{L}}{\mu_{L}} \equiv \text { Particle Reynolds Number }
\end{gathered}
$$

where $u_{*}$ is the shear velocity, $\tau_{B}$ is the shear stress acting on the solids surface layer, and $\tau_{C}$ is the critical shear stress for erosion.

The Shields Diagram (Vanoni 1975, Garcia 2008) provides the relationship between Equations (2.66) and (2.67). For non-cohesive solids, the Shields Diagram uniquely determines the critical shear stress. For fine cohesive solids, the Shields Diagram provides a qualitative trend of the critical shear stress, but does not provide specific critical shear stress values.

\subsubsection{Waste Properties That Affect Pump Suction of Solids}

As shown in Equations (2.66) and (2.67), the waste properties affecting the solids suction by a transfer pump are the density and the viscosity of the liquid and the size and the density of solids for a non-cohesive solid. Note that the solids density is needed to estimate the submerged solids weight, $\left(\rho_{S}-\rho_{L}\right) g$.

For a cohesive solid, the critical shear stress is not only a function of these four waste properties, but also of the cohesiveness of the solids and its history and condition. Thus, waste chemistry and history also play roles in critical shear stress. Critical shear stress is usually obtained through laboratory or field 
measurements and is related to shear strength (Dunn 1959, Pertheniades 1962, 1993, Teeter 1988, Mazurek et al. 2001). There are no reported measurements of critical shear stress for Hanford waste.

\subsubsection{Pipeline Critical Velocity}

Sludge and saltcake wastes at the Hanford Site will be transferred through pipelines within tank farms, between tank farms, from tank farms to the WTP, and between process vessels within the WTP (Julyk et al. 2000, Slurry Transport Expert Panel 2002, Onishi et al. 2002). It is important that solids do not deposit in pipelines during slurry pipeline transfer to avoid pipe plugging. Deposition is prevented by making certain that the pipeline velocity exceeds the deposition velocity.

The velocity of a slurry pipeline flow and its relationship to the pipeline pressure gradient are shown in Figure 2.6 (Govier and Aziz 1987), together with the velocity-pressure drop relationship of a pure liquid. As depicted in Figure 2.6, the following four regimes of slurry pipeline flow occur as the slurry velocity, $V_{M}$, is decreased.

- Regime 1 , symmetric suspension $\left(V_{M} \geq V_{M 1}\right)$

All solids of the slurry flow are transported as suspended load, and the vertical distribution of the solids concentration is uniform across the pipe cross-section.

- Regime 2, asymmetric suspension $\left(V_{M 2} \leq V_{M}<V_{M 1}\right)$

All solids are transported as suspended load, but the vertical distribution of the suspended solids concentrations is not uniform across the pipe cross-section.

- Regime 3, moving bed $\left(V_{M 3} \leq V_{M}<V_{M 2}\right)$

Solids in the slurry are transported as a bed load; solid particles are saltating (solids are rolling, sliding, hopping, and jumping along the pipeline bottom), and solids are not transported as suspended load. For slurry with mixed-size particles, some solids may be in suspension.

- Regime 4, stationary bed $\left(V_{M 4} \leq V_{M}<V_{M 3}\right)$

Some solids in the slurry are deposited on the bottom of the pipe. The upper part of the solid layers is in saltation, but the lower part of the solid layers is in stationary. With mixed-size particles, some solids can be in suspension. For the velocity below $V_{M 4}$, the pipe would be blocked. 


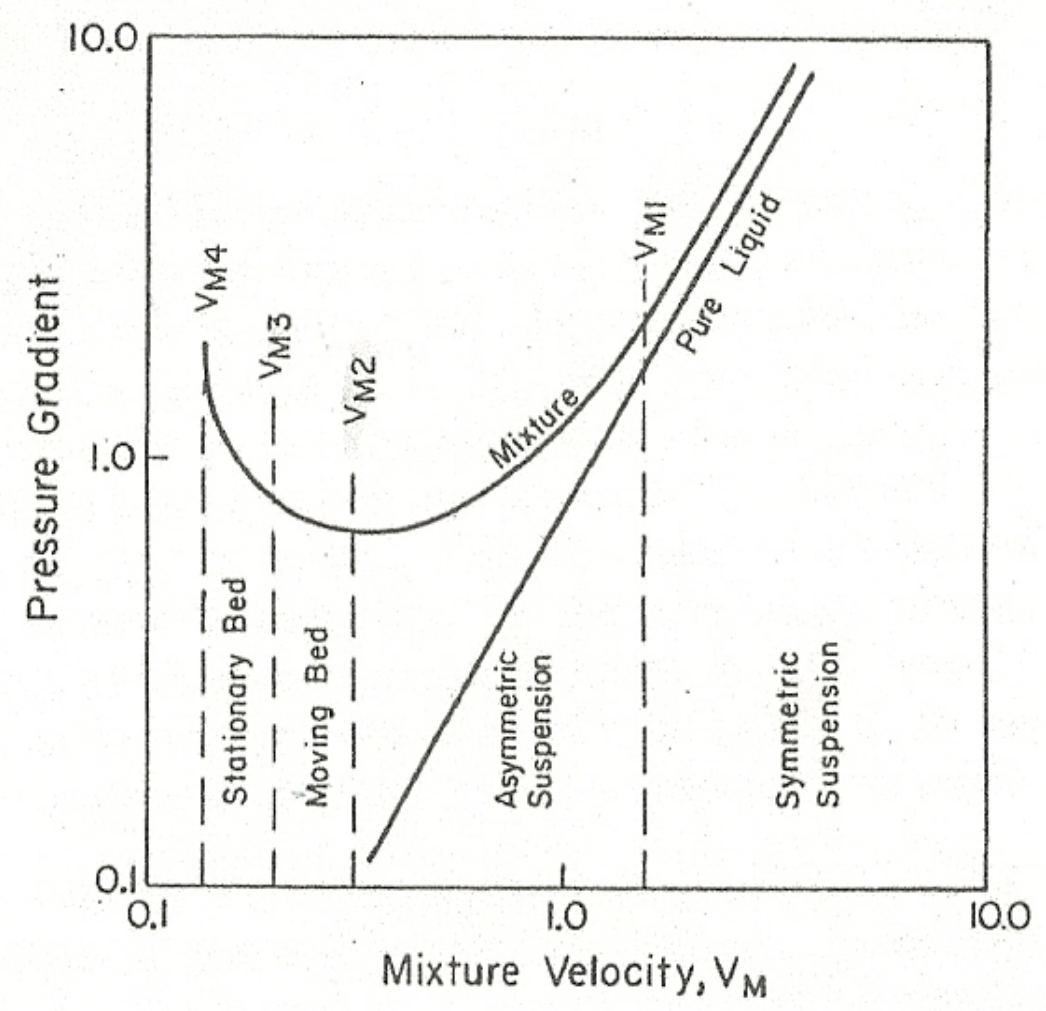

Figure 2.6. Pipeline Slurry Velocity and Pressure Gradient (Govier and Aziz 1987)

As shown in Figure 2.6, the pressure drop is the smallest at or around the slurry velocity of $V_{M 2}$. At or above that velocity, all solids are suspended if sizes, densities, and shapes of all solids are the same. A slurry with a velocity less than $V_{M 2}$ would be in danger of plugging a pipeline. Thus, slurry transport is usually operated at or above the velocity of $V_{M 2}$ (Wasp et al. 1977). This velocity, $V_{M 2}$, is defined here as the pipeline critical velocity.

The velocity must also be large enough to produce a turbulent flow. Note that the existence of the solids generally tends to reduce the turbulence. Wasp et al. (1977) discuss how to determine the transition from a laminar flow to a turbulent flow. Wasp et al. (1977) and Liu (2003) discuss how to handle non-Newtonian slurry, e.g., a Bingham flow.

\subsubsection{Examples of Pipeline Critical Velocity Calculation Models}

There are many formulas and models to estimate the critical velocity, $V_{M 2}$. The following subsections give examples of critical velocity calculation models.

\section{Models for Coarse, Non-Cohesive Solids}

The following two example models may be applied to estimate critical velocity for coarse, non-cohesive solids. 
Durand Model (Durand 1952)

$$
V_{M 2}=F_{L}\left[2 g D\left(\frac{\rho_{S}}{\rho_{L}}-1\right)\right]^{\frac{1}{2}}
$$

where $\quad D=$ pipe diameter $(\mathrm{m})$

$F_{L}=$ dimensionless factor (a densimetric Froude Number)

$g=$ gravitational acceleration constant $\left(\mathrm{m} / \mathrm{s}^{2}\right)$

$V_{M 2}=$ critical velocity $(\mathrm{m} / \mathrm{s})$

$\rho_{\mathrm{L}}=$ liquid density $\left(\mathrm{kg} / \mathrm{m}^{3}\right)$

$\rho_{S}=$ solid density $\left(\mathrm{kg} / \mathrm{m}^{3}\right)$.

The coefficient $F_{L}$ is affected by the particle size and solid concentration as shown in Figure 2.7, where $C_{V}$ is the volume fraction of UDS in the flow (Wasp et al. 1977). This classical model was developed with sand and coal slurry with particle diameters of 440 to 2,000 $\mu \mathrm{m}$ in water. The good agreement between measured and computed critical velocity incorporated in the value of $F_{L}$ is shown in Figure 2.8 (Wasp et al. 1977).

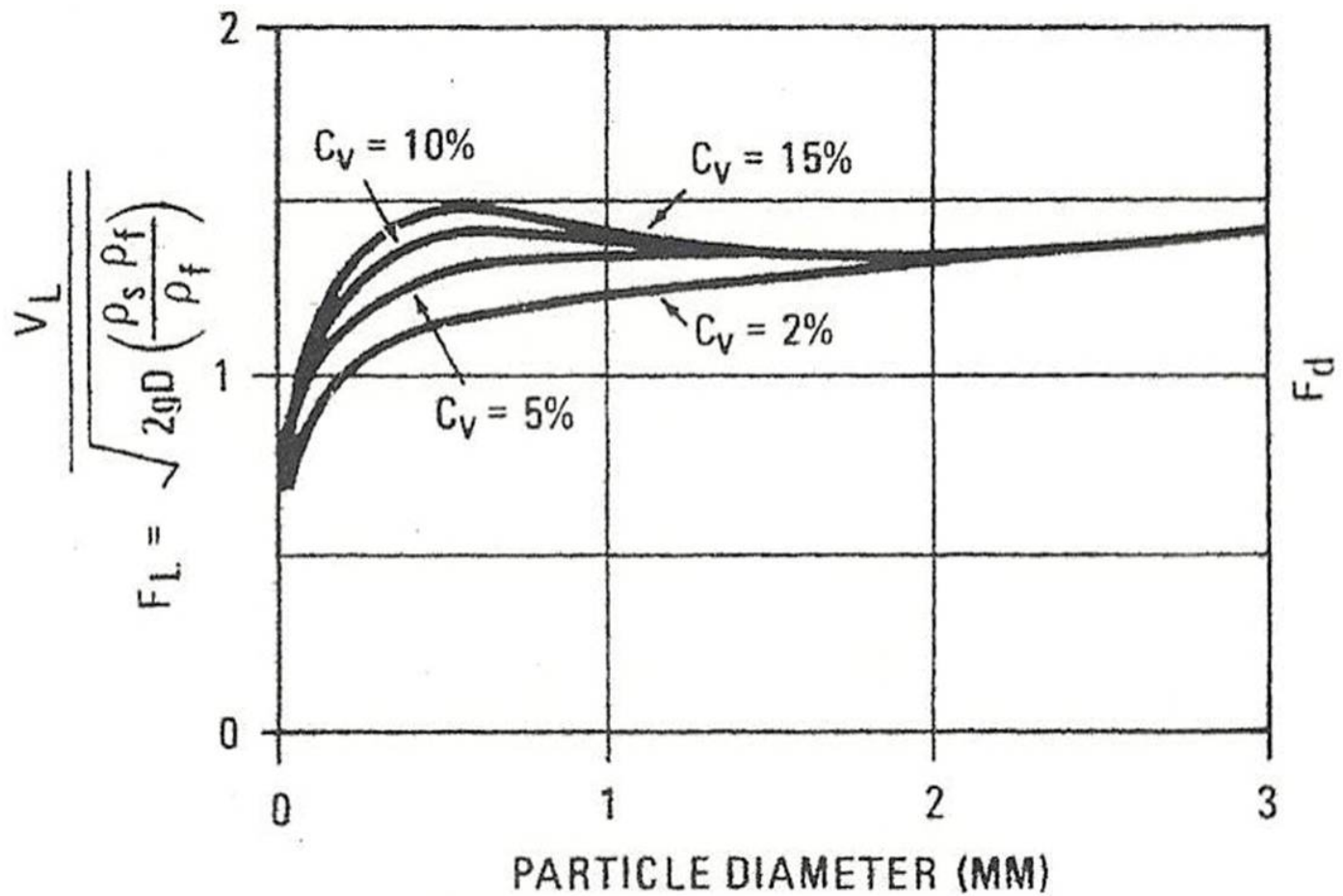

Figure 2.7. Parameter $F_{L}$ of the Durand Model (Wasp et al. 1977) 


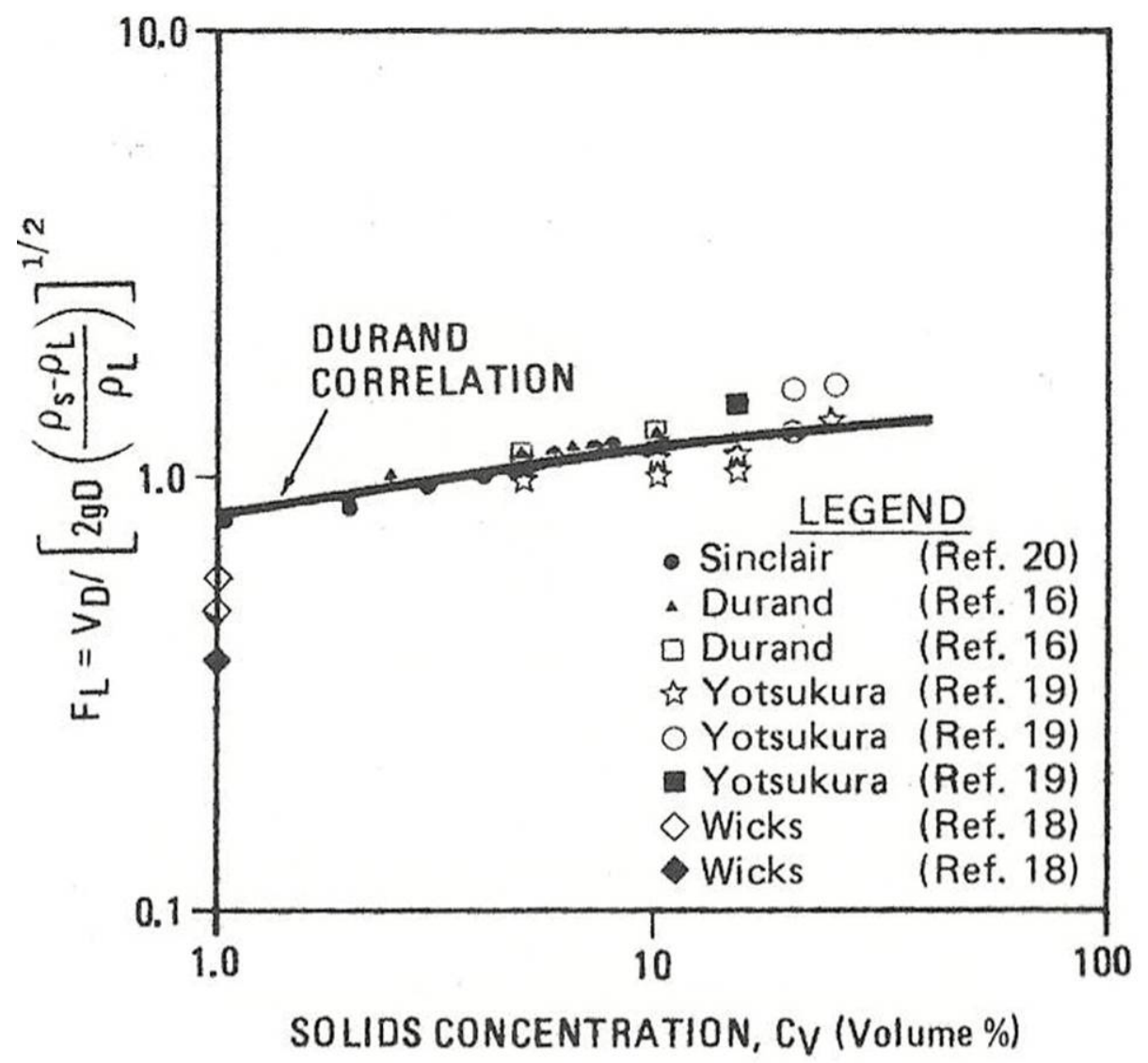

Figure 2.8. Comparison of Measured and Computed Critical Velocities Incorporated in the Value of $F_{L}$. (Wasp et al. 1977)

Oroskar-Turian Model (Oroskar and Turian 1980)

$$
\begin{gathered}
V_{M 2}=1.85 \sqrt{g d_{S}\left(\frac{\rho_{S}}{\rho_{L}}-1\right)} C_{V}^{0.1536}\left(1-C_{V}\right)^{0.3564}\left(\frac{d_{S}}{D}\right)^{-0.378}\left[\frac{D \rho_{L} \sqrt{g d_{S}\left(\frac{\rho_{S}}{\rho_{L}}-1\right)}}{\mu_{C}}\right]^{0.09} \chi^{0.30} \\
\chi=\frac{2}{\sqrt{\pi}}\left[\frac{2}{\sqrt{\pi}} \gamma \exp \left(-\frac{4 \gamma^{2}}{\pi}\right)+\int_{\gamma}^{\infty} \exp \left(-\frac{4 \gamma^{2}}{\pi}\right) d \gamma\right] \\
\gamma=\frac{V_{S H}}{V_{M 2}}
\end{gathered}
$$


where $\quad C_{V}=$ solid volume fraction

$d_{S}=$ particle size (median particle diameter, " $\left.\mathrm{d}_{50} "\right)(\mathrm{m})$

$V_{S H}=$ hindered settling velocity $(\mathrm{m} / \mathrm{s})$

$\chi=$ fraction of eddies having velocities equal to or greater than the settling velocity

$\mu_{C}=$ carrier fluid viscosity (Pa s).

Note that the viscosity in Equation (2.69) is that of a carrier fluid, which includes any uniformly suspended particles, and not the viscosity of the pure liquid. It is known that when different size particles are transported in a pipeline, some fine solids are vertically uniformly mixed and become a part of the carrier fluid (Wasp et al. 1963, 1977). Because the suspended particles make the carrier fluid denser and more viscous than the pure liquid, larger solids can be transported with less possibility of deposition.

Which slurry solids would become a part of a carrier fluid is a complex problem to determine, depending on many factors, e.g., liquid density, liquid viscosity, solid density and size, pipe diameter, pile flow velocity, solid concentration, slurry rheology, etc. Wasp et al. (1963) developed a method to determine which portion of solids becomes a part of the carrier.

The variation of $\chi$ as a function of $\gamma$ (Equation 2.70) is shown in Figure 2.9. The value of $\chi$ is about 0.96 for the slurry pipeline velocity of between $0.06 \mathrm{ft} / \mathrm{s}$ and $5.3 \mathrm{ft} / \mathrm{s}$ (Oroskar and Turian 1980).

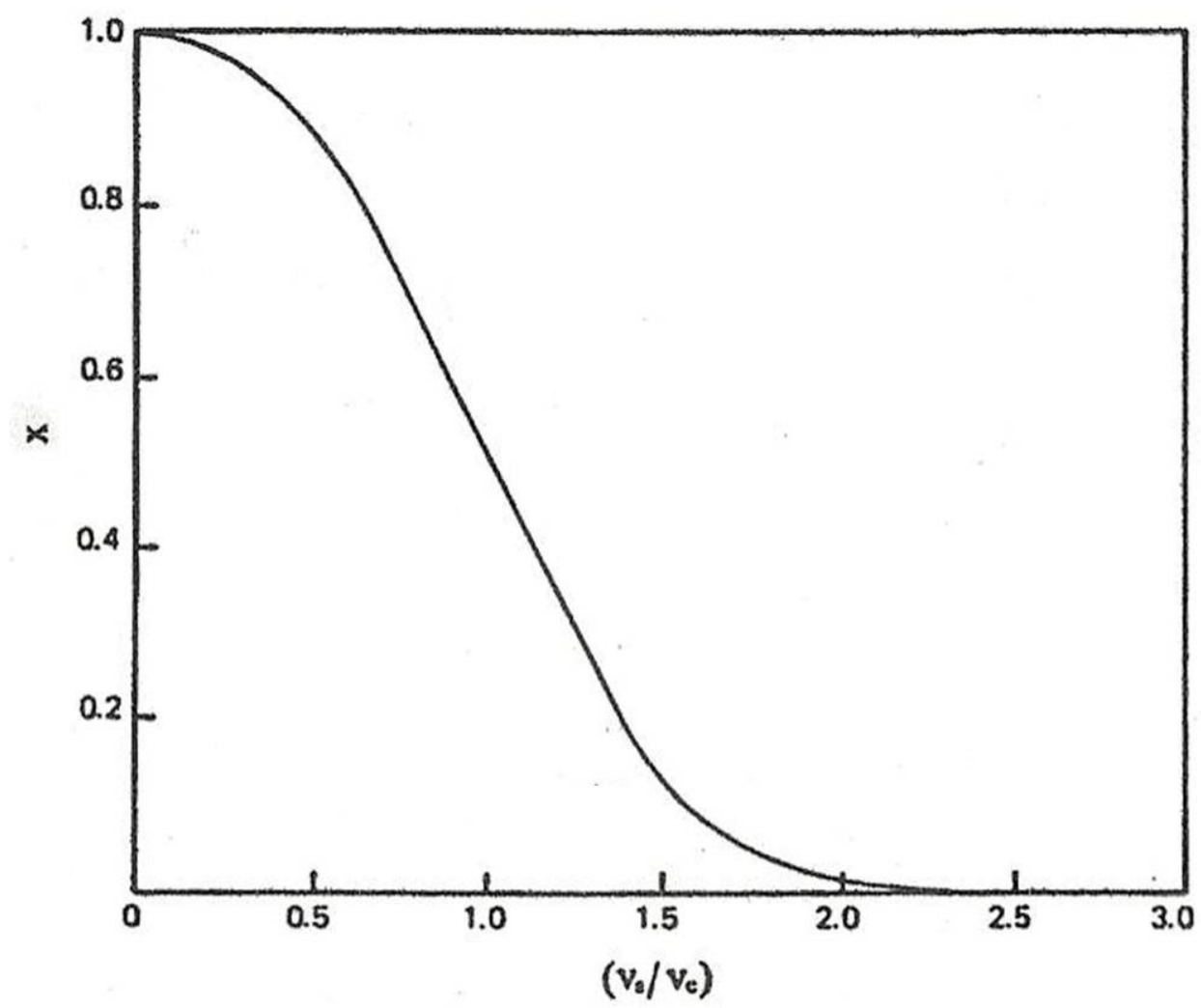

Figure 2.9. $\chi$ as a Function of the Ratio of $V_{S} / V_{C} . V_{S}$ is the slurry pipeline velocity and $V_{C}$ is the critical velocity. In this figure, $V_{C}=V_{M 2}$ (Oroskar and Turian 1980).

For slurry consisting of mixed size solids, the median diameter of the solids by volume, $d_{50}$, would be appropriate to use as the value of $d_{S}$ (Oroskar and Turian 1980). Beside this model, Turian developed 
many different models (Turian and Yuan 1977, Turian et al. 1987). Turian et al. (1987) states that a theoretical model developed by Oroskar and Turian (1980) and empirical models by Turian et al. (1987) have the best fit to the tested data. Oroskar and Turian (1980) reported that Equation (2.69) has a better overall fit with data than their theoretical model. The WTP design guide assigns Equation (2.69) as its slurry critical velocity model (Hall 2006).

Many slurry pipeline transport models have a particle diameter as one of the model parameters to determine the critical velocity. Some practitioners suggest the use of the median particle diameter, some others suggest different values, as large as a $95^{\text {th }}$ percentile value. Specific particle size selections also depend on the data that the model developers used to derive their critical velocity models. In some instances, a larger particle size is selected for design safety margins. In general, there is no universally accepted selection of the particle diameter for slurries with particles of various sizes.

\section{Models for Fine Solids}

The following three example models may be applied to estimate pipeline critical velocity for slurries of fine particles.

DG Thomas Model (Thomas 1962, Wasp and Slater 2004)

$$
\begin{gathered}
\frac{V_{M 2}}{u_{*}}=0.01\left(\frac{d_{S} u_{*} \rho_{C}}{\mu_{C}}\right)^{2.71} \\
u_{*}=\sqrt{\frac{\tau_{B}}{\rho_{m}}}
\end{gathered}
$$

where $u_{*}=$ friction velocity (or shear velocity) $(\mathrm{m} / \mathrm{s})$

$$
\begin{aligned}
\rho_{C} & =\text { density of carrier fluid }\left(\mathrm{kg} / \mathrm{m}^{3}\right) \\
\rho_{m} & =\text { density of slurry }\left(\mathrm{kg} / \mathrm{m}^{3}\right) \\
\tau_{\mathrm{B}} & =\text { wall shear stress }(\mathrm{Pa}) .
\end{aligned}
$$

This model is for fine particles. It was developed by experiments with ion exchange resins and glass beads with diameters of 0.75 to $66 \mu \mathrm{m}$ in water. Wasp et al. (1977) describe how to obtain the wall shear stress. In general, it is better to obtain the density and viscosity of the slurry by actual measurements than to estimate these values with mathematical formulas.

\section{$\underline{\text { AD Thomas Model (Thomas 1979) }}$}

$$
V_{M 3}=9.0\left[\frac{9.8 \mu_{C}\left(\frac{\rho_{S}}{\rho_{C}}-1\right)}{\rho_{C}}\right]^{0.37}\left(\frac{D \rho_{C}}{\mu_{C}}\right)^{0.11}
$$


This model is also for fine particles. Above this velocity of $V_{M 3}$, no stationary bed is formed, but there would be the moving bed in a pipeline. This model was based on experiments with 17 - and 26- $\mu \mathrm{m}$ silica sand in water and was derived from an equation similar to Equation (2.77) with some additional assumptions. This model uses S.I. units.

Wasp and Slater (Wasp and Slater 2004)

$$
V_{M 1}=0.18\left(\frac{\rho_{S}}{\rho_{C}}-1\right)^{0.5}\left(\frac{d_{95} \rho_{C} \sqrt{g D}}{\mu_{C}}\right)^{0.22} \exp \left(4.34 C_{V}\right)
$$

where $d_{95}$ is the particle diameter which is greater than the diameter of $95 \%$ of the particles by volume.

This model is for mixtures of mostly fine solids with some coarse particles. Note that for slurries of very fine particles, $V_{M 1}$ and $V_{M 2}$ values are almost the same. This model was developed with data for coal, copper, sulfur, phosphate magnetite having $62 \leq d_{95} \leq 850 \mu \mathrm{m}$ in water, and oil. The right-hand side of this equation is non-dimensional, except the coefficient, 0.18. Thus, as Wasp and Slater (2004) use S.I. units of $(\mathrm{m} / \mathrm{s})$, the units of the coefficient are likewise $(\mathrm{m} / \mathrm{s})$.

Poloski Model (Poloski et al. 2009b, c)

$$
F_{R}=0.59 A_{R}^{0.15}
$$

where,

$$
\begin{aligned}
& \text { Densimetric Froude Number, } F_{R}=\frac{V_{M 2}}{\sqrt{g D\left(\frac{\rho_{S}}{\rho_{L}}-1\right)}} \\
& \qquad A_{R}=\frac{4}{3} \frac{g d_{S}{ }^{3}\left(\frac{\rho_{S}}{\rho_{L}}-1\right) \rho_{L}{ }^{2}}{\mu_{L}{ }^{2}}
\end{aligned}
$$

This model was developed for slurries with $A_{R}<80$ comprising glass beads, alumina, and stainless steel in water.

\section{Summary of Models}

Equations (2.68) through (2.78) are examples of models used to determine the critical velocity of slurry pipe flow. These are summarized in Table 2.1.

As indicated in Section 2.3.2.1, the critical velocity must be such that the resulting slurry flow is turbulent and overcomes the resistance of the slurry, e.g., the yield stress of a Bingham plastic. 
Table 2.1. Summary of Some Examples of Critical Velocity Models

\begin{tabular}{|c|c|c|c|c|}
\hline \multirow{2}{*}{ Model } & Calculates & \multicolumn{2}{|c|}{ Solids } & \multirow{2}{*}{ Liquid } \\
\hline & Comments & Type & Size, $\mu \mathrm{m}$ & \\
\hline \multirow[b]{2}{*}{ Durand (1952) } & Velocity & Sand, Coal & $440 \sim 2000$ & Water \\
\hline & \multicolumn{4}{|c|}{$\begin{array}{l}\text { 1. Large solid sizes } \\
\text { 2. Narrow solid size distribution } \\
\text { 3. Slurry of the single solid density }\end{array}$} \\
\hline \multirow[b]{2}{*}{$\begin{array}{l}\text { Oroskar and Turian } \\
\qquad(1980)\end{array}$} & Velocity & Sand, Coal, Iron & $100 \sim 2040$ & $\begin{array}{c}\text { Water, Kerosene, } \\
\text { Ethylene glycol }\end{array}$ \\
\hline & \multicolumn{4}{|c|}{$\begin{array}{l}\text { 1. Extension of the Durand Model } \\
\text { 2. Theory based on the ratio of solid settling velocity to upward turbulent intensity } \\
\text { 3. Large solids } \\
\text { 4. Narrow solid size distribution } \\
\text { 5. Slurry of the single solid density }\end{array}$} \\
\hline \multirow{2}{*}{ DG Thomas (1962) } & Friction velocit & $\begin{array}{c}\text { Glass bead, } \\
\text { Ion exchange resins }\end{array}$ & $\mathrm{D}_{50}=0.75 \sim 66$ & Water \\
\hline & \multicolumn{4}{|c|}{$\begin{array}{l}\text { 1. Theory based on the ratio of solid settling velocity to upward turbulent intensity } \\
\text { 2. Fine particles }\end{array}$} \\
\hline \multirow[b]{2}{*}{ AD Thomas (1979) } & Velocity & Silica Sand & $\mathrm{D}_{50}=17,26$ & Water \\
\hline & \multicolumn{4}{|c|}{$\begin{array}{l}\text { 1. Theory based on the Sliding Bed concept and the ratio of settling velocity to upward turbulent } \\
\text { intensity } \\
\text { 2. Fine particles } \\
\text { 3. Wide size distributions } \\
\text { 4. Slurry of the single solid density } \\
\text { 5. Converts the deposition friction velocity to the deposition flow velocity }\end{array}$} \\
\hline \multirow[b]{2}{*}{$\begin{array}{l}\text { Wasp and Slatter } \\
\text { (2004) }\end{array}$} & Velocity & $\begin{array}{l}\text { Coal, Copper, Sulfur, } \\
\text { Phosphate Magnetite }\end{array}$ & $\mathrm{D}_{95}=62 \sim 850$ & Water, Oil \\
\hline & \multicolumn{4}{|c|}{$\begin{array}{l}\text { 1. Extension of the Durand Model } \\
\text { 2. Theory based on the ratio of solid settling velocity to upward turbulent intensity } \\
\text { 3. Fine particles } \\
\text { 3. Wide size distributions } \\
\text { 4. Slurry of the single solid density }\end{array}$} \\
\hline \multirow[b]{2}{*}{ Poloski (2009c) } & Velocity & $\begin{array}{l}\text { Glass bead, Alumina, } \\
\text { Stainless steel }\end{array}$ & $\mathrm{D}_{50}=6.2 \sim 140.3$ & Water \\
\hline & \multicolumn{4}{|c|}{$\begin{array}{l}\text { 1. Extension of the Shook et al. (2002) model } \\
\text { 2. Wide solid distribution } \\
\text { 3. Each slurry of single solid density }\end{array}$} \\
\hline
\end{tabular}

\subsubsection{Waste Properties That Affect Pipeline Critical Velocity}

For coarse non-cohesive solids, waste properties needed to use Equations (2.68) and (2.69) are the density and viscosity of liquid (or carrier fluid), the size and density of solids, and the solids settling velocity. With Equations (2.68) and (2.69), one may obtain the following equation for the order of magnitude of these waste properties:

$$
V_{M 2} \propto \rho_{S}^{\alpha} \rho_{L}^{\beta} d_{S}^{\gamma} \mu_{C}^{-0.09} \exp \left(V_{S}\right) C_{V}^{\delta} D^{\varepsilon}
$$

assuming that $F_{L}$ in the Durand model (Equation 2.67) may be expressed as

$$
F_{L} \propto d_{S}^{\phi} C_{V}^{\xi}
$$


where

$$
\begin{aligned}
\alpha & =0.5 \sim 0.545 \\
\beta & =0.455 \sim 0.5 \\
\gamma & =0.167 \sim \phi \\
\delta & =0.186 \sim 0.51 \sim \xi \\
\varepsilon & =0.33 \sim 0.468 \\
\phi & =\text { positive constant } \\
\xi & =\text { positive constant. }
\end{aligned}
$$

Equation (2.79) implies that key waste properties are, in order of their importance, densities of solid and liquid, solid diameter, and liquid or carrier fluid viscosity. The settling velocity also affects the critical velocity. Its relative importance with respect to these three key waste properties is discussed in Section 2.1.1.

For fine solids, Equation (2.74) reveals that the critical velocity to avoid formation of a stationary bed is

$$
V_{M 3} \propto \rho_{C}{ }^{-1.26} \rho_{S}^{0.37} \mu_{C}^{0.15} D^{0.11}
$$

Thus, the critical velocity above which no stationary bed exists is affected by carrier fluid density, solids density, carrier fluid viscosity, and pipe diameter, in that order of importance.

Because they depend on the amount of suspended solids, the carrier fluid density and viscosity are affected by many parameters, e.g., liquid density, liquid viscosity, solid density and size, pipe diameter, pipe flow velocity, solids concentration, etc. Thus, for a given slurry and specific operating conditions, it is best to determine carrier fluid properties by running a model such as given by Wasp et al. (1963) and supporting experiments. To gain a preliminary idea of carrier fluid properties for a given condition, one might arbitrarily assume that solids with diameters less than say, $10 \mu \mathrm{m}$, would be a part of a carrier fluid at several ft/s slurry flow velocity. However, this preliminary assumption needs to be tested by both modeling and experimental measurements.

For fine solids, Equation (2.75) indicates that the critical velocity to achieve the homogeneous solid concentration is

$$
V_{M 1} \propto \rho_{S}^{0.5} \rho_{C}^{-0.28} d_{95}^{0.22} \mu_{C}^{-0.22} \exp \left(C_{V}\right)
$$

The critical velocity for homogeneous vertical distribution of solids at a given solids concentration depends on solids density, carrier fluid density, and solids size and carrier fluid viscosity, in that order of importance. The key waste properties are solids density and solids size, in that order of importance. The carrier fluid density and viscosity are also important, so waste properties affecting the carrier fluid also need to be assessed.

Equation (2.76) indicates that the critical velocity is

$$
V_{M 2} \propto\left(\rho_{S}-\rho_{L}\right)^{0.65} d_{S}^{0.45} \rho_{L}^{-0.35} \mu_{L}^{-0.3}
$$


Thus, the key waste properties are the density difference between the solid and liquid, solid size, and density and viscosity of the liquid.

\subsubsection{Pipeline Erosion}

Wall surface erosion is a process in which a part of the wall material is removed by solids particle impingement on a wall surface. Two types of erosion processes are considered: 1) breaking loose pieces of surface material due to repeated surface deformation during collisions between particles and a surface, and 2) cutting a surface material due to impinging particles (Bitter 1963a, b).

To guide the design and maintenance of pipeline systems, a large number of models have been developed to predict wall erosion (Meng and Ludema 1995). However, the actual wall erosion processes are complex, and models to provide accurate predictions of wall surface erosion are still under development. The following subsection discusses several models that estimate wall surface erosion by jet and pipe flow.

\subsubsection{Examples of Surface Erosion Calculation Models}

Gupta et al. (1995) studied the effects of velocity, solids concentration, and particle size on erosion wear around the circumference of a horizontal mild steel pipeline conveying slurries of tailing materials. They derived the following reasonably accurate empirical correlation for the wear rate prediction of multi-sized particulate slurries:

$$
E_{\text {pipe }}=0.223 C_{W}^{0.556} d_{S}^{0.344} U^{2.148}
$$

where $E_{\text {pipe }}=$ erosion rate of horizontal mild steel straight pipe circumference ( $\mathrm{mm} /$ year)

$$
\begin{aligned}
U & =\text { slurry velocity }(\mathrm{m} / \mathrm{s}) \\
d_{S} & =\text { solids particle weighted-mean diameter }(\mathrm{m})
\end{aligned}
$$

$C_{W}=$ solids concentration (wt\%).

Gupta et al. (1995) reported that Equation (2.84) has an uncertainty margin of $\pm 14 \%$.

Wood (1999) obtained empirical correlations to predict the extent of carbon steel American Iron and Steel Institute (AISI) 1020 substrate erosion with $30^{\circ}$ and $90^{\circ}$ impingement angles of $2.1 \mathrm{wt} \%$ concentration sub-angular quart sand slurry flow as

$$
\begin{gathered}
E_{30^{\circ}}=17.711 \rho_{S}^{1.9812} r_{S}^{5.944} U^{3.962} \\
E_{90^{\circ}}=2.345 \rho_{S}^{1.169} r_{S}^{3.507} U^{2.338}
\end{gathered}
$$


where $E_{30^{\circ}}=$ erosion rate for with $30^{\circ}$ impingement angle (units of $\mu \mathrm{m}^{3} /$ solid impact, where solid impact $=$ (solids volumetric flow rate $)($ time $) /($ particle volume $)$

$E_{90^{\circ}}=$ erosion rate for with $90^{\circ}$ impingement angle ( $\mu \mathrm{m}^{3} /$ solid impact)

$U=$ flow velocity $(\mathrm{m} / \mathrm{s})$

$\rho_{S}=$ solids particle density $\left(\mathrm{kg} / \mathrm{m}^{3}\right)$

$r_{S}=$ solids particle radius $(\mathrm{cm})$.

\subsubsection{Waste Properties That Affect Surface Erosion}

The pipeline systems consist of pipes, pumps, valves, and fittings. Equation (2.84) through Equation (2.86) may be used to estimate the wall surface erosion of these components to the extent that the Hanford waste properties resemble those of tailing materials.

It is seen from Equation (2.85) that the erosion rate of mild steel straight pipe depends on slurry concentration, solids particle diameter, and slurry velocity. Velocity is the dominant factor, with the largest exponent of 2.148. The solids particle mean diameter is the only waste property in Equation (2.84). Thus, this equation implies that the straight pipe wall erosion depends only on the solids particle mean diameter as

$$
E_{\text {pipe }} \propto d_{S}^{0.344}
$$

Haugen et al. (1995) reported that the maximum erosion was obtained at the $30^{\circ}$ impingement angle of angular sand particles in an air stream. Therefore, Equation (2.85) is expected to provide conservative erosion prediction of pumps, valves, and fittings of a flow system. However, from Equation (2.85) and Equation (2.86), it is pointed out that the flow with $30^{\circ}$ particle impingement angle produces higher erosion than that of $90^{\circ}$ particle impingement angle only for the particle kinetic energy larger than $0.2 \mu \mathrm{J}$ (see Wood 1999). Therefore, both Equations (2.85) and (2.86) need to be used for conservative erosion estimations of pumps, valves, and fittings.

The solids particle density, solids particle radius, and slurry velocity are the parameters for the erosion caused by the slurry flow with $30^{\circ}$ and $90^{\circ}$ particle impingement angles against the wall of a pipeline system. The particle radius is the dominant factor for the erosion of this system because it has the largest exponent of 5.944 for a $30^{\circ}$ impingement angle and 3.507 for a $90^{\circ}$ impingement angle in Equations (2.85) and (2.86), respectively. From these equations, it is also found that the solids particle density and radius affect erosion of pumps and valves, giving the expressions of

$$
\begin{aligned}
& E_{30^{\circ}} \propto \rho_{S}^{1.9812} r_{S}^{5.944} \\
& E_{90^{\circ}} \propto \rho_{S}^{1.169} r_{S}^{3.507}
\end{aligned}
$$

Similar to wall erosion due to PJM operation as discussed in Section 2.2.5, while not apparent from these correlations, particle hardness is also expected to influence surface erosion. 


\subsection{Waste Processing}

The WTP will process (i.e., pretreat) waste received from tank farms to reduce non-radioactive species in the high-level glass stream that limit the waste loading of the glass. Specifically, $\mathrm{Al}$ and $\mathrm{Cr}$ sludge solids need to be dissolved and removed from the high-level stream to allow greater amounts of waste to be added to the high-level glass. Filtration will then be used to concentrate and wash the remaining solids (removing the dissolved $\mathrm{Al}$ and $\mathrm{Cr}$ as well as certain salts). Section 2.4.1 considers available filtration models and the waste characterization data needed by the models. Section 2.4.2 briefly addresses the thermodynamic models used to determine which solids may dissolve under a given set of process conditions.

\subsubsection{Solids Filtration}

The WTP plans to use filtration in pretreating waste to increase the solids concentration in a slurry by removing liquid. The driving force to move liquid through a filter is the pressure difference through a filter.

\subsubsection{Examples of Solids Filtration Calculation Models}

Flow through a filter is similar to groundwater flow through porous media. Groundwater flow is usually described by Darcy’s Law (Hemond and Fechner 1994):

$$
q=-K \frac{d h}{d x}=-\frac{k}{\mu_{L}} \frac{d p}{d x}
$$

where $h=$ hydraulic head

$K=$ hydraulic conductivity

$k=$ permeability

$p=$ pressure head

$q=$ specific discharge

$x=$ groundwater flow direction.

The specific discharge (Darcy flux) is the amount of liquid flowing across a unit area perpendicular to the flow direction per unit time.

A governing equation for filtration is (Green and Perry 2007)

$$
\frac{1}{A} \frac{d V}{d t}=\frac{P}{\mu_{L}\left[\lambda\left(\frac{w V}{A}+r\right)\right]}
$$

where $A=$ filtering surface area

$r=$ filter resistance including possible solids built-up on a filter

$t=$ time

$V=$ accumulated solids volume 
$w=$ weight of solids per unit volume of filtrate

$P=$ total pressure across a filter system

$\lambda=$ constant related to solids size.

\subsubsection{Relevant Waste Properties for Solids Filtration}

Darcy’s Law, Equation (2.90), shows that filter flux depends on liquid viscosity. Because fine solids may go through a filter, the solids size is also a relevant waste property to assess waste solids filtration. Equation (2.91) also indicates that liquid viscosity and solids size are the primary waste properties affecting filtration.

\subsubsection{Solids Dissolution}

The rate and extent to which waste solids dissolve are important to retrieval and processing operations. Chemical thermodynamics can be applied to determine the possible extent of dissolution, and two chemical equilibrium models have been used to predict Hanford waste solubilities. Establishing the extent of solids dissolution is important to leaching and washing operations in the WTP Pretreatment Facility. Solids dissolution may also occur when one waste is mixed with a second waste in a tank or when water or chemicals are added. This includes adding water to low-activity waste in DSTs to reduce the concentration of solids (Herting 1997, Onishi et al. 1999, 2003). It is not practical to predict solids dissolution in the very complex Hanford wastes without using chemical simulation computer codes to simulate all relevant chemical reactions.

\subsubsection{Waste Properties That Affect Solids Dissolution}

Relevant waste properties for chemical dissolution are aqueous species, gas and solid phases, and the concentration for each of these chemical species. The waste temperature is also important in many cases.

\subsection{Summary of Physical and Rheological Parameters}

Available models and correlations for waste storage, mobilization, transport, and processing were reviewed in Sections 2.1, 2.2, 2.3 and 2.4, respectively, to identify the waste parameters important to quantifying the phenomena. Table 2.2 summarizes the key waste properties that are considered to be important for these phenomena. The order of significance of waste properties was derived through the sensitivity of the models to the waste properties. The numbers in the table indicate their expected significance in order of their importance with 1 indicating the most important and 5 the least important.

As stated previously, the order of significance of the waste properties in Table 2.2 must be used in the context that some waste properties vary much more than some other waste properties do. For example, the solids size can vary several orders of magnitude (Section 3.2.5), while both the liquid viscosity (Section 3.2.2) and solid density (Sections 3.2.3 and 3.3) can vary by one order of magnitude, and the liquid density can increase by $50 \%$ from water to salt-saturated liquid (Section 3.2 .1 ). Thus, the order of importance of waste properties must also consider possible variations of these waste properties themselves. 
Table 2.2. Important Waste Properties

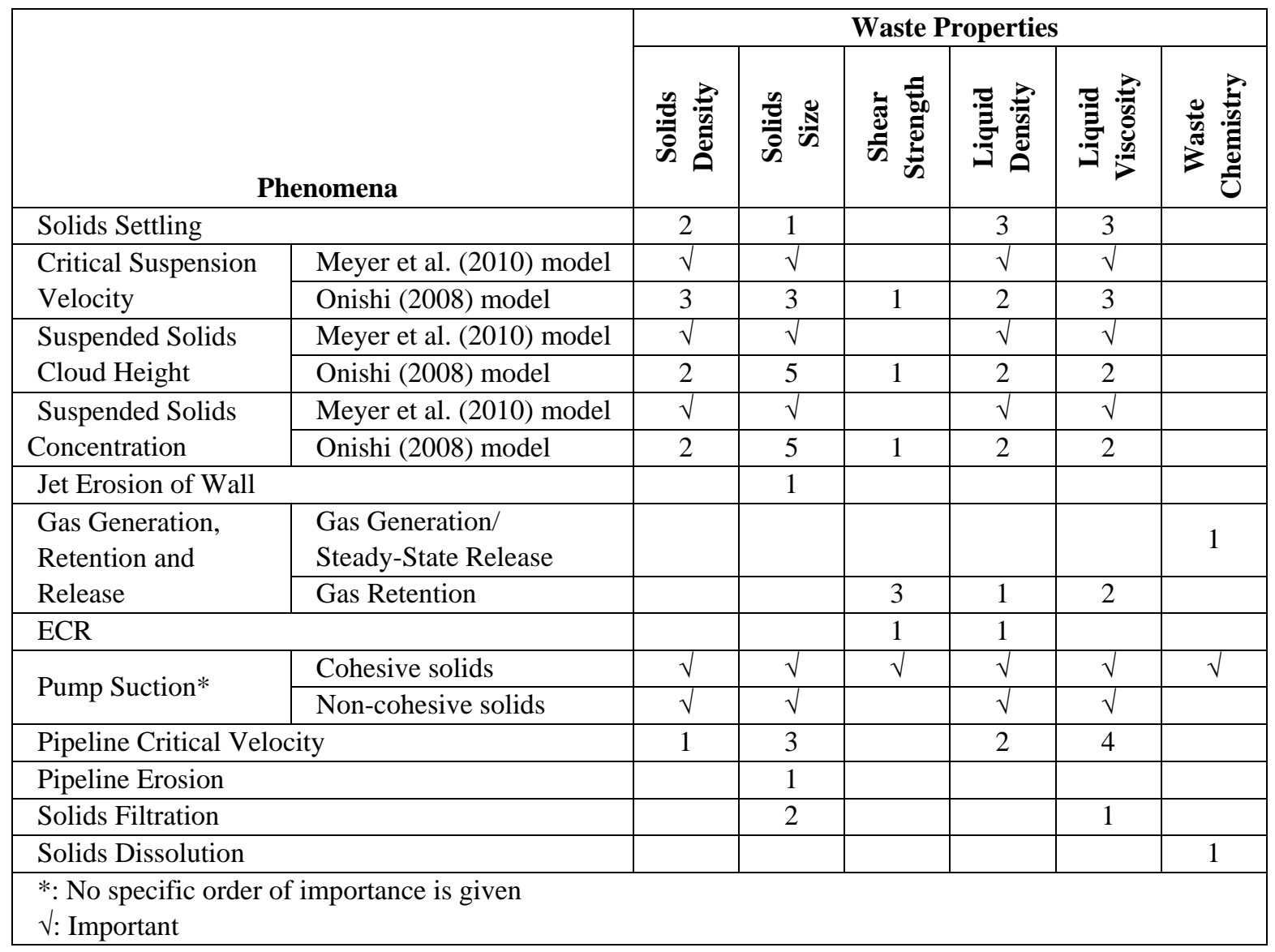




\subsection{Waste Performance Data}

Hanford waste characterization data are used to engineer safe storage, retrieval, transport, and processing operations. The specific waste parameters for evaluating process operations are identified in Section 2.0 by examining relevant example mathematical models of the phenomena.

The parameter characterizations of the as-stored Hanford waste, including both the liquid and solid phases, are presented in this section. Variations of these waste properties by tank and waste type are included. Data gaps with respect to 1) unquantified information (e.g., identification of amorphous solid phases, Section 3.2.3) and 2) waste volume represented for characterization of a specific parameter, are listed. Data allowing the evaluation of potential property changes due to retrieval and staging activities are provided. The effect of pretreatment processes on specific properties is considered in Section 4.0.

The data are from numerous and varied sources as referenced. It is assumed that the data from these references have been fully reviewed and documented in accordance with the analysts' QA programs.

\subsection{Waste Parameter Basis}

The Hanford waste parameters and data gaps are presented on bases of 1) tank, 2) waste type (general, i.e., sludge or saltcake, and specific), and 3) composite where possible and meaningful. Besides providing information for a specific tank, Basis 1 can be useful to investigate waste feed streams. Basis 2 provides an intermediary basis and allows for meaningful determination of data gaps, and Basis 3 is useful for broad considerations of waste properties.

The as-stored waste properties are presented for the characterizations made on various subsets of the 177 large underground storage tanks on the Hanford Site. The 177 storage tanks include 149 SSTs and 28 DSTs.

The general waste types, sludge and saltcake, are classified as such, based on the relative concentrations of soluble and insoluble UDS ${ }^{(a)}$. As specified in Weber (2009), a tank is classified as sludge if at least $75 \mathrm{vol} \%$ is sludge solids (insoluble UDS), and similarly, saltcake if it is at least 75 vol\% saltcake/salt slurry solids (soluble UDS).

Waste type definitions have evolved over time as additional information on the composition of wastes transferred to the Hanford tanks has been identified. The latest modifications were included in Revision 5 of the Hanford Defined Waste Model (Higley and Place 2004). As described in Section 3.2.3, the UDS composition information, following Wells et al. (2007) and Poloski et al. (2007), is based on the May 2002 Best Basis Inventories (BBIs). The majority of waste sampling was conducted before 2002, and thus the parameter characterizations from these samples are consistent with the $2002 \mathrm{BBI}$. Some tanks' wastes have been diluted or leached since 2002, changing both the solid and liquid compositions of the waste remaining in the tanks.

(a) UDS; undissolved solids. Those solids, whether soluble or insoluble, that are present as a solid phase and are not dissolved in the liquid phase of the waste. The UDS inventory (mass and volume) in each tank was determined by thermodynamic modeling that used 2002 Best Basis Inventory (BBI) data and by adjustments to modeling results, as described in Section 3.2.3.2. The UDS inventory does not include interstitial liquid. 
The primary and secondary waste types as specified in the 2002 BBI by volume of UDS (Section 3.2.3) for the individual tanks are provided in Table 3.1. Approximately $80 \%$ of the tanks have at least $95 \%$ of their UDS volume inventory characterized by the listed first and second most prevalent waste types. The waste type acronyms of Table 3.1 are defined in Table 3.2 as adopted from Agnew (1995), Meacham (2003), and Higley and Place (2004).

Table 3.1. Primary and Secondary Waste Types by Tank, 2002 BBI, UDS Volume Basis

\begin{tabular}{|c|c|c|c|c|}
\hline Tank & $\begin{array}{c}\text { Primary Waste } \\
\text { Type }^{1}\end{array}$ & $\begin{array}{c}\text { Volume } \\
\text { Fraction } \\
\text { Primary } \\
\text { Waste Type }^{3}\end{array}$ & $\begin{array}{c}\text { Secondary Waste } \\
\text { Type }^{2}\end{array}$ & $\begin{array}{c}\text { Volume } \\
\text { Fraction } \\
\text { Secondary } \\
\text { Waste Type }^{3}\end{array}$ \\
\hline A-101 & A1 saltcake & 0.99 & P2 sludge & 0.01 \\
\hline A-102 & A1 saltcake & 1.00 & $N / A^{4}$ & N/A \\
\hline A-103 & A1 saltcake & 0.99 & AR sludge & 0.01 \\
\hline A-104 & AR sludge & 0.96 & P1 sludge & 0.04 \\
\hline A-105 & P2 sludge & 1.00 & N/A & N/A \\
\hline A-106 & A1 saltcake & 0.37 & SRR sludge & 0.37 \\
\hline AN-101 & Liquid $^{5}$ & N/A & N/A & N/A \\
\hline AN-102 & A2 saltcake & 1.00 & N/A & N/A \\
\hline AN-103 & A2 saltcake & 1.00 & N/A & N/A \\
\hline AN-104 & A2 saltcake & 1.00 & N/A & N/A \\
\hline AN-105 & A2 saltcake & 1.00 & N/A & N/A \\
\hline AN-106 & A1 saltcake & 1.00 & N/A & N/A \\
\hline AN-107 & A2 saltcake & 1.00 & N/A & N/A \\
\hline AP-101 & Liquid & N/A & N/A & N/A \\
\hline AP-102 & Liquid & N/A & N/A & N/A \\
\hline AP-103 & Liquid & N/A & N/A & N/A \\
\hline AP-104 & Liquid & N/A & N/A & N/A \\
\hline AP-105 & A2 saltcake & 1.00 & N/A & N/A \\
\hline AP-106 & Liquid & N/A & N/A & N/A \\
\hline AP-107 & Liquid & N/A & N/A & N/A \\
\hline AP-108 & Liquid & N/A & N/A & N/A \\
\hline AW-101 & A2 saltcake & 1.00 & N/A & N/A \\
\hline AW-102 & CWP1 sludge & 1.00 & N/A & N/A \\
\hline AW-103 & CWZr2 sludge & 0.87 & A1 saltcake & 0.13 \\
\hline AW-104 & A2 saltcake & 0.71 & PL2 sludge & 0.29 \\
\hline AW-105 & CWZr2 sludge & 0.92 & PL2 sludge & 0.08 \\
\hline AW-106 & A2 saltcake & 1.00 & N/A & N/A \\
\hline AX-101 & A1 saltcake & 0.99 & SRR sludge & 0.01 \\
\hline AX-102 & A1 saltcake & 0.80 & BL sludge & 0.20 \\
\hline AX-103 & A1 saltcake & 0.90 & P2 sludge & 0.10 \\
\hline AX-104 & P2 sludge & 1.00 & N/A & N/A \\
\hline AY-101 & Unidentified sludge & 1.00 & N/A & N/A \\
\hline AY-102 & BL sludge & 1.00 & N/A & N/A \\
\hline AZ-101 & P3 sludge & 1.00 & N/A & N/A \\
\hline AZ-102 & P3 sludge & 1.00 & N/A & N/A \\
\hline
\end{tabular}


Table 3.1. (contd)

\begin{tabular}{|c|c|c|c|c|}
\hline Tank & $\begin{array}{c}\text { Primary Waste } \\
\text { Type }^{1}\end{array}$ & $\begin{array}{c}\text { Volume } \\
\text { Fraction } \\
\text { Primary } \\
\text { Waste Type }^{3}\end{array}$ & $\begin{array}{c}\text { Secondary Waste } \\
\text { Type }^{2}\end{array}$ & $\begin{array}{c}\text { Volume } \\
\text { Fraction } \\
\text { Secondary } \\
\text { Waste Type }^{3}\end{array}$ \\
\hline B-101 & B saltcake & 0.68 & BL sludge & 0.23 \\
\hline B-102 & B saltcake & 1.00 & N/A & N/A \\
\hline B-103 & B saltcake & 0.97 & $\begin{array}{l}\text { Mixed waste (MW) } \\
\text { sludge }\end{array}$ & 0.03 \\
\hline B-104 & 2C sludge & 0.51 & 1C sludge & 0.34 \\
\hline B-105 & B saltcake & 0.90 & 2C sludge & 0.06 \\
\hline B-106 & TBP sludge & 0.65 & 1C sludge & 0.35 \\
\hline B-107 & 1C sludge & 0.53 & B saltcake & 0.39 \\
\hline B-108 & B saltcake & 0.64 & CWP2 sludge & 0.36 \\
\hline B-109 & B saltcake & 0.53 & CWP2 sludge & 0.47 \\
\hline B-110 & 2C sludge & 0.99 & P2 sludge & 0.01 \\
\hline B-111 & 2C sludge & 0.89 & P2 sludge & 0.11 \\
\hline B-112 & 2C sludge & 0.53 & BY saltcake & 0.47 \\
\hline B-201 & 224 Pre-1949 sludge & 1.00 & N/A & N/A \\
\hline B-202 & 224 Post-1949 sludge & 1.00 & N/A & N/A \\
\hline B-203 & 224 Post-1949 sludge & 1.00 & N/A & N/A \\
\hline B-204 & 224 Post-1949 sludge & 1.00 & N/A & N/A \\
\hline BX-101 & CWP2 sludge & 0.74 & TBP sludge & 0.26 \\
\hline BX-102 & DE sludge & 0.61 & CWP2 sludge & 0.39 \\
\hline $\mathrm{BX}-103$ & CWP2 sludge & 0.67 & TBP sludge & 0.33 \\
\hline BX-104 & CWR1 sludge & 0.44 & MW sludge & 0.42 \\
\hline BX-105 & CWP1 sludge & 0.58 & TBP sludge & 0.29 \\
\hline BX-106 & CWP1 sludge & 0.50 & TBP sludge & 0.50 \\
\hline BX-107 & 1C sludge & 1.00 & N/A & N/A \\
\hline BX-108 & TBP sludge & 0.68 & 1C sludge & 0.32 \\
\hline BX-109 & TBP sludge & 1.00 & N/A & N/A \\
\hline $\mathrm{BX}-110$ & BY saltcake & 0.62 & A2 saltcake & 0.38 \\
\hline BX-111 & BY saltcake & 0.81 & 1C sludge & 0.19 \\
\hline BX-112 & 1C sludge & 1.00 & N/A & N/A \\
\hline BY-101 & BY saltcake & 0.89 & TFeCN sludge & 0.11 \\
\hline BY-102 & BY saltcake & 1.00 & N/A & N/A \\
\hline BY-103 & BY saltcake & 0.98 & CWP2 sludge & 0.02 \\
\hline BY-104 & BY saltcake & 0.86 & PFeCN sludge & 0.14 \\
\hline BY-105 & BY saltcake & 0.89 & PFeCN sludge & 0.09 \\
\hline BY-106 & BY saltcake & 0.93 & PFeCN sludge & 0.07 \\
\hline BY-107 & BY saltcake & 0.94 & PFeCN sludge & 0.06 \\
\hline BY-108 & BY saltcake & 0.80 & PFeCN sludge & 0.20 \\
\hline BY-109 & BY saltcake & 0.90 & CWP2 sludge & 0.10 \\
\hline BY-110 & BY saltcake & 0.88 & PFeCN sludge & 0.12 \\
\hline BY-111 & BY saltcake & 1.00 & N/A & N/A \\
\hline BY-112 & BY saltcake & 0.99 & MW sludge & 0.01 \\
\hline C-101 & CWP1 sludge & 0.62 & TBP sludge & 0.38 \\
\hline C-102 & CWP2 sludge & 0.71 & CWP1 sludge & 0.10 \\
\hline
\end{tabular}


Table 3.1. (contd)

\begin{tabular}{|c|c|c|c|c|}
\hline Tank & $\begin{array}{c}\text { Primary Waste } \\
\text { Type }^{1}\end{array}$ & $\begin{array}{c}\text { Volume } \\
\text { Fraction } \\
\text { Primary } \\
\text { Waste Type }^{3}\end{array}$ & $\begin{array}{c}\text { Secondary Waste } \\
\text { Type }^{2}\end{array}$ & $\begin{array}{c}\text { Volume } \\
\text { Fraction } \\
\text { Secondary } \\
\text { Waste Type }^{3}\end{array}$ \\
\hline C-103 & CWP1 sludge & 0.60 & AR sludge & 0.40 \\
\hline C-104 & CWP1 sludge & 0.34 & CWP2 sludge & 0.23 \\
\hline C-105 & CWP1 sludge & 0.90 & TBP sludge & 0.10 \\
\hline C-106 & AR sludge & 1.00 & N/A & N/A \\
\hline C-107 & 1C sludge & 0.54 & SRR sludge & 0.36 \\
\hline C-108 & 1C sludge & 0.44 & TBP sludge & 0.38 \\
\hline C-109 & TFeCN sludge & 0.38 & CWP1 sludge & 0.36 \\
\hline $\mathrm{C}-110$ & 1C sludge & 1.00 & N/A & N/A \\
\hline C-111 & TFeCN sludge & 0.41 & CWP1 sludge & 0.28 \\
\hline $\mathrm{C}-112$ & TFeCN sludge & 0.69 & CWP1 sludge & 0.16 \\
\hline C-201 & HS sludge & 1.00 & N/A & N/A \\
\hline C-202 & HS sludge & 1.00 & N/A & N/A \\
\hline C-203 & HS sludge & 1.00 & N/A & N/A \\
\hline $\mathrm{C}-204$ & HS sludge & 1.00 & N/A & N/A \\
\hline S-101 & S1 saltcake & 0.27 & R1 (boiling) sludge & 0.27 \\
\hline S-102 & S2 saltcake & 0.59 & S1 saltcake & 0.35 \\
\hline S-103 & S1 saltcake & 0.51 & S2 saltcake & 0.44 \\
\hline S-104 & R saltcake & 0.47 & R1 (boiling) sludge & 0.43 \\
\hline S-105 & S1 saltcake & 0.99 & R1 (non-boiling) sludge & 0.01 \\
\hline S-106 & S1 saltcake & 1.00 & N/A & N/A \\
\hline S-107 & R1 (boiling) sludge & 0.36 & CWR1 sludge & 0.35 \\
\hline S-108 & S1 saltcake & 0.99 & R1 (non-boiling) sludge & 0.01 \\
\hline S-109 & S1 saltcake & 0.97 & R1 (non-boiling) sludge & 0.03 \\
\hline S-110 & S1 saltcake & 0.73 & R1 (boiling) sludge & 0.21 \\
\hline S-111 & S1 saltcake & 0.77 & R1 (non-boiling) sludge & 0.19 \\
\hline S-112 & S1 saltcake & 0.99 & R1 (non-boiling) sludge & 0.01 \\
\hline SX-101 & R1 (non-boiling) sludge & 0.41 & R saltcake & 0.31 \\
\hline SX-102 & S1 saltcake & 0.78 & R1 (non-boiling) sludge & 0.14 \\
\hline SX-103 & S1 saltcake & 0.83 & R saltcake & 0.16 \\
\hline SX-104 & S1 saltcake & 0.59 & R1 (boiling) sludge & 0.32 \\
\hline SX-105 & S1 saltcake & 0.85 & R1 (non-boiling) sludge & 0.15 \\
\hline SX-106 & S2 saltcake & 0.82 & S1 saltcake & 0.18 \\
\hline SX-107 & R1 (boiling) sludge & 0.87 & R saltcake & 0.13 \\
\hline SX-108 & R1 (boiling) sludge & 1.00 & N/A & N/A \\
\hline SX-109 & R saltcake & 0.76 & R1 (boiling) sludge & 0.24 \\
\hline SX-110 & R1 (boiling) sludge & 0.51 & R saltcake & 0.49 \\
\hline SX-111 & R1 (boiling) sludge & 0.72 & R saltcake & 0.28 \\
\hline SX-112 & R1 (boiling) sludge & 0.80 & R saltcake & 0.20 \\
\hline SX-113 & DE sludge & 0.89 & R1 (boiling) sludge & 0.11 \\
\hline SX-114 & R saltcake & 0.67 & R1 (boiling) sludge & 0.33 \\
\hline SX-115 & R1 (boiling) sludge & 1.00 & N/A & N/A \\
\hline SY-101 & S2 saltcake & 1.00 & N/A & N/A \\
\hline SY-102 & Unidentified sludge & 0.51 & Z sludge & 0.49 \\
\hline
\end{tabular}


Table 3.1. (contd)

\begin{tabular}{|c|c|c|c|c|}
\hline Tank & $\begin{array}{c}\text { Primary Waste } \\
\text { Type }^{1}\end{array}$ & $\begin{array}{c}\text { Volume } \\
\text { Fraction } \\
\text { Primary } \\
\text { Waste Type }^{3}\end{array}$ & $\begin{array}{c}\text { Secondary Waste } \\
\text { Type }^{2}\end{array}$ & $\begin{array}{c}\text { Volume } \\
\text { Fraction } \\
\text { Secondary } \\
\text { Waste Type }^{3}\end{array}$ \\
\hline SY-103 & S2 saltcake & 1.00 & N/A & N/A \\
\hline $\mathrm{T}-101$ & T2 saltcake & 0.56 & CWR2 sludge & 0.44 \\
\hline T-102 & CWP2 sludge & 0.89 & MW sludge & 0.11 \\
\hline T-103 & CWP2 sludge & 0.74 & CWR1 sludge & 0.22 \\
\hline T-104 & 1C sludge & 1.00 & N/A & N/A \\
\hline T-105 & 2C sludge & 0.74 & CWR1 sludge & 0.12 \\
\hline T-106 & CWR1 sludge & 0.54 & 1C sludge & 0.46 \\
\hline $\mathrm{T}-107$ & 1C sludge & 0.85 & TBP sludge & 0.10 \\
\hline T-108 & T1 saltcake & 0.60 & 1C sludge & 0.40 \\
\hline T-109 & T1 saltcake & 1.00 & N/A & N/A \\
\hline $\mathrm{T}-110$ & 2C sludge & 0.97 & 224 Post-1949 sludge & 0.03 \\
\hline $\mathrm{T}-111$ & 2C sludge & 0.55 & 224 Post-1949 sludge & 0.45 \\
\hline T-112 & 2C sludge & 0.56 & 224 Post-1949 sludge & 0.44 \\
\hline T-201 & 224 Pre-1949 sludge & 1.00 & N/A & N/A \\
\hline $\mathrm{T}-202$ & 224 Post-1949 sludge & 1.00 & N/A & N/A \\
\hline T-203 & 224 Post-1949 sludge & 1.00 & N/A & N/A \\
\hline T-204 & 224 Post-1949 sludge & 1.00 & N/A & N/A \\
\hline TX-101 & R1 (non-boiling) sludge & 0.81 & T2 saltcake & 0.15 \\
\hline TX-102 & T2 saltcake & 0.99 & MW sludge & 0.01 \\
\hline TX-103 & T2 saltcake & 0.98 & T1 saltcake & 0.02 \\
\hline TX-104 & R1 (non-boiling) sludge & 0.59 & T2 saltcake & 0.41 \\
\hline TX-105 & T2 saltcake & 0.99 & MW sludge & 0.01 \\
\hline TX-106 & T2 saltcake & 0.98 & R1 (non-boiling) sludge & 0.01 \\
\hline TX-107 & T2 saltcake & 0.73 & R saltcake & 0.27 \\
\hline TX-108 & T2 saltcake & 0.95 & TBP sludge & 0.04 \\
\hline TX-109 & 1C sludge & 1.00 & N/A & N/A \\
\hline TX-110 & T2 saltcake & 0.92 & 1C sludge & 0.08 \\
\hline TX-111 & T2 saltcake & 0.88 & 1C sludge & 0.12 \\
\hline TX-112 & T2 saltcake & 0.97 & T1 saltcake & 0.03 \\
\hline TX-113 & T2 saltcake & 0.85 & 1C sludge & 0.15 \\
\hline TX-114 & T2 saltcake & 0.90 & T1 saltcake & 0.09 \\
\hline TX-115 & T2 saltcake & 0.98 & TBP sludge & 0.02 \\
\hline TX-116 & T1 saltcake & 0.48 & T2 saltcake & 0.41 \\
\hline TX-117 & T2 saltcake & 0.59 & T1 saltcake & 0.35 \\
\hline TX-118 & T2 saltcake & 0.69 & Unidentified saltcake & 0.31 \\
\hline TY-101 & 1CFeCN sludge & 0.61 & T1 saltcake & 0.39 \\
\hline TY-102 & T2 saltcake & 0.55 & T1 saltcake & 0.45 \\
\hline TY-103 & TBP sludge & 0.40 & 1CFeCN sludge & 0.31 \\
\hline TY-104 & 1CFeCN sludge & 0.70 & TBP sludge & 0.30 \\
\hline TY-105 & TBP sludge & 1.00 & N/A & N/A \\
\hline TY-106 & DE sludge & 0.76 & TBP sludge & 0.24 \\
\hline U-101 & R1 (non-boiling) sludge & 1.00 & N/A & N/A \\
\hline U-102 & T2 saltcake & 0.58 & S2 saltcake & 0.27 \\
\hline
\end{tabular}


Table 3.1. (contd)

\begin{tabular}{|c|c|c|c|c|}
\hline Tank & $\begin{array}{c}\text { Primary Waste } \\
\text { Type }^{1}\end{array}$ & $\begin{array}{c}\text { Volume } \\
\text { Fraction } \\
\text { Primary } \\
\text { Waste Type }^{3}\end{array}$ & $\begin{array}{c}\text { Secondary Waste } \\
\text { Type }^{2}\end{array}$ & $\begin{array}{c}\text { Volume } \\
\text { Fraction } \\
\text { Secondary } \\
\text { Waste Type }^{3}\end{array}$ \\
\hline U-103 & S1 saltcake & 0.69 & S2 saltcake & 0.28 \\
\hline U-104 & DE sludge & 0.72 & R1 (non-boiling) sludge & 0.28 \\
\hline U-105 & S2 saltcake & 0.71 & T2 saltcake & 0.19 \\
\hline U-106 & S1 saltcake & 1.00 & N/A & N/A \\
\hline U-107 & S2 saltcake & 0.91 & CWR1 sludge & 0.05 \\
\hline U-108 & S1 saltcake & 0.51 & S2 saltcake & 0.41 \\
\hline U-109 & S1 saltcake & 0.48 & S2 saltcake & 0.41 \\
\hline U-110 & R1 (boiling) sludge & 0.60 & CWR1 sludge & 0.22 \\
\hline U-111 & S2 saltcake & 0.59 & S1 saltcake & 0.32 \\
\hline U-112 & R1 (non-boiling) sludge & 0.39 & CWR1 sludge & 0.34 \\
\hline U-201 & CWR1 sludge & 1.00 & N/A & N/A \\
\hline U-202 & CWR1 sludge & 1.00 & N/A & N/A \\
\hline U-203 & CWR1 sludge & 1.00 & N/A & N/A \\
\hline U-204 & CWR1 sludge & 1.00 & N/A & N/A \\
\hline \multicolumn{5}{|c|}{$\begin{array}{l}\text { 1. The primary waste type indicates which waste is present in the highest volumetric quantity for the listed tank. } \\
\text { 2. Secondary wastes are any other wastes present in that tank. } \\
\text { 3. UDS volume basis. } \\
\text { 4. N/A, not applicable. }\end{array}$} \\
\hline
\end{tabular}


Table 3.2. Waste Type Definitions

\begin{tabular}{|c|c|}
\hline Waste Type & Definition \\
\hline 1C sludge & $\mathrm{BiPO}_{4}$ first cycle decontamination waste (1944-1956) \\
\hline $1 \mathrm{CFeCN}$ sludge & $\begin{array}{l}\text { Ferrocyanide sludge from in-farm scavenging of 1C supernatants in TY-Farm } \\
\text { (1955-1958) }\end{array}$ \\
\hline 224 Post-1949 sludge & Lanthanum fluoride process 224 Building waste (1950-1956) \\
\hline 224 Pre-1949 sludge & Lanthanum fluoride process 224 Building waste (1944-1949) \\
\hline 2C sludge & $\mathrm{BiPO}_{4}$ second cycle decontamination waste (1944-1956) \\
\hline A1 saltcake & $\begin{array}{l}\text { Saltcake from first 242-A Evaporator campaign using 241-A-102 feed tank } \\
\text { (1977-1980) }\end{array}$ \\
\hline A2 saltcake & Saltcake from the second 242-A Evaporator campaign (1981-1994) \\
\hline AR sludge & Washed plutonium uranium extraction (PUREX) sludge (1967-1976) \\
\hline B saltcake & Saltcake from 242-B Evaporator operation (1951-1953) \\
\hline BL sludge & Low-level waste from B Plant Sr and Cs recovery operations (1967-1976) \\
\hline BY saltcake & Saltcake from in-tank solidification (ITS) in BY-Farm (1965-1974) \\
\hline CWP1 sludge & PUREX cladding waste, aluminum clad fuel (1956-1960) \\
\hline CWP2 sludge & PUREX cladding waste, aluminum clad fuel (1961-1972) \\
\hline CWR1 sludge & Reduction oxidation (REDOX) cladding waste, aluminum clad fuel (1952-1960) \\
\hline CWR2 sludge & REDOX cladding waste, aluminum clad fuel (1961-1966) \\
\hline CWZr2 sludge & PUREX zirconium cladding waste (1983-1989) \\
\hline DE sludge & Diatomaceous earth \\
\hline HS sludge & Hot semi-works 90Sr recovery waste (1962-1967) \\
\hline MW sludge & $\mathrm{BiPO}_{4}$ process metal waste (1944-1956) \\
\hline P1 sludge & PUREX HLW (1955-1962) \\
\hline P2 sludge & PUREX HLW (1963-1967) \\
\hline P3 sludge & PUREX HLW (1983-1990) \\
\hline PFeCN sludge & Ferrocyanide sludge from in-plant scavenged supernatant \\
\hline PL2 sludge & PUREX LLW (1983-1988) \\
\hline R saltcake & Saltcake from self-concentration in S- and SX-Farms (1952-1966) \\
\hline R1 (boiling) sludge & Boiling REDOX HLW \\
\hline R1 (non-boiling) sludge & Non-boiling REDOX HLW \\
\hline S1 saltcake & $\begin{array}{l}\text { Saltcake from the first 242-S Evaporator campaign using 241-S-102 feed tank } \\
(1973-1976)\end{array}$ \\
\hline S2 saltcake & $\begin{array}{l}\text { Saltcake from the second 242-S Evaporator campaign using 241-S-102 feed tank } \\
(1977-1980)\end{array}$ \\
\hline SRR sludge & Sr recovery waste from sluiced P sludge \\
\hline T1 saltcake & Saltcake from the 242-T Evaporator campaign (1951-1956) \\
\hline T2 saltcake & Saltcake from the last 242-T Evaporator campaign (1965-1976) \\
\hline TBP sludge & Tributyl phosphate waste (from solvent based uranium recovery operations) \\
\hline TFeCN sludge & Ferrocyanide sludge produced by in-tank or in-farm scavenging \\
\hline Z sludge & Z Plant waste \\
\hline
\end{tabular}




\subsection{Data}

Parameter characterization data for as-stored Hanford waste are provided for liquid density and $\mathrm{pH}$, Section 3.2.1, liquid viscosity, Section 3.2.2, UDS particle composition and density, Section 3.2.3, UDS primary particle size and shape, Section 3.2.4, UDS particle size, Section 3.2.5, UDS particle settling, Section 3.6, and slurry rheology, Section 3.2.7. Gaps in the data are discussed and summarized in Section 5.0.

As specified previously, the data are from numerous and varied sources as referenced. It is assumed that the data from these references have been fully reviewed and documented in accordance with the analysts' QA programs.

\subsubsection{Liquid Density and pH}

The density and $\mathrm{pH}$ liquid properties of tank wastes have been quantified as listed in Appendix A. Predictions of these and other liquid properties had been made by the Environmental Simulation Program $(\text { ESP })^{(a)}$ chemical thermodynamic model for all 177 Hanford waste tanks based on 2002 BBI composition information. However, the $\mathrm{pH}$ predictions generated by ESP were frequently overestimates compared to measured values, as was determined for tanks where data were available to evaluate them (Table D-1, Cowley et al. 2003). For this reason, the $\mathrm{pH}$ and density data presented in this report are taken from other sources.

The liquid density data for each tank were taken from the liquid densities given by the gas release document (Table A-3, Meacham 2009) for the liquid in bulk waste. The reference gave data for both interstitial liquid (in the bulk solids layers) and supernatant liquid where present. The reference's data for densities of supernatant liquid were not used. The liquid densities used for the liquid present within sludge and saltcake waste types were then calculated from the densities for the tank or tanks representing the waste types. More information on selecting representative tanks for waste types is given in Section 3.2.3.4 where the selected representatives are listed.

The $\mathrm{pH}$ and hydroxide data were not available for all 177 tanks. Three different references were checked: the gas release document (Table A-1, Meacham 2009), the Tank Waste Information Network System (TWINS) database, and a document containing historical $\mathrm{pH}$ and hydroxide data for SSTs (Wodrich et al. 1992). This last reference contains $\mathrm{OH}$ and $\mathrm{pH}$ measurements from the 1960s through the 1980s. In this period there were a number of uncertainties in measurement because of the presence of $\mathrm{OH}$-complexing ions and the ionic strength of the solutions. In many cases, the $\mathrm{pH}$ was biased low because of glass electrode limitations. Some of the information in the historical $\mathrm{pH}$ report (Wodrich et al.) consisted of indirect estimates of $\mathrm{pH}$ based on concentrations of other species (e.g., dissolved aluminum) that were present. ${ }^{(b)}$

(a) ESP was supplied and developed by OLI Systems, Inc., Morris Plains, New Jersey (OLI 1998).

(b) The historical $\mathrm{pH} / \mathrm{OH}$ data are supplied strictly on the basis of being the only available data. Their uncertainty is substantial not only because of the analytical issues but because various reactions could have changed the hydroxide concentration since the time of measurement. 
The hydroxide concentrations from Meacham (2009) were the preferred source of data for all tanks where the concentrations were derived from measurements for liquid samples (not bulk solids samples). ${ }^{\text {(a) }}$ For the remaining tanks, the hydroxide data provided by Meacham were estimates, consistent with the BBI current at the time of the report, and were not based on measurements. For the present purpose, when tanks lacked actual measurements in TWINS, the estimates from Meacham (2009) were used when they were consistent either with TWINS data for other tanks that contained a similar waste type or with historical (pre-1992) data from Wodrich et al. (1992) for the tank. When the Meacham non-measurement-based estimates were not found to be consistent with those data sources, one of three approaches was followed: 1) pre-1992 historical data for the tank were used, 2) data from another tank containing similar waste were used, or 3 ) the $\mathrm{pH} / \mathrm{OH}$ were marked as not available.

In the present study, when the available data consisted of $\mathrm{pH}$ measured by a glass electrode, the hydroxide concentration was calculated using the standard relation for an aqueous solution wherein the activity coefficient for the hydroxyl anion is assumed to be equal to 1 :

$$
O H(\mu g / m L)=1000 * 17.08 * 10^{p H-14}
$$

When the available data consisted of the hydroxide concentration measured by titration, the same equation was used and rearranged to solve for $\mathrm{pH}$. This relation is expected to underestimate the $\mathrm{pH}$ at high ionic strength and high $\mathrm{pH}$.

As noted in Appendix G of Wodrich et al. (1992), many historical data were taken using glass $\mathrm{pH}$ electrodes, whose readings are lower than actual values for sodium concentrations greater than $2 \mathrm{M}$ and $\mathrm{pH}$ readings between 8 and 14. It was unclear whether any correction for sodium had been made for many of the historical measurements. The more recent data located in TWINS include warnings that at $\mathrm{pH}$ of 12 or greater, the hydroxide data obtained from titration are to be used instead of data from $\mathrm{pH}$ electrodes. This rule was followed in using TWINS data.

It should be noted that much of the information for liquid density and $\mathrm{pH}$ is derived from sources that are more recent than the 2002 BBI used to define the UDS composition and density (Section 3.2.3). Some tanks' wastes have been diluted or leached since 2002, changing both the solid and liquid compositions of the waste remaining in the tanks. This inconsistency between liquid- and solid-phase information is one of the gaps in the present study's characterization.

\subsubsection{Liquid Rheology}

Rheology data are available for the liquid of a limited number of Hanford tanks. The data were obtained from laboratory measurements of samples retrieved from the tanks, including push- and rotary-mode core samples, auger samples, and grab samples. A discussion of rheological characterization and measurement techniques is provided in Poloski et al. (2007).

Hanford liquid supernatant is generally a Newtonian fluid, so Newtonian viscosity is used to characterize the liquid rheology (Poloski et al. 2007). Liquid viscosity data of as-received samples at various temperatures are available for the tanks and waste types listed in Table 3.3. The data are included

(a) The Meacham (2009) values for hydroxide concentration were checked against the data in TWINS, as of October 2010, to determine whether or not values were based on measurements (those present in TWINS). 
as part of Appendix I. Diluted sample measurements are available in some cases. References are provided in the table.

The liquid temperature can have an effect on liquid viscosity. The Andrade correlation is often used when evaluating the relationship between increasing temperature and decreasing viscosity of liquids (Poloski et al. 2007). However, it is not accurate for high-temperature fluids or highly polar mixtures. The Andrade correlation can be written as

$$
\mu_{\mathrm{L}}=\mathrm{Ae}^{\frac{\mathrm{B}}{\mathrm{T}}}
$$

where $\mu_{\mathrm{L}}$ is the Newtonian viscosity (cP), T is the liquid temperature in (K), and A (in $\mathrm{CP}$ ) and $\mathrm{B}$ (in $\mathrm{K}$ ) are the fitting parameters.

All the tanks listed in Table 3.3 have liquid viscosity measurements over a range of temperatures, typically between 20 and $65^{\circ} \mathrm{C}$. The liquid comprises water and dissolved solids. In addition to temperature, the chemical composition and concentration of the dissolved solids in the liquid also may have a significant effect on the viscosity. The chemical composition and concentration thereof were represented by the liquid density in Poloski et al. (2007). Viscosity data as a function of liquid density is available for 7 of the 11 tanks listed in Table 3.3.

A liquid viscosity model accounting for both temperature and liquid density was developed in Poloski et al. (2007) as

$$
\mu_{\mathrm{L}}=\left(\mathrm{ae}^{\mathrm{b}\left(\rho_{\mathrm{L}}-1\right)}\right) \mathrm{e}^{\left(\mathrm{c}\left(\rho_{\mathrm{L}}-1\right)+\mathrm{d}\right) / \mathrm{T}}
$$

where $\rho_{\mathrm{L}}$ is the liquid density $(\mathrm{g} / \mathrm{mL})$, and a $(\mathrm{cP}), \mathrm{b}(\mathrm{g} / \mathrm{mL})^{-1}, \mathrm{c}(\mathrm{K} \cdot \mathrm{mL} / \mathrm{g})$, and $\mathrm{d}(\mathrm{K})$ are the fitting parameters.

Table 3.3. Liquid Viscosity Data Set

\begin{tabular}{|c|c|c|}
\hline Tank & Reference(s) & Primary Waste Type \\
\hline AN-103 & HNF-7153 & A2 saltcake \\
\hline AN-104 & WSRC-TR-2003-00295, Rev. 0, HNF-3352 & A2 saltcake \\
\hline AN-105 & HNF-SD-WM-DTR-046 Rev 0 & A2 saltcake \\
\hline AP-104 & WTP-RPT-069, Rev. 0, PNWD-3334 & N/A \\
\hline AW-101 & HNF-4964 Rev. 0 & A2 saltcake \\
\hline AY-102 & RPP-8909 & BL sludge \\
\hline AZ-101 & RPT-7078, Rev. 0, TWINS & P3 sludge \\
\hline C-104 & RPP-5798 & CWP1 sludge \\
\hline C-107 & RPP-18799, Rev 0; TWINS & 1C sludge \\
\hline S-112 & RPP-10984 Rev. 0 & S1 saltcake \\
\hline SY-101 & 82100-99-017 & S2 saltcake \\
\hline N/A Not applicable, liquid only tank & \\
\hline
\end{tabular}


In Table 3.4, the fitting parameters for Eqs. (3.2) and (3.3) are provided for the tanks that are dependent on the available data set (i.e., temperature only or both temperature and liquid density). The best fits were determined via a least-squares regression. The standard deviations of the fit parameters for primary waste type A2 saltcake, the only waste type with multiple tank characterization, are similar to the standard deviations across all of the represented waste types with the exception of parameter $b$. This result may suggest that, for the limited data set, the liquid viscosity functionality is not dependent on the UDS waste type. A comparison of the measured and predicted values is provided in Figure 3.1. At measured viscosities greater than approximately $15 \mathrm{cP}$, the model fits are shown to underpredict the measured data.

Due to the limited liquid viscosity data set, the functionality of viscosity with liquid temperature and density are evaluated for the data set as a whole. For the entire data set, as referenced above for the individual tanks, the model (fit parameters determined via least-squares regression, minimum viscosity set to unity) underpredicts the measured data for viscosities greater than approximately $15 \mathrm{cP}$, and overpredicts below (Figure 3.2). Within the limitations of the data set, this result may suggest an additional functionality not addressed by the model.

As described above, the chemical composition and concentration of the dissolved solids in the liquid are represented by the liquid density in Eq. (3.3). The functionality of the dissolved solids concentration and liquid density for Hanford liquid was shown by Onishi et al. (2005), shown herein as Figure 3.3. The linear trend of increasing density with increasing dissolved solids concentration (i.e., decreasing water concentration) is shown to level off starting at approximately $1.4 \mathrm{~g} / \mathrm{mL}$ liquid density. Thus, Eq. (3.3) was applied to the entire data set partitioned into two by liquid density.

The least-squares regression minimized the combined sum of the square of the errors for the liquid-density-partitioned data sets, and the liquid density at which the partition occurred was included as a fit parameter. The best fit, Figure 3.4, was achieved with the liquid density partition set to approximately $1.41 \mathrm{~g} / \mathrm{mL}$, which agrees well with the density-concentration functionality break indicated in Figure 3.3. This partitioning by density shows a reduction in the under-and-over-prediction of the model. The residual (measured viscosity minus predicted) of Figure 3.2, $-31.9 \mathrm{cP}$, is reduced to $-9.5 \mathrm{cP}$ in Figure 3.4. The Eq. (3.3) fit parameter estimates for the density-partitioned entire data set are provided in Table 3.4. These model parameter estimates can be used to calculate predicted liquid viscosity values for specific density and temperature combinations.

Statistical methods appropriate for the nonlinear model form in Eq. (3.3) were used to calculate 95\% lower and upper prediction limits associated with the predicted liquid viscosities that result from applying the model form in Eq. (3.3) and corresponding parameter estimates given in Table 3.4 to each density and temperature combination included in the sample data. These prediction limits were calculated using the formulas (Myers 1990)

$$
\begin{gathered}
\mathrm{LL}_{\mathrm{i}}=\hat{\mu}\left(\mathbf{x}_{\mathrm{i}}, \hat{\boldsymbol{\theta}}\right)-\mathrm{t}_{\alpha / 2, \mathrm{n}-\mathrm{p}} \sqrt{\hat{\sigma}^{2}\left(1+\mathbf{w}_{\mathrm{i}}^{\prime}\left(\mathbf{W}^{\prime} \mathbf{W}\right)^{-1} \mathbf{w}_{\mathrm{i}}\right)} \text { and } \\
\mathrm{UL}_{\mathrm{i}}=\hat{\mu}\left(\mathbf{x}_{\mathrm{i}}, \hat{\boldsymbol{\theta}}\right)+\mathrm{t}_{\alpha / 2, \mathrm{n}-\mathrm{p}} \sqrt{\hat{\sigma}^{2}\left(1+\mathbf{w}_{\mathrm{i}}^{\prime}\left(\mathbf{W}^{\prime} \mathbf{W}\right)^{-1} \mathbf{W}_{\mathrm{i}}\right)} .
\end{gathered}
$$


where

$$
\begin{array}{rl}
\mathrm{LL}_{\mathrm{i}}= & \text { lower prediction limit for the } \mathrm{i}^{\text {th }} \text { data point } \\
\mathrm{UL}_{\mathrm{i}}= & \text { upper prediction limit for the } \mathrm{i}^{\text {th }} \text { data point } \\
\hat{\mu}\left(\mathbf{x}_{\mathrm{i}}, \hat{\boldsymbol{\theta}}\right)= & \text { model-predicted liquid viscosity obtained using the estimated model coefficients } \\
& \hat{\boldsymbol{\theta}} \text { and the } \mathrm{i}^{\text {th }} \text { data point } \mathbf{x}_{\mathrm{i}} \text {, which is a vector containing the } \mathrm{i}^{\text {th }} \text { observed density } \\
& \text { and temperature pair } \\
\mathrm{t}_{\alpha / 2, \mathrm{n}-\mathrm{p}=} & \text { a student's } t \text { statistic based on the prediction interval confidence level of } \\
& 100(1-\alpha) \% \text { and degrees of freedom equal to } \mathrm{n}-\mathrm{p} \text {, where } \mathrm{n} \text { is the number of data } \\
& \text { points, } \mathrm{p} \text { is the number of estimated model parameters, and for the } 95 \% \\
& \text { prediction limits calculated, } \alpha=0.05 \\
\hat{\sigma}^{2}= & \text { estimated error variance calculated using } \hat{\sigma}^{2}=\text { MSE }=\sqrt{\frac{\mathrm{SSE}}{\mathrm{n}-\mathrm{p}}} \\
& \text { denotes the sum of squared residuals, and } \mathrm{n}-\mathrm{p} \text { is the associated degrees of freedom } \\
\mathbf{W}_{\mathbf{W}} & \mathrm{n} \times \mathrm{p} \text { matrix of partial derivatives of the nonlinear model in Eq. (3.2.2.2) with } \\
& \text { respect to the model parameters (i.e., a Jacobian matrix) evaluated at each of the } \\
& \mathrm{n} \text { data points and using the p estimated model parameters } \\
\mathbf{w}_{\mathrm{i}}= & \mathrm{i}^{\text {th }} \text { row of the matrix } \mathbf{W} \text {, which represents the } \mathrm{i}^{\mathrm{th}} \text { data point } \mathbf{x}_{\mathrm{i}} \text { evaluated in } \\
& \text { each of the partial derivatives using the parameter estimates. }
\end{array}
$$

Separate prediction limit calculations were conducted using the appropriate part of the model labeled "Entire Data Set” in Table 3.4. Thus, for a data point with liquid density greater than or equal to $1.41 \mathrm{~g} / \mathrm{mL}$, the parameter estimates listed in the final row of the table were used to calculate the corresponding predicted liquid viscosity value and corresponding prediction limits. Likewise, the values of $\mathbf{n}$ and $\hat{\sigma}^{2}$ and the matrix $\mathbf{W}$ used in the prediction limit calculations were determined using the data from the appropriate liquid-density-partitioned subset of the observed data. So again, in calculating the prediction limits for a data point having a liquid density of $1.41 \mathrm{~g} / \mathrm{mL}$ or higher, the values of $\mathrm{n}$ and $\hat{\sigma}^{2}$ and the matrix $\mathbf{W}$ were determined from the subset of data points that had observed liquid densities of $1.41 \mathrm{~g} / \mathrm{mL}$ or higher. Finally, the number of model parameters (denoted as $\mathrm{p}$ in the equations above) represented in the degrees of freedom involved in the prediction limit calculations was 5 for all cases.

As described above, the model fit parameter estimates for the density-partitioned data set associated with the nonlinear model in Eq. (3.3) were determined using an optimization routine that sought to minimize the sum of squared residuals. The process involved estimating nine parameters: a partition point among liquid density values, four model parameters to use when applying the model form to data points having density below the partition point, and four model parameters to use when applying the model form to data points having density at or above the partition point. However, the calculations relative to a given data point did not involve all of the nine parameter estimates. Based on the observed liquid density relative to the partition point at $1.41 \mathrm{~g} / \mathrm{mL}$, only the four parameter estimates included in the appropriate part of the model were used to calculate a predicted viscosity value and corresponding prediction limits. Of course, both parts of the model shared the $1.41 \mathrm{~g} / \mathrm{mL}$ as the partition point on liquid density. The number of estimated model parameters, $p$, was therefore assumed as $p=5$, and hence, a degrees of freedom of $n-5$ in all prediction limit calculations. The overall data set included 45 data points with liquid density values below $1.41 \mathrm{~g} / \mathrm{mL}$ and 18 data points with liquid density values greater than or equal to $1.41 \mathrm{~g} / \mathrm{mL}$. 
As Eq. (3.3) can be used to calculate predicted liquid viscosity values for new density and temperature pairs (over conditions and domains comparable to those seen in the data used to develop the liquid viscosity model), Eq. (3.4) can be used to calculate corresponding prediction limits. Alternatively, for this study, a much simpler approach was found for approximating the $95 \%$ prediction limits associated with a given predicted value. Because the estimated standard error of an individual predicted liquid viscosity value $\left(\sqrt{\hat{\sigma}^{2}\left(1+\mathbf{w}_{\mathrm{i}}^{\prime}\left(\mathbf{W}^{\prime} \mathbf{W}\right)^{-1} \mathbf{W}_{\mathrm{i}}\right)}\right.$ in Eq. [3.4]) was found to be relatively constant for each of the liquid-density-partitioned data subsets as shown in Figure 3.5, the lower and upper prediction interval limits can be accurately approximated with linear relationships involving the predicted viscosity. The linear relationships that approximate the 95\% prediction limits are given in Table 3.5.

The fact that these linear relationships have been shown to produce accurate approximations to calculated prediction limits for the observed data suggests that they offer a simple and reasonably accurate method for approximating the prediction interval limits associated with a given predicted viscosity, provided that the predicted value was the result of applying Eq. (3.3) to a reasonable (with respect to experimental conditions and domains represented by the model development data) density and temperature data point.

Table 3.4. Liquid Viscosity Model Parameters

\begin{tabular}{|c|c|c|c|c|c|c|c|}
\hline \multirow[t]{2}{*}{ Tank } & \multirow{2}{*}{$\begin{array}{c}\text { Primary Waste } \\
\text { Type }\end{array}$} & \multicolumn{2}{|c|}{ Eq. (3.2) } & \multicolumn{4}{|c|}{ Eq. (3.3) } \\
\hline & & A & B & $\mathbf{a}$ & b & C & d \\
\hline AN-103 & A2 saltcake & & $8.47 \mathrm{E}-04$ & $-2.15 \mathrm{E}+00$ & $3.65 \mathrm{E}+03$ & $1.66 \mathrm{E}+03$ \\
\hline AN-104 & A2 saltcake & & & 7.13E-04 & $5.97 \mathrm{E}-01$ & $9.90 \mathrm{E}+02$ & $2.54 \mathrm{E}+03$ \\
\hline AN-105 & A2 saltcake & & & 3.83E-03 & $-8.05 \mathrm{E}+00$ & $3.75 \mathrm{E}+03$ & $1.84 \mathrm{E}+03$ \\
\hline AP-104 & N/A & $3.75 \mathrm{E}-04$ & $2.73 \mathrm{E}+03$ & \\
\hline AW-101 & A2 saltcake & & $3.29 \mathrm{E}-04$ & $-5.22 \mathrm{E}+00$ & $3.93 \mathrm{E}+03$ & $2.18 \mathrm{E}+03$ \\
\hline AY-102 & BL sludge & $5.91 \mathrm{E}-04$ & $2.40 \mathrm{E}+03$ & \\
\hline AZ-101 & P3 sludge & $4.38 \mathrm{E}-02$ & $1.19 \mathrm{E}+03$ & & & & \\
\hline C-104 & CWP1 sludge & $2.69 \mathrm{E}+00$ & $-2.41 \mathrm{E}+02$ & & & & \\
\hline C-107 & 1C sludge & & $9.14 \mathrm{E}-04$ & $-9.95 E+01$ & $1.11 \mathrm{E}+03$ & $3.10 \mathrm{E}+03$ \\
\hline S-112 & S1 saltcake & & & $1.86 \mathrm{E}-03$ & $4.52 \mathrm{E}+00$ & $4.82 \mathrm{E}+02$ & $1.86 \mathrm{E}+03$ \\
\hline SY-101 & S2 saltcake & & & $1.45 \mathrm{E}-05$ & $4.32 \mathrm{E}+00$ & $1.11 \mathrm{E}+03$ & $3.10 \mathrm{E}+03$ \\
\hline \multirow{2}{*}{\multicolumn{2}{|c|}{ Entire Data Set ${ }^{(\mathrm{a})}$}} & \multicolumn{2}{|c|}{$\begin{array}{l}\text { Liquid Density }<1.41 \\
\mathrm{~g} / \mathrm{mL}\end{array}$} & $1.01 \mathrm{E}-01$ & $-6.06 \mathrm{E}+00$ & $3.49 \mathrm{E}+03$ & $7.46 \mathrm{E}+02$ \\
\hline & & \multicolumn{2}{|c|}{$\begin{array}{l}\text { Liquid Density } \geq 1.41 \\
\mathrm{~g} / \mathrm{mL}\end{array}$} & $1.25 \mathrm{E}-04$ & 8.16E-01 & $3.98 \mathrm{E}+03$ & $1.59 \mathrm{E}+03$ \\
\hline \multicolumn{8}{|c|}{$\begin{array}{l}\text { - See other model. } \\
\text { (a) In application, the liquid density at which the liquid viscosity of the Liquid Density }<1.41 \mathrm{~g} / \mathrm{mL} \text { Entire Data Set } \\
\text { model at } 1.41 \mathrm{~g} / \mathrm{mL} \text { and that of the Liquid Density } \geq 1.41 \mathrm{~g} / \mathrm{mL} \text { Entire Data Set model at the temperature of interest are } \\
\text { equivalent must be determined. If that density is } \geq 1.41 \mathrm{~g} / \mathrm{mL} \text {, the liquid held constant with increasing density above } \\
1.41 \mathrm{~g} / \mathrm{mL} \text { until the Liquid Density } \geq 1.41 \mathrm{~g} / \mathrm{mL} \text { Entire Data Set model viscosity is equivalent, and then the liquid } \\
\text { viscosity increases via the Liquid Density } \geq 1.41 \mathrm{~g} / \mathrm{mL} \text { Entire Data Set model as shown in Section } 3.4 \text {. }\end{array}$} \\
\hline
\end{tabular}


Table 3.5. Linear Relationships that Approximate 95\% Prediction Limits

\begin{tabular}{|l|c|c|}
\hline \multicolumn{1}{|c|}{ Model Partition } & Lower Prediction Limit $^{1}$ & Upper Prediction Limit $^{1}$ \\
\hline Liquid Density $<1.41 \mathrm{~g} / \mathrm{mL}$ & Maximum of $0.90 \mu-4.77$, or 1.0 & $1.02 \mu+5.41$ \\
\hline Liquid Density $\geq 1.41 \mathrm{~g} / \mathrm{mL}$ & Maximum of $0.96 \mu-7.09$, or 1.0 & $1.03 \mu+7.25$ \\
\hline 1. $\mu$ represents a predicted viscosity obtained using Eq. (3.3). \\
\hline
\end{tabular}

AN-103

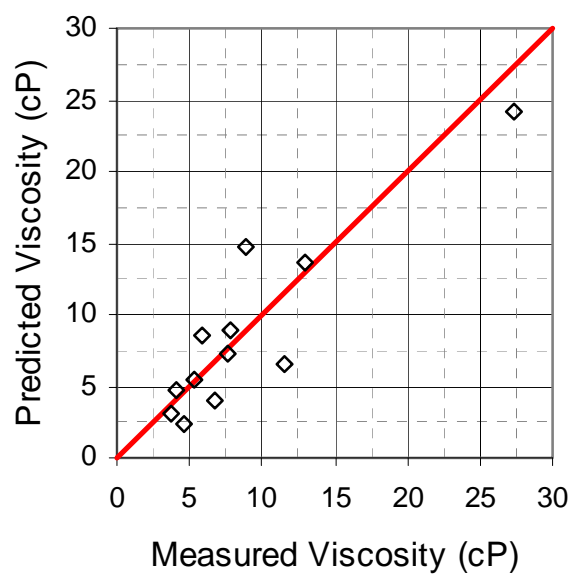

AN-105

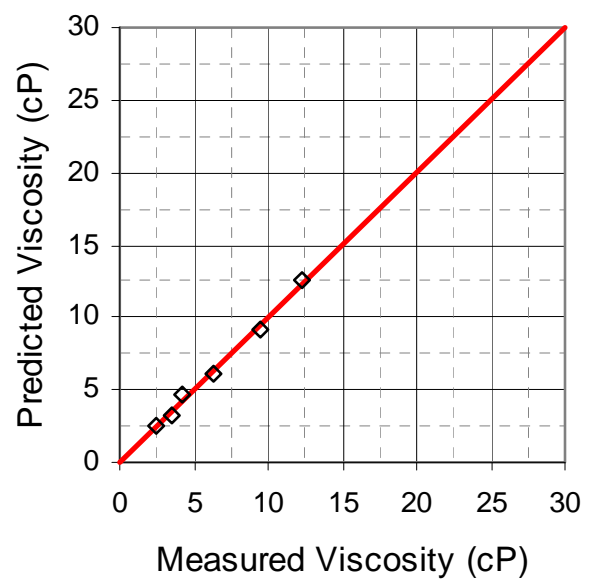

AN-104

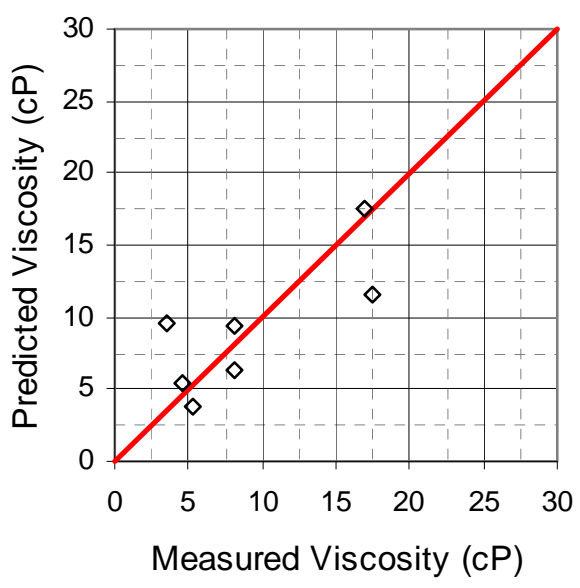

AP-104

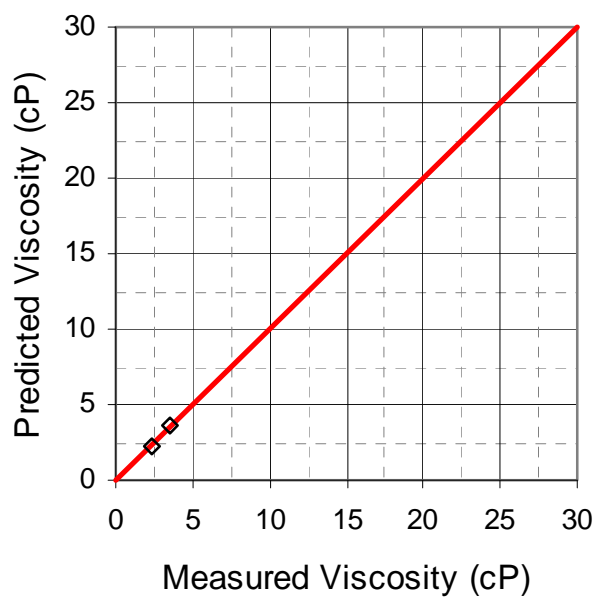

Figure 3.1. Comparison of Liquid Viscosity Measurements and Model Predictions by Tank. Diagonal lines indicate measurement and prediction parity. 


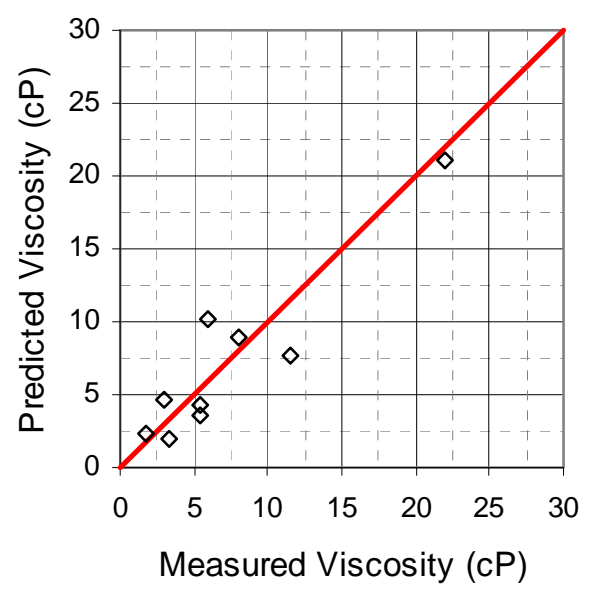

AZ-101

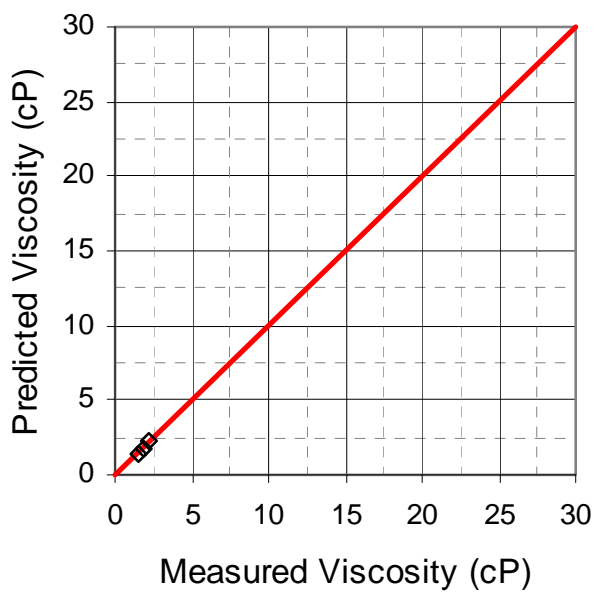

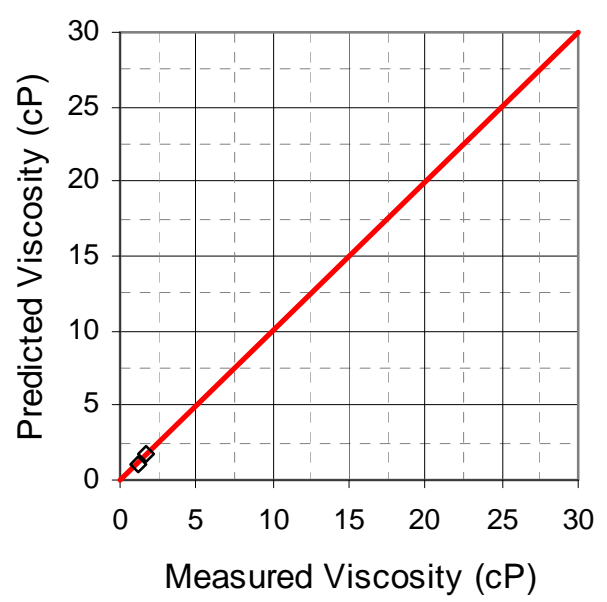

C-104

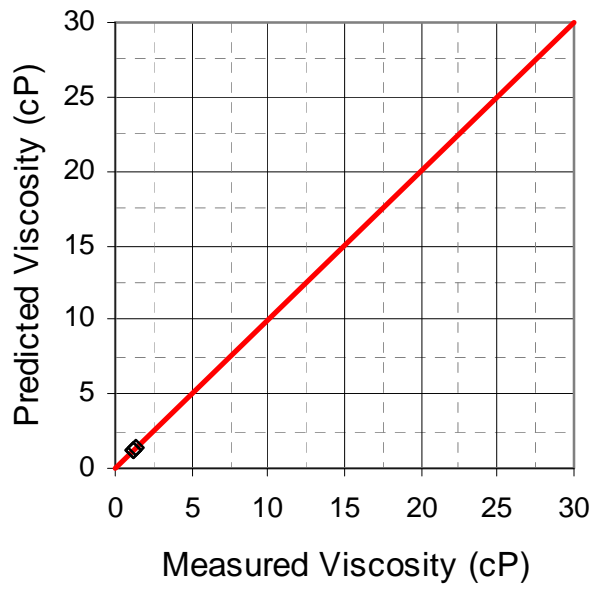

Figure 3.1. (contd) 
C-107

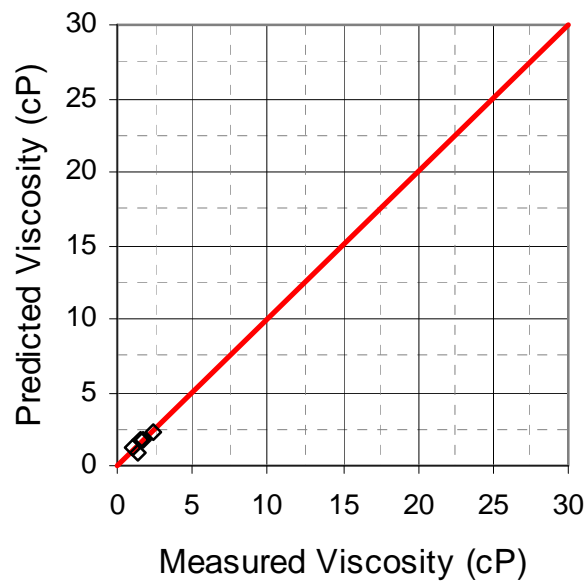

S-112

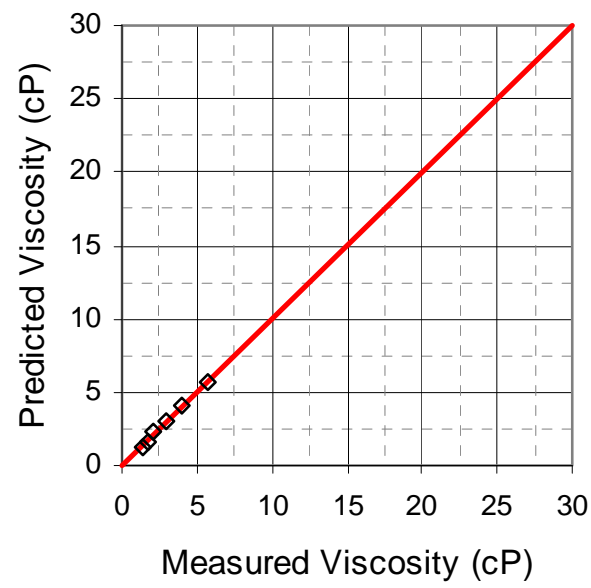

SY-101

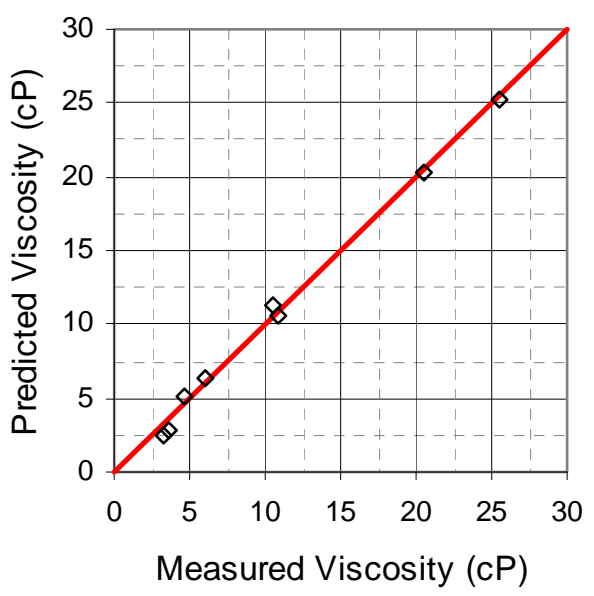

Figure 3.1. (contd) 


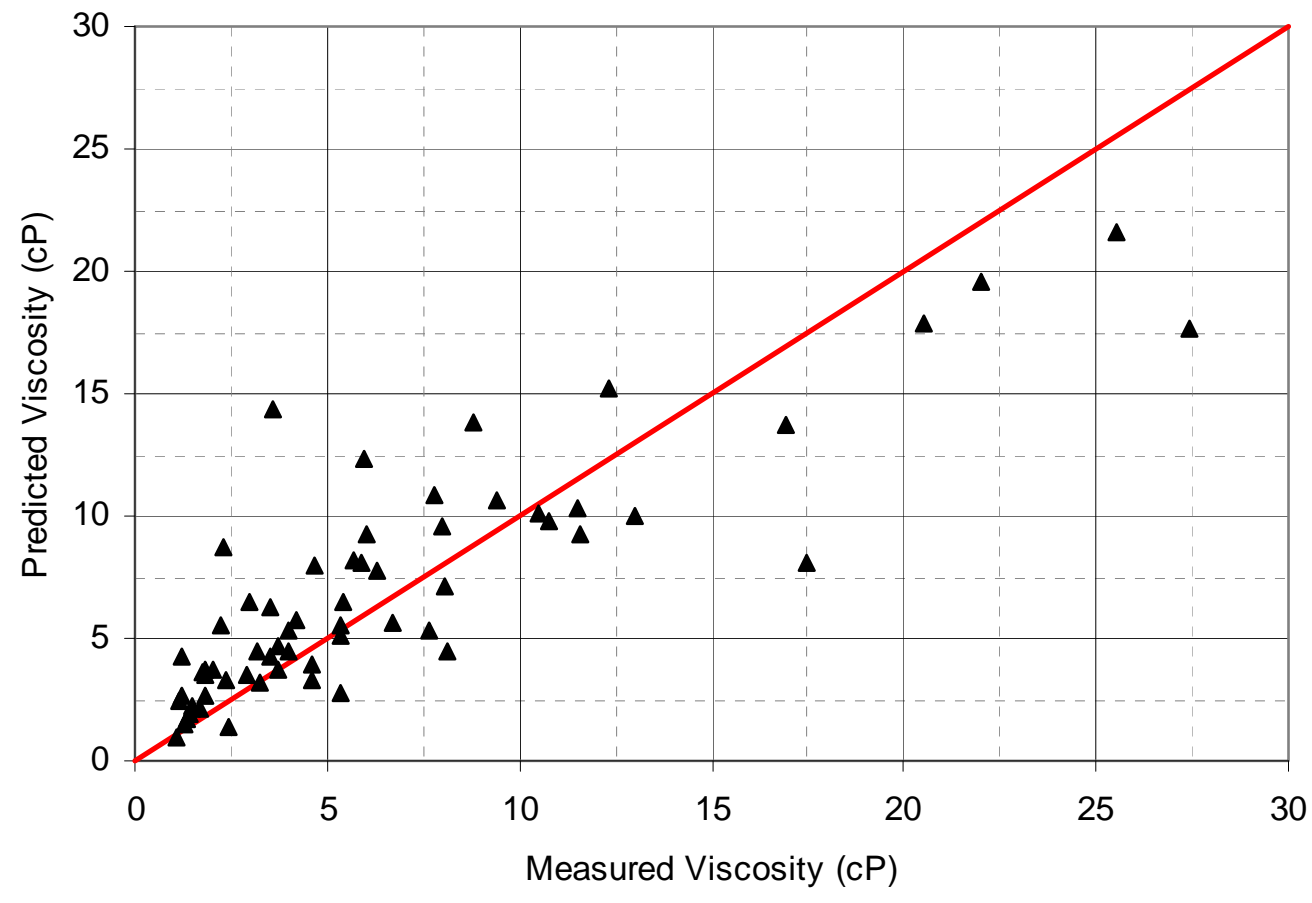

Figure 3.2. Comparison of Liquid Viscosity Measurements and Model Predictions for Entire Data Set. Diagonal line indicates measurement and prediction parity.

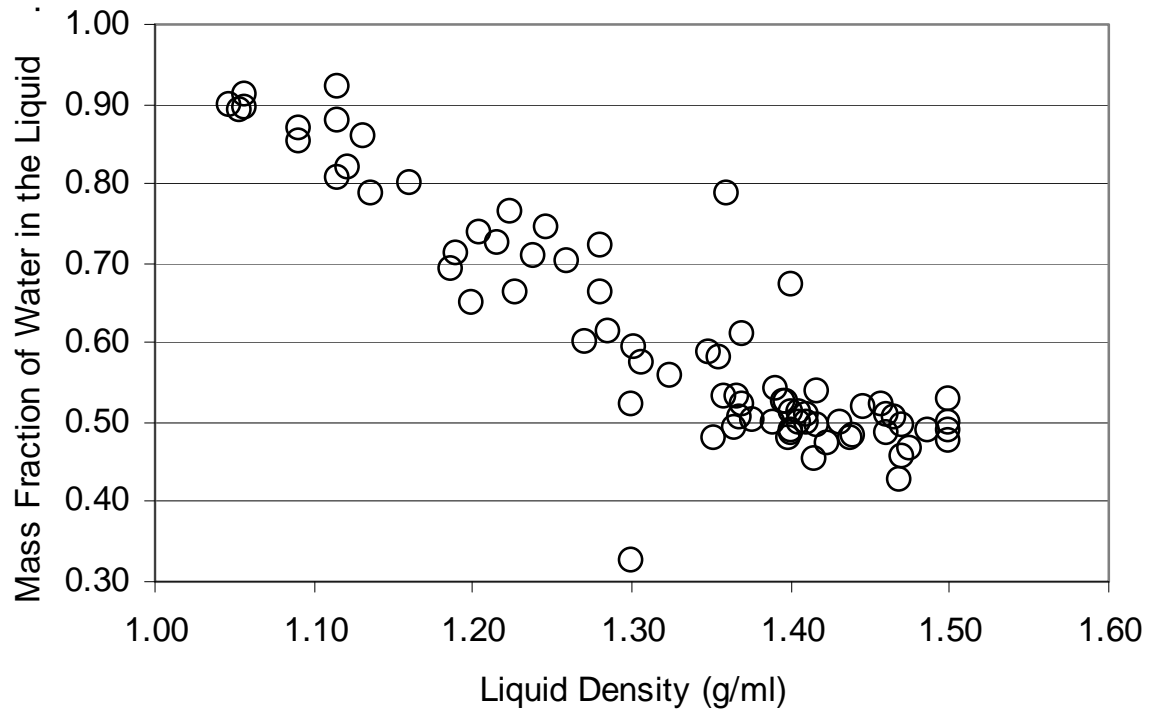

Figure 3.3. Hanford Liquid Density and Mass Fraction of Water. Figure 4.3, Onishi et al. (2005). 
\ Liquid Density $<1.41 \mathrm{~g} / \mathrm{mL} \Delta$ Liquid Density $\geq 1.41 \mathrm{~g} / \mathrm{mL}$

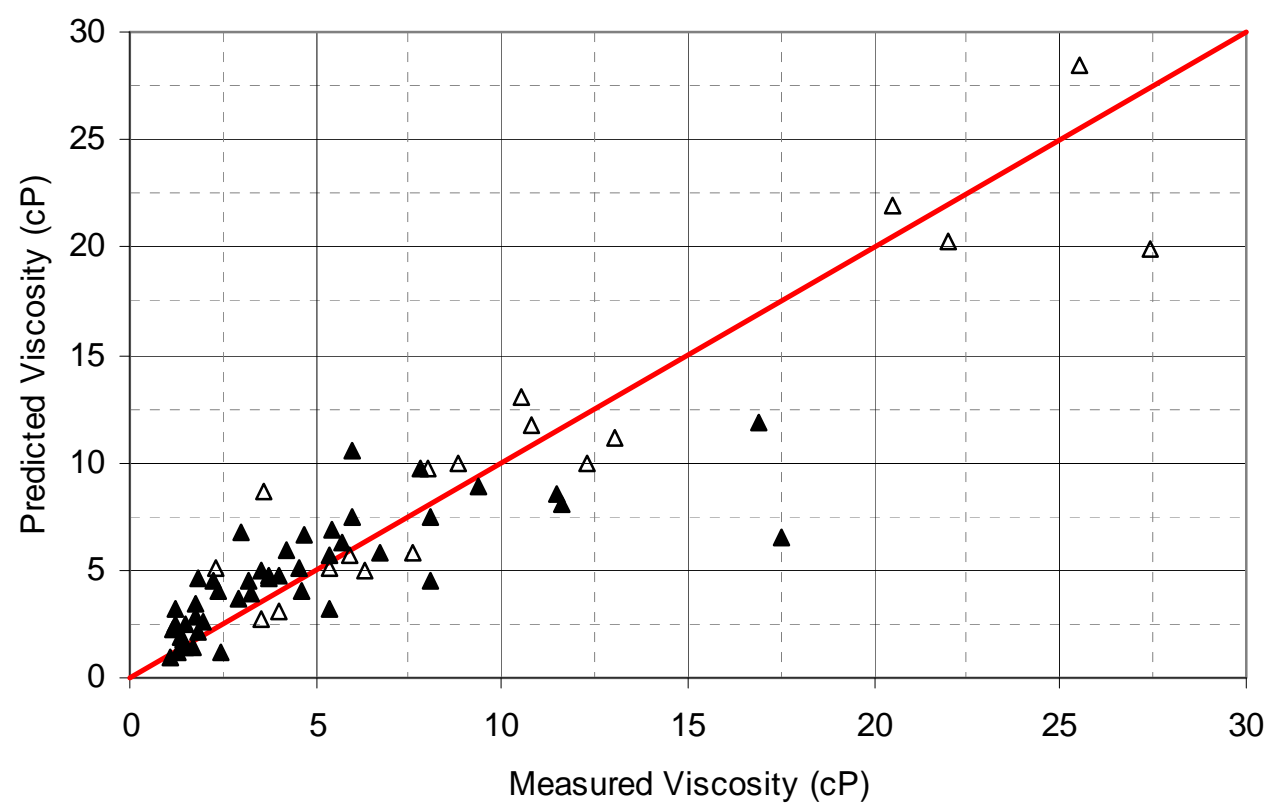

Figure 3.4. Comparison of Liquid Viscosity Measurements and Model Predictions for Entire Data Set with Segregation by Density. Diagonal line indicates measurement and prediction parity.

$\begin{array}{llll}\Delta \quad \text { UL, Liquid Density }<1.41 \mathrm{~g} / \mathrm{mL} & \square & \text { UL, Liquid Density } \geq 1.41 \mathrm{~g} / \mathrm{mL} \\ \Delta \quad \text { LL, Liquid Density }<1.41 \mathrm{~g} / \mathrm{mL} & \text { - } & \text { LL, Liquid Density } \geq 1.41 \mathrm{~g} / \mathrm{mL} \\ \text { - Predicted Value } & & \end{array}$

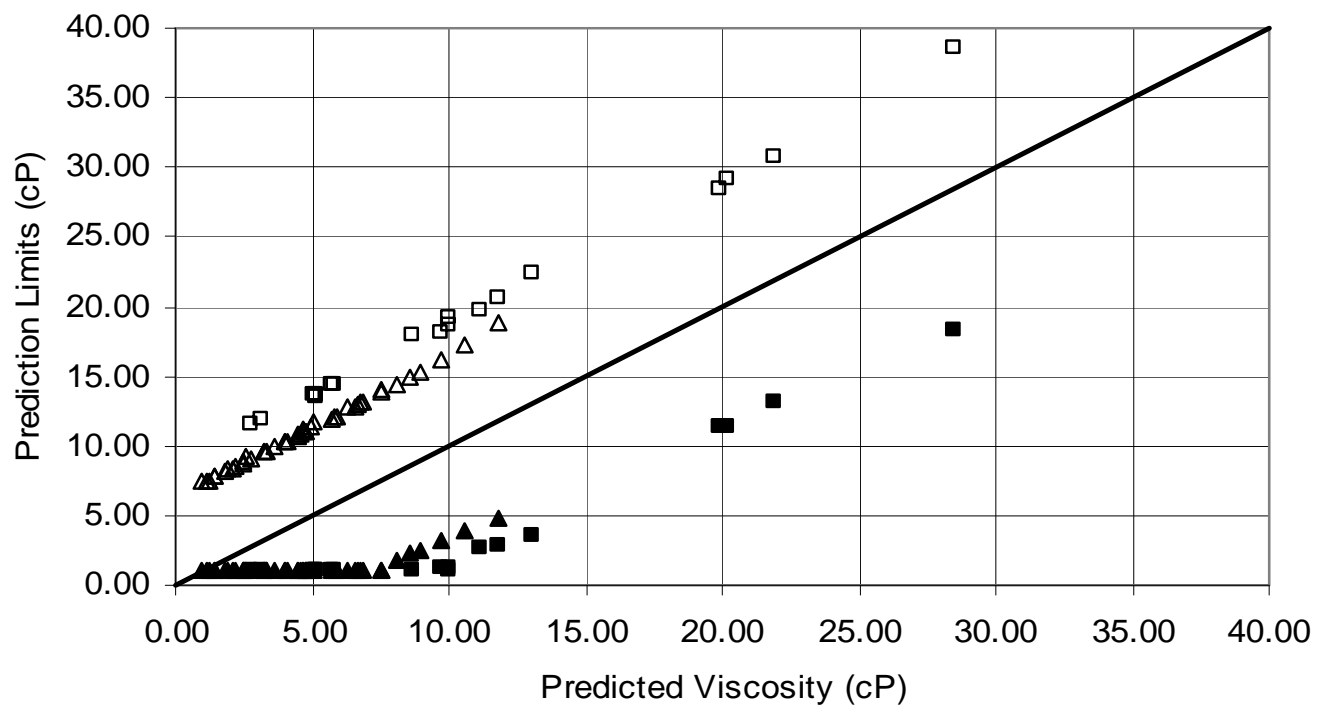

Figure 3.5. Liquid Viscosity Prediction Limits 


\subsubsection{Undissolved Solid Composition and Particle Density}

This section describes the approach for defining $\operatorname{UDS}^{(a)}$ phases present in the individual 177 Hanford tanks, in the Hanford defined waste types, and in the 177-tank composite waste. The definition of solid phases (UDS phases) is a major basis for the definition of particulate density. A summary table of the defined UDS phases and densities is provided.

This section includes both salt and non-salt solid phases, as in prior work (Wells et al. 2007, Poloski et al. 2007), with non-salt solids being the primary focus. New information has been used to update and improve the non-salt-solid-phase definitions used in prior work. In addition, this section discusses gaps in the information that is available to define solid phases.

A hybrid approach was taken to identify and quantify the UDS phases present in the Hanford tanks. The solids predicted by the ESP $^{(\mathrm{b})}$ chemical thermodynamic model from 2002 BBI composition information were taken as a first approximation of the phases present. Overview documents were then reviewed to find information on what solid phases were actually present in the tank wastes. These documents included Rapko and Lumetta (2000), the five solids characterization reports from the M12 project (Lumetta et al. 2009a, Snow et al. 2009, Fiskum et al. 2008, Edwards et al. 2009, Fiskum et al. 2009a), and the 2010 waste mineralogy report (Disselkamp 2010). The M12 project was performed in response to issue M12 (Undemonstrated Leaching Processes) as identified by the EFRT, which conducted an in-depth review of the process flowsheet of the WTP, have been used as a source of new data on sludge particle types. ${ }^{(c)}$ The UDS phase predictions that were made, based on the solids characterization information in the documents, were reviewed and revised by a panel of experts (Appendix B).

\subsubsection{Information Gaps}

The gaps in available data (or in data interpretation) that pertain to the definition of solid phases are summarized below. In some cases, existing observations and measurements might answer the questions posed, if the existing data were to be collected together and compared from tank to tank. In other cases, new measurements on existing or new samples, might be needed.

\section{Mixed Phases}

Many solid phases in the non-salt part of the waste are composed of mixtures of compounds of different metals. The fraction of the solids present in the mixed phases in each tank or waste type is not known, nor are the density and size distribution of the mixed solid phases. The mixtures of metals that have been observed in Hanford tank wastes include Al-Cr, Fe-Cr-Ni-Mn, Fe-Pb, Fe-Bi-P, Fe-Zr, and others.

(a) UDS; undissolved solids. Those solids, whether soluble or insoluble, that are present as a solid phase and are not dissolved in the liquid phase of the waste.

(b) ESP was supplied and developed by OLI Systems, Inc., Morris Plains, New Jersey (OLI 1998).

(c) CCN 132846. 2006. Comprehensive Review of the Hanford Waste Treatment Plant Flowsheet and Throughput - Assessment Conducted by an Independent Team of External Experts. Chartered by the Hanford Waste Treatment and Immobilization Plant Project at the Direction of the U.S. Department of Energy, Office of Environmental Management, Washington, DC. 
Some examples of mixed phases, though certainly not an exhaustive list, were found in the characterization studies carried out for the M12 task:

- High-bismuth $\mathrm{BiPO}_{4}$ sludge (Group 1, Lumetta et al. 2009a): Fe was a major part of the washed solids composition. No crystalline Fe phases were identified by X-ray diffraction (XRD), but an amorphous phase that was a dominant part of the solids was identified as a mixture of hydrous Fe(III) phosphate, $\mathrm{BiFeO}_{3}$, and small amounts of $\mathrm{BiPO}_{4}$ by using a combination of Fourier transform infrared spectroscopy (FTIR), mapping with scanning electron microscopy and energy dispersive X-ray spectroscopy (SEM/EDS), transmission electron microscopy (TEM), and thermogravimetric analysis (TGA) (Lumetta et al. 2009b).

- High-phosphate $\mathrm{BiPO}_{4}$ saltcake (Group 2, Lumetta et al. 2009a): SEM/EDS mapping of the washed solids showed an association of $\mathrm{Na}, \mathrm{Al}, \mathrm{Si}$, and $\mathrm{P}$ that was thought to indicate cancrinite (sodium aluminosilicate) with some entrained sodium mono-H phosphate. Some aluminosilicates were found to have incorporated transition metals ( $\mathrm{Cr}$ and $\mathrm{Fe}$ ). $\mathrm{Cr}, \mathrm{Ca}, \mathrm{U}$, and Fe were evenly distributed across SEM/EDS maps, suggesting that these were present as oxides or hydroxides mixed together. However, Fe and $\mathrm{U}$ were also observed in separate particles.

- High-aluminum plutonium uranium extraction (PUREX) cladding sludge (Group 3, Snow et al. 2009): A minimal amount of amorphous material was present in the washed solid. Different phases were usually found in separate particles, not mixed. One exception was $\mathrm{Zr}$, which was most often found associated with $\mathrm{U}$ and $\mathrm{Fe}$, with the $\mathrm{Zr} / \mathrm{U}$ ratio being variable.

- High-aluminum reduction oxidation (REDOX) cladding sludge (Group 4, Snow et al. 2009): The washed solids were primarily composed of gibbsite. EDS analyses indicated that some agglomerate particles contained a mixture of phases. One particle was a mixture of an amorphous Fe compound, $\mathrm{Ca}-\mathrm{U}$ oxide, and cancrinite. An EDS probe of a region of another material showed $\mathrm{Al}, \mathrm{Si}, \mathrm{Pb}, \mathrm{Fe}, \mathrm{Ca}$, $\mathrm{U}, \mathrm{Cr}, \mathrm{Mn}$, and P. It was not clear whether the U-containing phases that were found also contained other elements.

- High-aluminum REDOX sludge (Group 5, Fiskum et al. 2008): The report did not explicitly discuss any mixed phases in this waste, but stated that about $60 \%$ the $\mathrm{Cr}$ phase was apparently entrained in boehmite, based on parallel $\mathrm{Al}$ and $\mathrm{Cr}$ leach behavior.

- High-chromium REDOX saltcake (Group 6, Fiskum et al. 2008): The washed solids were described as being primarily a mixed amorphous agglomerate of Al-Cr phase with $\mathrm{Cr}$ (at small particle size) scattered throughout the matrix. In many cases, Fe and Mn were present at the same discrete locations as Cr.

- Tributyl phosphate (TBP) sludge (Group 7, Edwards et al. 2009): A significant number of the washed solid particles tended to form agglomerates with a complex composition including $\mathrm{O}, \mathrm{Na}, \mathrm{Al}$, $\mathrm{P}, \mathrm{Ca}$ and Fe. Gibbsite was also found in separate particles. TEM images showed that an Fe-Ca phosphate occurred as a finely divided phase that was tenaciously attached to larger crystals that were rich in Fe and U. TEM also showed iron and uranyl phosphates that were separate in some cases and mixed in others.

- FeCN sludge (Group 8, Fiskum et al. 2009a): The washed solid particles tended to form multi-component agglomerates with a range of particle densities, sizes, and morphologies represented in them. The major elements present, based on SEM/EDS analysis, were $\mathrm{O}, \mathrm{Al}$, and $\mathrm{Fe}$, with $\mathrm{Ca}, \mathrm{Na}$, $\mathrm{Si}, \mathrm{P}, \mathrm{U}, \mathrm{Ni}$, and sometimes $\mathrm{Sr}$ and $\mathrm{Bi}$ present in smaller proportions. Scanning transmission electron 
microscopy (STEM)/high-angle annular dark field (HAADF) identified Fe oxide, U oxide, Sr phosphate, and Fe-Ni phases.

Some of the mixed phases were formed by co-precipitation resulting in inclusion of impurities or occlusion of one particle within another. As a result, the mixed phases consist of non-stoichiometric ratios of various metals. Thus, the proportions of the compounds present may vary from tank to tank. The densities of the mixed phases, which are between those of the individual compounds in the mixture, will vary correspondingly. The presence of mixed phases therefore introduces a potentially large uncertainty in density. If the size distribution of mixed phases differs from the distribution expected for the average of the individual phases in the mixture, there could be an uncertainty in size distribution as well.

For example, $10 \mathrm{wt} \%$ of $\mathrm{Al}(\mathrm{OH})_{3}$ plus $10 \mathrm{wt} \%$ of $\mathrm{Cr}_{2} \mathrm{O}_{3}$ would have a mixture density of about $3.3 \mathrm{~g} / \mathrm{mL}$ and so would produce $20 \mathrm{wt} \%$ of solid that settles at a corresponding rate. If those two phases were completely separate, then $10 \mathrm{wt} \%$ each would settle at rates corresponding to particle densities of $2.4 \mathrm{~g} / \mathrm{mL}$ and $5.2 \mathrm{~g} / \mathrm{mL}$. The more mass that is present in mixtures rather than single compounds, the more the particles will tend to settle (or be mixed or be transported) at intermediate average densities rather than at the relatively low and high densities of the separate compounds.

Some questions that need to be addressed are

- What proportion of each metal is present in mixed phases and what proportion is in identified individual phases?

- How do these mixed phases differ in size distribution from the individual compounds present in them? Mixed-phase PSDs could differ from those of the individual compounds when not mixed because different processes could have formed them. Mixed-phases that are amorphous are likely to have been formed by very rapid precipitation, a process that does not allow crystals to form and produces sub-micron primary particles. A small particle size is not guaranteed, however, because particles may be cemented together.

- Are there any apparent mixing rules? For example, in $\mathrm{Al}-\mathrm{Cr}$ mixed phases, is the ratio of $\mathrm{Cr}$ to $\mathrm{Al}$ always small? Mixing rules, if they exist, would mean that mixed-phase densities could be estimated with less uncertainty.

To some extent, these questions could be addressed by reviewing existing observations and cross-comparing findings from particle observations (XRD, SEM/EDS, TEM, FTIR, etc.) with the results of dissolution experiments, TGA curves, and other indirect evidence. These types of information are often in reports written by different authors at different times for different purposes. To the extent that this is the case, existing information needs to be collated and viewed as a whole.

The M12 solids characterization reports provide a variety of detailed information on waste types that, taken together, represent about $75 \mathrm{wt} \%$ of the $\mathrm{Al}, \mathrm{Cr}$, and phosphate inventory that will remain in water-washed solids and require leaching. The information in the present report should be used to evaluate which waste types represent the greatest risk in terms of mixing and transport so that existing samples of those wastes can undergo the same type of study if information is not already available to be collated. 


\section{Extent of Hydration}

The present extent of hydration of some phases that originally precipitated as hydroxides has been questioned. $^{\text {(a) }}$ For example, iron is a major contributor to insoluble solids in Hanford tank wastes; it is present in all tanks and iron phases make up more than $20 \%$ of the insoluble solid mass in more than 30 tanks. Iron has been observed in wastes in various crystalline forms such as oxides $\left(\mathrm{Fe}_{2} \mathrm{O}_{3}\right.$, either hematite or magnetite) and partially hydrated goethite (FeOOH). In one case, Group 2 washed solids, TGA indicated that Fe was present in all three degrees of hydration, goethite, magnetite, and ferric hydroxide (Lumetta et al. 2009a). However, amorphous phases of Fe and other metal elements have also been observed and may be present in significant quantities.

Amorphous phases cannot be identified by XRD. They may be hydroxides, which are frequently amorphous or only slightly crystallized. Some of the phases in question are $\mathrm{Fe}(\mathrm{OH})_{3}, \mathrm{FeOOH}$, and $\mathrm{Fe}_{2} \mathrm{O}_{3}$ for iron, $\mathrm{Cr}(\mathrm{OH})_{3}, \mathrm{CrOOH}$, and $\mathrm{Cr}_{2} \mathrm{O}_{3}$ for chromium, $\mathrm{Bi}(\mathrm{OH})_{3}$, and $\mathrm{Bi}_{2} \mathrm{O}_{3}$ for bismuth, $\mathrm{Na}_{2} \mathrm{U}_{2} \mathrm{O}_{7}$, $\mathrm{NaUO}_{2} \mathrm{OOH}, \mathrm{UO}_{3}, \mathrm{U}_{3} \mathrm{O}_{8}$, and $\mathrm{UO}_{2}$ for uranium, and $\mathrm{Zr}(\mathrm{OH})_{4}, \mathrm{ZrO}(\mathrm{OH})_{2}$, and $\mathrm{ZrO}_{2}$ for zirconium. The densities range from 3 to $4 \mathrm{~g} / \mathrm{mL}$ for hydroxides up to $5.2 \mathrm{~g} / \mathrm{mL}$ for $\mathrm{Fe}_{2} \mathrm{O}_{3}, 8.9 \mathrm{~g} / \mathrm{mL}$ for $\mathrm{Bi}_{2} \mathrm{O}_{3}$, and 11.0 for $\mathrm{UO}_{2}$. Assuming that these amorphous phases are anhydrous oxides would significantly overestimate the densities of any hydroxides that are present. Conversely, assigning the metals to hydroxide phases would underestimate the densities of anhydrous oxide phases.

The relative importance of hydroxides versus oxyhydroxides versus oxides needs to be better defined. Many of the observed amorphous phases that might be hydroxides are also mixed phases, so an examination of the mixed phases should include a direct determination of their particle density, or some form of thermogravimetric testing that can define the extent of hydration. The compounds in mixed phases could be either more or less dehydrated than those in single compounds, depending on whether the mixed structure tends to interfere with solid-state reactions (such as $\mathrm{Fe}(\mathrm{OH})_{3} \rightarrow \mathrm{Fe}_{2} \mathrm{O}_{3}$ ) and dissolution/re-precipitation (such as $\mathrm{Fe}(\mathrm{OH})_{3} \rightarrow \mathrm{FeOOH}$ ).

It would be worth reviewing the tanks in which the oxide phases have been found to see whether a disproportionate number of them have histories of high waste temperature. The temperature increases the rate of the dehydration reactions that can occur during metal hydroxide aging.

\subsubsection{Definition of In-Tank Solid Phases}

As for the studies reported by Wells et al. (2007) and Poloski et al. (2007), the output of ESP modeling of the wastes in all 177 tanks was the basic data source. The May 2002 BBIs were used as whole-tank-average composition inputs to the ESP model, which uses thermodynamic data to calculate the liquid- and solid-phase compositions at equilibrium. This modeling effort (Cowley et al. 2003) was carried out to support the development of a tank-by-tank toxic source term for use in tank farm safety analyses.

In some cases, non-salt phases not predicted in ESP but observed by microscopic analysis were added in a manner that maintained the mass balances on the main analytes in the phases. In other cases, non-salt phases were removed because they were present only in trace quantities.

(a) In this report, "hydration" refers not only to bound waters of hydration but to the water that can potentially be removed by reactions that convert hydroxides to oxyhydroxides or oxyhydroxides to anhydrous oxides. 


\section{ESP Solids Basis}

The ESP predictions constitute the only solid-phase composition information that 1 ) is available for all 177 tanks, and 2) was prepared using a consistent method for all 177 tanks. Although it was advantageous to draw on this database, this application of ESP had certain limitations from a mineralogical point of view:

- Compositions were calculated on a whole-tank basis, as if all the different layers of waste had been mixed and allowed to come to equilibrium. The average composition that was input to ESP was a weighted average calculated from layer volumes, densities, and analyte concentrations.

- ESP required charge-balanced composition inputs, but the BBIs were not charge-balanced. Elements that were not of primary toxicological concern were adjusted to provide a charge balance (Appendix D of Cowley et al. 2003). The concentrations that were most often changed were those of $\mathrm{Na}, \mathrm{K}$, and $\mathrm{OH}$, but in some cases, other metals and anions were affected.

- Because the goal of the Cowley et al. study was to obtain toxicological risk factors, and some conservatism was considered acceptable, charge balances frequently erred in the direction of overpredicting hydroxide. The $\mathrm{OH}$ concentration had a relatively minor effect on toxicological risk, compared to other species present, but has more effect on the nature and density of the phases present in the solid.

- ESP, as an equilibrium model, is not expected to predict the correct concentration of any compounds that have not yet come to equilibrium with an in-tank chemical environment different from those in which they formed (e.g., different temperature, $\mathrm{pH}$, etc.). In this sense, ESP predicts the phases to be found in completely aged waste.

- Some compounds were excluded from precipitating to reflect kinetic limitations, to reduce computational time, or to avoid nonconvergence of the solution algorithm. As one significant example, boehmite was excluded. Boehmite is thermodynamically preferred to gibbsite only at low water concentrations and temperatures above $100^{\circ} \mathrm{C}$. It exists in tank wastes only because it was formed at historically high temperatures and has been kinetically limited from transforming to gibbsite. Because the presence of boehmite could not be modeled by ESP, which models thermodynamic equilibrium, it was excluded from the ESP-modeled solids database because including it would have increased computational time with no gain.

- Because of computational time constraints, the REDOX equilibrium was not calculated on a tank-by-tank basis in the 2002 study. Rather, expert judgment and generic-composition runs of ESP were used to fix the metal oxidation states in all tanks (Appendix C, Cowley et al. 2003). Iron was fixed as Fe(III), manganese as $\mathrm{Mn}(\mathrm{II})$, chromium as partly $\mathrm{Cr}(\mathrm{III})$ and partly $\mathrm{Cr}(\mathrm{VI})$, uranium as U(VI), and so forth. The ESP predictions could not include compounds formed by metals in any other oxidation states.

- The study assigned solid compounds to the trace analytes (including thorium, cadmium, copper, tin, and many others) without employing the ESP model; thus, these metals are not present in the compounds in the ESP-predictions database.

- Thermodynamic data were not available for all the compounds that could potentially form in the tank waste, which led to the omission of some compounds. 
The waste in a number of tanks has been retrieved since 2002, leading to changes in both the retrieved and receiver tank compositions. In cases where waste was diluted with $0.01 \mathrm{M} \mathrm{NaOH}$ but not leached with strong caustic or other chemicals, the composition of the non-salt part of the waste would not have been changed substantially, though much of the salt would have dissolved. In rare cases leaching - the oxalic acid addition to the C-106 heel or the 19 M caustic addition to the S-112 heel-is known to have changed the compositions of non-salt compounds. Because most of the leaching, dilution, and transfer took place after 2002, a prominent exception being SY-101, the non-salt solid compositions are more representative of the original waste types than post-retrieval compositions would be.

\section{Adjustments to ESP Solids}

The set of solid compounds predicted by the ESP model was recognized to be incomplete. Therefore, several reports were reviewed that gave overviews of the available information on waste mineralogy. Many tank-specific reports are available, but these were reviewed only in a few cases, not systematically.

The overview documents included Rapko and Lumetta (2000), the five solids characterization reports from the M12 task, and the 2010 waste mineralogy report (Disselkamp 2010). Table 3.6 summarizes the phases that were predicted and those that have been observed. The densities in the table are taken from Wells et al. (2007), CRC (1975), or (as a last resort) the website webmineral.com.

Table 3.6. Overview of Predicted and Observed Non-Salt Compounds and Their Densities

\begin{tabular}{|c|c|c|}
\hline Element & $\begin{array}{l}\text { Maximum ESP-Predicted wt\% in Non-Salt } \\
\text { Solids, and Tank Where Maximum is Predicted }\end{array}$ & Observed Phases $<$ Density $(\mathrm{g} / \mathrm{mL})>^{(\mathrm{a})}$ \\
\hline $\mathrm{Ag}$ & $\mathrm{Ag}_{2} \mathrm{CO}_{3}: 0.41 \%, \mathrm{AW}-105$ & $\begin{array}{l}\text { Ag oxide }<7.1> \\
\text { (Ag,Hg) oxide }<\text { NA }> \\
\text { Ag (zero-valence) }<10.5>\end{array}$ \\
\hline $\mathrm{Al}$ & $\begin{array}{l}\mathrm{Al}(\mathrm{OH})_{3}: 99.4 \%, \mathrm{AP}-108 \\
\left(\mathrm{NaAlO}_{2}\right)_{2} \cdot 2.5 \mathrm{H}_{2} \mathrm{O}: 63.0 \%, \mathrm{AN}-103 \\
\mathrm{NaAlCO}_{3}(\mathrm{OH})_{2}: 91.2 \%, \mathrm{TY}-102 \\
\mathrm{NaAlSiO}_{4}: 91.8 \%, \mathrm{BY}-111 \\
\mathrm{KAlSiO}_{4}: 34.7 \%, \mathrm{AW}-101\end{array}$ & $\begin{array}{l}\text { gibbsite }<2.4> \\
\text { aluminosilicates }<2.4-2.8> \\
\text { boehmite }<3.0> \\
\text { compounds of } \mathrm{Al} \text { and } \mathrm{Cr}, \mathrm{Fe}, \mathrm{U}, \mathrm{Bi} \text {, or } \\
\text { mixtures of their compounds }<\mathrm{NA}> \\
\mathrm{Al} \text { phosphates }<1.8-2.6> \\
\text { Na-Al phosphates }<\mathrm{NA}> \\
\text { other hydroxides }<2.4-2.5> \\
\text { diaspore }<3.4> \\
\mathrm{NaAlCO}_{3}(\mathrm{OH})_{2}<2.4> \\
\mathrm{Na}_{3} \mathrm{AlF}_{6}<3.0> \\
(\mathrm{NaAlO})_{2} \cdot 2.5 \mathrm{H}_{2} \mathrm{O}<2.5> \\
(\mathrm{Ca}, \mathrm{Sr})_{3}(\mathrm{Cr}, \mathrm{Al})_{2}(\mathrm{OH})_{12}<\sim 3>\end{array}$ \\
\hline $\mathrm{Bi}$ & $\begin{array}{l}\mathrm{Bi}_{2} \mathrm{O}_{3}: 53.1 \%, \mathrm{~T}-201 \\
\text { BiOCl: 32.5\%, B-104 }\end{array}$ & $\begin{array}{l}\mathrm{BiFeO}_{3}<7.9> \\
\mathrm{BiPO}_{4}<6.3> \\
\mathrm{Bi}_{2} \mathrm{O}_{3}<8.9> \\
\text { compounds of Bi combined with } \mathrm{Al}, \mathrm{Cr}, \mathrm{Fe} \\
\text { compounds }<\mathrm{NA}>\end{array}$ \\
\hline
\end{tabular}


Table 3.6. (contd)

\begin{tabular}{|c|c|c|}
\hline Element & $\begin{array}{l}\text { Maximum ESP-Predicted wt \% in Non-Salt } \\
\text { Solids, and Tank Where Maximum is Predicted }\end{array}$ & Observed Phases $<$ Density $(g / m L)>^{(a)}$ \\
\hline $\mathrm{Ca}$ & $\begin{array}{l}\mathrm{Ca}_{5} \mathrm{OH}\left(\mathrm{PO}_{4}\right)_{3}: 18.9 \%, \mathrm{C}-112 \\
\mathrm{CaCO}_{3}: 7.8 \%, \mathrm{C}-201 \\
\mathrm{CaC}_{2} \mathrm{O}_{4} \cdot \mathrm{H}_{2} \mathrm{O}: 2.5 \%, \mathrm{C}-203 \\
\mathrm{Ca}(\mathrm{OH})_{2}: 3.6 \%, \mathrm{~A}-104 \\
\mathrm{CaF}_{2}: 8.4 \%, \mathrm{~B}-202\end{array}$ & $\begin{array}{l}\mathrm{Ca}_{5} \mathrm{OH}\left(\mathrm{PO}_{4}\right)_{3}<3.1> \\
\text { Other apatites }<\mathrm{NA}> \\
\mathrm{CaCO}_{3}<2.7> \\
\mathrm{CaC}_{2} \mathrm{O}_{4} \bullet \mathrm{H}_{2} \mathrm{O}<2.2> \\
\mathrm{Ca} \text { hydroxide (portlandite) }<2.2> \\
\text { Ca phosphates }<2.4> \\
\text { Ca sulfates }<2.3-3.0> \\
\text { Ca-U compounds or mixtures }<4.1> \\
\text { Ca-Cr compounds or mixtures }<\sim 3> \\
\text { compounds of Ca combined with Al } \\
\text { compound } \\
(\mathrm{Ca}, \mathrm{Sr})_{3}(\mathrm{Cr}, \mathrm{Al})_{2}(\mathrm{OH})_{12}<\sim 3>\end{array}$ \\
\hline $\mathrm{Cr}$ & CrOOH: 44.8\%, S-109 & $\begin{array}{l}\mathrm{Cr}_{2} \mathrm{O}_{3}<5.2> \\
\mathrm{CrOOH}<4.1> \\
\mathrm{FeCr}_{2} \mathrm{O}_{4}<4.8> \\
\text { oxides of } \mathrm{Cr} \text { with Fe and } \mathrm{Mn}<\sim 5> \\
\text { compounds of } \mathrm{Cr} \text { combined with } \mathrm{Al}, \mathrm{Ca} \\
(\mathrm{Ca}, \mathrm{Sr})_{3}(\mathrm{Cr}, \mathrm{Al})_{2}(\mathrm{OH})_{12}<\sim 3> \\
\mathrm{Cr} \text { phosphate }<\mathrm{NA}> \\
\mathrm{Cr}(\mathrm{OH})_{3}<\mathrm{NA}>\end{array}$ \\
\hline $\mathrm{Fe}$ & FeOOH: 63.6\%, A-104 & $\begin{array}{l}\mathrm{Fe}_{2} \mathrm{O}_{3}, \mathrm{Fe}_{3} \mathrm{O}_{4}<4.9-5.2> \\
\text { goethite } \mathrm{FeOOH}<4.3> \\
\mathrm{FeCr}_{2} \mathrm{O}_{4}<4.8> \\
\mathrm{Fe}_{2} \mathrm{MnO}_{4}<4.8> \\
\mathrm{BiFeO}_{3}<7.9> \\
\text { oxides of Fe with } \mathrm{Cr} \text { and } \mathrm{Mn}<4.8-5.0> \\
\text { compounds of Fe combined with } \mathrm{Bi}, \mathrm{Pb} \text {, } \\
\mathrm{Cr}<\mathrm{NA}> \\
\mathrm{Fe} \text { phosphates }<\sim 3> \\
\mathrm{Fe}(\mathrm{OH})_{3}<\sim 3> \\
\mathrm{Fe} \text { oxalates }<2.3> \\
\text { lepidocrocite } \mathrm{FeOOH}<4.0>\end{array}$ \\
\hline $\mathrm{Hg}$ & HgO: $0.25 \%$, TY-105 & (Ag,Hg) oxide $<\mathrm{NA}>$ \\
\hline $\mathrm{La}$ & $\begin{array}{l}\mathrm{LaPO}_{4} \cdot 2 \mathrm{H}_{2} \mathrm{O}: 21.7 \%, \mathrm{~T}-203 \\
\mathrm{La}(\mathrm{OH})_{3}: 4.3 \%, \mathrm{~T}-201\end{array}$ & $\mathrm{La}_{4}\left(\mathrm{P}_{2} \mathrm{O}_{7}\right)_{3}<\mathrm{NA}>$ \\
\hline $\mathrm{Mn}$ & $\begin{array}{l}\mathrm{Mn}_{3}\left(\mathrm{PO}_{4}\right)_{2}: 19.2 \%, \mathrm{~T}-111 \\
\mathrm{Mn}(\mathrm{OH})_{2}: 21.6 \%, \mathrm{~B}-204 \\
\mathrm{MnCO}_{3}: 9.2 \%, \mathrm{~T}-109\end{array}$ & $\begin{array}{l}\mathrm{Fe}_{2} \mathrm{MnO}_{4}<4.8> \\
\text { other oxides and oxyhydroxides of } \mathrm{Mn} \\
\text { with } \mathrm{Fe} \\
<\mathrm{NA}> \\
\mathrm{Mn}_{2} \mathrm{CrO}_{4}, \mathrm{Mn}_{3} \mathrm{Cr}_{3} \mathrm{O}_{8}<5.0> \\
\mathrm{MnO}_{2}<5.0> \\
\mathrm{Mn}_{2} \mathrm{MnO}_{4}<5>\end{array}$ \\
\hline
\end{tabular}


Table 3.6. (contd)

\begin{tabular}{|c|c|c|}
\hline Element & $\begin{array}{l}\text { Maximum ESP-Predicted wt\% in Non-Salt } \\
\text { Solids, and Tank Where Maximum is Predicted }\end{array}$ & Observed Phases $<$ Density $(g / m L)>^{(a)}$ \\
\hline $\mathrm{Ni}$ & $\begin{array}{l}\mathrm{Ni}(\mathrm{OH})_{2}: 9.8 \%, \mathrm{AX}-102 \\
\mathrm{NiC}_{2} \mathrm{O}_{4} \cdot 2 \mathrm{H}_{2} \mathrm{O}: 4.3 \%, \mathrm{C}-203 \\
\mathrm{Ni}_{3}\left(\mathrm{PO}_{4}\right)_{2}: 1.0 \%, \mathrm{TY}-101\end{array}$ & $\begin{array}{l}\mathrm{Ni}_{3} \mathrm{O}_{2}(\mathrm{OH})_{4}<4> \\
\text { oxides or phosphates of Ni with Al, Cr, } \\
\mathrm{Fe}, \mathrm{Mn}<\mathrm{NA}>\end{array}$ \\
\hline $\mathrm{Pb}$ & $\begin{array}{l}\mathrm{Pb}_{3}\left(\mathrm{PO}_{4}\right)_{2}: 0.71 \%, \mathrm{C}-111 \\
\mathrm{~Pb}(\mathrm{OH})_{2}: 31.8 \%, \mathrm{C}-201 \\
\mathrm{PbCO}_{3}: 33.8 \%, \mathrm{C}-204\end{array}$ & $\begin{array}{l}\text { phosphates or oxides of } \mathrm{Pb} \text { with } \mathrm{Fe}, \mathrm{Mn}, \\
\mathrm{Ni}, \mathrm{Al}<\mathrm{NA}> \\
\mathrm{Pb}_{5}\left(\mathrm{PO}_{4}\right)_{3} \mathrm{OH}<7.2> \\
\mathrm{Pb}-\mathrm{Cl} \text { or } \mathrm{Pb}-\mathrm{O}<\mathrm{NA}>\end{array}$ \\
\hline $\mathrm{Pu}$ & $\mathrm{Pu}(\mathrm{OH})_{4}: 0.20 \%, \mathrm{TX}-118$ & $\mathrm{PuO}_{2}<11.4>$ \\
\hline Si & $\begin{array}{l}\mathrm{NaAlSiO}_{4}: 91.8 \%, \mathrm{BY}-111 \\
\mathrm{KAlSiO}_{4}: 34.7 \%, \mathrm{AW}-101 \\
\mathrm{SiO}_{2}: 54.4 \%, \mathrm{SX}-113\end{array}$ & $\begin{array}{l}\text { aluminosilicates }<2.4-2.8> \\
\mathrm{SiO}_{2}<2.6>\end{array}$ \\
\hline $\mathrm{Sr}$ & $\begin{array}{l}\mathrm{Sr}_{3}\left(\mathrm{PO}_{4}\right)_{2}: 1.5 \%, \mathrm{BX}-109 \\
\mathrm{SrCO}_{3}: 4.1 \%, \mathrm{BY}-104\end{array}$ & $\begin{array}{l}\mathrm{SrCO}_{3}<3.5> \\
\mathrm{NaSrPO}_{4} \cdot 9 \mathrm{H}_{2} \mathrm{O}<2.0> \\
\text { related phosphates }<\mathrm{NA}> \\
(\mathrm{Ca}, \mathrm{Sr})_{3}(\mathrm{Cr}, \mathrm{Al})_{2}(\mathrm{OH})_{12}<\sim 3>\end{array}$ \\
\hline Tc & $\mathrm{TcO}_{2}: 0.17 \%, \mathrm{~S}-109$ & not observed \\
\hline $\mathrm{U}$ & $\mathrm{Na}_{2} \mathrm{U}_{2} \mathrm{O}_{7}: 28.2 \%, \mathrm{BX}-109$ & $\begin{array}{l}\mathrm{Na}_{2} \mathrm{U}_{2} \mathrm{O}_{7}<5.6> \\
\mathrm{UO}_{2}, \mathrm{UO}_{3}, \mathrm{U}_{2} \mathrm{O}_{7}, \mathrm{U}_{3} \mathrm{O}_{8}<7.3-11.0> \\
\mathrm{NaUO}_{2} \mathrm{OOH}<6.4> \\
\mathrm{U} \text { and } \mathrm{U}-\mathrm{Al} \text { phosphates }<3.4-3.5> \\
\mathrm{Na}_{4} \mathrm{UO}_{2}\left(\mathrm{CO}_{3}\right)_{3}<3.8> \\
\mathrm{Ca}\left(\mathrm{UO}_{2}\right)_{3} \mathrm{CO}_{3} \mathrm{OH} \cdot 3 \mathrm{H}_{2} \mathrm{O}<4.1> \\
\mathrm{CaU}_{2} \mathrm{O}_{7}<\mathrm{NA}>\end{array}$ \\
\hline $\mathrm{Zr}$ & $\mathrm{ZrO}_{2}: 75.0 \%, \mathrm{AW}-105$ & $\begin{array}{l}\mathrm{ZrO}_{2}<5.7> \\
\text { oxides of } \mathrm{Zr} \text { with } \mathrm{Fe}, \mathrm{Mn}<\mathrm{NA}>\end{array}$ \\
\hline \multicolumn{3}{|c|}{$\begin{array}{l}\text { NA = "not available" } \\
\text { (a) Density is expressed to one decimal place, at most, because the purpose of this table is to show the range of } \\
\text { densities that have been observed. More precise densities are used in solid-phase calculations. Except where values } \\
\text { are noted as estimates with a “ " symbol, they are derived from measured values. }\end{array}$} \\
\hline
\end{tabular}

As a result of the mineralogical data review, the ESP-predicted, non-salt, solids set was modified to improve the match of the design-basis insoluble solids to observations. They do not address the limitations that were stated in Section 3.2.3.1 as information gaps, but provide an improved representation of the solids to the extent that non-mixed solid phases can do so.

The soluble salts were not modified, except that the quantities were changed as needed to account for modifications in non-salt species and to maintain an anion balance in the solids phase. However, in some cases, the modifications changed the solid-phase inventory of $\mathrm{Na}$, bound water, bound hydroxide, and/or $\mathrm{Cl}$. These changes in inventory are in addition to the changes that were made, for many tanks, in the original ESP inputs to obtain charge balance. Thus, the solid inventories of these species that are used in this study are not the same as those in the $2002 \mathrm{BBI}$. 
The most significant changes to solid phases were these:

- As in Wells et al. (2007), boehmite was substituted for gibbsite as the aluminum hydroxide/oxide phase in the tanks containing REDOX boiling waste.

- In tanks where most of the waste was bismuth phosphate (BP) sludge, $\mathrm{BiFeO}_{3}$ and $\mathrm{FePO}_{4} \cdot 2 \mathrm{H}_{2} \mathrm{O}$ were substituted for the ESP-predicted Bi and Fe phases.

- In tanks where most of the waste was BP saltcake, $\mathrm{FePO}_{4} \cdot 2 \mathrm{H}_{2} \mathrm{O}$ was substituted for the ESP-predicted Fe phase.

- In tanks where most of the waste was TBP sludge, $\mathrm{Na}_{2}\left(\mathrm{UO}_{2}\right)_{2}\left(\mathrm{PO}_{4}\right)_{2} \cdot 2 \mathrm{H}_{2} \mathrm{O}$ was substituted for the ESP-predicted U phase.

- $\mathrm{PuO}_{2}$ was substituted for $\mathrm{Pu}(\mathrm{OH})_{4}$ only in those tanks where Pu was known to have been added to the tank as $\mathrm{PuO}_{2}$, not in others where Pu co-precipitated as a trace contaminant of other phases.

The details of these and other conversions from the ESP solids set to the final set are tabulated in Table 3.7. A more detailed discussion of the bases for the final set of solid phases follows.

The modified set of non-salt solid phases was reviewed and revised by a panel of experts (Appendix B). The primary focus was on non-salt compounds; therefore, the salt phases predicted by ESP were used without expert review.

\section{Detailed Rationale for Solids Adjustments}

The tables in Appendix C contain lists of the non-salt phases that have been identified, or partially identified, for each of the metals that are part of the solids set. In general, the densities used in this section are taken from Wells et al. (2007), CRC (1975), or (as a last resort) the website webmineral.com.

Ag phases. Silver is a small contributor to sludge solids but was retained in the distribution because images of Ag-rich particles were available for three different tank wastes (AY-102, C-106, and SY-102) and because it is a high-density solid. In Wells et al. (2007), it was taken to be the ESP-predicted phase silver carbonate, with a crystal density of $6.077 \mathrm{~g} / \mathrm{mL}$. In this study, the predicted silver carbonate is modified to silver metal (zero valence) in tanks containing predominantly AR sludge and to silver oxide $\left(\mathrm{Ag}_{2} \mathrm{O}\right)$ in all other tanks. The AR tanks were A-104 and C-106.

Observations of Ag in solid phase are rare because it is a trace contributor. Zero-valence Ag was seen in as-received waste samples from C-106, which was 100\% AR waste type. Heel samples from C-103 and leached C-106 samples contained silver oxide.

Silver carbonate was converted to other forms by adding as much sodium carbonate salt to the solids as was needed to balance the loss of carbonate from the silver carbonate. No attempt was made to balance the sodium, oxide oxygen, or water of hydration that was added to the solids by the conversion.

Al phases. The treatment of $\mathrm{Al}$ phases, and the reasons for it, is nearly the same as given in Wells et al. (2007). The details can be found in that report. The following rules were used:

- As in Wells et al. (2007), dawsonite $\left(\mathrm{NaAlCO}_{3}(\mathrm{OH})_{2}\right)$, which was predicted by ESP, was left as is to reflect the chemical environment that made the carbonate thermodynamically possible. 
- As in Wells et al. (2007), the $\mathrm{Al}$ in the predicted $\mathrm{Al}(\mathrm{OH})_{3}$ was divided between gibbsite and boehmite (with the appropriate stoichiometric conversion to boehmite), depending on whether REDOX boiling waste had ever been added to the tank. The R1-boiling tanks were S-101, S-104, S-107, S-110, SX-103, SX-104, SX-107 through SX-115, and U-110 (Meacham 2003). In all other tanks, those without REDOX boiling waste, $\mathrm{Al}(\mathrm{OH})_{3}$ was considered to be gibbsite. The water lost from the solid phase by converting $\mathrm{Al}(\mathrm{OH})_{3}$ to $\mathrm{AlOOH}$ was not accounted for.

- Sodium aluminate, $\left(\mathrm{NaAlO}_{2}\right)_{2} \cdot 2.5 \mathrm{H}_{2} \mathrm{O}$, was predicted in only one tank (AN-103). As was done in Wells et al. (2007), it was assumed that on dilution, this phase would be transformed to an Al-equivalent amount of gibbsite, the $\mathrm{Al}$ phase present in wastes with lower hydroxide concentrations. The changes in water, sodium, and hydroxide balances implied by the conversion were not accounted for.

- The predicted sodium aluminosilicate $\left(\mathrm{NaAlSiO}_{4}\right)$ was left as is, in terms of its composition and mass, but was assigned the density of nitrate cancrinite $\left[\left(\mathrm{NaAlSiO}_{4}\right)_{6} \cdot\left(\mathrm{NaNO}_{3}\right)_{1.6} \cdot 2 \mathrm{H}_{2} \mathrm{O}\right.$ ], a phase whose composition and mass per mole $\mathrm{Al}$ are very similar to those of $\mathrm{NaAlSiO}_{4}$. In Wells et al. (2007), $\mathrm{NaAlSiO}_{4}$ was converted to an Al-equivalent mass of nitrate cancrinite. Several kinds of aluminosilicates have been observed in Hanford wastes, including nitrate cancrinite, carbonate cancrinites, and hydroxycancrinites. In general, these are expected to be less dense than the nominal $\mathrm{NaAlSiO}_{4}: 2.365 \mathrm{~g} / \mathrm{mL}$ for nitrate cancrinite, compared to $2.59 \mathrm{~g} / \mathrm{mL}$ for $\mathrm{NaAlSiO}_{4}$.

- The predicted potassium aluminosilicate $\left(\mathrm{KAlSiO}_{4}\right)$ was left as is. It was deleted from the 177-tank composition in Wells et al. (2007) because it was only a trace contributor in the all-tank composite. However, it is a significant contributor in some individual tanks and therefore is retained in this study.

It should be noted that boehmite has been observed in waste samples from tanks other than the R1-boiling set, including AN-102, AZ-101, AZ-102, C-106, C-109, and the Group 4 tanks (the U-200 tanks and U-105). One of these tanks (C-106) experienced steam bubbles before being emptied. It would be worth reviewing tank waste temperature histories to see whether there is a basis for expecting boehmite presence in tanks other than the R1-boiling set.

Aluminum-phosphorus phases have been observed in a number of tanks that contain $\mathrm{BiPO}_{4}$ or TBP sludge. The density of $\mathrm{AlPO}_{4}$ (as an example) is about $2.6 \mathrm{~g} / \mathrm{mL}$. The densities of aluminum phosphates and gibbsite are similar, so it was not felt to be important to convert gibbsite to an aluminum phosphate phase in the $\mathrm{BiPO}_{4}$ and TBP waste types.

Bi phases. The ESP model, as used in the 2002 study, predicted bismuth precipitation primarily as $\mathrm{Bi}_{2} \mathrm{O}_{3}$, with small amounts of $\mathrm{BiOCl}$ in some tanks. While a number of other bismuth phases have been observed in Hanford tank wastes, as shown in Table 3.6, BiOCl has not.

In Wells et al. (2007), it was assumed that $\mathrm{Bi}_{2} \mathrm{O}_{3}$ represented all the $\mathrm{Bi}$ phases present in the tanks. Since then, studies of $\mathrm{BiPO}_{4}$ sludge samples (Lumetta et al. 2009a, b) have established that an amorphous phase that was a dominant part of the solids in $\mathrm{BiPO}_{4}$ sludge samples was a mixture of hydrous $\mathrm{Fe}(\mathrm{III})$ phosphate, $\mathrm{BiFeO}_{3}$, and minor amounts of $\mathrm{BiPO}_{4}$. Small amounts of crystalline $\mathrm{BiPO}_{4}$ may also have been present, based on the XRD results.

Bismuth hydroxide (density $4.36 \mathrm{~g} / \mathrm{mL}$ ) has not been observed, but may be present, unidentified, in some amorphous solids. The hydroxide decomposes to oxide at $100^{\circ} \mathrm{C}$, a higher temperature than has 
been present in most Hanford waste tanks. The presence of anions can impede the conversion of bismuth hydroxide to $\mathrm{Bi}_{2} \mathrm{O}_{3}$ (Patil et al. 2005).

The present study assumes that $\mathrm{Bi}$ is present only as $\mathrm{Bi}_{2} \mathrm{O}_{3}$ except in tanks where $1 \mathrm{C}$ and $2 \mathrm{C}$ waste types (originally produced by the $\mathrm{BiPO}_{4}$ process) made up more than 60 vol\% of the bulk solid waste, according to the $2002 \mathrm{BBI}$. In those tanks, $\mathrm{Bi}_{2} \mathrm{O}_{3}$ and $\mathrm{BiOCl}$ were converted into a Bi-equivalent amount of $\mathrm{BiFeO}_{3}$. The amount of $\mathrm{BiFeO}_{3}$ that could be generated was limited by whether enough Fe was present in the tank solid-phase inventory to allow all the $\mathrm{Bi}$ to be converted. In other tanks, the $\mathrm{BiOCl}$ was converted into $\mathrm{Bi}_{2} \mathrm{O}_{3}$ and added to any $\mathrm{Bi}_{2} \mathrm{O}_{3}$ already present. The resulting changes in the oxygen and $\mathrm{Cl}$ inventories were not accounted for.

The conversion from $\mathrm{Bi}_{2} \mathrm{O}_{3}$ to $\mathrm{BiFeO}_{3}$ was not applied to tanks containing $\mathrm{BiPO}_{4}$ saltcake ( $\mathrm{BY}$ and $\mathrm{T}$ salt). The density of $\mathrm{BiFeO}_{3}$ is $7.9 \mathrm{~g} / \mathrm{mL}^{\text {(a) }} \mathrm{BiFeO}_{3}$ might be the main form of $\mathrm{Bi}$ in these saltcakes, but is not documented. Bismuth compounds were not observed in these saltcake types (Lumetta et al. 2009a), probably because $\mathrm{Bi}$ was a trace constituent in the solids remaining after the saltcake was washed for characterization tests. It was not considered necessary to modify the ESP-predicted Bi phase.

Ca phases. The Ca phases predicted by ESP are $\mathrm{Ca}_{5} \mathrm{OH}\left(\mathrm{PO}_{4}\right)_{3}, \mathrm{CaF}_{2}, \mathrm{CaCO}_{3}, \mathrm{Ca}(\mathrm{OH})_{2}$, and $\mathrm{CaC}_{2} \mathrm{O}_{4} \bullet \mathrm{H}_{2} \mathrm{O}$, in order of decreasing significance in tank inventories. Of these, $\mathrm{Ca}_{5} \mathrm{OH}\left(\mathrm{PO}_{4}\right)_{3}, \mathrm{CaCO}_{3}$, and $\mathrm{CaC}_{2} \mathrm{O}_{4} \cdot \mathrm{H}_{2} \mathrm{O}$ have been observed in the waste. In Wells et al. (2007), the species $\mathrm{Ca}_{5} \mathrm{OH}\left(\mathrm{PO}_{4}\right)_{3}$ and $\mathrm{CaF}_{2}$ were used to represent calcium phases. In the present study, all the Ca phases were left as predicted by ESP.

Cr phases. Chromium oxyhydroxide (CrOOH) was the only $\mathrm{Cr}(\mathrm{III})$ phase predicted by ESP to exist in the solid phase. This phase was a small contributor to the 177-tank composite; consequently, it was omitted from the set of phases in Wells et al. (2007). The phase is used as is in the present study.

Chromium is often found in mixed phases with other metals, particularly aluminum, iron, nickel, and manganese. Caustic leaching tests on REDOX sludge (Fiskum et al. 2008) suggested that about $60 \%$ of the solid-phase $\mathrm{Cr}$ was entrained in boehmite. The same report described the washed solids in S saltcake as being primarily a mixed amorphous agglomerate of Al-Cr phase with $\mathrm{Cr}$ (at small particle size) scattered throughout the matrix.

To the extent that $\mathrm{Cr}$ has been identified in single phases, it has appeared in all degrees of hydration: $\mathrm{Cr}(\mathrm{OH})_{3}, \mathrm{CrOOH}$, and $\mathrm{Cr}_{2} \mathrm{O}_{3}$ or $\mathrm{FeCr}_{2} \mathrm{O}_{4}$. Data taken in the $\mathrm{M} 12$ project tests also indicate that a range of $\mathrm{Cr}$ phases may be present, depending on waste type. Minimal $\mathrm{Cr}$ was removed from the washed $\mathrm{S}$ saltcake solids by caustic leaching with $3 \mathrm{M} \mathrm{NaOH}$ at $100^{\circ} \mathrm{C}$, and this unleached $\mathrm{Cr}$ was found by TGA to be at least $90 \%$ amorphous $\mathrm{Cr}_{2} \mathrm{O}_{3}$ (Fiskum et al. 2008). $\mathrm{BiPO}_{4}$ sludge also contained $\mathrm{Cr}$ that remained substantially undissolved (22\% dissolved) after caustic leaching with $3 \mathrm{M} \mathrm{NaOH}$ at $80^{\circ} \mathrm{C}$ (Lumetta et al. 2009a). Higher Cr caustic leach factors, above 60\%, were seen for REDOX cladding and PUREX cladding wastes (Snow et al. 2009) when leaching was carried out with $3 \mathrm{M} \mathrm{NaOH}$ at $80^{\circ} \mathrm{C}$. The higher leach factors could have been caused by $\mathrm{Cr}$ being present in a more leachable phase $\left[\mathrm{Cr}(\mathrm{OH})_{3}, \mathrm{CrOOH}\right.$, in smaller particles, or both.

(a) Fruth et al. (2007) state that $\mathrm{BiFeO}_{3}$ density is $7.495 \mathrm{~g} / \mathrm{mL}$ at $95 \%$ of theoretical density. 
There is no apparent rule for assigning degree of hydration according to waste type. A history of high waste temperatures could lead to less hydrated phases and should be checked to find out whether observations are in accord with this. As a compromise between low-density hydroxides and high-density oxides, all $\mathrm{Cr}$ is considered to be $\mathrm{CrOOH}$, an intermediate-density oxyhydroxide.

Fe phases. ESP uniformly predicted that Fe would precipitate as goethite (FeOOH). This prediction resulted from excluding $\mathrm{Fe}_{2} \mathrm{O}_{3}$ from the potential precipitates modeled by ESP because of the expectation that $\mathrm{FeOOH}$ was more commonly observed in tank sludges. Observations show Fe phases in all degrees of hydration and, in some cases, more than one valence: $\mathrm{Fe}(\mathrm{OH})_{3}$, $\mathrm{FeOOH}, \mathrm{Fe}_{2} \mathrm{O}_{3}, \mathrm{Fe}_{3} \mathrm{O}_{4}$, and spinels formed with $\mathrm{Mn}$ and $\mathrm{Cr}$. Observations of $\mathrm{Fe}(\mathrm{OH})_{3}$ are less frequent, possibly because it is amorphous and not identifiable by XRD. The low frequency of observation, therefore, does not prove that the compound occurs infrequently. The densities of the iron compounds range from about $3 \mathrm{~g} / \mathrm{mL}$ for $\mathrm{Fe}(\mathrm{OH})_{3}$ to $5.24 \mathrm{~g} / \mathrm{mL}$ for $\mathrm{Fe}_{2} \mathrm{O}_{3} \cdot{ }^{(a)}$

In general, rapid precipitation of ferric ion $\left(\mathrm{Fe}^{3+}\right)$ by base, which was the origin of much of the $\mathrm{Fe}$ precipitate in tank waste, leads to the formation of $\mathrm{Fe}(\mathrm{OH})_{3} \bullet \mathrm{xH}_{2} \mathrm{O}$. The hydroxide then ages into less hydrated forms, usually goethite $(\alpha-\mathrm{FeOOH})$ and hematite $\left(\alpha-\mathrm{Fe}_{2} \mathrm{O}_{3}\right)$. This aging proceeds more rapidly at higher temperatures and at higher $\mathrm{pH}(\mathrm{pH}=10$ or 11$)$. Hematite is favored over goethite for higher temperatures $\left(100^{\circ} \mathrm{C}\right.$ versus $\left.40^{\circ} \mathrm{C}\right)$, and goethite is favored at higher $\mathrm{pH}$. Increasing the $\mathrm{pH}$ from 7 to 11 increases the aging rate; that is, the rate at which ferric hydroxide changes to less hydrated forms (Baltpurvins et al. 1996).

Foreign ions (those other than ferric and hydroxide ions) have a variety of effects on precipitation and aging of ferric hydroxide, including direct precipitation of magnetite $\left(\mathrm{Fe}_{3} \mathrm{O}_{4}\right)$, the formation of magnetite by dissolution and reprecipitation, the hindrance or acceleration of $\mathrm{Fe}(\mathrm{OH})_{3}$ conversion to dehydrated phases, and so forth (Baltpurvins et al. 1996, Belleville et al. 1992, Kandori et al. 1992). Considering this complexity, it is probably not possible to use the in-tank chemical environment to make any universal rules about which phase of Fe would appear in each waste type.

Ferric phosphates and sodium iron phosphates have been observed in $\mathrm{BiPO}_{4}$ and TBP sludges. An amorphous $\mathrm{FePO}_{4} \cdot \mathrm{xH}_{2} \mathrm{O}$ phase was found to be the dominant form of iron in Group 1 samples, which were $\mathrm{BiPO}_{4}$ sludges (Lumetta et al. 2009a, b).

In Wells et al. (2007), Fe was assumed to be present as FeOOH. The current study assigns the Fe phase based on waste type. In tanks where 1C sludge, 2C sludge, BY saltcake, and/or T saltcake waste types (originally produced by the $\mathrm{BiPO}_{4}$ process) made up more than $60 \mathrm{vol} \%$ of the bulk solid waste, according to the $2002 \mathrm{BBI}$, the $\mathrm{Fe}$ in excess of that required for $\mathrm{BiFeO}_{3}$ is assumed to be present as $\mathrm{FePO}_{4} \cdot 2 \mathrm{H}_{2} \mathrm{O}$. In all other tanks, $\mathrm{Fe}$ is assumed to be $\mathrm{FeOOH}$ (a compromise between hydroxide and oxide).

The $\mathrm{PO}_{4}^{-3}$ that was used to convert $\mathrm{FeOOH}$ to $\mathrm{FePO}_{4} \cdot 2 \mathrm{H}_{2} \mathrm{O}$ was taken from phosphate and pyrophosphate salt phases to maintain a phosphate balance. The amount of ferric phosphate that could be produced by conversion of $\mathrm{FeOOH}$ was limited by the amount of $\mathrm{PO}_{4}^{-3} / \mathrm{P}_{2} \mathrm{O}_{7}^{-4}$ in the salt solid inventory.

(a) No reference was found for the density of $\mathrm{Fe}(\mathrm{OH})_{3}$. The density of $\mathrm{Fe}(\mathrm{OH})_{2}$ is $3.4 \mathrm{~g} / \mathrm{mL}$ (CRC 1975), and a comparison of $\mathrm{Fe}(\mathrm{II})$ chlorides and fluorides with the corresponding $\mathrm{Fe}(\mathrm{III})$ compounds shows that the $\mathrm{Fe}(\mathrm{III})$ compounds have lower densities. 
The changes in the inventories of water, hydroxide, oxide oxygen, and sodium that were caused by conversion were not accounted for.

Hg phases. Mercury, being a trace constituent, has seldom been observed in waste solids. It was observed once in the form of mercury oxide mixed with silver oxide in C-103 waste. It is plausible that mercury solid phases are not separate phases in tank waste, but are always present in mixed phases as a result of co-precipitation with the dominant solid phases. In this report, $\mathrm{Hg}$ is identified as the separate phase (HgO) predicted by ESP. It is assigned the density of $\mathrm{Ag}_{2} \mathrm{O}$, owing to observed co-precipitation. It was omitted from the solids set in Wells et al. (2007) because of its low concentration.

La phases. Lanthanum was predicted by ESP to exist in solid form as the phosphate $\left(\mathrm{LaPO}_{4} \cdot 2 \mathrm{H}_{2} \mathrm{O}\right)$ or hydroxide $\left(\mathrm{La}(\mathrm{OH})_{3}\right)$. The phosphate was chosen to represent La phases in Wells et al. (2007). In this study, the ESP-predicted La phases are maintained.

Mn phases. Three manganese phases were predicted by ESP: $\mathrm{Mn}_{3}\left(\mathrm{PO}_{4}\right)_{2}, \mathrm{Mn}(\mathrm{OH})_{2}$, and $\mathrm{MnCO}_{3}$. However, denser and more dehydrated Mn phases have been observed, including the Mn(IV) compound $\mathrm{MnO}_{2}$, the $\mathrm{Mn}$ (III) compound $\mathrm{MnOOH}$, manganese spinels such as $\mathrm{Mn}_{2} \mathrm{MnO}_{4}$, other spinels that include $\mathrm{Fe}$ or $\mathrm{Cr}$, and a mixed phase incorporating other metals and phosphorus.

In an aqueous environment containing no anions except hydroxide, one of the most stable solid phases of Mn over a wide $\mathrm{pH}$ range is $\mathrm{MnO}_{2}$ (Pourbaix 1974). $\mathrm{MnO}_{2}$ was not predicted by ESP to precipitate because $\mathrm{MnO}_{2}$ was excluded from the ESP allowable precipitate inputs to avoid the complexity of specifying redox reactions because $\mathrm{Mn}$ is only $\mathrm{Mn}(\mathrm{II})$ in solution but potentially other oxidation states in solid.

In Wells et al. (2007), all Mn was represented as the Mn-equivalent amount of $\mathrm{MnO}_{2}$. In the present study, the ESP-predicted $\mathrm{Mn}_{3}\left(\mathrm{PO}_{4}\right)_{2}$ is left as is; this reflects the direct observations of manganese in conjunction with phosphorus in a mixed phase. The predicted $\mathrm{Mn}(\mathrm{OH})_{2}$ and $\mathrm{MnCO}_{3}$ are converted to an Mn-equivalent amount of $\mathrm{MnO}_{2}$. The carbonate from $\mathrm{MnCO}_{3}$ is converted into $\mathrm{Na}_{2} \mathrm{CO}_{3} \cdot \mathrm{H}_{2} \mathrm{O}$ salt. The changes in the inventories of water, hydroxide, oxide oxygen, and sodium that were caused by conversion were not accounted for.

Ni phases. Three nickel phases were predicted by ESP: $\mathrm{Ni}(\mathrm{OH})_{2}, \mathrm{NiC}_{2} \mathrm{O}_{4} \cdot 2 \mathrm{H}_{2} \mathrm{O}$, and $\mathrm{Ni}_{3}\left(\mathrm{PO}_{4}\right)_{2}$. Observations of $\mathrm{Ni}$ phases are sparse but include $\mathrm{Ni}_{3} \mathrm{O}_{2}(\mathrm{OH})_{4}$, a mixed phase incorporating other metals and phosphorus, and another mixed phase identified as Fe-Cr-Ni-O-C. Thus, the observations provide partial support for all three of the ESP predictions. In Wells et al. (2007), all the Ni was represented as $\mathrm{Ni}(\mathrm{OH})_{2}$. In this study, the ESP-predicted phases are maintained, but the nickel oxalate (which has not been observed) is assigned the density of $\mathrm{FeOOH}$ because Ni and Fe phases are often mixed.

Pb phases. The $\mathrm{Pb}$ phases $\mathrm{PbCO}_{3}, \mathrm{~Pb}(\mathrm{OH})_{2}$, and $\mathrm{Pb}_{3}\left(\mathrm{PO}_{4}\right)_{2}$ were predicted by ESP. The few direct observations of $\mathrm{Pb}$ in waste solids include both mixed and single phases. The co-occurrence of $\mathrm{Pb}$ and $\mathrm{Fe}$ is common, and in tanks where wastes have low $\mathrm{Pb} / \mathrm{Fe}$ ratios, it would be plausible to treat the $\mathrm{Pb}$ phase as having (effectively) the density of the Fe phase. Lead phosphates and oxides or hydroxides, and possibly chloride, have been observed in tank waste solids. In Wells et al. (2007), the Pb phases were ignored as key sludge solids because they were trace contributors to the 177-tank composite. In the present study, the ESP-predicted $\mathrm{Pb}$ solid phases are maintained. 
Pu phases. The ESP-predicted plutonium solid was plutonium hydroxide, $\mathrm{Pu}(\mathrm{OH})_{4} ; \mathrm{PuO}_{2}$ was not included in the model's database. Plutonium solids have been observed only in SY-102. More than 70\% of the Pu mass found in the solids in SY-102 samples contained only $\mathrm{Pu}$ and $\mathrm{O}$, and was considered to be $\mathrm{PuO}_{2}$ (Callaway and Cooke 2004). The remainder of the Pu-bearing solids were mixed phases that frequently included bismuth, phosphorus, and/or aluminum. Another reference ${ }^{(a)}$ reviews the history of SY-102 and concludes that that tank, and possibly TX-farm tanks, were unusual cases in that they received $\mathrm{PuO}_{2}$ scrap material from the Plutonium Finishing Plant. In all other tanks, Pu was a trace constituent and, as such, would have come out of solution not as a separate phase but as a coprecipitate of dominant solid phases.

In Wells et al. (2007), all the $\mathrm{Pu}$ in the sludge solids was assumed to be $\mathrm{PuO}_{2}$. In this study, only the $\mathrm{Pu}$ in SY-102 and the TX-farm tanks is converted from the ESP-predicted $\mathrm{Pu}(\mathrm{OH})_{4}$ to $\mathrm{PuO}_{2}$. In all other tanks, the $\mathrm{Pu}(\mathrm{OH})_{4}$ is left as is and assigned the density of FeOOH. This assignment reflects co-precipitation with a phase that is present in much greater concentrations, as is the case for FeOOH. Calculations are simplified by the fact that $\mathrm{FeOOH}$ is found in almost all tanks. The changes in the inventories of hydroxide and oxide oxygen that were caused by conversion from $\mathrm{Pu}(\mathrm{OH})_{4}$ to $\mathrm{PuO}_{2}$ were not accounted for.

Si phases. Most of the Si present in solids was predicted to take the form of aluminosilicate; the remainder was predicted to be $\mathrm{SiO}_{2}$. This prediction was considered acceptable because the local soil minerals (quartz, feldspar, and others) are known to have entered the tanks. As in Wells et al. (2007), the ESP-predicted phases for Si are maintained.

Sr phases. ESP predicted that $\mathrm{Sr}$ would be present as $\mathrm{SrCO}_{3}$ or $\mathrm{Sr}_{3}\left(\mathrm{PO}_{4}\right)_{2}$. The few direct observations of $\mathrm{Sr}$ in tank waste solids have been identified as $\mathrm{NaSr}\left(\mathrm{PO}_{4}\right) \cdot 9 \mathrm{H}_{2} \mathrm{O}, \mathrm{Ca}_{\mathrm{x}} \mathrm{Sr}_{10-\mathrm{x}}\left(\mathrm{PO}_{4}\right)_{6}(\mathrm{OH})_{2}$, $(\mathrm{Ca}, \mathrm{Sr})_{3}(\mathrm{Cr}, \mathrm{Al})_{2}(\mathrm{OH})_{12}$, and $\mathrm{Sr}\left(\mathrm{H}_{2} \mathrm{PO}_{3}\right)_{3}$. In Wells et al. (2007), the $\mathrm{Sr}$ phases were ignored as key sludge solids because they were trace contributors to the 177-tank composite. In the present study, the ESP-predicted Sr solid phases are maintained.

U phases. Many uranium phases that have a wide range of densities, from about $4 \mathrm{~g} / \mathrm{mL}$ to about $11 \mathrm{~g} / \mathrm{mL}$, have been observed in sludge wastes. Few other elements are found in solid phases with such a wide density range. Of these observed $U$ solids, ESP predicted that only $\mathrm{Na}_{2} \mathrm{U}_{2} \mathrm{O}_{7}$ would precipitate, owing partly to input constraints on the oxidation states of $U$ and the input limitations on which solids in the ESP database were allowed to precipitate. The directly observed $U$ solids include $\mathrm{Na}_{2} \mathrm{U}_{2} \mathrm{O}_{7}, \mathrm{UO}_{3}$, $\mathrm{UO}_{2}, \mathrm{NaUO}_{2} \mathrm{PO}_{4} \cdot 3 \mathrm{H}_{2} \mathrm{O}$, and other uranyl and sodium and aluminum uranyl phosphates, $\mathrm{Ca}\left(\mathrm{UO}_{2}\right)_{3} \mathrm{CO}_{3} \mathrm{OH} \cdot 3 \mathrm{H}_{2} \mathrm{O}$, and $\mathrm{Na}_{4} \mathrm{UO}_{2}\left(\mathrm{CO}_{3}\right)_{3}$. Uranium was observed only in association with phosphorous in samples of TBP sludge (Edwards et al. 2009). These compounds were not completely identified, but may have included $\mathrm{Al}\left(\mathrm{UO}_{2}\right)_{2}\left(\mathrm{PO}_{4}\right)_{2} \mathrm{OH} \bullet 8 \mathrm{H}_{2} \mathrm{O}, \mathrm{UO}_{2}\left(\mathrm{H}_{2} \mathrm{PO}_{2}\right)_{2} \cdot \mathrm{H}_{2} \mathrm{O}$, autunite $\left[\mathrm{Ca}\left(\mathrm{UO}_{2}\right)_{2}\left(\mathrm{PO}_{4}\right)_{2} \bullet 10-12\left(\mathrm{H}_{2} \mathrm{O}\right)\right]$, and meta-autunite $\left[\mathrm{Ca}\left(\mathrm{UO}_{2}\right)_{2}\left(\mathrm{PO}_{4}\right)_{2} \bullet 2-6\left(\mathrm{H}_{2} \mathrm{O}\right)\right]$.

All $\mathrm{U}$ was represented as $\mathrm{Na}_{2} \mathrm{U}_{2} \mathrm{O}_{7}$ in Wells et al. (2007). The present study assigns the $\mathrm{U}$ phase based on waste type. In tanks where TBP sludge made up more than $60 \mathrm{vol} \%$ of the bulk solid waste, according to the $2002 \mathrm{BBI}$, the $\mathrm{U}$ is assumed to be present as $\mathrm{Na}_{2}\left(\mathrm{UO}_{2}\right)_{2}\left(\mathrm{PO}_{4}\right)_{2} \cdot 2 \mathrm{H}_{2} \mathrm{O}$. In all other tanks, $\mathrm{U}$ is assumed to be $\mathrm{Na}_{2} \mathrm{U}_{2} \mathrm{O}_{7}$, which is one of the more frequently observed phases. The compound

(a) Herting DL. 2/18/2010. "Evaluation of Plutonium Settling in Pretreatment Vessels." CCN 211814, to RE Edwards, RPP-WTP. 
$\mathrm{Na}_{2}\left(\mathrm{UO}_{2}\right)_{2}\left(\mathrm{PO}_{4}\right)_{2} \cdot 2 \mathrm{H}_{2} \mathrm{O}$ was not specifically observed in the TBP sludge waste, but contains the same ratio of $\mathrm{U}$ to $\mathrm{PO}_{4}$ as the compounds posited by Edwards et al. (2009) and has a similar density.

The $\mathrm{PO}_{4}{ }^{-3}$ that was used to convert $\mathrm{Na}_{2} \mathrm{U}_{2} \mathrm{O}_{7}$ to $\mathrm{Na}_{2}\left(\mathrm{UO}_{2}\right)_{2}\left(\mathrm{PO}_{4}\right)_{2} \cdot 2 \mathrm{H}_{2} \mathrm{O}$ was taken from phosphate and pyrophosphate salt phases to maintain a phosphate mass balance. The amount of sodium uranyl phosphate that could be produced by this conversion was limited by the total amount of $\mathrm{PO}_{4}{ }^{-3} / \mathrm{P}_{2} \mathrm{O}_{7}^{-4}$ in the salt solid inventory. The changes in the inventories of water, hydroxide, oxide oxygen, and sodium that were caused by conversion were not accounted for.

Some rule is needed for assigning uranium oxide phases to uranium in certain waste types, or perhaps in tanks with a history of high waste temperature. The high densities of the uranium oxides should be accounted for.

Zr phases. The ESP model predicted $\mathrm{ZrO}_{2}$ as the sole $\mathrm{Zr}$ solid phase that would precipitate in tank sludge. This phase has been observed in more than one tank waste; $\mathrm{Zr}$-Fe mixed phases have also been observed. Zirconium hydroxide, $\mathrm{Zr}(\mathrm{OH})_{4}$ (density $3.25 \mathrm{~g} / \mathrm{mL}$ ), and zirconyl hydroxide, $\mathrm{ZrO}(\mathrm{OH})_{2}$, have not been observed but may be present, unidentified, in some amorphous solids. The hydroxide must be heated to $500^{\circ} \mathrm{C}$ before it decomposes to the $\mathrm{Zr}$ oxide, which suggests that $\mathrm{Zr}$ is not present in tank waste in a fully dehydrated form.

As did Wells et al. (2007), this study treats all $\mathrm{Zr}$ as $\mathrm{ZrO}_{2}$, the ESP-predicted compound. The presence of lower density forms of $\mathrm{Zr}$ should be investigated to provide a better representation of tanks where $\mathrm{Zr}$ is the predominant metal.

Other phases. Although technetium phases were included in the ESP modeling results from the 2002 study, they were considered to be trace contributors and were omitted from the composition of solids in the present study. The same approach was taken in Wells et al. (2007).

Effect on sodium inventories. The conversions performed on $\mathrm{Ag}, \mathrm{Al}, \mathrm{Fe}, \mathrm{Mn}$, and $\mathrm{U}$ phases caused changes in the Na inventories in 91 tanks. The total change in Na over all tanks was a decrease of 125 MT. About $99 \%$ of the decrease in Na came from the T and TX farms and was caused by removing sodium phosphate salts associated with the conversion of $\mathrm{FeOOH}$ to $\mathrm{FePO}_{4} \cdot 2 \mathrm{H}_{2} \mathrm{O}$. In most of the affected tanks, that change was less than $5 \%$ of the pre-conversion Na inventory, less than $100 \mathrm{~kg}$ (0.1 MT), or both. The exceptions were the following five tanks:

T-104: loss of 4.94 MT Na, 7\% of the pre-conversion inventory

T-107: loss of 20.55 MT of Na, $47 \%$ of the pre-conversion inventory

T-110: loss of $6.81 \mathrm{MT}$ of Na, $78 \%$ of the pre-conversion inventory

T-203: gain of $0.42 \mathrm{MT}$ of Na, 29000\% of the pre-conversion inventory (caused by converting $\mathrm{MnCO}_{3}$ to $\mathrm{MnO}_{2}$ )

TX-109: loss of $13.74 \mathrm{MT}$ of Na, $10 \%$ of the pre-conversion inventory. 
In the cases of T-104 and TX-109, the pre-conversion Na inventory was greater than the $2002 \mathrm{BBI}$ value because of changes made to obtain a charge balance (Table D-2 of Cowley et al. 2003). The T-104 pre-conversion Na inventory was 35\% greater than the 2002 BBI value, and TX-109 was 40\% greater. Thus, the reductions resulting from compound conversion in the current study are changes toward the original BBI Na inventory for T-104 and TX-109. In the cases of T-107, T-110, and T-203, no change was made to obtain charge balance, and the changes in the current study are changes away from the original BBI Na inventory. 
Table 3.7. Non-Salt Solid Phases to be Used in This Report

\begin{tabular}{|c|c|c|}
\hline Solid Phase Predicted by ESP & Change from ESP Prediction & Reason for Change \\
\hline $\mathrm{Ag}_{2} \mathrm{CO}_{3}$ in $\mathrm{AR}$ tanks & $\mathrm{Ag}_{2} \mathrm{CO}_{3} \rightarrow 2 \mathrm{Ag}+\mathrm{Na}_{2} \mathrm{CO}_{3} \cdot \mathrm{H}_{2} \mathrm{O}^{(\mathrm{a}, \mathrm{b})}$ & $\begin{array}{l}\mathrm{Ag}_{2} \mathrm{CO}_{3} \text { has not been observed. } \mathrm{Ag} \text { (zero valence) has } \\
\text { been, but only in as-received C-106 (100\% AR waste } \\
\text { type). }\end{array}$ \\
\hline $\mathrm{Ag}_{2} \mathrm{CO}_{3}$ in other tanks & $\mathrm{Ag}_{2} \mathrm{CO}_{3} \rightarrow \mathrm{Ag}_{2} \mathrm{O}+\mathrm{Na}_{2} \mathrm{CO}_{3} \cdot \mathrm{H}_{2} \mathrm{O}^{(\mathrm{a}, \mathrm{b})}$ & The few other Ag-phase observations have been $\mathrm{Ag}_{2} \mathrm{O}$. \\
\hline$\left(\mathrm{NaAlO}_{2}\right)_{2} \cdot 2.5 \mathrm{H}_{2} \mathrm{O}^{(\mathrm{c})}$ & $\left(\mathrm{NaAlO}_{2}\right)_{2} \cdot 2.5 \mathrm{H}_{2} \mathrm{O} \rightarrow 2 *$ gibbsite $^{(\mathrm{d}, \mathrm{e}, \mathrm{f})}$ & $\begin{array}{l}\text { Conversion to gibbsite is applied to account for } \\
\text { re-precipitation of sodium aluminate as gibbsite after } \\
\text { slurry is diluted with water. }\end{array}$ \\
\hline $\mathrm{Al}(\mathrm{OH})_{3}$ in R1-boiling tanks & $\mathrm{Al}(\mathrm{OH})_{3} \rightarrow \mathrm{AlOOH}^{(\mathrm{f})}$ & $\begin{array}{l}\text { Boehmite has been observed in the R1-boiling waste } \\
\text { tanks. }\end{array}$ \\
\hline $\mathrm{Al}(\mathrm{OH})_{3}$ in other tanks & $\mathrm{Al}(\mathrm{OH})_{3}=$ gibbsite & $\begin{array}{l}\text { Bayerite and nordstrandite, other forms of } \mathrm{Al}(\mathrm{OH})_{3} \text {, are } \\
\text { rarely observed. }\end{array}$ \\
\hline $\mathrm{NaAlCO}_{3}(\mathrm{OH})_{2}$ & none & $\mathrm{n} / \mathrm{a}$ \\
\hline $\mathrm{NaAlSiO}_{4}$ & none & $\mathrm{n} / \mathrm{a}$ \\
\hline $\mathrm{KAlSiO}_{4}$ & none & $\mathrm{n} / \mathrm{a}$ \\
\hline $\mathrm{Bi}_{2} \mathrm{O}_{3}$ in $1 \mathrm{C}$ and $2 \mathrm{C}$ tanks & $\mathrm{Bi}_{2} \mathrm{O}_{3}+2 \mathrm{FeOOH}$ (etc.) $\rightarrow 2 \mathrm{BiFeO}_{3}{ }^{(\mathrm{e}, \mathrm{f})}$ & $\begin{array}{l}\text { Bismuth phosphate sludge samples were observed to } \\
\text { contain bismuth iron oxide, with no significant amount } \\
\text { of } \mathrm{Bi}_{2} \mathrm{O}_{3} \text { or } \mathrm{BiPO}_{4} \text {. }\end{array}$ \\
\hline $\mathrm{BiOCl}$ in $1 \mathrm{C}$ and $2 \mathrm{C}$ tanks & $\mathrm{BiOCl}+\mathrm{FeOOH}$ (etc.) $\rightarrow \mathrm{BiFeO}_{3}^{(e, f, g)}$ & BiOCl has not been observed. \\
\hline $\mathrm{Bi}_{2} \mathrm{O}_{3}$ in other tanks & none & $\mathrm{n} / \mathrm{a}$ \\
\hline $\mathrm{BiOCl}$ in other tanks & $2 \mathrm{BiOCl} \rightarrow \mathrm{Bi}_{2} \mathrm{O}_{3}{ }^{(e, g)}$ & $\begin{array}{l}\text { BiOCl has not been observed, even in tanks where high } \\
\text { fractions of it are predicted. }\end{array}$ \\
\hline $\mathrm{Ca}(\mathrm{OH})_{2}$ & none & \\
\hline $\mathrm{Ca}_{5} \mathrm{OH}\left(\mathrm{PO}_{4}\right)_{3}$ & none & \\
\hline $\mathrm{CaF}_{2}$ & none & \\
\hline $\mathrm{CaC}_{2} \mathrm{O}_{4} \cdot \mathrm{H}_{2} \mathrm{O}$ & none & \\
\hline $\mathrm{CaCO}_{3}$ & none & \\
\hline $\mathrm{CrOOH}$ & none & \\
\hline
\end{tabular}


Table 3.7. (contd)

\begin{tabular}{|c|c|c|}
\hline Solid Phase Predicted by ESP & Change from ESP Prediction & Reason for Change \\
\hline $\begin{array}{l}\text { FeOOH in } 1 \mathrm{C}, 2 \mathrm{C} \text {, BY salt, and } \mathrm{T} \text { salt } \\
\text { tanks }\end{array}$ & $\begin{array}{l}\mathrm{FeOOH}+\mathrm{Na}_{3} \mathrm{PO} 4 \cdot 12 \mathrm{H}_{2} \mathrm{O} \rightarrow \mathrm{FePO}_{4} \cdot 2 \mathrm{H}_{2} \mathrm{O} \\
\text { (other phosphate and pyrophosphate salts also used to } \\
\text { supply } \mathrm{PO}_{4}^{-3} \text { ) }\end{array}$ & $\begin{array}{l}\text { Substantial amounts of amorphous iron phosphate were } \\
\text { observed in BP sludge and salt tank samples. }\end{array}$ \\
\hline FeOOH in other tanks & none & \\
\hline $\mathrm{HgO}$ & Assigned $\mathrm{Ag}_{2} \mathrm{O}$ density & $\begin{array}{l}\text { Hg has not been observed, except in association with } \\
\text { Ag. }\end{array}$ \\
\hline $\mathrm{La}(\mathrm{OH})_{3}$ & none & \\
\hline $\mathrm{LaPO}_{4} \cdot 2 \mathrm{H}_{2} \mathrm{O}$ & none & \\
\hline $\mathrm{Mn}(\mathrm{OH})_{2}$ & $\mathrm{Mn}(\mathrm{OH})_{2} \rightarrow \mathrm{MnO}_{2}^{(\mathrm{f})}$ & \multirow{2}{*}{$\begin{array}{l}\text { Mn(IV) is one of the most stable Mn oxidation states in } \\
\text { an aqueous environment. }\end{array}$} \\
\hline $\mathrm{MnCO}_{3}$ & $\mathrm{MnCO}_{3} \rightarrow \mathrm{MnO}_{2}+\mathrm{Na}_{2} \mathrm{CO}_{3} \cdot \mathrm{H}_{2} \mathrm{O}^{(\mathrm{a}, \mathrm{b}, \mathrm{e})}$ & \\
\hline $\mathrm{Mn}_{3}\left(\mathrm{PO}_{4}\right)_{2}$ & None & $\begin{array}{l}\text { Mn phosphate was not converted to } \mathrm{MnO}_{2} \text { because } \mathrm{Mn} \\
\text { has been observed in mixed phases together with } \mathrm{P} \text {. }\end{array}$ \\
\hline $\mathrm{Ni}(\mathrm{OH})_{2}$ & None & \\
\hline $\mathrm{Ni}_{3}(\mathrm{PO} 4)_{2}$ & none & \\
\hline $\mathrm{NiC}_{2} \mathrm{O}_{4} \cdot 2 \mathrm{H}_{2} \mathrm{O}$ & assigned FeOOH density & $\begin{array}{l}\text { Ni oxalate has not been observed, suggesting that if it is } \\
\text { present, it is in a mixed phase. Ni-Fe mixed phases are } \\
\text { common. }\end{array}$ \\
\hline $\mathrm{Pb}(\mathrm{OH})_{2}$ & none & \\
\hline $\mathrm{Pb}_{3}\left(\mathrm{PO}_{4}\right)_{2}$ & none & \\
\hline $\mathrm{PbCO}_{3}$ & none & \\
\hline $\mathrm{Pu}(\mathrm{OH})_{4}$ in SY-102 and TX tanks & $\mathrm{Pu}(\mathrm{OH})_{4} \rightarrow \mathrm{PuO}_{2}^{(\mathrm{f})}$ & $\begin{array}{l}\text { There is historical evidence that } \mathrm{PuO}_{2} \text { scraps were added } \\
\text { to SY-102, TX-118, and possibly other TX tanks. }\end{array}$ \\
\hline $\mathrm{Pu}(\mathrm{OH})_{4}$ in other tanks & Assigned FeOOH density & $\begin{array}{l}\text { In most tanks, Pu should appear as a trace co-precipitate. } \\
\text { In this study, it is assumed to co-precipitate with Fe, that } \\
\text { element being present in nearly all tanks. }\end{array}$ \\
\hline $\mathrm{SiO}_{2}$ & none & \\
\hline $\mathrm{Sr}_{3}\left(\mathrm{PO}_{4}\right)_{2}$ & none & \\
\hline $\mathrm{SrCO}_{3}$ & none & \\
\hline $\mathrm{Na}_{2} \mathrm{U}_{2} \mathrm{O}_{7}$ in TBP tanks & $\begin{array}{l}\mathrm{Na}_{2} \mathrm{U}_{2} \mathrm{O}_{7}+2 \mathrm{Na}_{3} \mathrm{PO}_{4} \cdot 12 \mathrm{H}_{2} \mathrm{O} \text { (etc.) } \\
\mathrm{Na}_{2}\left(\mathrm{UO}_{2}\right)_{2}\left(\mathrm{PO}_{4}\right)_{2} \cdot 2 \mathrm{H}_{2} \mathrm{O}^{(\mathrm{d}, \mathrm{e}, \mathrm{f})} \\
\text { (other phosphate and pyrophosphate salts also used to } \\
\text { supply } \mathrm{PO}_{4}^{-3} \text { ) }\end{array}$ & $\begin{array}{l}\text { TBP waste samples were observed to contain } U \\
\text { phosphate phases, not oxides or diuranate. }\end{array}$ \\
\hline
\end{tabular}


Table 3.7. (contd)

\begin{tabular}{|l|l|l|}
\hline \multicolumn{1}{|c|}{ Solid Phase Predicted by ESP } & \multicolumn{1}{c|}{ Change from ESP Prediction } & Reason for Change \\
\hline $\mathrm{Na}_{2} \mathrm{U}_{2} \mathrm{O}_{7}$ in other tanks & none & \\
\hline $\mathrm{ZrO}_{2}$ & none \\
\hline (a) Sodium is not conserved; it is added to the solid phase as a salt. & \\
(b) Water is not conserved; it is added to the solid phase. & \\
(c) Sodium aluminate is a soluble salt that is classed as insoluble for handling and treatment purposes because dilution with water will first dissolve the \\
aluminate and then re-precipitate it as insoluble aluminum hydroxide. \\
(d) Sodium is not conserved; it is removed from the solid phase. \\
(e) Bound hydroxide is not conserved. \\
(f) Water is not conserved; it is removed from the solid phase. \\
(g) Chloride is not conserved; it is removed from the solid phase.
\end{tabular}


The changes in Na inventory, which are changes in the salt solid phases, are not considered substantial for the purpose of settling, mixing, and pipeline flow calculations. The differences from the 2002 BBI values should be kept in mind if the inventories generated by this study are used for chemistry-related calculations.

\subsubsection{Definition of Fully Washed Solid Phases}

In some cases, mixing and transfer equipment will be handling slurries that have been diluted or washed with water or $0.01 \mathrm{M} \mathrm{NaOH}$. The slurry from any given vessel can vary through a range of liquid compositions and solid phases in the process of being diluted and washed to a fully washed condition. Typically the first effect of dilution and washing is to remove interstitial liquid and dissolve sodium salt solids that are fairly soluble and contain a single sodium atom. The main example is $\mathrm{NaNO}_{3}$. The common-ion effect of $\mathrm{Na}$ tends to prevent low-solubility, one-sodium salts, like $\mathrm{NaF}$, and salts containing two or more sodium atoms from dissolving until all the $\mathrm{NaNO}_{3}$ has dissolved, at which point the $\mathrm{Na}$ concentration in the liquid begins to fall. As dissolution continues, salts with smaller numbers of sodium atoms tend to dissolve earlier in the process. Throughout dissolution, the non-salt solid phases, being subjected to a changing liquid composition, may dissolve and reprecipitate as different compounds of the same metal.

This study does not consider the solids phases present in the intermediate steps of dilution and washing, though these slurries will have a significant impact on processing. The solids phases that are formed by chemical leaching with strong $\mathrm{NaOH}$, oxalic acid, etc., are also not considered here. We define a fully washed solids composition as one in which all the original solid-phase metals are present in the form of oxide or hydroxide. The original liquid phase that was in contact with the solids is not considered to have contributed any solids as a result of dilution-produced precipitation; e.g., the precipitation of aluminum hydroxide from solution as a result of diluting dissolved aluminate is not considered. It should be noted that this composition is not the same as that which results from applying the BBI wash factors, which is also referred to as "fully washed" in other contexts.

The fully washed slurry is assumed to be composed of water and solids with the fully washed composition. The composition of the insoluble solids at any point during washing would fall somewhere between the extremes of the original in-tank, non-salt material and the fully washed composition. Table 3.9 shows the fully washed solids and how they relate to the solids present before washing. Results are tabulated in Appendix C. 
Table 3.8. Fully Washed ${ }^{(a)}$ Solid Phases for This Report

\begin{tabular}{|c|c|}
\hline Solid Phase Before Washing & Fully Washed Phase \\
\hline $\mathrm{Ag}, \mathrm{Ag}_{2} \mathrm{O}$ & $\mathrm{Ag}_{2} \mathrm{O}$ \\
\hline Gibbsite & Unchanged \\
\hline Boehmite (AlOOH) & Unchanged \\
\hline $\mathrm{NaAlCO}_{3}(\mathrm{OH})_{2}$ & Gibbsite \\
\hline $\mathrm{NaAlSiO}_{4}$ & Unchanged \\
\hline $\mathrm{KAlSiO}_{4}$ & Unchanged \\
\hline $\mathrm{BiFeO}_{3}$ & Unchanged \\
\hline$\overline{\mathrm{Bi}_{2} \mathrm{O}_{3}}$ & Unchanged \\
\hline $\mathrm{Ca}(\mathrm{OH})_{2}$ & Unchanged \\
\hline $\mathrm{Ca}_{5} \mathrm{OH}\left(\mathrm{PO}_{4}\right)_{3}, \mathrm{CaF}_{2}, \mathrm{CaC}_{2} \mathrm{O}_{4} \cdot \mathrm{H}_{2} \mathrm{O}, \mathrm{CaCO}_{3}$ & $\mathrm{Ca}(\mathrm{OH})_{2}$ \\
\hline $\mathrm{CrOOH}$ & Unchanged \\
\hline $\mathrm{FePO}_{4} \cdot 2 \mathrm{H}_{2} \mathrm{O}$ & $\mathrm{FeOOH}$ \\
\hline $\mathrm{FeOOH}$ & Unchanged \\
\hline $\mathrm{HgO}$ & Unchanged \\
\hline $\mathrm{La}(\mathrm{OH})_{3}$ & Unchanged \\
\hline $\mathrm{LaPO}_{4} \cdot 2 \mathrm{H}_{2} \mathrm{O}$ & $\mathrm{La}(\mathrm{OH})_{3}$ \\
\hline $\mathrm{MnO}_{2}$ & Unchanged \\
\hline $\mathrm{Mn}_{3}\left(\mathrm{PO}_{4}\right)_{2}$ & $\mathrm{MnO}_{2}$ \\
\hline $\mathrm{Ni}(\mathrm{OH})_{2}$ & Unchanged \\
\hline $\mathrm{Ni}_{3}(\mathrm{PO} 4)_{2}, \mathrm{NiC}_{2} \mathrm{O}_{4} \cdot 2 \mathrm{H}_{2} \mathrm{O}$ & $\mathrm{Ni}(\mathrm{OH})_{2}$ \\
\hline $\mathrm{Pb}(\mathrm{OH})_{2}$ & Unchanged \\
\hline $\mathrm{Pb}_{3}\left(\mathrm{PO}_{4}\right)_{2}, \mathrm{PbCO}_{3}$ & $\mathrm{~Pb}(\mathrm{OH})_{2}$ \\
\hline $\mathrm{Pu}(\mathrm{OH})_{4}$ & Unchanged \\
\hline $\mathrm{PuO}_{2}$ & Unchanged \\
\hline $\mathrm{SiO}_{2}$ & Unchanged \\
\hline $\mathrm{Sr}_{3}\left(\mathrm{PO}_{4}\right)_{2}, \mathrm{SrCO}_{3}$ & $\mathrm{Sr}(\mathrm{OH})_{2}$ \\
\hline $\mathrm{Na}_{2}\left(\mathrm{UO}_{2}\right)_{2}\left(\mathrm{PO}_{4}\right)_{2} \cdot 2 \mathrm{H}_{2} \mathrm{O}$ & $\mathrm{Na}_{2} \mathrm{U}_{2} \mathrm{O}_{7}$ \\
\hline $\mathrm{Na}_{2} \mathrm{U}_{2} \mathrm{O}_{7}$ & Unchanged \\
\hline $\mathrm{ZrO}_{2}$ & Unchanged \\
\hline
\end{tabular}

\subsubsection{Selection of Tanks to Represent Waste Types}

For each of the waste types present in Hanford tanks, one or more tanks were identified that would best represent the insoluble solids in the waste type, based on the 2002 BBI. All the data used in tank selection were from the May 2002 BBI. Results are tabulated in Appendix C.

The representative tank for each sludge type was chosen using these criteria:

1. The waste type was the only type in the tank, or the only type that was sludge. If it was impossible to find a tank whose waste solids were $100 \%$ of the type, tanks where the other waste was saltcake alone (containing little mass of insoluble solids) were preferred to tanks where the other waste was sludge. 
Although waste of any given type may not have exactly the same composition in one tank as in another, it is preferable to choose "high-purity" specimens of the waste type, where possible. This makes the ESP predictions, which are based on the average waste composition in the entire tank, more appropriate.

2. There were direct measurements of metals and anions in the solids of the tank, obtained from core samples. Core samples (if still available) could be used in laboratory tests, including solid-phase characterization, rheological behavior, and settling velocity tests. In addition, the BBI compositions used in ESP inputs are likely to be more appropriate for tanks where actual compositions were measured, compared to those where compositions were based on waste stream estimates or data for the same waste type in other tanks.

3. The $\mathrm{pH}$ predicted by ESP was the closest available approximation to a reasonable value for the waste. The ESP runs were originally made to determine waste toxicity. Because hydroxide did not have a strong effect on toxicity, compared to other species, the $\mathrm{pH}$ was not fine-tuned and was allowed to be higher than expected in many cases because that bias provided conservatism. The solid-phase compounds predicted by ESP are more likely to be correct where the ESP-predicted pH (which ESP calculated from the predicted hydronium ion concentration) was closer to being correct.

Table 3.9 provides more detail of tank selection for sludge waste types and states the caveats that apply for the less well-represented waste types. The results of a corresponding analysis for saltcake waste types are given in Table 3.10. The same three criteria were used to select representative tanks for saltcake waste types as for sludge waste types.

The approach that was used to select representative tanks is similar to that used in the M12 solids characterization studies that were already cited. However, the present study does not necessarily select the same tanks because the M12 studies had somewhat different constraints and goals. Firstly, the M12 studies focused on sample selection and so were not constrained to use only tanks where all the waste was a single type. On the other hand, the M12 studies were constrained to use tanks for which samples were still available. Secondly, the M12 studies included leach tests and therefore required waste samples that contained high concentrations of species whose leaching was a technical concern, such as $\mathrm{Al}$, $\mathrm{Cr}$, or phosphate. This latter requirement was not part of the present study.

Not all waste types have adequately representative tanks. Poloski et al. (2007) discussed the sludge types found in the Hanford tanks with respect to those for which rheological data were or were not available. The present review found that several sludge types (B, CEM, CWZr1, OWW3, P1, SRR, TH1, $\mathrm{TH} 2$, and Z) were not reasonably represented by the average non-salt composition in any tank, owing to the small amounts present in any given tank. The same is true for the R1 saltcake and unidentified saltcake types.

The solid-phase compositions and average solid-particle densities for each waste type were calculated from the representative tank or tanks for the waste. In cases where the waste type was represented by the average of more than one tank, the average mass fraction of each component was calculated by summing the solid-phase component masses from all the tanks and dividing by the sum of the masses of all solid phases. The non-salt density was calculated as the sum of the non-salt masses over all solid phases and all tanks that are being averaged divided by the sum of non-salt volumes over all solid phases and all tanks. The salt density was calculated from 


$$
\rho_{\text {salt }}=\left(\frac{\left(1-\omega_{\text {non }}\right) \phi_{\text {non }}}{\left(1-\phi_{\text {non }}\right) \omega_{\text {non }}}\right) \rho_{\text {non }}
$$

where $\rho_{\text {salt }}=$ density of salt portion of solid

$\omega_{\text {non }}=$ mass fraction of non-salt in the solid

$\phi_{\text {non }}=$ volume fraction of non-salt solid

$\rho_{\text {non }}=$ density of non-salt portion of solid. 
Table 3.9. Tanks Identified to Represent Sludge Waste Types (Based on 2002 BBI)

\begin{tabular}{|c|c|c|c|c|}
\hline $\begin{array}{c}2002 \text { BBI } \\
\text { Waste Type }\end{array}$ & $\begin{array}{c}\text { Number of } \\
\text { Tanks in Which } \\
\text { Waste Type is } \\
\text { Present }\end{array}$ & $\begin{array}{c}\text { Total Bulk } \\
\text { Volume of } \\
\text { Waste Type } \\
\text { (kL) } \\
\end{array}$ & $\begin{array}{l}\text { Selected Representative } \\
\text { Tank for Insolubles }\end{array}$ & $\begin{array}{l}\text { Tanks Where Sludge is Predominantly This Type: } \\
\text { Information on Which Tank Choice Was Based }\end{array}$ \\
\hline $1 \mathrm{C}$ & 27 & 8572 & $\begin{array}{l}\text { Average of BX-107, } \\
\text { C-110, and T-104 }\end{array}$ & $\begin{array}{l}\text { BX-107, BX-112, C-110, T-104, and TX-109 solids are all completely 1C. } \\
\text { Of these, only TX-109 has no data from cores. BX-107 had predicted } \\
\mathrm{pH}=11 \text { where the measured } \mathrm{pH} \text { was } 10 \text {; BX-112 had a high predicted } \\
\mathrm{pH}>13 \text {; C- } 110 \text { had a predicted } \mathrm{pH} \text { of } 12+\text { where the measured } \mathrm{pH} \text { was } 11 \text {; } \\
\mathrm{T}-104 \text { had a predicted } \mathrm{pH} \text { of } 11 \text { where the measured } \mathrm{pH} \text { was } 10 \text {. } \\
\text { For comparison, the samples of } 1 \mathrm{C} / 2 \mathrm{C} \text { waste used in Group } 1 \text { testing } \\
\text { (Lumetta et al. 2009a) were taken from BX-112 and T-104. }\end{array}$ \\
\hline $2 \mathrm{C}$ & 9 & 5230 & $\begin{array}{l}\text { Average of B-110 and } \\
\text { T-110 }\end{array}$ & $\begin{array}{l}\text { B-110 solids contain only sludge, which is } 99 \% 2 \mathrm{C} \text {. T-110 solids contain } \\
\text { only sludge, which is } 97 \% \text { 2C. In both cases, the measured } \mathrm{pH} \text { was about } 8 \text {, } \\
\text { but the predicted } \mathrm{pH} \text { was } 14 \text { for B-110 and } 10.5 \text { for T- } 110 \text {. } \\
\text { For comparison, the samples of } 1 \mathrm{C} / 2 \mathrm{C} \text { waste used in Group } 1 \text { testing } \\
\text { (Lumetta et al. 2009a) were taken from BX-112 and T-104. }\end{array}$ \\
\hline R1 (boiling) & 16 & 4058 & SX-108 & $\begin{array}{l}\text { The tanks in which this waste type predominates are SX-107 (all sludge is } \\
\text { R1, } 87 \% \text { of solids are sludge, no core data), SX-108 (all solids are R1, core } \\
\text { data), SX-111 (all sludge is R1, 72\% of solids are sludge, no core data), } \\
\text { SX-112 (all sludge is R1, 80\% of solids are sludge, no core data), and } \\
\text { SX-115 (all waste is R1, core data do not include metals or anion analysis). } \\
\text { The predicted pH in SX-108 is } 13.4 \text { (a free OH concentration of } \\
8100 \mathrm{mg} / \mathrm{kg} \text { liquid). } \\
\text { The sludge samples used in Group } 5 \text { studies (Fiskum et al. 2008) came } \\
\text { from S-101, S-107, S-110, and SX-103. There are core data for all of these } \\
\text { R1 (boiling) tanks, but in all these cases, saltcake makes up most of the } \\
\text { waste volume, and CWR sludge is also present. They are therefore not used } \\
\text { to represent the waste type. }\end{array}$ \\
\hline
\end{tabular}


Table 3.9. (contd)

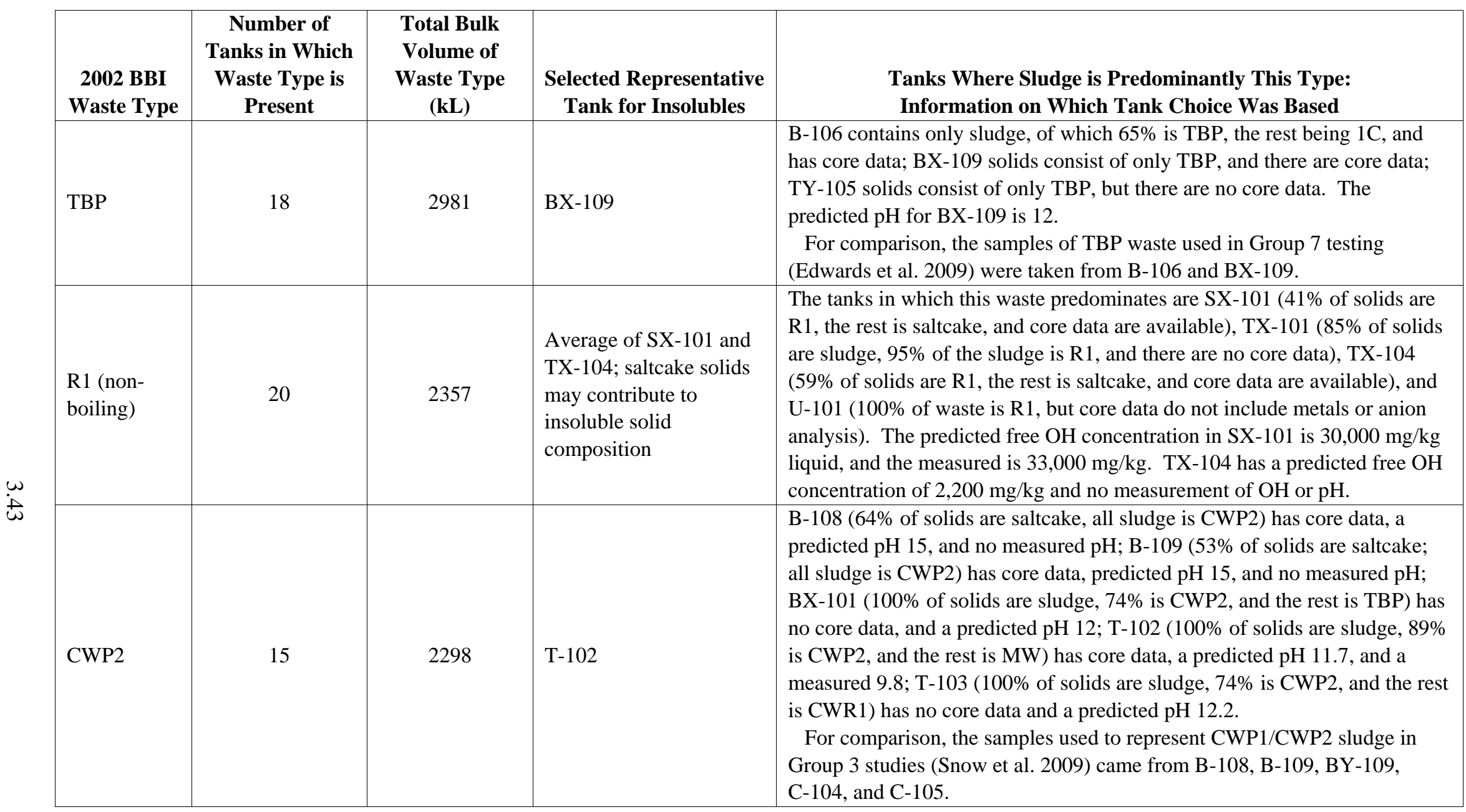


Table 3.9. (contd)

\begin{tabular}{|c|c|c|c|c|}
\hline $\begin{array}{c}2002 \text { BBI } \\
\text { Waste Type }\end{array}$ & $\begin{array}{c}\text { Number of } \\
\text { Tanks in Which } \\
\text { Waste Type is } \\
\text { Present }\end{array}$ & $\begin{array}{c}\text { Total Bulk } \\
\text { Volume of } \\
\text { Waste Type } \\
(\mathbf{k L})\end{array}$ & $\begin{array}{l}\text { Selected Representative } \\
\text { Tank for Insolubles }\end{array}$ & $\begin{array}{l}\text { Tanks Where Sludge is Predominantly This Type: } \\
\text { Information on Which Tank Choice Was Based }\end{array}$ \\
\hline CWP1 & 11 & 1939 & C-105 & $\begin{array}{l}\text { AW-102 solids are } 100 \% \text { sludge, of which all is CWP1. C- } 105 \text { solids are } \\
100 \% \text { sludge, of which } 90 \% \text { is CWP1 (the rest is TBP). AW- } 102 \text { contained } \\
\text { mostly liquid and was characterized only by grab samples; the predicted } \mathrm{pH} \\
\text { was } 15 \text {. C- } 105 \text { had a few constituents measured from cores, and its } \\
\text { predicted pH was a more moderate } 13.6 \text { (a free OH concentration of } \\
11,000 \mathrm{mg} / \mathrm{kg} \text { liquid). } \\
\text { For comparison, the samples used to represent CWP1/CWP2 sludge in } \\
\text { Group } 3 \text { studies (Snow et al. 2009) came from B-108, B-109, BY-109, } \\
\text { C-104, and C-105. }\end{array}$ \\
\hline CWZr2 & 2 & 1693 & $\begin{array}{l}\text { Average of AW-103 and } \\
\text { AW-105 }\end{array}$ & $\begin{array}{l}\text { CWZr2 appears in AW-103, where it makes up } 87 \% \text { of the waste (the rest } \\
\text { being saltcake), and in AW-105, which contains only sludge of which } 92 \% \\
\text { is CWZr2 (the rest is PL2). There are core data for both tanks. The } \\
\text { measured free OH concentration for AW- } 103 \text { is } 9,000 \mathrm{mg} / \mathrm{kg} \text { liquid, and the } \\
\text { predicted is } 13,000 \mathrm{mg} / \mathrm{kg} \text {; the measured free } \mathrm{OH} \text { concentration for } \\
\text { AW- } 105 \text { is } 5000 \mathrm{mg} / \mathrm{kg} \text {, and the predicted is } 5,300 \mathrm{mg} / \mathrm{kg} \text {. }\end{array}$ \\
\hline CWR1 & 18 & 1603 & $\begin{array}{l}\text { Average of U-109, } \\
\text { U-201, U-202, U-203, } \\
\text { and U-204; U-109 } \\
\text { saltcake solids may } \\
\text { contribute to insoluble } \\
\text { solid composition }\end{array}$ & $\begin{array}{l}100 \% \text { of U-201 solids are CWR1, but the core data do not include metals or } \\
\text { anions. U-202, U-203, and U-204 contain only CWR1 solids, but had no } \\
\text { core data as of 2002. U-105 and U-109 contain } 90 \% \text { saltcake and } 10 \% \\
\text { sludge, of which all is CWR1. Both U-105 and U-109 have core data, and } \\
\text { the predicted pH is } ~ 14 \text {, compared to a measured pH in U-105 of > } 13.5 \text {. } \\
\text { The solids from U-109 and the four U-200 tanks were averaged. } \\
\text { For comparison, the samples used to represent CWR1/CWR2 sludge in } \\
\text { Group } 4 \text { studies (Snow et al. 2009) came from U-105, U-201, U-202, } \\
\text { U-203, and U-204. }\end{array}$ \\
\hline
\end{tabular}


Table 3.9. (contd)

\begin{tabular}{|c|c|c|c|c|}
\hline $\begin{array}{c}2002 \text { BBI } \\
\text { Waste Type }\end{array}$ & $\begin{array}{c}\text { Number of } \\
\text { Tanks in Which } \\
\text { Waste Type is } \\
\text { Present }\end{array}$ & $\begin{array}{c}\text { Total Bulk } \\
\text { Volume of } \\
\text { Waste Type } \\
\text { (kL) }\end{array}$ & $\begin{array}{l}\text { Selected Representative } \\
\text { Tank for Insolubles }\end{array}$ & $\begin{array}{l}\text { Tanks Where Sludge is Predominantly This Type: } \\
\text { Information on Which Tank Choice Was Based }\end{array}$ \\
\hline $\mathrm{DE}$ & 6 & 1058 & $\begin{array}{l}\text { TX-116 (no core data } \\
\text { available) }\end{array}$ & $\begin{array}{l}\text { BX-102 contains only sludge, of which } 61 \% \text { is DE; TX-116 contains only } \\
\text { sludge, of which } 89 \% \text { is DE; TX-116 solids are } 88 \% \text { saltcake and } 12 \% \\
\text { sludge, of which all is DE; TX- } 117 \text { solids are } 94 \% \text { saltcake and } 6 \% \text { sludge, } \\
\text { of which all is DE; TY- } 106 \text { contains only sludge, of which } 76 \% \text { is DE; } \\
\text { U- } 104 \text { contains only sludge, of which } 72 \% \text { is DE. None of these tanks have } \\
\text { core data; TY- } 106 \text { has data from augered solids, but no analyses for metal } \\
\text { or anion compositions. }\end{array}$ \\
\hline PFeCN & 6 & 813 & $\begin{array}{l}\text { Average of BY-104 and } \\
\text { BY-108; saltcake solids } \\
\text { may contribute to } \\
\text { insoluble solid } \\
\text { composition. }\end{array}$ & $\begin{array}{l}\text { In BY-104, BY-106, BY-107, and BY-108, PFeCN makes up all of the } \\
\text { sludge, but most of the solids ( } 80 \text { to } 94 \% \text { ) are not sludge but saltcake. All } \\
\text { of these tanks have core data. BY-104 (14\% of solids are PFeCN) and } \\
\text { BY-108 ( } 20 \% \text { of solids are PFeCN) have core samples that contain high Fe. } \\
\text { There is no measured pH in either of these two tanks; the predicted pH is } \\
\text { about } 14 \text { in BY-104 and is > } 15 \text { in BY-108. } \\
\text { For comparison, the samples used to represent } 1 \text { CFeCN/PFeCN/TFeCN } \\
\text { sludge in Group } 8 \text { studies (Fiskum et al. 2009a) came from BY-104, } \\
\text { BY-105, BY-106, BY-108, and (in larger proportion) BY-110. }\end{array}$ \\
\hline BL & 3 & 647 & AY-102 & $\begin{array}{l}\text { AY-102 solids contain only BL. AX-102 solids are } 20 \% \text { sludge, of which } \\
\text { all is BL. B-101 solids are } 32 \% \text { sludge, of which } 72 \% \text { is BL. }\end{array}$ \\
\hline TFeCN & 5 & 636 & $\begin{array}{l}\text { Average of BY-101 and } \\
\text { C-112; however, other } \\
\text { wastes are present in } \\
\text { both tanks. }\end{array}$ & $\begin{array}{l}\text { BY-101 solids are } 89 \% \text { saltcake, the sludge is an all TFeCN type, and there } \\
\text { are two core samples characterized. C-112 solids are all sludge and are } \\
69 \% \text { TFeCN, the rest being } 1 \text { C and CWP1; the tank has core samples. An } \\
\text { average of the solids from BY-101 and C-112 is used, though neither is } \\
\text { ideal. } \\
\text { For comparison, the samples used to represent } 1 \text { CFeCN/PFeCN/TFeCN } \\
\text { sludge in Group } 8 \text { studies (Fiskum et al. 2009a) came from BY-104, } \\
\text { BY-105, BY-106, BY-108, and (in larger proportion) BY-110. }\end{array}$ \\
\hline
\end{tabular}


Table 3.9. (contd)

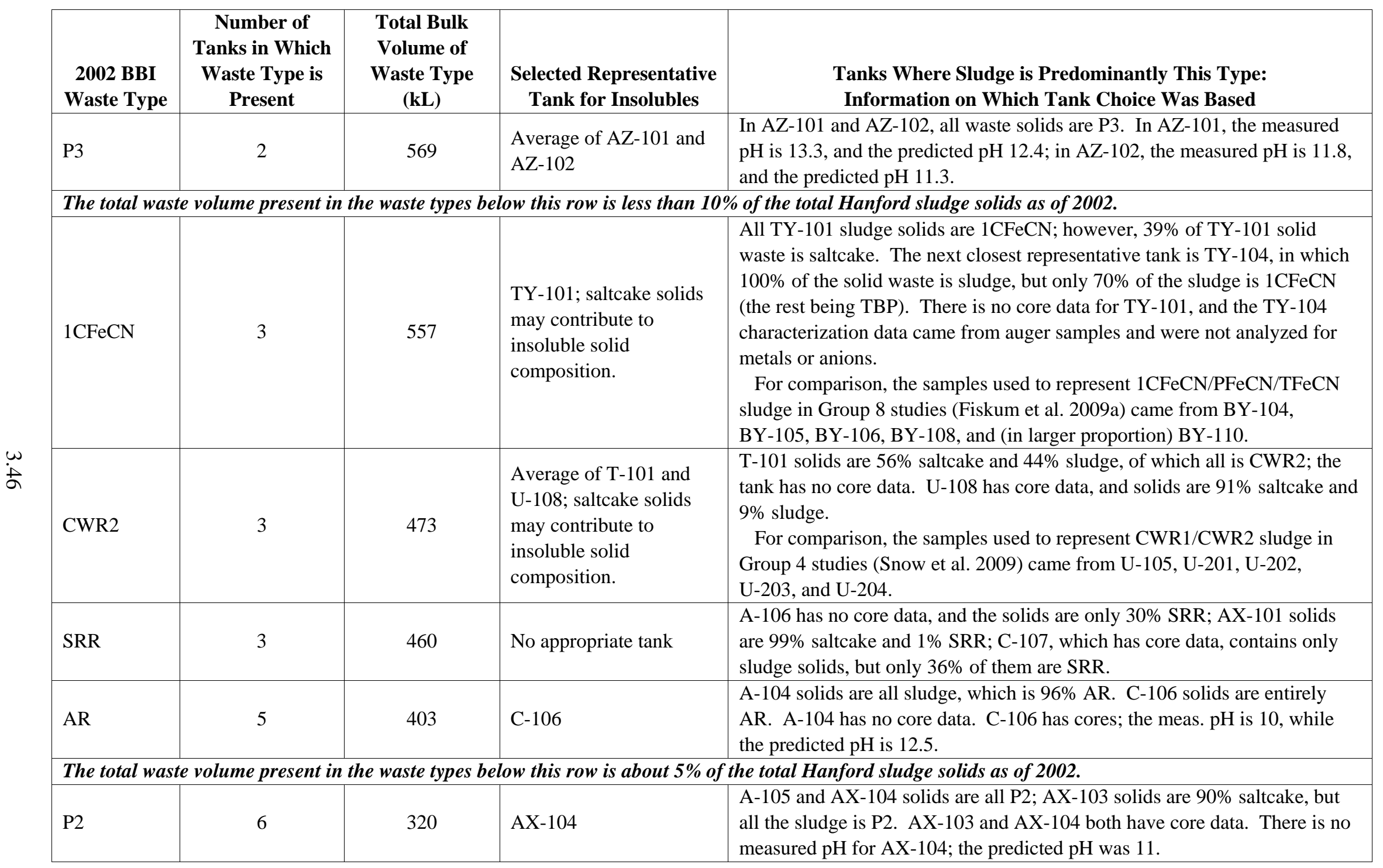


Table 3.9. (contd)

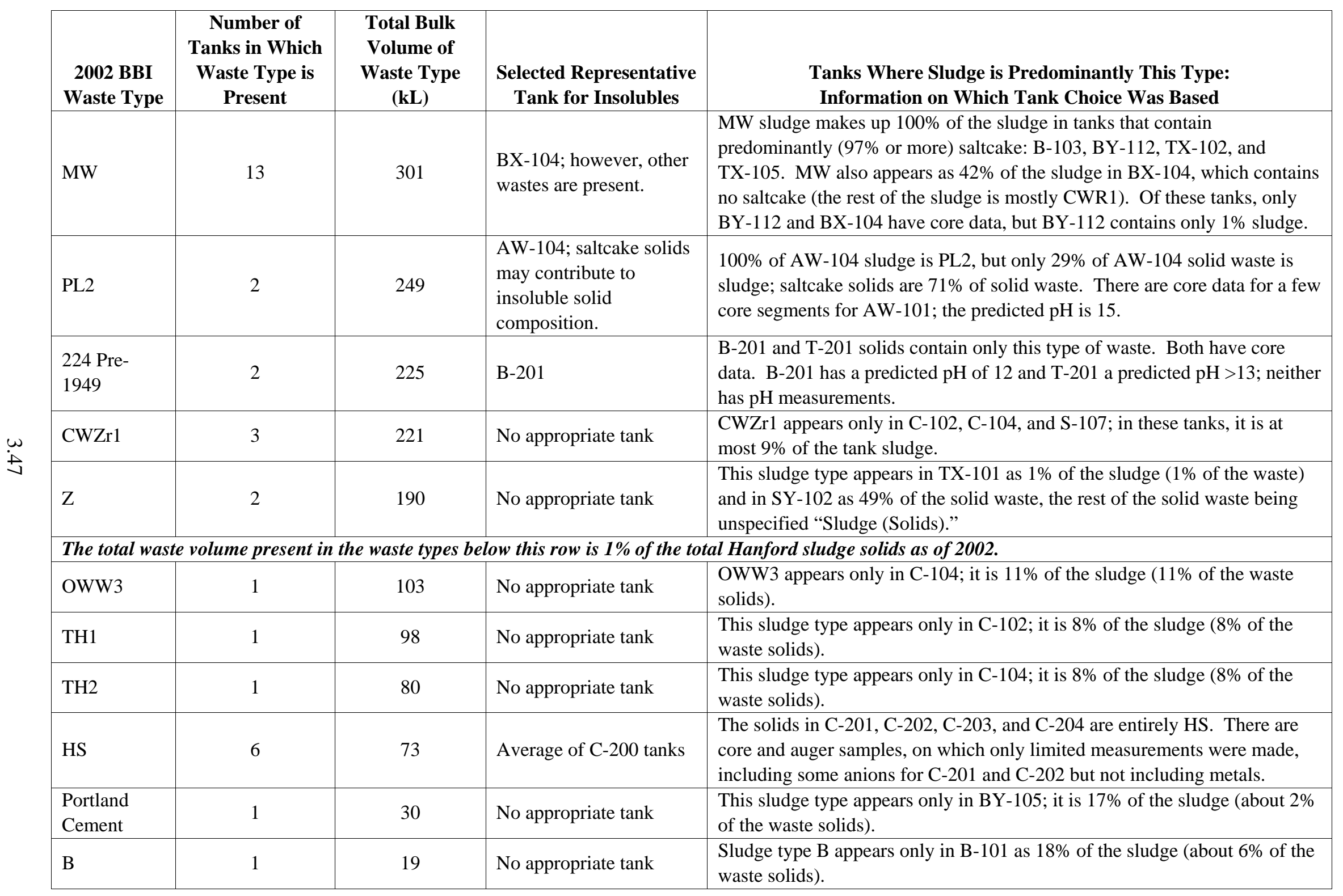


Table 3.9. (contd)

\begin{tabular}{|l|c|c|l|l|}
\hline $\begin{array}{c}\text { 2002 BBI } \\
\text { Waste Type }\end{array}$ & $\begin{array}{c}\text { Number of } \\
\text { Tanks in Which } \\
\text { Waste Type is } \\
\text { Present }\end{array}$ & $\begin{array}{c}\text { Total Bulk } \\
\text { Volume of } \\
\text { Waste Type } \\
(\mathbf{k L})\end{array}$ & $\begin{array}{c}\text { Selected Representative } \\
\text { Tank for Insolubles }\end{array}$ & $\begin{array}{c}\text { Tanks Where Sludge is Predominantly This Type: } \\
\text { Information on Which Tank Choice Was Based }\end{array}$ \\
\hline P1 & 1 & 4 & No appropriate tank & P1 appears only in A-104; it is 4\% of the sludge (4\% of the waste solids). \\
\hline Wastes are listed in decreasing order of the total bulk volume present in Hanford tanks. All percentages are in terms of bulk volume of waste.
\end{tabular}


Table 3.10. Tanks Identified to Represent Saltcake Waste Types (Based on 2002 BBI)

\begin{tabular}{|c|c|c|c|c|}
\hline S1 & 22 & 19759 & $\begin{array}{l}\text { Average of S-105, } \\
\text { S-106, and U-106 }\end{array}$ & $\begin{array}{l}\text { S1 saltcake is } 97 \% \text { or more of the waste in tanks S-105, S- } 106 \text {, S- } 108 \text {, } \\
\text { S-109, S-112, and U-106. In all these cases, the remainder of the waste is } \\
\text { sludge. Of these six tanks, core samples are available for five: S-105 (core } \\
\text { composite only), S-106, S- } 109 \text { (but only the top of the waste), U-106, and } \\
\text { U-108. The predicted pHs for the five tanks are } 15,15,15,13 \text {, and } 15 \text {; the } \\
\text { measured pH in S- } 105 \text { is } 12.8 \text {. The predicted free OH concentrations in the } \\
\text { six tanks are } 15,000,15,000,38,000,210 \text {, and } 32,000 \mathrm{mg} / \mathrm{kg} \text { liquid, } \\
\text { respectively. The measured free OH concentrations in S-106, S-109, } \\
\text { U-106, and U-108 were } 39,000,59,000,3,800 \text {, and } 35,000 \mathrm{mg} / \mathrm{kg} \text {. } \\
\text { For comparison, the salt samples used to represent S1/S2 saltcake in } \\
\text { Group } 6 \text { studies (Fiskum et al. 2008) came from S-106, S-111, SX-102, } \\
\text { SX-105, SX-106, SY-103, U-103, and U-106. }\end{array}$ \\
\hline
\end{tabular}


Table 3.10. (contd)

\begin{tabular}{|c|c|c|c|c|}
\hline $\begin{array}{c}2002 \text { BBI } \\
\text { Waste Type }\end{array}$ & $\begin{array}{c}\text { Number of } \\
\text { Tanks in Which } \\
\text { Waste Type is } \\
\text { Present }\end{array}$ & $\begin{array}{c}\text { Total Bulk } \\
\text { Volume of } \\
\text { Waste Type } \\
\text { (kL) }\end{array}$ & $\begin{array}{l}\text { Selected Representative } \\
\text { Tank for Saltcake }\end{array}$ & $\begin{array}{l}\text { Tanks Where Saltcake is Predominantly This Type: } \\
\text { Information on Which Tank Choice Was Based }\end{array}$ \\
\hline BY & 15 & 13824 & $\begin{array}{l}\text { Average of BY-102, } \\
\text { BY-111, and BY-112 }\end{array}$ & $\begin{array}{l}\text { The tanks in which BY saltcake predominates are BX-111 and BY-101 } \\
\text { through BY-112. Of these tanks, the ones in which the waste is more than } \\
99 \% \text { BY saltcake and for which core samples are available are BY-102, } \\
\text { BY-111, and BY-112. The predicted free OH concentration in BY-102 is } \\
3,600 \mathrm{mg} / \mathrm{kg} \text { liquid, and the predicted free OH concentrations are } \\
50,000 \mathrm{mg} / \mathrm{kg} \text { and } 43,000 \mathrm{mg} / \mathrm{kg} \text { in BY-111 and BY-112. No pH or free } \\
\text { OH measurements are available for these tanks. } \\
\text { For comparison, the samples used to represent BY/T saltcake in Group } 2 \\
\text { studies (Lumetta et al. 2009a) came from BX-110, BX-111, BY-104, } \\
\text { BY-105, BY-107, BY-108, BY-109, BY-110, BY-112, T-108, T-109, } \\
\text { TX-104, and TX-113. }\end{array}$ \\
\hline A2 & 10 & 9822 & $\begin{array}{l}\text { Average of AN-103, } \\
\text { AN-104, AN-105, and } \\
\text { AW-101 }\end{array}$ & $\begin{array}{l}\text { In AN-102 through AN-105, AN-107, AP-105, AW-101, and AW-106, the } \\
\text { solids are } 100 \% \text { A2 saltcake. Of these, AN-103, AN-104, AN-105, and } \\
\text { AW- } 101 \text { had core data that had not been rendered obsolete by subsequent } \\
\text { transfers. The predicted free OH concentrations were } 49,000,43,000 \text {, } \\
\text { 33,000, and 55,000 mg/kg liquid for these four tanks; the measured free OH } \\
\text { concentrations were } 57,000,51,000,41,000 \text {, and } 65,000 \mathrm{mg} / \mathrm{kg} \text {. }\end{array}$ \\
\hline S2 & 16 & 8631 & SY-103 & $\begin{array}{l}\text { S2 saltcake predominates in SX-106 ( } 82 \% \text { of solids are S2 and } 18 \% \text { are S1 } \\
\text { saltcake); and SY-103 ( } 100 \% \text { of solids are S2). The solids in SY-101 are } \\
\text { also } 100 \% \text { S2 saltcake, but were diluted as part of remediation operations in } \\
1999-2000 \text {. The predicted free OH concentration in SY-103 is } \\
24,000 \mathrm{mg} / \mathrm{kg} \text { liquid; the measured value was } 22,000 \mathrm{mg} / \mathrm{kg} \text {. } \\
\text { For comparison, the salt samples used to represent S1/S2 saltcake in } \\
\text { Group } 6 \text { studies (Fiskum et al. 2008) came from S-106, S-111, SX-102, } \\
\text { SX-105, SX-106, SY-103, U-103, and U-106. }\end{array}$ \\
\hline
\end{tabular}


Table 3.10. (contd)

\begin{tabular}{|c|c|c|c|c|}
\hline $\begin{array}{c}2002 \text { BBI } \\
\text { Waste Type }\end{array}$ & $\begin{array}{c}\text { Number of } \\
\text { Tanks in Which } \\
\text { Waste Type is } \\
\text { Present }\end{array}$ & $\begin{array}{c}\text { Total Bulk } \\
\text { Volume of } \\
\text { Waste Type } \\
\text { (kL) }\end{array}$ & $\begin{array}{l}\text { Selected Representative } \\
\text { Tank for Saltcake }\end{array}$ & $\begin{array}{l}\text { Tanks Where Saltcake is Predominantly This Type: } \\
\text { Information on Which Tank Choice Was Based }\end{array}$ \\
\hline A1 & 9 & 4683 & $\begin{array}{l}\text { Average of A-101 and } \\
\text { AX-101 }\end{array}$ & $\begin{array}{l}\text { The tanks in which A1 saltcake predominates in the solids are A-101 (all } \\
\text { saltcake is A1, 99\% of the solids are saltcake, and core data are available), } \\
\text { A- } 102 \text { (all solids are A1, and auger data are available), A-103 (all saltcake } \\
\text { is A1, } 99 \% \text { of the solids are saltcake, and there are no core data), AN-106 } \\
\text { (all waste is A1, and grab sample data are available), AX-101 (all saltcake } \\
\text { is A1, } 99 \% \text { of the solids are saltcake, and core data are available), AX-102 } \\
\text { (all saltcake is A1, } 80 \% \text { of the solids are saltcake, and auger data are } \\
\text { available), and AX-103 (all saltcake is A1, } 90 \% \text { of the solids are saltcake, } \\
\text { and core data are available). The A- } 101 \text { measured free OH is about } \\
29,000 \mathrm{mg} / \mathrm{kg} \text { liquid, compared to a predicted value of } 18,000 \mathrm{mg} / \mathrm{kg} \text {; the } \\
\text { AX-101 measured free OH is about } 26,000 \mathrm{mg} / \mathrm{kg} \text {, compared to a predicted } \\
\text { value of } 20,000 \mathrm{mg} / \mathrm{kg} \text {. }\end{array}$ \\
\hline \multicolumn{5}{|c|}{ The total waste volume present in the waste types below this row is about $10 \%$ of the total Hanford salt solids as of 2002.} \\
\hline $\mathrm{R}$ & 11 & 2613 & $\begin{array}{l}\text { SX-109 (however, } \\
\text { sludge is present in the } \\
\text { tank) }\end{array}$ & $\begin{array}{l}\text { In most of the tanks where R saltcake is present, it makes up less than } 50 \% \\
\text { of the waste. The two exceptions are SX-109 ( } 76 \% \text { R saltcake and the } \\
\text { remainder sludge) and SX-114 ( } 67 \% \text { R saltcake and the remainder sludge). } \\
\text { There are no core samples from either of these tanks. The predicted free } \\
\text { OH concentrations are } 25,000 \text { and 16,000 mg/kg liquid, respectively. }\end{array}$ \\
\hline $\mathrm{T} 1$ & 9 & 2374 & $\mathrm{~T}-109$ & $\begin{array}{l}\text { T1 saltcake predominates only in T-108 ( } 60 \% \text { of solids are T1, and the } \\
\text { remainder are sludge) and T- } 109 \text { ( } 100 \% \text { of the solids are T1). Both of these } \\
\text { tanks have auger samples. The predicted pH in T-109 is 11; there are no } \mathrm{pH} \\
\text { or free OH measurements. } \\
\text { For comparison, the samples used to represent BY/T saltcake in Group } 2 \\
\text { studies (Lumetta et al. 2009a) came from BX-110, BX-111, BY-104, } \\
\text { BY-105, BY-107, BY-108, BY-109, BY-110, BY-112, T-108, T-109, } \\
\text { TX-104, and TX-113. }\end{array}$ \\
\hline
\end{tabular}


Table 3.10. (contd)

\begin{tabular}{|c|c|c|c|c|}
\hline $\begin{array}{c}2002 \text { BBI } \\
\text { Waste Type }\end{array}$ & $\begin{array}{l}\text { Number of } \\
\text { Tanks in Which } \\
\text { Waste Type is } \\
\text { Present }\end{array}$ & $\begin{array}{c}\text { Total Bulk } \\
\text { Volume of } \\
\text { Waste Type } \\
(\mathbf{k L})\end{array}$ & $\begin{array}{l}\text { Selected Representative } \\
\text { Tank for Saltcake }\end{array}$ & $\begin{array}{l}\text { Tanks Where Saltcake is Predominantly This Type: } \\
\text { Information on Which Tank Choice Was Based }\end{array}$ \\
\hline B & 8 & 2203 & $\begin{array}{l}\text { B-108 (however, sludge } \\
\text { is present in the tank) }\end{array}$ & $\begin{array}{l}\text { The solids in Tanks B-102, B-103, and B- } 108 \text { are } 90 \% \text { or more B saltcake, } \\
\text { but the samples from these tanks (when available at all) were not analyzed } \\
\text { for metals and anions. B- } 101 \text { is } 68 \% \text { B saltcake, the rest of the solids being } \\
\text { sludge; core samples were not analyzed for metals. The best choice is } \\
\text { B-108, } 63 \% \text { B saltcake, the rest of the solids being sludge. The predicted } \\
\text { free OH concentration for B- } 108 \text { is } 16,000 \mathrm{mg} / \mathrm{kg} \text { liquid. }\end{array}$ \\
\hline \multicolumn{5}{|c|}{ The total waste volume present in the waste types below this row is less than 1\% of the total Hanford salt solids as of 2002.} \\
\hline R1 & 1 & 262 & $\begin{array}{l}\text { S-101 (however, S1 and } \\
\text { S2 saltcake dominate in } \\
\text { the composition, and } \\
\text { sludge is present) }\end{array}$ & $\begin{array}{l}\text { R1 saltcake is present only in S-101, where it is } 31 \% \text { of the saltcake, and the } \\
\text { waste is } 65 \% \text { saltcake; the other wastes in the tank are sludge and S1 and S2 } \\
\text { saltcake. The predicted free OH concentration in S-101 is } 26,000 \mathrm{mg} / \mathrm{kg} \\
\text { liquid, and the measured concentration is } 32,000 \mathrm{mg} / \mathrm{kg} \text {. }\end{array}$ \\
\hline $\begin{array}{l}\text { Identified } \\
\text { only as } \\
\text { saltcake }\end{array}$ & 1 & 256 & $\begin{array}{l}\text { Not really a waste type - } \\
\text { no representative tank }\end{array}$ & $\begin{array}{l}\text { This undesignated saltcake descriptor is given only to TX-118, where it is } \\
31 \% \text { of the solids (all the solids are saltcake); the other solids in the tank is } \\
\text { T2 saltcake. There are core data for this tank. The predicted pH is } 11 \text {, and } \\
\text { there is no pH measurement. }\end{array}$ \\
\hline
\end{tabular}




\subsubsection{UDS Solid Phase Summary}

As described, a hybrid approach was taken to identify and quantify the UDS phases present in the Hanford tanks. The solids predicted by the $\mathrm{ESP}^{(\mathrm{a})}$ chemical thermodynamic model from the $2002 \mathrm{BBI}$ composition information were taken as a first approximation of the phases present. Overview documents were then reviewed to find information on what solid phases were actually present in the tank wastes, and the predicted compositions were adjusted accordingly. The UDS phase selections were reviewed and revised by a panel of experts.

Table 3.11 provides a summary of the defined UDS phases and their respective density. A total of 52 compounds are defined for the Hanford waste, with 36 non-salt and 16 salt compounds. The concentration of these compounds is provided in Appendix C by tank, waste type, and composite. Not all compounds are present in each tank or waste type.

Table 3.11. UDS Phase and Density Summary

\begin{tabular}{|c|c|}
\hline Compound & Density $(\mathrm{g} / \mathrm{mL})$ \\
\hline $\mathrm{Ag}$ & 10.5 \\
\hline $\mathrm{Ag}_{2} \mathrm{O}$ & 7.143 \\
\hline $\mathrm{Bi}_{2} \mathrm{O}_{3}$ & 8.9 \\
\hline $\mathrm{BiFeO}_{3}$ & 7.9 \\
\hline $\mathrm{Ca}(\mathrm{OH})_{2}$ & 2.24 \\
\hline $\mathrm{Ca}_{5} \mathrm{OH}\left(\mathrm{PO}_{4}\right)_{3}$ & 3.14 \\
\hline $\mathrm{CaC}_{2} \mathrm{O}_{4} \cdot \mathrm{H}_{2} \mathrm{O}$ & 2.2 \\
\hline $\mathrm{CaCO}_{3}$ & 2.71 \\
\hline $\mathrm{CaF}_{2}$ & 3.18 \\
\hline $\mathrm{CrOOH}$ & 4.11 \\
\hline $\mathrm{FePO}_{4} \cdot 2 \mathrm{H}_{2} \mathrm{O}$ & 3.15 \\
\hline $\mathrm{FeOOH}$ & 4.26 \\
\hline Gibbsite & 2.42 \\
\hline Boehmite & 3.01 \\
\hline $\mathrm{HgO}$ (co-precipitated with $\mathrm{Ag}_{2} \mathrm{O}$ ) & 7.143 \\
\hline $\mathrm{KAlSiO}_{4}$ & 2.61 \\
\hline $\mathrm{La}(\mathrm{OH})_{3}$ & 2.3 \\
\hline $\mathrm{LaPO}_{4} \cdot 2 \mathrm{H}_{2} \mathrm{O}$ & 6.51 \\
\hline $\mathrm{Mn}_{3}\left(\mathrm{PO}_{4}\right)_{2}$ & 3.102 \\
\hline $\mathrm{MnO}_{2}$ & 5.026 \\
\hline $\mathrm{Na}_{2}\left(\mathrm{UO}_{2}\right)_{2}\left(\mathrm{PO}_{4}\right)_{2} \cdot 2 \mathrm{H}_{2} \mathrm{O}$ & 3.5 \\
\hline $\mathrm{Na}_{2} \mathrm{U}_{2} \mathrm{O}_{7}$ & 5.617 \\
\hline $\mathrm{NaAlCO}_{3}(\mathrm{OH})_{2}$ & 2.42 \\
\hline $\mathrm{NaAlSiO}_{4}$ & 2.365 \\
\hline $\mathrm{Ni}(\mathrm{OH})_{2}$ & 4.15 \\
\hline $\mathrm{Ni}_{3}\left(\mathrm{PO}_{4}\right)_{2}$ & 3.93 \\
\hline $\mathrm{NiC}_{2} \mathrm{O}_{4} \cdot 2 \mathrm{H}_{2} \mathrm{O}$ (co-precip, with FeOOH) & 4.26 \\
\hline
\end{tabular}

(a) ESP was supplied and developed by OLI Systems, Inc., Morris Plains, New Jersey. 
Table 3.11. (contd)

\begin{tabular}{|c|c|}
\hline Compound & Density $(\mathrm{g} / \mathrm{mL})$ \\
\hline $\mathrm{Pb}(\mathrm{OH})_{2}$ & 7.1 \\
\hline $\mathrm{Pb}_{3}\left(\mathrm{PO}_{4}\right)_{2}$ & 7.1 \\
\hline $\mathrm{PbCO}_{3}$ & 6.6 \\
\hline $\mathrm{Pu}(\mathrm{OH})_{4}$ (co-precip. on Fe phase) & 4.26 \\
\hline $\mathrm{PuO}_{2}$ & 11.43 \\
\hline $\mathrm{SiO}_{2}$ & 2.6 \\
\hline $\mathrm{Sr}_{3}\left(\mathrm{PO}_{4}\right)_{2}$ & 3.5 \\
\hline $\mathrm{SrCO}_{3}$ & 3.5 \\
\hline $\mathrm{ZrO}_{2}$ & 5.68 \\
\hline $\mathrm{KNO}_{3}$ & 2.109 \\
\hline $\mathrm{Na}_{2} \mathrm{C}_{2} \mathrm{O}_{4}$ & 2.34 \\
\hline $\mathrm{Na}_{2} \mathrm{CO}_{3} \cdot \mathrm{H}_{2} \mathrm{O}$ & 2.25 \\
\hline $\mathrm{Na}_{2} \mathrm{SO}_{4}$ & 2.68 \\
\hline $\mathrm{Na}_{2} \mathrm{SO}_{4} \cdot 10 \mathrm{H}_{2} \mathrm{O}$ & 1.464 \\
\hline $\mathrm{Na}_{3} \mathrm{FSO}_{4}$ & 2.65 \\
\hline $\mathrm{Na}_{3} \mathrm{NO}_{3} \mathrm{SO}_{4} \cdot \mathrm{H}_{2} \mathrm{O}$ & 2.3 \\
\hline $\mathrm{Na}_{3} \mathrm{PO}_{4} \cdot 0.25 \mathrm{NaOH} \bullet 12 \mathrm{H}_{2} \mathrm{O}$ & 1.62 \\
\hline $\mathrm{Na}_{3} \mathrm{PO}_{4} \bullet 8 \mathrm{H}_{2} \mathrm{O}$ & 1.8 \\
\hline $\mathrm{Na}_{4} \mathrm{P}_{2} \mathrm{O}_{7} \cdot 10 \mathrm{H}_{2} \mathrm{O}$ & 1.83 \\
\hline $\mathrm{Na}_{6}\left(\mathrm{SO}_{4}\right)_{2} \mathrm{CO}_{3}$ & 2.64 \\
\hline $\mathrm{NaF}$ & 2.78 \\
\hline $\mathrm{Na}_{7} \mathrm{~F}\left(\mathrm{PO}_{4}\right)_{2} \cdot 19 \mathrm{H}_{2} \mathrm{O}$ & 1.75 \\
\hline $\mathrm{NaHCO}_{3}$ & 2.159 \\
\hline $\mathrm{NaNO}_{2}$ & 2.168 \\
\hline $\mathrm{NaNO}_{3}$ & 2.26 \\
\hline
\end{tabular}

\subsubsection{UDS Primary Particle Size and Shape}

This section provides information on the size and shape of the primary particles of the UDS phase compounds from Table 3.11, Section 3.2.3, that were not addressed in Wells et al. (2007). A summary of the primary particle sizes is provided that incorporates the information from Wells et al. (2007). The waste sample analyses conducted during the response to issue M12. A notable finding from several of the tank waste groups from this study was the occurrence of amorphous phases.

Most previous characterization studies have used SEM and XRD as the major characterization tools. In the M12 study, TEM was used for the first time with an advanced TEM at the University of Nevada, Las Vegas. This instrument combined with the instrument at the Radiochemical Processing Laboratory (RPL) enabled elucidation of the nano-sized particles that dominate many of the Hanford tank sludges. Amorphous phases included Al-Cr oxides in Group 5 and 6 sludges and Bi-phosphate bearing solids found in Group 1 and 2. The amorphous phases are not readily detected with XRD and have remained poorly classified as they cannot be associated with a particular mineral type. In this section, using data sources such as the M12 series of studies, new information on the nature of the amorphous components in Hanford tank waste has been obtained. 
The methodology employed to determine particle size has been to measure individual particles on a calibrated image. An expert panel (Appendix D) was convened to review and revise the particle size estimates and determine consensus options for those compounds without information. When particles are well spaced, an automated analysis program has been used; however, in most Hanford tank sludges, particles are often agglomerated. The TEM images are more effective at identifying individual particles because the exact dimensions of the particles are visible in a "2D view." In contrast, the SEM images show large "3D" agglomerates where it is difficult to measure an accurate distance. The resolution of SEM is such that the overlapping particles can look like one large particle. The optical microscope is only useful for very large particles, and these were covered in Wells et al. (2007).

The effort to quantify the UDS primary particle size and shape for the defined UDS phase compounds was undertaken to provide the best estimates possible. However, the measurements must be treated as estimates only. As is described in this section, for those compounds where images were specifically identified, a finite set of images is available for the waste from a limited number of tanks. For other compounds, surrogate images, wherein the specific UDS phase is not replicated or certain, are used, with similar caveats regarding sample size. In addition, there are compounds with no images available, and expert judgment is used to assign the particle characteristics.

Particles in Hanford tank waste have been measured with optical microscopy, SEM, and TEM images. A selection of the images evaluated is included in Appendix D. These techniques have significant limits for making particle size determinations. Optical images are limited, in general, to particles several micrometers and do not provide compositional information. The technique has been applied to the identification of salt particle sizes only in some cases. SEM images represent the largest set of data available. However, particle size measurements are hampered by the visibility of individual particles, which can be related to the quality of the instrument used in the analysis, the beam voltage, and the detector type used for image formation. In Figure 3.6, an example of backscattered imaging and secondary imaging is shown. The material is typical of agglomerates exhibiting several particle types. Backscattered imaging has been used to highlight the high Z solids, in this case, the bismuth-bearing phases. A particle in the bottom right-hand corner of the BSE image was measured at $2.69 \mu \mathrm{m}$; however, the same particle in the secondary image implies that the particle consists of smaller particles, approximately $1 \mu \mathrm{m}$ in diameter. The particles measured at $2.22 \mu \mathrm{m}$ and $1.34 \mu \mathrm{m}$ in the BSE image are not clearly distinguishable as individual particles in the SE image. 


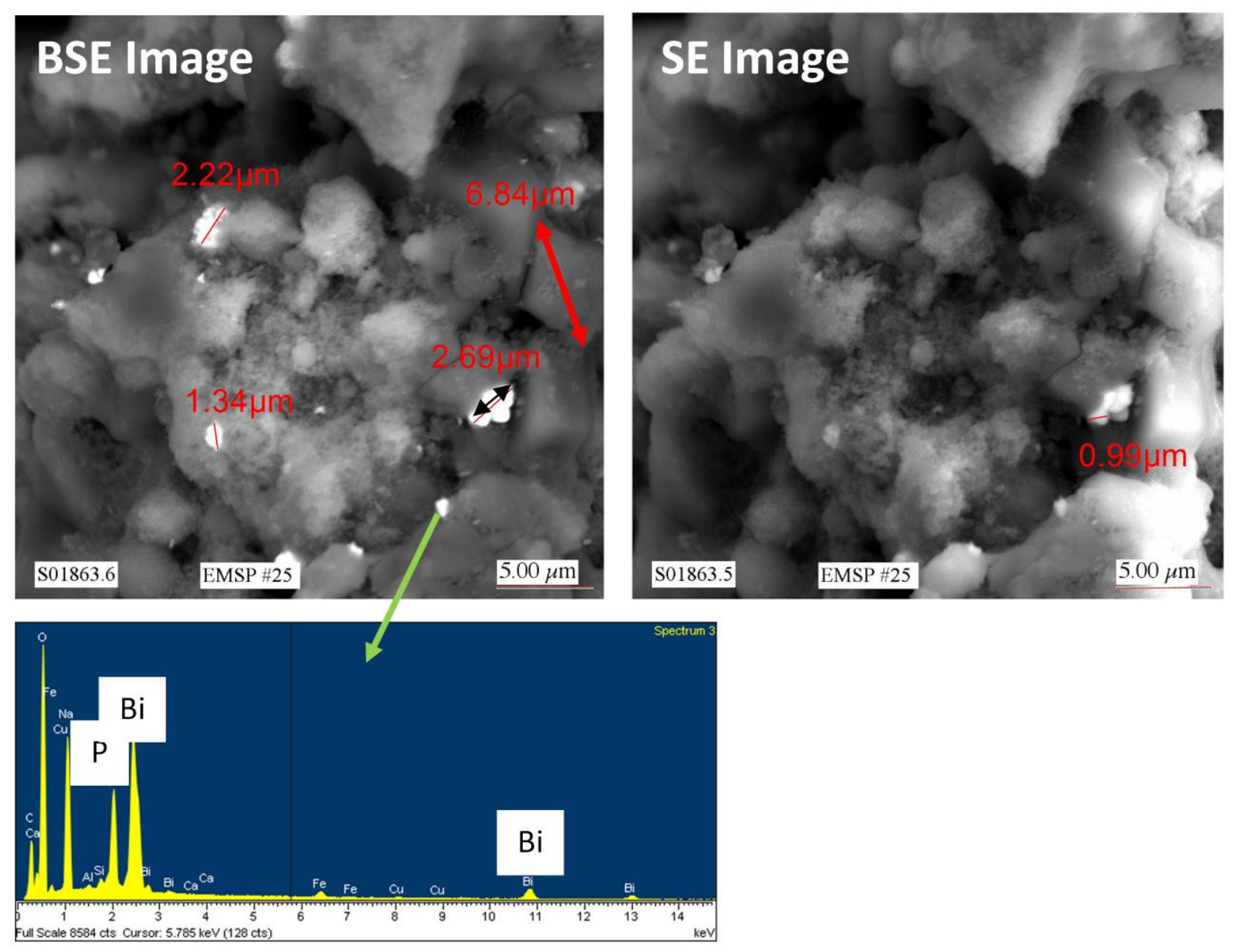

Figure 3.6. Measurement Techniques with SEM Example. For Information Only.

\subsubsection{Bismuth Phases}

Evidence for bismuth oxide, bismuth phosphate (BP), and a complex bismuth-iron, silico-phosphate phase is found in the Hanford tank wastes. The bismuth oxide phase occurred as plates or cubes, and the phosphate aggregates and the silico-phosphate phase occurred as a less distinct amorphous mass. La, Si, $\mathrm{K}, \mathrm{Cr}$, and $\mathrm{Mn}$ were also detected in this material, which was much better characterized with TEM. In Figure 3.7, an euhedral cubic $\mathrm{B}_{2} \mathrm{O}_{3}$ particle $~ 5$ to $7 \mu \mathrm{m}$ in size is shown together with botryoidal BP agglomerates with a much smaller particle size range. The bright white material is almost pure BP; however, the darker material also contains Si and Fe. There is a steady decrease in particle size and degree of obvious crystallinity going from bismuth oxide to BP to a mixed bismuth-iron silicate phosphate. An example of the different forms of bismuth found in Hanford waste is shown in Figure 3.8. 


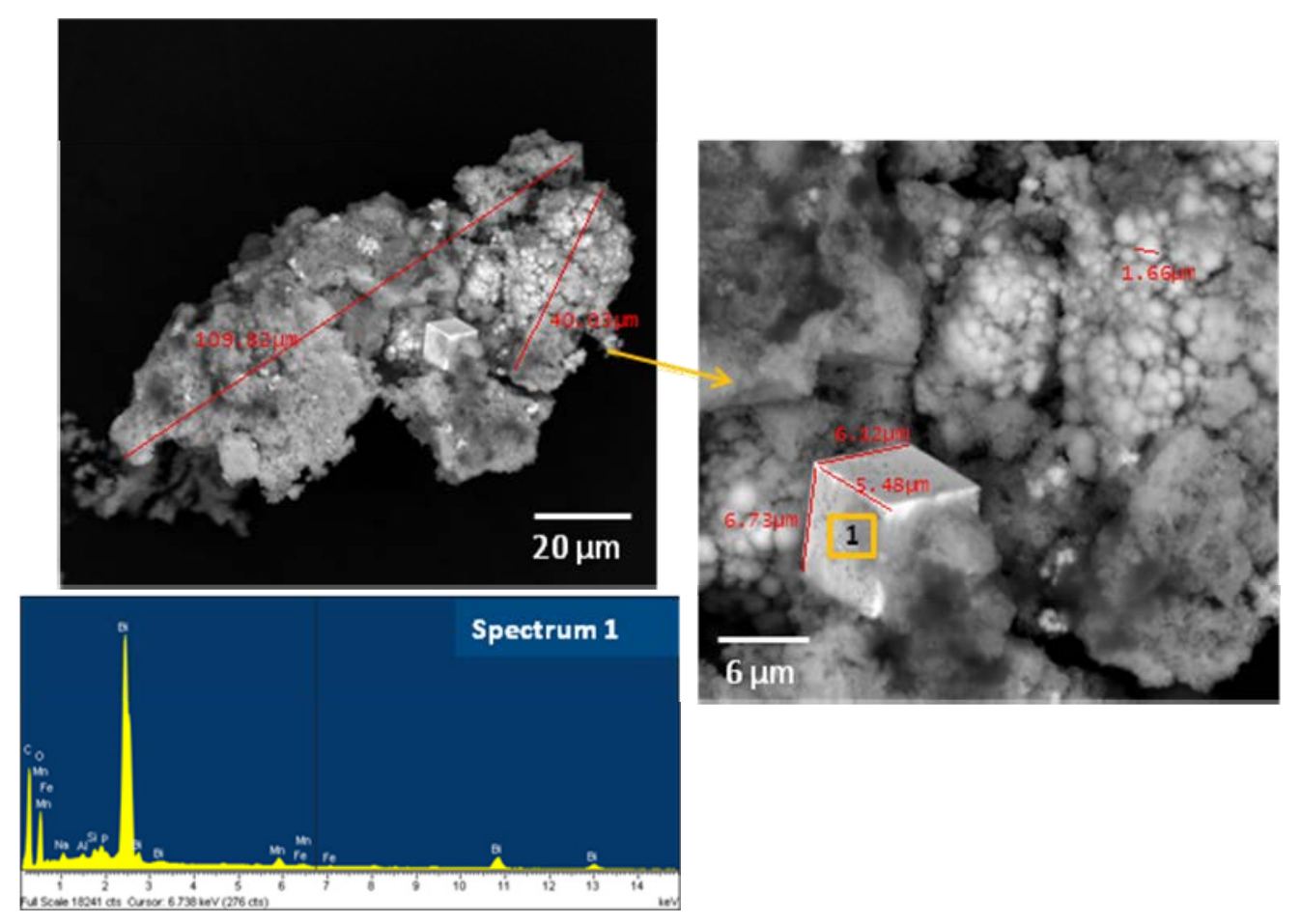

Figure 3.7. Euhedral Cubic $\mathrm{B}_{2} \mathrm{O}_{3}$ Particle Together with Botryoidal Bismuth Phosphate Agglomerates (taken from data collected from Environmental Management Science Program [EMSP] project 90162, 2006)

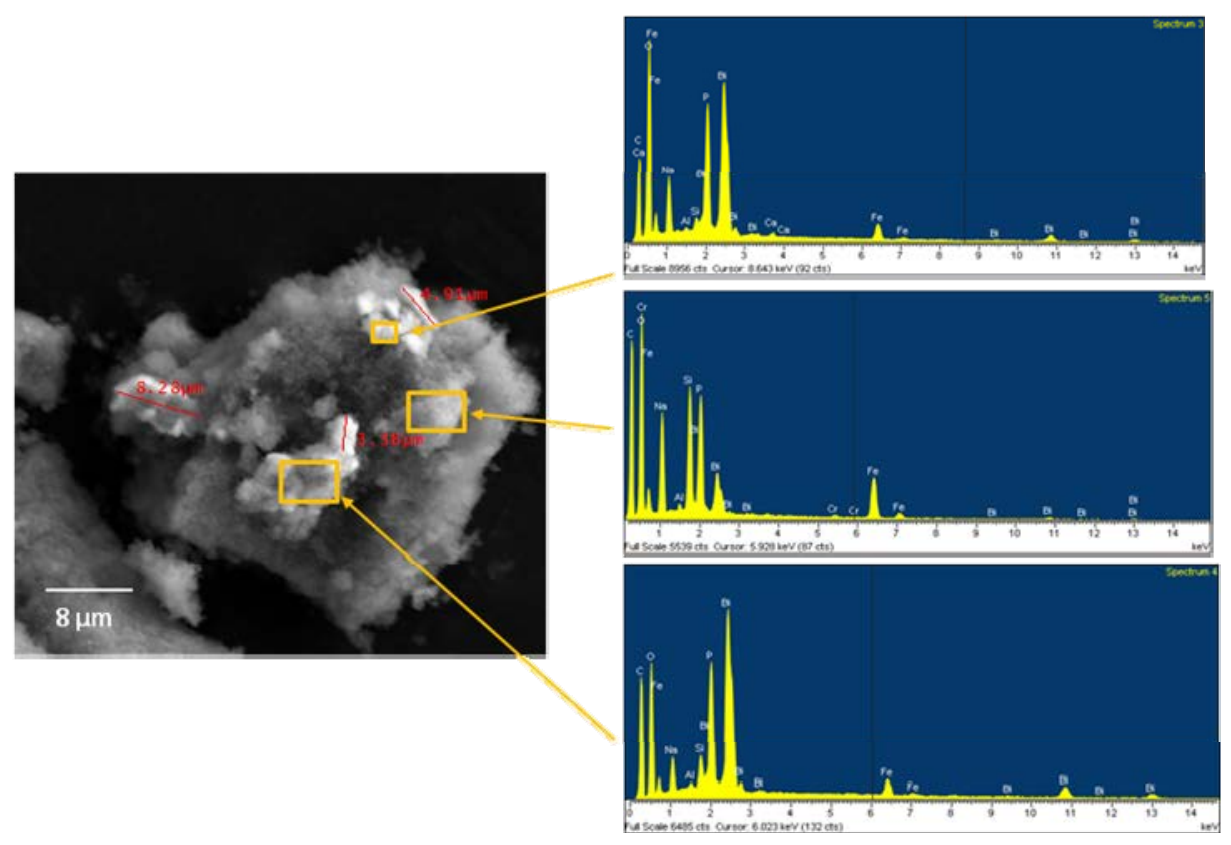

Figure 3.8. Different Forms of Bismuth in the Hanford Tank Waste (taken from data collected from EMSP project 90162, 2006) 
From the M12 study, BP sludge (Group 1 and 2) samples were observed to contain Bi-P phases but not bismuth oxide. Tanks that contain saltcake (Group 2) were excluded because high $\mathrm{OH}$ concentrations might convert $\mathrm{BiPO}_{4}$ to $\mathrm{Bi}_{2} \mathrm{O}_{3}$. Data from the microanalysis information of Lumetta et al. (2009a) on BP sludge and BP saltcake were analyzed for composition and morphology. The overall composition and structure, Figure 3.9, can be used to estimate a more reasonable density. Mixed leached BP sludge and BP saltcake contained an iron bismuth phase that was shown to be structurally related to bismuthoferrite $\left[\mathrm{BiFe}_{2}^{3+}{ }_{2}\left(\mathrm{SiO}_{4}\right)_{2}(\mathrm{OH})\right]$ Edwards et al. (2009). This phase was the most prominent particle in the sample. As it is X-ray amorphous, the phase was not identified in the XRD scans. It was present as agglomerates in various sizes (see Figure 3.10). There is a need to describe these aggregates better so that a more reasonable assessment of their density can be obtained. A PSD using the TEM images is shown in Figure 3.11.

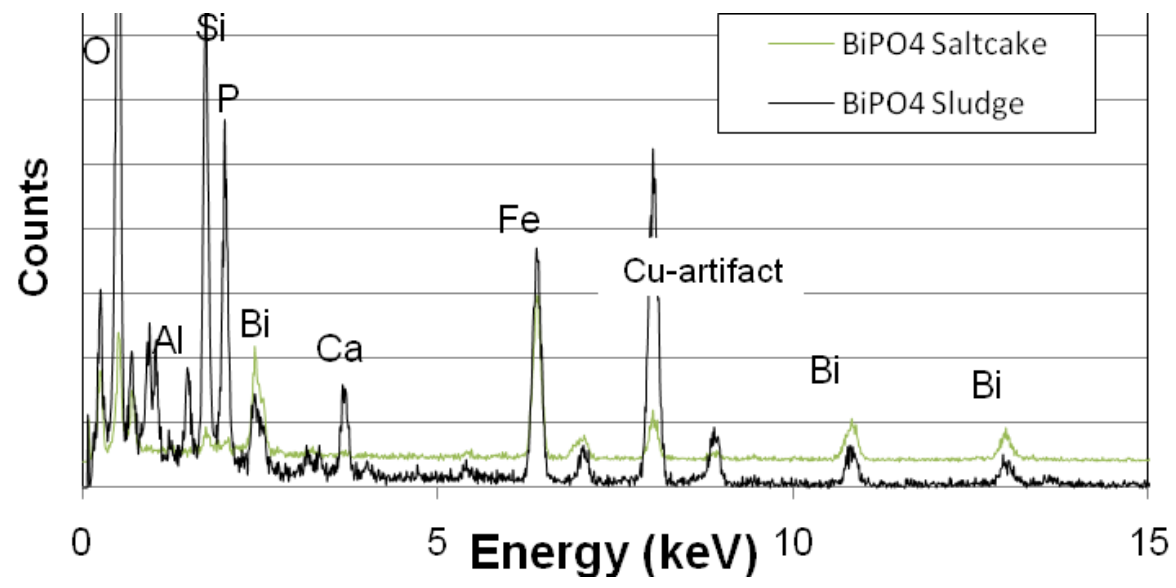

Figure 3.9. TEM-EDS Analysis of Main Amorphous Bismuth Phase Found in the BP Sludge and Saltcake. For Information Only.

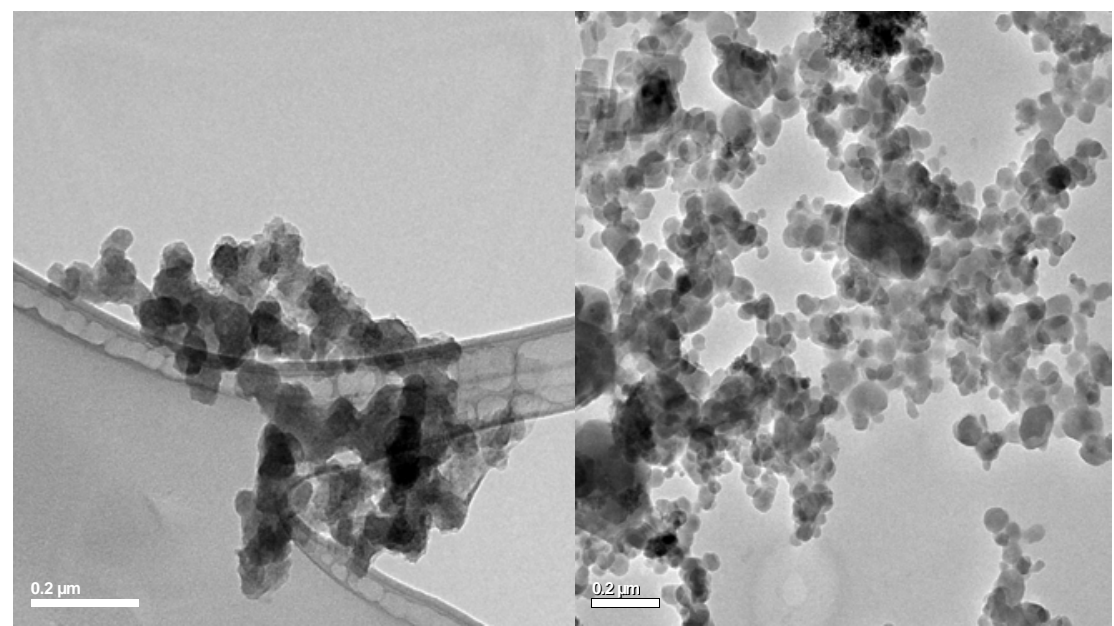

Figure 3.10. TEM Images of (a) BP Sludge and (b) Leached BP Sludge that Can Be Used for Estimating the Fractal Dimension (taken from Lumetta et al. 2009a) 


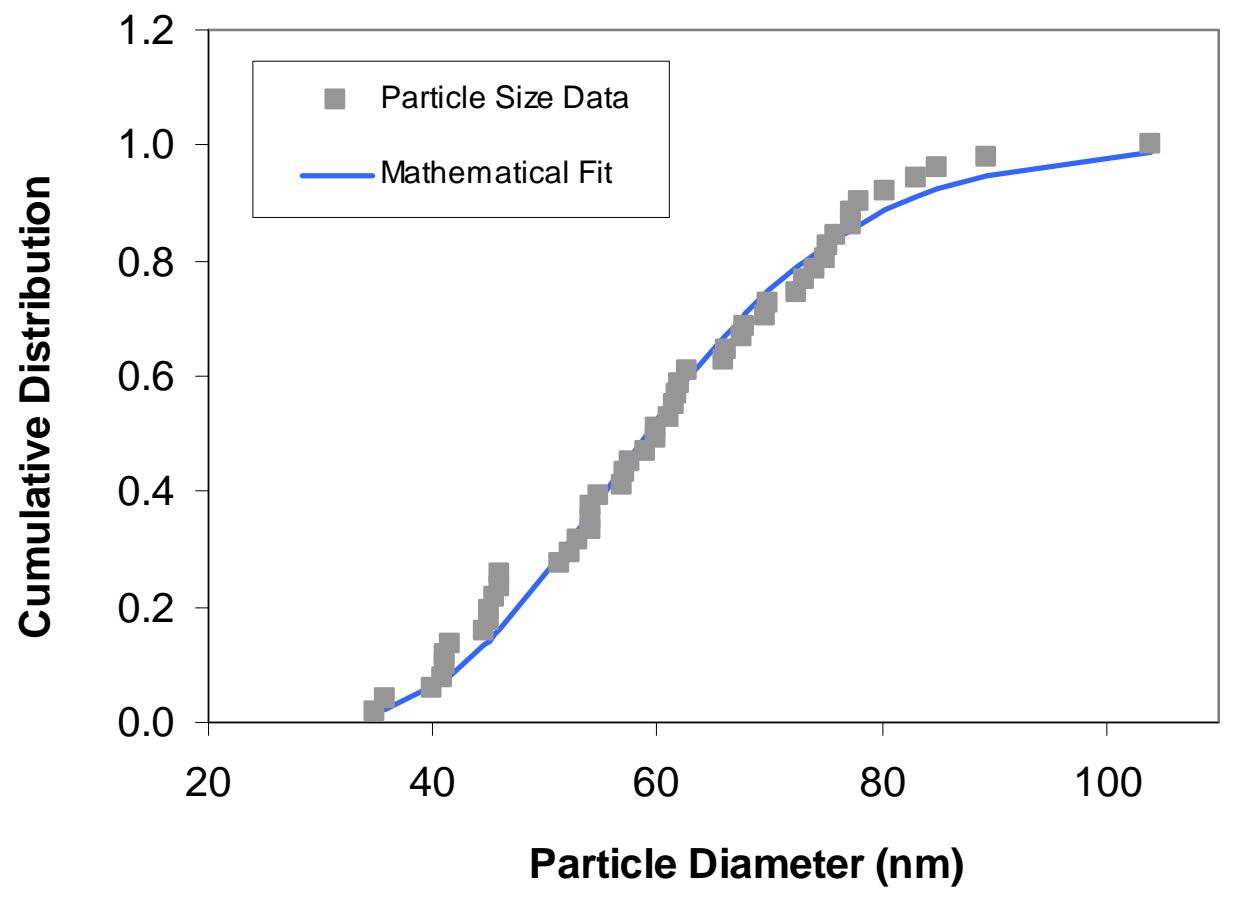

Figure 3.11. Cumulative Distribution of Particle Sizes for Bismuth-Iron Oxide Phase

Leaching resulted in particles that had much the same dimensions as the untreated bismuth-phosphate sludge, but were more enriched in uranium and hence were denser (see Table 3.12). SEM images of the bismuth phases were unable to resolve the particle size dimensions. Using values obtained from these images would result in an overestimation of the heavy particle fraction. The results for particle size were based on TEM data. The particles of bismuth should be considered spherical. Only a bismuth iron oxide and bismuth oxide were modeled for this report, based on assessments of the expert panel (panel meeting notes provided in Appendix D).

\subsubsection{Aluminum}

The major aluminum phases in Hanford tank waste include boehmite (AlOOH) and gibbsite as well as nitrate-cancrinite and dawsonite. The dominant aluminum phase in the PUREX cladding waste has been found to be gibbsite, based on XRD analyses. Gibbsite and other aluminum phase images are shown in Appendix D. Boehmite has been identified in TEM studies of sample Group 5 (REDOX), Figure 3.12. The phase was identified by its rhombohedral platelet-like morphology. Boehmite is sometimes observed to be fibrous or acicular, so such observations are not always diagnostic. In the presence of nitrate, boehmite is known to precipitate as hexagonal plates. The morphology of gibbsite crystals evolves from thin, rounded hexagons and faceted lozenges into faceted plates and blocks with well-formed basal prismatic faces. When boehmite is present in excess, dispersion and re-agglomeration processes "fluff-up" agglomerates, whereas when gibbsite is in excess, the small boehmite particles can become attached to the larger particles and will not contribute to changing the density of the solids. 
Table 3.12. Compositional Analysis of Bismuth Phosphate Sludge “As-Received.” (For Information Only.)

\begin{tabular}{|c|c|c|c|}
\hline \multicolumn{5}{|c|}{ Bismuth Phosphate Sludge } \\
\hline $\begin{array}{c}\text { Ratio } \\
(\mathrm{Bi} / \mathrm{Fe})\end{array}$ & $\begin{array}{c}\text { Ratio } \\
(\mathrm{Si} / \mathrm{P})\end{array}$ & $\begin{array}{c}\text { Atomic } \\
(\mathrm{Fe} / \mathrm{P})\end{array}$ & $\begin{array}{c}\text { Si/U } \\
(\text { Atomic })\end{array}$ \\
\hline 0.6 & 1.0 & 0.5 & 44.4 \\
\hline 0.9 & 0.9 & 0.7 & 28.4 \\
\hline 0.9 & 0.9 & 1.0 & 26.3 \\
\hline \multicolumn{4}{|c|}{ Caustic Leached Bismuth Phosphate Sludge } \\
\hline $\begin{array}{c}\text { Ratio } \\
(\mathrm{Bi} / \mathrm{Fe})\end{array}$ & $\begin{array}{c}\text { Ratio } \\
(\mathrm{Si} / \mathrm{P})\end{array}$ & $\begin{array}{c}\text { Atomic } \\
(\mathrm{Fe} / \mathrm{P})\end{array}$ & $\begin{array}{c}\text { Si/U } \\
(\text { Atomic })\end{array}$ \\
\hline 1.5 & 1.5 & 15.5 & 5.6 \\
\hline 1.5 & 1.9 & 21.5 & 0.0 \\
\hline 2.1 & 1.7 & 14.9 & 3.7 \\
\hline 1.9 & 2.8 & 37.0 & 2.2 \\
\hline 2.0 & 0.8 & 0.2 & 5.5 \\
\hline 2.5 & 0.8 & 0.1 & 5.2 \\
\hline 4.9 & 0.8 & 0.1 & 6.3 \\
\hline \multicolumn{5}{|c|}{} \\
\hline
\end{tabular}

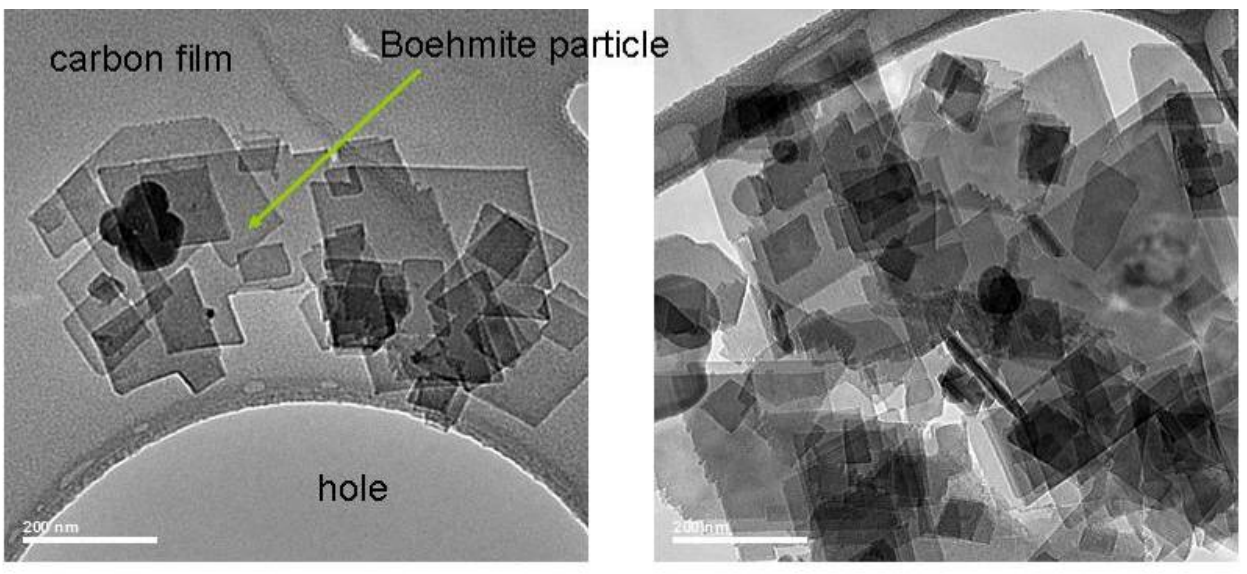

Figure 3.12. TEM Images of Boehmite Particles from Unleached REDOX Sludge (Fiskum et al. 2008, Figure 8.29, pg. 8.34)

Particle size data for boehmite was reported in Wells et al. (2007). The range was provided as $20 \mathrm{~nm}$ to $500 \mathrm{~nm}$ with a mode of $100 \mathrm{~nm}$. These data were based on a report by Lumetta et al. (1997). Based on the boehmite TEM work reported for REDOX tank sludge, Figure 3.13, the range was determined as 30 to $350 \mathrm{~nm}$ with a mode of $110 \mathrm{~nm}$, which is in very good agreement with the earlier study from a different set of Hanford tank sludges. The thicknesses of the boehmite crystals can be estimated based on their apparent transparency in TEM images obtained at $\sim 100 \mathrm{kV}$. This suggests that they cannot be much thicker than $30 \mathrm{~nm}$. 


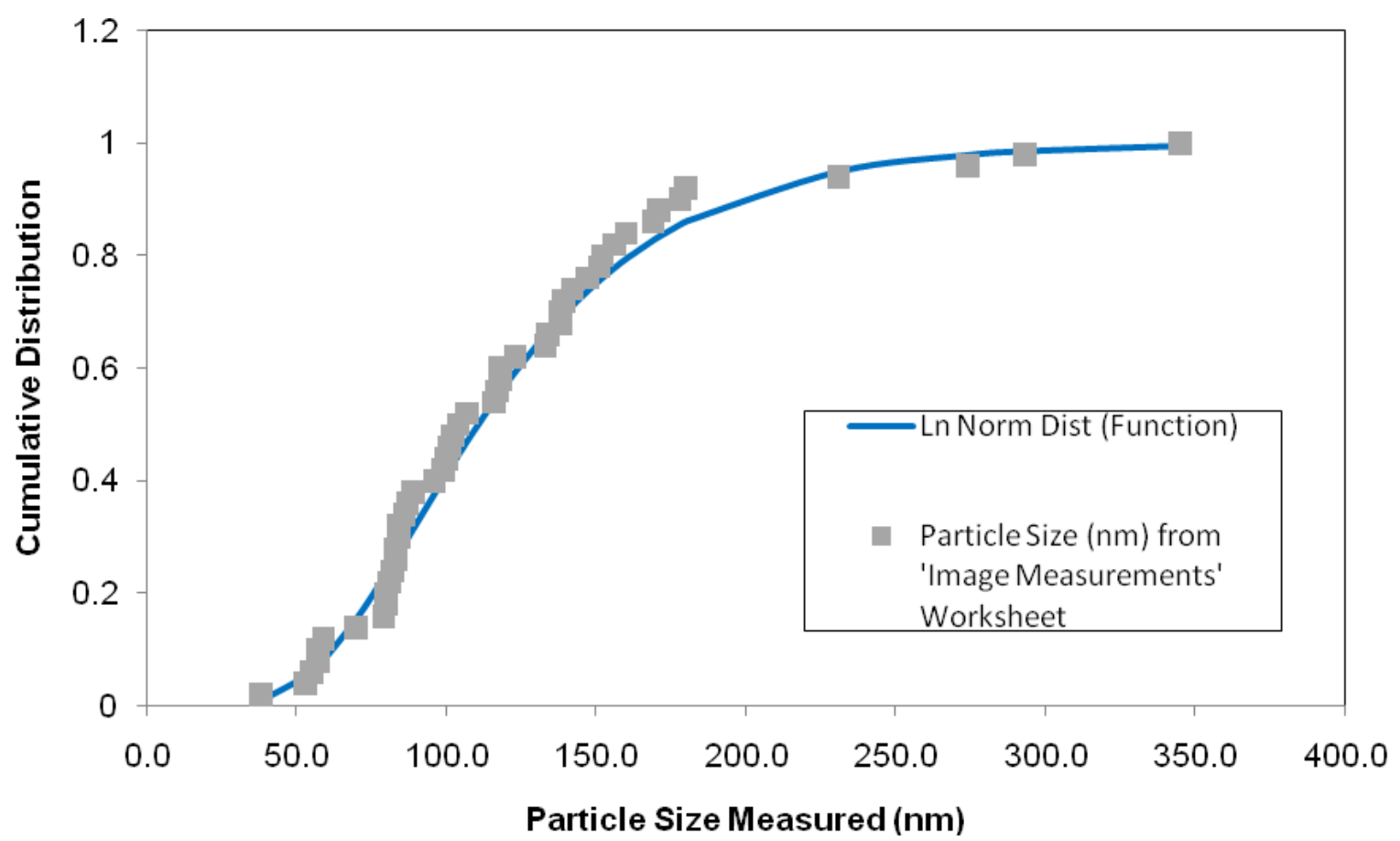

Figure 3.13. Cumulative Distribution of Particle Sizes for Boehmite from REDOX Tank Sludge (Fiskum et al. 2008, Figure 8.30, pg. 8.35). For Information Only.

A critical parameter that governs the rate of dissolution of aluminum during waste pre-treatment is the form and particle size of the aluminum species. The two primary insoluble forms of aluminum present in the waste are gibbsite and boehmite, although there are also numerous minor and mixed species as well, particularly nitrate-cancrinite. Boehmite is difficult to dissolve, and gibbsite is relatively easy to dissolve in caustic. The dissolution rate of boehmite is thought to be the rate-limiting step in aluminum dissolution. The particle size and degree of crystallinity of each species also impact the dissolution rate, and viscosity, settling velocity, and filtration rates impact the liquid-solid phase separation steps that follow dissolution.

\subsubsection{Chromium Phases}

Chromium oxyhydroxide $(\mathrm{CrOOH})$ is predicted to make up more than $5 \mathrm{wt} \%$ of the salt-free solid in 40 tanks, with the highest concentrations (about $15 \mathrm{wt} \%$ up to $45 \mathrm{wt} \%$ ) being present in A-101, AN-104, AN-105, BY-112, S-109, SX-101, and SX-106. Based on characterization studies, opinions differ whether the chromium phase is $\mathrm{CrOOH}$ or $\mathrm{Cr}_{2} \mathrm{O}_{3}$. As described in Section 3.2.3, all $\mathrm{Cr}$ in this work is considered to be $\mathrm{CrOOH}$. Hanford tank samples from M12 Group 6 contained a mixed Al-Cr phase with variable $\mathrm{Al}$ and $\mathrm{Cr}$ content. Some particles are mainly $\mathrm{Cr}$, based on TEM analyses. Analysis with fine electron probes has indicated that the nano-sized particles remain mixed Al-Cr down to very fine scales. The distribution of these particles is difficult to define and is variable, depending on the technique chosen to examine the particles. On the SEM, only large agglomerates can be observed, and it was not clear if these agglomerates represent the behavior within sludge. On the TEM, the individual particulates can be observed, and these are described in Figure 3.14 where the range is 22 to $420 \mathrm{~nm}$. 
The chromium phases are amorphous solids. In Figure 3.15, a TEM image of the particles shows the nano-sized nature of the materials and their tendency to form agglomerates. The round particle marked with the 120.04-nm distance is an alumino-silicate, and the other particles are Al-Cr phases. Similar TEM images of caustic-leached solids show the same basic morphology, although leached phases are mostly devoid of aluminum. The PSD is similar to the unleached material. Similar phases were observed by Herting et al. (2002) with SEM where the small scale of the particulates could not be resolved, but the observation that $\mathrm{Al}$ and $\mathrm{Cr}$ are closely associated in this phase is consistent as shown in Figure 3.16. The PSD for chromium phases was based on this very fine distribution observed in the TEM studies.

\subsubsection{Iron Oxides}

Iron oxides have been observed as both amorphous iron oxides or hydroxides and as hematite. The size distribution of iron oxide/hydroxide is important as it also defines the forms of plutonium in the tanks other than oxide. On occasion, hematite particles have been observed to be several microns in length. The data shown in Figure 3.17 were presented in Wells et al. (2007) and show the distribution of iron oxides. The expert panel (see Appendix D) determined that $\mathrm{Ni}_{3}\left(\mathrm{PO}_{4}\right)_{2}$ would have the same size distribution data as $\mathrm{Mn}_{3}\left(\mathrm{PO}_{4}\right)_{2}$ because the compounds are analogous whereas $\mathrm{NiC}_{2} \mathrm{O}_{4} \cdot 2 \mathrm{H}_{2} \mathrm{O}$ would be treated as having the size data and density from $\mathrm{Fe}_{2} \mathrm{O}_{3}$ from Wells et al. (2007). Hence, data on both $\mathrm{FeOOH}$ and $\mathrm{Fe}_{2} \mathrm{O}_{3}$ (hematite) were included in the analysis.

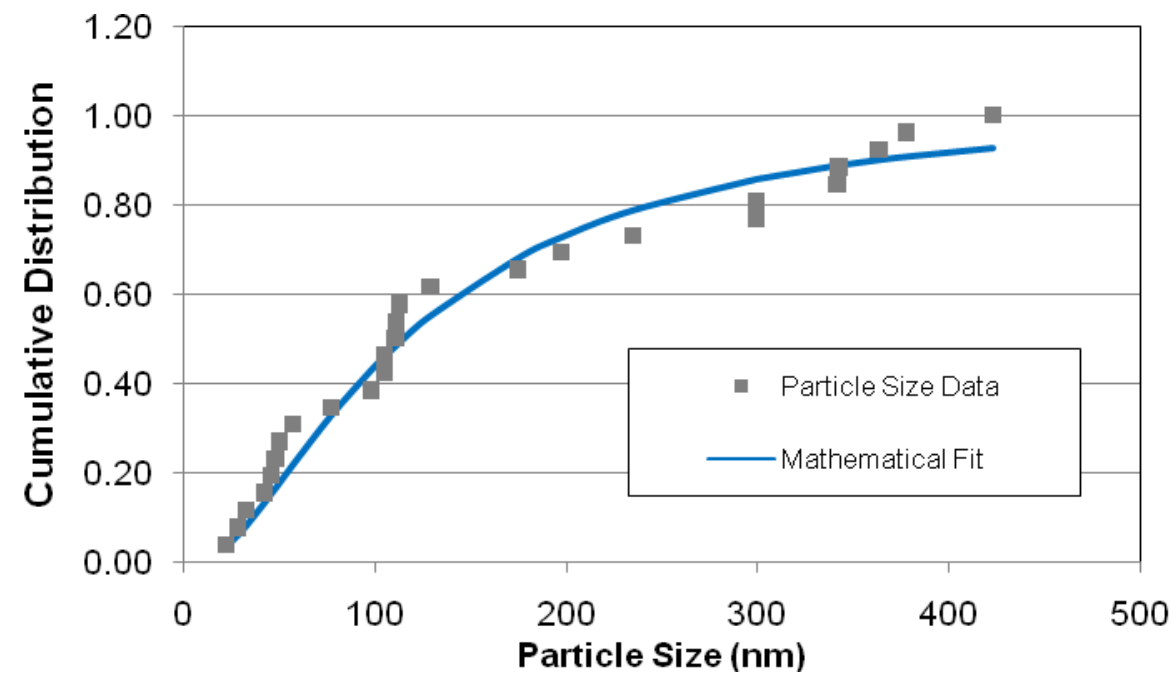

Figure 3.14. Cumulative Distribution of Particle Sizes for CrOOH from REDOX Tank Sludge (M12 Group 6, Fiskum et al. 2008) 


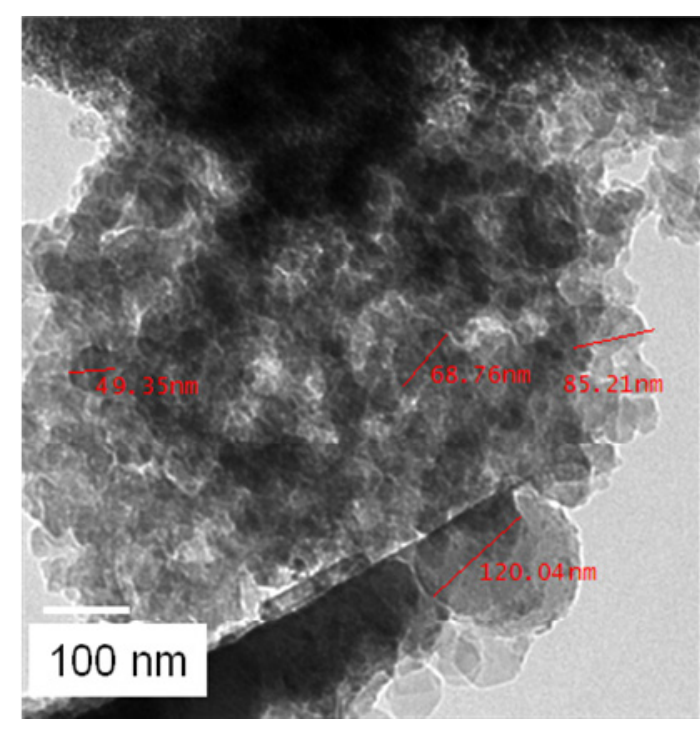

Figure 3.15. TEM Image of $\mathrm{CrOOH}$ Particles from Group 6 Tank Sludge (taken from Fiskum et al. 2008)

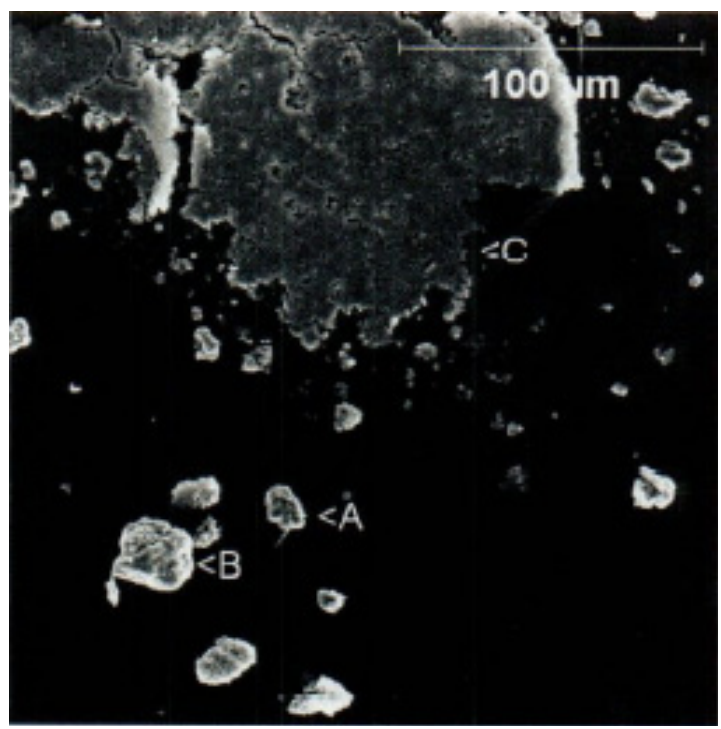

Figure 3.16. Particle Labeled (C) Consisted of a Fine Grained Aluminum and Chromium Bearing Particulate with Imbedded Uranium-Rich Particles (taken from Figure 5.7-2 from BY9Dil6, Herting et al. 2002).

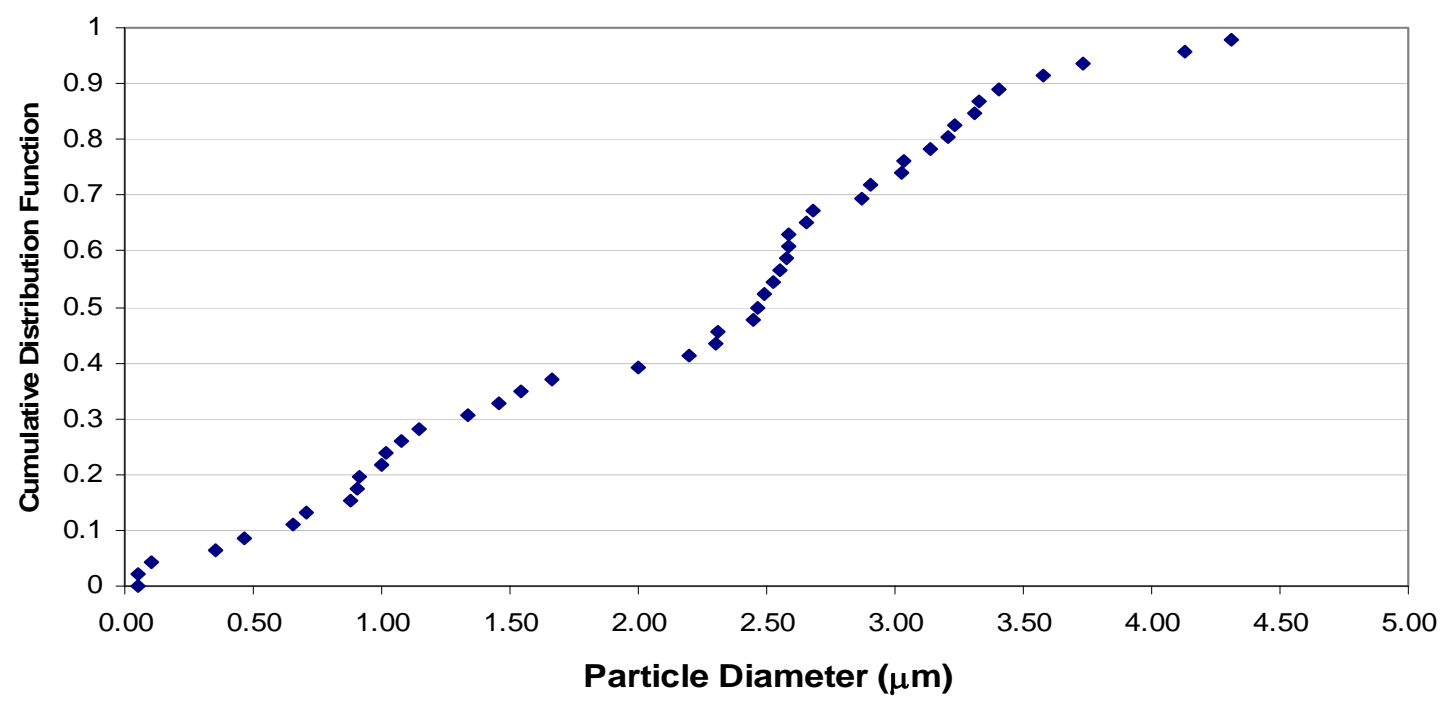

Figure 3.17. PSD for Hematite/ Ferrihydrite Phases (Wells et al. 2007). Notes: Ferrihydrite and hematite sizes taken from images ${ }^{(a)}$ AY102, AZ102, C101, C103, C106, SY102, and AN107, which were taken from the following (Frye 2005, Herting and Cooke 2004, Bechtold et al. 2002, Callaway and Cooke 2004, Herting et al. 2004) and Figure 4.11, pg. 4.14 (AZ-101) (Buck et al. 2003).

(a) (S06R000025-13a.jpg, S03T002066-2a.jpg, S05R000161-10a.jpg, C103FE-OXIDE.jpg, S03R000016A-14a.jpg, S02T002424-7a.jpg) 


\subsubsection{Strontium-Bearing Phases}

Strontium was reported as occurring in hydrouvarovite $\left[(\mathrm{Ca}, \mathrm{Sr})_{3}(\mathrm{Cr}, \mathrm{Al})_{2}(\mathrm{OH})_{12}\right]$, and $\mathrm{SrCO}_{3}$ (strontianite) may also be present in some wastes; indeed, a dissolution test seemed to indicate its presence in a saltcake waste sample (expert panel meeting notes, Appendix D). The expert panel also suggested that the $\mathrm{Sr}_{3}\left(\mathrm{PO}_{4}\right)_{2}$ particle size range should be changed to a larger one that reflects the nastrophite $\left(\mathrm{NaSrPO}_{4} \bullet 9 \mathrm{H}_{2} \mathrm{O}\right)$ observations. Nastrophite was been observed as extremely large $200-\mu \mathrm{m}$ particles in salt wastes. Wells et al. (2007) obtained a particle size range for the $\mathrm{Sr}_{3}\left(\mathrm{PO}_{4}\right)_{2}$ that would be used to describe $\mathrm{SrCO}_{3}$ in the final assessment. Actual TEM data on a strontium phosphate phase, which was found in ferrocyanide sludge (M12 Group 8), was identified as the apatite mineral phase, $\mathrm{Sr}_{5}\left(\mathrm{PO}_{4}\right)_{3}(\mathrm{OH})$ (see Fiskum et al. 2008). The determined PSD is shown in Figure 3.18. The particles were elongated, and the shape factor for these particles was calculated to be $0.44 \pm 0.10$ (Figure 3.19). The shape was determined assuming that the particle thickness was the same as the width, which is reasonable because the particles would be electron transparent below a thickness of 30 to $50 \mathrm{~nm}$. Given the analysis of the strontium phosphate with TEM as a sludge-based apatite-phase and the reported salt phases containing Sr, there is significant disagreement on the nature of the strontium phases in the Hanford sludge (see Section 3.2.3). The conservative approach has been to use the larger size ranges.

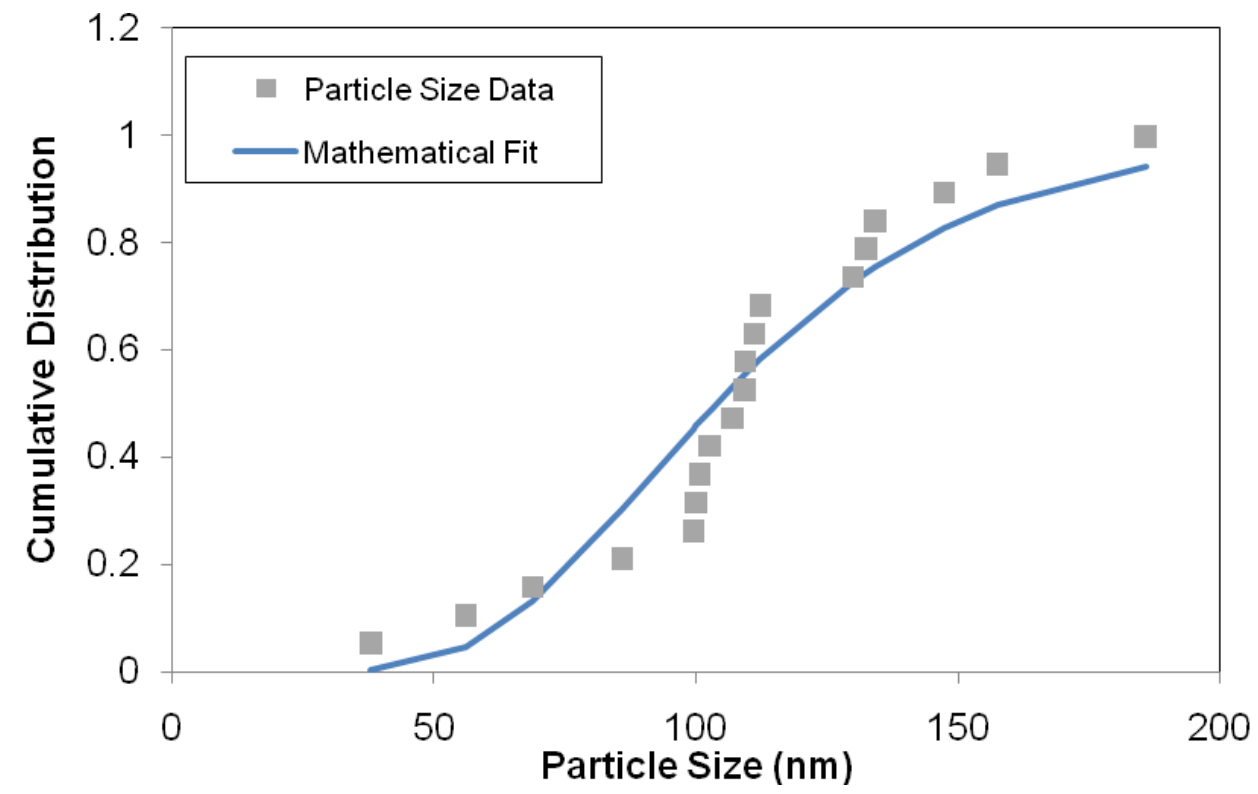

Figure 3.18. Cumulative Distribution of Particle Sizes for Strontium Phosphate Phase from Ferro-Cyanide Tank Sludge (M12 Group 8) 


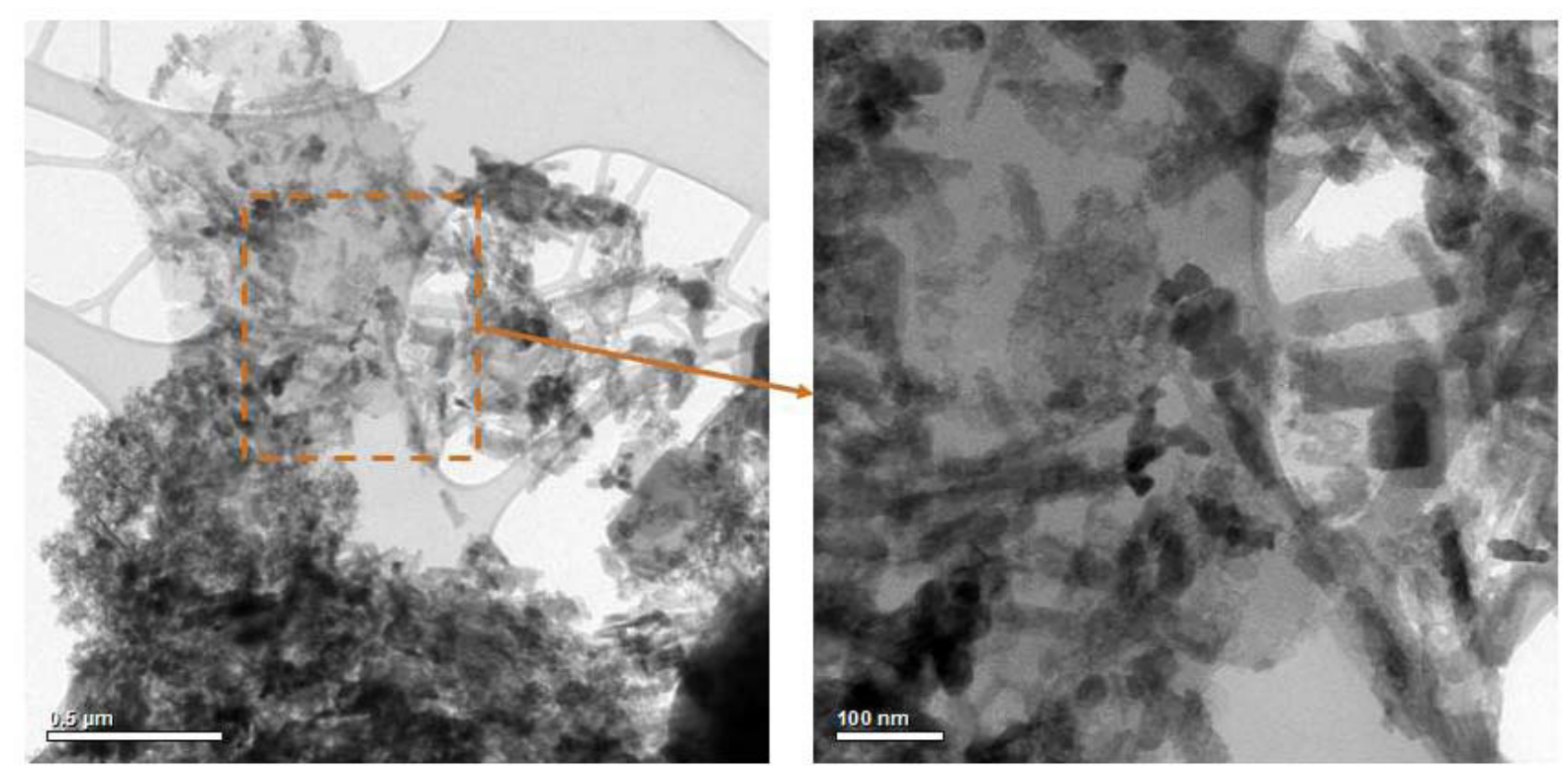

Figure 3.19. TEM Image of Strontium Phosphate Particles from Ferrocyanide Tank Sludge Group (taken from material reported in Fiskum et al. [2009a], Figure 3.21, pg. 3.34 and images contained in the M12 Group 8 TEM data).

\subsubsection{Uranium Phases}

In the Hanford tanks, $\mathrm{U}(\mathrm{VI})$ has been predicted to precipitate initially as sodium uranate $\left(\mathrm{Na}_{2} \mathrm{UO}_{4}\right)$ or as a soluble peroxide complex salt $\mathrm{Na}_{4} \mathrm{UO}_{2}\left(\mathrm{O}_{2}\right)_{3}$ (Krot et al. 1998). Under alkaline conditions (with heat and oxygen-free environments) the sodium uranate would be anticipated to transform to $\mathrm{U}_{3} \mathrm{O}_{8} \bullet \mathrm{nH}_{2} \mathrm{O}$ and $\mathrm{UO}_{2} \bullet \mathrm{nH}_{2} \mathrm{O}$ (Giammar and Herring 2004). Lumetta et al. (1997) reported the existence of a sodium clarkeite $\left\{\mathrm{Na}\left[\left(\mathrm{UO}_{2}\right) \mathrm{O}(\mathrm{OH})\right]\left(\mathrm{H}_{2} \mathrm{O}\right)_{0-1}\right\}$ in samples from Hanford tanks AN-102 and S-110. This observation is supported by Snow et al. (2008) who identified clarkeite in leached AN-101. The clarkeite XRD pattern is similar to that of sodium uranium oxide $\left[\mathrm{Na}_{2} \mathrm{U}_{2} \mathrm{O}_{7}\right]$, but in these cases, the clarkeite pattern resulted in an improved fit to the data. The size distribution for the round clarkeite phase is shown in Figure 3.20. In contrast, Krupka et al. (2006) were unable to confirm the occurrence of clarkeite in Hanford tanks C-203 and C-204 but identified čejkaite $\left[\mathrm{Na}_{4}\left(\mathrm{UO}_{2}\right)\left(\mathrm{CO}_{3}\right)_{3}\right]$. 


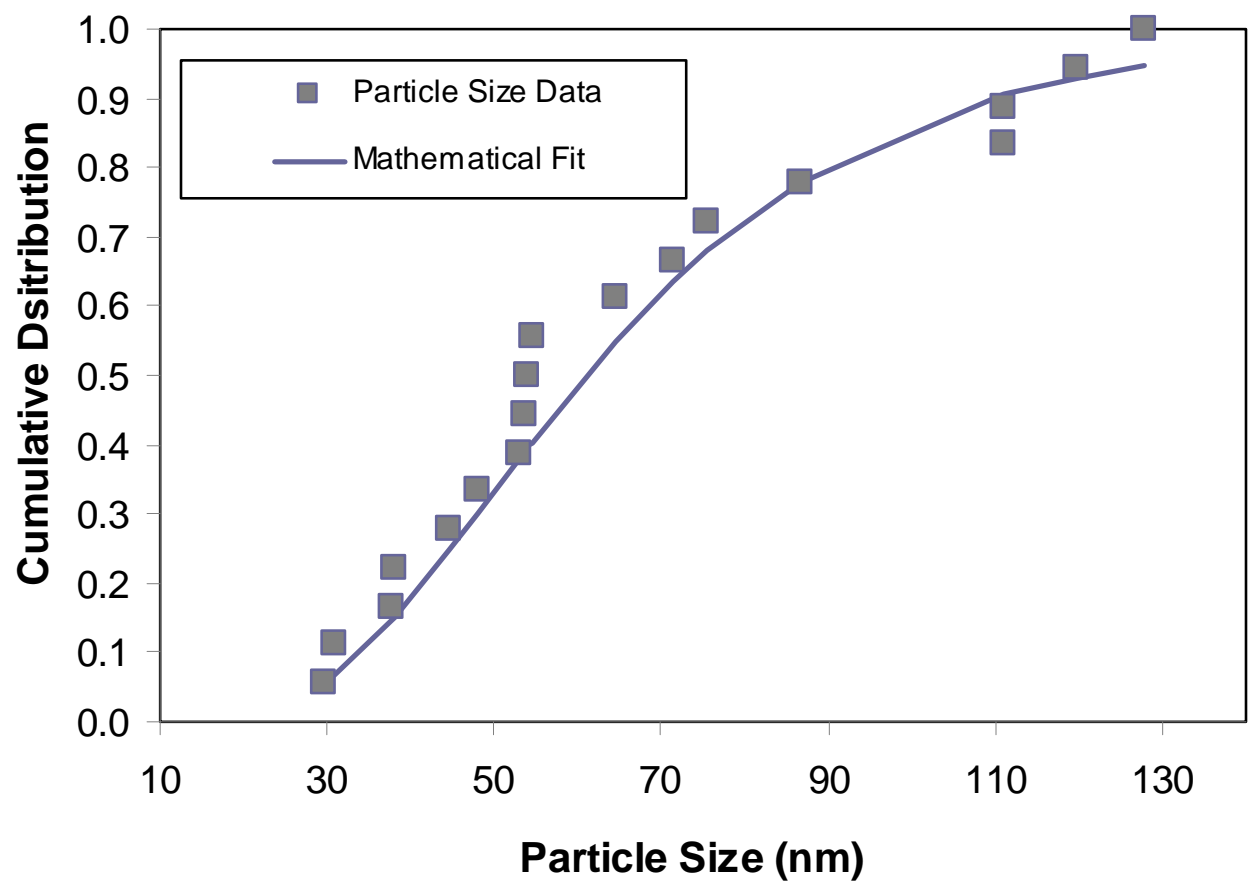

Figure 3.20. Cumulative Distribution of Particle Sizes for Uranium Oxide Phase from Tank Sludge (M12 Group 5). (Data taken from images reported in Shimskey et al. 2009b.)

The size distribution of čejkaite (uranium), plutonium phase particles, aluminum phases, cancrinite (zeolitic) phases, and iron oxide phases were determined for the current work. Three of the actinide phases had similar size ranges, except the uranium oxide type particles. However, cejkaite, the uranyl carbonate phase (both a natural and Tank-derived phase), is only considered stable under evaporative conditions, and hence, the large particle sizes observed for this phase are unlikely to occur during waste processing.

A clarkeite-related phase has been reported in several studies previously. In Wells et al. (2007), the maximum size for clarkeite was estimated at $15 \mu \mathrm{m}$; however, inspection of the cumulative distribution in Figure 3.21 suggests that this value is too high. The maximum value is around $5 \mu \mathrm{m}$ based on low-resolution SEM data. It is more likely that these particles are considerably smaller.

The uranium phases observed in the REDOX sludge (M12 Group 5) were also identified as clarkeite and ranged in size from a few nanometers up to $100 \mathrm{~nm}$. The particles were both round and elongated, as shown in Figure 3.22. In PUREX sludge samples, the uranium phases were extremely small, ranging in size from 2 to $10 \mathrm{~nm}$ in diameter (Figure 3.23). Based on the high-resolution images, the uranium particles were clearly crystalline. These were different from the clarkeite particles identified in REDOX sludge and may be consistent with the predictions of nano-particles made by Burns (2010). Nevertheless, as the current concern is with maximum particle sizes, the occurrence of these nano-phase particles of uranium need not be considered. The recommendation is that the present value of $15 \mu \mathrm{m}$ for the maximum size be reduced to $5 \mu \mathrm{m}$. This value remains extremely conservative based on the TEM analyses. 


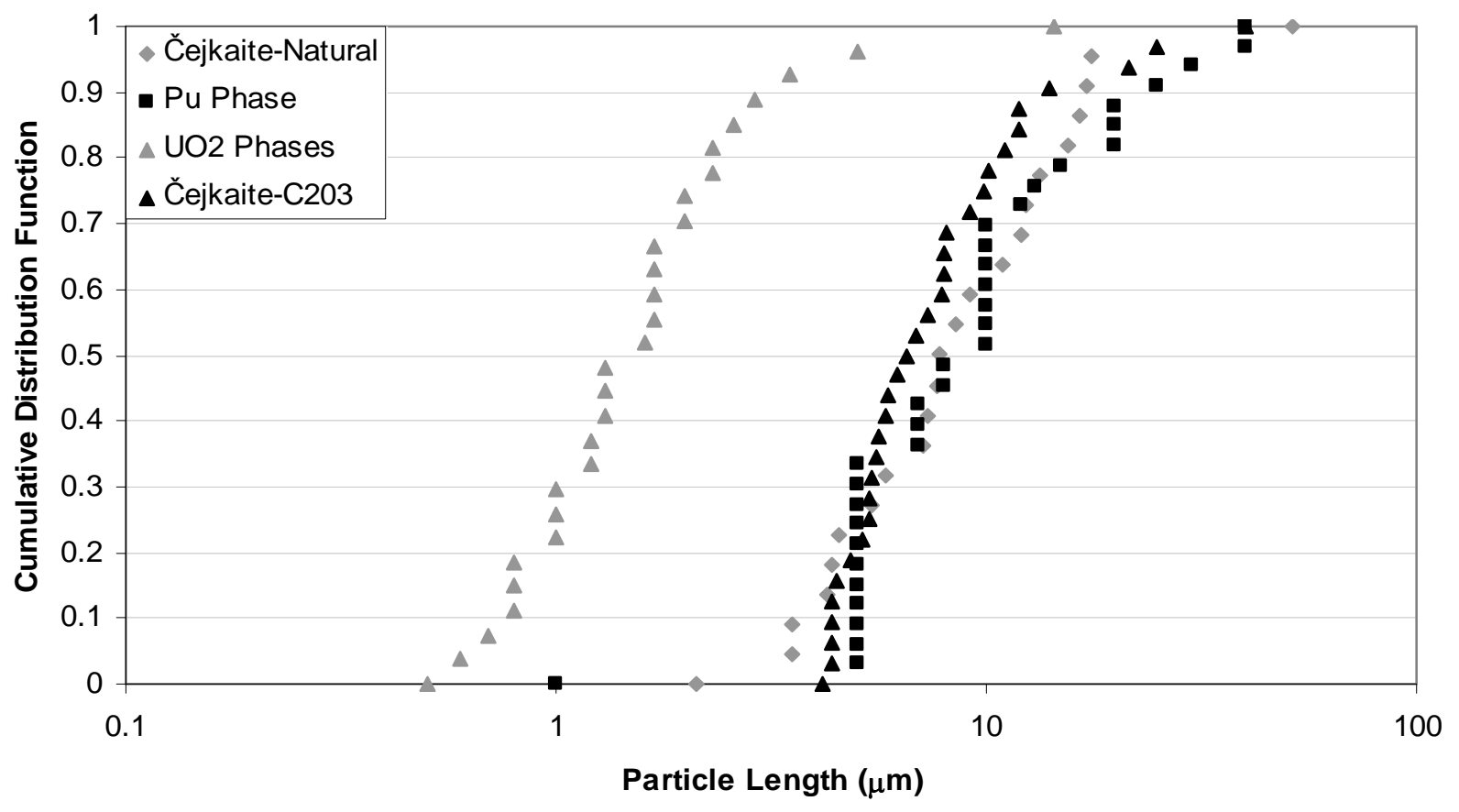

Figure 3.21. Cumulative Distribution of Particle Sizes for Actinide Phases (Wells et al. 2007). Source: Krupka et al. (2006); Callaway and Cooke (2004).

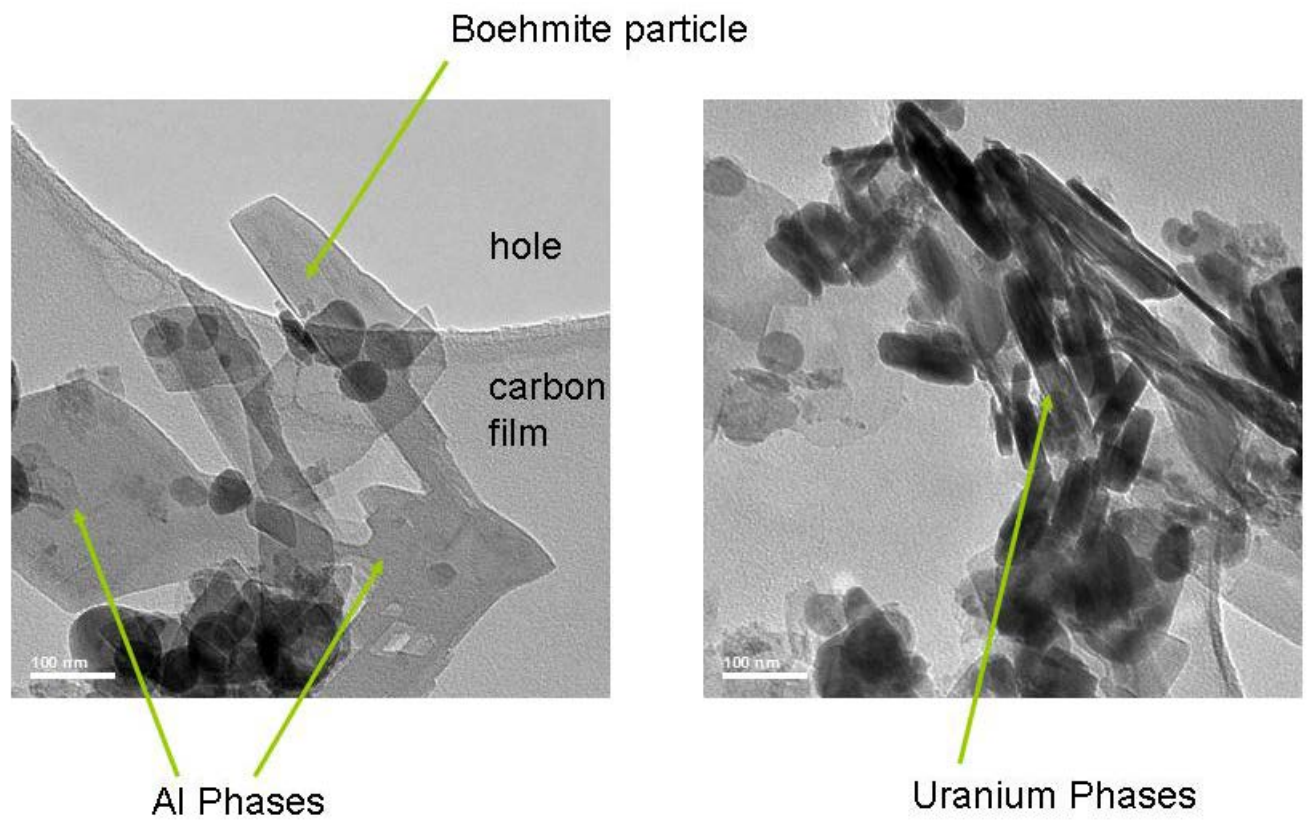

Figure 3.22. Bright Field Image of Elongated Uranium Phases in REDOX Sludge Samples Associated with Boehmite Crystallites. Taken from Fiskum et al., 2008, Figure 8.36, pg. 8.38. 


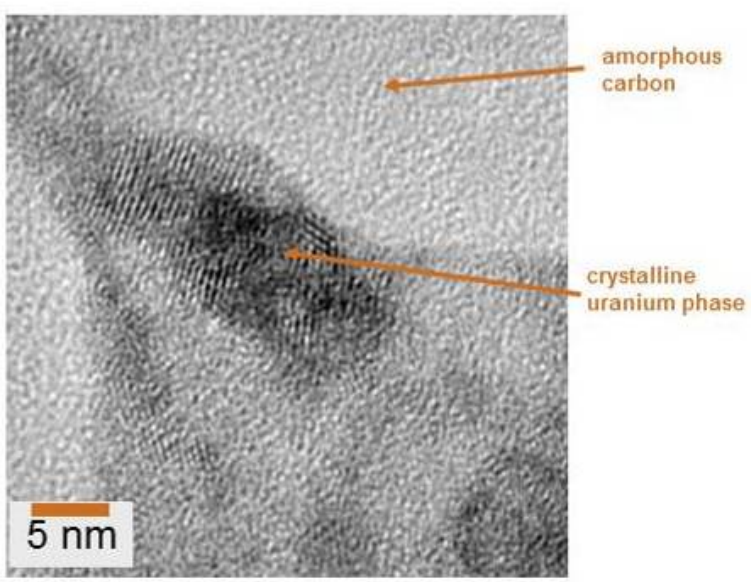

Figure 3.23. High-Resolution Images of Uranium Particles in M12 Group 3 (PUREX) (taken from material reported in Snow et al., 2009, Figure 3.20a, pg. 3.33)

Uranium phases in each of the tank sludge composites examined in the M-12 study had different morphologies, size ranges, and compositions. The uranium phases found in G3-S-WL appeared to be the smallest sized, uranium-bearing particles found.

Figure 3.24 shows highly heterogeneous particle agglomerates. Gibbsite, cancrinite, zirconium-rich phases, and uranium oxide particles are all present. Differentiating phases and determining particle sizes, is difficult using SEM images when multiple phases are present and one phase is significantly larger than the others in size (gibbsite in this case).

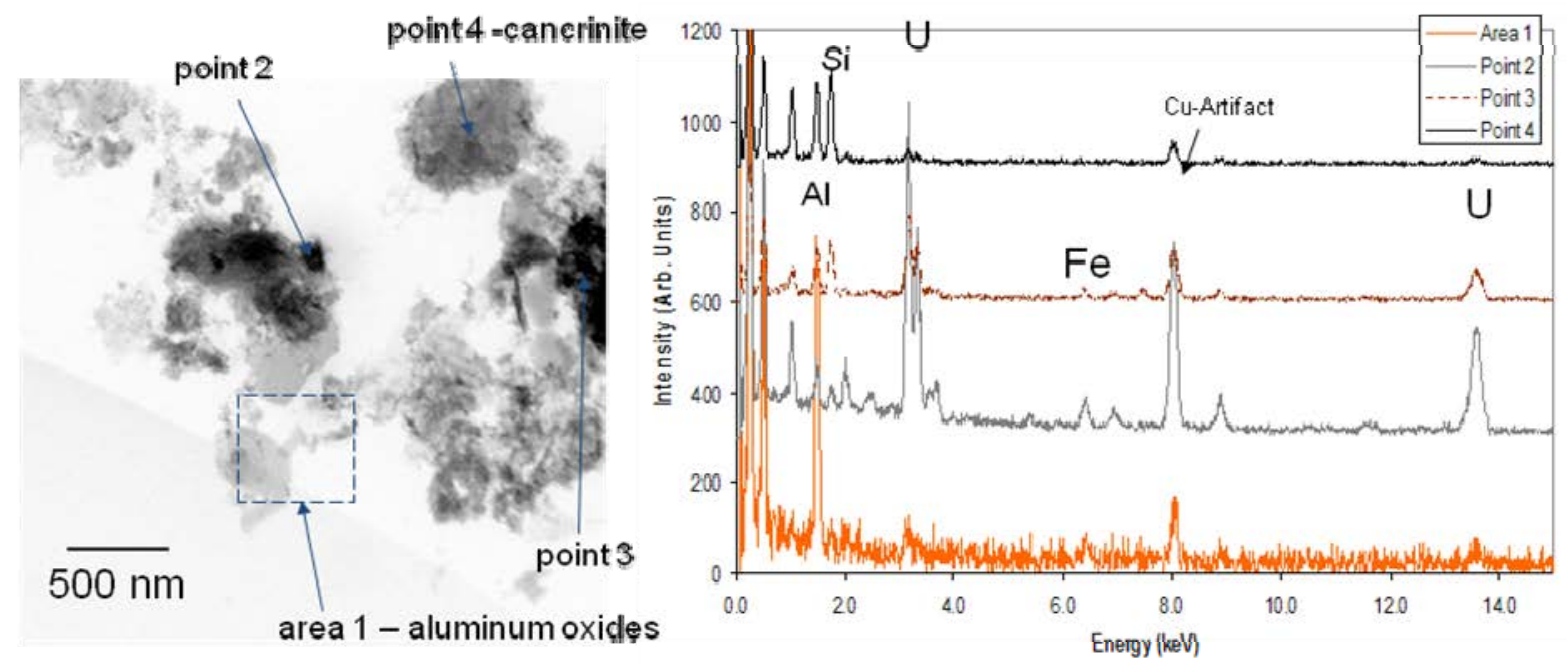

Figure 3.24. STEM-HAADF Image (inverted contrast) and EDS Analyses of Regions of Interest (taken from Snow et al. 2009, Figure 3.18, pg. 3.31)

\section{Uranyl Phosphates}

Very large crystals of a uranium phosphate have been reported in TBP sludge samples (see Edwards et al. 2009). These particles were several micrometers in length and up to 1 to $2 \mu \mathrm{m}$ in width. For the 
current work, these were assigned a particle length of $5 \mu \mathrm{m}$. In the TEM, many of the particles were electron transparent suggesting that they were $<50$ to $100 \mathrm{~nm}$ thick (see Figure 3.25). A few small precipitates were found on the surface of the acicular crystals. An EDS probe was used to determine the exact composition of the phase. Sodium was visible, but was removed rapidly by condensing down the probe even slightly. Electron diffraction on the platy axis revealed a well-formed rectangular lattice. The phase was indexed to a sodium uranyl phosphate reported by Locock et al. (2004).

There are many varieties of uranyl phosphates in nature, with autunite and meta-autunite being the most common. Sodium meta-autunite can be reversibly hydrated to form fully hydrated sodium autunite. Both minerals are tetragonal (Finch and Murakami 1999). Following caustic leaching of this sludge type, the uranyl phosphates dissolved and re-precipitated as much smaller amorphous particles.

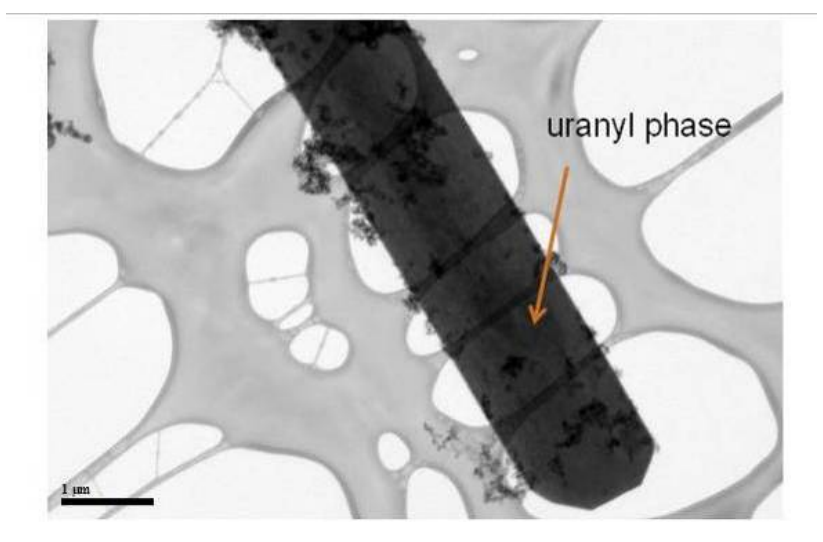

Figure 3.25. TEM Image of Large Uranyl Phosphate in TBP Sludge (taken from Edwards et al. 2009, Figure 3.23, pg. 3.31). Scale bar is $1 \mu \mathrm{m}$.

\subsubsection{Plutonium Phases}

Appendix D provides images of Pu particles as provided in Wells et al. (2007). A particle shape factor was used to adjust the likely size of these phases. The expert panel recommended applying $\mathrm{PuO}_{2}$ properties to SY-102 and TX farm Pu and assigning the $\mathrm{Pu}(\mathrm{OH})_{4}$ identity and FeOOH properties to $\mathrm{Pu}$ in all other tanks, thus treating it as co-precipitate (see Appendix D). The particle size range for FeOOH is therefore used to describe the density and size characteristics for most Pu in the Hanford inventory.

\subsubsection{Iron Phosphates}

Data on iron phosphates have been obtained from characterizing the M12 Group 7 (TBP) sludge samples. These phases were present as both single crystals and as a partially amorphous material (see Figure 3.26).

Plate-like sodium iron phosphates from TBP sludge were between 5 and $20 \mu \mathrm{m}$ in diameter with a mean diameter of $15 \mu \mathrm{m}$. The compositional analysis of the phase based on TEM-EDS analyses was $\mathrm{NaFe}_{2-3}\left(\mathrm{PO}_{4}\right)_{4-5}$. The Fe-phosphates, kidwellite $\left[\mathrm{Na}\left(\mathrm{Fe}^{3+}, \mathrm{Cu}\right)_{9+\mathrm{x}}\left(\mathrm{PO}_{4}\right)_{6}(\mathrm{OH})_{11} \cdot 3\left(\mathrm{H}_{2} \mathrm{O}\right)\right]$ and cyrilovite $\left[\mathrm{Na}\left(\mathrm{Fe}^{3+}\right)_{3}\left(\mathrm{PO}_{4}\right)_{2}(\mathrm{OH})_{4} \cdot 2\left(\mathrm{H}_{2} \mathrm{O}\right)\right]$ have been reported in nature in occurrence with autunites and other uranyl phosphates that were also observed in these specimens. Natrodufrenite $\left[\left(\mathrm{Fe}^{3+}, \mathrm{NaFe}^{2+}\right)\left(\mathrm{Fe}^{3+}\right.\right.$, 
$\left.\mathrm{Al})_{5}\left(\mathrm{PO}_{4}\right)_{4}(\mathrm{OH})_{6} \cdot 2\left(\mathrm{H}_{2} \mathrm{O}\right)\right]$ and ercitite $\left[\mathrm{Na}\left(\mathrm{Mn}^{3+}, \mathrm{Fe}^{3+}\right)\left(\mathrm{PO}_{4}\right)(\mathrm{OH}) \cdot 2\left(\mathrm{H}_{2} \mathrm{O}\right)\right]$ are formed under low-temperature hydrothermal conditions that may be similar to the environments found in the Hanford sludges. For example, cancrinite and the uranyl phosphates are known to form in laboratory tests under low temperature and high $\mathrm{pH}$ hydrothermal conditions and were found in these sludge samples from the tanks.

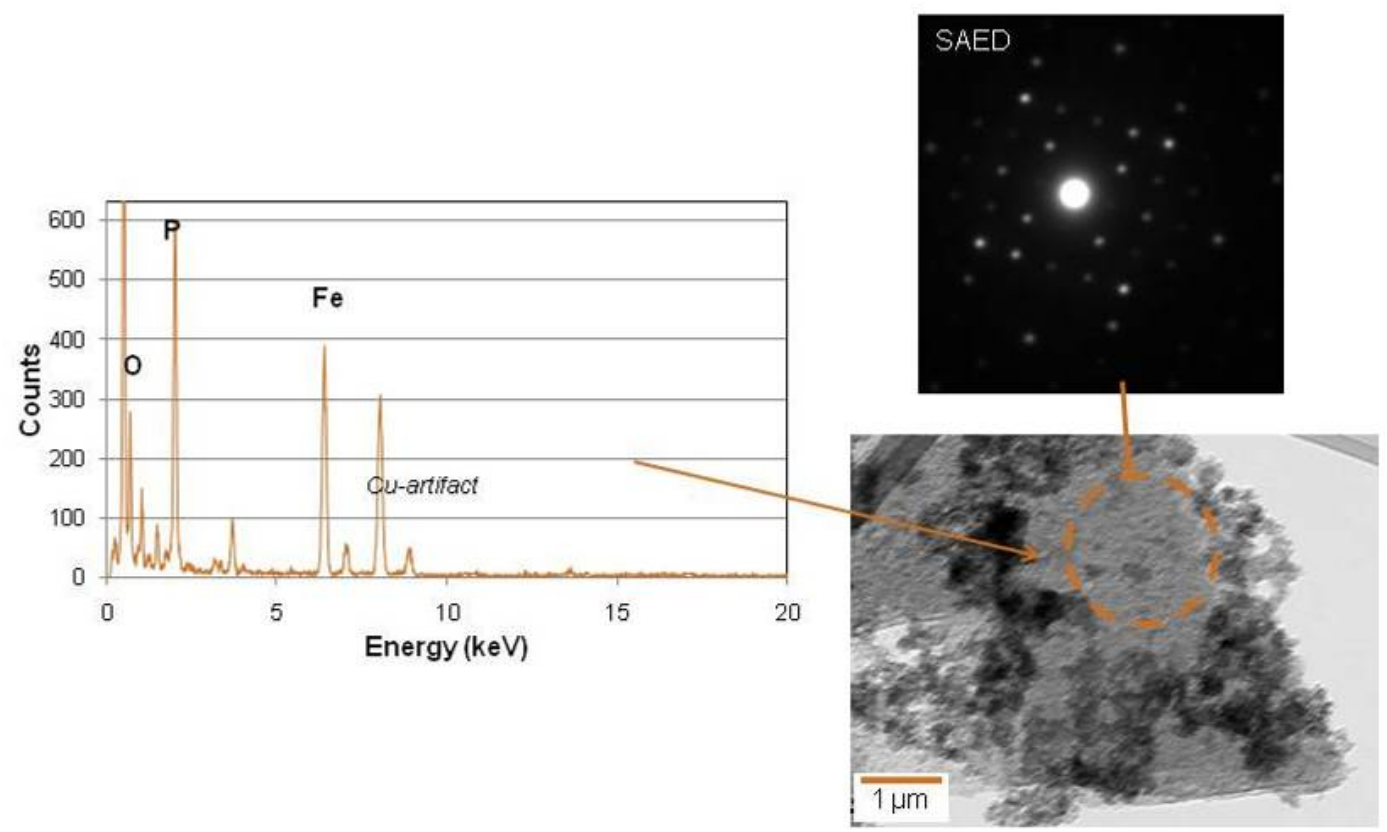

Figure 3.26. TEM-EDS Analysis of an Iron Phosphate Phase, Electron Diffraction of the Phase and a TEM Image (taken from Edwards et al. 2009, Figure 3.20, pg. 3.29)

\subsubsection{Zirconium Phases}

Zirconium phases were reported in the analysis of AZ-101 in Buck et al. (2003), but the image was not well resolved, and the individual crystallites could not be observed. The SEM image shown in Figure 3.27 was used to develop the distribution plot shown in Figure 3.28. The image was obtained at a low voltage to enable the particle shape to be observed, but the analysis to confirm the presence of $\mathrm{Zr}$ was performed at high voltage where image resolution is lost. This is a common problem in SEM when examining Hanford tank sludge.

Discrete zirconium oxide particles were observed in PUREX cladding waste sludge (M12 Group 3). These particles were between 50 and $100 \mathrm{~nm}$ based on TEM images. The example used for this UDS particle size study was taken from "as-received” material from C-103, which gives a large, more conservative distribution. The particles were elongated, but the precise shape factor value is indeterminate. 


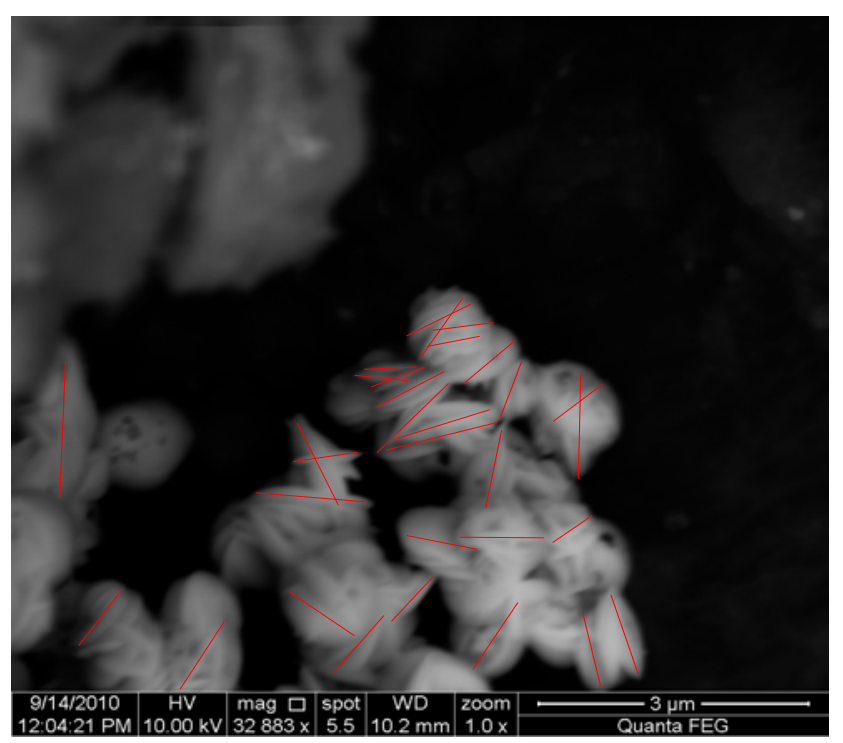

Figure 3.27. SEM Image of ZrOOH Particles from Hanford Tank Sludge Solids C-103. For Information Only.

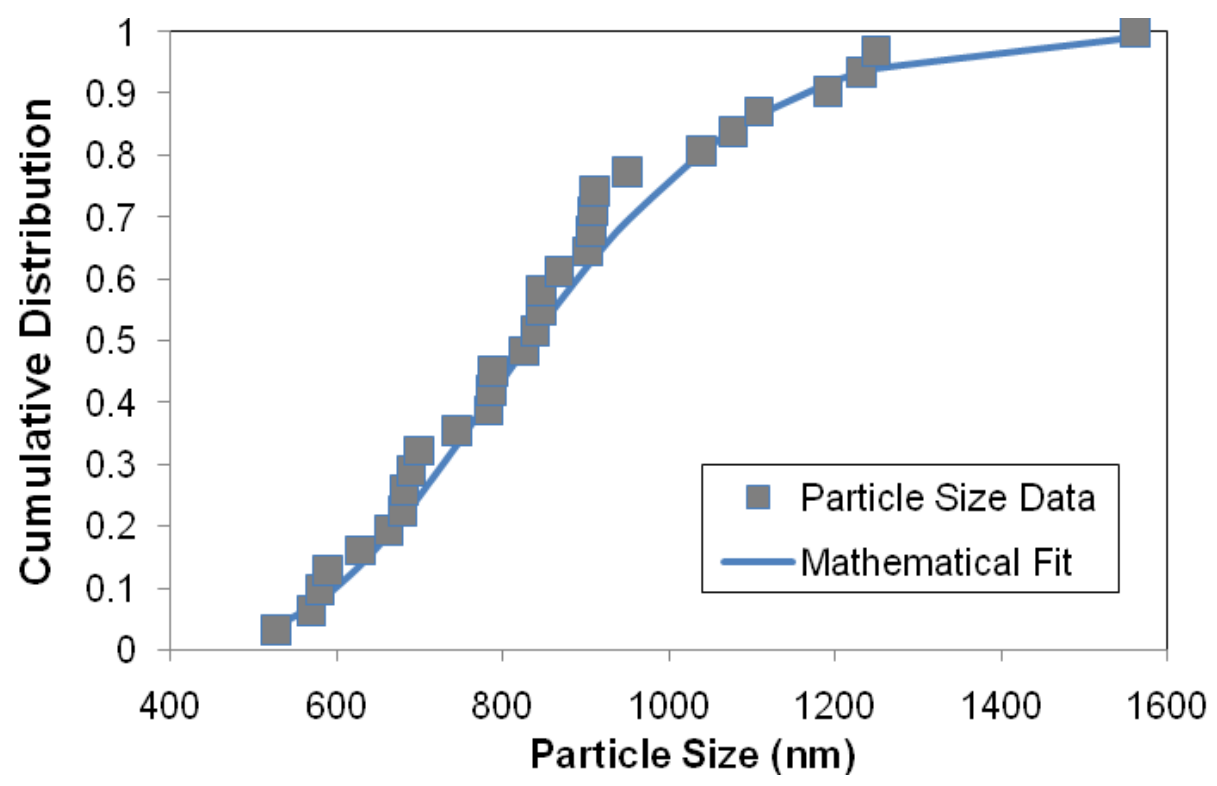

Figure 3.28. Cumulative Distribution of Particle Sizes for Zirconium Oxide (ZrOOH) Phase from Hanford Tank Sludge Solids (C-103)_SEM Image Data. For Information Only.

\subsubsection{Other Minor Phases}

Rare phases in the Hanford tanks have little useful particle size data. $\mathrm{Ag}_{2} \mathrm{CO}_{3}$ has not been observed, and it would be difficult to separate this phase from $\mathrm{AgO}_{2}$ without access to an energy-loss system on the TEM. Metallic Ag has been observed, but only in as-received C-106 (100\% AR waste type). In Figure 3.29, an example of an Ag-Hg alloy particle from C-103 sludge solids is shown. As the Ag density is high, it should be accounted for in the models as a metal. The rarity of Ag particles is such that PSDs could not be provided. Iron oxides may be an important phase for the sorption and/or 
co-precipitation of other elements, such as ruthenium (see Figure 3.30). Ruthenium is not included as a species of interest in the current evaluation.

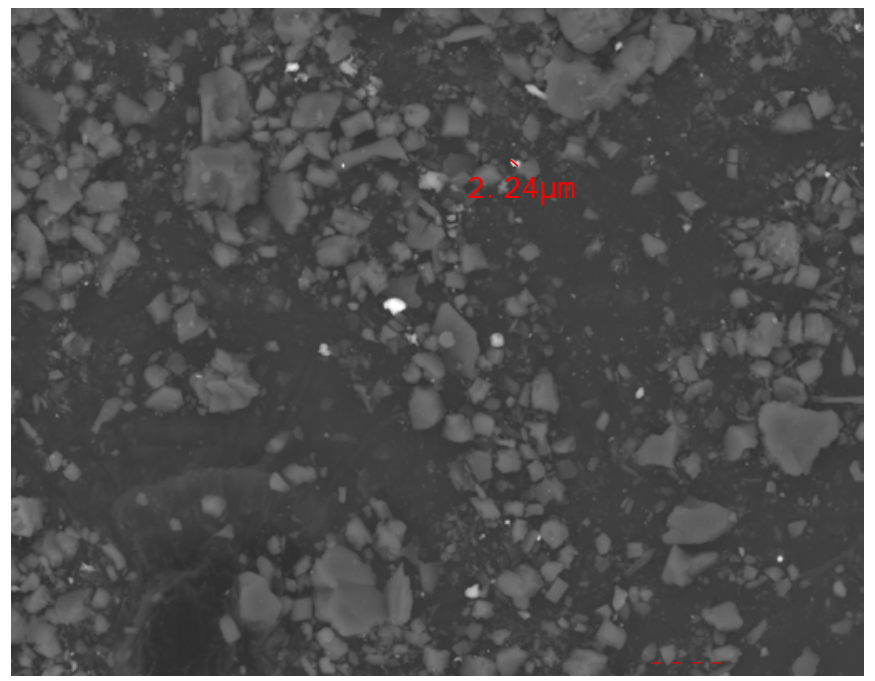

Figure 3.29. SEM Image of a Silver Particle from Hanford Tank C-103
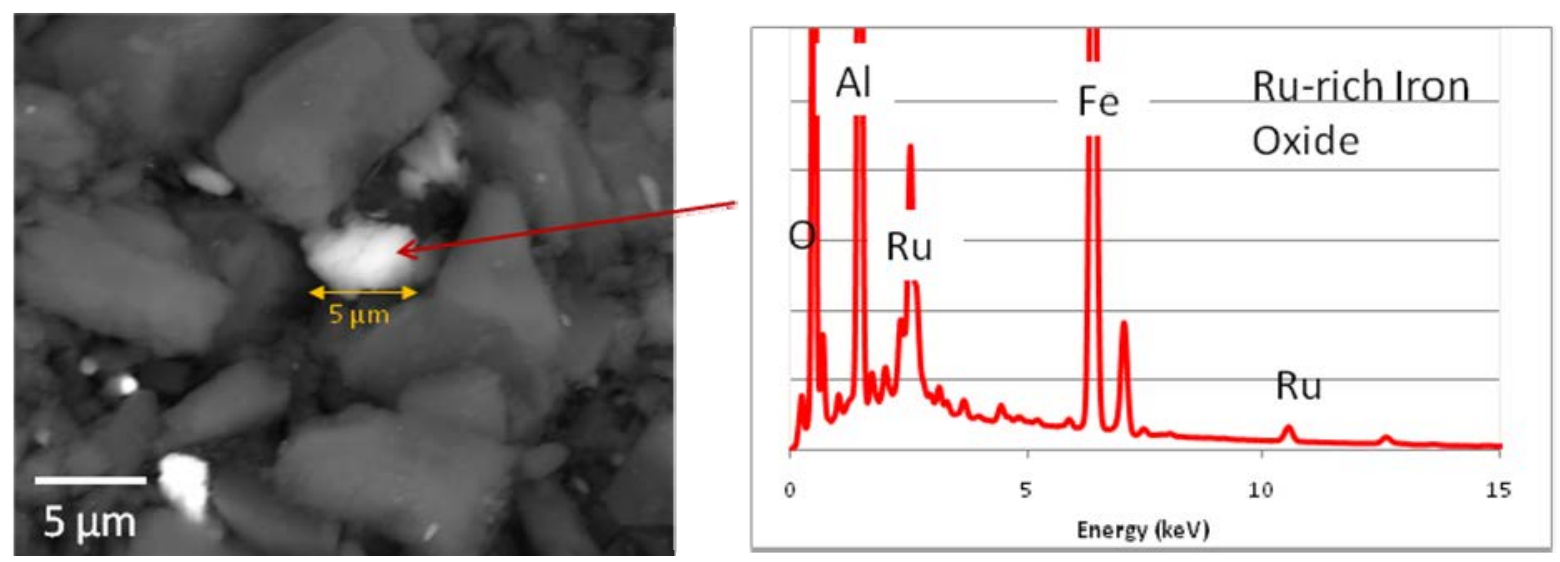

Figure 3.30. Ruthenium-Rich Iron Oxide Phase from Hanford Tank Sludge Solids C-103. For Information Only.

The occurrence of lead is of concern in the Hanford tank solids. It is unlikely that lead occurs as a discrete solid, and examples from the analysis of Hanford tank sludge solids C-103 indicate this. The co-existence of lead with iron oxides has been demonstrated through X-ray elemental mapping and analysis (see Figure 3.31). The expert panel (see Appendix D) recommended that the size-distribution data for $\mathrm{Pb}_{5} \mathrm{OH}\left(\mathrm{PO}_{4}\right)_{3}$ would be applied to all $\mathrm{Pb}$ compounds because those data show small sizes consistent with observations of $\mathrm{Pb}$-containing particles. 


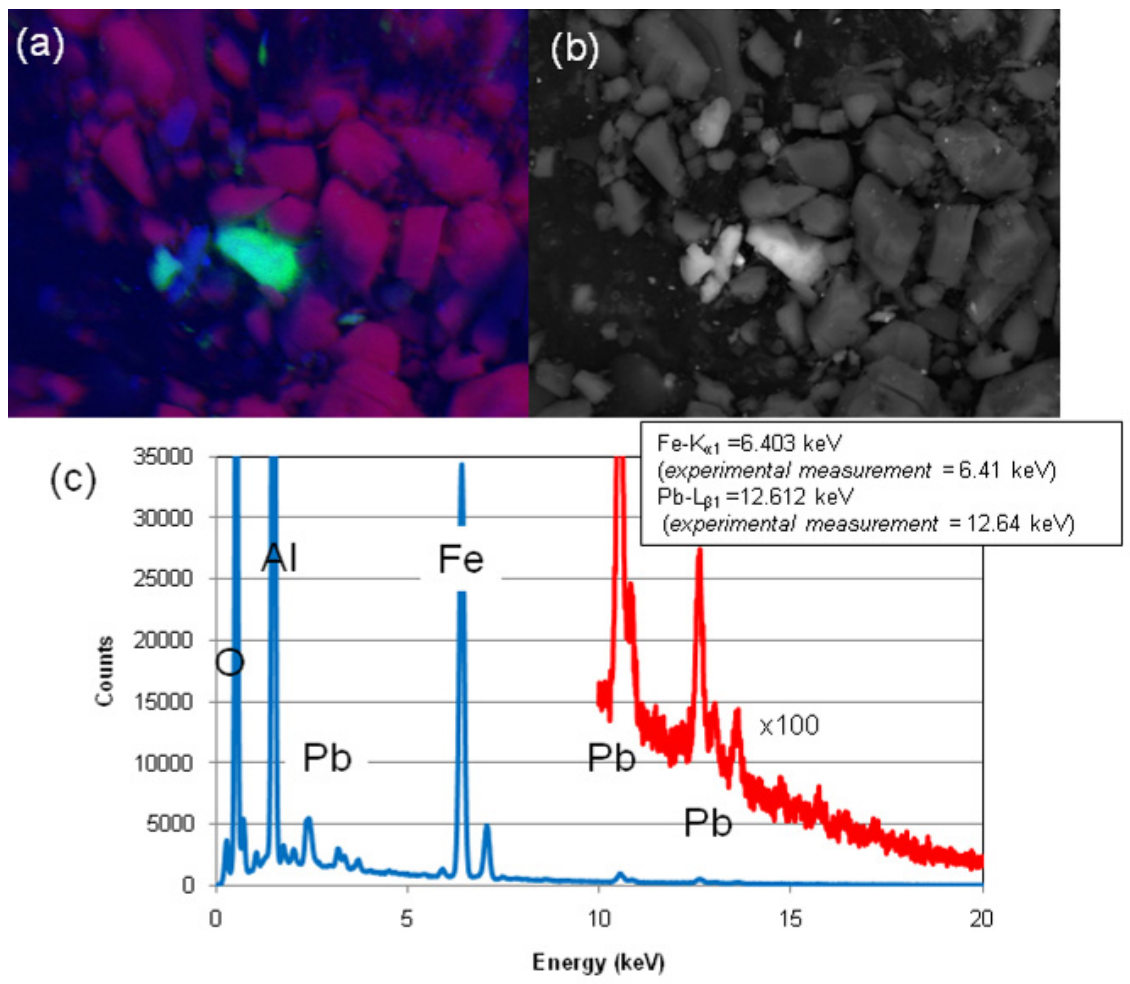

Figure 3.31. (a) Combined Elemental SEM Maps of Lead-Bearing Iron Oxide Phase from Hanford Tank Sludge Solids C-103, (b) Backscattered Image of Particle, and (c) EDS Analysis Showing the Presence of $\mathrm{Pb}$. For Information Only.

The PSD for hematite was discussed in Wells et al. (2007), which occurs as rose pellet precipitates. The TEM analyses performed for the M12 series provide some new data on iron oxyhydroxide. Much smaller amorphous particles were observed (see Figure 3.32).

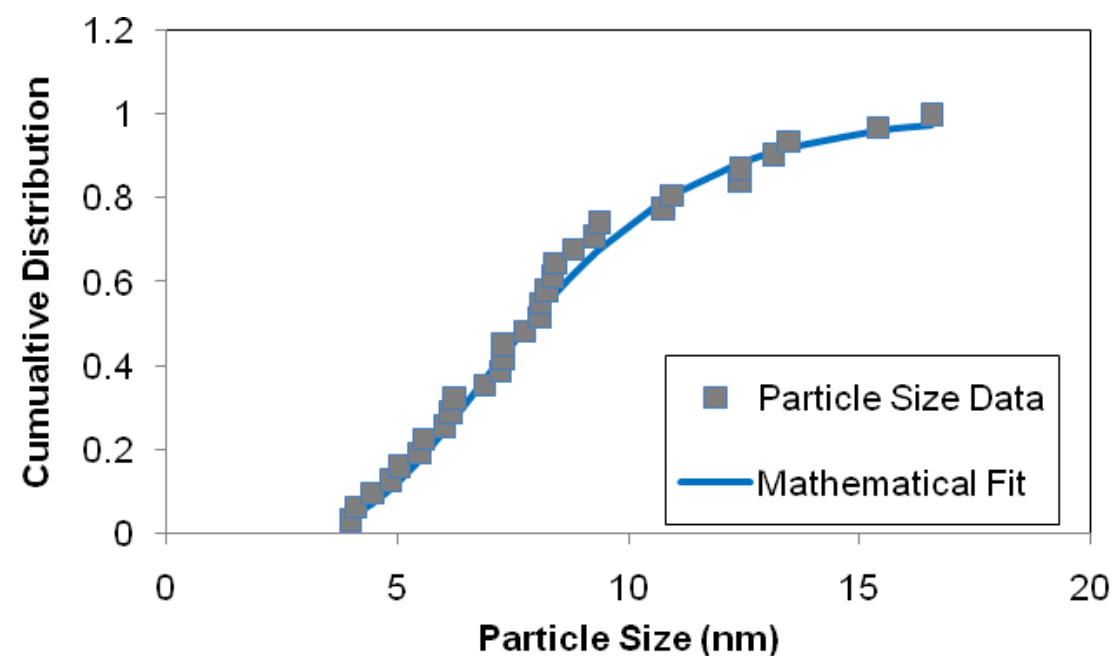

Figure 3.32. Cumulative Distribution of Particle Sizes for Iron Oxide (FeOOH) Phase from Hanford Tank Sludge Solids_-TEM Data 


\subsubsection{Salt Particles}

Salt particles in the Hanford tank wastes are known to be macroscopically large on occasion. Within the sodium sulfate-sodium carbonate system, the dominant sulfate salts are burkeite $\left(\mathrm{Na}_{6}\left(\mathrm{SO}_{4}\right)_{2} \mathrm{CO}_{3}\right)$ or schairerite $\left(\mathrm{Na}_{3} \mathrm{FSO}_{4}\right)$ (see Figure 3.33). The EDS analyses of these phases are not diagnostic; however, the identifications reported here are based on the assessments reported in Herting et al. (2002). Extremely large particles have been reported for natrophosphate in excess of $2 \mathrm{~mm}$ in length. Some hot cell video images have shown particles 5 to $10 \mathrm{~cm}$ in length. The identity of these particles is uncertain, so they have not been included in this assessment.
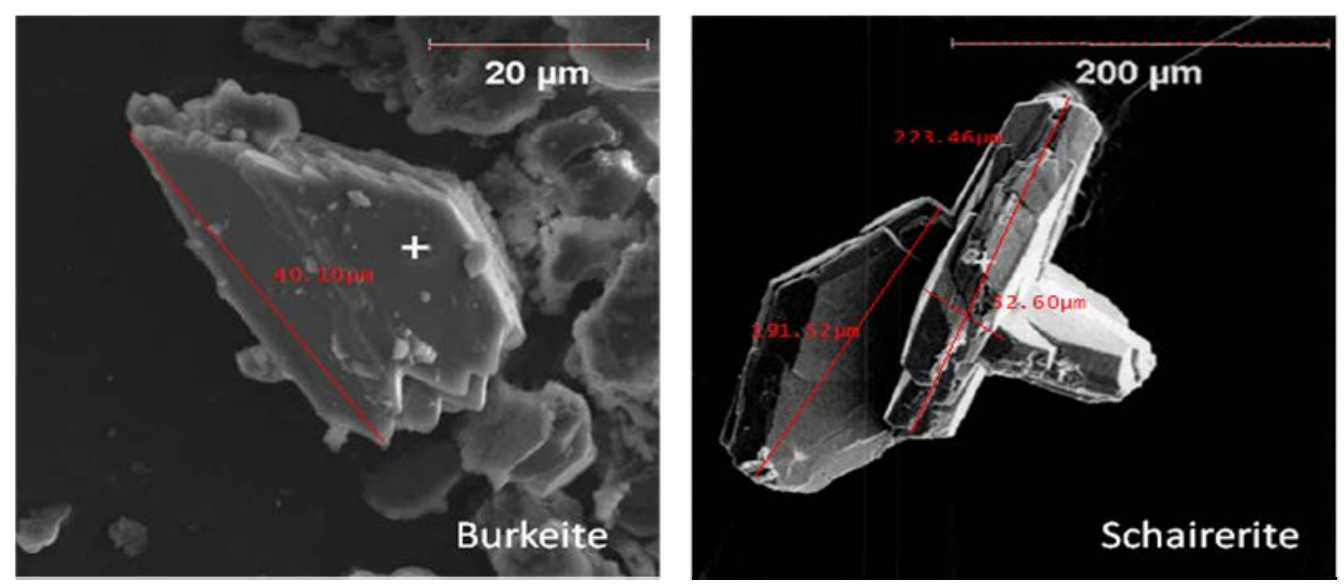

Figure 3.33. SEM Images from Hanford Tank Salts

Studies by Herting and co-workers have demonstrated that the sodium-carbonate-sulfate environments in Hanford salt-cakes are dominated by minerals such as trona $\left[\mathrm{Na}_{3} \mathrm{H}\left(\mathrm{CO}_{3}\right)_{2} \cdot 2 \mathrm{H}_{2} \mathrm{O}\right]$ and sodium oxalate $\left(\mathrm{Na}_{2} \mathrm{C}_{2} \mathrm{O}_{4}\right)$ (see Figure 3.34), although other common sulfate and carbonate minerals, including mirabilite $\left(\mathrm{Na}_{2} \mathrm{SO}_{4} \cdot 10 \mathrm{H}_{2} \mathrm{O}\right)$ and natron $\left(\mathrm{Na}_{2} \mathrm{CO}_{3} \bullet 10 \mathrm{H}_{2} \mathrm{O}\right)$, have not been reported.
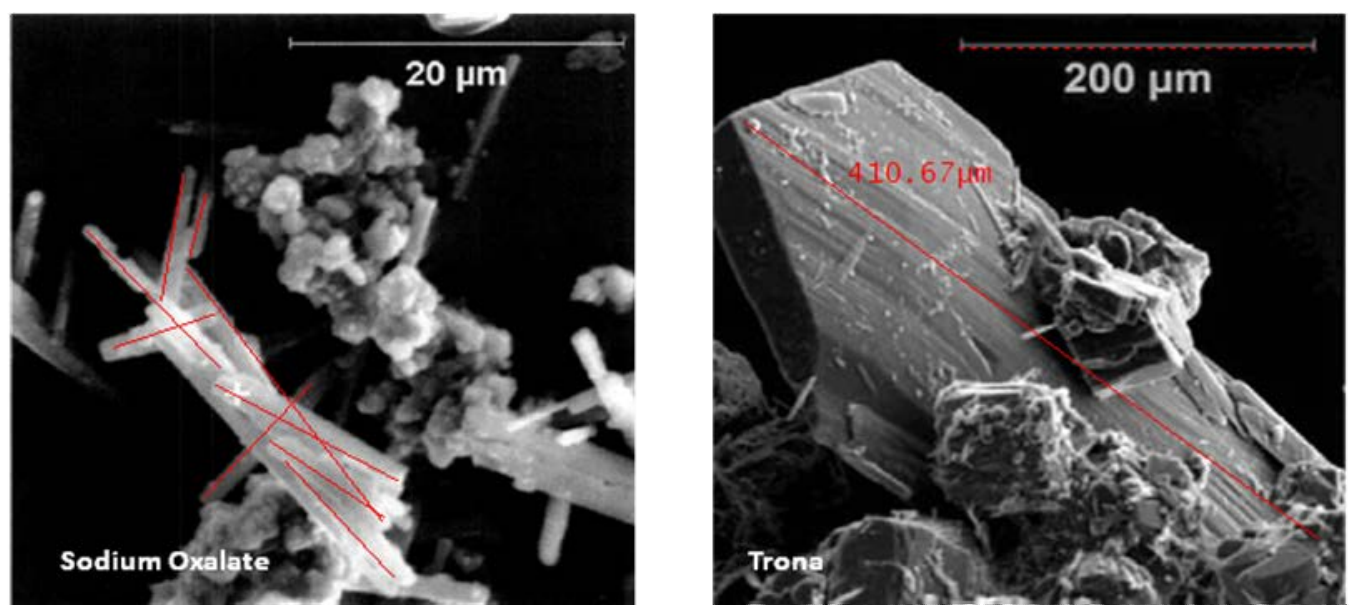

Figure 3.34. SEM Images of Oxalate and Carbonate Salts from Hanford Tank Salts 
Many of the phosphate particles can reach exceptional sizes. A notable example is natrophosphate (see Figure 3.35). This single particle is close to $2.5 \mathrm{~mm}$ in diameter. SEM images showing NaF crystals, hexagonal plates of $\mathrm{Na}_{3} \mathrm{FSO}_{4}$, hexagonal $\mathrm{Na}_{3} \mathrm{AlF}_{6}$ on a large hexagonal $\mathrm{Na}_{3} \mathrm{FSO}_{4}$, and laths or blades of $\mathrm{Na}_{2} \mathrm{C}_{2} \mathrm{O}_{4}$ are shown in Figure 3.36.

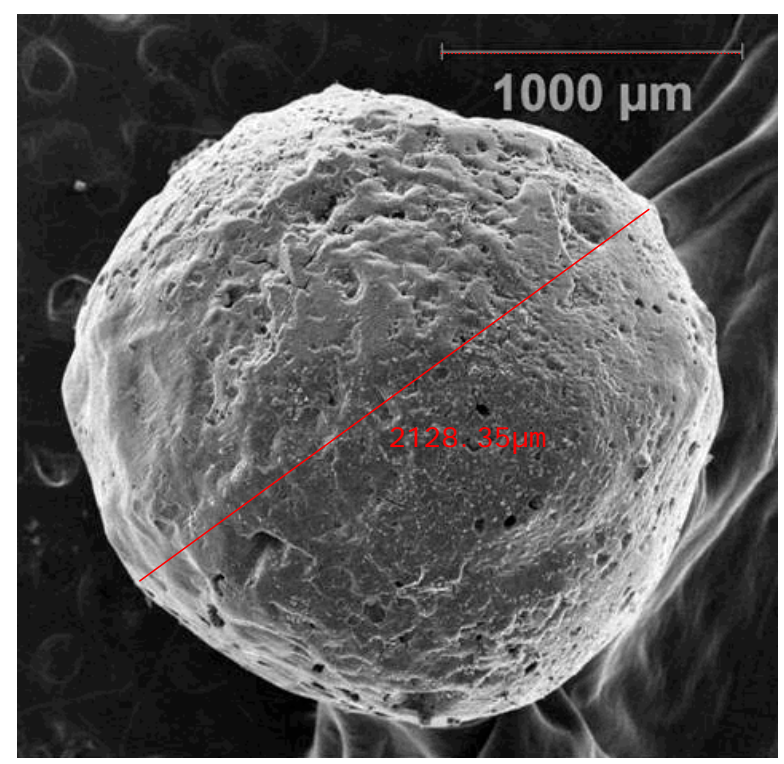

Figure 3.35. SEM Image of Natrophosphate

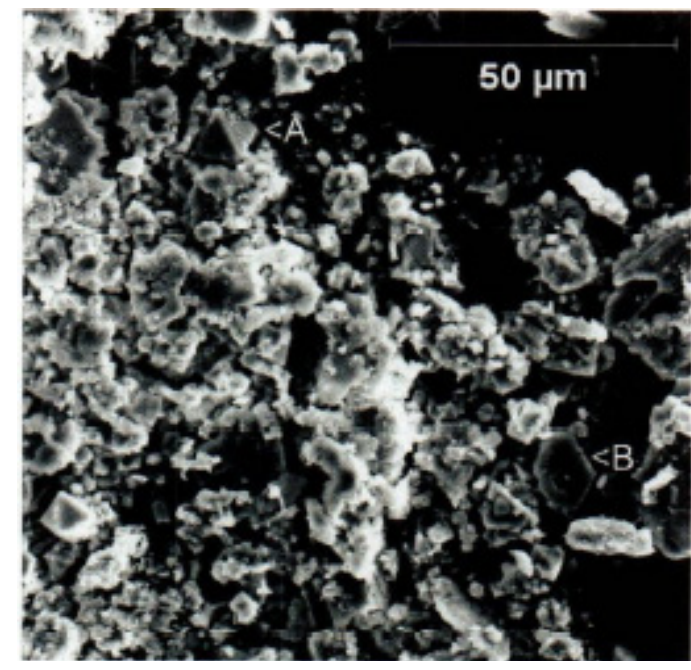

SEM image showing $\mathrm{NaF}$ crystals, labeled (A) and hexagonal plates of $\mathrm{Na}_{3} \mathrm{FSO}_{4}$ labeled (B) Some of the plates did not show sulfur so they were assumed to be $\mathrm{Na}_{2} \mathrm{C}_{2} \mathrm{O}_{4}$ (Herting et al. 2002).

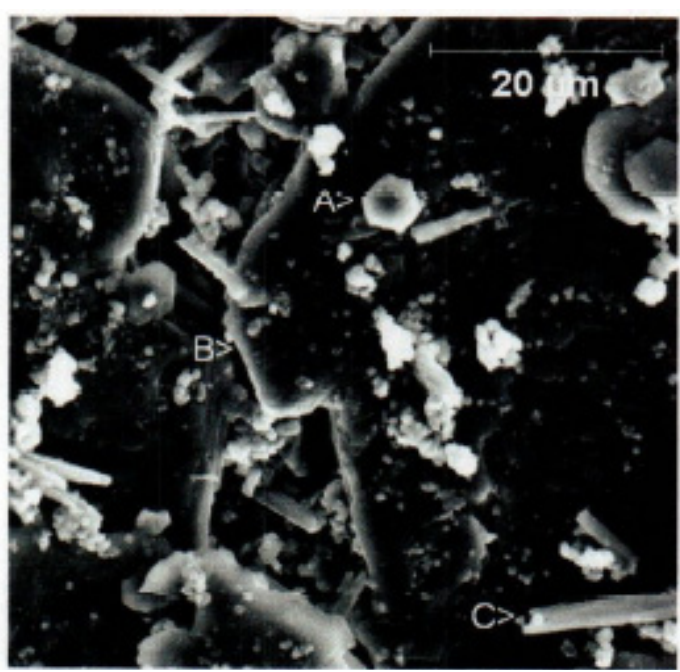

Showing hexagonal $\mathrm{Na}_{3} \mathrm{AlF}_{6}$ (crystal labeled $\mathbf{A}$ ) on a large hexagonal $\mathrm{Na}_{3} \mathrm{FSO}_{4}$ labeled (B). The laths or blades correspond to $\mathrm{Na}_{2} \mathrm{C}_{2} \mathrm{O}_{4}$ labeled (C) (Herting et al. 2002).

Figure 3.36. SEM Image of Various Salts 


\subsubsection{UDS Primary Particle Size Summary}

Particle sizes reported with TEM are, in general, smaller than those reported by other techniques. The TEM enables the individual particles to be resolved, and is the only technique available for investigating amorphous phases. The compositions are also more complex than the idealized compositions listed in Section 3.2.3. The particle sizes for salts are from SEM and optical microscopy analyses.

Particle-shape factors were difficult to define, so, depending on the perceived average shape, the size was multiplied by single value. The applied shape factors, Equation (2.10), Section 2.1.1.1, as determined for many of the salt phases and a limited number of the sludge particles in the current work and from that of Wells et al. (2007), are given in Table 3.13. The identified particle size information is tabulated in Appendix D, and a summary of the maximum primary particle sizes for the UDS phase compounds and densities of Table 3.11, Section 3.2.3, is provided in Table 3.14. Size information from Wells et al. (2007) is incorporated in this table. All sizes in Table 3.14 are provided on a spherical basis.

As previously described, the maximum primary particle sizes of Table 3.14 must be treated as estimates only. The given sizes are estimates only because 1) for those compounds where images were specifically identified, a finite set of images are available for the waste from a limited number of tanks, 2) for other compounds, surrogate images, wherein the specific UDS phase is not replicated or certain, are used, with similar caveats regarding sample size, and 3) for those compounds with no images available, expert judgment is used to assign the particle characteristics.

Table 3.13. Particle Shape Factor Summary

\begin{tabular}{|c|c|}
\hline Compound & Shape Factor \\
\hline Boehmite & 0.26 \\
\hline $\mathrm{NaAlCO}_{3}(\mathrm{OH})_{2}$ & 0.42 \\
\hline $\mathrm{Na}_{2}\left(\mathrm{UO}_{2}\right)_{2}\left(\mathrm{PO}_{4}\right)_{2} \cdot 2 \mathrm{H}_{2} \mathrm{O}$ & 0.5 \\
\hline $\mathrm{PuO}_{2}$ & 0.5 \\
\hline $\mathrm{Sr}_{3}\left(\mathrm{PO}_{4}\right)_{2}$ & 0.44 \\
\hline $\mathrm{Na}_{2} \mathrm{C}_{2} \mathrm{O}_{4}$ & 0.4 \\
\hline $\mathrm{Na}_{2} \mathrm{CO}_{3} \cdot\left(\mathrm{H}_{2} \mathrm{O}\right)$ & 0.8 \\
\hline $\mathrm{Na}_{2} \mathrm{SO}_{4}$ & 0.8 \\
\hline $\mathrm{Na}_{3} \mathrm{FSO}_{4}$ & 0.8 \\
\hline $\mathrm{Na}_{3} \mathrm{NO}_{3} \mathrm{SO}_{4} \cdot \mathrm{H}_{2} \mathrm{O}$ & 0.8 \\
\hline $\mathrm{Na}_{3} \mathrm{PO}_{4} \cdot 12 \mathrm{H}_{2} \mathrm{O}$ & 0.4 \\
\hline $\mathrm{Na}_{6} \mathrm{CO}_{3}\left(\mathrm{SO}_{4}\right)_{2}$ & 0.8 \\
\hline $\mathrm{Na}_{3}\left(\mathrm{CO}_{3}\right)\left(\mathrm{HCO}_{3}\right) \cdot 2\left(\mathrm{H}_{2} \mathrm{O}\right)$ & 0.8 \\
\hline
\end{tabular}


Table 3.14. UDS Phase and Maximum Primary Particle Size Summary

\begin{tabular}{|c|c|c|}
\hline Compound & Density $(\mathrm{g} / \mathrm{mL})$ & $\begin{array}{c}\text { Maximum Spherical } \\
\text { Primary Particle } \\
\text { Size }(\mu \mathrm{m})\end{array}$ \\
\hline $\mathrm{Ag}$ & 10.5 & 2 \\
\hline $\mathrm{Ag}_{2} \mathrm{O}$ & 7.143 & 2 \\
\hline $\mathrm{Bi}_{2} \mathrm{O}_{3}$ & 8.9 & 3 \\
\hline $\mathrm{BiFeO}_{3}$ & 7.9 & 0.1 \\
\hline $\mathrm{Ca}(\mathrm{OH})_{2}$ & 2.24 & 9 \\
\hline $\mathrm{Ca}_{5} \mathrm{OH}\left(\mathrm{PO}_{4}\right)_{3}$ & 3.14 & 9 \\
\hline $\mathrm{CaC}_{2} \mathrm{O}_{4} \cdot \mathrm{H}_{2} \mathrm{O}$ & 2.2 & 9 \\
\hline $\mathrm{CaCO}_{3}$ & 2.71 & 55 \\
\hline $\mathrm{CaF}_{2}$ & 3.18 & 15 \\
\hline $\mathrm{CrOOH}$ & 4.11 & 0.4 \\
\hline $\mathrm{FePO}_{4} \cdot 2 \mathrm{H}_{2} \mathrm{O}$ & 3.15 & 0.02 \\
\hline $\mathrm{FeOOH}$ & 4.26 & 0.015 \\
\hline Gibbsite & 2.42 & 200 \\
\hline Boehmite & 3.01 & 0.052 \\
\hline $\mathrm{HgO}$ & 7.143 & 2 \\
\hline $\mathrm{KAlSiO}_{4}$ & 2.61 & 8 \\
\hline $\mathrm{La}(\mathrm{OH})_{3}$ & 2.3 & 3 \\
\hline $\mathrm{LaPO}_{4} \cdot 2 \mathrm{H}_{2} \mathrm{O}$ & 6.51 & 3 \\
\hline $\mathrm{Mn}_{3}\left(\mathrm{PO}_{4}\right)_{2}$ & 3.102 & 8 \\
\hline $\mathrm{MnO}_{2}$ & 5.026 & 10 \\
\hline $\mathrm{Na}_{2}\left(\mathrm{UO}_{2}\right)_{2}\left(\mathrm{PO}_{4}\right)_{2} \cdot 2 \mathrm{H}_{2} \mathrm{O}$ & 3.5 & 5 \\
\hline $\mathrm{Na}_{2} \mathrm{U}_{2} \mathrm{O}_{7}$ & 5.617 & 5 \\
\hline $\mathrm{NaAlCO}_{3}(\mathrm{OH})_{2}$ & 2.42 & 4.2 \\
\hline $\mathrm{NaAlSiO}_{4}$ & 2.365 & 8 \\
\hline $\mathrm{Ni}(\mathrm{OH})_{2}$ & 4.15 & 0.5 \\
\hline $\mathrm{Ni}_{3}\left(\mathrm{PO}_{4}\right)_{2}$ & 3.93 & 8 \\
\hline $\mathrm{NiC}_{2} \mathrm{O}_{4} \cdot 2 \mathrm{H}_{2} \mathrm{O}$ & 4.26 & 1.6 \\
\hline $\mathrm{Pb}(\mathrm{OH})_{2}$ & 7.1 & 5 \\
\hline $\mathrm{Pb}_{3}\left(\mathrm{PO}_{4}\right)_{2}$ & 7.1 & 0.4 \\
\hline $\mathrm{PbCO}_{3}$ & 6.6 & 5 \\
\hline $\mathrm{Pu}(\mathrm{OH})_{4}$ (co-precip. on Fe phase) & 4.26 & 0.015 \\
\hline $\mathrm{PuO}_{2}$ & 11.43 & 20 \\
\hline $\mathrm{SiO}_{2}$ & 2.6 & 100 \\
\hline $\mathrm{Sr}_{3}\left(\mathrm{PO}_{4}\right)_{2}$ & 3.5 & 0.065 \\
\hline $\mathrm{SrCO}_{3}$ & 3.5 & 0.065 \\
\hline $\mathrm{ZrO}_{2}$ & 5.68 & 50 \\
\hline $\mathrm{KNO}_{3}$ & 2.109 & 2200 \\
\hline $\mathrm{Na}_{2} \mathrm{C}_{2} \mathrm{O}_{4}$ & 2.34 & 8 \\
\hline $\mathrm{Na}_{2} \mathrm{CO}_{3} \cdot \mathrm{H}_{2} \mathrm{O}$ & 2.25 & 80 \\
\hline $\mathrm{Na}_{2} \mathrm{SO}_{4}$ & 2.68 & 112 \\
\hline $\mathrm{Na}_{2} \mathrm{SO}_{4} \cdot 10 \mathrm{H}_{2} \mathrm{O}$ & 1.464 & 112 \\
\hline $\mathrm{Na}_{3} \mathrm{FSO}_{4}$ & 2.65 & 176 \\
\hline $\mathrm{Na}_{3} \mathrm{NO}_{3} \mathrm{SO}_{4} \cdot \mathrm{H}_{2} \mathrm{O}$ & 2.3 & 80 \\
\hline
\end{tabular}


Table 3.14. (contd)

\begin{tabular}{|l|c|c|}
\hline \multicolumn{1}{|c|}{ Compound } & Density $\mathbf{( g / m L )}$ & $\begin{array}{c}\text { Maximum Spherical } \\
\text { Primary Particle } \\
\text { Size }(\boldsymbol{\mu m})\end{array}$ \\
\hline $\mathrm{Na}_{3} \mathrm{PO}_{4.0} \cdot 25 \mathrm{NaOH} \cdot 12 \mathrm{H}_{2} \mathrm{O}$ & 1.62 & 440 \\
\hline $\mathrm{Na}_{3} \mathrm{PO}_{4} \cdot 8 \mathrm{H}_{2} \mathrm{O}$ & 1.8 & 2200 \\
\hline $\mathrm{Na}_{4} \mathrm{P}_{2} \mathrm{O}_{7} \cdot 10 \mathrm{H}_{2} \mathrm{O}$ & 1.83 & 2200 \\
\hline $\mathrm{Na}_{6}\left(\mathrm{SO}_{4}\right)_{2} \mathrm{CO}_{3}$ & 2.64 & 32 \\
\hline $\mathrm{NaF}$ & 2.78 & 12 \\
\hline $\mathrm{Na}_{7} \mathrm{~F}_{\left(\mathrm{PO}_{4}\right)_{2} \cdot 19 \mathrm{H}_{2} \mathrm{O}}$ & 1.75 & 2100 \\
\hline $\mathrm{NaHCO}_{3}$ & 2.159 & 328 \\
\hline $\mathrm{NaNO}_{2}$ & 2.168 & 2200 \\
\hline $\mathrm{NaNO}_{3}$ & 2.26 & 650 \\
\hline
\end{tabular}

\subsubsection{UDS PSDS}

Composite waste PSDs were originally developed in Wells et al. (2007) for two primary sample conditions: 1) minimal disturbance and 2) sonicated. The source sample PSDs used to generate the original composite PSDs were restricted to sludge tank solids analyzed under flow and/or recirculating conditions with and without sonication. The current report expands the source PSD data sets to enable the development of composite waste PSDs under both flow and no-flow conditions and for both sludge and saltcake solids. In the following sub-sections, the criteria used to down-select sample PSD data for the expanded database are given. The new data set includes both original entries in the database for Wells et al. (2007) and new data. The selection criteria originally reported in Wells et al. (2007) are included and have been updated with the additional PSD selection criteria used to incorporate new PSD data sets for flow and non-flow conditions for both sludge and saltcake PSDs.

Historical PSD measurements for different tanks and waste types were reviewed to develop representative PSDs from those measurements. Particle size analyses have been performed on core and auger samples from the Hanford tanks. The references containing tank waste PSD measurements and considered for this report are listed in Table 3.15. This list comprises the original reference list used for Wells et al. (2007), which consisted of a total of 53 references, along with an additional 23 references.

There is a larger set of references containing particle size measurements for various tank wastes. However, these additional references are primarily concerned with the influence of post-retrieval treatment processes, such as caustic leaching, on the PSD. The reports listed in Table 3.15 were selected for consideration because they contained PSD measurements for "as-received" material. In some cases, these reports also present PSDs for treated wastes. Post-retrieval processing PSD data included in the data set are from the waste sample analyses conducted during the response to issue M12.

The goal of any PSD analysis is to obtain a particle size measurement that is representative of the tested sample under specified conditions. However, the tank configuration and the chemistry and physical properties of the waste contained therein makes representative sampling difficult. A discussion of the general difficulties of PSD sampling and analysis is provided in Appendix G. Further, as evidenced in Table 3.15, PSD measurements are not available for all 177 tanks. In the cases where PSD 
measurements were taken, creating a single representative distribution was complicated by the fact that many of the measurements were not equivalent or directly comparable.

In Section 3.2.5.1, the PSD selection criteria from the references in Table 3.15 are described, and the selected data sets are listed in Section 3.2.5.2. The PSD generation methods are described in Section 3.2.5.3, and the representative PSDs are provided in Section 3.2.5.4.

Table 3.15. PSD References Considered

\begin{tabular}{|c|c|c|}
\hline \# & Reference & Tanks with PSD Data \\
\hline 1 & $\begin{array}{l}\text { 7S110-WSC-03-002, 2003, “Particle Size Distribution Analysis Results: Tank 241-AN-102, } \\
\text { Core 307.” Internal memo, WS Callaway to KG Carothers, October 7, 2003), CH2M Hill } \\
\text { Hanford Group, Inc., Richland, Washington. }\end{array}$ & AN-102 \\
\hline 2 & $\begin{array}{l}\text { 7S110-WSC-03-012, 2003, “Particle Size Distribution Analysis of Samples from Tank } \\
\text { 241-AZ-102, Core 310,” (internal memorandum from WS Callaway to KG Carothers dated } \\
\text { December 12), CH2M Hill Hanford Group, Inc., Richland, Washington. }\end{array}$ & AZ-102 \\
\hline 3 & $\begin{array}{l}\text { 7S110-WSC-05-011, 2005, “Particle Size Measurements in Support of the Tank } \\
\text { 241-AN-102 Chemistry Control Recovery Plan,” (internal memorandum from } \\
\text { WS Callaway, GA Cooke and DL Herting to KG Carothers dated March 18), CH2M Hill } \\
\text { Hanford Group, Inc., Richland, Washington. }\end{array}$ & AN-102 \\
\hline 4 & $\begin{array}{l}\text { 7S110-WSC-06-148, 2006, “Particle Size Distribution Measurements on Samples from } \\
\text { Tank 241-AY-102 Core 319,” (internal memorandum from WS Callaway to KG Carothers } \\
\text { dated January 24), CH2M Hill Hanford Group, Inc., Richland, Washington. }\end{array}$ & AY-102 \\
\hline 5 & $\begin{array}{l}\text { B3610-WSC-02-028, 2002, “Particle Size Distribution Analysis Results: Tank } \\
\text { 241-AN-107, Core 304,” (internal memorandum from WS Callaway to HL Baker dated } \\
\text { December 26), Fluor Hanford, Inc., Richland, Washington. }\end{array}$ & AN-107 \\
\hline 6 & $\begin{array}{l}\text { BNFL-RPT-030, 2000, Characterization, Washing, Leaching, and Filtration of C-104 } \\
\text { Sludge, Pacific Northwest National Laboratory, Richland, Washington. }\end{array}$ & C-104 \\
\hline 7 & $\begin{array}{l}\text { BNFL-RPT-038, 2000, Characterization, Washing, Leaching, and Filtration of AZ-102, } \\
\text { Pacific Northwest National Laboratory, Richland, Washington. }\end{array}$ & AZ-102 \\
\hline 8 & $\begin{array}{l}\text { Brooks et al. 1996. "Sludge Pretreatment Studies Using Hanford Tank C-107.” Letter } \\
\text { Report prepared for the Westinghouse Hanford Company by Pacific Northwest National } \\
\text { Laboratory, Richland, Washington. }\end{array}$ & C-107 \\
\hline 9 & $\begin{array}{l}\text { CH2M-0400872, 2004, “Distribution of Plutonium-Rich Particles in Tank 241-SY-102 } \\
\text { Sludge,” (external letter from WS Callaway and GA Cooke, CH2M Hill Hanford Group, } \\
\text { Inc., to KH Abel, BNI, dated May 17), CH2M Hill Hanford Group, Inc., Richland, } \\
\text { Washington. }\end{array}$ & SY-102 \\
\hline 10 & $\begin{array}{l}\text { FH-0201635, 2002, “Particle Size Distribution Analysis Results: Tank 241-AY-101, } \\
\text { Cores } 275 \text { and 277,” (external letter from WS Callaway, FH, to JH Baldwin, CH2M Hill } \\
\text { Hanford Group, Inc., dated April 9), Fluor Hanford, Inc., Richland, Washington. }\end{array}$ & AY-101 \\
\hline 11 & $\begin{array}{l}\text { FH-0201835, 2002, “Particle Size Distribution Analysis Results: Tank 241-C-107, } \\
\text { Cores } 287 \text { and 288,” (external letter from WS Callaway, FH, to JH Baldwin, CH2M Hill } \\
\text { Hanford Group, Inc., dated April 24), Fluor Hanford, Inc., Richland, Washington. }\end{array}$ & C-107 \\
\hline 12 & $\begin{array}{l}\text { FH-0202775, 2002, “Particle Size Distribution Analysis Results: Tank 241-SY-102, } \\
\text { Core 284,” (external letter from WS Callaway, FH, to JH Baldwin, CH2M Hill Hanford } \\
\text { Group, Inc., dated June 18), Fluor Hanford, Inc., Richland, Washington. }\end{array}$ & SY-102 \\
\hline 13 & $\begin{array}{l}\text { FH-0202392, 2002, “Particle Size Distribution Analysis Results: Tank 241-AY-102, } \\
\text { Cores } 289 \text { and 290,” (external letter from WS Callaway, FH, to JH Baldwin, CH2M Hill } \\
\text { Hanford Group, Inc., dated May 28), Fluor Hanford, Inc., Richland, Washington. }\end{array}$ & AY-102 \\
\hline 14 & $\begin{array}{l}\text { Gray et al. 1993, “Characterization of the First Core Sample of Neutralized Current Acid } \\
\text { Waste from Double-Shell Tank 102-AZ,” (unnumbered report), Pacific Northwest } \\
\text { Laboratory, Richland, Washington. }\end{array}$ & AZ-102 \\
\hline
\end{tabular}


Table 3.15. (contd)

\begin{tabular}{|c|c|c|}
\hline$\#$ & Reference & Tanks with PSD Data \\
\hline 15 & $\begin{array}{l}\text { Gray et al. 1993, “Characterization of the Second Core Sample of Neutralized Current Acid } \\
\text { Waste from Double-Shell Tank 101-AZ.” PNNL-13027 (Limited Distribution), Pacific } \\
\text { Northwest National Laboratory, Richland, Washington. }\end{array}$ & AZ-101 \\
\hline 16 & $\begin{array}{l}\text { HNF-1647, 1999, Tank 241-S-111, Core } 237 \text { Analytical Results for the Final Report, } \\
\text { Rev. 0A, Waste Management of Hanford, Inc., for Fluor Daniel Hanford, Inc., Richland, } \\
\text { Washington. }\end{array}$ & S-101 \\
\hline 17 & $\begin{array}{l}\text { HNF-1666, 1999, Tank 241-SY-101, Cores 255, 256, and 257, Analytical Results for the } \\
\text { Final Report, Rev. 0A, Waste Management of Hanford, Inc., for Fluor Daniel Hanford, Inc., } \\
\text { Richland, Washington. }\end{array}$ & SY-101 \\
\hline 18 & $\begin{array}{l}\text { HNF-3352 Rev. 0, 1998, Results of Dilution Studies with Waste from Tank 241-AN-104, } \\
\text { Fluor Daniel Hanford Inc., Richland, Washington. }\end{array}$ & AN-104 \\
\hline 19 & $\begin{array}{l}\text { HNF-4964 Rev. OA, 2000, Results of Dilution Studies with Waste from } \\
\text { Tank 211-AW-101, Fluor Hanford Inc., Richland, Washington. }\end{array}$ & AW-101 \\
\hline 20 & $\begin{array}{l}\text { HNF-7078 Rev. 0A, 2002, Results of Retrieval Testing of Sludge from Tank 241-AZ-101, } \\
\text { Fluor Hanford, Inc., Richland, Washington. }\end{array}$ & AZ-101 \\
\hline 21 & $\begin{array}{l}\text { HNF-8862 Rev. 0, 2002, Particle Property Analyses of High-Level Waste Tank Sludges, } \\
\text { Fluor Hanford, Inc., Richland, Washington. }\end{array}$ & $\begin{array}{c}\text { AW-103, AY-101, AY-102, } \\
\text { AZ-102, C-104, C-107, SY-102 }\end{array}$ \\
\hline 22 & $\begin{array}{l}\text { HNF-SD-WM-DTR-046 Rev. OA, 1999, Results of Dilution Studies with Waste from Tank } \\
\text { 241-AN-I 05, Fluor Daniel Hanford Inc., Richland, Washington. }\end{array}$ & AN-105 \\
\hline 23 & $\begin{array}{l}\text { LA-UR-95-2070, 1995, Sludge Washing and Alkaline Leaching Test son Actual Hanford } \\
\text { Tank Sludge: A Status Report, Los Alamos National Laboratory, Los Alamos, New } \\
\text { Mexico }\end{array}$ & $\begin{array}{c}\text { B-202, BX-105, C-108, S-104, } \\
\text { T-104, T-107 }\end{array}$ \\
\hline 24 & $\begin{array}{l}\text { LA-UR-96-2839, 1996, Sludge Water Washing and Alkaline Tests on Actual Hanford Tank } \\
\text { Sludge: FY } 1996 \text { Results, Los Alamos National Laboratory, Los Alamos, New Mexico. }\end{array}$ & B-104, BX-109, C-107, TY-104 \\
\hline 25 & $\begin{array}{l}\text { LA-UR 97-2889, 1997, Sludge Water Washing and Alkaline Tests on Actual Hanford Tank } \\
\text { Sludge: FY } 1997 \text { Results, Los Alamos National Laboratory, Los Alamos, New Mexico. }\end{array}$ & $\begin{array}{l}\text { B-106, BX-103, C-104, C-105, } \\
\text { SX-113 }\end{array}$ \\
\hline 26 & $\begin{array}{l}\text { Peters, 1988, Tank 101-AY Sludge Particle Size Analysis, (unnumbered memorandum to } \\
\text { RS Edrington, dated July 22), Westinghouse Hanford Company, Richland, Washington. }\end{array}$ & AY-101 \\
\hline 27 & $\begin{array}{l}\text { Peterson, 1990, Preliminary Results—Analysis of Waste Layers from DST 101-AZ Core } \\
\# 2 \text {, (letter 9001040 to AJ DiLiberto and LM Sasaki, dated February 28), Pacific Northwest } \\
\text { Laboratory, Richland, Washington. }\end{array}$ & AZ-101 \\
\hline 28 & $\begin{array}{l}\text { PNL-10078, 1995, Washing and Alkaline Leaching of Hanford Tank Sludges: A Status } \\
\text { Report, Pacific Northwest Laboratory, Richland, Washington. }\end{array}$ & B-201, U-110 \\
\hline 29 & $\begin{array}{l}\text { PNL-10099, 1994, Tank Characterization Report for Single-Shell Tank B-111, Pacific } \\
\text { Northwest Laboratory, Richland, Washington. }\end{array}$ & B-111 \\
\hline 30 & $\begin{array}{l}\text { PNL-10101, 1994, Tank Characterization Report for Single-Shell Tank T-102, Pacific } \\
\text { Northwest Laboratory, Richland, Washington. }\end{array}$ & $\mathrm{T}-102$ \\
\hline 31 & $\begin{array}{l}\text { PNL-10175, 1994, Ferrocyanide Safety Project: Comparison of Actual and Simulated } \\
\text { Ferrocyanide Waste Properties, Pacific Northwest Laboratory, Richland, Washington. }\end{array}$ & C-109, C-112 \\
\hline 32 & $\begin{array}{l}\text { PNL-10712, Washing and Caustic Leaching of Hanford Tank Sludges: Results of FY } 1995 \\
\text { Studies, 1995, Pacific Northwest Laboratory, Richland, Washington. }\end{array}$ & $\begin{array}{l}\text { B-111, BX-107, C-103, S-104, } \\
\text { SY-103, T-104, T-111 }\end{array}$ \\
\hline 33 & $\begin{array}{l}\text { PNNL-11025, 1996, Comparison of Simulants to Actual Neutralized Current Acid Waste: } \\
\text { Process and Product Testing of Three NCAW Core Samples from Tanks 101-AZ and } \\
\text { 102-AZ, Pacific Northwest National Laboratory, Richland, Washington. }\end{array}$ & AZ-101, AZ-102 \\
\hline 34 & $\begin{array}{l}\text { PNNL-11098, 1996, Comparison of Simulants to Actual Neutralized Current Acid Waste: } \\
\text { Process and Product Testing of Three NCAW Core Samples from Tanks 101-AZ and } \\
\text { 102-AZ, Pacific Northwest National Laboratory, Richland, Washington. }\end{array}$ & AZ-101, AZ-102 \\
\hline 35 & $\begin{array}{l}\text { PNNL-11278 Rev. 1, 1996, Washing and Leaching of Hanford Sludges: Results of FY } 1996 \\
\text { Studies, Pacific Northwest National Laboratory, Richland, Washington. }\end{array}$ & $\begin{array}{l}\text { BY-104, BY-110, C-107, S-107, } \\
\text { SX-108 }\end{array}$ \\
\hline 36 & $\begin{array}{l}\text { PNNL-11352, 1996, Tank SY-102 Waste Retrieval Assessment: Rheological } \\
\text { Measurements and Pump Jet Mixing Simulations, Pacific Northwest National Laboratory, } \\
\text { Richland, Washington. }\end{array}$ & SY-102 \\
\hline 37 & $\begin{array}{l}\text { PNNL-11381 Rev. 1, 1996, Washing and Caustic Leaching of Hanford Tank C-106 Sludge, } \\
\text { Pacific Northwest National Laboratory, Richland, Washington. }\end{array}$ & C-106 \\
\hline
\end{tabular}


Table 3.15. (contd)

\begin{tabular}{|c|c|c|}
\hline$\#$ & Reference & Tanks with PSD Data \\
\hline 38 & $\begin{array}{l}\text { PNNL-11580, 1997, Caustic Leaching of Composite AZ-101/AZ-102 Hanford Tank } \\
\text { Sludge, Pacific Northwest National Laboratory, Richland, Washington. }\end{array}$ & $\begin{array}{l}\text { AZ-101 / AZ-102 composition } \\
\text { sample }\end{array}$ \\
\hline 39 & $\begin{array}{l}\text { PNNL-11636, 1997, Washing and Leaching of Hanford Sludges: Results of FY } 1997 \\
\text { Studies, Pacific Northwest National Laboratory, Richland, Washington. }\end{array}$ & AN-104, BY-108, S-101, S-111 \\
\hline 40 & $\begin{array}{l}\text { PNNL-12010, 1998, Bench-Scale Enhanced Sludge Washing and Gravity Settling of } \\
\text { Hanford Tank C-107 Sludge, Pacific Northwest National Laboratory, Richland, } \\
\text { Washington. }\end{array}$ & S-107 \\
\hline 41 & $\begin{array}{l}\text { PNNL-13028, 1999, Characterization of the First Core Sample of Neutralized Current Acid } \\
\text { Waste from Double-Shell Tank 101-AZ, Pacific Northwest National Laboratory, Richland, } \\
\text { Washington. }\end{array}$ & AZ-101 \\
\hline 42 & $\begin{array}{l}\text { PNNL-16133, 2006, Characterization and Correlation of Particle-Level Interactions to the } \\
\text { Macroscopic Rheology of Powders, Granular Slurries, and Colloidal Suspensions, Pacific } \\
\text { Northwest National Laboratory, Richland, Washington. }\end{array}$ & B-203, T-110, T-203, T-204 \\
\hline 43 & $\begin{array}{l}\text { RPP-5798 Rev. 0, 2002, Results of Retrieval Studies with Waste from Tank 241-C-104, } \\
\text { Fluor Hanford, Inc., Richland, Washington. }\end{array}$ & C-104 \\
\hline 44 & $\begin{array}{l}\text { RPP-9806 Rev. 0, 2002, Results of Retrieval Testing of Sludge from Tank 241-AZ-102, } \\
\text { Fluor Hanford, Inc., Richland, Washington. }\end{array}$ & AZ-102 \\
\hline 45 & $\begin{array}{l}\text { WHC-EP-0643, 1993, Tank Characterization Report for Single-Shell Tank 241-U-110, } \\
\text { Westinghouse Hanford Company, Richland, Washington. }\end{array}$ & U-110 \\
\hline 46 & $\begin{array}{l}\text { WHC-EP-0668, 1993, Tank Characterization Report: Tank 241-C-109, Westinghouse } \\
\text { Hanford Company, Richland, Washington. }\end{array}$ & C-109 \\
\hline 47 & $\begin{array}{l}\text { WHC-EP-0739, 1994, Tank Characterization Report for Single-Shell Tank 241-BX-107, } \\
\text { Westinghouse Hanford Company, Richland, Washington. }\end{array}$ & BX-107 \\
\hline 48 & $\begin{array}{l}\text { WHC-SD-WM-ER-370, Tank Characterization Report for Single-Shell Tank 241-S-104, } \\
\text { Westinghouse Hanford Company, Richland, Washington. }\end{array}$ & S-104 \\
\hline 49 & $\begin{array}{l}\text { WHC-SD-WM-TI-540, 1992, Analysis Report for 241-BY-104 Auger Samples, } \\
\text { Westinghouse Hanford Company, Richland, Washington. }\end{array}$ & BY-104 \\
\hline 50 & $\begin{array}{l}\text { WTP-RPT-021, Rev. 1, 2002, Chemical and Physical Properties Testing of 241-AN-102 } \\
\text { Tank Waste Blended with 241-C-104 Wash/Leachate Solutions, Pacific Northwest National } \\
\text { Laboratory, Richland, Washington. }\end{array}$ & AN-102, C-104 \\
\hline 51 & $\begin{array}{l}\text { WTP-RPT-043 Rev. 1, 2003, Filtration, Washing, and Caustic Leaching of Hanford Tank } \\
\text { AZ-101 Sludge, Pacific Northwest National Laboratory, Richland, Washington. }\end{array}$ & AZ-101 \\
\hline 52 & $\begin{array}{l}\text { WTP-RPT-048 Rev. 1, 2004, Chemical Analysis and Physical Properties Testing of } \\
\text { 241-AZ-101 Tank Waste Supernatant and Centrifuged Solids, Pacific Northwest National } \\
\text { Laboratory, Richland Washington. }\end{array}$ & AZ-101 \\
\hline 53 & $\begin{array}{l}\text { WTP-RPT-076, 2003, Chemical Analysis and Physical Properties Testing of 241-AZ-101 } \\
\text { Tank Waste Supernatant and Centrifuged Solids, Pacific Northwest National Laboratory, } \\
\text { Richland Washington. }\end{array}$ & AZ-101 \\
\hline 54 & $\begin{array}{l}\text { BNFL-RPT-002, Ultrafiltration and Characterization of AW-101 Supernatant and Entrained } \\
\text { Solids, Pacific Northwest National Laboratory, Richland, Washington. }\end{array}$ & AW-101 \\
\hline 55 & $\begin{array}{l}\text { BNFL-RPT-033, Development of Inactive High-Level Waste Envelope D Simulants for } \\
\text { Scaled Crossflow Filtration Testing, Pacific Northwest National Laboratory, Richland, } \\
\text { Washington. }\end{array}$ & AZ-101, AZ-102, C-106 \\
\hline 56 & $\begin{array}{l}\text { BNFL-RPT-034, Rheological Studies on Pretreated Feed and Melter Feed from AW-101 } \\
\text { and AN-107. }\end{array}$ & AW-101, AN-107 \\
\hline 57 & $\begin{array}{l}\text { PNNL-11652, Bench-Scale Crossflow Filtration of Hanford Tank C-106, C-107, B-110 and } \\
\text { U-110 Sludge Slurries. }\end{array}$ & C-106, C-107, B-110, U-110 \\
\hline 58 & $\begin{array}{l}\text { PNNL-14144, Retrieval and Pipeline Transfer Assessment of Hanford Tank 241-AN-105 } \\
\text { Waste }\end{array}$ & AN-105 \\
\hline 59 & $\begin{array}{l}\text { WSRC-TR-2003-00205, Compositing and Characterization of Samples from Hanford Tank } \\
\text { 241-AY-102/C-106 }\end{array}$ & AY-102, C-106 \\
\hline 60 & $\begin{array}{l}\text { WTP-RPT-022, Chemical Analysis and Physical Property Testing of Diluted 241-AP-101 } \\
\text { Tank Waste }\end{array}$ & AP-101 \\
\hline 61 & $\begin{array}{l}\text { WTP-RPT-044, Combined Entrained Solids and Sr/TRU Removal from AN-102 Waste } \\
\text { Blended with C-104 Sludge Pretreatment Solutions }\end{array}$ & AN-102 \\
\hline
\end{tabular}


Table 3.15. (contd)

\begin{tabular}{|c|c|c|}
\hline \# & Reference & Tanks with PSD Data \\
\hline 62 & $\begin{array}{l}\text { WTP-RPT-095, Rheological and Physical Properties of AZ-101 LAW Pretreated Waste and } \\
\text { Melter Feed }\end{array}$ & AZ-101 \\
\hline 63 & $\begin{array}{l}\text { WTP-RPT-096, Rheological and Physical Properties of AZ-101 HLW Pretreated Sludge } \\
\text { and Melter Feed }\end{array}$ & AZ-101 \\
\hline 64 & $\begin{array}{l}\text { WTP-RPT-107, Rheological and Physical Properties of AP-104 LAW Pretreated Waste and } \\
\text { Melter Feed }\end{array}$ & AP-104 \\
\hline 65 & $\begin{array}{l}\text { WTP-RPT-157, Characterization and Leach Testing for REDOX Sludge and S-Saltcake } \\
\text { Actual Waste Sample Composites }\end{array}$ & M12 Composite \\
\hline 66 & $\begin{array}{l}\text { WTP-RPT-166, Characterization, Leaching, and Filtration Testing for Bismuth Phosphate } \\
\text { Sludge (Group 1) and Bismuth Phosphate Saltcake (Group 2) Actual Waste Sample } \\
\text { Composites }\end{array}$ & M12 Composite \\
\hline 67 & $\begin{array}{l}\text { WTP-RPT-167, Characterization and Leach Testing for PUREX Cladding Waste Sludge } \\
\text { (Group 3) and REDOX Cladding Waste Sludge (Group 4) Actual Waste Sample } \\
\text { Composites }\end{array}$ & M12 Composite \\
\hline 68 & $\begin{array}{l}\text { WTP-RPT-169, Characterization, Leaching, and Filtration Testing for Tributyl Phosphate } \\
\text { (TBP, Group 7) Actual Waste Sample Composites }\end{array}$ & M12 Composite \\
\hline 69 & $\begin{array}{l}\text { WTP-RPT-170, Characterization, Leaching, and Filtration Testing of Ferrocyanide Tank } \\
\text { Sludge (Group 8) Actual Waste Composite }\end{array}$ & M12 Composite \\
\hline 70 & $\begin{array}{l}\text { WTP-RPT-172, Filtration and Leach Testing for REDOX Sludge and S-Saltcake Actual } \\
\text { Waste Sample Composites }\end{array}$ & M12 Composite \\
\hline 71 & $\begin{array}{l}\text { WTP-RPT-181, Filtration and Leach Testing for PUREX Cladding Sludge and REDOX } \\
\text { Cladding Sludge Actual Waste Sample Composites }\end{array}$ & M12 Composite \\
\hline 72 & WHC-EP-0589, Tank 101-SY Window C Core Sample Results and Interpretation & SY-101 \\
\hline 73 & WHC-EP-0628, Tank 101-SY Window E Core Sample Results and Interpretation & SY-101 \\
\hline 74 & 12110-PCL92-018, Particle Size Analysis of 101-SY Window E Samples & SY-101 \\
\hline 75 & $\begin{array}{l}\text { HNF-6062, Tank 241-AZ-101 Grab Samples From Mixer Pump Test Events 5, 7, 8, and } 9 \\
\text { Analytical Results for the Final Results for the Report }\end{array}$ & AZ-101 \\
\hline 76 & RPP-8909, Results of Retrieval Testing of Sludge from Tank 241-AY-102 & AY-102 \\
\hline
\end{tabular}

\subsubsection{PSD Data Selection Criteria}

Because of the wide differences in sampling and measurement of tank waste PSDs, some of the referenced PSD measurements in Table 3.15 were not included. The final PSD measurements included in the data set are considered to provide the most reliable assessments of the size of particles in a given tank or waste type for a given flow/mixing regime. Exclusions were made with the intention of eliminating biased, flawed, or irrelevant PSD measurements and were based on tank waste type, treatment of the sample before PSD measurement, and the method and instrument used for measuring the PSD. In the following subsections, the basis for inclusion and exclusion of data sets as adopted from Wells et al. (2007) is outlined in detail.

\section{Types of PSD Considered}

In the reports listed in Table 3.15, PSDs are often reported using both volume and number bases. Number distributions provide information on the number of particles in a given size range, whereas volume distributions relate the volume fraction of material in a given size range. Only volume-based PSDs were included in the composite PSD data set. In the balance of this report, the term PSD indicates volume-based PSD. 


\section{Tank Samples Considered}

The influence of tank retrieval method and location of sample extraction was not considered. All "as-received" tanks wastes were treated equally and assumed to be sampled such that the particles and aggregates contained therein were equally representative of their source tank.

\section{Tank Waste Pretreatments Considered}

While some of the reports listed in Table 3.15 examine the influence of post-retrieval treatment processes, such as caustic leaching on PSD, only PSDs corresponding to as-received material were considered for the tank PSDs with the exception of washed saltcake data. With the exception of the post-retrieval processing PSD data for the M12 samples, results from samples formed by compositing multiple tank wastes into a single sample were excluded.

When received for analysis, the tank material was often reported to have phase segregated into a clear supernatant layer over a dense settled sludge layer. Samples were typically homogenized to resuspend the solids before subsampling aliquots for PSD and other measurements. As with the method and location of sample retrieval from the source tank, the influence of mechanical pretreatments like homogenization and subsampling on the measured PSD and its quality is neglected. This does not extend to the mechanical operations to disperse the sample during the particle size measurement (e.g., measurement flow conditions). Exclusions made on this basis are described in the PSD Analyzers Considered subsection below.

Tank waste samples used for PSD analyses must sometimes be diluted with an additional suspending phase to meet maximum allowable dispersion obscuration requirements of the particle size analyzer employed for the measurement. Most commercially available analyzers have dispersion limits of approximately $1 \%$ solids (by volume), whereas tank waste samples range anywhere from 5 to $15 \%$ solids. Diluting the tank waste material to facilitate measurement is not considered a chemical pretreatment. To minimize dissolution effects, preference is given to particle size measurements of tank waste diluted with actual waste supernatant for that tank or a high ionic strength suspending phase simulant (such as $1 \mathrm{M}$ $\mathrm{NaOH}, 1 \mathrm{M} \mathrm{NaNO}_{3}$, supernatant liquid, or surrogate supernatant). Particle size measurements of tank wastes diluted with inhibited water $(0.01 \mathrm{M} \mathrm{NaOH})$ or deionized (DI) water are provisionally included if no acceptable high-ionic strength dilutions exist with the understanding that some salt solids may dissolve.

\section{PSD Analyzers Considered}

The PSD measurements included in the reports listed in Table 3.15 were carried out using a range of instruments, all of which employ a light-scattering technique except the Brinkman device, which uses light obscuration. A complete list of the instruments used is given in Table 3.16. Within a particular report, most PSD analyses were carried out using a single instrument, although there were some exceptions. For example, Microtrac X-100 PSD measurements were accompanied by Microtrac ultrafine particle analyzer (UPA) measurements (which provide additional resolution for submicron particles) in some reports. In Bechtold et al. (2002), PSD measurements of material from the same tanks were taken using both the Horiba LA-910 and Microtrac X-100/UPA systems. In Lumetta et al. (1996), while most tank samples were sized using a Microtrac X-100 analyzer, the sample from Tank C-107 was only analyzed with a Brinkman PSA 2010. 
Table 3.16. Instruments Used for PSD Analysis in Reports Listed in Table 3.15

\begin{tabular}{|l|c|l|l|l||}
\hline \multicolumn{1}{|c|}{ Analyzer } & $\begin{array}{c}\text { Measurement } \\
\text { Range } \\
(\boldsymbol{\mu m})\end{array}$ & \multicolumn{1}{|c||}{ Stirring/Flow } & \multicolumn{1}{|c||}{ Sonication } & \multicolumn{1}{c||}{ Status } \\
\hline Brinkman PSA 2010 & $0.7-50$ & Magnetic Stir Bar & None & Provisionally Included \\
\hline HIAC/ROYCO & $4-225$ & NA $^{(a)}$ & NA & Excluded \\
\hline Horiba LA-910 & $0.02-1020$ & Stir in Reservoir/Flow & External/Internal & $\begin{array}{l}\text { Included-Flow Cell } \\
\text { Included_Fraction Cell }\end{array}$ \\
\hline Leeds \& Northrup UPA & $0.003-6.5$ & None & None & Excluded \\
\hline Malvern Mastersizer 2000 & $0.02-2000$ & Flow Cell & Internal & Included \\
\hline Microtrac UPA & $0.003-6.5$ & Magnetic Stir Bar & None & Excluded \\
\hline Microtrac X-100 & $0.04-700$ & Flow Cell & Internal & Included \\
\hline (a) NA = information not available. & & \\
\hline
\end{tabular}

The Microtrac UPA and Leeds \& Northrup UPA are nonflow systems useful for characterizing sub-micron particles. Because the measuring range of nonflow systems (0.003 to $6.5 \mu \mathrm{m})$ does not fully encompass the spread of particle sizes for tank wastes as determined by microscopy, including any PSD data set measured using these systems would preferentially bias the fines fraction in the final composite distribution. Thus, all UPA measurements were excluded from the composite distribution data set both in Wells et al. (2007) and in this work.

The measurement range of the HIAC/ROYCO system is limited to 4.5 to $225 \mu \mathrm{m}$. PSDs for typical tank wastes, which are expected to contain particles smaller than $4.5 \mu \mathrm{m}$ and/or larger than $225 \mu \mathrm{m}$, cannot be properly characterized using this system. Given that this instrument was only used for one data set (AZ-101) and the limited measuring range of the instrument, the data from this instrument were excluded.

The Brinkman PSA 2010 employs minimal stirring in a fraction cell during measurement. Because the mechanical agitation is weak relative to flow cell systems, there is some concern that large, dense particles may settle to the bottom of the cell before starting the measurement. Based on these concerns, all PSDs obtained by the Brinkman PSA 2010 were excluded from the composite size distribution in Wells et al. (2007). These data, where the $99^{\text {th }}$ percentile particle size is less than $150 \mu \mathrm{m}$, have been included in the present study as a less-energetic PSD measurement technique than the flowing configurations (see Section 3.2.5.2). Sludge tanks with only Brinkman data include AZ-102, B-111, B-201, BX-107, C-103, T-104, T-111 and U-110, and saltcake tanks include AN-104, AN-105, BY-104, S-104, and SY-103. Thus, as evident from Table 3.17, inclusion of the Brinkman data substantially increased the number of fraction cell type PSDs. Brinkman data was also included for AZ-101 (4 of the 17 PSD data sets, Table 3.17) and SY-101 (6 of the 8 PSD data sets, Table 3.17).

PSD measurements taken with the Horiba LA-910 have employed 1) a static fraction cell with external sample sonication and 2) a flow cell with the capability of internal sample sonication that continuously circulates the sample. For the first configuration, there are similar concerns as with the Brinkman PSA 2010. All PSDs employing the fraction cell were excluded in Wells et al. (2007). These data have been included in the present study similar to the Brinkman PSA 2010 data.

The flow cell configuration for the Horiba LA-910 includes a sample reservoir that is mechanically mixed and a pump that supplies a portion of the sample through the analyzer optics. This configuration 
also allows internal sonication during the measurement and control over the stirring and pumping speeds. Horiba LA-910 measurements in the reports referenced in Table 3.15 employ a diversity of pump and flow speeds. Like with the fraction cell, there is concern that the lower stir/pump speeds were not sufficient to suspend large, dense particles during the measurements. For the Horiba LA-910, the circulator pump speed setting is adjustable from 0 to 6 with " 6 " corresponding to a pump discharge rate of $14.4 \mathrm{~mL} / \mathrm{s}$. Stir speed settings range from 0 to 6 , with "6”"corresponding to a stirring speed of $1000 \mathrm{rpm}$. Experience has found that pump settings of " 5 " or greater combined with stir speeds of " 3 " provide adequate suspension of particles. As such, measurements using pump/stir speeds of " $6 / 3$ " or " $5 / 3$ " were included in the composite size distribution in Wells et al. (2007). Both unsonicated (referred to as minimal disturbance in Wells et al. 2007) and sonicated data sets were considered. In most cases, the samples were sonicated at least 2 minutes before analysis. Measurements at lower pump/stir settings, such as "4/2" and "6/1," were excluded from the composite size distribution in Wells et al. (2007). In the present study, all PSD data collected under various flow conditions have been included in the data set. Where multiple flow conditions were examined for any given sample, data obtained with flow condition closest to the "minimal disturbance" settings outlined above were used. This expanded the data set to include a pump/stir speed of $4 / 2$ which was previously excluded.

Bechtold et al. (2002) observed that PSDs from the Horiba LA-910 and Microtrac X-100/UPA systems of settled material from laboratory tests failed to identify very many large particles despite their being visible during the settling tests. As referenced in Appendix G, Bechtold et al. (2002) also noted that, in comparison to sieving analysis of particle size, the light-scattering particle size analyzer was poor at finding particles above $500 \mu \mathrm{m}$ in size. Thus, larger particulates may be underrepresented by these instruments.

Like the Horiba LA-910, the Microtrac X-100 is equipped with a flow cell and internal sonication capabilities that continuously circulate and agitate the sample dispersion during measurement. The X-100 is typically operated at a flow rate of 40 or $60 \mathrm{~mL} / \mathrm{s}$. Both are assumed to provide adequate sample dispersion; Microtrac X-100 measurements included in the composite PSD cover both unsonicated and sonicated size-distributions.

The Malvern Mastersizer 2000 is equipped with a Hydro $\mu \mathrm{P}$ sample dispersion unit that allows for internal sample sonication and includes a flow cell with speed settings that range from 500 to $5000 \mathrm{rpm}$. A pump setting of $3000 \mathrm{rpm}$ is considered to provide sufficient sample agitation to prevent the settling of large, dense particles. The manufacturer-specified upper particle size limits for the device are discussed in Appendix G. As with the observations for the Horiba LA-910 and Microtrac X-100/UPA, these limits indicate that large particles may be underrepresented. The Malvern Mastersizer 2000 measurements include both unsonicated and sonicated size-distributions.

\section{Sonicated Measurements Considered}

Sonicated PSD data sets are typically measured on the same sample aliquot after the initially unsonicated measurement is taken. Sonication data are typically measured after the first period of sonication has elapsed. Depending on the experimental protocol followed, the period of sonication before measurement ranges between 60 and 300 seconds. All subsequent reported PSD measurements performed after initial sample sonication and PSD measurement are discarded. No external sonication data are included. 


\section{Other Considerations}

Additional PSD measurements were included or excluded on a case-by-case basis. Duplicate measurements, when available, were typically included in the compiled data set as long as they satisfied the other criteria outlined above. The PSD data sets for some tanks include a large number of duplicates. Other tank data sets are composed of only one PSD observation. The intention of including duplicate measurements is to provide as much data as possible for subsequent statistical analysis. For a given report, PSDs for duplicate samples were only included if the sample dilution and treatment were equivalent to the primary sample.

Both sonicated and unsonicated data measured under flow conditions were examined for the presence of air bubbles. For flow systems, stirring, pumping, and sonication can generate or entrain air bubbles into the measurement cell in the form of submillimeter- to millimeter-sized bubbles. The light-scattering systems typically employed in these systems cannot distinguish air bubbles from actual particles in this size range. Even a single bubble can appreciably influence PSD measurements because of their large volume relative to micrometer-sized particles. For this reason, any transient peaks in the millimeter size range observed during the measurement should typically be noted and subsequently excluded. It is not known if this was the case for the measurements used herein. For this reason, any data showing large peaks around $1 \mathrm{~mm}$ are considered suspect (but not necessarily excluded). The following methodology is used to evaluate samples with large peaks:

- Unsonicated data showing peaks around 500 to $1000 \mu \mathrm{m}$ are considered suspect. The corresponding sonicated data set is examined. If the peak remains both during and after the application of ultrasonics, the data set is included. If the peak vanishes upon application of ultrasonics and is not observed in duplicate measurements, the PSD is excluded. The underlying assumptions are that sonication will dislodge any bubbles in the measurement cell and/or the sonication causes small bubbles to coalesce and the bigger bubbles to rise and pop in the open reservoir of the instrument. These assumptions may not be valid; thus, exclusions based on suspicion of bubbles are made only if duplicate samples do not show the large peaks in either their minimal disturbance or sonicated data. This is done to avoid potential exclusion of particles and flocs in the 500- to 1000 - $\mu \mathrm{m}$ range. If no duplicate measurements have been made or duplicates show the same behavior as the primary sample, the suspect sample is included.

- Sonicated data showing peaks around $1 \mathrm{~mm}$ are compared to the corresponding unsonicated data set. If no large peak exists in the unsonicated data set, the sonicated data are excluded from further consideration to avoid inclusion of unnatural agglomerates and/or bubbles in the data sets. Bubbles, flocs or instrument abnormalities caused by a change in the background resulting from sonication could all present themselves as peaks around $1 \mathrm{~mm}$. Bubbles are excluded because they are not insoluble waste particles, flocs generated from sonication are excluded because mechanisms required to generate them are unlikely in waste treatment and instrument abnormalities are an artifact of the size analysis and are thus excluded.

If corresponding data are not available to validate suspect sets, they are excluded.

\subsubsection{PSD Data Set}

Data from 19 tanks were originally included in the composite PSDs of Wells et al. (2007). For this study, the data set has been expanded to include data from a total of 34 tanks that encompass previously 
excluded flow regimes as well as saltcake tanks. Of the 34 tanks included, 22 are designated as sludge tanks while the other 12 are classified as saltcake tanks. From these 34 tanks, PSD measurements were further down-selected to generate eight types of PSDs, taking into account the waste type, measurement regime, and sample preparation. The data are presented, depending upon the instrumentation set-up as Flowing Sonicated, Flowing Unsonicated, or No-Flow Unsonicated.

Certain particle size analyzers (e.g., Horiba LA-910 and Microtrac X-100, see Section 3.2.5.1) suspend and circulate solid samples within the instruments during PSD measurements. This configuration is termed the "Flowing" condition. Certain particle size analyzers (e.g., Horiba LA-910 and Brinkman PSA 2010, see Section 3.2.5.1) can be operated with the sample contained in a "fraction cell," and the PSD measurements are designated as "No-Flow." Thus, the Flowing and No-Flow designations indicate that the instrumentation configuration is either:

- Flowing: A dilute suspension of sample UDS in a liquid dispersant is caused to flow through an optical-grade glass sample cell. The sample and dispersant liquid are introduced into the analyzer in a sloped-bottom tank ( $300 \mathrm{~mL}$ in volume for the Horiba LA-910). The suspension exit port is located in the lowest point of the sample tank, and the return port is elevated above the tank bottom. A mechanical adjustable-speed bladed stirrer rotating in a horizontal plane near the bottom of the sample tank is used to disperse and mix the UDS into the suspension. The specimen is circulated through the measurement flow loop, including the glass sample cell, via a variable-speed circulation pump.

- No-Flow: The sample is introduced into a "fraction cell" attachment. For the Horiba LA-90 the fraction cell is $\sim 11 \mathrm{~mL}$ in volume, approximately $70 \times 45 \times 3.5 \mathrm{~mm}, \mathrm{H} \times \mathrm{W} \times \mathrm{D}$. A magnetic stir bar (11 mm long by $2.5 \mathrm{~mm}$ diameter) can be rotated in the vertical plane in one of the bottom corners of the fraction cell to facilitate dispersion and circulation of the sample during PSD measurement.

As described in Section 3.2.5.1, sonication for the Flow condition is accomplished via an ultrasonic generator in the sample tank, which may be operated during the PSD measurement. It is stated in WTP Project Memorandum CCN 186332, “...sonication is a very high energy and irreproducible process...sonication is much more energy intensive than pumping or mixing for many hours and similar to grinding or milling. Its purpose is to reduce the particles to a primary particle size."(a) The solid suspension and circulation of the flowing condition also exert shear forces on the solid particulate. Thus it is assumed that the most energetic PSD measurements with respect to the potential break-up of agglomerates is Flowing Sonicated, followed by Flowing Unsonicated, and the least disturbance is provided by No-Flow Unsonicated.

This potential breakup of larger agglomerate particles for the higher energy PSDs is indicated by PSD data from AY-102 and AZ-101. As reported in Wells et al. (2007), experience has found that stir and pump settings at the upper range of the devices provide adequate suspension of large, dense particles. However, comparing the Flowing Sonicated and Flowing Unsonicated PSDs for Hanford tanks AZ-101 and AY-102 from Wells et al. (2007) shows, with the exception of one AY-102 PSD, that there is essentially no difference in the sonicated and unsonicated PSDs. This result suggests that either the particulate already exists as primary particles and does not break up because of the PSD measurement, or

(a) WTP Project Memorandum CCN 186332. Letter correspondence from AW Etchells, Dupont Technology Consulting to SA Saunders, WTP-Bechtel. January 29, 2007. Comments on the Input Particle Size Report. 
the upper range stir and pump settings for the flowing configuration break up agglomerates similar to the sonication. In Interoffice Memo 7S110-WSC-06-148, PSDs for AY-102 are reported at slightly reduced stir and pump settings (i.e., less energetic measurement condition) for both sonicated and unsonicated conditions. ${ }^{\text {(a) }}$ To a varying extent, each PSD from Interoffice Memo 7S110-WSC-06-148 was reduced in size by sonication (approximately 50\% to $5 \%$ reduction in size due to sonication). This observation also suggests that the upper range stir and pump settings for the Flowing configuration break up agglomerates similar to sonication.

The PSD type classifications are:

- Sludge, Flowing Unsonicated: previously defined in Wells et al. (2007) as minimal disturbance PSDs that were taken under flow conditions sufficient to suspend all of the particulate matter while minimizing any shear-induced breakage of flocs/aggregates. Although the sample conditions could influence the particle sizes as described above for AY-102 samples, the goal was to evaluate the presence of flocs or soft agglomerates in the waste samples while maintaining favorable suspending flows for dense/large particles. These data would be expected to include individual primary particles and both soft and hard agglomerates. This data set has been expanded to include other flow regimes, notably $4 / 2$ pump/stir speeds.

- Sludge, Flowing Sonicated: the sonicated PSDs correspond to particle size measurement data taken under flow conditions. The primary difference is that the sample is sonicated immediately before and during measurement. The goal was to evaluate distribution under conditions of maximal agitation. The data would be expected to include individual primary particles and hard agglomerates but not flocs or soft agglomerates.

- Sludge, No-Flow Unsonicated: the No-Flow PSD corresponds to the particle size measurement data taken under static flow conditions. These samples are mixed by means of an in-cell small stir bar. In the absence of flow, this condition is not considered sufficient to suspend all of the particulate matter present in the sample. The PSD measurement may bias lighter/smaller particles because heavy/large particles may settle to the bottom of the cell and not be measured. The data would be expected to include flocs and soft agglomerates present in the samples.

- Saltcake, Flowing Unsonicated: this is the same as for the Flowing Unsonicated sludge PSD above. The soluble particles could dissolve if the suspending solution was not saturated in the soluble constituents during analysis. These data would be expected to include individual primary particles and both soft and hard agglomerates.

- Saltcake, Flowing Sonicated: this is the same as for the Flowing Sonicated sludge PSD above. Dissolution rates of the soluble particles could be influenced by the sonication during analysis.

- Saltcake, No-Flow Unsonicated: this is the same as for No-Flow Unsonicated sludge PSD above. The soluble particles could dissolve if the suspending solution was not saturated in the soluble constituents during analysis. The data would be expected to include flocs and soft agglomerates present in the samples.

(a) 7S110-WSC-06-148. January 24, 2006. WS Callaway to KG Carothers. Particle Size Distribution Measurements on Samples from Tank 241-AY-102 Core 319. CH2M HILL Hanford Group, Richland, Washington. 
- Saltcake, Flowing Unsonicated, Washed: minimal disturbance PSDs for post-retrieval washed waste. The washing process eliminates readily soluble particulate matter that may be present in saltcake waste. These data would be expected to include insoluble individual primary particles and both soft and hard agglomerates.

- Saltcake, Flowing Sonicated, Washed: sonicated PSDs for post-retrieval washed waste. The washing process eliminates readily soluble particulate matter that may be present in saltcake waste. The data would be expected to include insoluble individual primary particles and hard agglomerates but not flocs or soft agglomerates.

The PSD data meeting the selection criteria described in Section 3.2.5.1 are listed by tank and PSD type in Table 3.17. Also included is the number of PSD data sets for that tank and PSD type. Composite PSDs are created for each of the PSD types and include the appropriate M12 waste group data as shown-see Section 3.2.5.3. Volume weighting factors for the PSD composites are determined by the UDS volume in the tank/M12 waste group and the total UDS volume in the PSD type (UDS volume, Section 3.2.3). The volume weighting factors sum to unity for each PSD type/composite.

The tank PSDs, together with the M12 data for as-received composite groups, are used for the waste type PSDs (see Section 3.2.5.3) as listed in Table 3.18. The volume weighting factors are developed similarly to those for the composite PSDs, and the volume weighting factors sum to unity for each waste type. Following Barker et al. (1999), only those tanks with greater than $70 \%$ by volume of a single waste type are included to represent a specific waste type. Of the 44 PSD type/waste type combinations, only 14 new PSDs are created (non-unity entries for the volume weight factors).

Table 3.17. PSD Data Set, Composite, and Tank PSDs

\begin{tabular}{|c|c|c|c|c|}
\hline \multirow{4}{*}{ PSD Type/Composite } & $\begin{array}{c}\text { Tank/M12 } \\
\text { Group }\end{array}$ & Data Reference(s) & $\begin{array}{c}\text { PSD Data } \\
\text { Set Count }\end{array}$ & $\begin{array}{c}\text { Volume } \\
\text { Weighting } \\
\text { Factors }\end{array}$ \\
\hline \multirow{5}{*}{$\begin{array}{l}\text { FW-103 } \\
\text { Sludge, Flowing }\end{array}$} & HNF-8862, Rev. 0 & 7 & $5.9 \mathrm{E}-02$ \\
\cline { 2 - 5 } & AY-101 & HNF-8862, Rev. 0 & 4 & $3.2 \mathrm{E}-02$ \\
\cline { 2 - 5 } & AY-102 & HNF-8862, Rev. 0, 7S110-WSC-06-148 & 10 & $2.6 \mathrm{E}-02$ \\
\cline { 2 - 5 } & AZ-101 & WTP-RPT-048 Rev. 1 & 1 & $1.2 \mathrm{E}-02$ \\
\cline { 2 - 5 } & AZ-102 & 7S110-WSC-03-012, BNFL-RPT-038, & 20 & \\
\cline { 2 - 5 } & B-203 & HNF-8862, Rev. 0, RPP-9806 & $1.4 \mathrm{E}-02$ \\
\cline { 2 - 5 } & C-104 & ENFL-RPT-030, HNF-8862, Rev. 0 & 6 & $1.2 \mathrm{E}-03$ \\
\cline { 2 - 5 } & C-106 & PNNL-11381 & 1 & $1.6 \mathrm{E}-02$ \\
\cline { 2 - 5 } & C-107 & HNF-8362, Rev. 0 & 4 & $5.6 \mathrm{E}-02$ \\
\cline { 2 - 5 } & S-107 & PNNL-11278 Rev. 1, PNNL-12010 & 2 & $4.3 \mathrm{E}-02$ \\
\cline { 2 - 5 } & SX-108 & PNNL-11278 Rev. 1 & 1 & $2.9 \mathrm{E}-02$ \\
\cline { 2 - 5 } & SY-102 & HNF-8862, Rev. 0, PNNL-11352 & 3 & $4.1 \mathrm{E}-02$ \\
\cline { 2 - 5 } & T-110 & EMSP 90162 & 1 & $6.3 \mathrm{E}-03$ \\
\cline { 2 - 5 } & T-203 & EMSP 90162 & 1 & $8.3 \mathrm{E}-04$ \\
\cline { 2 - 5 } & T-204 & EMSP 90162 & 1 & $8.7 \mathrm{E}-04$ \\
\cline { 2 - 5 } & M12 Group 1 & WTP-RPT-166 & 2 & $1.3 \mathrm{E}-01$ \\
\cline { 2 - 5 } & M12 Group 3 & WTP-RPT-167 & & $2.4 \mathrm{E}-01$ \\
\hline
\end{tabular}


Table 3.17. (contd)

\begin{tabular}{|c|c|c|c|c|}
\hline PSD Type/Composite & $\begin{array}{c}\text { Tank/M12 } \\
\text { Group }\end{array}$ & Data Reference(s) & $\begin{array}{l}\text { PSD Data } \\
\text { Set Count }\end{array}$ & $\begin{array}{c}\text { Volume } \\
\text { Weighting } \\
\text { Factors } \\
\end{array}$ \\
\hline & M12 Group 4 & WTP-RPT-167 & 2 & $2.3 \mathrm{E}-02$ \\
\hline & M12 Group 5 & WTP-RPT-157 & 2 & 8.7E-02 \\
\hline & M12 Group 7 & WTP-RPT-157 & 0 & $4.9 \mathrm{E}-02$ \\
\hline & M12 Group 8 & WTP-RPT-169 & 0 & $9.6 \mathrm{E}-02$ \\
\hline \multirow{21}{*}{$\begin{array}{l}\text { Sludge, Flowing } \\
\text { Unsonicated }\end{array}$} & AW-103 & HNF-8862, Rev. 0 & 8 & 5.9E-02 \\
\hline & AY-101 & HNF-8862, Rev. 0 & 6 & $3.2 \mathrm{E}-02$ \\
\hline & AY-102 & HNF-8862, Rev. 0, 7S110-WSC-06-148 & 10 & $2.6 \mathrm{E}-02$ \\
\hline & AZ-101 & WTP-RPT-048 Rev. 1 & 1 & $1.2 \mathrm{E}-02$ \\
\hline & AZ-102 & $\begin{array}{c}\text { BNFL-RPT-038, HNF-8862, Rev. 0, } \\
\text { RPP-9806 }\end{array}$ & 13 & $1.4 \mathrm{E}-02$ \\
\hline & B-203 & PNL-16133 & 1 & $1.2 \mathrm{E}-03$ \\
\hline & C-104 & BNFL-RPT-030, HNF-8862, Rev. 0 & 8 & 5.3E-02 \\
\hline & C-106 & PNNL-11381 & 1 & $1.6 \mathrm{E}-03$ \\
\hline & C-107 & HNF-8862, Rev. 0 & 4 & $5.6 \mathrm{E}-02$ \\
\hline & S-107 & PNNL-11278 Rev. 1, PNNL-12010 & 2 & 4.3E-02 \\
\hline & SX-108 & PNNL-11278 Rev. 1 & 1 & $2.9 \mathrm{E}-02$ \\
\hline & SY-102 & HNF-8862, Rev. 0, PNNL-11352 & 3 & $4.1 \mathrm{E}-02$ \\
\hline & $\mathrm{T}-110$ & PNL-16133 & 1 & $6.3 \mathrm{E}-03$ \\
\hline & $\mathrm{T}-203$ & PNL-16133 & 1 & 8.3E-04 \\
\hline & T-204 & PNL-16133 & 1 & 8.7E-04 \\
\hline & M12 Group 1 & WTP-RPT-166 & 2 & $1.3 \mathrm{E}-01$ \\
\hline & M12 Group 3 & WTP-RPT-167 & 2 & $2.4 \mathrm{E}-01$ \\
\hline & M12 Group 4 & WTP-RPT-167 & 2 & $2.3 \mathrm{E}-02$ \\
\hline & M12 Group 5 & WTP-RPT-157 & 2 & $8.7 \mathrm{E}-02$ \\
\hline & M12 Group 7 & WTP-RPT-157 & 2 & $4.9 \mathrm{E}-02$ \\
\hline & M12 Group 8 & WTP-RPT-169 & 2 & $9.6 \mathrm{E}-02$ \\
\hline \multirow{13}{*}{$\begin{array}{l}\text { Sludge, No-Flow } \\
\text { Unsonicated }\end{array}$} & AY-102 & RPP-8909 Rev. 0, FH 0202392 & 23 & $5.2 \mathrm{E}-02$ \\
\hline & AZ-101 & $\begin{array}{l}\text { PNL-11098, HNF-7078, HNF-6062, } \\
\text { PNNL-13027 }\end{array}$ & 17 & 2.4E-02 \\
\hline & AZ-102 & PNL-11098 & 1 & $2.7 \mathrm{E}-02$ \\
\hline & B-111 & PNL-10712 & 1 & $4.1 \mathrm{E}-02$ \\
\hline & B-201 & PNL-10078 & 2 & $4.4 \mathrm{E}-03$ \\
\hline & BX-107 & PNL-10712 & 1 & $1.8 \mathrm{E}-01$ \\
\hline & C-103 & PNL-10712 & 1 & $1.4 \mathrm{E}-01$ \\
\hline & C-104 & RPP-5798 & 1 & $1.1 \mathrm{E}-01$ \\
\hline & C-107 & FH-0201835 & 28 & $1.1 \mathrm{E}-01$ \\
\hline & SY-102 & FH-0202775 & 8 & $8.1 \mathrm{E}-02$ \\
\hline & $\mathrm{T}-104$ & PNL-10712 & 1 & $1.2 \mathrm{E}-01$ \\
\hline & $\mathrm{T}-111$ & PNL-10712 & 1 & $4.1 \mathrm{E}-02$ \\
\hline & U-110 & WHC-EP-0643 Rev. 1, PNL-10078 & 2 & 7.0E-02 \\
\hline \multirow{4}{*}{$\begin{array}{l}\text { Saltcake, Flowing } \\
\text { Sonicated }\end{array}$} & AN-102 & 7S110-WSC-03-002, 7S110-WSC-05-011 & 14 & $4.2 \mathrm{E}-02$ \\
\hline & AN-104 & PNNL-11636 & 2 & $4.2 \mathrm{E}-02$ \\
\hline & AN-107 & B3610-WSC-02-028 & 10 & $1.2 \mathrm{E}-02$ \\
\hline & BY-104 & PNNL-11278 Rev. 1 & 1 & $4.4 \mathrm{E}-02$ \\
\hline
\end{tabular}


Table 3.17. (contd)

\begin{tabular}{|c|c|c|c|c|}
\hline PSD Type/Composite & $\begin{array}{c}\text { Tank/M12 } \\
\text { Group }\end{array}$ & Data Reference(s) & $\begin{array}{l}\text { PSD Data } \\
\text { Set Count }\end{array}$ & $\begin{array}{c}\text { Volume } \\
\text { Weighting } \\
\text { Factors } \\
\end{array}$ \\
\hline & BY-108 & PNNL-11636 & 1 & 3.4E-02 \\
\hline & BY-110 & PNNL-11278 Rev. 1 & 1 & 3.9E-02 \\
\hline & S-104 & PNNL-11636 & 1 & $2.5 \mathrm{E}-02$ \\
\hline & M12 Group 2 & WTP-RPT-166 & 2 & 4.8E-01 \\
\hline & M12 Group 6 & WTP-RPT-157 & 2 & $2.9 \mathrm{E}-01$ \\
\hline \multirow{9}{*}{$\begin{array}{l}\text { Saltcake, Flowing } \\
\text { Unsonicated }\end{array}$} & AN-102 & 7S110-WSC-05-011 & 5 & $4.2 \mathrm{E}-02$ \\
\hline & AN-104 & PNNL-11636 & 2 & $4.1 \mathrm{E}-02$ \\
\hline & BY-104 & PNNL-11278 Rev. 1 & 1 & 4.3E-02 \\
\hline & BY-108 & PNNL-11636 & 1 & $3.3 \mathrm{E}-02$ \\
\hline & BY-110 & PNNL-11278 Rev. 1 & 1 & $3.9 \mathrm{E}-02$ \\
\hline & S-101 & PNNL-11636 & 1 & $2.5 \mathrm{E}-02$ \\
\hline & S-104 & PNNL-11636 & 1 & $2.5 \mathrm{E}-02$ \\
\hline & M12 Group 2 & WTP-RPT-166 & 2 & 4.7E-01 \\
\hline & M12 Group 6 & WTP-RPT-157 & 2 & $2.8 \mathrm{E}-01$ \\
\hline \multirow{7}{*}{$\begin{array}{l}\text { Saltcake, No-Flow } \\
\text { Unsonicated }\end{array}$} & AN-104 & HNF-3352 Rev. 0 & 1 & $1.8 \mathrm{E}-01$ \\
\hline & AN-105 & HNF-SD-WM-DTR-046 & 2 & $1.4 \mathrm{E}-01$ \\
\hline & AW-101 & HNF-4964 Rev. 0 & 1 & $1.8 \mathrm{E}-01$ \\
\hline & BY-104 & WHC-SD-WM-TI-540 & 4 & 1.9E-01 \\
\hline & S-104 & PNL-10712 & 1 & $1.1 \mathrm{E}-01$ \\
\hline & SY-101 & $\begin{array}{l}\text { 12110-PCL92-01B/WHC-EP-0628, } \\
\text { HNF-1666 Rev.0-A, } \\
\text { 12110-PCL92-01B/WHC-EP-0628 }\end{array}$ & 8 & 8.8E-02 \\
\hline & SY-103 & PNL-10712 & 1 & 1.2E-01 \\
\hline $\begin{array}{l}\text { Saltcake, Flowing } \\
\text { Sonicated, Washed }\end{array}$ & AN-102 & WTP-RPT-076 & 2 & $1.0 \mathrm{E}+00$ \\
\hline $\begin{array}{c}\text { Saltcake, Flowing } \\
\text { Unsonicated, Washed }\end{array}$ & AN-102 & WTP-RPT-076 & 2 & $1.0 \mathrm{E}+00$ \\
\hline
\end{tabular}

Table 3.18. Waste Type PSD Data Sets

\begin{tabular}{|c|c|c|c|}
\hline \multirow{4}{*}{ PSD Type } & Waste Type & PSD Data Set(s) & $\begin{array}{c}\text { Volume Weighting } \\
\text { Factors }\end{array}$ \\
\hline \multirow{5}{*}{ Sludge, Flowing Sonicated } & 224 Post-1949 sludge & B-203, T-203, T-204 & $0.41,0.29,0.3$ \\
\cline { 2 - 4 } & 1C and 2C sludge & M12 Group 1 & 1 \\
\cline { 2 - 4 } & 2C sludge & T-110 & 1 \\
\cline { 2 - 4 } & AR sludge & C-106 & 1 \\
\cline { 2 - 4 } & CWP1 and CWP2 sludge & A12 Group 3 & 1 \\
\cline { 2 - 4 } & CWR1 sludge & M12 Group 4 & 1 \\
\cline { 2 - 4 } & CWZr2 sludge & AW-103 & $0.48,0.52$ \\
\cline { 2 - 4 } & P3 sludge & AZ-101, AZ-102 & 1 \\
\cline { 2 - 4 } & PFeCN sludge & M12 Group 8 & \\
\hline
\end{tabular}


Table 3.18. (contd)

\begin{tabular}{|c|c|c|c|}
\hline PSD Type & Waste Type & PSD Data Set(s) & $\begin{array}{c}\text { Volume Weighting } \\
\text { Factors }\end{array}$ \\
\hline & R1 (boiling) sludge & SX-108, M12 Group 5 & $0.25,0.75$ \\
\hline & TBP sludge & M12 Group 7 & 1 \\
\hline & Unidentified sludge & AY-101 & 1 \\
\hline \multirow{13}{*}{$\begin{array}{l}\text { Sludge, Flowing } \\
\text { Unsonicated }\end{array}$} & 224 Post-1949 sludge & B-203, T-203, T-204 & $0.41,0.29,0.3$ \\
\hline & 1C and 2C sludge & M12 Group 1 & 1 \\
\hline & 2C sludge & $\mathrm{T}-110$ & 1 \\
\hline & AR sludge & C-106 & 1 \\
\hline & BL sludge & AY-102 & 1 \\
\hline & CWP1 and CWP2 sludge & M12 Group 3 & 1 \\
\hline & CWR1 sludge & M12 Group 4 & 1 \\
\hline & CWZr2 sludge & AW-103 & 1 \\
\hline & P3 sludge & AZ-101, AZ-102 & $0.48,0.52$ \\
\hline & PFeCN sludge & M12 Group 8 & 1 \\
\hline & R1 (boiling) sludge & SX-108, M12 Group 5 & $0.25,0.75$ \\
\hline & TBP sludge & M12 Group 7 & 1 \\
\hline & Unidentified sludge & AY-101 & 1 \\
\hline \multirow{5}{*}{$\begin{array}{l}\text { Sludge, No-Flow } \\
\text { Unsonicated }\end{array}$} & 224 Pre-1949 sludge & B-201 & 1 \\
\hline & 1C sludge & BX-107, T-104 & $0.59,0.41$ \\
\hline & 2C sludge & B-111 & 1 \\
\hline & BL sludge & AY-102 & 1 \\
\hline & P3 sludge & AZ-101, AZ-102 & $0.48,0.52$ \\
\hline \multirow{4}{*}{ Saltcake, Flowing Sonicated } & A2 saltcake & AN-104, AN-102, AN-107 & $0.43,0.44,0.13$ \\
\hline & BY saltcake & BY-104, BY-108, BY-110 & $0.38,0.27,0.35$ \\
\hline & BY, T1, and T2 saltcake & M12 Group 2 & 1 \\
\hline & S1 and S2 Saltcake & M12 Group 6 & 1 \\
\hline \multirow{4}{*}{$\begin{array}{l}\text { Saltcake, Flowing } \\
\text { Unsonicated }\end{array}$} & A2 saltcake & AN-104, AN-102 & $0.5,0.5$ \\
\hline & BY saltcake & BY-104, BY-108, BY-110 & $0.38,0.27,0.35$ \\
\hline & BY, T1, and T2 saltcake & M12 Group 2 & 1 \\
\hline & S1 and S2 Saltcake & M12 Group 6 & 1 \\
\hline \multirow{3}{*}{$\begin{array}{l}\text { Saltcake, No-Flow } \\
\text { Unsonicated }\end{array}$} & A2 saltcake & AW-101, AN-105, AN-104 & $0.36,0.28,0.36$ \\
\hline & BY saltcake & BY-104 & 1 \\
\hline & S2 saltcake & SY-101, SY-103 & $0.43,0.57$ \\
\hline $\begin{array}{c}\text { Saltcake, Flowing } \\
\text { Sonicated, Washed }\end{array}$ & A2 saltcake & AN-102 & 1 \\
\hline $\begin{array}{c}\text { Saltcake, Flowing } \\
\text { Unsonicated, Washed }\end{array}$ & A2 saltcake & AN-102 & 1 \\
\hline
\end{tabular}

If a tank waste is treated as "represented" with respect to particle size regardless of the number of measurements for a given tank, PSDs for approximately $40 \%$ of the Hanford waste UDS volume are represented for Flowing Sonicated, 58\% for Flowing Unsonicated, and 41\% for No-Flow Unsonicated. All PSD data from Table 3.17 are included in these tallies with the exception of the three M12 groups with multiple waste types (Table 3.18). The UDS volume in each tank (from Section 3.2.3) is multiplied by the fraction of the primary waste type in that tank. Thus, more waste types are classified as "represented" than are listed in Table 3.18, which includes, as previously specified, only those tanks with greater than $70 \%$ by volume of a single waste type. 
The fraction of a specific waste type for which PSD data are available is shown in the following figures for each of the PSD types (washed saltcake is not included). As a metric indicating the relative importance of the various waste types, the fraction of the waste type relative to the total UDS inventory ${ }^{(a)}$ is also shown. To show very small fractions, the vertical axis is logarithmic. From Figure 3.37, 5 of the 13 primary waste types for Sludge, Flowing Sonicated have greater than $50 \%$ by UDS volume representation for PSDs. Greater than $50 \%$ by UDS volume representation for PSDs is shown for 5 of the 13 primary waste types for Sludge, Flowing Unsonicated (Figure 3.38), 3 of 8 for Sludge, No-Flow Unsonicated (Figure 3.39), none of the 3 for Saltcake, Flowing Sonicated (Figure 3.40), none of the 4 for Saltcake, Flowing Unsonicated (Figure 3.41), and none of the 3 for Saltcake, No-Flow Unsonicated (Figure 3.42).

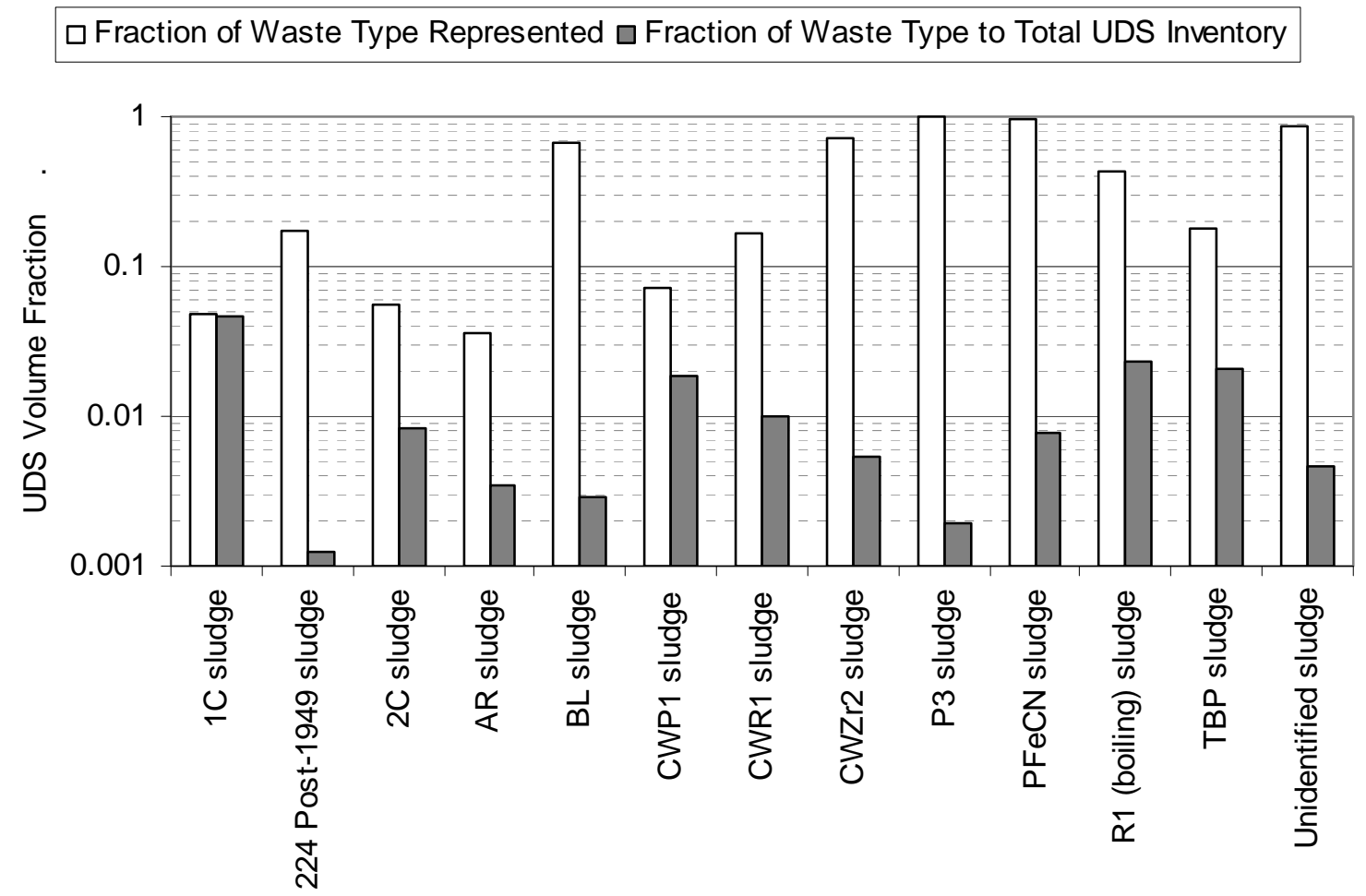

Figure 3.37. Waste Types Represented by Sludge, Flowing Sonicated PSDs

(a) The UDS inventory (mass and volume) in each tank was determined by thermodynamic modeling that used 2002 Best Basis Inventory (BBI) data and by adjustments to modeling results, as described in Section 3.2.3.2. The UDS inventory does not include interstitial liquid. 
Fraction of Waste Type Represented $\square$ Fraction of Waste Type to Total UDS Inventory

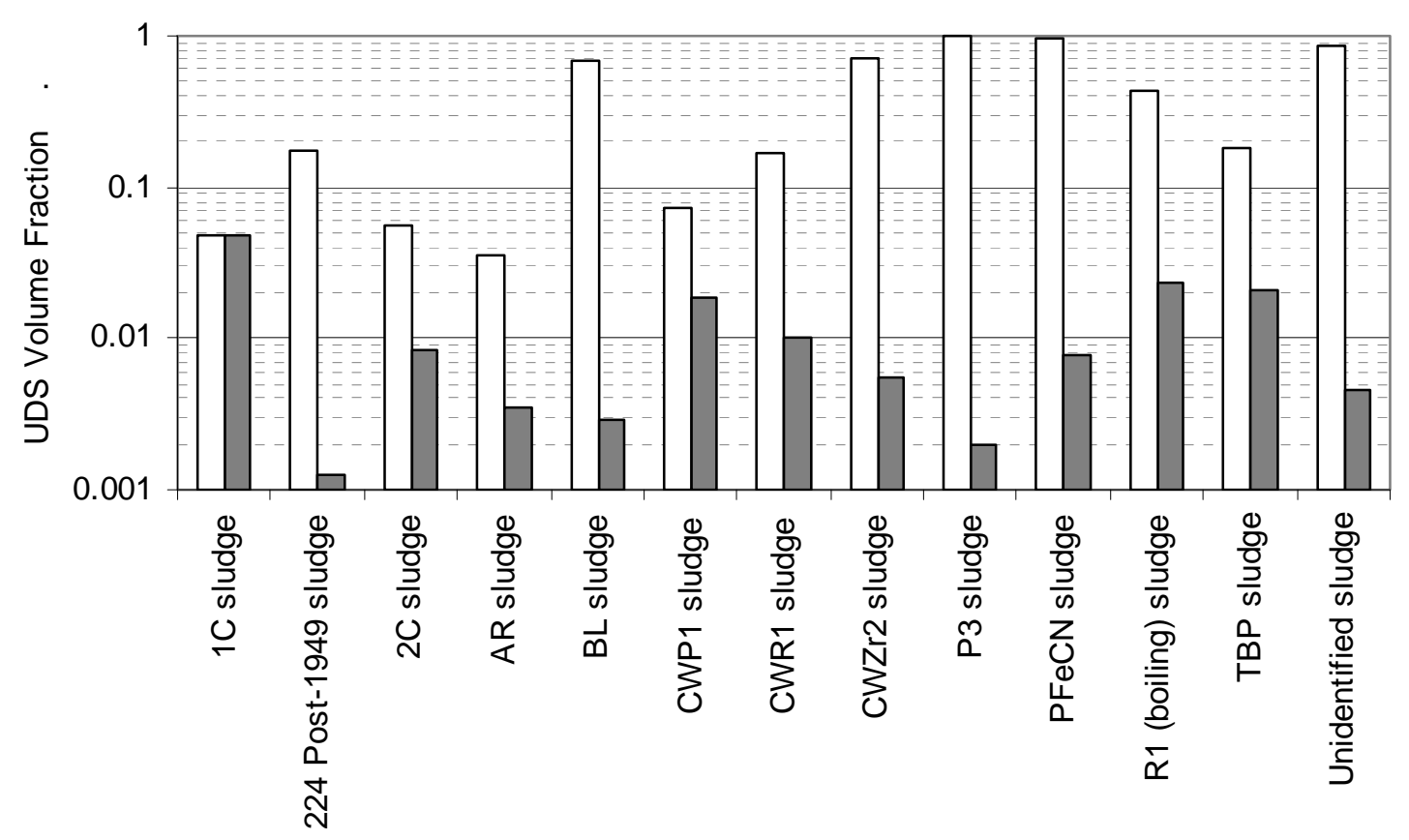

Figure 3.38. Waste Types Represented by Sludge, Flowing Unsonicated PSDs

$\square$ Fraction of Waste Type Represented $\square$ Fraction of Waste Type to Total UDS Inventory

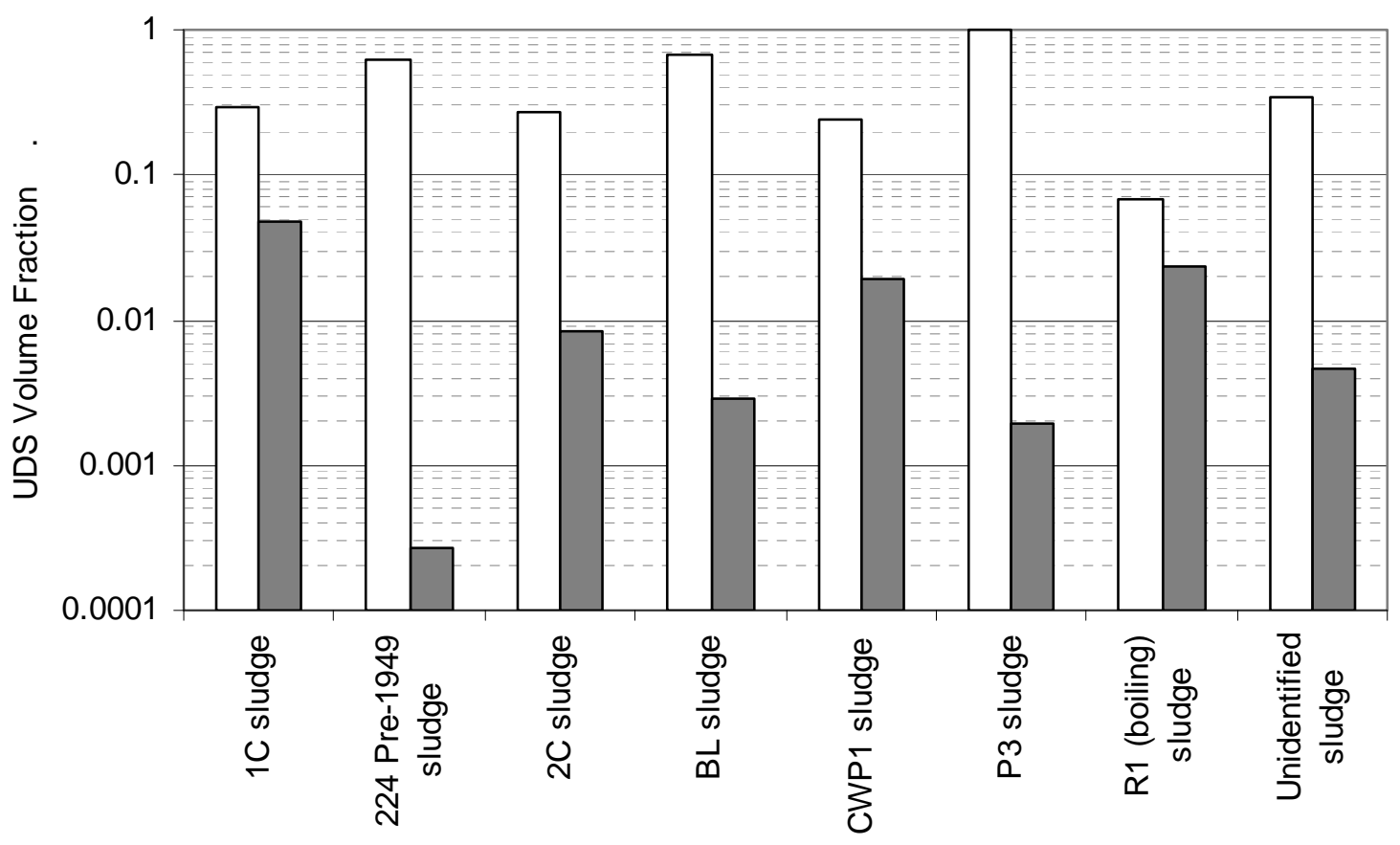

Figure 3.39. Waste Types Represented by Sludge, No-Flow Unsonicated PSDs 
$\square$ Fraction of Waste Type Represented $\square$ Fraction of Waste Type to Total UDS Inventory

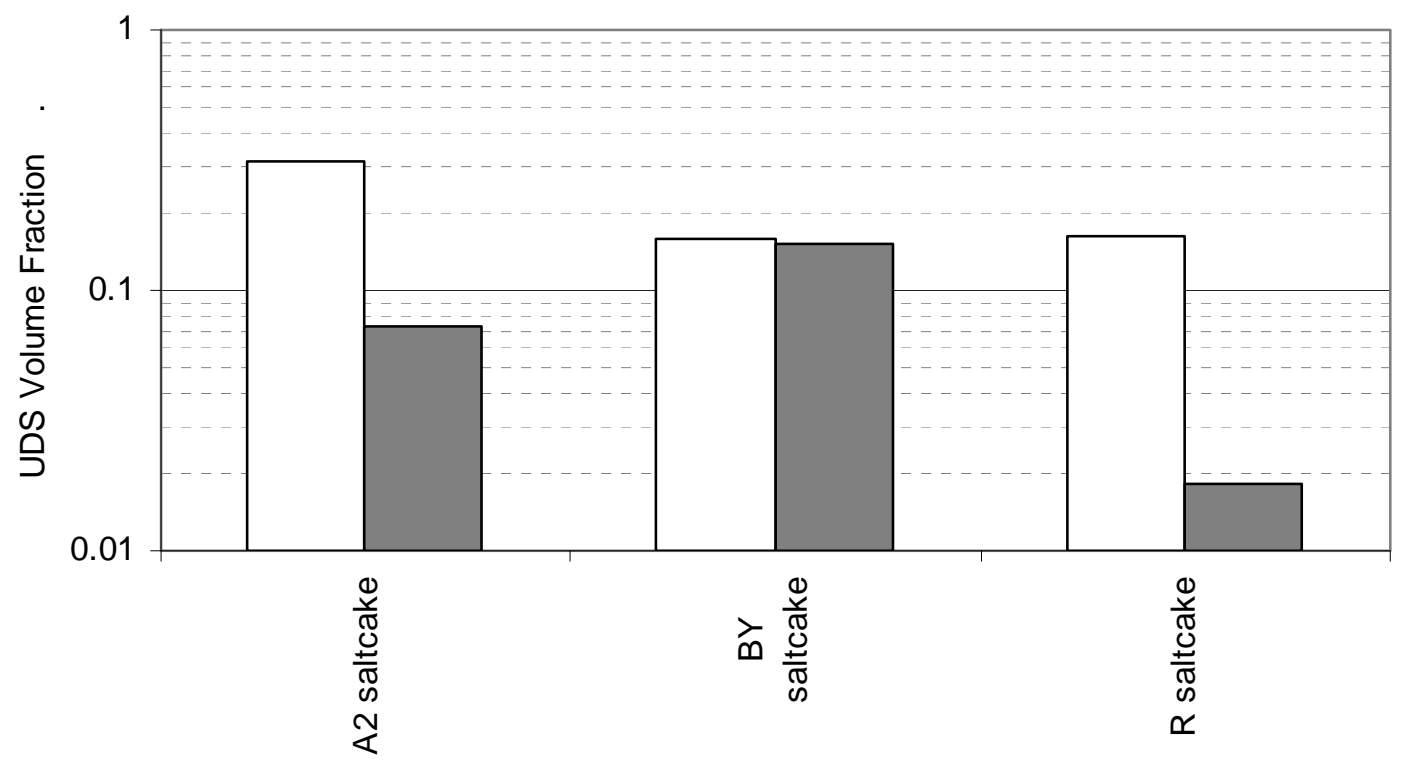

Figure 3.40. Waste Types Represented by Saltcake, Flowing Sonicated PSDs

$\square$ Fraction of Waste Type Represented $\square$ Fraction of Waste Type to Total UDS Inventory

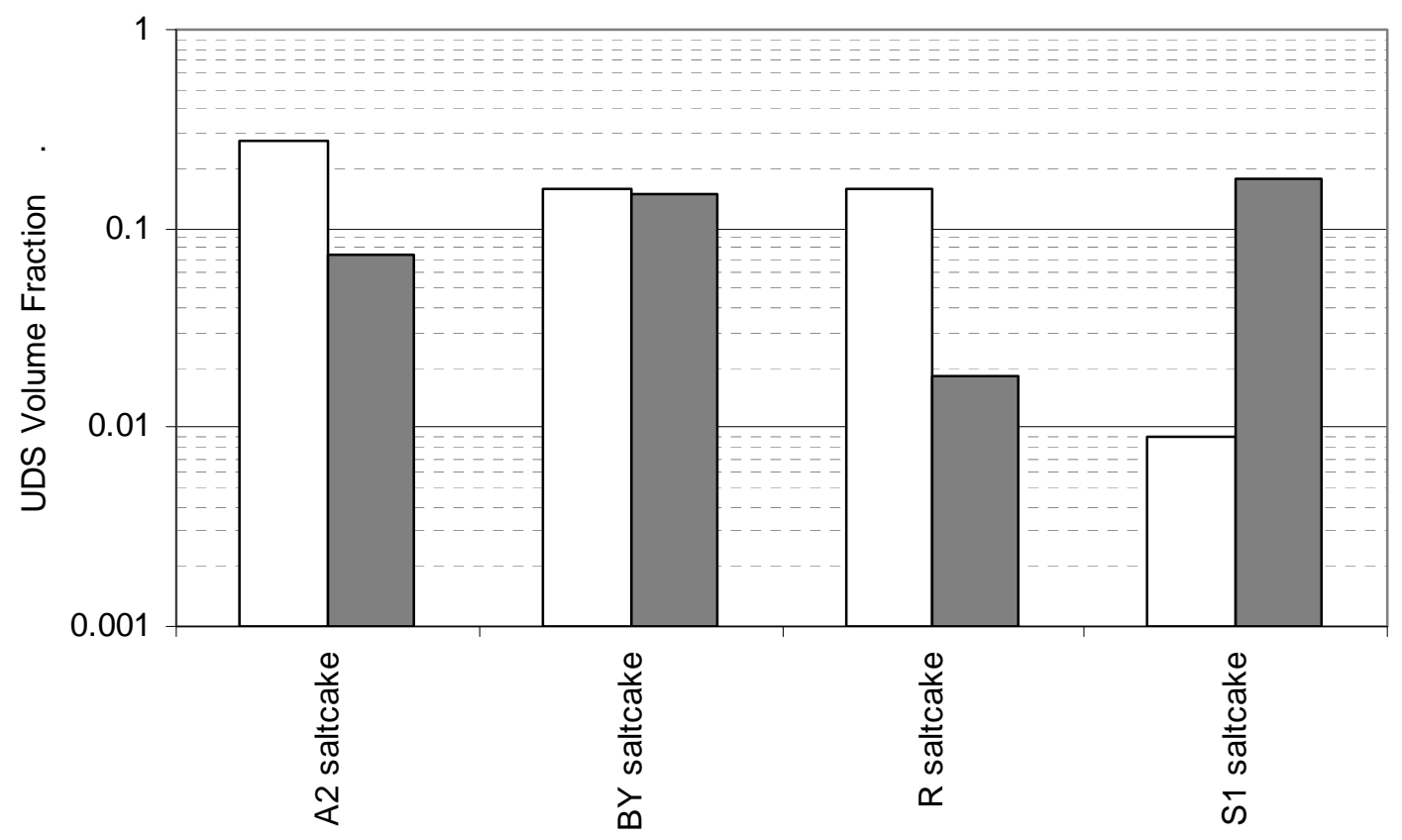

Figure 3.41. Waste Types Represented by Saltcake, Flowing Unsonicated PSDs 


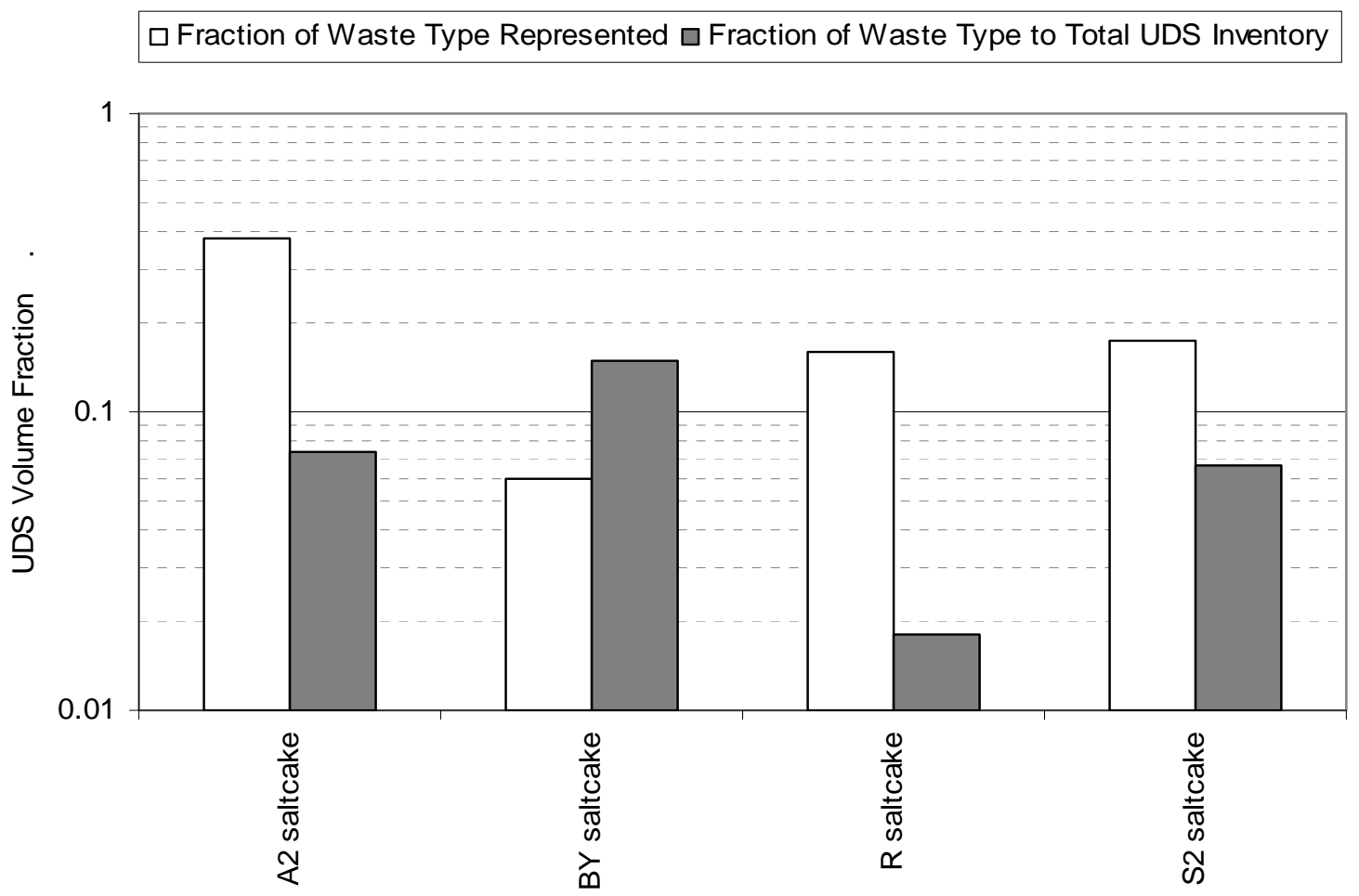

Figure 3.42. Waste Types Represented by Saltcake, No-Flow Unsonicated PSDs

\subsubsection{PSD Methods}

Statistical methods and the existing data as listed Section 3.2.5.2 were used to estimate PSDs for specific individual waste tanks, waste types (combinations of waste tanks), and waste composites (over a specified set of waste and slurry characteristics). The PSDs generated provide estimated particle sizes for the specified percentiles of the true PSD for a given waste tank, waste type, or composite. The standard percentiles of interest selected for this work are P01 (the first percentile), P05 (the fifth percentile), P10 (the tenth percentile, etc.), P20, P25, P30, P40, P50, P60, P70, P75, P80, P90, P95, P99, and P100. For example, in the PSD reported for an individual waste tank, the P75 value is the estimated particle size associated with the $75^{\text {th }}$ percentile, meaning that $75 \%$ of the particles in the tank are expected to be smaller than the reported P75 value. The cumulative percentile associated with the $75^{\text {th }}$ percentile is 0.75 .

The PSD summaries include estimates of "typical” particle sizes for the specified percentiles as well as lower and upper limits (ULs) on particle sizes for the specified percentiles. The PSDs generated consolidate available sample data and provide a unified summary for a given waste tank, waste type, or composite. A critical part of the PSD generation process was to determine P100 estimates for the different waste tanks, waste types, composites, or composite subsets. Although the reported P100 values are in fact only estimates of maximum particle sizes, they are likely to be of particular interest for subsequent work. The PSD summaries are reported in two formats, one that focuses on the percentiles of interest and the other based on designated size bins that cover a range from 0 to 10,000 $\mu \mathrm{m}$. This section describes the estimation methods used to determine P100 values as well as data combination and 
interpolation methods used to generate the reported PSDs. Specific aspects of the PSD development methods are described in the following subsections.

\section{Data}

As described in Section 3.2.5.1, the PSD data is from one or more samples taken from various waste tanks at the Hanford site, and the samples were analyzed using one of several different instruments. For each sample, analyzed particle sizes associated with some or all of the percentiles of interest were determined.

Regardless of which instrument was used, the analyses were conducted on slurry samples from the waste tanks as the sample units rather than individual waste particles. So while the objective of making inferences concerning the distribution of particle sizes within the waste tanks implies that the preferred population units would be individual waste particles, the sampling and analysis methods used to collect data force the population units for this study to be the different sub-quantities of the tanks' content that were available for sampling. Consequently, the particle sizes listed as the initial data for a given sample were not obtained from measurements on individual particles. They are a set of values, obtained through analysis, that together describe the collection of waste particles in the particular slurry sample. As discussed, an objective of the PSD development work was to consolidate sample information and generate PSDs that summarize and represent, to the extent possible, the distribution of waste particle sizes for a given waste tank, waste type, or composite. To that end, it must be assumed that the samples are representative of the waste tanks and that particle sizes are generally present in the tanks in the same proportions as seen in the respective slurry samples.

As an example, Table 3.19 contains PSDs from waste tank AZ-102. The first row of the table lists the cumulative percentiles considered in this study. The remaining rows of the table list the particle size analysis results for 13 slurry samples from this tank. The columns of the table are the cumulative percentiles of interest. Entries in the table have been rounded to 2 decimal places.

Table 3.19. Initial Data Matrix, AZ-102 Example (values are particle sizes in microns)

\begin{tabular}{|c|c|c|c|c|c|c|c|c|c|c|c|c|c|c|c|}
\hline Percentile & 0.01 & 0.05 & 0.10 & 0.20 & 0.25 & 0.30 & 0.40 & 0.50 & 0.60 & 0.70 & 0.75 & 0.80 & 0.90 & 0.95 & 0.99 \\
\hline Sample 1 & 0.71 & 1.26 & 1.99 & 3.75 & 4.66 & 5.59 & 7.71 & 10.87 & 15.42 & 20.38 & 23.09 & 26.13 & 34.62 & 42.43 & 60.57 \\
\hline Sample 2 & 0.68 & 1.21 & 1.89 & 3.56 & 4.40 & 5.29 & 7.21 & 10.04 & 14.65 & 19.89 & 22.64 & 25.67 & 33.96 & 41.64 & 60.22 \\
\hline Sample 3 & 1.29 & 2.62 & NA & NA & 8.37 & NA & NA & 20.60 & NA & NA & 63.40 & NA & NA & 214.70 & 306.60 \\
\hline Sample 4 & 1.66 & 3.06 & NA & NA & 9.71 & NA & NA & 24.36 & NA & NA & 105.50 & NA & NA & 624.80 & 769.50 \\
\hline Sample 5 & 1.34 & 2.15 & NA & NA & 5.44 & NA & NA & 10.06 & NA & NA & 15.70 & NA & NA & 24.60 & 29.80 \\
\hline Sample 6 & 1.53 & 2.74 & NA & NA & 8.72 & NA & NA & 20.51 & NA & NA & 208.60 & NA & NA & 558.00 & 721.40 \\
\hline Sample 7 & 1.33 & 2.12 & NA & NA & 5.29 & NA & NA & 9.82 & NA & NA & 15.40 & NA & NA & 24.30 & 29.60 \\
\hline Sample 8 & 1.35 & 2.21 & NA & NA & 5.81 & NA & NA & 11.07 & NA & NA & 17.80 & NA & NA & 28.10 & 36.90 \\
\hline Sample 9 & 1.41 & 2.35 & 3.18 & 4.83 & 5.73 & 6.70 & 8.99 & 12.21 & 18.46 & 74.76 & 103.75 & 121.29 & 158.13 & 192.47 & 275.37 \\
\hline Sample 10 & 1.39 & 2.27 & 3.04 & 4.51 & 5.33 & 6.20 & 8.25 & 11.06 & 16.01 & 53.95 & 101.17 & 121.64 & 161.77 & 197.06 & 282.80 \\
\hline Sample 11 & 1.37 & 2.25 & 3.04 & 4.55 & 5.38 & 6.29 & 8.44 & 11.43 & 17.07 & 72.32 & 105.18 & 123.63 & 161.95 & 196.39 & 281.38 \\
\hline Sample 12 & 1.62 & 2.86 & 4.07 & 6.99 & 8.97 & 11.60 & 20.81 & 37.08 & 53.23 & 69.48 & 78.39 & 88.95 & 119.94 & 151.27 & 226.72 \\
\hline Sample 13 & 1.54 & 2.62 & 3.62 & 5.85 & 7.18 & 8.78 & 13.37 & 22.62 & 46.76 & 78.14 & 92.31 & 106.39 & 137.97 & 167.21 & 241.67 \\
\hline
\end{tabular}

Some of the values listed in Table 3.19 were calculated by interpolation using data obtained directly from the sample analyses of Section 3.2.5.2. For some tanks, like AZ-102, the initial data matrix still included missing percentile values (denoted as NA in Table 3.19). Therefore, as a final data preparation step, missing entries in the initial data matrix were filled by interpolation, or extrapolation in the cases 
where empty cells occurred at the beginning or end of a row (lower or higher percentiles for a sample). The resulting final data matrix resulting from the initial data matrix for AZ-102 is provided in Table 3.20. Entries in this table have also been rounded to 2 decimal places.

Table 3.20. Final Data Matrix, AZ-102 Example (values are particle sizes in microns)

\begin{tabular}{|c|c|c|c|c|c|c|c|c|c|c|c|c|c|c|c|}
\hline Percentile & 0.01 & 0.05 & 0.10 & 0.20 & 0.25 & 0.30 & 0.40 & 0.50 & 0.60 & 0.70 & 0.75 & 0.80 & 0.90 & 0.95 & 0.99 \\
\hline Sample 1 & 0.71 & 1.26 & 1.99 & 3.75 & 4.66 & 5.59 & 7.71 & 10.87 & 15.42 & 20.38 & 23.09 & 26.13 & 34.62 & 42.43 & 60.57 \\
\hline Sample 2 & 0.68 & 1.21 & 1.89 & 3.56 & 4.40 & 5.29 & 7.21 & 10.04 & 14.65 & 19.89 & 22.64 & 25.67 & 33.96 & 41.64 & 60.22 \\
\hline Sample 3 & 1.29 & 2.62 & 3.50 & 6.26 & 8.37 & 10.02 & 14.37 & 20.60 & 32.30 & 50.63 & 63.40 & 86.01 & 158.27 & 214.70 & 306.60 \\
\hline Sample 4 & 1.66 & 3.06 & 4.08 & 7.28 & 9.71 & 11.67 & 16.86 & 24.36 & 43.78 & 78.69 & 105.50 & 164.58 & 400.52 & 624.80 & 769.50 \\
\hline Sample 5 & 1.34 & 2.15 & 2.71 & 4.31 & 5.44 & 6.15 & 7.87 & 10.06 & 12.02 & 14.36 & 15.70 & 17.57 & 21.99 & 24.60 & 29.80 \\
\hline Sample 6 & 1.53 & 2.74 & 3.66 & 6.53 & 8.72 & 10.35 & 14.57 & 20.51 & 51.87 & 131.17 & 208.60 & 266.77 & 436.32 & 558.00 & 721.40 \\
\hline Sample 7 & 1.33 & 2.12 & 2.66 & 4.21 & 5.29 & 5.99 & 7.67 & 9.82 & 11.76 & 14.07 & 15.40 & 17.26 & 21.68 & 24.30 & 29.60 \\
\hline Sample 8 & 1.35 & 2.21 & 2.81 & 4.56 & 5.81 & 6.61 & 8.55 & 11.07 & 13.39 & 16.19 & 17.80 & 19.95 & 25.07 & 28.10 & 36.90 \\
\hline Sample 9 & 1.41 & 2.35 & 3.18 & 4.83 & 5.73 & 6.70 & 8.99 & 12.21 & 18.46 & 74.76 & 103.75 & 121.29 & 158.13 & 192.47 & 275.37 \\
\hline Sample 10 & 1.39 & 2.27 & 3.04 & 4.51 & 5.33 & 6.20 & 8.25 & 11.06 & 16.01 & 53.95 & 101.17 & 121.64 & 161.77 & 197.06 & 282.80 \\
\hline Sample 11 & 1.37 & 2.25 & 3.04 & 4.55 & 5.38 & 6.29 & 8.44 & 11.43 & 17.07 & 72.32 & 105.18 & 123.63 & 161.95 & 196.39 & 281.38 \\
\hline Sample 12 & 1.62 & 2.86 & 4.07 & 6.99 & 8.97 & 11.60 & 20.81 & 37.08 & 53.23 & 69.48 & 78.39 & 88.95 & 119.94 & 151.27 & 226.72 \\
\hline Sample 13 & 1.54 & 2.62 & 3.62 & 5.85 & 7.18 & 8.78 & 13.37 & 22.62 & 46.76 & 78.14 & 92.31 & 106.39 & 137.97 & 167.21 & 241.67 \\
\hline
\end{tabular}

Two different interpolation approaches were used to fill missing entries based on the instrument used to analyze the particular sample. If a Brinkmann PSA 2010 was used, simple linear interpolation was used to fill in missing data values. Such interpolation best represents the process used by the Brinkmann instrument to analyze particle sizes for a slurry sample. If another instrument was used, such as the Horiba or Microtrac X-100, logarithmic interpolation $\left(\log _{10}\right)$ was used to fill in missing data values. Such interpolation best represents the analysis process employed by these instruments. Samples that included only one "measured" or analyzed percentile value were dropped from the PSD generation process because they provided no means to conduct interpolation or extrapolation to fill the missing values in those samples. The final data matrix, with any initially missing values having been filled, is referred to hereafter as the working data matrix. The working data matrices were used to generate PSD summaries for the different waste tanks, waste types, composites, and composite subsets.

As described in Section 3.2.5.2, PSD measurements were performed with different instrumentation configurations, and the PSDS are classified as either sludge or saltcake. Separate PSDs were generated based on these different PSD type classifications (Table 3.17 and Table 3.18).

With the exception of those PSDs identified by the M12 Group in Table 3.17 and Table 3.18, the data obtained from sample analyses only included "measured" values up to the $99^{\text {th }}$ percentile, P99. This can be seen in the example data from AZ-102 shown above. P100 values were provided for the M12 Group data, and P100 values were estimated for non-M12 samples. The P100 particle sizes for non-M12 samples were estimated as a separate step in the PSD generation process, not as part of the initial data preparation.

\section{Methods}

As previously stated, the generated PSDs provide estimates of "typical” particle sizes as well as lower limits and ULs on particle sizes for specific percentiles of interest. The "typical” particle sizes reported can be thought of as "average" particle sizes, although they were not obtained by averaging as in the 
calculation of a mean. They are considered as reasonable point estimates, providing a central estimate of particle size for a given percentile over relevant samples. Different approaches were used to obtain estimates of "typical" particle sizes than were used to estimate lower and ULs on particle sizes. In addition, different approaches were used to estimate P100 values than were used to estimate non-P100 values. Thus, four different estimation approaches were used to generate PSDs for this report: 1) “typical” particle sizes for non-P100 percentiles, 2) “typical” particle sizes for P100, 3) lower and ULs for non-P100 percentiles, and 4) lower and ULs for P100. The estimation approaches are described subsequently.

Additional steps were taken when generating PSDs for a particular waste type or composite compared to the simpler process involved when generating PSDs for individual waste tanks. The additional steps involved either one or both of the following:

- accounting for volume differences among the tanks included in the waste types or composites

- combining data from M12 samples and non-M12 samples for a given waste type or composite.

\section{Estimating "Typical” Particle Sizes for Non-P100 Percentiles}

Two methods were used to produce estimates of "typical” particle sizes corresponding to the non-P100 percentiles of interest. The first method involved forming a combined distribution over all samples for a particular waste tank, waste type, or composite. The second method involved determining the median value for each percentile of interest over the particle sizes (from analysis) contained in the working data matrix.

The combined distribution for a particular waste tank, waste type, or composite is formed using the working data matrix. For waste types, or composites that involve samples from multiple tanks, volume weights are also used in forming the combined distribution. The volume weights reflect the relative UDS volume of the waste tanks included in the particular waste type or composite and therefore sum to 1.0 or are normalized to sum to 1.0 over the tanks included in the waste type or composite. If the working data matrix contains data for a single waste tank, the volume weight for that tank is 1.0. The main steps taken to form the combined distribution are described below.

- The probability associated with each particle size is determined. Sizes corresponding to the first percentile (P01) from each sample have an incremental probability of 0.01 . The associated probability for P01 sizes is 0.01 (the incremental probability) multiplied by the volume weight for the tank from which the sample was taken and divided by the number of samples represented in the working data matrix. For the sizes corresponding to other percentiles (P05 through P99 for non-M12 sample, or P05 through P100 for M12 samples), the incremental probability is the difference between the proportion represented by the corresponding percentile and the proportion represented by the preceding percentile reported. Thus, the incremental probability for P75 is 0.05 because the preceding percentile reported is P70, so $0.75-0.70=0.05$. The associated probability for P75 is the incremental probability 0.05 multiplied by the volume weight for the tank from which the sample was taken and divided by the number of samples represented in the working data matrix. For size values that initially appear more than once in the combined distribution, only a single listing of the value is retained, and the associated probability for that size is the sum of the incremental probabilities associated with the initial listings. 
- The sizes listed in the working data matrix (representing all relevant samples) are ordered from smallest to largest.

- The cumulative probability associated with each size is determined. This is done by summing on the associated probabilities of the ordered sizes.

For cases involving only non-M12 samples, the combined distribution as initially formed only reflects a cumulative probability of 0.99 because the relevant samples only include "measured" or analyzed results for percentiles up to P99. The combined distribution is then used to estimate a "typical" P100 value for such cases. That estimation process is discussed in the next section. For cases involving only M12 samples, the combined distribution incorporates the "measured" or analyzed P100 values from the relevant samples. Thus, for such cases, the combined distribution reflects a cumulative probability of 1.0. For cases involving both non-M12 and M12 samples, formulating the combined distribution requires some additional steps. These additional steps are described below.

- A preliminary portion of the combined distribution is formed using the non-M12 samples. This preliminary portion is used to produce an estimate of P100 (discussed in the next section) representing the non-M12 samples. The P100 estimate is added to the preliminary portion. With this estimate of P100, the cumulative probability accounted for by the preliminary portion equals the sum of the volume weights for the non-M12 tanks represented.

- A secondary portion of the combined distribution is formed using the M12 samples. The cumulative probability accounted for by the secondary portion equals the sum of the volume weights for the M12 tanks represented.

- The preliminary and secondary portions are joined, and the sizes are re-ordered from smallest to largest. With the sizes re-ordered, the associated probabilities are summed to obtain cumulative probabilities, thereby completing the combined distribution.

Examples illustrating the calculations conducted to determine the probabilities associated with sizes included in a combined distribution are provided in Appendix F.

\section{Estimating "Typical” Particle Sizes for P100}

Like the methods described above for estimating "typical” particle sizes for non-P100 percentiles, the methods used to estimate "typical" particle sizes for P100 involved combined distributions as well as medians from the working data matrix.

When generating PSDs for waste types involving only M12 tanks, the methods used to estimate “typical” P100 values were quite simple. In such cases, the "typical” P100 estimate obtained from the combined distribution was simply the largest P100 value from the analyzed samples, which is the final entry in the combined distribution, and the "typical" P100 estimate obtained using medians was just the median over the P100 values from the analyzed samples.

When generating PSDs for cases involving any non-M12 tanks (so that "measured” or analyzed P100 values were not available for all samples), the process of estimating "typical” particle sizes for P100 was more complicated. In such cases, a probability distribution was sought that would accurately model the upper tail of a PSD. Candidate distributions used for similar purposes in the literature were the Weibull distribution, the log-normal distribution, and the Gumbel distribution. As discussed below, the Gumbel 
distribution was found to be the best choice for this study. Therefore, a method involving the Gumbel distribution was used to estimate "typical” P100 values for samples without reported P100 values Dierick et al. 2000).

A Gumbel distribution with location parameter $\mu$ and scale parameter $\beta$ is represented by the relatively simple cumulative distribution function

$$
F(x)=\exp \left(-\exp \left(\frac{-(x-\mu)}{\beta}\right)\right) \text { for any } x
$$

where $F$ denotes the cumulative percentile of the distribution up to a specified value $x$. For this current work, $x$ represents particle sizes, and values of $F$ are the corresponding cumulative percentiles. This cumulative distribution function can be converted to a linear equation in $x$ by applying the natural logarithm twice, then solving for $x$ :

$$
\begin{aligned}
& \ln (-\ln (F))=\frac{-(x-\mu)}{\beta} \\
& x=\mu-\beta \ln (-\ln (F))
\end{aligned}
$$

Note, however, that no finite value of $x$ results in a cumulative percentile of $F=1$. Still, an estimated size (an $x$ value) representing P100 can be obtained using an approximation of 1.0 for $F$ in the equation above. To do this, estimates of $\mu$ and $\beta$ must also be determined. Least-squares regression can be used to estimate $\mu$ and $\beta$ (as illustrated in Kinnison 1985).

Because the M12 data include “measured” P100 values, they provided "truth" data to compare different methods for estimating P100. Accordingly, several estimation methods were assessed by applying the methods to the reported percentile values, up to and including P99, from the M12 data to obtain corresponding estimates of P100. The estimated P100 values were then compared to the reported P100 values from the M12 samples. This exercise compared estimation methods based on the Weibull and log-normal distributions as well as several approaches involving the Gumbel distribution: the least-squares regression approach, a method of moments approach, and a graphical approach (Kinnison 1985). Based on these comparisons, it was determined that the Gumbel distribution, using the least-squares regression fit to the upper-tail percentiles from the reported data to estimate $\mu$ and $\beta$, generally provided the best estimates of P100 for these data. The exercise also supported the use of 6 to 8 data points for the regressions and the use of 0.999 as an approximation for 1.0 when calculating P100 with the regression equations.

The method involving medians to estimate "typical” particle sizes for P100 was conducted as follows. For samples from non-M12 tanks, the Gumbel distribution and least-squares regression were used to determine P100 estimates for each sample separately. Samples from M12 tanks included analyzed P100 values as part of the sample data. The median over the estimated and analyzed P100 values for the various samples included in the waste tank, waste type, composite, or composite subset was then determined and used in the PSD summary as an estimated "typical” particle size for P100. Thus, this method did not involve the combined distribution for the waste tank, waste type, composite, or composite 
subset. An example to illustrate the two methods used to obtain "typical” P100 estimates is provided in Appendix F.

\section{Estimating Lower and Upper Limits on Particle Sizes for Non-P100 Percentiles}

For a given percentile, the minimum particle size over all samples for a particular waste tank, waste type, or composite was used as an estimated lower limit for particle sizes associated with that percentile. Similarly, for a given percentile, the maximum particle size over all samples for a particular waste tank, waste type, or composite was used as an estimated UL for particle sizes associated with that percentile.

In Appendix F, an example is again used to illustrate how minimum and maximum values are used as lower and ULs for the non-P100 percentiles of interest. Note that these minimum and maximum values are not confidence bounds on particle sizes for the specified percentiles. The reasons that these minimum and maximum values cannot be viewed as confidence bounds are explained in Appendix G. Still, they do indicate how diverse the results were from the analyzed samples for this example.

\section{Estimating Lower and Upper Limits on Particle Sizes for P100}

For waste types involving only M12 tanks, “measured” or analyzed P100 values were available for each sample. For such cases, the lower and UL estimates on P100 were determined by taking the minimum and maximum of the analyzed P100 values, respectively. For cases involving only non-M12 tanks, the lower and UL estimates on P100 were determined by taking the minimum and maximum of the P100 estimates obtained by applying the Gumbel distribution and least-squares regression to each of the available samples for the particular waste tank, waste type, composite, or composite subset. For cases involving both M12 and non-M12 tanks, the lower and UL estimates on P100 were determined by taking the minimum and maximum of the set consisting of the minimum and maximum analyzed P100 values for the M12 samples and the minimum and maximum P100 estimates for the non-M12 samples.

\section{PSD Summaries}

Combining the estimates of "typical" particle sizes for the percentiles of interest with the lower and UL estimates for those percentiles, PSD summaries were generated for the different waste tanks, waste types, and composites. Two PSD summary formats were used, the first based on the percentiles of interest and the second based on certain size bins that represent a logarithmic range in particle size from 0 to $10,000 \mu \mathrm{m}$.

Estimates produced as described in the Methods section are compiled to form the first summary matrix for a given PSD. Table 3.21 shows the final PSD summary (rounded to 2 decimal places), using the first summary format, for the four samples from the AY-101 example of Appendix F. Figure 3.43 illustrates the PSD for AY-101 based on the first summary format. Note that the row labeled "Comb. Dist." lists the estimates of "typical” particle sizes for the different percentiles based on the combined distribution. The Median row lists estimates of "typical" particle sizes based on the percentile medians. The Minimum row lists estimated lower limits on particle sizes for the different percentiles based on the percentile minimums. The Maximum row lists estimated ULs on particle sizes for the different percentiles based on the percentile maximums. 
Table 3.21. PSD Summary for AY-101 (values are particle sizes in microns)

\begin{tabular}{|c|c|c|c|c|c|c|c|c|c|c|c|c|c|c|c|c|}
\hline Perce & 0.01 & 0.05 & 0.10 & 0.20 & 0.25 & 0.30 & 0.40 & 0.50 & 0.60 & 0.70 & 0.75 & 0.80 & 0.90 & 0.95 & 0.99 & 1.00 \\
\hline Minimum & 0.18 & 0.28 & 0.42 & 0.78 & 0.97 & 1.17 & 1.81 & 3.65 & 5.02 & 6.15 & 6.80 & 7.77 & 10.15 & 11.60 & 16.10 & 22.84 \\
\hline $\begin{array}{l}\text { Comb. } \\
\text { Dist. }\end{array}$ & 27 & 0.50 & 0.77 & 1.28 & 1.70 & 2.12 & 3.65 & 5.46 & 6.61 & 7.84 & 8.47 & 9.90 & 12.41 & 14.60 & 19.90 & 28.01 \\
\hline Maximum & 30 & 61 & 0.86 & 1.70 & 2.40 & 2.83 & 3.95 & 5.50 & 6.78 & 7.94 & 8.70 & 9.90 & 12.83 & 14.60 & 19.90 & 28.07 \\
\hline Median & 0.24 & 0.45 & 0.65 & 1.16 & 1.50 & 1.81 & 3.00 & 4.78 & 6.17 & 7.51 & 8.14 & 8.83 & 10.84 & 12.86 & 18.09 & 25.10 \\
\hline
\end{tabular}

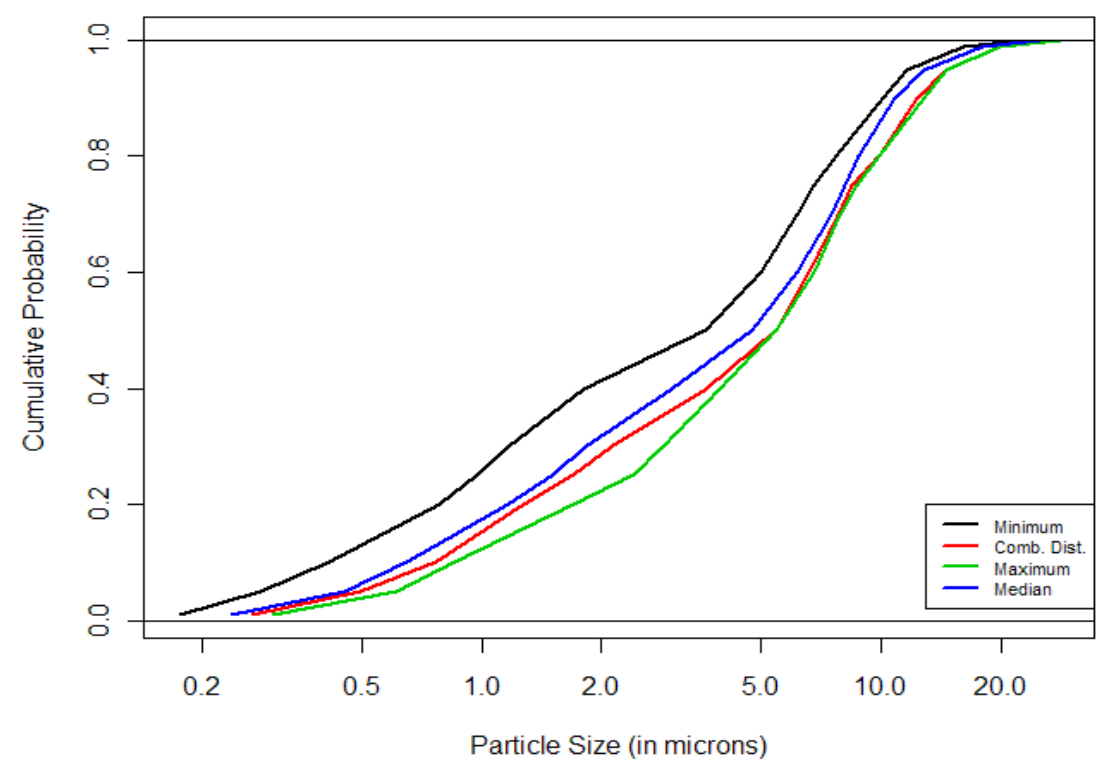

Figure 3.43. Graphical Representation of PSD Summary for AY-101

As mentioned, the second PSD summary format is based on certain size bins. Fifty-five bins are defined by specific boundaries ranging from 0 to $10,000 \mu \mathrm{m}$. Rather than cumulative probabilities, this second summary format gives estimated probabilities associated with each of the bins, representing the estimated relative frequency or proportion of particles in a given waste tank, waste type, composite, or composite subset that falls within the designated bin boundaries. Two linear interpolation approaches were used to determine these bin probabilities. For the minimums, maximums, and medians listed in the first PSD summary matrix, interpolation was conducted on the cumulative percentiles presented in the matrix (see Table 3.21) using, in turn, the corresponding minimums, the corresponding maximums, and the corresponding medians reported in the summary matrix. For the estimates of "typical” particle sizes for the percentiles of interest obtained using the combined distribution, the interpolation was conducted on the cumulative percentiles represented in the complete combined distribution, which includes the estimate of "typical” particle size for P100. Using the complete combined distribution to conduct interpolations was preferred because it included a fuller listing of percentile estimates than just the percentiles of interest. This was not the case for the minimums, maximums, and medians that only involved the percentiles of interest, having been determined from the samples represented in the working data matrix. Thus, the second PSD summary format also includes estimates of "typical” particle sizes and lower and UL estimates on particle sizes based on the combined distribution, medians, minimums, and maximums, but reported relative to the 55 designated particle size bins. Table 3.22 shows how the interpolation was conducted using the combined distribution formed from the four AY-101 samples with 
the corresponding estimate of P100 included. Entries shown in red in the table were determined by interpolation.

Table 3.22. Example Interpolations to Form PSD Summary Based on Designated Size Bins for AY-101 (bin boundaries and sizes are in microns)

\begin{tabular}{|c|c||c|c|}
\hline \multicolumn{2}{|c||}{ Bins for PSD Summary } & \multicolumn{2}{c|}{ Combined Distribution } \\
\hline \hline Bin Number & Upper Bin Boundary & Size & Cumulative Probability \\
\hline 1 & 0.01 & & \\
\hline$\ldots$ & & & \\
\hline 11 & 0.129 & & \\
\hline 12 & 0.167 & & \\
\hline & & 0.175 & 0.003 \\
\hline & & 0.213 & 0.005 \\
\hline 13 & 0.215 & & 0.005 \\
\hline & & 0.26 & 0.008 \\
\hline 14 & 0.278 & & 0.017 \\
\hline$\ldots$ & & & \\
\hline 55 & 10000 & & \\
\hline
\end{tabular}

Focusing on just the bins represented in the second summary format, Table 3.23 contains the cumulative probabilities for each of the bins.

Table 3.23. Example Designated Size Bins and Corresponding Cumulative Probabilities for AY-101 (upper bin boundaries are in microns)

\begin{tabular}{|c|c|c|}
\hline Bin Number & Upper Bin Boundary & Cumulative Probability \\
\hline 1 & 0.01 & 0 \\
\hline$\ldots$ & & 0 \\
\hline 11 & 0.129 & 0 \\
\hline 12 & 0.167 & 0.005 \\
\hline 13 & 0.215 & 0.017 \\
\hline 14 & 0.278 & \\
\hline$\ldots$ & & 1 \\
\hline 55 & 10000 & \\
\hline
\end{tabular}

The bin probabilities based on the combined distribution for AY-101 can be derived from Table 3.23 by subtracting adjacent cumulative probabilities to obtain the incremental probabilities associated with each of the bins. These results are given in Table 3.24. 
Table 3.24. Example Designated Size Bins and Corresponding Probabilities for AY-101 (upper bin boundaries are in microns)

\begin{tabular}{|c|c|c|}
\hline Bin Number & Upper Bin Boundary & Probability \\
\hline 1 & 0.01 & 0 \\
\hline$\ldots$ & & \\
\hline 11 & 0.129 & 0 \\
\hline 12 & 0.167 & 0 \\
\hline 13 & 0.215 & 0.005 \\
\hline 14 & 0.278 & 0.012 \\
\hline$\ldots$ & & \\
\hline 55 & 10000 & 0 \\
\hline
\end{tabular}

The interpolations relative to the minimums, maximums, and medians were conducted in a similar fashion but involved only the cumulative probabilities and sizes corresponding to the percentiles of interest. The final PSD summary probabilities using the second summary format for the four samples from AY-101 is given in Table 3.25.

Table 3.25. PSD Summary Based on Designated Size Bins for AY-101 (sizes are in microns; other columns, except bin number, are associated probabilities)

\begin{tabular}{|c|c|c|c|c|c|}
\hline Bin Number & Size & Minimum & Combined Distribution & Maximum & Median \\
\hline 1 & 0.01 & 0 & 0 & 0 & 0 \\
\hline 2 & 0.013 & 0 & 0 & 0 & 0 \\
\hline 3 & 0.017 & 0 & 0 & 0 & 0 \\
\hline 4 & 0.022 & 0 & 0 & 0 & 0 \\
\hline 5 & 0.028 & 0 & 0 & 0 & 0 \\
\hline 6 & 0.036 & 0 & 0 & 0 & 0 \\
\hline 7 & 0.046 & 0 & 0 & 0 & 0 \\
\hline 8 & 0.06 & 0 & 0 & 0 & 0 \\
\hline 9 & 0.077 & 0 & 0 & 0 & 0 \\
\hline 10 & 0.1 & 0 & 0 & 0 & 0 \\
\hline 11 & 0.13 & 0 & 0 & 0 & 0 \\
\hline 12 & 0.17 & 0 & 0 & 0 & 0.018 \\
\hline 13 & 0.22 & 0.026 & 0.005 & 0.018 & 0.015 \\
\hline 14 & 0.28 & 0.024 & 0.012 & 0.014 & 0.020 \\
\hline 15 & 0.36 & 0.030 & 0.015 & 0.017 & 0.035 \\
\hline 16 & 0.46 & 0.034 & 0.015 & 0.034 & 0.037 \\
\hline 17 & 0.6 & 0.037 & 0.026 & 0.034 & 0.044 \\
\hline 18 & 0.77 & 0.047 & 0.032 & 0.035 & 0.051 \\
\hline 19 & 1 & 0.060 & 0.059 & 0.045 & 0.058 \\
\hline 20 & 1.29 & 0.062 & 0.039 & 0.037 & 0.052 \\
\hline 21 & 1.67 & 0.059 & 0.043 & 0.062 & 0.053 \\
\hline 22 & 2.15 & 0.041 & 0.056 & 0.074 & 0.052 \\
\hline 23 & 2.78 & 0.034 & 0.022 & 0.076 & 0.059 \\
\hline 24 & 3.59 & 0.044 & 0.074 & 0.094 & 0.095 \\
\hline 25 & 4.64 & 0.075 & 0.067 & 0.145 & 0.131 \\
\hline 26 & 5.99 & 0.114 & 0.101 & 0.120 & 0.140 \\
\hline 27 & 7.74 & 0.112 & 0.109 & & \\
\hline 28 & 10 & 0.095 & 0.135 & & 0 \\
\hline
\end{tabular}


Table 3.25. (contd)

\begin{tabular}{|c|c|c|c|c|c|}
\hline Bin Number & Size & Minimum & Combined Distribution & Maximum & Median \\
\hline 29 & 12.9 & 0.068 & 0.117 & 0.099 & 0.092 \\
\hline 30 & 16.7 & 0.029 & 0.038 & 0.063 & 0.029 \\
\hline 31 & 21.5 & 0.007 & 0.027 & 0.026 & 0.016 \\
\hline 32 & 27.8 & 0.002 & 0.008 & 0.008 & 0.005 \\
\hline 33 & 35.9 & 0 & 0 & 0 & 0 \\
\hline 34 & 46.4 & 0 & 0 & 0 & 0 \\
\hline 35 & 59.9 & 0 & 0 & 0 & 0 \\
\hline 36 & 77.4 & 0 & 0 & 0 & 0 \\
\hline 37 & 100 & 0 & 0 & 0 & 0 \\
\hline 38 & 129 & 0 & 0 & 0 & 0 \\
\hline 39 & 167 & 0 & 0 & 0 & 0 \\
\hline 40 & 215 & 0 & 0 & 0 & 0 \\
\hline 41 & 278 & 0 & 0 & 0 & 0 \\
\hline 42 & 359 & 0 & 0 & 0 & 0 \\
\hline 43 & 464 & 0 & 0 & 0 & 0 \\
\hline 44 & 599 & 0 & 0 & 0 & 0 \\
\hline 45 & 774 & 0 & 0 & 0 & 0 \\
\hline 46 & 1000 & 0 & 0 & 0 & 0 \\
\hline 47 & 1292 & 0 & 0 & 0 & 0 \\
\hline 48 & 1668 & 0 & 0 & 0 & 0 \\
\hline 49 & 2154 & 0 & 0 & 0 & 0 \\
\hline 50 & 2783 & 0 & 0 & 0 & 0 \\
\hline 51 & 3594 & 0 & 0 & 0 & 0 \\
\hline 52 & 4642 & 0 & 0 & 0 & 0 \\
\hline 53 & 5995 & 0 & 0 & 0 & 0 \\
\hline 54 & 7743 & 0 & 0 & 0 & 0 \\
\hline 55 & 10000 & 0 & 0 & 0 & 0 \\
\hline
\end{tabular}

PSD summaries in both summary formats were generated for the individual waste tanks, waste types, composites, and composite subsets considered in this study.

\subsubsection{PSD Results}

As described in Section 3.2.5.3, PSD summaries were generated for the different waste tanks, waste types, and composites in two formats. The first PSD format is based on the percentiles of interest and is used to present the results. The second PSD format is based on certain size bins that represent a logarithmic range in particle size from 0 to $10,000 \mu \mathrm{m}$. The second PSD format is used for the PSDD modeling of Section 3.3.

As described based on sampling and measurement techniques, the derived PSDs provide a "best representation" of the size of the particulate matter in the tanks, waste types, or composites. The waste types and composite PSDs are, in some sense, "averaged” distributions. As such, and because they are generated from a limited data set, the waste type and composite PSDs should be expected to differ from the PSDs for specific tanks and should not be considered either bounding or conservative.

For each PSD format, the PSDs are provided as the "typical" particle sizes for the different percentiles based on the combined distribution, estimates of "typical” particle sizes based on the 
percentile medians, estimates of the lower limits on particle sizes for the different percentiles based on the percentile minimums, and estimates of the ULs on particle sizes for the different percentiles based on the percentile maximums, e.g., Table 3.21. Tank, waste type, and composite PSDs for the percentiles of interest are provided in Appendix $\mathrm{H}$ for the combined, median, minimum, and maximum distributions.

From Table 3.17, sludge Tank C-107 has multiple PSD data sets for Flowing Sonicated, Flowing Unsonicated, and No-Flow Unsonicated and is thus selected as the PSD result example shown in Figure 3.44, Figure 3.45, and Figure 3.46, respectively. For this example, little differentiation is seen between the minimum and maximum for the Flowing Sonicated and Flowing Unsonicated. Greater than one order of magnitude difference between the minimum and maximum is shown at a given probability for the No-Flow Unsonicated PSDs.

A size increase from Flowing Sonicated, to Flowing Unsonicated, to No-Flow Unsonicated is also shown. For example, the $90^{\text {th }}$ percentile of the combined distribution increases from approximately $10 \mu \mathrm{m}$ to $15 \mu \mathrm{m}$ to $129 \mu \mathrm{m}$, respectively. Aside from potential sample and handling differences, this size increase follows the expectation described in Section 3.2.5.2 that the most energetic PSD measurements with respect to the potential break-up of agglomerates is Flowing Sonicated, followed by Flowing Unsonicated, and the least disturbance is provided by No-Flow Unsonicated. This result is observed even though the No-Flow Unsonicated PSD measurement may bias lighter/smaller particles since heavy/large particles may settle to the bottom of the cell and not be measured (Section 3.2.5.2).

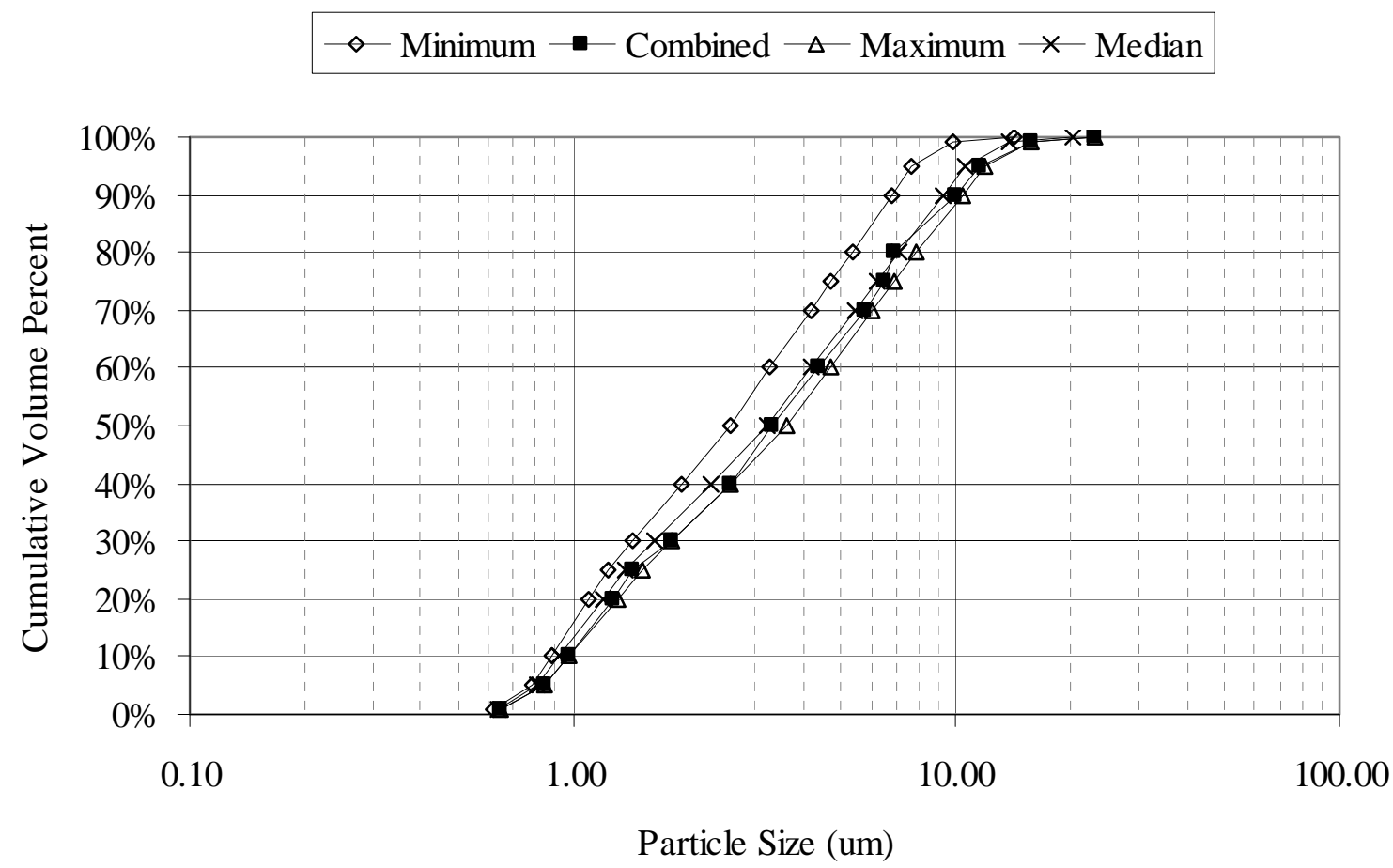

Figure 3.44. PSD Summary for C-107, Sludge, Flowing Sonicated 


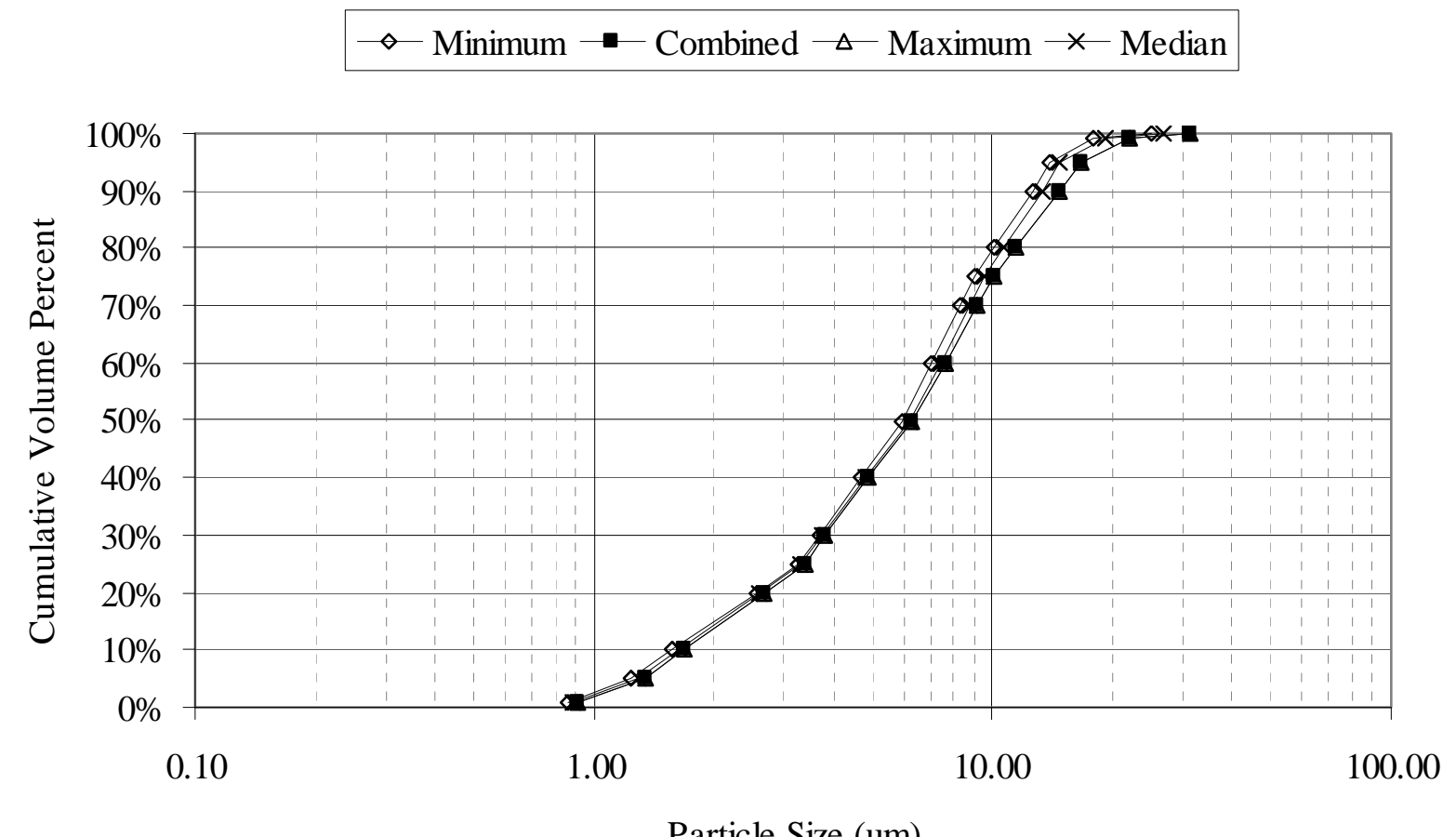

Particle Size (um)

Figure 3.45. PSD Summary for C-107, Sludge, Flowing Unsonicated

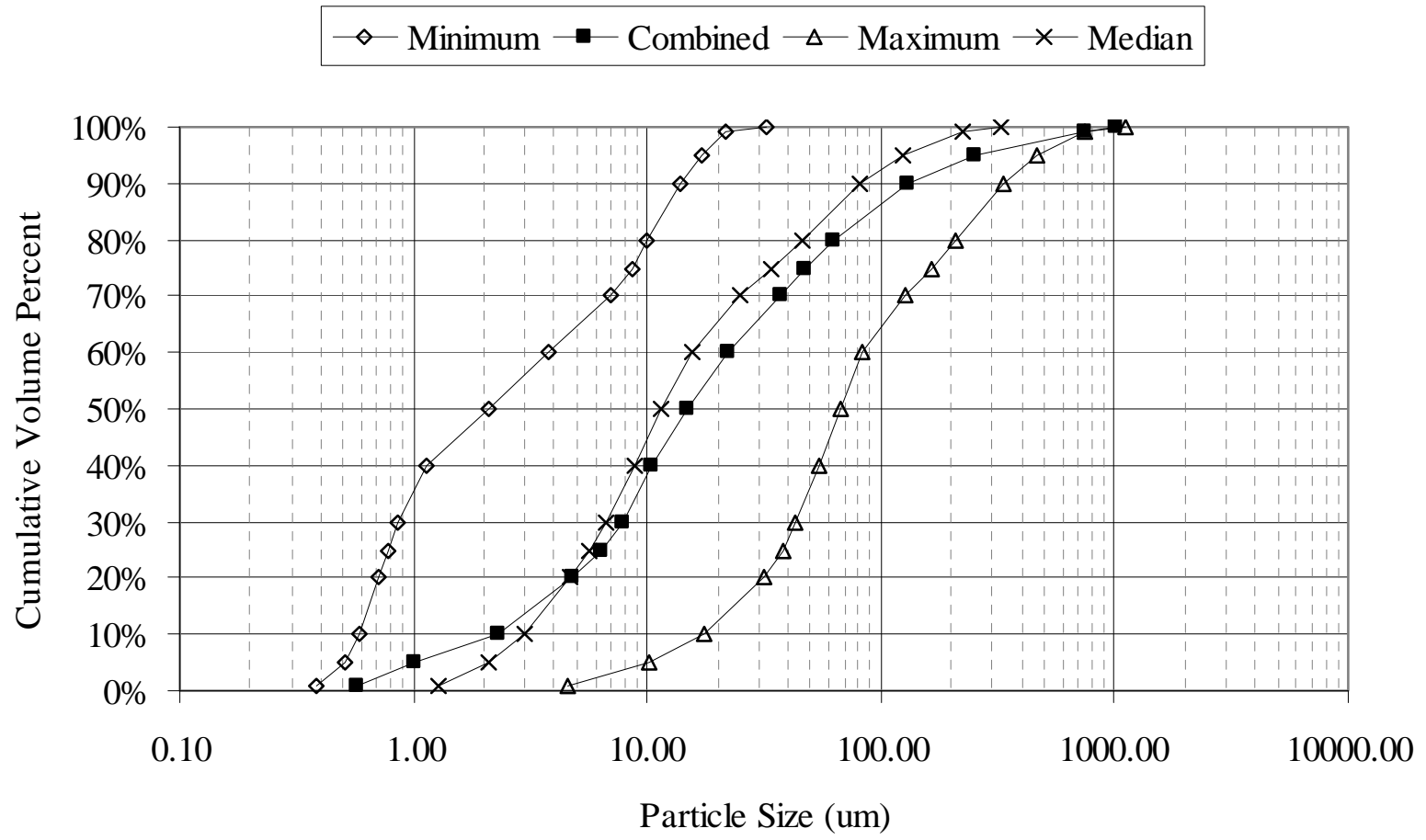

Figure 3.46. PSD Summary for C-107, Sludge, No-Flow Unsonicated 
The tank combined distributions are qualitatively compared in Figure 3.47 through Figure 3.52. The specific PSDs are provided in Appendix $\mathrm{H}$ for the combined, median, minimum, and maximum distributions. For both the sludge and saltcake tanks, the Flowing Sonicated and Flowing Unsonicated PSDs appear to have a more uniform distribution shape than the No-Flow Unsonicated PSDs. As shown in the figures, the $50^{\text {th }}$ percentile particle sizes for the Flowing Sonicated and Flowing Unsonicated PSDs are more tightly grouped (less than one order of magnitude difference) than those for the No-Flow Unsonicated PSDs (almost 2 orders of magnitude difference).

For sludge, AZ-102 has the largest PSD for the Flowing Sonicated and Flowing Unsonicated PSD, while AZ-101 is at or near to the smallest. It is interesting to note that these two tanks comprise $100 \%$ of the P3 waste type UDS volume. For the Sludge, No-Flow Unsonicated PSDs, C-104 has the largest PSD while C-103 has the smallest. Again, the largest and smallest PSDs are from the same waste type, both of which have a primary waste type of CWP1 (Section 3.1).

Tanks AN-102 and BY-104 are shown to have the largest PSDs for Saltcake, Flowing Sonicated, and Flowing Unsonicated PSDs, with AN-107 and AN-104 the smallest, respectively. The largest PSD for Saltcake, No-Flow Unsonicated is AW-101, and the smallest are S-104 and AN-104. Thus, there is some consistency across the PSD types for concurrent tanks. However, as with the sludge, tanks from the same waste type, A2, are shown to span the PSDs. 


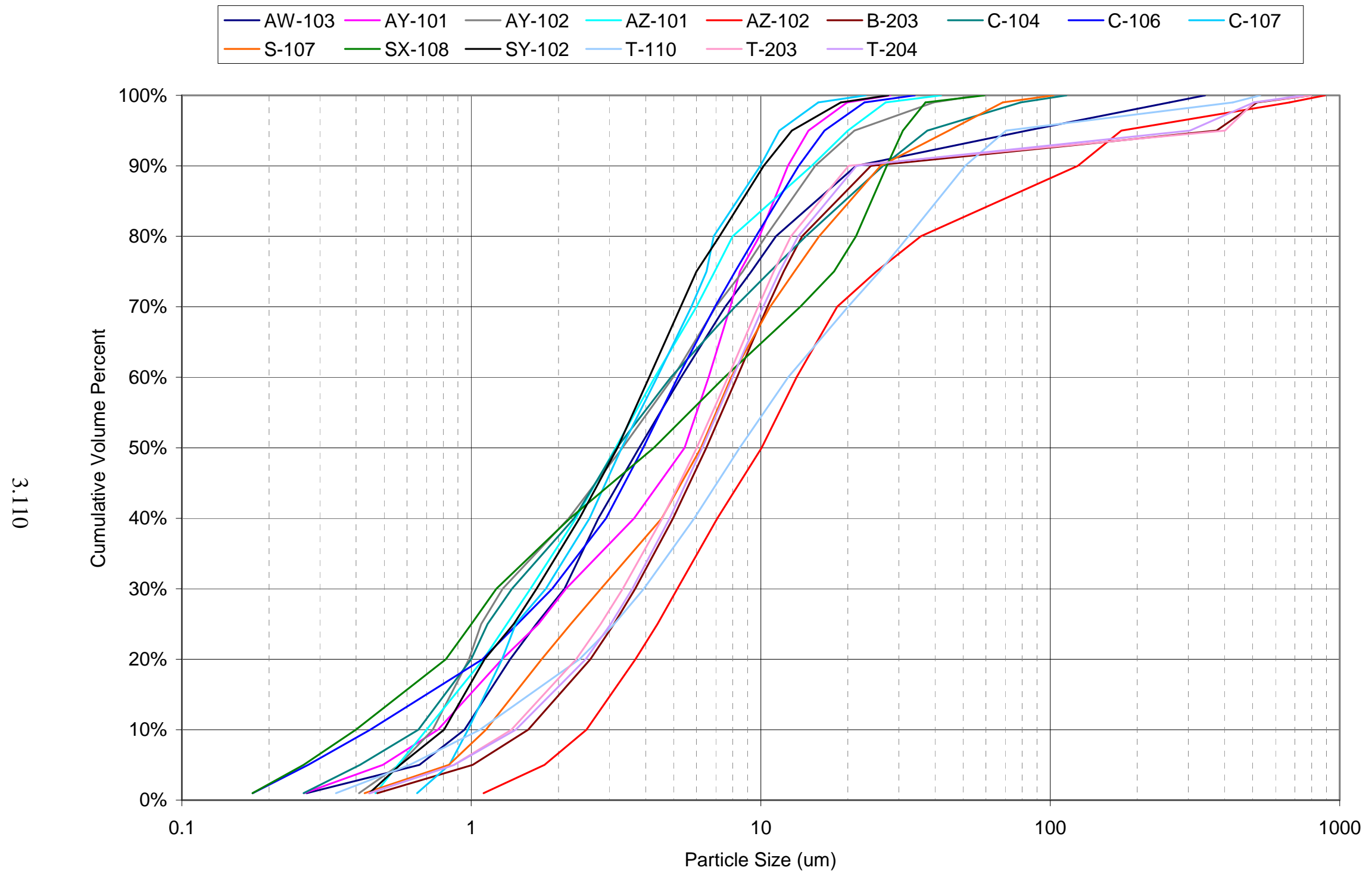

Figure 3.47. Tank Combined PSD Summary, Sludge, Flowing Sonicated 


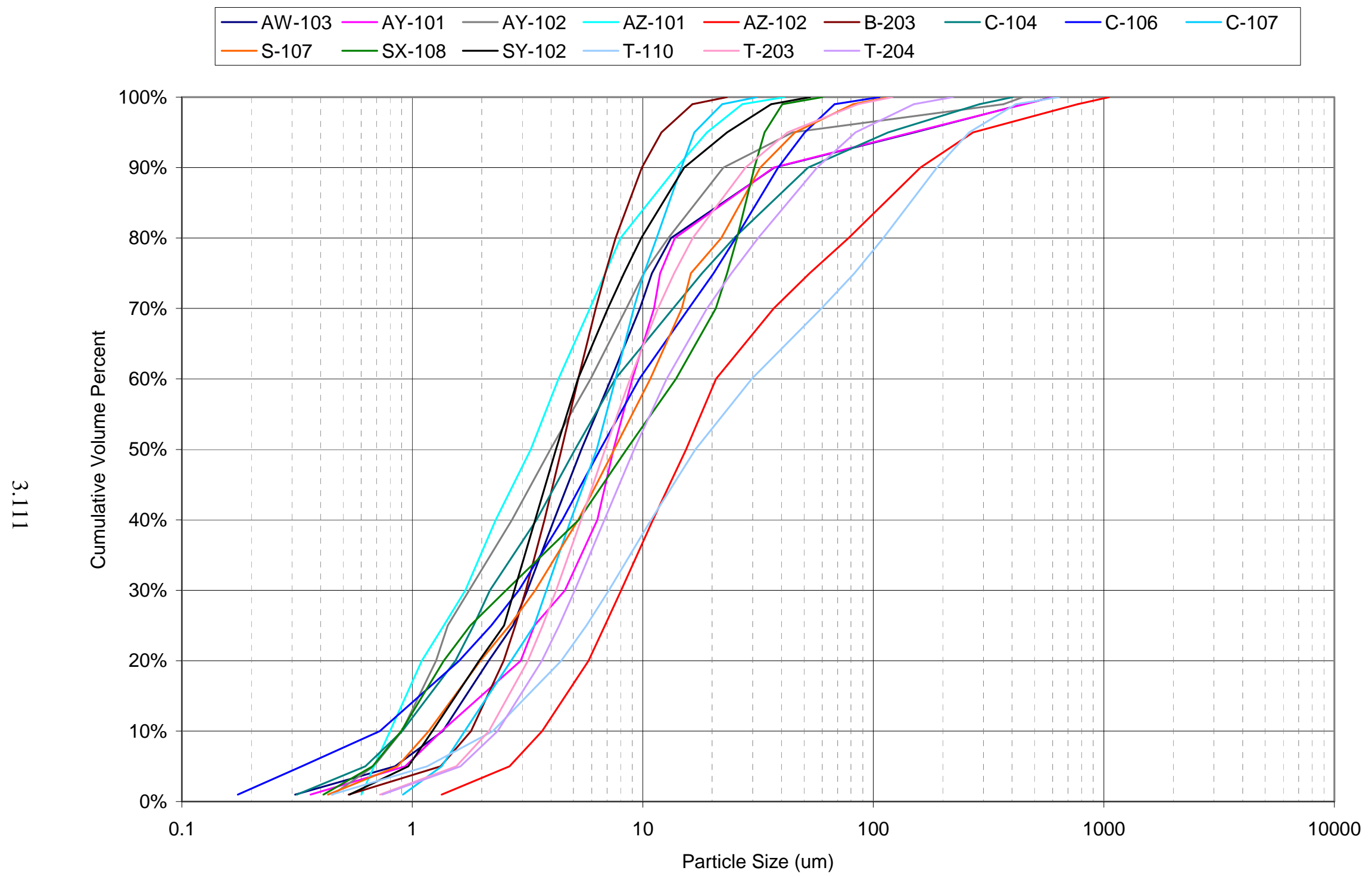

Figure 3.48. Tank Combined PSD Summary, Sludge, Flowing Unsonicated 


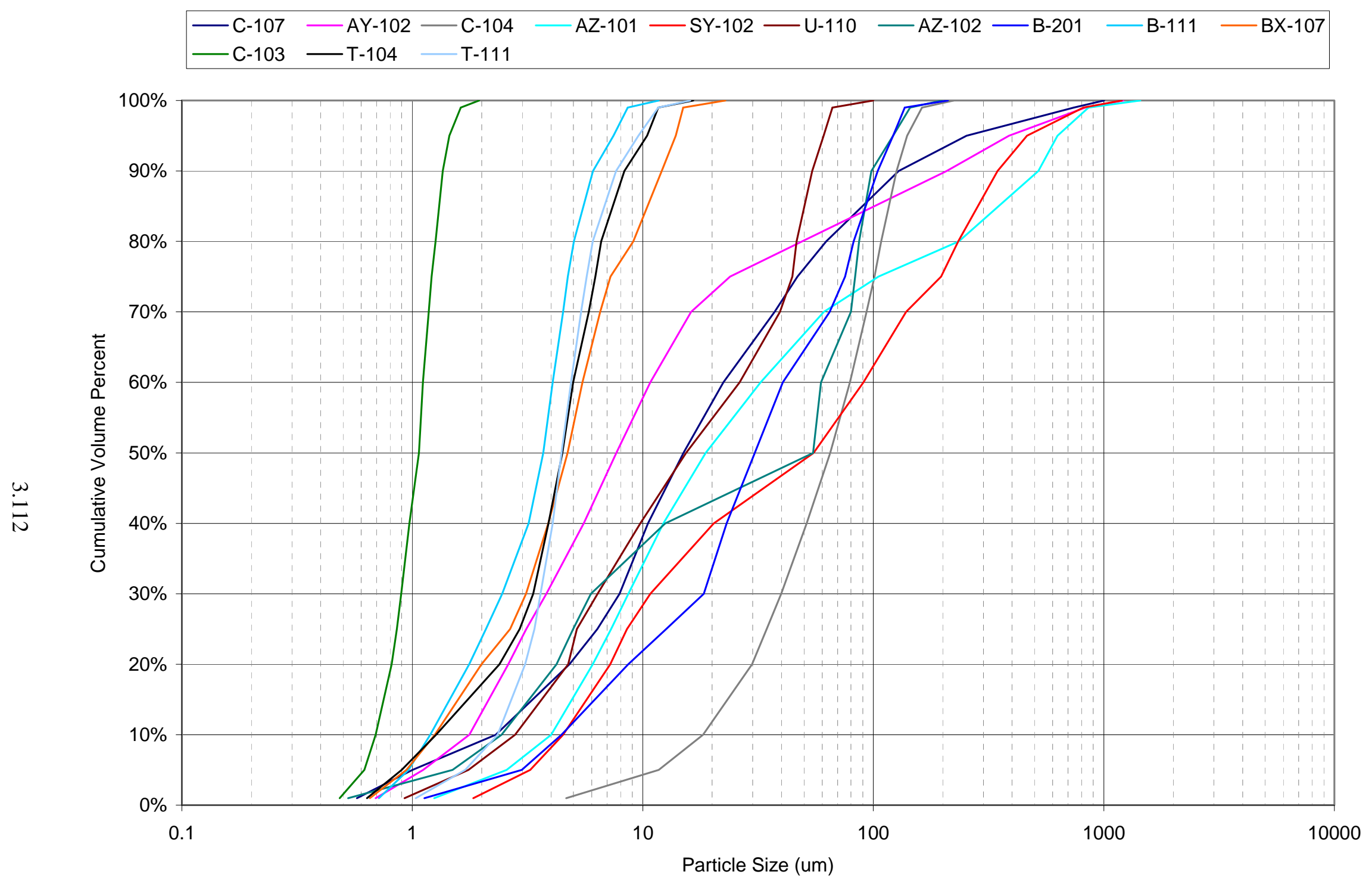

Figure 3.49. Tank Combined PSD Summary, Sludge, No-Flow Unsonicated 


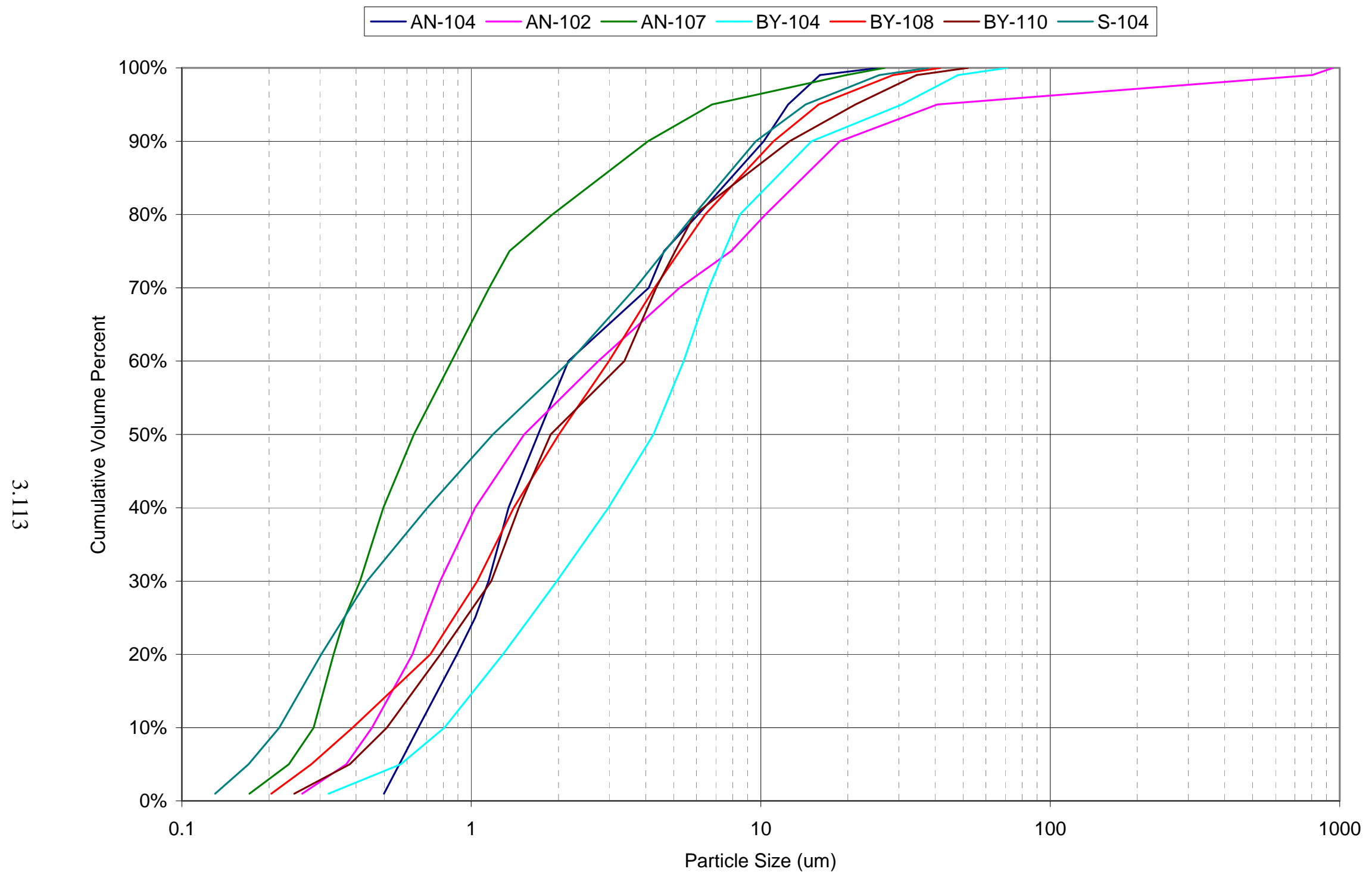

Figure 3.50. Tank Combined PSD Summary, Saltcake, Flowing Sonicated 


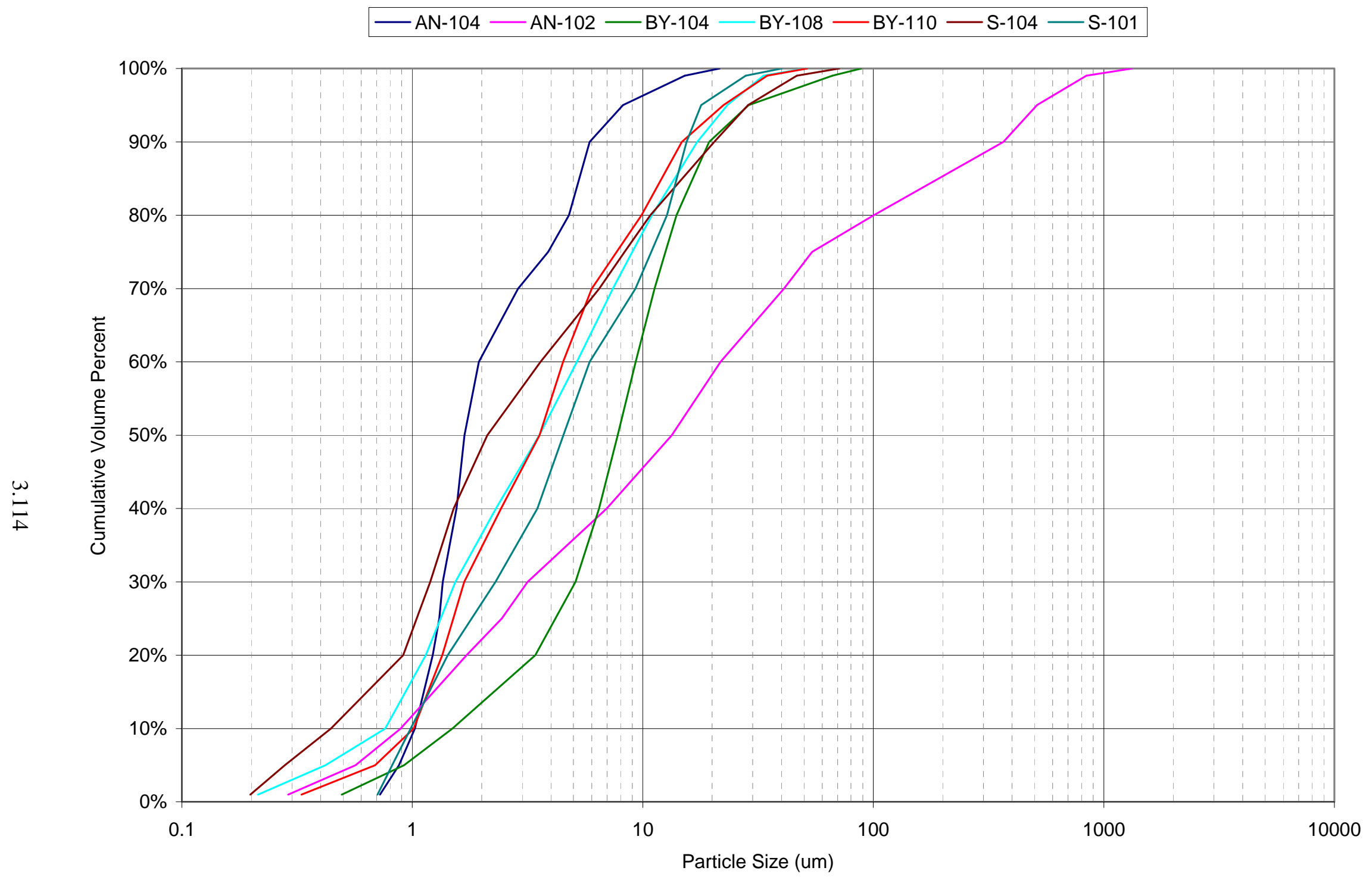

Figure 3.51. Tank Combined PSD Summary, Saltcake, Flowing Unsonicated 


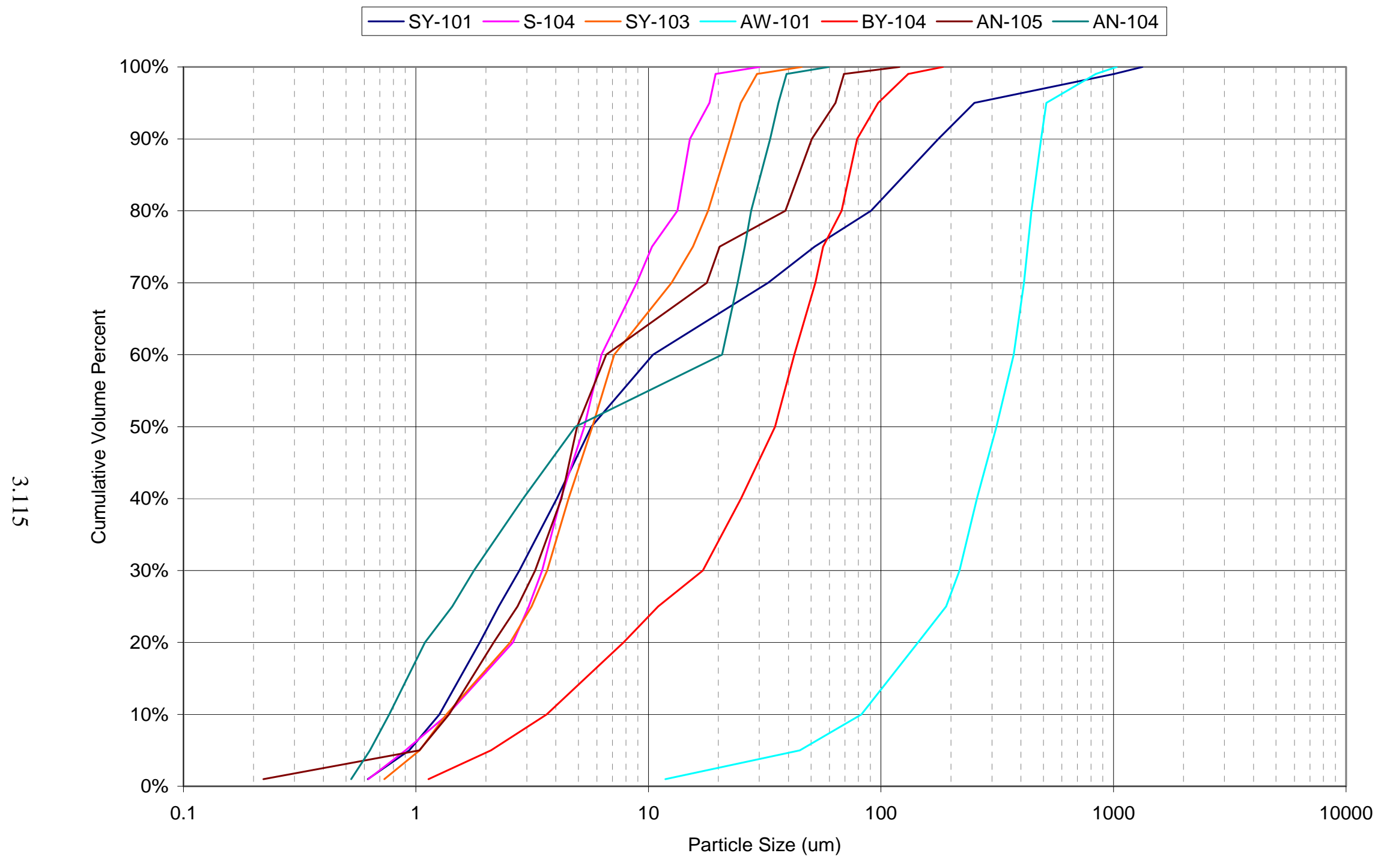

Figure 3.52. Tank Combined PSD Summary, Saltcake, No-Flow Unsonicated 
The waste type combined distributions are compared in Figure 3.53 through Figure 3.58. As with the tank PSDs, the Flowing Sonicated and Flowing Unsonicated PSDs appear to have a more uniform distribution shape than the No-Flow Unsonicated PSDs for both the sludge and saltcake waste types. The $50^{\text {th }}$ percentile particle sizes for the Flowing Sonicated and Flowing Unsonicated PSDs are more tightly grouped than those for the No-Flow Unsonicated PSDs.

Although dependent on the cumulative probability, 2C sludge generally has the largest PSDs for Flowing Sonicated and Flowing Unsonicated PSD types. BL sludge has the smallest. The 2C sludge is shown to have the smallest No-Flow Unsonicated PSD, with P3 as the biggest. For the limited data set, the largest and smallest saltcake waste type PSDs are dependent on the PSD type. 


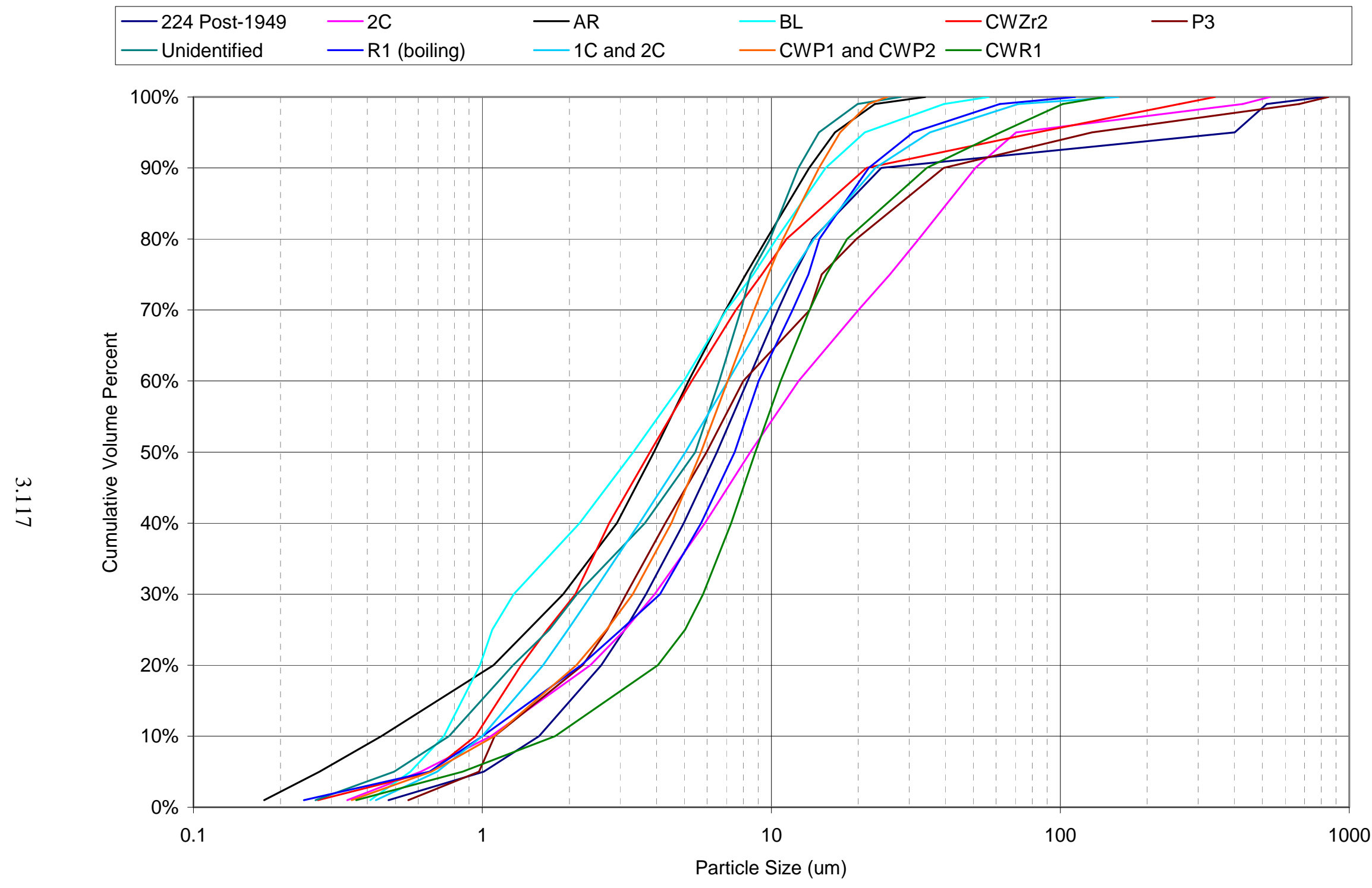

Figure 3.53. Waste Type Combined PSD Summary, Sludge, Flowing Sonicated 


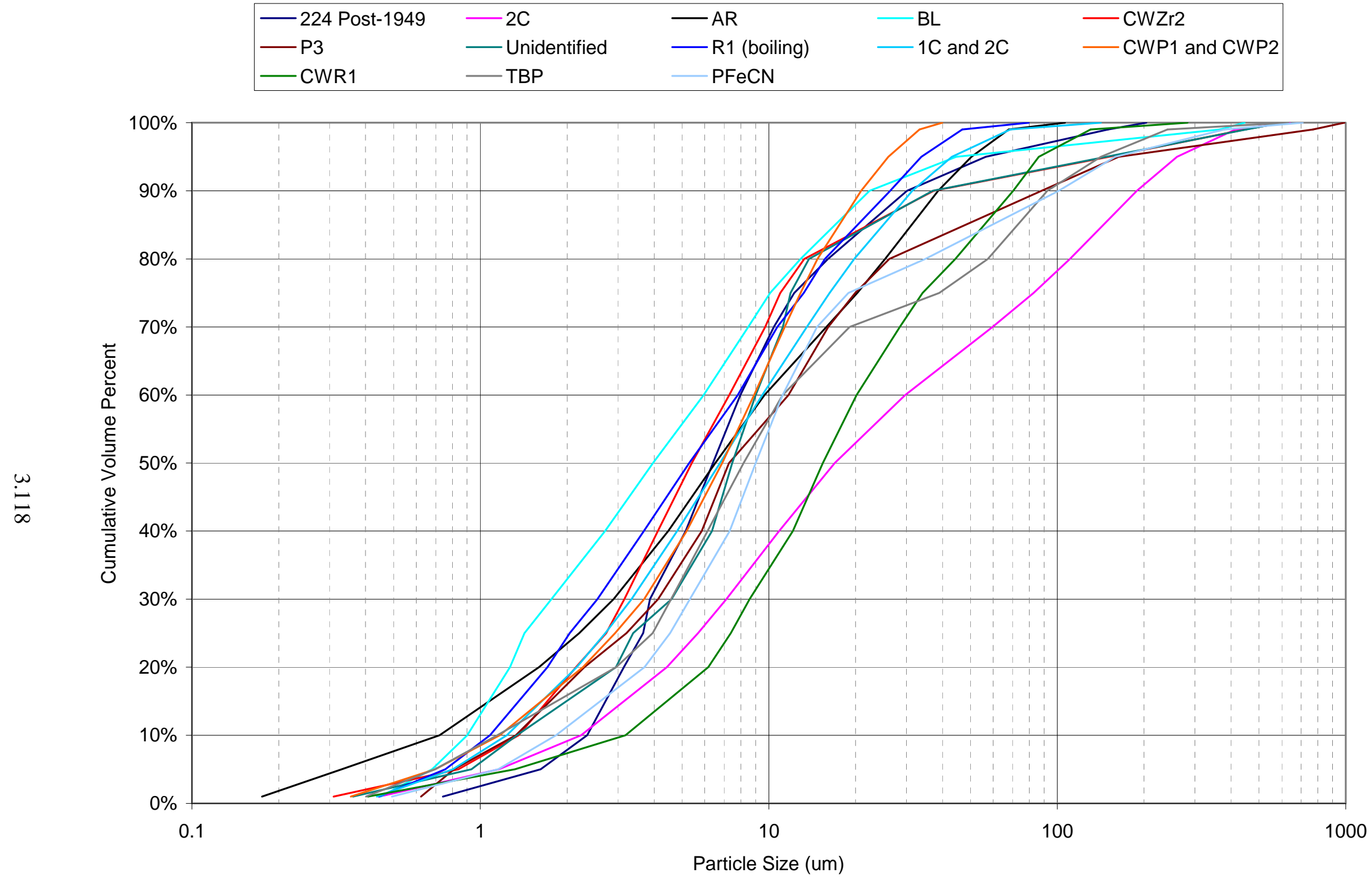

Figure 3.54. Waste Type Combined PSD Summary, Sludge, Flowing Unsonicated 


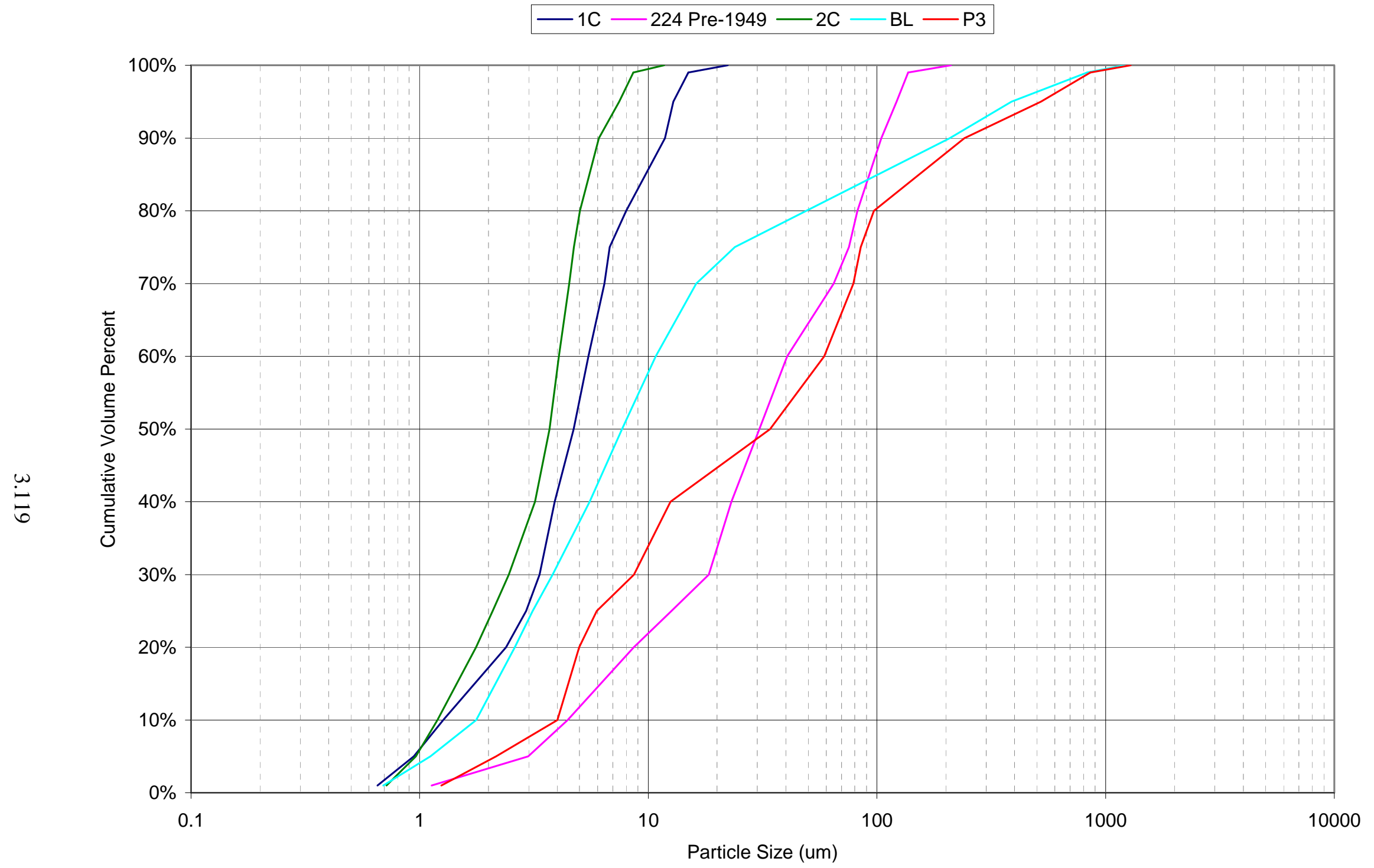

Figure 3.55. Waste Type Combined PSD Summary, Sludge, No-Flow Unsonicated 


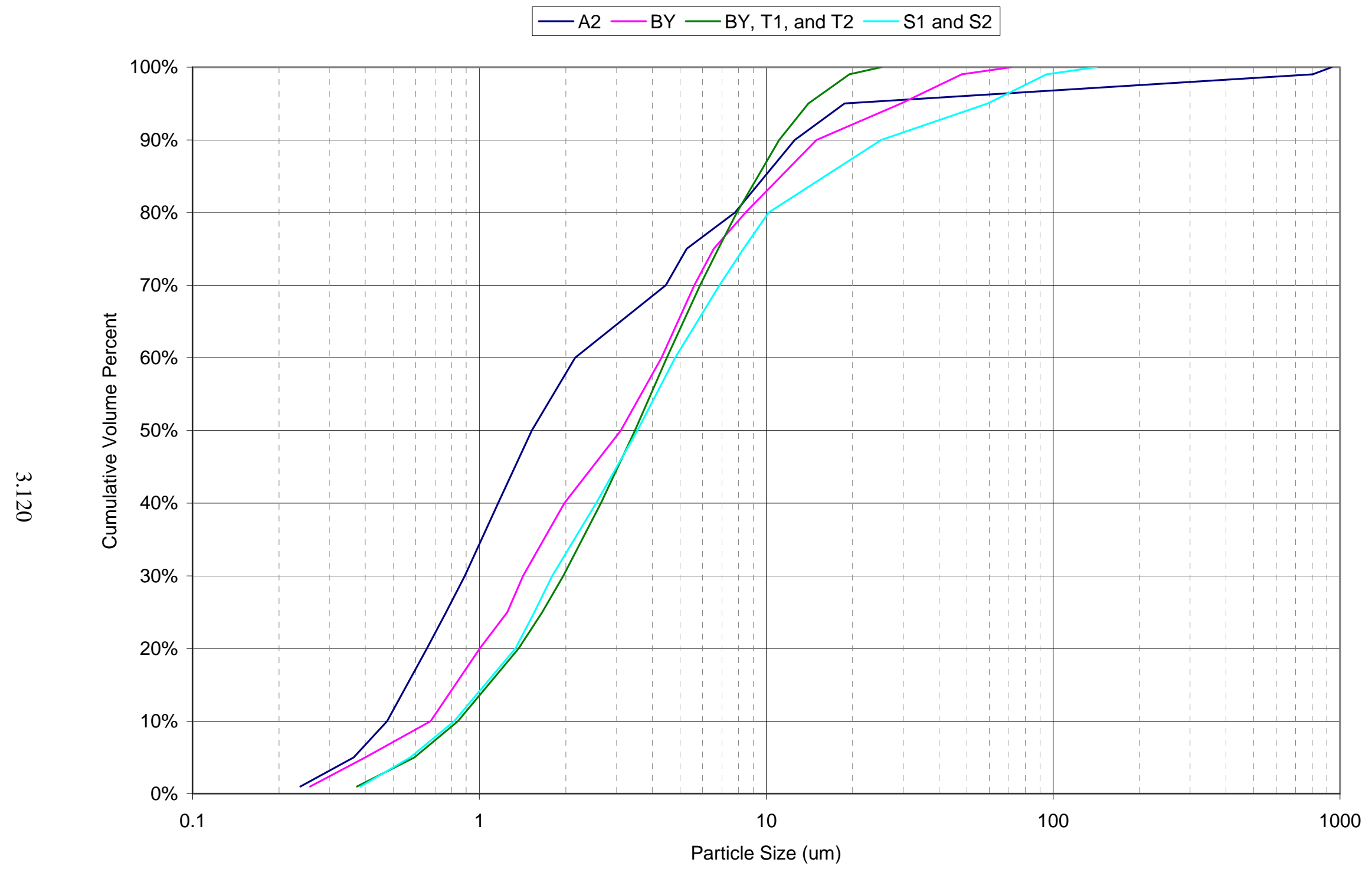

Figure 3.56. Waste Type Combined PSD Summary, Saltcake, Flowing Sonicated 


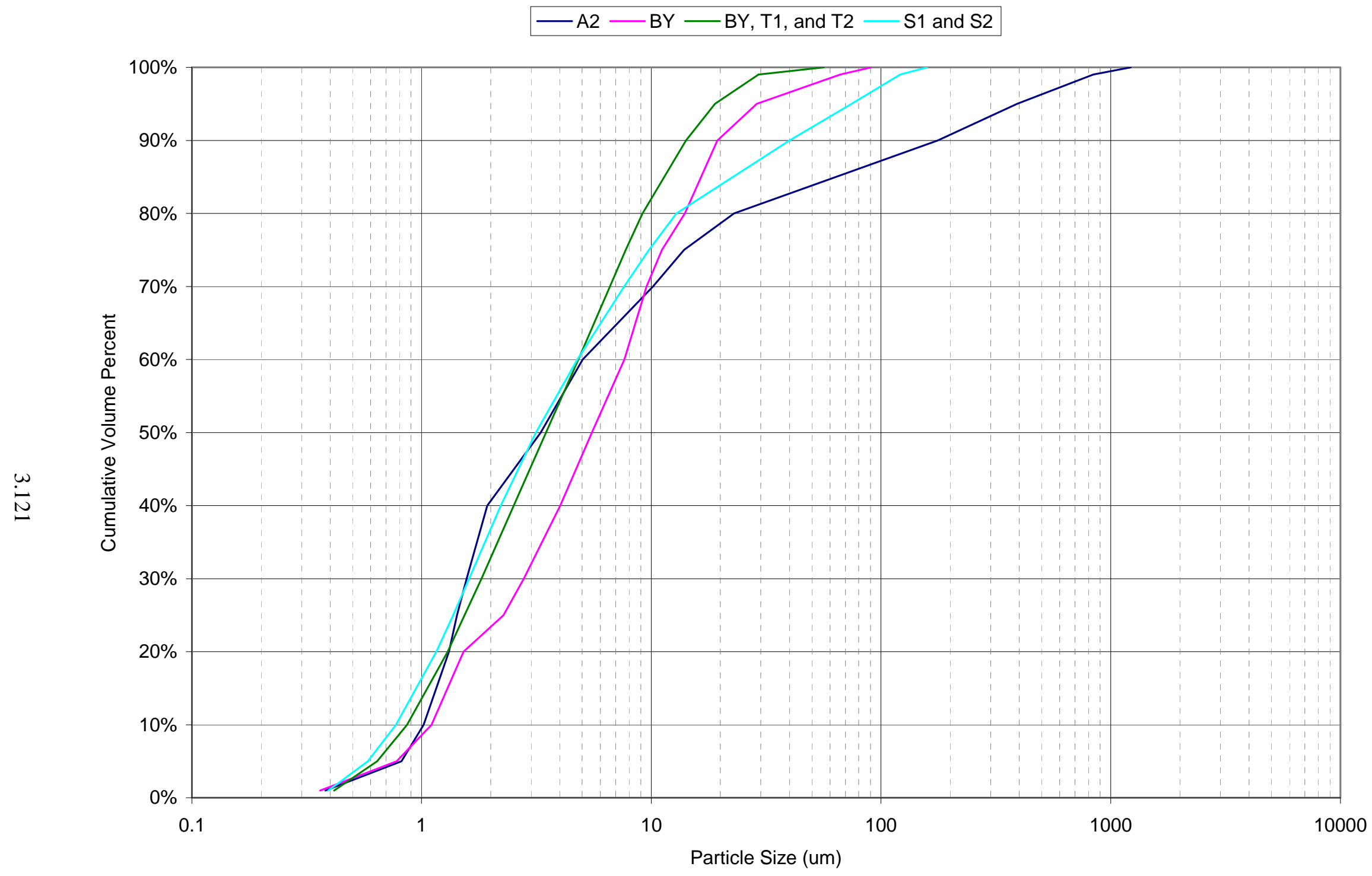

Figure 3.57. Waste Type Combined PSD Summary, Saltcake, Flowing Unsonicated 


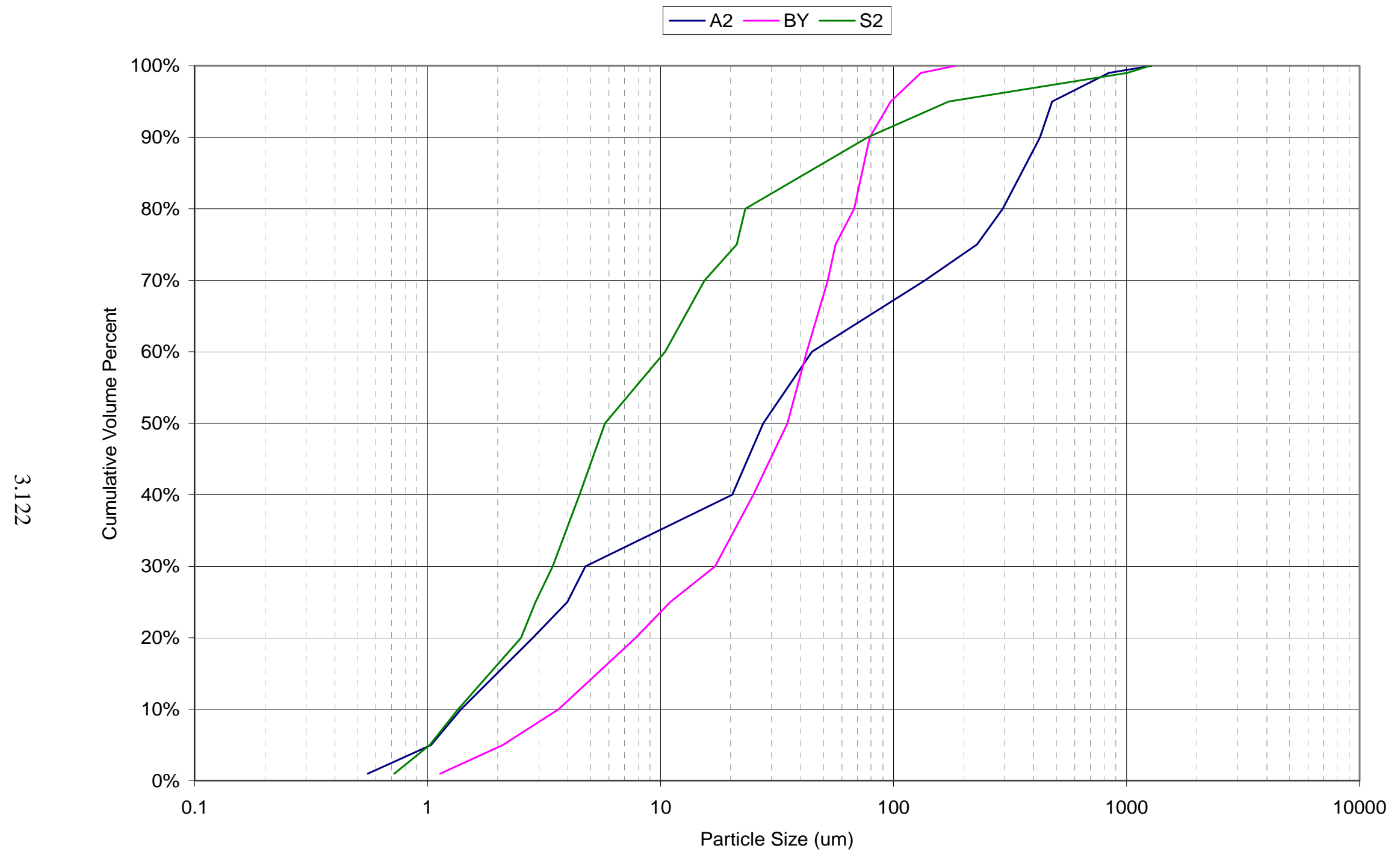

Figure 3.58. Waste Type Combined PSD Summary, Saltcake, No-Flow Unsonicated 
The composite combined PSDs are shown in Figure 3.59 and Figure 3.60. The Sludge, Flowing Sonicated and Sludge, Flowing Unsonicated composite PSDs are most comparable to the PSDs provided by Wells et al. (2007). In comparing the respective PSDs to those of Wells et al. (2007), there are changes in the PSD data set as described in Sections 3.2.5.1 and 3.2.5.2 as well as changes in the PSD generation method, described in Section 3.2.5.3. As shown in Table 3.26 and Figure 3.61, these changes resulted in minor differences in the PSDs.

Also included in Figure 3.61 is the mean PSD from Jewett et al. (2002). This mean PSD is based on the combination of PSDs from seven HLW tanks, and is most comparable to the composite Sludge, Flowing Unsonicated PSD from the current work. The seven tanks from Jewett et al. (2002) are included in the 15 tanks that are used together with the M12 Group data for the composite Sludge, Flowing Unsonicated PSD, however, the input PSDs for the initial seven tanks are different due to the data selection process. For the current work, the researcher that performed the sample analyses selected different sets of PSD results that most closely meet the Flowing Unsonicated condition and provide a representative measurement of the sample.

Note that the combination methodology employed to average particle sizes for the mean PSD of Jewett et al. (2002) did not adjust for the fact that particle diameters behave as the cube root of the volume of the particle. Thus, averaging multiple estimates of the particle size associated with the $99^{\text {th }}$ percentile by volume, for example, does not yield the same result as the single value obtained when the PSDs are combined using a volume weighting. In general, values calculated as the average of the individual percentiles (as in Jewett et al. 2002) will tend to overestimate the same percentile value from the combined volume-weighted PSDs. The magnitude of the difference between the approaches depends on the variability in the individual PSDs. In the current work, the use of a combined volume-weighted approach along with a selection of different PSD data results in a smaller PSD than reported in Jewett et. al. (2002).

A size increase from Flowing Sonicated, to Flowing Unsonicated, to No-Flow Unsonicated is shown. Again, this size increase follows the expectations described in Section 3.2.5.2 that the most energetic PSD measurements with respect to the potential break-up of agglomerates is Flowing Sonicated, followed by Flowing Unsonicated, and the least disturbance is provided by No-Flow Unsonicated. As discussed previously, this is evidenced even though the No-Flow Unsonicated PSD measurement may bias lighter/smaller particles because heavy/large particles may settle to the bottom of the cell and not be measured (Section 3.2.5.2).

With the exception of the No-Flow Unsonicated PSD, the saltcake is shown to have smaller PSDs than the sludge. This result is counter-intuitive based on the relative soluble and insoluble primary particle sizes provided in Section 3.2.4. 


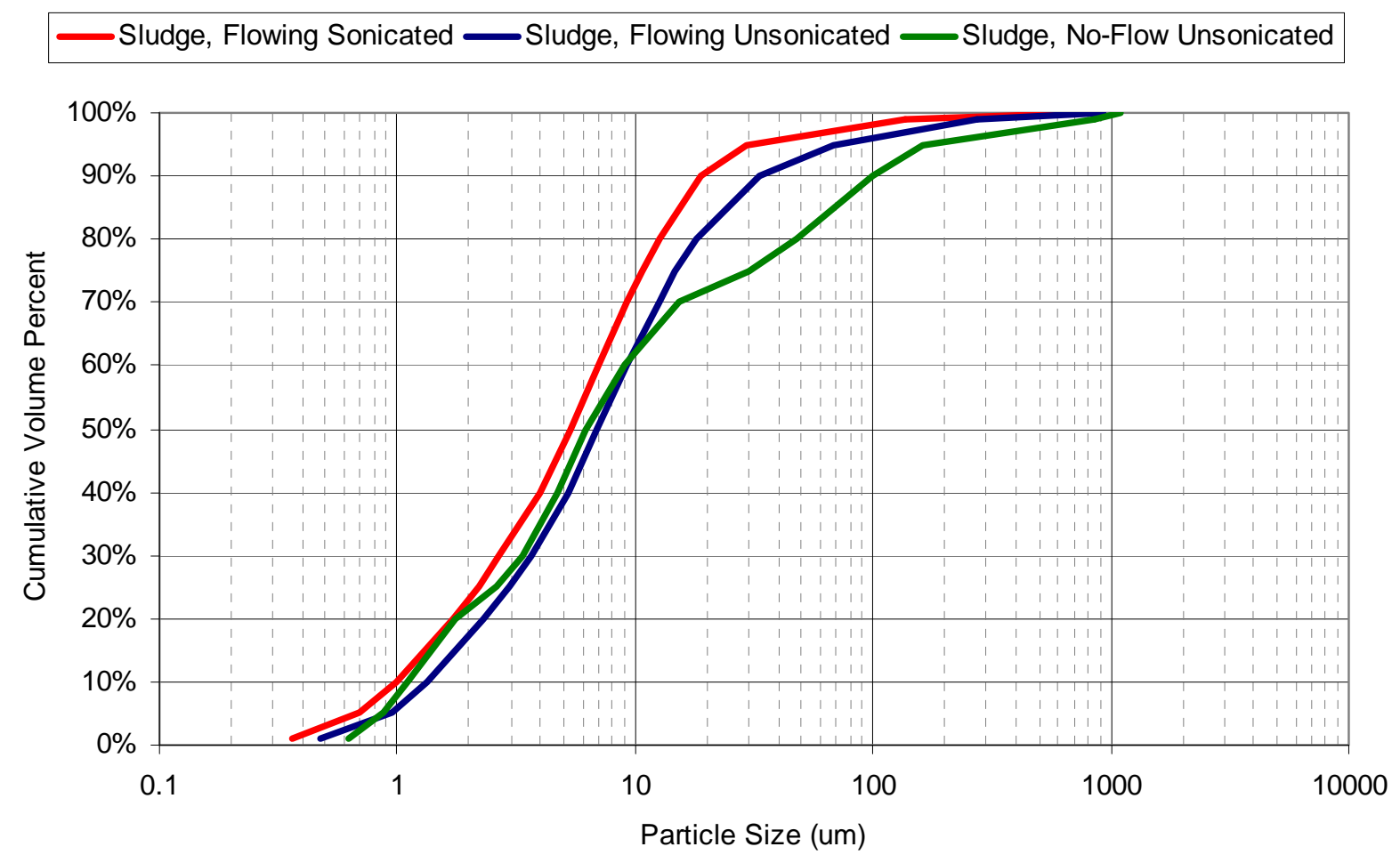

Figure 3.59. Composite Combined PSD Summary, Sludge

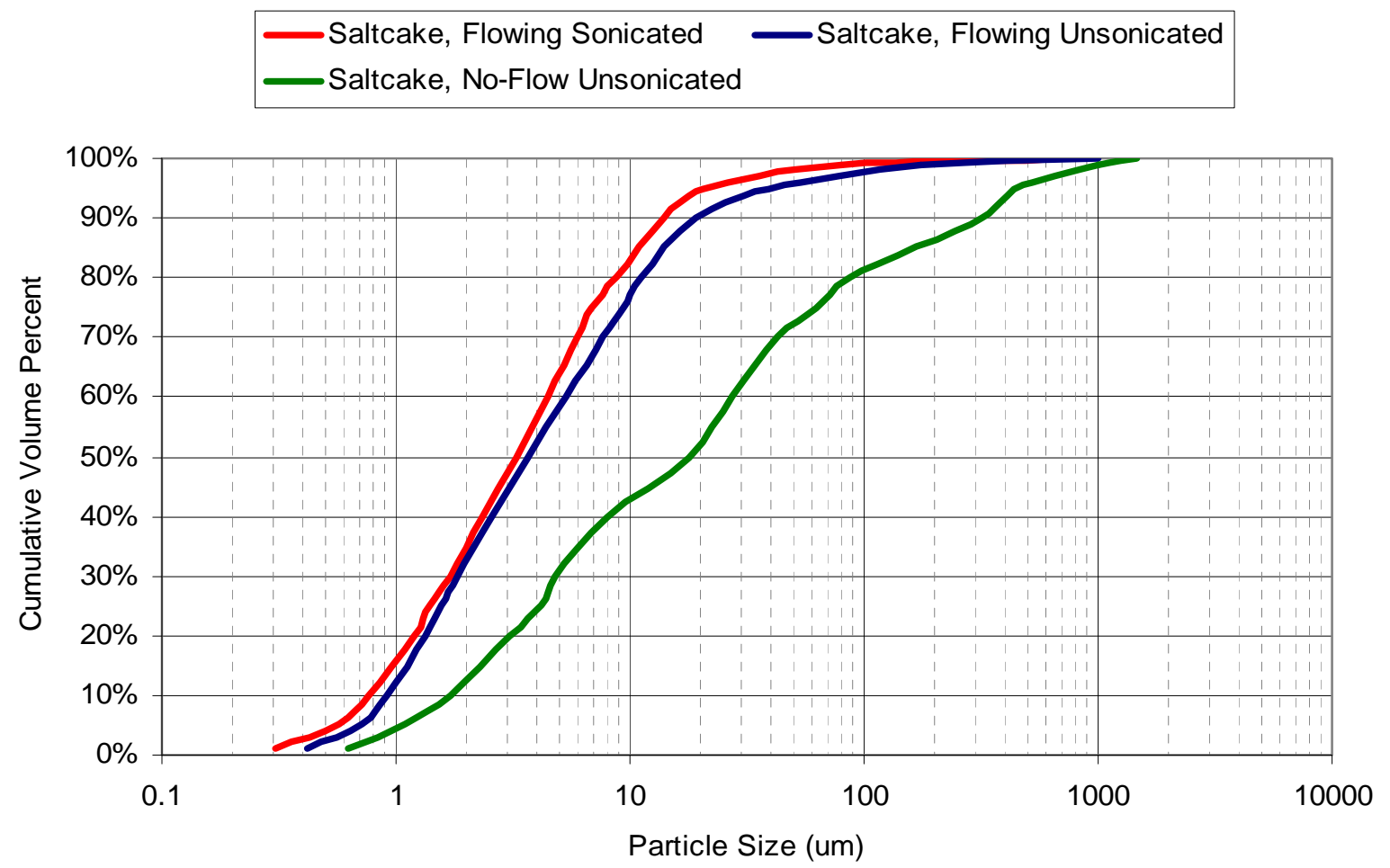

Figure 3.60. Composite Combined PSD Summary, Saltcake 
Table 3.26. Composite Combined PSDs

\begin{tabular}{|l|c|c|c|c|c|c|c|c|}
\hline & \multicolumn{9}{|c|}{ Particle Size Percentiles $(\boldsymbol{\mu m})$} \\
\cline { 2 - 10 } Composite PSD & $\mathbf{0 . 0 1}$ & $\mathbf{0 . 0 5}$ & $\mathbf{0 . 2 5}$ & $\mathbf{0 . 5}$ & $\mathbf{0 . 7 5}$ & $\mathbf{0 . 9 5}$ & $\mathbf{0 . 9 9}$ & $\mathbf{1}$ \\
\hline Sludge, Flowing Sonicated & 0.36 & 0.69 & 2.19 & 5.37 & 10.68 & 29.19 & 135.27 & 791.67 \\
\hline All Sonicated (Wells et al. 2007) & 0.39 & 0.7 & 1.63 & 4.39 & 10.1 & 33.4 & 112 & 774 \\
\hline Sludge, Flowing Unsonicated & 0.48 & 0.94 & 2.93 & 6.90 & 14.69 & 68.68 & 275.12 & 930.79 \\
\hline All Minimal Disturbance (Wells et al. 2007) & 0.65 & 1 & 2.8 & 6.31 & 14 & 58.6 & 256 & 1000 \\
\hline Jewett et al. (2002) & 0.7 & 1.2 & 3.7 & 7.5 & 31 & 140 & 210 & NA \\
\hline Sludge, No-Flow Unsonicated & 0.62 & 0.88 & 2.58 & 6.22 & 29.77 & 162.41 & 858.48 & 1093.55 \\
\hline Saltcake, Flowing Sonicated & 0.31 & 0.57 & 1.39 & 3.30 & 6.85 & 20.56 & 80.37 & 939.22 \\
\hline Saltcake, Flowing Unsonicated & 0.41 & 0.72 & 1.55 & 3.66 & 9.30 & 39.02 & 175.63 & 1003.44 \\
\hline Saltcake, No-Flow Unsonicated & 0.63 & 1.09 & 4.18 & 18.03 & 62.58 & 437.80 & 1006.49 & 1457.54 \\
\hline NA No da provichyyyyyyyyyyyy
\end{tabular}

NA No data provided in Jewett et al. (2002)

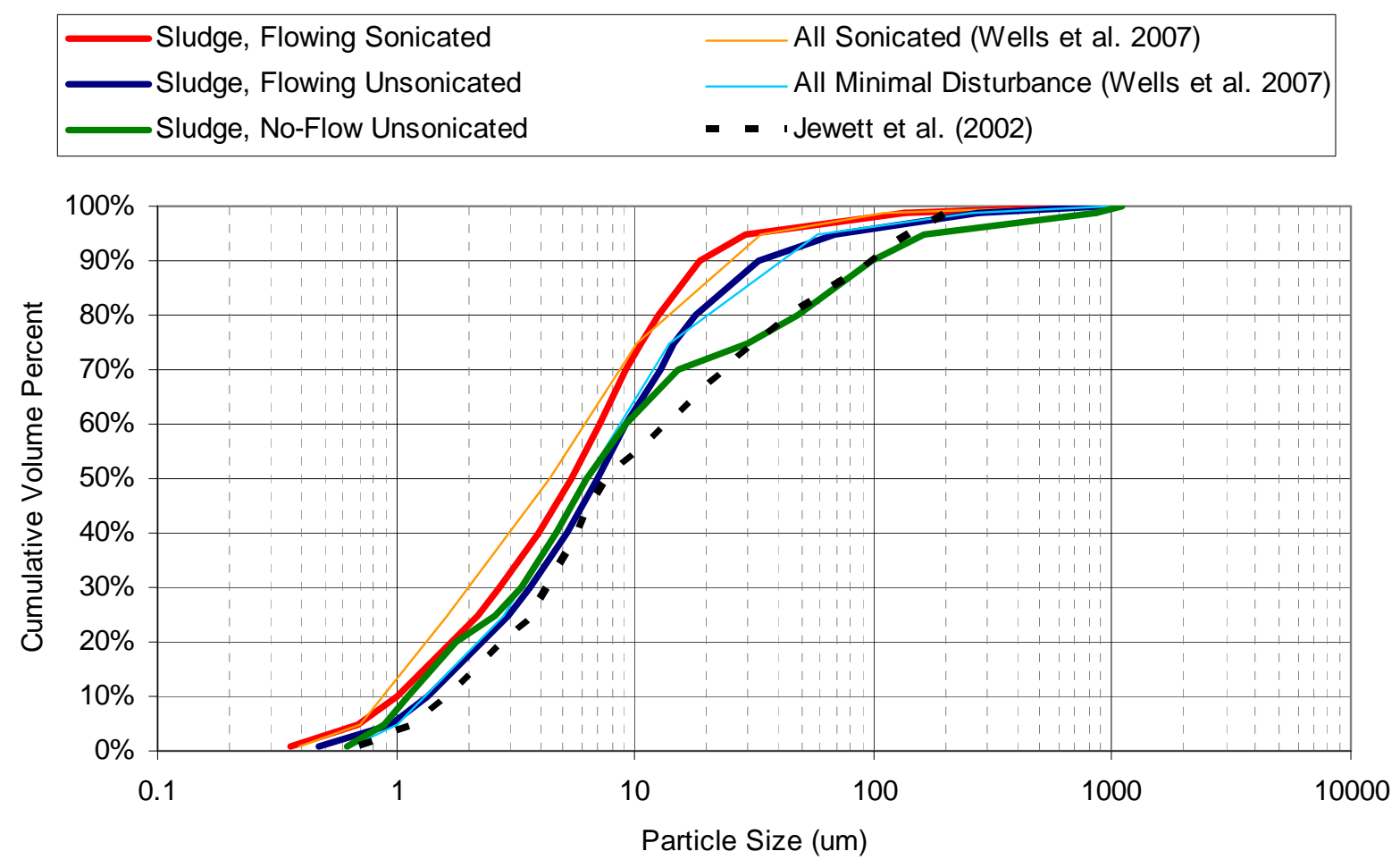

Figure 3.61. Sludge Composite Combined PSDs, Comparison to Wells et al. (2007)

\subsubsection{PSD Uncertainty}

A common request expressed by end users of PSD results is for some measure of uncertainty to be provided with the PSDs. This is driven by the need to use bounding particle sizes for developing simulants or for use in engineering calculations. An understanding of the size of the solid particles in a tank waste sample is crucial to estimating sedimentation rates, the ease with which the solids can be filtered, the flow behavior of the solids when pumped through a pipe, and the force required to suspend solids and keep the solids suspended in a pipe or tank. One approach to address this issue is to provide an estimate of the uncertainty based on the performance characteristics of the method and instruments. 
Another approach is to develop tolerance or confidence limits based on the actual sample results. These approaches are discussed in the following subsections. Unfortunately, neither approach results in quantifiable, technically defensible uncertainties for the PSDs.

\section{PSD Measurement Uncertainty}

Multiple methods have been developed to determine the size of particles in polydisperse systems. These methods include microscopy, wet and dry sieving, sedimentation, electrical pulse counting, hydrodynamics, electroacoustics, and light scattering. Multiple methods have been used in characterizing the particle size of tank wastes with the primary methods being microscopy, sieving, sedimentation, and light scattering. Methods based on light scattering provide a rapid method of determining the PSD of particles in the submicron to millimeter size range and is currently the dominant method for characterizing the PSD of tank wastes. This subsection contains a summary of a more extensive discussion on PSD uncertainty that is presented in Appendix G.

Light-scattering methods determine particle size by analyzing the scattering pattern (intensity of the scattered light as a function of angle from the incident light) created when light is scattered by particles. Solid particles in tank waste slurries and sludges are generally polydisperse; therefore, a mean particle size does not provide sufficient information to design systems for handling slurries and sludges of Hanford tank waste. Data on the distribution of particle sizes is necessary to characterize the solids and their resulting slurries and sludges. In light-scattering techniques, PSDs are calculated by comparing a sample's scattering pattern with an appropriate optical model using a mathematical inversion process. Mie theory has primarily been used when calculating the distribution of particle sizes in Hanford tank waste samples.

Mie theory can be applied to a suspension of particles provided the particles being measured are spherical, the suspension is dilute, and the particles are homogeneous. Hanford tank wastes contain irregular-shaped particles with a variety of sizes, presenting deviations from Mie theory. Additional empirical modeling and measurement procedures provide adjustments to handle these deviations. These adjustments provide more accurate results but are not rigorous solutions for these deviations; therefore, errors are encountered in PSD measurements of these non-ideal systems.

Error in these measurements can result from poor sampling, lack of detailed information on optical properties of the particles, deviations from spherical configurations of the particles, concentrated suspensions, and the presence of agglomerates. Care must be exercised in measuring the PSD, analyzing the data, and reporting the data to make certain that accurate information on PSDs is presented.

Measuring a PSD often requires selecting a dispersing phase, using surfactants, and numerous operational and analysis parameters. Operational experience and observations during PSD measurements of a wet dispersion are key to obtaining accurate results.

A PSD measured by light-scattering depends on the conditions under which the dispersion or powder is presented to the laser beam and the duration and frequency of the interactions between the particles in the dispersion or powder and the laser beam. Modern PSD analyzers typically allow control of operational parameters that impact the state of dispersion and the interaction time of particles with the laser beam, thereby impacting the measurement statistics. These operational parameters define how a given sample dispersion (or powder) is "run," and once executed, these run parameters cannot be changed without re-running the sample. Typical run parameters include, but are not limited to: 
- recirculation rate between the dispersion reservoir and observation/flow cell (if the two exist as separate entities)

- stirrer/agitator rate in either the dispersion reservoir and/or observation/flow cell

- dispersion sonication (either before or during measurement)

- frequency of scattering signal observation (i.e., the data collection rate)

- integration time (i.e., the total duration of observation or the number of observations that make up a single PSD measurement)

- choice of dispersing phase and use of surfactants/dispersants.

It should be noted that the limitations outlined above apply only to wet dispersion systems. Size analyzers that work with dry powders are available and have their own unique run parameters that affect measurement uncertainty.

Analysis parameters can be altered after the measurement is completed because they control the way averaged diffraction data are interpreted when calculating the PSD of the dispersion. Analysis parameters include (but are not limited to):

- interpretive model for the scattering pattern (e.g., single narrow distribution, multiple narrow distribution, and broad general distribution)

- refractive index of both the particle and suspending phase

- absorption index of the particle.

Hanford tank waste sludges and slurries are complex mixtures of crystalline and amorphous precipitated metal hydroxides. Typical speciation includes aluminum- and iron-bearing minerals of broad (several orders of magnitudes) size distributions. When applied to multicomponent systems with a broad size distribution, light-scattering models generally cannot resolve sharp peaks associated with individual species, especially when those peaks overlap. Even when the individual peaks are well separated, the analyzer may merge two peaks into a single peak if the peak intensities are on the same order of magnitude, especially when the broad PSD model is used. When peaks are well separated in size range, the analyzer may be able to distinguish the peaks separately, but may not characterize the range of particle sizes in each peak correctly. Even though peaks may be well-separated, there can be overlap of the diffraction patterns of large and small particles. In these cases, small-particle scattering may be confused with the stronger scattering from large-particles, especially if the small-particle species is present in limited amounts (e.g., 5 vol\% or less).

To illustrate these points, case studies (see Appendix G for discussion) for measuring mixed component (both with respect to chemical species and size) PSDs were performed. In these studies, a mixture of known composition was created from components whose size distribution had been determined by light scattering. The PSD of this mixture was measured and compared to the distribution calculated from the individual components. In this way, the difference between single-component and mixed systems can be assessed, and the uncertainty associated with mixed system PSD measurements evaluated. Despite the uncertainty with regard to PSD measurement accuracy, studying these multi-component, broad PSD dispersions has demonstrated that when measuring conditions are well-controlled, PSD measurements of complex systems are highly reproducible. Variations of 1 to 3\% 
in select percentiles were typical. The precision decreases for larger particles (greater than $70 \mu \mathrm{m}$ ) up to $14 \%$ at the $99^{\text {th }}$ percentile $(320 \mu \mathrm{m})$. Difficulty in obtaining a representative sampling of large particle morphologies in mixtures with broad PSDs results in these increased variances for larger particles. While PSD measurements may not always be accurate, they can be precise under controlled conditions.

A PSD measured by light-scattering methods may not accurately capture the true distribution of particle sizes in a given dispersion or powder with respect to either the size or volume contribution of particles. Light scattering by highly monodisperse, spherical particles with uniform well-known optical properties provide accurate PSDs as shown by measuring calibration standards where greater than $1 \%$ accuracy is often obtained. However, even the inclusion of a second monodisperse spherical particle of different size into this ideal system can yield unexpected results, especially if the model used to interpret the scattering pattern is not optimized for that system. For more complex systems, such as multicomponent systems with broad (several orders of magnitude) mixtures of particle size, PSD analysis by light scattering can provide a general guide as to the range, size, and relative volumes of particles; however, light scattering may not be able to detect all particle sizes and may incorrectly interpret the relative volume contributions of each species. The potential for misinterpreting even simple systems creates significant uncertainty with respect to what is the "true" PSD of the dispersion or powder. Although the light-scattering measurements of complex systems are best characterized as an apparent PSD they are arguably adequate to characterize the general sizes of particles present in the waste.

Measurement uncertainty derives from many sources, such as the state of particle agglomeration and how it responds to flow/mixing conditions and dispersing phase chemistry as well as difficulties in deconvoluting overlapping scattering patterns in multicomponent systems. Therefore, assigning an estimate to PSD measurement uncertainty is difficult. While it may be possible to develop a better understanding of uncertainty, it would require developing and studying model systems, such as well-characterized mixtures of particles that span the entire measuring range of the instrument (0.02 to $2000 \mu \mathrm{m}$ ) and encompass the range of particle optical properties, chemistries, and morphologies encountered in Hanford tank wastes. A thorough exploration of uncertainty would require a significant investment of resources. A smaller effort involving a relatively simple set of measurements with individual broad based particles and combinations of particles could probably provide reasonable estimates of the uncertainty.

\section{PSD Uncertainty Estimates}

This subsection discusses some possible approaches to estimating PSD uncertainties and the technical difficulties that make the use of tolerance or confidence limits questionable.

\section{PSD Summaries}

PSDs are summarized in this report by listings of particle size estimates associated with certain percentiles. For example, suppose that a particular waste tank has a PSD summarized in Table 3.27.

Table 3.27. Example PSD

\begin{tabular}{|l|c|c|c|c|c|c|c|c|c|c|c|c|c|c|c|c|}
\hline Percentile & 0.01 & 0.05 & 0.10 & 0.20 & 0.25 & 0.30 & 0.40 & 0.50 & 0.60 & 0.70 & 0.75 & 0.80 & 0.90 & 0.95 & 0.99 & 1.00 \\
\hline Size $(\mu \mathrm{m})$ & 0.53 & 1.49 & 2.44 & 4.23 & 4.98 & 5.95 & 12.5 & 54.7 & 59.3 & 80.0 & 83.4 & 86.7 & 98.2 & 121.7 & 144.3 & 198.5 \\
\hline
\end{tabular}


Then, for this example waste tank, a particle size of $83.4 \mu \mathrm{m}$ represents the $75^{\text {th }}$ percentile. This means that $75 \%$ of the particles in this tank should be less than $83.4 \mu \mathrm{m}$ in size. Of course, the $83.4 \mu \mathrm{m}$ is an estimate. Taken alone, the $83.4 \mu \mathrm{m}$ is a point estimate because it is a single value used to estimate an unknown population value of interest, in this case, the $75^{\text {th }}$ percentile of particle sizes. In general, interval estimates are preferred over point estimates whenever possible because they incorporate uncertainties and provide a range of values wherein the true (but unknown) population value might reasonably lie. A two-sided interval estimate involves lower and ULs. A one-sided interval estimate involves either a lower limit or an UL, with the other side of the interval being unbounded. Statistically derived interval estimates are generally determined based on a desired confidence level. When summarizing PSDs, it is reasonable to prefer some type of interval estimate to describe each percentile reported. However, as will be discussed herein, commonly used statistical interval methods were not appropriate for this study.

\section{Interval Estimates}

A tolerance interval (TI) is an appropriate confidence-based interval estimate to use when estimating bounds on a particular proportion of the values in a given population (the true values of a particular random variable). For example, a two-sided TI might be used to estimate lower and ULs that should capture 75\% (the central 75\%) of the values in a particular population. Or, a one-sided upper TI might be used to estimate an UL on the $75^{\text {th }}$ percentile for values in a particular population. In these examples, the $75 \%$ or 0.75 is referred to as the content level for the TI. There is also a confidence level associated with the TI. The confidence level reflects the objective that a stated proportion (the confidence level) of TIs formed in the same way and under the same conditions would capture a desired proportion (the content level) of the population values.

Another commonly used interval estimate is a confidence interval (CI). CIs are similar to TIs in that they are both confidence-based. But CIs differ from TIs in that they provide interval estimates of population parameters (e.g., the population mean or population variance) rather than some proportion of population values. Estimating population parameters was not a primary objective relative to the PSDs considered in this current study, so CIs were not used for PSD summaries in this report.

TIs can generally be calculated in cases where the population of interest has a known distribution with key parameter values (such as the mean and variance) that are also known. When such information is not known, TIs can at times be adequately approximated, provided that certain conditions are satisfied. A wide variety of TI approximation methods exist, each with their respective details. For the purposes of this report, it suffices to focus on some of the more common TI methods and related issues. Commonly used formulas for approximating one- and two-sided TIs for normal distributions are (Krishnamoorthy and Thomas 2009),

$$
\begin{aligned}
& \text { One-sided lower TI: }[L T L,+\infty) \text { where } \quad L T L=\hat{\mu}-k_{1} \hat{\sigma}, \\
& \text { One-sided upper TI: }(-\infty, U T L] \text { where } \quad U T L=\hat{\mu}+k_{1} \hat{\sigma} \text {, and }
\end{aligned}
$$

Two-sided TI: $[L T L, U T L]$ where $L T L=\hat{\mu}-k_{2} \hat{\sigma}$ and $U T L=\hat{\mu}+k_{2} \hat{\sigma}$. 
In the above formulas, $L T L$ denotes a lower tolerance limit, $U T L$ denotes an upper tolerance limit, $\hat{\mu}$ is an estimate of the population mean, $\hat{\sigma}$ is an estimate of the population standard deviation, and $k_{1}$ and $k_{2}$ are multiplying factors (for one-sided and two-sided TIs, respectively) determined so as to provide a desired confidence level that the TI captures a specified proportion of the population values. For the purposes of this work, one-sided upper TIs would be more meaningful (provided that TI methodology is even appropriate here) than two-sided TIs because one-sided upper TIs would provide ULs on the percentiles of a PSD. Two-sided TIs are estimates of central population proportions. This is apparent based on how the " \pm " is used in the two-sided TI formula. As such, two-sided TIs are not meaningful relative to the percentiles of a PSD.

\section{TI Methodology Requirements}

These commonly used TI formulas (given above) involve certain elements and are based on certain requirements concerning the data used to generate them. These elements and requirements are discussed below.

Population Units-These are the items for which random variable values can be obtained; the set of all possible such items. They are also the items to which the TI statement should apply.

Sample Units-These are a subset of the population units; those actually selected and analyzed to generate the sample statistics used to calculate the TIs.

Random Sample (Independent Observations) - Having a random sample of population units is generally required to justify the use of calculational properties that apply to independent realizations of a random variable. Measurements obtained from a random sample of population units are used to calculate the estimates of the population mean and standard deviation needed to construct the TIs.

Normality-A primary requirement of the TI formulas listed above is that the values used to determine the tolerance limits represent a random sample from a normally distributed population. Accordingly, the corresponding formulas are often referred to as normal theory formulas. The resulting TIs are formulated so as to capture a specified proportion of a normal distribution with a particular confidence level.

Estimate of Mean-The sample mean $\bar{x}=\hat{\mu}$ is generally used to estimate the true population mean $\mu$. The sample mean is an unbiased estimator of the population mean provided the sample can be considered as independent observations from the same distribution, the distribution representing the population of interest.

Estimate of Standard Deviation-The sample standard deviation $s=\hat{\sigma}$ is generally used to estimate the true but unknown population standard deviation $\sigma$. The sample standard deviation is calculated under the assumption that the sample represents some number of independent observations from a particular population. A more complicated version of the commonly used sample standard deviation formula would be needed if the sample does not meet this criterion. Furthermore, the sample standard deviation is actually a biased estimator of $\sigma$, even when the sample represents independent observations from the population of interest. Moreover, while $\sigma$ is a measure of the true variation among population values, the calculated value of $\hat{\sigma}$ may include other sources of variation and uncertainty. For example, measurement error, the magnitude of which can differ from one instrument to another or from one operator to another, and sampling error can cause $\hat{\sigma}$ to be an inflated estimate of $\sigma$. Ideally, measurements from sample units represent realizations of a random variable that are known without error. 
This must either be assumed, or again, a more complicated estimator of $\sigma$ must be determined and used. When variation and uncertainty from extraneous sources can be estimated, adjustments can potentially be made in the calculation of $\hat{\sigma}$ to obtain a better estimate of the true population variance $\sigma$.

The type of data available for this study raise some concerns regarding the required conditions listed above. In particular:

- For this study, the sample units are not a subset of the preferred population units. The desired TI statements for this study would provide further information concerning the reported PSDs. They would provide ULs on specified percentiles of particle sizes for the various waste tanks, waste types, composites, and composite subsets. Therefore, the preferred population units would be waste particles. However, the sample units were not waste particles; they were slurry samples from the various waste tanks. The slurry samples contained waste particles, but the particles themselves were not directly measured to obtain the sample values to be used to calculate the estimates needed to construct TIs. Instead, the slurry samples were analyzed and ultimately produced multiple values per slurry sample: the estimated particle sizes believed to represent certain percentiles of a population of particle sizes within a given waste tank, waste type, composite, and composite subset.

- While the slurry samples taken from a given waste tank could be considered independent if proper sampling methods were employed, the quantities obtained from the analysis of a given slurry sample are certainly not independent.

- For many of the waste tanks included in this study, very few samples were available. These small sample sizes make it difficult to assess the normality assumption. It was not uncommon to see outliers among the observations in a given sample, or an outlying sample when compared to other samples from a waste tank. Having outliers present in a small sample is not indicative of normal distributions.

When the necessary conditions and data requirements are not fully satisfied, the resulting TIs cannot be considered accurate or meaningful. That is, TIs calculated using data or under conditions other than those needed to apply a particular formula cannot be expected to capture the specified proportion of population values with the specified relative frequency. Either the content level or the confidence level, or both, associated with the TI will not be as stated. How inaccurate the stated content and/or confidence levels might be is difficult to say, and depends on the severity of the departures from the requirements.

\section{Example of TI Calculation}

Suppose that the samples from a particular waste tank result in the data matrix of Table 3.28.

Table 3.28. Example Data Matrix (particle size in microns)

\begin{tabular}{|l|c|c|c|c|c|c|c|c|c|c|c|c|c|c|c|}
\hline Percentile & 0.01 & 0.05 & 0.10 & 0.20 & 0.25 & 0.30 & 0.40 & 0.05 & 0.60 & 0.70 & 0.75 & 0.80 & 0.90 & 0.95 & 0.99 \\
\hline Sample 1 & 0.18 & 0.27 & 0.39 & 0.81 & 1.17 & 1.49 & 2.40 & 3.87 & 5.37 & 7.44 & 8.76 & 10.60 & 15.53 & 18.80 & 26.7 \\
\hline Sample 2 & 0.18 & 0.27 & 0.38 & 0.74 & 1.04 & 1.33 & 2.19 & 3.59 & 5.23 & 7.63 & 9.21 & 11.28 & 16.90 & 20.70 & 29.3 \\
\hline Sample 3 & 0.60 & 0.94 & 1.12 & 1.60 & 1.91 & 2.13 & 2.65 & 3.30 & 4.16 & 5.25 & 5.90 & 6.91 & 9.48 & 11.1 & 15.3 \\
\hline Sample 4 & 0.69 & 1.03 & 1.23 & 1.76 & 2.10 & 2.36 & 3.00 & 3.80 & 4.88 & 6.27 & 7.10 & 8.29 & 11.30 & 13.2 & 18.5 \\
\hline Sample 5 & 0.70 & 1.08 & 1.36 & 2.17 & 2.74 & 3.50 & 5.70 & 9.30 & 21.63 & 50.30 & 76.7 & 92.82 & 135.93 & 164.5 & 258.0 \\
\hline Sample 6 & 0.31 & 0.54 & 0.77 & 1.07 & 1.21 & 1.37 & 1.80 & 2.49 & 3.41 & 4.66 & 5.53 & 6.73 & 16.04 & 83.14 & 255.6 \\
\hline Sample 7 & 0.27 & 0.47 & 0.68 & 0.97 & 1.08 & 1.20 & 1.50 & 1.99 & 2.75 & 3.76 & 4.47 & 5.43 & 9.24 & 14.04 & 26.56 \\
\hline
\end{tabular}


If a TI is to be formed for a given percentile, say the $75^{\text {th }}$ percentile, then seven observations are available for use in forming the TI. The seven observations for the $75^{\text {th }}$ percentile are $8.76,9.21,5.90$, 7.10, 76.7, 5.53, and 4.47. The resulting sample mean is $\hat{\mu}=16.81$. The resulting sample standard deviation is $\hat{\sigma}=26.46$. A TI formed to provide an UL on the $75^{\text {th }}$ percentile of population values suggests that the content level for the interval would be 75\%. If the desired confidence level for such a one-sided upper TI is $95 \%$, then the multiplying factor for the interval is $k_{1}=1.732$, based on seven observations. Thus, a one-sided upper $95 \%$ tolerance limit on the $75^{\text {th }}$ percentile of population values is $U T L=\hat{\mu}+k_{1} \hat{\sigma}=16.81+1.732 * 26.46=62.64 \mu \mathrm{m}$.

Note that one of the observations, the 76.7, is clearly an outlier. It is actually beyond the calculated upper tolerance limit. Also, one might question whether these seven observations should be considered a random sample from a normal distribution.

\section{Interpretation of TI Estimates}

TIs are often misinterpreted. TIs calculated to describe PSDs for this study would be particularly susceptible to misinterpretation. For example, it might be tempting to interpret the UTL calculated in the example above as meaning that "we are $95 \%$ confident that $75 \%$ of the waste particles in this waste tank are less than $62.64 \mu \mathrm{m}$.” This is a poor interpretation for two main reasons. First, as explained previously, the sample units for this study were not waste particles, so the TI is technically not a statement about sizes of individual waste particles. To make a confidence statement about the sizes of individual waste particles, the seven observations used to calculate the UTL would have to be measured sizes from a random sample of seven individual waste particles. Instead, the TI is a confidence statement about the $75^{\text {th }}$ percentiles that could be obtained from a population of analyzed slurry samples from the waste tank. The UTL calculated in the example above provides a 95\% (in confidence) UL for the $75^{\text {th }}$ percentile of $75^{\text {th }}$ percentile values that would result from analyses of all possible slurry samples that could be taken from this waste tank. In fact, a UTL based on a 95\% confidence level for the $90^{\text {th }}$ percentile of observed $75^{\text {th }}$ percentile values could have been calculated in a similar fashion. Such an UL would be difficult to interpret from a practical point of view and would probably not be considered very useful. Second, no specific confidence is associated with the number 62.64. The confidence level associated with a TI refers to the confidence in the TI construction process. Thus, a more correct interpretation of the UTL calculated above is that " $95 \%$ of the UTLs calculated using the specified formula would capture the $75^{\text {th }}$ percentile of $75^{\text {th }}$ percentile values for all possible slurry samples from this waste tank.” Furthermore, the stated 95\% confidence level and 75\% content level for this UTL are dependent on how well the requirements associated with this normal theory TI formula and methodology are satisfied.

\section{Alternative Interval Estimates}

Given the potential departures from the conditions and data requirements that indicate the appropriate use of TIs, and given the potential for misinterpretation of TIs, it is natural to question whether some other methodology for estimating confidence-based intervals might be appropriately applied for this work, based on some additional assumptions. Due to the nature of the data available for this study, a CI might be preferred over a TI if a confidence statement about true percentiles of actual particle sizes is desired. Of course, key assumptions must be made in connection with the CI approach. For example, suppose that an upper confidence limit ( $U C L)$ on the true $75^{\text {th }}$ percentile for particles in a particular waste tank is desired. Denote the true $75^{\text {th }}$ percentile for individual waste particles in the tank as $P_{75}$. The $75^{\text {th }}$ 
percentile values obtained from analyzing some number of slurry samples (a random sample) from this tank could be used to calculate a UCL on the true mean of the population of such observed (from analysis) $75^{\text {th }}$ percentile values over all possible slurry samples from the tank. Normal theory formulas for calculating the $U C L$ are typically approximated using

$$
U C L=\bar{x}+t_{\alpha, n-1} \frac{s}{\sqrt{n}}
$$

$$
\text { where } \begin{aligned}
n= & \text { sample size } \\
\bar{x}= & \text { sample mean } \\
s= & \text { sample standard deviation } \\
100(1-\alpha) \%= & \text { confidence level associated with the } U C L \text { so that } t_{\alpha, n-1} \text { is a } t \text {-statistic } \\
& \text { with } n-1 \text { degrees of freedom and a cumulative probability of } 1-\alpha .
\end{aligned}
$$

Of course, this formula is based on the assumption that the underlying population has an approximately normal distribution. Furthermore, the calculated $U C L$ is an upper bound on the true mean of the population of observed (from analysis) $75^{\text {th }}$ percentile values over all possible slurry samples from the tank. Denote the true mean of the observed $75^{\text {th }}$ percentiles (from analysis) as $\mu_{\hat{p}_{75}}$. If it happens that $\mu_{\hat{p}_{75}}=P_{75}$, then the calculated $U C L$ can serve as an upper confidence limit on $P_{75}$ as well. The true difference between $\mu_{\hat{p}_{75}}$ and $P_{75}$ is, of course, unknown, so it would be difficult to assess the usefulness of a calculated UCL.

Non-parametric TI methods exist that do not require the underlying population to be normally distributed. They are not calculated using estimates of the population mean and standard deviation. Instead, they use the minimal and/or maximal values observed from a sample of a designated size. However, these non-parametric TIs require relatively large sample sizes to attain confidence levels that would typically be considered acceptable. For example, a random sample of 30 observations would be needed to achieve $95 \%$ confidence that the $90^{\text {th }}$ percentile of a population (not necessarily normally distributed) would be less than the maximal value in the sample. Samples of this size were not available for this study.

\section{PSD Uncertainty Conclusions}

Based on the objectives, available data, and methods considered for this current work, it was decided that confidence-based interval methods would not be used to generate lower and upper estimates associated with the percentiles of reported PSDs. Instead, the minimum and maximum of the observed (from analysis) values at the percentiles of interest, obtained from slurry samples from a given waste tank, waste type, composite, or composite subset, were reported. This approach was preferred because of the limitations in the data and potential violations of the requirements associated with normal theory methods. The minimum and maximum values do provide some indication of range, but are not confidence-based because the uncertainties associated with the PSD estimates are not understood sufficiently to support confidence statements. 


\subsubsection{UDS Particle Settling}

The tendency for solids in Hanford tank wastes to drop out during processing can be evaluated, to some extent, by using data from gravity-settling tests conducted on waste samples. In these tests, aliquots of the samples were mixed and then allowed to settle in graduated cylinders or graduated centrifuge cones. The sediment volume was measured as the volume from the bottom of the container to the visible interface between the clear supernatant and the opaque suspension. The sediment volume was recorded as a function of time. In a few reports, the height of the sediment bed was also recorded as a function of time.

The settling rate measured by this method is controlled by the settling rate of the most slowly settling particles in the suspension; these particles form the upper interface with the clarified supernatant. The interface velocity is constant until the point when the upper interface approaches the surface of the settled solids, at which point the motion of the interface between solids and liquid makes a transition to being governed by compaction of the solids under their own weight. In a monodisperse suspension (particles of uniform size and density), all particles settle at the same rate, and a sharp boundary exists between the clarified portion of the settling system and the settling particles.

Hanford tank waste solids vary in size and density (i.e., are polydisperse and polypycnic). Unless the solids concentration is great enough to allow smaller particles to be trapped in the lattice formed by large particles, ${ }^{(a)}$ each size fraction or material type (different particle densities) settles at its own characteristic velocity. The result resembles a chromatographic separation: there is a series of regions occupied by different velocity categories of particles and, correspondingly, there is a series of more or less distinct zone interfaces, each with its own velocity. Of these, only the uppermost is visible; the interior interfaces are difficult to observe visually.

The boundary between the clarified supernatant and a region of polydisperse settling solids may be diffuse; however, in Hanford waste settling tests, a distinct interface usually develops between the turbid suspension and clear supernatant. Unless there is a change in the properties of the particle type that makes up the interface because of agglomeration or for other reasons, the upper interface velocity would be expected to remain constant until it approaches the settled solids surface.

A number of mechanisms are involved in settling. Fine particles may form agglomerates or flocs (MacLean 1999), causing an increase in their settling velocity. In the polydisperse suspension, several different vertical zones will form as particles with different settling velocities separate from each other (Ha and Liu 2002). At any point in the settling suspension, the solids volumetric concentration may be large enough for particles or flocs to interact hydrodynamically with each other, causing hindered settling (and decreasing velocity) or allowing wake capture of smaller particles by larger particles (increasing the velocity of the fines). Convective structures may form in which the overall downward velocity is greater

(a) Davies and Kaye (1971) studied “interlocking” slurries, in which there was no size segregation because equal-density particles of different sizes were forced to settle at the same rate. When bidisperse spheres whose diameters differed by 20 to $25 \%$ were tested, size segregation was not seen for concentrations above 35 vol\%. The concentration limit above which size segregation was not present was only $17.5 \%$ for a mixture of cylinders and cubes, indicating that more angular particles may interlock more readily than spheres. However, when spheres of different sizes and different densities were tested, the concentration above which there was no size segregation increased to $63 \mathrm{vol} \%$. The authors theorized that the lattice was not strong enough to hold particles of a different density in place. 
than the settling velocity of unconvected particles (Biesheuvel et al. 2001). Finally, the zone of high solids concentration that forms at the bottom of the container grows upward, because of freshly settling material, at the same time it is being compacted by its own weight.

Settling rate data are available for 20 individual tanks and 7 waste-group composites representing 18 waste types, as shown in Table 3.29. The tanks and single waste type Groups of Table 3.29 represent 13 primary waste types as defined in Table 3.1. As shown in Figure 3.62, there are 3 of the 13 primary waste types for which $50 \%$ or more of the tanks containing the waste types have been characterized for settling, i.e., have had core or grab samples tested for settling rate. The solid phases in the 13 primary waste types make up approximately $23 \%$ by volume of the total solid-phase inventory.

\subsubsection{Data and Interpretation Gaps}

One significant question about settling data, or their interpretation, is that of the correct scale-up to tank conditions. A factor of 10 discrepancy between rapid interface settling in Tank AZ-101 and slower settling in the laboratory studies of AZ-101 composite samples was observed (Gauglitz et al. 2009, 2010b).

In addition, when velocity distributions were calculated from PSDDs and compared to interface velocities observed in Tank AZ-101, Tank AY-102, and a laboratory-scale column (3-inch ID) containing a three-component simulant, the interface velocities consistently lay at or above the $50^{\text {th }}$ percentile of the calculated distributions. Because the visible interface by definition consists of the slowest-settling particles, its velocity would be expected to be closer to the lowest-velocity percentile than to the median or higher percentiles of the distribution. The reason for the discrepancies is not clear, although the possible contributing causes include

- Experimentally based: interface velocities measured in the laboratory might have been low because of wall effects in the graduated cylinders ( 2-cm ID) or an initial suspension depth ( $20 \mathrm{~cm}$ or less) that was not great enough to allow velocity to be fully developed (see MacLean 1999). However, the data that were reviewed did not clearly demonstrate the effect of test vessel size.

- Difference in particles: there might have been more particle agglomeration in the tank than in the graduated cylinder in the laboratory. However, PSDDs that were calculated in a manner that accounted for agglomeration (fractal dimension less than 3) did not produce velocity distributions that were consistent with the higher observed velocities.

- Difference in the PSDDs: the distribution of particle crystal densities and sizes might be biased in a way that tends to underestimate the velocities calculated for individual particles, based on the PSDDs. However, the three-component simulant was well defined, being a simple set of known solid phases, so it is unlikely to have been biased but nevertheless showed higher than expected interface velocities.

- Differences in the PSDs: the difference in comparison depending on the PSD measurement technique may suggest that the characterization of the particulate via certain methodologies may not represent the particulate as it settles.

- Difference in the flow field: wake capture or particle-induced convection, such as "vertical streaming," might have pulled the interface particles down more rapidly in the tank or in the 3-inch-ID column than in the relatively confined graduated cylinders. 
Table 3.29. Settling Rate Data Set

\begin{tabular}{|c|c|c|}
\hline Tank & Reference(s) & Primary Waste Type \\
\hline AN-104 & HNF-3352 & A2 saltcake \\
\hline AN-105 & HNF-SD-WM-DTR-046 Rev 0 & A2 saltcake \\
\hline AY-102 & RPP-8909 & BL sludge \\
\hline AZ-101 & $\begin{array}{c}\text { RPT-7078 } \\
\text { RPP-6548, Rev } 1 \\
\text { WTP-RPT-177 } \\
\text { PNNL-13027 } \\
\end{array}$ & P3 sludge \\
\hline AZ-102 & RPP-9806 & P3 sludge \\
\hline B-202 & $\begin{array}{c}\text { PNNL-14221 } \\
\text { WHC-SD-WM-DP-034 } \\
\text { WHC-SD-WM-ER-371 }\end{array}$ & 224 Post-1949 sludge \\
\hline B-203 & $\begin{array}{l}\text { PNNL-14365 } \\
\text { PNNL-16133 }\end{array}$ & 224 Post-1949 sludge \\
\hline BX-107 & $\begin{array}{l}\text { SST BX-107 Cores } 40 \text { and } 41 \text { data package, } \\
\text { C04-001-7 }\end{array}$ & 1C sludge \\
\hline C-104 & RPP-5798 & CWP sludges $^{(a)}$ \\
\hline C-107 & RPP-18799 & 1C sludge \\
\hline C-109 & WHC-SD-WM-ER-402, Rev 0; Core 47 & TFeCN sludge \\
\hline $\mathrm{C}-110$ & C-110 Cores 37, 38 and 39 data package.pdf & 1C sludge \\
\hline $\mathrm{C}-112$ & SST C-112 Core 36 data package & TFeCN sludge \\
\hline S-104 & WHC-SD-WM-DP-031 & $\begin{array}{c}\text { R saltcake/ } \\
\text { R1(boiling) sludge }{ }^{(\mathrm{b})}\end{array}$ \\
\hline $\mathrm{T}-102$ & $\begin{array}{l}\text { WHC-SD-WM-DP-052 Rev } 0 \\
\text { HNF-SD-WM-ER-700 Rev. } 0\end{array}$ & CWP2 sludge \\
\hline T-107 & WHC-SD-WM-ER-382, Rev 0 & 1C sludge \\
\hline $\mathrm{T}-110$ & $\begin{array}{l}\text { PNNL-14365 } \\
\text { PNNL-16133 }\end{array}$ & 2C sludge \\
\hline $\mathrm{T}-111$ & SD-WM-DP-024 & 2C sludge \\
\hline T-203 & $\begin{array}{l}\text { PNNL-14365 } \\
\text { PNNL-16133 } \\
\end{array}$ & 224 Post-1949 sludge \\
\hline $\mathrm{T}-204$ & $\begin{array}{l}\text { PNNL-14365 } \\
\text { PNNL-16133 }\end{array}$ & 224 Post-1949 sludge \\
\hline Group 1 & WTP-RPT-166 & 1C/2C sludges ${ }^{(a)}$ \\
\hline Group 2 & WTP-RPT-166 & BY/T saltcakes \\
\hline Group 3 & WTP-RPT-167 & CWP sludges \\
\hline Group 4 & WTP-RPT-167 & CWR1 sludge \\
\hline Group 6 & WTP-RPT-157 & S saltcakes \\
\hline Group 7 & WTP-RPT-169 & TBP sludge \\
\hline Group 8 & WTP-RPT-170 & PFeCN sludge \\
\hline \multicolumn{3}{|c|}{$\begin{array}{l}\text { The UDS density data used in settling rate data were taken from modified ESP predictions } \\
\text { based on the } 2002 \mathrm{BBI} \text {; see Section 3.2.3.2. } \\
\text { (a) CWP sludge is a combination of CWP1 and CWP2 sludge, grouped together because of } \\
\text { their similar process source. } \\
\text { (b) R saltcake and R1 sludge are grouped together because both are REDOX wastes. }\end{array}$} \\
\hline
\end{tabular}




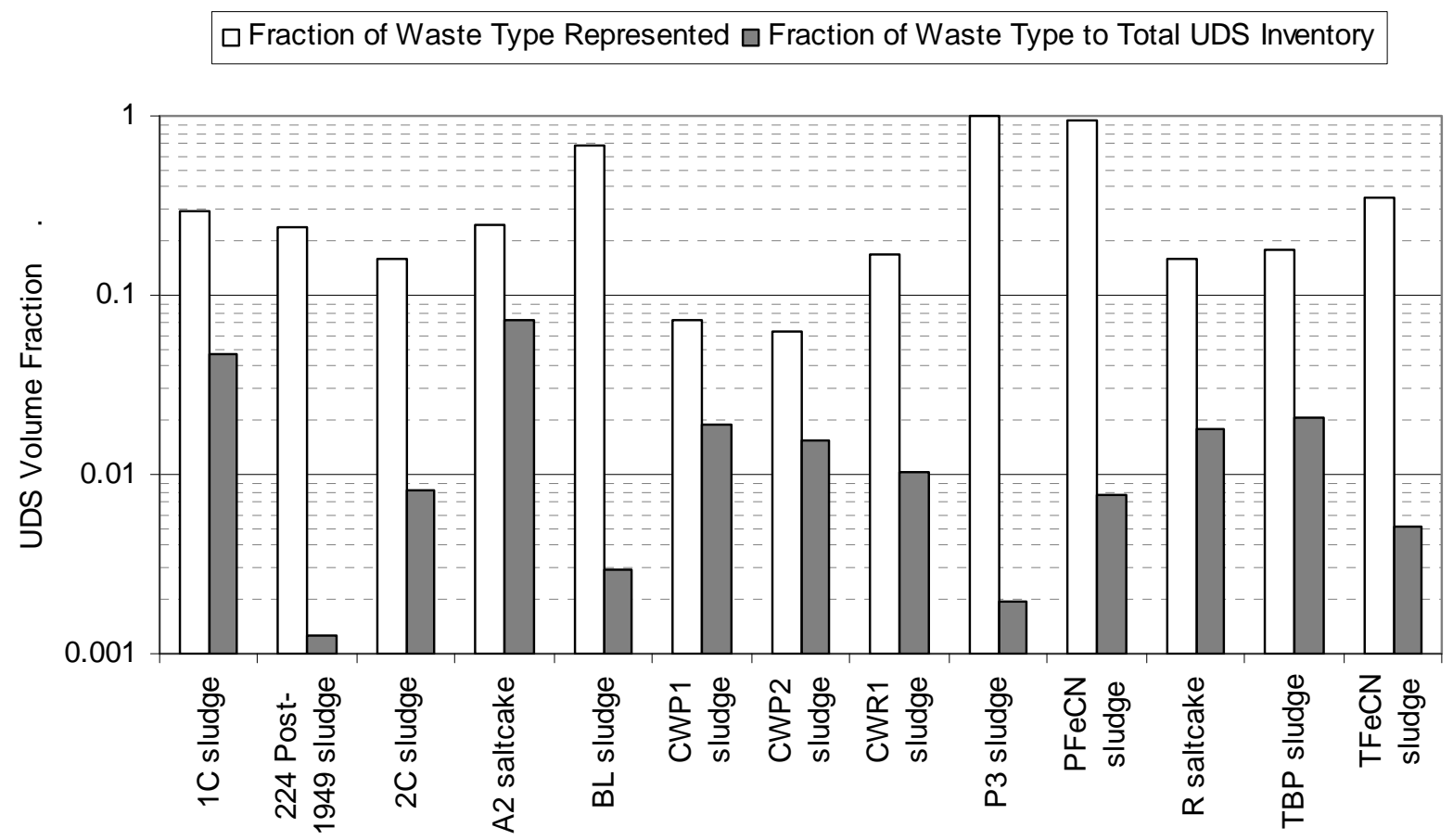

Figure 3.62. UDS Waste Types Represented by Settling Rate Data Set

The presence and effect of particle-induced convective structures has not been confirmed either in tanks or in laboratory conditions. A further review of existing literature would serve two purposes: 1) evaluate whether conditions could produce particle-induced convection; 2) suggest experimental methods that could detect and measure convection, since the opaque nature of the waste does not allow visual observation.

Visual observations can detect the interface between the slowest-settling particles and the clarified liquid above the settling suspension, but not the interior concentration interfaces caused by faster settling particles. These faster particles are those that are harder to mobilize and mix, so the absence of observing them is a gap that relates very directly to mobilization and mixing design. A literature search would suggest instruments and methods to measure the velocity of interior interfaces.

Another gap in data comes from the tendency of settling tests to be reported only in terms of relative initial sediment volume. This makes it difficult to interpret the data to provide settling velocity. Settling velocity is an absolute property of the solid/liquid system and can only be calculated from data for the absolute height of the interface. In this report, sediment heights (when not reported) have been calculated from the known sample volume and the inner diameters of graduated cylinders or centrifuge tubes that would be large enough to hold the sample volume. These are estimates only because centrifuge tubes and graduated cylinders are not standardized.

\subsubsection{Settling Velocity Models}

The relationship of the sedimentation interface velocity to particle settling velocities, which are defined by particle and liquid properties, is complex. Thus, it is useful to briefly discuss the approach for 
calculating the rate of single particles settling in a suspending phase before discussing complicating factors. A single particle under the influence of gravity in a quiescent medium will settle to the bottom of the container if the particle is more dense than the suspending phase. The settling rate is determined by a combination of thermal motion, gravitation force, and resisting drag force on the particle. Thermal motion tends to impact only colloidal systems where particle size is less than $1 \mu \mathrm{m}$ and is not considered here.

The rate at which a single particle settles in a suspending liquid depends on the size, shape, and density of the particle as well as the density and viscosity of the suspending medium. Camenen (2007) provided Eq. (2.14) for the terminal velocity, $V_{S O}$, of a spherical or non-spherical particle in a very dilute suspension in an infinite vessel, where it is effectively a single particle because there is no hydrodynamic interaction among particles and no effect from nearby walls. In more concentrated suspensions, there are hydrodynamic interactions between the settling particles and the liquid displaced by particles that flow upward, decreasing the average settling velocity ("hindered settling”) and making the particle velocity dependent on the local concentration. For a monodisperse suspension, the settling velocity under hindered settling conditions, $V_{S}$, can be expressed as a power function of the solids volume fraction, a reference solids volume fraction for the particles, ${ }^{(a)}$ and the single-particle settling velocity, as in Eq. (2.17). This equation describes the effects of the increased effective viscosity and liquid upwelling at higher particle concentrations.

In general practice, suspensions are not monodisperse, and simplified models are used to represent the bulk sedimentation rate, without any explicit correlation to the properties of individual particles. A recent very simplified sedimentation model that treated the settling rate as if it had a first-order dependence on solids concentration was described by Renko $(1996,1998)$. The model (also presented in Section 2.0, Eq. (2.18)) can be expressed in terms of the height, $z$, of the suspension interface at a given time:

$$
\frac{Z}{z_{0}}=\frac{C \phi_{0}}{\alpha}+\left(1-\frac{C \phi_{0}}{\alpha}\right) e^{-\frac{\alpha}{\phi_{0} z_{0}} t}
$$

The model does not account for the portion of the solids that have finished settling, but makes the approximating assumption that all solids are still in suspension at a uniform solids volume fraction, $\phi$, that increases with time. Thus Eq. (3.8) can be rearranged to give $\phi$ as a function of time:

$$
\phi=\left[\frac{C}{\alpha}+\left(\frac{1}{\phi_{0}}-\frac{C}{\alpha}\right) e^{-\frac{\alpha}{\phi_{0} z_{0}} t}\right]^{-1}
$$

Taking the derivative of Eq. (3.8) with time gives the interface velocity, $V$ :

$$
V=\left(\frac{\alpha}{\phi_{0}}-C\right) e^{-\frac{\alpha}{\phi_{0} z_{0}} t}
$$

(a) Some sources refer to the reference solids fraction that appears in the hindered settling equation as a maximum packing factor, but this invites confusion with the packing factor of the settled solids. The two parameters have their origin in different processes and so are not necessarily the same. 
where $z_{0}=$ initial interface height

$\phi_{0}=$ initial solid-phase volume fraction in the well-mixed suspension

$C=$ adjustable constant found by fitting the model, units of velocity

$\alpha=$ adjustable constant found by fitting the model, units of velocity

$t=$ time

The settling properties of the system, as described by the model above, are the following:

- The time constant for settling, $\tau_{s}$, is equal to $\phi_{0} z_{0} / \alpha$. The term $\alpha / \phi_{0}$ is a characteristic velocity related to the settling velocity of the particles that make up the interface. Barring any effects of the container dimensions or of hindered velocity, if the same solids were tested in the same liquid but at different initial concentrations, the settling velocity would be the same in all the tests, and $\alpha$ would presumably be proportional to $\phi_{0}$.

- The final solids volume fraction as time goes to infinity, $\phi_{f}$, is $\alpha / C$. As noted by MacLean (1999), the final solids volume fraction is a function of the total depth of the settled solids layer because a deeper layer weighs more and compacts itself further under its own weight. Because the depth of settled solids is a function of $z_{0}$ and $\phi_{0}, C$ can depend on both these properties. However, the dependence of $C$ on the depth of settled solids may not be fully apparent over the few-day period and small bed depth of most settling tests.

- The initial interface velocity, $V_{0}$, is equal to $\left(\alpha / \phi_{0}-C\right)$.

At the outset of gravity settling, the interface is usually observed to descend in a fast linear manner that is consistent with a nearly constant velocity. In addition, during the period when the almost-completely settled sediment is gradually compacted under its own weight, the slow interface motion is not constant-velocity but, for practical purposes, can be treated as such. These "fast" and "slow" interface velocities can be expressed in terms of the initial velocity, $V_{0}$, that is derived from Eq. (3.8). Although the exponential formulation of the Renko sedimentation model is not mathematically consistent with a constant velocity at any point in time, the "fast" and "slow" velocities provide a reasonable representation of the observed near-constant velocities at beginning and end. Figure 3.63 illustrates the model and its relationship to the fast and slow velocities calculated from it. 


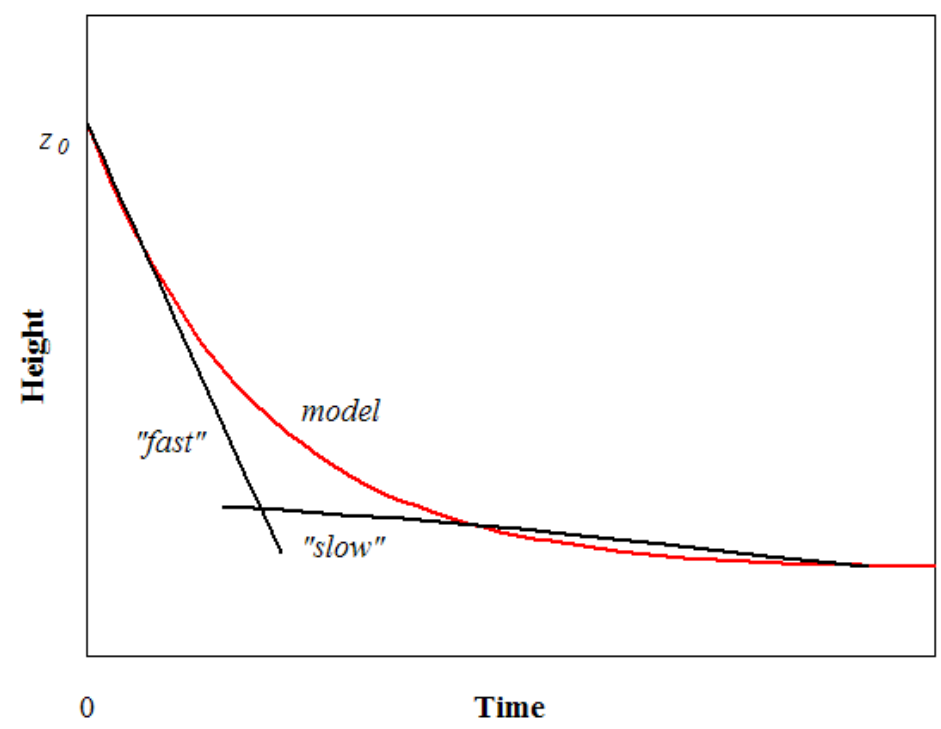

Figure 3.63. Sedimentation Model and Fast and Slow Velocities Based on It

The early fast stage of settling can be defined as that which takes place during the period over which the velocity decreases by $30 \%$. The average velocity calculated over this period is within $\sim 20 \%$ of the instantaneous velocity and therefore is approximately constant. By this definition, the average velocity of the fast portion of settling is $84.1 \%$ of $V_{0}$. During the period when the fast velocity is applicable, which is equal to $35.7 \%$ of the time constant, $\tau_{s}$, the interface descends from $z_{0}$ to $\left(0.3 \phi_{0} / \phi_{f}+0.7\right) z_{0}$, and the instantaneous solids concentration increases from $\phi_{0}$ to $\phi_{0} /\left(0.3 \phi_{0} / \phi_{f}+0.7\right)$.

The slow stage of settling can be defined as that which occurs after the interface level is $90 \%$ of the way to its final value. The time required to reach this point is $2.303 \tau_{s}$. At this time, the velocity is $10 \%$ of its initial value; it will drop down to $1 \%$ of the initial value (or effectively zero velocity) at $4.605 \tau_{s}$. The slow velocity is $3.9 \%$ of $V_{0}$.

A different empirically based model was described and used by MacLean (1999). MacLean defined a universal settling curve (in plotted rather than analytical form) in terms of dimensionless height and time. The non-dimensionalizing parameters were the rapid velocity observed during settling of a suspension dilute enough to have a constant interface velocity during early settling, the initial height of the suspension, and the final sediment height estimated from the maximum solids concentration produced by centrifuging at the slowest possible rate. The settling data on which the universal curve was based were primarily obtained from using a $\mathrm{Fe}(\mathrm{OH})_{3} / \mathrm{Al}(\mathrm{OH})_{3}$ simulant in $100-\mathrm{mL}, 500 \mathrm{~mL}$, and 2-L graduated cylinders, and in a column of about $30 \mathrm{ft}$ height and $1 \mathrm{ft}$ diameter. A multi-component precipitated-hydroxide simulant was also used in the graduated-cylinder-scale tests. The liquid had a $\mathrm{pH}$ of about 12 and a sodium concentration of about $5 \mathrm{M}$ in most of these simulants.

A third modeling approach was used in another set of modeling studies of waste settling (Brooks et al. 1997, 1998, 1999). The models used in those studies accounted for two stages of interface motion, hindered settling and sediment self-compression. The hindered settling mechanism given by Eq. (2.17) was applied at solids concentrations less than the gel point. The first term of the equation accounts for the effect of hydrodynamic interactions between settling particles on the slip velocity of particles (the liquid 
velocity at the particle surface). The second term accounts for the upflow of displaced liquid, i.e., the difference between the slip velocity and the velocity relative to a fixed frame of reference. The sediment self-compression regime applied to interface motion at solids concentrations greater than the gel point. Here the interface velocity was governed by the sediment weight and the compressive yield stress of the sediment aggregate network. Data from centrifuged samples, as well as gravity-settled samples, were used to determine the self-compression parameters. This more complex approach is potentially useful; however, the simpler model in Eq. (3.8) is used in this study because of the limitations of the available data.

\subsubsection{Settling Velocity Data Analysis}

The previous study (Poloski et al. 2007) included 58 sets of sedimentation data and each set was fitted to Eq. (3.8). The authors noted that values for the initial interface height $\left(z_{0}\right)$ were unknown for many of the data because the reports only gave volume (or vol\%) rather than height. Because most tests were performed in small graduated cylinders or graduated centrifuge cones with a total volume of 20 to $50 \mathrm{~mL}$, Poloski et al. assumed $z_{0}=10 \mathrm{~cm}$ in all calculations.

The present study makes different assumptions for the initial interface height and therefore includes new fits of Eq. (3.8) for the same 58 sets of sedimentation data considered by Poloski et al. The present study also adds model fits for 35 more data sets that have been generated or located since 2007 (Lumetta et al. 2009a, Snow et al. 2009, Fiskum et al. 2008, Edwards et al. 2009, Fiskum et al. 2009a, Callaway 2000, Warrant 2002, 2004, Gray et al. 1993). Among the new data are laboratory settling data from the M12 project and in-tank data for the settling test carried out in AZ-101 after mixing pump tests were complete.

As was done for the Poloski et al. study, data were selected only from tests where caustic leaching had not been performed and where measurements of suspension interface level were made. Gauglitz et al. (2009) list four tanks in which sedimentation was observed-AZ-101, AY-102, SY-101, and SY-102. However, AZ-101 was the only one for which the suspension interface level was measured; therefore, it is the only in-tank settling test that is included in the present model-fitting analysis.

In the present study, the values for initial sediment height, $z_{0}$, were chosen as follows for the laboratory settling tests:

1. Measured values of $z_{0}$ were the first choice, when available.

2. In those of the 58 previously used data sets where height data were lacking, it was assumed that the test container was the smallest standard size of graduated cylinder or centrifuge tube that would hold the initial volume of suspension. The initial height $z_{0}$ was calculated from the initial suspension volume and the diameter of the container. While variation in the dimensions of containers makes this approach approximate, values of $z_{0}$ should be closer, in cases where relatively large volumes of suspension were tested, than the previous assumption of $10 \mathrm{~cm} .{ }^{\text {(a) }}$

(a) A 100-mL graduated cylinder (inner diameter $2.5 \mathrm{~cm}$ ) was assumed for suspension volumes between 50 and $100 \mathrm{~mL}$; a 50-mL graduated cylinder (ID $2.0 \mathrm{~cm}$ ) was assumed for suspension volumes between 25 and $50 \mathrm{~mL}$; a 25-mL graduated cylinder (ID $1.6 \mathrm{~cm}$ ) was assumed for suspension volumes between 15 and $25 \mathrm{~mL}$; and a $15-\mathrm{mL}$ centrifuge tube (ID $1.5 \mathrm{~cm}$ ) was used for volumes less than $15 \mathrm{~mL}$. 
3. Some of the new data sets included sediment volume data for all times, but only had height data for times later than $t=0$. The initial height was calculated from the initial volume, using the height/volume ratio from later data.

4. In other new data sets, neither initial volume nor initial height were given. The model was fit with initial height as a third adjustable parameter.

Each data set was reviewed for signs of an initial delay in interface movement. Recall that the interface velocity is governed by the particles with the slowest settling velocities. If these particles are so small or low in density that their settling velocity is imperceptible until they form aggregates, then a delay can occur before the interface descends (suggested by MacLean 1999). The subsequent motion is then governed by the aggregate settling velocity. An initial delay may also occur when the upward velocity of liquid caused by rapid settling of a large volume fraction of particles is greater than the settling velocity of other particles (Dorrell and Hogg 2010). Under those circumstances, the slower particles may not begin to settle until the rapid ones have completed settling. In data sets where an initial delay was evident, the model was fit to the part of the data set after motion had started.

For the laboratory settling tests, the initial well-mixed solid volume fraction, $\phi_{0}$, was calculated from estimated solid-phase densities and from measured physical properties data that provided the mass fraction of UDS. Since there were no direct measurements of solid-phase density, the densities estimated from chemical modeling of the 2002 BBIs in the tanks were used (see Section 3.2.3.2).

The $z_{0}$ and $\phi_{0}$ values for the AZ-101 in-tank settling test were determined from information reported by Wells and Ressler (2009). At the end of mixer pump tests in AZ-101, all the solids in the tank had been mobilized, but only $32 \%$ of the solids had been suspended. In the present study, both $\phi_{0}$ and $z_{0}$ were assigned values that accounted for incomplete solids suspension. The volume fraction of the suspended solids was assumed to have been uniform. The initial interface height was set not at the elevation above the tank bottom, but at the elevation above the top of the unsuspended solids layer, which was assumed to have been $68 \%$ of the 17.5 -in. layer present before mixing.

Table 3.30 provides the model input parameters $\left(z_{0}\right.$ and $\left.\phi_{0}\right)$, the initial delay in interface motion, the dominant waste type in the tank(s) from which solids were taken, the extent of dilution of the sample, the fitted parameters ( $\alpha$ and $C$ ), and the final settled solids volume fraction $(\alpha / C)$ and "fast" interface velocity calculated from the fitted parameters. The fitted parameters are found by using the Solver module in Excel ${ }^{\circledR}$ to minimize the sum of the squares of the prediction errors, subject to the constraint that $\alpha>0$. The data sets in the table are arranged in groups by waste type. Within each group, the data are sorted by tank, and for each tank, the data are arranged in order of decreasing $\phi_{0}$. All tests were conducted at ambient temperature except for the few that are noted in the table. The $\mathrm{R}^{2}$ values for all the fits were greater than $94 \%$, except as noted. ${ }^{\text {(a) }}$

$\left({ }^{a}\right)$ The measure of correlation fit $\mathrm{R}^{2}$, which is calculated as 1 - (Sum of squares of prediction errors)/(Sum of squares of total errors). Here the prediction error is defined as (predicted - measured) and the total error is defined as (measured - average of measurements). 
Table 3.30. Results of Settling Data Analysis

\begin{tabular}{|c|c|c|c|c|c|c|c|c|c|}
\hline Tank & $\begin{array}{c}\text { Primary and } \\
\text { Secondary Waste } \\
\text { Types } \\
\end{array}$ & Dilution & $\begin{array}{c}z_{0} \\
(\mathbf{m m})\end{array}$ & $\begin{array}{c}\phi_{0} \\
\text { (vol\%) }\end{array}$ & $\begin{array}{c}\text { Delay } \\
\text { (hr) }\end{array}$ & $\begin{array}{c}\alpha \\
(\mathbf{m m} / \mathbf{h r})\end{array}$ & $\begin{array}{c}C \\
(\mathbf{m m} / \mathbf{h r}) \\
\end{array}$ & $\begin{array}{l}\text { Final } \\
\text { Solids } \\
\text { (vol\%) }\end{array}$ & $\begin{array}{c}\text { "Fast" } \\
\text { Interface } \\
\text { Velocity } \\
\text { (mm/hr) }\end{array}$ \\
\hline AN-104 settled solids ${ }^{(\mathrm{a}, \mathrm{e})}$ & A2 saltcake & none & 99.5 & 7.1 & $\mathrm{n} / \mathrm{d}$ & 1.64 & 19.7 & 8.3 & 2.9 \\
\hline AN-104 composite ${ }^{(a)}$ & A2 saltcake & none & 95.5 & 3.2 & $\mathrm{n} / \mathrm{d}$ & 1.12 & 19.8 & 5.6 & 13 \\
\hline AN-104 composite ${ }^{(a)}$ & A2 saltcake & DI water; 50 vol\% & 148 & 0.88 & $\mathrm{n} / \mathrm{d}$ & 1.01 & 10.9 & 9.2 & 88 \\
\hline AN-104 settled solids ${ }^{(a)}$ & A2 saltcake & DI water; 80 vol\% & 99.3 & 0.37 & $\mathrm{n} / \mathrm{d}$ & 0.140 & 6.07 & 2.3 & 27 \\
\hline AN-105 settled solids ${ }^{(\mathrm{f})}$ & A2 saltcake & none & 124 & 11 & $\mathrm{n} / \mathrm{d}$ & 1.23 & 4.91 & 25 & 5.7 \\
\hline AN-105 composite ${ }^{(\mathrm{a}, \mathrm{e})}$ & A2 saltcake & none & 99.5 & 8.2 & $\mathrm{n} / \mathrm{d}$ & 0.791 & 4.96 & 16 & 4.0 \\
\hline AN-105 settled solids ${ }^{(f)}$ & A2 saltcake & DI water; 80 vol\% & 124 & 4.2 & $\mathrm{n} / \mathrm{d}$ & 0.670 & 2.22 & 30 & 11 \\
\hline AN-105 composite ${ }^{(\mathrm{a}, \mathrm{f})}$ & A2 saltcake & DI water; 50 vol\% & 92.9 & 0.72 & $\mathrm{n} / \mathrm{d}$ & 0.447 & 7.40 & 6.0 & 46 \\
\hline AY-102 composite ${ }^{(\mathrm{c})}$ & $\mathrm{BL}$ & none & 181 & 6.3 & 0 & 1.36 & 12.8 & 11 & 7.3 \\
\hline AY-102 composite ${ }^{(c)}$ & $\mathrm{BL}$ & none & 179 & 5.1 & 0 & 0.946 & 10.8 & 8.8 & 6.4 \\
\hline $\begin{array}{l}\text { AZ-101 composite } \\
\text { (1993) }\end{array}$ & P3 & $70 \mathrm{wt} \%$ supernatant & 45.8 & 9.2 & 0 & 0.412 & 1.72 & 24 & 2.3 \\
\hline $\begin{array}{l}\text { AZ-101 composite } \\
\text { (1993) }\end{array}$ & P3 & $70 \mathrm{wt} \%$ supernatant & 46.2 & 9.2 & 0 & 0.238 & 0.860 & 28 & 1.5 \\
\hline $\begin{array}{l}\text { AZ-101 composite } \\
\text { (1993) }\end{array}$ & P3 & $90 \mathrm{wt} \%$ supernatant & 47.1 & 3.0 & 0 & 0.140 & 1.13 & 12 & 2.9 \\
\hline $\begin{array}{l}\text { AZ-101 composite } \\
\text { (1993) }\end{array}$ & P3 & $90 \mathrm{wt} \%$ supernatant & 46.5 & 3.0 & 0 & 0.123 & 0.957 & 13 & 2.6 \\
\hline $\begin{array}{l}\text { AZ-101 composite } \\
\text { (2000) }\end{array}$ & P3 & none & 188 & 1.3 & 0 & 1.54 & 18.7 & 8.2 & 86 \\
\hline $\begin{array}{l}\text { AZ-101 composite } \\
\text { (2000) }\end{array}$ & P3 & none & 188 & 1.2 & 0 & 1.57 & 17.2 & 9.1 & 96 \\
\hline $\begin{array}{l}\text { AZ-101, in-tank } \\
\text { settling }\end{array}$ & P3 & none & 8054 & 0.43 & 0 & 14.4 & 180 & 8.0 & 2670 \\
\hline AZ-102 composite & P3 & none & 190 & 2.4 & 0 & 4.15 & 48.8 & 8.5 & 104 \\
\hline AZ-102 composite & P3 & none & 190 & 2.4 & 0 & 4.81 & 54.3 & 8.9 & 123 \\
\hline B-202 Seg. 4 & 224 Post-1949 & DI water; $1: 1$ by vol. & 63.9 & 2.3 & 0.8 & 0.239 & 8.17 & 2.9 & 1.7 \\
\hline B-202 Seg. 6 & 224 Post-1949 & DI water; $1: 1$ by vol. & 69.0 & 2.3 & 1 & 0.254 & 9.66 & 2.6 & 1.2 \\
\hline B-202 Seg. 4 & 224 Post-1949 & DI water; $1: 1$ by vol. & 67.3 & 2.1 & 0.8 & 0.282 & 10.9 & 2.6 & 2.2 \\
\hline
\end{tabular}


Table 3.30. (contd)

\begin{tabular}{|c|c|c|c|c|c|c|c|c|c|}
\hline Tank & $\begin{array}{c}\text { Primary and } \\
\text { Secondary Waste } \\
\text { Types }\end{array}$ & Dilution & $\begin{array}{c}z_{0} \\
(\mathbf{m m})\end{array}$ & $\begin{array}{c}\phi_{0} \\
\text { (vol\%) }\end{array}$ & $\begin{array}{l}\text { Delay } \\
\text { (hr) }\end{array}$ & $\begin{array}{c}\alpha \\
(\mathbf{m m} / \mathbf{h r})\end{array}$ & $\begin{array}{c}C \\
(\mathbf{m m} / \mathbf{h r})\end{array}$ & $\begin{array}{l}\text { Final } \\
\text { Solids } \\
\text { (vol\%) }\end{array}$ & $\begin{array}{c}\text { "Fast” } \\
\text { Interface } \\
\text { Velocity } \\
\text { (mm/hr) }\end{array}$ \\
\hline B-202 Seg. 2 & 224 Post-1949 & DI water; $1: 1$ by vol. & 66.8 & 2.0 & 0.8 & 0.240 & 9.23 & 2.6 & 2.2 \\
\hline B-202 Seg. 6 & 224 Post-1949 & DI water; $1: 1$ by vol. & 70.2 & 2.0 & 0.8 & 0.298 & 12.7 & 2.3 & 1.7 \\
\hline B-202 Seg. 2 & 224 Post-1949 & DI water; $1: 1$ by vol. & 71.9 & 2.0 & 0.8 & 0.246 & 9.35 & 2.6 & 2.4 \\
\hline B-202 Seg. 2 & 224 Post-1949 & DI water; 3:1 by vol. & 62.2 & 1.0 & 0 & 0.401 & 15.7 & 2.6 & 20 \\
\hline B-202 Seg. 6 & 224 Post-1949 & DI water; 3:1 by vol. & 69.0 & 0.95 & 0 & 0.264 & 13.7 & 1.9 & 12 \\
\hline B-202 Seg. 6 & 224 Post-1949 & DI water; 3:1 by vol. & 68.5 & 0.88 & 0 & 0.218 & 11.8 & 1.9 & 11 \\
\hline B-202 Seg. 4 & 224 Post-1949 & DI water; 3:1 by vol. & 69.0 & 0.79 & 0 & 0.295 & 15.0 & 2.0 & 19 \\
\hline B-202 Seg. 4 & 224 Post-1949 & DI water; 3:1 by vol. & 69.0 & 0.79 & 0 & 0.232 & 11.7 & 2.0 & 15 \\
\hline B-202 Seg. 2 & 224 Post-1949 & DI water; 3:1 by vol. & 63.4 & 0.76 & 0 & 0.428 & 22.9 & 1.9 & 28 \\
\hline B-203 composite & 224 Post-1949 & DI water; $1: 1$ by mass & 75.3 & 2.7 & 1 & 0.184 & 6.02 & 3.1 & 0.68 \\
\hline B-203 composite & 224 Post-1949 & DI water; $4: 1$ by mass & 73.6 & 1.0 & 0 & 0.930 & 39.3 & 2.4 & 43 \\
\hline T-203 composite & 224 Post-1949 & DI water; $1: 1$ by mass & 77.5 & 2.0 & 2 & 0.118 & 5.35 & 2.2 & 0.36 \\
\hline T-203 composite & 224 Post-1949 & DI water; $4: 1$ by mass & 75.8 & 0.67 & 0 & 0.317 & 21.9 & 1.4 & 21 \\
\hline T-204 composite & 224 Post-1949 & DI water; $1: 1$ by mass & 77.0 & 1.1 & 1 & 0.0539 & 4.22 & 1.3 & 0.48 \\
\hline T-204 composite & 224 Post-1949 & DI water; $4: 1$ by mass & 74.1 & 0.44 & 0 & 0.589 & 68.0 & 0.87 & 56 \\
\hline BX-107 Seg. 4 & $1 \mathrm{C}$ & DI water; $1: 1$ & 204 & 2.7 & $\mathrm{n} / \mathrm{d}$ & 1.13 & 28.4 & 4.0 & 12 \\
\hline BX-107 Seg. 2 & $1 \mathrm{C}$ & DI water; $1: 1$ & 204 & 2.6 & $\mathrm{n} / \mathrm{d}$ & 1.49 & 37.5 & 4.0 & 17 \\
\hline BX-107 Seg. 6 & $1 \mathrm{C}$ & DI water; $1: 1$ & 204 & 2.2 & $\mathrm{n} / \mathrm{d}$ & 1.03 & 25.2 & 4.1 & 18 \\
\hline BX-107 Seg. 4 & $1 \mathrm{C}$ & DI water; 3:1 & 204 & 0.48 & $\mathrm{n} / \mathrm{d}$ & 0.541 & 42.6 & 1.3 & 59 \\
\hline BX-107 Seg. 2 & $1 \mathrm{C}$ & DI water; 3:1 & 204 & 0.46 & $\mathrm{n} / \mathrm{d}$ & 0.598 & 53.0 & 1.1 & 64 \\
\hline BX-107 Seg. 6 & $1 \mathrm{C}$ & DI water; 3:1 & 204 & 0.40 & $\mathrm{n} / \mathrm{d}$ & 0.347 & 27.4 & 1.3 & 50 \\
\hline C-107 composite & $\begin{array}{c}54 \text { vol\% } 1 \text { 1C, } 36 \text { vol\% } \\
\text { SRR }\end{array}$ & $\begin{array}{c}\text { DI water; } 140 \mathrm{~g} \\
\text { UDS/L }\end{array}$ & 192 & 8.1 & 0 & 2.85 & 11.9 & 24 & 20 \\
\hline C-107 composite & $\begin{array}{c}54 \text { vol\% 1C, } 36 \text { vol\% } \\
\text { SRR }\end{array}$ & $\begin{array}{c}\text { DI water; } 140 \mathrm{~g} \\
\text { UDS/L }\end{array}$ & 187 & 7.6 & 0 & 1.25 & 8.74 & 14 & 6.5 \\
\hline C-107 composite & $\begin{array}{c}54 \text { vol\% 1C, } 36 \text { vol\% } \\
\text { SRR }\end{array}$ & $\begin{array}{c}\text { DI water; } 100 \mathrm{~g} \\
\text { UDS/L }\end{array}$ & 192 & 5.4 & 0 & 1.93 & 8.86 & 22 & 22 \\
\hline
\end{tabular}


Table 3.30. (contd)

\begin{tabular}{|c|c|c|c|c|c|c|c|c|c|}
\hline Tank & $\begin{array}{c}\text { Primary and } \\
\text { Secondary Waste } \\
\text { Types }\end{array}$ & Dilution & $\begin{array}{c}z_{0} \\
(\mathbf{m m})\end{array}$ & $\begin{array}{c}\phi_{0} \\
(\mathrm{vol} \%)\end{array}$ & $\begin{array}{l}\text { Delay } \\
\text { (hr) }\end{array}$ & $\begin{array}{c}\alpha \\
(\mathbf{m m} / \mathbf{h r})\end{array}$ & $\begin{array}{c}C \\
(\mathbf{m m} / \mathbf{h r})\end{array}$ & $\begin{array}{l}\text { Final } \\
\text { Solids } \\
\text { (vol\%) }\end{array}$ & $\begin{array}{c}\text { "Fast" } \\
\text { Interface } \\
\text { Velocity } \\
\text { (mm/hr) }\end{array}$ \\
\hline C-107 composite & $\begin{array}{c}54 \text { vol\% 1C, } 36 \text { vol\% } \\
\text { SRR }\end{array}$ & $\begin{array}{c}\text { DI water; } 100 \mathrm{~g} \\
\text { UDS/L }\end{array}$ & 190 & 5.1 & 0 & 1.58 & 12.4 & 13 & 16 \\
\hline C-107 composite & $\begin{array}{c}54 \text { vol\% 1C, } 36 \text { vol\% } \\
\text { SRR }\end{array}$ & $\begin{array}{c}\text { DI water; } 60 \mathrm{~g} \\
\text { UDS/L }\end{array}$ & 190 & 3.5 & 0 & 2.49 & 9.57 & 26 & 53 \\
\hline C-107 composite & $\begin{array}{c}54 \text { vol\% 1C, } 36 \text { vol\% } \\
\text { SRR }\end{array}$ & $\begin{array}{c}\text { DI water; } 60 \mathrm{~g} \\
\text { UDS/L }\end{array}$ & 190 & 3.3 & 0 & 1.20 & 9.48 & 13 & 23 \\
\hline C-110 Seg. 2 & $1 \mathrm{C}$ & DI water; $1: 1$ & 204 & 2.5 & 0 & 0.934 & 22.5 & 4.2 & 12 \\
\hline C-110 Seg. $4^{(\mathrm{g})}$ & $1 \mathrm{C}$ & DI water; $1: 1$ & 204 & 1.5 & 0 & 0.349 & 15.8 & 2.2 & 6.9 \\
\hline C-110 Seg. 2 & $1 \mathrm{C}$ & DI water; 3:1 & 204 & 0.47 & 0 & 0.264 & 18.7 & 1.4 & 31 \\
\hline C-110 Seg. 4 & $1 \mathrm{C}$ & DI water; 3:1 & 204 & 0.27 & 0 & 0.159 & 19.7 & 0.81 & 33 \\
\hline Group $1^{(\mathrm{c})}$ & high-Bi 1C and 2C & none & 55.9 & 6.3 & 0 & 0.709 & 9.08 & 7.8 & 1.9 \\
\hline Group $1^{(\mathrm{c})}$ & high-Bi 1C and 2C & none & 62.9 & 4.6 & 0 & 0.621 & 10.7 & 5.8 & 2.4 \\
\hline Group $1^{(\mathrm{c})}$ & high-Bi 1C and 2C & none & 46.4 & 3.8 & 0 & 0.157 & 2.96 & 5.3 & 0.99 \\
\hline $\mathrm{T}-107$ & 85 vol\% 1C & DI water; $1: 1$ & 204 & 7.5 & 0 & 1.21 & 10.9 & 11 & 4.5 \\
\hline $\mathrm{T}-107$ & 85 vol\% $1 \mathrm{C}$ & DI water; 3:1 & 204 & 3.4 & 0 & 2.08 & 21.7 & 9.6 & 33 \\
\hline T-110 composite & 97 vol\% $2 \mathrm{C}$ & $\begin{array}{l}\text { DI water; } 30 \text { wt } \% \\
\text { dilution }\end{array}$ & 77.5 & 6.2 & 4 & 0.128 & 1.74 & 7.3 & 0.27 \\
\hline T-110 composite & 97 vol\% 2C & DI water; $1: 1$ & 77.0 & 2.5 & 0.2 & 0.587 & 10.9 & 5.4 & 10 \\
\hline T-110 composite & 97 vol\% $2 \mathrm{C}$ & DI water; $4: 1$ & 76.4 & 0.98 & 0 & 0.644 & 15.4 & 4.2 & 43 \\
\hline T-111 Seg. $8^{(\mathrm{c})}$ & $\begin{array}{c}55 \text { vol\% 2C, } 45 \% 224 \\
\text { Post-1949 }\end{array}$ & DI water; 1:1 & 66.8 & 1.9 & 0.5 & 0.0350 & 1.37 & 2.6 & 0.41 \\
\hline T-111 Seg. 2 & $\begin{array}{c}55 \text { vol\% 2C, } 45 \% 224 \\
\text { Post- } 1949\end{array}$ & DI water; 3:1 & 67.3 & 0.17 & 0 & 0.0156 & 4.69 & 0.33 & 3.7 \\
\hline T-111 Seg. 2 & $\begin{array}{c}55 \text { vol\% 2C, } 45 \% 224 \\
\text { Post-1949 }\end{array}$ & DI water; 1:1 & 62.2 & 0.12 & 0.5 & 0.00919 & 6.76 & 0.14 & 1.0 \\
\hline C-104 & $\begin{array}{c}57 \text { vol\% CWP, } 15 \% \\
\text { unidentified }\end{array}$ & $\begin{array}{c}\text { DI water; } 140 \mathrm{~g} \\
\text { UDS/L }\end{array}$ & 204 & 5.1 & 0 & 0.840 & 7.83 & 11 & 7.2 \\
\hline C-104 & $\begin{array}{c}57 \text { vol\% CWP, } 15 \% \\
\text { unidentified }\end{array}$ & $\begin{array}{c}\text { DI water; } 100 \mathrm{~g} \\
\text { UDS/L }\end{array}$ & 202 & 3.0 & 0.7 & 0.686 & 8.75 & 7.8 & 12 \\
\hline
\end{tabular}


Table 3.30. (contd)

\begin{tabular}{|c|c|c|c|c|c|c|c|c|c|}
\hline Tank & $\begin{array}{c}\text { Primary and } \\
\text { Secondary Waste } \\
\text { Types } \\
\end{array}$ & Dilution & $\begin{array}{c}z_{0} \\
(\mathbf{m m})\end{array}$ & $\begin{array}{c}\phi_{0} \\
(\mathrm{vol} \%)\end{array}$ & $\begin{array}{l}\text { Delay } \\
\text { (hr) }\end{array}$ & $\begin{array}{c}\alpha \\
(\mathbf{m m} / \mathrm{hr})\end{array}$ & $\begin{array}{c}C \\
(\mathrm{~mm} / \mathrm{hr})\end{array}$ & $\begin{array}{c}\text { Final } \\
\text { Solids } \\
\text { (vol\%) }\end{array}$ & $\begin{array}{c}\text { "Fast" } \\
\text { Interface } \\
\text { Velocity } \\
\text { (mm/hr) }\end{array}$ \\
\hline C-104 & $\begin{array}{c}57 \text { vol\% CWP, } 15 \% \\
\text { unidentified }\end{array}$ & $\begin{array}{c}\text { DI water; } 60 \mathrm{~g} \\
\text { UDS/L }\end{array}$ & 204 & 2.1 & 0 & 0.904 & 9.31 & 9.7 & 28 \\
\hline Group 3 & high-Al CWP & none & 60.7 & 18 & 0 & 18.1 & 63.7 & 28 & 31 \\
\hline Group 3 & high-Al CWP & none & 79.7 & 17 & 0 & 20.5 & 70.2 & 29 & 42 \\
\hline Group 3 & high-Al CWP & none & 71.8 & 16 & 0 & 19.5 & 71.1 & 27 & 41 \\
\hline T-102 Seg. $2^{\text {(d) }}$ & 89 vol\% CWP2 & DI water; $1: 1$ & 204 & 4.7 & 0 & 21.0 & 76.5 & 28 & 314 \\
\hline T-102 Seg. $2^{\text {(d) }}$ & 89 vol\% CWP2 & DI water; 3:1 & 204 & 2.1 & 0 & 9.49 & 45.8 & 21 & 347 \\
\hline C-109 & $\begin{array}{c}38 \text { vol\% TFeCN, 36\% } \\
\text { CWP1 }\end{array}$ & DI water; $1: 1$ & 204 & 3.5 & 0 & 1.18 & 29.0 & 4.1 & 3.7 \\
\hline C-109 & $\begin{array}{c}38 \text { vol\% TFeCN, 36\% } \\
\text { CWP1 }\end{array}$ & DI water; 3:1 & 204 & 2.2 & 0 & 1.67 & 32.9 & 5.1 & 37 \\
\hline C-112 composite & 69 vol\% TFeCN & DI water; 1:1 & 204 & 5.9 & 0 & 0.350 & 3.97 & 8.8 & 1.6 \\
\hline C-112 composite & 69 vol\% TFeCN & DI water; 3:1 & 204 & 2.2 & 0 & 0.858 & 17.6 & 4.9 & 18 \\
\hline Group $8^{(\mathrm{c})}$ & high-Fe PFeCN & none & 79.2 & 5.6 & 0 & 0.885 & 9.36 & 9.5 & 5.5 \\
\hline Group 8 & high-Fe PFeCN & none & 85.7 & 5.2 & 0 & 2.58 & 26.8 & 9.6 & 19 \\
\hline Group $8^{(\mathrm{c})}$ & high-Fe PFeCN & none & 81.0 & 5.0 & 0 & 1.45 & 16.0 & 9.1 & 11 \\
\hline S-104 Seg. 2 & $\begin{array}{l}47 \text { vol\% R saltcake, } \\
\text { 43\% R1 (boiling) }\end{array}$ & DI water; 1:1 & 204 & 8.7 & 0 & 1.02 & 10.4 & 9.8 & 1.1 \\
\hline S-104 Seg. 2 & $\begin{array}{c}47 \text { vol\% R saltcake, } \\
\text { 43\% R1 (boiling) }\end{array}$ & DI water; 3:1 & 204 & 2.8 & 0 & 0.758 & 17.5 & 4.3 & 8.4 \\
\hline S-104 Seg. 4 & $\begin{array}{l}47 \text { vol\% R saltcake, } \\
\text { 43\% R1 (boiling) }\end{array}$ & DI water; 3:1 & 204 & 2.3 & 0 & 0.506 & 18.4 & 2.7 & 3.3 \\
\hline Group 6 $6^{(\mathrm{c})}$ & high-Cr S saltcake & none & 82.3 & 9.2 & 1 & 1.36 & 10.5 & 13 & 3.5 \\
\hline Group $6^{(\mathrm{c})}$ & high-Cr S saltcake & none & 80.7 & 9.0 & 0.4 & 1.02 & 7.53 & 14 & 3.2 \\
\hline Group $6^{(\mathrm{c})}$ & high-Cr S saltcake & none & 86.0 & 8.9 & 0.8 & 1.29 & 9.65 & 13 & 4.0 \\
\hline Group $7^{(\mathrm{c})}$ & TBP & none & 83.8 & 7.9 & 3 & 0.226 & 2.49 & 9.1 & 0.31 \\
\hline Group 7 & TBP & none & 82.3 & 7.7 & 0.4 & 0.765 & 8.61 & 8.9 & 1.2 \\
\hline Group $7^{(\mathrm{c})}$ & TBP & none & 73.2 & 4.5 & 3 & 0.122 & 2.40 & 5.1 & 0.27 \\
\hline
\end{tabular}


Table 3.30. (contd)

\begin{tabular}{|c|c|c|c|c|c|c|c|c|c|}
\hline Tank & $\begin{array}{c}\text { Primary and } \\
\text { Secondary Waste } \\
\text { Types }\end{array}$ & Dilution & $\begin{array}{c}z_{0} \\
(\mathbf{m m})\end{array}$ & $\begin{array}{c}\phi_{0} \\
(\operatorname{vol} \%)\end{array}$ & $\begin{array}{c}\text { Delay } \\
\text { (hr) }\end{array}$ & $\begin{array}{c}\alpha \\
(\mathrm{mm} / \mathrm{hr})\end{array}$ & $\begin{array}{c}C \\
(\mathrm{~mm} / \mathrm{hr})\end{array}$ & $\begin{array}{l}\text { Final } \\
\text { Solids } \\
\text { (vol\%) }\end{array}$ & $\begin{array}{c}\text { "Fast" } \\
\text { Interface } \\
\text { Velocity } \\
\text { (mm/hr) }\end{array}$ \\
\hline Group 2 & $\begin{array}{l}\text { high-PO4 BY and T } \\
\text { saltcake }\end{array}$ & none & 80.0 & 37 & 1 & 4.36 & 11.2 & 39 & 0.51 \\
\hline Group 2 & $\begin{array}{l}\text { high-PO4 BY and T } \\
\text { saltcake }\end{array}$ & none & 76.2 & 25 & 1 & 1.56 & 5.76 & 27 & 0.47 \\
\hline Group $2^{\text {(c) }}$ & $\begin{array}{l}\text { high-PO4 BY and T } \\
\text { saltcake }\end{array}$ & none & 87.6 & 21 & 0.5 & 1.40 & 5.51 & 25 & 0.99 \\
\hline Group 4 & high-Al CWR & none & 98.4 & 19 & 0 & 26.2 & 73.8 & 36 & 51 \\
\hline Group 4 & high-Al CWR & none & 94.7 & 18 & 0 & 24.6 & 74.1 & 33 & 53 \\
\hline Group 4 & high-Al CWR & none & 95.8 & 18 & 0 & 25.1 & 74.5 & 34 & 55 \\
\hline \multicolumn{10}{|c|}{$\begin{array}{l}\text { n/d: not determined because data were insufficient. } \\
\text { (a) Test was conducted at } 45^{\circ} \mathrm{C} \text {. } \\
\text { (b) Test was conducted at AZ- } 101 \text { in-tank temperature of } 60^{\circ} \mathrm{C} \text { (Carlson et al. 2001, p. 5-17). } \\
\text { (c) Data trends suggest settling was not complete at the test end time, } 72 \mathrm{hr} \text { or more. } \\
\text { (d) Settling was complete by the time the first data point (after the initial one) was taken at } 2 \text { hr; the settling velocity may be greater than indicated here. } \\
\text { (e) } \mathrm{R}^{2} \text { is about } 80 \% \text {. } \\
\text { (f) There were only three data points in this set, so parameters are not well-determined. } \\
\text { (g) } \mathrm{R}^{2} \text { is about } 90 \% \text {. }\end{array}$} \\
\hline
\end{tabular}




\section{AZ-101: Difference Between In-Lab and In-Tank Settling Velocities}

AZ-101 is the only tank in which the motion of the suspension interface has been studied. ${ }^{(a)}$ The interface measurements were made using the suspended solids profiler (a laser-reflectance turbidity probe), gamma-monitoring probes, and ultrasonic interface level analyzer, and were confirmed with grab samples (Carlson et al. 2001). These measurements, which were confirmed by grab-sample analysis, indicated that settling was substantially complete in half a day. The discrepancy between the rapid interface settling in the tank and the slower settling of the visible interfaces in the laboratory studies (Gauglitz et al. 2009, 2010b) raises a significant question about the extent to which laboratory settling data can be scaled up to tank settling. ${ }^{(b)}$

As can be seen in Table 3.30, the in-tank values of fast velocity that were measured in Tank AZ-101 at the end of the mixing-pump tests (Carlson et al. 2001) are more than 20 times greater than those measured in the laboratory test whose initial solids concentration was closest to tank conditions (Callaway 2000). The discrepancy is not merely a feature of the model fit. The average interface velocities over the first $1.5 \mathrm{hr}$, calculated directly from data at $0 \mathrm{hr}$ and $1.5 \mathrm{hr}$, were $6.5 \times 10^{-4} \mathrm{~m} / \mathrm{s}$ for in-tank settling and $2.0 \times 10^{-5} \mathrm{~m} / \mathrm{s}$ for laboratory settling. According to Eq. (2.17), when used with the Stokes' Law exponent of 4.65, hindered settling cannot cause the observed difference in hindered settling velocities between $\phi_{0}=1.2 \mathrm{vol} \%$ (laboratory tests) and $\phi_{0}=0.43 \mathrm{vol} \%$ (tank) unless the $C_{V \max }$ parameter was about 2.0 vol\%, which is quite low compared to the final solids fractions observed in all the tests of AZ-101 waste (Table 3.30). Some effect beyond hindered settling was apparently present.

One speculative explanation of the discrepancy is that different types of particles (with different velocity ranges) were being measured by the instruments in Tank AZ-101 than by the visual measurements in the laboratory. A polydisperse, multiple-density material can produce a number of different interfaces, one of which (the topmost) is visible. Visual interface observations are based on the slowest-settling particles that cause enough turbidity to be seen. The in-tank instruments might have "seen" not the uppermost interface, but the more concentrated interior interface(s) produced by faster settling particles.

The mixed concentration of the suspended solids in AZ-101 for the in situ settling test was approximately 0.43 vol\% solids. This volume concentration approximates the limit of visual detection of turbidity for the waste materials. The suspended solids profiler had a lower calibration bound of $2.48 \mathrm{~g}$ solids per liter, approximately 1 vol\%; although the test concentration was less than the lower calibration

(a) Settling has also been observed in other tanks, including SY-101, SY-102, and AY-102 (Gauglitz et al. 2009). However, the only data available for these tanks are the settled solids levels, not the levels of the upper suspension interfaces. The motion of the settled solids interface is not directly related to the motion of the upper suspension interface because different particle species are involved and compaction rather than settling is the driving mechanism for the settled solids interface. In the cases of the receiver tanks, AY-102 and SY-102, solids were added in several phases. The motion of the settled solids surface was difficult to interpret in that it was caused by the simultaneous compaction of solids from several different additions, each of a different age and a different stage of compaction. AY-102 interface velocity estimates, which are expected to be underestimates, are discussed in a later section.

(b) A dimensionless scaling approach was used by Carlson et al. (2001) to fit the laboratory settling data to the in-tank data. Their model provided a better overall fit to the entire in-tank data set than did the dimensionless model of MacLean et al. (1999). However, the Carlson et al. fit used the initial velocity from the laboratory tests as the in-tank velocity and so did not resolve the discrepancy in early settling. 
limit, the solids were still apparently detectable. The uppermost interface, potentially undetectable via visual observation, might have been composed only of solids whose concentration was too dilute to be measured by the profiler or detected in the grab samples, which instead captured a faster settling category of particles that had a higher concentration.

The same speculation can be made for the ultrasonic instruments, whose calibration range was not stated by Carlson et al. (2001). The gamma detectors were used to measure particles emitting gamma rays above $850 \mathrm{keV}$, such as europium isotopes. These would not necessarily be the same as the naked-eye interface particles. However, the gamma data clearly show that the gamma-ray-emitting particles settled at the rapid rate.

Leaving aside the speculative differences in perceived solids, there were some known differences between the laboratory and in-tank solids. The laboratory settling test was made on a composite of AZ-101 solids, whereas the in-tank settling test included only the estimated 32\% of the solid that was suspended by the mixer pumps. There is no way to tell whether the limited suspension capability of the jet mixer pumps affected the size distribution of the suspended material. One possibility is that only the smallest or least dense particles were suspended —at least, at the time measurements were made-starting approximately 10 minutes after the cessation of a single mixer pump operation. Another is that the jet suspended solids over the whole size and density distribution, but had only enough power to lift $32 \%$ of the mass and mix it through the supernatant liquid. The latter possibility may be supported by the similarity of PSD results for samples from the in situ settling test and tank composites (Figure E.11, Appendix E).

The slowest-settling solids determine the interface velocity. These solids would probably have been present in the suspended 32\% in Tank AZ-101 as well as in the laboratory sample. Supposing they predominated, the in-tank and in-laboratory velocities should have been more similar than they were. On the other hand, that conclusion is only correct if the settling velocity of the predominating slow fraction was independent of the higher velocity material. As will be discussed later, the velocities of different fractions of the solids are unlikely to be independent for a variety of reasons, including particle collision/capture and wake capture.

The size of the vessel is another possible cause of the discrepancy. Other studies of Hanford waste have expressed varying opinions as to the effect of container size on settling velocity. MacLean (1999) found, using caustic iron/aluminum hydroxide suspensions, that the early "fast" velocity of the interface differed relatively little between a $30 \mathrm{ft}$ high, $1 \mathrm{ft}$ diameter column and three sizes of graduated cylinders (2 L, $500 \mathrm{~mL}$, and $100 \mathrm{~mL}$ ). The velocities measured at different sizes were all within $\pm 20 \%$ of the average over all four sizes. However, a neutralized current acid nuclear waste (NCAW) simulant did show an apparent slight decrease of the fast velocity as the graduated-cylinder diameter decreased. Brooks et al. $(1997,1998)$ compared tank-waste settling tests that were conducted at "bench scale" with earlier "laboratory scale" tests ( $\sim 10 \mathrm{~g}$ of waste). The trends in settling velocity versus diameter were not consistent from one waste to another. The authors believed that wall effects and bridging probably decreased the observed settling velocity at "laboratory scale." At "bench scale," tests with kaolin and C-106 simulant showed no difference in interface settling velocity between columns with inner diameters of $10 \mathrm{~cm}$ and $30 \mathrm{~cm}$ (Appendix A, Brooks 1997).

A reading of the literature on settling and sedimentation indicates that possible reasons for the discrepancy between in-tank and in-laboratory interface velocities of AZ-101 solids include both particle 
properties and system properties. First, faster settling can occur as a result of non-spherical particle shapes. The settling rate for non-spherical particles depends on the orientation of the particle with respect to flow. According to Green and Perry (2008), the drag coefficient for cylindrical particles may be as much as one order of magnitude lower than that of a spherical particle of equivalent volume. This translates into a terminal settling velocity, for the cylindrical particle, that is approximately three times the velocity of the spherical particle. It should be noted that the great majority of size distributions measured for tank wastes (such as those given in Section 3.2.5) were determined by laser diffraction, which presents particle size results in terms of the diameters of the spheres of equal volume. As such, higher-than-expected settling rates relative to calculations from the equal-volume spheres may occur because of incorrect assumptions about particle shape.

The shape factor probably has some bearing on the settling behavior of AZ-101 solids. The predominant solids in AZ-101 (based on the estimates made from the $2002 \mathrm{BBI}$ ) were aluminum and iron compounds. Disselkamp (2010) lists gibbsite, boehmite, and hematite as having been observed, and hematite was determined to be the prevalent solid. Boehmite was observed, but not in abundance. As described in Section 3.2.4, hematite and boehmite exhibit very elongated (acicular) particles. The non-spherical shape of major constituents of AZ-101 could help to explain measured in-tank velocities that were higher than estimated velocities based on PSDDs and spherical particles. However, it is not clear that particle shape can explain differences in velocity between interface velocities measured under laboratory conditions and those measured under tank conditions, unless sample handling tended to break long in-tank particles into smaller particles with lower aspect ratios. This explanation seems unlikely since the acicular particles already mentioned were found in "handled" samples.

The interface velocity can also be affected by particle interactions that are partly related to particle properties and partly related to system properties (e.g., shear rates that affect agglomeration).

- Particle agglomeration-yields a composite structure that is larger and less dense than the original primary particles from which the agglomerate was formed. Stokes' Law indicates that the particle settling rate depends on the first power of the difference between the liquid density and the solid (or agglomerate) density and on the square of the particle diameter. As stated in Berg (2001), the formation of agglomerates or flocs may greatly increase the sedimentation rate.

- Particle collision and capture-refers to particle interactions that arise when particles or particle agglomerates settle at different rates, causing an increase in the overall rate of settling for the system. Here, fast settling particles collide with and pull down particles in their settling path that would settle more slowly otherwise. This effect can occur for systems with broad distributions of size and density. It can also occur because of particle agglomeration. With regard to the latter, Berg (2001) states that in some circumstances, "voluminous flocs may be produced which have a sweeping effect as they settle, collecting unaggregated particles and small flocs.”

- Wake capture - refers to entrainment of smaller particles in the wake that is generated by a large settling particle. These small particles are then dragged down with the larger particles, effecting faster settling of the suspension. Lovell and Rose (1991) argue that wake capture was responsible for their test results: the observed settling velocity of sand less than $140 \mu \mathrm{m}$ diameter was increased by $87 \%$ over the estimated single-particle terminal velocity, and larger particles also showed increases. The increase in velocity decreased with particle size. In their tests, with particle Reynolds number $>1$, settling velocity increases occurred at low solids concentration (about 0.6 vol\%). 
The question is whether conditions, or particles, in Tank AZ-101 were different enough from those in the laboratory tests to cause the observed difference in initial velocity. If differences in agglomeration caused the AZ-101 in-tank interface velocity to be higher than that in the laboratory, it would have to be because the agglomerates in the laboratory were smaller. Sample handling could have broken up the agglomerates before they were introduced into the cylinder. Hypothetically, there might have been higher shear rates experienced by agglomerates, on average, within the graduated cylinder because its $2.5 \mathrm{~cm}$ inner diameter put the wall closer to the in-cylinder particle population than the tank wall was to most of the in-tank population. However, this seems unlikely. There was vigorous mixing in Tank AZ-101 immediately before the settling test: the flow velocity through the pumps was approximately $18 \mathrm{~m} / \mathrm{s}$ for a period of time that was equivalent to 72 waste volumes passing through the pumps (Wells and Ressler 2009).

As for particle collision/capture and wake capture, it is not clear whether the tank conditions were more conducive to capture than the laboratory conditions. Agglomerates may have been larger in the tank than in the cylinder, depending on whether the shear caused by pump mixing broke them up. It was previously mentioned that the particles suspended in Tank AZ-101 for the settling test included only part (32\%) of the solids present in the laboratory samples. Since the most rapidly settling particles, the ones more likely to capture fines in their wake, may not have been included among the measured suspended particles in the tank, this mechanism seems inconsistent with the higher settling velocity in the tank.

One more phenomenon, "vertical streaming," has been observed to increase settling velocities in some experiments. This is a convective phenomenon (possibly related to wake capture) where particles migrate laterally into separate vertical columns of rapidly settling particles, which can convect particles downward at up to 10 times the single-particle settling rate. Tong and Ackerson (1998) show that the governing equations for local fluctuations in particle concentration and velocity are the same as those in turbulent buoyant convection of a fluid, implying that similar instability behavior is possible. Lovell and Rose (1991) provide several references to studies where this phenomenon has been observed and state that this had typically been observed at high concentrations (i.e., greater than 15 vol\% particles) and $R>1$. Biesheuvel (2001) presents a method for predicting particle size ratios and density ratios that would lead to convective structures (lateral non-uniformity) in concentrated bidisperse suspensions. These references do not exhaust the literature on the subject. A more complete review might provide a way to gauge whether particle-induced convection is a more important effect in tank or in laboratory equipment.

\section{Effect of Initial Volume Fraction}

In the Renko interface velocity model, Eqs. (3.8) through (3.10), the model fitting parameters $\alpha$ and $C$ are specific to the suspension properties and possibly to the dimensions of the system, and they provide estimates of the interface velocity (from the "fast" step) and the final solids fraction. In the case of AZ-101, there were indications that the fast velocity varied with solid volume fraction. The final solids volume fraction $\phi_{f}=\alpha / C$ was surprisingly close to constant, considering the greater depth of the in-tank solids layer and the fact that only the most easily suspended portion of the tank solids were involved in settling.

This section reviews the fast velocities and final solids fractions (Table 3.30) for laboratory settling tests on some of the wastes from tanks other than AZ-101. The focus is on data for two waste types, 
1C/2C (BP process) and 224 Post-1949, for which a large number of settling tests have been conducted at different initial solids volume fractions. One point to keep in mind is that, where the different volume fractions in the AZ-101 tests were produced by dilution with waste supernatant, the different volume fractions in the $1 \mathrm{C} / 2 \mathrm{C}$ and 224 waste settling tests were generated by adding DI water. This form of dilution probably dissolved some solids and certainly would have changed the $\mathrm{pH}$ and ionic strength of the liquid. As a result, solids concentration effects cannot be separated from composition effects in comparing the parameters.

Figure 3.64 and Figure 3.65 show the variation of "fast" velocity and $\phi_{f}$ with the initial solids volume fraction $\phi_{0}$ of 224 Post-1949 sludge. All of the tests in these figures were carried out to a 72-hr settling time. Figure 3.64 shows a consistent downward trend in the fast velocity as initial solids increase (and water dilution decreases). Hindered settling is thought to be the cause of this downtrend.

MacLean (1999) comments that the test vessel must be tall enough to allow a distinct rapid settling period to develop and that the height required will depend on the suspension properties. A 100-mL graduated cylinder was adequate for the NCAW simulant used in that study, while the caustic $\mathrm{Fe}(\mathrm{OH})_{3} / \mathrm{Al}(\mathrm{OH})_{3}$ slurry required that a cylinder larger than $500 \mathrm{~mL}$ be used. The tests in Figure 3.64 were all carried out in $15-\mathrm{mL}$ centrifuge cones with $z_{0}$ of about $7 \mathrm{~cm}$. The low values of velocity in some of the tests may be related to the shortness of the column, but this theory can only be verified by testing waste samples or simulants with a set of columns of different heights.

The distinct upward trend of $\phi_{f}$ with increasing $\phi_{0}$ (Figure 3.65) is nearly 1:1. Although this suggests the kind of dependence on $\phi_{0}$ that would result from self-compaction and the associated dependence on settled solids bed depth (i.e., on $\phi_{0}$ ), it is surprising that self-compaction, a relatively slow process, could become significant in three days of test duration. 


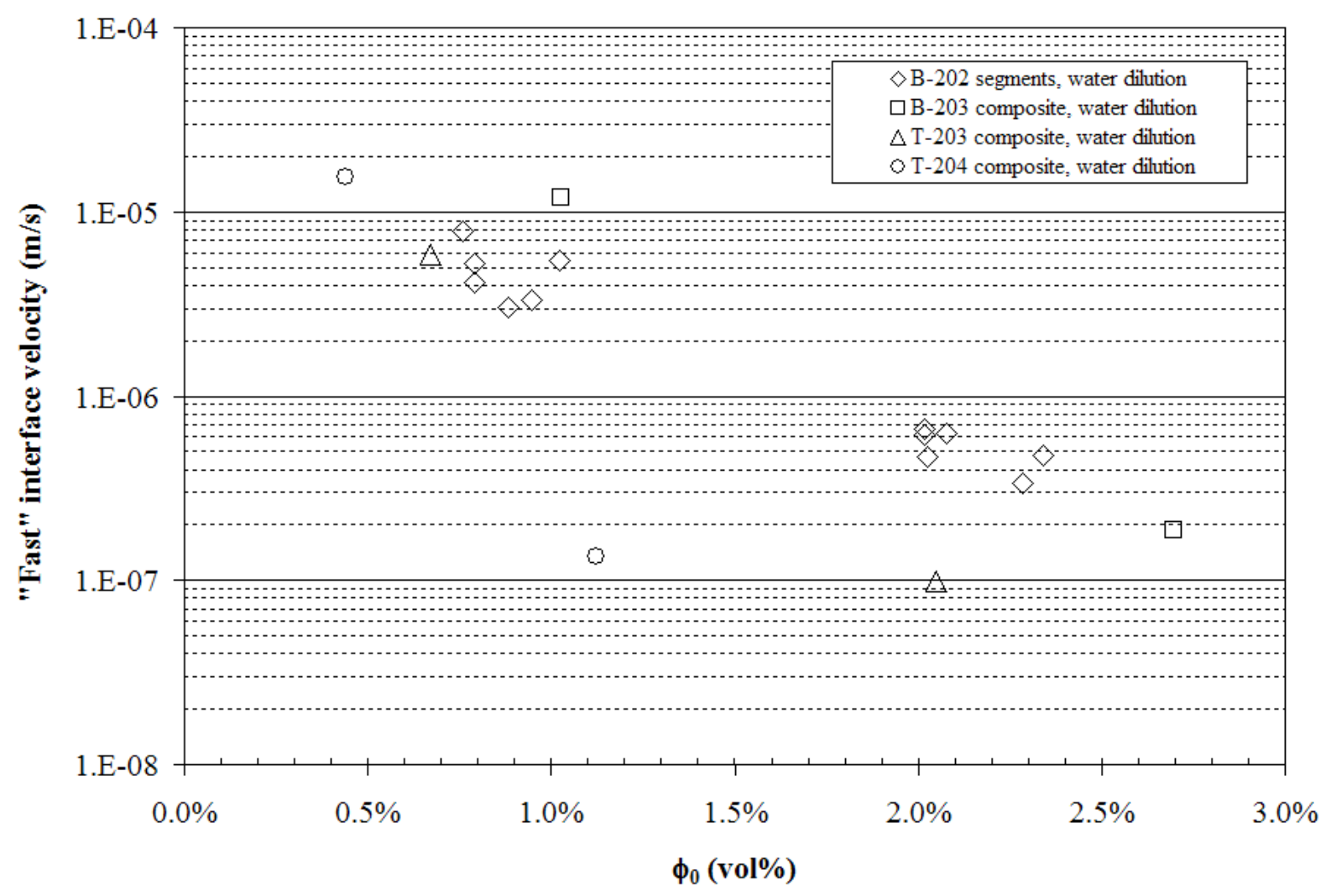

Figure 3.64. Variation of the Fast Velocity with Initial Solids Fraction Concentration in 224 Post-1949 Sludges 


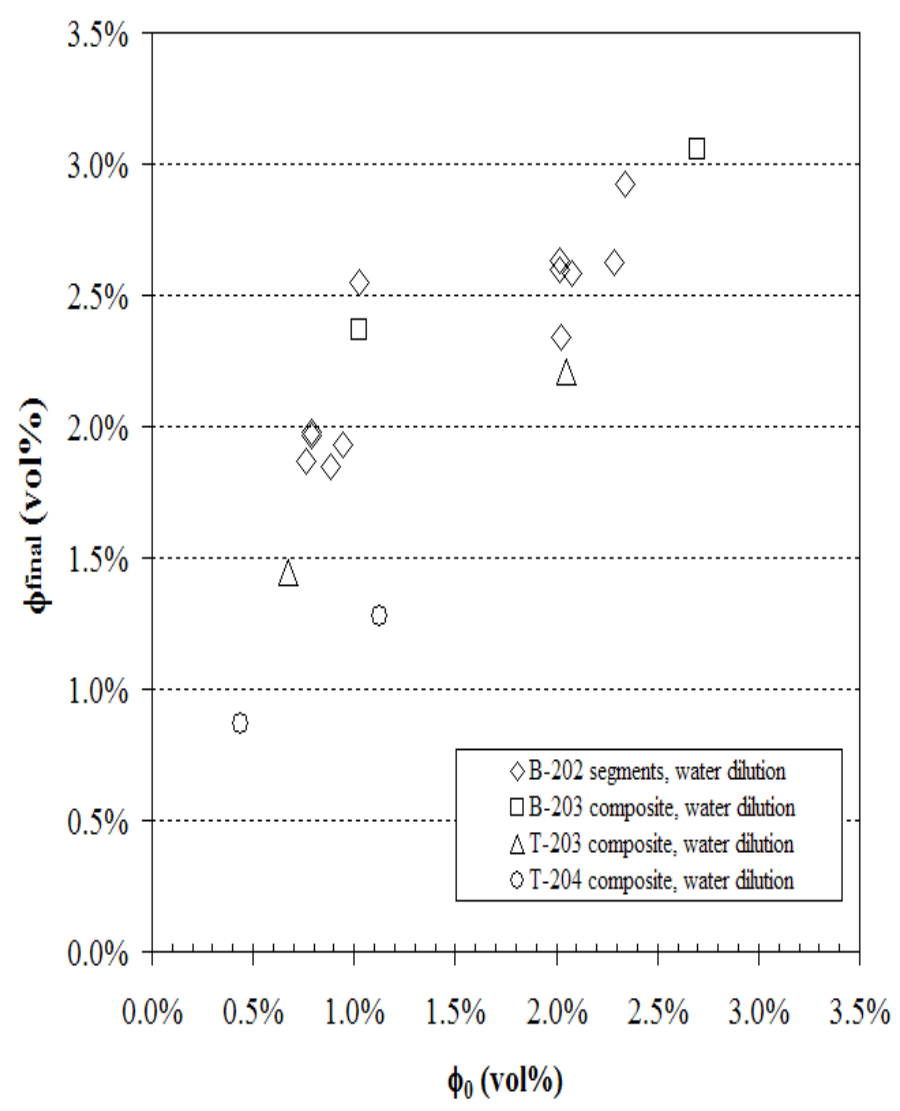

Figure 3.65. Variation of the Final Solids Concentration $\phi_{\text {final }}$ with Initial Solids Fraction Concentration in 224 Post-1949 Sludges

Figure 3.66 and Figure 3.67 show the variation of 1C/2C sludge "fast" velocity and $\phi_{f}$ with $\phi_{0}$. All of the tests in these figures were carried out to at least a 56-hr settling time; the C-107 tests were carried out to $215 \mathrm{hr}$, a significantly longer time that would be expected to affect self-compaction and the final solid volume fraction. The tests also were carried out with different amounts of sample and different initial heights: the Group 1, T-110, and T-111 samples were small, and initial heights were 50 to $80 \mathrm{~mm}$ (corresponding to centrifuge cones), while the other samples were larger and had initial heights of about $200 \mathrm{~mm}$ (corresponding to 100-mL graduated cylinders). 


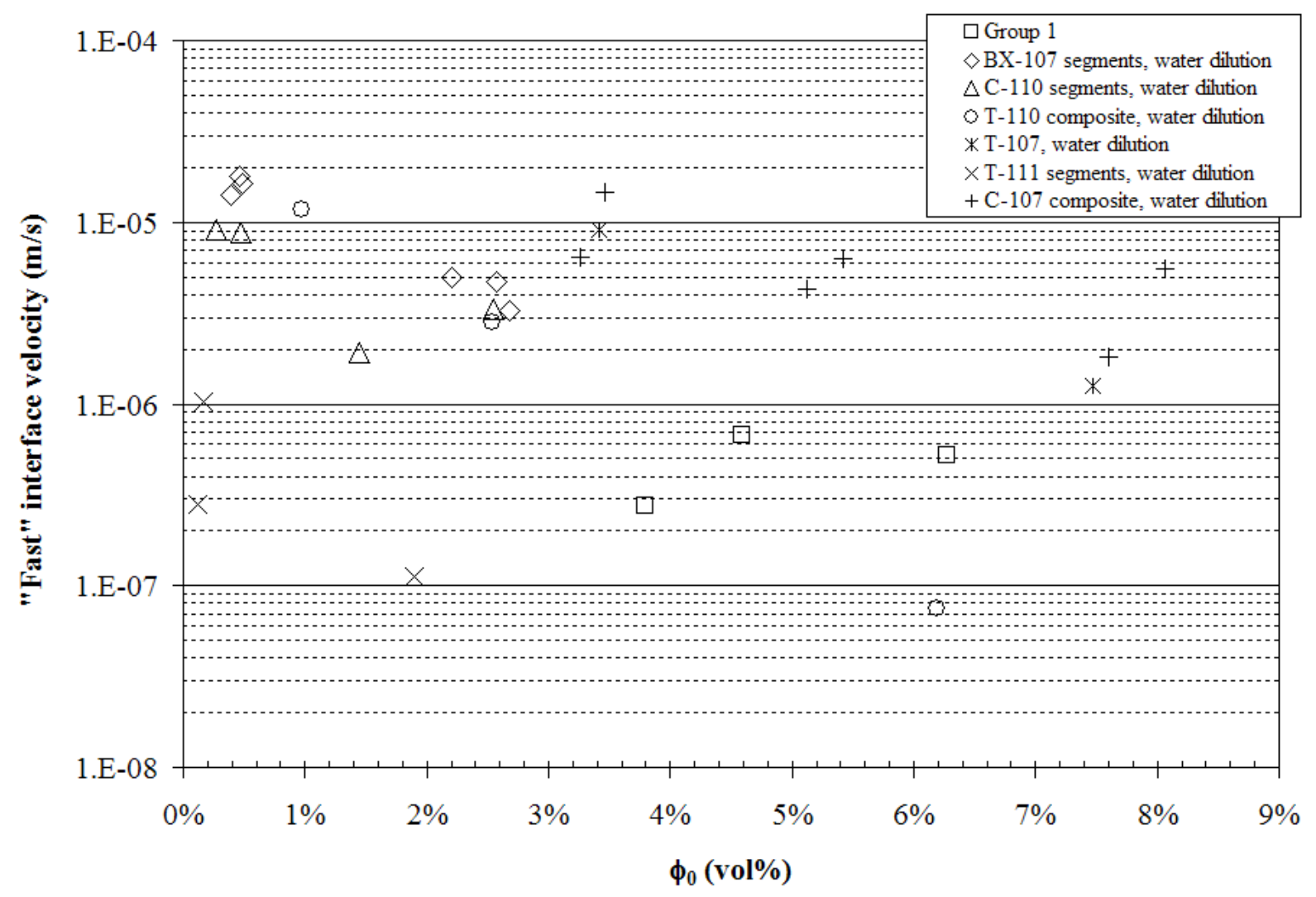

Figure 3.66. Variation of the Fast Velocity with Initial Solids Fraction Concentration in 1C/2C Wastes

In general, the velocities decrease with initial solids fraction, as expected because of hindered settling. As can be seen in Figure 3.66, the T-111 segments, tested with 50- to 70-mm initial height and coming from a tank where waste is 55 vol\% 1C/2C and 45 vol\% 224 Post-1949 waste, have much lower velocity than other samples. These tests may not be representative of $1 \mathrm{C} / 2 \mathrm{C}$ waste. The other tank where the 1C/2C content is relatively low, 54 vol\%, was C-107. For this tank, the secondary waste type was SRR, samples were core composites rather than single segments, and the test was conducted at 200-mm initial height. The velocity values for C-107 differ by core (which is not identified in Figure 3.66 or in Table 3.30).

The wastes that are pure or nearly pure 1C/2C waste are Group 1, BX-107, C-110, T-107, and T-110. When comparing the velocities from the two tests that were carried out with 50- to 70-mm initial height, Group 1 and T-110 composites, to the remainder of the tests that were conducted with 200-mm initial height (BX-107, C-110, and T-107), it is difficult to see a clear distinction. Group 1, with the shorter column, does have some of the lowest velocities. The same cannot be said for all the T-110 measurements, however. The data do not seem to support any conclusions about the effect of column height in the $70-\mathrm{mm}$ to $200-\mathrm{mm}$ range.

Figure 3.67 shows the variation of $\phi_{f}$ with $\phi_{0}$ in $1 \mathrm{C} / 2 \mathrm{C}$ wastes. The increasing trend is evident. Most of the wastes fall along a 1:1 line, as did the 224 Post-1949 waste samples. The T-107 samples show somewhat higher compaction, while C-107 compaction is considerably higher. The much higher $\phi_{f}$ for C-107 might be related to the presence of another waste type, SRR, because the two cores give differing 
results for $\phi_{f}$ as they did for velocity. However, the long test duration (three or four times as long as for any of the other tests plotted) is certainly part of the cause of the greater compaction.

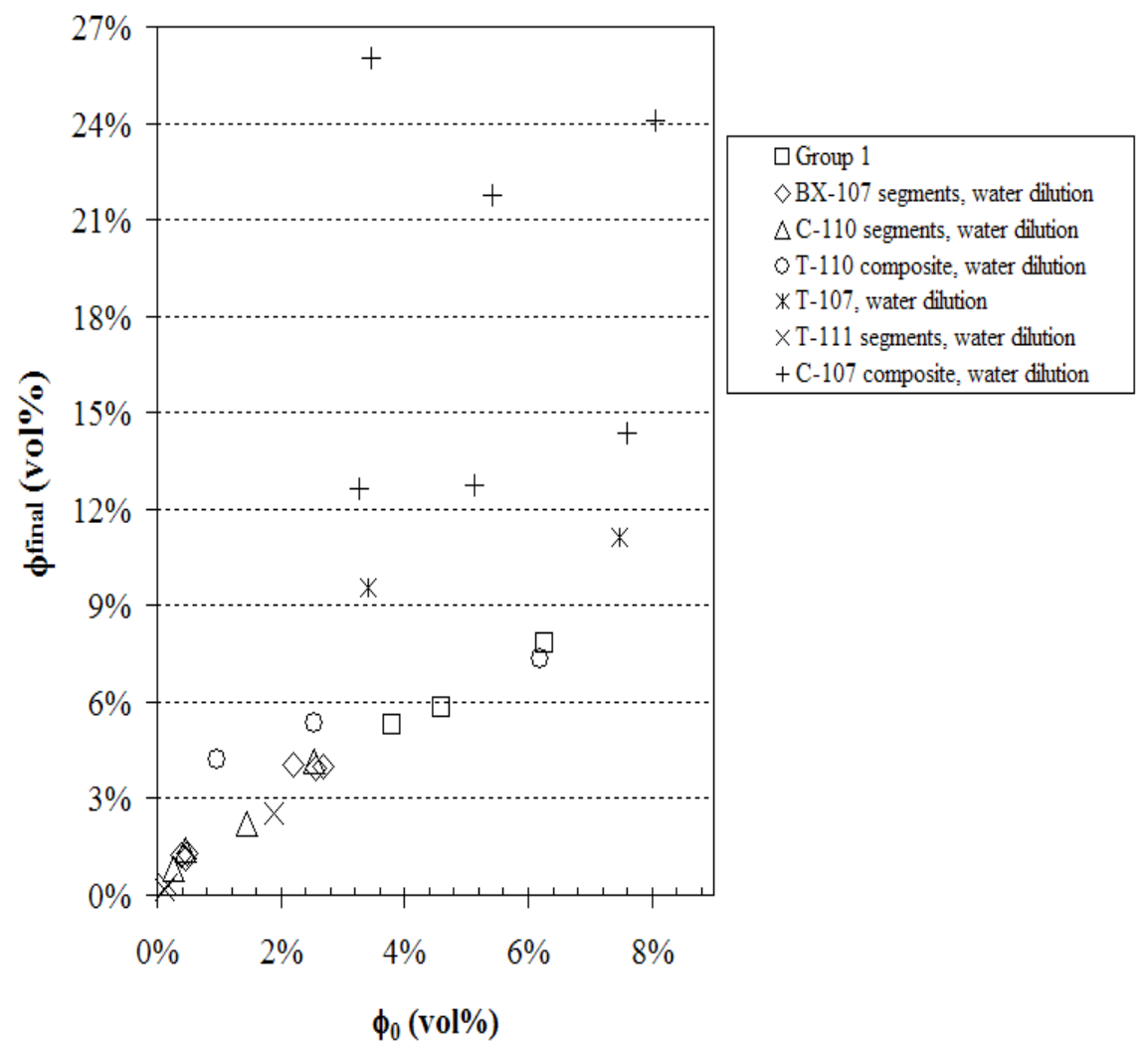

Figure 3.67. Variation of the Final Solids Concentration $\phi_{\text {final }}$ with Initial Solids Fraction Concentration in $1 \mathrm{C} / 2 \mathrm{C}$ Wastes

\section{Waste Types}

As shown in Table 3.30, the wastes that have undergone laboratory settling tests represent A2 saltcake, BL sludge, P3 sludge, 224 Post-1949 sludge, 1C/2C sludge, CWP sludge, FeCN sludge, R/S sludge/saltcake, TBP sludge, BY/T saltcake, and CWR sludge. The properties and dependence on initial solids volume fraction of the 224 Post-1949 and 1C/2C sludges have already been described. There are fewer measurements for other waste types, and tests performed with different initial height and test durations may not be strictly comparable. Therefore, only extremes of settling behavior will be noted here.

The waste types in which there was most often an initial delay before settling became visible were 224 Post-1949 sludges, S saltcake, BY and T saltcake, and TBP sludge. The longest delays (3 hr, in two of the three samples) were seen in TBP sludge. In 224 Post-1949 sludge, delays were consistently seen at 1:1 water dilution but not at the higher dilutions. A minority of $1 \mathrm{C} / 2 \mathrm{C}$ samples also showed initial settling delays, with the longest one being seen in T-110 waste at low dilution (30 wt\% water). 
Figure 3.68 gives an overview of the "fast" interface velocities that have been found in laboratory tests and in-tank observations. (The in-tank AY-102 velocities are approximations and are expected to be underestimates of the actual interface velocities; the method by which they were inferred from data is discussed in a later section.) The highest laboratory velocities were in CWP and CWR sludge and P3 waste (AZ-101 and AZ-102). The laboratory velocities for Group 2 (BY and T salt) and Group 7 (TBP sludges) wastes showed the most consistent tendency to be among the lowest values.

Some samples of A2 saltcake and all three BY/T saltcake samples had final solid volume fractions greater than 20\%. The CWR waste and the lowest-dilution AZ-101 sample also had high final solid fractions. However, the BY/T saltcake samples also started from volume fractions greater than $20 \%$. The lowest final solid fractions were seen in 224 Post-1949 waste, some of the 1C/2C wastes, and T-111 (in particular).

\subsubsection{Calculation of Settling Velocity from PSDDs}

This section makes comparisons between the settling velocity distributions calculated from particle size-density distributions (PSDDs, Section 3.3) and the settling velocities observed in actual experiments. These comparisons between behavior computed from the characterization of the particulate and actual measurements of the behavior are discussed with respect to the apparent accuracy of the characterization.

Because there is some doubt about the applicability of laboratory-scale experiments (conducted in graduated cylinders and centrifuge tubes) to tank conditions, the focus of this section is on larger scale tests. These include settling of AZ-101 solids in Tank AZ-101 after mixer pump operation (Carlson et al. 2001), settling of three-component waste simulant in a 4-ft-tall column, ${ }^{\left({ }^{a}\right)}$ and settling in Tank AY-102 of solids transferred from C-106 (Cuta et al. 2000). Although the three-component simulant was not designed to be a full simulant of tank waste, it is of interest as a multicomponent suspension whose PSDD is completely based on measurements.

(a) Beeman GH. 2010. "Recipes for Simulant Strengths," project letter WTP/RPP-MOA-PNNL-00494 from GH Beeman (PNNL) to HR Hazen (BNI). March 12, 2010. Pacific Northwest National Laboratory, Richland, Washington. 


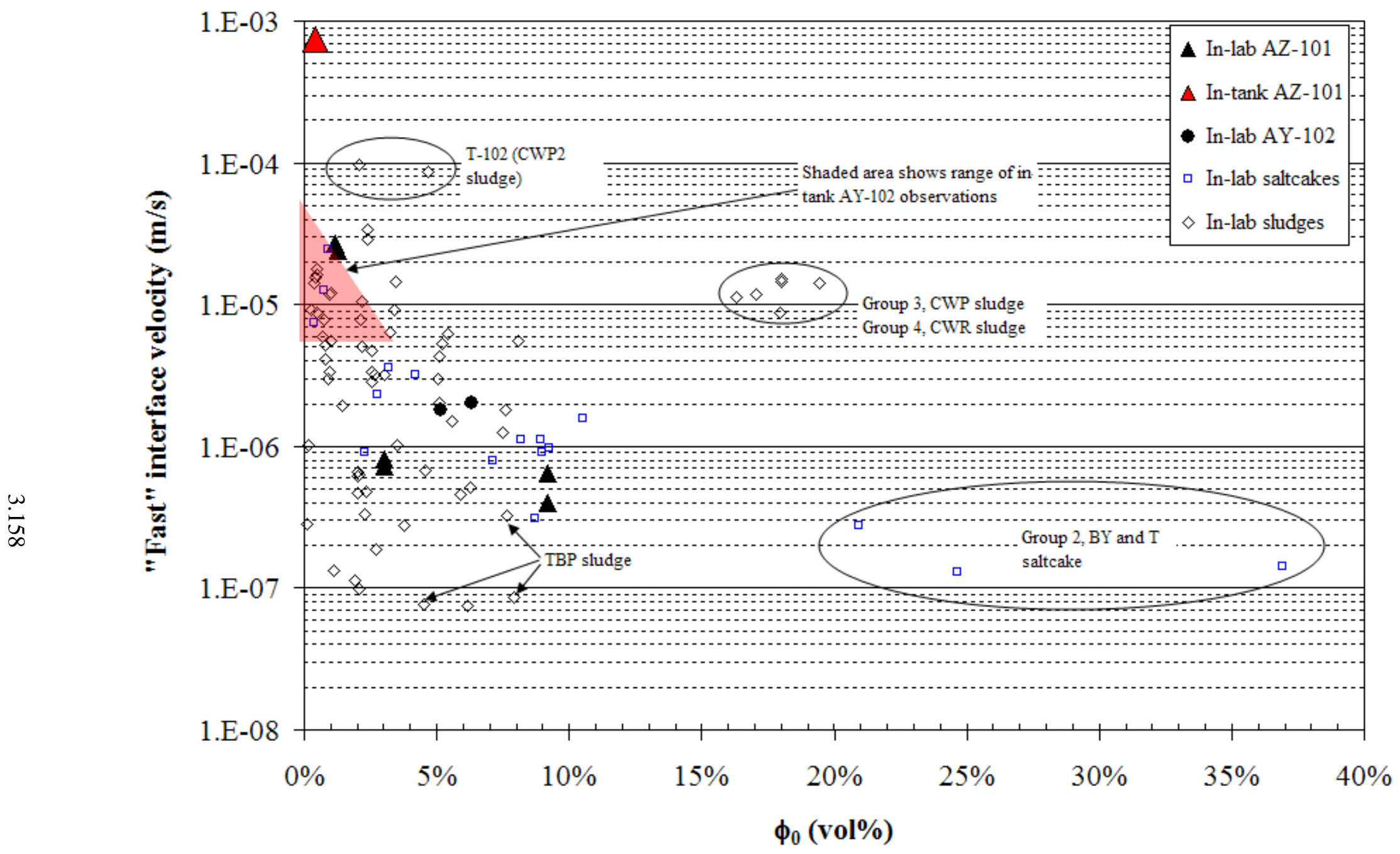

Figure 3.68. Overview of Interface Velocities 


\section{PSDDs: AZ-101 In-Tank Settling}

As discussed in Section 3.2.6.3.1, interface movement measurements were made in Tank AZ-101 after mixer pump tests had suspended about $32 \%$ of the solids throughout the supernatant liquid. The solids concentration at the time settling began was 0.43 vol\% (Table 3.30). The average interface velocity observed over the first $1.5 \mathrm{hr}$ was $6.5 \times 10^{-4} \mathrm{~m} / \mathrm{s}$. The interface velocity calculated for the "fast" step was $7.4 \times 10^{-4} \mathrm{~m} / \mathrm{s}$, which was the average velocity over the first $0.86 \mathrm{hr}$. (Because the averaging time was shorter, the "fast" velocity was greater than the 1.5 -hr average of $6.5 \times 10^{-4} \mathrm{~m} / \mathrm{s}$.) The corresponding unhindered interface velocity was calculated to be $9.6 \times 10^{-4} \mathrm{~m} / \mathrm{s}$. Eq. (2.17) was used to calculate the unhindered velocity, with $C_{V \max }$ set equal to the final solids volume fraction of 0.080 shown in Table 3.30.

Figure 3.69 and Figure 3.70 plot the measured "fast" interface velocity and the estimated unhindered velocity in AZ-101 (red lines) and the distribution of unhindered particle terminal velocities calculated from different types of PSDDs for the waste. The Camenen equation (Eq. 2.14), with parameters appropriate for spherical particles, was used to estimate the velocity for each combination of size bin and density.

The PSDDs used to create Figure 3.69 assume that all particles, of whatever size, are primary particles (fractal dimension $\mathrm{D}_{\mathrm{F}}=3$ and density equal to crystal density; see Section 3.3). The three PSDD velocity distributions that are shown represent different degrees of energy applied during PSD measurement, that is, different degrees of potential particle breakup. As described in Section 3.2.5, the PSD data are taken under conditions referred to as No-Flow, Flowing Unsonicated, or Flowing Sonicated. It should be noted that the No-Flow PSDDs are the only ones that include PSD measurements from the grab samples taken during the AZ-101 settling test.

As shown in Figure 3.69, there is essentially no difference in the Flowing Sonicated and Flowing Unsonicated particle velocity distributions, which results from the similarity in the PSDs (see Section 3.2.5). This similarity suggests that either the particulate exists as primary particles, or the upper range stir and pump settings for the Flowing configuration breaks up agglomerates as completely as does sonication. The No-Flow velocity distribution includes higher velocities because of the substantial fraction of larger particulate (Section 3.2.5), suggesting that agglomerates are present in the solids and that flow breaks up those agglomerates. (Because the Flowing Sonicated and Unsonicated distributions are so similar, the effect of sonication alone on agglomerates cannot be judged.) The particle sizes that are present in the No-Flow condition, but not in the Flowing condition, are between approximately 50 and $1700 \mu \mathrm{m}$ (Section 3.2.5).

The PSDDs used to create Figure 3.70 assume "No-Flow" conditions (minimum breakup) and a range of different fractal dimensions. As described in Section 3.3, at $\mathrm{D}_{\mathrm{F}}=3$, particles of all sizes are assumed to be solid primary particles or agglomerates without any pore space and thus have a crystal density appropriate for the particular solid phase. For lower $\mathrm{D}_{\mathrm{F}}$, the particles above a certain defined size are assigned a density as a function of the crystal density, the liquid density, and the agglomerate porosity that is calculated from $\mathrm{D}_{\mathrm{F}}$. 


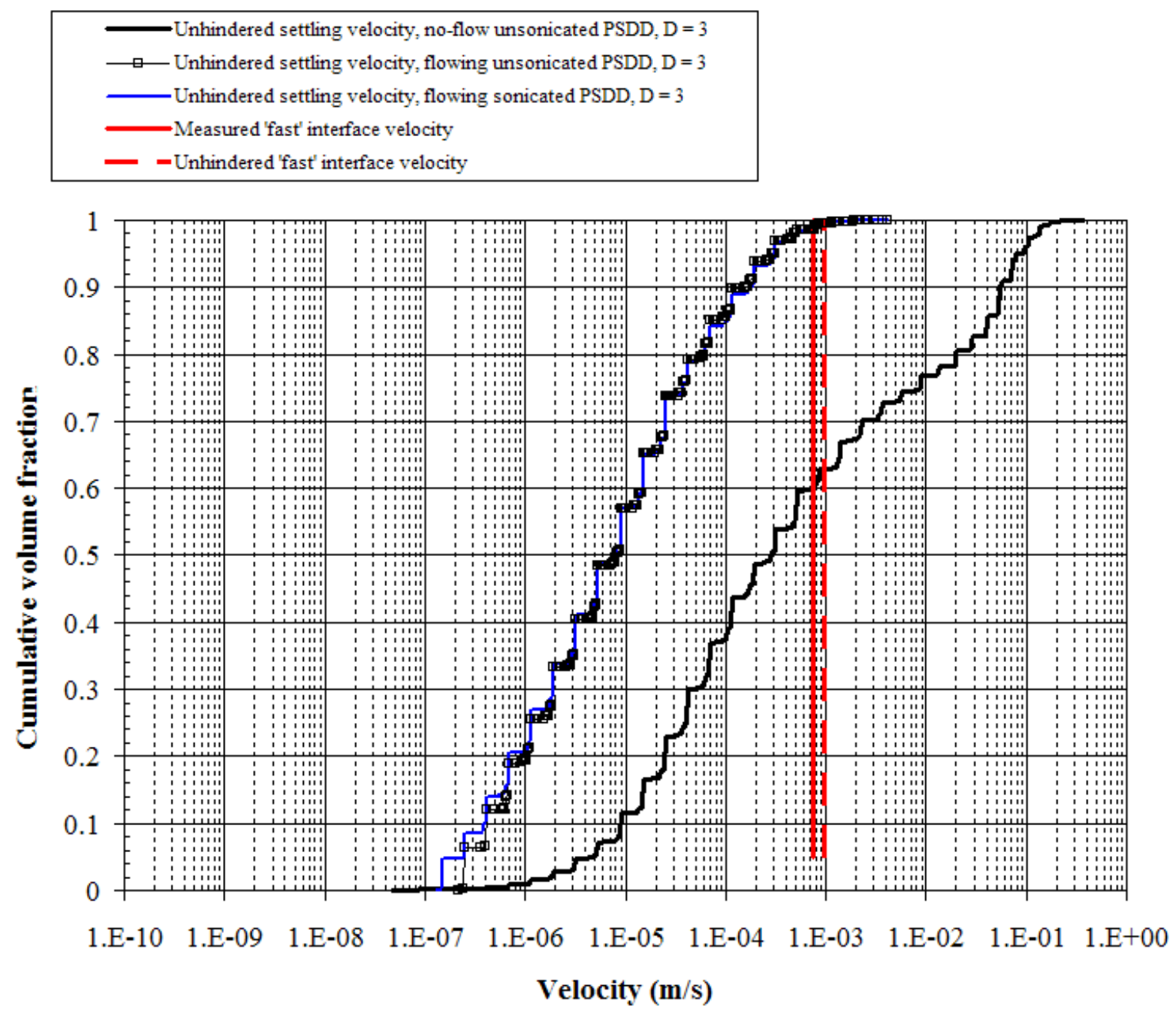

Figure 3.69. Interface Velocity for In-Tank Suspended AZ-101 Solids and Particle Velocity Distributions for All AZ-101 Solids (with all particles treated as primary particles) 


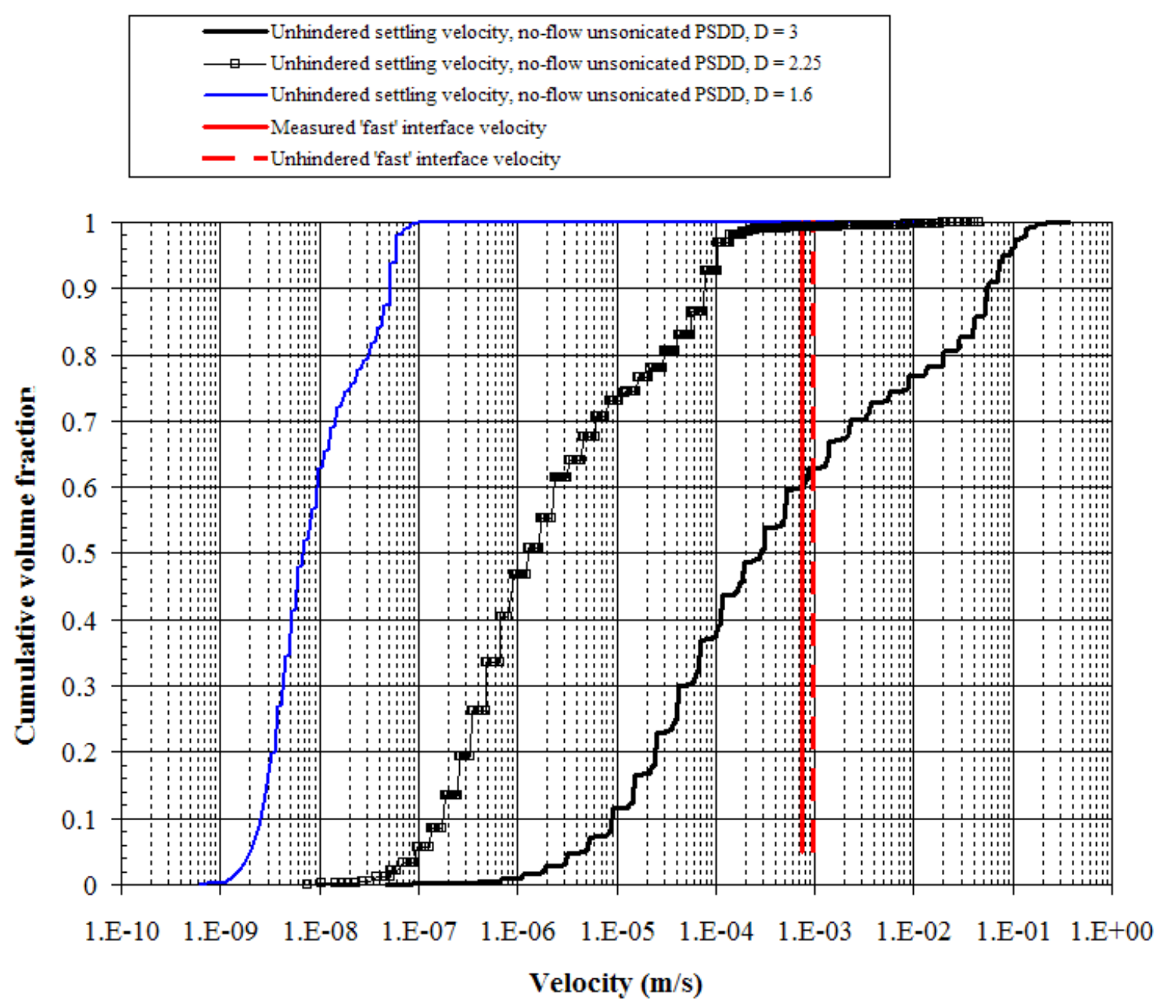

Figure 3.70. Interface Velocity for In-Tank Suspended AZ-101 Solids and Particle Velocity Distributions for All AZ-101 Solids (at No-Flow condition, varied fractal dimension)

The unhindered interface velocity in Tank AZ-101 lies at about the $98^{\text {th }}$ percentile of the Flowing Sonicated and Unsonicated velocity distributions in Figure 3.69, and at the $63^{\text {rd }}$ percentile of the "No-Flow" distribution. Therefore, the lowest velocities calculated from particle properties do not seem to determine the interface velocity. By definition, the observed top interface travels at the settling velocity of the slowest visible particles, but under AZ-101 in-tank conditions, the slowest particles were not settling as slowly as the sizes and densities in the PSDD dictate. Other examples of observed settling rates that greatly exceed the calculated settling rate of primary particles are discussed in Section 3.2.4.6.2 and Section 3.2.6.4.3. This suggests the presence of one or more phenomena that accelerate the settling rate, such as wake capture of smaller particles by larger ones, agglomeration followed by the formation of convective structures, or the particulate of the measured settling rate is not accurately characterized by some or all of the PSDDs.

It should be noted that the high interface velocity of the suspended solids in the AZ-101 tank, relative to the velocity distribution calculated from PSDDs, is not necessarily characteristic of all tank wastes. For example, mixer pump operations that were carried out in saltcake Tank SY-101 before remediation 
produced particulate that "remain(ed) in suspension with little stratification over periods of a few days to over a week between mixer pump runs.” The slow settling was not the result of gelling or any type of immobilization of the supernatant layer in which the solids were suspended. This layer consistently behaved as a fluid in the tank, based on the uniform temperature profiles (consistent with convection) that were observed (Gauglitz et al. 2010b). Although core samples taken from this region were thick, this can be attributed to precipitation at laboratory temperature of species that were dissolved at tank temperature.

Figure 3.70 shows that for the No-Flow AZ-101 size distribution the effect of agglomeration is to decrease rather than increase the velocities at the lower end of the distribution. Three different fractal dimensions are shown: $D_{F}=3$ for solid primary particles, and $D_{F}=2.25$ and $D_{F}=1.6$ for agglomerates. The decrease in velocity with decreasing fractal dimension results from the increasing porosity of the agglomerates, which gives them a density very close to that of the liquid at the largest sizes. The conclusion is that if the measured PSDs are accepted as representing the in-tank particulate, then treating the particles as agglomerates, i.e., reducing the particle density with increasing particle size, produces results that are further from expected, in that the observed velocities are further from the expected low end of the velocity distribution.

Bechtold et al. (2002) postulated that PSDs of settled material from laboratory tests failed to identify very many large particles despite their being visible during the settling tests because the particle size analyzer conditions broke up the large particulate. As referenced in Appendix G, Bechtold et al. (2002) also noted that, in comparison to sieving analysis of particle size, the light-scattering particle size analyzer was poor at finding particles above $500 \mu \mathrm{m}$ in size. Thus, it may be that the results shown in Figure 3.69 and Figure 3.70, which demonstrate a gap in the current understanding of settling velocity, are influenced by an under-representation of larger agglomerates in the PSDs than were present during settling.

\section{PSDDs: Three-Component Simulant}

In support of the M3 PJM testing program, tests of three-component simulants were conducted in a column $4 \mathrm{ft}$ tall and 3 inches in inner diameter. ${ }^{\text {(a) }}$ Settling data (interface heights) were measured over a period of about $24 \mathrm{hr}$. Three of the tests used the same simulant at three different concentrations of total solids, $5 \mathrm{wt} \%, 10 \mathrm{wt} \%$, and $15 \mathrm{wt} \%$. The solids in the simulant were $90 \mathrm{wt} \%$ boehmite (volume $\left.\mathrm{d}_{50}=8.9 \mu \mathrm{m}\right), 5 \mathrm{wt} \%$ stainless steel (volume $\left.\mathrm{d}_{50}=140 \mu \mathrm{m}\right)$, and $5 \mathrm{wt} \%$ sand (volume $\left.\mathrm{d}_{50}=262 \mu \mathrm{m}\right){ }^{(\mathrm{b})}$ In all of these simulants, the liquid was $0.25 \mathrm{M} \mathrm{NaCl}$.

All three of the level versus time profiles exhibited a long linear decrease in level (constant interface velocity during settling), followed by a period of smaller change in level as compaction proceeded. In order to smooth the data and obtain a single interface velocity to characterize the settling in each test, the sedimentation model, Eq. (3.8), was fitted to the level/time profiles from the three tests, and the "fast" interface velocities of the tests were calculated from the fit parameters. These velocities were

(a) Letter from GH Beeman (PNNL) to HR Hazen (BNI), March 12, 2010. "Subcontract No. 24590-QL-HC49-00001 - Project 55753 (WA \#028) Recipes for Simulant Strengths”, WTP/RPP-MOA-PNNL-00494.

(b) PSDs of stainless steel and sand came from a personal communication, handed out by Sundar (BNI) to PNNL staff at a meeting on 1/14/2010. The PSD of boehmite was taken from TDP-WTPSP-590. The stainless steel PSD stated the material had been sonicated. No statement about sonication, or lack of it, was made for the other two materials. 
$5.0 \times 10^{-4} \mathrm{~m} / \mathrm{s}$ for the $5 \mathrm{wt} \%$ simulant, $3.6 \times 10^{-4} \mathrm{~m} / \mathrm{s}$ for the $10 \mathrm{wt} \%$ simulant, and $1.8 \times 10^{-4} \mathrm{~m} / \mathrm{s}$ for the $15 \mathrm{wt} \%$ simulant.

The interface velocities in the three tests were assumed to be three distinct velocities only because the interface particles were in the presence of three different solids concentrations. This amounts to assuming that the same type of particle made up the interface in all three tests and leads to the conclusion that in unhindered conditions, the three tests would have exhibited the same interface velocity. Therefore, the three interface velocities were fit to a hindered-settling equation, Eq. (2.17), to estimate the unhindered velocity, $V_{S O}$. The total solids concentration at the interface was assumed to be equal to the initial well-mixed concentration, for lack of other information. ${ }^{(a)}$ The maximum solids volume fraction $C_{V \max }$ was used as the adjustable parameter to obtain the closest possible match between the unhindered velocity estimates for the three tests. This approach assumes that the velocities are affected by hindrance (increased effective viscosity and upwelling of the liquid) but not by wake capture, convective streaming, and so forth. The resulting estimates of the unhindered velocity of the interface particles were between $8.2 \times 10^{-4} \mathrm{~m} / \mathrm{s}$ and $1.1 \times 10^{-3} \mathrm{~m} / \mathrm{s}$. The fact that a reasonably close match between the three tests' estimates of unhindered velocity could be obtained suggests that hindrance was the main effect leading to differences in interface velocities.

Figure 3.71 plots the estimates of unhindered interface velocity (red lines) and the distribution of unhindered particle terminal velocities calculated from the PSDDs of the boehmite, stainless steel, and sand particles. Of the PSDs used in these PSDDs, that for stainless steel is known to have been based on sonicated particles; the other two materials are not identified as sonicated or not, but sonication is not expected to have had much effect given the constituents. The Camenen equation (Eq. 2.14), with parameters appropriate for spherical particles, was used to estimate the velocity for each combination of size bin and density.

(a) It is unlikely that the interface concentration was equal to the well-mixed total concentration, but probable that it was proportional to the total concentration. If that were the case, fitting the model by using the correct (unknown) interface solids concentration would have given a fit-parameter $C_{V \max }$ that was smaller in the same proportion, and would have produced the same set of unhindered velocities. 


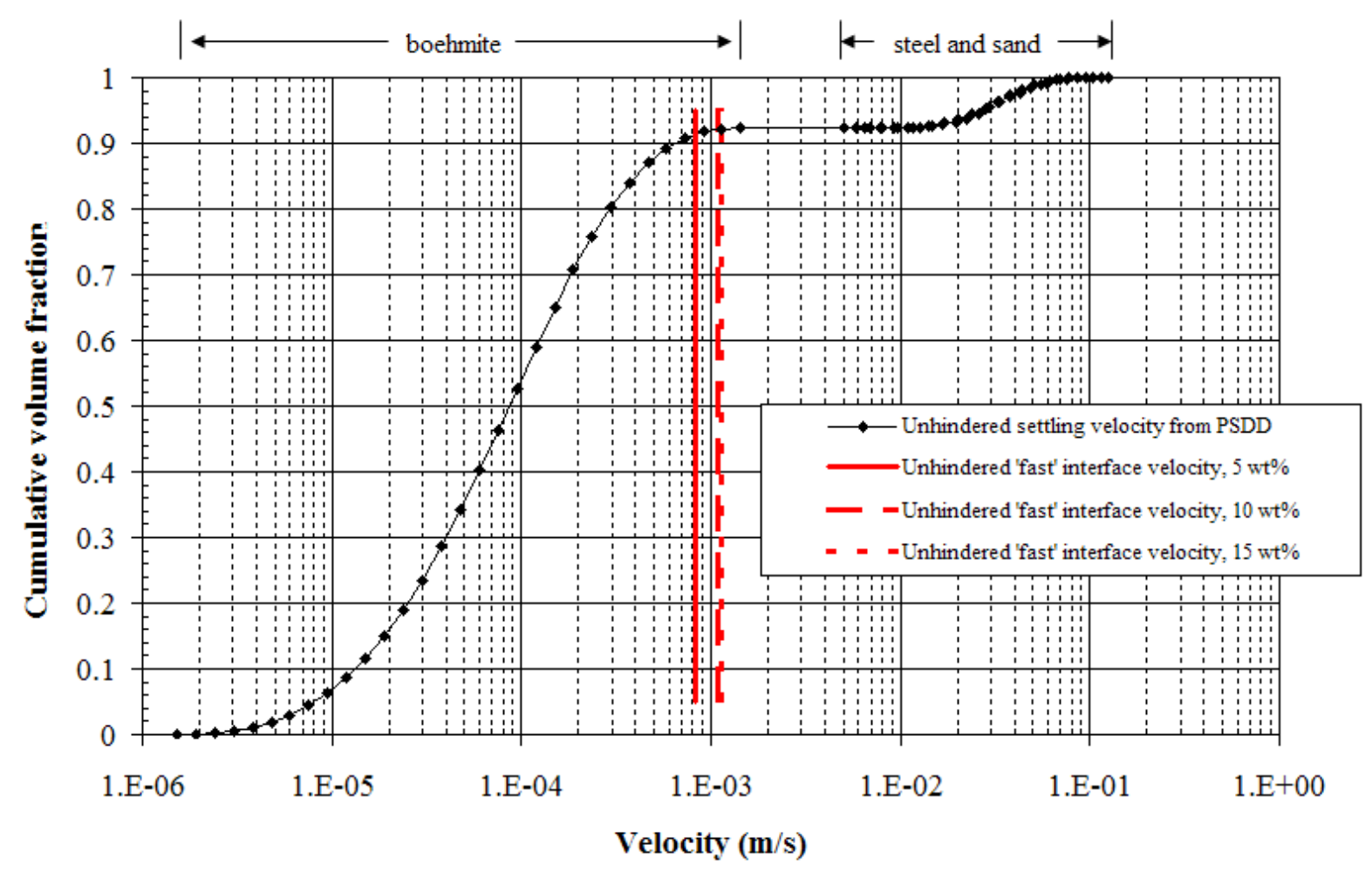

Figure 3.71. Interface Velocity and Particle Velocity Distribution for a Three-Component Simulant

The estimated unhindered interface velocity lies at about the $92^{\text {nd }}$ percentile of the particle velocity distribution, which is nearly equal to the velocity of the largest gibbsite particles but well below the lowest velocity of the steel and sand particles. As in the case of in-tank settling in AZ-101, the calculated velocities of the slowest individual particles are too low to be consistent with the estimated unhindered interface velocity (from measured rates). In this respect, the behavior of the smaller scale, simpler simulant is similar to that of the suspended solids in Tank AZ-101, even though it cannot be proven that the mechanisms are the same.

As noted, the similarity between the three-component simulant and the AZ-101 waste may be due to different mechanisms. Further, the three-component simulant may not accurately represent actual waste, and different simulants and wastes may reasonably be expected to have different behavior. If it is assumed however that the observed relation of the calculated and measured settling velocities of the three-component simulant (i.e., approximately the $92^{\text {nd }}$ percentile) applies to AZ-101 waste, the Flowing Sonicated and Flowing Unsonicated PSDDs under-represent the actual settling velocity of the AZ-101 particulate, even with a fractal dimension 3, thereby indicating that the PSDs are too small. The No-Flow Unsonicated PSDD with $\mathrm{D}_{\mathrm{F}}=3$ over-represents the particulate, and under-represents with $\mathrm{D}_{\mathrm{F}}=2.25$. The No-Flow Unsonicated PSD may therefore characterize the particulate, but the exercise of determining the exact fractal dimension for the AZ-101 No-Flow Unsonicated PSDD at which the calculated and measured settling velocities coincide at the $92^{\text {nd }}$ percentile is rendered moot by the necessary assumptions. 


\section{PSDDs: AY-102 In-Tank Settling}

Through 1998 and 1999, sluicing operations were carried out to transfer most of the sludge in Tank C-106 to Tank AY-102. Supernatant liquid from AY-102 was used as the sluicing fluid. The data gathered during this campaign included sediment level and total volumes of the transfers (Cuta et al. 2000). These data can be used to make rough estimates of the settling velocity by assuming that sediment growth, for any batch, ended only when the particle that had been highest up in the suspension at the end of the batch transfer finally settled all the way down to the top of the sediment layer. The interface velocity is therefore estimated as the distance from the surface of the sediment to the surface of the waste, divided by the time from the end of the transfer to the time when the sediment layer stopped growing. In addition, if the solid volume fraction in the sediment is known, then the solid volume fraction in the transfer can also be calculated from the increase in the height of the sediment. If the concentration of solids is assumed to be uniform in the suspension that was produced by transfer, the transfer solid fraction can be used to estimate the unhindered interface velocity of the interface particles, based on the estimate of the presumably hindered interface velocity.

The assumptions involved in the calculation make it an approximation of the true interface velocity, which is the "fast" step of settling and compaction. In fact, the velocity calculated from the data available for AY-102 are likely to produce an underestimate of the "fast" velocity because the period of time involved in settling probably includes some compaction time as well as settling time.

Calculations of unhindered interface velocity were performed by Cuta et al. (2000). The present study recalculates the velocities using the same basic approach, but using information for the solid volume fraction in the sediment that was not available for the earlier study. In addition, the present study uses a different expression for calculating the hindered settling velocity (Eq. 2.17) than was used in the earlier report.

The sediment solid volume fraction was found to be 0.16 , based on calculations of solids composition, solids volume, and sludge layer bulk volume in the 2002 BBI for AY-102 (as described in Section 3.2.3). ${ }^{\text {(a) }}$ The interface velocities were fit to the hindered-settling equation to estimate the unhindered interface velocity. The total solids concentration at the interface was assumed to be equal to the initial well-mixed, post-transfer concentration. The maximum solids volume fraction $C_{V \max }$ in Eq. (2.17) was used as the adjustable parameter to obtain the closest possible match between the unhindered velocity estimates for the transfers (assuming all had interface velocities governed by the same type of particle). The resulting estimates of unhindered interface velocity ranged from $8 \times 10^{-6} \mathrm{~m} / \mathrm{s}$ to $5 \times 10^{-5} \mathrm{~m} / \mathrm{s}$, with the median value being $\sim 2 \times 10^{-5} \mathrm{~m} / \mathrm{s}$. The wide range of velocities suggests that the interface was not made up of the same size or density of particle in all batches transferred. Because the model fit was carried out on the apparently incorrect assumption that all unhindered velocities were based on the same particle type, the estimates of unhindered velocity must be considered approximations and therefore are represented as a range, not a single value.

By way of comparison, the laboratory settling tests indicated a "fast" interface velocity of $1.9 \times 10^{-6} \mathrm{~m} / \mathrm{s}(7 \mathrm{~mm} / \mathrm{hr})$, consistent with the sedimentation model fit parameters for AY-102 that are

(a) The difference between the sediment solid volume fraction determined here, 0.16 , and that reported in Gauglitz et al. (2010b), 0.29, can primarily be explained by the difference in the average undissolved solid density, $3.4 \mathrm{~g} / \mathrm{mL}$, Section 3.2.3, and $2.5 \mathrm{~g} / \mathrm{mL}$ (Gauglitz et al. 2010b). 
given in Table 3.30. ${ }^{\text {(a) }}$ This is effectively a hindered velocity. The unhindered velocity can be estimated by using the initial concentration, an average of 0.057 solid fraction, with the same equation and $C_{V \max }$ (equal to 0.124 ) as for the in-tank settling. The result is an unhindered interface velocity estimate of $3.4 \times 10^{-5} \mathrm{~m} / \mathrm{s}$. This is in the same range as the estimated unhindered interface velocities, which themselves are probably underestimates of the true interface velocity.

Figure 3.72 and Figure 3.73 show the approximate range of unhindered interface velocities in the AY-102 in situ settling. The figures also show the distribution of unhindered particle terminal velocities calculated from different types of PSDDs for the waste. The Camenen equation (Eq. 2.14), with parameters appropriate for spherical particles, was used to estimate the unhindered velocity for each combination of size bin and density.

The PSDDs used to create Figure 3.72 assume that all particles, of whatever size, are primary particles (fractal dimension of 3, density equal to the phase crystal density) and show the effects of different degrees of energy applied during PSD measurement. Unhindered particle velocity distributions are presented for PSDDs based on PSD data taken under No-Flow conditions, Flowing Unsonicated conditions, and Flowing Sonicated conditions. The PSDDs used to create Figure 3.73 all assume the lowest energy applied ("No-Flow”) and the least particle breakup, but make different assumptions as to whether the larger particles, for any given solid phase, have crystal density or floc density. As described previously, decreasing the fractal dimension $\mathrm{D}_{\mathrm{F}}$ corresponds to lowering the density of the larger particles.

As shown in Figure 3.72, the Flowing Unsonicated particle velocity distributions are slightly higher than those for Flowing Sonicated conditions. Sonication plus flow breaks up the agglomerates, whose presence is indicated by the substantially higher velocities in the No-Flow distribution more completely than does flow alone. The maximum particle size under Flowing Sonicated conditions was $60 \mu \mathrm{m}$; under Flowing Unsonicated conditions, the maximum was $464 \mu \mathrm{m}$; and under conditions of no flow and no sonication, the maximum was $1292 \mu \mathrm{m}$ (Section 3.2.5). The discussion of the Bechtold et al. (2002) results made above regarding the AZ-101 comparison may also influence the AY-102 results. Even though the unhindered interface velocities for AY-102 are underestimates of the actual interface velocity, for reasons already discussed, they lie well above the low ends of all the velocity distributions calculated from PSDDs. This behavior is consistent with what was seen for AZ-101 in-tank settling and the three-component simulant.

\section{PSDDs: Summary}

As stated, the comparisons between the settling velocity distributions calculated from the PSDDs and measured settling velocities suggest either the presence of one or more phenomena that accelerate the settling rate or the settling behavior of the particulate is not accurately characterized by certain PSDDs. Thus, to accurately represent the settling behavior of the waste via characterization of the particulate, either the phenomena that accelerates the settling rate must be understood and accounted for or process data must be considered for the selection of a representative PSDD.

(a) The samples used in the laboratory tests were originally taken from AY-102 in December 1999 (Warrant 2001), about 2 months after C-106 waste had last been added to the tank. 


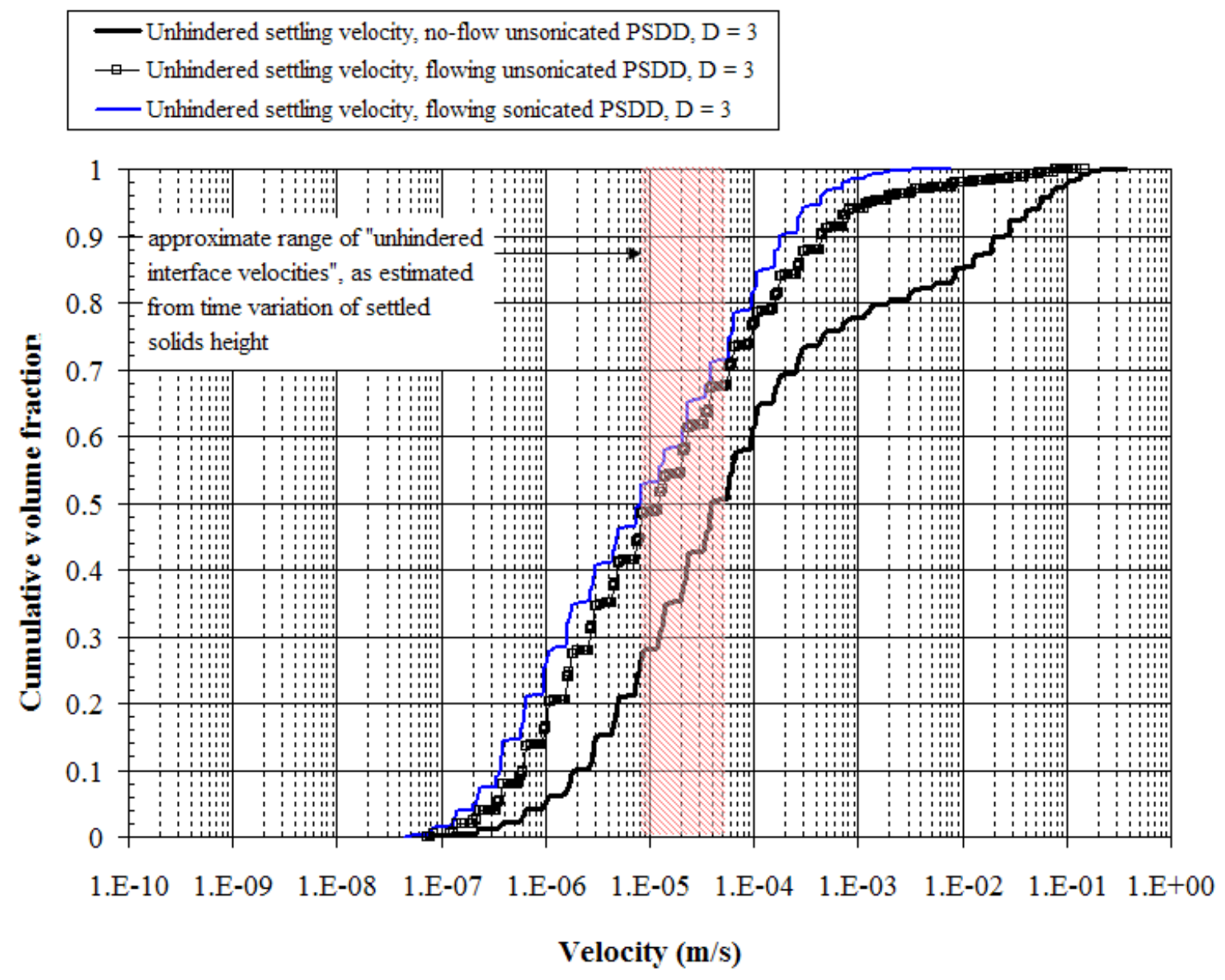

Figure 3.72. Interface Velocity and Particle Velocity Distributions for AY-102 Solids (with all particles treated as primary particles) 


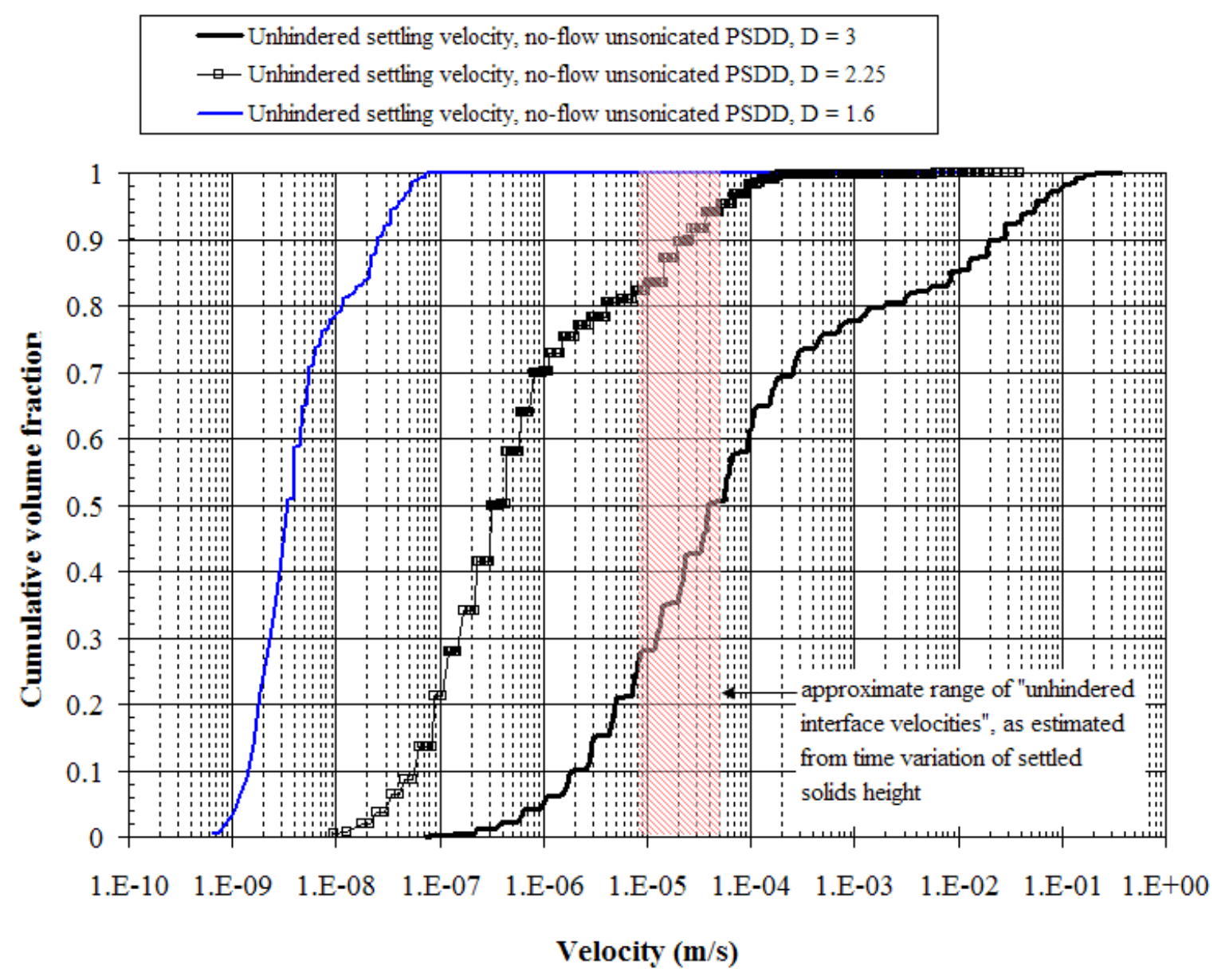

Figure 3.73. Interface Velocity and Particle Velocity Distributions for AY-102 Solids (at No-Flow condition, varied fractal dimension)

\subsubsection{Slurry Rheology}

Rheology data are available for the slurry of a limited number of Hanford tanks. The majority of the rheology data are obtained from laboratory measurements of samples retrieved from the tanks. These retrieved samples included push- and rotary-mode core samples, auger samples, and grab samples. A limited set of data is available from in situ measurements. Slurry rheological properties obtained by the measurements include the Bingham rheological model consistency and yield stress and shear strength. A discussion of rheological characterization and measurement techniques is provided in Poloski et al. (2007).

The shear-strength data are presented in Section 3.2.7.1 and the Bingham rheological model data in Section 3.2.7.2. The functionalities of the rheological parameters with respect to waste properties are described where possible. Waste storage, mobilization, and transport issues are impacted by these relationships. The data are presented on by-tank, by-waste type, and overall bases. The rheological characterization is compared where possible to the limited process data. The rheological data sets are included as Appendix I. 


\subsubsection{Shear Strength}

Shear strength is a common term used to describe a material's yield stress in shear as measured directly, e.g., via a shear vane. Shear-strength measurements are available for concentrated slurries from 37 individual tanks representing 15 primary waste types (see Table 3.31). The majority of these measurements are from waste sediments. References are listed. The "Group” waste samples of Table 3.31 encompass an additional eight waste types, and are composed of samples from numerous waste tanks (see reference listed in Table 3.31). The complete data set is provided in Appendix I.

Shear-strength measurements are available from laboratory sediment sample measurements as well as in situ sediment measurements from a limited number of tanks. The shear-strength measurement techniques employed for the samples are also provided in Table 3.31. The variation in technique is due to laboratory (shear vane) vs. in situ (ball rheometer) measurements and "opportunistic" techniques (waste extrusion). The methods are discussed below, and the data from each technique are summarized.

A slurry material's shear strength depends on the characteristics of the system, including UDS concentration, particle size and distribution, particle shape, $\mathrm{pH},{ }^{(a)}$ quiescent time, elevation within the sediment, and retained gas content. Information, although limited, is available for Hanford slurries to consider a number of these functionalities. The effects of UDS concentration, quiescent time, particle size, elevation within the sediment, and retained gas content on shear strength are discussed.

Table 3.31. Shear-Strength Data Set

\begin{tabular}{|c|c|c|c|c|}
\hline Tank & Reference(s) & Primary Waste Type & $\begin{array}{c}\text { Primary } \\
\text { Waste Type } \\
\text { Fraction }\end{array}$ & $\begin{array}{c}\text { Measurement } \\
\text { Technique }\end{array}$ \\
\hline Group 1 & WTP-RPT-166 & 1C and 2C sludge & N/A & shear vane \\
\hline BX-107 & $\begin{array}{l}\text { SST BX-107 Cores } 40 \text { and } \\
41 \text { data package }\end{array}$ & \multirow{4}{*}{ 1C sludge } & 1 & shear vane \\
\hline C-107 & TWINS & & 0.54 & shear vane \\
\hline C-110 & $\begin{array}{c}\text { C-110 Cores } 37,38 \text { and } 39 \\
\text { data package.pdf }\end{array}$ & & 1 & shear vane \\
\hline T-107 & $\begin{array}{c}\text { WHC-SD-WM-ER-382, rev } \\
\text { 0, TWINS }\end{array}$ & & 0.85 & shear vane \\
\hline B-202 & $\begin{array}{c}\text { PNNL-14221, } \\
\text { WHC-SD-WM-DP-034 }\end{array}$ & \multirow{4}{*}{224 Post-1949 sludge } & 1 & shear vane \\
\hline B-203 & PNNL-14221, PNNL-14365 & & 1 & waste extrusion \\
\hline B-204 & \multirow{2}{*}{ PNNL-14221 } & & 1 & waste extrusion \\
\hline $\mathrm{T}-202$ & & & 1 & waste extrusion \\
\hline
\end{tabular}

(a) The pH of the Hanford waste is intentionally kept basic to inhibit corrosion in the carbon steel vessels. Examples of shear strength being affected by the $\mathrm{pH}$ of the liquid, and UDS systems are given by Ancey and Jorrot (2001) and Zhou et al. (1999). 
Table 3.31. (contd)

\begin{tabular}{|c|c|c|c|c|}
\hline Tank & Reference(s) & Primary Waste Type & $\begin{array}{c}\text { Primary } \\
\text { Waste Type } \\
\text { Fraction } \\
\end{array}$ & $\begin{array}{c}\text { Measurement } \\
\text { Technique }\end{array}$ \\
\hline \multirow{2}{*}{$\mathrm{T}-203$} & \multirow{4}{*}{ PNNL-14221, PNNL-14365 } & & \multirow{2}{*}{1} & waste extrusion \\
\hline & & & & shear vane \\
\hline \multirow{2}{*}{$\mathrm{T}-204$} & & & \multirow{2}{*}{1} & waste extrusion \\
\hline & & & & shear vane \\
\hline B-201 & $\begin{array}{l}\text { PNNL-14221, WHC-SD- } \\
\text { WM-DP-037, TWINS }\end{array}$ & \multirow{2}{*}{224 Pre-1949 sludge } & 1 & shear vane \\
\hline $\mathrm{T}-201$ & PNNL-14221 & & 1 & waste extrusion \\
\hline B-111 & $\begin{array}{c}\text { Segment 5; WHC-SD-WM- } \\
\text { ER-382, Rev 0, TWINS }\end{array}$ & \multirow{4}{*}{ 2C sludge } & 0.89 & shear vane \\
\hline \multirow{2}{*}{$\mathrm{T}-110$} & \multirow{2}{*}{ PNNL-14221, PNNL-14365 } & & \multirow{2}{*}{0.97} & waste extrusion \\
\hline & & & & shear vane \\
\hline $\mathrm{T}-111$ & $\begin{array}{l}\text { SD-WM-DP-024, Personal } \\
\text { Communication with } \\
\text { JM Tingey }\end{array}$ & & 0.55 & shear vane \\
\hline \multirow[b]{2}{*}{ AY-102 } & \multirow{2}{*}{$\begin{array}{c}\text { PNNL-14763, } \\
\text { WSRC-TR-2003-00205 } \\
\text { Rev 0, 8D500-DBB-01-018 }\end{array}$} & \multirow[b]{2}{*}{ BL sludge } & 1 & waste extrusion \\
\hline & & & 1 & shear vane \\
\hline Group 3 & WTP-RPT-167 & CWP1 and CWP2 sludge & N/A & shear vane \\
\hline C-104 & $\begin{array}{l}\text { RPP-5798, Rev 0B, } \\
\text { 8D500-DBB-01-018 }\end{array}$ & CWP1 sludge & 0.34 & shear vane \\
\hline Group 4 & WTP-RPT-167 & CWR1 sludge & N/A & shear vane \\
\hline AW-103 & 8D500-DBB-01-018 & CWZr2 sludge & 0.87 & shear vane \\
\hline AZ-101 & $\begin{array}{c}\text { PNNL-13027, } \\
\text { 8D500-DBB-01-018 }\end{array}$ & \multirow[t]{2}{*}{ P3 sludge } & 1 & shear vane \\
\hline AZ-102 & 8D500-DBB-01-018 & & 1 & shear vane \\
\hline Group 8 & WTP-RPT-170 & PFeCN sludge (Group 8) & N/A & shear vane \\
\hline S-104 & WHC-SD-WM-DP-031 & R saltcake & 0.47 & shear vane \\
\hline Group 5 & WTP-RPT-157 & R1 (boiling) sludge & N/A & shear vane \\
\hline Group 7 & WTP-RPT-169 & TBP sludge & N/A & shear vane \\
\hline C-109 & $\begin{array}{c}\text { SG McKinley et al. } \\
\text { Characterization Report } \\
\text { March 30, } 1993 \\
\end{array}$ & \multirow[t]{2}{*}{ TFeCN sludge } & 0.38 & shear vane \\
\hline C-112 & $\begin{array}{l}\text { SST C-112 Core } 36 \text { data } \\
\text { package }\end{array}$ & & 0.69 & shear vane \\
\hline AY-101 & 8D500-DBB-01-018 & \multirow{2}{*}{ Unidentified sludge } & 1 & shear vane \\
\hline SY-102 & PNNL-11352, TWINS & & 0.51 & shear vane \\
\hline A-101 & RPP-7249 & A1 saltcake & 0.99 & waste extrusion \\
\hline AN-102 & TWINS & \multirow{7}{*}{ A2 saltcake } & 1 & shear vane \\
\hline \multirow[b]{2}{*}{ AN-103 } & PNNL-11706, & & & ball rheometer \\
\hline & $\begin{array}{l}\text { PNNL-14221, RPP-6655, } \\
\text { PNNL-11296 }\end{array}$ & & 1 & $\begin{array}{c}\text { waste extrusion } \\
\text { shear vane }\end{array}$ \\
\hline \multirow{2}{*}{ AN-104 } & PNNL-14221, RPP-6655, & & & ball rheometer \\
\hline & PNNL-11296 & & 1 & waste extrusion \\
\hline AN-105 & RPP-6655, PNNL-11296 & & 1 & ball rheometer \\
\hline AN-107 & TWINS & & 1 & shear vane \\
\hline
\end{tabular}


Table 3.31. (contd)

\begin{tabular}{|c|c|c|c|c|}
\hline Tank & Reference(s) & Primary Waste Type & $\begin{array}{c}\text { Primary } \\
\text { Waste Type } \\
\text { Fraction }\end{array}$ & $\begin{array}{c}\text { Measurement } \\
\text { Technique }\end{array}$ \\
\hline \multirow{3}{*}{$\begin{array}{l}\text { AW-101 } \\
\text { AW-101 }\end{array}$} & \multirow{3}{*}{$\begin{array}{c}\text { PNNL-11706, } \\
\text { PNNL-14221, RPP-6655 }\end{array}$} & & \multirow{3}{*}{1} & ball rheometer \\
\hline & & & & waste extrusion \\
\hline & & & & shear vane \\
\hline Group 2 & WTP-RPT-166 & BY, T1, and T2 saltcake & N/A & shear vane \\
\hline U-103 & RPP-7249 & S1 saltcake & 0.69 & waste extrusion \\
\hline S-102 & PNNL-11706, RPP-7249 & \multirow{7}{*}{ S2 saltcake } & 0.59 & waste extrusion \\
\hline SY-101 & $\begin{array}{l}\text { HNF-1666 Rev 0A, HNF- } \\
\text { 1666 Rev 0B, WHC-EP- } \\
\text { 0628, WHC-SD-WM-DRT- } \\
\text { 026, RPP-6655, PNNL- } \\
\text { 11296, PNL-10198, TWINS }\end{array}$ & & 1 & shear vane \\
\hline \multirow{3}{*}{ SY-103 } & \multirow{3}{*}{$\begin{array}{c}\text { PNNL-11706, } \\
\text { PNNL-14221, RPP-6655, } \\
\text { PNNL-11296 }\end{array}$} & & \multirow{3}{*}{1} & ball rheometer \\
\hline & & & & waste extrusion \\
\hline & & & & shear vane \\
\hline \multirow{2}{*}{ U-107 } & \multirow{2}{*}{ RPP-7249, TWINS } & & \multirow{2}{*}{0.91} & waste extrusion \\
\hline & & & & shear vane \\
\hline Group 6 & WTP-RPT-157 & S1 and S2 Saltcake & N/A & shear vane \\
\hline
\end{tabular}

\section{Shear Strength-Measurement Techniques and Data Summary}

The shear-strength measurement techniques employed for Hanford waste, discussed in more detail in Poloski et al. (2007), are

- Ball Rheometer. In situ measurement. The rheology of the waste can be estimated directly from the drag force on a ball as it is moved vertically through the waste at various speeds.

- Waste Extrusion. Video images recorded during the horizontal waste core extrusions after sample retrieval are analyzed to estimate the shear strength. Estimates are based on the extrusion behavior.

- Shear Vane. Laboratory sample measurement. Measured directly by slowly rotating a vane immersed in a sample and recording the resulting torque as a function of time.

The ball rheometer measurements, being in situ, may thus be expected to be most representative of the shear strength of the sediment as the material exists in the tanks. However, as described in Stewart et al. (1996a), the ball rheometer technique relies on an approach similar to the extrapolation of shear stress-shear rate data to zero shear rate, and is thus an indirect measurement of shear strength (Dzuy and Boger 1992). The maximum shear-strength values reported for the ball rheometer, $900 \mathrm{~Pa}$ (see Appendix I), are based on the strength required to support the ball. Typically, indirect measurements do not produce shear-strength values that are intrinsic and instrument-geometry/operation indifferent (Dzuy and Boger 1983 and 1992, Chhabra 1992, Barnes 1999, Turian et al. 2002).

Shear strength is determined from the shear vane measurement of the maximum torque required to rotate the vane (and thus a "cylinder" of the material) within the sample. The shear vane technique is a direct assessment of the point at which the material yields or starts to flow (Dzuy and Boger 1992). 
However, the shear vane measurements are taken on laboratory samples, so the samples, relative to in situ conditions, are disturbed and may be at a different temperature or may have been cycled through different temperatures, are at a different pressure, etc. The magnitude of the effect of changes from in situ conditions is dependent on the waste type.

Waste extrusion estimates of a sample's shear strength are made by comparing the sample extrusion behavior to that of simulants of known shear vane-measured shear strength (Gauglitz and Aiken 1997, Rassat et al. 2003). Although the waste-extrusion techniques rely on the behavior of ex-tank core extrusion, they may be as representative of in situ conditions as available ex-tank samples (Gauglitz et al. 2009).

Differences in shear-strength results from the ball rheometer and shear vane methods are not unexpected given the different techniques (Gauglitz and Aiken 1997, from Heath 1987). It follows, based on this possible difference and the technique itself, that waste extrusion estimates may have different shear-strength results as well. Therefore, the shear-strength measurements are presented by technique. Individual tank results by measurement technique are provided in the Shear Strength—Elevation Within the Sediment sub-section below.

Gauglitz et al. (2009) provided cumulative sediment shear-strength data by measurement technique wherein the probabilities were strictly based on measurement count. Consideration was given to improving this analysis via a reasonable statistical combination. However, it was determined that disparity between measurement techniques and results as well as incomplete waste characterization made meaningful combinations difficult. Thus, a summary of the data is presented following Gauglitz et al. (2009).

Shear-strength measurements are summarized by measurement technique; not accounted for are measurement number, location, representativeness beyond initial sample conditions, the length of time the shear strength has developed, and the relative fraction of Hanford inventory. The data sets for the different techniques encompass different waste tanks as listed in Table 3.31. In some instances, multiple measurements are available throughout the depth and/or at different radial locations in the tank. In others, single measurements are reported. Further, the data set itself represents only a part of the Hanford inventory. The sample history can also have a significant impact on results, and this may vary depending on the waste type. Shear strength is a function of time, and some of the measurements are from sludge material that has been stored undisturbed for decades. High shear strength values that result from long storage times are not likely to represent the shear strength of recently retrieved wastes. Data availability is also affected by the measurement technique. In addition, some tanks contain significantly greater fractions of the Hanford UDS inventory than others.

If a tank waste is treated as "represented" with respect to shear strength regardless of the number of measurements for a given tank, sediment shear strength for approximately $24 \%$ of the Hanford waste UDS volume is represented (47\% if the "Group" characterizations of Table 3.31 are included). As shown in Figure 3.74, 7 of the 15 primary waste types with shear-strength data have greater than $50 \%$ by UDS volume representation. The 15 primary waste types comprise approximately $52 \%$ by volume of the total UDS inventory.

Cumulative shear-strength distributions for sludge waste (primary waste type) shear vane and waste extrusion data are provided in Figure 3.75 and Figure 3.76, respectively. Shear vane, waste extrusion, and 
ball rheometer data for saltcake waste (primary waste type) are provided in Figure 3.77, Figure 3.78, and Figure 3.79.

Approximate $5^{\text {th }}, 50^{\text {th }}$ (median), and $95^{\text {th }}$ percentiles are listed in Table 3.32 . The $95^{\text {th }}$ percentile is a conservative indicator of the UL of a data set. The shear vane percentiles for sludge and saltcake are relatively similar while the waste extrusion percentiles, especially at the median, are different. The ball rheometer was not deployed in sludge waste. The ball rheometer results for saltcake show the limit of the technique, $900 \mathrm{~Pa}$.

While the analysis of the shear-strength data has limitations, the cumulative probability distributions provide estimates for shear-strength limits and range. Shear vane results exceed those for the other techniques, and $95^{\text {th }}$ percentiles of approximately 6,400 $\mathrm{Pa}$ (sludge) and 5,700 Pa (saltcake) are indicated. The $95^{\text {th }}$ percentile of combined (both sludge and saltcake) shear vane results is approximately $\left.6,200 \mathrm{~Pa} .{ }^{(\mathrm{a}}\right)$ The $95^{\text {th }}$ and $5^{\text {th }}$ percentiles are nominally factors of 10 around the $50^{\text {th }}$ percentile.

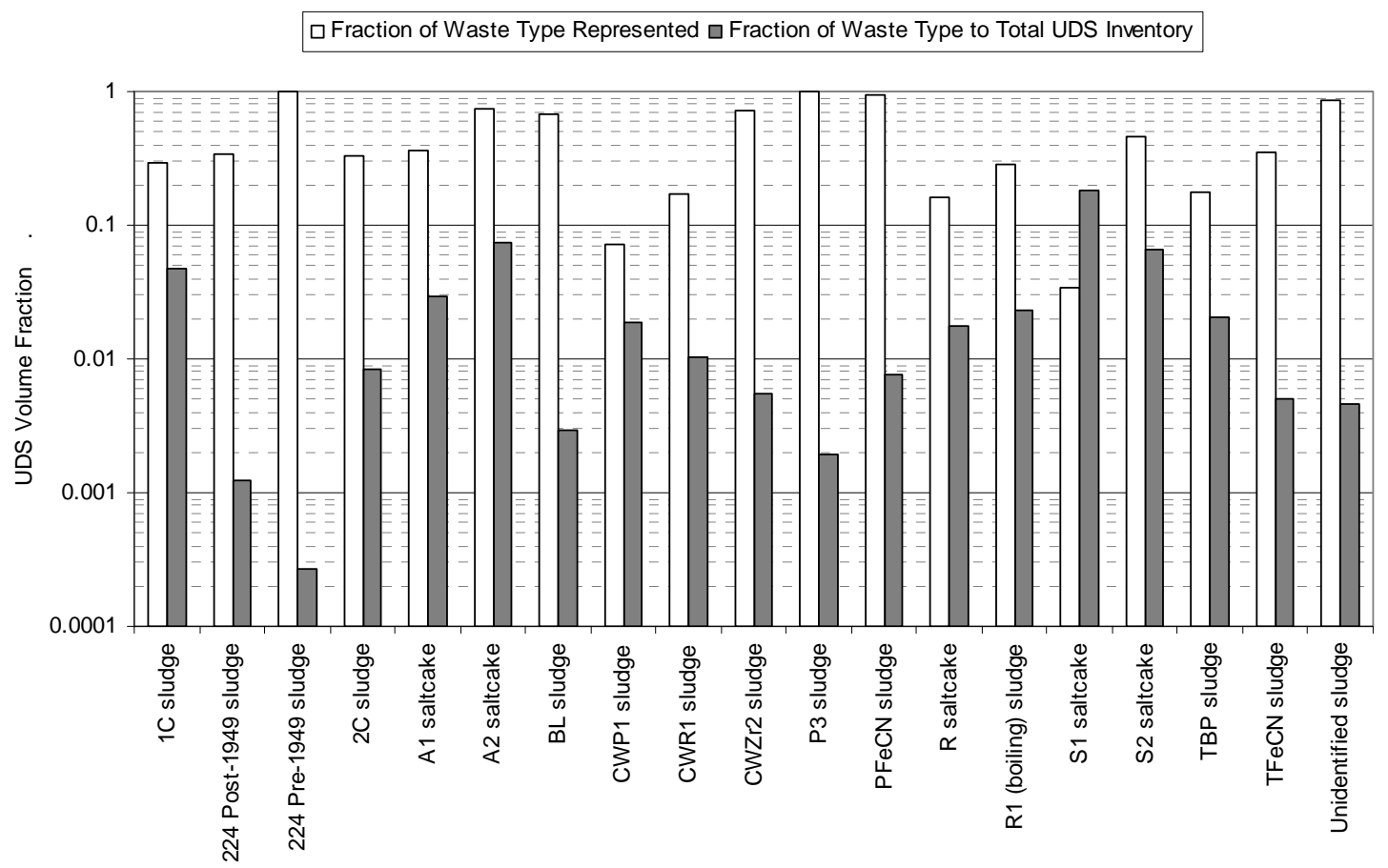

Figure 3.74. Waste Types Represented by Shear-Strength Data Set. Multiple waste types Groups (Table 3.31) are not included.

(a) Gauglitz et al. (2009) reported approximately 7,000 Pa; the difference is due to the expanded data set in the current work. 


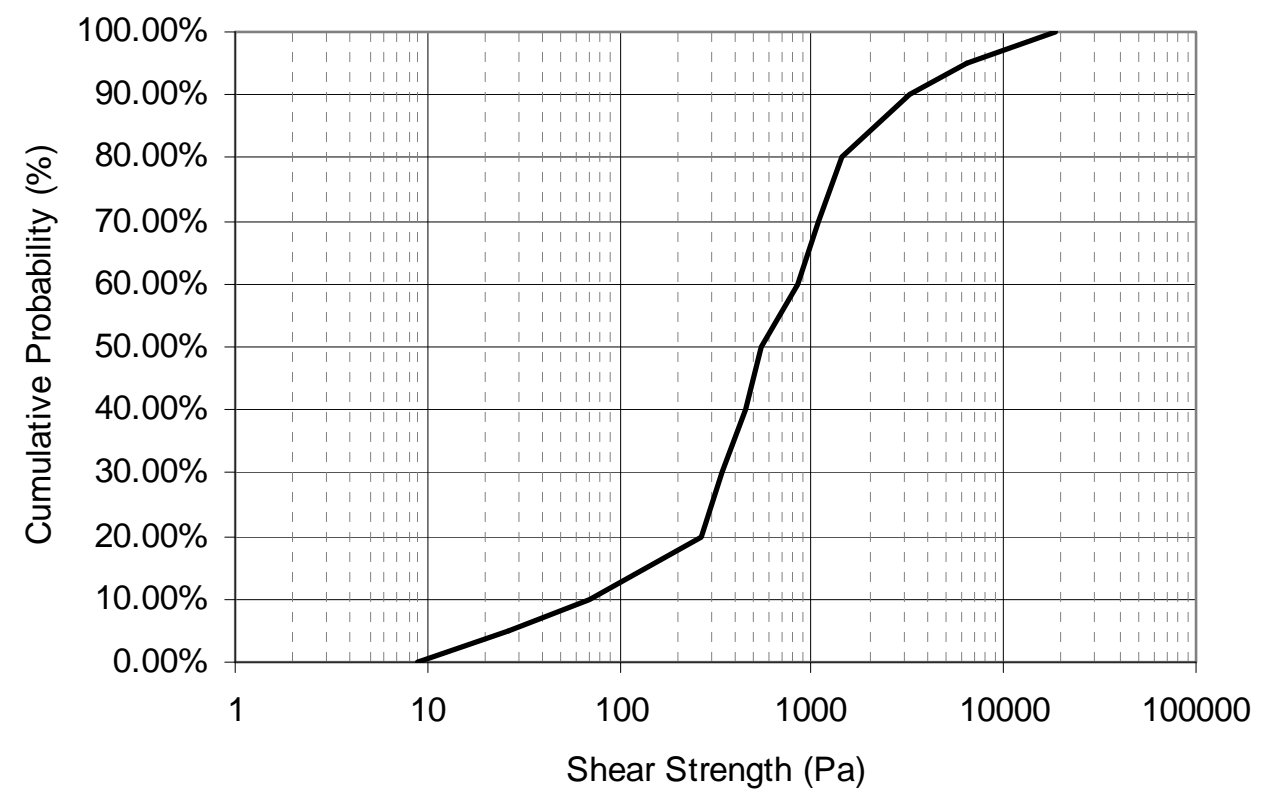

Figure 3.75. Data Summary of Sludge Shear Vane Shear Strength, 132 Measurements

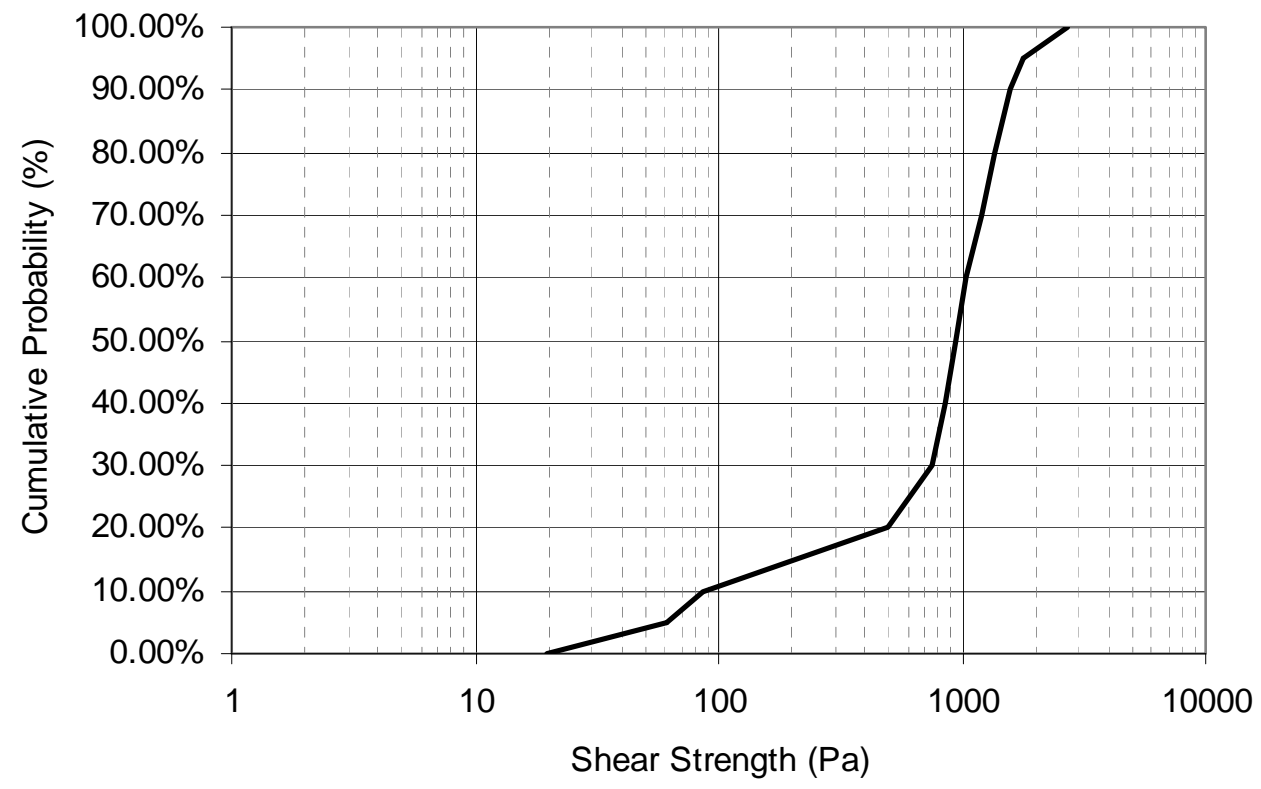

Figure 3.76. Data Summary of Sludge Waste Extrusion Shear Strength, 124 Measurements 


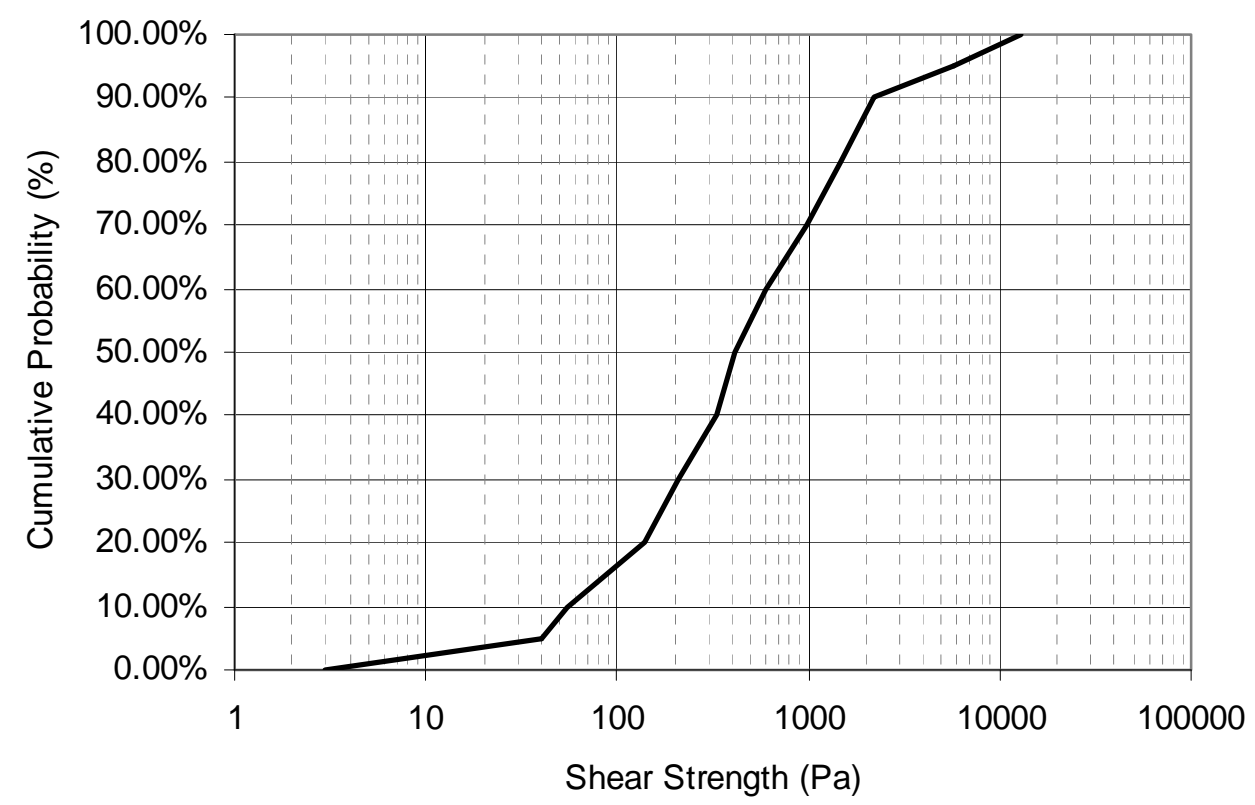

Figure 3.77. Data Summary of Saltcake Shear Vane Shear Strength, 87 Measurements

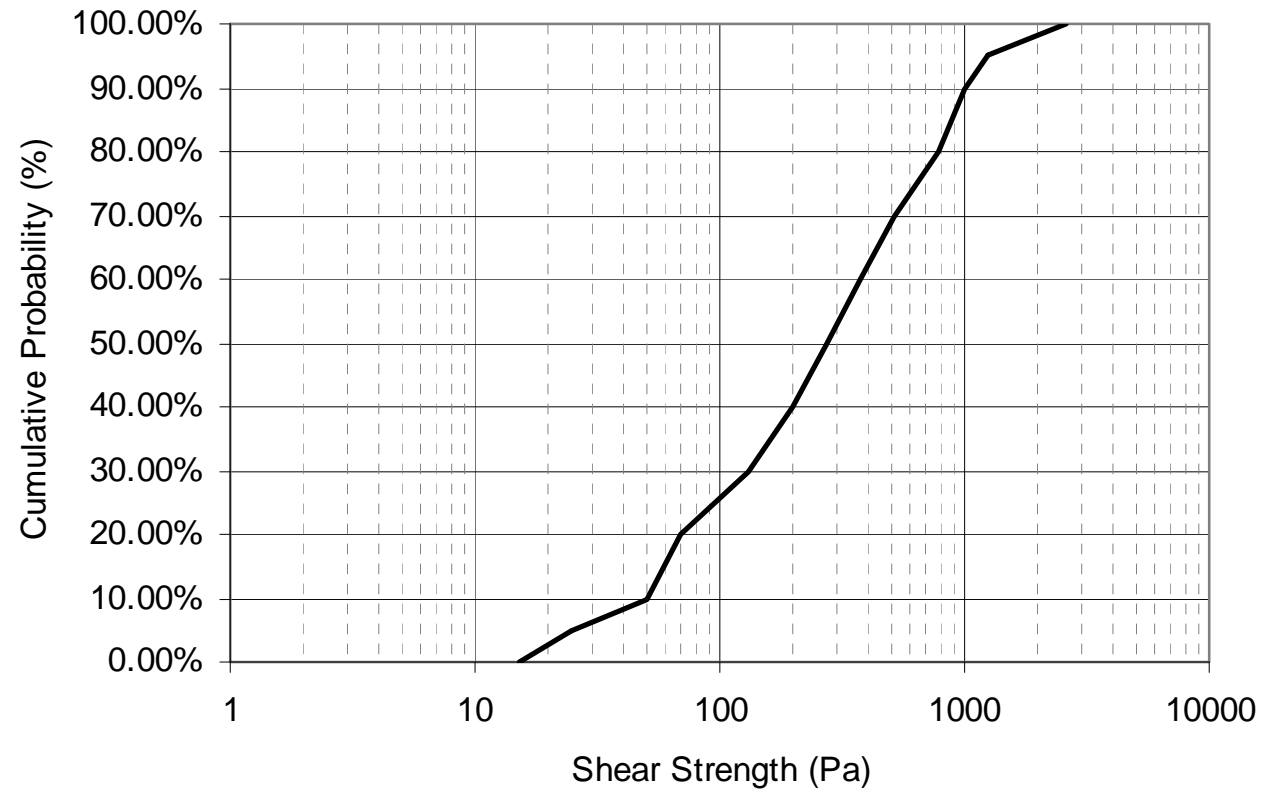

Figure 3.78. Data Summary of Saltcake Waste Extrusion Shear Strength, 147 Measurements 


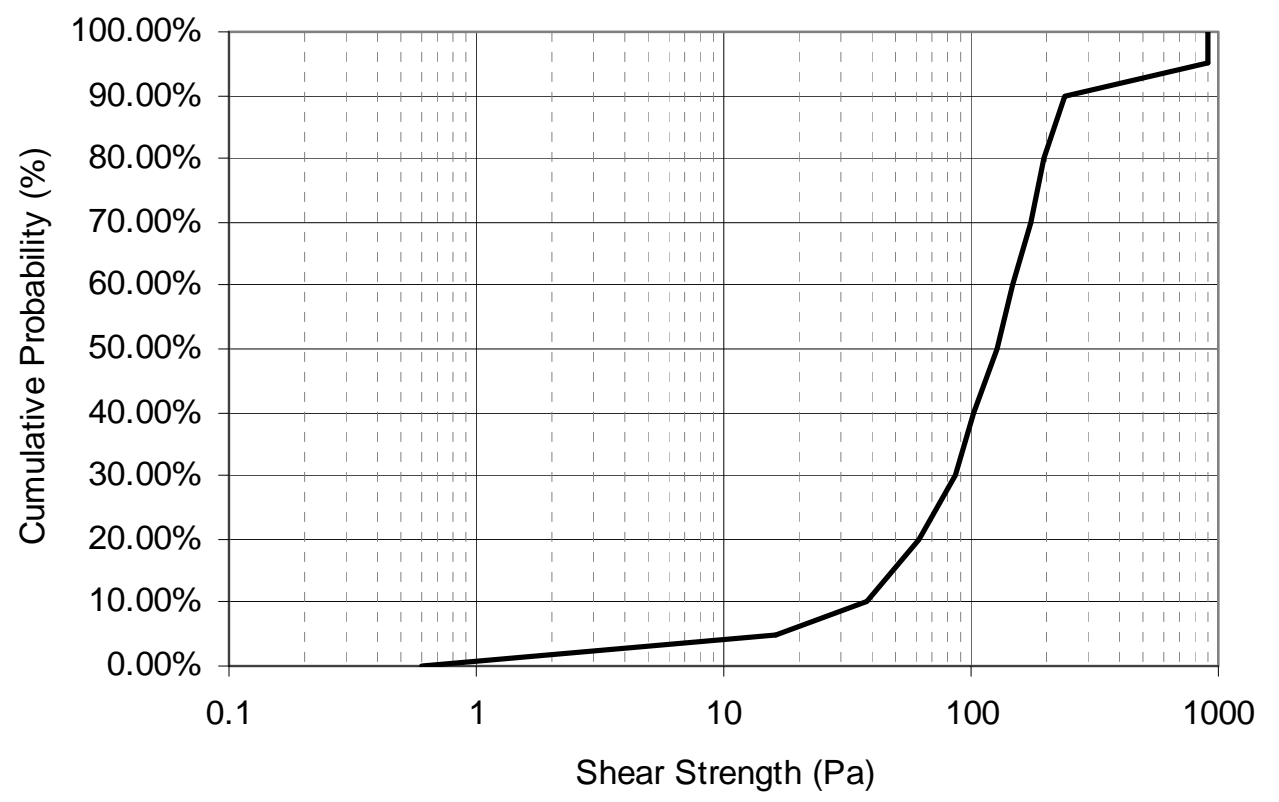

Figure 3.79. Data Summary of Saltcake Ball Rheometer Shear Strength, 170 Measurements 
Table 3.32. Shear-Strength Percentiles

\begin{tabular}{|c|c|c|c|c|}
\hline $\begin{array}{c}\text { Primary Waste } \\
\text { Type Form }\end{array}$ & $\begin{array}{c}\text { Measurement } \\
\text { Technique }\end{array}$ & $\mathbf{5}^{\text {th }}$ Percentile $^{(\mathbf{a})}$ & $\begin{array}{c}\mathbf{5 0}^{\text {th }} \\
\text { Percentile }^{(\mathbf{a})}\end{array}$ & $\mathbf{9 5}^{\text {th }}$ Percentile $^{\text {(a) }}$ \\
\hline \multirow{2}{*}{ Sludge } & shear vane & 27 & 541 & 6439 \\
\cline { 2 - 5 } & core extrusion & 62 & 931 & 1786 \\
\hline \multirow{3}{*}{ Saltcake } & shear vane & 41 & 411 & 5700 \\
\cline { 2 - 5 } & core extrusion & 25 & 270 & 1238 \\
\cline { 2 - 5 } & ball rheometer & 16 & 126 & 900 \\
\hline \multirow{2}{*}{ All } & shear vane & 33 & 507 & 6208 \\
\cline { 2 - 5 } & core extrusion & 36 & 594 & 1595 \\
\hline \multicolumn{2}{|c|}{ (a) Probabilities are solely based on measurement count. } \\
\hline
\end{tabular}

\section{Shear Strength-UDS Concentration}

A slurry material's shear strength is a function of the UDS concentration. The literature abounds with data for shear strength as a function of solids concentration for numerous different slurries (e.g., Schatzmann et al. 2003, Turian et al. 2002, Ancey and Jorrot 2001, Zhou et al. 1999 and 2001, Channell and Zukoski 1997, Buscall et al. 1987). Limited shear vane data as a function of UDS concentration are available for Hanford waste.

Hanford waste shear-strength and UDS-concentration data for core composites and dilutions thereof (see Tingey et al. 2003) for B-203 and T-204 (UDS concentrations from Gauglitz et al. 2009), and T-203 (UDS concentrations from Poloski et al. 2006) are shown in Figure 3.80.

There may be discrepancy in the measured composite UDS concentration and that estimated for in situ conditions for these B-203, T-204, and T-203 measurements. The core composites for these tanks were composed of re-hydrated samples from storage (Tingey et al. 2003). The typical total fractional water content in the as-received samples used to make the composites of Tingey et al. (2003) was approximately 0.80 by mass (Rassat et al. 2003). To achieve the lowest UDS mass concentration reported in Poloski et al. (2006) for the undiluted composite with this water content, T-204 at 0.17 (Figure 3.80), the concentration of water in the liquid must be approximately 0.95 by mass. This result is not in agreement with the liquid density of $1.17 \mathrm{~g} / \mathrm{mL}$ in T-204 (Section 3.2.1), which has an approximate maximum water concentration at that density of 0.85 by mass (Onishi et al. 2005). That is, at a water content of 0.85 in the liquid by mass, the UDS concentration in T-204 would be 0.06.

Also included in Figure 3.80 are data for AZ-101. The highest UDS concentration (0.48) is the average sediment mass fraction (Wells and Ressler 2009), and the next lower (0.40) was determined from the specific sample characterization (Gray et al. 1993). The minimum concentration value, following Gauglitz et al. (2009), is from measurements on a pretreated sample (Poloski et al. 2003, 2007). ${ }^{\text {(a) }}$ The lines in Figure 3.80 are power-law fits determined with a least squares regression using the Solver module

(a) The pretreated sample was caustic leached and washed, Geeting et al. (2003). 
in Excel ${ }^{\circledR}$ to minimize the sum of the squares of the prediction errors. ${ }^{(a)}$ The fit parameters for the power-law equation,

$$
\tau=\mathrm{Aw}_{\mathrm{S}}{ }^{\mathrm{B}}
$$

where $\tau$ is the shear strength (Pa), and $\mathrm{w}_{\mathrm{S}}$ is the mass fraction UDS, are provided in Table 3.32 .

The sample UDS concentration was reduced via dilution (B-203, T-203, and T-204) and pretreatment (AZ-101). Although the UDS of the tank waste considered is composed primary of insoluble solids, the dilutions altered the liquid properties at a minimum, and thus the data represent additional changes beyond UDS concentration alone.

As summarized in Gauglitz et al. (2009), the data in Figure 3.80 show that even small changes in the UDS content, such as through settling, stratification, and compaction, result in dramatic changes in the shear strength. The data also show that at the same UDS concentration, the shear strength for different actual wastes can differ by a factor of 100 or more.

From Table 3.31, the waste in B-203, T-203, and T-204 is the 224 Post-1949 sludge waste type, while AZ-101 is P3 sludge. These waste types comprise less than $0.3 \%$ of the UDS inventory at Hanford by volume. B-203, T-203, and T-204 comprise $17 \%$ by volume of the 224 Post-1949 sludge waste type Hanford UDS inventory, and AZ-101 comprises 48\% of P3 sludge. Thus, the functionality of shear strength with UDS concentration is described for an extremely limited fraction of the waste.

Within the 224 Post-1949 sludge waste type, there is substantial variation of the shear strength as a function of UDS concentration relations. Thus, it is plausible to expect, as supported by the difference in the P3 sludge, that a broad range of behavior may be expected for the Hanford UDS inventory.

(a) Power-law dependencies for shear properties are exhibited for a wide range of suspensions in the literature. 


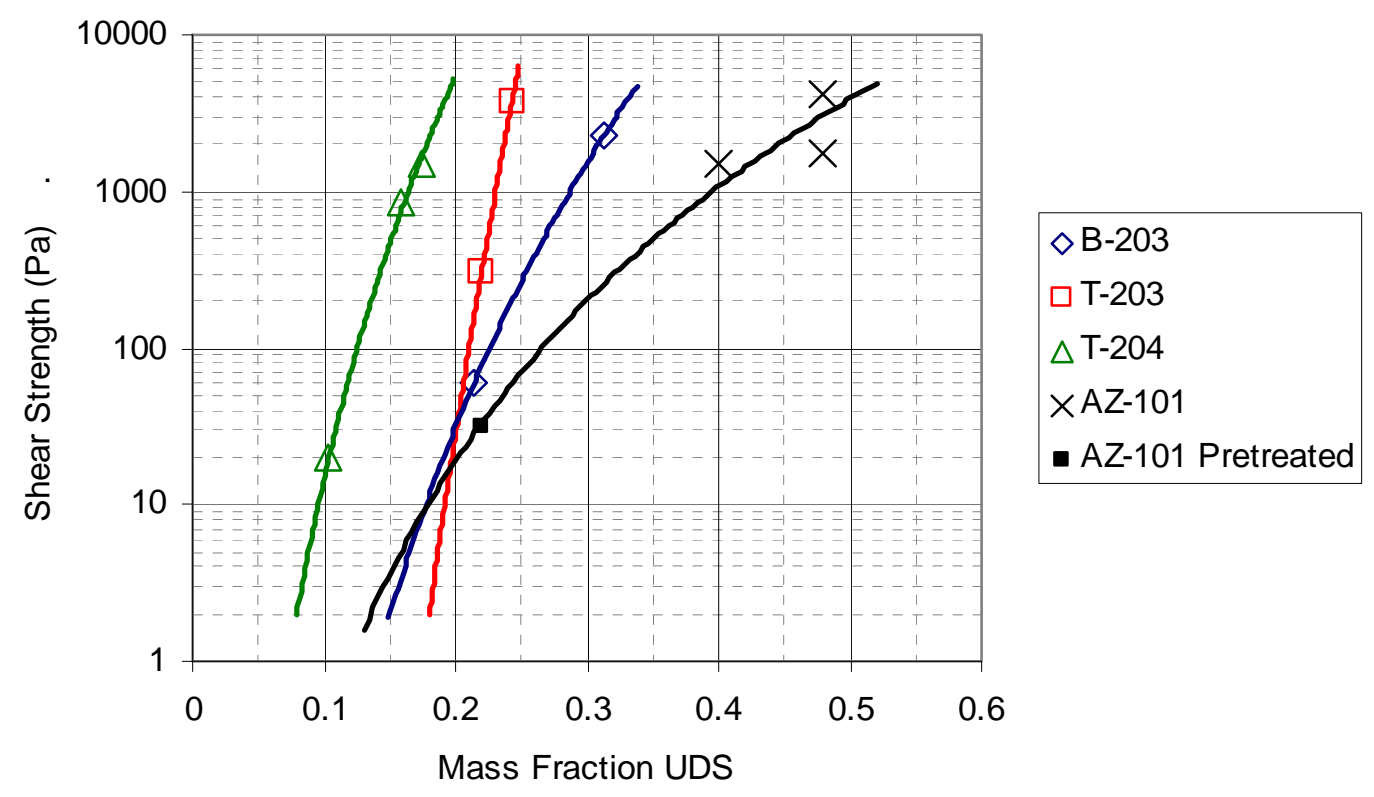

Figure 3.80. Shear Strength as a Function of UDS Concentration, Shear Vane Data

Table 3.33. Power-Law Parameters for Shear Strength as a Function of UDS Concentration Available for Hanford Waste

\begin{tabular}{|c|c|c|c|}
\hline Tank & A & B & $\mathbf{R}^{\mathbf{2}}$ \\
\hline B-203 & 1.53E8 & 9.57 & 1.0 \\
\hline T-203 & $1.03 \mathrm{E} 19$ & 25.1 & 1.0 \\
\hline T-204 & 4.77E9 & 8.51 & 0.999 \\
\hline AZ-101 & $2.18 E 5$ & 5.80 & 0.964 \\
\hline
\end{tabular}

Shear strength as a function of UDS concentration relations for materials with some similarity to Hanford waste are provided in Figure 3.81. The figure includes data for chemical simulants specifically designed to represent certain physical and chemical properties of Hanford sludge, clay slurries commonly employed for Hanford waste simulants, and metal oxide solutions.

The data for Simulants 1 and 2 are taken from Wells et al. (2010a). Wells et al. (2010b) showed Simulant 1 to represent the Hanford sludge as a whole and Simulant 2 the resultant sludge from a retrieval scenario (a combination of C-104, C-111, and C-112 waste in AN-101). The simulants are unique with respect to each other for some of the properties considered.

Numerous experimental studies related to the storage and retrieval of waste from the large underground Hanford storage tanks have employed clay slurries as simulants to represent the waste of interest. These studies have included investigations of gas retention and release (Gauglitz et al. 1994, 1995, 1996, Stewart et al. 1996b), sediment mobilization (Powell et al. 1995b, Enderlin et al. 2003, 
Bontha et al. 2005, Kurath et al. 2007), and slurry transport (Poloski et al. 2009a, Bontha et al. 2010). The waste extrusion shear strength method development of Gauglitz and Aiken (1997) used bentonite slurry to mimic ductile waste behavior. Correlations for EPK kaolin (Gauglitz et al. 2010a), kaolin/bentonite from Rassat et al. (2003), and bentonite (Alderman et al. 1991) are presented in Figure 3.81.

The "Red Mud" data are a vane average from Dzuy and Boger (1983). Red mud is a waste product in the Bayer process for extracting alumina from bauxite and comprises large quantities of $\mathrm{Fe}_{2} \mathrm{O}_{3}, \mathrm{SiO}_{2}$, and $\mathrm{Al}_{2} \mathrm{O}_{3}$ with various other metal oxides. The suspending liquid contains a large amount of soluble salts, and, owing to the high caustic content, the $\mathrm{pH}$ can be as high as 12 . About 70 to $80 \%$ by weight of the red mud solid particles are less than $10 \mu \mathrm{m}$. The maximum and minimum $\mathrm{d}_{50}$ (median by volume) particle size $\mathrm{Al}_{2} \mathrm{O}_{3}$ particulate and DI water slurry data are determined from Zhou et al. (1999) at a pH of 8.9. Shear-strength measurements were taken with a shear vane.

Bentonite clay is shown to be similar to the T-204 data, and the kaolin/bentonite slurry and Simulant 2 to the AZ-101 data. Simulant 2, EPK kaolin slurry, and metal oxide systems are shown to require higher UDS concentrations to achieve equivalent shear strengths to the Hanford waste. These results suggest that a broad range of shear strength as a function of UDS concentration behavior may be expected for the different wastes comprising the Hanford UDS inventory.
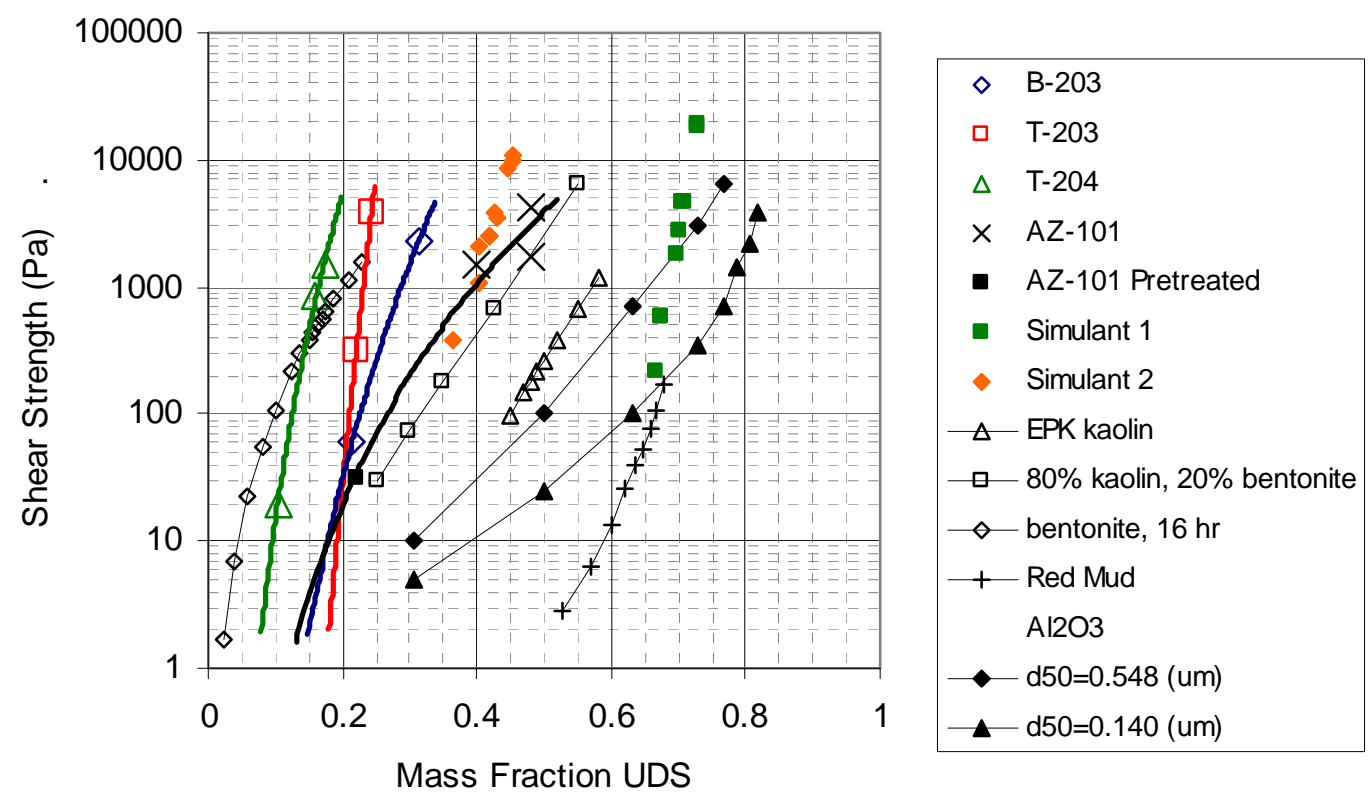

Figure 3.81. Shear Strength as a Function of UDS Concentration; Shear Vane Data for Hanford Waste and Various Slurries

Data for AY-102 sediment are considered for shear strength as a function of UDS concentration as sampled in situ (samples represent in situ locations) as opposed to the diluted/pretreated samples of Figure 3.80. Approximately $186 \mathrm{kgal}$ of sludge was retrieved from C-106 and transferred to AY-102 via three sluicing campaigns from November 1998 through October 1999 (Cuta et al. 2000). AY-102 contained approximately 9 inches of sediment initially and had nominally 67 inches of sediment after the 
completion of the sluicing campaigns. There were seven major periods of retrieval over the nominal 1 year total retrieval time. As such, the sediment layer in AY-102 was sequentially loaded. ${ }^{(a)}$

Percent water data, providing a representation of the UDS concentration with the expected uniform-with-depth interstitial liquid, and the shear strength for AY-102 sediment cores 270 and 273 are shown in Figure 3.82 (Gauglitz et al. 2009). These two cores were taken via outer-radius risers that are approximately opposite each other. There is no indication that water content and shear strength are correlated. The influences of quiescent time until measurement and elevation in the sediment on the AY-102 shear-strength measurements are discussed in subsequent sub-sections.

Nominally 1000 Pa shear-strength measurements are reported in Figure 3.82 at water contents of the total sample of approximately 30 to $45 \%$. With a water content in the interstitial liquid of approximately $84 \%$ by mass (Poloski et al. 2007), these total-sample water contents correspond approximately to UDS mass fractions of 0.64 and 0.46 , respectively. The range of UDS mass fraction at $1000 \mathrm{~Pa}$ shear strength for AY-102 sediment therefore approximates the mid-range of the UDS mass fraction range at $1000 \mathrm{~Pa}$ of the data in Figure 3.81, thereby reinforcing the fact that a broad range of shear strength as a function of UDS concentration behavior may be expected for the different wastes comprising the Hanford UDS inventory.

- Core 270, Shear Strength $\Delta$ Core 273, Shear Strength

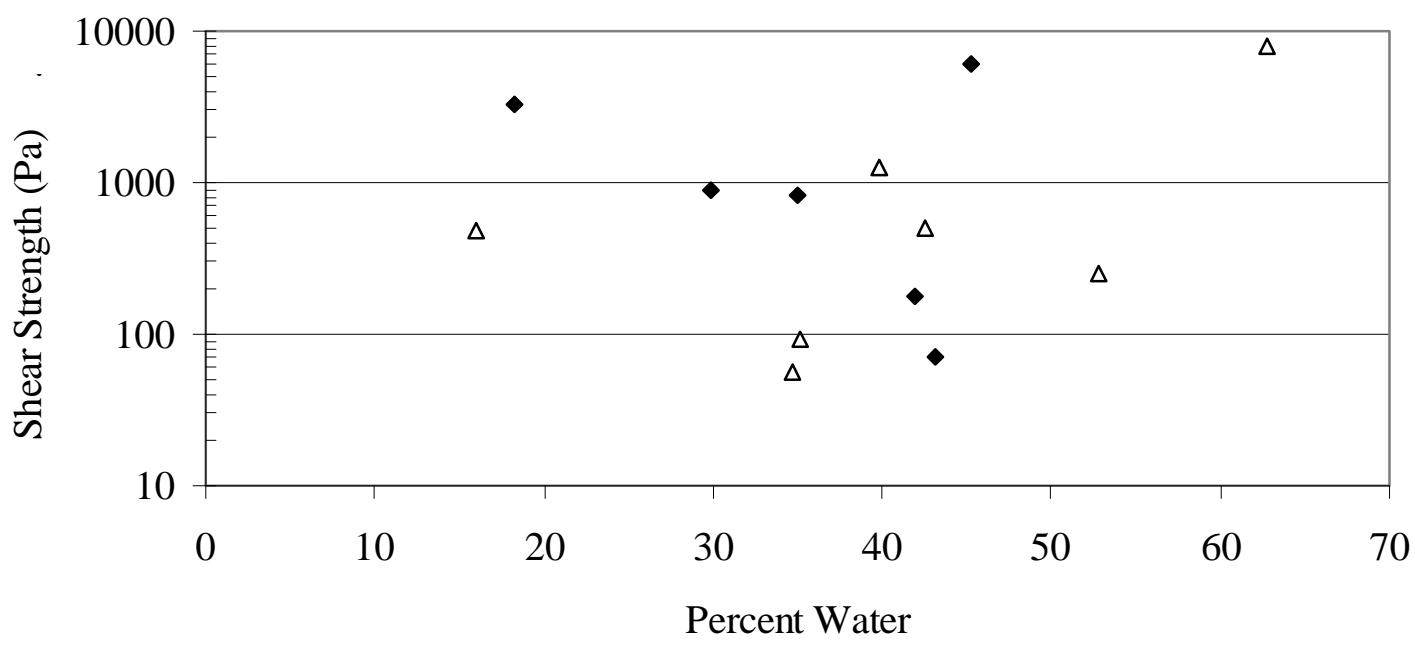

Figure 3.82. AY-102 Sediment Shear Strength as a Function of Percent Water, Shear Vane Data (Gauglitz et al. 2009)

As presented, the Hanford waste data set for shear strength as a function of UDS concentration is limited. Additional consideration of this relationship is made by approximating an average UDS concentration of the sediments with shear-strength measurements. Average UDS concentrations by mass are determined similarly to Gauglitz et al. (2010b) wherein the sediment and liquid densities are taken

(a) $100 \%$ of the UDS volume in AY-102 is classified as BL sludge, Table 3.1. 
from Weber (2009), and the solid density is taken from Appendix C. ${ }^{\text {(a) }}$ The data are presented by assigning the average sediment UDS concentration to all shear-strength measurements for given tank (with the exception of data for diluted samples, e.g., the tanks of Figure 3.80).

The shear-strength, average UDS concentration relationships can be presented by waste type or larger composites. As shown in Table 3.31, 10 of the 15 primary waste types have multiple tanks represented. In those waste types with multiple tanks, there are limited cases with substantial data sets that have significant differences in average UDS concentration. Thus shear-strength measurements by measurement type grouped for primary waste type as sludge (Figure 3.83) or saltcake (Figure 3.84) waste are presented. With a broad range of UDS concentrations providing similar shear strengths, the data again suggest that different shear-strength-UDS concentration behavior may be expected for the different wastes comprising the Hanford UDS inventory.

A least squares regression fit of Eq. (3.11) is shown in Figure 3.83 and Figure 3.84. The fits, made using the Solver module in Excel ${ }^{\circledR}$ to minimize the sum of the squares of the prediction errors, are to the complete data sets, in which, as shown in Figure 3.80, there can be substantial variation of the shear strength as a function of UDS concentration dependent on the waste considered, and are independent of measurement technique. Thus, the fits are useful only to provide an indication of a typical shear-strength result at a given UDS concentration. The results for sludge wastes range from approximately $500 \mathrm{~Pa}$ at the lower UDS concentration up to $2000 \mathrm{~Pa}$. The expected sludge shear strength is shown to exceed that of saltcake.

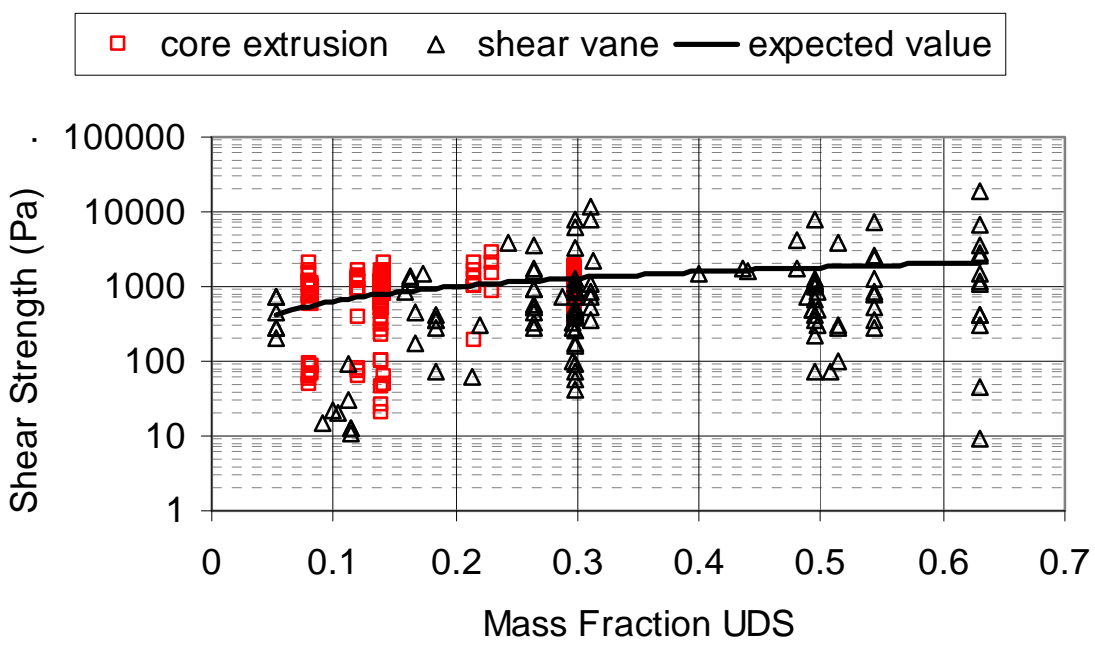

Figure 3.83. Shear Strength as a Function of Average UDS Concentration, Tanks with Primary Waste Type Sludge

(a) Layer densities for tanks that have been substantially diluted and retrieved since characterization of shear strength, e.g. SY-101, have been adjusted to reflect the conditions at the time of sampling. 


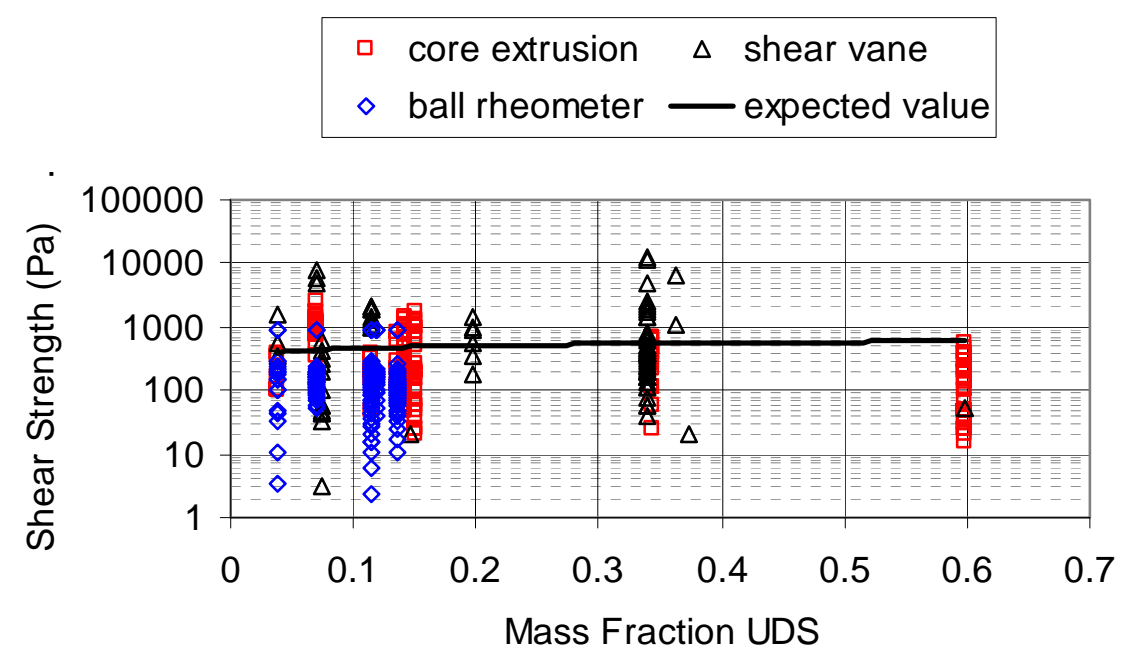

Figure 3.84. Shear Strength as a Function of Average UDS Concentration, Tanks with Primary Waste Type Saltcake

\section{Shear Strength-Quiescent Time}

A slurry's shear strength may increase with quiescent time independently of changes in UDS concentration due to settling or compaction. Poloski et al. (2007) applied the shear-strength rebuild model of Speers et al. (1987),

$$
\frac{G_{t}-G_{\infty}}{G_{0}-G_{\infty}}=e^{-k t}
$$

$$
\text { where } \begin{aligned}
\mathrm{G}_{\mathrm{t}} & =\text { shear strength } \\
\mathrm{t} & =\text { gel time } \\
\mathrm{G}_{0} & =\text { initial shear strength } \\
\mathrm{G}_{\infty} & =\text { equilibrium shear strength } \\
\mathrm{k} & =\text { gel time constant, }
\end{aligned}
$$

to the laboratory transient shear-strength data for AZ-101 pretreated sludge (the only Hanford slurry data identified for determining the gel time constant) as well as to other industrial and Hanford sludge simulant slurries. The shear strength of the AZ-101 pretreated sludge was shown to reach 95\% of the equilibrium shear strength in approximately 10 hours (gel time constant of $0.262 \mathrm{hr}^{-1}$ ), while the majority of the other slurries considered required days, see Figure 3.85 (from Poloski et al. 2007). ${ }^{\text {(a) }}$ As shown in Figure 3.85, the dispersed gel-chemical and East Coast biovert show decreased time to reach equilibrium strength with increasing temperature.

From Table 3.31, the waste in AZ-101 is P3 sludge. This waste type comprises less than $0.2 \%$ of the UDS inventory at Hanford by volume. AZ-101 comprises 48\% of P3 sludge. Thus, the functionality of

(a) Time to reach $95 \%$ of the equilibrium shear strength approximated by $-\ln (0.05) / \mathrm{k}$ (Poloski et al. 2007). 
shear strength with quiescent time is described for an extremely limited fraction of the waste, and that which is described is for a pretreated sample. The AZ-101 pretreated sludge gel time constant $0.262 \mathrm{hr}^{-1}$ (and thereby the approximately 10-hour period to reach 95\% of the equilibrium shear strength) was recommended by Poloski et al. (2007) to be applied to Hanford waste in the absence of additional transient shear-strength information.

The maximum shear strength from any measurement technique, representing a maximum equilibrium shear strength attainable in the nominally 10-hour period described by the AZ-101 pretreated sludge data by tank and waste type from the Appendix I data, are provided in Figure 3.86 and Figure 3.87 for sludge and saltcake waste types, respectively. The maximum shear-strength values are indiscriminate of measurement technique. A broad range of maximum shear strength is indicated, with approximately $4000 \mathrm{~Pa}$ for actual AZ-101 waste. As discussed below, the limited waste data indicate that the AZ-101 pretreated sludge gel time constant may be applicable to sludge wastes, but may significantly overestimate the shear-strength re-growth rate for saltcake wastes.

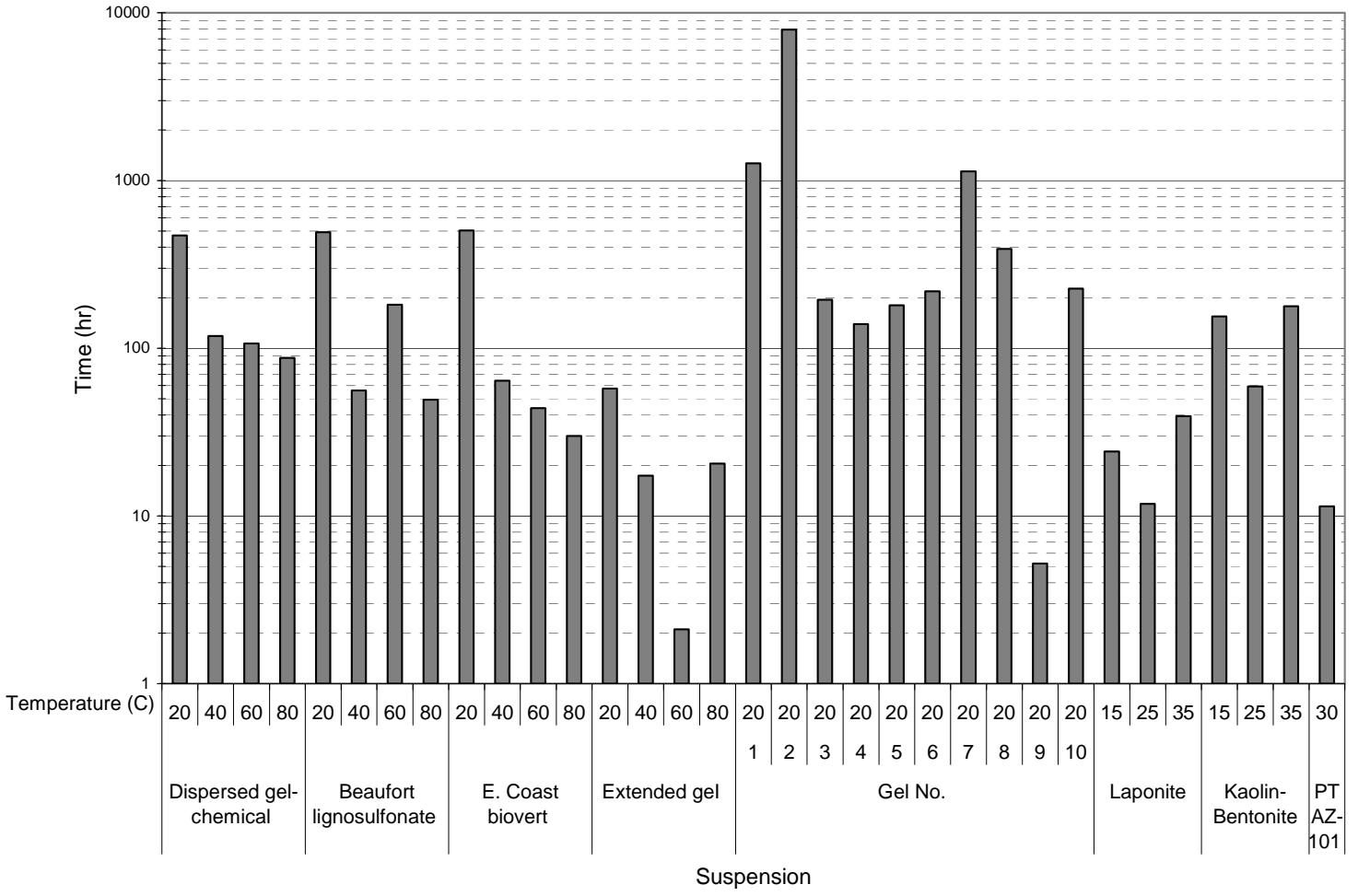

Figure 3.85. Time to Reach Equilibrium Shear Strength, Comparison of Various Suspensions (Poloski et al. 2007) 


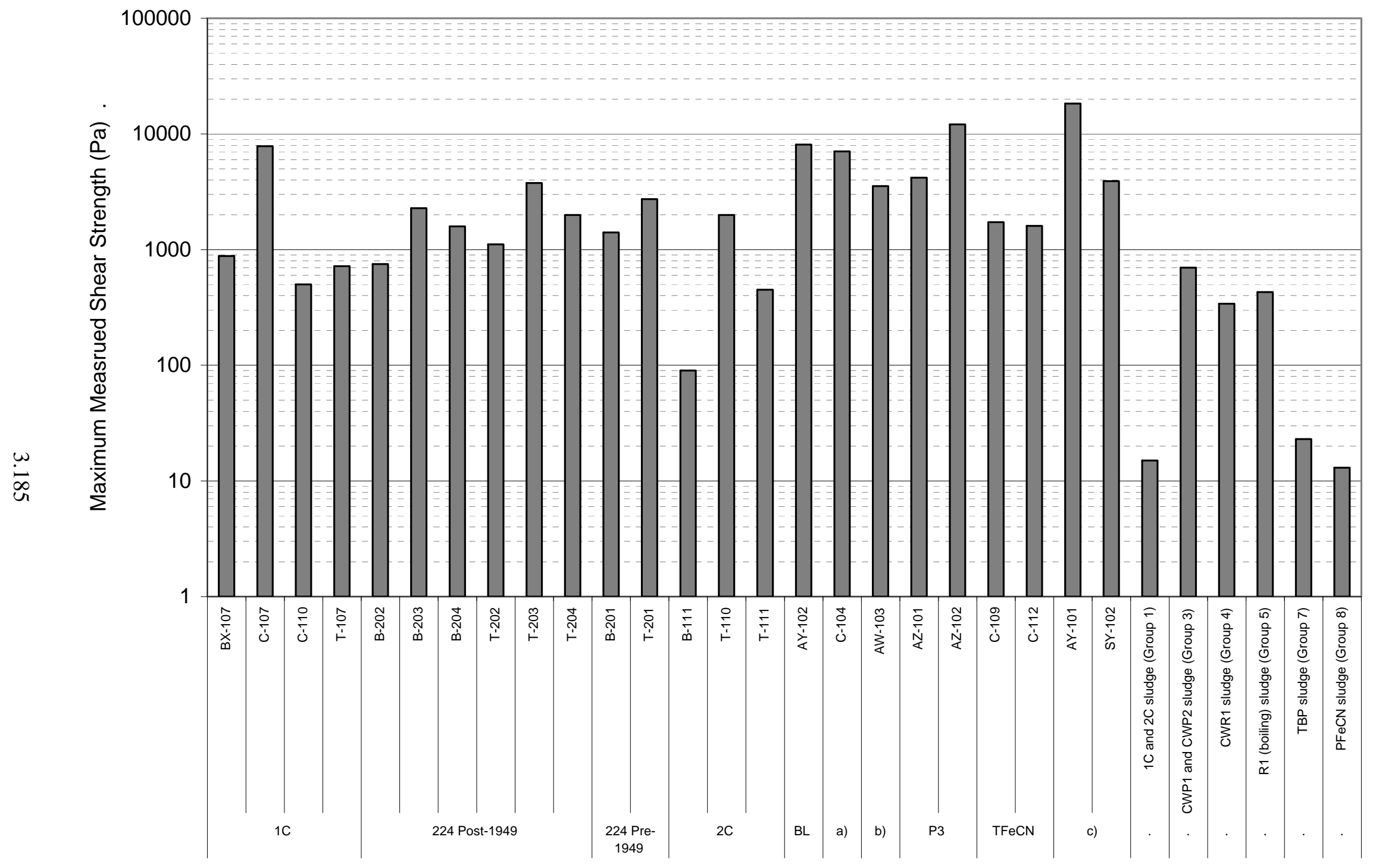

Figure 3.86. Maximum Measured Shear Strength, Sludge: a) CWP1, b) CWZr2, c) Unidentified 


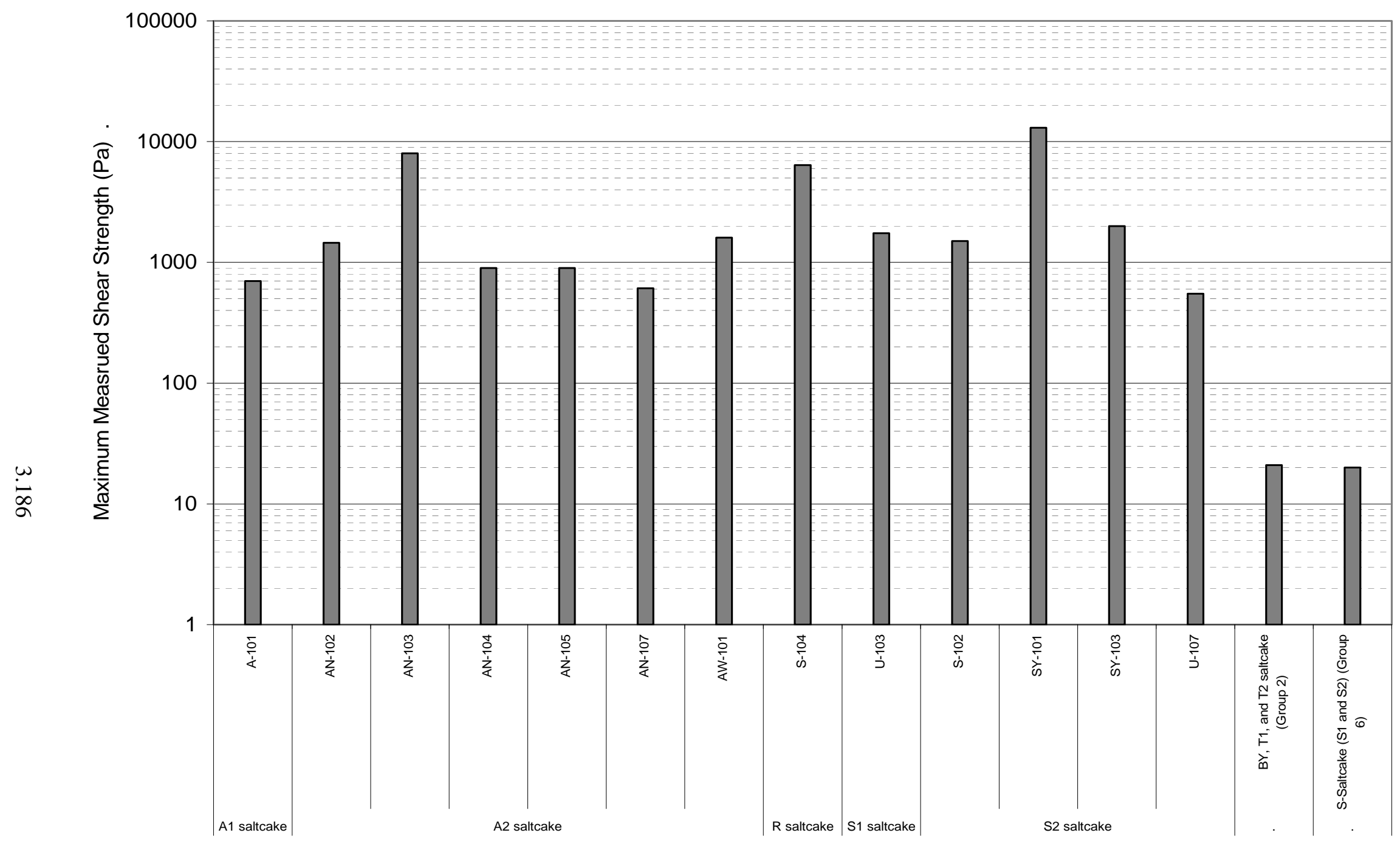

Figure 3.87. Maximum Measured Shear Strength, Saltcake 
The shear strength of settled in situ Hanford sediments following any combination of mixing, transfer, and other disturbances are considered in relation to the approximate 10-hour shear-strength re-growth period of the AZ-101 pretreated sludge. Insightful results from two sludge tanks are considered, AZ-101 and AY-102, and from three saltcake tanks, SY-101, AN-104, and AN-105.

The shear strength of the sediment layer in AZ-101 approximately 80 days after essentially complete mobilization via the mixer pumps (Carlson et al. 2001) was apparently equivalent to the material's original shear strength (Gauglitz et al. 2009). In addition, Gauglitz et al. (2009) noted that sludge-weight measurements show, depending on the riser where the sludge weight was deployed, that there was a settled layer with a shear strength of at least 200 Pa beginning as early as 1 day after mixing ceased. Thus, the AZ-101 actual waste data are consistent with the AZ-101 pretreated sludge gel time constant.

The sequential retrieval of C-106 sediment into AY-102 (see above) resulted in different "aged" settled layers in the sediment. In Figure 3.88, the shear-strength data from AY-102 core samples are plotted as a function of time since they were deposited (Gauglitz et al. 2009). The shear-strength measurements are grouped by approximate nominal elevations. Based on this figure, the shear strength appears to be independent of time. Gauglitz et al. (2009) suggest that the minimum time for a given elevation, approximately 100 days, was sufficient for the material to reach its "final" shear strength. It is not possible to determine at what point during the initial 100 days the "final" shear strength was attained. The applicable sludge tank data, therefore, do not provide evidence that the AZ-101 pretreated sludge gel time constant under-represents the shear-strength re-growth period.

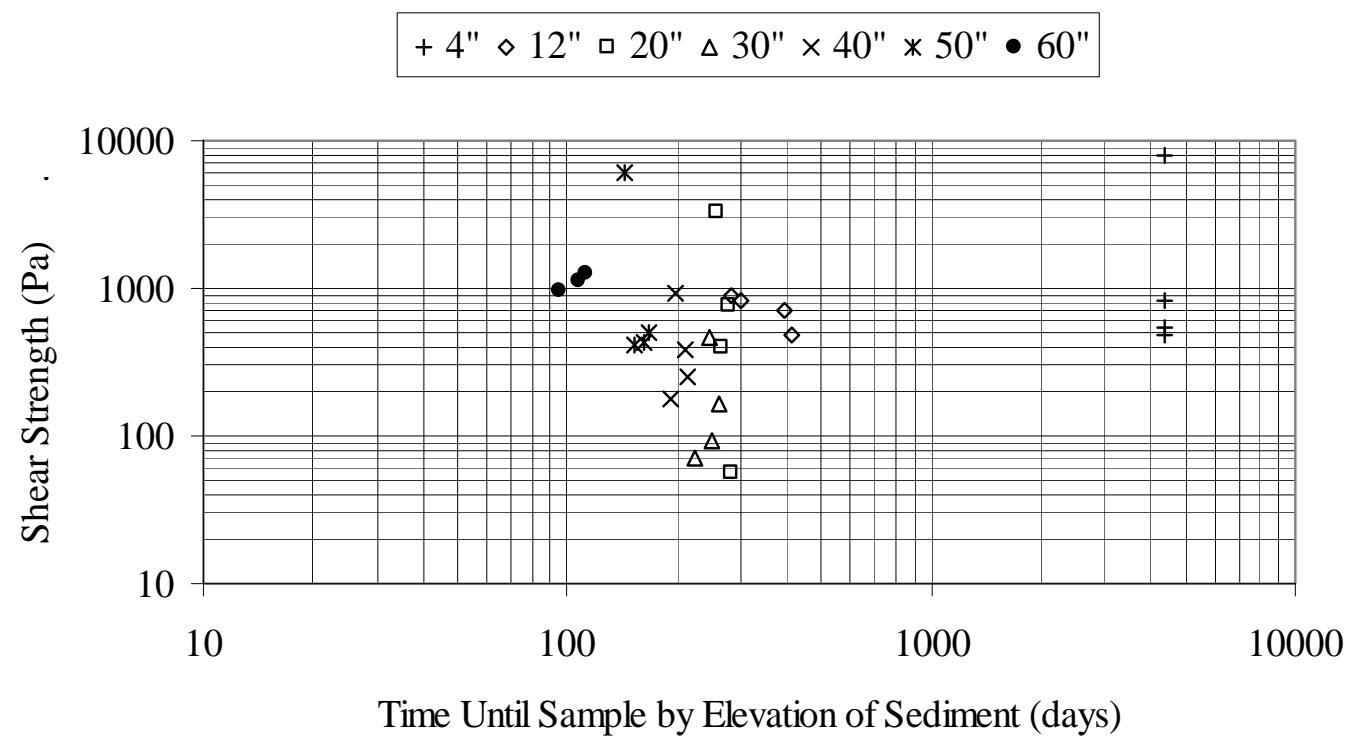

Figure 3.88. AY-102 Sediment Shear Strength for Waste Cores 270 Through 273 as a Function of Time. Legend indicates approximate elevation from tank bottom (Gauglitz et al. 2009).

Starting in 1993 and continuing to April 2000, the sediment layer in SY-101 was mobilized by a mixer pump, typically on a tri-weekly schedule that resulted in regions away from the orientation of the mixer pump being "undisturbed” for nominal 30-day periods. As reported in Gauglitz et al. (2009), the shear strength of a recently settled layer approximately 10 days after the mixer pump was directed at that 
location, as measured with the ball rheometer, was about $10 \mathrm{~Pa}$ at 24 inches above the tank bottom and $30 \mathrm{~Pa}$ at 6 inches.

Further, slurry from the mobilized SY-101 sediment was transported into SY-102. It is acknowledged that soluble solids most certainly were dissolved by water dilutions during the retrieval and transfer process as well as in the more dilute supernate of SY-102. However, the resultant shear strength of the settled material in SY-102 is still of interest. The sediment shear strength in SY-102 was measured with a shear vane on a core sample that was taken 235 days after the final waste transfer from SY-101 (Gauglitz et al. 2009). The median of the measurements is $279 \mathrm{~Pa}$, which, as with the 10 and $24 \mathrm{~Pa}$ in situ measurements, is substantially lower than the SY-101 maximum of approximately 10,000 Pa from Figure 3.87. Thus, it is indicated that the AZ-101 pretreated sludge gel time constant may significantly overestimate the shear-strength re-growth rate for the pre-1999 SY-101 sediment.

Onishi et al. (2003) evaluated sequential-pass ball rheometer data (the ball was repeatedly lowered and raised through the sediment at the same radial location) from AN-104 and AN-105 to investigate how the shear strength changes as the waste is disturbed. The time elapsed between each measurement at a given elevation is estimated to be at least 50 minutes.

As shown in Figure 3.89, the shear strength was reduced as the ball rheometer repeatedly passed through the AN-105 sediment. The shear strength is indicated to be sensitive to shear history and approaches an asymptotic limit with several repeated shearings. The AN-104 shear-strength magnitude and changes with subsequent passes are similar to those of AN-105 (Onishi et al. 2003).

Clearly, the at-least 50-minute interval between ball passes at a given elevation was insufficient for the AN-104 and AN-105 sediment to regain its original shear strength. Thus, for the available saltcake tank data, the AZ-101 pretreated sludge gel time constant overestimates the shear-strength re-growth rate. These results are in agreement with the supposition of Poloski et al. (2007), wherein large shear-strength values in saltcake wastes were attributed to precipitated salt bridges between particles that would likely require excessive time to reform.

A summary of laboratory shear strength measurements made on settled layers that formed in laboratory-scale settling experiments is provided in Gauglitz et al. (2009). During the extensive laboratory testing conducted on eight waste group composites in support of the WTP effort to resolve the EFRT issue M12, composite samples were gravity settled over a period of a couple days, creating a settled solids layer under a supernatant layer, and the shear strength of the settled layer was measured. For four samples with less than $15 \mathrm{wt} \%$ UDS in the bulk samples, the shear strength of the settled solids varied between 11 and $23 \mathrm{~Pa}$ and the settled sample volume ranged from $38.9 \%$ to $85.7 \%$ of the total sample. This is a relatively deep layer of settled waste with a shear strength in the range of $20 \mathrm{~Pa}$. The two strongest samples, which are plutonium-uranium extraction (PUREX) cladding waste sludge (Group 3 ) and reduction-oxidation (REDOX) cladding waste sludge (Group 4), had a total UDS in the bulk sample of about $30 \mathrm{wt} \%$ UDS and strengths between 100 and $700 \mathrm{~Pa}$. These samples were measured after sitting undisturbed for a 72-hour period, and it was proposed that the shear strength values were low because both measurements were performed with the rotary vane of the instrument only half immersed into the sample. 


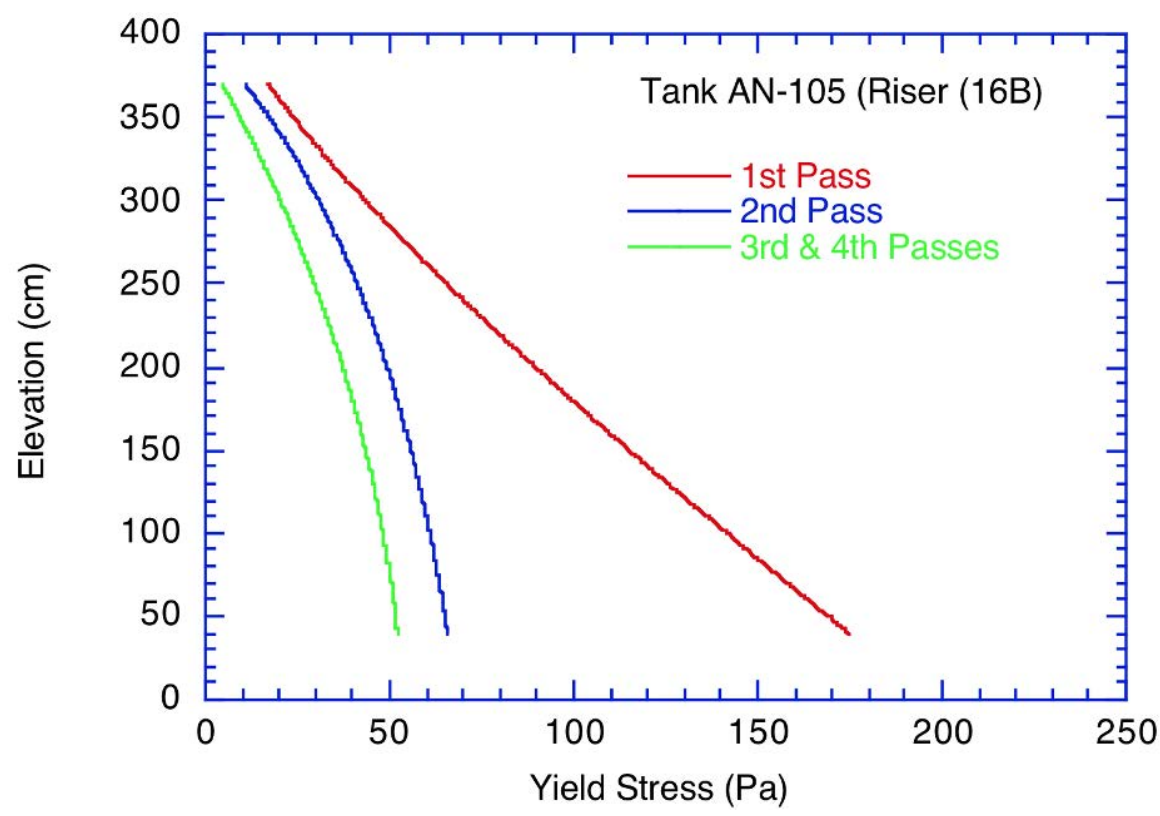

Figure 3.89. AN-105 Sediment Shear Strength from Subsequent Ball Rheometer Passes (Onishi et al. 2003)

\section{Shear Strength-Particle Size}

The shear strength of a slurry is influenced by the particle size and distribution of the UDS particulate. Discussions regarding the functionality of shear strength with particle size and distribution for different slurries can be found in Naeini and Baziar (2004), Turian et al. (2002), Ancey and Jorrot (2001), Zhou et al. (1999 and 2001), Buscal et al. (1987), Schatzmann et al. (2003), etc.

Naeini and Baziar (2004) investigated the effect of size distribution by varying the concentration of a "fine" component, silt with median size $25 \mu \mathrm{m}$, maximum size of $74 \mu \mathrm{m}$, in sand with a median size of $190 \mu \mathrm{m}$ and $100 \%$ greater than $74 \mu \mathrm{m}$. As the silt content increases up to $35 \%$, the strength (via triaxial compression tests) decreases. With further increase in the silt content, the strength is increased.

The shear strength (as determined from the slump method) increases as the concentration of glass beads and sand (300 to $1200 \mathrm{~mm}$ ) is increased in kaolin clay (Ancey and Jorrot 2001). The grain shape was also a key parameter in determining the shear strength; the more irregular the particle shape, the larger the shear strength.

With coal particulate slurries, Turian et al. (2002) found that narrowly distributed larger sized particulate slurries showed no shear strength (shear vane measurements) while broadly distributed finer sized slurries did. Zhou et al. (1999) found that the shear strength (shear vane measurements on $\mathrm{Al}_{2} \mathrm{O}_{3}$ slurries; see Figure 3.81) is dependent on the inverse square of the diameter, or

$$
\tau=\frac{\mathrm{K}}{\mathrm{d}^{2}} \phi^{\mathrm{c}}
$$


where $\mathrm{K}=$ a constant that relates bond strength to material properties and surface chemistry condition

$\mathrm{d}=$ particle diameter

$\phi=$ UDS concentration by volume

$\mathrm{c}=\mathrm{a}$ fitting parameter.

Poloski et al. (2007) considered the functionality of the maximum shear strength in a tank with respect to particle size based on Zhou et al. (2001). The relationship with slurry composition for waste types and shear strength was analyzed and it was found that, with the exception of the saltcake wastes, the shear strength increases with increasing concentration of the following species (in order of significance): zirconium compounds $>$ boehmite $>$ bismuth compounds $>$ gibbsite $>$ iron compounds. The silica and aluminosilicate content did not show a strong effect on shear strength.

The observation was explained by a particle size argument. The zirconium compounds, boehmite, bismuth compounds, gibbsite, and iron hydroxide particles are generally submicron, colloidal particles with a large surface-to-mass ratio (see Section 3.2.4). The silica present in the tank waste is generally thought to come from Hanford sand with a much larger particle size (Wells et al. 2007). In this situation, increases in the relative amounts of $\mathrm{ZrO}_{2}$, boehmite, gibbsite, and iron hydroxide would reduce the overall particle diameter and increase the shear strength. Adding silica would raise the particle diameter and reduce the shear strength of the mixture.

\section{Shear Strength-Elevation Within the Sediment}

As described in Section 2.0, sediment shear strength is a significant parameter for sediment mobilization. Gas retention and release behavior also depends on shear strength (e.g., Meacham 2010, Weber 2009, Stewart et al. 2005). The relation of shear strength with elevation in the sediment can affect these dependencies. Shear strength as a function of elevation is considered for the Appendix I data set.

In Figure 3.90 through Figure 3.101, the shear strength is provided as a function of height for waste tanks as grouped by primary waste type. Compaction or consolidation of deeper sediment due to the increased lithostatic load may increase the shear strength because of increased UDS concentration. Although exceptions exist, including the previously discussed BL sludge AY-102, the prevalent trend is that shear strength increases with depth into the sediment. Wells et al. (2010a) demonstrated that, for the two chemical sludge simulants described in the Shear Strength-UDS Concentration sub-section, the shear strength of consolidated samples at a given UDS concentration was the same as that of unconsolidated samples at the same UDS concentration.

As noted in Gauglitz et al. (2009), the shear vane and waste-extrusion shear-strength results are significantly different in saltcake waste tanks and compare more favorably to each other in the sludge waste tanks. It was postulated that the difference in the results for the saltcake wastes is due to solids precipitation ex-tank.

The waste-extrusion, shear-strength results for saltcake wastes in AW-101, AN-103, AN-104, and SY-103 are similar in magnitude (within a factor of two or better) and reproduce the same trends with shear strength and sample location as the in situ ball rheometer results (Gauglitz and Aiken 1997, Rassat et al. 2003). The shear vane results for saltcake Tanks SY-103, AN-103, and AW-101 are in some instances up to an order of magnitude greater for concurrent elevations than the waste-extrusion and ball 
rheometer results. A comparison of the AY-102 shear vane and waste-extrusion shear-strength results is made in Onishi and Wells (2004). For concurrent core samples, the median shear vane results are typically $67 \%$ of the median waste-extrusion results.

The differences in the shear vane and core extrusion results for 224 Post-1949 and 2C sludge may illustrate the potential impacts of sample representativeness beyond initial sample conditions, as the shear vane results are single measurements on composited samples that were analyzed a minimum of 6 years after the sampling event, and the waste-extrusion results are from individual core segment extrusions (extrusions are typically performed days to months after sampling). Differences in sample concentration are discussed above in the Shear Strength-UDS Concentration sub-section.

The primary waste types with sediment shear strength as a function of elevation encompass $39 \%$ of the Hanford UDS volume inventory with the largest representation by saltcake tanks (see Table 3.34). Accounting for the fraction of a specific waste type in a given tank in a particular waste type, only three sludge types have representation greater than $50 \%$ by volume UDS. 


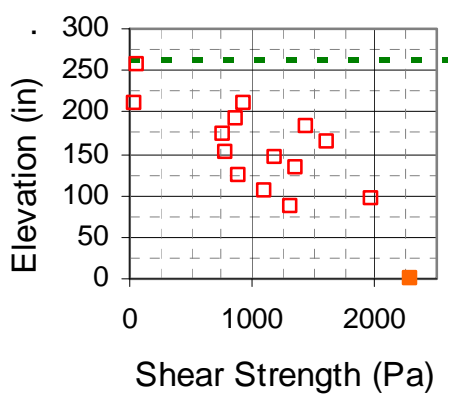

T-202

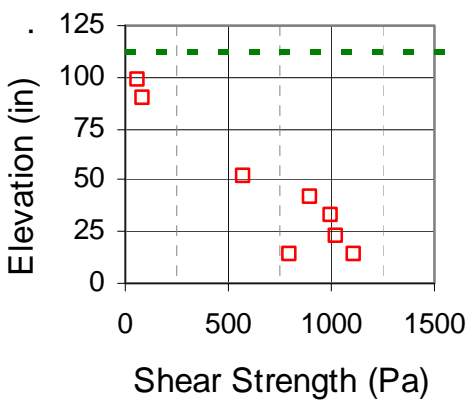

B-204

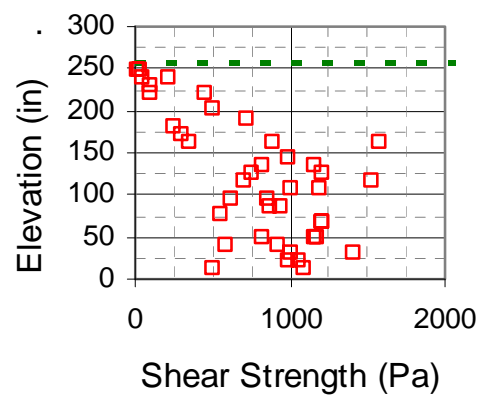

T-203

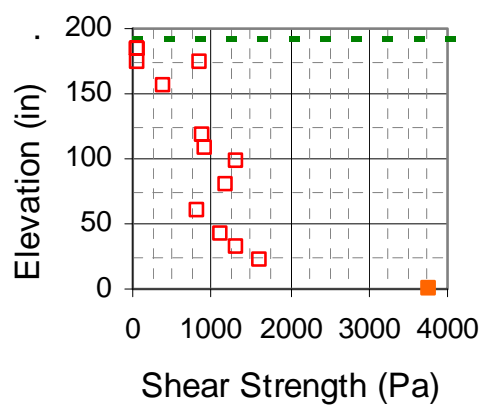

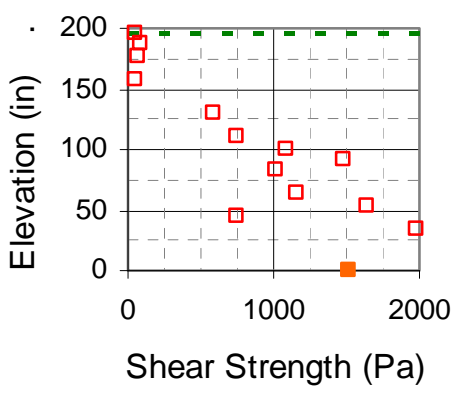

Figure 3.90. 224 Post-1949 Sludge Shear Strength as a Function of Elevation. Green dashed linesediment height; red squares-waste extrusion; orange solid square-shear vane on composite. 


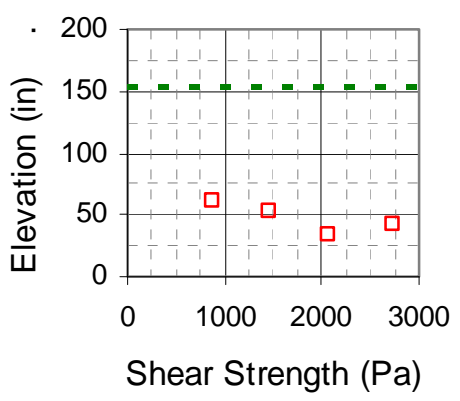

Figure 3.91. 224 Pre-1949 Sludge Shear Strength as a Function of Elevation. Green dashed linesediment height; red squares—waste extrusion.

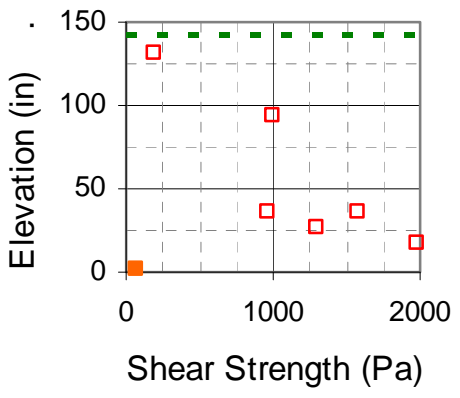

Figure 3.92. 2C Sludge Shear Strength as a Function of Elevation. Green dashed line—sediment height; red squares — waste extrusion; orange solid square — shear vane on composite.

A-101

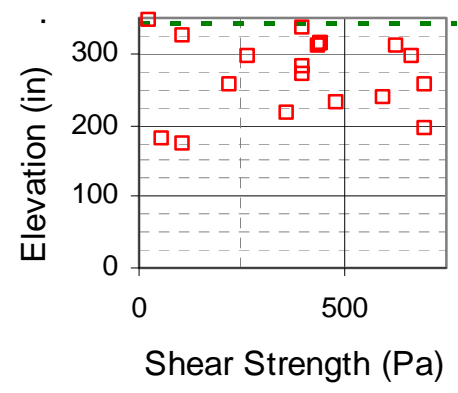

Figure 3.93. A1 Saltcake Shear Strength as a Function of Elevation. Green dashed line-waste height; red squares-waste extrusion. 
AN-103

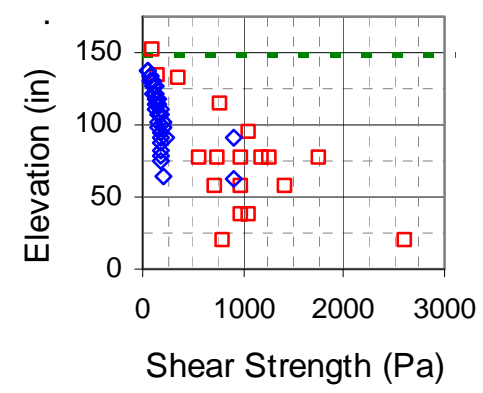

AN-104

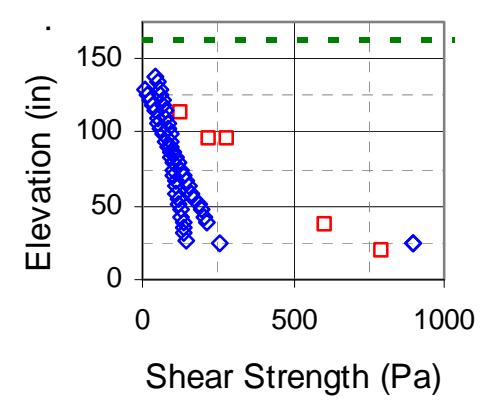

AW-101

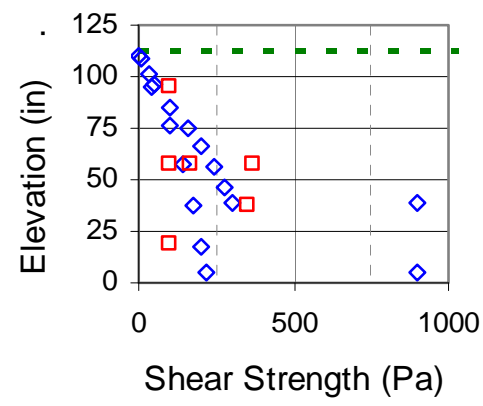

AN-103

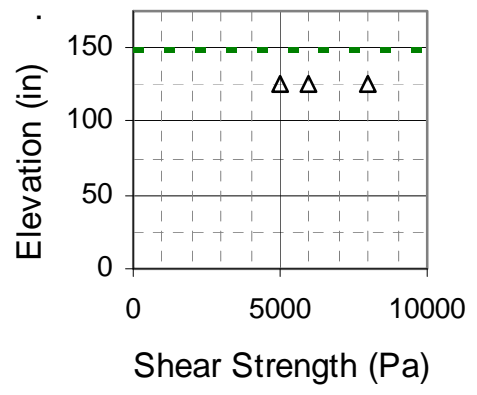

AN-105

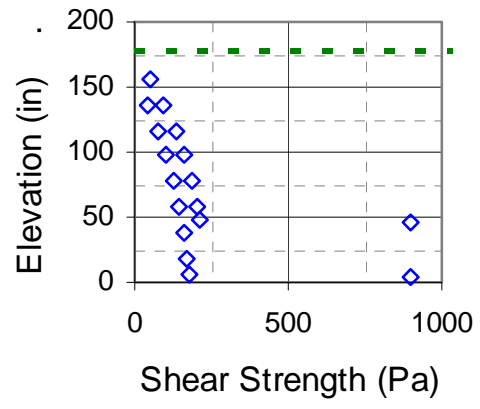

AW-101

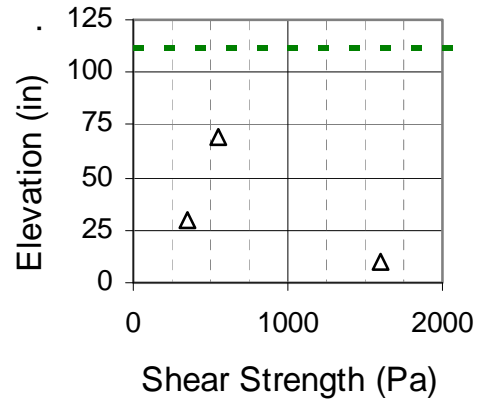

Figure 3.94. A2 Saltcake Shear Strength as a Function of Elevation. Green dashed line—sediment height; red squares—waste extrusion; black triangles—shear vane; blue diamonds—ball rheometer. 


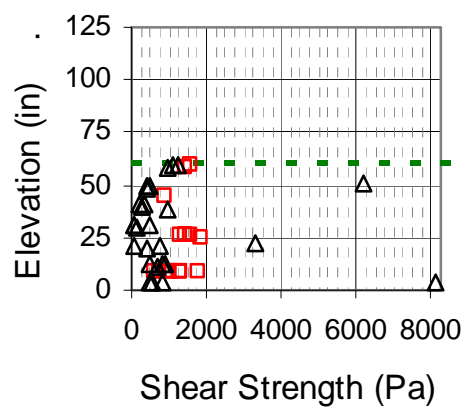

Figure 3.95. BL Sludge Shear Strength as a Function of Elevation. Green dashed line-sediment height; red squares—waste extrusion; black triangles—shear vane.

C-104

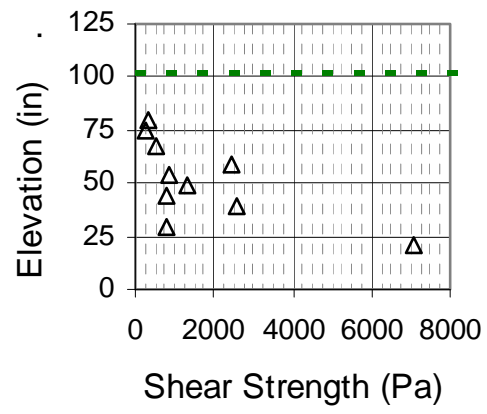

Figure 3.96. CWP1 Sludge Shear Strength as a Function of Elevation. Green dashed line—sediment height; black triangles — shear vane.

AW-103

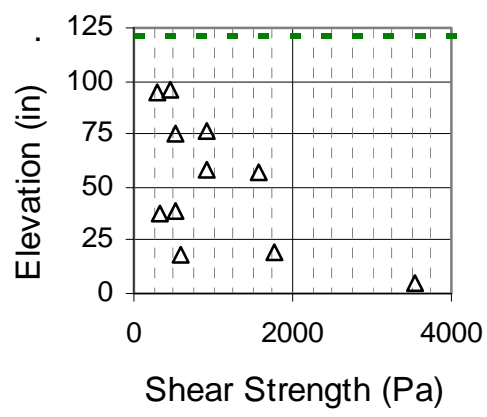

Figure 3.97. CWZr2 Sludge Shear Strength as a Function of Elevation. Green dashed line-sediment height; black triangles—-shear vane. 
AZ-101

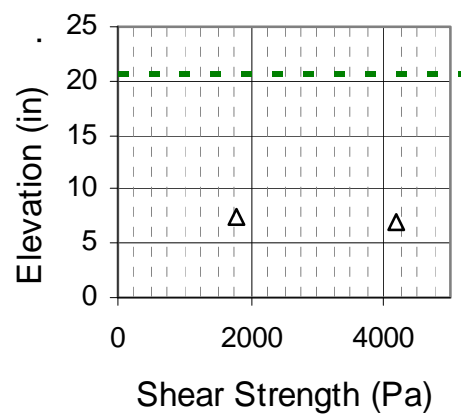

AZ-102

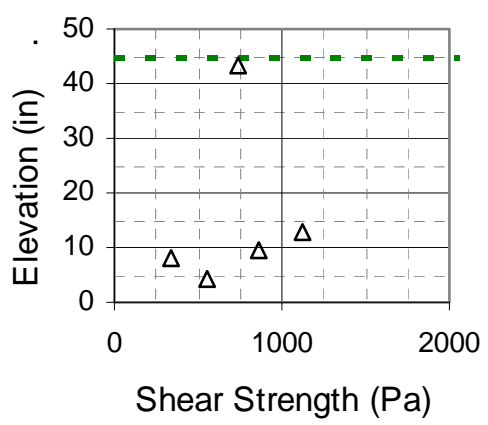

Figure 3.98. P3 Sludge Shear Strength as a Function of Elevation. Green dashed line—sediment height; black triangles—-shear vane.

U-103

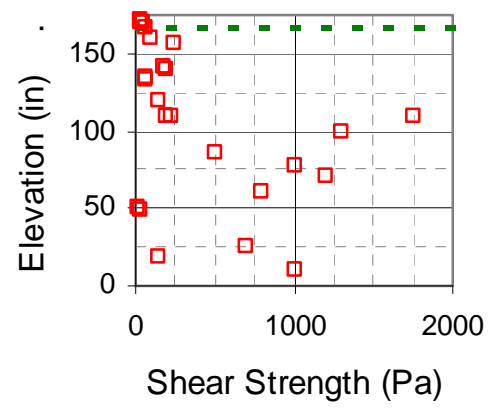

Figure 3.99. S1 Saltcake Shear Strength as a Function of Elevation. Green dashed line-sediment height; red squares—-waste extrusion. 


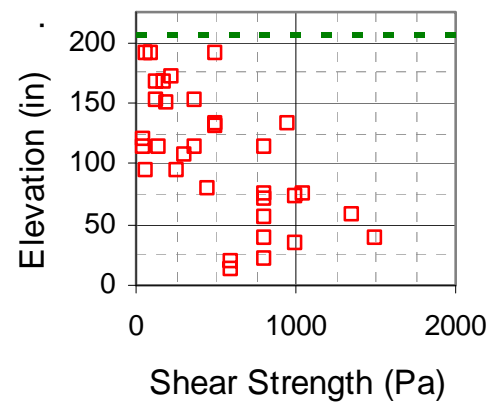

SY-103

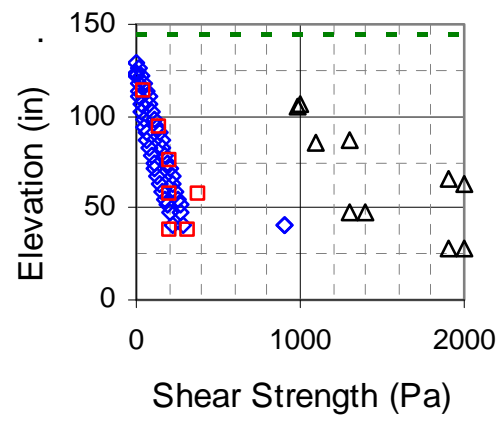

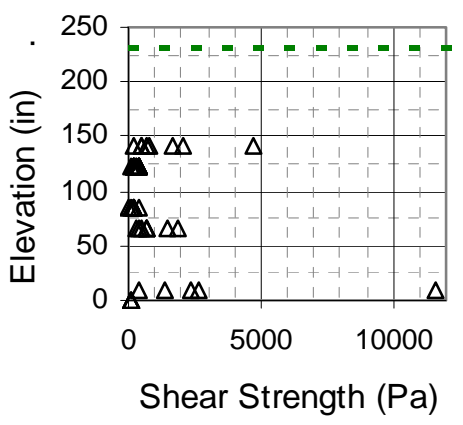

U-107

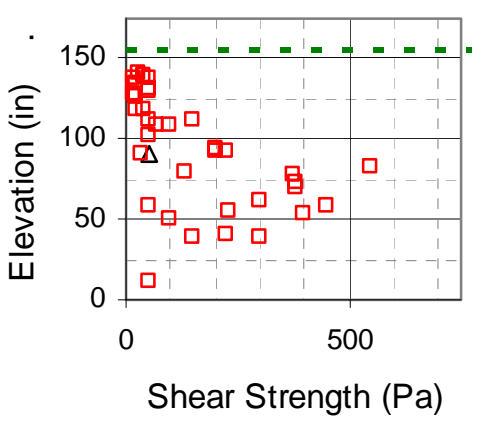

Figure 3.100. S2 Saltcake Shear Strength as a Function of Elevation. Green dashed line-sediment height; red squares—-waste extrusion; black triangles—shear vane; blue diamonds_-ball rheometer.

AY-101

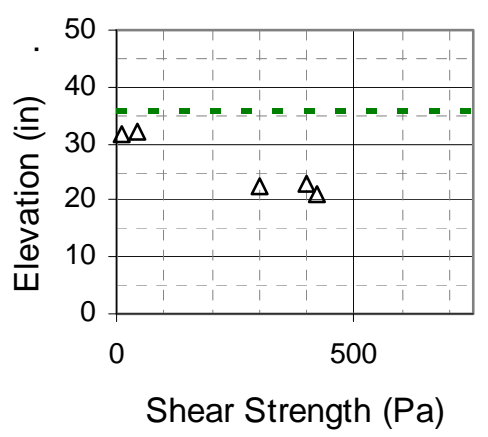

Figure 3.101. Unidentified Sludge Shear Strength as a Function of Elevation. Green dashed linesediment height; black triangles_-shear vane. 
Table 3.34. Waste Type Representation of Shear Strength as a Function of Elevation

\begin{tabular}{|c|c|c|}
\hline Primary Waste Type & $\begin{array}{c}\text { Waste Type Fraction by } \\
\text { Volume of Hanford UDS } \\
\text { Inventory }\end{array}$ & $\begin{array}{c}\text { Fraction by Volume of } \\
\text { Waste Type } \\
\text { Represented }\end{array}$ \\
\hline 224 Post-1949 sludge & $1.2 \mathrm{E}-03$ & 0.27 \\
\hline 224 Pre-1949 sludge & $2.7 \mathrm{E}-04$ & 0.38 \\
\hline 2C Sludge & $8.3 \mathrm{E}-03$ & 0.06 \\
\hline A1 saltcake & $2.9 \mathrm{E}-02$ & 0.36 \\
\hline A2 saltcake & $7.3 \mathrm{E}-02$ & 0.57 \\
\hline BL sludge & $2.9 \mathrm{E}-03$ & 0.68 \\
\hline CWP1 sludge & $1.9 \mathrm{E}-02$ & 0.07 \\
\hline CWZr2 sludge & $5.4 \mathrm{E}-03$ & 0.71 \\
\hline P3 sludge & $2.0 \mathrm{E}-03$ & 1.00 \\
\hline S1 saltcake & $1.8 \mathrm{E}-01$ & 0.03 \\
\hline S2 saltcake & $6.6 \mathrm{E}-02$ & 0.43 \\
\hline Unidentified sludge & $4.6 \mathrm{E}-03$ & 0.52 \\
\hline
\end{tabular}

\section{Shear Strength-Effect of Retained Gas Content}

The retained gas content in a sediment affects the material's measured shear strength. Gauglitz et al. (1995) showed that the shear strength decreased proportionally to the gas content for clay-based sludge simulants. For glass bead simulants, however, they determined larger shear strengths with the presence of gas bubbles. No actual waste data are available.

\subsubsection{Comparison of Measured Shear Strength to Process Data}

The shear-strength data discussed in Section 3.2.7.1 are compared to limited available process data in this section. A qualitative comparison is made based on results of mixer pump operation in Tanks AZ-101 and SY-101, and sludge weight sediment depths are considered.

As described in Section 2.0, the ECR is the distance between the exit of the mixer pump nozzle and the base of the sediment eroded by the mixer pump. The scaled ECR data from Powell et al. (1997) are provided in Figure 3.102 together with the full-scale ECR data from AZ-101 and SY-101 (Gauglitz et al. 2010b). Averaged shear vane data were used to describe the AZ-101 and SY-101 sediment shear strength. As described by Gauglitz et al. (2010b), these ECR data show remarkable agreement between the scaled clay testing and the full-scale in situ Hanford waste mobilization. Although certainly not definitive, this comparison suggests that shear vane measurements on waste samples are representative of in situ conditions. 


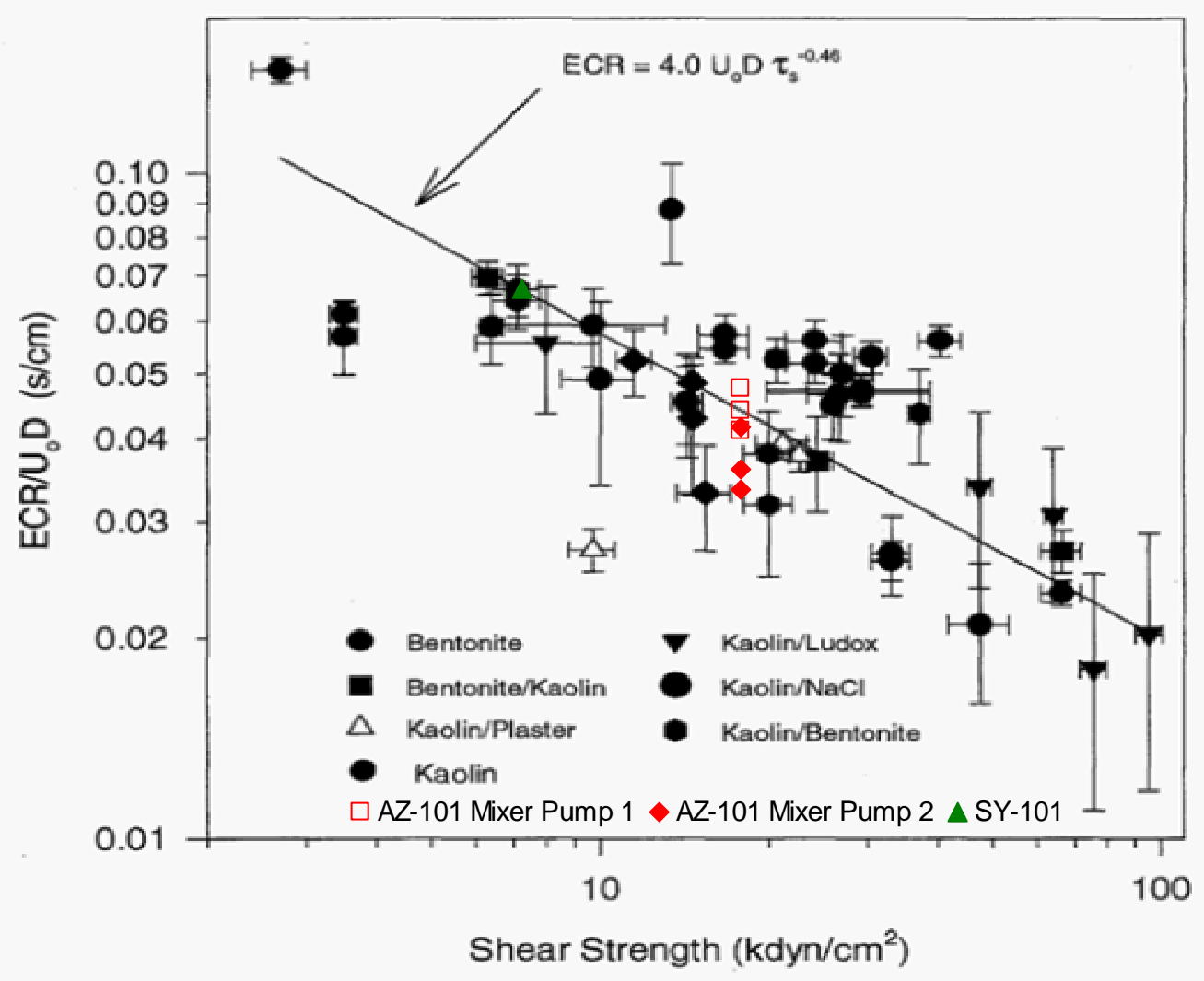

Figure 3.102. ECR, AZ-101 and SY-101 Results Compared to Scaled Tests (Gauglitz et al. 2010b)

Sludge weights, which are essentially a metal weight hung on a tape measure, are routinely used in the Hanford tank farms to measure the level of solids inside underground waste tanks. The weight is lowered into the waste until it is supported by the sediment. As described in Appendix B of Gauglitz et al. (2009), the sediment shear strength needed to support a sludge weight has been investigated. For the limited available data set, comparing sludge weight information to sediment shear-strength measurements showed that 1 ) strong sediment, as measured with a shear vane on waste samples, supports the sludge weight in two sludge tanks (AZ-101 and AY-102), and 2) analytical approaches for estimating the shear strength required to support the sludge weight were in good agreement with in situ ball rheometer results in a saltcake tank (AW-101).

\subsubsection{Bingham Parameters}

Hanford slurries can be characterized rheologically as non-Newtonian, Bingham plastic fluids (Poloski et al. 2007). The Bingham rheological model parameters consistency (viscosity) and yield stress for 29 individual tanks representing 15 primary waste types are listed in Table 3.35. References are also listed. The "Group" waste samples of Table 3.35 encompass an additional 8 waste types and comprise samples from numerous waste tanks (for details regarding group composition, see reference listed in Table 3.35 for the respective group). The data set is provided in Appendix I, and data for a single tank typically includes supernatant, composite, and diluted sample analysis. 
The measurements given in Appendix I are from laboratory samples. Similar to shear strength, the Bingham plastic rheological parameters of a slurry are dependent on the characteristics of the system, including UDS concentration, particle size and distribution, particle shape, temperature, and $\mathrm{pH}$. The effects of UDS concentration and temperature on the Bingham parameters are discussed.

Table 3.35. Bingham Rheology Data Set

\begin{tabular}{|c|c|c|c|}
\hline Tank & Reference(s) & Primary Waste Type & $\begin{array}{c}\text { Primary Waste Type } \\
\text { Volume Fraction }\end{array}$ \\
\hline AP-104 & $\begin{array}{c}\text { WTP-RPT-069, Rev. 0, } \\
\text { PNWD-3334 }\end{array}$ & Not Classified & Not Classified \\
\hline Group 1 & WTP-RPT-166 & 1C and 2C sludge & N/A \\
\hline BX-107 & $\begin{array}{l}\text { SST BX-107 Cores } 40 \text { and } \\
41 \text { data package }\end{array}$ & 1C sludge & 1 \\
\hline C-107 & $\begin{array}{c}\text { RPP-18799, } \\
\text { B3600-DBB-03-005 }\end{array}$ & 1C sludge & 0.54 \\
\hline C-110 & $\begin{array}{c}\text { C-110 Cores 37, } 38 \text { and } 39 \\
\text { data package.pdf }\end{array}$ & $1 \mathrm{C}$ sludge & 1 \\
\hline $\mathrm{T}-107$ & $\begin{array}{l}\text { Fit from hardcopies in } \\
\text { Tingey archive file }\end{array}$ & 1C sludge & 0.85 \\
\hline B-202 & $\begin{array}{c}\text { PNNL-14221, } \\
\text { WHC-SD-WM-DP-034, } \\
\text { WHC-SD-WM-ER-371 }\end{array}$ & 224 Post-1949 sludge & 1 \\
\hline B-203 & PNNL-14365 & 224 Post-1949 sludge & 1 \\
\hline $\mathrm{T}-203$ & PNNL-14365 & 224 Post-1949 sludge & 1 \\
\hline $\mathrm{T}-204$ & PNNL-14365 & 224 Post-1949 sludge & 1 \\
\hline B-201 & $\begin{array}{c}\text { PNNL-14221, } \\
\text { WHC-SD-WM-DP-037 }\end{array}$ & 224 Pre-1949 sludge & 1 \\
\hline $\mathrm{T}-110$ & PNNL-14365 & 2C sludge & 0.97 \\
\hline $\mathrm{T}-111$ & SD-WM-DP-024 & 2C sludge & 0.55 \\
\hline $\mathrm{AN}-102^{(\mathrm{a})}$ & WTP-RPT-021 Rev. 1 & A2 saltcake & 1 \\
\hline $\mathrm{AN}-103^{(\mathrm{a})}$ & HNF-7153 & A2 saltcake & 1 \\
\hline AN-104 & $\begin{array}{c}\text { WSRC-TR-2003-00295, } \\
\text { REV. 0, HNF-3352 }\end{array}$ & A2 saltcake & 1 \\
\hline AN-105 & $\begin{array}{c}\text { HNF-SD-WM-DTR-046 } \\
\text { rev } 0\end{array}$ & A2 saltcake & 1 \\
\hline AN-107 & $\begin{array}{l}\text { WSRC-TR-2003-00210, } \\
\text { REV } 0\end{array}$ & A2 saltcake & 1 \\
\hline AW-101 & HNF-4964 Rev. 0 & A2 saltcake & 1 \\
\hline AY-102 & $\begin{array}{l}\text { WSRC-TR-2003-00205, } \\
\text { REVISION 0, RPP-8909 }\end{array}$ & BL sludge & 1 \\
\hline Group 2 & WTP-RPT-166 & BY, T1, and T2 saltcake & N/A \\
\hline Group 3 & WTP-RPT-167 & CWP1 and CWP2 sludge & N/A \\
\hline C-104 & RPP-5798 & CWP1 sludge & 0.34 \\
\hline T-102 & $\begin{array}{l}\text { HNF-SD-WM-ER-700 } \\
\text { Rev. 0, } \\
\text { WHC-SD-WM-DP-052 } \\
\text { Rev 0 }\end{array}$ & CWP2 sludge & 0.89 \\
\hline
\end{tabular}


Table 3.35. (contd)

\begin{tabular}{|c|c|c|c|}
\hline Tank & Reference(s) & Primary Waste Type & $\begin{array}{c}\text { Primary Waste Type } \\
\text { Volume Fraction }\end{array}$ \\
\hline Group 4 & WTP-RPT-167 & CWR1 sludge & N/A \\
\hline AZ-101 & WTP-RPT-043, Rev 1 & P3 sludge & 1 \\
\hline AZ-102 ${ }^{\text {(a) }}$ & BNFL-RPT-038 Rev. 0 & P3 sludge & 1 \\
\hline Group 8 & WTP-RPT-170 & PFeCN sludge & N/A \\
\hline S-104 & WHC-SD-WM-DP-031 & R saltcake & 0.47 \\
\hline Group 5 & WTP-RPT-157 & R1 (boiling) sludge & N/A \\
\hline S-112 & RPP-10984 Rev. 0 & S1 saltcake & 0.99 \\
\hline SY-101 & WHC-EP-0628 & S2 saltcake & 1 \\
\hline Group 6 & WTP-RPT-157 & S-Saltcake (S1 and S2) & N/A \\
\hline Group 7 & WTP-RPT-169 & TBP sludge & N/A \\
\hline C-109 & $\begin{array}{c}\text { WHC-SD-WM-ER-402, } \\
\text { Rev 0; Core } 47\end{array}$ & TFeCN sludge & 0.38 \\
\hline C-112 & $\begin{array}{c}\text { SST C-112 Core } 36 \text { data } \\
\text { package }\end{array}$ & TFeCN sludge & 0.69 \\
\hline SY-102 & PNNL-11352 & Unidentified sludge & 0.51 \\
\hline
\end{tabular}

\section{Bingham Parameters-Data Summary}

Poloski et al. (2007) showed the range of Bingham parameters (Bingham yield stress and consistency) for 28 waste tanks from 12 sludge types covering a range of temperatures from $20^{\circ}$ to $95^{\circ} \mathrm{C}$. These data, in combination with the additional data that are included in Appendix I for one additional tank and the eight waste groups, are plotted in Figure 3.103. A general trend of increasing yield stress with consistency is observed. A Bingham yield stress of $1 \mathrm{~Pa}$ is sufficient to impact jet mixing (Gauglitz et al. (2010b) and particle settling as well as minimally influence gas bubble retention (Gauglitz et al. 1996). Measurements $<<1$ Pa may be considered as essentially zero yield stress. Results of essentially zero yield stress at the viscosity of water, $1 \mathrm{cP}$, are shown in Figure 3.103. Single data points are shown Figure 3.103, but each point typically is one of a series from a dilution curve as will be presented below. 


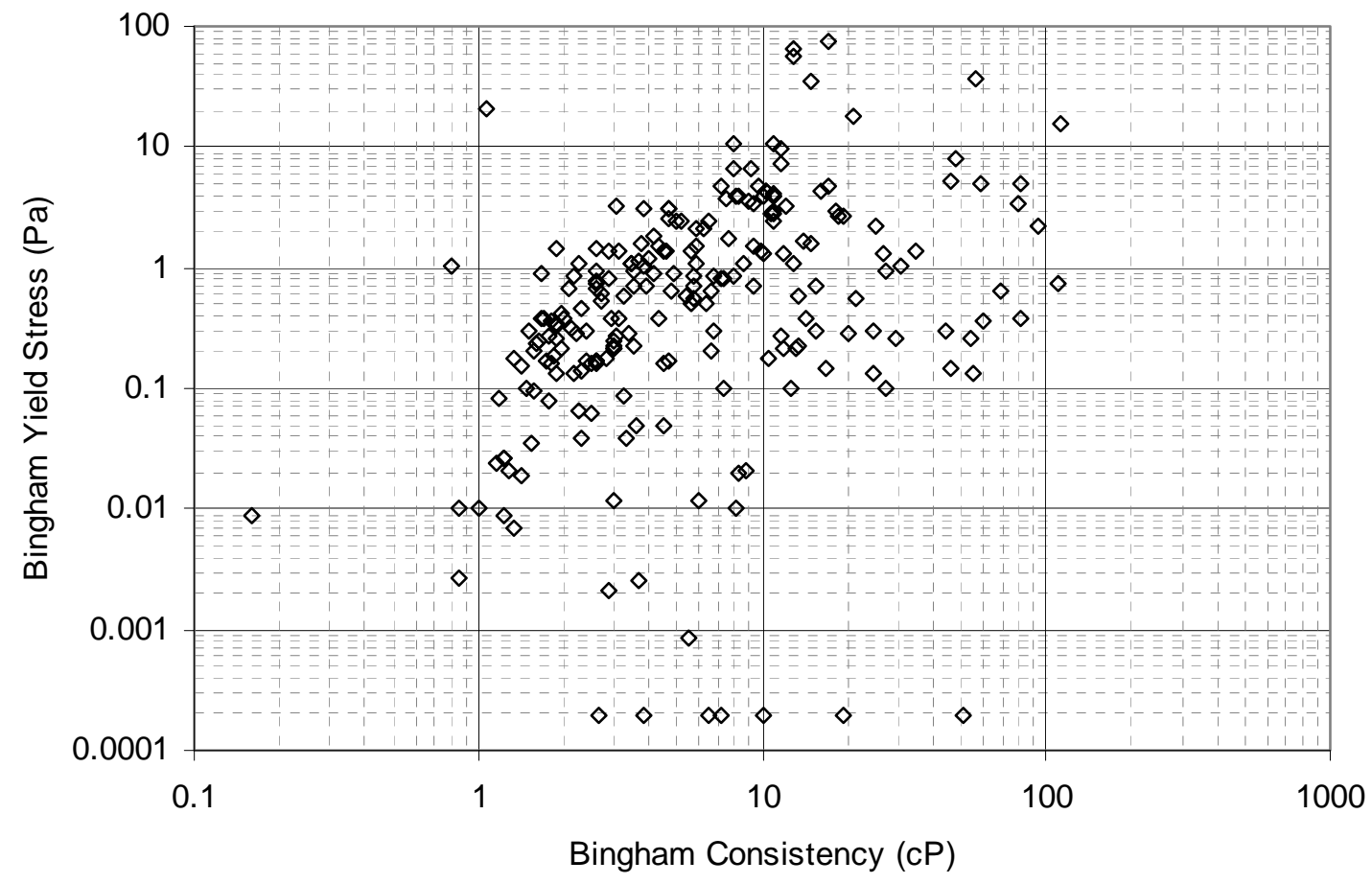

Figure 3.103. Bingham Parameters

If the tanks listed in Table 3.35 are treated as represented with respect to Bingham model parameters regardless of the number of measurements for a given tank or dilution level, Bingham parameters for approximately $13 \%$ of the Hanford waste UDS volume are represented (37\% if "Group" characterizations [Table 3.35] are included). As shown in Figure 3.104, 5 of the 18 primary waste types with Bingham parameter data have greater than $50 \%$ representation for the primary waste type UDS volume, as indicated in Figure 3.74. The 18 primary waste types comprise approximately $51 \%$ by volume of the total UDS inventory. 


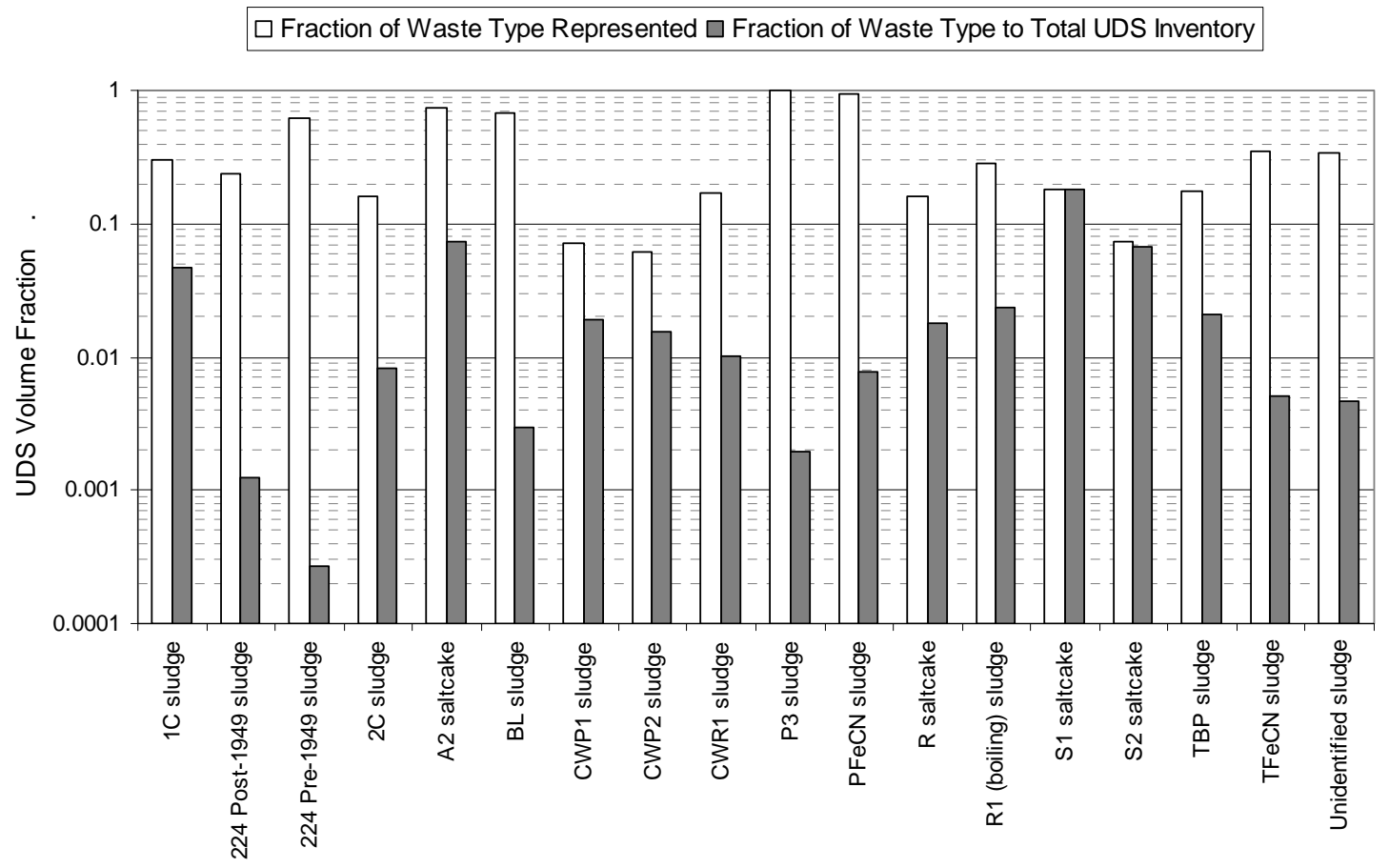

Figure 3.104. Waste Types Represented by Bingham Rheological Model Data Set. Multiple waste type Groups (Table 3.35) are not included.

To gain an understanding of how the rheological parameters in the database vary by Hanford waste tank and waste type, Poloski et al. (2007) considered the maximum measured Bingham parameters for each waste tank. The maximum Bingham parameters for a given tank and waste type from the Appendix I data are provided in Figure 3.105 and Figure 3.106 for sludge and Figure 3.107 and Figure 3.108 for saltcake waste types, respectively. Tanks that have consistency values but do not appear on the Bingham yield stress plots have no measured yield stress. This may be due to the slurry acting as a Newtonian fluid, or it may be the case that there were no measurements made on UDS-containing samples. The functionality of the Bingham parameters with UDS concentration is discussed below. Independent of sample conditions and properties, the yield stress of sludge is generally greater than the saltcake, whereas the saltcake generally has greater consistency values. 


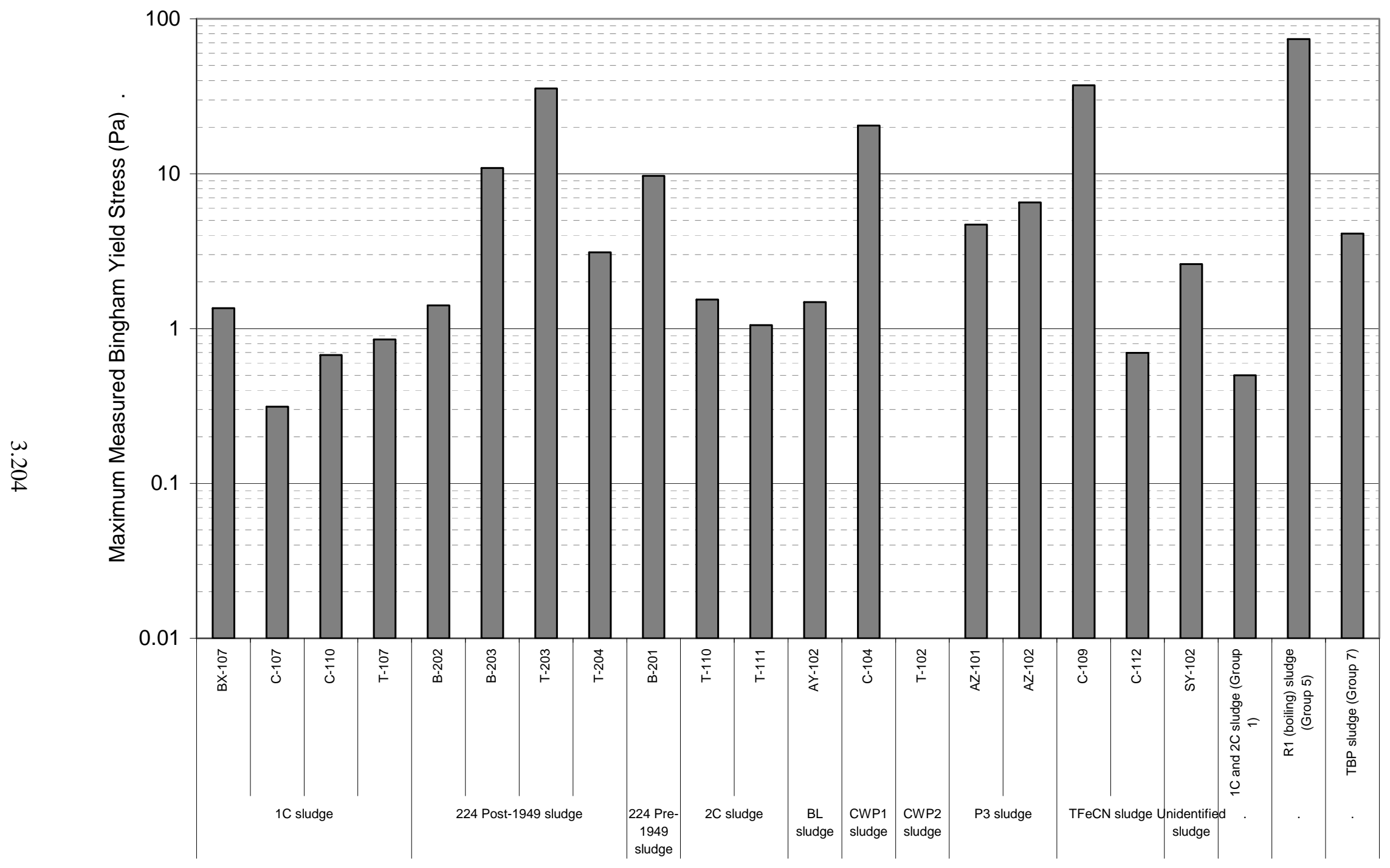

Figure 3.105. Maximum Measured Bingham Yield Stress, Sludge 


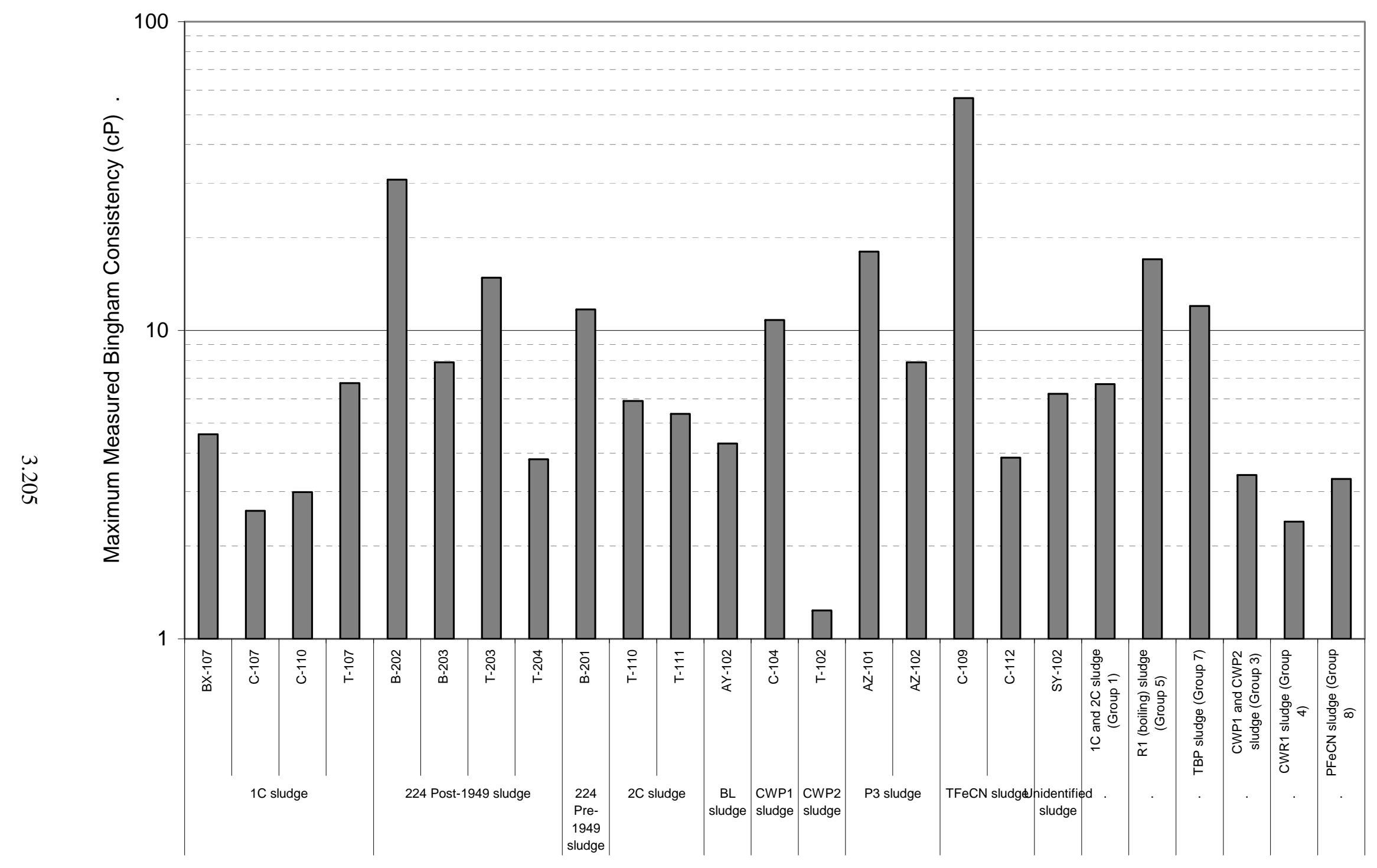

Figure 3.106. Maximum Measured Bingham Consistency, Sludge 


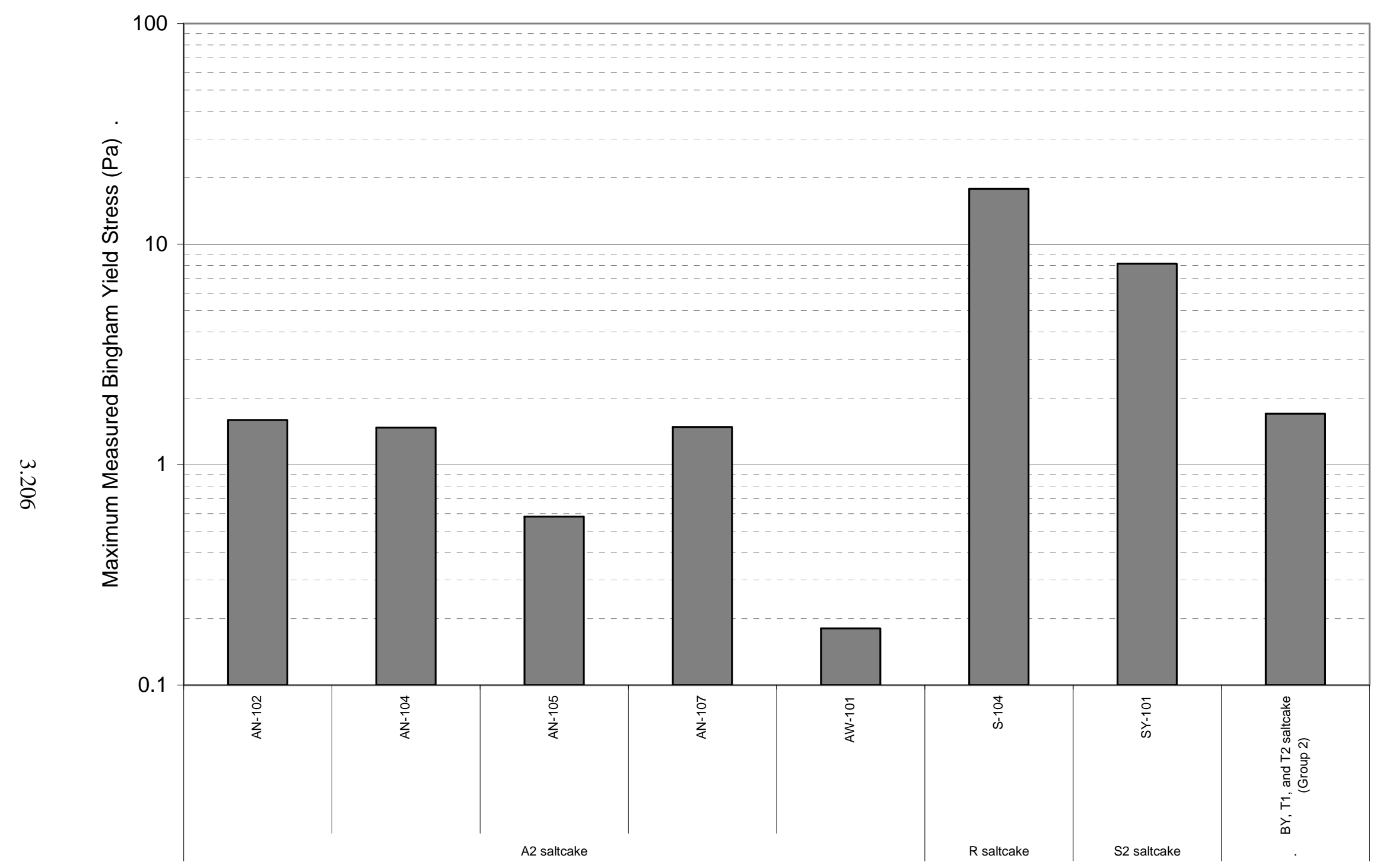

Figure 3.107. Maximum Measured Bingham Yield Stress, Saltcake 


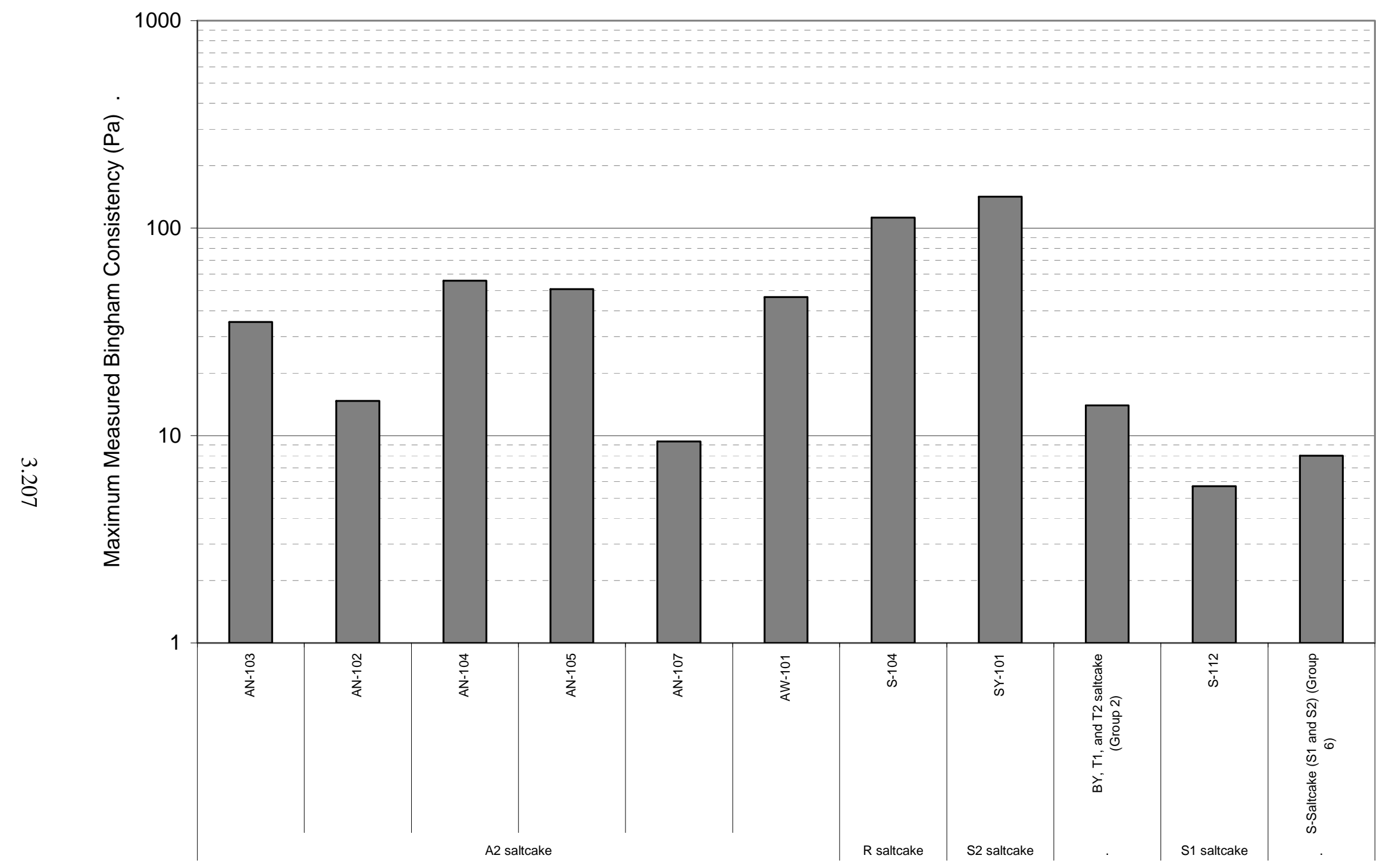

Figure 3.108. Maximum Measured Bingham Consistency, Saltcake 


\section{Bingham Parameters-UDS Concentration}

The Bingham rheological parameters are a strong function of the UDS concentration. Similarly, as described above in the Shear Strength-UDS Concentration sub-section, a slurry's Bingham parameters are a function of the UDS concentration. Data for the Bingham parameters as a function of UDS concentration are available for 23 of the 29 tanks (Table 3.35) for 13 primary waste types.

Bingham parameters as a function of UDS concentration data are available for approximately $8 \%$ of the Hanford waste by UDS volume. Four of the 13 primary UDS waste types have greater than $50 \%$ representation for the primary UDS waste type volume (Figure 3.109). The 13 primary waste types comprise approximately $26 \%$ by volume of the total UDS inventory.

The Bingham parameter variations with UDS concentration for the 23 represented tanks are provided in Figure 3.110 and Figure 3.111. Sludge and saltcake waste, as indicated by the primary waste type, are shown separately. The temperature range of the measurements is 20 to $95^{\circ} \mathrm{C}$. Even for the disparate data set, a trend of increasing Bingham parameters with increasing UDS concentration is observable. The data indicate, for sludge waste at a UDS mass fraction of 0.1, for example, that the Bingham yield stress and viscosity data limits may vary by nominally two orders of magnitude. This extreme variation may be attributed to the varied waste and sample conditions represented.

Saltcake is shown to have higher yield stress at lower concentrations than sludge, with nominally $1 \mathrm{~Pa}$ Bingham yield stress reported for saltcake at a UDS concentration of less than $1 \%$ by mass, while $1 \mathrm{~Pa}$ is not reported for sludge below a UDS concentration of approximately $6 \%$ by mass. Saltcake is shown to have measured Bingham viscosities higher than sludge at equivalent UDS concentrations. 


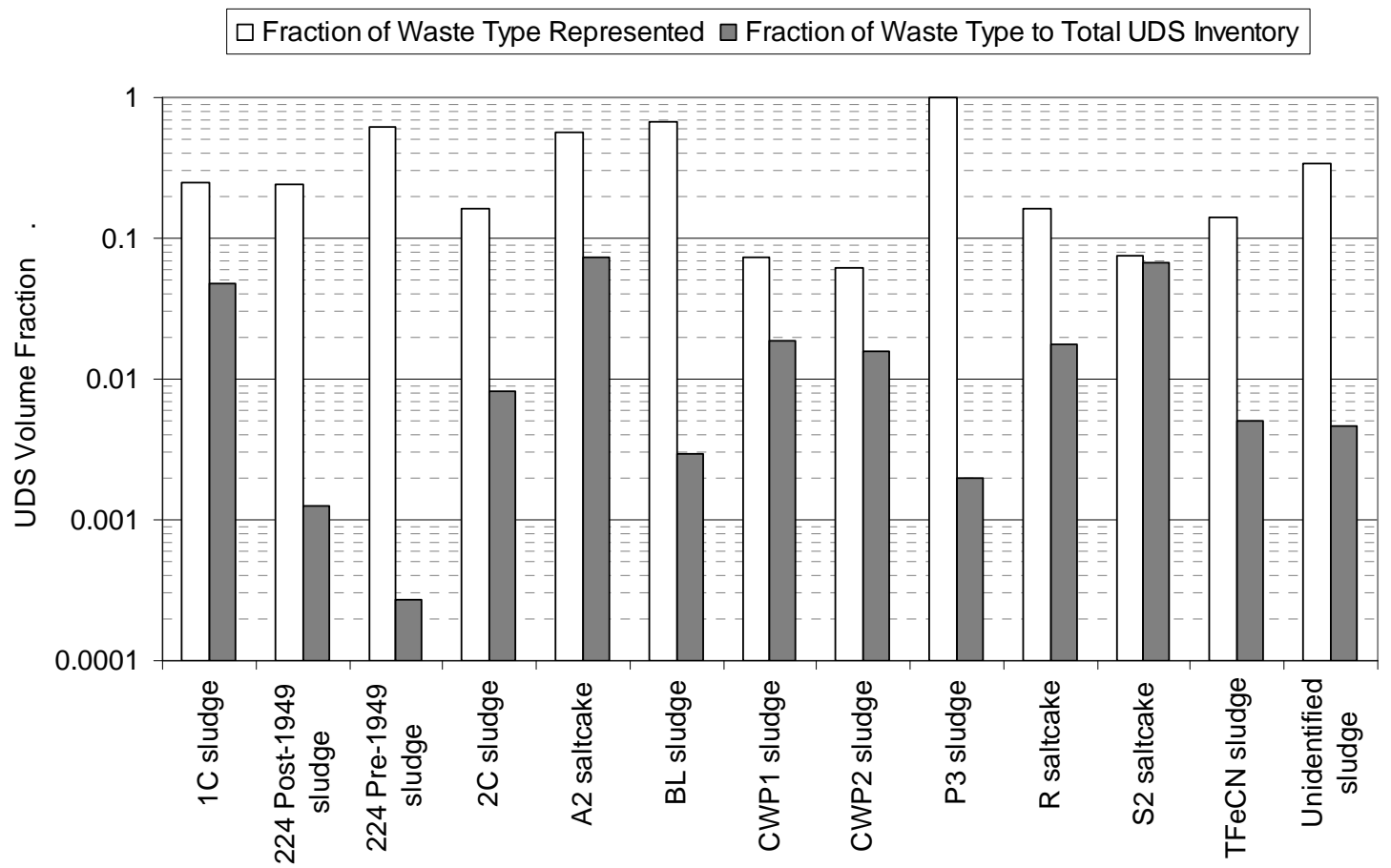

Figure 3.109. Primary Waste Types with Bingham Parameter Characterization as a Function of UDS Concentration

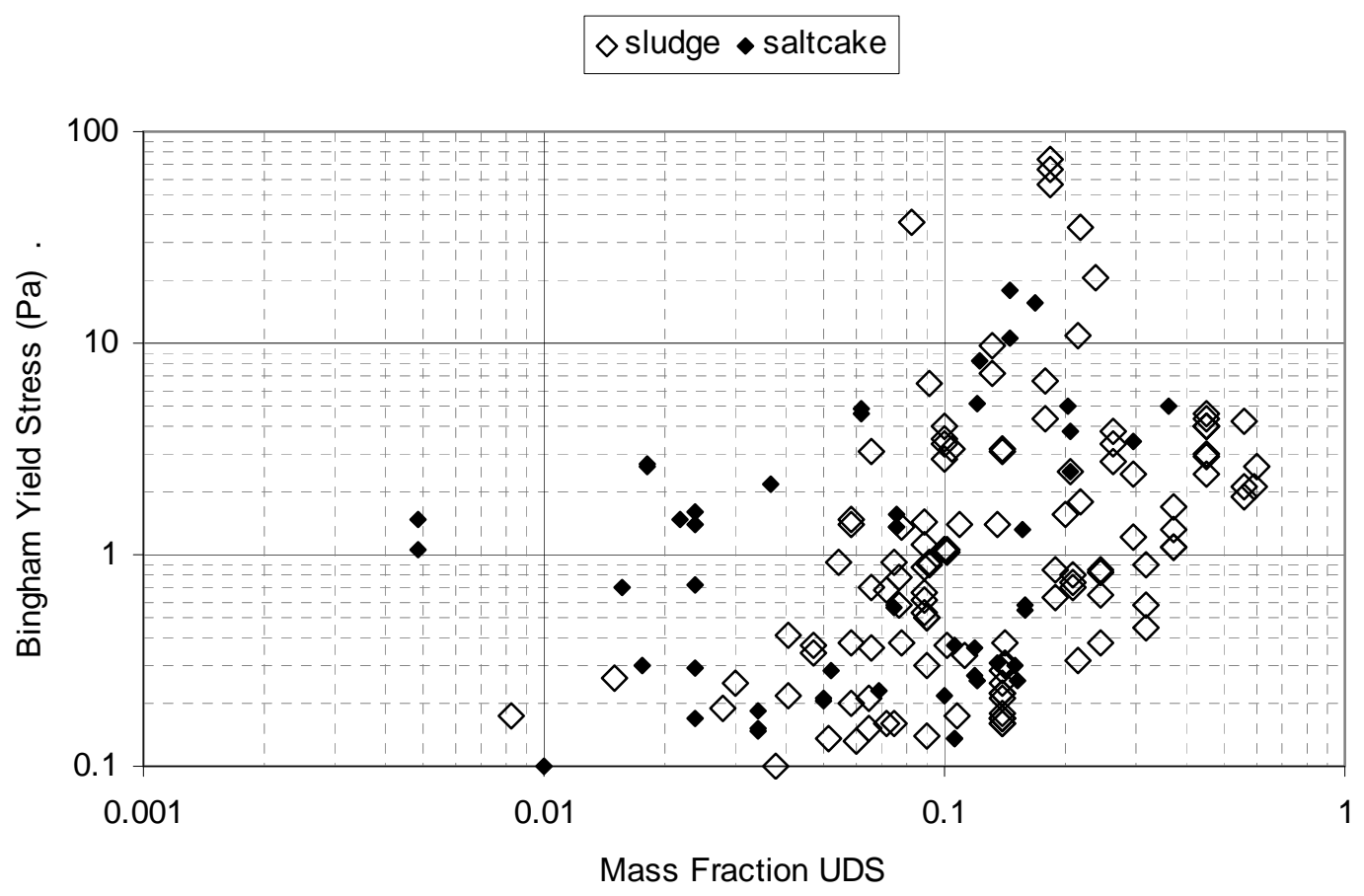

Figure 3.110. Sludge and Saltcake Bingham Yield Stress as a Function of UDS Concentration, 20 to $95^{\circ} \mathrm{C}$ 


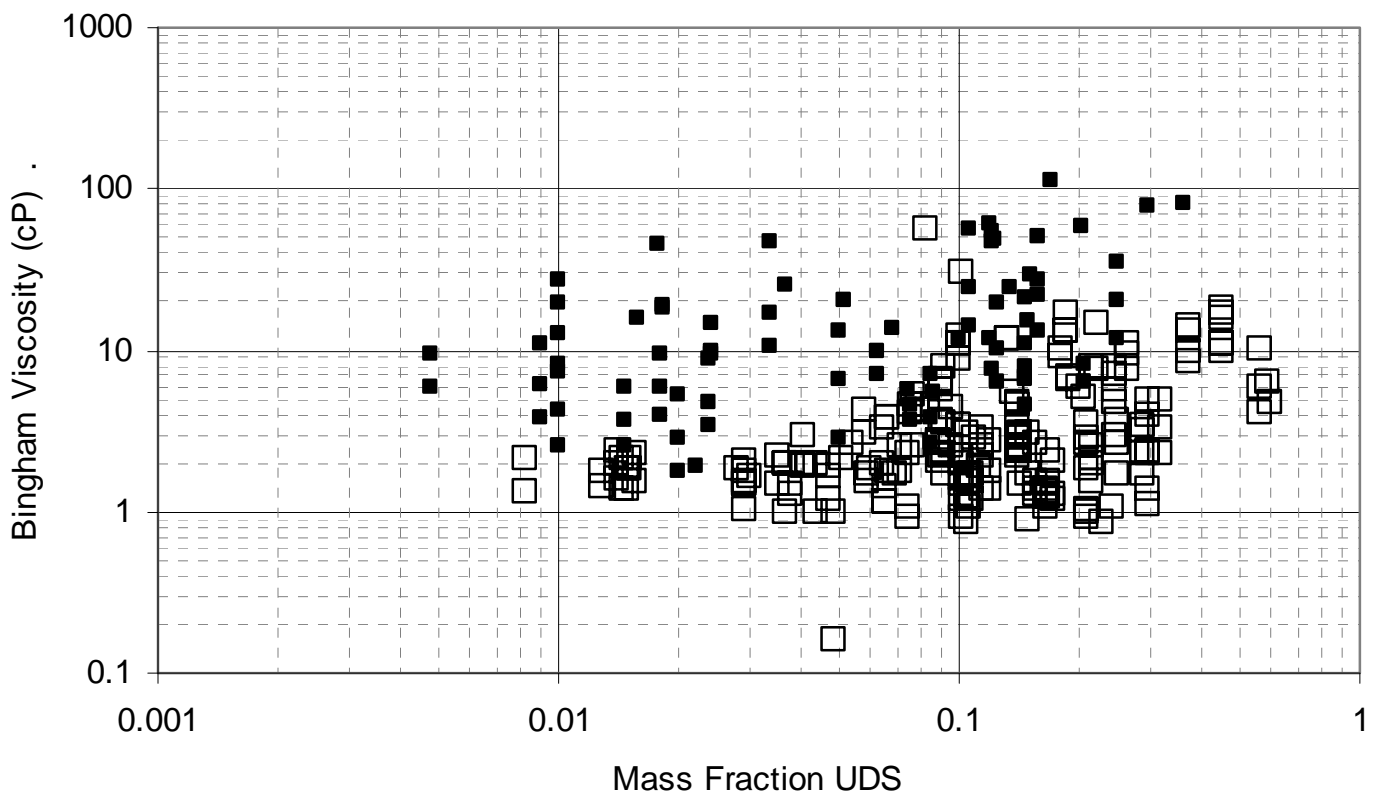

Figure 3.111. Sludge and Saltcake Bingham Viscosity as a Function of UDS Concentration, 20 to $95^{\circ} \mathrm{C}$

Power-law dependencies for shear properties are exhibited for a wide range of suspensions in the literature (see Section 3.2.7.1). Equation 3.11, can be re-written as

$$
\tau_{\mathrm{B}}=\mathrm{Ew}_{\mathrm{S}}{ }^{\mathrm{F}}
$$

where $\mathrm{E}$ and $\mathrm{F}$ are fitting parameters that are applied to the Bingham yield stress, $\tau_{\mathrm{B}}(\mathrm{Pa})$. The Bingham viscosity, $\mu_{\mathrm{B}}(\mathrm{cP})$, is written as

$$
\mu_{\mathrm{B}}=\mathrm{Ge}^{\mathrm{Hw} \mathrm{s}_{\mathrm{s}}}
$$

where $\mathrm{G}$ and $\mathrm{H}$ are fitting parameters following Poloski et al. (2007).

These equations are typically valid only when the chemistry of the slurry is held constant while varying the solids loading. This can be achieved by diluting the slurry with liquid that is at equilibrium with the slurry UDS. Both water and supernatant liquid were used as the diluent for the Bingham data cited in this report.

Data for individual tanks and primary waste types are shown in Figure 3.112 through Figure 3.124. Equations (3.14) and (3.15) were applied to the data sets when appropriate (increasing trend indicated, Bingham yield stress $>1 \mathrm{~Pa}$, single dilution medium). The data are grouped by waste type, and separate plots are shown for the measurement temperatures. The model fits, found by either using Trendline 
function or the Solver module in Excel ${ }^{\circledR}$ to minimize the sum of the squares of the prediction errors, are included in the figures. The reported $\mathrm{R}^{2}$ values are from the Trendline function in Excel ${ }^{\circledR}$.

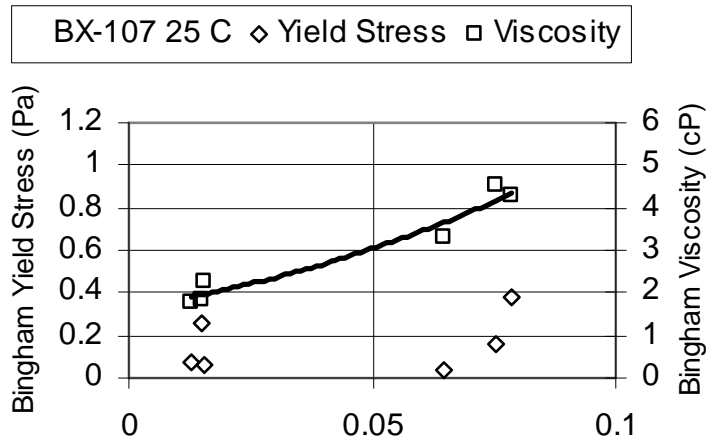

Mass Fraction UDS

$$
\begin{gathered}
y=1.62 e^{12.50 x} \\
R^{2}=0.95
\end{gathered}
$$

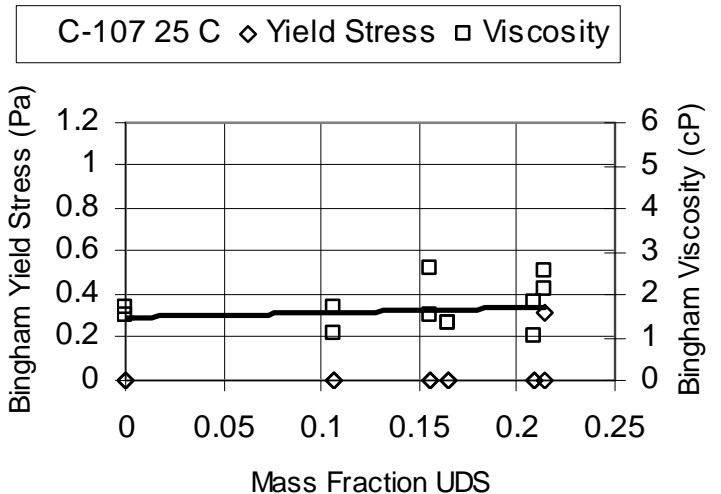

$$
\begin{gathered}
y=1.47 e^{0.69 x} \\
R^{2}=0.03
\end{gathered}
$$

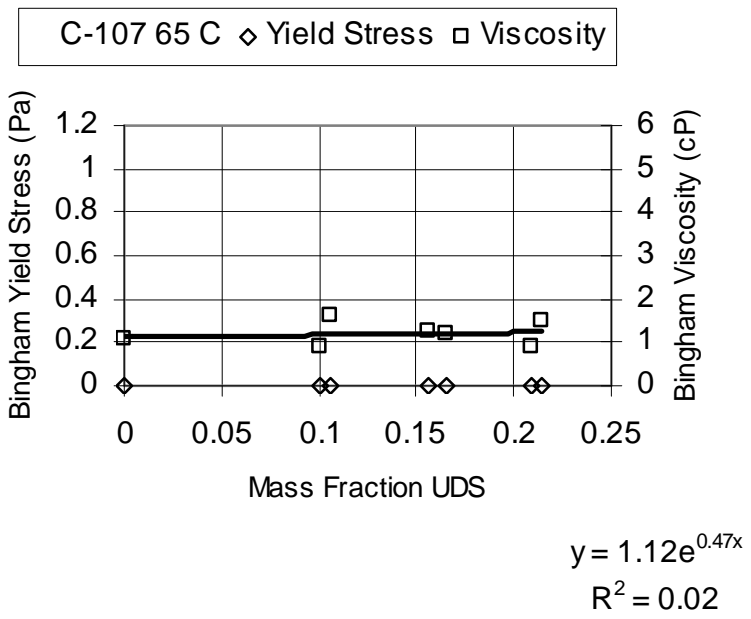

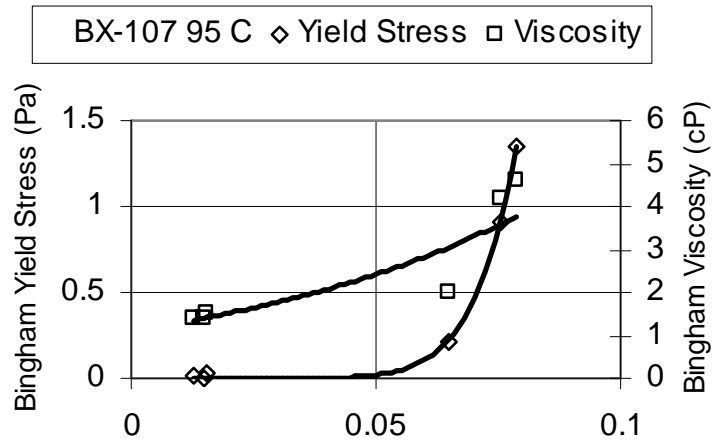

Mass Fraction UDS

$$
\begin{gathered}
y=1.12 e^{15.51 x} \\
R^{2}=0.83
\end{gathered}
$$
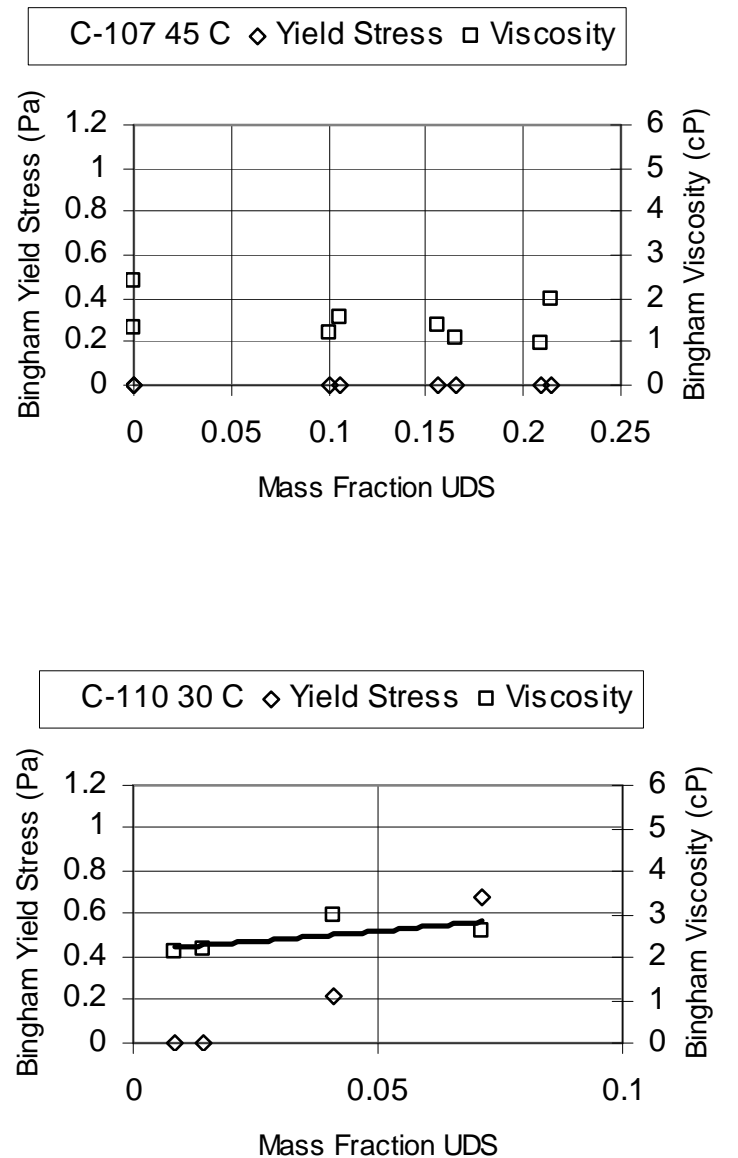

$$
\begin{gathered}
y=2.15 e^{3.83 x} \\
R^{2}=0.47
\end{gathered}
$$

Figure 3.112. 1C Sludge Bingham Parameters as Functions of UDS Concentration 

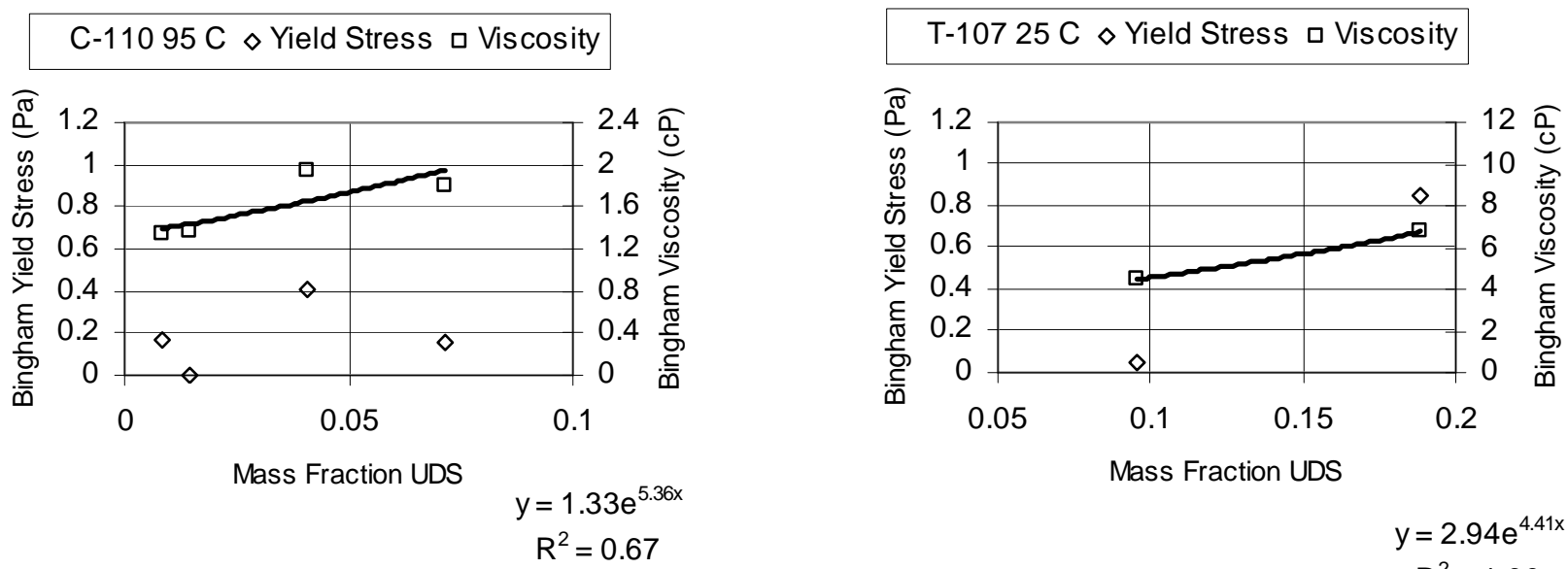

Mass Fraction UDS

$$
\begin{gathered}
y=2.94 e^{4.41 x} \\
R^{2}=1.00
\end{gathered}
$$
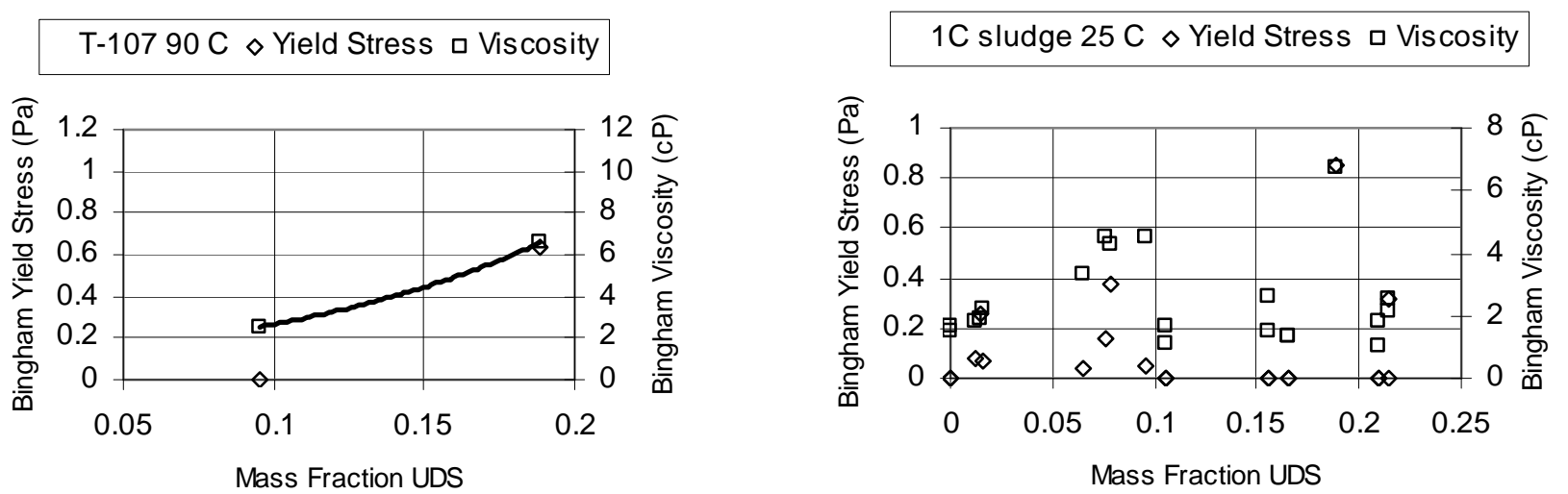

Mass Fraction UDS

$$
\begin{gathered}
y=0.97 e^{10.16 x} \\
R^{2}=1.00
\end{gathered}
$$

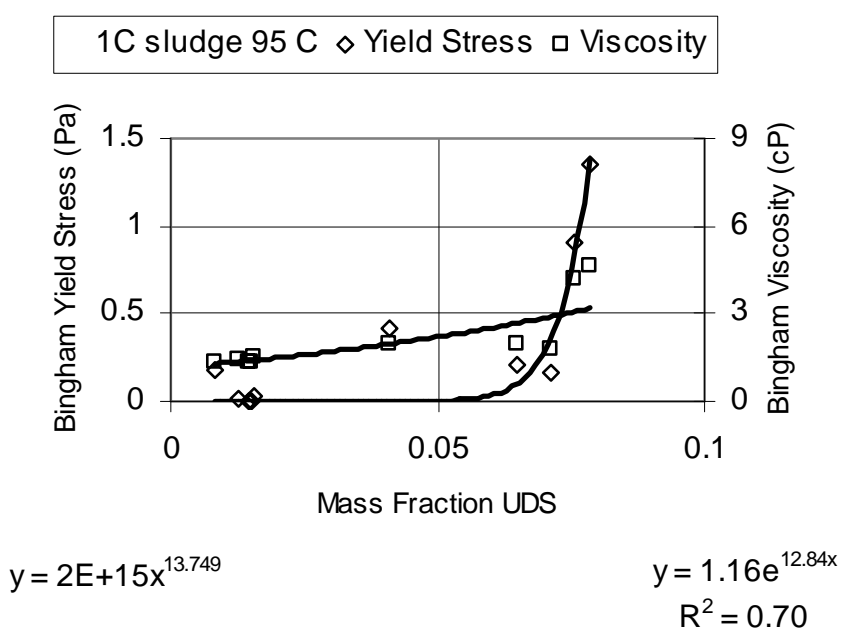

Figure 3.112. (contd) 


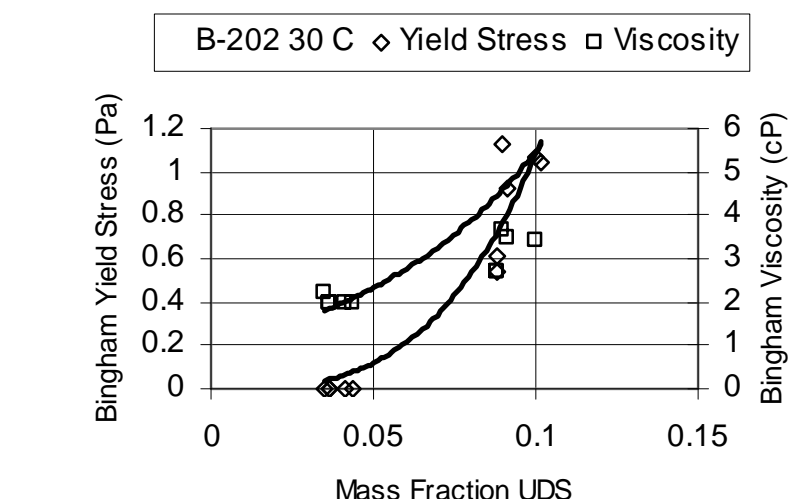

$y=1642.4 x^{3.1829}$

Mass Fraction UDS

$y=0.99 e^{17.02 x}$

$\mathrm{R}^{2}=0.39$

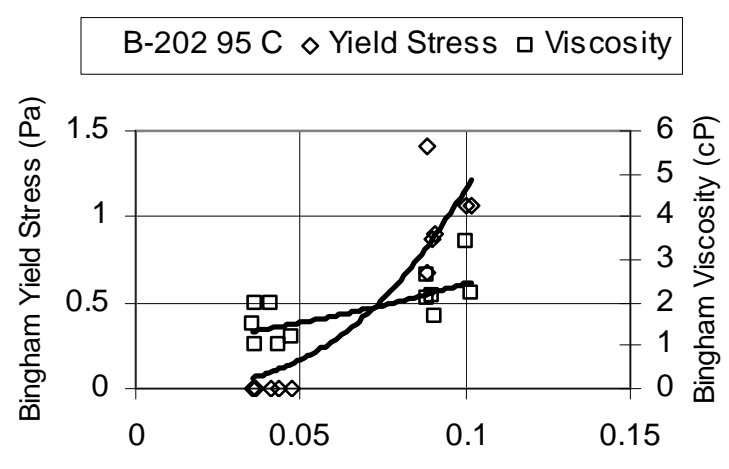

Mass Fraction UDS

$y=709.79 x^{2.7868}$

$$
\begin{gathered}
y=0.96 e^{9.36 x} \\
R^{2}=0.49
\end{gathered}
$$

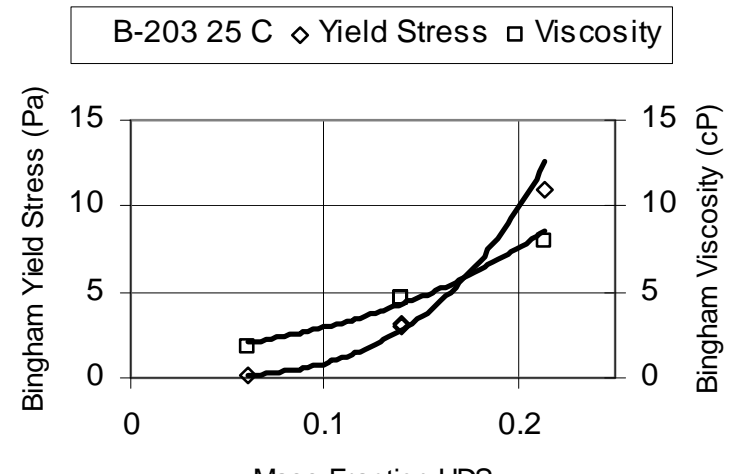

Mass Fraction UDS

$$
\begin{array}{cc}
y=2937.66 x^{3.54} & y=1.16 e^{9.38 x} \\
R^{2}=1.00 & R^{2}=0.97
\end{array}
$$

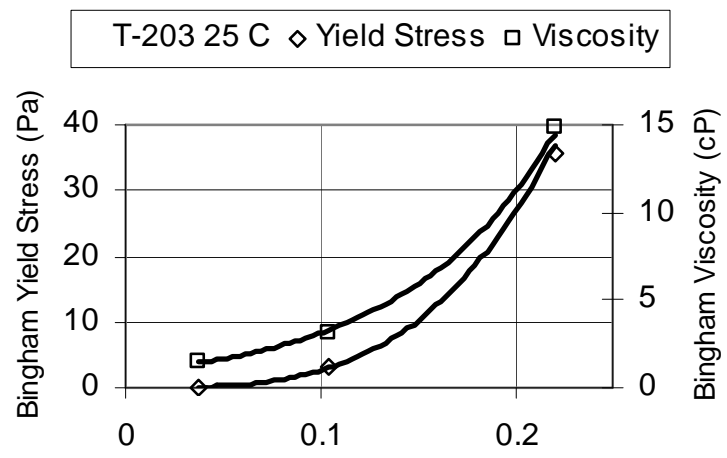

$$
\begin{array}{ccc}
y=5858.63 x^{3.35} & \text { Mass Fraction UDS } & y=0.87 e^{12.76 x} \\
R^{2}=1.00 & R^{2}=1.00
\end{array}
$$

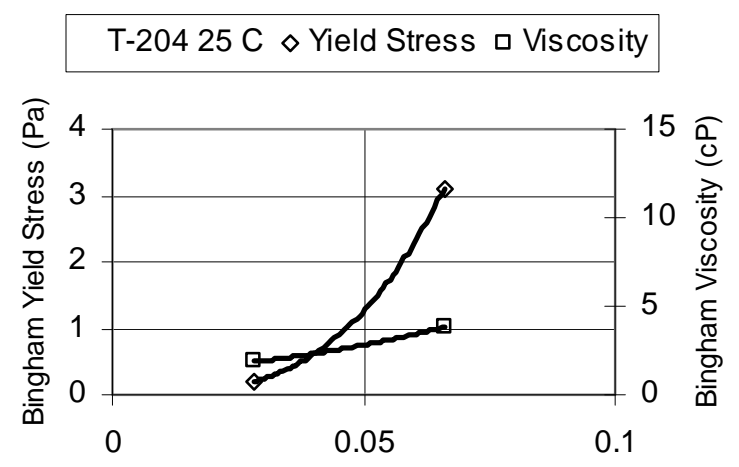

Mass Fraction UDS

\section{Post-1949 sludge 25 C $\diamond$ Yield Stress $\square$ Viscosity}

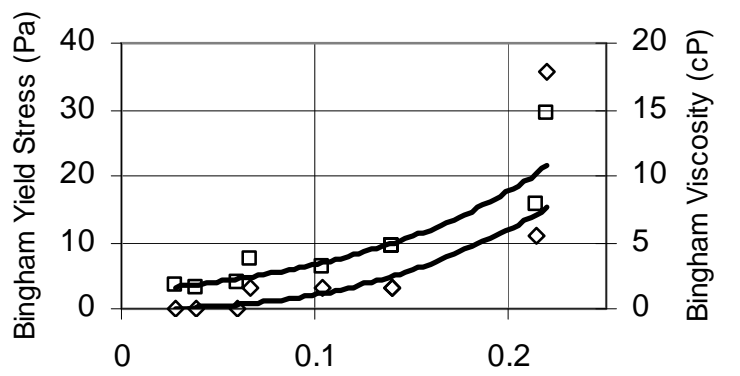

Mass Fraction UDS

$$
\begin{gathered}
y=667.39 x^{2.50} \\
R^{2}=0.80
\end{gathered}
$$

Figure 3.113. 224 Post-1949 Sludge Bingham Parameters as Functions of UDS Concentration 


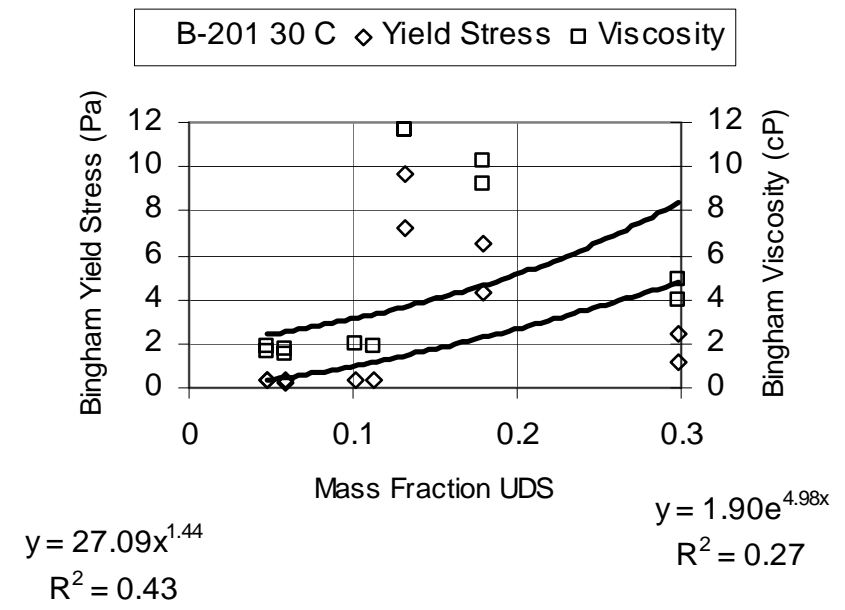

Figure 3.114. 224 Pre-1949 Sludge Bingham Parameters as Functions of UDS Concentration 


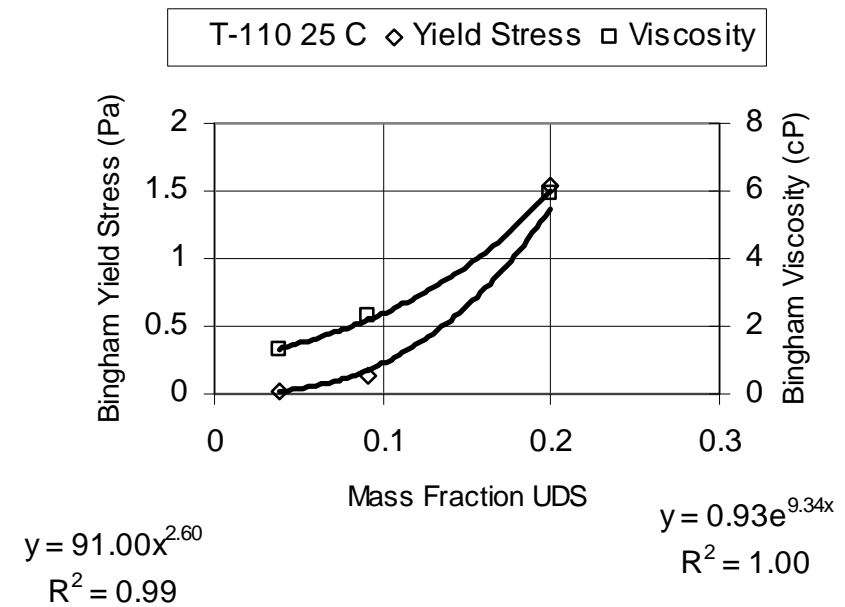

T-111 27 C $\diamond$ Yield Stress $\square$ Viscosity

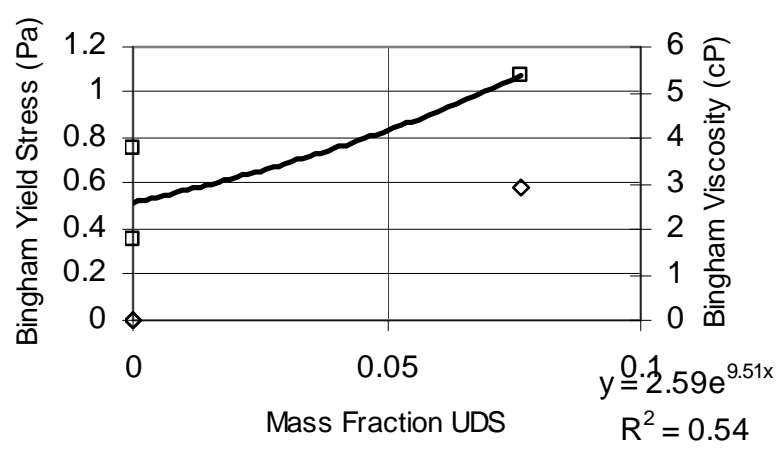

T-11195 C $\diamond$ Yield Stress $\square$ Viscosity

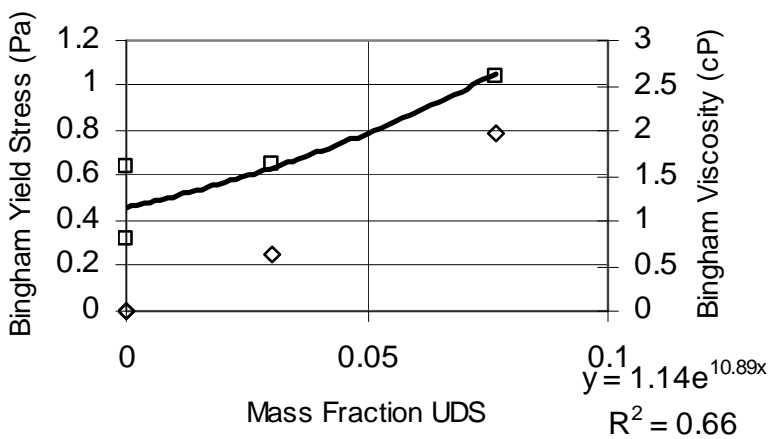

Figure 3.115. 2C Sludge Bingham Parameters as Functions of UDS Concentration 

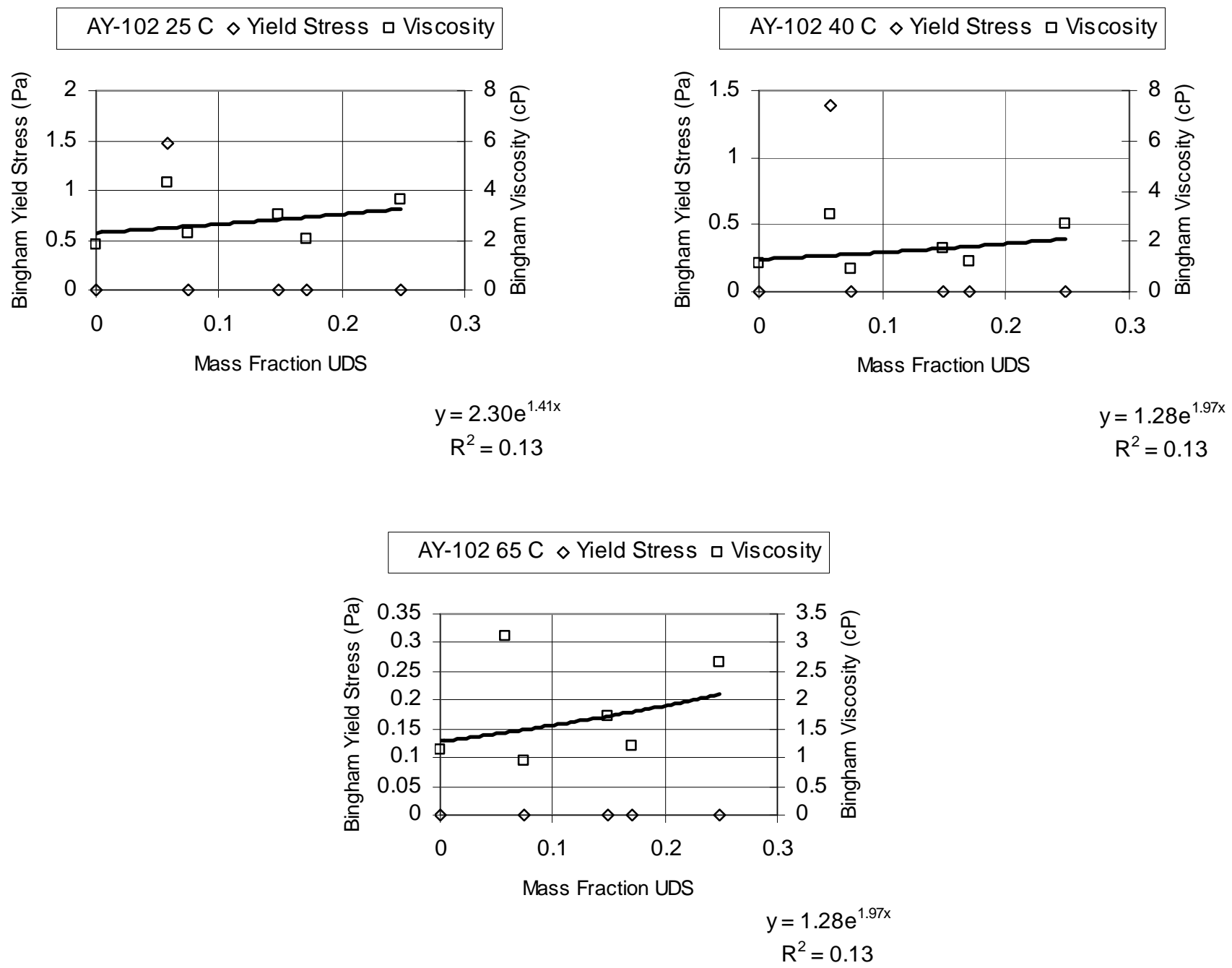

Figure 3.116. BL Sludge Bingham Parameters as Functions of UDS Concentration 


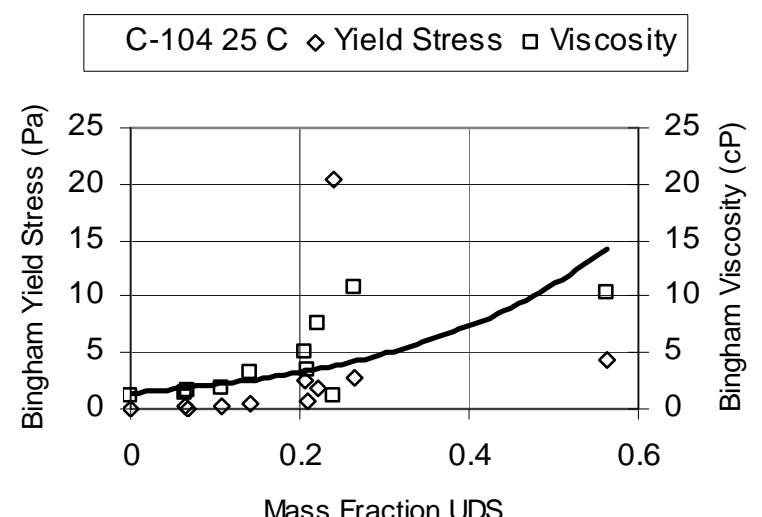

$$
\begin{gathered}
y=1.43 e^{4.10 x} \\
R^{2}=0.51
\end{gathered}
$$

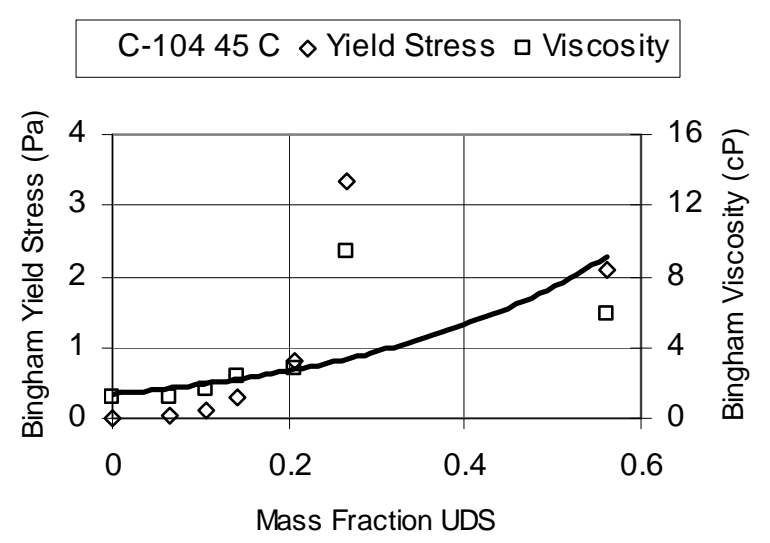

$$
\begin{gathered}
y=1.36 e^{3.39 x} \\
R^{2}=0.62
\end{gathered}
$$

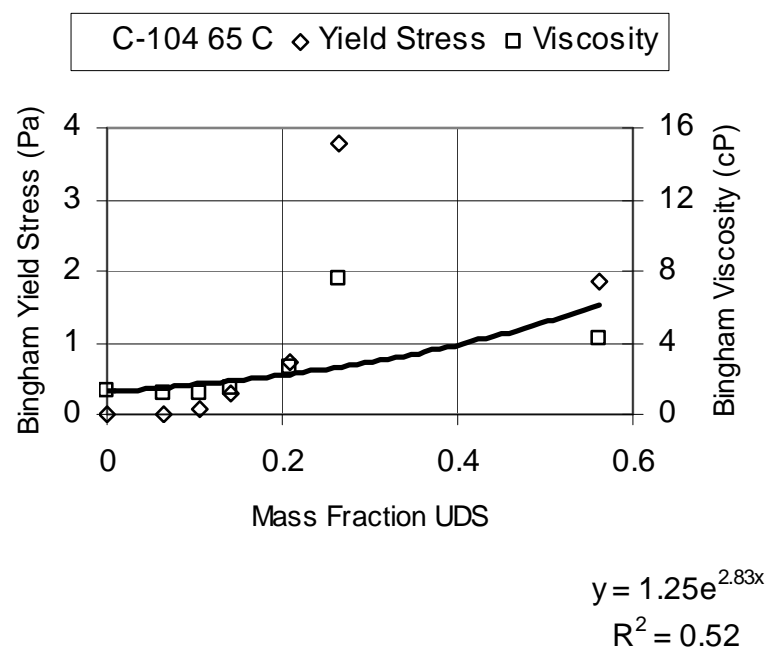

Figure 3.117. CWP1 Sludge Bingham Parameters as Functions of UDS Concentration 


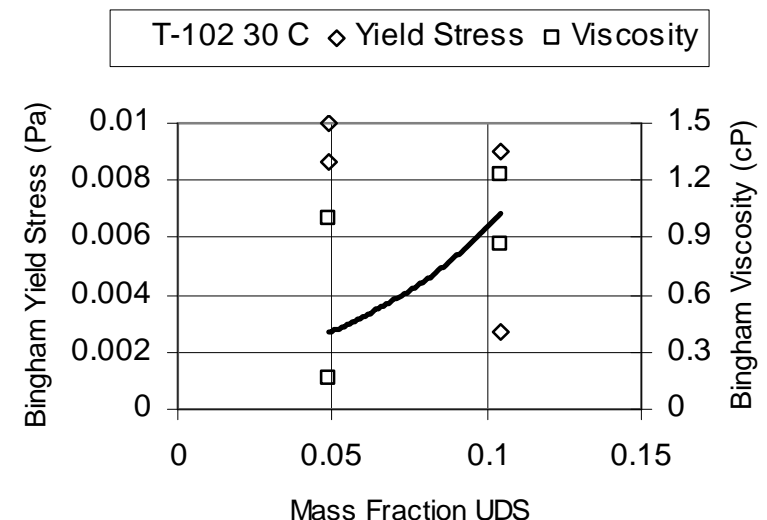

$$
\begin{gathered}
y=0.17 e^{16.98 x} \\
R^{2}=0.34
\end{gathered}
$$

Figure 3.118. CWP2 Sludge Bingham Parameters as Functions of UDS Concentration

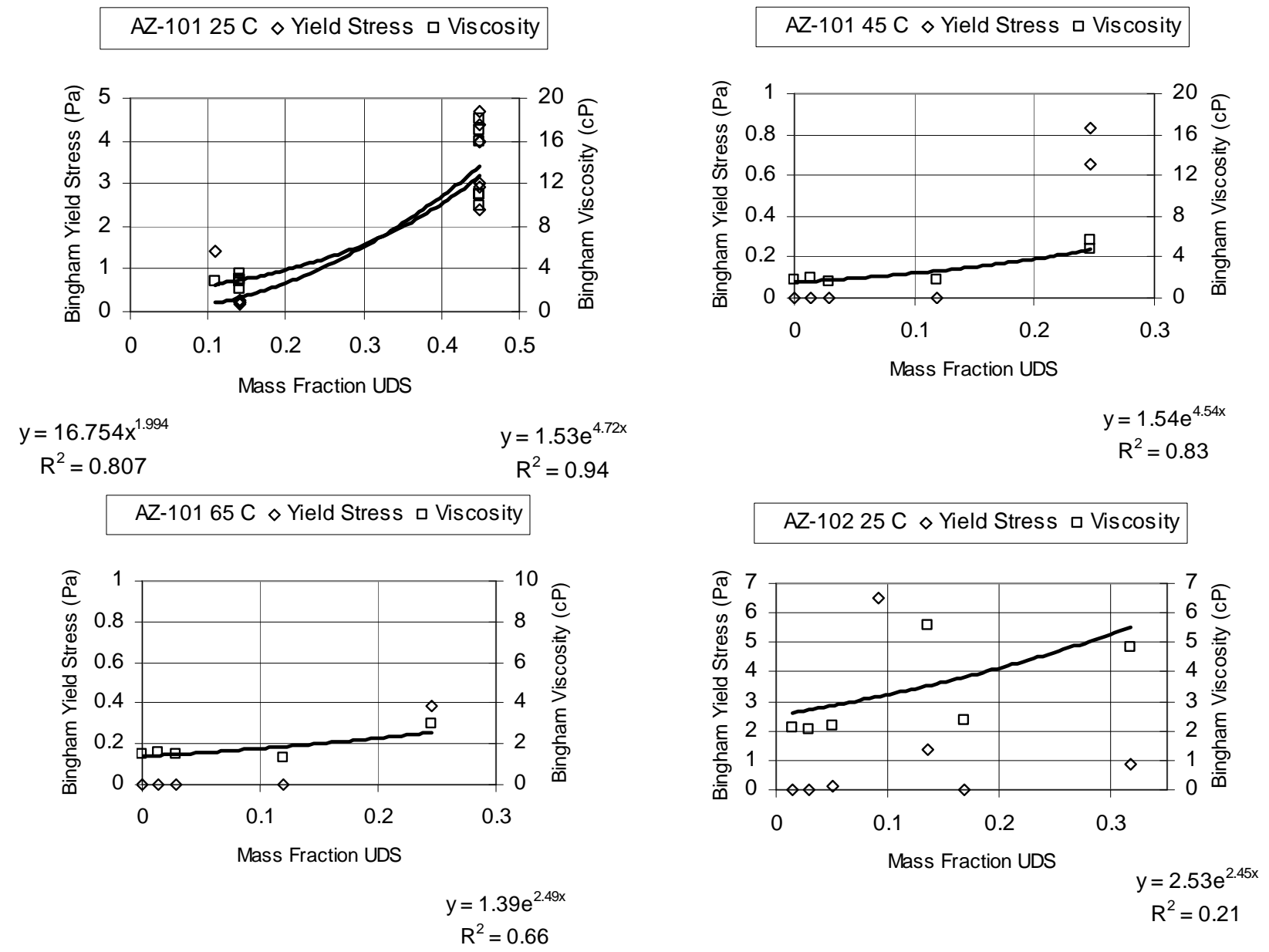

Figure 3.119. P3 Sludge Bingham Parameters as Functions of UDS Concentration 


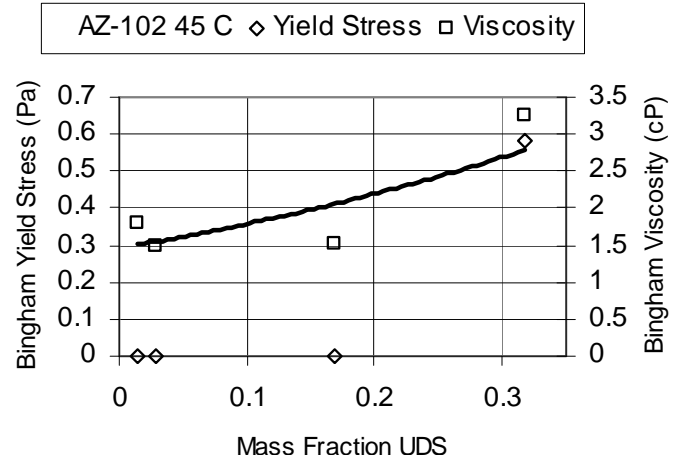

$$
\begin{gathered}
y=1.46 e^{2.04 x} \\
R^{2}=0.63
\end{gathered}
$$

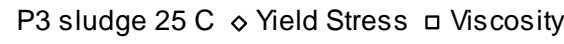

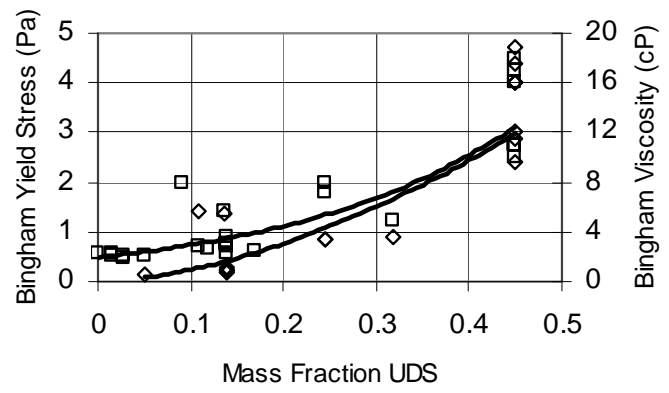

$y=11.29 x^{1.67}$

$\mathrm{R}^{2}=0.74$

$$
\begin{gathered}
y=1.96 e^{4.12 x} \\
R^{2}=0.82
\end{gathered}
$$

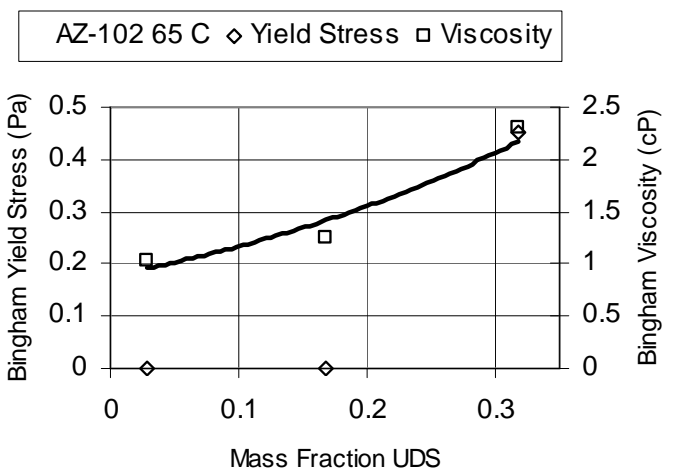

$y=0.88 e^{2.84 x}$
$R^{2}=0.93$

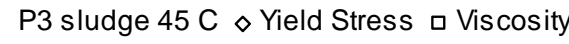

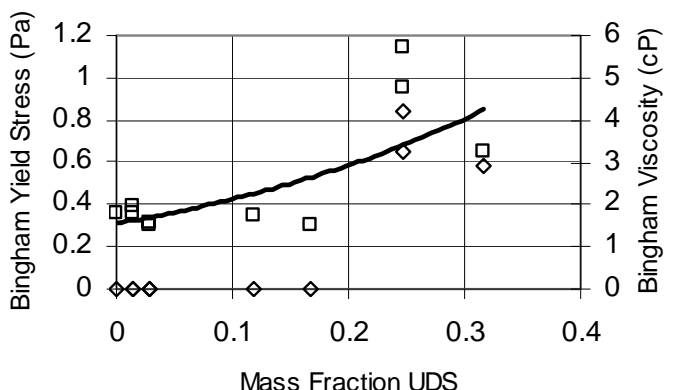

$$
\begin{gathered}
y=1.55 e^{3.18 x} \\
R^{2}=0.58
\end{gathered}
$$

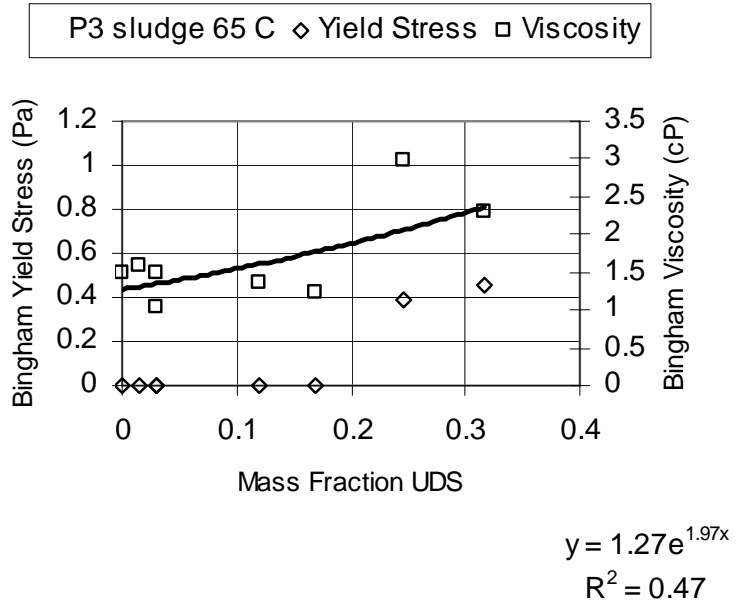

Figure 3.119. (contd) 


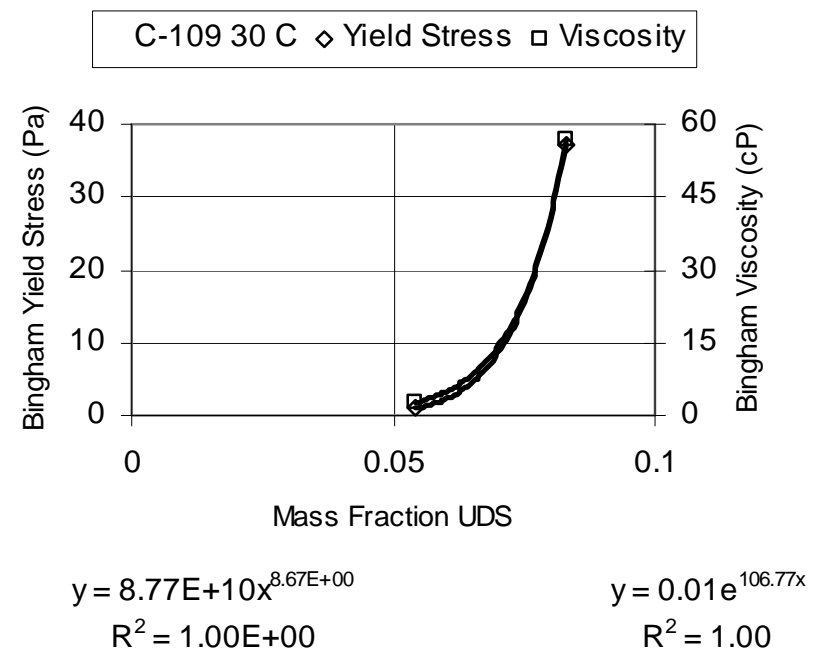

Figure 3.120. TFeCN Sludge Bingham Parameters as Functions of UDS Concentration

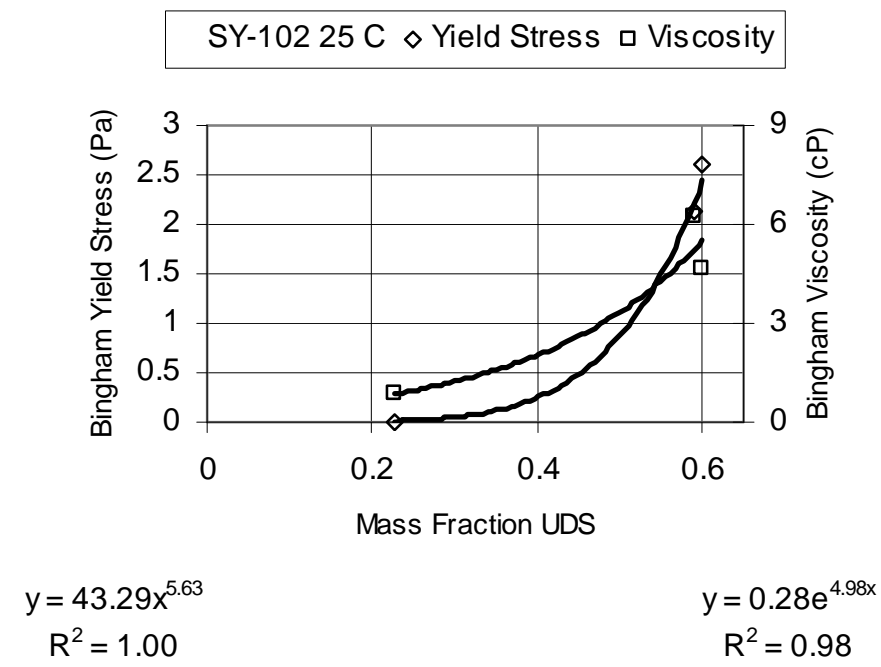

Figure 3.121. Unidentified Sludge Bingham Parameters as Functions of UDS Concentration 


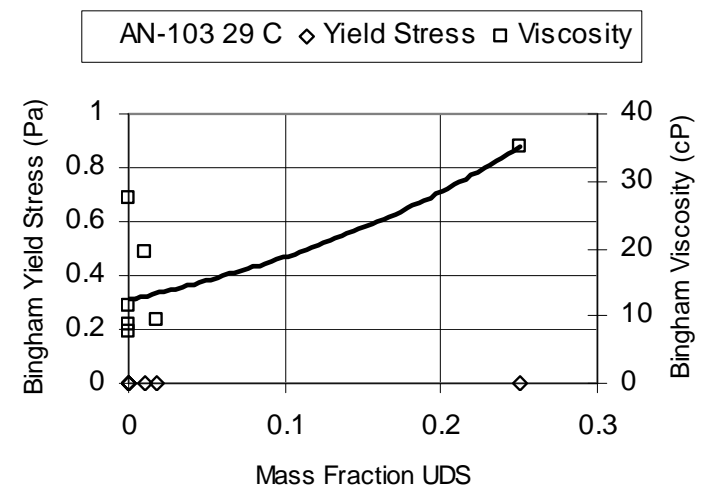

$$
\begin{gathered}
y=12.40 e^{4.15 x} \\
R^{2}=0.42
\end{gathered}
$$

AN-103 65 C $\diamond$ Yield Stress $\square$ Viscosity

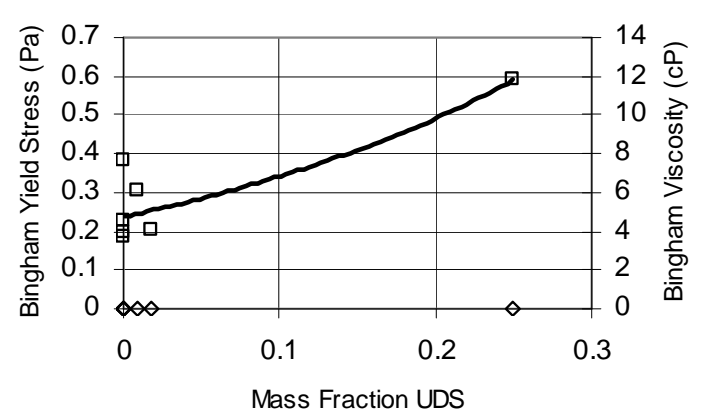

$$
\begin{gathered}
y=4.75 e^{3.63 x} \\
R^{2}=0.62
\end{gathered}
$$

AN-104 45 C $\diamond$ Yield Stress $\square$ Viscosity

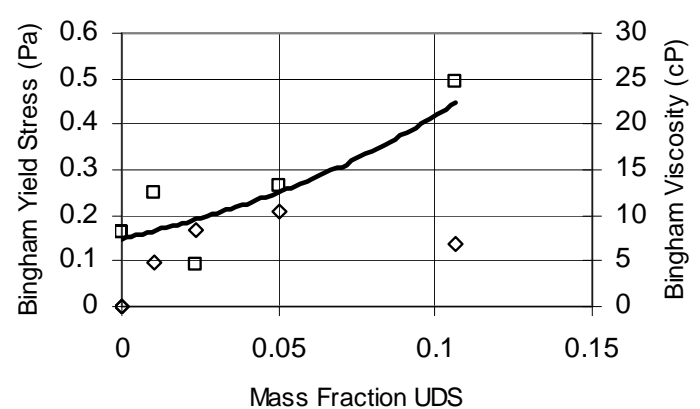

$$
\begin{gathered}
y=7.48 e^{10.30 x} \\
R^{2}=0.56
\end{gathered}
$$

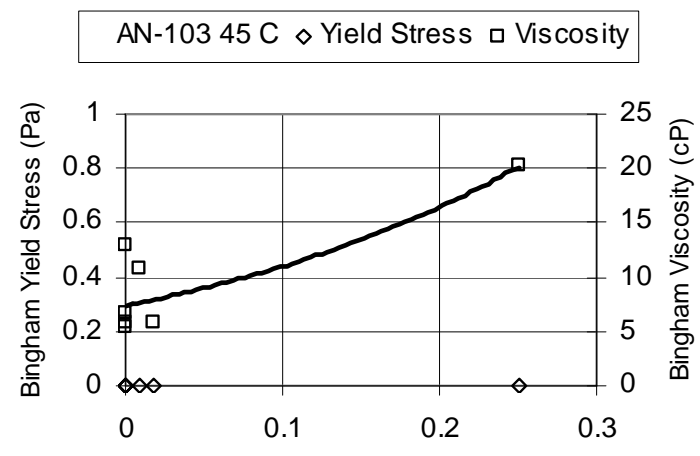

Mass Fraction UDS

$$
\begin{gathered}
y=7.35 e^{4.02 x} \\
R^{2}=0.55
\end{gathered}
$$
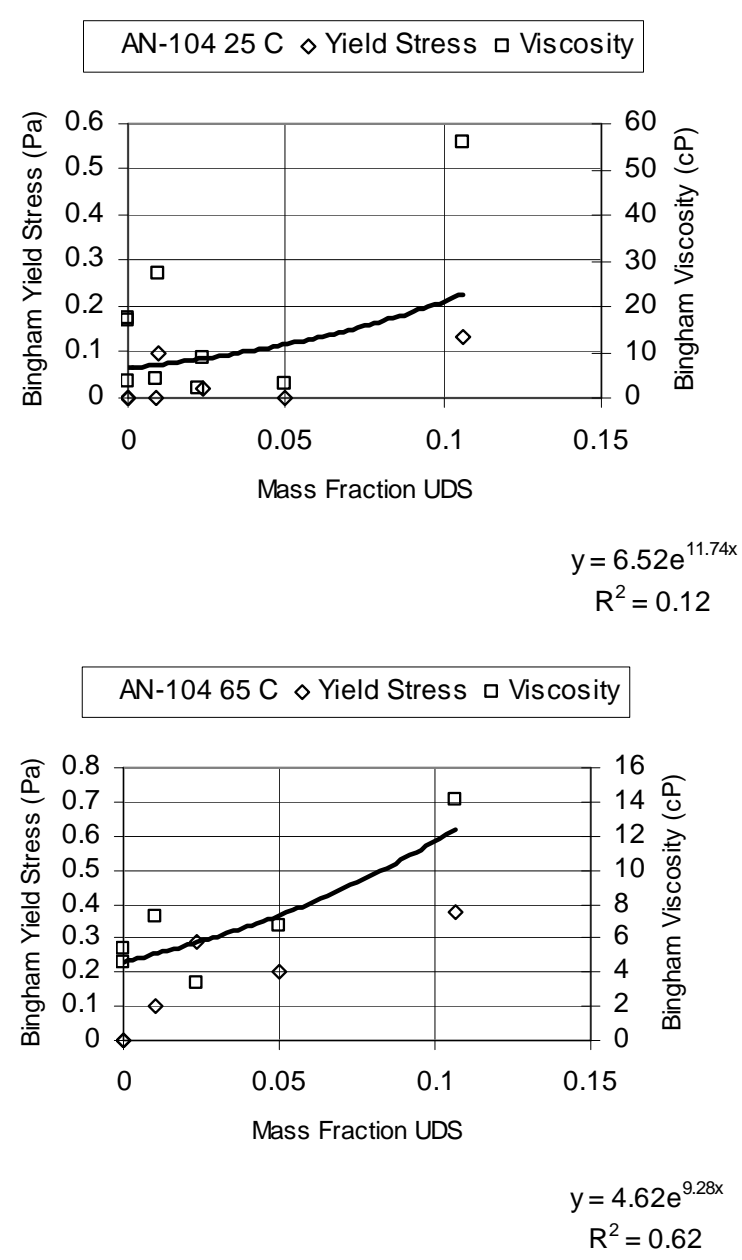

Figure 3.122. A2 Saltcake Bingham Parameters as Functions of UDS Concentration 


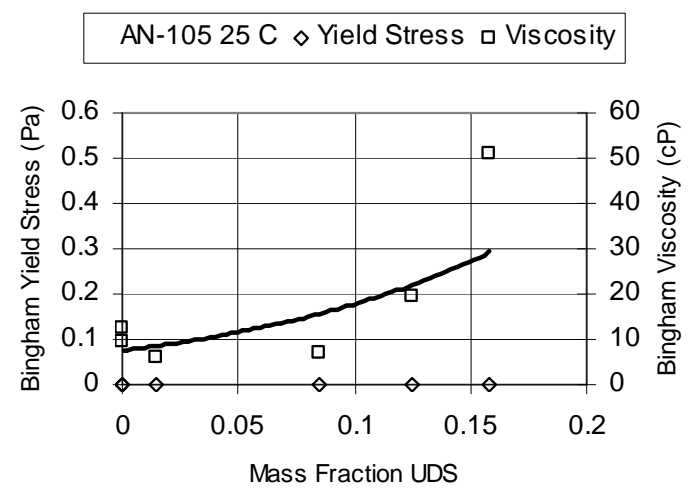

$$
\begin{gathered}
y=7.54 e^{8.57 x} \\
R^{2}=0.56
\end{gathered}
$$

AN-105 65 C $\diamond$ Yield Stress $\square$ Viscosity

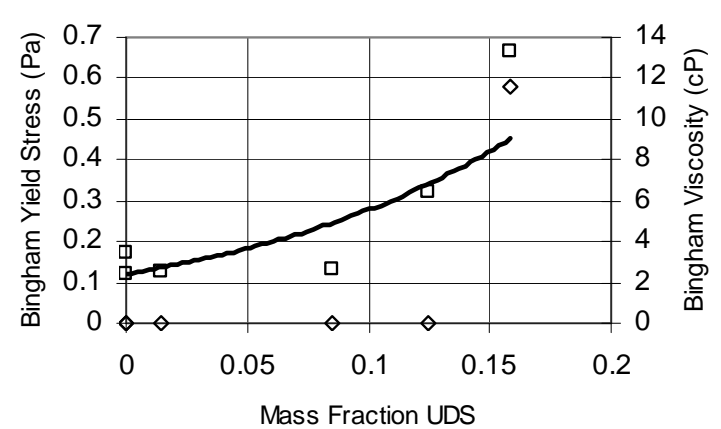

$$
\begin{gathered}
y=2.42 e^{8.33 x} \\
R^{2}=0.71
\end{gathered}
$$

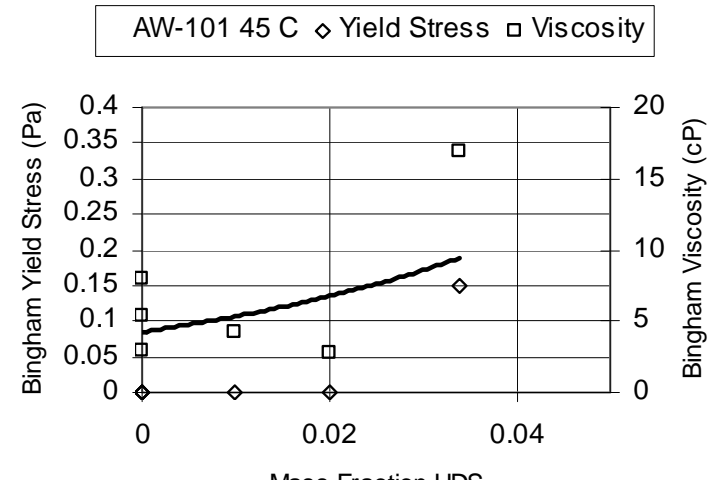

$$
\begin{gathered}
y=4.22 e^{23.59 x} \\
R^{2}=0.24
\end{gathered}
$$

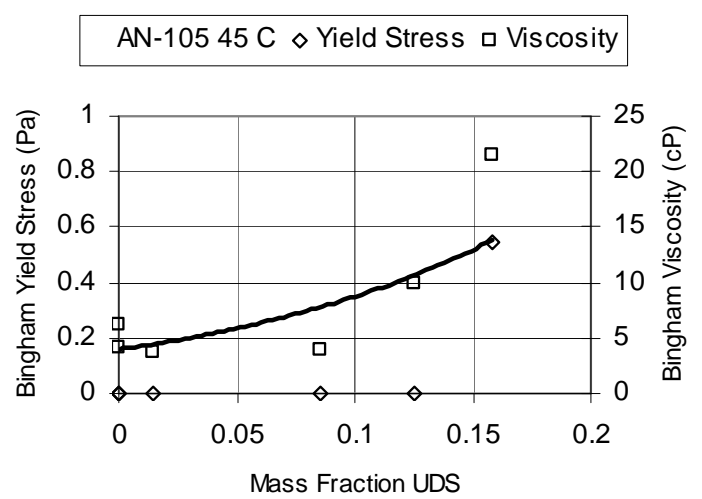

$$
\begin{gathered}
y=3.97 e^{7.89 x} \\
R^{2}=0.61
\end{gathered}
$$

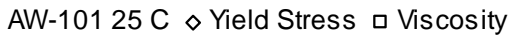

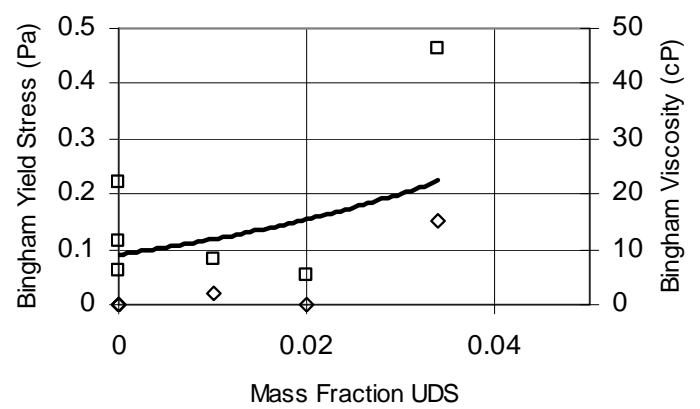

$$
\begin{gathered}
y=9.09 e^{26.51 x} \\
R^{2}=0.20
\end{gathered}
$$

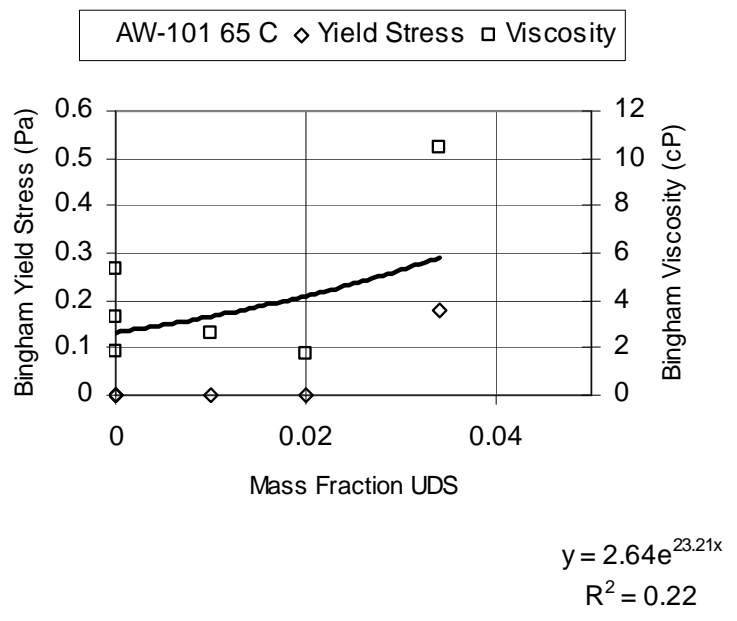

Figure 3.122. (contd) 


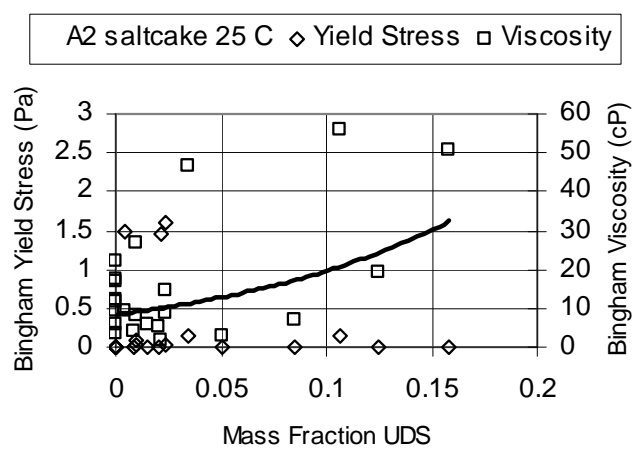

$$
\begin{gathered}
y=8.33 e^{8.56 x} \\
R^{2}=0.18
\end{gathered}
$$

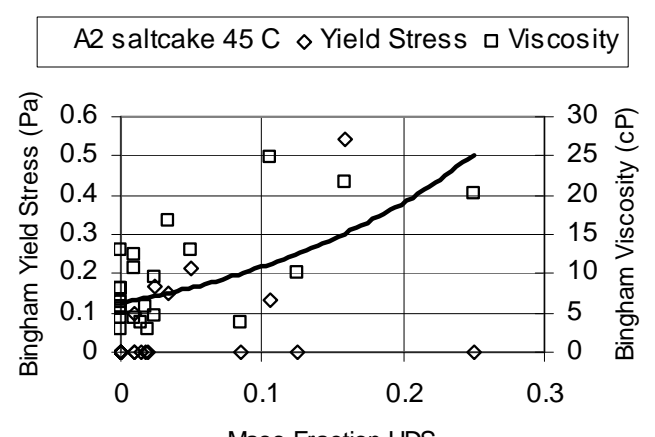

Mass Fraction UDS

$$
\begin{gathered}
y=6.23 e^{5.59 x} \\
R^{2}=0.31
\end{gathered}
$$

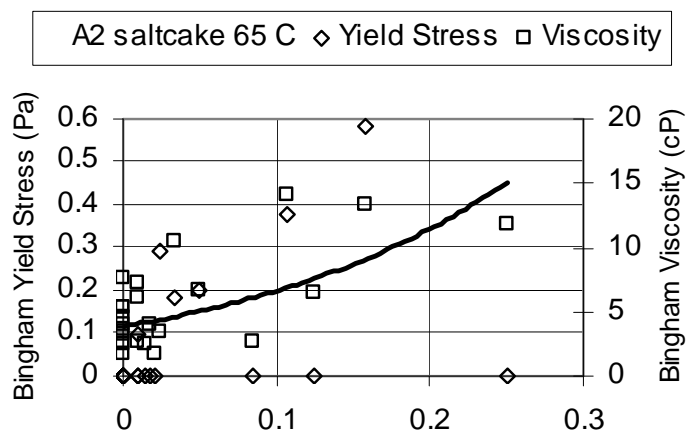

Mass Fraction UDS

$$
\begin{gathered}
y=3.84 e^{5.46 x} \\
R^{2}=0.33
\end{gathered}
$$

Figure 3.122. (contd)

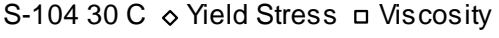

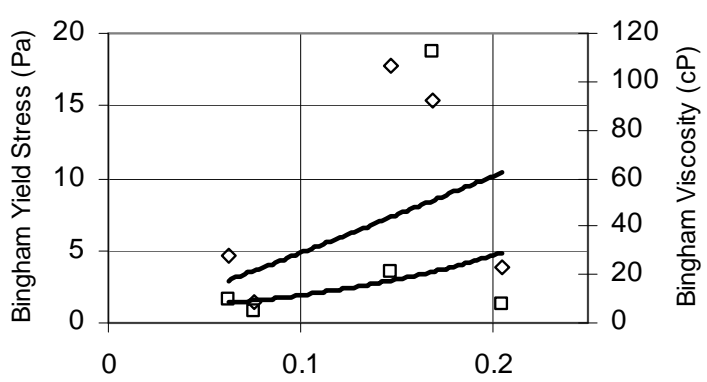

Mass Fraction UDS

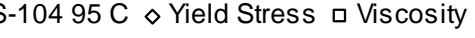

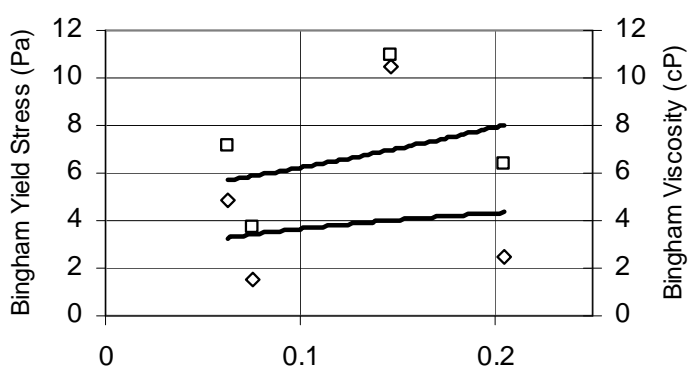

Mass Fraction UDS

$$
\begin{gathered}
y=6.31 x^{0.24} \\
R^{2}=0.03
\end{gathered}
$$

$$
\begin{gathered}
y=4.90 e^{2.41 x} \\
R^{2}=0.13
\end{gathered}
$$

$y=56.25 x^{1.07}$

$\mathrm{R}^{2}=0.27$

$$
\begin{gathered}
y=4.73 e^{8.88 x} \\
R^{2}=0.19
\end{gathered}
$$

Figure 3.123. R Saltcake Bingham Parameters as Functions of UDS Concentration 

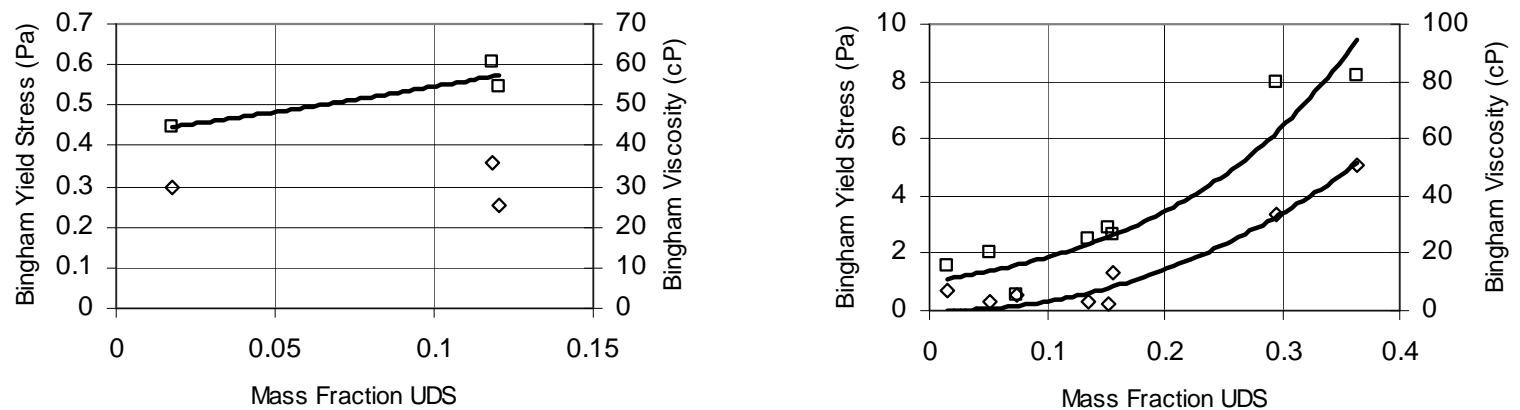

$$
\begin{gathered}
y=42.81 e^{2.44 x} \\
R^{2}=0.87
\end{gathered}
$$

$$
\begin{gathered}
y=10.10 e^{6.15 x} \\
R^{2}=0.73
\end{gathered}
$$

SY-101 65 C $\diamond$ Yield Stress 口 Viscosity

\section{SY-101 70 C $\diamond$ Yield Stress $\square$ Viscosity}
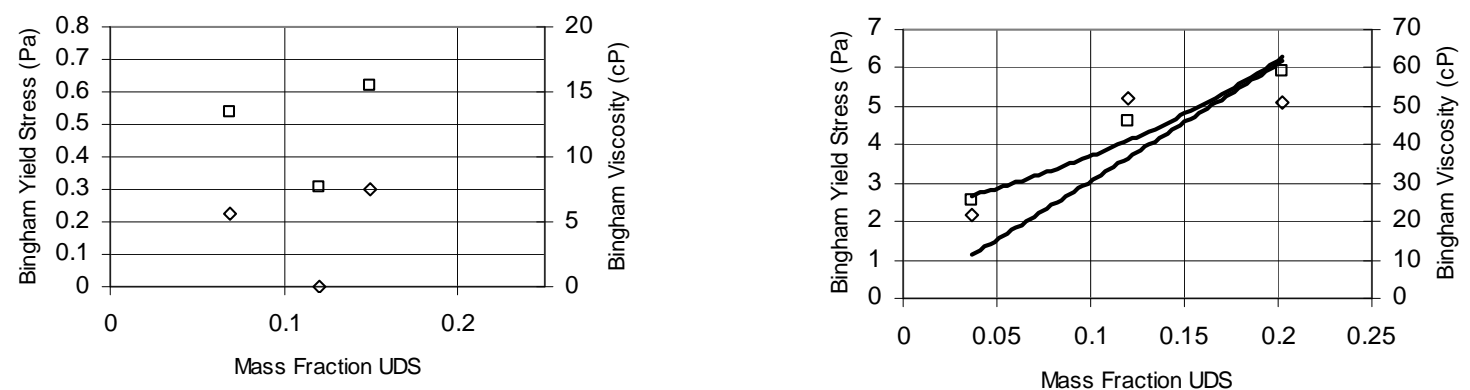

$y=30.504 x$

$$
\begin{gathered}
y=22.19 e^{5.14 x} \\
R^{2}=0.94
\end{gathered}
$$

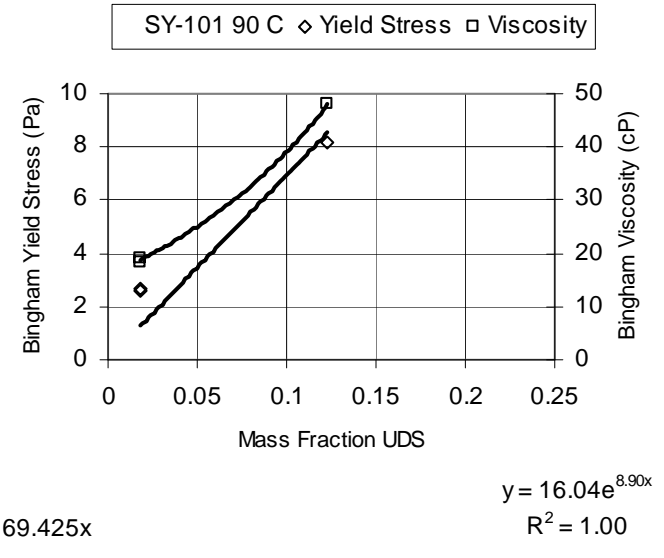

Figure 3.124. S2 Saltcake Bingham Parameters as Functions of UDS Concentration 
Correlations for both Bingham parameters as a function of UDS concentration were found for a subset of the 23 tanks and for 4 of the primary waste types with multiple tanks represented. For those tanks and primary waste types wherein correlations were found, there are some instances where the model fits may be seen to represent the data well, e.g., Figure 3.113, 224 Post-1949 sludge, and others where the correlations are very poor, e.g., Figure 3.122, A2 saltcake (no yield stress correlation, low correlation coefficients for the viscosity models).

Figure 3.112 through Figure 3.124 present the Bingham parameter-UDS concentration data independent of the methodology used to alter the UDS concentration. In Figure 3.117 (C-104, CWP1 sludge at $25^{\circ} \mathrm{C}$ ) the data include as-received, water-washed, and caustic leached and washed (from cross-flow filtration studies) as well as water-diluted material at different UDS concentrations. As discussed in Poloski et al. (2007), a peak in the yield stress trend and a minimum in the consistency trend are observed. The peak shows the importance of sample process history as the abnormally high yield stress value corresponds to a sample that was processed during cross-flow filtration. The sample underwent similar chemical changes as it was dewatered and water washed to a certain solids loading. However, the use of the filtration equipment may have altered the slurry's physical properties through dissolution of saltcake particles, a lowered ionic strength (zeta potential), and potential particle attrition from the pump/filter loop. This demonstrates that sample process history can significantly influence the waste rheology as discussed in Section 4.0.

As described for shear strength in Section 3.2.7.1, the Bingham parameters of a slurry, in addition to changing with the UDS concentration, can also vary significantly with slurry chemical and physical properties such as $\mathrm{pH}$, UDS and liquid chemistry, and particle size and shape. As dilution with water occurs, all of these properties may change as soluble particles dissolve, changing particle size and chemistry, and aqueous species are diluted while soluble UDS are added to the liquid until equilibrium is reached.

Poloski et al. (2007) described the rheological changes due solely to UDS concentration change as "mechanically dominated” and the latter changes as "chemically dominated." "Mechanically dominated" behavior was postulated to be shown when the rheological properties increase with increasing UDS concentration per Eqs. (3.14) and (3.15) (e.g., B-203, T-203, Figure 3.113), and “chemically dominated” when the rheological properties increase to a maximum value with water dilution. Examples that show this type of behavior include C-104, Figure 3.117 and B-201, Figure 3.114.

\section{Bingham Parameters-Temperature}

From the individual tank and primary waste type figures in the preceding sub-section, it can be seen that temperature can influence the Bingham parameters. In general, from Figure 3.112 through Figure 3.124, the Bingham viscosity decreases with temperature while the Bingham yield stress response varies.

A specific consideration of temperature and Bingham parameters is made for the waste type Groups 1 through 8 of Table 3.35. For those waste group samples with a non-zero Bingham yield stress, the yield

stress is essentially shown to be independent of the temperature of the slurry (Figure 3.125). As shown in Figure 3.126, Bingham viscosity, with the exception of waste Group 5, decreases with increasing temperature. 
$\neg$ Group 1, 9 wt\% UDS $\rightarrow$ Group 2, 37.4 wt\% UDS

*—Group 5, 18.5 wt\% UDS $\rightarrow-$ Group 7,10 wt\% UDS

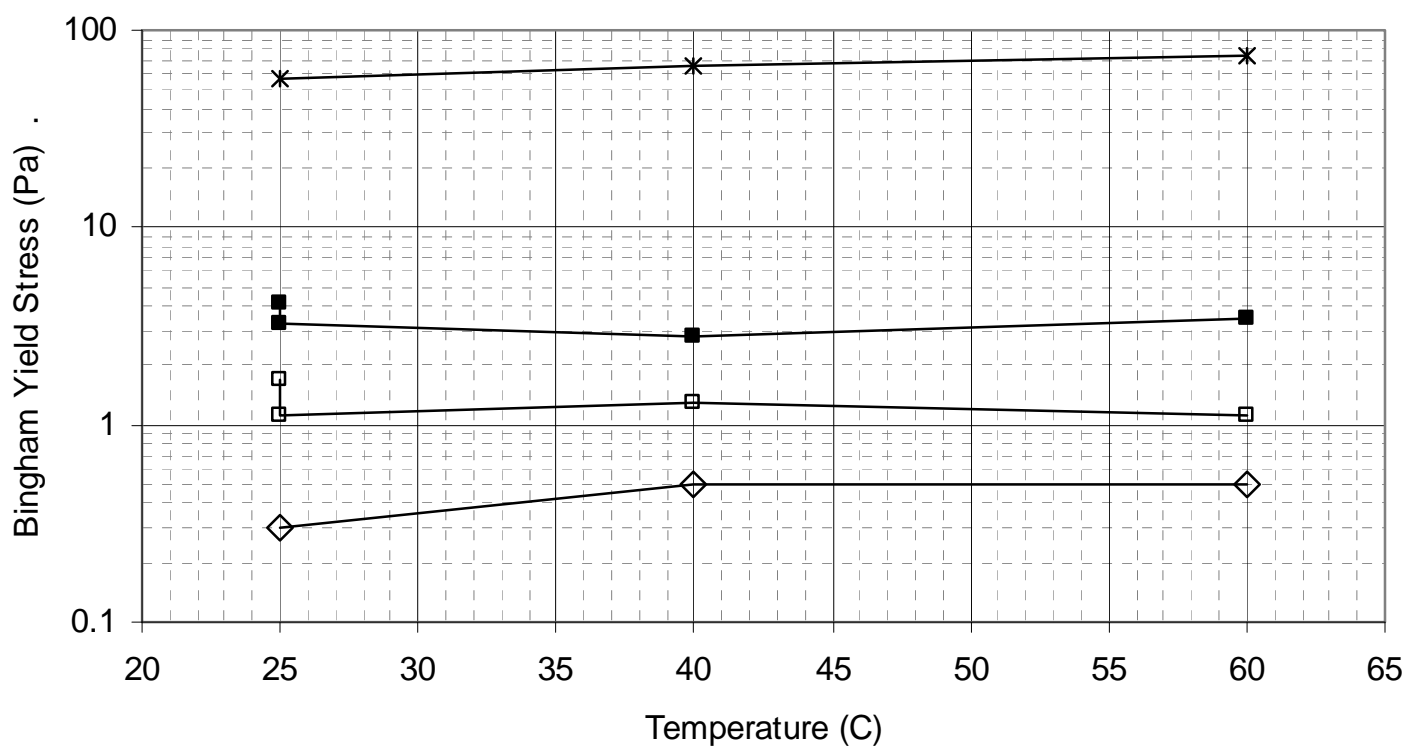

Figure 3.125. Bingham Yield Stress as a Function of Temperature
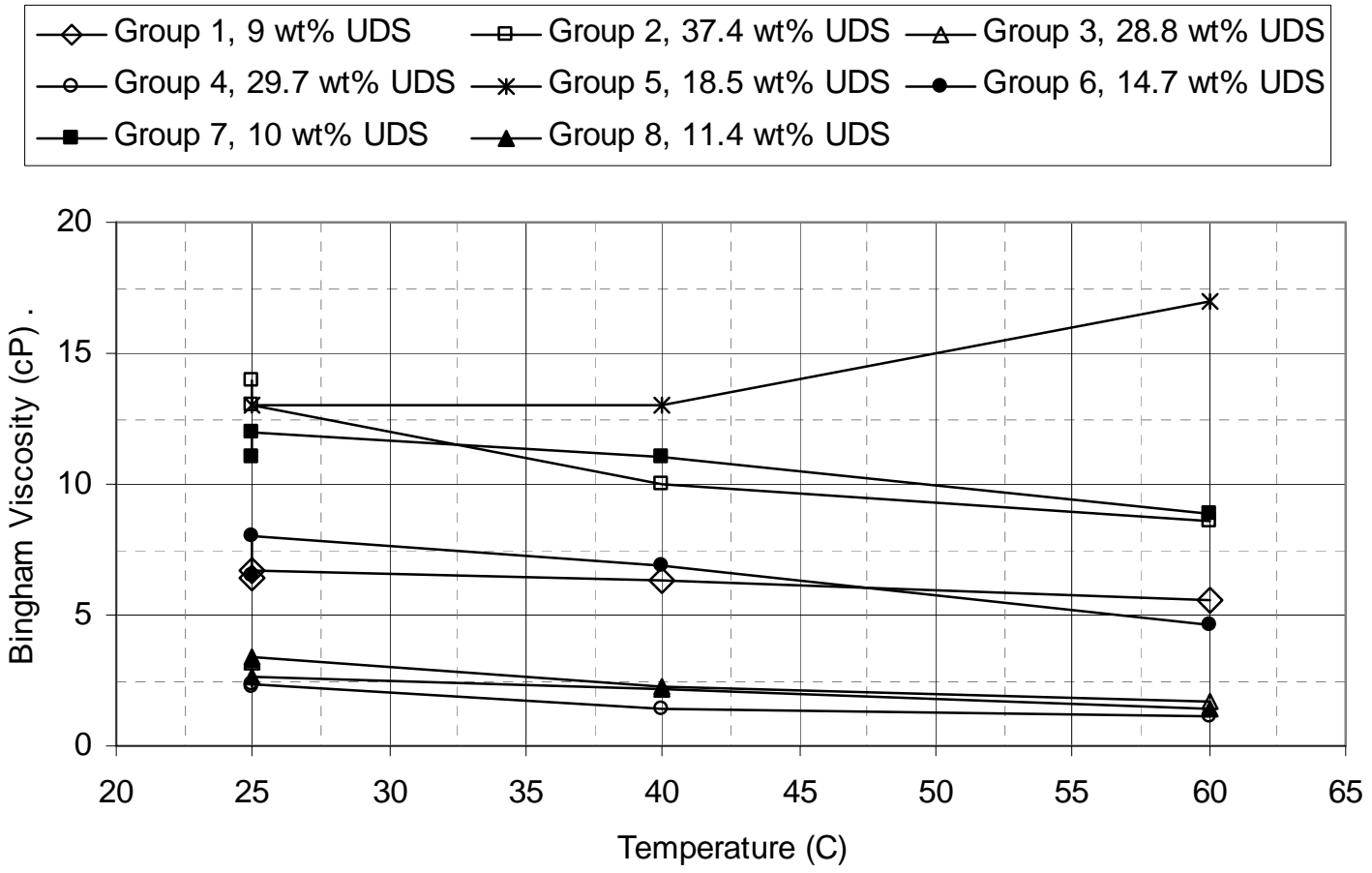

Figure 3.126. Bingham Viscosity as a Function of Temperature 


\section{Comparison of Bingham Yield Stress and Shear Strength}

As summarized in Wells et al. (2010b), the Bingham yield stress and shear strength at similar sample conditions can be compared. Note that the Bingham yield stress does not necessarily represent the value of the yield stress in shear as an intrinsic, instrument-geometry indifferent, rheological property of a slurry, while the shear strength (i.e., the yield stress in shear measured with a shear vane) is a direct measurement (Turian et al. 2002, Nguyen and Boger 1983, Nguyen and Boger 1992, Barnes 1999).

The Bingham yield stress and shear strength of pretreated Hanford sludge waste is compared in Poloski et al. (2004), and the shear strength is shown to be larger by about a factor of three. Gauglitz et al. (2009) reported comparisons for Hanford sludge waste. The ratio of shear strength to Bingham yield stress for diluted B-203 and T-203 waste is 5 and 8, respectively (Tingey et al. 2003). A ratio approaching 1000 may be determined from core-sample analyses of AZ-101 waste (Urie et al. 2002). ${ }^{\text {(a) }}$

Overshoot behavior has been observed for rheograms of Hanford sludge slurries (Tingey et al. 2003). Speers et al. (1987) refer to "gel strength" as "the shear strength overshoot which occurs in a previously resting fluid which is suddenly sheared." For analysis of Hanford sludge simulants in Wells et al. (2010b), the material was completely mobilized immediately before being introduced into the rheometer, and the measurements were immediately taken once the sample was in the rheometer. In a study pertaining to sludge rheograms, Baudez (2006) concluded that the initial stress overshoot is highly dependent on the shear-rate increment profile and the data sampling.

The Bingham yield stress (low UDS concentrations) or approximate peak shear stress (high UDS concentrations) from the simulant rheogram ramp-up flow curves for the waste simulants of Wells et al. (2010b) is provided in Table 3.36 together with the Bingham yield stress and shear strength at 0 hours (the 0 hour shear strength was measured with a shear vane on material that was completely mobilized immediately prior to the measurement). As described in the Shear Strength-UDS Concentration sub-section, Simulant 1 represents the Hanford sludge as a whole and Simulant 2 the resultant sludge from a retrieval scenario (a combination of C-104, C-111, and C-112 waste in AN-101). Good agreement (Bingham yield stress to shear strength ratio is $\sim 1$ ) is shown for the lower UDS concentration conditions. These comparisons potentially show that at the lower UDS concentrations, the simulants behave as a Bingham plastic since both the indirect and direct measures of the yield stress in shear are in close agreement.

The peak shear stress (flow curve ramp-up) is shown to compare much more favorably with the shear strength than the Bingham yield stress (flow curve ramp-down) for the higher UDS concentrations. The rheogram peak shear-stress to shear-strength ratios for Simulants 1 and 2 are in remarkable agreement at 1.25 and 1.35, respectively. This comparison may suggest that the overshoot behavior from the rheograms is not solely an artifact of the indirect measurement method.

(a) Memorandum from DB Bechtold to KE Bell, RA Esch, and FH Steen. Correction of Shear Strength Measurements Reported by 222S Laboratory. March 28, 2001. 8D500-DBB-01-018, Fluor Hanford, Richland, Washington. 
Table 3.36. Bingham Yield Stress and Shear-Strength Comparison, Wells et al. (2010b)

\begin{tabular}{lcccc}
\hline \multicolumn{1}{c}{ Material } & Mass Fraction UDS & $\begin{array}{c}\text { Bingham Yield } \\
\text { Stress }(\mathrm{Pa})\end{array}$ & $\begin{array}{c}\text { Ramp-up Shear } \\
\text { Stress (Pa) }\end{array}$ & $\begin{array}{c}\text { Shear Strength } \\
(\mathrm{Pa})\end{array}$ \\
\hline Simulant 1 & 0.31 & 30 & 31.5 & 34.3 \\
Simulant 1 & 0.40 & $16^{(\mathrm{b})}$ & $735^{(\mathrm{b})}$ & 921 \\
\hline Simulant 2 & 0.23 & 6.5 & 7.4 & 8.7 \\
Simulant 2 & 0.35 & 185 & 575 & 775 \\
\hline (a) Approximate values from flow curve ramp-ups. Simulant 1 and 2 low UDS concentrations, Bingham yield \\
stress, Simulant 1 and 2 high UDS concentrations, peak shear stress. \\
(b) Average of two measurements.
\end{tabular}

\subsection{Estimated Particle Size and Density Distributions}

The UDS composition and particle density, Section 3.2.3, the UDS primary particle size and shape, Section 3.2.4, and the UDS PSDs, Section 3.2.5, are used to estimate particle size and density distributions (PSDDs) following the approach of Wells et al. (2007). PSDDs are created for the individual tanks with PSD data available, for the primary waste types represented by PSD data, and for sludge and saltcake waste type composites created from the available data. Although a limited fraction of the waste is characterized, see Section 3.2.5 regarding the PSD characterization for example, the individual tank PSDDs show variability in the waste. Both sludge and saltcake PSDDs may be pertinent to waste storage and handling operations within the tanks farms, while the sludge waste PSDDs are more representative of diluted waste such as the waste feed delivery to the WTP.

The PSDDs provide a "best representation" of a volume-based probability for the solid particles in terms of particle size and density. Any application of the provided PSDDs must acknowledge the limitations of the available data as described in the preceding sections. In Section 3.3.1, the PSDD modeling approach is presented, and the PSSD runs are summarized in Section 3.3.2. The PSDD results are discussed in Section 3.3.2.

\subsubsection{PSDD Modeling Approach}

To define UDS PSDDs, the UDS compounds are modeled with the PSDs such that the PSD is reproduced, and the UDS compound mass is conserved as per Wells et al. (2007). A least-squares optimization problem was formulated by solving for a set of weighting factors that minimizes the error between the input UDS compound mass fraction and the calculated mass fraction of the compound.

The optimization, provided in more detail in Wells et al. (2007), is summarized:

- The PSD is described as a series of bins of particle size. For each bin/compound combination, the volume fraction of that bin that falls below the maximum observed size for the given compound is defined. 
- Normalized UDS compound volume fractions are multiplied by the volume probability of size, thus distributing the particulate volume across size and compound.

- The resultant volume fractions are converted to mass. When a fractal dimension (see Section 3.3.1.1) less than 3 is used the liquid fraction of the particulate is accounted for.

- The resultant compound masses are normalized, and the solid phase compound mass fractions are summed across the PSD. The sum of the squares of the difference between the computed and input solid phase compound mass fractions is minimized.

- The solid phase compound densities are computed as a function of the particle size and fractal dimension. No limit to maximum agglomeration size is assigned relative to the PSD.

Wells et al. (2007) provided representative PSDDs that were generated via Monte Carlo simulations that included variation in the input PSD, UDS compound density and concentration as well as primary particle size. The representative PSDDs were centroid results of the simulations.

A Monte Carlo simulation approach was not used for the current work for the following three reasons:

1) The PSD development for the current effort does not rely on data sampling (see Section 3.2.5),

2) The variation in the UDS compound density and concentration of Wells et al. (2007) is reduced with the increased number of included compounds (see Section 3.2.3), and

3) The uniform variation in primary particle size assigned in Wells et al. (2007) necessarily had a centroid of approximately $50 \%$ of the maximum primary particle size.

Thus, because of reasons 1) and 2), the PSD and UDS compound data, which is already expected centroid values, was input to a single optimization. For fractal dimensions other than 3 , the maximum primary size is set to $50 \%$ of the maximum observed primary particle size based on reason 3 ).

\subsubsection{Fractal Dimension Basis}

As described in Wells et al. (2007), the Hanford waste solid particles range in size and density from small, dense, primary particles to large, low-density, diffuse flocs or soft agglomerates and large, relatively dense, cemented aggregates and stable agglomerates (collectively termed hard agglomerates). Figure 3.127 depicts these different particles. 


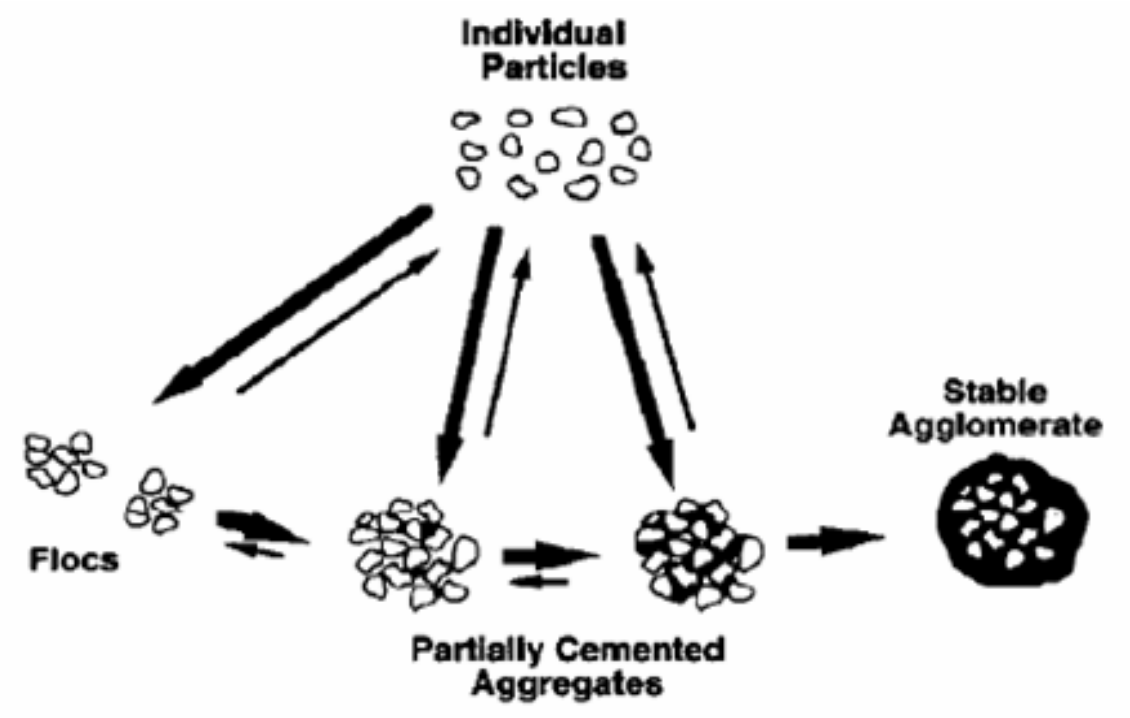

Figure 3.127. Solid Particles and Soft and Hard Agglomerates (from Ilievski and White 1994)

The individual particle size in a waste stream may vary by five orders of magnitude and is affected by the constituents present. Because the individual primary particles vary in size and density, the agglomerates formed from them also vary in density. Thus, because tank waste usually contains mixtures of primary particles and their composite agglomerates, it is reasonable to account for both primary particles and agglomerates to evaluate the sludge particulate characteristics. The functional relationship between the agglomerate size and its density provided in Section 2.0,

$$
\rho=\left(\frac{R}{r}\right)^{D_{F}-3}\left(\rho_{S}-\rho_{L}\right)+\rho_{L}
$$

can be used to determine the particle density based on its size

$$
\text { where } \begin{aligned}
\rho & =\text { agglomerate density } \\
D_{\mathrm{F}} & =\text { fractal dimension } \\
\mathrm{R} & =\text { agglomerate size } \\
\mathrm{r} & =\text { primary particle size } \\
\rho_{\mathrm{L}} & =\text { liquid density } \\
\rho_{\mathrm{S}} & =\text { primary particle density. }
\end{aligned}
$$

Agglomerate density therefore decreases as the size increases for a constant value of $\mathrm{D}_{\mathrm{F}}$. The fractal dimension, $\mathrm{D}_{\mathrm{F}}$, is between 0 and 3 . At $\mathrm{D}_{\mathrm{F}}=3$, the particulate is a primary particle or an agglomerate without any pore space. In general, a higher solid concentration forms larger agglomerates, but not in all cases (Selomnlya et al. 2002). When the driving force for agglomeration is very high (diffusion-limited aggregation), agglomerates are not very dense, having $\mathrm{D}_{\mathrm{F}} \approx 1.6 \sim 1.8$. When the driving force for agglomeration is weak (reaction-limited aggregation), agglomerates are more dense, with $\mathrm{D}_{\mathrm{F}}=1.8 \sim 2.25$. High-packing dense agglomerates may have $\mathrm{D}_{\mathrm{F}} \geq 2.5$. Although Hanford tank waste conditions (i.e., high $\mathrm{pH}$ and ion strength) favor reaction-limited agglomeration, a wide range of primary particles in the Hanford waste may form agglomerates in sediment that has been compressed by overlaying solids. 
Wells et al. (2007) further summarized the limited evaluations of the fractal dimension relevant to Hanford tank waste that are available. Rector and Bunker (1995b) reported that most colloidal agglomerates form a fractal structure with a fractal dimension, $\mathrm{D}_{\mathrm{F}}$, of $1.6 \sim 2.5$. A colloidal study assumed $1.8 \sim 2.5$ for the fractal dimension of an agglomerate of $\mathrm{Fe}(\mathrm{OH})^{3}$ (a) $^{\text {(a) }}$ Rector and Bunker (1995b) simulated an agglomeration process, resulting in a fractal dimension of 2.25. Bunker et al. (2000) also used the fractal dimension of 2.25 for an experimental study conducted to provide insight into the Hanford tank waste. They reported that Hanford sludge waste has a wide range of primary particles forming agglomerates, and the overlaying solids in the sediment layer exerts the normal and shear force that rearranges the agglomerates, possibly increasing their fractal dimension. Thus, the fractal dimension value may be greater than $2.25 \sim 2.5$.

Table 3.37 presents some measured fractal dimension values reported in the literature ranging from 1.6 to 2.8 (Kramer and Clark 1999, Kim and Kramer 2005). Most of these experiments have been performed on agglomerates with single-sized primary particles. There is also work indicating that in some cases, the fractal dimension decreases as the agglomerate size increases (Khelifa and Hill 2006), and there is a maximum agglomerate size that can be attained under shear (Selomnlya et al. 2003). Models relating the effect of shearing on the maximum agglomerate size are presented in Wells et al. (2007).

(a) Bunker BC, PJ Bruinsma, GL Gruff, CR Hymas, XS Li, JR Phillips, DR Rector, PA Smith, L Song, JM Tingey, and Y Wang. 1995. “Colloidal Studies for Solid/Liquid Separation.” TWRSPP-95-045, Pacific Northwest National Laboratory, Richland, Washington. 
Table 3.37. Measured Fractal Dimensions (from Wells et al. 2007)

\begin{tabular}{|c|c|}
\hline \multicolumn{1}{|c|}{ System } & Fractal Dimension \\
\hline $\begin{array}{c}\text { Ferric sulfate flocs } \\
<0.65 \mu \mathrm{m}\end{array}$ & 2.3 \\
$>0.65 \mu \mathrm{m}$ & 2.8 \\
\hline Ferric chloride $(<0.75 \mu \mathrm{m})$ & 2.3 \\
\hline$\alpha$-FeOOH & \\
(a) Diffusion limited aggregation & 1.6 \\
(b) Reaction limited aggregation & 2.0 \\
\hline Kaolin with aluminum sulfate & $1.6 \sim 1.95$ \\
\hline Nickel hydrocarbonate microsphere & \\
without shear & $1.7 \sim 1.8$ \\
with shear & $2.2 \sim 2.7$ \\
\hline Latex sphere & 2.1 \\
(a) Small flocs & 2.5 \\
(b) large flocs & $1.75 \sim 2.25$ \\
\hline Latex sphere with sodium chloride & 2.48 \\
\hline Polystyrene spheres & \\
\hline
\end{tabular}

Because a fractal analysis is usually based on the "self similitude" concept imposing constant fractal dimension, and because there is a lack of specific Hanford waste data to support the different or varying fractal dimensions for the sludge agglomerates, Wells et al. (2007) assumed that the fractal dimension was uniform and constant over the entire range of compounds and primary particle sizes forming agglomerates. An approach based on the expected volume-weighted average solid density and total agglomerate volume fraction was used to estimate the fractal dimension. However, as was acknowledged in Wells et al. (2007), there is uncertainty in the methodologies used to estimate the average solid density from actual waste data.

For the current work, the fractal dimension is varied from 3, which is used for all runs, to 2.25 (Rector and Bunker 1995b and Bunker et al. 2000) and 1.6 (minimum value from Table 3.37) for limited cases. When a fractal dimension of 3 is used, the input compound and size volume and mass distributions are preserved. When the fractal dimension is less than 3 , the input compound mass distribution and size volume distribution are preserved. Application of a fractal dimension other than 3 has several limitations and assumptions, including:

- Each primary particle forms its own agglomerates.

- A single fractal dimension value was used for agglomerates of different compounds and primary particle sizes.

- The same single fractal dimension value was used for all agglomerate sizes.

- The diameter of an equilibrium sphere was used to represent a non-spherical primary particle.

- All primary particles form agglomerates of any size within a measured solid size range, regardless of the size of primary particles. 
- Due to shear and normal forces acting on it, waste may have different agglomerate sizes and associated fractal dimensions during retrieval and transport processes as compared to in situ conditions.

\subsubsection{PSDD Runs}

The PSD data are partitioned by measurement technique as described in Section 3.2.5. PSDD runs are based on these PSD measurement techniques as shown in Table 3.38 for sludge waste and Table 3.39 for saltcake. For example, there are 15 PSDDs for sludge tanks with "Flowing Sonicated” PSDs. From these 15 PSDDs and including the waste group data, there are 13 waste-type PSDDs and the Sludge, Flowing Sonicated composite PSDD. The effect of the different PSD techniques relative to particle settling characteristics is discussed in Section 3.2.6.

In Table 3.38, the tanks and groups that comprise the Flowing Sonicated and Flowing Unsonicated PSSDs are the same, while a different set (some tanks are concurrent) is available for the No-Flow Unsonicated PSDDs. Unique tanks and groups with some concurrence comprise the saltcake PSDDs of Table 3.39. The tank, waste type, and composite UDS compositions for the PSDDs are provided in Appendix C.

As described in Section 3.3.1, all PSDD run cases are modeled with a fractal dimension of 3. PSDDs with fractal dimensions of 2.25 and 1.6 are modeled for the two tanks with in situ settling rate information (AZ-101 and AY-102, see Section 3.2.6) as well as for the composites.

The Sludge, Flowing Sonicated and Sludge, Flowing Unsonicated composite PSDDs are most comparable to the PSDDs provided by Wells et al. (2007). Data differences include changes in the PSD data set (see Section 3.2.5), and the sludge composite UDS inventory for the current work includes the quantities of salt compounds present in the PSD-characterized sludge wastes (see Section 3.2.3). In Wells et al. (2007), all soluble salt species were removed from the UDS inventory estimates even though these materials were likely represented in the PSDs. In addition, as referenced in Section .3.3.1, the number of UDS compounds represented was increased for the current work. 
Table 3.38. Sludge Waste PSDDs

\begin{tabular}{|c|c|c|c|}
\hline PSD Technique & Tank & Primary Waste Type & Composite \\
\hline \multirow{20}{*}{$\begin{array}{l}\text { Flowing Sonicated/ } \\
\text { Flowing Unsonicated }\end{array}$} & C-107 & $\mathrm{n} / \mathrm{a}^{1}$ & \multirow{20}{*}{ Sludge, Flowing Sonicated } \\
\hline & B-203 & \multirow{3}{*}{224 Post-1949 sludge } & \\
\hline & T-203 & & \\
\hline & T-204 & & \\
\hline & $\mathrm{T}-110$ & 2C sludge & \\
\hline & C-106 & AR sludge & \\
\hline & AY-102 & BL sludge & \\
\hline & C-104 & $\mathrm{n} / \mathrm{a}$ & \\
\hline & AW-103 & CWZr2 sludge & \\
\hline & AZ-101 & & \\
\hline & AZ-102 & P3 sludge & \\
\hline & AY-101 & Unidentified sludge & \\
\hline & SY-102 & $\mathrm{n} / \mathrm{a}$ & \\
\hline & S-107 & $\mathrm{n} / \mathrm{a}$ & \\
\hline & SX-108 & R1 (boiling) sludge & \\
\hline & \multirow{5}{*}{ Groups $^{2}$} & $1 \mathrm{C}$ and $2 \mathrm{C}$ sludge & \\
\hline & & CWP1 and CWP2 sludge & \\
\hline & & CWR1 sludge & \\
\hline & & TBP sludge & \\
\hline & & PFeCN sludge & \\
\hline \multirow{13}{*}{ No-Flow Unsonicated } & C-107 & $\mathrm{n} / \mathrm{a}$ & \multirow{13}{*}{ Sludge, No-Flow Unsonicated } \\
\hline & BX-107 & & \\
\hline & $\mathrm{T}-104$ & 1C sludge & \\
\hline & B-201 & 224 Pre-1949 sludge & \\
\hline & B-111 & 2C sludge & \\
\hline & $\mathrm{T}-111$ & $\mathrm{n} / \mathrm{a}$ & \\
\hline & AY-102 & BL sludge & \\
\hline & C-104 & $\mathrm{n} / \mathrm{a}$ & \\
\hline & C-103 & $\mathrm{n} / \mathrm{a}$ & \\
\hline & AZ-101 & \multirow{2}{*}{ P3 sludge } & \\
\hline & AZ-102 & & \\
\hline & U-110 & $\mathrm{n} / \mathrm{a}$ & \\
\hline & SY-102 & $\mathrm{n} / \mathrm{a}$ & \\
\hline
\end{tabular}


Table 3.39. Saltcake Waste PSDDs

\begin{tabular}{|c|c|c|c|}
\hline PSD Technique & Tank & Primary Waste Type & Composite \\
\hline \multirow{9}{*}{ Flowing Sonicated } & AN-104 & \multirow{3}{*}{ A2 saltcake } & \multirow{9}{*}{ Saltcake, Flowing Sonicated } \\
\hline & AN-102 & & \\
\hline & AN-107 & & \\
\hline & BY-104 & \multirow{3}{*}{ BY saltcake } & \\
\hline & BY-108 & & \\
\hline & BY-110 & & \\
\hline & S-104 & $\mathrm{n} / \mathrm{a}^{1}$ & \\
\hline & Grounc ${ }^{2}$ & BY, T1, and T2 saltcake & \\
\hline & Groups & S-Saltcake (S1 and S2) & \\
\hline \multirow{9}{*}{ Flowing Unsonicated } & AN-104 & \multirow{2}{*}{ A2 saltcake } & \multirow{9}{*}{ Saltcake, Flowing Unsonicated } \\
\hline & AN-102 & & \\
\hline & BY-104 & & \\
\hline & BY-108 & BY saltcake & \\
\hline & BY-110 & & \\
\hline & S-104 & $\mathrm{n} / \mathrm{a}$ & \\
\hline & S-101 & $\mathrm{n} / \mathrm{a}$ & \\
\hline & \multirow{2}{*}{ Groups } & BY, T1, and T2 saltcake & \\
\hline & & S-Saltcake (S1 and S2) & \\
\hline \multirow{7}{*}{ No-Flow Unsonicated } & AW-101 & \multirow{3}{*}{ A2 saltcake } & \multirow{7}{*}{ Saltcake, No-Flow Unsonicated } \\
\hline & AN-105 & & \\
\hline & AN-104 & & \\
\hline & BY-104 & BY saltcake & \\
\hline & S-104 & $\mathrm{n} / \mathrm{a}$ & \\
\hline & SY-101 & \multirow{2}{*}{ S2 saltcake } & \\
\hline & SY-103 & & \\
\hline Flowing Sonicated, Washed & AN-102 & A2 saltcake & - \\
\hline Flowing Unsonicated, Washed & AN-102 & A2 saltcake & - \\
\hline
\end{tabular}

\subsubsection{PSDD Results}

The PSDD approach summarized in Section 3.3.1 to relate measured PSDs to UDS compounds was applied for the PSDD runs of Table 3.38 and Table 3.39. As described, there may be specific uncertainty in the PSDs as a result of waste sampling technique, location, and handling. These considerations also apply to the UDS compound information, including phases and density, relative concentrations, and maximum primary particle size. The PSDDs are presented in tabular form in Section 3.3.3.1, the PSDDs are compared with each other in Section 3.3.3.2, and approaches for selecting particulate representing a specific probability in the PSDD are discussed in Section 3.3.3.3. 


\subsubsection{PSDDs}

The PSDDs are 3-dimensional matrices of volume-based probability of each UDS compound in a PSD "bin" and its density in that bin. Sludge, Flowing Unsonicated composite PSDDs are provided as examples in Table 3.40 to Table 3.44. In each table, the PSDD bins represent the upper and lower size limit of the particles in each bin. For the Sludge, Flowing Unsonicated composite PSDD of Table 3.40, a fractal dimension of 3 is used, and the particulate density of the UDS compounds is listed in the top row are constant for all particle sizes at the respective crystal density. The second row of Table 3.40, volume fraction, is the summation of the volume fraction of each compound. The remainder of the table entries are volume fraction. For example, gibbsite $\left[\mathrm{Al}(\mathrm{OH})_{3}\right]$ comprises $21 \%$ of the solids particulate by volume, and gibbsite particles $>7.7 \mu \mathrm{m}$ and less than or equal to $10 \mu \mathrm{m}$ have a density of $2.42 \mathrm{~g} / \mathrm{mL}$ and make up $2 \%$ of the solids by volume. This Sludge, Flowing Unsonicated composite PSDD with a fractal dimension of 3 is most comparable to the Case 3 PSDD from Wells et al. (2007). The Case 3 PSDD is stated in WTP Project Memorandum CCN 186332 to be the “...the most accurate and most conservative” PSDD approach in Wells et al. (2007). ${ }^{\text {(a) }}$

With a fractal dimension less than 3, the particle density decreases with size. Thus, the volume fraction of each UDS compound by particle size for the Sludge, Flowing Unsonicated composite PSDD with a fractal dimension of 2.25 is presented in Table 3.41, and the density of each UDS compound by size in Table 3.42. To interpret, consider the example of compound Ag at a particle size of $>7.7 \mu \mathrm{m}$ and less than or equal to $10 \mu \mathrm{m}$. Nominally, $0.0002 \%$ of the solid particulate by volume (Table 3.41 ) is at this size and has a density of $2.83 \mathrm{~g} / \mathrm{mL}$ (Table 3.42). The solid particulate by volume of Ag at a particle size of $>10 \mu \mathrm{m}$ and less than or equal to $13 \mu \mathrm{m}$ is again approximately $0.0002 \%$ (Table 3.41 ) but the density is reduced to $2.54 \mathrm{~g} / \mathrm{mL}$ (Table 3.42). The volume fraction (Table 3.43) and density (Table 3.44) are provided for the Sludge, Flowing Unsonicated composite PSDD with a fractal dimension of 1.6. The particle density decreases more rapidly with particle size for the lower fractal dimension.

The Sludge, Flowing Unsonicated composite PSDDs with a fractal dimension less than 3 are most comparable to the Case 4 PSDD from Wells et al. (2007). As described in Section 3.3.1.1, there are limitations and assumptions associated with these PSDDs due to the uncertainty of the fractal dimension. Regarding the Case 4 PSDD of Wells et al. (2007), it is stated in WTP Project Memorandum CCN 186332 that "The use of a fractal dimension [less than 3] is probably more descriptive of the actual system than crystal density [i.e., fractal dimension of 3]. However the fractal dimension used [2.6 in Wells et al. (2007)] is based on very little data and is used generally when in actuality it would probably vary with chemical species.”(a) The bracketed and italicized text has been added.

(a) WTP Project Memorandum CCN 186332. Letter correspondence from AW Etchells, Dupont Technology Consulting to SA Saunders, WTP-Bechtel. January 29, 2007. Comments on the Input Particle Size Report. 
Table 3.40. Sludge, Flowing Unsonicated PSDD, Fractal Dimension 3

\begin{tabular}{|c|c|c|c|c|c|c|c|c|c|c|c|c|c|c|c|c|c|c|}
\hline $\begin{array}{l}\text { Density } \\
(\mathrm{g} / \mathrm{mL})\end{array}$ & 10.5 & 7.143 & 8.9 & 7.9 & 3.14 & 2.71 & 3.18 & 4.11 & 3.15 & 4.26 & 2.42 & 3.01 & 7.143 & 2.61 & 2.3 & 6.51 & 3.102 & 5.026 \\
\hline \begin{tabular}{|l|} 
Volume \\
Fraction \\
\end{tabular} & 2.7E-07 & 9.4E-05 & 5.7E-04 & $2.4 \mathrm{E}-03$ & $7.0 \mathrm{E}-03$ & $9.8 \mathrm{E}-04$ & $9.2 \mathrm{E}-04$ & $4.1 \mathrm{E}-03$ & 7.5E-03 & 2.3E-02 & $2.1 \mathrm{E}-01$ & 4.3E-02 & $1.0 \mathrm{E}-05$ & $1.1 \mathrm{E}-03$ & $9.2 \mathrm{E}-06$ & $2.4 \mathrm{E}-04$ & 7.5E-04 & $1.9 \mathrm{E}-03$ \\
\hline $\mathrm{d}_{\mathrm{S}}(\mu \mathrm{m})$ & $\mathrm{Ag}$ & Ag2O & $\mathrm{Bi} 2 \mathrm{O} 3$ & $\mathrm{BiFeO} 3$ & $\mathrm{Ca} 5 \mathrm{OH}(\mathrm{PO} 4) 3$ & $\mathrm{CaCO} 3$ & $\mathrm{CaF} 2$ & $\mathrm{CrOOH}$ & $\mathrm{FePO} 4.2 \mathrm{H} 2 \mathrm{O}$ & $\mathrm{FeOOH}$ & Gibbsite & Boehmite & $\mathrm{HgO}$ & KAlSiO4 & $\mathrm{La}(\mathrm{OH}) 3$ & $\mathrm{LaPO} 4 \cdot 2 \mathrm{H} 2 \mathrm{O}$ & \begin{tabular}{|l|}
$\mathrm{Mn} 3(\mathrm{PO} 4) 2$ \\
\end{tabular} & $\mathrm{MnO} 2$ \\
\hline 0.22 & $1 \mathrm{E}-10$ & $3 \mathrm{E}-08$ & $2 \mathrm{E}-07$ & 9E-07 & $3 \mathrm{E}-06$ & 4E-07 & $3 \mathrm{E}-07$ & $1 \mathrm{E}-06$ & $3 \mathrm{E}-06$ & $8 \mathrm{E}-06$ & 7E-05 & $2 \mathrm{E}-05$ & 4E-09 & 4E-07 & 3E-09 & 9E-08 & $3 \mathrm{E}-07$ & $7 \mathrm{E}-07$ \\
\hline 0.28 & $1 \mathrm{E}-10$ & $5 \mathrm{E}-08$ & $3 \mathrm{E}-07$ & $1 \mathrm{E}-06$ & 4E-06 & 5E-07 & 5E-07 & 2E-06 & $4 \mathrm{E}-06$ & $1 \mathrm{E}-05$ & $1 \mathrm{E}-04$ & $2 \mathrm{E}-05$ & 6E-09 & 6E-07 & 5E-09 & 1E-07 & 4E-07 & $1 \mathrm{E}-06$ \\
\hline 0.36 & $1 \mathrm{E}-09$ & $3 \mathrm{E}-07$ & $2 \mathrm{E}-06$ & 9E-06 & 3E-05 & $4 \mathrm{E}-06$ & 3E-06 & $2 \mathrm{E}-05$ & 3E-05 & $9 \mathrm{E}-05$ & $8 \mathrm{E}-04$ & $2 \mathrm{E}-04$ & $4 \mathrm{E}-08$ & $4 \mathrm{E}-06$ & 3E-08 & $9 \mathrm{E}-07$ & 3E-06 & 7E-06 \\
\hline 0.46 & $1 \mathrm{E}-09$ & $5 \mathrm{E}-07$ & $3 \mathrm{E}-06$ & $1 \mathrm{E}-05$ & $4 \mathrm{E}-05$ & $5 \mathrm{E}-06$ & $5 \mathrm{E}-06$ & 2E-05 & $4 \mathrm{E}-05$ & $1 \mathrm{E}-04$ & $1 \mathrm{E}-03$ & $2 \mathrm{E}-04$ & $5 \mathrm{E}-08$ & $6 \mathrm{E}-06$ & $5 \mathrm{E}-08$ & $1 \mathrm{E}-06$ & 4E-06 & $1 \mathrm{E}-05$ \\
\hline 0.60 & $1 \mathrm{E}-09$ & $4 \mathrm{E}-07$ & $3 \mathrm{E}-06$ & $1 \mathrm{E}-05$ & $3 \mathrm{E}-05$ & $5 \mathrm{E}-06$ & $4 \mathrm{E}-06$ & 2E-05 & $3 \mathrm{E}-05$ & $1 \mathrm{E}-04$ & $1 \mathrm{E}-03$ & $2 \mathrm{E}-04$ & $5 \mathrm{E}-08$ & $5 \mathrm{E}-06$ & $4 \mathrm{E}-08$ & \begin{tabular}{l|l}
$1 \mathrm{E}-06$ \\
\end{tabular} & $3 \mathrm{E}-06$ & $9 \mathrm{E}-06$ \\
\hline 0.77 & $7 \mathrm{E}-09$ & $2 \mathrm{E}-06$ & $1 \mathrm{E}-05$ & 6E-05 & $2 \mathrm{E}-04$ & $2 \mathrm{E}-05$ & $2 \mathrm{E}-05$ & $1 \mathrm{E}-04$ & $2 \mathrm{E}-04$ & $5 \mathrm{E}-04$ & $5 \mathrm{E}-03$ & $1 \mathrm{E}-03$ & 2E-07 & $3 \mathrm{E}-05$ & $2 \mathrm{E}-07$ & $6 \mathrm{E}-06$ & $2 \mathrm{E}-05$ & $4 \mathrm{E}-05$ \\
\hline 1 & $4 \mathrm{E}-09$ & $1 \mathrm{E}-06$ & $8 \mathrm{E}-06$ & 3E-05 & 9E-05 & $1 \mathrm{E}-05$ & $1 \mathrm{E}-05$ & $5 \mathrm{E}-05$ & $1 \mathrm{E}-04$ & $3 \mathrm{E}-04$ & $3 \mathrm{E}-03$ & $6 \mathrm{E}-04$ & $1 \mathrm{E}-07$ & $2 \mathrm{E}-05$ & $1 \mathrm{E}-07$ & $3 \mathrm{E}-06$ & $1 \mathrm{E}-05$ & $3 \mathrm{E}-05$ \\
\hline 1.3 & $1 \mathrm{E}-08$ & 4E-06 & $2 \mathrm{E}-05$ & $1 \mathrm{E}-04$ & $3 \mathrm{E}-04$ & $4 \mathrm{E}-05$ & $4 \mathrm{E}-05$ & $2 \mathrm{E}-04$ & $3 \mathrm{E}-04$ & $1 \mathrm{E}-03$ & 9E-03 & $2 \mathrm{E}-03$ & 5E-07 & $5 \mathrm{E}-05$ & $4 \mathrm{E}-07$ & $1 \mathrm{E}-05$ & $3 \mathrm{E}-05$ & $8 \mathrm{E}-05$ \\
\hline 1.7 & $1 \mathrm{E}-08$ & $3 \mathrm{E}-06$ & $2 \mathrm{E}-05$ & $9 \mathrm{E}-05$ & $2 \mathrm{E}-04$ & 3E-05 & $3 \mathrm{E}-05$ & $1 \mathrm{E}-04$ & $3 \mathrm{E}-04$ & $8 \mathrm{E}-04$ & 7E-03 & $2 \mathrm{E}-03$ & 4E-07 & 4E-05 & 3E-07 & $9 \mathrm{E}-06$ & $3 \mathrm{E}-05$ & 7E-05 \\
\hline 2.2 & $1 \mathrm{E}-08$ & $5 \mathrm{E}-06$ & $3 \mathrm{E}-05$ & $1 \mathrm{E}-04$ & $4 \mathrm{E}-04$ & 5E-05 & $5 \mathrm{E}-05$ & $2 \mathrm{E}-04$ & $4 \mathrm{E}-04$ & $1 \mathrm{E}-03$ & $1 \mathrm{E}-02$ & $2 \mathrm{E}-03$ & $5 \mathrm{E}-07$ & $6 \mathrm{E}-05$ & $5 \mathrm{E}-07$ & $1 \mathrm{E}-05$ & $4 \mathrm{E}-05$ & $1 \mathrm{E}-04$ \\
\hline 2.8 & $2 \mathrm{E}-08$ & $5 \mathrm{E}-06$ & $3 \mathrm{E}-05$ & $1 \mathrm{E}-04$ & $4 \mathrm{E}-04$ & 6E-05 & $5 \mathrm{E}-05$ & $2 \mathrm{E}-04$ & $4 \mathrm{E}-04$ & $1 \mathrm{E}-03$ & $1 \mathrm{E}-02$ & $2 \mathrm{E}-03$ & 6E-07 & $7 \mathrm{E}-05$ & $5 \mathrm{E}-07$ & $1 \mathrm{E}-05$ & $4 \mathrm{E}-05$ & $1 \mathrm{E}-04$ \\
\hline 3.6 & $2 \mathrm{E}-08$ & 6E-06 & $3 \mathrm{E}-05$ & $1 \mathrm{E}-04$ & $4 \mathrm{E}-04$ & $6 \mathrm{E}-05$ & $6 \mathrm{E}-05$ & $2 \mathrm{E}-04$ & $5 \mathrm{E}-04$ & $1 \mathrm{E}-03$ & $1 \mathrm{E}-02$ & $3 \mathrm{E}-03$ & 6E-07 & $7 \mathrm{E}-05$ & 6E-07 & $1 \mathrm{E}-05$ & 5E-05 & $1 \mathrm{E}-04$ \\
\hline 4.6 & $2 \mathrm{E}-08$ & 7E-06 & $4 \mathrm{E}-05$ & $2 \mathrm{E}-04$ & $5 \mathrm{E}-04$ & 7E-05 & $6 \mathrm{E}-05$ & 3E-04 & $5 \mathrm{E}-04$ & $2 \mathrm{E}-03$ & $1 \mathrm{E}-02$ & $3 \mathrm{E}-03$ & 7E-07 & $8 \mathrm{E}-05$ & 6E-07 & $2 \mathrm{E}-05$ & $5 \mathrm{E}-05$ & $1 \mathrm{E}-04$ \\
\hline 6.0 & $2 \mathrm{E}-08$ & 7E-06 & $4 \mathrm{E}-05$ & $2 \mathrm{E}-04$ & $5 \mathrm{E}-04$ & $8 \mathrm{E}-05$ & $7 \mathrm{E}-05$ & 3E-04 & $6 \mathrm{E}-04$ & $2 \mathrm{E}-03$ & $2 \mathrm{E}-02$ & $3 \mathrm{E}-03$ & $8 \mathrm{E}-07$ & 9E-05 & $7 \mathrm{E}-07$ & $2 \mathrm{E}-05$ & $6 \mathrm{E}-05$ & $1 \mathrm{E}-04$ \\
\hline 7.7 & $3 \mathrm{E}-08$ & 9E-06 & $5 \mathrm{E}-05$ & $2 \mathrm{E}-04$ & $7 \mathrm{E}-04$ & $9 \mathrm{E}-05$ & $9 \mathrm{E}-05$ & $4 \mathrm{E}-04$ & $7 \mathrm{E}-04$ & $2 \mathrm{E}-03$ & $2 \mathrm{E}-02$ & $4 \mathrm{E}-03$ & $1 \mathrm{E}-06$ & $1 \mathrm{E}-04$ & $9 \mathrm{E}-07$ & $2 \mathrm{E}-05$ & 7E-05 & $2 \mathrm{E}-04$ \\
\hline 10 & 2E-08 & 8E-06 & 5E-05 & $2 \mathrm{E}-04$ & 6E-04 & 8E-05 & $8 \mathrm{E}-05$ & $3 \mathrm{E}-04$ & 6E-04 & $2 \mathrm{E}-03$ & $2 \mathrm{E}-02$ & 3E-03 & $9 \mathrm{E}-07$ & 9E-05 & $8 \mathrm{E}-07$ & 2E-05 & \begin{tabular}{l|l|}
$6 \mathrm{E}-05$ & \\
\end{tabular} & $2 \mathrm{E}-04$ \\
\hline 13 & $2 \mathrm{E}-08$ & 8E-06 & 5E-05 & $2 \mathrm{E}-04$ & 6E-04 & 9E-05 & $8 \mathrm{E}-05$ & 4E-04 & 7E-04 & 2E-03 & $2 \mathrm{E}-02$ & 4E-03 & 9E-07 & $1 \mathrm{E}-04$ & $8 \mathrm{E}-07$ & $2 \mathrm{E}-05$ & 7E-05 & $2 \mathrm{E}-04$ \\
\hline 17 & $2 \mathrm{E}-08$ & 7E-06 & 4E-05 & $2 \mathrm{E}-04$ & 5E-04 & 7E-05 & $7 \mathrm{E}-05$ & 3E-04 & 6E-04 & 2E-03 & $2 \mathrm{E}-02$ & 3E-03 & $8 \mathrm{E}-07$ & 9E-05 & 7E-07 & $2 \mathrm{E}-05$ & \begin{tabular}{l|l|}
$6 \mathrm{E}-05$ \\
\end{tabular} & $1 \mathrm{E}-04$ \\
\hline 22 & $2 \mathrm{E}-08$ & $6 \mathrm{E}-06$ & 3E-05 & 1E-04 & 4E-04 & $6 \mathrm{E}-05$ & 6E-05 & 2E-04 & 5E-04 & 1E-03 & $1 \mathrm{E}-02$ & 3E-03 & 6E-07 & 7E-05 & 6E-07 & $1 \mathrm{E}-05$ & \begin{tabular}{l|l|}
$5 \mathrm{E}-05$ \\
\end{tabular} & $\overline{1 \mathrm{E}-04}$ \\
\hline 28 & $1 \mathrm{E}-08$ & $4 \mathrm{E}-06$ & $2 \mathrm{E}-05$ & 9E-05 & 3E-04 & $4 \mathrm{E}-05$ & $4 \mathrm{E}-05$ & 2E-04 & $3 \mathrm{E}-04$ & 9E-04 & $8 \mathrm{E}-03$ & $2 \mathrm{E}-03$ & $4 \mathrm{E}-07$ & $4 \mathrm{E}-05$ & $4 \mathrm{E}-07$ & \begin{tabular}{|l|}
$9 \mathrm{E}-06$ \\
\end{tabular} & \begin{tabular}{|l|}
$3 \mathrm{E}-05$ \\
\end{tabular} & $7 \mathrm{E}-05$ \\
\hline 36 & $8 \mathrm{E}-09$ & $3 \mathrm{E}-06$ & $2 \mathrm{E}-05$ & $7 \mathrm{E}-05$ & 2E-04 & $3 \mathrm{E}-05$ & $3 \mathrm{E}-05$ & $1 \mathrm{E}-04$ & $2 \mathrm{E}-04$ & 6E-04 & 6E-03 & 1E-03 & $3 \mathrm{E}-07$ & $3 \mathrm{E}-05$ & 3E-07 & \begin{tabular}{|c|}
$\mathrm{E}-06$ \\
\end{tabular} & $2 \mathrm{E}-05$ & $\overline{5 \mathrm{E}-05}$ \\
\hline 46 & 5E-09 & $2 \mathrm{E}-06$ & $1 \mathrm{E}-05$ & $4 \mathrm{E}-05$ & $1 \mathrm{E}-04$ & $2 \mathrm{E}-05$ & $2 \mathrm{E}-05$ & 7E-05 & $1 \mathrm{E}-04$ & $4 \mathrm{E}-04$ & $4 \mathrm{E}-03$ & $8 \mathrm{E}-04$ & 2E-07 & 2E-05 & $2 \mathrm{E}-07$ & $4 \mathrm{E}-06$ & $1 \mathrm{E}-05$ & $3 \mathrm{E}-05$ \\
\hline 60 & $3 \mathrm{E}-09$ & $1 \mathrm{E}-06$ & $7 \mathrm{E}-06$ & $3 \mathrm{E}-05$ & $8 \mathrm{E}-05$ & $1 \mathrm{E}-05$ & $1 \mathrm{E}-05$ & $5 \mathrm{E}-05$ & $9 \mathrm{E}-05$ & 3E-04 & $2 \mathrm{E}-03$ & 5E-04 & $1 \mathrm{E}-07$ & $1 \mathrm{E}-05$ & $1 \mathrm{E}-07$ & $3 \mathrm{E}-06$ & 9E-06 & $2 \mathrm{E}-05$ \\
\hline 77 & 3E-09 & $1 \mathrm{E}-06$ & 7E-06 & 3E-05 & $8 \mathrm{E}-05$ & $1 \mathrm{E}-05$ & $1 \mathrm{E}-05$ & 5E-05 & 9E-05 & 3E-04 & $2 \mathrm{E}-03$ & 5E-04 & $1 \mathrm{E}-07$ & $1 \mathrm{E}-05$ & $1 \mathrm{E}-07$ & $3 \mathrm{E}-06$ & $9 \mathrm{E}-06$ & 2E-05 \\
\hline 100 & $4 \mathrm{E}-09$ & $1 \mathrm{E}-06$ & $7 \mathrm{E}-06$ & $3 \mathrm{E}-05$ & 9E-05 & $1 \mathrm{E}-05$ & $1 \mathrm{E}-05$ & 5E-05 & $1 \mathrm{E}-04$ & 3E-04 & 3E-03 & 5E-04 & $1 \mathrm{E}-07$ & $1 \mathrm{E}-05$ & $1 \mathrm{E}-07$ & $3 \mathrm{E}-06$ & $1 \mathrm{E}-05$ & 2E-05 \\
\hline 129 & 3E-09 & $9 \mathrm{E}-07$ & $5 \mathrm{E}-06$ & 2E-05 & 6E-05 & 9E-06 & 8E-06 & $4 \mathrm{E}-05$ & $7 \mathrm{E}-05$ & $2 \mathrm{E}-04$ & $2 \mathrm{E}-03$ & $4 \mathrm{E}-04$ & $9 \mathrm{E}-08$ & $1 \mathrm{E}-05$ & $8 \mathrm{E}-08$ & $2 \mathrm{E}-06$ & 7E-06 & $2 \mathrm{E}-05$ \\
\hline 167 & $2 \mathrm{E}-09$ & $7 \mathrm{E}-07$ & $4 \mathrm{E}-06$ & $2 \mathrm{E}-05$ & $5 \mathrm{E}-05$ & $8 \mathrm{E}-06$ & $7 \mathrm{E}-06$ & $3 \mathrm{E}-05$ & $6 \mathrm{E}-05$ & $2 \mathrm{E}-04$ & $2 \mathrm{E}-03$ & 3E-04 & $8 \mathrm{E}-08$ & $9 \mathrm{E}-06$ & $7 \mathrm{E}-08$ & $2 \mathrm{E}-06$ & \begin{tabular}{l|l}
$6 \mathrm{E}-06$ \\
\end{tabular} & $1 \mathrm{E}-05$ \\
\hline 215 & $1 \mathrm{E}-09$ & $4 \mathrm{E}-07$ & $3 \mathrm{E}-06$ & $1 \mathrm{E}-05$ & $3 \mathrm{E}-05$ & $4 \mathrm{E}-06$ & $4 \mathrm{E}-06$ & $2 \mathrm{E}-05$ & $3 \mathrm{E}-05$ & $1 \mathrm{E}-04$ & $9 \mathrm{E}-04$ & $2 \mathrm{E}-04$ & $5 \mathrm{E}-08$ & $5 \mathrm{E}-06$ & $4 \mathrm{E}-08$ & $1 \mathrm{E}-06$ & $3 \mathrm{E}-06$ & $9 \mathrm{E}-06$ \\
\hline 278 & $8 \mathrm{E}-10$ & $3 \mathrm{E}-07$ & $2 \mathrm{E}-06$ & 7E-06 & $2 \mathrm{E}-05$ & 3E-06 & $3 \mathrm{E}-06$ & $1 \mathrm{E}-05$ & $2 \mathrm{E}-05$ & 7E-05 & $6 \mathrm{E}-04$ & $1 \mathrm{E}-04$ & $3 \mathrm{E}-08$ & $3 \mathrm{E}-06$ & $3 \mathrm{E}-08$ & 7E-07 & $2 \mathrm{E}-06$ & $6 \mathrm{E}-06$ \\
\hline 359 & $5 \mathrm{E}-10$ & $2 \mathrm{E}-07$ & $1 \mathrm{E}-06$ & $5 \mathrm{E}-06$ & $1 \mathrm{E}-05$ & 2E-06 & $2 \mathrm{E}-06$ & $8 \mathrm{E}-06$ & $1 \mathrm{E}-05$ & $4 \mathrm{E}-05$ & 4E-04 & $8 \mathrm{E}-05$ & $2 \mathrm{E}-08$ & $2 \mathrm{E}-06$ & $2 \mathrm{E}-08$ & $5 \mathrm{E}-07$ & \begin{tabular}{|l|}
$\mathrm{E}-06$ \\
\end{tabular} & $4 \mathrm{E}-06$ \\
\hline 464 & $8 \mathrm{E}-10$ & 3E-07 & $2 \mathrm{E}-06$ & 7E-06 & 2E-05 & 3E-06 & $3 \mathrm{E}-06$ & $1 \mathrm{E}-05$ & $2 \mathrm{E}-05$ & 7E-05 & 6E-04 & $1 \mathrm{E}-04$ & 3E-08 & $3 \mathrm{E}-06$ & $3 \mathrm{E}-08$ & $7 \mathrm{E}-07$ & $2 \mathrm{E}-06$ & 6E-06 \\
\hline 599 & $2 \mathrm{E}-11$ & 7E-09 & $4 \mathrm{E}-08$ & $2 \mathrm{E}-07$ & 5E-07 & $8 \mathrm{E}-08$ & 7E-08 & 3E-07 & \begin{tabular}{|c|}
$6 \mathrm{E}-07$ \\
\end{tabular} & $2 \mathrm{E}-06$ & 2E-05 & $3 \mathrm{E}-06$ & $8 \mathrm{E}-10$ & 9E-08 & $7 \mathrm{E}-10$ & $2 \mathrm{E}-08$ & \begin{tabular}{ll|}
$6 \mathrm{E}-08$ \\
\end{tabular} & $1 \mathrm{E}-07$ \\
\hline 774 & $3 \mathrm{E}-10$ & 9E-08 & 5E-07 & $2 \mathrm{E}-06$ & $7 \mathrm{E}-06$ & 9E-07 & 9E-07 & 4E-06 & 7E-06 & $2 \mathrm{E}-05$ & $2 \mathrm{E}-04$ & 4E-05 & $1 \mathrm{E}-08$ & 1E-06 & 9E-09 & 2E-07 & \begin{tabular}{|c|}
$7 \mathrm{E}-07$ \\
\end{tabular} & $2 \mathrm{E}-06$ \\
\hline 1000 & 1E-09 & \begin{tabular}{|c|}
$3 \mathrm{E}-07$ \\
\end{tabular} & $2 \mathrm{E}-06$ & 9E-06 & 3E-05 & 4E-06 & 3E-06 & 1E-05 & 3E-05 & $8 \mathrm{E}-05$ & 8E-04 & $2 \mathrm{E}-04$ & 4E-08 & 4E-06 & $3 \mathrm{E}-08$ & \begin{tabular}{|c|}
$9 \mathrm{E}-07$ \\
\end{tabular} & 3E-06 & $\overline{7 \mathrm{E}-06}$ \\
\hline
\end{tabular}


Table 3.40. (contd)

\begin{tabular}{|c|c|c|c|c|c|c|c|c|c|c|c|c|c|c|c|}
\hline \begin{tabular}{|l} 
Density \\
$(\mathrm{g} / \mathrm{mL})$
\end{tabular} & 3.5 & 5617 & 242 & $2365]$ & 4.5 & 7.1 & 4.26 & 1143 & 3.5 & 35 & 568 & 234 & $225]$ & 1464 & 265 \\
\hline$(\mathrm{g} / \mathrm{mL})$ & (3.5) & 3.017 & 2.42 & 2.005 & 4.13 & 1.1 & 4.20 & 11.45 & 3.0 & 3.5 & 5.68 & 2.34 & 2.25 & 1.464 & 2.65 \\
\hline Volume & & & & & & & & & & & & & & & \\
\hline Fraction & $1.2 \mathrm{E}-03$ & $8.0 \mathrm{E}-03$ & $5.4 \mathrm{E}-03$ & $5.3 \mathrm{E}-02$ & $2.1 \mathrm{E}-03$ & $6.7 \mathrm{E}-04$ & $2.4 \mathrm{E}-05$ & $9.9 \mathrm{E}-07$ & $5.9 \mathrm{E}-05$ & $1.2 \mathrm{E}-03$ & \begin{tabular}{|l|l|}
$1.6 \mathrm{E}-02$ \\
\end{tabular} & $2.6 \mathrm{E}-02$ & $5.4 \mathrm{E}-02$ & $2.4 \mathrm{E}-02$ & $1.3 \mathrm{E}-02$ \\
\hline$d_{S}(\mu \mathrm{m})$ & $\mathrm{Na} 2(\mathrm{UO} 2) 2(\mathrm{PO} 4) 2.2 \mathrm{H} 2 \mathrm{O}$ & $\mathrm{Na} 2 \mathrm{U} 2 \mathrm{O} 7$ & NaAlCO3(OH)2 & NaAlSiO4 & $\mathrm{Ni}(\mathrm{OH}) 2$ & $\mathrm{~Pb}(\mathrm{OH}) 2$ & $\mathrm{Pu}(\mathrm{OH}) 4$ (co-precip. on Fe phase) & PuO2 & Sr3(PO4)2 & SrCO3 & ZrO2 & $\mathrm{Na} 2 \mathrm{C} 2 \mathrm{O} 4$ & \begin{tabular}{|l|l}
$\mathrm{Na} 2 \mathrm{CO} 3 . \mathrm{H} 2 \mathrm{O}$ & $\mathrm{I}$ \\
\end{tabular} & $\mathrm{Na} 2 \mathrm{SO} 4.10 \mathrm{H} 2 \mathrm{O}$ & Na3FSO4 \\
\hline 0.22 & $4 \mathrm{E}-07$ & $3 \mathrm{E}-06$ & $2 \mathrm{E}-06$ & $2 \mathrm{E}-05$ & $8 \mathrm{E}-07$ & $2 \mathrm{E}-07$ & $9 \mathrm{E}-09$ & $4 \mathrm{E}-10$ & $2 \mathrm{E}-08$ & $4 \mathrm{E}-07$ & $6 \mathrm{E}-06$ & $9 \mathrm{E}-06$ & $2 \mathrm{E}-05$ & $9 \mathrm{E}-06$ & $5 \mathrm{E}-06$ \\
\hline 0.28 & $7 \mathrm{E}-07$ & $4 \mathrm{E}-06$ & $3 \mathrm{E}-06$ & $3 \mathrm{E}-05$ & $1 \mathrm{E}-06$ & $4 \mathrm{E}-07$ & $1 \mathrm{E}-08$ & $5 \mathrm{E}-10$ & $3 \mathrm{E}-08$ & $6 \mathrm{E}-07$ & $9 \mathrm{E}-06$ & $1 \mathrm{E}-05$ & $3 \mathrm{E}-05$ & $1 \mathrm{E}-05$ & $7 \mathrm{E}-06$ \\
\hline 0.36 & $5 \mathrm{E}-06$ & $3 \mathrm{E}-05$ & $2 \mathrm{E}-05$ & $2 \mathrm{E}-04$ & $8 \mathrm{E}-06$ & $2 \mathrm{E}-06$ & $9 \mathrm{E}-08$ & $4 \mathrm{E}-09$ & $2 \mathrm{E}-07$ & $4 \mathrm{E}-06$ & $6 \mathrm{E}-05$ & $1 \mathrm{E}-04$ & $2 \mathrm{E}-04$ & $9 \mathrm{E}-05$ & $5 \mathrm{E}-05$ \\
\hline 0.46 & $6 \mathrm{E}-06$ & $4 \mathrm{E}-05$ & $3 \mathrm{E}-05$ & $3 \mathrm{E}-04$ & $1 \mathrm{E}-05$ & $3 \mathrm{E}-06$ & $1 \mathrm{E}-07$ & $5 \mathrm{E}-09$ & $3 \mathrm{E}-07$ & 6E-06 & \begin{tabular}{|l|l} 
\\
\end{tabular} & $1 \mathrm{E}-04$ & 3E-04 & $1 \mathrm{E}-04$ & 7E-05 \\
\hline 0.60 & 6E-06 & $4 \mathrm{E}-05$ & 3E-05 & 2E-04 & $1 \mathrm{E}-05$ & 3E-06 & $1 \mathrm{E}-07$ & $5 \mathrm{E}-09$ & 3E-07 & 5E-06 & \begin{tabular}{|l|l|} 
\\
\end{tabular} & $1 \mathrm{E}-04$ & 3E-04 & $1 \mathrm{E}-04$ & $6 \mathrm{E}-05$ \\
\hline 0.77 & 3E-05 & 2E-04 & $1 \mathrm{E}-04$ & $1 \mathrm{E}-03$ & 5E-05 & $2 \mathrm{E}-05$ & $6 \mathrm{E}-07$ & $2 \mathrm{E}-08$ & $1 \mathrm{E}-06$ & 3E-05 & $4 \mathrm{E}-04$ & 6E-04 & $1 \mathrm{E}-03$ & 6E-04 & 3E-04 \\
\hline 1 & 2E-05 & $1 \mathrm{E}-04$ & 7E-05 & 7E-04 & 3E-05 & 9E-06 & $3 \mathrm{E}-07$ & $1 \mathrm{E}-08$ & $8 \mathrm{E}-07$ & $2 \mathrm{E}-05$ & 2E-04 & 3E-04 & 7E-04 & 3E-04 & $2 \mathrm{E}-04$ \\
\hline 1.3 & $5 \mathrm{E}-05$ & 3E-04 & $2 \mathrm{E}-04$ & $2 \mathrm{E}-03$ & 9E-05 & $3 \mathrm{E}-05$ & $1 \mathrm{E}-06$ & $4 \mathrm{E}-08$ & 3E-06 & $5 \mathrm{E}-05$ & $7 \mathrm{E}-04$ & $1 \mathrm{E}-03$ & $2 \mathrm{E}-03$ & $1 \mathrm{E}-03$ & $6 \mathrm{E}-04$ \\
\hline 1.7 & 4E-05 & 3E-04 & $2 \mathrm{E}-04$ & $2 \mathrm{E}-03$ & 7E-05 & $2 \mathrm{E}-05$ & $9 \mathrm{E}-07$ & $3 \mathrm{E}-08$ & $2 \mathrm{E}-06$ & $4 \mathrm{E}-05$ & 6E-04 & 9E-04 & $2 \mathrm{E}-03$ & $9 \mathrm{E}-04$ & $5 \mathrm{E}-04$ \\
\hline 2.2 & 6E-05 & $4 \mathrm{E}-04$ & 3E-04 & 3E-03 & $1 \mathrm{E}-04$ & 3E-05 & $1 \mathrm{E}-06$ & $5 \mathrm{E}-08$ & 3E-06 & 6E-05 & 8E-04 & $1 \mathrm{E}-03$ & 3E-03 & $1 \mathrm{E}-03$ & 7E-04 \\
\hline 2.8 & 7E-05 & 5E-04 & 3E-04 & 3E-03 & $1 \mathrm{E}-04$ & 4E-05 & $1 \mathrm{E}-06$ & $6 \mathrm{E}-08$ & 3E-06 & 7E-05 & 9E-04 & $1 \mathrm{E}-03$ & 3E-03 & $1 \mathrm{E}-03$ & 7E-04 \\
\hline 3.6 & 7E-05 & 5E-04 & 3E-04 & 3E-03 & $1 \mathrm{E}-04$ & 4E-05 & $1 \mathrm{E}-06$ & $6 \mathrm{E}-08$ & 4E-06 & 7E-05 & $1 \mathrm{E}-03$ & $2 \mathrm{E}-03$ & 3E-03 & $1 \mathrm{E}-03$ & $8 \mathrm{E}-04$ \\
\hline 4.6 & 9E-05 & 6E-04 & 4E-04 & $4 \mathrm{E}-03$ & $1 \mathrm{E}-04$ & 5E-05 & $2 \mathrm{E}-06$ & $7 \mathrm{E}-08$ & $4 \mathrm{E}-06$ & $8 \mathrm{E}-05$ & $1 \mathrm{E}-03$ & $2 \mathrm{E}-03$ & $4 \mathrm{E}-03$ & $2 \mathrm{E}-03$ & $9 \mathrm{E}-04$ \\
\hline 6.0 & $1 \mathrm{E}-04$ & 6E-04 & 4E-04 & $4 \mathrm{E}-03$ & $2 \mathrm{E}-04$ & 5E-05 & $2 \mathrm{E}-06$ & $8 \mathrm{E}-08$ & 5E-06 & 9E-05 & $1 \mathrm{E}-03$ & $2 \mathrm{E}-03$ & \begin{tabular}{|l|}
$\mathrm{E}-03$ \\
\end{tabular} & $2 \mathrm{E}-03$ & $1 \mathrm{E}-03$ \\
\hline 7.7 & $1 \mathrm{E}-04$ & 7E-04 & 5E-04 & 5E-03 & 2E-04 & 6E-05 & $2 \mathrm{E}-06$ & 9E- 08 & 6E-06 & 1E-04 & 2E-03 & $2 \mathrm{E}-03$ & \begin{tabular}{|l|}
$5 \mathrm{E}-03$ \\
\end{tabular} & $2 \mathrm{E}-03$ & $1 \mathrm{E}-03$ \\
\hline 10 & $1 \mathrm{E}-04$ & 7E-04 & 4E-04 & $4 \mathrm{E}-03$ & $2 \mathrm{E}-04$ & 6E-05 & $2 \mathrm{E}-06$ & $8 \mathrm{E}-08$ & 5E-06 & $1 \mathrm{E}-04$ & $1 \mathrm{E}-03$ & $2 \mathrm{E}-03$ & 4E-03 & $2 \mathrm{E}-03$ & $1 \mathrm{E}-03$ \\
\hline 13 & $1 \mathrm{E}-04$ & 7E-04 & 5E-04 & 5E-03 & 2E-04 & 6E-05 & $2 \mathrm{E}-06$ & $9 \mathrm{E}-08$ & 5E-06 & $1 \mathrm{E}-04$ & $1 \mathrm{E}-03$ & $2 \mathrm{E}-03$ & 5E-03 & 2E-03 & $1 \mathrm{E}-03$ \\
\hline 17 & 9E-05 & 6E-04 & 4E-04 & 4E-03 & 2E-04 & 5E-05 & $2 \mathrm{E}-06$ & 7E-08 & 4E-06 & 9E-05 & $1 \mathrm{E}-03$ & $2 \mathrm{E}-03$ & 4E-03 & 2E-03 & $1 \mathrm{E}-03$ \\
\hline 22 & 7E-05 & 5E-04 & 3E-04 & 3E-03 & $1 \mathrm{E}-04$ & 4E-05 & $1 \mathrm{E}-06$ & $6 \mathrm{E}-08$ & $4 \mathrm{E}-06$ & 7E-05 & $1 \mathrm{E}-03$ & $2 \mathrm{E}-03$ & 3E-03 & $1 \mathrm{E}-03$ & $8 \mathrm{E}-04$ \\
\hline 28 & 5E-05 & 3E-04 & 2E-04 & $2 \mathrm{E}-03$ & $8 \mathrm{E}-05$ & $3 \mathrm{E}-05$ & $9 \mathrm{E}-07$ & $4 \mathrm{E}-08$ & $2 \mathrm{E}-06$ & $5 \mathrm{E}-05$ & $6 \mathrm{E}-04$ & $1 \mathrm{E}-03$ & $2 \mathrm{E}-03$ & $9 \mathrm{E}-04$ & $5 \mathrm{E}-04$ \\
\hline 36 & 4E-05 & $2 \mathrm{E}-04$ & $2 \mathrm{E}-04$ & $1 \mathrm{E}-03$ & $6 \mathrm{E}-05$ & $2 \mathrm{E}-05$ & $7 \mathrm{E}-07$ & $3 \mathrm{E}-08$ & $2 \mathrm{E}-06$ & 3E-05 & $5 \mathrm{E}-04$ & 7E-04 & $2 \mathrm{E}-03$ & 7E-04 & $4 \mathrm{E}-04$ \\
\hline 46 & 2E-05 & $1 \mathrm{E}-04$ & 1E-04 & $1 \mathrm{E}-03$ & $4 \mathrm{E}-05$ & $1 \mathrm{E}-05$ & $4 \mathrm{E}-07$ & $2 \mathrm{E}-08$ & $1 \mathrm{E}-06$ & $2 \mathrm{E}-05$ & 3E-04 & 5E-04 & 1E-03 & 4E-04 & $2 \mathrm{E}-04$ \\
\hline 60 & $1 \mathrm{E}-05$ & 9E-05 & 6E-05 & 6E-04 & 2E-05 & $8 \mathrm{E}-06$ & 3E-07 & $1 \mathrm{E}-08$ & 7E-07 & $1 \mathrm{E}-05$ & 2E-04 & 3E-04 & 6E-04 & 3E-04 & $2 \mathrm{E}-04$ \\
\hline 77 & $1 \mathrm{E}-05$ & $9 \mathrm{E}-05$ & 6E-05 & 6E-04 & $2 \mathrm{E}-05$ & $8 \mathrm{E}-06$ & 3E-07 & $1 \mathrm{E}-08$ & 7E-07 & $1 \mathrm{E}-05$ & 2E-04 & 3E-04 & 6E-04 & 3E-04 & 2E-04 \\
\hline 100 & 2E-05 & $1 \mathrm{E}-04$ & 7E-05 & 7E-04 & 3E-05 & 9E-06 & 3E-07 & $1 \mathrm{E}-08$ & $8 \mathrm{E}-07$ & $2 \mathrm{E}-05$ & $2 \mathrm{E}-04$ & 3E-04 & 7E-04 & 3E-04 & $2 \mathrm{E}-04$ \\
\hline 129 & $1 \mathrm{E}-05$ & $7 \mathrm{E}-05$ & 5E-05 & 5E-04 & $2 \mathrm{E}-05$ & 6E-06 & $2 \mathrm{E}-07$ & $9 \mathrm{E}-09$ & 5E-07 & $1 \mathrm{E}-05$ & $1 \mathrm{E}-04$ & 2E-04 & 5E-04 & $2 \mathrm{E}-04$ & $1 \mathrm{E}-04$ \\
\hline 167 & 1E-05 & 6E-05 & $4 \mathrm{E}-05$ & $4 \mathrm{E}-04$ & $2 \mathrm{E}-05$ & 5E-06 & $2 \mathrm{E}-07$ & $8 \mathrm{E}-09$ & $5 \mathrm{E}-07$ & $9 \mathrm{E}-06$ & $1 \mathrm{E}-04$ & $2 \mathrm{E}-04$ & $4 \mathrm{E}-04$ & $2 \mathrm{E}-04$ & $1 \mathrm{E}-04$ \\
\hline 215 & 6E-06 & 4E-05 & 2E-05 & $2 \mathrm{E}-04$ & $9 \mathrm{E}-06$ & $3 \mathrm{E}-06$ & $1 \mathrm{E}-07$ & $4 \mathrm{E}-09$ & 3E-07 & 5E-06 & 7E-05 & $1 \mathrm{E}-04$ & 2E-04 & $1 \mathrm{E}-04$ & $6 \mathrm{E}-05$ \\
\hline 278 & $4 \mathrm{E}-06$ & 2E-05 & 2E-05 & 2E-04 & 6E-06 & 2E-06 & $7 \mathrm{E}-08$ & 3E-09 & 2E-07 & 3E-06 & 5E-05 & 7E-05 & 2E-04 & 7E-05 & 4E-05 \\
\hline 359 & $2 \mathrm{E}-06$ & 2E-05 & $1 \mathrm{E}-05$ & $1 \mathrm{E}-04$ & 4E-06 & $1 \mathrm{E}-06$ & $5 \mathrm{E}-08$ & 2E-09 & $1 \mathrm{E}-07$ & 2E-06 & 3E-05 & 5E-05 & $1 \mathrm{E}-04$ & $5 \mathrm{E}-05$ & $2 \mathrm{E}-05$ \\
\hline 464 & $4 \mathrm{E}-06$ & 2E-05 & 2E-05 & $2 \mathrm{E}-04$ & $6 \mathrm{E}-06$ & $2 \mathrm{E}-06$ & $7 \mathrm{E}-08$ & 3E-09 & $2 \mathrm{E}-07$ & $3 \mathrm{E}-06$ & 5E-05 & 7E-05 & 2E-04 & 7E-05 & $4 \mathrm{E}-05$ \\
\hline 599 & $1 \mathrm{E}-07$ & 6E-07 & $4 \mathrm{E}-07$ & $4 \mathrm{E}-06$ & $2 \mathrm{E}-07$ & 5E-08 & $2 \mathrm{E}-09$ & $8 \mathrm{E}-11$ & 5E-09 & 9E-08 & $1 \mathrm{E}-06$ & $2 \mathrm{E}-06$ & \begin{tabular}{|l|}
$4 \mathrm{E}-06$ \\
\end{tabular} & $2 \mathrm{E}-06$ & $1 \mathrm{E}-06$ \\
\hline 774 & $1 \mathrm{E}-06$ & 7E-06 & 5E-06 & 5E-05 & $2 \mathrm{E}-06$ & 6E-07 & $2 \mathrm{E}-08$ & $9 \mathrm{E}-10$ & $6 \mathrm{E}-08$ & $1 \mathrm{E}-06$ & $2 \mathrm{E}-05$ & $2 \mathrm{E}-05$ & 5E-05 & $2 \mathrm{E}-05$ & $1 \mathrm{E}-05$ \\
\hline 1000 & 5E-06 & 3E-05 & 2E-05 & 2E-04 & $8 \mathrm{E}-06$ & 2E-06 & $9 \mathrm{E}-08$ & 4E-09 & 2E-07 & $4 \mathrm{E}-06$ & 6E-05 & 9E-05 & $2 \mathrm{E}-04$ & $9 \mathrm{E}-05$ & 5E-05 \\
\hline
\end{tabular}


Table 3.40 (contd)

\begin{tabular}{|c|c|c|c|c|c|c|c|c|}
\hline $\begin{array}{l}\text { Density } \\
(\mathrm{g} / \mathrm{mL})\end{array}$ & 2.3 & 1.62 & 1.83 & 2.64 & 2.78 & 1.75 & 2.168 & 2.26 \\
\hline Volume & & & & & & & & \\
\hline Fraction & $2.2 \mathrm{E}-05$ & $3.4 \mathrm{E}-02$ & $1.2 \mathrm{E}-02$ & $5.2 \mathrm{E}-03$ & $4.0 \mathrm{E}-02$ & $1.1 \mathrm{E}-01$ & $4.8 \mathrm{E}-04$ & $2.9 \mathrm{E}-01$ \\
\hline$d_{\mathrm{s}}(\mu \mathrm{m})$ & Na3NO3SO4.H2O & Na3PO4.0.25NaOH.12H2O & Na4P2O7.10H2O & \begin{tabular}{|l|}
$\mathrm{Na} 6(\mathrm{SO} 4) 2 \mathrm{CO} 3$ \\
\end{tabular} & $\mathrm{NaF}$ & Na7F(PO4)2.19H2O & NaNO2 & NaNO3 \\
\hline 0.22 & $8 \mathrm{E}-09$ & $1 \mathrm{E}-05$ & 4E-06 & $2 \mathrm{E}-06$ & $1 \mathrm{E}-05$ & $4 \mathrm{E}-05$ & $2 \mathrm{E}-07$ & $1 \mathrm{E}-04$ \\
\hline 0.28 & $1 \mathrm{E}-08$ & $2 \mathrm{E}-05$ & $7 \mathrm{E}-06$ & $3 \mathrm{E}-06$ & $2 \mathrm{E}-05$ & $6 \mathrm{E}-05$ & $3 \mathrm{E}-07$ & $2 \mathrm{E}-04$ \\
\hline 0.36 & $8 \mathrm{E}-08$ & $1 \mathrm{E}-04$ & $5 \mathrm{E}-05$ & $2 \mathrm{E}-05$ & $1 \mathrm{E}-04$ & $4 \mathrm{E}-04$ & $2 \mathrm{E}-06$ & $1 \mathrm{E}-03$ \\
\hline 0.46 & $1 \mathrm{E}-07$ & $2 \mathrm{E}-04$ & $6 \mathrm{E}-05$ & $3 \mathrm{E}-05$ & $2 \mathrm{E}-04$ & $6 \mathrm{E}-04$ & $2 \mathrm{E}-06$ & $2 \mathrm{E}-03$ \\
\hline 0.60 & $1 \mathrm{E}-07$ & 2E-04 & $6 \mathrm{E}-05$ & $2 \mathrm{E}-05$ & 2E-04 & $5 \mathrm{E}-04$ & $2 \mathrm{E}-06$ & $1 \mathrm{E}-03$ \\
\hline 0.77 & $5 \mathrm{E}-07$ & $8 \mathrm{E}-04$ & $3 \mathrm{E}-04$ & $1 \mathrm{E}-04$ & $1 \mathrm{E}-03$ & $3 \mathrm{E}-03$ & $1 \mathrm{E}-05$ & $7 \mathrm{E}-03$ \\
\hline 1 & $3 \mathrm{E}-07$ & 5E-04 & $2 \mathrm{E}-04$ & $7 \mathrm{E}-05$ & 5E-04 & $1 \mathrm{E}-03$ & $6 \mathrm{E}-06$ & $4 \mathrm{E}-03$ \\
\hline 1.3 & $1 \mathrm{E}-06$ & $1 \mathrm{E}-03$ & 5E-04 & $2 \mathrm{E}-04$ & $2 \mathrm{E}-03$ & $5 \mathrm{E}-03$ & $2 \mathrm{E}-05$ & $1 \mathrm{E}-02$ \\
\hline 1.7 & $8 \mathrm{E}-07$ & $1 \mathrm{E}-03$ & $4 \mathrm{E}-04$ & $2 \mathrm{E}-04$ & $1 \mathrm{E}-03$ & $4 \mathrm{E}-03$ & $2 \mathrm{E}-05$ & $1 \mathrm{E}-02$ \\
\hline 2.2 & 1E-06 & 2E-03 & 6E-04 & $3 \mathrm{E}-04$ & 2E-03 & 6E-03 & 2E-05 & $2 \mathrm{E}-02$ \\
\hline 2.8 & $1 \mathrm{E}-06$ & $2 \mathrm{E}-03$ & 7E-04 & $3 \mathrm{E}-04$ & $2 \mathrm{E}-03$ & 6E-03 & 3E-05 & $2 \mathrm{E}-02$ \\
\hline 3.6 & $1 \mathrm{E}-06$ & 2E-03 & 7E-04 & $3 \mathrm{E}-04$ & 2E-03 & $7 \mathrm{E}-03$ & 3E-05 & $2 \mathrm{E}-02$ \\
\hline 4.6 & $2 \mathrm{E}-06$ & $2 \mathrm{E}-03$ & 9E-04 & $4 \mathrm{E}-04$ & 3E-03 & \begin{tabular}{c|}
$8 \mathrm{E}-03$ \\
\end{tabular} & 3E-05 & $2 \mathrm{E}-02$ \\
\hline 6.0 & 2E-06 & 3E-03 & 9E-04 & $4 \mathrm{E}-04$ & 3E-03 & 9E-03 & $4 \mathrm{E}-05$ & $2 \mathrm{E}-02$ \\
\hline 7.7 & 2E-06 & 3E-03 & 1E-03 & 5E-04 & 4E-03 & $1 \mathrm{E}-02$ & 4E- 05 & $3 \mathrm{E}-02$ \\
\hline 10 & 2E-06 & 3E-03 & 1E-03 & $4 \mathrm{E}-04$ & 3E-03 & $9 \mathrm{E}-03$ & 4E- 05 & $2 \mathrm{E}-02$ \\
\hline 13 & 2E-06 & 3E-03 & $1 \mathrm{E}-03$ & 5E-04 & 4E- 03 & $1 \mathrm{E}-02$ & 4E- 05 & $3 \mathrm{E}-02$ \\
\hline 17 & 2E-06 & 3E-03 & 9E-04 & $4 \mathrm{E}-04$ & 3E-03 & $8 \mathrm{E}-03$ & 4E- 05 & $2 \mathrm{E}-02$ \\
\hline 22 & $1 \mathrm{E}-06$ & $2 \mathrm{E}-03$ & 7E-04 & $3 \mathrm{E}-04$ & $2 \mathrm{E}-03$ & $7 \mathrm{E}-03$ & 3E-05 & $2 \mathrm{E}-02$ \\
\hline 28 & 9E-07 & $1 \mathrm{E}-03$ & 5E-04 & $2 \mathrm{E}-04$ & $2 \mathrm{E}-03$ & $4 \mathrm{E}-03$ & $2 \mathrm{E}-05$ & $1 \mathrm{E}-02$ \\
\hline 36 & 6E-07 & 1E-03 & 3E-04 & $1 \mathrm{E}-04$ & 1E-03 & $3 \mathrm{E}-03$ & 1E-05 & $8 \mathrm{E}-03$ \\
\hline 46 & 4E-07 & 6E-04 & 2E-04 & $9 \mathrm{E}-05$ & 7E-04 & $2 \mathrm{E}-03$ & 9E-06 & $5 \mathrm{E}-03$ \\
\hline 60 & $3 \mathrm{E}-07$ & 4E-04 & $1 \mathrm{E}-04$ & $6 \mathrm{E}-05$ & 5E-04 & $1 \mathrm{E}-03$ & 6E-06 & $3 \mathrm{E}-03$ \\
\hline 77 & 3E-07 & 4E-04 & $1 \mathrm{E}-04$ & 6E-05 & 5E-04 & $1 \mathrm{E}-03$ & 6E-06 & $3 \mathrm{E}-03$ \\
\hline 100 & 3E-07 & $4 \mathrm{E}-04$ & $2 \mathrm{E}-04$ & 7E-05 & 5E-04 & $1 \mathrm{E}-03$ & 6E-06 & $4 \mathrm{E}-03$ \\
\hline 129 & 2E-07 & 3E-04 & $1 \mathrm{E}-04$ & 5E-05 & 4E-04 & $1 \mathrm{E}-03$ & 4E-06 & $3 \mathrm{E}-03$ \\
\hline 167 & 2E-07 & 3E-04 & 1E-04 & 4E-05 & 3E-04 & $9 \mathrm{E}-04$ & 4E-06 & $2 \mathrm{E}-03$ \\
\hline 215 & $1 \mathrm{E}-07$ & $2 \mathrm{E}-04$ & 6E-05 & 2E-05 & $2 \mathrm{E}-04$ & 5E-04 & $2 \mathrm{E}-06$ & $1 \mathrm{E}-03$ \\
\hline 278 & 6E-08 & 1E-04 & 4E-05 & 2E-05 & 1E-04 & $3 \mathrm{E}-04$ & 1E-06 & $9 \mathrm{E}-04$ \\
\hline 359 & $4 \mathrm{E}-08$ & $7 \mathrm{E}-05$ & $2 \mathrm{E}-05$ & $1 \mathrm{E}-05$ & 8E-05 & $2 \mathrm{E}-04$ & 9E-07 & $6 \mathrm{E}-04$ \\
\hline 464 & 6E-08 & $1 \mathrm{E}-04$ & $4 \mathrm{E}-05$ & 2E-05 & $1 \mathrm{E}-04$ & $3 \mathrm{E}-04$ & 1E-06 & $9 \mathrm{E}-04$ \\
\hline 599 & $2 \mathrm{E}-09$ & 3E-06 & 1E-06 & 4E-07 & 3E-06 & $9 \mathrm{E}-06$ & 4E-08 & 2E-05 \\
\hline 774 & 2E-08 & 3E-05 & $1 \mathrm{E}-05$ & 5E-06 & 4E- 05 & $1 \mathrm{E}-04$ & 4E-07 & $3 \mathrm{E}-04$ \\
\hline 1000 & $8 \mathrm{E}-08$ & 1E-04 & 4E-05 & 2E-05 & 1E-04 & $4 \mathrm{E}-04$ & 2E-06 & $1 \mathrm{E}-03$ \\
\hline
\end{tabular}


Table 3.41. Sludge, Flowing Unsonicated PSDD Volume Fraction, Fractal Dimension 2.25

\begin{tabular}{|c|c|c|c|c|c|c|c|c|c|c|c|c|c|c|c|c|c|c|}
\hline \begin{tabular}{|l|} 
Volume \\
Fraction
\end{tabular} & $2.5 \mathrm{E}-05$ & 8.8E-05 & $2.3 \mathrm{E}-04$ & $1.1 \mathrm{E}-02$ & $1.7 \mathrm{E}-03$ & $1.8 \mathrm{E}-04$ & $2.1 \mathrm{E}-04$ & 6.7E-03 & $9.6 \mathrm{E}-02$ & $3.7 \mathrm{E}-01$ & $3.3 \mathrm{E}-02$ & $3.4 \mathrm{E}-01$ & 3.7E- 05 & $2.9 \mathrm{E}-04$ & $6.0 \mathrm{E}-05$ & $1.1 \mathrm{E}-04$ & $2.2 \mathrm{E}-04$ & 4.3E- 04 \\
\hline$d_{S}(\mu \mathrm{m})$ & $\mathrm{Ag}$ & Ag2O & $\mathrm{Bi} 2 \mathrm{O} 3$ & $\mathrm{BiFeO} 3$ & $\mathrm{Ca} 5 \mathrm{OH}(\mathrm{PO} 4) 3$ & $\mathrm{CaCO} 3$ & CaF2 & $\mathrm{CrOOH}$ & $\mathrm{FePO} 4.2 \mathrm{H} 2 \mathrm{O}$ & $\mathrm{FeOOH}$ & Gibbsite & Boehmite & $\mathrm{HgO}$ & KAlSiO4 & $\mathrm{La}(\mathrm{OH}) 3$ & $\mathrm{LaPO} 4 \cdot 2 \mathrm{H} 2 \mathrm{O}$ & Mn3(PO4)2 & $\mathrm{MnO} 2$ \\
\hline 0.22 & 9E-09 & $3 \mathrm{E}-08$ & $8 \mathrm{E}-08$ & 4E-06 & 6E-07 & 6E-08 & 7E-08 & $2 \mathrm{E}-06$ & $3 \mathrm{E}-05$ & 1E-04 & $1 \mathrm{E}-05$ & 1E-04 & $1 \mathrm{E}-08$ & 1E-07 & $2 \mathrm{E}-08$ & $4 \mathrm{E}-08$ & $8 \mathrm{E}-08$ & 2E-07 \\
\hline 0.28 & $1 \mathrm{E}-08$ & 5E-08 & 1E-07 & 6E-06 & $9 \mathrm{E}-07$ & 1E-07 & $1 \mathrm{E}-07$ & 4E-06 & $5 \mathrm{E}-05$ & 2E-04 & $2 \mathrm{E}-05$ & 2E-04 & $2 \mathrm{E}-08$ & 2E-07 & 3E-08 & $6 \mathrm{E}-08$ & 1E-07 & $2 \mathrm{E}-07$ \\
\hline 0.36 & $9 \mathrm{E}-08$ & $3 \mathrm{E}-07$ & $8 \mathrm{E}-07$ & $4 \mathrm{E}-05$ & $6 \mathrm{E}-06$ & $7 \mathrm{E}-07$ & $8 \mathrm{E}-07$ & $2 \mathrm{E}-05$ & $4 \mathrm{E}-04$ & $1 \mathrm{E}-03$ & $1 \mathrm{E}-04$ & $1 \mathrm{E}-03$ & $1 \mathrm{E}-07$ & $1 \mathrm{E}-06$ & $2 \mathrm{E}-07$ & $4 \mathrm{E}-07$ & $8 \mathrm{E}-07$ & $2 \mathrm{E}-06$ \\
\hline 0.46 & $1 \mathrm{E}-07$ & $5 \mathrm{E}-07$ & $1 \mathrm{E}-06$ & $6 \mathrm{E}-05$ & 9E-06 & 9E-07 & $1 \mathrm{E}-06$ & $3 \mathrm{E}-05$ & $5 \mathrm{E}-04$ & $2 \mathrm{E}-03$ & $2 \mathrm{E}-04$ & $2 \mathrm{E}-03$ & $2 \mathrm{E}-07$ & $2 \mathrm{E}-06$ & $3 \mathrm{E}-07$ & $6 \mathrm{E}-07$ & $1 \mathrm{E}-06$ & $2 \mathrm{E}-06$ \\
\hline 0.60 & $1 \mathrm{E}-07$ & 4E-07 & 1E-06 & 5E-05 & $8 \mathrm{E}-06$ & $8 \mathrm{E}-07$ & 1E-06 & $3 \mathrm{E}-05$ & $4 \mathrm{E}-04$ & $2 \mathrm{E}-03$ & 2E-04 & 2E-03 & $2 \mathrm{E}-07$ & 1E-06 & $3 \mathrm{E}-07$ & 5E-07 & 1E-06 & $2 \mathrm{E}-06$ \\
\hline 0.77 & 6E-07 & $2 \mathrm{E}-06$ & 5E-06 & 3E-04 & $4 \mathrm{E}-05$ & 4E-06 & 5E-06 & $2 \mathrm{E}-04$ & $2 \mathrm{E}-03$ & 9E-03 & $8 \mathrm{E}-04$ & 8E-03 & 9E-07 & 7E-06 & 1E-06 & $3 \mathrm{E}-06$ & 5E-06 & $1 \mathrm{E}-05$ \\
\hline 1 & 3E-07 & $1 \mathrm{E}-06$ & $3 \mathrm{E}-06$ & $2 \mathrm{E}-04$ & $2 \mathrm{E}-05$ & $2 \mathrm{E}-06$ & $3 \mathrm{E}-06$ & 9E-05 & $1 \mathrm{E}-03$ & 5E-03 & $4 \mathrm{E}-04$ & $5 \mathrm{E}-03$ & 5E-07 & 4E-06 & $8 \mathrm{E}-07$ & $2 \mathrm{E}-06$ & $3 \mathrm{E}-06$ & $6 \mathrm{E}-06$ \\
\hline 1.3 & $1 \mathrm{E}-06$ & $4 \mathrm{E}-06$ & $1 \mathrm{E}-05$ & $5 \mathrm{E}-04$ & $7 \mathrm{E}-05$ & $8 \mathrm{E}-06$ & $9 \mathrm{E}-06$ & $3 \mathrm{E}-04$ & $4 \mathrm{E}-03$ & $2 \mathrm{E}-02$ & $1 \mathrm{E}-03$ & 1E-02 & $2 \mathrm{E}-06$ & $1 \mathrm{E}-05$ & $3 \mathrm{E}-06$ & $5 \mathrm{E}-06$ & $9 \mathrm{E}-06$ & $2 \mathrm{E}-05$ \\
\hline 1.7 & 9E-07 & $3 \mathrm{E}-06$ & $8 \mathrm{E}-06$ & $4 \mathrm{E}-04$ & $6 \mathrm{E}-05$ & 6E-06 & $7 \mathrm{E}-06$ & $2 \mathrm{E}-04$ & $3 \mathrm{E}-03$ & $1 \mathrm{E}-02$ & $1 \mathrm{E}-03$ & $1 \mathrm{E}-02$ & $1 \mathrm{E}-06$ & $1 \mathrm{E}-05$ & $2 \mathrm{E}-06$ & 4E-06 & $8 \mathrm{E}-06$ & 2E-05 \\
\hline 2.2 & $1 \mathrm{E}-06$ & $5 \mathrm{E}-06$ & $1 \mathrm{E}-05$ & $6 \mathrm{E}-04$ & $9 \mathrm{E}-05$ & $9 \mathrm{E}-06$ & $1 \mathrm{E}-05$ & $3 \mathrm{E}-04$ & $5 \mathrm{E}-03$ & $2 \mathrm{E}-02$ & $2 \mathrm{E}-03$ & $2 \mathrm{E}-02$ & $2 \mathrm{E}-06$ & $2 \mathrm{E}-05$ & $3 \mathrm{E}-06$ & 6E-06 & $1 \mathrm{E}-05$ & 2E-05 \\
\hline 2.8 & $1 \mathrm{E}-06$ & $5 \mathrm{E}-06$ & $1 \mathrm{E}-05$ & $6 \mathrm{E}-04$ & $1 \mathrm{E}-04$ & $1 \mathrm{E}-05$ & $1 \mathrm{E}-05$ & $4 \mathrm{E}-04$ & $5 \mathrm{E}-03$ & $2 \mathrm{E}-02$ & $2 \mathrm{E}-03$ & $2 \mathrm{E}-02$ & $2 \mathrm{E}-06$ & $2 \mathrm{E}-05$ & $3 \mathrm{E}-06$ & $7 \mathrm{E}-06$ & $1 \mathrm{E}-05$ & $2 \mathrm{E}-05$ \\
\hline 3.6 & $1 \mathrm{E}-06$ & 5E-06 & $1 \mathrm{E}-05$ & $7 \mathrm{E}-04$ & $1 \mathrm{E}-04$ & $1 \mathrm{E}-05$ & $1 \mathrm{E}-05$ & 4E-04 & 6E-03 & $2 \mathrm{E}-02$ & 2E-03 & $2 \mathrm{E}-02$ & 2E-06 & 2E-05 & $4 \mathrm{E}-06$ & 7E-06 & $1 \mathrm{E}-05$ & 3E-05 \\
\hline 4.6 & $2 \mathrm{E}-06$ & 6E-06 & $2 \mathrm{E}-05$ & $8 \mathrm{E}-04$ & $1 \mathrm{E}-04$ & 1E-05 & 1E-05 & 5E-04 & $7 \mathrm{E}-03$ & $3 \mathrm{E}-02$ & $2 \mathrm{E}-03$ & 2E-02 & 3E-06 & $2 \mathrm{E}-05$ & \begin{tabular}{ll|}
$\mathrm{E}-06$ \\
\end{tabular} & $8 \mathrm{E}-06$ & $2 \mathrm{E}-05$ & $3 \mathrm{E}-05$ \\
\hline 6.0 & 2E-06 & 7E-06 & 2E-05 & 9E-04 & 1E-04 & 1E-05 & 2E-05 & 5E-04 & 7E-03 & 3E-02 & 3E-03 & $3 \mathrm{E}-02$ & 3E-06 & 2E-05 & 5E-06 & 9E-06 & $2 \mathrm{E}-05$ & 3E-05 \\
\hline 7.7 & $2 \mathrm{E}-06$ & $8 \mathrm{E}-06$ & $2 \mathrm{E}-05$ & 1E-03 & $2 \mathrm{E}-04$ & 2E-05 & $2 \mathrm{E}-05$ & $6 \mathrm{E}-04$ & 9E-03 & 3E-02 & $3 \mathrm{E}-03$ & 3E-02 & 3E-06 & 3E-05 & 6E-06 & $1 \mathrm{E}-05$ & 2E-05 & $4 \mathrm{E}-05$ \\
\hline 10 & $2 \mathrm{E}-06$ & 7E-06 & $2 \mathrm{E}-05$ & 9E-04 & $1 \mathrm{E}-04$ & 1E-05 & $2 \mathrm{E}-05$ & 5E-04 & $8 \mathrm{E}-03$ & 3E-02 & $3 \mathrm{E}-03$ & 3E-02 & $3 \mathrm{E}-06$ & $2 \mathrm{E}-05$ & 5E-06 & 9E-06 & $2 \mathrm{E}-05$ & 3E-05 \\
\hline 13 & 2E-06 & $8 \mathrm{E}-06$ & 2E-05 & $1 \mathrm{E}-03$ & $2 \mathrm{E}-04$ & 2E-05 & 2E-05 & $6 \mathrm{E}-04$ & 9E-03 & $3 \mathrm{E}-02$ & $3 \mathrm{E}-03$ & $3 \mathrm{E}-02$ & $3 \mathrm{E}-06$ & $3 \mathrm{E}-05$ & 5E-06 & $1 \mathrm{E}-05$ & $2 \mathrm{E}-05$ & $4 \mathrm{E}-05$ \\
\hline 17 & $2 \mathrm{E}-06$ & 7E-06 & 2E-05 & $8 \mathrm{E}-04$ & $1 \mathrm{E}-04$ & 1E-05 & 2E-05 & 5E-04 & $7 \mathrm{E}-03$ & 3E-02 & 2E-03 & $3 \mathrm{E}-02$ & 3E-06 & 2E-05 & 4E-06 & 9E-06 & $2 \mathrm{E}-05$ & 3E-05 \\
\hline 22 & 1E-06 & 5E-06 & 1E-05 & 7E-04 & $1 \mathrm{E}-04$ & 1E-05 & 1E-05 & 4E-04 & 6E-03 & 2E-02 & 2E-03 & $2 \mathrm{E}-02$ & 2E-06 & 2E-05 & \begin{tabular}{ll|}
$4 \mathrm{E}-06$ \\
\end{tabular} & 7E-06 & 1E-05 & $3 \mathrm{E}-05$ \\
\hline 28 & 9E-07 & 3E-06 & 9E-06 & $4 \mathrm{E}-04$ & $7 \mathrm{E}-05$ & $7 \mathrm{E}-06$ & $8 \mathrm{E}-06$ & $3 \mathrm{E}-04$ & $4 \mathrm{E}-03$ & $1 \mathrm{E}-02$ & $1 \mathrm{E}-03$ & $1 \mathrm{E}-02$ & $1 \mathrm{E}-06$ & $1 \mathrm{E}-05$ & $2 \mathrm{E}-06$ & $4 \mathrm{E}-06$ & $8 \mathrm{E}-06$ & 2E-05 \\
\hline 36 & 7E-07 & 2E-06 & $6 \mathrm{E}-06$ & 3E-04 & 5E-05 & 5E-06 & 6E-06 & $2 \mathrm{E}-04$ & 3E-03 & $1 \mathrm{E}-02$ & 9E-04 & $1 \mathrm{E}-02$ & $1 \mathrm{E}-06$ & $8 \mathrm{E}-06$ & $2 \mathrm{E}-06$ & 3E-06 & $6 \mathrm{E}-06$ & $1 \mathrm{E}-05$ \\
\hline 46 & 4E-07 & $2 \mathrm{E}-06$ & 4E-06 & $2 \mathrm{E}-04$ & $3 \mathrm{E}-05$ & 3E-06 & 4E-06 & $1 \mathrm{E}-04$ & 2E-03 & 7E-03 & 6E-04 & 6E-03 & 7E-07 & 5E-06 & 1E-06 & $2 \mathrm{E}-06$ & $4 \mathrm{E}-06$ & $8 \mathrm{E}-06$ \\
\hline 60 & 3E-07 & $1 \mathrm{E}-06$ & 3E-06 & 1E-04 & $2 \mathrm{E}-05$ & 2E-06 & $2 \mathrm{E}-06$ & $8 \mathrm{E}-05$ & $1 \mathrm{E}-03$ & $4 \mathrm{E}-03$ & 4E-04 & 4E-03 & 4E-07 & 3E-06 & 7E-07 & $1 \mathrm{E}-06$ & $3 \mathrm{E}-06$ & 5E-06 \\
\hline 77 & 3E-07 & $1 \mathrm{E}-06$ & 3E-06 & 1E-04 & $2 \mathrm{E}-05$ & 2E-06 & 2E-06 & $8 \mathrm{E}-05$ & $1 \mathrm{E}-03$ & $4 \mathrm{E}-03$ & 4E-04 & 4E-03 & $4 \mathrm{E}-07$ & 3E-06 & 7E-07 & $1 \mathrm{E}-06$ & 3E-06 & 5E-06 \\
\hline 100 & $3 \mathrm{E}-07$ & 1E-06 & 3E-06 & 1E-04 & $2 \mathrm{E}-05$ & 2E-06 & 3E-06 & 9E-05 & $1 \mathrm{E}-03$ & 5E-03 & 4E-04 & 4E-03 & 5E-07 & 4E-06 & $8 \mathrm{E}-07$ & $1 \mathrm{E}-06$ & $3 \mathrm{E}-06$ & 6E-06 \\
\hline 129 & $2 \mathrm{E}-07$ & $8 \mathrm{E}-07$ & $2 \mathrm{E}-06$ & $1 \mathrm{E}-04$ & $2 \mathrm{E}-05$ & $2 \mathrm{E}-06$ & $2 \mathrm{E}-06$ & 6E-05 & 9E-04 & $3 \mathrm{E}-03$ & $3 \mathrm{E}-04$ & $3 \mathrm{E}-03$ & $3 \mathrm{E}-07$ & 3E-06 & 5E-07 & $1 \mathrm{E}-06$ & $2 \mathrm{E}-06$ & 4E-06 \\
\hline 167 & 2E-07 & 7E-07 & $2 \mathrm{E}-06$ & 9E-05 & $1 \mathrm{E}-05$ & $1 \mathrm{E}-06$ & 2E-06 & $5 \mathrm{E}-05$ & 7E-04 & 3E-03 & $3 \mathrm{E}-04$ & $3 \mathrm{E}-03$ & $3 \mathrm{E}-07$ & 2E-06 & 5E-07 & 9E-07 & $2 \mathrm{E}-06$ & 3E-06 \\
\hline 215 & 1E-07 & 4E-07 & 1E-06 & 5E-05 & $8 \mathrm{E}-06$ & $8 \mathrm{E}-07$ & 9E-07 & $3 \mathrm{E}-05$ & 4E-04 & $2 \mathrm{E}-03$ & 1E-04 & $2 \mathrm{E}-03$ & $2 \mathrm{E}-07$ & 1E-06 & \begin{tabular}{|c|}
$3 \mathrm{E}-07$ \\
\end{tabular} & $5 \mathrm{E}-07$ & 1E-06 & 2E-06 \\
\hline 278 & $7 \mathrm{E}-08$ & 3E-07 & $7 \mathrm{E}-07$ & 3E-05 & 5E-06 & $5 \mathrm{E}-07$ & $6 \mathrm{E}-07$ & $2 \mathrm{E}-05$ & $3 \mathrm{E}-04$ & 1E-03 & $1 \mathrm{E}-04$ & 1E-03 & $1 \mathrm{E}-07$ & 9E-07 & $2 \mathrm{E}-07$ & 3E-07 & 6E-07 & $1 \mathrm{E}-06$ \\
\hline 359 & 5E-08 & $2 \mathrm{E}-07$ & $4 \mathrm{E}-07$ & $2 \mathrm{E}-05$ & 3E-06 & 3E-07 & $4 \mathrm{E}-07$ & $1 \mathrm{E}-05$ & 2E-04 & 7E-04 & 6E-05 & 6E-04 & 7E-08 & 6E-07 & $1 \mathrm{E}-07$ & $2 \mathrm{E}-07$ & $4 \mathrm{E}-07$ & $8 \mathrm{E}-07$ \\
\hline 464 & $7 \mathrm{E}-08$ & $3 \mathrm{E}-07$ & $7 \mathrm{E}-07$ & $3 \mathrm{E}-05$ & 5E-06 & $5 \mathrm{E}-07$ & $6 \mathrm{E}-07$ & $2 \mathrm{E}-05$ & $3 \mathrm{E}-04$ & $1 \mathrm{E}-03$ & $1 \mathrm{E}-04$ & 1E-03 & $1 \mathrm{E}-07$ & 9E-07 & $2 \mathrm{E}-07$ & 3E-07 & $6 \mathrm{E}-07$ & $1 \mathrm{E}-06$ \\
\hline 599 & 2E-09 & 7E-09 & $2 \mathrm{E}-08$ & 9E-07 & $1 \mathrm{E}-07$ & 1E-08 & $2 \mathrm{E}-08$ & 5E-07 & $8 \mathrm{E}-06$ & 3E-05 & 3E-06 & $3 \mathrm{E}-05$ & 3E-09 & $2 \mathrm{E}-08$ & 5E-09 & 9E-09 & $2 \mathrm{E}-08$ & 3E-08 \\
\hline 774 & $2 \mathrm{E}-08$ & $8 \mathrm{E}-08$ & 2E-07 & 1E-05 & $2 \mathrm{E}-06$ & 2E-07 & 2E-07 & 6E-06 & 9E-05 & 3E-04 & 3E-05 & 3E-04 & 3E-08 & $3 \mathrm{E}-07$ & 6E-08 & $1 \mathrm{E}-07$ & $2 \mathrm{E}-07$ & 4E-07 \\
\hline 1000 & 9E-08 & 3E-07 & $8 \mathrm{E}-07$ & $4 \mathrm{E}-05$ & 6E-06 & $7 \mathrm{E}-07$ & $8 \mathrm{E}-07$ & $2 \mathrm{E}-05$ & $4 \mathrm{E}-04$ & 1E-03 & $1 \mathrm{E}-04$ & $1 \mathrm{E}-03$ & $1 \mathrm{E}-07$ & 1E-06 & $2 \mathrm{E}-07$ & $4 \mathrm{E}-07$ & $8 \mathrm{E}-07$ & 2E-06 \\
\hline
\end{tabular}


Table 3.41. (contd)

\begin{tabular}{|c|c|c|c|c|c|c|c|c|c|c|c|c|c|c|c|}
\hline \begin{tabular}{|l|} 
Volume \\
Fraction
\end{tabular} & $3.9 \mathrm{E}-04$ & $2.5 \mathrm{E}-03$ & $1.8 \mathrm{E}-03$ & $1.3 \mathrm{E}-02$ & $3.0 \mathrm{E}-03$ & $1.7 \mathrm{E}-04$ & 8.7E-03 & $1.5 \mathrm{E}-06$ & $6.5 \mathrm{E}-04$ & $8.2 \mathrm{E}-03$ & 2.7E-03 & $6.4 \mathrm{E}-03$ & $8.8 \mathrm{E}-03$ & 3.9E-03 & $2.1 \mathrm{E}-03$ \\
\hline $\mathrm{d}_{\mathrm{S}}(\mu \mathrm{m})$ & $\mathrm{Na} 2(\mathrm{UO} 2) 2(\mathrm{PO} 4) 2.2 \mathrm{H} 2 \mathrm{O}$ & Na2U2O7 & NaAlCO3(OH)2 & NaAlSiO4 & $\mathrm{Ni}(\mathrm{OH}) 2$ & $\mathrm{~Pb}(\mathrm{OH}) 2$ & $\mathrm{Pu}(\mathrm{OH}) 4$ (co-precip. on Fe phase) & $\mathrm{PuO2}$ & Sr3(PO4)2 & $\mathrm{SrCO} 3$ & $\mathrm{ZrO} 2$ & $\mathrm{Na} 2 \mathrm{C} 2 \mathrm{O} 4$ & Na2CO3.H2O & \begin{tabular}{|l|}
$\mathrm{Na} 2 \mathrm{SO} 4.10 \mathrm{H} 2 \mathrm{O}$ \\
\end{tabular} & Na3FSO4 \\
\hline 0.22 & $1 \mathrm{E}-07$ & $9 \mathrm{E}-07$ & $7 \mathrm{E}-07$ & $5 \mathrm{E}-06$ & $1 \mathrm{E}-06$ & $6 \mathrm{E}-08$ & $3 \mathrm{E}-06$ & $5 \mathrm{E}-10$ & $2 \mathrm{E}-07$ & $3 \mathrm{E}-06$ & $1 \mathrm{E}-06$ & $2 \mathrm{E}-06$ & $3 \mathrm{E}-06$ & $1 \mathrm{E}-06$ & $8 \mathrm{E}-07$ \\
\hline 0.28 & $2 \mathrm{E}-07$ & $1 \mathrm{E}-06$ & $1 \mathrm{E}-06$ & $7 \mathrm{E}-06$ & $2 \mathrm{E}-06$ & $9 \mathrm{E}-08$ & $5 \mathrm{E}-06$ & $8 \mathrm{E}-10$ & $4 \mathrm{E}-07$ & $4 \mathrm{E}-06$ & $1 \mathrm{E}-06$ & $3 \mathrm{E}-06$ & $5 \mathrm{E}-06$ & $2 \mathrm{E}-06$ & $1 \mathrm{E}-06$ \\
\hline 0.36 & $1 \mathrm{E}-06$ & $9 \mathrm{E}-06$ & $7 \mathrm{E}-06$ & $5 \mathrm{E}-05$ & $1 \mathrm{E}-05$ & $6 \mathrm{E}-07$ & $3 \mathrm{E}-05$ & $6 \mathrm{E}-09$ & $2 \mathrm{E}-06$ & $3 \mathrm{E}-05$ & $1 \mathrm{E}-05$ & $2 \mathrm{E}-05$ & $3 \mathrm{E}-05$ & $1 \mathrm{E}-05$ & $8 \mathrm{E}-06$ \\
\hline 0.46 & $2 \mathrm{E}-06$ & $1 \mathrm{E}-05$ & $1 \mathrm{E}-05$ & $7 \mathrm{E}-05$ & $2 \mathrm{E}-05$ & $9 \mathrm{E}-07$ & $5 \mathrm{E}-05$ & $8 \mathrm{E}-09$ & $3 \mathrm{E}-06$ & $4 \mathrm{E}-05$ & $1 \mathrm{E}-05$ & $3 \mathrm{E}-05$ & $5 \mathrm{E}-05$ & $2 \mathrm{E}-05$ & $1 \mathrm{E}-05$ \\
\hline 0.60 & $2 \mathrm{E}-06$ & $1 \mathrm{E}-05$ & $9 \mathrm{E}-06$ & $6 \mathrm{E}-05$ & $1 \mathrm{E}-05$ & $8 \mathrm{E}-07$ & $4 \mathrm{E}-05$ & $7 \mathrm{E}-09$ & $3 \mathrm{E}-06$ & $4 \mathrm{E}-05$ & $1 \mathrm{E}-05$ & 3E-05 & $4 \mathrm{E}-05$ & $2 \mathrm{E}-05$ & $1 \mathrm{E}-05$ \\
\hline 0.77 & 9E-06 & 6E-05 & $4 \mathrm{E}-05$ & $3 \mathrm{E}-04$ & $7 \mathrm{E}-05$ & $4 \mathrm{E}-06$ & $2 \mathrm{E}-04$ & $4 \mathrm{E}-08$ & $2 \mathrm{E}-05$ & $2 \mathrm{E}-04$ & $6 \mathrm{E}-05$ & 2E-04 & $2 \mathrm{E}-04$ & $9 \mathrm{E}-05$ & $5 \mathrm{E}-05$ \\
\hline 1 & $5 \mathrm{E}-06$ & $3 \mathrm{E}-05$ & $2 \mathrm{E}-05$ & $2 \mathrm{E}-04$ & $4 \mathrm{E}-05$ & $2 \mathrm{E}-06$ & $1 \mathrm{E}-04$ & $2 \mathrm{E}-08$ & $9 \mathrm{E}-06$ & $1 \mathrm{E}-04$ & $4 \mathrm{E}-05$ & 9E- 05 & $1 \mathrm{E}-04$ & $5 \mathrm{E}-05$ & $3 \mathrm{E}-05$ \\
\hline 1.3 & $2 \mathrm{E}-05$ & $1 \mathrm{E}-04$ & $8 \mathrm{E}-05$ & $6 \mathrm{E}-04$ & $1 \mathrm{E}-04$ & $7 \mathrm{E}-06$ & $4 \mathrm{E}-04$ & $6 \mathrm{E}-08$ & $3 \mathrm{E}-05$ & $4 \mathrm{E}-04$ & $1 \mathrm{E}-04$ & 3E- 04 & $4 \mathrm{E}-04$ & $2 \mathrm{E}-04$ & $9 \mathrm{E}-05$ \\
\hline 1.7 & $1 \mathrm{E}-05$ & $9 \mathrm{E}-05$ & $6 \mathrm{E}-05$ & $5 \mathrm{E}-04$ & $1 \mathrm{E}-04$ & $6 \mathrm{E}-06$ & $3 \mathrm{E}-04$ & 5E-08 & $2 \mathrm{E}-05$ & $3 \mathrm{E}-04$ & $1 \mathrm{E}-04$ & $2 \mathrm{E}-04$ & 3E-04 & $1 \mathrm{E}-04$ & $7 \mathrm{E}-05$ \\
\hline 2.2 & $2 \mathrm{E}-05$ & $1 \mathrm{E}-04$ & $1 \mathrm{E}-04$ & 7E-04 & $2 \mathrm{E}-04$ & $9 \mathrm{E}-06$ & 5E-04 & $8 \mathrm{E}-08$ & 3E-05 & $4 \mathrm{E}-04$ & $1 \mathrm{E}-04$ & 3E-04 & 5E-04 & $2 \mathrm{E}-04$ & $1 \mathrm{E}-04$ \\
\hline 2.8 & 2E-05 & $1 \mathrm{E}-04$ & $1 \mathrm{E}-04$ & $8 \mathrm{E}-04$ & $2 \mathrm{E}-04$ & $1 \mathrm{E}-05$ & 5E-04 & $8 \mathrm{E}-08$ & 4E- 05 & $5 \mathrm{E}-04$ & 2E-04 & 4E- 04 & 5E-04 & $2 \mathrm{E}-04$ & $1 \mathrm{E}-04$ \\
\hline 3.6 & 2E-05 & $1 \mathrm{E}-04$ & $1 \mathrm{E}-04$ & $8 \mathrm{E}-04$ & $2 \mathrm{E}-04$ & $1 \mathrm{E}-05$ & 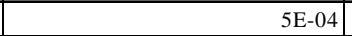 & $9 \mathrm{E}-08$ & 4E- 05 & $5 \mathrm{E}-04$ & 2E-04 & $4 \mathrm{E}-04$ & 5E-04 & $2 \mathrm{E}-04$ & $1 \mathrm{E}-04$ \\
\hline 4.6 & $3 \mathrm{E}-05$ & $2 \mathrm{E}-04$ & $1 \mathrm{E}-04$ & $9 \mathrm{E}-04$ & $2 \mathrm{E}-04$ & $1 \mathrm{E}-05$ & $6 \mathrm{E}-04$ & $1 \mathrm{E}-07$ & $5 \mathrm{E}-05$ & $6 \mathrm{E}-04$ & $2 \mathrm{E}-04$ & 4E- 04 & $6 \mathrm{E}-04$ & $3 \mathrm{E}-04$ & $1 \mathrm{E}-04$ \\
\hline 6.0 & $3 \mathrm{E}-05$ & $2 \mathrm{E}-04$ & $1 \mathrm{E}-04$ & $1 \mathrm{E}-03$ & $2 \mathrm{E}-04$ & $1 \mathrm{E}-05$ & $7 \mathrm{E}-04$ & $1 \mathrm{E}-07$ & $5 \mathrm{E}-05$ & $6 \mathrm{E}-04$ & $2 \mathrm{E}-04$ & $5 \mathrm{E}-04$ & $7 \mathrm{E}-04$ & $3 \mathrm{E}-04$ & $2 \mathrm{E}-04$ \\
\hline 7.7 & 4E- 05 & 2E-04 & $2 \mathrm{E}-04$ & $1 \mathrm{E}-03$ & 3E-04 & $2 \mathrm{E}-05$ & $8 \mathrm{E}-04$ & $1 \mathrm{E}-07$ & $6 \mathrm{E}-05$ & $8 \mathrm{E}-04$ & 3E-04 & $6 \mathrm{E}-04$ & $8 \mathrm{E}-04$ & $4 \mathrm{E}-04$ & $2 \mathrm{E}-04$ \\
\hline 10 & 3E-05 & $2 \mathrm{E}-04$ & $2 \mathrm{E}-04$ & $1 \mathrm{E}-03$ & $2 \mathrm{E}-04$ & $1 \mathrm{E}-05$ & $7 \mathrm{E}-04$ & $1 \mathrm{E}-07$ & $5 \mathrm{E}-05$ & $7 \mathrm{E}-04$ & $2 \mathrm{E}-04$ & $5 \mathrm{E}-04$ & $7 \mathrm{E}-04$ & $3 \mathrm{E}-04$ & $2 \mathrm{E}-04$ \\
\hline 13 & 3E-05 & $2 \mathrm{E}-04$ & $2 \mathrm{E}-04$ & $1 \mathrm{E}-03$ & 3E-04 & $2 \mathrm{E}-05$ & $8 \mathrm{E}-04$ & $1 \mathrm{E}-07$ & $6 \mathrm{E}-05$ & 7E-04 & $2 \mathrm{E}-04$ & $6 \mathrm{E}-04$ & $8 \mathrm{E}-04$ & $4 \mathrm{E}-04$ & $2 \mathrm{E}-04$ \\
\hline 17 & $3 \mathrm{E}-05$ & $2 \mathrm{E}-04$ & $1 \mathrm{E}-04$ & $1 \mathrm{E}-03$ & $2 \mathrm{E}-04$ & $1 \mathrm{E}-05$ & $6 \mathrm{E}-04$ & $1 \mathrm{E}-07$ & $5 \mathrm{E}-05$ & $6 \mathrm{E}-04$ & $2 \mathrm{E}-04$ & $5 \mathrm{E}-04$ & $7 \mathrm{E}-04$ & $3 \mathrm{E}-04$ & $2 \mathrm{E}-04$ \\
\hline 22 & $2 \mathrm{E}-05$ & $1 \mathrm{E}-04$ & $1 \mathrm{E}-04$ & $8 \mathrm{E}-04$ & $2 \mathrm{E}-04$ & $1 \mathrm{E}-05$ & 5E-04 & $9 \mathrm{E}-08$ & $4 \mathrm{E}-05$ & $5 \mathrm{E}-04$ & $2 \mathrm{E}-04$ & $4 \mathrm{E}-04$ & 5E-04 & $2 \mathrm{E}-04$ & $1 \mathrm{E}-04$ \\
\hline 28 & $2 \mathrm{E}-05$ & $9 \mathrm{E}-05$ & $7 \mathrm{E}-05$ & 5E-04 & $1 \mathrm{E}-04$ & $7 \mathrm{E}-06$ & $3 \mathrm{E}-04$ & $6 \mathrm{E}-08$ & $3 \mathrm{E}-05$ & $3 \mathrm{E}-04$ & $1 \mathrm{E}-04$ & $2 \mathrm{E}-04$ & 3E-04 & $2 \mathrm{E}-04$ & $8 \mathrm{E}-05$ \\
\hline 36 & $1 \mathrm{E}-05$ & $7 \mathrm{E}-05$ & $5 \mathrm{E}-05$ & $4 \mathrm{E}-04$ & $8 \mathrm{E}-05$ & $5 \mathrm{E}-06$ & $2 \mathrm{E}-04$ & $4 \mathrm{E}-08$ & $2 \mathrm{E}-05$ & $2 \mathrm{E}-04$ & $8 \mathrm{E}-05$ & $2 \mathrm{E}-04$ & $2 \mathrm{E}-04$ & $1 \mathrm{E}-04$ & $6 \mathrm{E}-05$ \\
\hline 46 & 7E-06 & 4E-05 & $3 \mathrm{E}-05$ & $2 \mathrm{E}-04$ & 5E-05 & $3 \mathrm{E}-06$ & $2 \mathrm{E}-04$ & $3 \mathrm{E}-08$ & $1 \mathrm{E}-05$ & $2 \mathrm{E}-04$ & 5E-05 & $1 \mathrm{E}-04$ & $2 \mathrm{E}-04$ & $7 \mathrm{E}-05$ & $4 \mathrm{E}-05$ \\
\hline 60 & $5 \mathrm{E}-06$ & $3 \mathrm{E}-05$ & $2 \mathrm{E}-05$ & $2 \mathrm{E}-04$ & $3 \mathrm{E}-05$ & $2 \mathrm{E}-06$ & $1 \mathrm{E}-04$ & $2 \mathrm{E}-08$ & $8 \mathrm{E}-06$ & $1 \mathrm{E}-04$ & 3E-05 & $7 \mathrm{E}-05$ & $1 \mathrm{E}-04$ & $5 \mathrm{E}-05$ & $2 \mathrm{E}-05$ \\
\hline 77 & $5 \mathrm{E}-06$ & $3 \mathrm{E}-05$ & $2 \mathrm{E}-05$ & 2E-04 & $3 \mathrm{E}-05$ & $2 \mathrm{E}-06$ & $1 \mathrm{E}-04$ & $2 \mathrm{E}-08$ & $8 \mathrm{E}-06$ & $1 \mathrm{E}-04$ & $3 \mathrm{E}-05$ & $7 \mathrm{E}-05$ & $1 \mathrm{E}-04$ & \begin{tabular}{|c|}
$\mathrm{E}-05$ \\
\end{tabular} & $2 \mathrm{E}-05$ \\
\hline 100 & $5 \mathrm{E}-06$ & $3 \mathrm{E}-05$ & $2 \mathrm{E}-05$ & $2 \mathrm{E}-04$ & 4E-05 & $2 \mathrm{E}-06$ & $1 \mathrm{E}-04$ & $2 \mathrm{E}-08$ & $8 \mathrm{E}-06$ & $1 \mathrm{E}-04$ & 3E-05 & $8 \mathrm{E}-05$ & $1 \mathrm{E}-04$ & $5 \mathrm{E}-05$ & $3 \mathrm{E}-05$ \\
\hline 129 & $4 \mathrm{E}-06$ & 2E-05 & $2 \mathrm{E}-05$ & $1 \mathrm{E}-04$ & 3E-05 & $2 \mathrm{E}-06$ & $8 \mathrm{E}-05$ & $1 \mathrm{E}-08$ & $6 \mathrm{E}-06$ & $8 \mathrm{E}-05$ & $2 \mathrm{E}-05$ & $6 \mathrm{E}-05$ & $8 \mathrm{E}-05$ & $4 \mathrm{E}-05$ & $2 \mathrm{E}-05$ \\
\hline 167 & $3 \mathrm{E}-06$ & $2 \mathrm{E}-05$ & $1 \mathrm{E}-05$ & $1 \mathrm{E}-04$ & $2 \mathrm{E}-05$ & $1 \mathrm{E}-06$ & $7 \mathrm{E}-05$ & $1 \mathrm{E}-08$ & $5 \mathrm{E}-06$ & $6 \mathrm{E}-05$ & $2 \mathrm{E}-05$ & 5E-05 & 7E-05 & $3 \mathrm{E}-05$ & $2 \mathrm{E}-05$ \\
\hline 215 & $2 \mathrm{E}-06$ & $1 \mathrm{E}-05$ & $8 \mathrm{E}-06$ & 6E-05 & $1 \mathrm{E}-05$ & $8 \mathrm{E}-07$ & $4 \mathrm{E}-05$ & 7E-09 & 3E-06 & $4 \mathrm{E}-05$ & $1 \mathrm{E}-05$ & 3E-05 & $4 \mathrm{E}-05$ & $2 \mathrm{E}-05$ & $9 \mathrm{E}-06$ \\
\hline 278 & $1 \mathrm{E}-06$ & 7E-06 & $5 \mathrm{E}-06$ & $4 \mathrm{E}-05$ & 9E-06 & $5 \mathrm{E}-07$ & $3 \mathrm{E}-05$ & 4E-09 & $2 \mathrm{E}-06$ & $2 \mathrm{E}-05$ & $8 \mathrm{E}-06$ & $2 \mathrm{E}-05$ & 3E-05 & $1 \mathrm{E}-05$ & $6 \mathrm{E}-06$ \\
\hline 359 & $7 \mathrm{E}-07$ & 5E-06 & $3 \mathrm{E}-06$ & $3 \mathrm{E}-05$ & $6 \mathrm{E}-06$ & $3 \mathrm{E}-07$ & $2 \mathrm{E}-05$ & $3 \mathrm{E}-09$ & $1 \mathrm{E}-06$ & $2 \mathrm{E}-05$ & $5 \mathrm{E}-06$ & $1 \mathrm{E}-05$ & $2 \mathrm{E}-05$ & $7 \mathrm{E}-06$ & $4 \mathrm{E}-06$ \\
\hline 464 & $1 \mathrm{E}-06$ & $7 \mathrm{E}-06$ & $5 \mathrm{E}-06$ & $4 \mathrm{E}-05$ & $9 \mathrm{E}-06$ & $5 \mathrm{E}-07$ & $3 \mathrm{E}-05$ & 4E-09 & $2 \mathrm{E}-06$ & $2 \mathrm{E}-05$ & $8 \mathrm{E}-06$ & $2 \mathrm{E}-05$ & $3 \mathrm{E}-05$ & $1 \mathrm{E}-05$ & $6 \mathrm{E}-06$ \\
\hline 599 & $3 \mathrm{E}-08$ & $2 \mathrm{E}-07$ & $1 \mathrm{E}-07$ & $1 \mathrm{E}-06$ & $2 \mathrm{E}-07$ & $1 \mathrm{E}-08$ & $7 \mathrm{E}-07$ & $1 \mathrm{E}-10$ & $5 \mathrm{E}-08$ & $6 \mathrm{E}-07$ & $2 \mathrm{E}-07$ & $5 \mathrm{E}-07$ & $7 \mathrm{E}-07$ & $3 \mathrm{E}-07$ & $2 \mathrm{E}-07$ \\
\hline 774 & $4 \mathrm{E}-07$ & $2 \mathrm{E}-06$ & $2 \mathrm{E}-06$ & $1 \mathrm{E}-05$ & $3 \mathrm{E}-06$ & $2 \mathrm{E}-07$ & $8 \mathrm{E}-06$ & $1 \mathrm{E}-09$ & $6 \mathrm{E}-07$ & $8 \mathrm{E}-06$ & $3 \mathrm{E}-06$ & $6 \mathrm{E}-06$ & $8 \mathrm{E}-06$ & $4 \mathrm{E}-06$ & $2 \mathrm{E}-06$ \\
\hline 1000 & $1 \mathrm{E}-06$ & $9 \mathrm{E}-06$ & $7 \mathrm{E}-06$ & $5 \mathrm{E}-05$ & $1 \mathrm{E}-05$ & $6 \mathrm{E}-07$ & $3 \mathrm{E}-05$ & 5E-09 & $2 \mathrm{E}-06$ & $3 \mathrm{E}-05$ & $1 \mathrm{E}-05$ & 2E-05 & $3 \mathrm{E}-05$ & $1 \mathrm{E}-05$ & $8 \mathrm{E}-06$ \\
\hline
\end{tabular}


Table 3.41. (contd)

\begin{tabular}{|c|c|c|c|c|c|c|c|c|}
\hline \multicolumn{9}{|l|}{ Volume } \\
\hline Fraction & $1.6 \mathrm{E}-05$ & $5.4 \mathrm{E}-03$ & $1.9 \mathrm{E}-03$ & $9.3 \mathrm{E}-04$ & $8.5 \mathrm{E}-03$ & $1.7 \mathrm{E}-02$ & $1.6 \mathrm{E}-04$ & $4.6 \mathrm{E}-02$ \\
\hline $\mathrm{d}_{\mathrm{S}}(\mu \mathrm{m})$ & Na3NO3SO4.H2O & Na3PO4.0.25NaOH.12H2O & $\mathrm{Na} 4 \mathrm{P} 2 \mathrm{O} 7.10 \mathrm{H} 2 \mathrm{O}$ & $\mathrm{Na} 6(\mathrm{SO} 4) 2 \mathrm{CO} 3$ & $\mathrm{NaF}$ & Na7F(PO4)2.19H2O & NaNO2 & NaNO3 \\
\hline 0.22 & 6E-09 & $2 \mathrm{E}-06$ & $7 \mathrm{E}-07$ & $3 \mathrm{E}-07$ & $3 \mathrm{E}-06$ & $6 \mathrm{E}-06$ & $6 \mathrm{E}-08$ & $2 \mathrm{E}-05$ \\
\hline 0.28 & $8 \mathrm{E}-09$ & $3 \mathrm{E}-06$ & $1 \mathrm{E}-06$ & $5 \mathrm{E}-07$ & $5 E-06$ & 9E-06 & $9 \mathrm{E}-08$ & $2 \mathrm{E}-05$ \\
\hline 0.36 & $6 \mathrm{E}-08$ & $2 \mathrm{E}-05$ & $7 \mathrm{E}-06$ & $3 \mathrm{E}-06$ & $3 \mathrm{E}-05$ & $6 \mathrm{E}-05$ & $6 \mathrm{E}-07$ & $2 \mathrm{E}-04$ \\
\hline 0.46 & $8 \mathrm{E}-08$ & $3 \mathrm{E}-05$ & $1 \mathrm{E}-05$ & $5 \mathrm{E}-06$ & $4 \mathrm{E}-05$ & 9E-05 & $8 \mathrm{E}-07$ & $2 \mathrm{E}-04$ \\
\hline 0.60 & $7 \mathrm{E}-08$ & $3 \mathrm{E}-05$ & $9 \mathrm{E}-06$ & $4 \mathrm{E}-06$ & $4 \mathrm{E}-05$ & $8 \mathrm{E}-05$ & $7 \mathrm{E}-07$ & $2 \mathrm{E}-04$ \\
\hline 0.77 & $4 \mathrm{E}-07$ & 1E-04 & 5E-05 & $2 \mathrm{E}-05$ & $2 \mathrm{E}-04$ & 4E-04 & 4E-06 & $1 \mathrm{E}-03$ \\
\hline 1 & 2E-07 & 7E-05 & 3E-05 & $1 \mathrm{E}-05$ & $1 \mathrm{E}-04$ & 2E-04 & 2E-06 & 6E-04 \\
\hline 1.3 & $7 \mathrm{E}-07$ & $2 \mathrm{E}-04$ & $8 \mathrm{E}-05$ & 4E-05 & $4 \mathrm{E}-04$ & $7 \mathrm{E}-04$ & $7 \mathrm{E}-06$ & $2 \mathrm{E}-03$ \\
\hline 1.7 & $6 \mathrm{E}-07$ & $2 \mathrm{E}-04$ & $7 \mathrm{E}-05$ & $3 \mathrm{E}-05$ & $3 \mathrm{E}-04$ & $6 \mathrm{E}-04$ & $6 \mathrm{E}-06$ & $2 \mathrm{E}-03$ \\
\hline 2.2 & $8 \mathrm{E}-07$ & $3 \mathrm{E}-04$ & $1 \mathrm{E}-04$ & $5 \mathrm{E}-05$ & $4 \mathrm{E}-04$ & 9E-04 & $8 \mathrm{E}-06$ & $2 \mathrm{E}-03$ \\
\hline 2.8 & $9 \mathrm{E}-07$ & $3 \mathrm{E}-04$ & $1 \mathrm{E}-04$ & $5 \mathrm{E}-05$ & $5 E-04$ & $1 \mathrm{E}-03$ & 9E-06 & $3 \mathrm{E}-03$ \\
\hline 3.6 & 9E-07 & 3E-04 & 1E-04 & 6E-05 & 5E-04 & $1 \mathrm{E}-03$ & $1 \mathrm{E}-05$ & $3 \mathrm{E}-03$ \\
\hline 4.6 & $1 \mathrm{E}-06$ & $4 \mathrm{E}-04$ & $1 \mathrm{E}-04$ & $6 \mathrm{E}-05$ & $6 \mathrm{E}-04$ & $1 \mathrm{E}-03$ & $1 \mathrm{E}-05$ & $3 \mathrm{E}-03$ \\
\hline 6.0 & $1 \mathrm{E}-06$ & 4E-04 & $1 \mathrm{E}-04$ & $7 \mathrm{E}-05$ & 7E-04 & $1 \mathrm{E}-03$ & $1 \mathrm{E}-05$ & $4 \mathrm{E}-03$ \\
\hline 7.7 & $1 \mathrm{E}-06$ & 5E-04 & $2 \mathrm{E}-04$ & 9E-05 & $8 \mathrm{E}-04$ & 2E-03 & $1 \mathrm{E}-05$ & $4 \mathrm{E}-03$ \\
\hline 10 & $1 \mathrm{E}-06$ & $4 \mathrm{E}-04$ & $2 \mathrm{E}-04$ & $8 \mathrm{E}-05$ & $7 \mathrm{E}-04$ & $1 \mathrm{E}-03$ & $1 \mathrm{E}-05$ & $4 \mathrm{E}-03$ \\
\hline 13 & $1 \mathrm{E}-06$ & 5E-04 & 2E-04 & 8E-05 & $8 \mathrm{E}-04$ & 2E-03 & $1 \mathrm{E}-05$ & $4 \mathrm{E}-03$ \\
\hline 17 & $1 \mathrm{E}-06$ & $4 \mathrm{E}-04$ & $1 \mathrm{E}-04$ & 7E-05 & $6 \mathrm{E}-04$ & $1 \mathrm{E}-03$ & $1 \mathrm{E}-05$ & $3 \mathrm{E}-03$ \\
\hline 22 & 9E-07 & $3 \mathrm{E}-04$ & $1 \mathrm{E}-04$ & $6 \mathrm{E}-05$ & $5 \mathrm{E}-04$ & $1 \mathrm{E}-03$ & $1 \mathrm{E}-05$ & $3 \mathrm{E}-03$ \\
\hline 28 & 6E-07 & 2E-04 & 7E-05 & 4E-05 & $3 \mathrm{E}-04$ & 7E-04 & 6E-06 & 2E-03 \\
\hline 36 & 4E-07 & 2E-04 & 5E-05 & 3E-05 & $2 \mathrm{E}-04$ & 5E-04 & 4E-06 & $1 \mathrm{E}-03$ \\
\hline 46 & 3E-07 & 1E-04 & 3E-05 & $2 \mathrm{E}-05$ & $2 \mathrm{E}-04$ & 3E-04 & 3E-06 & $8 \mathrm{E}-04$ \\
\hline 60 & $2 \mathrm{E}-07$ & 6E-05 & $2 \mathrm{E}-05$ & $1 \mathrm{E}-05$ & $1 \mathrm{E}-04$ & 2E-04 & 2E-06 & 5E-04 \\
\hline 77 & 2E-07 & $6 \mathrm{E}-05$ & 2E-05 & 1E-05 & $1 \mathrm{E}-04$ & 2E-04 & $2 \mathrm{E}-06$ & $5 \mathrm{E}-04$ \\
\hline 100 & 2E-07 & 7E-05 & 2E-05 & $1 \mathrm{E}-05$ & $1 \mathrm{E}-04$ & 2E-04 & 2E-06 & $6 \mathrm{E}-04$ \\
\hline 129 & $1 \mathrm{E}-07$ & 5E-05 & 2E-05 & 8E-06 & $8 \mathrm{E}-05$ & 2E-04 & $1 \mathrm{E}-06$ & $4 \mathrm{E}-04$ \\
\hline 167 & $1 \mathrm{E}-07$ & $4 \mathrm{E}-05$ & $1 \mathrm{E}-05$ & 7E-06 & $7 \mathrm{E}-05$ & $1 \mathrm{E}-04$ & $1 \mathrm{E}-06$ & $4 \mathrm{E}-04$ \\
\hline 215 & $7 \mathrm{E}-08$ & 2E-05 & 9E-06 & $4 \mathrm{E}-06$ & $4 \mathrm{E}-05$ & $8 \mathrm{E}-05$ & $7 \mathrm{E}-07$ & $2 \mathrm{E}-04$ \\
\hline 278 & 5E-08 & 2E-05 & 6E-06 & 3E-06 & $2 \mathrm{E}-05$ & 5E-05 & 5E-07 & $1 \mathrm{E}-04$ \\
\hline 359 & $3 \mathrm{E}-08$ & $1 \mathrm{E}-05$ & 4E-06 & $2 \mathrm{E}-06$ & $2 \mathrm{E}-05$ & 3E-05 & 3E-07 & 9E-05 \\
\hline 464 & 5E-08 & 2E-05 & 6E-06 & 3E-06 & $2 \mathrm{E}-05$ & 5E-05 & 5E-07 & $1 \mathrm{E}-04$ \\
\hline 599 & $1 \mathrm{E}-09$ & $4 \mathrm{E}-07$ & $1 \mathrm{E}-07$ & 7E-08 & $7 \mathrm{E}-07$ & 1E-06 & $1 \mathrm{E}-08$ & $4 \mathrm{E}-06$ \\
\hline 774 & $1 \mathrm{E}-08$ & 5E-06 & $2 \mathrm{E}-06$ & 9E-07 & $8 \mathrm{E}-06$ & 2E-05 & $1 \mathrm{E}-07$ & $4 \mathrm{E}-05$ \\
\hline 1000 & 6E-08 & 2E-05 & 7E-06 & 3E-06 & 3E-05 & 6E-05 & 6E-07 & $2 \mathrm{E}-04$ \\
\hline
\end{tabular}


Table 3.42. Sludge, Flowing Unsonicated PSDD Density, Fractal Dimension 2.25

\begin{tabular}{|c|c|c|c|c|c|c|c|c|c|c|c|c|c|c|c|c|c|c|}
\hline $\mathrm{d}_{\mathrm{s}}(\mu \mathrm{m})$ & $\mathrm{Ag}$ & Ag2O & $\mathrm{Bi} 2 \mathrm{O} 3$ & $\mathrm{BiFeO3}$ & $\mathrm{Ca} 5 \mathrm{OH}(\mathrm{PO} 4) 3$ & $\mathrm{CaCO} 3$ & $\mathrm{CaF} 2$ & $\mathrm{CrOOH}$ & $\mathrm{FePO} 4.2 \mathrm{H} 2 \mathrm{O}$ & $\mathrm{FeOOH}$ & Gibbsite & Boehmite & $\mathrm{HgO}$ & KAlSiO4 & $\mathrm{La}(\mathrm{OH}) 3$ & $\mathrm{LaPO} 4 \cdot 2 \mathrm{H} 2 \mathrm{O}$ & Mn3(PO4)2 & $\mathrm{MnO} 2$ \\
\hline 0.22 & 10.50 & 7.14 & 8.90 & 3.42 & 3.14 & 2.71 & 3.18 & 4.11 & 1.37 & 1.42 & 2.42 & 1.55 & 7.14 & 2.61 & 2.30 & 6.51 & 3.10 & 5.03 \\
\hline 0.28 & 10.50 & 7.14 & 8.90 & 3.03 & 3.14 & 2.71 & 3.18 & 3.47 & 1.33 & 1.38 & 2.42 & 1.48 & 7.14 & 2.61 & 2.30 & 6.51 & 3.10 & 5.03 \\
\hline 0.36 & 10.50 & 7.14 & 8.90 & 2.70 & 3.14 & 2.71 & 3.18 & 3.06 & 1.30 & 1.34 & 2.42 & 1.43 & 7.14 & 2.61 & 2.30 & 6.51 & 3.10 & 5.03 \\
\hline 0.46 & 10.50 & 7.14 & 8.90 & 2.44 & 3.14 & 2.71 & 3.18 & 2.73 & 1.28 & 1.31 & 2.42 & 1.38 & 7.14 & 2.61 & 2.30 & 6.51 & 3.10 & 5.03 \\
\hline 0.60 & 10.50 & 7.14 & 8.90 & 2.21 & 3.14 & 2.71 & 3.18 & 2.46 & 1.26 & 1.29 & 2.42 & 1.34 & 7.14 & 2.61 & 2.30 & 6.51 & 3.10 & 5.03 \\
\hline 0.77 & 10.50 & 7.14 & 8.90 & 2.03 & 3.14 & 2.71 & 3.18 & 2.24 & 1.25 & 1.27 & 2.42 & 1.31 & 7.14 & 2.61 & 2.30 & 6.51 & 3.10 & 5.03 \\
\hline 1 & 10.50 & 7.14 & 8.90 & 1.88 & 3.14 & 2.71 & 3.18 & 2.05 & 1.23 & 1.25 & 2.42 & 1.29 & 7.14 & 2.61 & 2.30 & 6.51 & 3.10 & 5.03 \\
\hline 1.3 & 10.50 & 7.14 & 8.90 & 1.76 & 3.14 & 2.71 & 3.18 & 1.90 & 1.22 & 1.24 & 2.42 & 1.27 & 7.14 & 2.61 & 2.30 & 6.51 & 3.10 & 5.03 \\
\hline 1.7 & 7.53 & 5.24 & 8.90 & 1.65 & 3.14 & 2.71 & 3.18 & 1.77 & 1.21 & 1.22 & 2.42 & 1.25 & 5.24 & 2.61 & 2.30 & 6.51 & 3.10 & 5.03 \\
\hline 2.2 & 6.42 & 4.53 & 7.06 & 1.57 & 3.14 & 2.71 & 3.18 & 1.66 & 1.21 & 1.21 & 2.42 & 1.24 & 4.53 & 2.61 & 2.03 & 5.24 & 3.10 & 5.03 \\
\hline 2.8 & 5.50 & 3.94 & 6.03 & 1.50 & 3.14 & 2.71 & 3.18 & 1.58 & 1.20 & 1.21 & 2.42 & 1.23 & 3.94 & 2.61 & 1.88 & 4.53 & 3.10 & 5.03 \\
\hline 3.6 & 4.74 & 3.46 & 5.18 & 1.44 & 3.14 & 2.71 & 3.18 & 1.51 & 1.19 & 1.20 & 2.42 & 1.22 & 3.46 & 2.61 & 1.76 & 3.94 & 3.10 & 5.03 \\
\hline 4.6 & 4.12 & 3.06 & 4.48 & 1.40 & 3.14 & 2.71 & 3.18 & 1.45 & 1.19 & 1.19 & 2.42 & 1.21 & 3.06 & 2.61 & 1.65 & 3.46 & 3.10 & 5.03 \\
\hline 6.0 & 3.61 & 2.73 & 3.90 & 1.36 & 2.76 & 2.71 & 3.18 & 1.40 & 1.19 & 1.19 & 2.42 & 1.20 & 2.73 & 2.23 & 1.57 & 3.06 & 2.60 & 5.03 \\
\hline 7.7 & 3.18 & 2.46 & 3.43 & 1.32 & 2.48 & 2.71 & 3.18 & 1.36 & 1.18 & 1.19 & 2.42 & 1.20 & 2.46 & 2.05 & 1.50 & 2.73 & 2.35 & 3.95 \\
\hline 10 & 2.83 & 2.23 & 3.03 & 1.30 & 2.25 & 2.71 & 2.79 & 1.33 & 1.18 & 1.18 & 2.42 & 1.19 & 2.23 & 1.89 & 1.44 & 2.46 & 2.14 & 3.46 \\
\hline 13 & 2.54 & 2.05 & 2.71 & 1.27 & 2.06 & 2.71 & 2.51 & 1.30 & 1.18 & 1.18 & 2.42 & 1.19 & 2.05 & 1.77 & 1.39 & 2.23 & 1.97 & 3.06 \\
\hline 17 & 2.30 & 1.89 & 2.44 & 1.26 & 1.91 & 2.71 & 2.27 & 1.28 & 1.18 & 1.18 & 2.42 & 1.18 & 1.89 & 1.66 & 1.36 & 2.05 & 1.83 & 2.73 \\
\hline 22 & 2.10 & 1.77 & 2.22 & 1.24 & 1.78 & 2.71 & 2.08 & 1.26 & 1.18 & 1.18 & 2.42 & 1.18 & 1.77 & 1.58 & 1.32 & 1.89 & 1.72 & 2.46 \\
\hline 28 & 1.94 & 1.66 & 2.03 & 1.23 & 1.67 & 2.71 & 1.92 & 1.24 & 1.18 & 1.18 & 2.42 & 1.18 & 1.66 & 1.51 & 1.30 & 1.77 & 1.62 & 2.23 \\
\hline 36 & 1.81 & 1.58 & 1.88 & 1.22 & 1.58 & 2.43 & 1.79 & 1.23 & 1.17 & 1.18 & 2.42 & 1.18 & 1.58 & 1.45 & 1.27 & 1.66 & 1.54 & 2.05 \\
\hline 46 & 1.69 & 1.51 & 1.76 & 1.21 & 1.51 & 2.21 & 1.68 & 1.22 & 1.17 & 1.17 & 2.42 & 1.18 & 1.51 & 1.40 & 1.26 & 1.58 & 1.48 & 1.90 \\
\hline 60 & 1.60 & 1.45 & 1.66 & 1.20 & 1.45 & 2.03 & 1.59 & 1.21 & 1.17 & 1.17 & 2.42 & 1.18 & 1.45 & 1.36 & 1.24 & 1.51 & 1.42 & 1.77 \\
\hline 77 & 1.53 & 1.40 & 1.57 & 1.20 & 1.40 & 1.88 & 1.52 & 1.20 & 1.17 & 1.17 & 2.42 & 1.17 & 1.40 & 1.33 & 1.23 & 1.45 & 1.38 & 1.66 \\
\hline 100 & 1.47 & 1.36 & 1.50 & 1.19 & 1.36 & 1.75 & 1.46 & 1.20 & 1.17 & 1.17 & 2.42 & 1.17 & 1.36 & 1.30 & 1.22 & 1.40 & 1.34 & 1.58 \\
\hline 129 & 1.41 & 1.33 & 1.44 & 1.19 & 1.33 & 1.65 & 1.41 & 1.19 & $\begin{array}{ll}1.17 \\
\end{array}$ & 1.17 & 2.42 & 1.17 & 1.33 & 1.28 & 1.21 & 1.36 & 1.31 & 1.51 \\
\hline 167 & 1.37 & 1.30 & 1.40 & 1.19 & 1.30 & 1.57 & 1.37 & 1.19 & 1.17 & 1.17 & 2.02 & 1.17 & 1.30 & 1.26 & 1.20 & 1.33 & 1.29 & 1.45 \\
\hline 215 & 1.34 & 1.28 & 1.36 & $\begin{array}{ll}1.18 \\
\end{array}$ & 1.28 & 1.50 & 1.33 & 1.19 & 1.17 & 1.17 & 1.87 & 1.17 & 1.28 & 1.24 & 1.20 & 1.30 & 1.27 & 1.40 \\
\hline 278 & 1.31 & 1.26 & 1.32 & 1.18 & 1.26 & 1.44 & 1.30 & 1.18 & 1.17 & 1.17 & 1.75 & 1.17 & 1.26 & 1.23 & 1.19 & 1.28 & 1.25 & 1.36 \\
\hline 359 & 1.28 & 1.24 & 1.30 & 1.18 & 1.24 & 1.39 & 1.28 & 1.18 & $\begin{array}{ll}1.17 \\
\end{array}$ & $\begin{array}{ll}1.17 \\
\end{array}$ & 1.65 & 1.17 & 1.24 & 1.22 & 1.19 & 1.26 & 1.24 & 1.33 \\
\hline 464 & 1.26 & 1.23 & 1.27 & 1.18 & 1.23 & 1.35 & 1.26 & 1.18 & $\begin{array}{ll}1.17 \\
\end{array}$ & 1.17 & 1.57 & 1.17 & 1.23 & 1.21 & 1.19 & 1.24 & 1.22 & 1.30 \\
\hline 599 & 1.25 & 1.22 & 1.26 & 1.18 & 1.22 & 1.32 & 1.25 & 1.18 & $\begin{array}{ll}1.17 \\
\end{array}$ & 1.17 & 1.50 & 1.17 & 1.22 & 1.20 & 1.18 & 1.23 & 1.22 & 1.28 \\
\hline 774 & 1.23 & 1.21 & 1.24 & 1.17 & 1.21 & 1.30 & 1.23 & 1.18 & \begin{tabular}{ll|}
1.17 \\
\end{tabular} & 1.17 & 1.44 & 1.17 & 1.21 & 1.20 & 1.18 & 1.22 & 1.21 & 1.26 \\
\hline 1000 & 1.22 & 1.20 & 1.23 & 1.17 & 1.20 & 1.27 & 1.22 & 1.17 & 1.17 & 1.17 & 1.39 & 1.17 & 1.20 & 1.19 & 1.18 & 1.21 & 1.20 & 1.24 \\
\hline
\end{tabular}


Table 3.42. (contd)

\begin{tabular}{|c|c|c|c|c|c|c|c|c|c|c|c|c|c|c|c|}
\hline$d_{S}(\mu \mathrm{m})$ & $\mathrm{Na} 2(\mathrm{UO} 2) 2(\mathrm{PO} 4) 2.2 \mathrm{H} 2 \mathrm{O}$ & \begin{tabular}{|l|}
$\mathrm{Na} 2 \mathrm{U} 2 \mathrm{O} 7$ \\
\end{tabular} & \begin{tabular}{|l|}
$\mathrm{NaAlCO} 3(\mathrm{OH}) 2$ \\
\end{tabular} & NaAlSiO4 & \begin{tabular}{|l|}
$\mathrm{Ni}(\mathrm{OH}) 2$ \\
\end{tabular} & $\mathrm{~Pb}(\mathrm{OH}) 2$ & $\mathrm{Pu}(\mathrm{OH}) 4$ (co-precip. on Fe phase) & $\mathrm{PuO2}$ & \begin{tabular}{|l|}
$\mathrm{Sr} 3(\mathrm{PO} 4) 2$ \\
$\mathrm{~s}$
\end{tabular} & SrCO3 & $\mathrm{ZrO} 2$ & Na2C2O4 & Na2CO3.H2O & $\mathrm{Na} 2 \mathrm{SO} 4.10 \mathrm{H} 2 \mathrm{O}$ & Na3FSO4 \\
\hline 0.22 & 3.50 & 5.62 & 2.42 & 2.37 & 4.15 & 7.10 & 1.42 & 11.43 & 1.73 & 1.73 & 5.68 & 2.34 & 2.25 & 1.46 & 2.65 \\
\hline 0.28 & 3.50 & 5.62 & 2.42 & 2.37 & 4.15 & 7.10 & 1.38 & 11.43 & 1.64 & 1.64 & 5.68 & 2.34 & 2.25 & 1.46 & 2.65 \\
\hline 0.36 & 3.50 & 5.62 & 2.42 & 2.37 & 3.44 & 7.10 & 1.34 & 11.43 & 1.55 & 1.55 & 5.68 & 2.34 & 2.25 & 1.46 & 2.65 \\
\hline 0.46 & 3.50 & 5.62 & 2.42 & 2.37 & 3.04 & 7.10 & 1.31 & 11.43 & 1.49 & 1.49 & 5.68 & 2.34 & 2.25 & 1.46 & 2.65 \\
\hline 0.60 & 3.50 & 5.62 & 2.42 & 2.37 & 2.72 & 7.10 & 1.29 & 11.43 & 1.43 & 1.43 & 5.68 & 2.34 & 2.25 & 1.46 & 2.65 \\
\hline 0.77 & 3.50 & 5.62 & 2.42 & 2.37 & 2.45 & 7.10 & 1.27 & 11.43 & 1.39 & 1.39 & 5.68 & 2.34 & 2.25 & 1.46 & 2.65 \\
\hline 1 & 3.50 & 5.62 & 2.42 & 2.37 & 2.22 & 7.10 & 1.25 & 11.43 & 1.35 & 1.35 & 5.68 & 2.34 & 2.25 & 1.46 & 2.65 \\
\hline 1.3 & 3.50 & 5.62 & 2.42 & 2.37 & 2.04 & 7.10 & 1.24 & 11.43 & 1.32 & 1.32 & 5.68 & 2.34 & 2.25 & 1.46 & 2.65 \\
\hline 1.7 & 3.50 & 5.62 & 2.42 & 2.37 & 1.89 & 7.10 & 1.22 & 11.43 & 1.29 & 1.29 & 5.68 & 2.34 & 2.25 & 1.46 & 2.65 \\
\hline 2.2 & 3.50 & 5.62 & 2.42 & 2.37 & 1.76 & 7.10 & 1.21 & 11.43 & 1.27 & 1.27 & 5.68 & 2.34 & 2.25 & 1.46 & 2.65 \\
\hline 2.8 & 3.50 & 5.62 & 2.18 & 2.37 & 1.66 & 7.10 & 1.21 & 11.43 & 1.25 & 1.25 & 5.68 & 2.34 & 2.25 & 1.46 & 2.65 \\
\hline 3.6 & 2.94 & 4.56 & 2.01 & 2.37 & 1.57 & 5.69 & 1.20 & 11.43 & \begin{tabular}{|l|}
1.24 \\
\end{tabular} & 1.24 & 5.68 & 2.34 & 2.25 & 1.46 & 2.65 \\
\hline 4.6 & 2.63 & 3.97 & 1.86 & 2.37 & 1.50 & 4.90 & 1.19 & 11.43 & \begin{tabular}{|l|}
1.23 \\
\end{tabular} & 1.23 & 5.68 & 2.34 & 2.25 & 1.46 & 2.65 \\
\hline 6.0 & 2.38 & \begin{tabular}{|l|}
3.48 \\
\end{tabular} & 1.74 & 2.05 & 1.45 & 4.25 & 1.19 & 11.43 & \begin{tabular}{l|l|}
1.22 \\
\end{tabular} & 1.22 & 5.68 & 2.03 & 2.25 & 1.46 & 2.65 \\
\hline $\begin{array}{l}7.7 \\
\end{array}$ & 2.17 & 3.07 & 1.64 & 1.90 & 1.40 & \begin{tabular}{|l|}
3.71 \\
\end{tabular} & 1.19 & 11.43 & \begin{tabular}{|l|l|}
1.21 \\
\end{tabular} & 1.21 & 5.68 & 1.88 & 2.25 & 1.46 & 2.65 \\
\hline 10 & 1.99 & 2.74 & 1.56 & 1.77 & $\begin{array}{ll}1.36 \\
\end{array}$ & 3.27 & \begin{tabular}{|l|}
1.18 \\
\end{tabular} & 11.43 & 1.20 & 1.20 & 5.68 & 1.76 & 2.25 & 1.46 & 2.65 \\
\hline 13 & 1.85 & 2.47 & 1.49 & 1.67 & 1.32 & 2.90 & 1.18 & 11.43 & \begin{tabular}{ll|}
1.20 \\
\end{tabular} & 1.20 & \begin{tabular}{|l|}
5.68 \\
\end{tabular} & $\begin{array}{ll}1.66 \\
\end{array}$ & 2.25 & 1.46 & 2.65 \\
\hline 17 & 1.73 & 2.24 & 1.43 & 1.58 & 1.30 & 2.60 & 1.18 & 8.16 & 1.19 & 1.19 & 5.68 & 1.57 & 2.25 & 1.46 & 2.65 \\
\hline 22 & 1.63 & 2.05 & 1.39 & 1.51 & 1.28 & 2.35 & 1.18 & 6.94 & 1.19 & 1.19 & 5.68 & 1.50 & 2.25 & 1.46 & 2.65 \\
\hline 28 & 1.55 & 1.90 & 1.35 & 1.45 & 1.26 & 2.14 & 1.18 & 5.93 & 1.18 & 1.18 & 5.68 & 1.44 & 2.25 & 1.46 & 2.65 \\
\hline 36 & 1.49 & 1.77 & 1.32 & 1.40 & 1.24 & 1.97 & 1.18 & 5.10 & 1.18 & 1.18 & 4.61 & 1.40 & 2.25 & 1.46 & 2.65 \\
\hline 46 & 1.43 & 1.67 & 1.29 & 1.36 & 1.23 & 1.83 & 1.17 & 4.41 & 1.18 & 1.18 & 4.01 & 1.36 & 2.25 & 1.46 & 2.65 \\
\hline 60 & \begin{tabular}{|l|}
1.39 \\
\end{tabular} & 1.58 & 1.27 & 1.33 & 1.22 & 1.72 & 1.17 & 3.85 & $\begin{array}{ll}1.18 \\
\end{array}$ & 1.18 & 3.51 & 1.32 & \begin{tabular}{|l|}
1.97 \\
\end{tabular} & 1.46 & 2.65 \\
\hline 77 & 1.35 & $\begin{array}{ll}1.51 \\
\end{array}$ & 1.25 & 1.30 & 1.21 & $\begin{array}{ll}1.62 \\
\end{array}$ & 1.17 & \begin{tabular}{|l|}
3.38 \\
\end{tabular} & $\begin{array}{ll}1.18 \\
\end{array}$ & \begin{tabular}{|l|}
1.18 \\
\end{tabular} & 3.10 & \begin{tabular}{ll|}
1.30 \\
\end{tabular} & \begin{tabular}{|l|}
1.83 \\
\end{tabular} & 1.40 & 2.65 \\
\hline 100 & 1.32 & 1.45 & 1.24 & 1.28 & 1.20 & 1.54 & 1.17 & 2.99 & 1.18 & 1.18 & 2.76 & 1.27 & 1.71 & 1.36 & 2.65 \\
\hline 129 & 1.29 & 1.40 & 1.23 & 1.26 & 1.20 & 1.48 & 1.17 & 2.68 & 1.17 & 1.17 & 2.49 & 1.26 & 1.62 & 1.33 & 2.28 \\
\hline 167 & 1.27 & 1.36 & 1.22 & 1.24 & 1.19 & 1.42 & 1.17 & 2.41 & 1.17 & 1.17 & 2.26 & 1.24 & 1.54 & 1.30 & 2.09 \\
\hline 215 & 1.25 & 1.33 & 1.21 & 1.23 & 1.19 & 1.38 & 1.17 & 2.20 & 1.17 & 1.17 & 2.07 & 1.23 & 1.48 & 1.28 & 1.93 \\
\hline 278 & 1.24 & 1.30 & 1.20 & 1.22 & 1.19 & 1.34 & 1.17 & 2.02 & 1.17 & 1.17 & 1.91 & 1.22 & 1.42 & 1.26 & 1.79 \\
\hline 359 & 1.23 & 1.28 & 1.20 & 1.21 & 1.18 & 1.31 & 1.17 & 1.87 & 1.17 & 1.17 & 1.78 & 1.21 & 1.38 & 1.24 & 1.69 \\
\hline 464 & 1.22 & 1.26 & 1.19 & 1.20 & 1.18 & 1.29 & 1.17 & 1.75 & 1.17 & 1.17 & 1.67 & 1.20 & 1.34 & 1.23 & 1.60 \\
\hline 599 & 1.21 & 1.24 & 1.19 & 1.20 & 1.18 & 1.27 & 1.17 & 1.65 & 1.17 & 1.17 & 1.59 & 1.20 & 1.31 & 1.22 & 1.52 \\
\hline 774 & 1.20 & 1.23 & 1.18 & 1.19 & 1.18 & 1.25 & 1.17 & 1.56 & 1.17 & 1.17 & 1.51 & 1.19 & 1.29 & 1.21 & 1.46 \\
\hline 1000 & 1.20 & 1.22 & 1.18 & 1.19 & 1.18 & 1.24 & 1.17 & 1.49 & 1.17 & 1.17 & 1.45 & 1.19 & 1.27 & 1.20 & 1.41 \\
\hline
\end{tabular}


Table 3.42. (contd)

\begin{tabular}{|c|c|c|c|c|c|c|c|c|}
\hline $\mathrm{d}_{\mathrm{s}}(\mu \mathrm{m})$ & Na3NO3SO4.H2O & Na3PO4.0.25NaOH.12H2O & $\mathrm{Na} 4 \mathrm{P} 2 \mathrm{O} 7.10 \mathrm{H} 2 \mathrm{O}$ & Na6(SO4)2CO3 & $\mathrm{NaF}$ & Na7F(PO4)2.19H2O & NaNO2 & NaNO3 \\
\hline 0.22 & 2.30 & 1.62 & 1.83 & 2.64 & 2.78 & 1.75 & 2.17 & 2.26 \\
\hline 0.28 & 2.30 & 1.62 & 1.83 & 2.64 & 2.78 & 1.75 & 2.17 & 2.26 \\
\hline 0.36 & 2.30 & 1.62 & 1.83 & 2.64 & 2.78 & 1.75 & 2.17 & 2.26 \\
\hline 0.46 & 2.30 & 1.62 & 1.83 & 2.64 & 2.78 & 1.75 & 2.17 & 2.26 \\
\hline 0.60 & 2.30 & 1.62 & 1.83 & 2.64 & 2.78 & 1.75 & 2.17 & 2.26 \\
\hline 0.77 & 2.30 & 1.62 & 1.83 & 2.64 & 2.78 & 1.75 & 2.17 & 2.26 \\
\hline 1 & 2.30 & 1.62 & 1.83 & 2.64 & 2.78 & 1.75 & 2.17 & 2.26 \\
\hline 1.3 & 2.30 & 1.62 & 1.83 & 2.64 & 2.78 & 1.75 & 2.17 & 2.26 \\
\hline 1.7 & 2.30 & 1.62 & 1.83 & 2.64 & 2.78 & 1.75 & 2.17 & 2.26 \\
\hline 2.2 & 2.30 & 1.62 & 1.83 & 2.64 & 2.78 & 1.75 & 2.17 & 2.26 \\
\hline 2.8 & 2.30 & 1.62 & 1.83 & 2.64 & 2.78 & 1.75 & 2.17 & 2.26 \\
\hline 3.6 & 2.30 & 1.62 & 1.83 & 2.64 & 2.78 & 1.75 & 2.17 & 2.26 \\
\hline 4.6 & 2.30 & 1.62 & 1.83 & 2.64 & 2.78 & 1.75 & 2.17 & 2.26 \\
\hline 6.0 & 2.30 & 1.62 & 1.83 & 2.64 & 2.78 & 1.75 & 2.17 & 2.26 \\
\hline 7.7 & 2.30 & 1.62 & 1.83 & 2.64 & 2.78 & 1.75 & 2.17 & 2.26 \\
\hline 10 & 2.30 & 1.62 & 1.83 & 2.64 & 2.27 & 1.75 & 2.17 & 2.26 \\
\hline 13 & 2.30 & 1.62 & 1.83 & 2.64 & 2.08 & 1.75 & 2.17 & 2.26 \\
\hline 17 & 2.30 & 1.62 & 1.83 & 2.64 & 1.92 & 1.75 & 2.17 & 2.26 \\
\hline 22 & 2.30 & 1.62 & 1.83 & 2.35 & 1.79 & 1.75 & 2.17 & 2.26 \\
\hline 28 & 2.30 & 1.62 & 1.83 & 2.14 & 1.68 & 1.75 & 2.17 & 2.26 \\
\hline 36 & 2.30 & 1.62 & 1.83 & 1.97 & 1.59 & 1.75 & 2.17 & 2.26 \\
\hline 46 & 2.30 & 1.62 & 1.83 & 1.83 & 1.52 & 1.75 & 2.17 & 2.26 \\
\hline 60 & 2.00 & 1.62 & 1.83 & 1.72 & 1.46 & 1.75 & 2.17 & 2.26 \\
\hline 77 & 1.86 & 1.62 & 1.83 & 1.62 & 1.41 & 1.75 & 2.17 & 2.26 \\
\hline 100 & 1.74 & 1.62 & 1.83 & 1.54 & 1.37 & 1.75 & 2.17 & 2.26 \\
\hline 129 & 1.64 & 1.62 & 1.83 & 1.48 & 1.33 & 1.75 & 2.17 & 2.26 \\
\hline 167 & 1.56 & 1.62 & 1.83 & 1.42 & 1.30 & 1.75 & 2.17 & 2.26 \\
\hline 215 & 1.49 & 1.62 & 1.83 & 1.38 & 1.28 & 1.75 & 2.17 & 2.26 \\
\hline 278 & 1.43 & 1.62 & 1.83 & 1.34 & 1.26 & 1.75 & 2.17 & 2.26 \\
\hline 359 & 1.39 & 1.48 & 1.83 & 1.31 & 1.24 & 1.75 & 2.17 & 2.26 \\
\hline 464 & 1.35 & 1.43 & 1.83 & 1.29 & 1.23 & 1.75 & 2.17 & 2.00 \\
\hline 599 & 1.32 & 1.38 & 1.83 & 1.27 & 1.22 & 1.75 & 2.17 & 1.86 \\
\hline 774 & 1.29 & 1.35 & 1.83 & 1.25 & 1.21 & 1.75 & 2.17 & 1.74 \\
\hline 1000 & 1.27 & 1.31 & 1.83 & 1.24 & 1.20 & 1.75 & 2.17 & 1.64 \\
\hline
\end{tabular}


Table 3.43. Sludge, Flowing Unsonicated PSDD Volume Fraction, Fractal Dimension 1.6

\begin{tabular}{|c|c|c|c|c|c|c|c|c|c|c|c|c|c|c|c|c|c|c|}
\hline \begin{tabular}{|l|} 
Volume \\
Fraction
\end{tabular} & 7.0E-07| & $4.5 \mathrm{E}-06$ & $1.6 \mathrm{E}-05$ & $5.2 \mathrm{E}-03$ & $9.0 \mathrm{E}-05$ & 7.7E-06 & 9.7E-06 & $1.3 \mathrm{E}-03$ & $1.5 \mathrm{E}-01$ & $5.7 \mathrm{E}-01$ & $1.4 \mathrm{E}-03$ & $2.5 \mathrm{E}-01$ & $1.7 \mathrm{E}-06$ & $1.6 \mathrm{E}-05$ & $2.4 \mathrm{E}-06$ & 7.2E-06 & $1.1 \mathrm{E}-05$ & $2.3 \mathrm{E}-05$ \\
\hline $\mathrm{d}_{\mathrm{S}}(\mu \mathrm{m})$ & $\mathrm{Ag}$ & Ag2O & $\mathrm{Bi} 2 \mathrm{O} 3$ & $\mathrm{BiFeO3}$ & $\mathrm{Ca} 5 \mathrm{OH}(\mathrm{PO} 4) 3$ & $\mathrm{CaCO} 3$ & $\mathrm{CaF} 2$ & $\mathrm{CrOOH}$ & FePO4.2H2O & FeOOH & Gibbsite & Boehmite & $\mathrm{HgO}$ & KAlSiO4 & $\mathrm{La}(\mathrm{OH}) 3$ & $\mathrm{LaPO} \cdot 2 \mathrm{H} 2 \mathrm{O}$ & Mn3(PO4)2 & $\mathrm{MnO} 2$ \\
\hline 0.22 & $3 \mathrm{E}-10$ & $2 \mathrm{E}-09$ & 6E-09 & $2 \mathrm{E}-06$ & 3E-08 & 3E-09 & 3E-09 & 4E-07 & 5E-05 & $2 \mathrm{E}-04$ & 5E-07 & 9E-05 & $6 \mathrm{E}-10$ & 6E-09 & 9E-10 & $3 \mathrm{E}-09$ & 4E-09 & $8 \mathrm{E}-09$ \\
\hline 0.28 & $4 \mathrm{E}-10$ & $2 \mathrm{E}-09$ & $9 \mathrm{E}-09$ & $3 \mathrm{E}-06$ & 5E-08 & 4E-09 & 5E-09 & 7E-07 & $8 \mathrm{E}-05$ & 3E-04 & $8 \mathrm{E}-07$ & $1 \mathrm{E}-04$ & $9 \mathrm{E}-10$ & $9 \mathrm{E}-09$ & $1 \mathrm{E}-09$ & 4E-09 & 6E-09 & $1 \mathrm{E}-08$ \\
\hline 0.36 & 3E-09 & $2 \mathrm{E}-08$ & 6E-08 & $2 \mathrm{E}-05$ & 3E-07 & 3E-08 & 4E-08 & 5E-06 & 6E-04 & $2 \mathrm{E}-03$ & 5E-06 & 9E-04 & 6E-09 & 6E-08 & 9E-09 & $3 \mathrm{E}-08$ & $4 \mathrm{E}-08$ & $8 \mathrm{E}-08$ \\
\hline 0.46 & 4E-09 & 2E-08 & 8E-08 & 3E-05 & 5E-07 & 4E-08 & 5E-08 & 7E-06 & $8 \mathrm{E}-04$ & 3E-03 & 7E-06 & $1 \mathrm{E}-03$ & 9E-09 & 8E-08 & 1E-08 & 4E-08 & 6E-08 & 1E-07 \\
\hline 0.60 & 3E-09 & $2 \mathrm{E}-08$ & 7E-08 & $2 \mathrm{E}-05$ & 4E-07 & $4 \mathrm{E}-08$ & 5E-08 & 6E-06 & 7E-04 & 3E-03 & 7E-06 & $1 \mathrm{E}-03$ & 8E-09 & 7E-08 & $1 \mathrm{E}-08$ & 3E-08 & 5E-08 & $1 \mathrm{E}-07$ \\
\hline 0.77 & $2 \mathrm{E}-08$ & $1 \mathrm{E}-07$ & $4 \mathrm{E}-07$ & $1 \mathrm{E}-04$ & $2 \mathrm{E}-06$ & 2E-07 & $2 \mathrm{E}-07$ & 3E-05 & $4 \mathrm{E}-03$ & $1 \mathrm{E}-02$ & $3 \mathrm{E}-05$ & 6E-03 & 4E-08 & $4 \mathrm{E}-07$ & $6 \mathrm{E}-08$ & 2E-07 & 3E-07 & $5 \mathrm{E}-07$ \\
\hline 1 & 9E-09 & 6E-08 & 2E-07 & 7E-05 & 1E-06 & 1E-07 & 1E-07 & 2E-05 & $2 \mathrm{E}-03$ & 8E-03 & 2E-05 & 3E-03 & 2E-08 & 2E-07 & 3E-08 & 1E-07 & 1E-07 & 3E-07 \\
\hline 1.3 & 3E-08 & $2 \mathrm{E}-07$ & 7E-07 & 2E-04 & $4 \mathrm{E}-06$ & 3E-07 & $4 \mathrm{E}-07$ & 5E-05 & 7E-03 & $2 \mathrm{E}-02$ & 6E-05 & $1 \mathrm{E}-02$ & 7E-08 & $7 \mathrm{E}-07$ & $1 \mathrm{E}-07$ & 3E-07 & 5E-07 & $1 \mathrm{E}-06$ \\
\hline 1.7 & 2E-08 & 2E-07 & 6E-07 & $2 \mathrm{E}-04$ & 3E-06 & 3E-07 & 3E-07 & 4E-05 & 5E-03 & $2 \mathrm{E}-02$ & 5E-05 & 9E-03 & 6E-08 & 6E-07 & 8E-08 & 3E-07 & 4E-07 & 8E-07 \\
\hline 2.2 & $4 \mathrm{E}-08$ & $2 \mathrm{E}-07$ & $8 \mathrm{E}-07$ & $3 \mathrm{E}-04$ & 5E-06 & $4 \mathrm{E}-07$ & $5 \mathrm{E}-07$ & 7E-05 & $8 \mathrm{E}-03$ & $3 \mathrm{E}-02$ & $8 \mathrm{E}-05$ & $1 \mathrm{E}-02$ & 9E-08 & $8 \mathrm{E}-07$ & $1 \mathrm{E}-07$ & $4 \mathrm{E}-07$ & 6E-07 & $1 \mathrm{E}-06$ \\
\hline 2.8 & 4E-08 & 3E-07 & 9E-07 & 3E-04 & 5E-06 & 4E-07 & 6E-07 & 7E-05 & 9E-03 & $3 \mathrm{E}-02$ & 8E-05 & $1 \mathrm{E}-02$ & $1 \mathrm{E}-07$ & 9E-07 & $1 \mathrm{E}-07$ & $4 \mathrm{E}-07$ & 6E-07 & 1E-06 \\
\hline 3.6 & $4 \mathrm{E}-08$ & $3 \mathrm{E}-07$ & $1 \mathrm{E}-06$ & 3E-04 & $5 \mathrm{E}-06$ & $5 \mathrm{E}-07$ & $6 \mathrm{E}-07$ & $8 \mathrm{E}-05$ & 9E-03 & $3 \mathrm{E}-02$ & 9E-05 & $1 \mathrm{E}-02$ & $1 \mathrm{E}-07$ & $1 \mathrm{E}-06$ & 1E-07 & 4E-07 & 6E-07 & $1 \mathrm{E}-06$ \\
\hline 4.6 & 5E-08 & 3E-07 & $1 \mathrm{E}-06$ & $4 \mathrm{E}-04$ & 6E-06 & 5E-07 & 7E-07 & 9E-05 & $1 \mathrm{E}-02$ & $4 \mathrm{E}-02$ & $1 \mathrm{E}-04$ & $2 \mathrm{E}-02$ & $1 \mathrm{E}-07$ & $1 \mathrm{E}-06$ & $2 \mathrm{E}-07$ & 5E-07 & $7 \mathrm{E}-07$ & $2 \mathrm{E}-06$ \\
\hline 6.0 & 5E-08 & 3E-07 & 1E-06 & 4E-04 & 7E-06 & 6E-07 & 7E-07 & 1E-04 & $1 \mathrm{E}-02$ & 4E-02 & $1 \mathrm{E}-04$ & 2E-02 & 1E-07 & 1E-06 & 2E-07 & 6E-07 & 8E-07 & 2E-06 \\
\hline 7.7 & $6 \mathrm{E}-08$ & $4 \mathrm{E}-07$ & $1 \mathrm{E}-06$ & $5 \mathrm{E}-04$ & $8 \mathrm{E}-06$ & 7E-07 & 9E-07 & $1 \mathrm{E}-04$ & $1 \mathrm{E}-02$ & $5 \mathrm{E}-02$ & $1 \mathrm{E}-04$ & $2 \mathrm{E}-02$ & $2 \mathrm{E}-07$ & $1 \mathrm{E}-06$ & $2 \mathrm{E}-07$ & $7 \mathrm{E}-07$ & $1 \mathrm{E}-06$ & $2 \mathrm{E}-06$ \\
\hline 10 & 6E-08 & 4E-07 & 1E-06 & 4E-04 & 7E-06 & 6E-07 & $8 \mathrm{E}-07$ & 1E-04 & $1 \mathrm{E}-02$ & 5E-02 & 1E-04 & 2E-02 & 1E-07 & 1E-06 & 2E-07 & 6E-07 & 9E-07 & $2 \mathrm{E}-06$ \\
\hline 13 & $6 \mathrm{E}-08$ & $4 \mathrm{E}-07$ & $1 \mathrm{E}-06$ & 5E-04 & 8E-06 & 7E-07 & $9 \mathrm{E}-07$ & $1 \mathrm{E}-04$ & $1 \mathrm{E}-02$ & $5 \mathrm{E}-02$ & $1 \mathrm{E}-04$ & $2 \mathrm{E}-02$ & $2 \mathrm{E}-07$ & $1 \mathrm{E}-06$ & $2 \mathrm{E}-07$ & 6E-07 & $1 \mathrm{E}-06$ & $2 \mathrm{E}-06$ \\
\hline 17 & 5E-08 & 3E-07 & $1 \mathrm{E}-06$ & 4E-04 & 7E-06 & 6E-07 & 7E-07 & 9E-05 & $1 \mathrm{E}-02$ & 4E-02 & 1E-04 & $2 \mathrm{E}-02$ & 1E-07 & 1E-06 & $2 \mathrm{E}-07$ & 5E-07 & $8 \mathrm{E}-07$ & $2 \mathrm{E}-06$ \\
\hline 22 & $4 \mathrm{E}-08$ & $3 \mathrm{E}-07$ & $1 \mathrm{E}-06$ & $3 \mathrm{E}-04$ & 5E-06 & 5E-07 & 6E-07 & $8 \mathrm{E}-05$ & 9E-03 & $3 \mathrm{E}-02$ & 9E-05 & $1 \mathrm{E}-02$ & $1 \mathrm{E}-07$ & $1 \mathrm{E}-06$ & $1 \mathrm{E}-07$ & 4E-07 & 6E-07 & $1 \mathrm{E}-06$ \\
\hline 28 & 3E-08 & 2E-07 & 6E-07 & 2E-04 & 3E-06 & 3E-07 & 4E-07 & 5E-05 & 6E-03 & $2 \mathrm{E}-02$ & 6E-05 & 9E-03 & 7E-08 & 6E-07 & 9E-08 & 3E-07 & 4E-07 & 9E-07 \\
\hline 36 & $2 \mathrm{E}-08$ & $1 \mathrm{E}-07$ & $5 \mathrm{E}-07$ & $1 \mathrm{E}-04$ & 3E-06 & $2 \mathrm{E}-07$ & $3 \mathrm{E}-07$ & $4 \mathrm{E}-05$ & $4 \mathrm{E}-03$ & $2 \mathrm{E}-02$ & $4 \mathrm{E}-05$ & $7 \mathrm{E}-03$ & 5E-08 & 5E-07 & $7 \mathrm{E}-08$ & $2 \mathrm{E}-07$ & $3 \mathrm{E}-07$ & 6E-07 \\
\hline 46 & $1 \mathrm{E}-08$ & $8 \mathrm{E}-08$ & 3E-07 & 9E-05 & 2E-06 & $1 \mathrm{E}-07$ & $2 \mathrm{E}-07$ & 2E-05 & $3 \mathrm{E}-03$ & $1 \mathrm{E}-02$ & 3E-05 & 4E-03 & 3E-08 & 3E-07 & $4 \mathrm{E}-08$ & $1 \mathrm{E}-07$ & 2E-07 & $4 \mathrm{E}-07$ \\
\hline 60 & $8 \mathrm{E}-09$ & $5 \mathrm{E}-08$ & $2 \mathrm{E}-07$ & $6 \mathrm{E}-05$ & 1E-06 & 9E-08 & 1E-07 & $1 \mathrm{E}-05$ & $2 \mathrm{E}-03$ & $7 \mathrm{E}-03$ & $2 \mathrm{E}-05$ & $3 \mathrm{E}-03$ & $2 \mathrm{E}-08$ & $2 \mathrm{E}-07$ & $3 \mathrm{E}-08$ & $8 \mathrm{E}-08$ & $1 \mathrm{E}-07$ & $3 \mathrm{E}-07$ \\
\hline 77 & $8 \mathrm{E}-09$ & 5E-08 & $2 \mathrm{E}-07$ & 6E-05 & 1E-06 & $9 \mathrm{E}-08$ & 1E-07 & $1 \mathrm{E}-05$ & $2 \mathrm{E}-03$ & 7E-03 & $2 \mathrm{E}-05$ & 3E-03 & $2 \mathrm{E}-08$ & $2 \mathrm{E}-07$ & 3E-08 & $8 \mathrm{E}-08$ & 1E-07 & $3 \mathrm{E}-07$ \\
\hline 100 & 9E-09 & 6E-08 & 2E-07 & 7E-05 & 1E-06 & 1E-07 & 1E-07 & 2E-05 & $2 \mathrm{E}-03$ & 7E-03 & 2E-05 & 3E-03 & 2E-08 & 2E-07 & 3E-08 & 9E-08 & 1E-07 & 3E-07 \\
\hline 129 & 6E-09 & $4 \mathrm{E}-08$ & $1 \mathrm{E}-07$ & 5E-05 & $8 \mathrm{E}-07$ & 7E-08 & 9E-08 & $1 \mathrm{E}-05$ & $1 \mathrm{E}-03$ & $5 \mathrm{E}-03$ & $1 \mathrm{E}-05$ & 2E-03 & $2 \mathrm{E}-08$ & $1 \mathrm{E}-07$ & $2 \mathrm{E}-08$ & 7E-08 & $1 \mathrm{E}-07$ & $2 \mathrm{E}-07$ \\
\hline 167 & 5E-09 & 4E-08 & $1 \mathrm{E}-07$ & 4E-05 & 7E-07 & 6E-08 & 7E-08 & 1E-05 & $1 \mathrm{E}-03$ & 4E-03 & 1E-05 & $2 \mathrm{E}-03$ & 1E-08 & $1 \mathrm{E}-07$ & $2 \mathrm{E}-08$ & $6 \mathrm{E}-08$ & $8 \mathrm{E}-08$ & $2 \mathrm{E}-07$ \\
\hline 215 & 3E-09 & $2 \mathrm{E}-08$ & $7 \mathrm{E}-08$ & 2E-05 & $4 \mathrm{E}-07$ & $3 \mathrm{E}-08$ & $4 \mathrm{E}-08$ & 6E-06 & $7 \mathrm{E}-04$ & $3 \mathrm{E}-03$ & 6E-06 & $1 \mathrm{E}-03$ & 8E-09 & $7 \mathrm{E}-08$ & $1 \mathrm{E}-08$ & 3E-08 & $5 \mathrm{E}-08$ & $1 \mathrm{E}-07$ \\
\hline 278 & 2E-09 & $1 \mathrm{E}-08$ & $5 \mathrm{E}-08$ & 2E-05 & 3E-07 & $2 \mathrm{E}-08$ & 3E-08 & 4E-06 & \begin{tabular}{l|l|}
$\mathrm{E}-04$ \\
\end{tabular} & $2 \mathrm{E}-03$ & $4 \mathrm{E}-06$ & 7E-04 & 5E-09 & 5E-08 & 7E-09 & 2E-08 & 3E-08 & 7E-08 \\
\hline 359 & 1E-09 & 9E-09 & $3 \mathrm{E}-08$ & $1 \mathrm{E}-05$ & 2E-07 & 1E-08 & $2 \mathrm{E}-08$ & 2E-06 & \begin{tabular}{|c|}
$3 \mathrm{E}-04$ \\
\end{tabular} & 1E-03 & 3E-06 & 5E-04 & 3E-09 & 3E-08 & 5E-09 & 1E-08 & 2E-08 & $4 \mathrm{E}-08$ \\
\hline 464 & $2 \mathrm{E}-09$ & $1 \mathrm{E}-08$ & $5 \mathrm{E}-08$ & 2E-05 & 3E-07 & 2E-08 & $3 \mathrm{E}-08$ & $4 \mathrm{E}-06$ & $4 \mathrm{E}-04$ & $2 \mathrm{E}-03$ & $4 \mathrm{E}-06$ & $7 \mathrm{E}-04$ & 5E-09 & $5 \mathrm{E}-08$ & 7E-09 & 2E-08 & $3 \mathrm{E}-08$ & 7E-08 \\
\hline 599 & 5E-11 & 4E-10 & 1E-09 & 4E-07 & 7E-09 & 6E-10 & $8 \mathrm{E}-10$ & $1 \mathrm{E}-07$ & 1E-05 & $4 \mathrm{E}-05$ & 1E-07 & $2 \mathrm{E}-05$ & $1 \mathrm{E}-10$ & 1E-09 & 2E-10 & 6E-10 & $8 \mathrm{E}-10$ & $2 \mathrm{E}-09$ \\
\hline 774 & 7E-10 & 4E-09 & 1E-08 & 5E-06 & $8 \mathrm{E}-08$ & 7E-09 & 9E-09 & 1E-06 & 1E-04 & 5E-04 & 1E-06 & $2 \mathrm{E}-04$ & 2E-09 & 1E-08 & 2E-09 & 7E-09 & 1E-08 & $2 \mathrm{E}-08$ \\
\hline 1000 & 3E-09 & $2 \mathrm{E}-08$ & 6E-08 & 2E-05 & 3E-07 & 3E-08 & $4 \mathrm{E}-08$ & 5E-06 & 6E-04 & $2 \mathrm{E}-03$ & 5E-06 & 9E-04 & 6E-09 & 6E-08 & 9E-09 & $3 \mathrm{E}-08$ & $4 \mathrm{E}-08$ & $8 \mathrm{E}-08$ \\
\hline
\end{tabular}


Table 3.43. (contd)

\begin{tabular}{|c|c|c|c|c|c|c|c|c|c|c|c|c|c|c|c|}
\hline Volume & & & & & & & & & & & & & & & \\
\hline Fraction & $2.3 \mathrm{E}-05$ & $1.4 \mathrm{E}-04$ & $1.1 \mathrm{E}-04$ & $7.1 \mathrm{E}-04$ & 4.4E-04 & $1.2 \mathrm{E}-05$ & $1.0 \mathrm{E}-02$ & $6.3 \mathrm{E}-08$ & $0.0 \mathrm{E}+00$ & $6.2 \mathrm{E}-03$ & $1.2 \mathrm{E}-04$ & $3.4 \mathrm{E}-04$ & $3.9 \mathrm{E}-04$ & $1.7 \mathrm{E}-04$ & $9.1 \mathrm{E}-05$ \\
\hline$d_{S}(\mu \mathrm{m})$ & $\mathrm{Na} 2(\mathrm{UO} 2) 2(\mathrm{PO} 4) 2.2 \mathrm{H} 2 \mathrm{O}$ & $\mathrm{Na} 2 \mathrm{U} 2 \mathrm{O} 7$ & NaAlCO3(OH)2 & NaAlSiO4 & $\mathrm{Ni}(\mathrm{OH}) 2$ & $\mathrm{~Pb}(\mathrm{OH}) 2$ & $\mathrm{Pu}(\mathrm{OH}) 4$ (co-precip. on Fe phase) & $\mathrm{PuO2}$ & Sr3(PO4)2 & SrCO3 & $\mathrm{ZrO} 2$ & $\mathrm{Na} 2 \mathrm{C} 2 \mathrm{O} 4$ & Na2CO3.H2O & $\mathrm{Na} 2 \mathrm{SO} 4.10 \mathrm{H} 2 \mathrm{O}$ & Na3FSO4 \\
\hline 0.22 & 8E-09 & $5 \mathrm{E}-08$ & $4 \mathrm{E}-08$ & $3 \mathrm{E}-07$ & $2 \mathrm{E}-07$ & $4 \mathrm{E}-09$ & $4 \mathrm{E}-06$ & $2 \mathrm{E}-11$ & $0 \mathrm{E}+00$ & $2 \mathrm{E}-06$ & $4 \mathrm{E}-08$ & $1 \mathrm{E}-07$ & $1 \mathrm{E}-07$ & $6 \mathrm{E}-08$ & $3 \mathrm{E}-08$ \\
\hline 0.28 & $1 \mathrm{E}-08$ & $8 \mathrm{E}-08$ & $6 \mathrm{E}-08$ & $4 \mathrm{E}-07$ & $2 \mathrm{E}-07$ & $7 \mathrm{E}-09$ & $5 \mathrm{E}-06$ & $3 \mathrm{E}-11$ & $0 \mathrm{E}+00$ & $3 \mathrm{E}-06$ & $7 \mathrm{E}-08$ & $2 \mathrm{E}-07$ & $2 \mathrm{E}-07$ & $9 \mathrm{E}-08$ & $5 \mathrm{E}-08$ \\
\hline 0.36 & $9 \mathrm{E}-08$ & $5 \mathrm{E}-07$ & $4 \mathrm{E}-07$ & $3 \mathrm{E}-06$ & $2 \mathrm{E}-06$ & $5 \mathrm{E}-08$ & $4 \mathrm{E}-05$ & $2 \mathrm{E}-10$ & $0 \mathrm{E}+00$ & $2 \mathrm{E}-05$ & $5 \mathrm{E}-07$ & $1 \mathrm{E}-06$ & $1 \mathrm{E}-06$ & $6 \mathrm{E}-07$ & $3 \mathrm{E}-07$ \\
\hline 0.46 & $1 \mathrm{E}-07$ & $8 \mathrm{E}-07$ & $6 \mathrm{E}-07$ & $4 \mathrm{E}-06$ & $2 \mathrm{E}-06$ & $7 \mathrm{E}-08$ & $5 \mathrm{E}-05$ & $3 \mathrm{E}-10$ & $0 \mathrm{E}+00$ & $3 \mathrm{E}-05$ & $6 \mathrm{E}-07$ & $2 \mathrm{E}-06$ & $2 \mathrm{E}-06$ & $9 \mathrm{E}-07$ & $5 \mathrm{E}-07$ \\
\hline 0.60 & $1 \mathrm{E}-07$ & $7 \mathrm{E}-07$ & $5 \mathrm{E}-07$ & $3 \mathrm{E}-06$ & $2 \mathrm{E}-06$ & $6 \mathrm{E}-08$ & $5 \mathrm{E}-05$ & $3 \mathrm{E}-10$ & $0 \mathrm{E}+00$ & $3 \mathrm{E}-05$ & \begin{tabular}{|c|}
$6 \mathrm{E}-07$ \\
\end{tabular} & $2 \mathrm{E}-06$ & $2 \mathrm{E}-06$ & $8 \mathrm{E}-07$ & $4 \mathrm{E}-07$ \\
\hline 0.77 & $6 \mathrm{E}-07$ & $3 \mathrm{E}-06$ & $3 \mathrm{E}-06$ & $2 \mathrm{E}-05$ & 1E-05 & 3E-07 & $2 \mathrm{E}-04$ & $2 \mathrm{E}-09$ & $0 \mathrm{E}+00$ & $1 \mathrm{E}-04$ & 3E-06 & $8 \mathrm{E}-06$ & 9E-06 & $4 \mathrm{E}-06$ & $2 \mathrm{E}-06$ \\
\hline 1 & 3E-07 & $2 \mathrm{E}-06$ & $2 \mathrm{E}-06$ & 1E-05 & $6 \mathrm{E}-06$ & $2 \mathrm{E}-07$ & $1 \mathrm{E}-04$ & $9 \mathrm{E}-10$ & $0 \mathrm{E}+00$ & $8 \mathrm{E}-05$ & $2 \mathrm{E}-06$ & $5 \mathrm{E}-06$ & $5 \mathrm{E}-06$ & $2 \mathrm{E}-06$ & $1 \mathrm{E}-06$ \\
\hline 1.3 & $1 \mathrm{E}-06$ & $6 \mathrm{E}-06$ & $5 \mathrm{E}-06$ & $3 \mathrm{E}-05$ & $2 \mathrm{E}-05$ & $5 \mathrm{E}-07$ & $4 \mathrm{E}-04$ & $3 \mathrm{E}-09$ & $0 \mathrm{E}+00$ & 3E-04 & $5 \mathrm{E}-06$ & $1 \mathrm{E}-05$ & $2 \mathrm{E}-05$ & $8 \mathrm{E}-06$ & $4 \mathrm{E}-06$ \\
\hline 1.7 & $8 \mathrm{E}-07$ & $5 \mathrm{E}-06$ & $4 \mathrm{E}-06$ & $3 \mathrm{E}-05$ & $2 \mathrm{E}-05$ & 4E-07 & $4 \mathrm{E}-04$ & $2 \mathrm{E}-09$ & $0 \mathrm{E}+00$ & $2 \mathrm{E}-04$ & $4 \mathrm{E}-06$ & $1 \mathrm{E}-05$ & $1 \mathrm{E}-05$ & $6 \mathrm{E}-06$ & $3 \mathrm{E}-06$ \\
\hline 2.2 & 1E-06 & $8 \mathrm{E}-06$ & 6E-06 & 4E-05 & 2E-05 & 7E-07 & $5 \mathrm{E}-04$ & $3 \mathrm{E}-09$ & $0 \mathrm{E}+00$ & $3 \mathrm{E}-04$ & 6E- -6 & $2 \mathrm{E}-05$ & 2E-05 & $9 \mathrm{E}-06$ & $5 \mathrm{E}-06$ \\
\hline 2.8 & 1E-06 & $8 \mathrm{E}-06$ & $6 \mathrm{E}-06$ & 4E-05 & 3E-05 & $7 \mathrm{E}-07$ & $6 \mathrm{E}-04$ & $4 \mathrm{E}-09$ & $0 \mathrm{E}+00$ & $4 \mathrm{E}-04$ & $7 \mathrm{E}-06$ & $2 \mathrm{E}-05$ & $2 \mathrm{E}-05$ & $1 \mathrm{E}-05$ & $5 \mathrm{E}-06$ \\
\hline 3.6 & 1E-06 & 9E-06 & $7 \mathrm{E}-06$ & 4E-05 & 3E-05 & $8 \mathrm{E}-07$ & $6 \mathrm{E}-04$ & $4 \mathrm{E}-09$ & $0 \mathrm{E}+00$ & 4E- 04 & 7E-06 & $2 \mathrm{E}-05$ & 2E-05 & $1 \mathrm{E}-05$ & $6 \mathrm{E}-06$ \\
\hline 4.6 & $2 \mathrm{E}-06$ & $1 \mathrm{E}-05$ & $8 \mathrm{E}-06$ & 5E-05 & $3 \mathrm{E}-05$ & $9 \mathrm{E}-07$ & $7 \mathrm{E}-04$ & $4 \mathrm{E}-09$ & $0 \mathrm{E}+00$ & 4E- 04 & $8 \mathrm{E}-06$ & $2 \mathrm{E}-05$ & 3E-05 & $1 \mathrm{E}-05$ & $6 \mathrm{E}-06$ \\
\hline 6.0 & $2 \mathrm{E}-06$ & $1 \mathrm{E}-05$ & \begin{tabular}{|c|}
$\mathrm{E}-06$ \\
\end{tabular} & $5 \mathrm{E}-05$ & $3 \mathrm{E}-05$ & $1 \mathrm{E}-06$ & $8 \mathrm{E}-04$ & 5E-09 & $0 \mathrm{E}+00$ & 5E-04 & 9E-06 & 3E-05 & 3E-05 & $1 \mathrm{E}-05$ & $7 \mathrm{E}-06$ \\
\hline 7.7 & $2 \mathrm{E}-06$ & $1 \mathrm{E}-05$ & $1 \mathrm{E}-05$ & $7 \mathrm{E}-05$ & $4 \mathrm{E}-05$ & $1 \mathrm{E}-06$ & $9 \mathrm{E}-04$ & $6 \mathrm{E}-09$ & $0 \mathrm{E}+00$ & $6 \mathrm{E}-04$ & $1 \mathrm{E}-05$ & $3 \mathrm{E}-05$ & 4E-05 & $2 \mathrm{E}-05$ & $8 \mathrm{E}-06$ \\
\hline 10 & $2 \mathrm{E}-06$ & $1 \mathrm{E}-05$ & \begin{tabular}{|c|}
$\mathrm{E}-06$ \\
\end{tabular} & $6 \mathrm{E}-05$ & $4 \mathrm{E}-05$ & $1 \mathrm{E}-06$ & $8 \mathrm{E}-04$ & $5 \mathrm{E}-09$ & $0 \mathrm{E}+00$ & 5E-04 & $1 \mathrm{E}-05$ & $3 \mathrm{E}-05$ & $3 \mathrm{E}-05$ & $1 \mathrm{E}-05$ & $8 \mathrm{E}-06$ \\
\hline 13 & $2 \mathrm{E}-06$ & $1 \mathrm{E}-05$ & $1 \mathrm{E}-05$ & $6 \mathrm{E}-05$ & $4 \mathrm{E}-05$ & $1 \mathrm{E}-06$ & $9 \mathrm{E}-04$ & $6 \mathrm{E}-09$ & $0 \mathrm{E}+00$ & $6 \mathrm{E}-04$ & $1 \mathrm{E}-05$ & 3E-05 & $3 \mathrm{E}-05$ & $2 \mathrm{E}-05$ & $8 \mathrm{E}-06$ \\
\hline 17 & $2 \mathrm{E}-06$ & $1 \mathrm{E}-05$ & $8 \mathrm{E}-06$ & 5E-05 & $3 \mathrm{E}-05$ & \begin{tabular}{|c|}
$\mathrm{E}-07$ \\
\end{tabular} & $7 \mathrm{E}-04$ & 5E-09 & $0 \mathrm{E}+00$ & $5 \mathrm{E}-04$ & 9E-06 & 3E-05 & $3 \mathrm{E}-05$ & $1 \mathrm{E}-05$ & $7 \mathrm{E}-06$ \\
\hline 22 & $1 \mathrm{E}-06$ & $9 \mathrm{E}-06$ & $7 \mathrm{E}-06$ & 4E-05 & $3 \mathrm{E}-05$ & $8 \mathrm{E}-07$ & $6 \mathrm{E}-04$ & $4 \mathrm{E}-09$ & $0 \mathrm{E}+00$ & 4E- 04 & 7E-06 & $2 \mathrm{E}-05$ & $2 \mathrm{E}-05$ & $1 \mathrm{E}-05$ & $6 \mathrm{E}-06$ \\
\hline 28 & $9 \mathrm{E}-07$ & $6 \mathrm{E}-06$ & $4 \mathrm{E}-06$ & $3 \mathrm{E}-05$ & $2 \mathrm{E}-05$ & $5 \mathrm{E}-07$ & $4 \mathrm{E}-04$ & $2 \mathrm{E}-09$ & $0 \mathrm{E}+00$ & 2E-04 & $5 \mathrm{E}-06$ & $1 \mathrm{E}-05$ & $2 \mathrm{E}-05$ & $7 \mathrm{E}-06$ & $4 \mathrm{E}-06$ \\
\hline 36 & $7 \mathrm{E}-07$ & $4 \mathrm{E}-06$ & $3 \mathrm{E}-06$ & $2 \mathrm{E}-05$ & $1 \mathrm{E}-05$ & $4 \mathrm{E}-07$ & $3 \mathrm{E}-04$ & $2 \mathrm{E}-09$ & $0 \mathrm{E}+00$ & $2 \mathrm{E}-04$ & $3 \mathrm{E}-06$ & $1 \mathrm{E}-05$ & $1 \mathrm{E}-05$ & $5 E-06$ & $3 \mathrm{E}-06$ \\
\hline 46 & $4 \mathrm{E}-07$ & $3 \mathrm{E}-06$ & $2 \mathrm{E}-06$ & $1 \mathrm{E}-05$ & $8 \mathrm{E}-06$ & $2 \mathrm{E}-07$ & $2 \mathrm{E}-04$ & $1 \mathrm{E}-09$ & $0 \mathrm{E}+00$ & $1 \mathrm{E}-04$ & $2 \mathrm{E}-06$ & $6 \mathrm{E}-06$ & $7 \mathrm{E}-06$ & $3 \mathrm{E}-06$ & $2 \mathrm{E}-06$ \\
\hline 60 & 3E-07 & 2E-06 & $1 \mathrm{E}-06$ & 8E-06 & 5E-06 & $1 \mathrm{E}-07$ & $1 \mathrm{E}-04$ & 7E-10 & $0 \mathrm{E}+00$ & $7 \mathrm{E}-05$ & 1E-06 & 4E-06 & 5E-06 & $2 \mathrm{E}-06$ & $1 \mathrm{E}-06$ \\
\hline 77 & $3 \mathrm{E}-07$ & $2 \mathrm{E}-06$ & $1 \mathrm{E}-06$ & $8 \mathrm{E}-06$ & 5E-06 & 1E-07 & $1 \mathrm{E}-04$ & $7 \mathrm{E}-10$ & $0 \mathrm{E}+00$ & $7 \mathrm{E}-05$ & 1E-06 & 4E-06 & 5E-06 & $2 \mathrm{E}-06$ & $1 \mathrm{E}-06$ \\
\hline 100 & $3 \mathrm{E}-07$ & $2 \mathrm{E}-06$ & $1 \mathrm{E}-06$ & $9 \mathrm{E}-06$ & $6 \mathrm{E}-06$ & $2 \mathrm{E}-07$ & $1 \mathrm{E}-04$ & $8 \mathrm{E}-10$ & $0 \mathrm{E}+00$ & $8 \mathrm{E}-05$ & 2E-06 & \begin{tabular}{|c|}
$\mathrm{E}-06$ \\
\end{tabular} & $5 \mathrm{E}-06$ & $2 \mathrm{E}-06$ & $1 \mathrm{E}-06$ \\
\hline 129 & $2 \mathrm{E}-07$ & $1 \mathrm{E}-06$ & $1 \mathrm{E}-06$ & $7 \mathrm{E}-06$ & $4 \mathrm{E}-06$ & 1E-07 & $9 \mathrm{E}-05$ & $6 \mathrm{E}-10$ & $0 \mathrm{E}+00$ & $6 \mathrm{E}-05$ & $1 \mathrm{E}-06$ & $3 \mathrm{E}-06$ & $4 \mathrm{E}-06$ & $2 \mathrm{E}-06$ & $8 \mathrm{E}-07$ \\
\hline 167 & $2 \mathrm{E}-07$ & $1 \mathrm{E}-06$ & $9 \mathrm{E}-07$ & $6 \mathrm{E}-06$ & $3 \mathrm{E}-06$ & $1 \mathrm{E}-07$ & $8 \mathrm{E}-05$ & $5 \mathrm{E}-10$ & $0 \mathrm{E}+00$ & 5E-05 & $9 \mathrm{E}-07$ & $3 \mathrm{E}-06$ & $3 \mathrm{E}-06$ & $1 \mathrm{E}-06$ & $7 \mathrm{E}-07$ \\
\hline 215 & $1 \mathrm{E}-07$ & $6 \mathrm{E}-07$ & $5 \mathrm{E}-07$ & $3 \mathrm{E}-06$ & $2 \mathrm{E}-06$ & $6 \mathrm{E}-08$ & $4 \mathrm{E}-05$ & $3 \mathrm{E}-10$ & $0 \mathrm{E}+00$ & $3 \mathrm{E}-05$ & $5 \mathrm{E}-07$ & $2 \mathrm{E}-06$ & $2 \mathrm{E}-06$ & $8 \mathrm{E}-07$ & $4 \mathrm{E}-07$ \\
\hline 278 & $7 \mathrm{E}-08$ & $4 \mathrm{E}-07$ & $3 \mathrm{E}-07$ & $2 \mathrm{E}-06$ & $1 \mathrm{E}-06$ & $4 \mathrm{E}-08$ & $3 \mathrm{E}-05$ & $2 \mathrm{E}-10$ & $0 \mathrm{E}+00$ & $2 \mathrm{E}-05$ & 4E- 07 & $1 \mathrm{E}-06$ & 1E-06 & 5E-07 & 3E-07 \\
\hline 359 & 4E-08 & 3E-07 & $2 \mathrm{E}-07$ & 1E-06 & $8 \mathrm{E}-07$ & 2E-08 & $2 \mathrm{E}-05$ & $1 \mathrm{E}-10$ & $0 \mathrm{E}+00$ & 1E-05 & $2 \mathrm{E}-07$ & $7 \mathrm{E}-07$ & $7 \mathrm{E}-07$ & $3 \mathrm{E}-07$ & $2 \mathrm{E}-07$ \\
\hline 464 & $7 \mathrm{E}-08$ & $4 \mathrm{E}-07$ & \begin{tabular}{|c|}
$\mathrm{E}-07$ \\
\end{tabular} & $2 \mathrm{E}-06$ & $1 \mathrm{E}-06$ & 4E-08 & $3 \mathrm{E}-05$ & $2 \mathrm{E}-10$ & $0 \mathrm{E}+00$ & $2 \mathrm{E}-05$ & 4E- 07 & $1 \mathrm{E}-06$ & $1 \mathrm{E}-06$ & $5 \mathrm{E}-07$ & $3 \mathrm{E}-07$ \\
\hline 599 & $2 \mathrm{E}-09$ & $1 \mathrm{E}-08$ & $9 \mathrm{E}-09$ & $6 \mathrm{E}-08$ & $3 \mathrm{E}-08$ & 1E-09 & $8 \mathrm{E}-07$ & $5 \mathrm{E}-12$ & $0 \mathrm{E}+00$ & $5 \mathrm{E}-07$ & 9E-09 & $3 \mathrm{E}-08$ & $3 \mathrm{E}-08$ & $1 \mathrm{E}-08$ & $7 \mathrm{E}-09$ \\
\hline 774 & $2 \mathrm{E}-08$ & $1 \mathrm{E}-07$ & $1 \mathrm{E}-07$ & $7 \mathrm{E}-07$ & $4 \mathrm{E}-07$ & $1 \mathrm{E}-08$ & $9 \mathrm{E}-06$ & $6 \mathrm{E}-11$ & $0 \mathrm{E}+00$ & $6 \mathrm{E}-06$ & $1 \mathrm{E}-07$ & $3 \mathrm{E}-07$ & $4 \mathrm{E}-07$ & $2 \mathrm{E}-07$ & $9 \mathrm{E}-08$ \\
\hline 1000 & $9 \mathrm{E}-08$ & $5 \mathrm{E}-07$ & $4 \mathrm{E}-07$ & $3 \mathrm{E}-06$ & $2 \mathrm{E}-06$ & $5 \mathrm{E}-08$ & $4 \mathrm{E}-05$ & $2 \mathrm{E}-10$ & $0 \mathrm{E}+00$ & $2 \mathrm{E}-05$ & $4 \mathrm{E}-07$ & $1 \mathrm{E}-06$ & $1 \mathrm{E}-06$ & $6 \mathrm{E}-07$ & $3 \mathrm{E}-07$ \\
\hline
\end{tabular}


Table 3.43. (contd)

\begin{tabular}{|c|c|c|c|c|c|c|c|c|}
\hline \multicolumn{9}{|l|}{ Volume } \\
\hline Fraction & $6.3 \mathrm{E}-07$ & $2.4 \mathrm{E}-04$ & $8.5 \mathrm{E}-05$ & $4.2 \mathrm{E}-05$ & $4.3 \mathrm{E}-04$ & $7.6 \mathrm{E}-04$ & $3.6 \mathrm{E}-06$ & $2.0 \mathrm{E}-03$ \\
\hline$d_{S}(\mu \mathrm{m})$ & Na3NO3SO4.H2O & $\mathrm{Na} 3 \mathrm{PO} 4.0 .25 \mathrm{NaOH} .12 \mathrm{H} 2 \mathrm{O}$ & Na4P2O7.10H2O & Na6(SO4)2CO3 & $\mathrm{NaF}$ & $\mathrm{Na} 7 \mathrm{~F}(\mathrm{PO} 4) 2.19 \mathrm{H} 2 \mathrm{O}$ & NaNO2 & NaNO3 \\
\hline 0.22 & $2 \mathrm{E}-10$ & $9 \mathrm{E}-08$ & $3 \mathrm{E}-08$ & $1 \mathrm{E}-08$ & $2 \mathrm{E}-07$ & $3 \mathrm{E}-07$ & $1 \mathrm{E}-09$ & $7 \mathrm{E}-07$ \\
\hline 0.28 & $3 \mathrm{E}-10$ & $1 \mathrm{E}-07$ & $5 \mathrm{E}-08$ & $2 \mathrm{E}-08$ & $2 \mathrm{E}-07$ & $4 \mathrm{E}-07$ & $2 \mathrm{E}-09$ & $1 \mathrm{E}-06$ \\
\hline 0.36 & $2 \mathrm{E}-09$ & 9E-07 & $3 \mathrm{E}-07$ & $2 \mathrm{E}-07$ & $2 \mathrm{E}-06$ & $3 \mathrm{E}-06$ & $1 \mathrm{E}-08$ & $7 \mathrm{E}-06$ \\
\hline 0.46 & $3 \mathrm{E}-09$ & $1 \mathrm{E}-06$ & $4 \mathrm{E}-07$ & $2 \mathrm{E}-07$ & $2 \mathrm{E}-06$ & $4 \mathrm{E}-06$ & $2 \mathrm{E}-08$ & $1 \mathrm{E}-05$ \\
\hline 0.60 & $3 \mathrm{E}-09$ & $1 \mathrm{E}-06$ & $4 \mathrm{E}-07$ & $2 \mathrm{E}-07$ & $2 \mathrm{E}-06$ & $4 \mathrm{E}-06$ & $2 \mathrm{E}-08$ & $9 \mathrm{E}-06$ \\
\hline 0.77 & $1 \mathrm{E}-08$ & 6E-06 & $2 \mathrm{E}-06$ & $1 \mathrm{E}-06$ & $1 \mathrm{E}-05$ & $2 \mathrm{E}-05$ & $9 \mathrm{E}-08$ & $5 \mathrm{E}-05$ \\
\hline 1 & $8 \mathrm{E}-09$ & $3 \mathrm{E}-06$ & $1 \mathrm{E}-06$ & $6 \mathrm{E}-07$ & $6 \mathrm{E}-06$ & $1 \mathrm{E}-05$ & $5 \mathrm{E}-08$ & $3 \mathrm{E}-05$ \\
\hline 1.3 & $3 \mathrm{E}-08$ & $1 \mathrm{E}-05$ & $4 \mathrm{E}-06$ & $2 \mathrm{E}-06$ & $2 \mathrm{E}-05$ & $3 \mathrm{E}-05$ & $2 \mathrm{E}-07$ & $9 \mathrm{E}-05$ \\
\hline 1.7 & $2 \mathrm{E}-08$ & $8 \mathrm{E}-06$ & $3 \mathrm{E}-06$ & $1 \mathrm{E}-06$ & $2 \mathrm{E}-05$ & $3 \mathrm{E}-05$ & $1 \mathrm{E}-07$ & $7 \mathrm{E}-05$ \\
\hline 2.2 & $3 \mathrm{E}-08$ & $1 \mathrm{E}-05$ & $4 \mathrm{E}-06$ & $2 \mathrm{E}-06$ & $2 \mathrm{E}-05$ & $4 \mathrm{E}-05$ & $2 \mathrm{E}-07$ & $1 \mathrm{E}-04$ \\
\hline 2.8 & $4 \mathrm{E}-08$ & $1 \mathrm{E}-05$ & $5 \mathrm{E}-06$ & $2 \mathrm{E}-06$ & $2 \mathrm{E}-05$ & $4 \mathrm{E}-05$ & $2 \mathrm{E}-07$ & $1 \mathrm{E}-04$ \\
\hline 3.6 & $4 \mathrm{E}-08$ & $1 \mathrm{E}-05$ & $5 \mathrm{E}-06$ & $3 \mathrm{E}-06$ & $3 \mathrm{E}-05$ & 5E-05 & $2 \mathrm{E}-07$ & $1 \mathrm{E}-04$ \\
\hline 4.6 & $4 \mathrm{E}-08$ & $2 \mathrm{E}-05$ & $6 \mathrm{E}-06$ & $3 \mathrm{E}-06$ & $3 \mathrm{E}-05$ & 5E-05 & $3 \mathrm{E}-07$ & $1 \mathrm{E}-04$ \\
\hline 6.0 & $5 \mathrm{E}-08$ & $2 \mathrm{E}-05$ & $7 \mathrm{E}-06$ & $3 \mathrm{E}-06$ & $3 \mathrm{E}-05$ & $6 \mathrm{E}-05$ & $3 \mathrm{E}-07$ & $2 \mathrm{E}-04$ \\
\hline 7.7 & $6 \mathrm{E}-08$ & $2 \mathrm{E}-05$ & $8 \mathrm{E}-06$ & $4 \mathrm{E}-06$ & $4 \mathrm{E}-05$ & 7E-05 & $3 \mathrm{E}-07$ & $2 \mathrm{E}-04$ \\
\hline 10 & $5 \mathrm{E}-08$ & $2 \mathrm{E}-05$ & $7 \mathrm{E}-06$ & $3 \mathrm{E}-06$ & $3 \mathrm{E}-05$ & 6E-05 & $3 \mathrm{E}-07$ & $2 \mathrm{E}-04$ \\
\hline 13 & $6 \mathrm{E}-08$ & $2 \mathrm{E}-05$ & $8 \mathrm{E}-06$ & $4 \mathrm{E}-06$ & $4 \mathrm{E}-05$ & $7 \mathrm{E}-05$ & $3 \mathrm{E}-07$ & $2 \mathrm{E}-04$ \\
\hline 17 & $5 \mathrm{E}-08$ & $2 \mathrm{E}-05$ & $6 \mathrm{E}-06$ & $3 \mathrm{E}-06$ & $3 \mathrm{E}-05$ & 6E-05 & $3 \mathrm{E}-07$ & $1 \mathrm{E}-04$ \\
\hline 22 & $4 \mathrm{E}-08$ & $1 \mathrm{E}-05$ & $5 \mathrm{E}-06$ & $3 \mathrm{E}-06$ & $3 \mathrm{E}-05$ & 5E-05 & $2 \mathrm{E}-07$ & $1 \mathrm{E}-04$ \\
\hline 28 & $2 \mathrm{E}-08$ & $9 \mathrm{E}-06$ & $3 \mathrm{E}-06$ & $2 \mathrm{E}-06$ & $2 \mathrm{E}-05$ & 3E-05 & $1 \mathrm{E}-07$ & $8 \mathrm{E}-05$ \\
\hline 36 & $2 \mathrm{E}-08$ & $7 \mathrm{E}-06$ & $2 \mathrm{E}-06$ & $1 \mathrm{E}-06$ & $1 \mathrm{E}-05$ & 2E-05 & $1 \mathrm{E}-07$ & $6 \mathrm{E}-05$ \\
\hline 46 & $1 \mathrm{E}-08$ & \begin{tabular}{|l|}
$\mathrm{E}-06$ \\
\end{tabular} & $2 \mathrm{E}-06$ & $8 \mathrm{E}-07$ & $8 \mathrm{E}-06$ & $1 \mathrm{E}-05$ & $7 \mathrm{E}-08$ & $4 \mathrm{E}-05$ \\
\hline 60 & $7 \mathrm{E}-09$ & $3 \mathrm{E}-06$ & $1 \mathrm{E}-06$ & $5 \mathrm{E}-07$ & $5 \mathrm{E}-06$ & 9E-06 & $4 \mathrm{E}-08$ & $2 \mathrm{E}-05$ \\
\hline 77 & $7 \mathrm{E}-09$ & 3E-06 & $1 \mathrm{E}-06$ & $5 \mathrm{E}-07$ & $5 \mathrm{E}-06$ & 9E-06 & $4 \mathrm{E}-08$ & $2 \mathrm{E}-05$ \\
\hline 100 & $8 \mathrm{E}-09$ & \begin{tabular}{|c|}
$\mathrm{E}-06$ \\
\end{tabular} & $1 \mathrm{E}-06$ & $5 \mathrm{E}-07$ & $5 \mathrm{E}-06$ & $1 \mathrm{E}-05$ & $5 \mathrm{E}-08$ & $3 \mathrm{E}-05$ \\
\hline 129 & 6E-09 & $2 \mathrm{E}-06$ & $8 \mathrm{E}-07$ & $4 \mathrm{E}-07$ & $4 \mathrm{E}-06$ & $7 \mathrm{E}-06$ & $3 \mathrm{E}-08$ & $2 \mathrm{E}-05$ \\
\hline 167 & $5 \mathrm{E}-09$ & $2 \mathrm{E}-06$ & $7 \mathrm{E}-07$ & $3 \mathrm{E}-07$ & $3 \mathrm{E}-06$ & 6E-06 & $3 \mathrm{E}-08$ & $2 \mathrm{E}-05$ \\
\hline 215 & $3 \mathrm{E}-09$ & $1 \mathrm{E}-06$ & $4 \mathrm{E}-07$ & $2 \mathrm{E}-07$ & $2 \mathrm{E}-06$ & $3 \mathrm{E}-06$ & $2 \mathrm{E}-08$ & $9 \mathrm{E}-06$ \\
\hline 278 & $2 \mathrm{E}-09$ & 7E-07 & 2E-07 & $1 \mathrm{E}-07$ & $1 \mathrm{E}-06$ & $2 \mathrm{E}-06$ & $1 \mathrm{E}-08$ & $6 \mathrm{E}-06$ \\
\hline 359 & $1 \mathrm{E}-09$ & 5E-07 & $2 \mathrm{E}-07$ & $8 \mathrm{E}-08$ & $8 \mathrm{E}-07$ & $1 \mathrm{E}-06$ & 7E-09 & $4 \mathrm{E}-06$ \\
\hline 464 & $2 \mathrm{E}-09$ & $7 \mathrm{E}-07$ & 2E-07 & $1 \mathrm{E}-07$ & $1 \mathrm{E}-06$ & $2 \mathrm{E}-06$ & $1 \mathrm{E}-08$ & $6 \mathrm{E}-06$ \\
\hline 599 & $5 \mathrm{E}-11$ & $2 \mathrm{E}-08$ & 7E-09 & 3E-09 & $3 \mathrm{E}-08$ & $6 \mathrm{E}-08$ & $3 \mathrm{E}-10$ & $2 \mathrm{E}-07$ \\
\hline \begin{tabular}{|l|}
774 \\
\end{tabular} & $6 \mathrm{E}-10$ & $2 \mathrm{E}-07$ & $8 \mathrm{E}-08$ & $4 \mathrm{E}-08$ & $4 \mathrm{E}-07$ & $7 \mathrm{E}-07$ & $3 \mathrm{E}-09$ & $2 \mathrm{E}-06$ \\
\hline 1000 & 2E-09 & 9E-07 & 3E-07 & $2 \mathrm{E}-07$ & $2 \mathrm{E}-06$ & 3E-06 & 1E-08 & $7 \mathrm{E}-06$ \\
\hline
\end{tabular}


Table 3.44. Sludge, Flowing Unsonicated PSDD Density, Fractal Dimension 1.6

\begin{tabular}{|c|c|c|c|c|c|c|c|c|c|c|c|c|c|c|c|c|c|c|}
\hline $\mathrm{d}_{\mathrm{s}}(\mu \mathrm{m})$ & $\mathrm{Ag}$ & Ag2O & $\mathrm{Bi} 2 \mathrm{O} 3$ & $\mathrm{BiFeO3}$ & $\mathrm{Ca} 5 \mathrm{OH}(\mathrm{PO} 4) 3$ & $\mathrm{CaCO} 3$ & $\mathrm{CaF} 2$ & $\mathrm{CrOOH}$ & $\mathrm{FePO} 4.2 \mathrm{H} 2 \mathrm{O}$ & |FeOOH & Gibbsite & Boehmite & $\mathrm{HgO}$ & KAlSiO4 & \begin{tabular}{|l|}
$\mathrm{La}(\mathrm{OH}) 3$ \\
\end{tabular} & $\mathrm{LaPO} 4 \cdot 2 \mathrm{H} 2 \mathrm{O}$ & $\mathrm{Mn3(PO4)2}$ & $\mathrm{MnO2}$ \\
\hline 0.22 & 10.50 & 7.14 & 8.90 & 2.04 & 3.14 & 2.71 & 3.18 & 4.11 & 1.20 & 1.20 & 2.42 & 1.27 & 7.14 & 2.61 & 2.30 & 6.51 & 3.10 & 5.03 \\
\hline 0.28 & 10.50 & 7.14 & 8.90 & 1.78 & 3.14 & 2.71 & 3.18 & 3.02 & 1.19 & 1.19 & 2.42 & 1.24 & 7.14 & 2.61 & 2.30 & 6.51 & 3.10 & 5.03 \\
\hline 0.36 & 10.50 & 7.14 & 8.90 & 1.60 & 3.14 & 2.71 & 3.18 & 2.46 & 1.18 & 1.18 & 2.42 & 1.22 & 7.14 & 2.61 & 2.30 & 6.51 & 3.10 & 5.03 \\
\hline 0.46 & 10.50 & 7.14 & 8.90 & 1.47 & 3.14 & 2.71 & 3.18 & 2.07 & 1.18 & 1.18 & 2.42 & 1.20 & 7.14 & 2.61 & 2.30 & 6.51 & 3.10 & 5.03 \\
\hline 0.60 & 10.50 & 7.14 & 8.90 & 1.38 & 3.14 & 2.71 & 3.18 & 1.80 & 1.18 & 1.18 & 2.42 & 1.19 & 7.14 & 2.61 & 2.30 & 6.51 & 3.10 & 5.03 \\
\hline 0.77 & 10.50 & 7.14 & 8.90 & 1.32 & 3.14 & 2.71 & 3.18 & 1.61 & 1.17 & 1.17 & 2.42 & 1.19 & 7.14 & 2.61 & 2.30 & 6.51 & 3.10 & 5.03 \\
\hline 1 & 10.50 & 7.14 & 8.90 & 1.27 & 3.14 & 2.71 & 3.18 & 1.48 & 1.17 & 1.17 & 2.42 & 1.18 & 7.14 & 2.61 & 2.30 & 6.51 & 3.10 & 5.03 \\
\hline 1.3 & 10.50 & 7.14 & 8.90 & 1.24 & 3.14 & 2.71 & 3.18 & 1.39 & 1.17 & 1.17 & 2.42 & 1.18 & 7.14 & 2.61 & 2.30 & 6.51 & 3.10 & 5.03 \\
\hline 1.7 & 5.73 & 4.09 & 8.90 & 1.22 & 3.14 & 2.71 & 3.18 & 1.32 & 1.17 & 1.17 & 2.42 & 1.18 & 4.09 & 2.61 & 2.30 & 6.51 & 3.10 & 5.03 \\
\hline 2.2 & 4.36 & 3.21 & 5.83 & 1.20 & 3.14 & 2.71 & 3.18 & 1.28 & 1.17 & \begin{tabular}{|l|}
1.17 \\
\end{tabular} & 2.42 & 1.17 & \begin{tabular}{|l|}
3.21 \\
\end{tabular} & 2.61 & 1.85 & 4.39 & 3.10 & 5.03 \\
\hline 2.8 & 3.40 & 2.60 & 4.42 & 1.19 & 3.14 & 2.71 & 3.18 & 1.24 & 1.17 & 1.17 & 2.42 & 1.17 & 2.60 & 2.61 & 1.65 & 3.42 & 3.10 & 5.03 \\
\hline 3.6 & 2.73 & 2.17 & 3.44 & 1.19 & 3.14 & 2.71 & 3.18 & 1.22 & 1.17 & 1.17 & 2.42 & 1.17 & 2.17 & 2.61 & 1.50 & 2.74 & 3.10 & 5.03 \\
\hline 4.6 & 2.26 & 1.87 & 2.76 & 1.18 & 3.14 & 2.71 & 3.18 & 1.21 & 1.17 & 1.17 & 2.42 & 1.17 & 1.87 & 2.61 & 1.40 & 2.27 & 3.10 & 5.03 \\
\hline 6.0 & 1.93 & 1.66 & 2.28 & 1.18 & 2.49 & 2.71 & 3.18 & 1.20 & 1.17 & 1.17 & 2.42 & 1.17 & 1.66 & 1.99 & 1.33 & 1.94 & 2.27 & 5.03 \\
\hline 7.7 & 1.70 & 1.51 & 1.95 & 1.18 & 2.09 & 2.71 & 3.18 & 1.19 & 1.17 & 1.17 & 2.42 & 1.17 & 1.51 & 1.74 & 1.28 & 1.71 & 1.94 & 3.26 \\
\hline 10 & 1.54 & 1.41 & 1.71 & 1.17 & 1.81 & 2.71 & 2.51 & 1.18 & 1.17 & 1.17 & 2.42 & 1.17 & 1.41 & 1.57 & 1.25 & 1.55 & 1.71 & 2.63 \\
\hline 13 & 1.43 & 1.34 & 1.55 & 1.17 & 1.62 & 2.71 & 2.11 & 1.18 & 1.17 & 1.17 & 2.42 & 1.17 & 1.34 & 1.45 & 1.23 & 1.43 & 1.54 & 2.19 \\
\hline 17 & 1.35 & 1.29 & 1.44 & 1.17 & 1.48 & 2.71 & 1.83 & 1.18 & 1.17 & \begin{tabular}{|c|}
1.17 \\
\end{tabular} & 2.42 & 1.17 & \begin{tabular}{|c|}
1.29 \\
\end{tabular} & 1.37 & 1.21 & 1.35 & 1.43 & 1.88 \\
\hline 22 & 1.30 & 1.25 & 1.36 & 1.17 & 1.39 & 2.71 & 1.63 & 1.17 & 1.17 & 1.17 & 2.42 & 1.17 & 1.25 & 1.31 & 1.20 & 1.30 & 1.35 & 1.67 \\
\hline 28 & 1.26 & 1.23 & 1.30 & 1.17 & 1.32 & 2.71 & 1.49 & 1.17 & 1.17 & 1.17 & 2.42 & 1.17 & 1.23 & 1.27 & 1.19 & 1.26 & 1.30 & 1.52 \\
\hline 36 & 1.23 & 1.21 & 1.26 & 1.17 & 1.28 & 2.23 & 1.39 & 1.17 & 1.17 & 1.17 & 2.42 & 1.17 & 1.21 & 1.24 & 1.18 & 1.23 & 1.26 & 1.41 \\
\hline 46 & 1.21 & 1.20 & 1.23 & 1.17 & 1.25 & 1.91 & 1.33 & 1.17 & 1.17 & 1.17 & 2.42 & 1.17 & 1.20 & 1.22 & 1.18 & 1.21 & 1.23 & 1.34 \\
\hline 60 & 1.20 & 1.19 & 1.21 & 1.17 & 1.22 & 1.69 & 1.28 & 1.17 & 1.17 & 1.17 & 2.42 & 1.17 & \begin{tabular}{|c|}
1.19 \\
\end{tabular} & 1.20 & 1.18 & 1.20 & 1.21 & 1.29 \\
\hline 77 & 1.19 & 1.18 & 1.20 & 1.17 & 1.21 & 1.53 & 1.25 & 1.17 & 1.17 & 1.17 & 2.42 & 1.17 & 1.18 & 1.19 & 1.17 & 1.19 & 1.20 & 1.25 \\
\hline 100 & 1.18 & 1.18 & \begin{tabular}{|c|}
1.19 \\
\end{tabular} & 1.17 & 1.20 & 1.42 & 1.22 & 1.17 & 1.17 & 1.17 & 2.42 & 1.17 & $\mid$\begin{tabular}{|l|}
1.18 \\
\end{tabular} & 1.19 & $\begin{array}{ll}1.17 \\
\end{array}$ & 1.18 & 1.19 & 1.23 \\
\hline 129 & 1.18 & 1.18 & \begin{tabular}{|c|}
1.19 \\
\end{tabular} & 1.17 & 1.19 & 1.35 & 1.21 & 1.17 & 1.17 & 1.17 & 2.42 & 1.17 & \begin{tabular}{|c|}
1.18 \\
\end{tabular} & 1.18 & 1.17 & 1.18 & 1.18 & 1.21 \\
\hline 167 & 1.18 & 1.17 & 1.18 & 1.17 & 1.18 & 1.29 & 1.20 & 1.17 & 1.17 & 1.17 & 1.78 & 1.17 & 1.17 & 1.18 & 1.17 & 1.18 & 1.18 & 1.20 \\
\hline 215 & 1.18 & 1.17 & 1.18 & 1.17 & 1.18 & 1.26 & 1.19 & 1.17 & 1.17 & 1.17 & 1.60 & 1.17 & 1.17 & 1.18 & 1.17 & 1.18 & 1.18 & 1.19 \\
\hline 278 & 1.17 & 1.17 & \begin{tabular}{|c|}
1.18 \\
\end{tabular} & 1.17 & 1.18 & 1.23 & 1.18 & 1.17 & 1.17 & 1.17 & 1.47 & 1.17 & \begin{tabular}{|c|}
1.17 \\
\end{tabular} & 1.17 & 1.17 & 1.17 & 1.18 & 1.18 \\
\hline 359 & 1.17 & 1.17 & 1.17 & 1.17 & 1.17 & 1.21 & 1.18 & 1.17 & 1.17 & 1.17 & 1.38 & 1.17 & 1.17 & 1.17 & 1.17 & 1.17 & 1.17 & 1.18 \\
\hline 464 & 1.17 & 1.17 & 1.17 & 1.17 & 1.17 & 1.20 & 1.18 & 1.17 & 1.17 & 1.17 & 1.32 & 1.17 & 1.17 & 1.17 & 1.17 & 1.17 & 1.17 & 1.18 \\
\hline 599 & 1.17 & 1.17 & 1.17 & 1.17 & 1.17 & 1.19 & 1.17 & 1.17 & 1.17 & 1.17 & 1.27 & 1.17 & 1.17 & 1.17 & 1.17 & 1.17 & 1.17 & 1.17 \\
\hline 774 & 1.17 & 1.17 & 1.17 & 1.17 & 1.17 & 1.18 & 1.17 & 1.17 & 1.17 & 1.17 & 1.24 & 1.17 & 1.17 & 1.17 & 1.17 & 1.17 & 1.17 & 1.17 \\
\hline 1000 & 1.17 & 1.17 & 1.17 & 1.17 & 1.17 & 1.18 & 1.17 & 1.17 & 1.17 & 1.17 & 1.22 & 1.17 & 1.17 & 1.17 & 1.17 & 1.17 & 1.17 & 1.17 \\
\hline
\end{tabular}


Table 3.44. (contd)

\begin{tabular}{|c|c|c|c|c|c|c|c|c|c|c|c|c|c|c|c|}
\hline $\mathrm{d}_{\mathrm{s}}(\mu \mathrm{m})$ & $\mathrm{Na} 2(\mathrm{UO} 2) 2(\mathrm{PO} 4) 2.2 \mathrm{H} 2 \mathrm{O}$ & \begin{tabular}{|l|}
$\mathrm{Na} 2 \mathrm{U} 2 \mathrm{O} 7$ \\
\end{tabular} & $\mathrm{NaAlCO}(\mathrm{OH}) 2$ & $\mathrm{NaAlSiO4}$ & $\mathrm{Ni}(\mathrm{OH}) 2$ & $\mathrm{~Pb}(\mathrm{OH}) 2$ & $\mathrm{Pu}(\mathrm{OH}) 4$ (co-precip. on Fe phase) & PuO2 & Sr3(PO4)2 & SrCO3 & $\mathrm{ZrO} 2$ & $\mathrm{Na} 2 \mathrm{C} 2 \mathrm{O} 4$ & Na2CO3.H2O & $\mathrm{Na} 2 \mathrm{SO} 4.10 \mathrm{H} 2 \mathrm{O}$ & Na3FSO4 \\
\hline 0.22 & 3.50 & 5.62 & 2.42 & 2.37 & 4.15 & 7.10 & 1.20 & 11.43 & 1.33 & 1.33 & 5.68 & 2.34 & 2.25 & 1.46 & 2.65 \\
\hline 0.28 & 3.50 & 5.62 & 2.42 & 2.37 & 4.15 & 7.10 & 1.19 & 11.43 & 1.29 & 1.29 & 5.68 & 2.34 & 2.25 & 1.46 & 2.65 \\
\hline 0.36 & 3.50 & 5.62 & 2.42 & 2.37 & 2.96 & 7.10 & 1.18 & 11.43 & 1.25 & 1.25 & 5.68 & 2.34 & 2.25 & 1.46 & 2.65 \\
\hline 0.46 & 3.50 & 5.62 & 2.42 & 2.37 & 2.42 & 7.10 & 1.18 & 11.43 & 1.23 & 1.23 & 5.68 & 2.34 & 2.25 & 1.46 & 2.65 \\
\hline 0.60 & 3.50 & 5.62 & 2.42 & 2.37 & 2.05 & 7.10 & 1.18 & 11.43 & 1.21 & 1.21 & 5.68 & 2.34 & 2.25 & 1.46 & 2.65 \\
\hline 0.77 & 3.50 & 5.62 & 2.42 & 2.37 & 1.78 & 7.10 & 1.17 & 11.43 & 1.20 & 1.20 & 5.68 & 2.34 & 2.25 & 1.46 & 2.65 \\
\hline 1 & 3.50 & 5.62 & 2.42 & 2.37 & 1.60 & 7.10 & 1.17 & 11.43 & 1.19 & 1.19 & 5.68 & 2.34 & 2.25 & 1.46 & 2.65 \\
\hline 1.3 & 3.50 & 5.62 & 2.42 & 2.37 & 1.47 & 7.10 & 1.17 & 11.43 & 1.18 & 1.18 & 5.68 & 2.34 & 2.25 & 1.46 & 2.65 \\
\hline 1.7 & 3.50 & 5.62 & 2.42 & 2.37 & 1.38 & 7.10 & 1.17 & 11.43 & 1.18 & 1.18 & 5.68 & 2.34 & 2.25 & 1.46 & 2.65 \\
\hline 2.2 & 3.50 & 5.62 & 2.42 & 2.37 & 1.32 & 7.10 & 1.17 & 11.43 & 1.18 & 1.18 & 5.68 & 2.34 & 2.25 & 1.46 & 2.65 \\
\hline 2.8 & 3.50 & 5.62 & 2.01 & 2.37 & 1.27 & 7.10 & 1.17 & 11.43 & 1.17 & 1.17 & 5.68 & 2.34 & 2.25 & 1.46 & 2.65 \\
\hline 3.6 & 2.57 & 3.85 & 1.76 & 2.37 & 1.24 & 4.74 & 1.17 & 11.43 & 1.17 & 1.17 & 5.68 & 2.34 & 2.25 & 1.46 & 2.65 \\
\hline 4.6 & 2.15 & 3.04 & 1.58 & 2.37 & 1.22 & 3.66 & 1.17 & 11.43 & 1.17 & 1.17 & 5.68 & 2.34 & 2.25 & 1.46 & 2.65 \\
\hline 6.0 & 1.85 & 2.48 & 1.46 & 1.85 & 1.20 & 2.91 & 1.17 & 11.43 & 1.17 & 1.17 & 5.68 & 1.83 & 2.25 & 1.46 & 2.65 \\
\hline 7.7 & 1.65 & 2.08 & 1.37 & 1.64 & 1.19 & 2.39 & 1.17 & 11.43 & 1.17 & 1.17 & 5.68 & 1.63 & 2.25 & 1.46 & 2.65 \\
\hline 10 & 1.50 & 1.81 & 1.31 & 1.50 & 1.19 & 2.02 & 1.17 & 11.43 & 1.17 & 1.17 & 5.68 & 1.49 & 2.25 & 1.46 & 2.65 \\
\hline 13 & 1.40 & 1.62 & 1.27 & 1.40 & 1.18 & 1.77 & 1.17 & 11.43 & 1.17 & 1.17 & 5.68 & 1.40 & 2.25 & 1.46 & 2.65 \\
\hline 17 & 1.33 & 1.48 & 1.24 & 1.33 & 1.18 & 1.59 & 1.17 & 6.18 & 1.17 & 1.17 & 5.68 & 1.33 & 2.25 & 1.46 & 2.65 \\
\hline 22 & 1.28 & 1.39 & 1.22 & 1.28 & 1.18 & 1.46 & 1.17 & 4.67 & 1.17 & 1.17 & 5.68 & 1.28 & 2.25 & 1.46 & 2.65 \\
\hline 28 & 1.25 & 1.32 & 1.20 & 1.25 & 1.17 & 1.37 & 1.17 & 3.62 & 1.17 & 1.17 & 5.68 & 1.25 & 2.25 & 1.46 & 2.65 \\
\hline 36 & 1.23 & 1.28 & 1.19 & 1.23 & 1.17 & 1.31 & 1.17 & 2.88 & 1.17 & 1.17 & 3.88 & 1.22 & 2.25 & 1.46 & 2.65 \\
\hline 46 & 1.21 & 1.24 & 1.19 & 1.21 & 1.17 & 1.27 & 1.17 & 2.37 & 1.17 & 1.17 & 3.07 & 1.21 & 2.25 & 1.46 & 2.65 \\
\hline 60 & 1.20 & 1.22 & 1.18 & 1.20 & 1.17 & 1.24 & 1.17 & 2.01 & 1.17 & 1.17 & 2.50 & 1.20 & 1.78 & 1.46 & 2.65 \\
\hline 77 & 1.19 & 1.21 & 1.18 & 1.19 & 1.17 & 1.22 & 1.17 & 1.75 & 1.17 & 1.17 & 2.10 & 1.19 & 1.60 & 1.36 & 2.65 \\
\hline 100 & 1.18 & 1.20 & 1.18 & 1.18 & 1.17 & 1.20 & 1.17 & 1.58 & 1.17 & 1.17 & 1.82 & 1.18 & 1.47 & 1.30 & 2.65 \\
\hline 129 & 1.18 & 1.19 & 1.17 & 1.18 & 1.17 & 1.19 & 1.17 & 1.46 & 1.17 & 1.17 & 1.62 & 1.18 & 1.38 & 1.26 & 2.03 \\
\hline 167 & 1.18 & 1.18 & 1.17 & 1.18 & 1.17 & 1.19 & 1.17 & 1.37 & 1.17 & 1.17 & 1.49 & 1.18 & 1.32 & 1.23 & 1.77 \\
\hline 215 & 1.17 & 1.18 & 1.17 & 1.17 & 1.17 & 1.18 & 1.17 & 1.31 & 1.17 & 1.17 & 1.39 & 1.17 & 1.27 & 1.21 & 1.59 \\
\hline 278 & 1.17 & 1.18 & 1.17 & 1.17 & 1.17 & 1.18 & 1.17 & 1.27 & 1.17 & 1.17 & 1.32 & 1.17 & 1.24 & 1.20 & 1.47 \\
\hline 359 & 1.17 & 1.17 & 1.17 & 1.17 & 1.17 & 1.18 & 1.17 & 1.24 & 1.17 & 1.17 & 1.28 & 1.17 & 1.22 & 1.19 & 1.38 \\
\hline 464 & 1.17 & 1.17 & 1.17 & 1.17 & 1.17 & 1.17 & 1.17 & 1.22 & 1.17 & 1.17 & 1.25 & 1.17 & 1.20 & 1.19 & 1.31 \\
\hline 599 & 1.17 & 1.17 & 1.17 & 1.17 & 1.17 & 1.17 & 1.17 & 1.20 & 1.17 & 1.17 & 1.22 & 1.17 & 1.19 & 1.18 & 1.27 \\
\hline 774 & 1.17 & 1.17 & 1.17 & 1.17 & 1.17 & 1.17 & 1.17 & 1.19 & 1.17 & 1.17 & 1.21 & 1.17 & 1.19 & 1.18 & 1.24 \\
\hline 1000 & 1.17 & 1.17 & 1.17 & 1.17 & 1.17 & 1.17 & 1.17 & 1.19 & 1.17 & 1.17 & 1.20 & 1.17 & 1.18 & 1.18 & 1.22 \\
\hline
\end{tabular}


Table 3.44. (contd)

\begin{tabular}{|c|c|c|c|c|c|c|c|c|}
\hline $\mathrm{d}_{\mathrm{s}}(\mu \mathrm{m})$ & Na3NO3SO4.H2O & $\mathrm{Na} 3 \mathrm{PO} 4.0 .25 \mathrm{NaOH} .12 \mathrm{H} 2 \mathrm{O}$ & $\mathrm{Na} 4 \mathrm{P} 2 \mathrm{O} 7.10 \mathrm{H} 2 \mathrm{O}$ & Na6(SO4)2CO3 & $\mathrm{NaF}$ & Na7F(PO4)2.19H2O & NaNO2 & $\mathrm{NaNO3}$ \\
\hline 0.22 & 2.30 & 1.62 & 1.83 & 2.64 & 2.78 & 1.75 & 2.17 & 2.26 \\
\hline \begin{tabular}{|c|}
0.28 \\
\end{tabular} & 2.30 & 1.62 & 1.83 & 2.64 & 2.78 & 1.75 & 2.17 & 2.26 \\
\hline 0.36 & 2.30 & 1.62 & 1.83 & 2.64 & 2.78 & 1.75 & 2.17 & 2.26 \\
\hline 0.46 & 2.30 & 1.62 & 1.83 & 2.64 & 2.78 & 1.75 & 2.17 & 2.26 \\
\hline 0 & 2.30 & 1.62 & 1.83 & 2.64 & 2.78 & 1.75 & 2.17 & 2.26 \\
\hline 0.77 & 2.30 & 1.62 & 1.83 & 2.64 & 2.78 & 1.75 & 2.17 & 2.26 \\
\hline 1 & 2.30 & 1.62 & 1.83 & 2.64 & 2.78 & 1.75 & 2.17 & 2.26 \\
\hline \begin{tabular}{|l|}
1.3 \\
\end{tabular} & 2.30 & 1.62 & 1.83 & 2.64 & 2.78 & 1.75 & 2.17 & 2.26 \\
\hline \begin{tabular}{|l|}
1.7 \\
\end{tabular} & 2.30 & 1.62 & 1.83 & 2.64 & 2.78 & 1.75 & 2.17 & 2.26 \\
\hline 2.2 & 2.30 & 1.62 & 1.83 & 2.64 & 2.78 & 1.75 & 2.17 & 2.26 \\
\hline 2.8 & 2.30 & 1.62 & 1.83 & 2.64 & 2.78 & 1.75 & 2.17 & 2.26 \\
\hline 3.6 & 2.30 & 1.62 & 1.83 & 2.64 & 2.78 & 1.75 & 2.17 & 2.26 \\
\hline 4.6 & 2.30 & 1.62 & 1.83 & 2.64 & 2.78 & 1.75 & 2.17 & 2.26 \\
\hline \begin{tabular}{ll|}
6.0 \\
\end{tabular} & 2.30 & 1.62 & 1.83 & 2.64 & 2.78 & 1.75 & 2.17 & 2.26 \\
\hline \begin{tabular}{|l|}
7.7 \\
\end{tabular} & 2.30 & 1.62 & 1.83 & 2.64 & 2.78 & 1.75 & 2.17 & 2.26 \\
\hline 10 & 2.30 & 1.62 & 1.83 & 2.64 & 1.96 & 1.75 & 2.17 & 2.26 \\
\hline 13 & 2.30 & 1.62 & 1.83 & 2.64 & 1.72 & 1.75 & 2.17 & 2.26 \\
\hline 17 & 2.30 & 1.62 & 1.83 & 2.64 & 1.55 & 1.75 & 2.17 & 2.26 \\
\hline 22 & 2.30 & 1.62 & 1.83 & 2.14 & 1.44 & 1.75 & 2.17 & 2.26 \\
\hline 28 & 2.30 & 1.62 & 1.83 & 1.85 & 1.36 & 1.75 & 2.17 & 2.26 \\
\hline 36 & 2.30 & 1.62 & 1.83 & 1.64 & 1.30 & 1.75 & 2.17 & 2.26 \\
\hline 46 & 2.30 & 1.62 & 1.83 & 1.50 & 1.26 & 1.75 & 2.17 & 2.26 \\
\hline 60 & 1.81 & 1.62 & 1.83 & 1.40 & 1.23 & 1.75 & 2.17 & 2.26 \\
\hline 77 & 1.62 & 1.62 & 1.83 & 1.33 & 1.21 & 1.75 & 2.17 & 2.26 \\
\hline 100 & 1.48 & 1.62 & 1.83 & 1.28 & 1.20 & 1.75 & 2.17 & 2.26 \\
\hline 129 & 1.39 & 1.62 & 1.83 & 1.25 & 1.19 & 1.75 & 2.17 & 2.26 \\
\hline 167 & 1.32 & 1.62 & 1.83 & 1.23 & 1.19 & 1.75 & 2.17 & 2.26 \\
\hline 215 & 1.28 & 1.62 & 1.83 & 1.21 & 1.18 & 1.75 & 2.17 & 2.26 \\
\hline 278 & 1.24 & 1.62 & 1.83 & 1.20 & 1.18 & 1.75 & 2.17 & 2.26 \\
\hline 359 & 1.22 & 1.40 & 1.83 & 1.19 & 1.18 & 1.75 & 2.17 & 2.26 \\
\hline 464 & 1.21 & 1.33 & 1.83 & 1.18 & 1.17 & 1.75 & 2.17 & 1.83 \\
\hline 599 & 1.20 & 1.28 & 1.83 & 1.18 & 1.17 & 1.75 & 2.17 & 1.63 \\
\hline 774 & \begin{tabular}{|l|}
1.19 \\
\end{tabular} & 1.25 & 1.83 & 1.18 & 1.17 & 1.75 & 2.17 & 1.49 \\
\hline 1000 & \begin{tabular}{|l|}
1.18 \\
\end{tabular} & 1.22 & 1.83 & 1.17 & 1.17 & 1.75 & 2.17 & 1.40 \\
\hline
\end{tabular}



Although representation of the PSDDs in the preceding tabular form is amenable to presentation, the PSDDs as columnar arrays of size, density, and volume fraction are more useful for calculations and comparison. Due to the extensive size of the PSDDs and since electronic files are most useful to potential end users, columnar PSDDs for the runs of Table 3.38 and Table 3.39 are provided electronically in the attached CD. The columnar PSDDs can be used as described in Sections 3.3.3.2 and 3.3.3.3.

\subsubsection{PSDD Comparison}

To select representative solid particles based on a PSDD, a waste operation model such as those presented in Section 2.0 may be applied. For simulants used in PJM mixing analysis, Meyer et al. (2009) applied the unhindered particle settling velocity calculation of Camenen (2007), provided in Section 2.0, to the Case 3 PSDD from Wells et al. (2007). Application of the Camenen (2007) equation produced a cumulative volume-based probability of the unhindered settling velocity of the particulate comprising the PSDD.

An approach following Meyer et al. (2009) is used to provide a graphical means of volume-based probabilistic comparison of the PSDDs (as opposed to the size, density, and volume fraction-tabulated form presented above). All settling velocities are calculated for the PSDD particulate in water $(1 \mathrm{~g} / \mathrm{mL}$, $1 \mathrm{cP})$. The first PSDD-based settling velocity comparison is made for the Sludge, Flowing Unsonicated PSDD of Table 3.40 through Table 3.44. The PSD remains constant, but the UDS density at particle sizes larger than the maximum primary particle size is reduced, so the settling velocity is reduced with decreasing fractal dimension (Figure 3.128). This comparison is similar to that made in Section 3.2.6 for the AZ-101 PSDDs with respect to the in situ measured settling velocity. In Figure 3.129, comparison is made for the Sludge, Flowing Sonicated, Sludge, Flowing Unsonicated, and Sludge, No-Flow Unsonicated PSDDs. Although the magnitude of the difference is reduced in comparison with the referenced AZ-101 example, the No-Flow Unsonicated PSDD again shows the highest settling velocities.

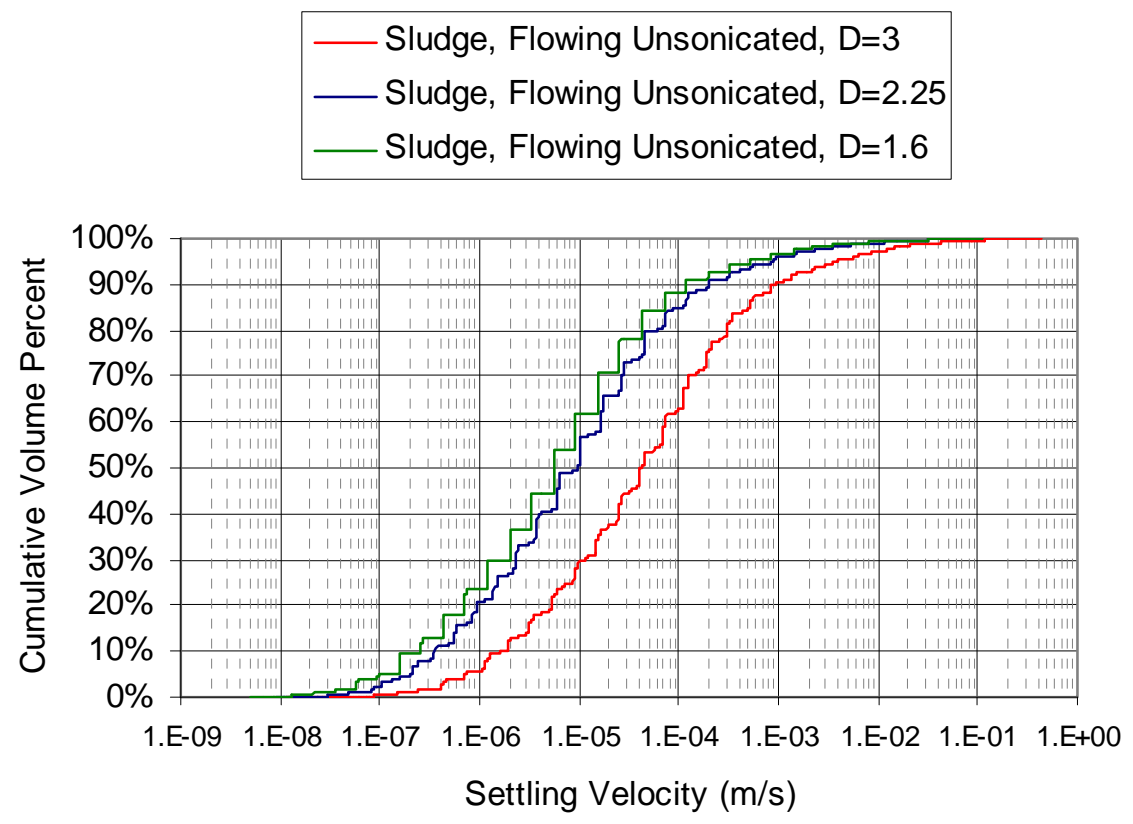

Figure 3.128. Sludge, Flowing Unsonicated Composite PSDD Calculated Settling Velocity as a Function of Fractal Dimension 


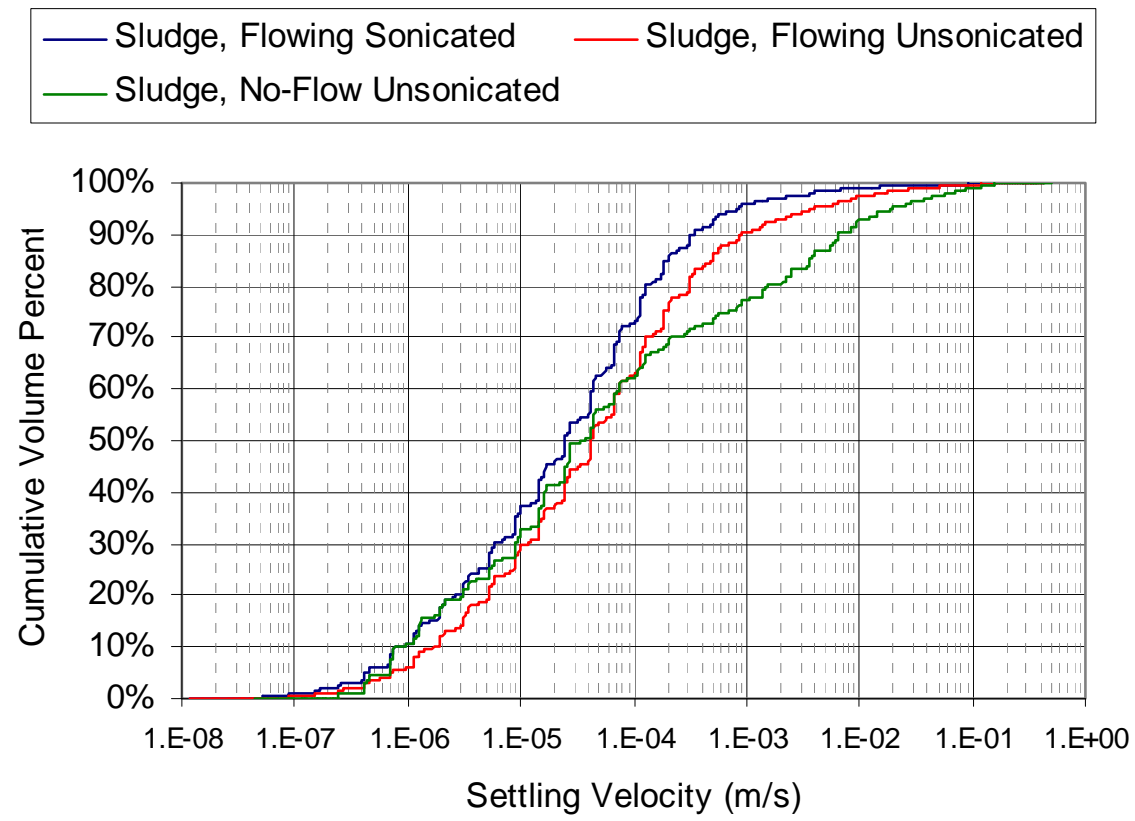

Figure 3.129. Sludge Composite PSDD Calculated Settling Velocity as a Function of PSD Measurement, Fractal Dimension $=3$

Generalized comparison of the tank PSDDs can be made based on this settling velocity approach. For sludge tank Flowing Unsonicated PSDDs with a fractal dimension of 3, the fastest calculated unhindered settling velocity is in T-110, and the slowest is in AZ-101 (Figure 3.130) at the $50^{\text {th }}$ percentile. Variations in this "ranking" occur dependent on the cumulative probability considered. Also shown in Figure 3.130 is the composite Sludge, Flowing Unsonicated PSDD.

The calculated unhindered settling velocity for the sludge tank No-Flow Unsonicated PSDDs with a fractal dimension of 3 are shown in Figure 3.131. Concurrent tanks between Figure 3.130 and Figure 3.131 are depicted by the bold lines in the figures. For the No-Flow Unsonicated PSDDs at the $50^{\text {th }}$ percentile, AZ-101 is no longer depicted with the slowest calculated unhindered settling velocity: C-104 is the fastest, and C-103 is the slowest. The Flowing Unsonicated PSDDs appear to have a more uniform distribution shape than the No-Flow Unsonicated PSDDs. Given the UDS composition and relative concentrations of individual tanks (i.e., Section 3.2.3), disparate PSDDs may be more representative of in situ waste conditions. As shown in the figures, the $50^{\text {th }}$ percentile calculated settling velocities for the Flowing Unsonicated PSDDs are more tightly grouped (approximately 1.5 order of magnitude difference) than those for the No-Flow Unsonicated PSDDs (approximately 3.3 order of magnitude difference). The composite Sludge, No-Flow Unsonicated PSDD is also shown in Figure 3.131.

Comparison of the Flowing Unsonicated and No-Flow Unsonicated PSDD calculated unhindered settling velocities for saltcake tanks in Figure 3.132 and Figure 3.133 shows similar distribution shape and grouping results. As for the sludge, concurrent tanks in the saltcake figures are depicted by the bold lines, and the composite PSDDs are included. 
The indications of fastest to slowest PSDD calculated unhindered settling velocity for the sludge and saltcake tanks at the $50^{\text {th }}$ percentile are compared in relative terms (i.e., slower or faster; quantitative comparison of measured and PSDD calculated settling rates are made in Section 3.2.6) to the "fast" interface velocities measured in laboratory tests and given in Table 3.30, Section 3.2.6. The comparison is made to determine if there is relative (i.e., slower or faster) agreement with laboratory-measured settling rates and PSDD-based calculations to provide a level of confidence in the representativeness of the PSDDs with respect to settling data.

For concurrent tanks in the Flowing Unsonicated and No-Flow Unsonicated PSDDs with measured laboratory settling rates at nominally equivalent initial UDS concentration of $3 \mathrm{vol} \%$ :

- C-107 has relatively (with respect to concurrent tanks at 3 vol\% UDS) faster laboratory rates and medium to low PSDD rates.

- C-104 has relatively medium laboratory rates, and the PSDD rates are the lowest (Figure 3.130) and the fastest (Figure 3.131).

- AZ-101 has the relatively slowest laboratory rates, and the PSDD rates are the slowest (Figure 3.130) and medium (Figure 3.131).

- AN-104 and S-104 have relatively similar laboratory and PSDD rates; both are slow.

$\mathrm{T}-110$, indicated as the fastest calculated unhindered settling velocity in Figure 3.130, has slower relative "fast" laboratory interface velocities at 3 vol\% UDS (Table 3.30). There is no "fast" laboratory interface velocity data for AY-102 at 3 vol\% UDS and no interface velocity data for C-104 (slowest calculated unhindered settling velocity in Figure 3.131), AN-102 (fastest in Figure 3.132), or AW-101 (fastest in Figure 3.133). Thus, there is limited indication of relative (i.e., slower or faster) agreement with laboratory-measured settling rates and PSDD-based calculations.

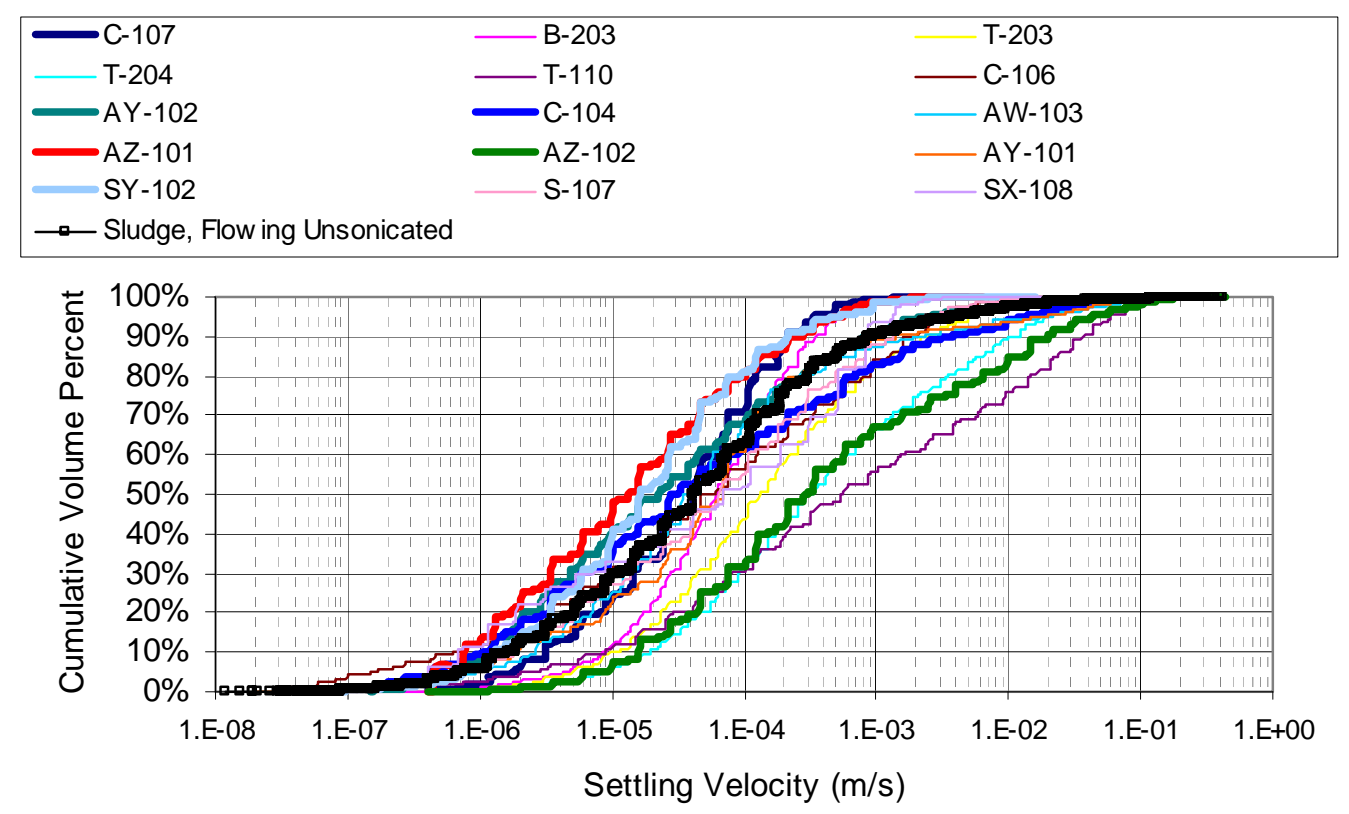

Figure 3.130. Sludge Tank Flowing Unsonicated PSDDs, Calculated Settling Velocity Comparison, Fractal Dimension = 3. Bold lines denote concurrent tanks with No-Flow Unsonicated PSDDs. 


\begin{tabular}{|c|c|c|}
\hline C-107 & BX-107 & T-104 \\
\hline B-201 & B-111 & $-\mathrm{T}-111$ \\
\hline -AY-102 & $\longrightarrow$ C-104 & $-\mathrm{C}-103$ \\
\hline$A Z-101$ & $\longrightarrow A Z-102$ & $-\mathrm{U}-110$ \\
\hline SY-102 & $\rightarrow$ Sludge, No-Flow Unsonicated & \\
\hline
\end{tabular}

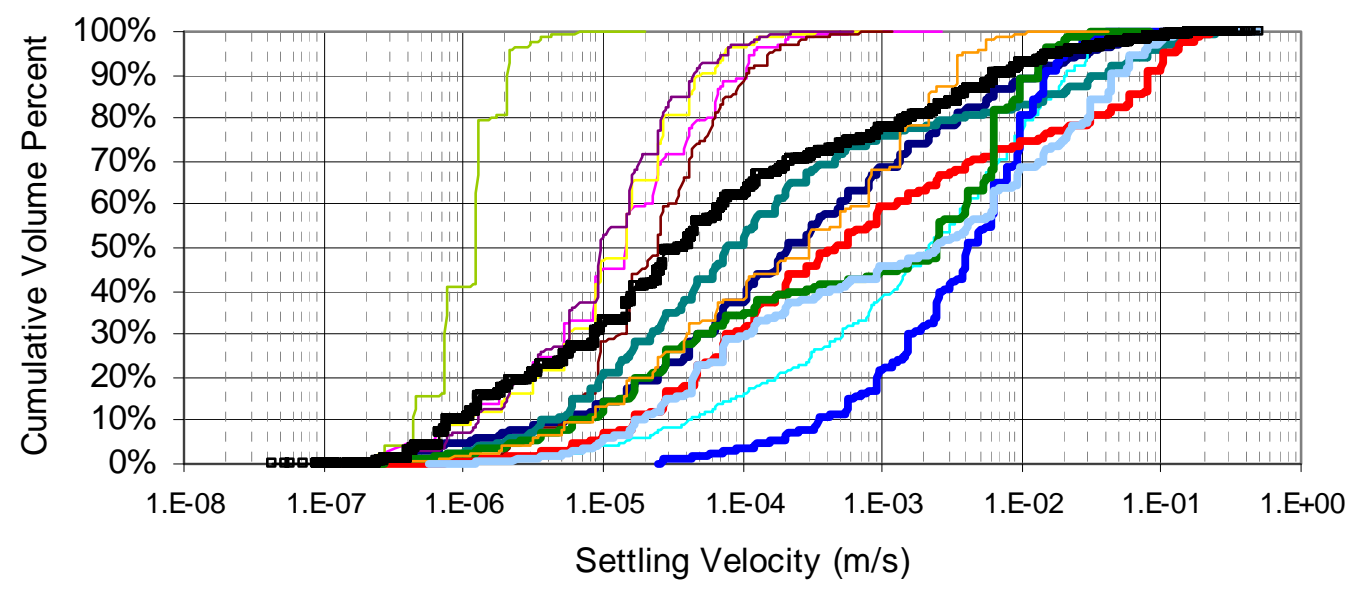

Figure 3.131. Sludge Tank No-Flow Unsonicated PSDDs, Calculated Settling Velocity Comparison, Fractal Dimension $=3$. Bold lines denote concurrent tanks with Flowing Unsonicated PSDDs.

\begin{tabular}{|c|c|}
\hline AN-104 & - AN-102 \\
\hline BY -104 & BY -108 \\
\hline - BY -110 & $\longrightarrow$ S-104 \\
\hline - S-101 & $\rightarrow$ Saltcake, Flow ing Unsonicated \\
\hline
\end{tabular}

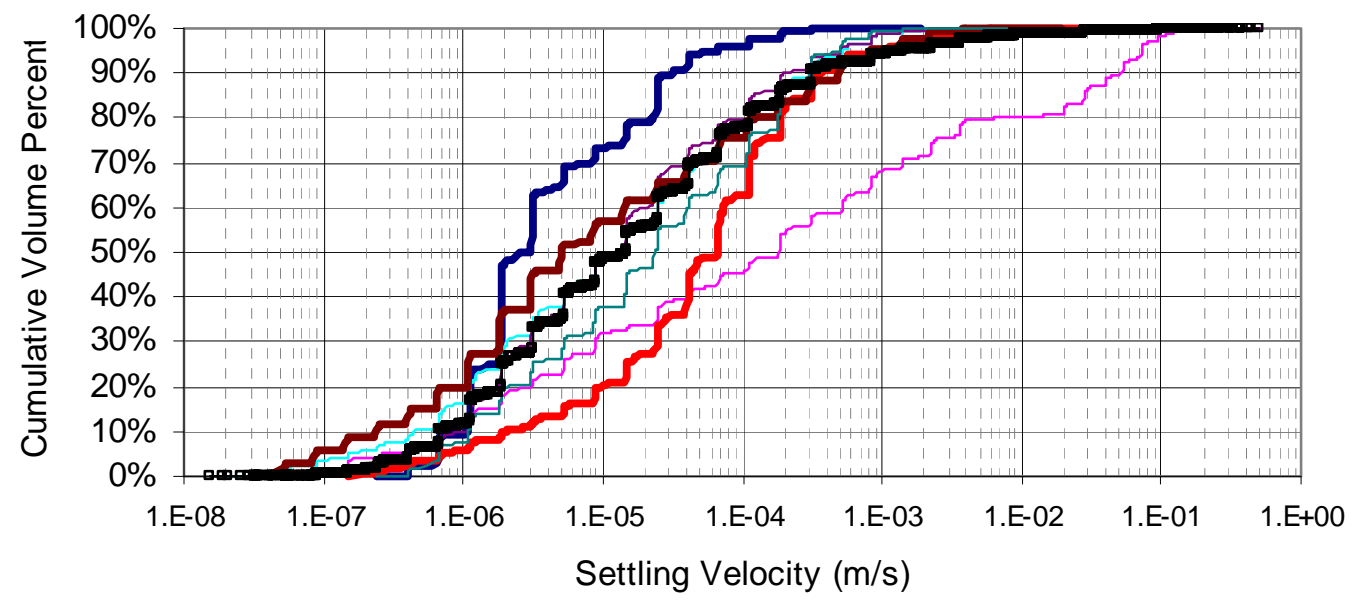

Figure 3.132. Saltcake Tank Flowing Unsonicated PSDDs, Calculated Settling Velocity Comparison, Fractal Dimension $=3$. Bold lines denote concurrent tanks with No-Flow Unsonicated PSDDs. 

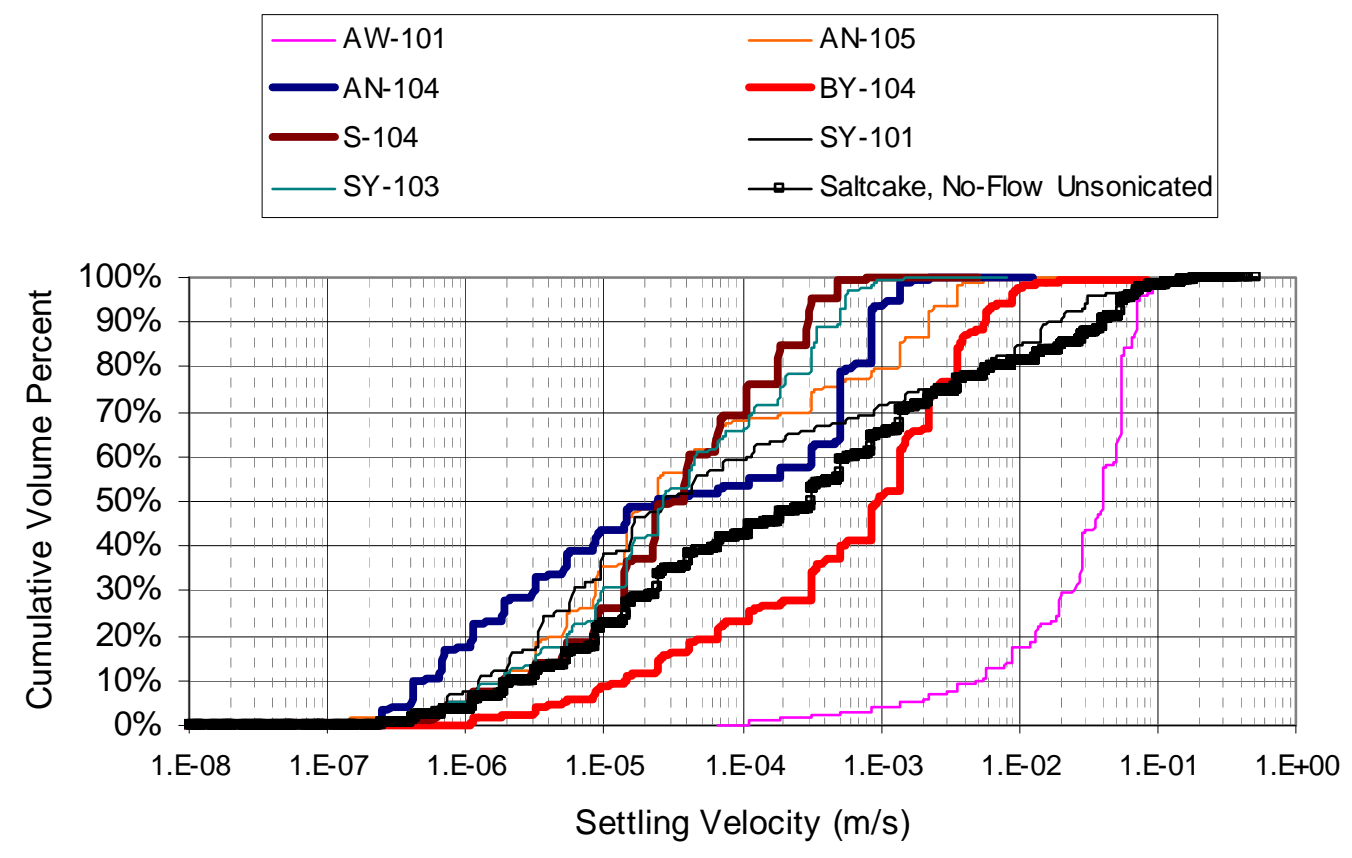

Figure 3.133. Saltcake Tank No-Flow Unsonicated PSDDs, Calculated Settling Velocity Comparison, Fractal Dimension $=3$. Bold lines denote concurrent tanks with Flowing Unsonicated PSDDs.

Comparison of relative in situ settling rates to the PSDDs can also be made. As presented in Section 3.2.6, there are in situ interface settling velocity data for AZ-101 and approximate estimates of the in situ settling velocity for AY-102. Within this limited and disparate data set, AZ-101 has a rate 10 to 100 times faster than AY-102 (see Figure 3.68). Comparison of the Flowing Unsonicated and No-Flow Unsonicated PSDD calculated settling velocities of AZ-101 and AY-102 shows a reversed relation; AY-102 is "faster" settling than AZ-101 (Figure 3.130) Flowing Unsonicated, and AZ-101 is up to a factor of 10 "faster" settling than AY-102 (Figure 3.131) No-Flow Unsonicated. Thus, the differences in the available in situ data are not represented by the Flowing Unsonicated PSDD, and the least amount of difference is approximated by the No-Flow Unsonicated PSDD.

\subsubsection{PSDDs, Examples of Use}

The PSDD-based settling velocity probabilities can be used to identify particles representing a specific probability in the PSDD. Meyer et al. (2009) determined that applying the $90^{\text {th }}$ percentile of the PSDD-based settling velocity for a chemical simulant into solid suspension models provided the best approximation to the solid suspension measured during mixing tests with that simulant. Although the actual mixing is a function of the slurry as a whole, the $90^{\text {th }}$ percentile can be considered as an example for representative particle selection for a specific probability from a PSDD. As noted in Paul et al. (2004), other percentiles may be used for various applications. This section simply provides an example of how the PSDD data may applied. 
The approach of using a single percentile from a distribution to represent the material is specified by Hall (2006) to determine the minimum flow velocity for slurry in a pipeline. For the particle size, Hall (2006) states:

"If the PSD is very narrow...the upper limit (UL) of the median $d_{50}$ particle size...is a good approximation of most of the slurry solids mass. The term ' $\mathrm{d}_{50}$ ' means, $50 \%$ of the particles comprising the slurry have a diameter equal to or smaller than the $\mathrm{d}_{50}$ diameter value.”

and

"If the PSD is broad, as is the case with as-received waste from the tank farm complex, the $\mathrm{d}_{95}$ UL particle size is a better representation of the majority of the solids mass.”

The terminology " $50 \%$ of the particles comprising the slurry have a diameter equal to or smaller than the d50 diameter value" is assumed to refer to d50 by volume, and thus the $\mathrm{d} 95$ is treated similarly. The terminology "d95 UL” implies a combination of individual PSDs (i.e., unique PSDs for combined process streams, tank wastes, etc.) has been made.

For the particle density, Hall (2006) states:

"If the PDD [particle density distribution] is known, use the $\mathrm{d}_{95}$ density for critical velocity calculations. If the PDD is unknown, such as is the case with as-received waste, but the bulk density and sludge volume fraction are known, the particle density can be estimated using...[the bulk sludge parameters of sludge density, interstitial liquid density, and solid volume fraction in the sludge]."

The bracketed and italicized text has been added. The term "d95 density" may be interpreted as the $95^{\text {th }}$ percentile of the PDD. It is assumed herein, as with the particle size, that the distribution is volume based. If the PDD is unknown, Hall (2006) specifies the use of the solid density that would conserve mass with the bulk (i.e., sludge) density, interstitial liquid density, and solid volume fraction in the sludge. This latter approach does not use a particulate density, only an average thereof.

As described, the methodology specified in Hall (2006) for a representative particle from a distribution for slurry transport velocity can be interpreted to require either the $95^{\text {th }}$ percentiles of both the particle size and density distributions or the $95^{\text {th }}$ percentile of the PSD and an average estimate of the particle density to be used to compute the critical velocity. Comparison of these options relative to PSDD probability approaches is provided below.

Based on the single particle selection examples of Meyer et al. (2009) and Hall (2006), the 95 ${ }^{\text {th }}$ percentile of the PSDD-based settling velocity probability is used as an example for identifying representative particles. The use of other percentiles, e.g., the $50^{\text {th }}$, may be reasonable depending on the application. For the Sludge, Flowing Unsonicated PSDD of Table 3.40 (fractal dimension of 3), the $95^{\text {th }}$ percentile of the settling velocity is approximately 3.9E-3 m/s (Figure 3.130). The particle size and density combinations that have this $95^{\text {th }}$ percentile settling velocity are shown in Figure 3.134. Particles that have terminal settling velocities at this rate settle faster than or equal to $95 \%$ of the individual solid particles represented by the Sludge, Flowing Unsonicated PSDD. The comparisons made of PSDD-based 
settling velocity to measured solid-liquid interface rates in Section 3.2.6 should be evaluated in conjunction with this approach.

As shown in Section 2.0, the Archimedes number is a parameter in some of the calculations for terminal settling velocity (specifically Camenen 2007), pipeline critical velocity (Poloski et al. 2009), critical suspension velocity (Meyer et al. 2009), and suspended particle cloud height (Meyer et al. 2009). The Archimedes number $95^{\text {th }}$ percentile particle size and density combinations are therefore also shown in Figure 3.134, with the $95^{\text {th }}$ percentile Ar for the Sludge, Flowing Unsonicated PSDD being approximately 6.5. As indicated, the Archimedes number is a parameter for the settling velocity calculation of Camenen (2007), so the results are similar.

Also shown in Figure 3.134 are $95^{\text {th }}$ percentile particle size and density combinations for pipeline critical velocity. The pipeline critical velocity particle determination follows the Wells et al. (2007) approach using the Oroskar and Turian (1980) equation (provided in Section 2.0). The functionality of this equation with UDS size and density is given in Wells et al. (2007) as

$$
\beta=\left[d^{0.167}(S-1)^{0.545}\right]
$$

where $S$ is the solid-to-liquid density ratio. The $95^{\text {th }}$ percentile $\beta$ for the Sludge, Flowing Unsonicated PSDD is approximately $0.29 \mathrm{~m}^{0.167}$.

The different functionalities for different approaches and applications are readily apparent. For example, a particle of nominally size $1 \mu \mathrm{m}$ and density $8 \mathrm{~g} / \mathrm{mL}$ has a $\beta$ value equivalent to the $95^{\text {th }}$ percentile, but this "particle," as it is to the left/below the $95^{\text {th }}$ percentile settling velocity and Ar number lines, is less than the $95^{\text {th }}$ percentile of those parameters. Therefore, the particle may be limiting or bounding for one calculation of pipeline transport, but not for other calculations for the same and specific calculations for settling, critical suspension velocity, and suspended particle cloud height.

Concurrence is achieved for the represented applications at a particle of approximately $70 \mu \mathrm{m}$ and $2.9 \mathrm{~g} / \mathrm{mL}$ (Figure 3.134). That is, a particle of this size and density is representative of the $95^{\text {th }}$ percentile by volume of the Sludge, Flowing Unsonicated PSDD for certain calculations of terminal settling velocity, pipeline critical velocity, critical suspension velocity, and suspended particle cloud height. As presented in Section 2.0, other calculations of this type as well as other applications have different functionalities. In addition, the applications are likely a function of the slurry as a whole. Finally, the representativeness of the PSDD itself must be considered (as in the comparisons with measured settling velocity).

As discussed above, Hall (2006) specifies either the $95^{\text {th }}$ percentiles of both the particle size and density distributions or the $95^{\text {th }}$ percentile of the PSD and an average estimate of the particle density for critical velocity calculation. For the Sludge, Flowing Unsonicated composite PSDD with a fractal dimension of 3 , the $95^{\text {th }}$ percentile size is $69 \mu \mathrm{m}$ (Section 3.2.5), the $95^{\text {th }}$ percentile density (i.e., the $95^{\text {th }}$ percentile of the UDS crystal densities for the UDS compounds of the Sludge, Flowing Unsonicated composite PSDD) is $8.9 \mathrm{~g} / \mathrm{mL}$ (Section 3.2.3), and the mass-weighted average UDS density (Sludge, Flowing Unsonicated composite PSDD) is approximately $2.4 \mathrm{~g} / \mathrm{mL}$ (Section 3.2.3). These combinations are provided in Figure 3.134 as "Hall (2006) 95-95" (69 $\mu \mathrm{m}, 8.9 \mathrm{~g} / \mathrm{mL})$ and "Hall (2006) 95-bulk" 
(69 $\mu \mathrm{m}, 2.4 \mathrm{~g} / \mathrm{mL}$ ), respectively. The 95-95 particle exceeds the $95^{\text {th }}$ percentiles of the represented applications (approximately the $98^{\text {th }}$ percentile settling velocity, $97^{\text {th }}$ percentile Ar, and $>99^{\text {th }}$ percentile $\beta$ ), and the 95-bulk particle under-represents the $95^{\text {th }}$ percentiles of the represented applications (approximately the $94^{\text {th }}$ percentile settling velocity and $\mathrm{Ar}$, and $91^{\text {st }}$ percentile $\beta$ ).

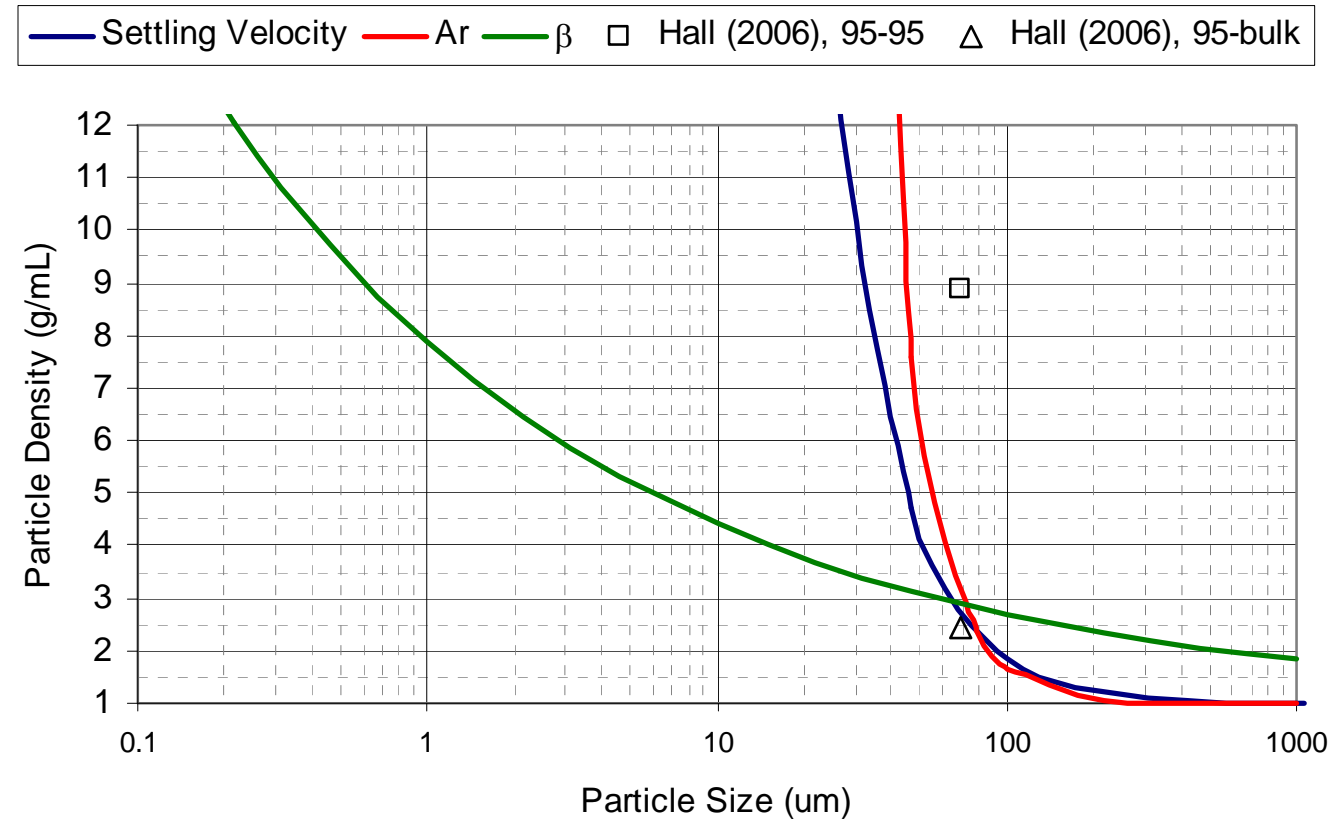

Figure 3.134. $95^{\text {th }}$ Percentile Particle Identification, Example Applications, Sludge, Flowing Unsonicated PSDD with a Fractal Dimension of 3

\subsection{Data Update Summary}

The work reported in this document differs from the prior work, Wells et al. (2007) and Poloski et al. (2007), generally via the addition of data and extension of the results, not alteration of the prior work. Since this document contains the most up-to-date and extensive data and analyses, it is recommended for use over the prior work.

The data sets in Wells et al. (2007) (UDS composition and particle density, UDS primary particle size and shape, UDS particle size distributions [PSDs], and estimated particle size and density distributions [PSDDs]) and Poloski et al. (2007) (liquid and slurry rheology, and UDS particle settling) were updated as described in Section 3.2 with additional data. The primary source of additional data is from a recent series of tests sponsored by the WTP. These tests involved an extensive suite of characterization and bench-scale process testing of 8 waste groups representing approximately $75 \%$ of the waste expected to be processed through the WTP.

A summary of the updates from Wells et al. (2007) and Poloski et al. (2007) to the current work is provided in Table 3.45. General data differences for those variables highlighted in Table 3.45 are summarized in Section 3.4.1. 
Table 3.45. Data Update Difference Summary

\begin{tabular}{|c|c|c|c|c|}
\hline \multicolumn{2}{|c|}{ Properties in Update } & $\begin{array}{l}\text { Wells et al. } \\
\text { (2007) }\end{array}$ & $\begin{array}{c}\text { Poloski et al. } \\
\text { (2007) }\end{array}$ & Update Difference \\
\hline \multirow{3}{*}{ Liquid } & Density & $\mathrm{n} / \mathrm{a}$ & $\mathrm{n} / \mathrm{a}$ & not included in prior work \\
\hline & $\mathrm{pH}$ & $\mathrm{n} / \mathrm{a}$ & $\mathrm{n} / \mathrm{a}$ & not included in prior work \\
\hline & Rheology & $\mathrm{n} / \mathrm{a}$ & included & additional data \\
\hline \multirow{7}{*}{ Solids } & Particle Density & included & $\mathrm{n} / \mathrm{a}$ & 52 solid phase compounds from 16 \\
\hline & $\begin{array}{l}\text { Primary Particle } \\
\text { Size and Shape }\end{array}$ & included & $\mathrm{n} / \mathrm{a}$ & 52 solid phase compounds from 16 \\
\hline & $\begin{array}{c}\text { Particle Size } \\
\text { Distribution (PSD) }\end{array}$ & included & $\mathrm{n} / \mathrm{a}$ & $\begin{array}{c}\text { additional data, } \\
\text { alternate instrument configuration, } \\
\text { combined, maximum, and minimum } \\
\text { PSDs } \\
\text { by tank, waste type, and composites }\end{array}$ \\
\hline & $\begin{array}{l}\text { Particle Size and } \\
\text { Density } \\
\text { Distribution } \\
\text { (PSDD) }\end{array}$ & included & $\mathrm{n} / \mathrm{a}$ & by tank, waste type, and composites \\
\hline & Particle Settling & $\mathrm{n} / \mathrm{a}$ & included & $\begin{array}{c}\text { additional data, } \\
\text { comparison to PSDDs }\end{array}$ \\
\hline & Shear Strength & $\mathrm{n} / \mathrm{a}$ & included & additional data \\
\hline & Bingham Rheology & $\mathrm{n} / \mathrm{a}$ & included & additional data \\
\hline
\end{tabular}

\subsubsection{General Data Differences}

General data differences between the current work and Wells et al. (2007), Poloski et al. (2007), and Jewett et al. (2002) are summarized for those variables highlighted in Table 3.45. Examples are provided of the data differences.

\section{Liquid Rheology}

Limited additional data was added to the liquid rheology data set, Section 3.2.2. Liquid rheology models for individual tanks are provided as well as a generalized model that accounts for additional compositional functionality in comparison to the Poloski et al. (2007) model. The generalized liquid viscosity model for the entire data set, Equation (3.3) and Table 3.4, is compared to the model of Poloski et al. (2007) in Figure 3.135. The liquid viscosity data at 25, 45, and $65^{\circ} \mathrm{C}$ (Appendix I) is also shown. The updated models are shown to predict elevated liquid viscosity results. 


\section{Particle Density and Primary Particle Size}

The 16 solid phase compounds of Wells et al. (2007) are expanded to 52 for the current work (see Section 3.2.3). Comparison of the solid phase compounds and density as well as the primary particle size (see Section 3.2.4) is made in Table 3.46. The increased number of solid phase compounds accounts for the expected variability in the waste For example, the silver compound of Wells et al. (2007), $\mathrm{Ag}_{2} \mathrm{CO}_{3}$, density $6.077 \mathrm{~g} / \mathrm{mL}$, maximum primary particle size of $4 \mu \mathrm{m}$, is replaced with Ag, $10.5 \mathrm{~g} / \mathrm{mL}, 2 \mu \mathrm{m}$, and $\mathrm{Ag}_{2} \mathrm{O}, 7.143 \mathrm{~g} / \mathrm{mL}, 2 \mu \mathrm{m}$. With the increased number of solid phase compounds, tank-by-tank comparisons can be made.

\section{Particle Size Distributions}

A comparison of the Flowing Unsonicated composite sludge PSDs of the current work is made to the previous results from Wells et al. (2007), and Jewett et al. (2002) in Figure 3.136. The Sludge, Flowing Unsonicated composite results are quite similar to the previous results from Wells et al. and Jewett et al. as indicated by the two solid blue lines and the dashed black line. This suggests that the additional data was not substantially different.

The dashed red and green lines represent the minimum and maximum values, respectively, of the measured PSDs comprising the Sludge, Flowing Unsonicated composite of the current work. As

discussed in Section 3.2.5, these minimum and maximum values cannot be viewed as confidence bounds. Confidence-based interval methods for PSD uncertainty bounds are challenged by limitations in the data and potential violations of the requirements associated with normal theory methods.

The differences of the data set and combination methodology between the current work and Jewett et al. (2002) are discussed in Section 3.2.5. It is therefore of note that the Sludge, Flowing Unsonicated, Max results from the current work and the 95/95 TL from Jewett et al. (2002) are shown as approximately equivalent. In addition to composite PSDs for alternate PSD instrumentation configurations, tank and waste type PSDs for sludge and saltcake wastes are also provided in Section 3.2.5, whereas Wells et al. (2007) only provided composite results for sludge. 

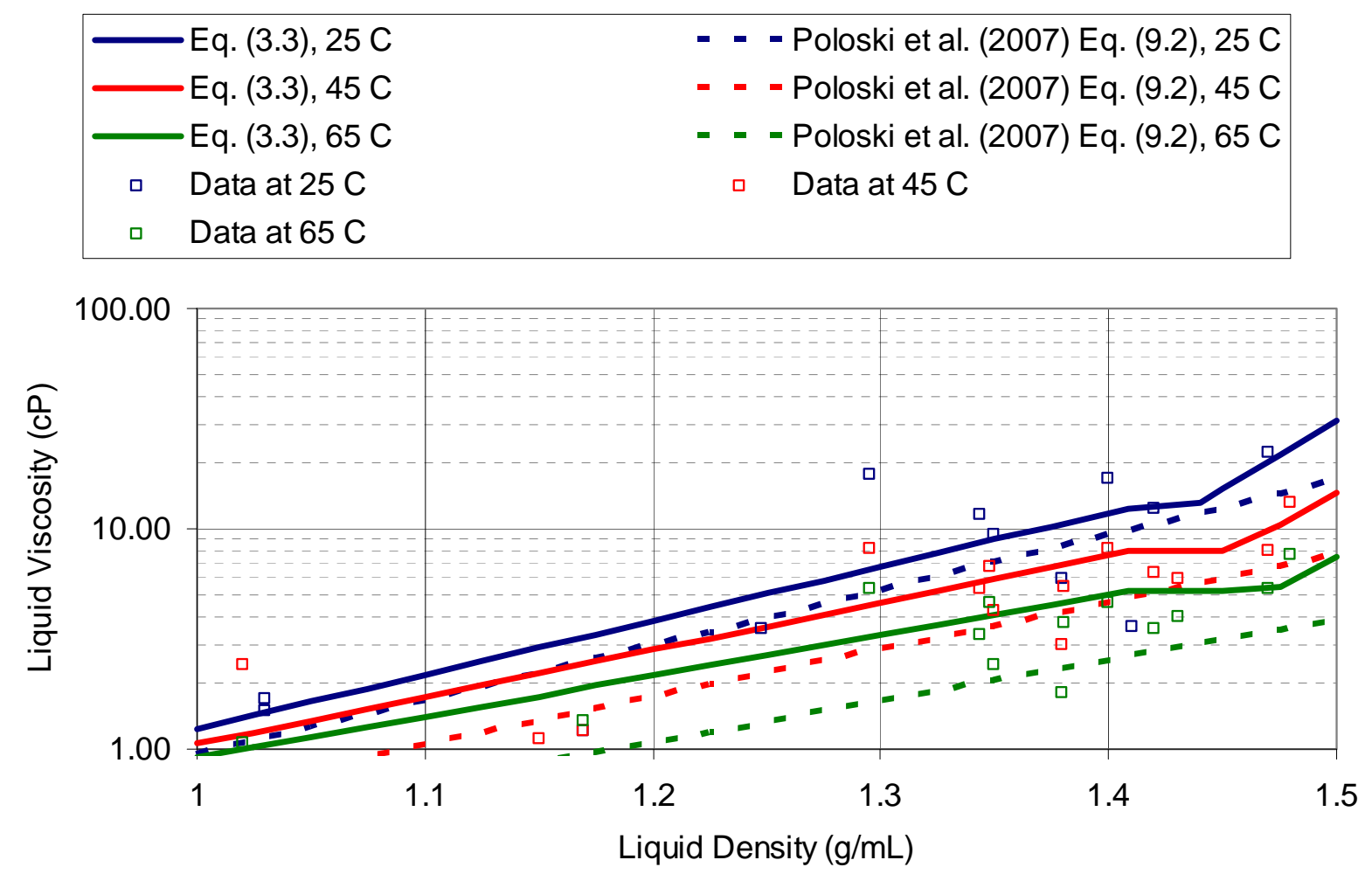

Figure 3.135. Liquid Viscosity Data Comparison 
Table 3.46. Primary Particle Characteristics of Hanford Sludge Wastes (Wells et al. 2007)

\begin{tabular}{|c|c|c|c|c|c|}
\hline \multicolumn{3}{|c|}{ Wells et al. (2007) } & \multicolumn{3}{|c|}{ Update } \\
\hline Compound & $\begin{array}{l}\text { Density } \\
\text { (g/mL) }\end{array}$ & $\begin{array}{c}\text { Maximum } \\
\text { Spherical } \\
\text { Primary } \\
\text { Particle } \\
\text { Size }(\mu \mathrm{m})\end{array}$ & Compound & $\begin{array}{l}\text { Density } \\
(\mathrm{g} / \mathrm{mL})\end{array}$ & $\begin{array}{c}\text { Maximum } \\
\text { Spherical } \\
\text { Primary } \\
\text { Particle } \\
\text { Size }(\mu \mathrm{m})\end{array}$ \\
\hline \multirow{2}{*}{$\mathrm{Ag}_{2} \mathrm{CO}_{3}$} & \multirow{2}{*}{6.077} & \multirow{2}{*}{4} & $\mathrm{Ag}$ & 10.5 & 2 \\
\hline & & & $\mathrm{Ag}_{2} \mathrm{O}$ & 7.143 & 2 \\
\hline \multirow{2}{*}{$\mathrm{Bi}_{2} \mathrm{O}_{3}$} & \multirow{2}{*}{8.9} & \multirow{2}{*}{10} & $\mathrm{Bi}_{2} \mathrm{O}_{3}$ & 8.9 & 3 \\
\hline & & & $\mathrm{BiFeO}_{3}$ & 7.9 & 0.1 \\
\hline \multirow{6}{*}{$\begin{array}{c}\mathrm{Ca}_{5} \mathrm{OH}\left(\mathrm{PO}_{4}\right)_{3} \\
\mathrm{CaF}_{2}\end{array}$} & \multirow{6}{*}{$\begin{array}{l}3.14 \\
3.18\end{array}$} & \multirow{6}{*}{$\begin{array}{l}0.1 \\
15\end{array}$} & $\mathrm{Ca}(\mathrm{OH})_{2}$ & 2.24 & 9 \\
\hline & & & $\mathrm{Ca}_{5} \mathrm{OH}\left(\mathrm{PO}_{4}\right)_{3}$ & 3.14 & 9 \\
\hline & & & $\mathrm{CaC}_{2} \mathrm{O}_{4} \bullet \mathrm{H}_{2} \mathrm{O}$ & 2.2 & 9 \\
\hline & & & $\mathrm{CaCO}_{3}$ & 2.71 & 55 \\
\hline & & & $\mathrm{CaF}_{2}$ & 3.18 & 15 \\
\hline & & & $\mathrm{CrOOH}$ & 4.11 & 0.4 \\
\hline \multirow{2}{*}{$\mathrm{Fe}_{2} \mathrm{O}_{3}$} & \multirow{2}{*}{5.24} & \multirow{2}{*}{1.6} & $\mathrm{FePO}_{4} \cdot 2 \mathrm{H}_{2} \mathrm{O}$ & 3.15 & 0.02 \\
\hline & & & $\mathrm{FeOOH}$ & 4.26 & 0.015 \\
\hline Boehmite & 3.01 & 0.05 & Boehmite & 3.01 & 0.052 \\
\hline Gibbsite & 2.42 & 20 & Gibbsite & 2.42 & 200 \\
\hline $\mathrm{NaAlCO}_{3}(\mathrm{OH})_{2}$ & 2.42 & 4.2 & $\mathrm{NaAlCO}_{3}(\mathrm{OH})_{2}$ & 2.42 & 4.2 \\
\hline$\left(\mathrm{NaAlSiO}_{4}\right)_{6} \cdot\left(\mathrm{NaNO}_{3}\right)_{1.6} \cdot 2 \mathrm{H}_{2} \mathrm{O}$ & 2.365 & 8 & $\mathrm{NaAlSiO}_{4}$ & 2.365 & 8 \\
\hline & $\mathrm{HgO}$ & 7.143 & 2 \\
\hline & & & $\mathrm{KAlSiO}_{4}$ & 2.61 & 8 \\
\hline \multirow{2}{*}{$\mathrm{LaPO}_{4} \cdot 2 \mathrm{H}_{2} \mathrm{O}$} & \multirow{2}{*}{6.51} & \multirow{2}{*}{3} & $\mathrm{La}(\mathrm{OH})_{3}$ & 2.3 & 3 \\
\hline & & & $\mathrm{LaPO}_{4} \cdot 2 \mathrm{H}_{2} \mathrm{O}$ & 6.51 & 3 \\
\hline \multirow{2}{*}{$\mathrm{MnO}_{2}$} & \multirow{2}{*}{5.026} & \multirow{2}{*}{10} & $\mathrm{Mn}_{3}\left(\mathrm{PO}_{4}\right)_{2}$ & 3.102 & 8 \\
\hline & & & $\mathrm{MnO}_{2}$ & 5.026 & 10 \\
\hline \multirow{2}{*}{$\mathrm{Na}_{2} \mathrm{U}_{2} \mathrm{O}_{7}$} & \multirow{2}{*}{5.617} & \multirow{2}{*}{15} & $\mathrm{Na}_{2}\left(\mathrm{UO}_{2}\right)_{2}\left(\mathrm{PO}_{4}\right)_{2} \cdot 2 \mathrm{H}_{2} \mathrm{O}$ & 3.5 & 5 \\
\hline & & & $\mathrm{Na}_{2} \mathrm{U}_{2} \mathrm{O}_{7}$ & 5.617 & 5 \\
\hline \multirow{3}{*}{$\mathrm{Ni}(\mathrm{OH})_{2}$} & \multirow{3}{*}{4.1} & \multirow{3}{*}{0.5} & $\mathrm{Ni}(\mathrm{OH})_{2}$ & 4.15 & 0.5 \\
\hline & & & $\mathrm{Ni}_{3}\left(\mathrm{PO}_{4}\right)_{2}$ & 3.93 & 8 \\
\hline & & & $\mathrm{NiC}_{2} \mathrm{O}_{4} \cdot 2 \mathrm{H}_{2} \mathrm{O}$ & 4.26 & 1.6 \\
\hline $\mathrm{PuO}_{2}$ & 11.43 & 40 & $\begin{array}{c}\mathrm{Pu}(\mathrm{OH})_{4} \text { (co-precip. on Fe } \\
\text { phase) }\end{array}$ & 4.26 & 0.015 \\
\hline & & & $\mathrm{PuO}_{2}$ & 11.43 & 20 \\
\hline & & & $\mathrm{Pb}(\mathrm{OH})_{2}$ & 7.1 & 5 \\
\hline & & & $\mathrm{Pb}_{3}\left(\mathrm{PO}_{4}\right)_{2}$ & 7.1 & 0.4 \\
\hline & & & $\mathrm{PbCO}_{3}$ & 6.6 & 5 \\
\hline $\mathrm{SiO}_{2}$ & 2.6 & 100 & $\mathrm{SiO}_{2}$ & 2.6 & 100 \\
\hline & & & $\mathrm{Sr}_{3}\left(\mathrm{PO}_{4}\right)_{2}$ & 3.5 & 0.065 \\
\hline & & & $\mathrm{SrCO}_{3}$ & 3.5 & 0.065 \\
\hline $\mathrm{ZrO}_{2}$ & 5.68 & 50 & $\mathrm{ZrO}_{2}$ & 5.68 & 50 \\
\hline
\end{tabular}


Table 3.46. (contd)

\begin{tabular}{|c|c|c|c|c|c|}
\hline \multicolumn{3}{|c|}{ Wells et al. (2007) } & \multicolumn{3}{|c|}{ Update } \\
\hline Compound & $\begin{array}{l}\text { Density } \\
\text { (g/mL) }\end{array}$ & $\begin{array}{c}\text { Maximum } \\
\text { Spherical } \\
\text { Primary } \\
\text { Particle } \\
\text { Size }(\mu \mathrm{m})\end{array}$ & Compound & $\begin{array}{l}\text { Density } \\
(\mathrm{g} / \mathrm{mL})\end{array}$ & $\begin{array}{c}\text { Maximum } \\
\text { Spherical } \\
\text { Primary } \\
\text { Particle } \\
\text { Size }(\mu \mathrm{m})\end{array}$ \\
\hline \multirow{16}{*}{\multicolumn{3}{|c|}{ no salts }} & $\mathrm{KNO}_{3}$ & 2.109 & 2200 \\
\hline & & & $\mathrm{Na}_{2} \mathrm{C}_{2} \mathrm{O}_{4}$ & 2.34 & 8 \\
\hline & & & $\mathrm{Na}_{2} \mathrm{CO}_{3} \cdot \mathrm{H}_{2} \mathrm{O}$ & 2.25 & 80 \\
\hline & & & $\mathrm{Na}_{2} \mathrm{SO}_{4}$ & 2.68 & 112 \\
\hline & & & $\mathrm{Na}_{2} \mathrm{SO}_{4} \cdot 10 \mathrm{H}_{2} \mathrm{O}$ & 1.464 & 112 \\
\hline & & & $\mathrm{Na}_{3} \mathrm{FSO}_{4}$ & 2.65 & 176 \\
\hline & & & $\mathrm{Na}_{3} \mathrm{NO}_{3} \mathrm{SO}_{4} \cdot \mathrm{H}_{2} \mathrm{O}$ & 2.3 & 80 \\
\hline & & & $\mathrm{Na}_{3} \mathrm{PO}_{4.0} \cdot 25 \mathrm{NaOH} \cdot 12 \mathrm{H}_{2} \mathrm{O}$ & 1.62 & 440 \\
\hline & & & $\mathrm{Na}_{3} \mathrm{PO}_{4} \bullet 8 \mathrm{H}_{2} \mathrm{O}$ & 1.8 & 2200 \\
\hline & & & $\mathrm{Na}_{4} \mathrm{P}_{2} \mathrm{O}_{7} \cdot 10 \mathrm{H}_{2} \mathrm{O}$ & 1.83 & 2200 \\
\hline & & & $\mathrm{Na}_{6}\left(\mathrm{SO}_{4}\right)_{2} \mathrm{CO}_{3}$ & 2.64 & 32 \\
\hline & & & $\mathrm{NaF}$ & 2.78 & 12 \\
\hline & & & $\mathrm{Na}_{7} \mathrm{~F}\left(\mathrm{PO}_{4}\right)_{2} \cdot 19 \mathrm{H}_{2} \mathrm{O}$ & 1.75 & 2100 \\
\hline & & & $\mathrm{NaHCO}_{3}$ & 2.159 & 328 \\
\hline & & & $\mathrm{NaNO}_{2}$ & 2.168 & 2200 \\
\hline & & & $\mathrm{NaNO}_{3}$ & 2.26 & 650 \\
\hline
\end{tabular}

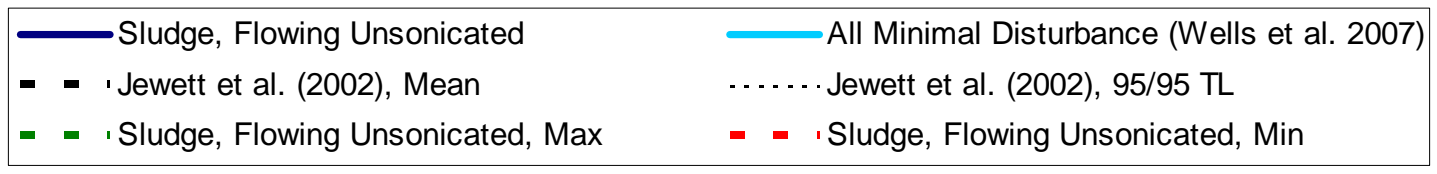

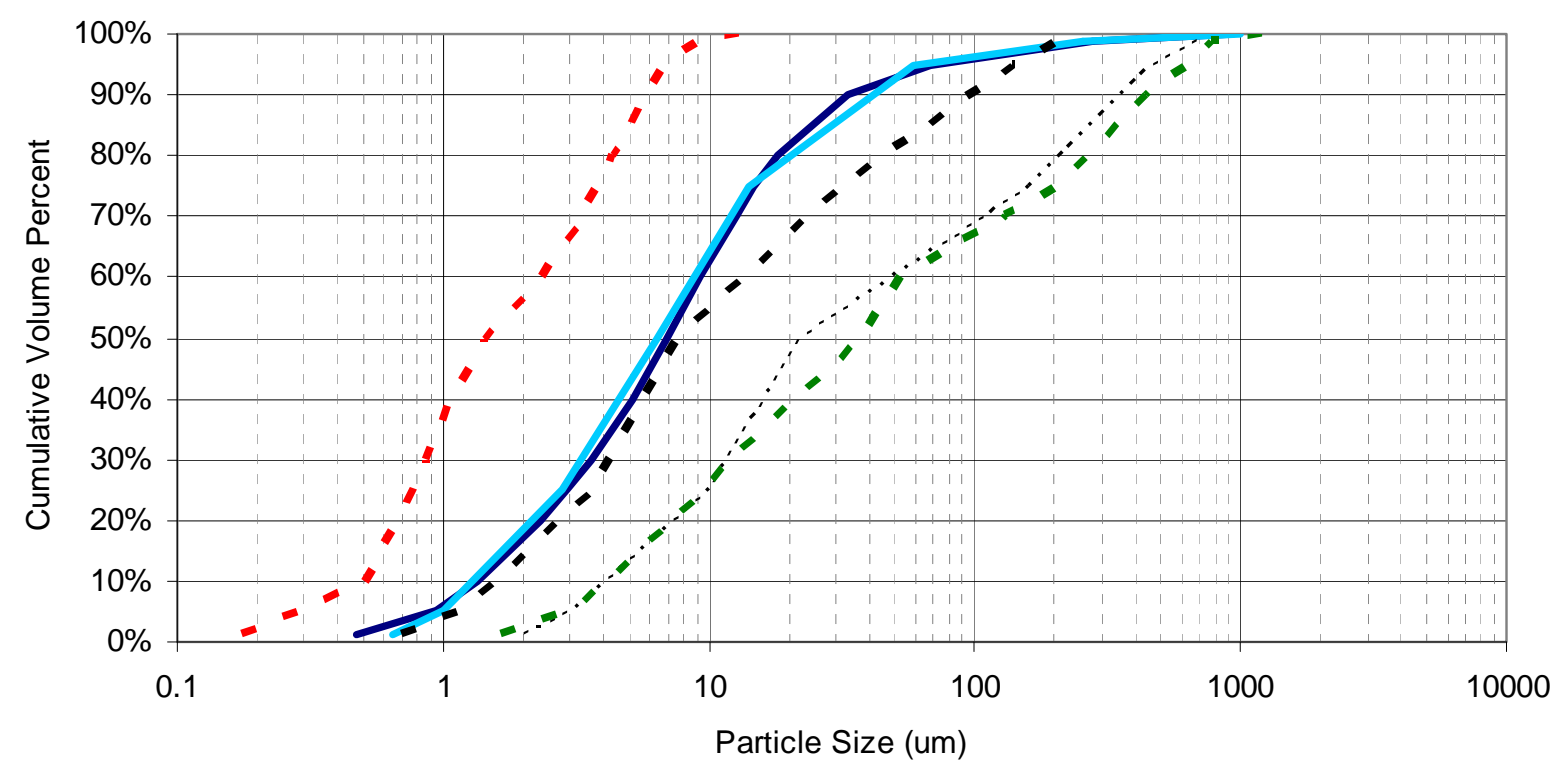

Figure 3.136. Composite Sludge PSD Comparison. Flowing Unsonicated Condition. 


\section{Particle Size and Density Distributions}

Resulting from the substantial increase of tank, waste type, and composite solid phase compound summaries and PSDs in the current work as compared to Wells et al. (2007), PSDDs are provided for sludge and saltcake tanks, waste types, and composites, Section 3.3. Thus, size, density, and concentration estimates, and therefore PSDDs, are now available for a significantly increased fraction of the Hanford waste in comparison to Wells et al. (2007).

Direct comparison of the Sludge, Flowing Unsonicated, D=3 PSDD, Section 3.3, is made to the Case 3 PSDD of Wells et al. (2007) in Figure 3.137. As done in Section 3.3, the comparison is made using the computed settling velocity of the particles in water. As with the PSDs in Figure 3.136, there are limited differences, indicating that the changes in the particle densities, Table 3.46, do not substantially alter the PSDD. Variation of the individual tank PSDDs in comparison to the composites is shown in Section 3.3.

Sludge, Flowing Unsonicated, D=3 —Wells et al. (2007), Case 3

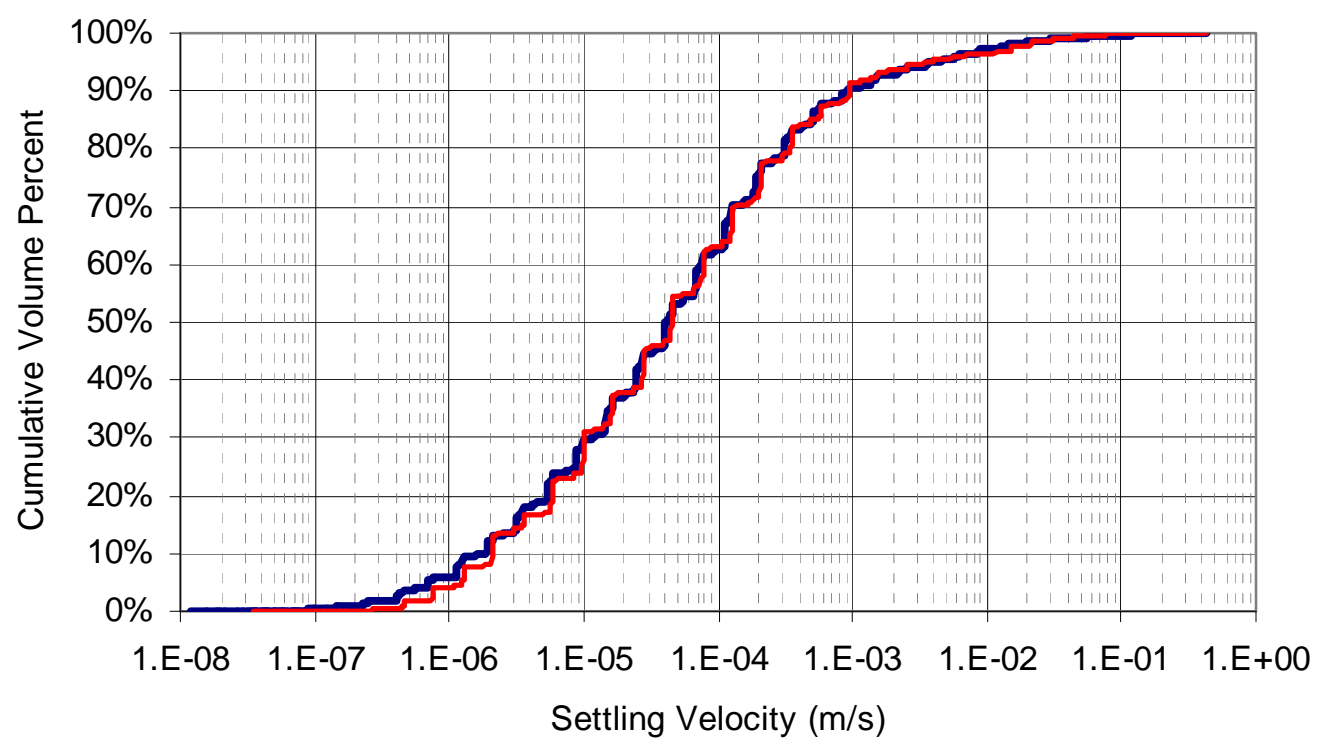

Figure 3.137. PSDD Comparison. Flowing Unsonicated Condition.

\section{Particle Settling}

In Section 3.2.6, the laboratory particle settling data of Poloski et al. (2007) is evaluated together with additional data, and the results are discussed with respect to in-tank settling data. These data are compared to settling velocities computed from the PSDDs. Thus, laboratory particle settling data is compared to in situ behavior as well as the characterization of the solid particulate.

The additional laboratory data shows similar settling rates as the laboratory data of Poloski et al. (2007). The discrepancy between the rapid interface settling in the tank and the slower settling of the visible interfaces in the laboratory studies referenced in Gauglitz et al. (2009) and (2010b) is discussed and speculative explanations are provided. Comparison of the particulate characterization (PSDD) to in 
situ settling data suggests that either the phenomena that accelerates the settling rate not yet understood and must be accounted for or process data must be considered for the selection of a representative PSDD.

\section{Rheology}

The shear strength data of Poloski et al. (2007) is enhanced with additional data as well as comparison to the limited process data for mobilization and the rebuild of shear strength with time after mobilization in Section 3.2.7. Other functionalities such as the effect of varied UDS concentration and elevation within the sediment are considered as well.

The shear strength data from the core extrusion length estimates, excluded from Poloski et al. (2007), are now included, which increases the fraction of characterized waste. Comparison of cumulative distributions from the shear vane, core extrusion, and ball rheometer data, Table 3.47, shows that while higher results have been measured with the shear vane, the median core extrusion results may exceed those from the other techniques.

Limited additional data were included for the Binham rheology data set, Figure 3.137. Elevated rheology results are shown from the additional data at a UDS concentration by mass of approximately 20\%. As with Poloski et al. (2007), functionalities on temperature and concentration for individual tanks and waste types are provided. Given the similarities in the data shown in Figure 3.137, the functionalities are essentially equivalent.

Hall (2006) specifies the use of Equation (5-12) of Jewett et al. (2002) to determine the carrier fluid viscosity for the prediction of slurry line flow critical velocity in the WTP. This model, developed in Jewett et al. (2002) from a limited data set as a function of slurry UDS concentration and strain rate, is compared to the Bingham rheology data of the current work in Figure 3.138. The apparent viscosity for both the data and the Jewett et al. (2002) model is determined at an approximated strain rate (e.g., see Chhabra and Richardson 2008) from a $6 \mathrm{ft} / \mathrm{s}$ transfer velocity in a 3-inch-diameter pipe. ${ }^{\text {(a) }}$ The wide variability in the physical and chemical properties of the Hanford UDS leads to the Bingham rheology variability at a given UDS concentration. This variability makes it difficult to apply a single simple correlation such as the Jewett et al. (2002) model to represent Hanford waste viscosity as a function of UDS concentration.

(a) Equation (5-12) of Jewett et al. (2002) uses the UDS concentration by volume, whereas the Bingham rheology data in Section 3.2.7 is presented by UDS mass concentration. To apply the model, conversion between mass and volume is accomplished using the average liquid and UDS density values listed in Jewet et al. (2002), 1.1 and $2.18 \mathrm{~g} / \mathrm{mL}$ respectively. The apparent viscosity trend with UDS concentration from the model relative to the data is not significantly impacted by these inputs. 
Table 3.47. Shear-Strength Percentiles (Table 3.32, Section 3.2.7)

\begin{tabular}{|c|c|c|c|c|}
\hline $\begin{array}{c}\text { Primary Waste } \\
\text { Type Form }\end{array}$ & $\begin{array}{c}\text { Measurement } \\
\text { Technique }\end{array}$ & $\mathbf{5}^{\text {th }}$ Percentile $^{(\mathbf{a})}$ & $\mathbf{5 0}^{\text {th }}$ Percentile $^{(\text {a) }}$ & $\mathbf{9 5}^{\text {th }}$ Percentile $^{\text {(a) }}$ \\
\hline \multirow{2}{*}{ Sludge } & shear vane & 27 & 541 & 6439 \\
\cline { 2 - 5 } & core extrusion & 62 & 931 & 1786 \\
\hline \multirow{3}{*}{ Saltcake } & shear vane & 41 & 411 & 5700 \\
\cline { 2 - 5 } & core extrusion & 25 & 270 & 1238 \\
\cline { 2 - 5 } & ball rheometer & 16 & 126 & 900 \\
\hline \multirow{2}{*}{ All } & shear vane & 33 & 507 & 6208 \\
\cline { 2 - 5 } & core extrusion & 36 & 594 & 1595 \\
\hline (a) Probabilities are solely based on measurement count.
\end{tabular}

$\diamond$ Current Work + Poloski et al. (2007)

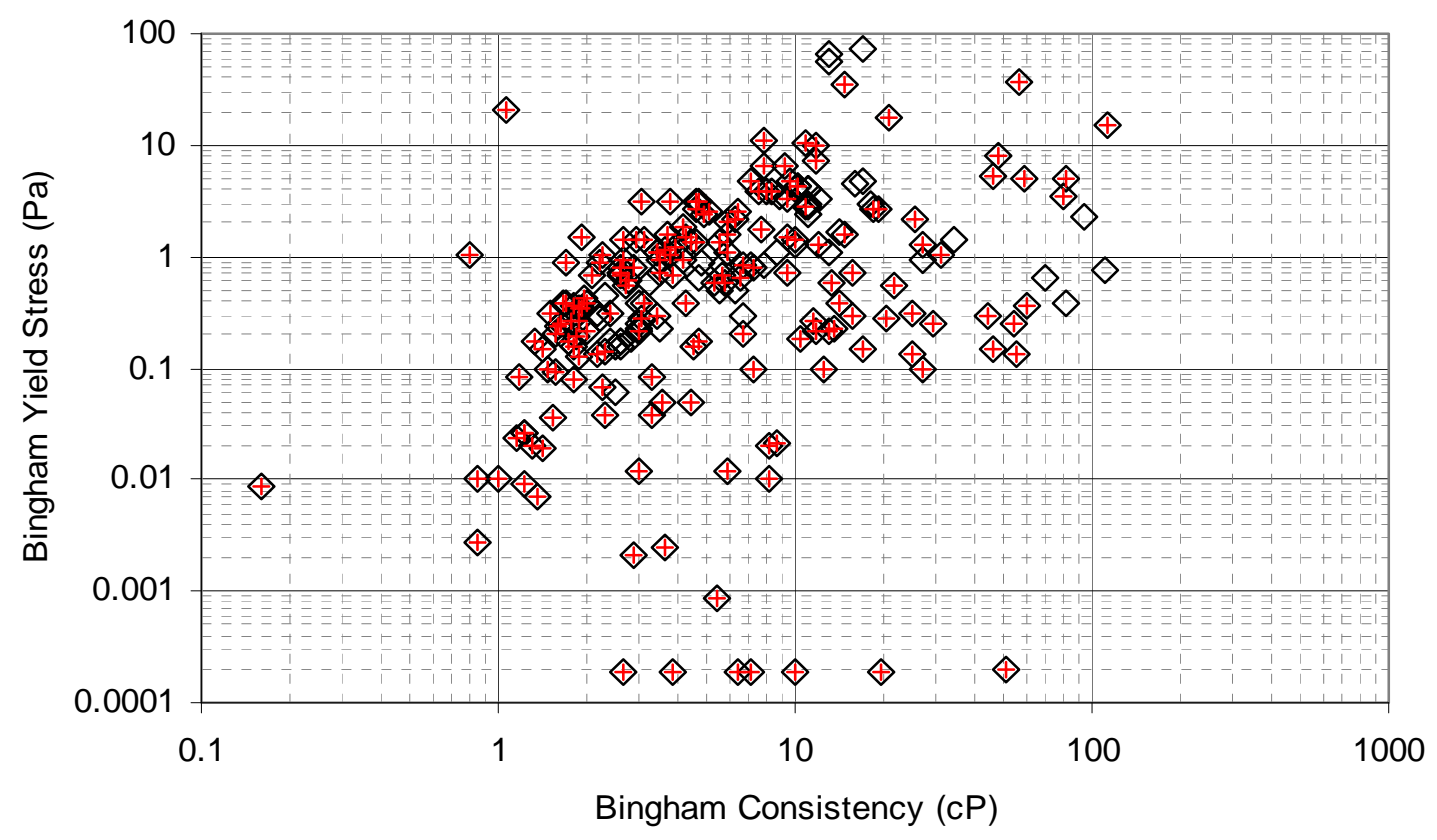

Figure 3.137. Bingham Rheology Comparison 


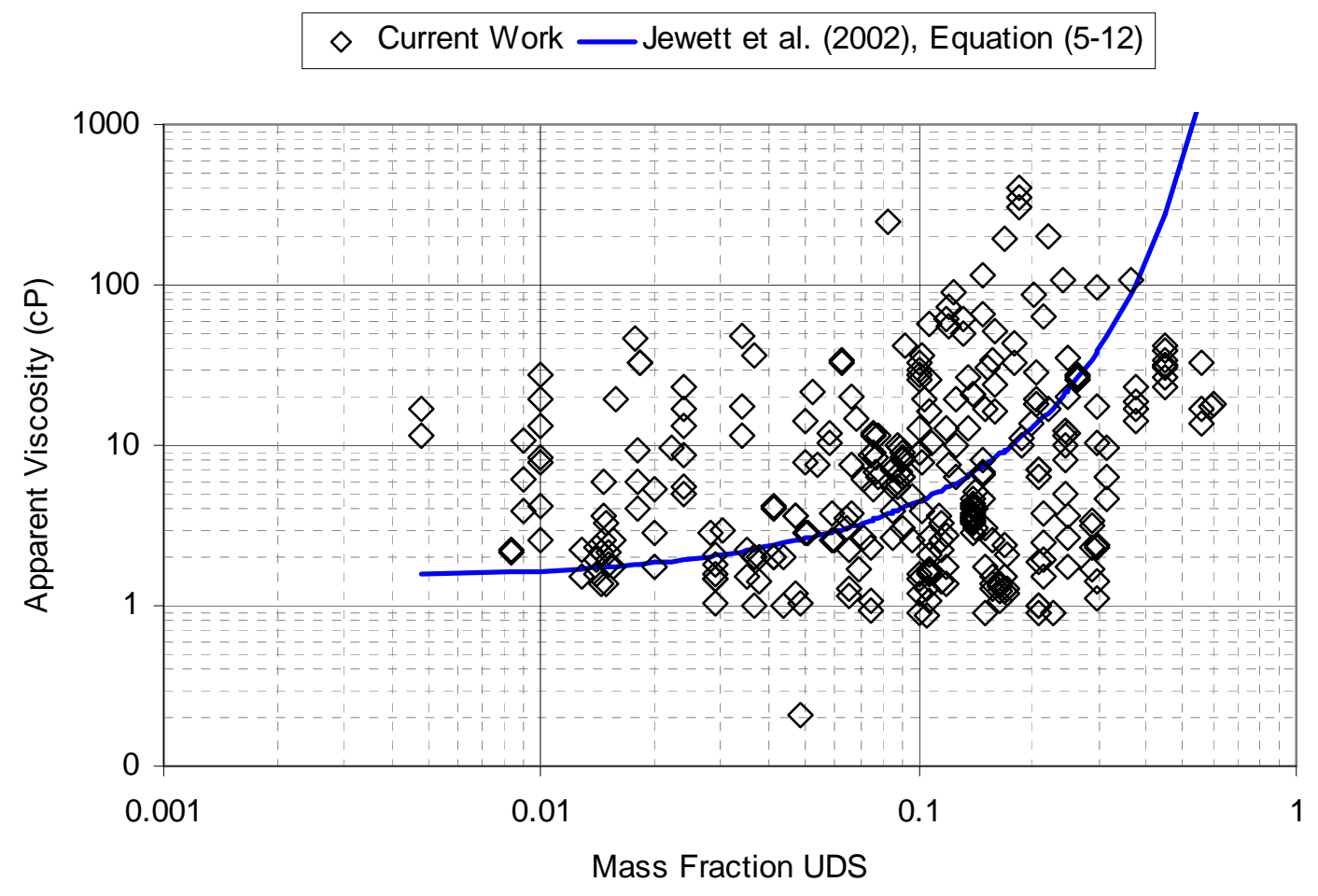

Figure 3.138. Apparent Viscosity Comparison 



\subsection{Effect of Pretreatment Processes on Waste Parameter Data}

The EFRT was assembled in October of 2005 to conduct an in-depth review of the WTP process flowsheet of the WTP. The EFRT identified 17 major issues, including issue M12-that neither the caustic leaching nor the oxidative leaching processes planned for the WTP Pretreatment Facility had been demonstrated at greater than bench scale. To address M12, ORP and WTP developed a pilot-scale leaching and ultrafiltration facility and demonstrated the effectiveness of the leaching and ultrafiltration processes (Kurath et al. 2009). In advance of and to support the pilot-scale demonstration of these leaching processes, eight waste types were identified, existing waste samples were selected and composited to obtain actual waste composites that would represent these eight waste groups, and extensive laboratory testing was conducted on the composites. These eight waste groups represented $\sim 75 \%$ of the HLW mass expected to be processed through the WTP (Fiskum et al. 2009a). The primary waste types of these M12 Groups are listed in Section 3.0.

Both parametric and laboratory-scale tests were conducted on the M12 waste composites, with attention given to both chemical and physical (e.g., PSD, rheology) changes due to the pretreatment processes. This section provides examples of the PSD and rheological changes due to the pretreatment processes observed for the M12 waste groups, with references for more detailed information.

\subsection{Groups 1 and 2-Bismuth Phosphate Sludge and Saltcake Wastes}

The BP sludge (Group 1) and BP saltcake (Group 2) waste sample composites were combined to obtain enough solids to conduct leaching, washing, and ultrafiltration tests in the hot cells unit filter (CUF). Lumetta et al. (2009a) provide details of the characterization of the mixture and the hot CUF tests conducted. PSD and rheology data obtained before and after key process operations are summarized below.

Initial parametric filtration tests were conducted on the mixture of Group 1 and Group 2 composites, with the final filtration step resulting in $14 \mathrm{wt} \%$ UDS slurry. The WTP target of $20 \mathrm{wt} \%$ UDS was not achieved. Samples of the $14 \mathrm{wt} \%$ slurry were collected and analyzed for chemical composition, PSD, and rheology. The differential PSD is shown in Figure 4.1 as the curve labeled "High-Solids, Pre-Leach." All of the PSDs in Figure 4.1 were measured with the Malvern Mastersizer (see Section 3.2.5.1) circulation pump running at $2000 \mathrm{rpm}$ and its ultrasonic agitator turned on to allow comparison of primary particles and hard agglomerates. The $d_{50}$ for this PSD was $2.2 \mu \mathrm{m}$, and particle diameters ranged from about 0.2 to $25 \mu \mathrm{m}$. The PSD data is included in Appendix H. Rheograms indicated that the slurry was non-Newtonian at 25,40 , and $60^{\circ} \mathrm{C}$, with a yield stress of 2 to $4 \mathrm{~Pa}$. Table 4.1 lists fitted rheology parameters for the Bingham plastic model (see Appendix B of Lumetta et al. 2009a for brief descriptions the rheometer used and the Bingham rheology model).

Caustic leaching was initiated by adding $7.6 \mathrm{M} \mathrm{NaOH}$ to the $14 \mathrm{wt} \%$ slurry and raising the mixture temperature to $100^{\circ} \mathrm{C}$ over a 5.3-h period. The amount of caustic added was calculated to make certain that all dissolved aluminum would remain in solution after caustic leaching was complete and the slurry had been cooled to $25^{\circ} \mathrm{C}$. The concentration of caustic $(7.6 \mathrm{M})$ was chosen to simulate the $19 \mathrm{M} \mathrm{NaOH}$ to 
be added in the WTP, plus steam condensate accumulated while heating the WTP vessel. Mixing (via rotating impeller) was continuous during caustic addition, heat-up, the 8 -h leach at $100^{\circ} \mathrm{C}$, and the $12-\mathrm{h}$ cool-down period. Evaporation of water from the reaction vessel during caustic leaching was significant, so the slurry level was monitored and water added periodically to maintain the initial level. Samples collected from the slurry indicated the soluble aluminum (gibbsite) had all been dissolved by the end of the 5.3-h, heat-up period.

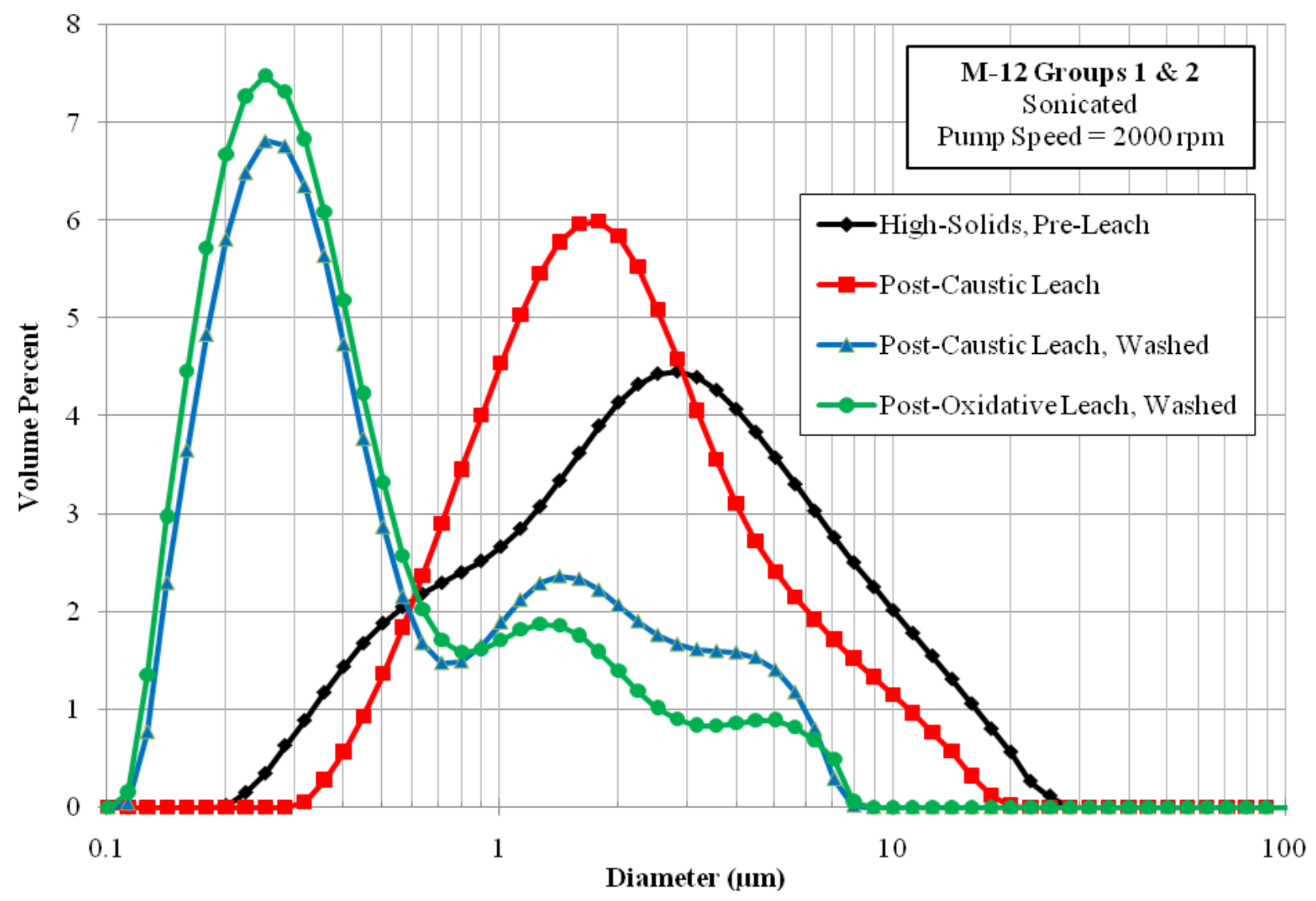

Figure 4.1. M-12 Waste Composite Groups 1 and 2 Leached and Washed Slurry PSDs

Table 4.1. M-12 Waste Composite Groups 1 and 2 Leached and Washed Slurry Rheology Values ${ }^{(a)}$

\begin{tabular}{|c|c|c|c|c|c|c|c|}
\hline \multirow[b]{2}{*}{ Sample } & \multirow{2}{*}{$\begin{array}{l}\text { UDS } \\
(w t \%)\end{array}$} & \multicolumn{3}{|c|}{ Bingham Yield Stress (Pa) } & \multicolumn{3}{|c|}{$\begin{array}{c}\text { Bingham Consistency } \\
(\mathbf{P a} \cdot \mathbf{s})\end{array}$} \\
\hline & & $25^{\circ} \mathrm{C}$ & $40^{\circ} \mathrm{C}$ & $60^{\circ} \mathrm{C}$ & $25^{\circ} \mathrm{C}$ & $40^{\circ} \mathrm{C}$ & $60^{\circ} \mathrm{C}$ \\
\hline High-Solids, Pre-Leach & 14.4 & 3.1 & 3.1 & 2.7 & 0.013 & 0.011 & 0.0090 \\
\hline Post-Caustic Leach, De-watered & 17.6 & 34 & 44 & 57 & 0.027 & 0.025 & 0.023 \\
\hline Post-Caustic Leach, Washed & 11.5 & 7.8 & 5.9 & 5.0 & 0.011 & 0.0057 & 0.0054 \\
\hline \multirow{2}{*}{ Sample } & \multirow{2}{*}{$\begin{array}{c}\text { UDS } \\
(\mathrm{wt} \%)\end{array}$} & \multicolumn{3}{|c|}{ Newtonian Viscosity (cP) } & \multirow{3}{*}{\multicolumn{3}{|c|}{ n.a. }} \\
\hline & & $25^{\circ} \mathrm{C}$ & $40^{\circ} \mathrm{C}$ & $60^{\circ} \mathrm{C}$ & & & \\
\hline Post-Oxidative Leach, Washed & 6.5 & 2.4 & 2.0 & 1.6 & & & \\
\hline
\end{tabular}


After caustic leaching was complete and the slurry cooled to $25^{\circ} \mathrm{C}$, it was re-concentrated to about $18 \mathrm{wt} \%$ UDS by filtration and sampled. The PSD for this sample is shown in Figure 4.1 as the "Post-Caustic Leach" curve. Other test data suggest that insoluble phosphates present in the waste composites were dissolved by the caustic leaching process, but were re-precipitated as sodium phosphate. Adding caustic also raised the concentration of sodium in the liquid phase and may have caused the precipitation of sodium salts that were at or near their saturated concentrations (e.g., sodium oxalate). The changes in PSD from feed (High-Solids, Pre-Leach curve) to leached waste (Post-Caustic Leach curve) are therefore thought to be due to dissolution of gibbsite and insoluble phosphates as well as the precipitation of sodium phosphate and possibly other sodium salts. Rheograms indicate the post-caustic leach to be non-Newtonian with a yield stress of $25 \mathrm{~Pa}$ at $25^{\circ} \mathrm{C}$, increasing to $50 \mathrm{~Pa}$ at $60^{\circ} \mathrm{C}$. Table 4.1 lists the Bingham yield stress and consistency values obtained.

The concentrated post-caustic leach slurry was washed five times to remove soluble species and reduce the concentration of caustic to about $0.25 \mathrm{M}$ (as required for oxidative leaching). Note that the hot CUF washing process was not prototypic of the WTP, where washing will be automated and involve many relatively small wash solution additions. Each wash involved adding about the same mass of caustic wash solution, mixing, and re-concentrating the slurry to its original volume. The concentration of caustic added was specified to prevent re-precipitation of aluminum, and decreased in each successive wash. At the end of the post-caustic leach wash, changes in liquid density and dissolution of solids resulted in a $20 \mathrm{wt} \%$ UDS slurry. This slurry was sampled and characterized for chemical composition, PSD, and rheology. The PSD is shown in Figure 4.1 as the "Post-Caustic Leach, Washed" curve. Note the significant decrease in particle sizes from the previous PSD, evidently caused when soluble sodium salts (e.g., sodium phosphate) dissolved. Here the largest particles were around $7 \mu \mathrm{m}$, and the $\mathrm{d}_{50}$ particle was about $0.4 \mu \mathrm{m}$. Though only about one third of the initial mass of pre-caustic leach solids has been dissolved at this point, the $d_{50}$ particle diameter has decreased by over a factor of 5 . Rheograms again indicate the slurry to be non-Newtonian, though in this case, the yield stress decreased with increasing temperature. Specific Bingham plastic model values are listed in Table 4.1.

Oxidative leaching to dissolve certain chromium III species was then conducted by adding a sodium permanganate solution to the slurry and mixing for 6 hours. The oxidatively leached slurry was then washed three times with $0.01 \mathrm{M}$ caustic to remove dissolved chromium. The post-oxidative leach, washed slurry was then sampled and characterized. The PSD, shown in Figure 4.1 as the "Post-Oxidative Leach, Washed" curve, differs little from the PSD of the slurry before oxidative leaching. This is consistent with the expectation of little change; the mass of chromium solids dissolved is small compared to the total mass of solids. Rheograms indicate the slurry is now Newtonian, with only small changes in its viscosity between 25,40 , and $60^{\circ} \mathrm{C}$. Table 4.1 lists the rheological parameters.

\subsection{Groups 3 and 4-PUREX Cladding and REDOX Cladding Wastes}

The PUREX cladding waste (Group 3) and REDOX cladding waste (Group 4) sample composites were combined to obtain enough solids to conduct leaching, washing, and ultrafiltration tests in the hot CUF. Shimskey et al. (2009a) provide details of the characterization of the mixture and the hot CUF tests conducted. Testing was similar to that described above for the Group 1 and Group 2 composite, except that no oxidative leaching was conducted (the low chromium levels in these wastes would make oxidative leaching unnecessary in the WTP). PSD and rheology data obtained before and after key process operations are summarized below. 
Figure 4.2 indicates that caustic leaching had relatively little effect on the PSD of the composite, despite the reported 94\% dissolution of aluminum compounds, which initially represented about 98\% of the solids (Shimskey et al. 2009a). The post-caustic leach wash, which primarily removed soluble phosphate salts, had a larger impact on the PSD (PSD data are included in Appendix H). As indicated in Table 4.2, at the low solids concentrations that were reached after caustic leaching and dissolution of aluminum compounds (primarily gibbsite), the slurry behaved as a Newtonian fluid.

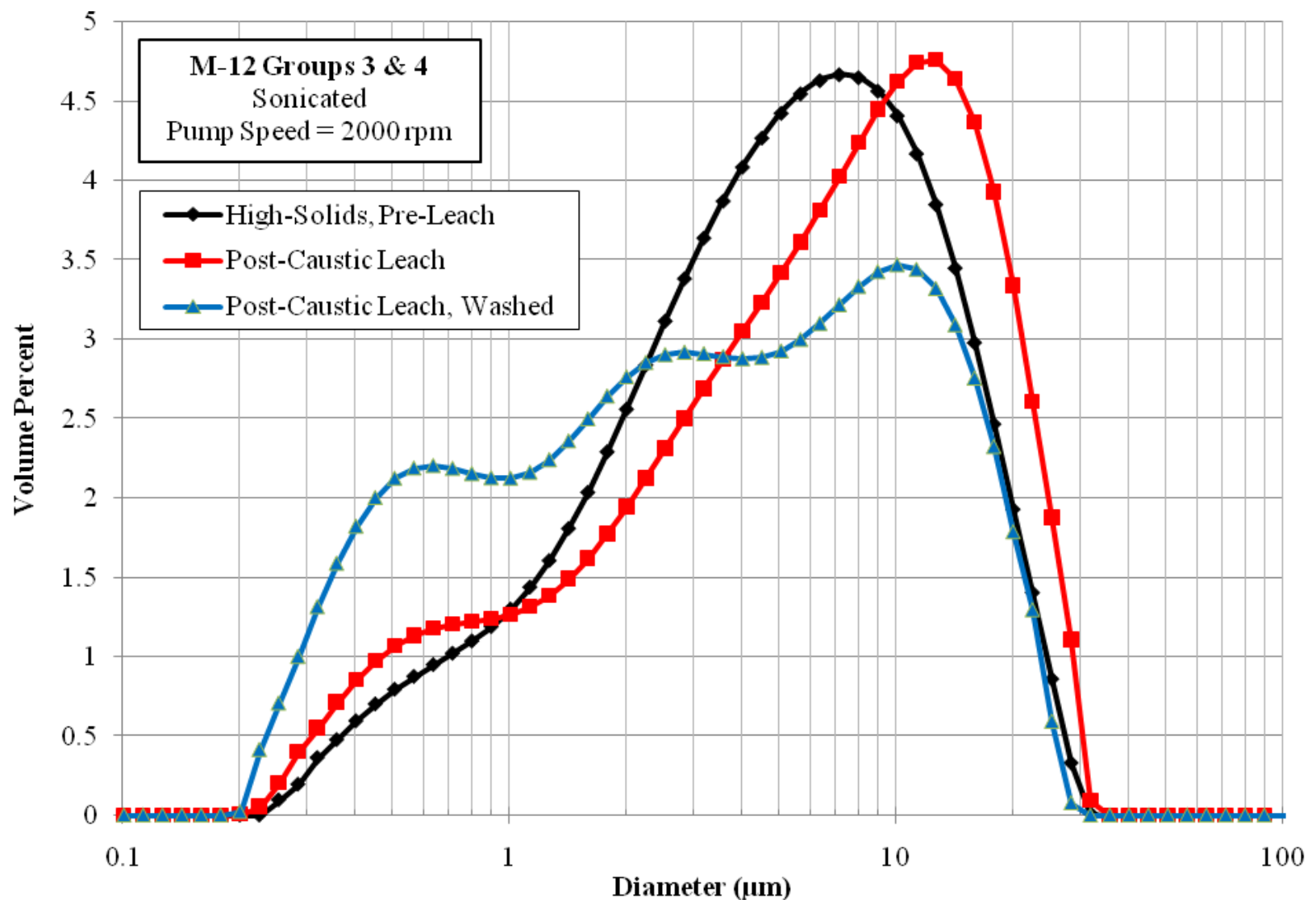

Figure 4.2. M-12 Waste Composite Groups 3 and 4 Leached and Washed Slurry PSDs

Table 4.2. M-12 Waste Composite Groups 3 and 4 Leached and Washed Slurry Rheology Values ${ }^{(a)}$

\begin{tabular}{|c|c|c|c|c|c|c|c|}
\hline \multirow[b]{2}{*}{ Sample } & \multirow{2}{*}{$\begin{array}{c}\text { UDS } \\
\text { (wt\%) }\end{array}$} & \multicolumn{3}{|c|}{ Bingham Yield Stress (Pa) } & \multicolumn{3}{|c|}{$\begin{array}{c}\text { Bingham Consistency } \\
(\mathbf{P a} \cdot \mathbf{s})\end{array}$} \\
\hline & & $25^{\circ} \mathrm{C}$ & $40^{\circ} \mathrm{C}$ & $60^{\circ} \mathrm{C}$ & $25^{\circ} \mathrm{C}$ & $40^{\circ} \mathrm{C}$ & $60^{\circ} \mathrm{C}$ \\
\hline High-Solids, Pre-Leach & 12.7 & 3.2 & 3.2 & 2.3 & 0.0074 & 0.0070 & 0.0052 \\
\hline \multirow{2}{*}{ Sample } & \multirow{2}{*}{$\begin{array}{c}\text { UDS } \\
\text { (wt \%) }\end{array}$} & \multicolumn{3}{|c|}{ Newtonian Viscosity (cP) } & \multirow{4}{*}{\multicolumn{3}{|c|}{ n.a. }} \\
\hline & & $25^{\circ} \mathrm{C}$ & $40^{\circ} \mathrm{C}$ & $60^{\circ} \mathrm{C}$ & & & \\
\hline Post-Caustic Leach, De-Watered & 2.6 & 15.3 & 9.5 & 5.4 & & & \\
\hline Post-Caustic Leach, Washed & 2.4 & 2.5 & 1.4 & 0.7 & & & \\
\hline
\end{tabular}




\subsection{Groups 5 and 6-REDOX Sludge and S Saltcake Wastes}

The REDOX sludge (Group 5) and S saltcake (Group 6) waste sample composites were combined to obtain enough solids to conduct leaching, washing, and ultrafiltration tests in the hot CUF. Shimskey et al. (2009b) provide details of the characterization of the mixture and the hot CUF tests conducted.

Testing was similar to that described above for the Group 1 and Group 2 composite. PSD and rheology data obtained before and after key process operations are summarized below.

Figure 4.3 illustrates the PSDs of the composited material before and after caustic leaching, after the post-caustic leach wash, and after the post-oxidative leach wash. Caustic leaching evidently dissolved the majority of the largest particles, shifting the PSD peak size from about $6 \mu \mathrm{m}$ to about $2 \mu \mathrm{m}$ (PSD data are included in Appendix H). The post-caustic leach wash had little effect on PSD (suggesting no soluble salt solids remained after caustic leaching), and oxidative leaching shifted the peak size of the PSD to about $0.7 \mu \mathrm{m}$ (possibly because the oxidative leaching with permanganate results in the formation of sub-micron $\mathrm{MnO}_{2}$ particles). Rheology measurements given in Table 4.3 suggest that caustic leaching increased yield stress (despite a decrease in the UDS concentration of the sample tested), and that at the measured UDS concentrations, the composite remained non-Newtonian throughout the testing.

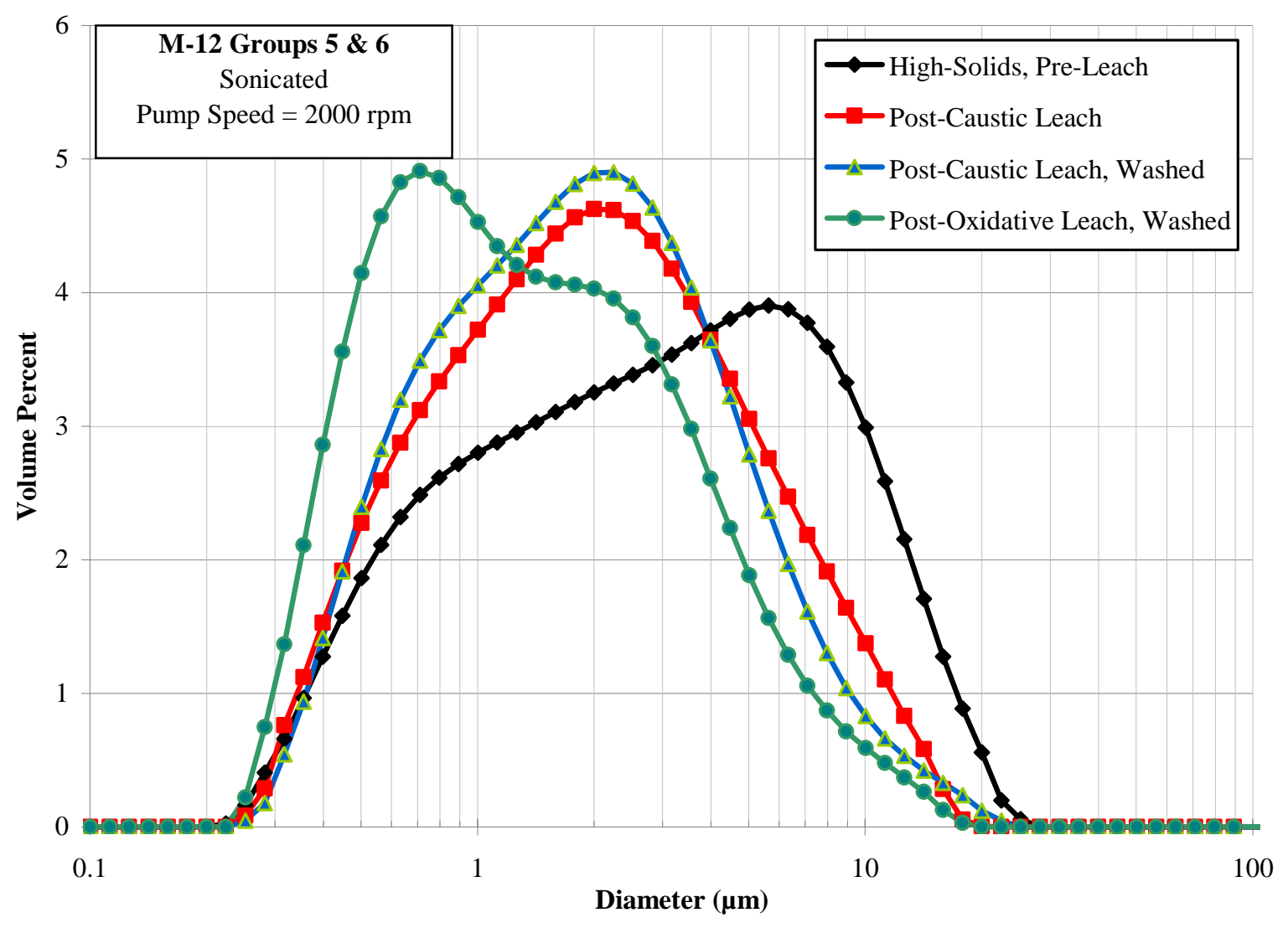

Figure 4.3. M-12 Waste Composite Groups 5 and 6 Leached and Washed Slurry PSDs 
Table 4.3. M-12 Waste Composite Groups 5 and 6 Leached and Washed Slurry Rheology Values ${ }^{(a)}$

\begin{tabular}{|c|c|c|c|c|c|c|c|}
\hline \multirow[b]{2}{*}{ Sample } & \multirow{2}{*}{$\begin{array}{l}\text { UDS } \\
(w t \%)\end{array}$} & \multicolumn{3}{|c|}{ Bingham Yield Stress (Pa) } & \multicolumn{3}{|c|}{$\begin{array}{c}\text { Bingham Consistency } \\
(\mathbf{P a} \cdot \mathbf{s})\end{array}$} \\
\hline & & $25^{\circ} \mathrm{C}$ & $40^{\circ} \mathrm{C}$ & $60^{\circ} \mathrm{C}$ & $25^{\circ} \mathrm{C}$ & $40^{\circ} \mathrm{C}$ & $60^{\circ} \mathrm{C}$ \\
\hline High-Solids, Pre-Leach & 13.2 & 6.7 & 7.2 & 11.4 & 0.014 & 0.011 & 0.010 \\
\hline Post-Caustic Leach, De-Watered & 8.9 & 10 & 21 & 23 & 0.029 & 0.020 & 0.015 \\
\hline Post-Caustic Leach, Washed & 12.8 & 20 & 22 & 24 & 0.015 & 0.012 & 0.012 \\
\hline Post-Oxidative Leach, Washed & 9.7 & 0.74 & 0.72 & 1.4 & 0.0051 & 0.0031 & 0.0031 \\
\hline
\end{tabular}

\subsection{Group 7 with AY-102 Tank Waste-TBP Sludge and Tank AY-102 Wastes}

Edwards et al. (2009) describe the testing of the M12 Group 7 waste composite. This waste composite contained high concentrations of both phosphate and aluminum (primarily as gibbsite) for caustic leach tests, but insufficient chromium to warrant an oxidative leaching step. The quantity of Group 7 waste composite was insufficient for hot-cell leaching and filtration tests, so available AY-102 waste samples were combined with the Group 7 composite for those tests. Because the radiation field from the samples exceeded limits, PSDs were not run on the hot cell test samples. Table 4.4 lists the measured Bingham yield stress and consistency parameters for the composite before and after caustic leaching, and after the post-caustic leach wash. The relatively small differences in yield stress observed in testing may be attributable to the changes in UDS.

Table 4.4. M-12 Waste Composite Group 7 and Tank AY-102 Leached and Washed Slurry Rheology Values $^{(a)}$

\begin{tabular}{|l|c|c|c|c|c|c|c|}
\hline \multirow{2}{*}{\multicolumn{1}{c|}{ Sample }} & \multirow{2}{*}{$\begin{array}{c}\text { UDS } \\
\mathbf{( w t \% )}\end{array}$} & \multicolumn{2}{c|}{ Bingham Yield Stress (Pa) } & \multicolumn{3}{c|}{$\begin{array}{c}\text { Bingham Consistency } \\
(\mathbf{P a} \cdot \mathbf{s})\end{array}$} \\
\cline { 3 - 8 } & & $\mathbf{2 5}^{\circ} \mathbf{C}$ & $\mathbf{4 0}^{\circ} \mathbf{C}$ & $\mathbf{6 0}^{\circ} \mathbf{C}$ & $\mathbf{2 5}^{\circ} \mathbf{C}$ & $\mathbf{4 0}^{\circ} \mathbf{C}$ & $\mathbf{6 0}^{\circ} \mathbf{C}$ \\
\hline High-Solids, Pre-Leach & 25.6 & 4.9 & 4.8 & 6.1 & 0.010 & 0.0086 & 0.0083 \\
\hline Post-Caustic Leach, De-Watered & 20.1 & 2.6 & 1.5 & 1.4 & 0.011 & 0.0089 & 0.0071 \\
\hline Post-Caustic Leach, Washed & 14.0 & 0.48 & 0.32 & 0.39 & 0.0029 & 0.0023 & 0.0014 \\
\hline
\end{tabular}

(a) Values at $25^{\circ} \mathrm{C}$ are averages of the primary and duplicate measurements.

\subsection{Group 8-Ferrocyanide Wastes}

The M12 Group 8 composite was composed of ferrocyanide waste samples. Fiskum et al. (2009a) provide details of the sample selection, waste compositing, and hot cell tests conducted. The Group 8 composite was subjected to caustic leaching at $60^{\circ} \mathrm{C}$ to dissolve aluminum (present mainly as gibbsite) and phosphate solids, but not oxidative leaching. Only about 55 to $60 \%$ of the aluminum solids were dissolved, and effectively none of the insoluble phosphate was dissolved. Figure 4.4 gives PSDs measured before caustic leaching and after post-caustic leach washing (no PSD was taken immediately before washing). The PSDs suggest that the leaching and washing steps decreased the volume fraction of particles below about $15 \mu \mathrm{m}$ (PSD data are included in Appendix H). Table 4.5 lists the Bingham 
rheology parameters measured during hot cell tests. Values in the table suggest that the caustic leach had little effect on yield stress, but washing increased yield stress significantly.

\subsection{Summary}

The WTP leaching and washing processes were observed to have different effects on the PSDs and rheology of each waste grouping. The largest changes in average particle size were observed for the Group 1 and 2 composite and the Group 5 and 6 composite, which experienced about an order of magnitude decrease in average particle size during the leaching and washing processes. An approximate factor of two increase in the particle size mode is observed after caustic leaching for the Group 3 and Group 4 composite. The group 8 PSDs were relatively unchanged during the leaching and washing process. Other authors (Poloski et al. 2009) have noted that the PSDs generally (but not always) increase during leaching and washing and that the particle density may increase as less dense materials are washed and leached from the solids. The variability in the wastes along with variations in experimental and measurement techniques makes it difficult to draw general conclusions about the impact of the pretreatment process on the PSDs of the slurries.

In both the Group 1 and Group 2, and Group 5 and Group 6 composites, pretreatment samples with reduced solids concentrations in comparison to the initial untreated samples are shown to have increased rheology. The leaching and washing processes remove the aluminum and soluble solids, which, as listed in Section 3.2, are generally lower-density solids. Thus, the fraction of higher-density particulate is increased after the pretreatment processes. 


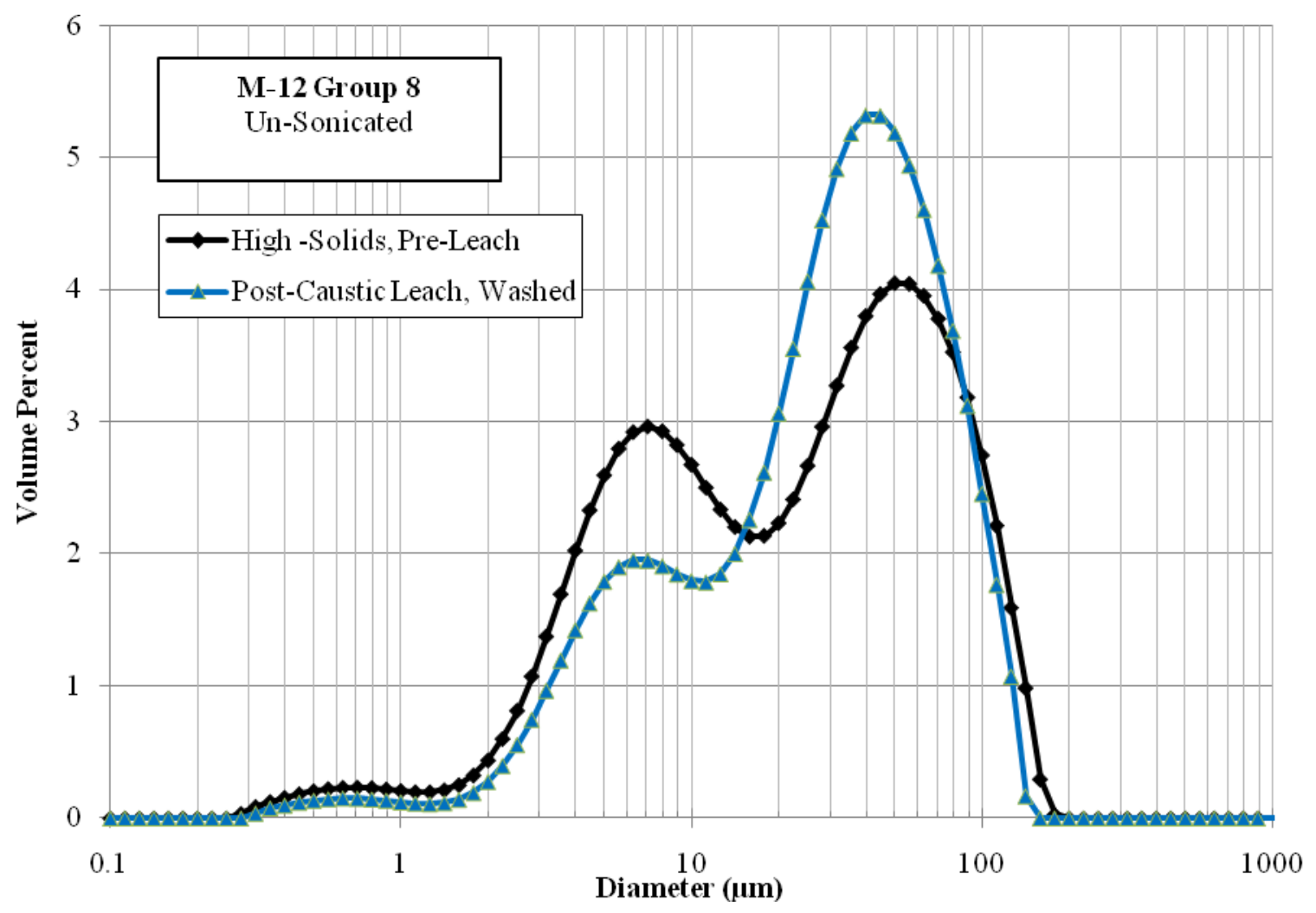

Figure 4.4. M-12 Waste Composite Group 8 Leached and Washed Slurry PSDs

Table 4.5. M-12 Waste Composite Group 8 Leached and Washed Slurry Rheology Values ${ }^{(a)}$

\begin{tabular}{|c|c|c|c|c|c|c|c|}
\hline \multirow[b]{2}{*}{ Sample } & \multirow{2}{*}{$\begin{array}{c}\text { UDS } \\
\text { (wt\%) }\end{array}$} & \multicolumn{3}{|c|}{ Bingham Yield Stress (Pa) } & \multicolumn{3}{|c|}{$\begin{array}{c}\text { Bingham Consistency } \\
(\mathbf{P a} \cdot \mathbf{s})\end{array}$} \\
\hline & & $25^{\circ} \mathrm{C}$ & $40^{\circ} \mathrm{C}$ & $60^{\circ} \mathrm{C}$ & $25^{\circ} \mathrm{C}$ & $40^{\circ} \mathrm{C}$ & $60^{\circ} \mathrm{C}$ \\
\hline High-Solids, Pre-Leach & 12.7 & 0.77 & 0.71 & 0.64 & 0.0050 & 0.0040 & 0.0027 \\
\hline Post-Caustic Leach, De-Watered & 23.3 & 1.1 & 0.95 & 1.0 & 0.0066 & 0.0056 & 0.0054 \\
\hline Post-Caustic Leach, Washed & 20.3 & 6.1 & 5.3 & 5.4 & 0.0067 & 0.0057 & 0.0051 \\
\hline
\end{tabular}

(a) Values at $25^{\circ} \mathrm{C}$ are averages of the primary and duplicate measurements. 


\subsection{Summary of Hanford Waste Physical and Rheological Property Data Gaps}

This section contains a summary of gaps in the Hanford waste property data as identified in the course of the work completed for this report. The primary focus is on data gaps identified by considering the fraction of the waste represented by the parameter characterizations (Section 5.1). Data gaps that may be addressed by improved or new analytical methods are discussed in Section 5.2. Gaps in scale-up, data interpretation, and analysis discussed in Section 5.3 are also noted, but they do not represent a comprehensive list.

\subsection{Data Gaps in Physical and Rheological Properties}

In this section, the gaps in the Hanford waste parameter characterization data are discussed. The gaps are presented in terms of the level of characterization by tanks, waste types and the overall inventory.

In Section 3.0, where possible and meaningful, the waste parameter measurements are related to the Hanford UDS inventory. For the purpose of defining data gaps, a tank waste is treated as "represented" if the parameter of interest has been measured for at least one sample of that tank's waste. In this approach, a number of important factors are not considered beyond those used to select specific data for the overall data set. These factors include the extent to which a sample represents the tank contents, the number of samples, the number of measurements made on a sample or the measurement technique.

A summary of the number of tanks and waste types for which a given parameter is characterized as given in Section 3.0 is provided in Table 5.1. The report sections in which each parameter is described are listed, as well as general categories of waste handling operations identified in Section 2.0 that are dependent on the parameter. The percentage of the Hanford UDS volume represented is determined from the primary waste types represented and their relative fractions to the waste inventory (primary waste types described Section 3.1). In general, less than $50 \%$ of the Hanford UDS volume is represented by the waste parameter data. The primary waste type of a given tank is defined as the most prevalent waste type as discussed in Section 3.0. The number of tanks and primary waste types are listed as well as the number of waste types that are considered to have more than $50 \%$ of the UDS volume represented by the data.

For example, for solids - shear strength - general data, 52\% of the Hanford UDS volume is represented by the primary waste types with shear strength measurements, indicating a data gap in shear strength of $48 \%$ of the Hanford UDS volume. However, only 7 of the 15 waste types represented (from the data for 36 tanks) have more than $50 \%$ characterization. Thus, a general shear strength data gap of $48 \mathrm{vol} \%$ of the UDS inventory is indicted only under an assumption that wastes with the same primary waste type are adequately represented by the existing data.

The waste characterization determined to have the most representation is Flowing Unsonicated PSDs at $58 \%$, leaving a data gap of $42 \mathrm{vol} \%$ (Table 5.1). The data sets with the lowest representation are those that describe property functionalities. For example, there was limited data where shear strength was obtained as a function of time.

The availability of data is shown in Table 5.2 by waste type and parameter. The 44 waste types and the percent of the Hanford UDS inventory represented by each waste type are identified in a manner 
consistent with the 2002 BBI described in Section 3.1. For each waste type and parameter a "yes" indicates that at least one analysis is available while a dash indicates that no data is available. For the applicable characterizations, the entries in "Hanford UDS Volume Represented", Table 5.1, are the summation of the "Percent of Hanford UDS Inventory" designated with a "yes" in Table 5.2.

It should be noted that some of the information available from the WTP work to support resolution of the M12 issue is not reflected in the data gaps identified in Tables 5.1 and 5.2. This is due to the fact that the composite samples used to represent the 8 sample groups were composed of a number of different but similar waste types. The composite nature of the samples made it difficult to attribute the results to a particular waste type. The eight waste groups represented $~ 75 \%$ of the HLW mass expected to be processed through the WTP. Of the eight waste groups, results from Groups 1 (1C and 2C sludge), 2 (BY, T1, and T2 saltcake), 3 (CWP1 and CWP2 sludge), and 6 (S1 and S2 Saltcake) are not reflected in the gap analysis in Table 5.1 and Table 5.2. 
Table 5.1. Summary of Waste Physical and Rheological Property Data Gaps

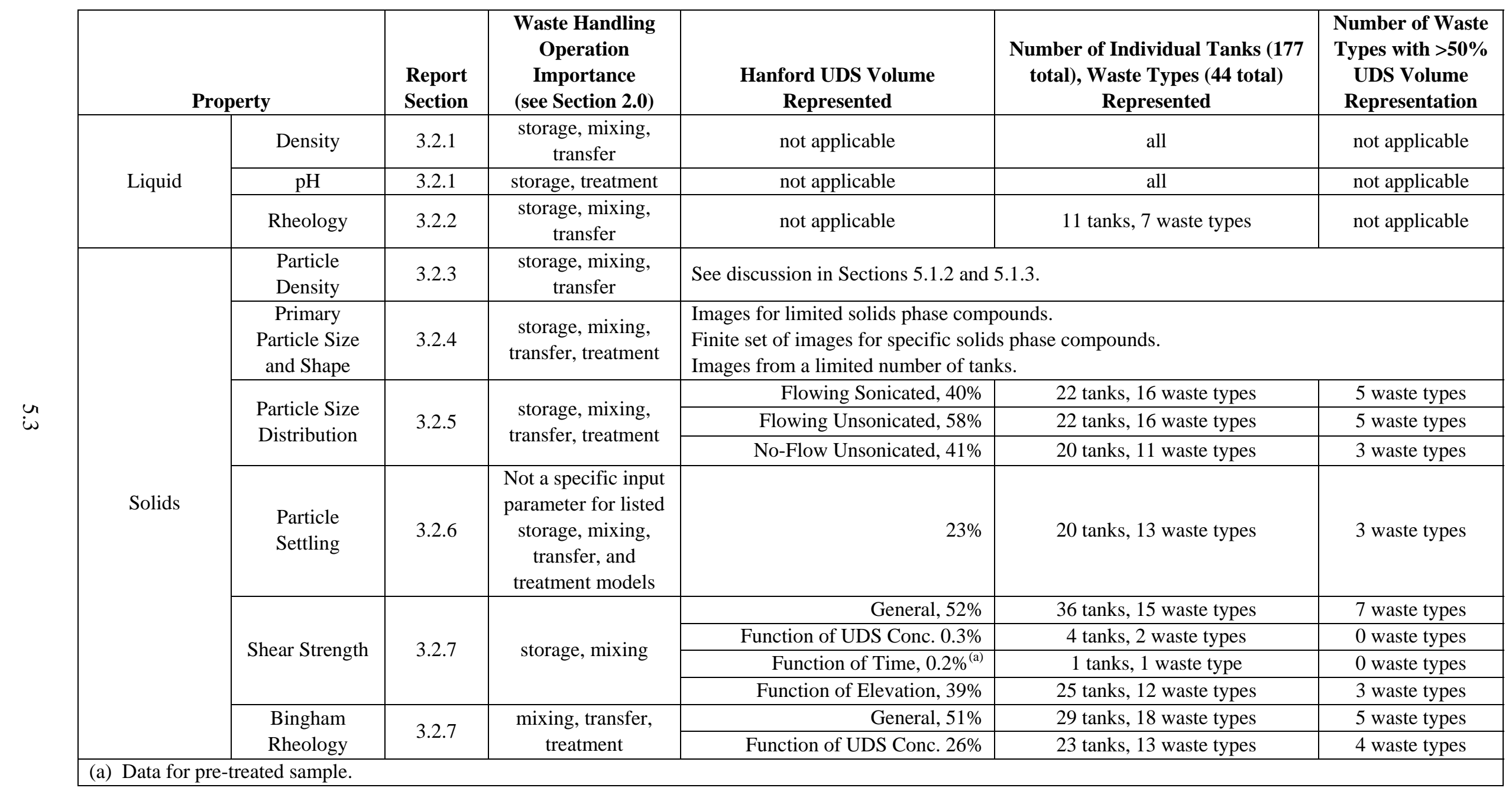


Table 5.2. Waste Type and Parameter Data (by Primary Waste Type)

\begin{tabular}{|c|c|c|c|c|c|c|c|c|}
\hline \multirow[b]{4}{*}{ Waste Type } & \multirow{4}{*}{$\begin{array}{c}\text { Percent } \\
\text { of } \\
\text { Hanford } \\
\text { UDS } \\
\text { Inventory }\end{array}$} & \multicolumn{7}{|c|}{ Data Available } \\
\hline & & \multirow{3}{*}{$\begin{array}{c}\text { Liquid } \\
\text { Rheology }\end{array}$} & \multicolumn{6}{|c|}{ Solids } \\
\hline & & & \multicolumn{3}{|c|}{ Particle Size Distribution } & \multirow[b]{2}{*}{$\begin{array}{l}\text { Particle } \\
\text { Settling }\end{array}$} & \multirow[b]{2}{*}{$\begin{array}{c}\text { Shear } \\
\text { Strength }\end{array}$} & \multirow[b]{2}{*}{$\begin{array}{l}\text { Bingham } \\
\text { Rheology }\end{array}$} \\
\hline & & & $\begin{array}{c}\text { Flowing } \\
\text { Sonicated }\end{array}$ & $\begin{array}{c}\text { Flowing } \\
\text { Unsonicated }\end{array}$ & $\begin{array}{c}\text { No-Flow } \\
\text { Unsonicated }\end{array}$ & & & \\
\hline Unidentified saltcake & $0.3 \%$ & - & - & - & - & - & - & - \\
\hline A1 saltcake & $2.9 \%$ & - & - & - & - & - & yes & - \\
\hline A2 saltcake & $7.3 \%$ & yes & yes & yes & yes & yes & yes & yes \\
\hline B saltcake & $3.1 \%$ & - & - & - & - & - & - & - \\
\hline BY saltcake & $15 \%$ & - & yes & yes & yes & - & - & - \\
\hline R1 saltcake & $0.1 \%$ & - & - & - & - & - & - & - \\
\hline R saltcake & $1.8 \%$ & - & yes & yes & yes & yes & yes & yes \\
\hline S1 saltcake & $18 \%$ & yes & - & yes & - & - & yes & yes \\
\hline S2 saltcake & $6.6 \%$ & yes & - & - & yes & - & yes & yes \\
\hline T1 saltcake & $2.6 \%$ & - & - & - & - & - & - & - \\
\hline T2 saltcake & $20 \%$ & - & - & - & - & - & - & - \\
\hline Unidentified sludge & $0.5 \%$ & - & yes & yes & yes & - & yes & yes \\
\hline 1C sludge & $4.7 \%$ & yes & yes & yes & yes & yes & yes & yes \\
\hline 1CFeCN sludge & $0.4 \%$ & - & - & - & - & - & - & - \\
\hline 224 Post-1949 sludge & $0.1 \%$ & - & yes & yes & - & yes & yes & yes \\
\hline 224 Pre-1949 sludge & $0.03 \%$ & - & - & - & yes & - & yes & yes \\
\hline 2C sludge & $0.8 \%$ & - & yes & yes & yes & yes & yes & yes \\
\hline AR sludge & $0.3 \%$ & - & yes & yes & - & - & - & - \\
\hline B sludge & $0.02 \%$ & - & - & - & - & - & - & - \\
\hline BL sludge & $0.3 \%$ & yes & yes & yes & yes & yes & yes & yes \\
\hline CWP1 sludge & $1.9 \%$ & yes & yes & yes & yes & yes & yes & yes \\
\hline CWP2 sludge & $1.6 \%$ & - & - & - & - & yes & - & yes \\
\hline CWR1 sludge & $1.0 \%$ & - & yes & yes & - & yes & yes & yes \\
\hline CWR2 sludge & $0.2 \%$ & - & - & - & - & - & - & - \\
\hline CWZr1 sludge & $0.1 \%$ & - & - & - & - & - & - & - \\
\hline CWZr2 sludge & $0.5 \%$ & - & yes & yes & - & - & yes & - \\
\hline
\end{tabular}


Table 5.2. (contd)

\begin{tabular}{|c|c|c|c|c|c|c|c|c|}
\hline \multirow[b]{4}{*}{ Waste Type } & \multirow{4}{*}{$\begin{array}{c}\begin{array}{c}\text { Percent } \\
\text { of }\end{array} \\
\text { Hanford } \\
\text { UDS } \\
\text { Inventory } \\
\end{array}$} & \multicolumn{7}{|c|}{ Data Available } \\
\hline & & \multirow{3}{*}{$\begin{array}{c}\text { Liquid } \\
\text { Rheology }\end{array}$} & \multicolumn{6}{|c|}{ Solids } \\
\hline & & & \multicolumn{3}{|c|}{ Particle Size Distribution } & \multirow[b]{2}{*}{$\begin{array}{l}\text { Particle } \\
\text { Settling }\end{array}$} & \multirow[b]{2}{*}{$\begin{array}{c}\text { Shear } \\
\text { Strength }\end{array}$} & \multirow[b]{2}{*}{$\begin{array}{l}\text { Bingham } \\
\text { Rheology }\end{array}$} \\
\hline & & & $\begin{array}{c}\text { Flowing } \\
\text { Sonicated }\end{array}$ & $\begin{array}{c}\text { Flowing } \\
\text { Unsonicated }\end{array}$ & $\begin{array}{c}\text { No-Flow } \\
\text { Unsonicated }\end{array}$ & & & \\
\hline DE sludge & $0.7 \%$ & - & - & - & - & - & - & - \\
\hline HS sludge & $0.1 \%$ & - & - & - & - & - & - & - \\
\hline MW sludge & $0.3 \%$ & - & - & - & - & - & - & - \\
\hline OWW3 sludge & $0.04 \%$ & - & - & - & - & - & - & - \\
\hline P1 sludge & $0.002 \%$ & - & - & - & - & - & - & - \\
\hline P2 sludge & $0.2 \%$ & - & - & - & - & - & - & - \\
\hline P3 sludge & $0.2 \%$ & yes & yes & yes & yes & yes & yes & yes \\
\hline PFeCN sludge & $0.8 \%$ & - & yes & yes & - & yes & yes & yes \\
\hline PL2 sludge & $0.1 \%$ & - & - & - & - & - & - & - \\
\hline Portland Cement sludge & $0.04 \%$ & - & - & - & - & - & - & - \\
\hline R1 (non-boiling) sludge & $1.8 \%$ & - & - & - & - & - & - & - \\
\hline R1 (boiling) sludge & $2.3 \%$ & - & yes & yes & yes & - & yes & yes \\
\hline SRR sludge & $0.2 \%$ & - & - & - & - & - & - & - \\
\hline TBP sludge & $2.1 \%$ & - & yes & yes & - & yes & yes & yes \\
\hline TFeCN sludge & $0.5 \%$ & - & - & - & - & yes & yes & yes \\
\hline TH1 sludge & $0.03 \%$ & - & - & - & - & - & - & - \\
\hline TH2 sludge & $0.03 \%$ & - & - & - & - & - & - & - \\
\hline Z sludge & $0.2 \%$ & - & - & - & - & - & - & - \\
\hline
\end{tabular}




\subsubsection{Variation of Parameters as a Function of Waste Type, UDS Concentration, and Processing Steps}

As shown repeatedly in Section 3.0, waste parameters can vary considerably for different waste types and there can be considerable variability within a given waste type. From Table 5.1, the data for the variation of rheological parameters as a function of UDS concentration, time, and storage conditions are relatively limited. The functionality of waste rheology and particle size distribution with pretreatment processing steps may have opposite trends depending on waste type as summarized in Section 4.0. The implication is that these variables can impact the waste properties in a complex way and that each tank or batch of waste may need to be individually characterized during retrieval and treatment.

\subsubsection{Particle Morphology}

The expert group assembled to examine information on the actual particles and morphology estimated that at least $50 \%$ of the solids are amorphous and are not well characterized. Many solid phases in the non-salt part of the waste are composed of mixtures of compounds of different metals. The fraction of the solids present in the mixed phases in each tank or waste type is not known, nor are the density and size distribution of the mixed solid phases. The mixtures of metals that have been observed in Hanford tank wastes include Al-Cr, Fe-Cr-Ni-Mn, Fe-Pb, Fe-Bi-P, Fe-Zr, and others.

Some of the mixed phases were probably formed by co-precipitation resulting in inclusion of impurities or occlusion of one particle within another. As a result, the mixed phases consist of non-stoichiometric ratios of various metals given the complex and variable history of the wastes in each tank. Thus, the proportions of the compounds present likely vary from tank to tank. The densities of the mixed phases, which are between those of the individual compounds in the mixture, will vary correspondingly. The presence of mixed phases, therefore, introduces a potentially large uncertainty in particle density. If the size distribution of mixed phases differs from the distribution expected for the average of the individual phases in the mixture, there could be an uncertainty in size distribution as well.

Mixed-phase PSDs could differ from those of the individual compounds because they may have been formed by different chemical processes. Mixed-phases that are amorphous are likely to have been formed by very rapid precipitation, a process that does not allow crystals to form and produces sub-micron primary particles. The effective particle size may be increased because the particles may be cemented together to form hard agglomerates.

To some extent, these gaps may be addressed by reviewing existing observations from solids analytical techniques (XRD, SEM/EDS, TEM, FTIR, etc.) and correlating these results with the results of dissolution experiments, TGA curves, and other indirect evidence. These types of information are often in reports written by different authors at different times for different purposes. To the extent that this is the case, existing information needs to be collated and viewed as a whole.

A potential source of information on particle morphology and relatively large particles is the information developed during the characterization of solids in residual wastes from single-shell tanks. The characterization of the residual solids is conducted using X-ray diffraction, scanning electron microscopy/energy dispersive X-ray spectroscopy and/or transmission electron microscopy plus other 
techniques (Krupka et al. 2010). This characterization information is obtained to develop models that simulate contaminate release mechanisms from the residual solids reacting with water infiltrating the tanks. While some of this information was incorporated into the current study a complete review of the available information was not completed. Analysis of the samples for particle size distribution could provide additional information on some of the larger particles that may challenge the transport and mixing systems. The extent of hydration of some phases that originally precipitated as hydroxides has been questioned and represents a knowledge gap. For example, iron is a major contributor to insoluble solids in Hanford tank wastes; it is present in all tanks and iron phases make up more than $20 \%$ of the insoluble solid mass in more than 30 tanks. Iron has been observed in wastes in various crystalline forms, such as oxides $\left(\mathrm{Fe}_{2} \mathrm{O}_{3}\right.$, either hematite or magnetite) and partially hydrated goethite (FeOOH). In one case, TGA indicated that Fe was present in all three degrees of hydration: goethite, magnetite, and ferric hydroxide (Lumetta et al. 2009a). However, amorphous phases of Fe and other metal elements have also been observed and may be present in significant quantities. The uncertainty in the extent of hydration is a knowledge gap that results in uncertainty in the distribution of particle densities.

An improved understanding of the relative abundance of hydroxides, oxyhydroxides, and oxides would reduce the uncertainty in the distribution of particle densities. Many of the observed amorphous phases that might be hydroxides are also mixed phases, so an examination of the mixed phases should include a direct determination of their particle density, or some form of thermogravimetric testing that can define the extent of hydration. The compounds in mixed phases could be either more or less dehydrated than those in single compounds, depending on whether the mixed structure tends to interfere with solid-state reactions (such as $\mathrm{Fe}(\mathrm{OH})_{3} \rightarrow \mathrm{Fe}_{2} \mathrm{O}_{3}$ ) and dissolution/re-precipitation (such as $\left.\mathrm{Fe}(\mathrm{OH})_{3} \rightarrow \mathrm{FeOOH}\right)$.

It would be worth reviewing the tanks in which the oxide phases have been found to see whether a disproportionate number of them have histories of high waste temperature. The temperature increases the rate of the dehydration reactions that can occur during metal hydroxide aging.

\subsubsection{Dry Solids Density}

The dry solids density is an important parameter used in simulant development and mixing assessments. During the simulant development process for mixing assessments it provides a target for the overall dry particle density for polydisperse simulants.

While estimates and a limited number of measurements are available this parameter has not been generally measured directly on samples. Some data are available for individual components where the morphology has been identified. Density values are obtained from the literature. As discussed in Section 5.1.2, less than 50\% of the solid phase morphology has been characterized. In addition, little is known about how the dry solids density changes during pretreatment as sodium is washed from the sludge solids by dissolving sodium salts.

As discussed in Section 5.2.2, the methods currently available to determine solids density include the use of gas pycnometers, a displacement method based on the use of dodecane (Tingey 2004), and calculating it as part of the UDS analyses (Smith and Prindiville 2002). The dry solid density does not necessarily represent the particles as they exist in the waste as interparticle liquid is not included. Section 5.2.3 describes a methodology to determine the solid density which includes potential interparticle liquid. 


\subsubsection{Shear Strength as a Function of Time}

Changes in shear strength in settled solids layers with an emphasis on shorter settling times and shear strength as a function of solids depth is not well quantified (Gauglitz et al. 2009, 2010b). An accurate understanding of the shear strength formation is needed so mixing systems are designed to prevent settled solids layers that may exceed the remobilization capabilities.

\subsubsection{Impact of Storage on Sample properties}

The impact of storage on the sample properties has not been systematically quantified. Some of the results presented in this report are based on analysis of samples that were obtained from archives at the 222-S Laboratory. In some cases these samples have been stored for up to 15 years. While significant effort is made to minimize the effect of storage, the long storage time may result in altered sample characteristics due to aging and drying.

\subsection{Gaps in Analytical Methods}

In this section, data gaps that may be addressed by improved or new analytical methods are summarized.

\subsubsection{Particle Size and Shape}

As noted in Section 3.2.5, light scattering is currently the dominant method for characterizing the PSD of tank wastes. Due to the nature of the technique and the many potential sources of error, the PSD results from this method are best characterized as an apparent PSD although the technique is arguably adequate to characterize the general particle sizes in the waste. In addition, the method does not provide information on the nature of agglomeration or particle shape and in some applications may underestimate the abundance of large particles. Other methods based on optical techniques are available that have the potential to provide more direct information on agglomeration and particle shape for individual particles. An example is the Particle Insight Dynamic Image Analyzer ${ }^{(a)}$ or the Malvern's Morphology G (optical particle size \& shape \& Raman). Results from this type of instrument appear to be more directly applicable to particles in pipeline transport or mixing systems.

\subsubsection{Volume Fraction of Solids in Slurry and Dry Solids Density}

The volume fraction of solids is an important parameter used in estimating slurry viscosity and pipeline critical velocity as well as performing hindered settling calculations. To obtain the volume fraction of dry solids in a slurry, it is generally necessary to determine the dry solids density and calculate the volume by dividing by the dry solid mass. Note that the solids volume fraction discussed here is the dry solids volume, and, as discussed in Section 5.1.3, potential interparticle liquid is not included. The dry solid volume fraction should not be confused with similar terminology that refers to a ratio of settled solids volume to a reference volume. Methods currently available to determine dry solids density include the use of gas pycnometers, a displacement method based on the use of dodecane (Tingey 2004), and calculating it as part of the UDS analyses (Smith and Prindiville 2002). A methodology to determine the

(a) Particulate Systems, 4356 Communications Dr. Norcross, GA 30093; www.particulatesystems.com. 
solid density which includes potential interparticle liquid is described in Section 5.2.3. The small amounts of actual waste sample typically used for the UDS analyses along with the difficulty working in hot cells provide density results that are not accurate enough for practical use. This gap could be addressed by additional method development applied to the UDS method to use larger samples or more accurate centrifuge cones. The easiest and most accurate method is gas pycnometry.

\subsubsection{Particle Size and Density Distributions}

Particle size and density distributions for Hanford wastes are important to waste transport and mixing and for developing simulants for evaluating these processes. PSD and particle density data are currently developed with separate analytical techniques. These attributes are combined in Section 3.3 to provide estimates of particle size and density distributions by making assumptions about the waste properties. This approach results in considerable uncertainty that may lead to:

- underestimating of waste characteristics (e.g., particle size measurements under-representing particles greater than $500 \mu \mathrm{m}$ in size, Bechtold et al. 2002)

- misrepresentation of the particle size for a particular application due to PSD instrument configuration (see PSD instrumentation configuration and results in Section 3.2.5 and comparison to process data, Sections 3.2.6 and Section 3.3), or

- excessive conservatism in simulant development and designs for waste transport and mixing equipment.

Consequently, there is a need for a method that has the capability to determine the particle size and corresponding density of individual particles simultaneously.

One concept currently under development and sponsored by EM-31 is a Particle Size and Velocity System. The method uses a settling column in which particulates settle through an appropriate fluid. Cameras obtain images of the settling particles with a sufficient magnification and frame rate to allow a determination of the settling rate. Particle size and shape information is also obtained. From these data, settling correlations can be used to determine the effective particle density for the sample conditions.

\subsubsection{Fractal Dimension}

As discussed in Section 3.3, assumptions about the fractal dimension of the waste agglomerates were necessary for estimating the particle size and density distributions of the wastes. The fractal dimension describes the fraction of an agglomerate that is dry solid as opposed to interparticle liquid. At this point, the detailed information on the fractal dimension of the waste agglomerates is limited with a likely range of 1.6 2.25. A maximum value of 3, equivalent to all solids throughout the size distribution having a crystal density, was assumed for the work reported in Section 3.3. This results in considerable uncertainty in the PSDDs and a large range of estimated values. Additional characterization of the waste samples could reduce this uncertainty. Determination of the fractal dimension using electron microscopy is generally only suitable for agglomerates with fractal dimension less than 2. For higher values, overlapping of primary particles results in distortion. This suggests that additional methods are needed for a more complete determination of agglomerate fractal dimension. 


\subsubsection{Abrasivity}

The abrasion properties of the waste are expected to have an impact on the erosion rates of processing equipment, but there are little data on the abrasivity of actual wastes. Most of the existing literature on the abrasivity of tanks wastes is based on testing with Hanford tank waste simulants. Only one value for a Miller Number obtained from actual was identified (Hodgson 1995). The value provided was a Miller Number of 8.4 obtained from a core sample taken from Tank 241-AZ-101. The lack of data suggests the need for a method to measure abrasivity in a radioactive environment with actual tank waste samples.

\subsubsection{Solids Settling Rate in Concentrated Slurries}

The current method of measuring the solids settling rates involves observing the interface of the settling solids and the clarified liquid above the solids. This approach provides a settling rate of the interface that generally represents the slowest settling solids. The larger, more dense particles likely settle at greater rates through the slurry. Data on these particulates are of interest for retrieval and mixing operations since they present a greater challenge. A method that could determine the settling rates over a wide range of particles and densities in concentrated slurries would provide useful data for assessing retrieval and mixing equipment.

\subsection{Gaps in Scale-Up, Data Interpretation and Analysis}

In this section, gaps in scale-up data interpretation and analysis are discussed.

\subsubsection{Interpretation and Scale-up of Settling Data}

One significant question about settling data, or their interpretation, is that of the correct scale-up to tank conditions. A factor of 10 discrepancy between rapid interface settling in Tank AZ-101 and slower settling in the laboratory studies of AZ-101 composite samples was observed (Gauglitz et al. 2009, 2010b). In addition, when velocity distributions were calculated from PSDDs and compared to interface velocities observed in Tank AZ-101, Tank AY-102, and a laboratory-scale column (3-inch ID) containing three-component simulant, the interface velocities consistently lay at or above the $50^{\text {th }}$ percentile of the calculated distributions. Because the visible interface by definition consists of the slowest-settling particles, its velocity would be expected to be closer to the lowest-velocity percentiles than to the median or higher percentiles of the distribution. The reason for these discrepancies is not clear, although the possible contributing causes include

- Experimentally based: interface velocities measured in the laboratory might have been low because of wall effects in the graduated cylinders ( 2-cm ID) or an initial suspension depth ( $20 \mathrm{~cm}$ or less) that was not great enough to allow the velocity to be fully developed (see Maclean 1999). However, the data that were reviewed did not clearly demonstrate the effect of test vessel size.

- Difference in particles: there might have been more particle agglomeration in the tank than in the graduated cylinder in the laboratory. Studies conducted with ocean sediments have shown that aggregate sizes and settling rates measured in the laboratory can be one to two orders of magnitude smaller than values obtained by in situ techniques (Mantovanelli and Ridd 2006). Aggregates are easily damaged when sampled and manipulated. However, PSDDs that were calculated in a manner 
that accounted for agglomeration (fractal dimension less than 3) did not produce velocity distributions that were consistent with the higher observed velocities.

- Difference in the PSDDs: the distribution of particle crystal densities and sizes might be biased in a way that tends to underestimate the velocities calculated for individual particles, based on the PSDDs. However, the three-component simulant was well defined, so it is not clear how the bias might have arisen.

- Difference in the flow field: wake capture or particle-induced convection, such as "vertical streaming," might have pulled the interface particles down more rapidly in the tank or in the 3-inch-ID column than in the relatively confined graduated cylinders.

The presence of or magnitude of the effect from particle-induced convective structures has not been confirmed either in tanks or in laboratory conditions. A further review of existing literature would serve two purposes: 1) evaluate whether conditions could produce particle-induced convection, and 2) suggest experimental methods that could detect and measure convection because the opaque nature of the waste does not allow visual observation.

Visual observations can detect the interface between the slowest-settling particles and the clarified liquid above the settling suspension, but not the interior concentration interfaces caused by faster-settling particles. These faster particles are those that are harder to mobilize and mix, so the absence of observing them is a gap that relates very directly to mobilization and mixing design. A literature search would suggest instruments and methods to measure the velocity of interior interfaces.

Another gap in data comes from the tendency of settling tests to be reported only in terms of relative initial sediment volume. This makes it difficult to interpret the data to provide settling velocity. Settling velocity is an absolute property of the solid/liquid system and can only be calculated from data for the absolute height of the interface. In this report, sediment heights (when not reported) have been calculated from the known sample volume and the inner diameters of graduated cylinders or centrifuge tubes that would be large enough to hold the sample volume. These are estimates only because centrifuge tubes and graduated cylinders are not standardized.

\subsubsection{PSD Uncertainty}

A common request expressed by end users of PSD results is for some measure of uncertainty to be provided with the PSDs. This desire is driven by the need to use bounding particle sizes for developing simulants or for use in engineering calculations. An understanding of the size of the solid particles in a tank waste sample is crucial in determining sedimentation rates, the ease with which the solids can be filtered, flow behavior of the solids when pumped through a pipe, and the force required to suspend solids and keep the solids suspended in a pipe or tank. One approach to address this issue is to provide an estimate of the uncertainty based on the performance characteristics of the method and instruments. Another approach is to develop tolerance or confidence limits based on the actual samples results. Unfortunately neither approach results in quantifiable, technically defensible uncertainties for the PSDs for the light-scattering methods currently in use.

Non-parametric tolerance interval methods exist that can provide technically defensible estimates of PSD uncertainty but these methods require sample sizes larger than the sample sizes currently available. This suggests that a larger number of samples be analyzed although the number required to attain 
acceptable confidence levels may be prohibitively large. For example, a random sample of 30 observations would be needed to achieve $95 \%$ confidence that the $90^{\text {th }}$ percentile of a population (not necessarily normally distributed) would be less than the maximal value in the sample.

Perhaps the most immediate approach to addressing this gap is to use the range of the actual data combined with knowledge of the wastes and the processes that generated them. For the existing data, the PSDs at a given percentile typically range over a factor of 5-10. Estimates of the maximum particle sizes have been provided in various tables and plots in Section 3.2.5. The topic of primary particle size and shape is addressed in Section 3.2.4 and Appendix D. Collectively this body of information may be used to form reasonable estimates of the bounding PSDs for the characterized waste.

\subsubsection{Critical Shear Stress for Erosion}

The critical shear stress for erosion is ideally the applied shear stress above which particulate will be removed from a surface or body. This parameter is pertinent to tank farm and WTP mixing and line flushing scenarios and is material dependent.

As described in Wells et al. (2009), the critical shear stress for a given material can be predicted from in situ or laboratory erosion measurements. There are different measurement techniques that may be used, but all require multiple data points such that the critical shear stress at zero-erosion (corresponding to the predicted onset of erosion) can be identified. Some of these erosion measurement techniques allow the erosion rate to be determined. For a shear stress beyond the critical shear stress applied to a material, two states of erosion will dominate, surface and bulk erosion. While bulk erosion may be initiated at applied shear stresses below a material's measured shear strength, it will occur if the applied stress is equal to or exceeds the measured shear strength.

Methods for determining the critical shear stress for the onset bulk erosion over a range of cohesive materials have not been successfully developed outside of experimental mapping of the erosion process as a function of applied shear stress for the specific material of interest.

\subsection{Recommendations}

Prioritizing the gaps identified in this section depends on the current state of knowledge, the priority of the applications for which the information is needed, and the timing with which information can be obtained. Recognizing that the authors of this document are not the decision makers concerning efforts to address the gaps identified in this section, some guidance is nevertheless offered on where additional efforts should be focused. In developing these observations the focus was on gaps for which relatively small amounts of data are available, data are relatively uncertain, and waste properties that are not readily modified by processing (e.g., rheology may be modified by diluting the solids concentration).

While the characterization of the tank wastes is not complete, additional characterization of the waste parameters with the methods currently available may not have a great impact on the average or median properties reported in this document. This suggestion is based on the observation that the results developed in this report are fairly similar to those of previous efforts even though a significant amount of new data has been obtained. Indeed the recent effort sponsored by WTP characterized samples from eight waste groups representing approximately $\sim 75 \%$ of the HLW mass expected to be processed through the 
WTP. What may be missing from this body of results is information on the outlying properties or extremes. Design and waste processing operations are often controlled by the extreme or most difficult wastes to be processed. Consideration should be given to focusing characterization efforts on samples suspected of having extreme values of the waste properties.

The dry solids density is an important parameter used in simulant development and mixing assessments. During the simulant development process for mixing assessments it provides a target for the overall dry particle density for polydisperse simulants. The dry solids density also has a role in determining the volume fraction of solids, which is an important parameter used in estimating slurry viscosity and pipeline critical velocity as well as performing hindered settling calculations. This gap appears to be relatively easy to fill by using a gas pycnometry method or extending the existing protocols for the determination of undissolved solids fractions. This gap can likely be filled as part of on-going waste characterization efforts.

An accurate understanding of the shear strength formation is needed so mixing systems are designed to prevent settled solids layers that may exceed the remobilization capabilities. Given the small amount of data available on this topic, filling this important gap would allow an assessment of the length of time settled sludges could remain undisturbed before challenging the mixing systems.

The light-scattering methods currently in use for measuring PSDs have some limitations. Some of these limitations can be overcome by supplementing the light-scattering techniques with optical-based PSD methods. While this would require the procurement of a new instrument, it would offer a direct measurement of the PSDs as well as direct information on the particle and agglomerate shapes.

Particle size and density distributions are important to waste transport and mixing operations and for developing simulants for evaluating these processes. The PSD and particle density data are currently obtained with separate analytical techniques and the separate measurements linked by making assumptions about the waste properties. This approach has several disadvantages that result in considerable uncertainty in the particle size and density distributions. Consequently the development of a new method that has the capability to simultaneously determine the size and corresponding density of individual particles would greatly reduce the uncertainty in the particle size and density distributions. This would likely result in more representative simulants and less-conservative designs for the mixing and transport systems.

The abrasion properties of the waste are expected to have an impact on the erosion rates of processing equipment, but there is little data on abrasivity. This data gap is due to the lack of a developed and accepted method for application to radioactive samples. Given the lack of data on abrasivity of the tank waste, existing methods should be examined to evaluate whether they can be adapted and qualified for radioactive waste samples. Alternatively, a new method may be required. 



\subsection{References}

Agnew SF. 1995. Hanford Defined Wastes: Chemical and Radionuclide Compositions. LA-UR-94-2657 Rev. 2, Los Alamos National Laboratory, Los Alamos, New Mexico.

Alderman NJ, GH Meeten, and JD Sherwood. 1991. “Vane Rheometry of Bentonite Gels.” Journal of Non-Newtonian Fluid Mechanics 39:291-310.

Ancey C and H Jorrot. 2001. "Yield Stress for Particle Suspensions Within a Clay Dispersion.” Journal of Rheology 45(2):297-319.

Baltpurvins KA, RC Burns, GA Lawrance, and AD Stuart. 1996. "Effect of pH and Anion Type on the Aging of Freshly Precipitated Iron(III) Hydroxide Sludges.” Environmental Science \& Technology 30:939-944.

Barker SA, WB Barton, DR Bratzel, M Epstein, PA Gauglitz, GD Johnson, SN Maruvada, CE Olson, ML Sauer, SE Slezak, CW Stewart, and J Young. 1999. Flammable Gas Safety Analysis Data Review. SNL000198, Savannah River National Laboratory, Aiken, South Carolina.

Barnes HA. 1999. “The Yield Stress - A Review or ' $\pi \alpha \nu \tau \alpha$ $\rho \varepsilon 1$ ' - Everything Flows?” Journal of Non-Newtonian Fluid Mechanics 81:133-178.

Baudez J-C. 2006. “About Peak and Loop in Sludge Rheograms.” Journal of Environmental Management 78:232-239.

Bechtold DB, WS Callaway GA Cooke, JB Duncan, DL Herting, JR Jewett, JC Person, and JM Tingey. 2002. Particle Property Analyses of High-Level Waste Tank Sludges. HNF-8862 Rev. 0, Fluor Hanford, Inc., Richland, Washington.

Bechtold DB, GA Cooke, DL Herting, JC Person, RS Viswanath, and RW Warrant. 2003. Laboratory Testing of Oxalic Acid Dissolution of Tank 241-C-106 Sludge. RPP-17158 Rev. 0, Fluor Hanford, Inc., Richland, Washington.

Belleville P, J-P Jolivet, E Tronc, and J Livage. 1992. “Crystallization of Ferric Hydroxide into Spinel by Adsorption on Colloidal Magnetite.” Journal of Colloid and Interface Science 150:453-460.

Berg JC. 2001. Course Handbook: Surface and Colloid Science. University of Washington, Seattle, Washington.

Biesheuvel PM, H Verweij, and V Breedveld. 2001. "Evaluation of Instability Criterion for Bidisperse Sedimentation.” AIChE J 47(1): 45-52.

Bitter JGA. 1963a. “A Study of Erosion Phenomena.” Part I. Wear 6:5-21.

Bitter JGA. 1963b. “A Study of Erosion Phenomena.” Part II. Wear 6:169-190.

Bontha JR, CW Stewart, DE Kurath, PA Meyer, ST Arm, CE Guzman-Leong, MS Fountain, M Friedrich, SA Hartley, LK Jagoda, CD Johnson, KS Koschik, DL Lessor, F Nigl, RL Russell, GL Smith, 
W Yantasee, and ST Yokuda. 2005. Technical Basis for Predicting Mixing and Flammable Gas Behavior in the Ultrafiltration Feed Process and High-Level Waste Lag Storage Vessels with Non-Newtonian Slurries. PNWD-3676 (WTP-RPT-132, Rev. 0), Battelle - Pacific Northwest Division, Richland, Washington.

Bontha JR, HE Adkins, KM Denslow, JJ Jenks, CA Burns, PP Schonewill, GP Morgan, MS Greenwood, J Blanchard, TJ Peters, PJ MacFarlan, EB Baer, and WA Wilcox. 2010. Test Loop Demonstration and Evaluation of Slurry Transfer Line Critical Velocity Measurement Instruments. PNNL-19441, Pacific Northwest National Laboratory, Richland, Washington.

Brooks KP, RL Myers, and KG Rappe. 1997. Bench-Scale Enhanced Sludge Washing and Gravity Settling of Tank C-106 Sludge. PNNL-11432, Pacific Northwest National Laboratory, Richland, Washington.

Brooks KP, JR Bontha, GR Golcar, RL Myers, KG Rappe, and DR Rector. 1998. Bench-Scale Enhanced Sludge Washing and Gravity Settling of Tank S-107 Sludge. PNNL-12010, Pacific Northwest National Laboratory, Richland, Washington.

Brooks KP, DR Rector, and PA Smith. 1999. “Gravity Settling of Hanford Single-Shell Tank Sludges.” Separation Science and Technology 34(6):1351-1370.

Buck ECB, W Arey, SK Fiskum, JGH Geeting, ED Jensen, BK McNamara, and AP Poloski. 2003. Identification of Washed Solids from Hanford Tanks 241-AN-102 and 241-AZ-101 with X-ray Diffraction, Scanning Electron Microscopy, and Light-Scattering Particle Analysis. PNWD-3300 (WTP-RPT-076 Rev 0), Battelle-Pacific Northwest Division.

Bunker BC, PJ Bruinsma, GL Gruff, CR Hymas, XS Li, JR Phillips, DR Rector, PA Smith, L Song, JM Tingey, and Y Wang. 1995. Colloidal Studies for Solid/Liquid Separation. TWRSPP-95-045, Pacific Northwest National Laboratory, Richland, Washington.

Bunker BC, J Liu, DR Rector, GL Graff, PA Smith, PJ Bruinsma, L Song, XS Li, JD Virden, NG Colton, JR Phillips, KD Keefer, and JM Tingey. 2000. "Fractal Colloidal Agglomerates in Nuclear Tank Wastes: Impact on Waste Properties and Processing.” Radioactive Waste Management and Environmental Research 38:97-133.

Burns PC. 2010. "From Extended Solids to Nano-Scale Actinide Clusters.” Comptes Rendus Chimie DOI:10.1016/j.crci2010.01.014.

Buscall R, IJ McGowan, PDA Mills, RF Stewart, D Sutton, LR White, and GE Yates. 1987. "The Rheology of Strongly Flocculated Suspensions.” Journal of Non-Newtonian Fluid Mechanics 24:183-202.

Callaway WS. 2000. Results of Retrieval Testing of Sludge from Tank 241-AZ-101. HNF-7078, Rev. 1, Fluor Hanford, Inc., Richland, Washington.

Callaway WS and GA Cooke. 2004. SY102 Images from: CH2M-0400872, to KH Abel, 05/17/2004. Distribution of Plutonium-Rich Particles in Tank 241-SY-102 Sludge. CH2M Hill Hanford Group, Inc., Richland, Washington. 
Callaway WS, GA Cooke, and DL Herting. Particle Size Measurements in Support of the Tank 241-AN-102 Chemistry Control Recovery Plan. 7S110-WSC-05-011, 3/18/2005, CH2M HILL, Hanford Group Inc., Richland, Washington.

Callaway WS and HJ Huber. 2010. Results of physicochemical characterization and caustic dissolution tests on Tank 241-C-108 Heel Solids, LAB-RPT-10-00001 Rev 0, CH2M HILL, Hanford Group Inc., Richland, Washington.

Camenen B. 2007. "Simple and General Formula for the Settling Velocity of Particles.” Journal of Hydraulic Engineering - American Society of Civil Engineers 133(2):229-233.

Cantrell KJ, KM Krupka, KN Geiszler, MJ Lindberg, BW Arey, and HT Schaef. 2008a. Hanford Tank 241-S-112 Residual Waste Composition and Leach Test Data. PNNL-17593, Pacific Northwest National Laboratory, Richland, Washington.

Cantrell KJ, KM Krupka, WJ Deutsch, MJ Lindberg, HT Schaef, KN Geiszler, and BW Arey. 2008b. Hanford Tank 241-C-103 Residual Waste Contaminant Release Models and Supporting Data. PNNL-16738, Pacific Northwest National Laboratory, Richland, Washington.

Carlson AB, JR Bellomy III, KG Carothers, JM Conner, BK Everrett, JD Guberski, EI Husa. DJ McCain, GW Reddick Jr., GR Tardiff, AM Templeton, MS Tiffany, SM Merry, PJ Fuller, RE Mendoza, MM Jennings, DM Stenkamp, and DG Douglas. 2000. Preliminary Test Report, 241-AZ-101 Mixer Pump Test. RPP-6548, Rev. 0, CH2M Hill Hanford Group, Inc., Richland, Washington.

Carlson AB, PJ Certa, TM Hohl, JR Bellomy III, TW Crawford, DC Hedengren, AM Templeton, HS Fisher, SJ Greenwood, DG Douglas, and WJ Ulbright Jr. 2001. Test Report, 241-AZ-101 Mixer Pump Test. RPP-6548, Rev. 1, Numatec Hanford Corporation, Richland, Washington.

Channell GM and CF Zukoski. 1997. "Shear and Compressive Rheology of Aggregated Alumina Suspensions.” AIChE Journal 43(7):1700-1708.

Chhabra RP. 1992. Bubbles, Drops, and Particles in Non-Newtonian Fluids. CRC Press, Inc., Boca Raton, Florida.

Clark LA and TM Wynn. 2007. "Methods for Determining Streambank Critical Shear Stress and Soil Erodibility: Implications for Erosion Rate Predictions.” Transactions of the American Society of Civil Engineers 50(1):95-106.

Cowley WL, JL Huckaby, LA Mahoney, GM Golcar, KM Krupka, DS Sklarew, and DJ Geniesse. 2003. Chemical Source Terms for Tank Farms Safety Analyses. RPP-8369, Rev. 2, CH2M Hill Hanford Group, Inc., Richland, Washington.

CRC. 1975. Handbook of Chemistry and Physics. $56^{\text {th }}$ Edition, RC Weast, Editor. CRC Press, Cleveland, Ohio.

Cuta JM, KG Carothers, DW Damschen, WL Kuhn, JA Lechelt, K Sathyanarayana, and LA Stauffer. 2000. Review of Waste Retrieval Sluicing System Operations and Data for Tanks 241-C-016 and 241-AY-102. PNNL-13319, Pacific Northwest National Laboratory, Richland, Washington. 
Davies R and BH Kaye. 1971. "Experimental Investigation into the Settling Behavior of Suspensions." Powder Technology 5:61-68.

Dierickx D, B Basu, J Vleugels, and O Van der Biest. 2000. "Statistical Extreme Value Modeling of Particle Size Distributions: Experimental Grain Size Distribution Type Estimation and Parameterization of Sintered Zirconia.” Materials Characterization 45:61-70.

Disselkamp RS. 2010. Hanford Waste Mineralogy Reference Report. RPP-RPT-46618 Rev. 1, Washington River Protection Solutions, LLC, Richland, Washington.

Dorrell R and AJ Hogg. 2010. “Sedimentation of bidisperse suspensions.” International Journal of Multiphase Flow 36:481-490.

Dunn IS. 1959. “Tractive Resistance of Cohesive Channels.” Journal of Soil Mechanics and Foundations Division, the American Society of Civil Engineers 85(SM3):1-24.

Durand R. 1952. "Hydraulic Transport of Coal and Solid materials in Pipes.” In: Proceedings of a Colloquium on the Hydraulic Transport of Coal, National Coal Board, London, United Kingdom, pp. 39-52.

Dzuy NQ and DV Boger. 1983. "Yield Stress Measurement for Concentrated Suspensions." Journal of Rheology 27 (4): 321-349.

Dzuy NQ and DV Boger. 1992. "Measuring the Flow Properties of Yield Stress Fluids." Annual Review of Fluid Mechanics 24: 47-88.

Edwards MK, JM Billing, DL Blanchard, EC Buck, AJ Casella, AM Casella, JV Crum, RC Daniel, KE Draper, SK Fiskum, LK Jagoda, ED Jenson, AE Kozelisky, PJ MacFarlan, RA Peterson, RW Shimskey, LA Snow, and RG Swoboda. 2009. Characterization, Leaching, and Filtration Testing for Tributyl Phosphate (TBP, Group 7) Actual Waste Sample Composites. PNNL-18119 (WTP-RPT-169 Rev. 0), Pacific Northwest National Laboratory, Richland, Washington.

Enderlin CW, G Terrones, CJ Bates, BK Hatchell, and B Adkins. 2003. Recommendations for Advanced Design Mixer Pump Operation in Savannah River Site Tank 18F. PNNL-14443, Pacific Northwest National Laboratory, Richland, Washington.

Finch R and T Murakami. 1999. Systematics and Paragenesis of Uranium Minerals. In: Burns PC and R Finch (eds.), Reviews in Mineralogy Uranium: Mineralogy, Geochemistry, and the Environment, Vol. 38, Mineralogical Society of America, Washington DC, pp. 91-179.

Fiskum SK, EC Buck, RC Daniel, K Draper, MK Edwards, TL Hubler, LK Jagoda, ED Jenson, AE Kozelisky, GJ Lumetta, PJ MacFarlan, BK McNamara, RA Peterson, SI Sinkov, LA Snow, and RG Swoboda. 2008. Characterization and Leach Testing for REDOX Sludge and S-Saltcake Actual Waste Sample Composites. PNNL-17368 (WTP-RPT-157, Rev. 0), Pacific Northwest National Laboratory, Richland, Washington.

Fiskum SK, JM Billing, EC Buck, JV Crum, RC Daniel, KE Draper, MK Edwards, AE Kozelisky, PJ MacFarlan, RA Peterson, and RW Shimskey. 2009a. Characterization, Leaching, and Filtration 
Testing of Ferrocyanide Tank Sludge (Group 8) Actual Waste Composite. PNNL-18120 (WTP-RPT-170 Rev. 0), Pacific Northwest National Laboratory, Richland, Washington.

Fiskum SK, JM Billing, EC Buck, RC Daniel, KE Draper, MK Edwards, ED Jenson, AE Kozelisky, PJ MacFarlan, RA Peterson, RW Shimskey, and LA Snow. 2009b. Laboratory Demonstration of the Pretreatment Process with Caustic and Oxidative Leaching Using Actual Hanford Tank Waste . PNNL 18007, Pacific Northwest National Laboratory, Richland, Washington.

Fruth V, L Mitoseriu, D Berger, A Ianculescu, C Matei, S Preda, and M Zaharescu. 2007. "Preparation and Characterization of $\mathrm{BiFeO}_{3}$ Ceramic.” Progress in Solid-State Chemistry 35:193-202.

Frye JM. 2005. C101 and C107 Images from 7S110-JMF-05-015, 4/29/2005. Results of Caustic Testing of Tank 241-C-101 \& 241-C-107 CH2M Hill Hanford Group, Inc., Richland, Washington.

Garcia MH, Editor. 2008. "Sedimentation Engineering - Processes, Measurements, Modeling, and Practice.” ASCE Manual and Reports on Engineering Practice, No. 110. American Society of Civil Engineering, Reston, Virginia.

Gauglitz PA and JT Aikin. 1997. Waste Behavior During Horizontal Extrusion: Effect of Waste Strength for Bentonite and Kaolin/Ludox Simulants and Strength Estimates for Wastes from Hanford Waste Tanks 241-SY-103, AW-101, AN-103, and S-102. PNNL-11706, Pacific Northwest National Laboratory, Richland, Washington.

Gauglitz PA, LA Mahoney, DP Mendoza, and MC Miller. 1994. Mechanisms of Gas Bubble Retention. PNL-10120, Pacific Northwest Laboratory, Richland, Washington.

Gauglitz PA, RR Shah, SD Rassat, LA Mahoney, and MR Powell. 1995. Gas Bubble Retention and Its Effects on Waste Properties: Retention Mechanisms, Viscosity, and Tensile and Shear Strengths. PNL-10740, Pacific Northwest Laboratory, Richland, Washington.

Gauglitz PA, SD Rassat, PR Bredt, JH Konynenbelt, SM Tingey, and DP Mendoza. 1996. Mechanisms of Gas Bubble Retention and Release: Results for Hanford Waste Tanks 241 S 102 and 241 SY 103 and Single Shell Tank Simulants. PNNL-11298, Pacific Northwest National Laboratory, Richland, Washington.

Gauglitz PA, BE Wells, JA Fort, and PA Meyer. 2009. An Approach to Understanding Cohesive Slurry Settling, Mobilization, and Hydrogen Gas Retention in Pulsed Jet Mixed Vessels. PNNL-17707 (WTP-RPT-177 Rev. 0), Pacific Northwest National Laboratory, Richland, Washington.

Gauglitz PA, B Buchmiller, JJ Jenks, J Chun, RL Russell, AJ Schmidt, and MM Mastor. 2010a. The Disruption of Vessel-Spanning Bubbles with Sloped Fins in Flat-Bottom and 2:1 Elliptical-Bottom Vessels. PNNL-19345, Pacific Northwest National Laboratory, Richland, Washington.

Gauglitz PA, BE Wells, JA Bamberger, JA Fort, J Chun, and JJ Jenks. 2010b. The Role of Cohesive Particle Interactions on Solids Uniformity and Mobilization During Jet Mixing: Test Recommendations. PNNL-19245, Pacific Northwest National Laboratory, Richland, Washington. 
Geeting JGH, RT Hallen, LK Jagoda, AP Poloski, RD Scheele, and DR Weier. 2003. Filtration, Washing, and Caustic Leaching of Hanford Tank AZ-101 Sludge. PNWD-3206, Rev. 1 (WTP-RPT-043, Rev. 1), Battelle - Pacific Northwest Division, Richland, Washington.

Giammar DE and JG Hering. 2004. "Influence of Dissolved Sodium and Cesium on Uranyl Oxide Hydrate Solubility.” Environmental Science \& Technology 38:171-179.

Govier GW and K Aziz. 1987. The Flow of Complex Mixtures in Pipes, Robert E. Krieger Publishing Company, Malabar, Florida.

Gray WJ, ME Peterson, RD Scheele, and JM Tingey. 1993. Characterization of the Second Core Sample of Neutralized Current Acid Waste From Double-Shell Tank 101-AZ. PNNL-13027, Pacific Northwest National Laboratory, Richland, Washington.

Green DW and RH Perry. 2007. Perry’s Chemical Engineering Handbook, McGraw-Hill, New York.

Green DW and RH Perry. 2008. Perry's Chemical Engineers' Handbook (8th Edition). McGraw-Hill, New York.

Gupta R, SN Singh, and V Sehadri. 1995. "Prediction of Uneven Wear in a Slurry Pipeline on the Basis of Measurements in a Pot Tester.” Wear 184:169-178.

Ha Z and S Liu. 2002. “Settling Velocities of Polydisperse Concentrated Suspensions.” Canadian Journal of Chemical Engineering 80:784-790.

Hall MN. 2006. Design Guide: Minimum Flow Velocity for Slurry Lines. WTP Project Doc. No. 24590-WTP-GPG-M-0058, Rev 0. Bechtel National, Inc., Richland, Washington.

Hanson GJ and A Simon. 2001. "Erodibility of Cohesive Streambeds in the Loess Area of the Midwestern USA.” Hydrological Processes 15:23-38.

Haugen K, O Kvernvold, A Ronold, and R Sandberg. 1995. “Sand Erosion of Wear-Resistant Materials: Erosion in Choke Valves.” Wear 186-187:179-188.

Heath WO. 1987. Development of an In Situ Method to Define the Rheological Properties of Slurries and Sludges Stored in Underground Tanks. PNL-6083, Pacific Northwest National Laboratory, Richland, Washington.

Hemond HF and EJ Fechner. 1994. Chemical Fate and Transport in the Environment. Academic Press, New York.

Herting DL. 1997. Results of Dilution Studies with Waste from Tank 241-AN-105, HNF-SD-WM-DTR-046. Numatec Hanford Corp., Richland, Washington.

Herting DL and GA Cooke. 2004. C103 Images from: 7S110-DLH-04-015, to KG Carothers, 05/05/2004. Caustic Demand Test Results Tank 241-C-103 Sludge, CH2M Hill Hanford Group, Inc., Richland, Washington. 
Herting DL, RW Warrant, and GA Cooke. 2002. Identification of Solid Phases in Saltcake from Hanford Site Waste Tanks. HNF- 11585, Rev. 0 (HNF-EDC-02-12376); Fluor Hanford, Inc., Richland, Washington.

Herting DL, GA Cooke, and RW Warrant. 2004. AN107 Images from: RPP-20018, 03/10/2004. Hanford Tank 241AN107 Solid Phase Characterization. CH2M Hill Hanford Group, Inc., Richland, Washington.

Higley BA and DE Place. 2004. Hanford Defined Waste Model - Revision 5. RPP-19822, CH2M Hill Hanford Group, Inc., Richland, Washington.

Hodgson, KM. 1995. Tank Characterization Report for Double Shell Tank 241-AZ-101, WHC-SD-WM-ER-410 Rev 0, Westinghouse Hanford Company, Richland Washington.

Hu TA. 2004. Empirical Rate Equation Model and Rate Calculations of Hydrogen Generation for Hanford Tank Waste. HNF-3851, Rev. 1, CH2M Hill Hanford Group, Inc., Richland, Washington.

Ilievski D and ET White. 1994. “Agglomeration During Precipitation: Agglomeration Mechanism Identification for $\mathrm{Al}(\mathrm{OH})_{3}$ Crystals in Stirred Caustic Aluminate Solutions.” Chemical Engineering Science 49(19):3227-3239.

Jewett JR, SD Estey, L Jensen, NW Kirch, DA Reynolds, and Y Onishi. 2002. Values of Particle Size, Particle Density, and Slurry Viscosity to Use in Waste Feed Delivery Transfer System Analysis.

RPP-9805 Rev. 1, Numatec Hanford Corporation, Richland, Washington.

Julyk LJ, TC Oten, and WL Wollis. 2000. "Waste Feed Delivery Transport System Analysis.” RPP-5346 Rev. 1, CH2M Hill Hanford Group, Inc., Richland, Washington.

Kandori K, S Uchida, S Kataoka, and T Ishikawa. 1992. "Effects of Silicate and Phosphate Ions on the Formation of Ferric Oxide Hydroxide Particles.” Journal of Material Science 27:719-728.

Khelifa A and PS Hill. 2006. "Models for Effective Density and Settling Velocity of Flocs.” Journal of Hydraulic Research 44(3):390-401.

Kim J-W and TA Kramer. 2005. “Improved Models for Fractal Colloidal Agglomeration: Computationally Efficient Algorithms.” Colloids and Surfaces A: Physicochem Engineering Aspects 253:33-49.

Kinnison RR. 1985. Applied Extreme Value Statistics. Macmillan Publishing Company, New York.

Kramer TA and MM Clark. 1999. "Incorporation of Agglomerate Breakup in the Simulation of Orthokinetic Coagulation.” Journal of Colloid and Interface Science 216:116-126.

Krishnamoorthy K and M Thomas. 2009. Statistical Tolerance Regions: Theory, Applications, and Computation. John Wiley \& Sons, Inc., Hoboken, New Jersey.

Krone RB. 1962. Flume Studies of the Transport of Sediment in Estuarine Shoaling Processes. Technical Report, Hydraulic Engineering Laboratory, University of California, Berkeley, California. 
Krone RB. 1993. "Sediment Revisited.” In: Nearshore and Estuarine Cohesive Sediment Transport, AJ Merta, Editor, Coastal Estuarine Studies, No. 42, American Geophysical Union, pp. 108-125.

Krot NN, VP Shilov, AM Fedoseev, AB Yussov, AA Bessonov, NA Budantseva, SI Nikitenko, GM Plavnik, TP Puraeva, MS Grigoriev, A Garnov A Yu., AV Gelis, VP Perminov, LN Astafurova, and CH Delegard. 1998. Alkaline Treatment of Acidic Solution from Hanford K Basin Sludge Dissolution. PNNL-11944, Pacific Northwest National Laboratory, Richland, Washington.

Krupka KM, HT Schaef, BW Arey, SM Heald, WJ Deutsch, MJ Lindberg, and KJ Cantrell. 2006. Residual Waste from Hanford Tanks 241-C-203 and 241-C-204. 1. Solids Characterization. Environmental Science \& Technology 40(12):3749-3754.

Krupka, KM, KJ Cantrell. HT Schaef, BW Arey, SM Healdm WJ Deutsch, and MJ Lindberg. 2010. Characterizing Solids in Residual Wastes from Single-Shell Tanks at the Hanford Site. Radwaste Solutions, 17(2):64-75.

Kurath DE, PA Meyer, JR Bontha, AP Poloski, JA Fort, WH Combs, WC Buchmiller, ID Welch, and MD Bleich. 2007. Assessment of Pulse Tube Mixing for Vessels Containing Non-Newtonian Slurries. PNWD-3827 (WTP-RPT-155, Rev. 0), Battelle - Pacific Northwest Division, Richland, Washington.

Kurath DE, BD Hanson, MJ Minette, DL Baldwin, BM Rapko, LA Mahoney, PP Schonewill, PW Eslinger, JL Huckaby, JM Billing, PS Sundar, GJ Josephson, JJ Toth, ST Yokuda, EBK Baer, SM Barnes, EC Golovich, SD Rassat, CF Brown, JGH Geeting, GJ Sevigny, AJ Casella, JR Bontha, RL Aaberg, PM Aker, CE Guzman-Leong, ML Kimura, SK Sundaram, RP Pires, BE Wells, and OP Bredt. 2009. Pretreatment Engineering Platform Phase 1 Final Test Report. PNNL-18894 (WTP-RPT-197 Rev. 0), Pacific Northwest National Laboratory, Richland, Washington.

Lansen PH, van Bendegom, J van den Berg, M Vries, and A Zanen. 1979. Principles of River Engineering: The Non-Tidal Alluvial River. Pitman Publishing, Limited. London, United Kingdom.

Liu H. 2003. Pipeline Engineering. Lewis Publishers of CRC Press Company, Boca Raton, Florida.

Locock AJ, PC Burns, MJM Duke, and TM Flynn. 2004. "Monovalent Cations in Structures of the Meta-Autunite Group.” Canada Mineralogist 42:973-996.

Lovell CJ and CW Rose. 1991. "Wake-Capture Effects Observed in a Comparison of Methods to Measure Particle Settling Velocity Beyond Stokes ‘Range.” Journal of Sedimentary Petrology 61(4): 575-582.

Lumetta GJ, BM Rapko, MJ Wagner, J Liu, and YL Chen. 1996. Washing and Caustic Leaching of Hanford Tank Sludge: Results of FY1996 Studies. PNNL-11636, Pacific Northwest National Laboratory, Richland, Washington.

Lumetta GJ, I Burgeson, MJ Wagner, J Liu, and YL Chen. 1997. Washing and Caustic Leaching of Hanford Tank Sludge: Results of FY1997 Studies. PNNL-11636, Rev. 1, Pacific Northwest National Laboratory, Richland, Washington. 
Lumetta GJ, EC Buck, RC Daniel, K Draper, MK Edwards, SK Fiskum, RT Hallen, LK Jagoda, ED Jenson, AE Kozelisky, PJ MacFarlan, RA Peterson, RW Shimskey, SI Sinkov, and LA Snow. 2009a. Characterization, Leaching, and Filtration Testing for Bismuth Phosphate Sludge (Group 1) and Bismuth Phosphate Saltcake (Group 2) Actual Waste Sample Composites. PNNL-17992 (WTP-RPT-166, Rev. 0), Pacific Northwest National Laboratory, Richland, Washington.

Lumetta GJ, BK McNamara, EC Buck, SK Fiskum, and LA Snow. 2009b. "Characteristics of High Phosphate Tank Waste and Simulant Development.” Environmental Science \& Technology 43:7843-7848.

MacLean GT. 1999. The Settling and Compaction of Nuclear Waste Slurries. HNF-5177, Rev. 0, Fluor Daniel Northwest, Richland, Washington.

Mantovanelli A. and P.V. Ridd. 2006. Devices to measure settling velocities of cohesive sediment aggregates: A review of the in situ technology. Journal of Sea Research, vol 56 p 199-226. Published by Elsevier B.V.

Mazurek KA, N Rajaratnam, and DC Sego. 2001. "Scour of Cohesive Soil by Submerged Circular Turbulent Impinging Jets.” Journal of Hydraulic Engineering of the American Society of Civil Engineers 127:598-606.

McNown JS, J Malaika, and HR Praminak. 1951. "Particle Shape and Settling Velocity.” In: the 4th Meeting of the International Association for Hydraulic Research, Bombay, India.

Meacham JE. 2003. Aluminum Wash and Leach Factors. RPP-11079, CH2M Hill Hanford Group, Inc., Richland, Washington.

Meacham JE. 2009. Steady-State Flammable Gas Release Rate Calculation and Lower Flammability Level Evaluation for Hanford Tank Waste. RPP-5926, Rev. 9, Washington River Protection Solutions, LLC, Richland, Washington.

Meacham JE. 2010. Gas Retention and Release from Hanford Site High Shear Strength Waste. RPP-RPT-26836, Rev. 0, Washington River Protection Solutions, LLC, Richland, Washington.

Mehta AJ and E Pertheniades. 1973. "Depositional Behavior of Cohesive Sediments.” PB-218-854, Technical Report No. 16, Florida University, Gainesville, Florida.

Meng HC and KC Ludema. 1995. "Wear Models and Predictive Equations: Their Form and Content." Wear 181-183:443-457.

Meyer PA, JA Bamberger, CW Enderlin, JA Fort, BE Wells, SK Sundaram, PA Scott, MJ Minette, GL Smith, CA Burns, MS Greenwood, GP Morgen, EBK Baer, SF Snyder, M White, GF Piepel, BG Amidan, and A Heredia-Langner. 2009. Pulse Jet Mixing Tests with Noncohesive Solids. PNNL-18098 (WTP-RPT-182, Rev. 0), Pacific Northwest National Laboratory, Richland Washington.

Meyer PA, EBK Baer, JA Bamberger, JA Fort, and MJ Minette. 2010. Assessment of Differences in Phase 1 and Phase 2 Test Observations for Waste Treatment Plant Pulse Jet Mixer Tests with 
Non-Cohesive Solids. PNNL-19085 (WTP-RPT-208, Rev. 0), Pacific Northwest National Laboratory, Richland Washington.

Myers RH. 1990. Classical and Modern Regression with Applications. Second Edition. Duxbury Press, Belmont, California.

Naeini SA and MH Baziar. 2004. "Effect of Fines Content on Steady-State Strength of Mixed and Layered Samples of Sand.” Soil Dynamics and Earthquake Engineering 24:181-187.

Nguyen QD and DV Boger. 1983. "Yield Stress Measurement for Concentrated Suspensions.” Journal of Rheology 27(4):321-349.

Nguyen QD and DV Boger. 1992. "Measuring the Flow Properties of Yield Stress Fluids.” Annual Review of Fluid Mechanics 24:47-88.

OLI. 1998. A Guide to Using the OLI Engine. OLI Systems, Inc., Morris Plains, New Jersey.

Onishi Y. 2008. "Estimates of Critical Suspension Velocities and Cloud Heights of the Hanford Waste Treatment and Immobilization Plant Vessels Based on Similarity Analysis.” YOC-104, Yasuo Onishi Consulting, LLC, Richland, Washington.

Onishi Y and KP Recknagle. 1997. Tank 241-AZ-101 Criticality Assessment Resulting from Pump Jet Mixing Sludge Mixing Simulations. PNNL-11486, Pacific Northwest National Laboratory, Richland Washington.

Onishi Y and BE Wells. 2004. Feasibility Study on Using Two Mixer Pumps for Tank 241-AY-102 Waste Mixing. PNNL-14763, Pacific Northwest National Laboratory, Richland, Washington.

Onishi Y, HC Graber, and DS Trent. 1993. "Preliminary Modeling of Wave-Enhanced Sediment and Contaminant Transport in New Bedford Harbor.” Book Series 42 of Estuarine and Coastal Water Cohesive Sediment Transport, pp. 541-557, AJ Mehta, Editor, American Geophysical Union.

Onishi Y, DS Trent, TE Michener, JE Van Beek, and CA Rieck. 1999. "Simulation of Radioactive Tank Waste Mixing with Chemical Reactions.” FEDSM99-7786 in: Proceedings of the 3rd ASME/JSME Joint Fluids Engineering Conference, July 18-23, San Francisco, California.

Onishi Y, KP Recknagle, and BE Wells. 2000. "Pump Jet Mixing and Pipeline Transfer Assessment for High-Activity Radioactive Wastes in Hanford Tank 241-AZ-102.” PNNL-13275, Pacific Northwest National Laboratory, Richland Washington.

Onishi Y, BE Wells, SA Hartley, and SK Cooley. 2002. Pipeline Cross-Site Transfer Assessment for Tank 241-SY-101. PNNL-13650, Pacific Northwest National Laboratory, Richland Washington.

Onishi Y, JM Tingey, BE Wells, J Lui, G Terrones, KP Recknagle, ST Yokuda, and M Quinn. 2003. Retrieval and Pipeline Transfer Assessment of Hanford Tank 241-AN-105. PNNL-14144, Pacific Northwest National Laboratory, Richland Washington. 
Onishi Y, BE Wells, AR Felmy, CW Enderlin, JM Tingey, TT Taylor, RW Goles, and GR Smith. 2005. How to Fill Sellafield Waste Data and Assessment Needs in Light of Hanford Experiences. PNWD-3543, Battelle, Pacific Northwest Division, Richland, Washington.

Onishi Y, ST Yokuda, and AJ Schmidt. 2009. Evaluation of Shear Strength Threshold of Concern for Retrieval of Interim-Stored K-Basin Sludge in the Hanford Site. PNNL-19946, 53451-RPT12, Pacific Northwest National Laboratory, Richland Washington.

Oroskar AR and RM Turian. 1980. "The Critical Velocity in Pipeline Flow of Slurries.” AIChE Journal 26(4): 551-558.

Papp IG. 2008. EFRT Issue M2 Closure Report. 24590-WTP-RPT-PET-08-008, Rev 0; DOE Contract DE-AC27-01RV14136, Pacific Northwest National Laboratory, Richland, Washington.

Papp IG and GM Duncan. 2009. Investigation of Erosion from High-Level Waste Slurries at the Hanford Waste Treatment and Immobilization Plant. December 2009 Bechtel Technology Journal, Vol. 2, No. 1, Bechtel Corporation.

Patil MM, VV Deshpande, SR Dhage and V Ravi. 2005. "Synthesis of Bismuth Oxide Nanoparticles at 100C.” Materials Letters 59:2523-2525.

Paul EL, VA Atiemo_Obeng, and SM Kresta. 2004. Handbook of Industrial Mixing Science and Practice. John Wiley \& Sons, Inc., Hoboken, New Jersey.

Perry RH and CH Chilton. 1973. Chemical Engineers’ Handbook. McGraw-Hill, New York.

Pertheniades E. 1962. A Study of Erosion and Deposition of Cohesive Solids in Salt Water. Ph.D. Thesis, Civil Engineering Department, University of California, Berkeley, California.

Pertheniades E. 1993. “Turbulence, Flocculation, and Cohesive Sediment Dynamics.” In: Nearshore and Estuarine Cohesive Sediment Transport, AJ Merta, Editor, Coastal Estuarine Studies, No. 42, American Geophysical Union, pp. 40-59.

Poirier MR. 2004. Mixing in SRS Closure Business Unit Applications. WSRC-TR-2004-00153, Savannah River Laboratory, Aiken, South Carolina.

Poloski AP, PR Bredt, JW Chenualt, RG Swoboda. 2003. Rheological and Physical Properties of AZ-101 HLW Pretreated Sludge and Melter Feed. PNWD-3366 (WTP-RPT-096, Rev. 0), Battelle Pacific Northwest Division, Richland, Washington.

Poloski AP, PA Meyer, LK Jagoda, and PR Hrma. 2004. Non-Newtonian Slurry Development and Selection for Pulse Jet Mixer Testing. PNWD-3495, WTP-RPT-111, Rev. 0, Battelle-Pacific Northwest Division, Richland, Washington.

Poloski AP, RC Daniel, DR Rector, PR Bredt, and BC Buck. 2006. Characterization and Correlation of Particle-Level Interactions to the Macroscopic Rheology of Powders, Granular Slurries, and Colloidal Suspensions. PNNL-16133, Pacific Northwest National Laboratory, Richland, Washington. 
Poloski AP, BE Wells, JM Tingey, LA Mahoney, MN Hall, SL Thomson, GL Smith, ME Johnson, JE Meacham, MA Knight, MG Thien, JJ Davis, and Y Onishi. 2007. Estimate of Hanford Waste Rheology and Settling Behavior. PNNL-16857 (WTP-RPT-154 Rev. 0), Pacific Northwest National Laboratory, Richland, Washington.

Poloski AP, ML Bonebrake, AM Casella, MD Johnson, JJ Toth, HE Adkins, Jr, J Chun, KM Denslow, M Luna, and JM Tingey. 2009a. Deposition Velocities of Non-Newtonian Slurries in Pipelines: Complex Simulant Testing. PNNL-18316 (WTP-RPT-189 Rev. 0), Pacific Northwest National Laboratory, Richland, Washington.

Poloski AP, HE Adkins, JAbrefah, AM Casella, RE Hohimer, F Nigl, MJ Minette, TT Toth, JM Tingey, and ST Yokuda. 2009b. Deposition Velocities of Newtonian and Non-Newtonian Slurries in Pipelines. PNNL-17639 (WTP-RPT-175, Rev. 0), Pacific Northwest National Laboratory, Richland, Washington.

Poloski AP, AW Etchells, J Chun, HE Adkins, AM Casella, MJ Minette, and ST Yokuda. 2009c. “A Pipeline Transport Correlation for Slurries With Small But Dense Particles.” Canadian Journal of Chemical Engineering 9999:1-8.

Pourbaix M. 1974. Atlas of Electrochemical Equilibria in Aqueous Solutions. National Association of Electrochemical Engineers, Houston, Texas.

Powell MR, GR Golcar, CR Hymas, and R McKay. 1995a. Fiscal Year 1993 1/25-Scale Sludge Mobilization Testing. PNL-10464, Pacific Northwest National Laboratory, Richland, Washington.

Powell MR, CM Gates, CR Hymas, MA Sprecher, and NJ Morter. 1995b. Fiscal Year 1994 1/25 ${ }^{\text {th }}$-Scale Sludge Mobilization Testing. PNL-10582, Pacific Northwest National Laboratory, Richland, Washington.

Powell MR, Y Onishi, and R Shekarriz. 1997. Research on Jet Mixing of Settled Sludges in Nuclear Waste Tanks at Hanford and Other DOE Sites: A Historical Perspective. PNNL-11686, Pacific Northwest National Laboratory, Richland, Washington.

Rapko BM and GJ Lumetta. 2000. Status Report on Phase Identification in Hanford Phase Sludges. PNWD-3065, Battelle - Pacific Northwest Division, Richland, Washington.

Rassat SD, LA Mahoney, BE Wells, DP Mendoza, and DD Caldwell. 2003. Assessment of Physical Properties of Transuranic Waste in Hanford Single-Shell Tanks. PNNL-14221, Pacific Northwest National Laboratory, Richland, Washington.

Rector DR and BC Bunker. 1995a. Effects of Colloidal Aggregation on the Sedimentation and Rheological Properties of Tank Waste. PNL-10761, Pacific Northwest National Laboratory, Richland Washington.

Rector DR and BC Bunker. 1995b. Sedimentation Modeling. PNL-10754, Pacific Northwest Laboratory, Richland, Washington.

Renko EK. 1996. “A Model for Batch Settling Curve.” Water SA, South African Journal of Science 22(4)339-344. 
Renko EK. 1998. "Modeling Hindered Batch Settling Part II: A Model for Computing Solids Profile of Calcium Carbonate Slurry.” Water SA, South African Journal of Science 24(4)331-336.

Reshma R, M Daas, R Srivastava, and B Tansel. 2007. "Resuspension of Non-Newtonian Slurries by Submerged Jet-Nozzles.” Experimental Thermal and Fluid Science 31:771-778.

Rouse H. 1961. Fluid Mechanics for Hydraulic Engineers. Dover Publications, Inc., New York.

Schatzmann M, P Ficher, and GR Bezzola. 2003. "Rheological Behavior of Fine and Large Particle Suspensions.” Journal of Hydraulic Engineering ASCE 129(10):796-803.

Selomnlya C, G Bushell, R Amal, and TD Waste. 2002. “Aggregation Mechanisms of Latex of Different Particle Sizes in a Controlled Shear Environment.” Langmuir 18:1974-1984.

Selomnlya C, G Bushell, R Amal, and TD Waste. 2003. "Understanding the Role of Restructuring in Flocculation: The Application of a Population Balance Model.” Chemical Engineering Science 58:327338.

Shimskey RW, JM Billing, EC Buck, AJ Casella, JV Crum, RC Daniel, K Draper, MK Edwards, RT Hallen, AE Kozelisky, PJ MacFarlan, RA Peterson and RG Swoboda. 2009a. Filtration and Leach Testing for PUREX Cladding Sludge and REDOX Cladding Sludge Actual Waste Sample Composites. PNNL-18048 (WTP-RPT-181 Rev. 0), Pacific Northwest National Laboratory, Richland, Washington.

Shimskey RW, JM Billing, EC Buck, RC Daniel, K Draper, MK Edwards, JGH Geeting, RT Hallen, ED Jenson, AE Kozelisky, PJ MacFarlan, RA Peterson, LA Snow, and RG Swoboda. 2009b. Filtration and Leach Testing for REDOX Sludge and S-Saltcake Actual Waste Sample Composites. PNNL-17965 (WTP-RPT-172 Rev. 0), Pacific Northwest National Laboratory, Richland, Washington.

Shook CA, RG Gillies, and RS Sanders. 2002. Pipeline Hydrotransport with Applications in the OilSand Industry. SRC Publication No. 11508-1E02, Saskatchewan Research Council, Saskatoon, Canada.

Simons DB and F Senturk. 1977. Sediment Transport Technology. Water Resources Publications, Fort Collins, Colorado.

Slurry Transport Expert Panel (Schlosser RL, Y Onishi, G Piot, MR Poirier, DA Reynolds, and EJ Wasp). 2002. Slurry Transport Expert Panel Final Report. RPP-13651, CH2M Hill Hanford Group, Inc., Richland, Washington.

Smith GL and K Prindiville. 2002. Guidelines for Performing Chemical, Physical, and Rheological Properties Measurements. 24590-WTP-GPG-RTD-001 Rev. 0, Bechtel National, Inc., Richland Washington.

Smith GL, AW Etchells, AP Poloski, BE Lewis Jr. MW Rinker, SL Marra (SRNL), RL Demmer. 2009. Slurry Retrieval, Pipeline Transport \& Plugging and Mixing Workshop. PNNL-18751, Pacific Northwest National Laboratory, Richland, Washington.

Snow LA, GJ Lumetta, SK Fiskum, and RA Peterson. 2008. "Boehmite Actual Waste Dissolution Studies.” Separation Science and Technology 43:2900-2916. 
Snow LA, EC Buck, AJ Casella, JV Crum, RC Daniel, K Draper, MK Edwards, SK Fiskum, LK Jagoda, ED Jenson, AE Kozelisky, PJ MacFarlan, RA Peterson, RG Swoboda. 2009. Characterization and Leach Testing for PUREX Cladding Waste Sludge (Group 3) and REDOX Cladding Waste Sludge (Group 4) Actual Waste Sample Composites. PNNL-18054 (WTP-RPT-167, Rev. 0), Pacific Northwest National Laboratory, Richland, Washington.

Spears RA, KR Holme, MA Tung, and WT Williamson. 1987. "Drilling Fluid Shear Stress Overshoot Behavior.” Rheologica Acta 26:447-452.

Stewart CW, JM Alzheimer, ME Brewster, G Chen, RE Mendoza, HC Reid, CL Shepard, and G Terrones. 1996a. In Situ Rheology and Gas Volume in Hanford Double-Shell Waste Tanks. PNNL-11296, Pacific Northwest National Laboratory, Richland, Washington.

Stewart CW, PA Meyer, ME Brewster, KP Recknagle, PA Gauglitz, HC Reid, and LA Mahoney. 1996b. Gas Retention and Release Behavior in Hanford Single-Shell Waste Tanks. PNNL-11391, Pacific Northwest National Laboratory, Richland, Washington.

Stewart CW, SA Hartley, PA Meyer, and BE Wells. 2005. Predicting Peak Hydrogen Concentrations from Spontaneous Gas Releases in Hanford Waste Tanks. PNNL-15238, Pacific Northwest National Laboratory, Richland, Washington.

Stewart CW, PA Meyer, DE Kurath, and SM Barnes. 2006. "Scaling Laws for Reduced-Scale A Test of Pulse Jet Mixing Systems in Non-Newtonian Slurries: Gas Retention and Release Behavior.” WM06 Conference, February 26-March 2, Tucson, Arizona.

Tedeschi AR. 2000. Deriving Requirements for Double-Shell Tank High-Level Waste Auxiliary Solids Mobilization. RPP-5664 CH2M Hill Hanford Group, Inc., Richland, Washington.

Teeter AM. 1988. New Bedford Harbor Project - Acushnet River Estuary Engineering Feasibility Study of Dredging and Dredged materials Disposal Alternatives - Report 2, Sediment and Contaminant Hydraulic Transport Investigations. U.S. Army Corps of Engineers, Waterway Experimental Station, Vicksburg, Mississippi.

Thomas AD. 1979. “The Role of Laminar/Turbulent Transition in Determining the Critical Deposit Velocity and the Operating Pressure Gradient for Long Distance Slurry Pipelines.” In: Proceedings of the 6th International Conference on the Hydraulic Transport of Solids in Pipes, September 26-28, Canterbury, United Kingdom, pp. 13-26.

Thomas DG. 1962. "Transport Characteristics of Suspensions: II. Minimum Transport Velocity for Flocculated Suspension in Horizontal Pipes.” AIChE Journal 7(3):423-430.

Tingey JM, J Gao, CH Delegard, LM Bagaasen, and BE Wells. 2003. Physical Property and Rheological Testing of Actual Transuranic Waste from Hanford Single-Shell Tanks. PNNL-14365, Pacific Northwest National Laboratory, Richland, Washington.

Tingey JM. 2004. Vacuum Drying of Actual Transuranic Waste from Hanford Tanks. PNNL-14670, Pacific Northwest National Laboratory, Richland, Washington. 
Tong P and BJ Ackerson. 1998. “Analogies between Colloidal Sedimentation and Turbulent Convection at High Prandtl Numbers.” Physical Review E 58(6):R6931-R6934.

Turian RM, JF Attal, DJ Sung, and LE Wedgewood. 2002. "Properties and Rheology of Coal-Water Mixtures Using Different Coals.” Fuel 81:2019-2033.

Turian RM, FL Hsu, and TW Ma. 1987 . Estimation of the Critical Velocity in Pipeline Flow of Slurries. Elsevier Sequoia.

Turian RM and Yuan TF. 1977. Flow of Slurries in Pipelines. AIChE Journal., 23(1977) 232-243.

Urie MW, GM Mong, PR Bredt, AP Poloski, JA Campbell, RD Scheele, OT Farmer, CZ Soderquist, SK Fiskum, RG Swoboda, LR Greenwood, MP Thomas, EW Hoppe, JJ Wagner, and LK Jagoda. 2002. Chemical Analysis and Physical Property Testing of 241-AZ-101-Tank Waste-Supernatant and Centrifuged Solids. PNWD-3215, Rev 1 (WTP-RPT-048, Rev 1), Battelle - Pacific Northwest Division, Richland, Washington.

van Kessel T and WGM van Kesteren. 2002. "Gas Production and Transport in Artificial Sludge Depots.” Waste Management 22:19-28.

Vanoni AA, Editor. 1975. Sedimentation Engineering, the ASCE Task Committee for the Preparation of the Manual on Sedimentation of the Sedimentation Committee of the Hydraulic Division. The American Society of Civil Engineers, New York.

Warrant RW. 2001. Results of Retrieval Testing of Sludge from Tank 241-AY-102. RPP-8909 Rev. 0, Fluor Hanford Inc., Richland, Washington.

Warrant RW. 2002. Results of Retrieval Testing of Sludge from Tank 241-AZ-102. RPP-9806 Rev. 0, Fluor Hanford Inc., Richland, Washington.

Warrant RW. 2004. Results of Retrieval Testing of Sludge from Tank 241-C-107. RPP-18799 Rev. 0, CH2M Hill Hanford Group, Inc., Richland, Washington.

Warrant RW. Results of Caustic Testing of Tank 241-AY-102 Core 319 Sludge Solids.

7S110-RWW-06-080, November 2006, CH2M HILL Hanford Group Inc., Richland, Washington.

Wasp EJ and PT Slatter. 2004. “Deposition Velocities for Small Particles in Large Pipes.” In: Proceedings of $12^{\text {th }}$ International Conference on the Hydraulic Transport of Solids in Pipes, September 20-24, Prague, Czech Republic, pp. 671-680.

Wasp EJ, TJ Regan, J Wither, PAC Cook, and JT Clancey. 1963. "Cross Country Coal Pipe Line Hydraulics.” Pipe Line News, Engineering, pp. 20-28.

Wasp EJ, JP Kenny, and RL Gandhi. 1977. Solid-Liquid Flow: Slurry Pipeline Transportation, Vol. 1, No. 4, Series on Bulk Materials Handling, Trans Tech Publications, Glausthal, Germany. 
Weber RA. 2009. Methodology and Calculations for the Assignment of Waste Groups for the Large Underground Waste Storage Tanks at the Hanford Site. RPP-10006, Rev. 8, Washington River Protection Solutions LLC, Richland, Washington.

Wells BE and JJ Ressler. 2009. Estimate of the Distribution of Solids Within Mixed Hanford Double-Shell Tank AZ-101: Implications for AY-102. PNNL-18327, Pacific Northwest National Laboratory, Richland, Washington.

Wells BE, MA Knight, EC Buck, SK Cooley, RC Daniel, LA Mahoney, PA Meyer, AP Poloski, JM Tingey, WS Callaway III, GA Cooke, ME Johnson, MG Thien, DJ Washenfelder, JJ Davis, MN Hall, GL Smith, SL Thomson, and Y Onishi. 2007. Estimate of Hanford Waste Insoluble Solid Particle Size and Density Distribution. PNWD-3824 (WTP-RPT-153, Rev. 0), Battelle-Pacific Northwest Division, Richland, Washington.

Wells BE, CW Enderlin, PA Gauglitz, and RA Peterson. 2009. Assessment of Jet Erosion for Potential Post-Retrieval K-Basin Settled Sludge. PNNL-18831, Pacific Northwest National Laboratory, Richland, Washington.

Wells BE, JJ Jenks, G Boeringa, NN Bauman, and AD Guzman. 2010a. Lateral Earth Pressure at Rest and Shear Modulus Measurements on Hanford Sludge Simulants. PNNL-19829, Pacific Northwest National Laboratory, Richland, Washington.

Wells BE, RL Russell, LA Mahoney, GN Brown, DE Rinehart, WC Buchmiller, EC Golovich, and JV Crum. 2010b. Hanford Sludge Simulant Selection for Soil Mechanics Property Measurement. PNNL-19250, Pacific Northwest National Laboratory, Richland, Washington.

Wodrich DD, GS Barney, GL Borsheim, DL Becker, WC Carlos, MJ Klem, RE Van der Cook, and JL Ryan. 1992. Summary of Single-Shell Waste Tank Stability. WHC-EP-0347 Supplement, Westinghouse Hanford Company, Richland, Washington.

Wood RJK. 1999. "The Erosion Performance of Candidate Internal Coatings for Slurry Handling and Pipeline Transport.” In: The 14th International Conference on Slurry Handling and Pipeline Transport - Hydrotransport 14, BHR Group Conference Series Publication No. 36, pp. 699-718.

Wu W and SY Wang. 2006. "Formulas for Sediment Porosity and Settling Velocity.” Journal of Hydraulic Division 132(8):858-862.

Zhou Z, MJ Solomon, PJ Scales, and DV Boger. 1999. "The Yield Stress of Concentrated Flocculated Suspensions of Size Distributed Particles.” Journal of Rheology 43(3):651-671.

Zhou Z, PJ Scales, and DV Boger. 2001. "Chemical and Physical Control of the Rheology of Concentrated Metal Oxide Suspensions." Chemical Engineering Science 56: 2901-2920. 
Appendix A

Density and pH of Liquid by Tank and by Waste Type 



\section{Appendix A: Density and pH of Liquid by Tank and by Waste Type}

Table A.1 and Table A.2 contain the liquid density, $\mathrm{pH}$, and hydroxide concentration information that was generated by the methods described in Section 3.2.1.

Table A.1 provides information for each tank and for the all-tank composite liquid, which is the volume-weighted average over all tank liquid inventories. The liquid mass and volume were determined by thermodynamic modeling that used 2002 Best Basis Inventory (BBI) data. Both supernatant liquid and the interstitial liquid in the bulk solid are included. The liquid density, $\mathrm{pH}$, and hydroxide concentration are not based on the thermodynamic modeling results from 2002, but on more recent data, or (when unavoidable) on historical (pre-1992) data. More details can be found in Section 3.2.1. For up-to-date data, the reader is directed to the current BBI.

Table A.2 gives the density, $\mathrm{pH}$, and hydroxide concentration for the saltcake and sludge waste types that were defined in the 2002 BBI. These were obtained by volume-averaging the data for the tanks identified in Section 3.2.3.4 as representatives of each waste type. Again, note that the waste types are as defined in 2002, but the liquid properties are taken from other sources. 
Table A.1. As-Is Liquid Compositions of Individual Tanks ("n/a" = not available)

\begin{tabular}{|l|c|c|c|c|c|c|}
\hline & 241-A-101 & 241-A-102 & 241-A-103 & 241-A-104 & 241-A-105 & 241-A-106 \\
\hline Total kg liquid for tank & $4.35 \mathrm{E}+06$ & $2.09 \mathrm{E}+05$ & $1.79 \mathrm{E}+06$ & $9.81 \mathrm{E}+04$ & $1.17 \mathrm{E}+05$ & $2.35 \mathrm{E}+05$ \\
\hline Total kL liquid for tank & 2917 & 133 & 1187 & 84 & 100 & 201 \\
\hline Liquid phase g/mL & 1.490 & 1.570 & 1.510 & 1.170 & 1.170 & 1.170 \\
\hline $\begin{array}{l}\text { OH (ug/mL) chosen from } \\
\text { data or calculated from } \\
\text { pH }\end{array}$ & 42700 & 34 & 48900 & 5.4 & 7245 & 42700 \\
\hline $\begin{array}{l}\text { pH chosen from data or } \\
\text { calculated from OH }\end{array}$ & 14.4 & 11.3 & 14.5 & 10.5 & 13.6 & 14.4 \\
\hline
\end{tabular}

\begin{tabular}{|l|c|c|c|c|c|c|}
\hline & 241-AN-101 & 241-AN-102 & 241-AN-103 & 241-AN-104 & 241-AN-105 & 241-AN-106 \\
\hline Total kg liquid for tank & $1.37 \mathrm{E}+06$ & $5.16 \mathrm{E}+06$ & $4.17 \mathrm{E}+06$ & $4.74 \mathrm{E}+06$ & $5.45 \mathrm{E}+06$ & $1.55 \mathrm{E}+05$ \\
\hline Total kL liquid for tank & 947 & 3659 & 2821 & 3385 & 3836 & 134 \\
\hline Liquid phase g/mL & 1.450 & 1.410 & 1.480 & 1.400 & 1.420 & 1.150 \\
\hline $\begin{array}{l}\text { OH (ug/mL) chosen from } \\
\text { data or calculated from } \\
\text { pH }\end{array}$ & 32800 & 8180 & 67300 & 65300 & 59800 & 6450 \\
\hline $\begin{array}{l}\text { pH chosen from data or } \\
\text { calculated from OH }\end{array}$ & 14.3 & 13.7 & 14.6 & 14.6 & 14.5 & 13.6 \\
\hline
\end{tabular}

\begin{tabular}{|l|c|c|c|c|c|c|}
\hline & 241-AN-107 & 241-AP-101 & 241-AP-102 & 241-AP-103 & 241-AP-104 & 241-AP-105 \\
\hline Total kg liquid for tank & $5.45 \mathrm{E}+06$ & $5.22 \mathrm{E}+06$ & $5.51 \mathrm{E}+05$ & $1.47 \mathrm{E}+06$ & $5.83 \mathrm{E}+06$ & $4.97 \mathrm{E}+06$ \\
\hline Total kL liquid for tank & 3813 & 4211 & 402 & 1055 & 4135 & 3916 \\
\hline Liquid phase g/mL & 1.430 & 1.240 & 1.370 & 1.390 & 1.410 & 1.270 \\
\hline $\begin{array}{l}\text { OH (ug/mL) chosen from } \\
\text { data or calculated from } \\
\text { pH }\end{array}$ & 18000 & 17900 & 34200 & 23100 & 26900 & 10400 \\
\hline $\begin{array}{l}\text { pH chosen from data or } \\
\text { calculated from OH }\end{array}$ & 14.0 & 14.0 & 14.3 & 14.1 & 14.2 & 13.8 \\
\hline
\end{tabular}


Table A.1. (contd)

\begin{tabular}{|l|c|c|c|c|c|c|}
\hline & 241-AP-106 & 241-AP-107 & 241-AP-108 & 241-AW-101 & 241-AW-102 & 241-AW-103 \\
\hline Total kg liquid for tank & $5.08 \mathrm{E}+06$ & $1.97 \mathrm{E}+05$ & $4.12 \mathrm{E}+06$ & $5.49 \mathrm{E}+06$ & $4.67 \mathrm{E}+06$ & $5.65 \mathrm{E}+06$ \\
\hline Total kL liquid for tank & 4200 & 154 & 2923 & 3733 & 3767 & 3978 \\
\hline Liquid phase g/mL & 1.210 & 1.280 & 1.410 & 1.470 & 1.240 & 1.420 \\
\hline $\begin{array}{l}\text { OH (ug/mL) chosen from } \\
\text { data or calculated from } \\
\text { pH }\end{array}$ & 7370 & 18800 & 36600 & 99500 & 16500 & 13100 \\
\hline $\begin{array}{l}\text { pH chosen from data or } \\
\text { calculated from OH }\end{array}$ & 13.6 & 14.0 & 14.3 & 14.8 & & 14.0 \\
\hline
\end{tabular}

\begin{tabular}{|c|c|c|c|c|c|c|}
\hline & 241-AW-104 & $241-\mathrm{AW}-105$ & 241-AW-106 & $241-\mathrm{AX}-101$ & $241-\mathrm{AX}-102$ & 241-AX-103 \\
\hline Total kg liquid for tank & $1.58 \mathrm{E}+06$ & $1.63 \mathrm{E}+06$ & $1.13 \mathrm{E}+06$ & $3.19 \mathrm{E}+06$ & $1.15 \mathrm{E}+05$ & $5.47 \mathrm{E}+05$ \\
\hline Total kL liquid for tank & 1128 & 1536 & 928 & 2086 & 82 & 377 \\
\hline Liquid phase $g / \mathrm{mL}$ & 1.400 & 1.060 & 1.220 & 1.530 & 1.390 & 1.450 \\
\hline $\begin{array}{l}\mathrm{OH}(\mathrm{ug} / \mathrm{mL}) \text { chosen from } \\
\text { data or calculated from } \\
\mathrm{pH}\end{array}$ & 25600 & 4810 & 11700 & 39000 & 38900 & 38900 \\
\hline $\begin{array}{l}\mathrm{pH} \text { chosen from data or } \\
\text { calculated from } \mathrm{OH}\end{array}$ & 14.2 & 13.5 & 13.8 & 14.4 & 14.4 & 14.4 \\
\hline
\end{tabular}

\begin{tabular}{|l|c|c|c|c|c|c|}
\hline & 241-AX-104 & 241-AY-101 & 241-AY-102 & 241-AZ-101 & 241-AZ-102 & 241-B-101 \\
\hline Total kg liquid for tank & $1.96 \mathrm{E}+04$ & $7.61 \mathrm{E}+05$ & $2.86 \mathrm{E}+06$ & $4.56 \mathrm{E}+06$ & $4.24 \mathrm{E}+06$ & $3.97 \mathrm{E}+05$ \\
\hline Total kL liquid for tank & 17 & 590 & 2483 & 3674 & 3721 & 259 \\
\hline Liquid phase g/mL & 1.170 & 1.290 & 1.150 & 1.240 & 1.140 & 1.530 \\
\hline $\begin{array}{l}\text { OH (ug/mL) chosen from } \\
\text { data or calculated from } \\
\text { pH }\end{array}$ & 7245 & 9580 & 38500 & 12400 & 2090 & $\mathrm{n} / \mathbf{a}$ \\
\hline $\begin{array}{l}\text { pH chosen from data or } \\
\text { calculated from OH }\end{array}$ & 13.6 & 13.8 & 14.4 & 13.9 & 13.1 & $\mathrm{n} / \mathbf{a}$ \\
\hline
\end{tabular}


Table A.1. (contd)

\begin{tabular}{|l|c|c|c|c|c|c|}
\hline & 241-B-102 & 241-B-103 & 241-B-104 & 241-B-105 & 241-B-106 & 241-B-107 \\
\hline Total kg liquid for tank & $7.72 \mathrm{E}+04$ & $1.21 \mathrm{E}+05$ & $1.63 \mathrm{E}+06$ & $5.03 \mathrm{E}+05$ & $4.82 \mathrm{E}+05$ & $5.27 \mathrm{E}+05$ \\
\hline Total kL liquid for tank & 61 & 96 & 1297 & 399 & 383 & 393 \\
\hline Liquid phase g/mL & 1.260 & 1.260 & 1.260 & 1.260 & 1.260 & 1.340 \\
\hline $\begin{array}{l}\text { OH (ug/mL) chosen from } \\
\text { data or calculated from } \\
\text { pH }\end{array}$ & $\mathrm{n} / \mathrm{a}$ & $\mathrm{n} / \mathrm{a}$ & $\mathrm{n} / \mathrm{a}$ & $\mathrm{n} / \mathrm{a}$ & $\mathrm{n} / \mathrm{a}$ & $\mathrm{n} / \mathrm{a}$ \\
\hline $\begin{array}{l}\mathrm{pH} \text { chosen from data or } \\
\text { calculated from OH }\end{array}$ & $\mathrm{n} / \mathrm{a}$ & $\mathrm{n} / \mathrm{a}$ & $\mathrm{n} / \mathrm{a}$ & $\mathrm{nl} / \mathrm{a}$ & $\mathrm{n} / \mathrm{a}$ & $\mathrm{n} / \mathrm{a}$ \\
\hline
\end{tabular}

\begin{tabular}{|l|c|c|c|c|c|c|}
\hline & 241-B-108 & 241-B-109 & 241-B-110 & 241-B-111 & 241-B-112 & 241-B-201 \\
\hline Total kg liquid for tank & $1.87 \mathrm{E}+05$ & $1.08 \mathrm{E}+05$ & $9.97 \mathrm{E}+05$ & $1.01 \mathrm{E}+06$ & $1.73 \mathrm{E}+05$ & $1.26 \mathrm{E}+05$ \\
\hline Total kL liquid for tank & 135 & 85 & 837 & 846 & 114 & 108 \\
\hline Liquid phase $\mathrm{g} / \mathrm{mL}$ & 1.380 & 1.260 & 1.190 & 1.190 & 1.510 & 1.170 \\
\hline $\begin{array}{l}\text { OH (ug/mL) chosen from } \\
\text { data or calculated from } \\
\mathrm{pH}\end{array}$ & $\mathrm{n} / \mathrm{a}$ & $\mathrm{n} / \mathrm{a}$ & 2.7 & & & \\
\hline $\begin{array}{l}\mathrm{pH} \text { chosen from data or } \\
\text { calculated from } \mathrm{OH}\end{array}$ & $\mathrm{n} / \mathrm{a}$ & $\mathrm{n} / \mathrm{a}$ & 10.2 & 10.2 & 35200 & $\mathrm{n} / \mathrm{a}$ \\
\hline
\end{tabular}

\begin{tabular}{|l|c|c|c|c|c|c|}
\hline & 241-B-202 & 241-B-203 & 241-B-204 & 241-BX-101 & 241-BX-102 & 241-BX-103 \\
\hline Total kg liquid for tank & $1.26 \mathrm{E}+05$ & $2.01 \mathrm{E}+05$ & $1.98 \mathrm{E}+05$ & $1.68 \mathrm{E}+05$ & $3.92 \mathrm{E}+05$ & $2.46 \mathrm{E}+05$ \\
\hline Total kL liquid for tank & 108 & 191 & 188 & 131 & 335 & 230 \\
\hline Liquid phase g/mL & 1.170 & 1.050 & 1.050 & 1.280 & 1.170 & 1.070 \\
\hline $\begin{array}{l}\text { OH (ug } / \mathrm{mL} \text { ) chosen from } \\
\text { data or calculated from } \\
\mathrm{pH}\end{array}$ & 0.54 & 0.54 & 0.54 & 0.85 & 17008 & 0.85 \\
\hline $\begin{array}{l}\mathrm{pH} \text { chosen from data or } \\
\text { calculated from OH }\end{array}$ & 9.5 & 9.5 & 9.5 & 9.7 & 14 & 9.7 \\
\hline
\end{tabular}


Table A.1. (contd)

\begin{tabular}{|l|c|c|c|c|c|c|}
\hline & 241-BX-104 & 241-BX-105 & 241-BX-106 & 241-BX-107 & 241-BX-108 & 241-BX-109 \\
\hline Total kg liquid for tank & $3.24 \mathrm{E}+05$ & $2.48 \mathrm{E}+05$ & $1.05 \mathrm{E}+05$ & $1.20 \mathrm{E}+06$ & $7.75 \mathrm{E}+04$ & $7.30 \mathrm{E}+05$ \\
\hline Total kL liquid for tank & 253 & 192 & 69 & 1024 & 66 & 624 \\
\hline Liquid phase g/mL & 1.280 & 1.290 & 1.510 & 1.170 & 1.170 & 1.170 \\
\hline $\begin{array}{l}\text { OH (ug/mL) chosen from } \\
\text { data or calculated from pH }\end{array}$ & 1701 & 1701 & 0.85 & 2.7 & 67.7 & 170 \\
\hline $\begin{array}{l}\text { pH chosen from data or } \\
\text { calculated from OH }\end{array}$ & 13 & 13 & 9.7 & 10.2 & 11.6 & 12 \\
\hline
\end{tabular}

\begin{tabular}{|l|c|c|c|c|c|c|}
\hline & 241-BX-110 & 241-BX-111 & 241-BX-112 & 241-BY-101 & 241-BY-102 & 241-BY-103 \\
\hline Total kg liquid for tank & $4.35 \mathrm{E}+05$ & $4.91 \mathrm{E}+05$ & $5.87 \mathrm{E}+05$ & $6.98 \mathrm{E}+05$ & $1.14 \mathrm{E}+06$ & $9.98 \mathrm{E}+05$ \\
\hline Total kL liquid for tank & 302 & 338 & 497 & 463 & 784 & 773 \\
\hline Liquid phase $\mathrm{g} / \mathrm{mL}$ & 1.440 & 1.450 & 1.180 & 1.510 & 1.460 & 1.290 \\
\hline $\begin{array}{l}\text { OH (ug/mL) chosen from } \\
\text { data or calculated from } \mathrm{pH}\end{array}$ & 35200 & 53784 & 4.3 & 38300 & 38300 & 31500 \\
\hline $\begin{array}{l}\text { pH chosen from data or } \\
\text { calculated from } \mathrm{OH}\end{array}$ & 14.3 & 14.5 & 10.4 & 14.4 & 14.4 & 14.3 \\
\hline
\end{tabular}

\begin{tabular}{|l|c|c|c|c|c|c|}
\hline & 241-BY-104 & $\mathbf{2 4 1 - B Y - 1 0 5}$ & $\mathbf{2 4 1 - B Y - 1 0 6}$ & $\mathbf{2 4 1 - B Y - 1 0 7}$ & 241-BY-108 & 241-BY-109 \\
\hline Total kg liquid for tank & $1.36 \mathrm{E}+06$ & $1.27 \mathrm{E}+06$ & $2.04 \mathrm{E}+06$ & $1.12 \mathrm{E}+06$ & $6.54 \mathrm{E}+05$ & $1.07 \mathrm{E}+06$ \\
\hline Total kL liquid for tank & 902 & 881 & 1554 & 764 & 492 & 714 \\
\hline Liquid phase g/mL & 1.510 & 1.440 & 1.310 & 1.460 & 1.330 & 1.500 \\
\hline $\begin{array}{l}\text { OH (ug/mL) chosen from } \\
\text { data or calculated from pH }\end{array}$ & 38300 & 38300 & 35800 & 38300 & 38300 & 38300 \\
\hline $\begin{array}{l}\text { pH chosen from data or } \\
\text { calculated from OH }\end{array}$ & 14.4 & 14.4 & 14.3 & 14.4 & 14.4 & 14.4 \\
\hline
\end{tabular}


Table A.1. (contd)

\begin{tabular}{|l|c|c|c|c|c|c|}
\hline & 241-BY-110 & 241-BY-111 & 241-BY-112 & 241-C-101 & 241-C-102 & 241-C-103 \\
\hline Total kg liquid for tank & $1.41 \mathrm{E}+06$ & $7.98 \mathrm{E}+05$ & $8.20 \mathrm{E}+05$ & $1.91 \mathrm{E}+05$ & $1.23 \mathrm{E}+06$ & $5.09 \mathrm{E}+05$ \\
\hline Total kL liquid for tank & 978 & 562 & 558 & 163 & 1055 & 519 \\
\hline Liquid phase g/mL & 1.440 & 1.420 & 1.470 & 1.170 & 1.170 & 0.980 \\
\hline $\begin{array}{l}\text { OH (ug/mL) chosen from } \\
\text { data or calculated from } \\
\text { pH }\end{array}$ & 38300 & 38300 & 38300 & 2.1 & 3394 & 0.101 \\
\hline $\begin{array}{l}\text { pH chosen from data or } \\
\text { calculated from OH }\end{array}$ & 14.4 & 14.4 & 14.4 & 10.1 & 13.3 & 8.8 \\
\hline
\end{tabular}

\begin{tabular}{|l|c|c|c|c|c|c|}
\hline & 241-C-104 & 241-C-105 & 241-C-106 & 241-C-107 & 241-C-108 & 241-C-109 \\
\hline Total kg liquid for tank & $9.42 \mathrm{E}+05$ & $2.79 \mathrm{E}+05$ & $1.35 \mathrm{E}+05$ & $8.85 \mathrm{E}+05$ & $2.08 \mathrm{E}+05$ & $1.85 \mathrm{E}+05$ \\
\hline Total kL liquid for tank & 806 & 227 & 133 & 756 & 185 & 158 \\
\hline Liquid phase $\mathrm{g} / \mathrm{mL}$ & 1.170 & 1.230 & 1.020 & 1.170 & 1.120 & 1.170 \\
\hline $\begin{array}{l}\text { OH (ug/mL) chosen from } \\
\text { data or calculated from } \\
\text { pH }\end{array}$ & 14000 & 0.85 & 7020 & 0.68 & 6830 & 214 \\
\hline $\begin{array}{l}\text { pH chosen from data or } \\
\text { calculated from OH }\end{array}$ & 13.9 & 9.7 & 13.6 & 9.6 & 13.6 & 12.1 \\
\hline
\end{tabular}

\begin{tabular}{|l|c|c|c|c|c|c|}
\hline & $\mathbf{2 4 1 - C - 1 1 0}$ & $\mathbf{2 4 1 - C}-\mathbf{1 1 1}$ & $\mathbf{2 4 1 - C}-\mathbf{1 1 2}$ & $\mathbf{2 4 1 - C - 2 0 1}$ & $\mathbf{2 4 1 - C}-202$ & $\mathbf{2 4 1 - C - 2 0 3}$ \\
\hline Total kg liquid for tank & $6.03 \mathrm{E}+05$ & $1.64 \mathrm{E}+05$ & $3.83 \mathrm{E}+05$ & $1.81 \mathrm{E}+03$ & $2.32 \mathrm{E}+03$ & $6.04 \mathrm{E}+03$ \\
\hline Total kL liquid for tank & 548 & 140 & 327 & 2 & 2 & 6 \\
\hline Liquid phase g/mL & 1.100 & 1.170 & 1.170 & 1.000 & 1.000 & 1.000 \\
\hline $\begin{array}{l}\text { OH (ug/mL) chosen from } \\
\text { data or calculated from } \\
\text { pH }\end{array}$ & 4.3 & 214 & 4.3 & 0.101 & 0.101 & 0.101 \\
\hline $\begin{array}{l}\text { pH chosen from data or } \\
\text { calculated from OH }\end{array}$ & 10.4 & 12.1 & 10.4 & 8.8 & 8.8 & 8.8 \\
\hline
\end{tabular}


Table A.1. (contd)

\begin{tabular}{|l|c|c|c|c|c|c|}
\hline & 241-C-204 & 241-S-101 & 241-S-102 & 241-S-103 & 241-S-104 & 241-S-105 \\
\hline Total kg liquid for tank & $8.62 \mathrm{E}+03$ & $1.98 \mathrm{E}+06$ & $1.32 \mathrm{E}+06$ & $9.65 \mathrm{E}+05$ & $1.13 \mathrm{E}+06$ & $1.40 \mathrm{E}+06$ \\
\hline Total kL liquid for tank & 9 & 1348 & 905 & 657 & 828 & 965 \\
\hline Liquid phase g/mL & 1.000 & 1.470 & 1.460 & 1.470 & 1.370 & 1.450 \\
\hline $\begin{array}{l}\text { OH (ug/mL) chosen from } \\
\text { data or calculated from } \\
\text { pH }\end{array}$ & 0.101 & 47600 & 37600 & 36500 & 5100 & 81800 \\
\hline $\begin{array}{l}\text { pH chosen from data or } \\
\text { calculated from OH }\end{array}$ & 8.8 & 14.4 & 14.3 & 14.3 & 13.5 & 14.7 \\
\hline
\end{tabular}

\begin{tabular}{|l|c|c|c|c|c|c|}
\hline & 241-S-106 & 241-S-107 & 241-S-108 & 241-S-109 & 241-S-110 & 241-S-111 \\
\hline Total kg liquid for tank & $1.46 \mathrm{E}+06$ & $1.68 \mathrm{E}+06$ & $1.89 \mathrm{E}+06$ & $1.52 \mathrm{E}+06$ & $1.05 \mathrm{E}+06$ & $1.76 \mathrm{E}+06$ \\
\hline Total kL liquid for tank & 1018 & 1282 & 1303 & 1019 & 735 & 1213 \\
\hline Liquid phase $\mathrm{g} / \mathrm{mL}$ & 1.430 & 1.310 & 1.450 & 1.490 & 1.430 & 1.450 \\
\hline $\begin{array}{l}\text { OH (ug/mL) chosen from } \\
\text { data or calculated from } \\
\mathrm{pH}\end{array}$ & 55500 & 29900 & 48100 & 81900 & 81800 & 51000 \\
\hline $\begin{array}{l}\mathrm{pH} \text { chosen from data or } \\
\text { calculated from } \mathrm{OH}\end{array}$ & 14.5 & 14.2 & 14.5 & 14.7 & 14.7 & 14.5 \\
\hline
\end{tabular}

\begin{tabular}{|l|c|c|c|c|c|c|}
\hline & 241-S-112 & 241-SX-101 & 241-SX-102 & 241-SX-103 & 241-SX-104 & 241-SX-105 \\
\hline Total kg liquid for tank & $1.19 \mathrm{E}+06$ & $1.62 \mathrm{E}+06$ & $2.43 \mathrm{E}+06$ & $2.04 \mathrm{E}+06$ & $1.81 \mathrm{E}+06$ & $1.52 \mathrm{E}+06$ \\
\hline Total kL liquid for tank & 935 & 1093 & 1663 & 1384 & 1231 & 1037 \\
\hline Liquid phase g/mL & 1.270 & 1.480 & 1.460 & 1.470 & 1.470 & 1.470 \\
\hline $\begin{array}{l}\text { OH (ug/mL) chosen from } \\
\text { data or calculated from } \\
\text { pH }\end{array}$ & 124000 & 48700 & 50100 & 45200 & 30500 & 40900 \\
\hline $\begin{array}{l}\mathrm{pH} \text { chosen from data or } \\
\text { calculated from OH }\end{array}$ & 14.9 & 14.5 & 14.5 & 14.4 & 14.3 & 14.4 \\
\hline
\end{tabular}


Table A.1. (contd)

\begin{tabular}{|l|c|c|c|c|c|c|}
\hline & 241-SX-106 & 241-SX-107 & 241-SX-108 & 241-SX-109 & 241-SX-110 & 241-SX-111 \\
\hline Total kg liquid for tank & $1.52 \mathrm{E}+06$ & $4.41 \mathrm{E}+05$ & $2.12 \mathrm{E}+05$ & $8.50 \mathrm{E}+05$ & $1.87 \mathrm{E}+05$ & $5.49 \mathrm{E}+05$ \\
\hline Total kL liquid for tank & 1176 & 294 & 181 & 611 & 159 & 366 \\
\hline Liquid phase g/mL & 1.290 & 1.500 & 1.170 & 1.390 & 1.170 & 1.500 \\
\hline $\begin{array}{l}\text { OH (ug/mL) chosen from } \\
\text { data or calculated from } \\
\text { pH }\end{array}$ & 31900 & $\mathrm{n} / \mathrm{a}$ & $\mathrm{n} / \mathrm{a}$ & $\mathrm{n} / \mathrm{a}$ & $\mathrm{n} / \mathrm{a}$ & 60700 \\
\hline $\begin{array}{l}\text { pH chosen from data or } \\
\text { calculated from OH }\end{array}$ & 14.3 & $\mathrm{n} / \mathrm{a}$ & $\mathrm{n} / \mathrm{a}$ & $\mathrm{n} / \mathbf{a}$ & $\mathrm{n} / \mathrm{a}$ & 14.6 \\
\hline
\end{tabular}

\begin{tabular}{|l|c|c|c|c|c|c|}
\hline & 241-SX-112 & 241-SX-113 & 241-SX-114 & 241-SX-115 & 241-SY-101 & 241-SY-102 \\
\hline Total kg liquid for tank & $3.36 \mathrm{E}+05$ & $6.40 \mathrm{E}+04$ & $7.13 \mathrm{E}+05$ & $1.38 \mathrm{E}+04$ & $3.84 \mathrm{E}+06$ & $5.00 \mathrm{E}+06$ \\
\hline Total kL liquid for tank & 224 & 55 & 475 & 12 & 3398 & 3815 \\
\hline Liquid phase $\mathrm{g} / \mathrm{mL}$ & 1.500 & 1.170 & 1.500 & 1.170 & 1.130 & 1.310 \\
\hline $\begin{array}{l}\text { OH (ug/mL) chosen from } \\
\text { data or calculated from } \\
\mathrm{pH}\end{array}$ & $\mathrm{n} / \mathrm{a}$ & $\mathrm{n} / \mathrm{a}$ & $\mathrm{n} / \mathrm{a}$ & 60700 & 11100 & 8830 \\
\hline $\begin{array}{l}\mathrm{pH} \text { chosen from data or } \\
\text { calculated from OH }\end{array}$ & $\mathrm{n} / \mathrm{a}$ & $\mathrm{n} / \mathrm{a}$ & $\mathrm{n} / \mathrm{a}$ & 14.6 & 13.8 & 13.7 \\
\hline
\end{tabular}

\begin{tabular}{|l|c|c|c|c|c|c|}
\hline & 241-SY-103 & 241-T-101 & 241-T-102 & 241-T-103 & 241-T-104 & 241-T-105 \\
\hline Total kg liquid for tank & $3.69 \mathrm{E}+06$ & $4.27 \mathrm{E}+05$ & $8.45 \mathrm{E}+04$ & $9.31 \mathrm{E}+04$ & $1.16 \mathrm{E}+06$ & $3.87 \mathrm{E}+05$ \\
\hline Total kL liquid for tank & 2474 & 295 & 74 & 78 & 995 & 328 \\
\hline Liquid phase g/mL & 1.490 & 1.450 & 1.140 & 1.190 & 1.170 & 1.180 \\
\hline $\begin{array}{l}\text { OH (ug/mL) chosen from } \\
\text { data or calculated from } \\
\text { pH }\end{array}$ & 37400 & 48100 & 62.5 & 1073 & 1.7 & 360 \\
\hline $\begin{array}{l}\text { pH chosen from data or } \\
\text { calculated from OH }\end{array}$ & 14.3 & 14.5 & 11.6 & 12.8 & 10 & 12.3 \\
\hline
\end{tabular}


Table A.1. (contd)

\begin{tabular}{|l|c|c|c|c|c|c|}
\hline & 241-T-106 & 241-T-107 & 241-T-108 & 241-T-109 & 241-T-110 & 241-T-111 \\
\hline Total kg liquid for tank & $5.58 \mathrm{E}+04$ & $6.41 \mathrm{E}+05$ & $3.70 \mathrm{E}+04$ & $1.66 \mathrm{E}+05$ & $1.45 \mathrm{E}+06$ & $1.90 \mathrm{E}+06$ \\
\hline Total kL liquid for tank & 48 & 548 & 29 & 132 & 1379 & 1624 \\
\hline Liquid phase g/mL & 1.170 & 1.170 & 1.260 & 1.260 & 1.050 & 1.170 \\
\hline $\begin{array}{l}\text { OH (ug/mL) chosen from } \\
\text { data or calculated from } \\
\text { pH }\end{array}$ & 135 & 3.4 & 107 & 170 & 0.043 & 1351 \\
\hline $\begin{array}{l}\text { pH chosen from data or } \\
\text { calculated from OH }\end{array}$ & 11.9 & 10.3 & 11.8 & 12.0 & 8.4 & 12.9 \\
\hline
\end{tabular}

\begin{tabular}{|l|c|c|c|c|c|c|}
\hline & $\mathbf{2 4 1 - T - 1 1 2}$ & $\mathbf{2 4 1 - T - 2 0 1}$ & $\mathbf{2 4 1 - T - 2 0 2}$ & $\mathbf{2 4 1 - T - 2 0 3}$ & 241-T-204 & 241-TX-101 \\
\hline Total kg liquid for tank & $2.65 \mathrm{E}+05$ & $1.20 \mathrm{E}+05$ & $9.31 \mathrm{E}+04$ & $1.61 \mathrm{E}+05$ & $1.60 \mathrm{E}+05$ & $3.24 \mathrm{E}+05$ \\
\hline Total kL liquid for tank & 241 & 114 & 80 & 137 & 137 & 223 \\
\hline Liquid phase $\mathrm{g} / \mathrm{mL}$ & 1.100 & 1.060 & 1.170 & 1.170 & 1.170 & 1.450 \\
\hline $\begin{array}{l}\text { OH (ug } / \mathrm{mL} \text { ) chosen from } \\
\text { data or calculated from } \\
\mathrm{pH}\end{array}$ & 8524 & $\mathrm{n} / \mathbf{a}$ & 2.7 & 2.7 & 2.7 & 17000 \\
\hline $\begin{array}{l}\mathrm{pH} \text { chosen from data or } \\
\text { calculated from } \mathrm{OH}\end{array}$ & 13.7 & $\mathrm{n} / \mathrm{a}$ & 10.2 & 10.2 & 10.2 & 14.0 \\
\hline
\end{tabular}

\begin{tabular}{|l|c|c|c|c|c|c|}
\hline & 241-TX-102 & 241-TX-103 & 241-TX-104 & 241-TX-105 & 241-TX-106 & 241-TX-107 \\
\hline Total kg liquid for tank & $7.67 \mathrm{E}+05$ & $4.77 \mathrm{E}+05$ & $2.31 \mathrm{E}+05$ & $1.75 \mathrm{E}+06$ & $1.20 \mathrm{E}+06$ & $8.58 \mathrm{E}+04$ \\
\hline Total kL liquid for tank & 529 & 331 & 160 & 1207 & 826 & 59 \\
\hline Liquid phase g/mL & 1.450 & 1.440 & 1.440 & 1.450 & 1.450 & 1.460 \\
\hline $\begin{array}{l}\text { OH (ug/mL) chosen from } \\
\text { data or calculated from } \\
\text { pH }\end{array}$ & 17000 & 17000 & $\mathrm{n} / \mathrm{a}$ & 17000 & 17000 & $\mathrm{n} / \mathrm{a}$ \\
\hline $\begin{array}{l}\text { pH chosen from data or } \\
\text { calculated from OH }\end{array}$ & 14.0 & 14.0 & $\mathrm{n} / \mathrm{a}$ & 14.0 & 14.0 & $\mathrm{n} / \mathrm{a}$ \\
\hline
\end{tabular}


Table A.1. (contd)

\begin{tabular}{|l|c|c|c|c|c|c|}
\hline & 241-TX-108 & 241-TX-109 & 241-TX-110 & 241-TX-111 & 241-TX-112 & 241-TX-113 \\
\hline Total kg liquid for tank & $4.44 \mathrm{E}+05$ & $1.23 \mathrm{E}+06$ & $1.61 \mathrm{E}+06$ & $1.27 \mathrm{E}+06$ & $1.95 \mathrm{E}+06$ & $1.41 \mathrm{E}+06$ \\
\hline Total kL liquid for tank & 306 & 1054 & 1108 & 873 & 1371 & 975 \\
\hline Liquid phase g/mL & 1.450 & 1.170 & 1.450 & 1.450 & 1.420 & 1.450 \\
\hline $\begin{array}{l}\text { OH (ug/mL) chosen from } \\
\text { data or calculated from } \\
\text { pH }\end{array}$ & 4272 & 17000 & 17000 & 17000 & 48100 & $n$ \\
\hline $\begin{array}{l}\text { pH chosen from data or } \\
\text { calculated from OH }\end{array}$ & 13.4 & 14.0 & 14.0 & 14.0 & 14.5 & $n$ \\
\hline
\end{tabular}

\begin{tabular}{|l|c|c|c|c|c|c|}
\hline & $\mathbf{2 4 1 - T X - 1 1 4}$ & $\mathbf{2 4 1 - T X - 1 1 5}$ & $\mathbf{2 4 1 - T X - 1 1 6}$ & $\mathbf{2 4 1 - T X - 1 1 7}$ & 241-TX-118 & 241-TY-101 \\
\hline Total kg liquid for tank & $1.56 \mathrm{E}+06$ & $1.78 \mathrm{E}+06$ & $1.65 \mathrm{E}+06$ & $1.29 \mathrm{E}+06$ & $7.31 \mathrm{E}+05$ & $3.71 \mathrm{E}+05$ \\
\hline Total kL liquid for tank & 1177 & 1225 & 1187 & 1024 & 504 & 294 \\
\hline Liquid phase $\mathrm{g} / \mathrm{mL}$ & 1.330 & 1.450 & 1.390 & 1.260 & 1.450 & 1.260 \\
\hline $\begin{array}{l}\text { OH }(\mathrm{ug} / \mathrm{mL} \text { ) chosen from } \\
\text { data or calculated from } \\
\mathrm{pH}\end{array}$ & 17000 & 3394 & 2540 & $\mathrm{n} / \mathrm{a}$ & 339 & $\mathrm{n} / \mathrm{a}$ \\
\hline $\begin{array}{l}\mathrm{pH} \text { chosen from data or } \\
\text { calculated from OH }\end{array}$ & 14.0 & 13.3 & 13.2 & $\mathrm{n} / \mathrm{a}$ & 12.3 & $\mathrm{n} / \mathrm{a}$ \\
\hline
\end{tabular}

\begin{tabular}{|l|c|c|c|c|c|c|}
\hline & $\mathbf{2 4 1 - T Y - 1 0 2}$ & $\mathbf{2 4 1 - T Y - 1 0 3}$ & $\mathbf{2 4 1 - T Y - 1 0 4}$ & $\mathbf{2 4 1 - T Y - 1 0 5}$ & $\mathbf{2 4 1 - T Y - 1 0 6}$ & $\mathbf{2 4 1 - U - 1 0 1}$ \\
\hline Total kg liquid for tank & $2.10 \mathrm{E}+05$ & $6.96 \mathrm{E}+05$ & $1.51 \mathrm{E}+05$ & $5.56 \mathrm{E}+\mathbf{0 5}$ & $4.07 \mathrm{E}+04$ & $7.12 \mathrm{E}+04$ \\
\hline Total kL liquid for tank & $\mathbf{1 5 4}$ & 480 & 128 & 475 & 35 & 61 \\
\hline Liquid phase g/mL & 1.360 & 1.450 & 1.180 & 1.170 & 1.170 & 1.170 \\
\hline $\begin{array}{l}\text { OH (ug/mL) chosen from } \\
\text { data or calculated from } \\
\text { pH }\end{array}$ & $\mathrm{n} / \mathrm{a}$ & 2.1 & 214.1 & $\mathrm{n} / \mathrm{a}$ & $\mathrm{n} / \mathrm{a}$ & $\mathrm{n} / \mathrm{a}$ \\
\hline $\begin{array}{l}\text { pH chosen from data or } \\
\text { calculated from OH }\end{array}$ & $\mathrm{n} / \mathrm{a}$ & 10.1 & 12.1 & $\mathrm{n} / \mathrm{a}$ & $\mathrm{n} / \mathrm{a}$ & $\mathrm{n} / \mathrm{a}$ \\
\hline
\end{tabular}


Table A.1. (contd)

\begin{tabular}{|l|c|c|c|c|c|c|}
\hline & $\mathbf{2 4 1 - U - 1 0 2}$ & $\mathbf{2 4 1 - U - 1 0 3}$ & $\mathbf{2 4 1 - U - 1 0 4}$ & $\mathbf{2 4 1 - U - 1 0 5}$ & $\mathbf{2 4 1 - U - 1 0 6}$ & $\mathbf{2 4 1 - U - 1 0 7}$ \\
\hline Total kg liquid for tank & $1.24 \mathrm{E}+06$ & $1.48 \mathrm{E}+06$ & $5.56 \mathrm{E}+05$ & $1.10 \mathrm{E}+06$ & $7.81 \mathrm{E}+05$ & $1.31 \mathrm{E}+06$ \\
\hline Total kL liquid for tank & 839 & 1026 & 397 & 751 & 583 & 916 \\
\hline Liquid phase g/mL & 1.480 & 1.440 & 1.400 & 1.460 & 1.340 & 1.430 \\
\hline $\begin{array}{l}\text { OH (ug/mL) chosen from } \\
\text { data or calculated from } \\
\mathrm{pH}\end{array}$ & 29300 & 30800 & $\mathrm{n} / \mathrm{a}$ & 20900 & 5110 & 31300 \\
\hline $\begin{array}{l}\mathrm{pH} \text { chosen from data or } \\
\text { calculatcd from OH }\end{array}$ & 14.2 & 14.3 & $\mathrm{n} / \mathrm{a}$ & 14.1 & 13.5 & 14.3 \\
\hline
\end{tabular}

\begin{tabular}{|l|c|c|c|c|c|c|}
\hline & $\mathbf{2 4 1 - U - 1 0 8}$ & $\mathbf{2 4 1 - U - 1 0 9}$ & $\mathbf{2 4 1 - U - 1 1 0}$ & $\mathbf{2 4 1 - U - 1 1 1}$ & $\mathbf{2 4 1 - U - 1 1 2}$ & $\mathbf{2 4 1 - U - 2 0 1}$ \\
\hline Total kg liquid for tank & $1.76 \mathrm{E}+06$ & $9.86 \mathrm{E}+05$ & $6.43 \mathrm{E}+05$ & $1.38 \mathrm{E}+06$ & $1.16 \mathrm{E}+05$ & $1.95 \mathrm{E}+04$ \\
\hline Total kL liquid for tank & 1260 & 671 & 550 & 986 & 99 & 15.5 \\
\hline Liquid phase g/mL & 1.400 & 1.470 & 1.170 & 1.400 & 1.170 & 1.260 \\
\hline $\begin{array}{l}\text { OH (ug/mL) chosen from } \\
\text { data or calculated from } \\
\text { pH }\end{array}$ & 49200 & 44200 & 42.7 & 33900 & $\mathrm{n} / \mathrm{a}$ & 12600 \\
\hline $\begin{array}{l}\mathrm{pH} \text { chosen from data or } \\
\text { calculated from OH }\end{array}$ & 14.5 & 14.4 & 11.4 & 14.3 & $\mathrm{n} / \mathrm{a}$ & 13.9 \\
\hline
\end{tabular}

\begin{tabular}{|l|c|c|c|c|}
\hline & $\mathbf{2 4 1 - U - 2 0 2}$ & $\mathbf{2 4 1 - U - 2 0 3}$ & $\mathbf{2 4 1 - U - 2 0 4}$ & $\begin{array}{c}\text { All-tank } \\
\text { composite }\end{array}$ \\
\hline Total kg liquid for tank & $1.80 \mathrm{E}+04$ & $1.88 \mathrm{E}+04$ & $1.11 \mathrm{E}+04$ & $2.13 \mathrm{E}+08$ \\
\hline Total kL liquid for tank & 14.0 & 14.7 & 10.0 & 158330.4 \\
\hline Liquid phase g/mL & 1.280 & 1.280 & 1.110 & 1.344 \\
\hline $\begin{array}{l}\text { OH (ug/mL) chosen from } \\
\text { data or calculated from } \\
\mathrm{pH}\end{array}$ & 17900 & 4750 & 1610 & 26519 \\
\hline $\begin{array}{l}\mathrm{pH} \text { chosen from data or } \\
\text { calculated from OH }\end{array}$ & 14.0 & 13.4 & 13.0 & $\mathbf{n} / \mathbf{a}$ \\
\hline
\end{tabular}


Table A.2. As-Is Liquid Compositions of Waste Types ("---" = not present, "n/a" = insufficient data)

\begin{tabular}{|l|c|c|c|c|c|c|}
\hline & $\begin{array}{c}\text { Sludge } \\
\text { 1C }\end{array}$ & $\begin{array}{c}\text { Sludge } \\
\text { R1 (boiling) }\end{array}$ & $\begin{array}{c}\text { Sludge } \\
\text { 2C }\end{array}$ & $\begin{array}{c}\text { Sludge } \\
\text { TBP }\end{array}$ & $\begin{array}{c}\text { Sludge } \\
\text { CWP2 }\end{array}$ & $\begin{array}{c}\text { Sludge } \\
\mathbf{2 2 4} \text { Post-1949 }\end{array}$ \\
\hline Avg g/mL, liquid & 1.155 & 1.170 & 1.103 & 1.170 & 1.140 & 1.100 \\
\hline Avg ug/mL OH & 2.6 & $\mathrm{n} / \mathrm{a}$ & 1.0 & 170 & 62.5 & 1.4 \\
\hline Avg pH & 10.2 & $\mathrm{n} / \mathrm{a}$ & 9.8 & 12.0 & 11.6 & 9.9 \\
\hline
\end{tabular}

\begin{tabular}{|l|c|c|c|c|c|c|}
\hline & $\begin{array}{c}\text { Sludge } \\
\text { CWP1 }\end{array}$ & $\begin{array}{c}\text { Sludge } \\
\text { CWZr2 }\end{array}$ & $\begin{array}{c}\text { Sludge } \\
\text { CWR1 }\end{array}$ & $\begin{array}{c}\text { Sludge } \\
\text { DE }\end{array}$ & $\begin{array}{c}\text { Sludge } \\
\text { R1 (non-boiling) }\end{array}$ & $\begin{array}{c}\text { Sludge } \\
\text { PFeCN }\end{array}$ \\
\hline Avg g/mL, liquid & 1.230 & 1.320 & 1.453 & 1.390 & 1.475 & 1.447 \\
\hline Avg ug/mL OH & 0.85 & 10790 & 41628 & 2540 & 48700 & 38300 \\
\hline Avg pH & 9.7 & 13.8 & 14.4 & 13.2 & 14.5 & 14.4 \\
\hline
\end{tabular}

\begin{tabular}{|l|c|c|c|c|c|c|}
\hline & $\begin{array}{c}\text { Sludge } \\
\text { BL }\end{array}$ & $\begin{array}{c}\text { Sludge } \\
\text { TFeCN }\end{array}$ & $\begin{array}{c}\text { Sludge } \\
\text { P3 }\end{array}$ & $\begin{array}{c}\text { Sludge } \\
\text { 1CFeCN }\end{array}$ & $\begin{array}{c}\text { Sludge } \\
\text { CWR2 }\end{array}$ & $\begin{array}{c}\text { Sludge } \\
\text { SRR }\end{array}$ \\
\hline Avg g/mL, liquid & 1.150 & 1.369 & 1.190 & 1.260 & 1.409 & $\mathrm{n} / \mathrm{a}$ \\
\hline Avg ug/mL OH & 38500 & 22437 & 7212 & $\mathrm{n} / \mathrm{a}$ & 48991 & $\mathrm{n} / \mathrm{a}$ \\
\hline Avg $\mathrm{pH}$ & 14.4 & 14.1 & 13.6 & $\mathrm{n} / \mathrm{a}$ & 14.5 & $\mathrm{n} / \mathrm{a}$ \\
\hline
\end{tabular}

\begin{tabular}{|l|c|c|c|c|c|c|}
\hline & $\begin{array}{c}\text { Sludge } \\
\text { AR }\end{array}$ & $\begin{array}{c}\text { Sludge } \\
\text { P2 }\end{array}$ & $\begin{array}{c}\text { Sludge } \\
\text { MW }\end{array}$ & $\begin{array}{c}\text { Sludge } \\
\text { PL2 }\end{array}$ & $\begin{array}{c}\text { Sludge } \\
\text { 224 Pre-1949 }\end{array}$ & $\begin{array}{c}\text { Sludge } \\
\text { CWZr1 }\end{array}$ \\
\hline Avg g/mL, liquid & 1.020 & 1.170 & 1.280 & 1.400 & 1.170 & $\mathrm{n} / \mathrm{a}$ \\
\hline Avg ug/mL OH & 7020 & 7245 & 1701 & 25600 & n/a & n/a \\
\hline Avg pH & 13.6 & 13.6 & 13.0 & 14.2 & n/a & n/a \\
\hline
\end{tabular}


Table A.2. (contd)

\begin{tabular}{|l|c|c|c|c|c|c|}
\hline & $\begin{array}{c}\text { Sludge } \\
\text { Z }\end{array}$ & $\begin{array}{c}\text { Sludge } \\
\text { OWW3 }\end{array}$ & $\begin{array}{c}\text { Sludge } \\
\text { TH1 }\end{array}$ & $\begin{array}{c}\text { Sludge } \\
\text { HS }\end{array}$ & $\begin{array}{c}\text { Sludge } \\
\text { Portland Cement }\end{array}$ & $\begin{array}{c}\text { Sludge } \\
\text { B }\end{array}$ \\
\hline Avg g/mL, liquid & $\mathrm{n} / \mathrm{a}$ & $\mathrm{n} / \mathrm{a}$ & $\mathrm{n} / \mathrm{a}$ & 1.000 & $\mathrm{n} / \mathrm{a}$ & $\mathrm{n} / \mathrm{a}$ \\
\hline Avg $\mathrm{ug} / \mathrm{mL} \mathrm{OH}$ & $\mathrm{n} / \mathrm{a}$ & $\mathrm{n} / \mathrm{a}$ & $\mathrm{n} / \mathrm{a}$ & 0.101 & $\mathrm{n} / \mathrm{a}$ & $\mathrm{n} / \mathrm{a}$ \\
\hline Avg $\mathrm{pH}$ & $\mathrm{n} / \mathrm{a}$ & $\mathrm{n} / \mathrm{a}$ & $\mathrm{n} / \mathrm{a}$ & 8.8 & $\mathrm{n} / \mathrm{a}$ & $\mathrm{n} / \mathrm{a}$ \\
\hline
\end{tabular}

\begin{tabular}{|l|c|c|c|c|c|c|}
\hline & $\begin{array}{c}\text { Sludge } \\
\text { P1 }\end{array}$ & $\begin{array}{c}\text { Salt } \\
\text { S1 }\end{array}$ & $\begin{array}{c}\text { Salt } \\
\text { T2 }\end{array}$ & $\begin{array}{c}\text { Salt } \\
\text { BY }\end{array}$ & $\begin{array}{c}\text { Salt } \\
\text { A2 }\end{array}$ & $\begin{array}{c}\text { Salt } \\
\text { S2 }\end{array}$ \\
\hline Avg g/mL, liquid & n/a & 1.417 & 1.450 & 1.451 & 1.441 & 1.490 \\
\hline Avg ug/mL OH & n/a & 53952 & n/a & 38300 & 73446 & 37400 \\
\hline Avg $\mathrm{pH}$ & $\mathrm{n} / \mathrm{a}$ & 14.5 & n/a & 14.4 & 14.6 & 14.3 \\
\hline
\end{tabular}

\begin{tabular}{|l|c|c|c|c|c|}
\hline & $\begin{array}{c}\text { Salt } \\
\text { A1 }\end{array}$ & $\begin{array}{c}\text { Salt } \\
\text { R }\end{array}$ & $\begin{array}{c}\text { Salt } \\
\text { T1 }\end{array}$ & $\begin{array}{c}\text { Salt } \\
\text { B }\end{array}$ & $\begin{array}{c}\text { Salt } \\
\text { R1 }\end{array}$ \\
\hline Avg g/mL, liquid & 1.507 & 1.390 & 1.260 & 1.380 & 1.470 \\
\hline Avg $\mathrm{ug} / \mathrm{mL} \mathrm{OH}$ & 41157 & $\mathrm{n} / \mathrm{a}$ & 170 & $\mathrm{n} / \mathrm{a}$ & 47600 \\
\hline Avg $\mathrm{pH}$ & 14.4 & $\mathrm{n} / \mathrm{a}$ & 12.0 & $\mathrm{n} / \mathrm{a}$ & 14.4 \\
\hline
\end{tabular}



Appendix B

\section{Expert Elicitation, Solid Phase Identification}





\section{Appendix B: Expert Elicitation, Solid Phase Identification}

A series of meetings were held to consult a team of experts in solid-phase identification and solids-handling process requirements. Their input provided valuable guidance in defining solid phases. The team included:

$\begin{array}{ll}\text { S Barnes } & \text { WTP } \\ \text { EC Buck } & \text { PNNL } \\ \text { R Burk } & \text { WTP } \\ \text { WS Callaway III } & \text { WRPS 222-S Laboratory } \\ \text { GA Cooke } & \text { WRPS 222-S Laboratory } \\ \text { RC Daniel } & \text { PNNL Radiochem. Proc. Laboratory } \\ \text { DA Greer } & \text { WRPS } \\ \text { DL Herting } & \text { WTP and WRPS 222-S Laboratory } \\ \text { JL Huckaby } & \text { WTP and PNNL } \\ \text { DE Kurath } & \text { PNNL } \\ \text { GJ Lumetta } & \text { PNNL Radiochem. Proc. Laboratory } \\ \text { LA Mahoney } & \text { PNNL } \\ \text { Y Onishi } & \text { PNNL } \\ \text { RA Peterson } & \text { PNNL } \\ \text { BM Rapko } & \text { PNNL Radiochem. Proc. Laboratory } \\ \text { JH Rasmussen } & \text { WRPS } \\ \text { JG Reynolds } & \text { WRPS } \\ \text { JM Tingey } & \text { PNNL Radiochem. Proc. Laboratory } \\ \text { BE Wells } & \text { PNNL }\end{array}$





\section{Appendix C}

Density and Composition of Existing and Fully-Washed Solid Phases by Tank, Waste Type, and Composite 



\section{Appendix C: Density and Composition of Existing and Fully-Washed Solid Phases by Tank, Waste Type, and Composite}

Table C. 1 through Table C. 4 contain the UDS composition and density information that was generated by the methods described in Section 3.2.3.2 (in-tank solids) and Section 3.2.3.3 (fully-washed solids).

Table C.1 provides information for each tank and for the all-tank composite UDS, which is the mass-weighted average over all tank UDS inventories. The UDS mass and composition in each tank were determined by thermodynamic modeling that used 2002 Best Basis Inventory (BBI) data and by adjustments to modeling results, as described in Section 3.2.3.2. The UDS densities that are shown in the table were applied to obtain solid phase volumes. Some low-concentration constituents, which are noted in the table, are considered to co-precipitate with more abundant phases and therefore are assigned the density of the more dominant phase.

At the end of Table C.1 are "saltcake" and "sludge" UDS compositions, which were calculated for composites of all tanks whose bulk solid waste was more than 70 vol\% saltcake or 70 vol\% sludge in 2002. PSD-composite compositions for several categories of particle size distribution (PSD) measurement (as described in Section 3.2.5.2) are also included: sludge under flow conditions (whether with or without sonication), sludge under No-Flow Unsonicated conditions, saltcake under Flow Sonicated conditions, saltcake under Flow Unsonicated conditions, and saltcake under No-Flow Unsonicated conditions. These PSD-composite compositions were calculated as volume-weighted averages of the UDS compositions in all the tanks whose measured PSDs were included in the PSD category (listed in Table 3.2.5.2). The tank weighting factors used in calculating the compositions were the same as those used in calculating the composite PSDs for the PSD categories.

The composite PSDs included PSD data from samples from single tanks and from the multi-tank composites used in M12 Group waste characterization. Samples from single tanks were assumed to come from whole-core composites; therefore, they were considered to represent the whole UDS volume of the tank. Samples used in M12 Group composites were selected to represent only the particular waste types of interest in the tanks from which samples were chosen. Therefore, the Group-related samples from a given tank were considered to represent only a fraction of the UDS volume in the tank, with the fraction being equal to the bulk solids volume of the waste type (or types) in the tank divided by the total bulk volume of solids in the tank. The PSD-composite weighting factor for each tank was calculated as the sum of the UDS volumes in the tank that were represented by single-tank samples and by Group composite samples, divided by the sum of UDS volumes in single-tank samples and Group samples over all the tanks and Groups whose PSD data were used in the composite PSD.

Table C. 2 gives the compositions and densities for the saltcake and sludge waste types that were defined in the 2002 BBI. These were obtained by volume-averaging the data for the tanks identified in Section 3.2.3.4 as representatives of each waste type. 
Table C.3 and Table C.4 provide much the same kind of information as Table C.1 and Table C.2, but are based on the calculated masses and compositions of fully-washed solids as defined in Section 3.2.3.3. No PSD-composite compositions are provided for fully-washed solids. Note that this "fully-washed" composition is not the same as that which results from applying the BBI wash factors, which is also referred to as "fully washed" in other contexts. 
Table C.1. As-Is Solid-Phase Compositions of Individual Tanks Based on 2002 BBI ("---" = not present)

\begin{tabular}{|c|c|c|c|c|c|c|c|c|c|c|}
\hline & $\begin{array}{l}\text { Density } \\
(\mathrm{g} / \mathrm{mL})\end{array}$ & $\begin{array}{c}\text { Mol. wt. } \\
\text { (g/mol) }\end{array}$ & $241-\mathrm{A}-101$ & $241-A-102$ & 241-A-103 & 241-A-104 & 241-A-105 & 241-A-106 & $241-\mathrm{AN}-101$ & 241-AN-102 \\
\hline Total kg solids for tank & \multirow{9}{*}{$\mathrm{n} / \mathrm{a}$} & \multirow{9}{*}{$n / a$} & $1.07 \mathrm{E}+06$ & $3.96 \mathrm{E}+04$ & $5.12 \mathrm{E}+05$ & $7.96 \mathrm{E}+04$ & $1.09 \mathrm{E}+05$ & $2.51 \mathrm{E}+05$ & $2.28 \mathrm{E}+04$ & $1.03 \mathrm{E}+06$ \\
\hline Total kL solids for tank & & & $4.60 \mathrm{E}+02$ & $1.29 \mathrm{E}+01$ & $2.18 \mathrm{E}+02$ & $2.21 \mathrm{E}+01$ & $3.86 \mathrm{E}+01$ & $9.83 \mathrm{E}+01$ & $9.40 \mathrm{E}+00$ & $4.40 \mathrm{E}+02$ \\
\hline Vol. frac. solid phase in tank & & & 0.136 & 0.088 & 0.155 & 0.209 & 0.278 & 0.329 & 0.010 & 0.107 \\
\hline Avg $\mathrm{g} / \mathrm{mL}$, all solid & & & 2.328 & 3.070 & 2.351 & 3.597 & 2.818 & 2.555 & 2.430 & 2.341 \\
\hline Total kg non-salt solids for tank & & & $5.93 \mathrm{E}+04$ & $3.46 \mathrm{E}+04$ & 1. $16 \mathrm{E}+05$ & $7.96 \mathrm{E}+04$ & $6.61 \mathrm{E}+04$ & $1.67 \mathrm{E}+05$ & $2.28 \mathrm{E}+04$ & $1.83 \mathrm{E}+05$ \\
\hline Total kL non-salt solids for tank & & & $1.98 \mathrm{E}+01$ & $1.09 \mathrm{E}+0 \mathrm{I}$ & $4.59 \mathrm{E}+01$ & $2.2 \mathrm{IE}+0 \mathrm{I}$ & $1.99 \mathrm{E}+01$ & $6.02 \mathrm{E}+01$ & $9.39 \mathrm{E}+00$ & $7.24 \mathrm{E}+01$ \\
\hline Wt. frac. non-salts in solid & & & 0.055 & 0.874 & 0.226 & 1.000 & 0.607 & 0.665 & 1.000 & 0.177 \\
\hline Avg $g / m L$, non-salt solid & & & 2.988 & 3.184 & 2.527 & 3.597 & 3.319 & 2.771 & 2.430 & 2.519 \\
\hline Vol. frac. non-salts in solid & & & 0.043 & 0.843 & 0.211 & 1.000 & 0.515 & 0.613 & 1.000 & 0.165 \\
\hline \multicolumn{11}{|l|}{ non-salts: mass fracs in total solid } \\
\hline$\overline{\mathrm{Ag}}$ & 10.5 & 107.87 & $-\cdots$ & --- & --- & --- & -- & --- & $-\cdots$ & -- \\
\hline $\mathrm{Ag}_{2} \mathrm{O}$ & 7.143 & 231.74 & -- & $0.02 \%$ & -- & --- & -- & --- & $0.03 \%$ & -- \\
\hline $\mathrm{Bi}_{2} \mathrm{O}_{3}$ & 8.9 & 465.96 & $0.03 \%$ & $0.15 \%$ & $0.0 .3 \%$ & --- & --- & $0.07 \%$ & $0.12 \%$ & $0.03 \%$ \\
\hline $\mathrm{BiFeO}_{3}$ & 7.9 & 312.83 & -.- & -.. & -.. &.- & $\ldots$ & -.- & $\ldots$ & $\ldots$ \\
\hline $\mathrm{Ca}(\mathrm{OH})_{2}$ & 2.24 & 74.09 & $\cdots$ & $\cdots$ & $\cdots$ & $3.64 \%$ & $\cdots$ & $\cdots$ & $\cdots$ & $\cdots$ \\
\hline $\mathrm{Ca}_{5} \mathrm{OH}\left(\mathrm{PO}_{4}\right)_{3}$ & 3.14 & 502.31 & $0.27 \%$ & $0.71 \%$ & $1.45 \%$ & $0.98 \%$ & $4.28 \%$ & $4.67 \%$ & $0.15 \%$ & $0.83 \%$ \\
\hline $\mathrm{CaC}_{2} \mathrm{O}_{4} \cdot \mathrm{H}_{2} \mathrm{O}$ & 2.2 & 146.11 & -- & -- & -- & -- & --- & -- & --- & -- \\
\hline $\mathrm{CaCO}_{3}$ & 2.71 & 100.09 & --- & -- & -- & -- & -- & -- & -- & -- \\
\hline $\mathrm{CaF}_{2}$ & 3.18 & 78.07 & --- & -- & --- & --- & --- & --- & --- & --- \\
\hline $\mathrm{CrOOH}$ & 4.11 & 85 & $1.35 \%$ & $5.91 \%$ & $0.74 \%$ & -- & $\cdots$ & $1.46 \%$ & $\cdots$ & $0.18 \%$ \\
\hline $\mathrm{FePO}_{4} \cdot 2 \mathrm{H}_{2} \mathrm{O}$ & 3.15 & 186.852 & --- & --- & --- & --- & -- & --- & -- & -- \\
\hline $\mathrm{FeOOH}$ & 4.26 & 88.85 & $0.25 \%$ & $12.80 \%$ & $0.52 \%$ & $63.70 \%$ & $26.76 \%$ & $14.85 \%$ & $0.05 \%$ & $0.23 \%$ \\
\hline Gibbsite & 2.42 & 78 & $1.79 \%$ & $37.23 \%$ & $0.83 \%$ & $3.43 \%$ & --- & --- & $99.21 \%$ & $15.81 \%$ \\
\hline Boehmite & 3.01 & 59.99 & --- & --- & --- & --- & -- & -- & --- & --- \\
\hline $\mathrm{HgO}$ (co-precip. with $\mathrm{Ag}_{2} \mathrm{O}$ ) & 7.143 & 216.59 & -- & -- & -- & $0.06 \%$ & $0.05 \%$ & $0.02 \%$ & --- & --- \\
\hline $\mathrm{KAlSiO}_{4}$ & 2.61 & 158.16 & $--\cdot$ & $-\cdots$ & -- & $\cdots$ & $--\cdot$ & --- & -- & -- \\
\hline $\mathrm{La}(\mathrm{OH})_{3}$ & 2.3 & 189.93 & -- & $-\cdots$ & -- & --- & --- & 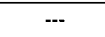 & -- & --- \\
\hline $\mathrm{LaPO}_{4} \cdot 2 \mathrm{H}_{2} \mathrm{O}$ & 6.51 & 269.91 & $0.03 \%$ & $0.08 \%$ & $0.04 \%$ & $0.03 \%$ & $0.62 \%$ & $0.06 \%$ & $0.10 \%$ & $0.01 \%$ \\
\hline $\mathrm{Mn}_{3}\left(\mathrm{PO}_{4}\right)_{2}$ & 3.102 & 354.76 & -- & -- & --- & -- & -- & $1.08 \%$ & -- & -- \\
\hline $\mathrm{MnO}_{2}$ & 5.026 & 86.94 & $--\cdot$ & $2.17 \%$ &.- & $8.85 \%$ & $0.70 \%$ & -.- & -- & $0.01 \%$ \\
\hline $\mathrm{Na}_{2}\left(\mathrm{UO}_{2}\right)_{2}\left(\mathrm{PO}_{4}\right)_{2} \cdot 2 \mathrm{H}_{2} \mathrm{O}$ & 3.5 & 811.952 & --- & --- & -- & --- & --- & --- & --- & --- \\
\hline $\mathrm{Na}_{2} \mathrm{U}_{2} \mathrm{O}_{7}$ & 5.617 & 634.03 & $0.38 \%$ & $19.27 \%$ & $0.62 \%$ & $0.11 \%$ & $0.00 \%$ & $0.38 \%$ & $0.17 \%$ & $0.14 \%$ \\
\hline $\mathrm{NaAICO}_{3}(\mathrm{OH})_{2}$ & 2.42 & 144 & --- & --- & --- & -- & --- & -- & --- & -- \\
\hline $\mathrm{NaAlSiO}_{4}$ & 2.365 & 142.05 & $1.25 \%$ & $7.77 \%$ & $18.20 \%$ & $16.93 \%$ & $21.38 \%$ & $42.49 \%$ & -- & -- \\
\hline $\mathrm{Ni}(\mathrm{OH})_{2}$ & 4.15 & 92.71 & $0.07 \%$ & $0.27 \%$ & $0.08 \%$ & $1.93 \%$ & $3.21 \%$ & $0.35 \%$ & $0.01 \%$ & $0.32 \%$ \\
\hline $\mathrm{Ni}_{3}\left(\mathrm{PO}_{4}\right)$ & 3.93 & 366.02 & --- & -- & -- & -- & --- & --- & -- & -- \\
\hline $\mathrm{NiC}_{2} \mathrm{O}_{4} \cdot 2 \mathrm{H}_{2} \mathrm{O}$ (on Fe phase) & 4.26 & 182.74 & -- & --- & -- & --- & -- & -- & -- & --- \\
\hline $\mathrm{Pb}(\mathrm{OH})_{2}$ & 7.1 & 241.21 & $0.08 \%$ & $0.72 \%$ & $0.05 \%$ & $0.11 \%$ & $2.36 \%$ & $0.43 \%$ & $0.13 \%$ & $0.10 \%$ \\
\hline $\mathrm{Pb}_{3}\left(\mathrm{PO}_{4}\right)_{2}$ & 7.1 & 811.54 & --- & -- & -- & --- & --- & --- & --- & --- \\
\hline $\mathrm{PbCO}_{3}$ & 6.6 & 267.21 & --- & --- & --- & --- & --- & --- & --- & --- \\
\hline $\mathrm{Pu}(\mathrm{OH})_{4}$ (co-precip. on Fe phase) & 4.26 & 307.03 & $0.00 \%$ & $0.02 \%$ & $0.00 \%$ & $0.02 \%$ & $0.01 \%$ & $0.01 \%$ & $0.00 \%$ & $0.00 \%$ \\
\hline $\mathrm{PuO}_{2}$ & 11.43 & 271 & --- & --- & --- & -- & --- & --- & --- & -- \\
\hline $\mathrm{SiO}_{2}$ & 2.6 & 60.08 & --- & --- & --- & -- & -- & --- & -- & --- \\
\hline $\mathrm{Sr}_{3}\left(\mathrm{PO}_{4}\right)_{2}$ & 3.5 & 452.8 & -- & -- & -- & -- & $0.12 \%$ & $0.03 \%$ & -- & -- \\
\hline $\mathrm{SrCO}_{3}$ & 3.5 & 147.63 & $0.01 \%$ & $0.02 \%$ & $0.01 \%$ & $0.16 \%$ & --- & -- & $0.02 \%$ & $0.01 \%$ \\
\hline $\mathrm{ZrO}_{2}$ & 5.68 & 123.22 & $0.02 \%$ & $0.27 \%$ & $0.08 \%$ & $0.05 \%$ & $1.16 \%$ & $0.58 \%$ & -- & $0.04 \%$ \\
\hline \multicolumn{11}{|l|}{ salts: mass fracs in total solid } \\
\hline $\mathrm{KNO}_{3}$ & 2.109 & 101.1 & $-\cdots$ & 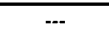 & $-\cdots$ & $-\cdots$ & $\cdots$ & $-\cdots$ & $\cdots$ & $-\cdots$ \\
\hline $\mathrm{Na}_{2} \mathrm{C}_{2} \mathrm{O}_{4}$ & 2.34 & 134 & $6.23 \%$ & $7.28 \%$ & $7.23 \%$ & -- & -- & $4.39 \%$ & -- & $7,90 \%$ \\
\hline $\mathrm{Na}_{2} \mathrm{CO}_{3} \cdot \mathrm{H}_{2} \mathrm{O}$ & 2.25 & 124 & $36.66 \%$ & $0.01 \%$ & $41.21 \%$ & -- & $22.68 \%$ & $5.29 \%$ & $0.01 \%$ & $20.33 \%$ \\
\hline $\mathrm{Na}_{2} \mathrm{SO}_{4}$ & 2.68 & 142.04 & -- & -- & -- & -- & -- & -- & -- & -- \\
\hline $\mathrm{Na}_{2} \mathrm{SO}_{4} \cdot 10 \mathrm{H}_{2} \mathrm{O}$ & 1.464 & 322.2 & --- & --- & +-- & --- & --- & --- & --- & --- \\
\hline $\mathrm{Na}_{3} \mathrm{FSO}_{4}$ & 2.65 & 184.03 & --- & $1.67 \%$ & $3.47 \%$ & --- & --- & --- & --- & $6.28 \%$ \\
\hline $\mathrm{Na}_{3} \mathrm{NO}_{3} \mathrm{SO}_{4} \cdot \mathrm{H}_{2} \mathrm{O}$ & 2.3 & 245.05 & --- & --- & --- & --- & -- & --- & --- & -- \\
\hline $\mathrm{Na}_{3} \mathrm{PO}_{4} \cdot 0.25 \mathrm{NaOH} \cdot 12 \mathrm{H}_{2} \mathrm{O}$ & 1.62 & 390.13 & -- & --- & --- & --- & -- & --- & --- & --- \\
\hline $\mathrm{Na}_{3} \mathrm{PO}_{4} \cdot 8 \mathrm{H}_{2} \mathrm{O}$ & 1.8 & 308.06 & --- & -- & +- & --- & $3.82 \%$ & --- & --- & --- \\
\hline $\mathrm{Na}_{4} \mathrm{P}_{2} \mathrm{O}_{7} \cdot 10 \mathrm{H}_{2} \mathrm{O}$ & 1.83 & 446.06 & --- & -- & --- & --- & --- & -- & --- & --- \\
\hline $\mathrm{Na}_{6}\left(\mathrm{SO}_{4}\right)_{2} \mathrm{CO}_{3}$ & 2,64 & 390,08 & $10.46 \%$ & $3.61 \%$ & $9.13 \%$ & -- & $12.10 \%$ & $0.90 \%$ & -- & $3.59 \%$ \\
\hline $\mathrm{NaF}$ & 2.78 & 41.99 & --- & --- & --- & --- & --- & --- & -- & --- \\
\hline $\mathrm{Na}_{7} \mathrm{~F}\left(\mathrm{PO}_{4}\right)_{2} \cdot 19 \mathrm{H}_{2} \mathrm{O}$ & 1.75 & 712.16 & --- & --- & $1.35 \%$ & --- & $0.74 \%$ & $2.21 \%$ & --- & --- \\
\hline $\mathrm{NaIICO}_{3}$ & 2.159 & 84.01 & -.- & -- & -.- & -- & -.- & ..- &.- & -- \\
\hline $\mathrm{NaNO}_{2}$ & 2.168 & 69 & --. & -- & -.. &.- & -.. & $7.73 \%$ & -.. & -. \\
\hline $\mathrm{NaNO}_{3}$ & 2.26 & 84.99 & $41.11 \%$ & -- & $14.95 \%$ & -- & -- & $13.02 \%$ & -- & $44.19 \%$ \\
\hline
\end{tabular}


Table C.1. (contd)

\begin{tabular}{|c|c|c|c|c|c|c|c|c|c|}
\hline & $\begin{array}{l}\text { Density } \\
(\mathrm{g} / \mathrm{mL})\end{array}$ & 241-AN-103 & 241-AN-104 & $241-\mathrm{AN}-105$ & $241-\mathrm{AN}-106$ & $241-\mathrm{AN}-107$ & 241-AP-101 & 241-AP-102 & 241-AP-103 \\
\hline Total kg solids for tank & \multirow{9}{*}{ 11/a } & $1.33 \mathrm{E}+06$ & $9.88 \mathrm{E}+05$ & $7.79 \mathrm{E}+05$ & $1.86 \mathrm{E}+04$ & $3.3 ! E+05$ & $1.30 \mathrm{E}+04$ & $2.03 \mathrm{E}+04$ & $2.74 \mathrm{E}+04$ \\
\hline Total kL solids for tank & & $5.95 \mathrm{E}+02$ & $4.34 \mathrm{E}+02$ & $3.43 \mathrm{E}+02$ & $8.60 \mathrm{E}+00$ & $1.26 \mathrm{E}+02$ & $5.05 \mathrm{E}+00$ & $9.91 \mathrm{E}+00$ & $1.21 \mathrm{E}+01$ \\
\hline Vol. frac. solid phase in tank & & 0.174 & 0.114 & 0.082 & 0.060 & 0.032 & 0.001 & 0.024 & 0.011 \\
\hline Avg g/mL, all solid & & 2.242 & 2.275 & 2.273 & 2.166 & 2.631 & 2.580 & 2.044 & 2.272 \\
\hline Total kg non-salt solids for tank & & $3.52 \mathrm{E}+04$ & $1.84 \mathrm{E}+04$ & $7.46 \mathrm{E}+04$ & $9.10 \mathrm{E}+03$ & $2.31 \mathrm{E}+05$ & $8.80 \mathrm{E}+03$ & $1.26 \mathrm{E}+04$ & $1.91 \mathrm{E}+04$ \\
\hline Total kL non-salt solids for tank & & $1.33 \mathrm{E}+01$ & $5.79 \mathrm{E}+00$ & $2.82 \mathrm{E}+0 \mathrm{I}$ & $3.56 \mathrm{E}+00$ & $8.81 \mathrm{E}+0 \mathrm{l}$ & $3.53 \mathrm{E}+00$ & $5.20 \mathrm{E}+00$ & $7.78 \mathrm{E}+00$ \\
\hline Wt. frac. non-salts in solid & & 0.026 & 0.019 & 0.096 & 0.488 & 0.697 & 0.676 & 0.624 & 0.697 \\
\hline Avg g/mL, non-salt solid & & 2.647 & 3.184 & 2.649 & 2.557 & 2.619 & 2.494 & 2.428 & 2.458 \\
\hline Vol. frac. non-salts in solid & & 0.022 & 0.013 & 0.082 & 0.414 & 0.700 & 0.699 & 0.525 & 0.644 \\
\hline \multicolumn{10}{|l|}{ non-salts: mass fracs in total solid } \\
\hline$\overline{A g}$ & 10.5 & -- & --- & --- & --- & --- & --- & --- & -- \\
\hline $\mathrm{Ag}_{2} \mathrm{O}$ & 7.143 & --- & --- & --- & -- & $0.00 \%$ & --- & $0.00 \%$ & $0.05 \%$ \\
\hline $\mathrm{Bi}_{2} \mathrm{O}_{3}$ & 8.9 & $0.01 \%$ & $0.01 \%$ & $0.02 \%$ & $0.12 \%$ & $0.01 \%$ & --- & $0.03 \%$ & $0.25 \%$ \\
\hline $\mathrm{BiFeO}_{3}$ & 7.9 & $\cdots$ & $\ldots$ & -- & $\cdots$ & $--\cdot$ & $-\cdots$ & $\cdots$ & $\cdots$ \\
\hline $\mathrm{Ca}(\mathrm{OH})_{2}$ & 2.24 & $\cdots$ & $\cdots$ & $\cdots$ & $\cdots$ & $\cdots$ & $\cdots$ & $\cdots$ & $\cdots$ \\
\hline $\mathrm{Ca}_{5} \mathrm{OH}\left(\mathrm{PO}_{4}\right)_{3}$ & 3.14 & $0.10 \%$ & $0.19 \%$ & $0.61 \%$ & --- & -- & $--\cdot$ & $0.32 \%$ & $0.60 \%$ \\
\hline $\mathrm{CaC}_{2} \mathrm{O}_{4} \cdot \mathrm{H}_{2} \mathrm{O}$ & 2.2 & -- & --- & --- & --- & --- & --- & -- & -- \\
\hline $\mathrm{CaCO}_{3}$ & 2.71 & -- & --- & --- & --- & --- & --- & --- & -- \\
\hline $\mathrm{CaF}_{2}$ & 3.18 & -- & --- & --- & $0.49 \%$ & $1.64 \%$ & $0.47 \%$ & --- & -- \\
\hline $\mathrm{CrOOH}$ & 4.11 & $0.23 \%$ & $0.77 \%$ & $1.39 \%$ & $4,04 \%$ & $0.38 \%$ & $\ldots$ & $\cdots$ & $\cdots$ \\
\hline $\mathrm{FePO}_{4} \cdot 2 \mathrm{H}_{2} \mathrm{O}$ & 3.15 & -- & --- & -- & --- & --- & --- & -- & -- \\
\hline $\mathrm{FeOOH}$ & 4.26 & $0.03 \%$ & $0.06 \%$ & $0.08 \%$ & $0.30 \%$ & $6.75 \%$ & $0.13 \%$ & $0.02 \%$ & $0.19 \%$ \\
\hline Gibbsite & 2.42 & $1.48 \%$ & --- & $7.35 \%$ & $42.47 \%$ & -- & $63.78 \%$ & $61.79 \%$ & $67.29 \%$ \\
\hline Boehmite & 3.01 & --- & --- & --- & --- & --- & --- & --- & -- \\
\hline $\mathrm{HgO}$ (co-precip. with $\mathrm{Ag}_{2} \mathrm{O}$ ) & 7.143 & --- & --- & --- & --- & --- & --- & --- & --- \\
\hline $\mathrm{KAlSiO}_{4}$ & 2.61 & $0.72 \%$ & --- & --- & $0.28 \%$ & --- & --- & --- & $\ldots$ \\
\hline $\mathrm{La}(\mathrm{OH})_{3}$ & 2.3 & $0.01 \%$ & $0.02 \%$ & --- & --- & $\begin{array}{ll}-- \\
\end{array}$ & --- & --- & -- \\
\hline $\mathrm{LaPO}_{4} \cdot 2 \mathrm{H}_{2} \mathrm{O}$ & 6.51 & --- & --- & $0.01 \%$ & $0.07 \%$ & $0.10 \%$ & $0.08 \%$ & $0.01 \%$ & $0.01 \%$ \\
\hline $\mathrm{Mn}_{3}\left(\mathrm{PO}_{4}\right)_{2}$ & 3.102 & -- & --- & --- & -- & -- & --- & --- & -- \\
\hline $\mathrm{MnO}_{2}$ & 5.026 &.-- & --- & --- & $0.04 \%$ & $1.38 \%$ & $\ldots$ & $0.05 \%$ & $\ldots$ \\
\hline $\mathrm{Na}_{2}\left(\mathrm{UO}_{2}\right)_{2}\left(\mathrm{PO}_{4}\right)_{2} \cdot 2 \mathrm{H}_{2} \mathrm{O}$ & 3.5 & --- & $\begin{array}{l}-- \\
\end{array}$ & --- & --- & $\begin{array}{ll}-- \\
\end{array}$ & --- & +-- & --- \\
\hline $\mathrm{Na}_{2} \mathrm{U}_{2} \mathrm{O}_{7}$ & 5.617 & $0.01 \%$ & $0.07 \%$ & $0.04 \%$ & $0.63 \%$ & --- & $2.35 \%$ & $0.02 \%$ & $0.18 \%$ \\
\hline $\mathrm{NaAICO}_{3}(\mathrm{OH})_{2}$ & 2.42 & -- & --- & -- & --- & $57.37 \%$ & --- & --- & -- \\
\hline $\mathrm{NaAlSiO}_{4}$ & 2.365 & -- & $0.69 \%$ & $0.00 \%$ & --- & --- & --- & --- & -- \\
\hline $\mathrm{Ni}(\mathrm{OH})_{2}$ & 4.15 & $0.00 \%$ & $0.03 \%$ & $0.02 \%$ & $0.23 \%$ & $1.16 \%$ & $0.23 \%$ & $0.06 \%$ & $0.82 \%$ \\
\hline $\mathrm{Ni}_{3}\left(\mathrm{PO}_{4}\right)$ & 3.93 & -- & -- & -- & -- &.- & $\cdots$ & $\cdots$ & $\cdots$ \\
\hline $\mathrm{NiC}_{2} \mathrm{O}_{4} \cdot 2 \mathrm{H}_{2} \mathrm{O}$ (on Fe phase) & 4.26 & -- & --- & --- & --- & --- & --- & --- & -- \\
\hline $\mathrm{Pb}(\mathrm{OH})_{2}$ & 7.1 & $0.04 \%$ & $0.03 \%$ & $0.04 \%$ & $0.07 \%$ & $0.67 \%$ & $0.51 \%$ & $0.04 \%$ & $0.28 \%$ \\
\hline $\mathrm{Pb}_{3}\left(\mathrm{PO}_{4}\right)_{2}$ & 7.1 & --- & --- & --- & --- & --- & --- & --- & --- \\
\hline $\mathrm{PbCO}_{3}$ & 6.6 & -- & --- & -- & --- & --- & --- & --- & -- \\
\hline $\mathrm{Pu}(\mathrm{OH})_{4}$ (co-precip. on Fe phase) & 4.26 & $0.00 \%$ & $0.00 \%$ & $0.00 \%$ & $0.01 \%$ & $0.00 \%$ & $0.00 \%$ & $0.00 \%$ & $0.00 \%$ \\
\hline $\mathrm{PuO}_{2}$ & 11.43 & -- & --- & -- & -- & --- & --- & --- & -- \\
\hline $\mathrm{SiO}_{2}$ & 2.6 & $\begin{array}{l}-- \\
\end{array}$ & --- & --- & $\begin{array}{l}-- \\
\end{array}$ & --- & --- & --- & --- \\
\hline $\mathrm{Sr}_{3}\left(\mathrm{PO}_{4}\right)_{2}$ & 3.5 & --- & --- & --- & --- & --- & --- & --- & --- \\
\hline $\mathrm{SrCO}_{3}$ & 3.5 & $0.00 \%$ & $0.00 \%$ & $0.00 \%$ & $0.02 \%$ & $0.01 \%$ & $0.01 \%$ & --- & $0.04 \%$ \\
\hline $\mathrm{ZrO}_{2}$ & 5.68 & -- & $0.00 \%$ & --- & $0.06 \%$ & $0.19 \%$ & -.- & -- & -- \\
\hline \multicolumn{10}{|l|}{ salts: mass fracs in total solid } \\
\hline $\mathrm{KNO}_{3}$ & 2.109 & $-\cdots$ & $\cdots$ & $-\cdots$ & $\begin{array}{ll}-\cdot \\
\end{array}$ & $-\cdots$ & $\cdots$ & $\cdots$ & $-\cdots$ \\
\hline $\mathrm{Na}_{2} \mathrm{C}_{2} \mathrm{O}_{4}$ & 2.34 & $1.73 \%$ & $3.96 \%$ & $4.05 \%$ & $22.74 \%$ & -- & --- & --- & -- \\
\hline $\mathrm{Na}_{2} \mathrm{CO}_{3} \cdot \mathrm{H}_{2} \mathrm{O}$ & 2.25 & $9.28 \%$ & $29.24 \%$ & $36.16 \%$ & $-\cdot$ & $1.97 \%$ & $\cdots$ & $0.00 \%$ & $0.02 \%$ \\
\hline $\mathrm{Na}_{2} \mathrm{SO}_{4}$ & 2.68 & -- & --- & --- & --- & --- & --- & --- & -- \\
\hline $\mathrm{Na}_{2} \mathrm{SO}_{4} \cdot 10 \mathrm{H}_{2} \mathrm{O}$ & 1.464 & -- & $\begin{array}{ll}-- \\
\end{array}$ & --- & --- & $\begin{array}{ll}-- \\
\end{array}$ & --- & --- & -- \\
\hline $\mathrm{Na}_{3} \mathrm{FSO}_{4}$ & 2.65 & $1.18 \%$ & $0.39 \%$ & $4.30 \%$ & --- & $18.78 \%$ & --- & --- & $8.51 \%$ \\
\hline $\mathrm{Na}_{3} \mathrm{NO}_{3} \mathrm{SO}_{4} \cdot \mathrm{H}_{2} \mathrm{O}$ & 2.3 & -- & -- & -- & -- & --- & --- & --- & -- \\
\hline $\mathrm{Na}_{3} \mathrm{PO}_{4} \bullet 0.25 \mathrm{NaOH} \bullet 12 \mathrm{H}_{2} \mathrm{O}$ & 1.62 & --- & --- & --- & $27.68 \%$ & --- & --- & $37.64 \%$ & --- \\
\hline $\mathrm{Na}_{3} \mathrm{PO}_{4} \bullet 8 \mathrm{H}_{2} \mathrm{O}$ & 1.8 & -- & --- & -- & -- & --- & --- & --- & -- \\
\hline $\mathrm{Na}_{4} \mathrm{P}_{2} \mathrm{O}_{7} \cdot 10 \mathrm{H}_{2} \mathrm{O}$ & 1.83 & --- & --- & --- & --- & --- & --- & --- & -- \\
\hline $\mathrm{Na}_{6}\left(\mathrm{SO}_{4}\right)_{2} \mathrm{CO}_{3}$ & 2.64 & --- & $6.82 \%$ & --- & -- & --. & --- & -- & --- \\
\hline $\mathrm{NaF}$ & 2.78 & -- & --- & --- & $0.76 \%$ & $9.59 \%$ & $32.44 \%$ & --- & -- \\
\hline $\mathrm{Na}_{7} \mathrm{~F}\left(\mathrm{PO}_{4}\right)_{2} \cdot 19 \mathrm{H}_{2} \mathrm{O}$ & 1.75 & $0.46 \%$ & $3.03 \%$ & $4.99 \%$ & --- & --- & --- & --- & $21.76 \%$ \\
\hline $\mathrm{Nal} \mathrm{ICO}{ }_{3}$ & 2.159 & -- & --. & -- & $--\cdot$ & --- & $-\cdots$ & $\cdots$ & -- \\
\hline $\mathrm{NaNO}_{2}$ & 2.168 & $28.94 \%$ & $0.78 \%$ & --- & -- & --- & --- & --- & -- \\
\hline $\mathrm{NaNO}_{3}$ & 2.26 & $55.77 \%$ & $53.91 \%$ & $40.92 \%$ & -- & -- & --- & --- & --- \\
\hline
\end{tabular}


Table C.1. (contd)

\begin{tabular}{|c|c|c|c|c|c|c|c|c|c|}
\hline & $\begin{array}{l}\text { Density } \\
(\mathrm{g} / \mathrm{mL})\end{array}$ & 241-AP-104 & 241-AP-105 & 241-AP-106 & 241-AP-107 & 241-AP-108 & 241-AW-101 & 241-AW-102 & $241-\mathrm{AW}-103$ \\
\hline Total kg solids for tank & \multirow{9}{*}{ 11/a } & $1.39 \mathrm{E}+05$ & $8.84 \mathrm{E}+05$ & $2.34 \mathrm{E}+05$ & $6.75 \mathrm{E}+03$ & $1.13 \mathrm{E}+05$ & $9.85 \mathrm{E}+05$ & $2.40 \mathrm{E}+05$ & $6.49 \mathrm{E}+05$ \\
\hline Total kL solids for tank & & $6.03 \mathbf{E}+01$ & $3.80 \mathrm{E}+02$ & $1.20 \mathrm{E}+02$ & $3.11 \mathrm{E}+00$ & $4.66 \mathrm{E}+01$ & $4.39 \mathrm{E}+02$ & $1.06 \mathrm{E}+02$ & $1.92 \mathrm{E}+02$ \\
\hline Vol. frac. solid phase in tank & & 0.014 & 0.088 & 0.028 & 0.020 & 0.016 & 0.105 & 0.027 & 0.046 \\
\hline Avg g/mL, all solid & & 2.303 & 2.327 & 1.951 & 2.171 & 2.426 & 2.242 & 2.276 & 3.387 \\
\hline Total kg non-salt solids for tank & & 1.24E+05 & $2.75 \mathrm{E}+05$ & $1.19 \mathrm{E}+05$ & $5.15 \mathrm{E}+03$ & $1.13 \mathrm{E}+05$ & $1.39 \mathrm{E}+04$ & $1.94 \mathrm{E}+05$ & $3.76 \mathrm{E}+05$ \\
\hline Total kL non-salt solids for tank & & $5.09 \mathrm{E}+01$ & 1. $13 \mathrm{E}+02$ & $4.90 \mathrm{E}+01$ & $2.12 \mathrm{E}+00$ & $4.66 \mathrm{E}+01$ & $3.88 \mathrm{E}+00$ & $7.73 \mathrm{E}+01$ & $9.26 \mathrm{E}+01$ \\
\hline Wt. frac. non-salts in solid & & 0.890 & 0.311 & 0.509 & 0.762 & 1.000 & 0.014 & 0.806 & 0.579 \\
\hline Avg g/mL, non-salt solid & & 2.429 & 2.434 & 2.430 & 2.428 & 2.426 & 3.586 & 2.505 & 4.055 \\
\hline Vol. frac. non-salts in solid & & 0.844 & 0.297 & 0.408 & 0.682 & 1.000 & 0.009 & $0.7 \hat{2} 2$ & 0.483 \\
\hline \multicolumn{10}{|l|}{ non-salts: mass fracs in total solid } \\
\hline $\mathrm{Ag}$ & 10.5 & --- & --- & -- & --- & --- & --- & --- & -- \\
\hline $\mathrm{Ag}_{2} \mathrm{O}$ & 7.143 & --- & --- & --- & -- & $0.01 \%$ & --- & -- & $0.11 \%$ \\
\hline $\mathrm{Bi}_{2} \mathrm{O}_{3}$ & 8.9 & $0.07 \%$ & $0.02 \%$ & --- & $0.05 \%$ & $0.06 \%$ & --- & $0.12 \%$ & $0.09 \%$ \\
\hline $\mathrm{BiFeO}_{3}$ & 7.9 & $\cdots$ & $\ldots$ & $--\cdot$ & $\cdots$ & $--\cdot$ & $-\cdots$ & $\cdots$ & $\cdots$ \\
\hline $\mathrm{Ca}(\mathrm{OH})_{2}$ & 2.24 & $\cdots$ & $\cdots$ & $\cdots$ & $\cdots$ & $\cdots$ & $\cdots$ & $\cdots$ & $\cdots$ \\
\hline $\mathrm{Ca}_{5} \mathrm{OH}\left(\mathrm{PO}_{4}\right)_{3}$ & 3.14 & $0.42 \%$ & $0.17 \%$ & $0.36 \%$ & $0.33 \%$ & $0.25 \%$ & $0.20 \%$ & $1.29 \%$ & -- \\
\hline $\mathrm{CaC}_{2} \mathrm{O}_{4} \cdot \mathrm{H}_{2} \mathrm{O}$ & 2.2 & -- & --- & --- & -- & --- & --- & -- & -- \\
\hline $\mathrm{CaCO}_{3}$ & 2.71 & -- & --- & --- & --- & --- & --- & --- & -- \\
\hline $\mathrm{CaF}_{2}$ & 3.18 & --- & --- & --- & --- & --- & --- & --- & $0.16 \%$ \\
\hline $\mathrm{CrOOH}$ & 4.11 & --- & -.. & $\cdots$ &.-- & -.. & $0.13 \%$ & $0.06 \%$ & $0.83 \%$ \\
\hline $\mathrm{FePO}_{4} \cdot 2 \mathrm{H}_{2} \mathrm{O}$ & 3.15 & -- & --- & -- & -- & --- & --- & --- & -- \\
\hline $\mathrm{FeOOH}$ & 4.26 & $0.06 \%$ & $0.07 \%$ & $0.01 \%$ & $0.05 \%$ & $0.05 \%$ & $0.13 \%$ & $2.38 \%$ & $0.40 \%$ \\
\hline Gibbsite & 2.42 & $88.17 \%$ & $30.65 \%$ & $50.26 \%$ & $75.55 \%$ & $99.39 \%$ & --- & $74.40 \%$ & $15.41 \%$ \\
\hline Boehmite & 3.01 & --- & --- & --- & --- & --- & --- & --- & -- \\
\hline $\mathrm{HgO}$ (co-precip. with $\mathrm{Ag}_{2} \mathrm{O}$ ) & 7.143 & --- & --- & --- & --- & --- & --- & --- & --- \\
\hline $\mathrm{KAlSiO}_{4}$ & 2.61 & --- & --- & --- & --- & --- & $0.49 \%$ & --- & $1.61 \%$ \\
\hline $\mathrm{La}(\mathrm{OH})_{3}$ & 2.3 & -- & $\begin{array}{ll}-- \\
\end{array}$ & --- & $\begin{array}{ll}-- \\
\end{array}$ & $\begin{array}{ll}-- \\
\end{array}$ & $0.00 \%$ & --- & -- \\
\hline $\mathrm{LaPO}_{4} \cdot 2 \mathrm{H}_{2} \mathrm{O}$ & 6.51 & $0.00 \%$ & $0.01 \%$ & $0.01 \%$ & $0.02 \%$ & $0.02 \%$ & --- & $0.07 \%$ & $0.14 \%$ \\
\hline $\mathrm{Mn}_{3}\left(\mathrm{PO}_{4}\right)_{2}$ & 3.102 & -- & --- & --- & -- & -- & --- & -- & -- \\
\hline $\mathrm{MnO}_{2}$ & 5.026 &.-- & --. & $0.10 \%$ & --. & -.. & $\ldots$ & --- & $\ldots$ \\
\hline $\mathrm{Na}_{2}\left(\mathrm{UO}_{2}\right)_{2}\left(\mathrm{PO}_{4}\right)_{2} \cdot 2 \mathrm{H}_{2} \mathrm{O}$ & 3.5 & --- & $\begin{array}{l}-- \\
\end{array}$ & --- & --- & --- & --- & --- & --- \\
\hline $\mathrm{Na}_{2} \mathrm{U}_{2} \mathrm{O}_{7}$ & 5.617 & $0.04 \%$ & $0.09 \%$ & $0.01 \%$ & $0.01 \%$ & $0.01 \%$ & $0.36 \%$ & $1.77 \%$ & $2.53 \%$ \\
\hline $\mathrm{NaAICO}_{3}(\mathrm{OH})_{2}$ & 2.42 & -- & --- & -- & -- & --- & --- & --- & -- \\
\hline $\mathrm{NaAlSiO}_{4}$ & 2.365 & -- & --- & --- & --- & --- & --- & --- & -- \\
\hline $\mathrm{Ni}(\mathrm{OH})_{2}$ & 4.15 & $0.18 \%$ & $0.04 \%$ & $0.08 \%$ & $0.16 \%$ & $0.13 \%$ & $0.01 \%$ & $0.30 \%$ & $0.03 \%$ \\
\hline $\mathrm{Ni}_{3}\left(\mathrm{PO}_{4}\right)$ & 3.93 & -- & -- & -- & -- & -.- & $\cdots$ & $\cdots$ & $\cdots$ \\
\hline $\mathrm{NiC}_{2} \mathrm{O}_{4} \cdot 2 \mathrm{H}_{2} \mathrm{O}$ (on Fe phase) & 4.26 & -- & --- & --- & --- & --- & --- & -- & -- \\
\hline $\mathrm{Pb}(\mathrm{OH})_{2}$ & 7.1 & $0.09 \%$ & $0.04 \%$ & $0.01 \%$ & $0.07 \%$ & $0.06 \%$ & $0.08 \%$ & $0.16 \%$ & $0.03 \%$ \\
\hline $\mathrm{Pb}_{3}\left(\mathrm{PO}_{4}\right)_{2}$ & 7.1 & --- & --- & --- & --- & --- & --- & --- & --- \\
\hline $\mathrm{PbCO}_{3}$ & 6.6 & -- & --- & -- & --- & --- & --- & --- & -- \\
\hline $\mathrm{Pu}(\mathrm{OH})_{4}$ (co-precip. on Fe phase) & 4.26 & $0.00 \%$ & $0.00 \%$ & $0.00 \%$ & $0.00 \%$ & $0.00 \%$ & $0.00 \%$ & $0.00 \%$ & $0.00 \%$ \\
\hline $\mathrm{PuO}_{2}$ & 11.43 & -- & --- & -- & -- & --- & --- & --- & -- \\
\hline $\mathrm{SiO}_{2}$ & 2.6 & $\begin{array}{l}-- \\
\end{array}$ & $\begin{array}{l}-- \\
\end{array}$ & --- & $\begin{array}{l}-- \\
\end{array}$ & --- & --- & --- & --- \\
\hline $\mathrm{Sr}_{3}\left(\mathrm{PO}_{4}\right)_{2}$ & 3.5 & --- & --- & --- & --- & --- & --- & $0.02 \%$ & --- \\
\hline $\mathrm{SrCO}_{3}$ & 3.5 & $0.01 \%$ & $0.00 \%$ & $0.00 \%$ & $0.01 \%$ & $0.01 \%$ & $0.00 \%$ & --- & $0.00 \%$ \\
\hline $\mathrm{ZrO}_{2}$ & 5.68 & -- & --. & $0.03 \%$ & -.- & $--\cdot$ & $0.01 \%$ & -- & $36.53 \%$ \\
\hline \multicolumn{10}{|l|}{ salts: mass fracs in total solid } \\
\hline $\mathrm{KNO}_{3}$ & 2.109 & $\overline{c--}$ & $\cdots$ & $\cdots$ & $\cdots$ & $-\cdots$ & $10.14 \%$ & $\cdots$ & $-\cdots$ \\
\hline $\mathrm{Na}_{2} \mathrm{C}_{2} \mathrm{O}_{4}$ & 2.34 & --- & $0.48 \%$ & -- & --- & --- & $5.68 \%$ & $1.21 \%$ & $1.48 \%$ \\
\hline $\mathrm{Na}_{2} \mathrm{CO}_{3} \cdot \mathrm{H}_{2} \mathrm{O}$ & 2.25 & -- & $42.17 \%$ & -- & $-\cdot$ & $0.01 \%$ & $14.32 \%$ & -.- & $0.06 \%$ \\
\hline $\mathrm{Na}_{2} \mathrm{SO}_{4}$ & 2.68 & -- & --- & --- & --- & --- & --- & --- & -- \\
\hline $\mathrm{Na}_{2} \mathrm{SO}_{4} \cdot 10 \mathrm{H}_{2} \mathrm{O}$ & 1.464 & -- & $\begin{array}{ll}-- \\
\end{array}$ & -- & --- & --- & $\begin{array}{ll}-- \\
\end{array}$ & --- & -- \\
\hline $\mathrm{Na}_{3} \mathrm{FSO}_{4}$ & 2.65 & -- & $7.38 \%$ & --- & --- & --- & $2.15 \%$ & --- & -- \\
\hline $\mathrm{Na}_{3} \mathrm{NO}_{3} \mathrm{SO}_{4} \cdot \mathrm{H}_{2} \mathrm{O}$ & 2.3 & -- & -- & -- & -- & --- & --- & --- & -- \\
\hline $\mathrm{Na}_{3} \mathrm{PO}_{4} \bullet 0.25 \mathrm{NaOH} \bullet 12 \mathrm{H}_{2} \mathrm{O}$ & 1.62 & $10.95 \%$ & --- & $49.13 \%$ & $23.76 \%$ & --- & --- & $18.22 \%$ & --- \\
\hline $\mathrm{Na}_{3} \mathrm{PO}_{4} \bullet 8 \mathrm{H}_{2} \mathrm{O}$ & 1.8 & -- & --- & -- & -- & --- & --- & --- & -- \\
\hline $\mathrm{Na}_{4} \mathrm{P}_{2} \mathrm{O}_{7} \cdot 10 \mathrm{H}_{2} \mathrm{O}$ & 1.83 & --- & --- & --- & --- & --- & --- & --- & -- \\
\hline $\mathrm{Na}_{6}\left(\mathrm{SO}_{4}\right)_{2} \mathrm{CO}_{3}$ & 2.64 & --- & --- & --- & --- & --- & --- & -- & --- \\
\hline $\mathrm{NaF}$ & 2.78 & -- & $4.32 \%$ & -- & --- & --- & --- & --- & $40.57 \%$ \\
\hline $\mathrm{Na}_{7} \mathrm{~F}\left(\mathrm{PO}_{4}\right)_{2} \cdot 19 \mathrm{H}_{2} \mathrm{O}$ & 1.75 & --- & $3.64 \%$ & -- & --- & --- & $2.69 \%$ & --- & --- \\
\hline $\mathrm{NaI} \mathrm{ICO}_{3}$ & 2.159 & -- & $\cdots$ & -- & $--\cdot$ & --. & --. & $--\cdot$ & -- \\
\hline $\mathrm{NaNO}_{2}$ & 2.168 & --- & --- & --- & -- & --- & $6.33 \%$ & --- & -- \\
\hline $\mathrm{NaNO}_{3}$ & 2.26 & --- & $10.92 \%$ & --- & -- & -- & $57.26 \%$ & --- & --- \\
\hline
\end{tabular}


Table C.1. (contd)

\begin{tabular}{|c|c|c|c|c|c|c|c|c|c|}
\hline & $\begin{array}{l}\text { Density } \\
(\mathrm{g} / \mathrm{mL})\end{array}$ & 241-AW-104 & $241-\mathrm{AW}-105$ & 241-AW-106 & $241-\mathrm{AX}-101$ & $241-\mathrm{AX}-102$ & 241-AX-103 & 241-AX-104 & 241-AY-101 \\
\hline Total kg solids for tank & \multirow{9}{*}{$11 / a$} & $1.71 \mathrm{E}+05$ & $2.84 \mathrm{E}+05$ & $4.24 \mathrm{E}+05$ & $1.04 \mathrm{E}+06$ & $7.89 \mathrm{E}+04$ & $8.33 \mathrm{E}+04$ & $3.85 \mathrm{E}+04$ & $2.85 \mathrm{E}+05$ \\
\hline Total kL solids for tank & & $6.15 \mathrm{E}+01$ & $7.45 \mathrm{E}+01$ & $1.87 \mathrm{E}+02$ & $4.49 \mathrm{E}+02$ & $3.16 \mathrm{E}+01$ & $3.30 \mathrm{E}+01$ & 1. $12 \mathrm{E}+01$ & $1.04 \mathrm{E}+02$ \\
\hline Vol. frac. solid phase in tank & & 0.052 & 0.046 & 0.168 & 0.177 & 0.277 & 0.080 & 0.402 & 0.150 \\
\hline Avg $\mathrm{g} / \mathrm{mL}$, all solid & & 2.784 & 3.818 & 2.268 & 2.325 & 2.499 & 2.524 & 3.426 & 2.732 \\
\hline Total kg non-salt solids for tank & & $6.27 \mathrm{E}+04$ & $1.72 \mathrm{E}+05$ & $1.09 \mathrm{E}+05$ & $1.32 \mathrm{E}+05$ & $3.12 \mathrm{E}+04$ & $3.72 \mathrm{E}+04$ & $3.80 \mathrm{E}+04$ & $1.69 \mathrm{E}+05$ \\
\hline Total kL non-salt solids for tank & & $2.06 \mathrm{E}+01$ & $3.39 \mathrm{E}+01$ & $4.26 \mathrm{E}+0 \mathrm{I}$ & $5.18 \mathrm{E}+01$ & $1.09 \mathrm{E}+01$ & $1.32 \mathrm{E}+01$ & $1.10 \mathrm{E}+01$ & $5.34 \mathrm{E}+01$ \\
\hline Wt. frac. non-salts in solid & & 0.366 & 0.604 & 0.258 & 0.126 & 0.396 & 0.446 & 0.986 & 0.593 \\
\hline Avg g/mL, non-salt solid & & 3.047 & 5.069 & 2.568 & 2.544 & 2.852 & 2.822 & 3.452 & 3.166 \\
\hline Vol. frac. non-salts in solid & & 0.334 & 0.455 & 0.228 & 0.115 & 0.347 & 0.399 & 0.978 & 0.512 \\
\hline \multicolumn{10}{|l|}{ non-salts: mass fracs in total solid } \\
\hline Ag & 10.5 & --- & --- & --- & --- & --- & --- & --- & --- \\
\hline $\mathrm{Ag}_{2} \mathrm{O}$ & 7.143 & $0.02 \%$ & $0.21 \%$ & --- & -- & -- & --- & --- & $0.05 \%$ \\
\hline $\mathrm{Bi}_{2} \mathrm{O}_{3}$ & 8.9 & $0.02 \%$ & $0.06 \%$ & $0.07 \%$ & $0.02 \%$ & $0.01 \%$ & $0.02 \%$ & --- & -- \\
\hline $\mathrm{BiFeO}_{3}$ & 7.9 & $\ldots$ & $\ldots$ & -.- & $\ldots$ & -.. & $\ldots$ & $\ldots$ & $\ldots$ \\
\hline $\mathrm{Ca}(\mathrm{OH})_{2}$ & 2.24 & $\cdots$ & $\cdots$ & $\cdots$ & $\cdots$ & 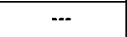 & $\cdots$ & 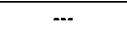 & $\cdots$ \\
\hline $\mathrm{Ca}_{5} \mathrm{OH}\left(\mathrm{PO}_{4}\right)_{3}$ & 3.14 & $1.83 \%$ & $\cdots$ & $0.39 \%$ & $0.22 \%$ & $0.61 \%$ & $0.99 \%$ & $0.35 \%$ & $1.17 \%$ \\
\hline $\mathrm{CaC}_{2} \mathrm{O}_{+} \cdot \mathrm{H}_{2} \mathrm{O}$ & 2.2 & -- & --- & --- & --- & -- & --- & -- & -- \\
\hline $\mathrm{CaCO}_{3}$ & 2.71 & --- & --- & -- & --- & $0.26 \%$ & --- & $3.69 \%$ & --- \\
\hline $\mathrm{CaF}_{2}$ & 3.18 & --- & $0.55 \%$ & --- & --- & -- & --- & $0.02 \%$ & --- \\
\hline $\mathrm{CrOOH}$ & 4.11 & $2.46 \%$ & $0.51 \%$ & $2.22 \%$ & $0.85 \%$ & $\cdots$ & $2.32 \%$ & -.. & $0.72 \%$ \\
\hline $\mathrm{FePO}_{4} \cdot 2 \mathrm{H}_{2} \mathrm{O}$ & 3.15 & -- & --- & --- & --- & --- & --- & -- & -- \\
\hline $\mathrm{FeOOH}$ & 4.26 & $2.44 \%$ & $1.42 \%$ & $0.19 \%$ & $0.20 \%$ & $8.92 \%$ & $8.24 \%$ & $58.23 \%$ & $25.96 \%$ \\
\hline Gibbsite & 2.42 & $18.45 \%$ & $1.32 \%$ & $21.90 \%$ & $10.61 \%$ & $18.18 \%$ & $23.65 \%$ & $10.98 \%$ & $27.09 \%$ \\
\hline Boehmite & 3.01 & --- & --- & --- & --- & --- & --- & --- & --- \\
\hline $\mathrm{HgO}$ (co-precip. with $\mathrm{Ag}_{2} \mathrm{O}$ ) & 7.143 & --- & --- & --- & --- & $0.02 \%$ & --- & $0.04 \%$ & --- \\
\hline $\mathrm{KAlSiO}_{4}$ & 2.61 & $2.87 \%$ & $3.68 \%$ & $-\cdots$ & -- & -- & --- & -- & $\ldots$ \\
\hline $\mathrm{La}(\mathrm{OH})_{3}$ & 2.3 & --- & --- & --- & --- & --- & --- & --- & --- \\
\hline $\mathrm{LaPO}_{4} \cdot 2 \mathrm{H}_{2} \mathrm{O}$ & 6.51 & $0.05 \%$ & $0.48 \%$ & $0.04 \%$ & $0.02 \%$ & $0.01 \%$ & $0.20 \%$ & $0.38 \%$ & $0.93 \%$ \\
\hline $\mathrm{Mn}_{3}\left(\mathrm{PO}_{4}\right)_{2}$ & 3.102 & -- & --- & --- & -- & -- & --- & -- & -- \\
\hline $\mathrm{MnO}_{2}$ & 5.026 & $0.65 \%$ & $0.69 \%$ & --- & --. & $0.69 \%$ & $0.94 \%$ & $1.00 \%$ & $1.17 \%$ \\
\hline $\mathrm{Na}_{2}\left(\mathrm{UO}_{2}\right)_{2}\left(\mathrm{PO}_{4}\right)_{2} \cdot 2 \mathrm{H}_{2} \mathrm{O}$ & 3.5 & -- & --- & --- & --- & --- & --- & $\begin{array}{ll}-- \\
\end{array}$ & --- \\
\hline $\mathrm{Na}_{2} \mathrm{U}_{2} \mathrm{O}_{7}$ & 5.617 & $5.69 \%$ & $6.01 \%$ & $0.38 \%$ & $0.13 \%$ & $0.13 \%$ & $0.38 \%$ & $0.58 \%$ & $0.30 \%$ \\
\hline $\mathrm{NaAICO}_{3}(\mathrm{OH})_{2}$ & 2.42 & -- & --- & -- & --- & $0.95 \%$ & --- & $17.30 \%$ & -- \\
\hline $\mathrm{NaAlSiO}_{4}$ & 2.365 & -- & --- & $0.37 \%$ & $0.50 \%$ & $5.85 \%$ & $6.23 \%$ & $0.49 \%$ & $0.34 \%$ \\
\hline $\mathrm{Ni}(\mathrm{OH})_{2}$ & 4.15 & $0.08 \%$ & $0.11 \%$ & $0.14 \%$ & $0.03 \%$ & $3.87 \%$ & $0.49 \%$ & $3.11 \%$ & $0.82 \%$ \\
\hline $\mathrm{Ni}_{3}\left(\mathrm{PO}_{4}\right)$ & 3.93 &.- & $-\cdots$ & $-\cdots$ & -- & -.. & $-\cdot$ & -. & $\ldots$ \\
\hline $\mathrm{NiC}_{2} \mathrm{O}_{4} \cdot 2 \mathrm{H}_{2} \mathrm{O}$ (on Fe phase) & 4.26 & --- & --- & --- & --- & --- & --- & --- & --- \\
\hline $\mathrm{Pb}(\mathrm{OH})_{2}$ & 7.1 & $0.09 \%$ & $0.02 \%$ & $0.04 \%$ & $0.04 \%$ & $0.05 \%$ & $0.75 \%$ & $1.45 \%$ & $0.43 \%$ \\
\hline $\mathrm{Pb}_{3}\left(\mathrm{PO}_{4}\right)_{2}$ & 7.1 & --- & --- & --- & $\begin{array}{c}-- \\
-\end{array}$ & --- & 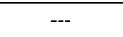 & $\begin{array}{l}-- \\
-1\end{array}$ & -- \\
\hline $\mathrm{PbCO}_{3}$ & 6.6 & -- & --- & -- & --- & --- & --- & --- & -- \\
\hline $\mathrm{Pu}(\mathrm{OH})_{4}$ (co-precip. on Fe phase) & 4.26 & $0.01 \%$ & $0.00 \%$ & $0.00 \%$ & $0.00 \%$ & $0.01 \%$ & $0.00 \%$ & $0.02 \%$ & $0.00 \%$ \\
\hline $\mathrm{PuO}_{2}$ & 11.43 & -- & --- & -- & --- & --- & --- & --- & -- \\
\hline $\mathrm{SiO}_{2}$ & 2.6 & $\begin{array}{l}-- \\
\end{array}$ & $\begin{array}{l}-- \\
\end{array}$ & --- & $\begin{array}{l}-- \\
\end{array}$ & --- & --- & --- & --- \\
\hline $\mathrm{Sr}_{3}\left(\mathrm{PO}_{4}\right)_{2}$ & 3.5 & --- & --- & --- & --- & --- & --- & --- & --- \\
\hline $\mathrm{SrCO}_{3}$ & 3.5 & $0.01 \%$ & $0.00 \%$ & $0.02 \%$ & $0.00 \%$ & $0.00 \%$ & $0.10 \%$ & $0.22 \%$ & $0.04 \%$ \\
\hline $\mathrm{ZrO}_{2}$ & 5.68 & $1.92 \%$ & $45.37 \%$ & $0.03 \%$ & $0.01 \%$ & $0.01 \%$ & $0.33 \%$ & $0.72 \%$ & $0.31 \%$ \\
\hline \multicolumn{10}{|l|}{ salts: mass fracs in total solid } \\
\hline $\mathrm{KNO}_{3}$ & 2.109 & -- & $\cdots$ & $\begin{array}{ll}-\cdots \\
\end{array}$ & $+\cdots$ & $\begin{array}{ll}-- \\
\end{array}$ & $\cdots$ & $\cdots$ & $-\cdots$ \\
\hline $\mathrm{Na}_{2} \mathrm{C}_{2} \mathrm{O}_{4}$ & 2.34 & $7.93 \%$ & $0.48 \%$ & $13.45 \%$ & $3.59 \%$ & $40.01 \%$ & --- & --- & $9.83 \%$ \\
\hline $\mathrm{Na}_{2} \mathrm{CO}_{3} \cdot \mathrm{H}_{2} \mathrm{O}$ & 2.25 & $0.01 \%$ & $0.11 \%$ & $37.37 \%$ & $32.07 \%$ & $4.41 \%$ & $34.62 \%$ & $1.42 \%$ & $29.52 \%$ \\
\hline $\mathrm{Na}_{2} \mathrm{SO}_{4}$ & 2.68 & -- & --- & --- & --- & --- & --- & --- & -- \\
\hline $\mathrm{Na}_{2} \mathrm{SO}_{4} \cdot 10 \mathrm{H}_{2} \mathrm{O}$ & 1.464 & -- & --- & --- & --- & --- & --- & --- & -- \\
\hline $\mathrm{Na}_{3} \mathrm{FSO}_{4}$ & 2.65 & $18.88 \%$ & --- & --- & $0.54 \%$ & $0.01 \%$ & $6.81 \%$ & --- & $1.06 \%$ \\
\hline $\mathrm{Na}_{3} \mathrm{NO}_{3} \mathrm{SO}_{4} \cdot \mathrm{H}_{2} \mathrm{O}$ & 2.3 & -- & --- & -- & --- & -- & --- & --- & -- \\
\hline $\mathrm{Na}_{3} \mathrm{PO}_{4} \bullet 0.25 \mathrm{NaOH} \bullet 12 \mathrm{H}_{2} \mathrm{O}$ & 1.62 & --- & --- & $2.24 \%$ & --- & --- & --- & --- & --- \\
\hline $\mathrm{Na}_{3} \mathrm{PO}_{4} \bullet 8 \mathrm{H}_{2} \mathrm{O}$ & 1.8 & -- & --- & -- & $\begin{array}{c}-- \\
-1\end{array}$ & --- & --- & --- & -- \\
\hline $\mathrm{Na}_{4} \mathrm{P}_{2} \mathrm{O}_{7} \cdot 10 \mathrm{H}_{2} \mathrm{O}$ & 1.83 & --- & --- & --- & --- & --- & --- & --- & --- \\
\hline $\mathrm{Na}_{6}\left(\mathrm{SO}_{4}\right)_{2} \mathrm{CO}_{3}$ & 2.64 & -- & --- & $7.79 \%$ & $9.13 \%$ & --- & $11.00 \%$ & --- & -- \\
\hline $\mathrm{NaF}$ & 2.78 & $35.50 \%$ & $38.97 \%$ & -- & --- & --- & --- & --- & --- \\
\hline $\mathrm{Na}_{7} \mathrm{~F}\left(\mathrm{PO}_{4}\right)_{2} \cdot 19 \mathrm{H}_{2} \mathrm{O}$ & 1.75 & $1.08 \%$ & --- & $11.17 \%$ & --- & --- & $2.93 \%$ & --- & $0.24 \%$ \\
\hline $\mathrm{NaIICO}_{3}$ & 2.159 & -- & $--\cdot$ & -- & $--\cdot$ & --. & $\cdots$ & -- & -- \\
\hline $\mathrm{NaNO}_{2}$ & 2.168 & --- & --- & --- & --- & -- & --- & --- & --- \\
\hline $\mathrm{NaNO}_{3}$ & 2.26 & -- & --- & $2.18 \%$ & $42.04 \%$ & $16.01 \%$ & --- & --- & --- \\
\hline
\end{tabular}


Table C.1. (contd)

\begin{tabular}{|c|c|c|c|c|c|c|c|c|c|c|}
\hline & $\begin{array}{l}\text { Density } \\
(\mathrm{g} / \mathbf{m L})\end{array}$ & 241-AY-102 & 241-AZ-101 & $241-A Z-102$ & 241-B-101 & 241-B-102 & 241-B-103 & 241-B-104 & 24I-B-105 & 241-B-106 \\
\hline Total kg solids for tank & \multirow{9}{*}{$\mathbf{n} / \mathbf{a}$} & $2.91 \mathrm{E}+05$ & $1.2 \mathrm{IE}+05$ & $1.31 E+05$ & $3.87 \mathrm{E}+05$ & $1.07 \mathrm{E}+05$ & $2.06 \mathrm{E}+05$ & $3.12 \mathrm{E}+05$ & $1.27 \mathrm{E}+06$ & $1.68 \mathrm{E}+05$ \\
\hline Total kL solids for tank & & $8.60 \mathrm{E}+01$ & $4.02 \mathrm{E}+01$ & $4.43 \mathrm{E}+01$ & $1.52 \mathrm{E}+02$ & $5.97 \mathrm{E}+01$ & $1.15 E+02$ & $1.20 \mathrm{E}+02$ & $6.99 \mathrm{E}+02$ & $8.14 \mathrm{E}+01$ \\
\hline Vol. frac. solid phase in tank & & 0.033 & 0.011 & 0.012 & 0.369 & 0.493 & 0.545 & 0.085 & 0.636 & 0.175 \\
\hline Avg $g / m L$, all solid & & 3.390 & 3.016 & 2.953 & 2.554 & 1.799 & 1.793 & 2.605 & 1.823 & 2.062 \\
\hline Total kg non-salt solids for tank & & $2.83 \mathrm{E}+05$ & $1.2 \mathrm{IE}+05$ & $1.31 E+05$ & $1.61 \mathrm{E}+05$ & I. $76 \mathrm{E}+04$ & $3.32 \mathrm{E}+04$ & $1.17 \mathrm{E}+05$ & $1.99 \mathrm{E}+05$ & $4.72 \mathrm{E}+04$ \\
\hline Total kL non-salt solids for tank & & $8.25 \mathrm{E}+01$ & $4.02 \mathrm{E}+01$ & $4.42 \mathrm{E}+01$ & $5.77 \mathrm{E}+01$ & $6.90 \mathrm{E}+00$ & $1.28 \mathrm{E}+01$ & $3.18 \mathrm{E}+01$ & $7.67 \mathrm{E}+01$ & $1.33 \mathrm{E}+01$ \\
\hline Wt. frac. non-salts in solid & & 0.972 & 1.000 & 0.999 & 0.417 & 0.164 & 0.161 & 0.374 & 0.156 & 0.281 \\
\hline Avg $\mathrm{g} / \mathrm{mL}$, non-salt solid & & 3.433 & 3.017 & 2.954 & 2.801 & 2.549 & 2.582 & 3.675 & 2.591 & 3.545 \\
\hline Vol. frac. non-salts in solid & & 0.960 & 1.000 & 0.999 & 0.380 & 0.116 & 0.112 & 0.265 & 0.110 & 0.163 \\
\hline \multicolumn{11}{|l|}{ non-salts: mass fracs in total solid } \\
\hline $\mathrm{Ag}$ & 10.5 & $\cdots$ & $-\cdots$ & $\cdots$ & $\cdots$ & $\cdots$ & $\cdots$ & $\cdots$ & --- & $\cdots$ \\
\hline $\mathrm{Ag}_{2} \mathrm{O}$ & 7.143 & $0.01 \%$ & $0.06 \%$ & $0.20 \%$ & --- & --- & --- & --- & --- & $0.02 \%$ \\
\hline $\mathrm{Bi}_{2} \mathrm{O}_{3}$ & 8.9 & $0.05 \%$ & --- & $0.02 \%$ & --- & $0.35 \%$ & $0.34 \%$ & --- & $0.55 \%$ & $3.24 \%$ \\
\hline $\mathrm{BilleO}_{3}$ & 7.9 & --- & --- & --- & --- & --- & --- & $11.66 \%$ & --- & --- \\
\hline $\mathrm{Ca}(\mathrm{OH})_{2}$ & 2.24 & --- & --- & --- & --- & -- & --- & --- & --- & --- \\
\hline $\mathrm{Ca}_{5} \mathrm{OH}\left(\mathrm{PO}_{4}\right)_{3}$ & 3.14 & $4.12 \%$ & $1.33 \%$ & $1.33 \%$ & $1.55 \%$ & $0.43 \%$ & $0.42 \%$ & --- & $0.41 \%$ & $1.24 \%$ \\
\hline $\mathrm{CaC}_{2} \mathrm{O}_{4} \cdot \mathrm{H}_{2} \mathrm{O}$ & 2.2 & --- & --- & --- & --- & --- & --- & --- & --- & --- \\
\hline $\mathrm{CaCO}_{3}$ & 2.71 & --- & --- & --- & --- & --- & --- & --- & --- & --- \\
\hline $\mathrm{CaF}_{2}$ & 3.18 & --- & --- & --- & --- & --- & --- & $0.44 \%$ & --- & $0.33 \%$ \\
\hline $\mathrm{CrOOH}$ & 4.11 & $0.86 \%$ & $0.06 \%$ & $0.29 \%$ & $0.18 \%$ & $0.21 \%$ & $0.22 \%$ & $0.35 \%$ & $0.21 \%$ & $0.14 \%$ \\
\hline $\mathrm{FePO}_{4} \cdot 2 \mathrm{H}_{2} \mathrm{O}$ & 3.15 & -- & --- & --- & --- & -- & -- & $17.15 \%$ & --- & --- \\
\hline $\mathrm{FeOOH}$ & 4.26 & $49.10 \%$ & $27.39 \%$ & $28.91 \%$ & $8.25 \%$ & $0.40 \%$ & $0.41 \%$ & --- & $0.59 \%$ & $8.77 \%$ \\
\hline Gibbsite & 2.42 & $31.64 \%$ & $55.72 \%$ & $30.04 \%$ & $11.89 \%$ & $13.34 \%$ & $12.72 \%$ & --- & $11.92 \%$ & $6.41 \%$ \\
\hline Boehmite & 3.01 & --- & --- & --- & --- & -- & --- & --- & --- & --- \\
\hline $\mathrm{HgO}$ (co-precip. with $\mathrm{Ag}_{2} \mathrm{O}$ ) & 7.143 & $0.00 \%$ & --- & --- & $0.03 \%$ & -- & --- & --- & --- & $0.02 \%$ \\
\hline $\mathrm{KAlSiO}_{4}$ & 2.61 & --- & --- & --- & --- & --- & --- & --- & --- & --- \\
\hline $\mathrm{La}(\mathrm{OH})_{3}$ & 2.3 & --- & --- & --- & --- & --- & --- & --- & --- & --- \\
\hline $\mathrm{LaPO}_{4} \cdot 2 \mathrm{H}_{2} \mathrm{O}$ & 6.51 & $0.77 \%$ & $1.48 \%$ & $1.13 \%$ & $0.00 \%$ & -- & $0.00 \%$ & $0.04 \%$ & $0.00 \%$ & $0.03 \%$ \\
\hline $\mathrm{Mn}_{3}\left(\mathrm{PO}_{4}\right)_{2}$ & 3.102 & $\cdots$ & $--\cdot$ & $-\cdot$ & $\cdots$ & $\cdots$ & $\cdots$ & --- & $--\cdot$ & $0.11 \%$ \\
\hline $\mathrm{MnO}_{2}$ & 5.026 & $6.45 \%$ & $0.35 \%$ & $0.59 \%$ & $0.01 \%$ & $0.04 \%$ & $0.05 \%$ & $0.14 \%$ & $0.02 \%$ & $--\cdot$ \\
\hline $\mathrm{Na}_{2}\left(\mathrm{UO}_{2}\right)_{2}\left(\mathrm{PO}_{4}\right)_{2} \cdot 2 \mathrm{H}_{2} \mathrm{O}$ & 3.5 & --- & --- & --- & $\cdots$ & $-\cdots$ & $\cdots$ & --- & --- & $6.78 \%$ \\
\hline $\mathrm{Na}_{2} \mathrm{U}_{2} \mathrm{O}_{7}$ & 5.617 & $1.41 \%$ & $1.69 \%$ & $2.17 \%$ & $0.86 \%$ & $0.32 \%$ & $0.63 \%$ & --- & $0.32 \%$ & --- \\
\hline $\mathrm{NaAlCO}_{3}(\mathrm{OH})_{2}$ & 2.42 & --- & --- & $21.63 \%$ & --- & -- & --- & --- & --- & $0.03 \%$ \\
\hline $\mathrm{NaAlSiO}_{4}$ & 2.365 & --- & $1.72 \%$ & $8.02 \%$ & $15.30 \%$ & $1.21 \%$ & $1.22 \%$ & $6.67 \%$ & $1.52 \%$ & $0.53 \%$ \\
\hline $\mathrm{Ni}(\mathrm{OH})_{2}$ & 4.15 & $1.18 \%$ & $1.68 \%$ & $1.70 \%$ & $3.53 \%$ & $0.01 \%$ & $0.01 \%$ & $0.06 \%$ & $0.01 \%$ & $0.03 \%$ \\
\hline $\mathrm{Ni}_{3}\left(\mathrm{PO}_{4}\right)_{2}$ & 3.93 & --- & --- & --- & -- & -- & --- & --- & --- & --- \\
\hline $\mathrm{NiC}_{2} \mathrm{O}_{1} * 2 \mathrm{H}_{2} \mathrm{O}$ (on Fe phase) & 4.26 & --- & --- & --- & $\cdots$ & --- & $--\cdot$ & --- & --- & $--\cdot$ \\
\hline $\mathrm{Pb}(\mathrm{OH})_{2}$ & 7.1 & $1.45 \%$ & $0.08 \%$ & $0.26 \%$ & $0.05 \%$ & $0.04 \%$ & $0.05 \%$ & $0.58 \%$ & $0.05 \%$ & $0.15 \%$ \\
\hline $\mathrm{Pb}_{3}\left(\mathrm{PO}_{4}\right)_{2}$ & 7.1 & -- & --- & --- & --- & -- & --- & --- & --- & --- \\
\hline $\mathrm{PbCO}_{3}$ & 6.6 & --- & --- & --- & --- & --- & --- & --- & --- & --- \\
\hline $\mathrm{Pu}\left(\mathrm{OH}_{4}\right.$ (co-precip. on Fe phase) & 4.26 & $0.01 \%$ & $0.02 \%$ & $0.03 \%$ & $0.00 \%$ & $0.00 \%$ & $0.00 \%$ & $0.00 \%$ & $0.00 \%$ & $0.00 \%$ \\
\hline $\mathrm{PuO}_{2}$ & 11.43 & --- & --- & --- & --- & -- & --- & --- & --- & --- \\
\hline $\mathrm{SiO}_{2}$ & 2.6 & --- & --- & --- & --- & -- & --- & --- & --- & --- \\
\hline $\mathrm{Sr}_{3}\left(\mathrm{PO}_{4}\right)_{2}$ & 3.5 & -- & --- & --- & -- & -- & -- & --- & --- & --- \\
\hline $\mathrm{SrCO}_{3}$ & 3.5 & $0.14 \%$ & $0.14 \%$ & $0.06 \%$ & $0.02 \%$ & $0.01 \%$ & $0.01 \%$ & $0.28 \%$ & $0.02 \%$ & $0.23 \%$ \\
\hline $\mathrm{ZrO}_{2}$ & 5.68 & $0.01 \%$ & $8.24 \%$ & $3.53 \%$ & $0.01 \%$ & $0.01 \%$ & $0.01 \%$ & $0.06 \%$ & $0.01 \%$ & $0.04 \%$ \\
\hline \multicolumn{11}{|l|}{ salts: mass fracs in total solid } \\
\hline $\mathrm{KNO}_{3}$ & 2.109 & 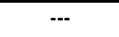 & $-\cdots$ & 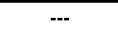 & $\cdots$ & $\cdots$ & $\cdots$ & $\begin{array}{ll}-- \\
\end{array}$ & --- & 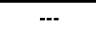 \\
\hline $\mathrm{Na}_{2} \mathrm{C}_{2} \mathrm{O}_{4}$ & 2.34 & $2.76 \%$ & --- & --- & $0.43 \%$ & $4.02 \%$ & $0.47 \%$ & $1.22 \%$ & $0.53 \%$ & $--\cdot$ \\
\hline $\mathrm{Na}_{2} \mathrm{CO}_{3} \cdot \mathrm{H}_{2} \mathrm{O}$ & 2.25 & $0.01 \%$ & $0.03 \%$ & $0.11 \%$ & --- & -- & --- & $0.20 \%$ & --- & $0.01 \%$ \\
\hline $\mathrm{Na}_{2} \mathrm{SO}_{4}$ & 2.68 & --- & --- & --- & --- & --- & --- & --- & --- & --- \\
\hline $\mathrm{Na}_{2} \mathrm{SO}_{4} \cdot 10 \mathrm{H}_{2} \mathrm{O}$ & 1.464 & --- & --- & --- & --- & $23.84 \%$ & $24.78 \%$ & --- & $22.29 \%$ & --- \\
\hline $\mathrm{Na}_{3} \mathrm{FSO}_{4}$ & 2.65 & --- & --- & --- & $0.33 \%$ & -- & --- & --- & --- & --- \\
\hline $\mathrm{Na}_{3} \mathrm{NO}_{3} \mathrm{SO}_{4} * \mathrm{H}_{2} \mathrm{O}$ & 2.3 & --- & --- & --- & --- & -- & --- & --- & --- & --- \\
\hline $\mathrm{Na}_{3} \mathrm{PO}_{+} \cdot 0.25 \mathrm{NaOH} \cdot 12 \mathrm{H}_{2} \mathrm{O}$ & 1.62 & --- & --- & --- & --- & --- & --- & --- & --- & --- \\
\hline $\mathrm{Na}_{3} \mathrm{PO}_{4} * 8 \mathrm{H}_{2} \mathrm{O}$ & 1.8 & --- & --- & --- & --- & -- & --- & --- & --- & --- \\
\hline $\mathrm{Na}_{4} \mathrm{P}_{2} \mathrm{O}_{7} \cdot 10 \mathrm{H}_{2} \mathrm{O}$ & 1.83 & --- & --- & --- & --- & -- & --- & --- & --- & $20.53 \%$ \\
\hline $\mathrm{Na}_{6}\left(\mathrm{SO}_{4}\right)_{2} \mathrm{CO}_{3}$ & 2.64 & --- & --- & --- & $23.66 \%$ & -- & --- & --- & --- & --- \\
\hline $\mathrm{NaF}$ & 2.78 & --- & --- & --- & --- & $0.76 \%$ & $0.66 \%$ & $2.40 \%$ & $0.67 \%$ & --- \\
\hline $\mathrm{Na}_{7} \mathrm{~F}\left(\mathrm{PO}_{4}\right)_{2} \bullet 19 \mathrm{H}_{2} \mathrm{O}$ & 1.75 & --- & --- & --- & --- & $50.74 \%$ & $50.44 \%$ & $5.67 \%$ & $48.05 \%$ & $51.36 \%$ \\
\hline $\mathrm{NaHCO}_{3}$ & 2.159 & --- & --- & --- & --- & -- & --- & --- & --- & --- \\
\hline $\mathrm{NaNO}_{2}$ & 2.168 & --- & --- & --- & --- & -- & --- & --- & --- & --- \\
\hline $\mathrm{NaNO}_{3}$ & 2.26 & --- & --- & --- & $33.88 \%$ & $4.27 \%$ & $7.57 \%$ & $53.08 \%$ & $12.85 \%$ & --- \\
\hline
\end{tabular}


Table C.1. (contd)

\begin{tabular}{|c|c|c|c|c|c|c|c|c|c|c|}
\hline & $\begin{array}{l}\text { Density } \\
(\mathrm{g} / \mathrm{mL})\end{array}$ & 241-B-107 & 241-B-108 & 241-B-109 & 241-B-110 & 241-B-111 & $241-B-112$ & 241-B-201 & 241-B-202 & 241-B-203 \\
\hline Total kg solids for tank & \multirow{9}{*}{ 11/a } & $4.44 \mathrm{E}+05$ & $3.93 \mathrm{E}+05$ & $7.37 \mathrm{E}+05$ & $2.22 \mathrm{E}+05$ & $1.84 \mathrm{E}+05$ & $3.92 \mathrm{E}+04$ & $3.63 \mathrm{E}+04$ & $1.75 \mathrm{E}+04$ & $2.34 \mathrm{E}+04$ \\
\hline Total kL solids for tank & & 2. $18 \mathrm{E}+02$ & $2.14 \mathrm{E}+02$ & $3.88 \mathrm{E}+02$ & $9.15 \mathrm{E}+01$ & $6.77 \mathrm{E}+01$ & $1.77 \mathrm{E}+01$ & $7.19 \mathrm{E}+00$ & $3.49 \mathrm{E}+00$ & $3.82 \mathrm{E}+00$ \\
\hline Vol. frac. solid phase in tank & & 0.357 & 0.612 & 0.819 & 0.099 & 0.074 & 0.134 & 0.062 & 0.031 & 0.020 \\
\hline Avg g/mL, all solid & & 2.036 & 1.839 & 1.901 & 2.425 & 2.717 & 2.221 & 5.056 & 5.026 & 6.142 \\
\hline Total $\mathrm{kg}$ non-salt solids for tank & & $8.87 \mathrm{E}+04$ & $8.35 \mathrm{E}+04$ & $1.57 \mathrm{E}+05$ & $1.14 \mathrm{E}+05$ & $1.18 \mathrm{E}+05$ & $1.81 \mathrm{E}+04$ & $3.28 \mathrm{E}+04$ & $1.70 \mathrm{E}+04$ & $2.31 \mathrm{E}+04$ \\
\hline Total kL non-salt solids for tank & & $3.02 \mathrm{E}+01$ & $3.35 \mathrm{E}+01$ & $5.92 \mathrm{E}+01$ & $2.93 \mathrm{E}+01$ & $3.23 \mathrm{E}+0 \mathrm{I}$ & $5.74 \mathrm{E}+00$ & $5.60 \mathrm{E}+00$ & $3.24 \mathrm{E}+00$ & $3.66 \mathrm{E}+00$ \\
\hline Wt. frac. non-salts in solid & & 0.200 & 0.213 & 0.214 & 0.513 & 0.641 & 0.460 & 0.902 & 0.967 & 0.985 \\
\hline Avg g/mL, non-salt solid & & 2.932 & 2.493 & 2.658 & 3.886 & 3.647 & 3.148 & 5.847 & 5.234 & 6.309 \\
\hline Vol. frac. non-salts in solid & & 0.139 & 0.157 & 0.153 & 0.320 & 0.477 & 0.325 & 0.780 & 0.928 & 0.959 \\
\hline \multicolumn{11}{|l|}{ non-salts: mass fracs in total solid } \\
\hline $\mathrm{Ag}$ & 10.5 & --- & --- & --- & --- & --- & --- & --- & --- & --- \\
\hline $\mathrm{Ag}_{2} \mathrm{O}$ & 7.143 & $0.00 \%$ & --- & --- & $0.03 \%$ & $0.00 \%$ & --- & $0.00 \%$ & $0.00 \%$ & $0.00 \%$ \\
\hline $\mathrm{Bi}_{2} \mathrm{O}_{3}$ & 8.9 & $1.94 \%$ & $0.25 \%$ & $0.47 \%$ & --- & -- & $8.04 \%$ & $44.05 \%$ & $29.75 \%$ & $45.07 \%$ \\
\hline $\mathrm{BiFeO}_{3}$ & 7.9 & $\ldots$ & $\ldots$ & $\ldots$ & $17.24 \%$ & $19.09 \%$ & $\ldots$ & $\ldots$ & -- & $\ldots$ \\
\hline $\mathrm{Ca}(\mathrm{OH})_{2}$ & 2.24 & $\cdots$ & $\cdots$ & $\cdots$ & $\cdots$ & $\cdots$ & 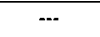 & 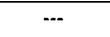 & $\cdots$ & $\cdots$ \\
\hline $\mathrm{Ca}_{5} \mathrm{OH}\left(\mathrm{PO}_{4}\right)_{3}$ & 3.14 & -- & $0.38 \%$ & $0.32 \%$ & $1.26 \%$ & -- & $1.38 \%$ & $0.63 \%$ & $1.96 \%$ & --. \\
\hline $\mathrm{CaC}_{2} \mathrm{O}_{4} * \mathrm{H}_{2} \mathrm{O}$ & 2.2 & --- & --- & --- & -- & --- & -- & --- & --- & --- \\
\hline $\mathrm{CaCO}_{3}$ & 2.71 & --- & --- & --- & --- & --- & --- & --- & --- & --- \\
\hline $\mathrm{CaF}_{2}$ & 3.18 & $0.18 \%$ & --- & --- & --- & $0.89 \%$ & --- & $4.57 \%$ & $8.15 \%$ & $0.42 \%$ \\
\hline $\mathrm{CrOOH}$ & 4.11 & $0.07 \%$ & $0.02 \%$ & $0.29 \%$ & $0.78 \%$ & $1.03 \%$ & $0.66 \%$ & $1.89 \%$ & $2.64 \%$ & $4.83 \%$ \\
\hline $\mathrm{FePO}_{4} \cdot 2 \mathrm{H}_{2} \mathrm{O}$ & 3.15 & --- & --- & --- & $27.41 \%$ & $24.94 \%$ & --- & --- & --- & --- \\
\hline $\mathrm{FeOOH}$ & 4.26 & $4.03 \%$ & $0.18 \%$ & $0.89 \%$ & --- & -- & $9.32 \%$ & $7.79 \%$ & $14.79 \%$ & $5.12 \%$ \\
\hline Gibbsite & 2.42 & $3.35 \%$ & $19.43 \%$ & $14.73 \%$ & --- & --- & $11.20 \%$ & --- & $2.46 \%$ & --- \\
\hline Boehmite & 3.01 & --- & --- & -- & --- & --- & --- & --- & -- & --- \\
\hline $\mathrm{HgO}$ (co-precip. with $\mathrm{Ag}_{2} \mathrm{O}$ ) & 7.143 & --- & --- & --- & --- & --- & --- & --- & --- & --- \\
\hline $\mathrm{KAISiO}_{4}$ & 2.61 & -- & --- & $0.05 \%$ & --- & --- & --- & $2.71 \%$ & $1.43 \%$ & $0.29 \%$ \\
\hline $\mathrm{La}(\mathrm{OH})_{3}$ & 2.3 & --- & --- & 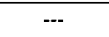 & --- & --- & --- & --- & --- & $0.83 \%$ \\
\hline $\mathrm{LaPO}_{4} \cdot 2 \mathrm{H}_{2} \mathrm{O}$ & 6.51 & $0.00 \%$ & $0.01 \%$ & $0.01 \%$ & $0.04 \%$ & $0.01 \%$ & $0.03 \%$ & $11.00 \%$ & $18.18 \%$ & $18.50 \%$ \\
\hline $\mathrm{Mn}_{3}\left(\mathrm{PO}_{4}\right)_{2}$ & 3.102 & $0.04 \%$ & --- & -- & -- & -- & -- & $2.20 \%$ & -- & -- \\
\hline $\mathrm{MnO}_{2}$ & 5.026 & -- & $0.00 \%$ & $0.08 \%$ & $0.06 \%$ & $0.08 \%$ & $0.07 \%$ & $14.10 \%$ & $15.45 \%$ & $21.82 \%$ \\
\hline $\mathrm{Na}_{2}\left(\mathrm{UO}_{2}\right)_{2}\left(\mathrm{PO}_{4}\right)_{2} \cdot 2 \mathrm{H}_{2} \mathrm{O}$ & 3.5 & --- & --- & --- & --- & --- & --- & --- & --- & --- \\
\hline $\mathrm{Na}_{2} \mathrm{U}_{2} \mathrm{O}_{7}$ & 5.617 & $0.47 \%$ & $0.27 \%$ & $1.54 \%$ & $0.17 \%$ & --- & $0.15 \%$ & $0.08 \%$ & $0.34 \%$ & $0.23 \%$ \\
\hline $\mathrm{NaAICO}_{3}(\mathrm{OH})_{2}$ & 2.42 & $5.10 \%$ & --- & --- & --- & $3.17 \%$ & --- & --- & -- & --- \\
\hline $\mathrm{NaAlSiO}_{4}$ & 2.365 & $4.64 \%$ & $0.49 \%$ & $2.74 \%$ & $3.65 \%$ & -- & $14.48 \%$ & --- & -- & -- \\
\hline $\mathrm{Ni}(\mathrm{OH})_{2}$ & 4.15 & $0.01 \%$ & $0.11 \%$ & $0.11 \%$ & $0.02 \%$ & -- & $0.09 \%$ & $0.30 \%$ & $0.26 \%$ & $0.28 \%$ \\
\hline $\mathrm{Ni}_{3}\left(\mathrm{PO}_{4}\right)$ & 3.93 & -- & $--\cdot$ & $\cdots$ & -- & --- & -- & $--\cdot$ & $\cdots$ & -- \\
\hline $\mathrm{NiC}_{2} \mathrm{O}_{4} \cdot 2 \mathrm{H}_{2} \mathrm{O}$ (on Fe phase) & 4.26 & --- & --- & --- & --- & $0.03 \%$ & --- & --- & --- & --- \\
\hline $\mathrm{Pb}(\mathrm{OH})_{2}$ & 7.1 & $0.10 \%$ & $0.11 \%$ & $0.10 \%$ & $0.38 \%$ & --- & $0.41 \%$ & $0.15 \%$ & $0.60 \%$ & $0.28 \%$ \\
\hline $\mathrm{Pb}_{3}\left(\mathrm{PO}_{4}\right)_{2}$ & 7.1 & -- & --- & --- & $\begin{array}{ll}-- \\
-\end{array}$ & --- & $\begin{array}{ll}-- \\
-1\end{array}$ & --- & -- & --- \\
\hline $\mathrm{PbCO}_{3}$ & 6.6 & -- & --- & --- & --- & $1.33 \%$ & --- & --- & --- & --- \\
\hline $\mathrm{Pu}(\mathrm{OH})_{4}$ (co-precip. on Fe phase) & 4.26 & $0.00 \%$ & $0.00 \%$ & $0.00 \%$ & $0.00 \%$ & $0.00 \%$ & $0.00 \%$ & $0.01 \%$ & $0.00 \%$ & $0.00 \%$ \\
\hline $\mathrm{PuO}_{2}$ & 11.43 & -- & -- & --- & --- & -- & -- & --- & -- & -- \\
\hline $\mathrm{SiO}_{2}$ & 2.6 & --- & $\begin{array}{l}-- \\
\end{array}$ & --- & --- & $13.27 \%$ & --- & --- & --- & --- \\
\hline $\mathrm{Sr}_{3}\left(\mathrm{PO}_{4}\right)_{2}$ & 3.5 & --- & --- & --- & --- & --- & --- & --- & --- & -- \\
\hline $\mathrm{SrCO}_{3}$ & 3.5 & $0.03 \%$ & $0.01 \%$ & $0.01 \%$ & $0.22 \%$ & $0.24 \%$ & $0.19 \%$ & $0.65 \%$ & $0.65 \%$ & $0.82 \%$ \\
\hline $\mathrm{ZrO}_{2}$ & 5.68 & $0.03 \%$ & $0.01 \%$ & $0.02 \%$ & $0.00 \%$ & $0.01 \%$ & $0.00 \%$ & $0.01 \%$ & -- & $0.00 \%$ \\
\hline \multicolumn{11}{|l|}{ salts: mass fracs in total solid } \\
\hline $\mathrm{KNO}_{3}$ & 2.109 & $-\cdots$ & $\begin{array}{ll}-\cdot \\
\end{array}$ & $\begin{array}{ll}\cdots \\
\end{array}$ & $-\cdots$ & 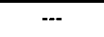 & $\begin{array}{c}-\cdot \\
\end{array}$ & 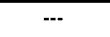 & $\begin{array}{c}-- \\
\end{array}$ & 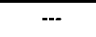 \\
\hline $\mathrm{Na}_{2} \mathrm{C}_{2} \mathrm{O}_{4}$ & 2.34 & $0.71 \%$ & $0.48 \%$ & $0.81 \%$ & $1.02 \%$ & $3.12 \%$ & $1.76 \%$ & $1.03 \%$ & $3.34 \%$ & --- \\
\hline $\mathrm{Na}_{2} \mathrm{CO}_{3} \cdot \mathrm{H}_{2} \mathrm{O}$ & 2.25 & $0.00 \%$ & $\cdots$ & $1.25 \%$ & $0.02 \%$ & $0.12 \%$ & -- & $8.81 \%$ & $0.00 \%$ & $1.50 \%$ \\
\hline $\mathrm{Na}_{2} \mathrm{SO}_{4}$ & 2.68 & -- & --- & --- & --- & -- & --- & --- & --- & -- \\
\hline $\mathrm{Na}_{2} \mathrm{SO}_{4} \cdot 10 \mathrm{H}_{2} \mathrm{O}$ & 1.464 & $2.88 \%$ & $26.90 \%$ & $16.02 \%$ & --- & --- & --- & --- & --- & --- \\
\hline $\mathrm{Na}_{3} \mathrm{FSO}_{4}$ & 2.65 & --- & --- & --- & --- & --- & --- & --- & --- & --- \\
\hline $\mathrm{Na}_{3} \mathrm{NO}_{3} \mathrm{SO}_{4} \cdot \mathrm{H}_{2} \mathrm{O}$ & 2.3 & --- & --- & --- & -- & -- & -- & --- & -- & --- \\
\hline $\mathrm{Na}_{3} \mathrm{PO}_{4} \cdot 0.25 \mathrm{NaOH} \cdot 12 \mathrm{H}_{2} \mathrm{O}$ & 1.62 & --- & --- & $22.79 \%$ & $7.42 \%$ & --- & --- & --- & --- & --- \\
\hline $\mathrm{Na}_{3} \mathrm{PO}_{4} \cdot 8 \mathrm{H}_{2} \mathrm{O}$ & 1.8 & --- & --- & --- & --- & --- & --- & --- & --- & --- \\
\hline $\mathrm{Na}_{4} \mathrm{P}_{2} \mathrm{O}_{7} \cdot 10 \mathrm{H}_{2} \mathrm{O}$ & 1.83 & --- & --- & --- & --- & $32.67 \%$ & --- & --- & --- & --- \\
\hline $\mathrm{Na}_{6}\left(\mathrm{SO}_{4}\right)_{2} \mathrm{CO}_{3}$ & 2.64 & --- &.- &.-- & --- & --- & --- & $\cdots$ & -- & --- \\
\hline $\mathrm{NaF}$ & 2.78 & -- & $5.56 \%$ & $4.35 \%$ & --- & --- & $0.90 \%$ & --- & --- & --- \\
\hline $\mathrm{Na}_{7} \mathrm{~F}\left(\mathrm{PO}_{4}\right)_{2} \cdot 19 \mathrm{H}_{2} \mathrm{O}$ & 1.75 & $48.12 \%$ & $38.77 \%$ & $17.84 \%$ & $40.28 \%$ & --- & $51.31 \%$ & --- & --- & --- \\
\hline $\mathrm{NaIICO}_{3}$ & 2.159 & -- & -.- & -.. & -.- & --- & -.. & $\cdots$ & --. & ..- \\
\hline $\mathrm{NaNO}_{2}$ & 2.168 & --- & --- & --- & --- & --- & --- & --- & --- & --- \\
\hline $\mathrm{NaNO}_{3}$ & 2.26 & $28.30 \%$ & $7.02 \%$ & $15.59 \%$ & --- & --- & -- & --- & --- & -- \\
\hline
\end{tabular}


Table C.1. (contd)

\begin{tabular}{|c|c|c|c|c|c|c|c|c|c|}
\hline & $\begin{array}{l}\text { Density } \\
(\mathrm{g} / \mathrm{mL})\end{array}$ & 241-B-204 & 241-BX-101 & 241-BX-102 & 241-BX-103 & 241-BX-104 & 241-BX-105 & 241-BX-106 & 241-BX-107 \\
\hline Total kg solids for tank & \multirow{9}{*}{ 11/a } & $2.52 \mathrm{E}+04$ & $1.20 \mathrm{E}+05$ & $2.26 \mathrm{E}+05$ & $1.16 \mathrm{E}+05$ & $3.38 \mathrm{E}+05$ & $1.57 \mathrm{E}+05$ & $1.48 \mathrm{E}+05$ & $6.06 \mathrm{E}+05$ \\
\hline Total kL solids for tank & & $3.89 \mathrm{E}+00$ & $4.86 \mathrm{E}+01$ & $8.90 \mathrm{E}+01$ & $4.68 \mathrm{E}+01$ & $1.26 \mathrm{E}+02$ & $7.90 \mathrm{E}+01$ & $7.27 \mathrm{E}+01$ & $2.89 \mathrm{E}+02$ \\
\hline Vol. frac. solid phase in tank & & 0.020 & 0.270 & 0.210 & 0.169 & 0.332 & 0.291 & 0.512 & 0.220 \\
\hline Avg g/mL, all solid & & 6.472 & 2.468 & 2.544 & 2.487 & 2.685 & 1.985 & 2.042 & 2.098 \\
\hline Total kg non-salt solids for tank & & $2.52 \mathrm{E}+04$ & $1.09 \mathrm{E}+05$ & $2.26 \mathrm{E}+05$ & $9.69 \mathrm{E}+04$ & $3.21 \mathrm{E}+05$ & $6.41 \mathrm{E}+04$ & $7.88 \mathrm{E}+04$ & $2.09 \mathrm{E}+05$ \\
\hline Total kL non-salt solids for tank & & $3.89 \mathrm{E}+00$ & $4.21 \mathrm{E}+0 \mathrm{l}$ & $8.90 \mathrm{E}+01$ & $3.72 \mathrm{E}+01$ & I. $18 \mathrm{E}+02$ & $2.21 \mathrm{E}+01$ & $3.05 \mathrm{E}+0 \mathrm{I}$ & $6.35 \mathrm{E}+0 \mathrm{l}$ \\
\hline Wt. frac. non-salts in solid & & 1.000 & 0.904 & 1.000 & 0.832 & 0.950 & 0.409 & 0.531 & 0.345 \\
\hline Avg g/mL, non-salt solid & & 6.472 & 2.580 & 2.544 & 2.603 & 2.724 & 2.906 & 2.583 & 3.297 \\
\hline Vol. frac. non-salts in solid & & 1.000 & 0.865 & 1.000 & 0.795 & 0.937 & 0.279 & 0.420 & 0.220 \\
\hline \multicolumn{10}{|l|}{ non-salts: mass fracs in total solid } \\
\hline $\mathrm{Ag}$ & 10.5 & --- & --- & --- & --- & -- & --- & --- & $\begin{array}{l}-- \\
\end{array}$ \\
\hline $\mathrm{Ag}_{2} \mathrm{O}$ & 7.143 & $0.01 \%$ & -- & --- & --- & $0.01 \%$ & --- & $0.01 \%$ & -- \\
\hline $\mathrm{Bi}_{2} \mathrm{O}_{3}$ & 8.9 & $48.50 \%$ & $0.51 \%$ & $0.36 \%$ & $0.41 \%$ & $0.12 \%$ & $0.26 \%$ & $0.20 \%$ & --- \\
\hline $\mathrm{BiFeO}_{3}$ & 7.9 & $\cdots$ & $\cdots$ & $\cdots$ & $\ldots$ & $--\cdot$ & $\ldots$ & $\cdots$ & $10.99 \%$ \\
\hline $\mathrm{Ca}(\mathrm{OH})_{2}$ & 2.24 & $\cdots$ & $\cdots$ & $\cdots$ & $\cdots$ & $\cdots$ & $\cdots$ & $\cdots$ & $\cdots$ \\
\hline $\mathrm{Ca}_{5} \mathrm{OH}\left(\mathrm{PO}_{4}\right)_{3}$ & 3.14 & -- & $0.98 \%$ & $0.75 \%$ & $1.16 \%$ & $0.85 \%$ & $2.86 \%$ & $3.69 \%$ & -- \\
\hline $\mathrm{CaC}_{2} \mathrm{O}_{4} * \mathrm{H}_{2} \mathrm{O}$ & 2.2 & --- & --- & --- & --- & --- & --- & --- & --- \\
\hline $\mathrm{CaCO}_{3}$ & 2.71 & --- & --- & --- & --- & -- & --- & -- & --- \\
\hline $\mathrm{CaF}_{2}$ & 3.18 & $0.47 \%$ & --- & $0.32 \%$ & --- & --- & --- & --- & $0.61 \%$ \\
\hline $\mathrm{CrOOH}$ & 4.11 & $4.72 \%$ & $\cdots$ & -.. & $0.05 \%$ & $1.14 \%$ & $4.23 \%$ & $2.49 \%$ & -.- \\
\hline $\mathrm{FePO}_{4} \cdot 2 \mathrm{H}_{2} \mathrm{O}$ & 3.15 & --- & --- & --- & --- & -- & --- & -- & $4.62 \%$ \\
\hline $\mathrm{FeOOH}$ & 4.26 & $5.31 \%$ & $8.91 \%$ & $6.25 \%$ & $8.79 \%$ & $1.53 \%$ & $3.00 \%$ & $2.24 \%$ & --- \\
\hline Gibbsite & 2.42 & --- & $60.05 \%$ & $32.76 \%$ & $44.67 \%$ & $73.17 \%$ & --- & --- & $6.86 \%$ \\
\hline Boehmite & 3.01 & --- & --- & --- & --- & --- & --- & -- & --- \\
\hline $\mathrm{HgO}$ (co-precip. with $\mathrm{Ag}_{2} \mathrm{O}$ ) & 7.143 & --- & $0.00 \%$ & --- & $0.01 \%$ & --- & $0.01 \%$ & $0.01 \%$ & --- \\
\hline $\mathrm{KAlSiO}_{4}$ & 2.61 & $0.29 \%$ & --- & $0.21 \%$ & -- & --- & $0.10 \%$ & $0.54 \%$ & --- \\
\hline $\mathrm{La}(\mathrm{OH})_{3}$ & 2.3 & $0.08 \%$ & $\begin{array}{ll}-- \\
\end{array}$ & --- & $\begin{array}{ll}-- \\
\end{array}$ & --- & --- & --- & --- \\
\hline $\mathrm{LaPO}_{4} \cdot 2 \mathrm{H}_{2} \mathrm{O}$ & 6.51 & $18.04 \%$ & $0.03 \%$ & $0.03 \%$ & $0.03 \%$ & $0.05 \%$ & $0.16 \%$ & $0.04 \%$ & $0.00 \%$ \\
\hline $\mathrm{Mn}_{3}\left(\mathrm{PO}_{4}\right)_{2}$ & 3.102 & -- & $0.45 \%$ & $0.34 \%$ & -- & -- & --- & --- & $0.04 \%$ \\
\hline $\mathrm{MnO}_{2}$ & 5.026 & $21.22 \%$ & $0.02 \%$ & $--\cdot$ & $0.29 \%$ & $0.35 \%$ & $0.74 \%$ & $0.55 \%$ & -- \\
\hline $\mathrm{Na}_{2}\left(\mathrm{UO}_{2}\right)_{2}\left(\mathrm{PO}_{4}\right)_{2} \cdot 2 \mathrm{H}_{2} \mathrm{O}$ & 3.5 & $\begin{array}{ll}-- \\
\end{array}$ & --- & --- & $\begin{array}{ll}-- \\
\end{array}$ & --- & --- & --- & --- \\
\hline $\mathrm{Na}_{2} \mathrm{U}_{2} \mathrm{O}_{7}$ & 5.617 & $0.20 \%$ & $0.96 \%$ & $0.05 \%$ & $1.45 \%$ & $15.39 \%$ & $4.87 \%$ & $1.37 \%$ & $0.84 \%$ \\
\hline $\mathrm{NaAICO}_{3}(\mathrm{OH})_{2}$ & 2.42 & -- & $9.38 \%$ & $9.90 \%$ & $19.10 \%$ & -- & --- & -- & $0.32 \%$ \\
\hline $\mathrm{NaAlSiO}_{4}$ & 2.365 & --- & $7.83 \%$ & $25.69 \%$ & $6.07 \%$ & $1.95 \%$ & $24.22 \%$ & $41.61 \%$ & $10.03 \%$ \\
\hline $\mathrm{Ni}(\mathrm{OH})_{2}$ & 4.15 & $0.33 \%$ & $0.46 \%$ & $0.17 \%$ & $0.38 \%$ & $0.05 \%$ & $0.07 \%$ & $0.05 \%$ & $0.01 \%$ \\
\hline $\mathrm{Ni}_{3}\left(\mathrm{PO}_{4}\right)$ & 3.93 & $\cdots$ & -- & -- & -- & -- & $\cdots$ & -- & -- \\
\hline $\mathrm{NiC}_{2} \mathrm{O}_{4} \cdot 2 \mathrm{H}_{2} \mathrm{O}$ (on Fe phase) & 4.26 & -- & --- & $0.32 \%$ & --- & --- & --- & --- & --- \\
\hline $\mathrm{Pb}(\mathrm{OH})_{2}$ & 7.1 & $0.24 \%$ & $0.39 \%$ & --- & $0.36 \%$ & $0.18 \%$ & $0.20 \%$ & $0.16 \%$ & $0.02 \%$ \\
\hline $\mathrm{Pb}_{3}\left(\mathrm{PO}_{4}\right)_{2}$ & 7.1 & -- & --- & $0.29 \%$ & --- & -- & --- & -- & --- \\
\hline $\mathrm{PbCO}_{3}$ & 6.6 & -- & --- & --- & --- & -- & --- & -- & --- \\
\hline $\mathrm{Pu}(\mathrm{OH})_{4}$ (co-precip. on Fe phase) & 4.26 & $0.00 \%$ & $0.00 \%$ & $0.00 \%$ & $0.01 \%$ & $0.00 \%$ & $0.00 \%$ & $0.00 \%$ & $0.00 \%$ \\
\hline $\mathrm{PuO}_{2}$ & 11.43 & --- & --- & --- & --- & --- & --- & -- & --- \\
\hline $\mathrm{SiO}_{2}$ & 2.6 & --- & --- & $22.28 \%$ & --- & --- & --- & --- & --- \\
\hline $\mathrm{Sr}_{3}\left(\mathrm{PO}_{4}\right)_{2}$ & 3.5 & --- & --- & $0.01 \%$ & --- & -- & --- & --- & $0.09 \%$ \\
\hline $\mathrm{SrCO}_{3}$ & 3.5 & $0.58 \%$ & $0.07 \%$ & --- & $0.11 \%$ & $0.02 \%$ & $0.02 \%$ & $0.02 \%$ & --- \\
\hline $\mathrm{ZrO}_{2}$ & 5.68 & $0.00 \%$ & $0.36 \%$ & $0.27 \%$ & $0.29 \%$ & $0.22 \%$ & $0.14 \%$ & $0.11 \%$ & $0.06 \%$ \\
\hline \multicolumn{10}{|l|}{ salts: mass fracs in total solid } \\
\hline $\mathrm{KNO}_{3}$ & 2.109 & $\overline{c--}$ & $\bar{c}$ & 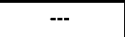 & $\cdots$ & $\cdots$ & $\cdots$ & $-\cdots$ & $-\cdots$ \\
\hline $\mathrm{Na}_{2} \mathrm{C}_{2} \mathrm{O}_{4}$ & 2.34 & --- & --- & --- & $9.47 \%$ & $3.33 \%$ & --- & $1.80 \%$ & $0.29 \%$ \\
\hline $\mathrm{Na}_{2} \mathrm{CO}_{3} \cdot \mathrm{H}_{2} \mathrm{O}$ & 2.25 & $0.00 \%$ & -- & $--\cdot$ & -.- & $0.01 \%$ & $\cdots$ & $0.02 \%$ & $-\cdot$ \\
\hline $\mathrm{Na}_{2} \mathrm{SO}_{4}$ & 2.68 & -- & --- & --- & --- & -- & --- & -- & --- \\
\hline $\mathrm{Na}_{2} \mathrm{SO}_{4} \cdot 10 \mathrm{H}_{2} \mathrm{O}$ & 1.464 & --- & --- & --- & --- & --- & --- & --- & --- \\
\hline $\mathrm{Na}_{3} \mathrm{FSO}_{4}$ & 2.65 & --- & --- & --- & --- & --- & --- & --- & --- \\
\hline $\mathrm{Na}_{3} \mathrm{NO}_{3} \mathrm{SO}_{4} \cdot \mathrm{H}_{2} \mathrm{O}$ & 2.3 & -- & --- & --- & --- & -- & --- & -- & --- \\
\hline $\mathrm{Na}_{3} \mathrm{PO}_{4} \cdot 0.25 \mathrm{NaOH} \cdot 12 \mathrm{H}_{2} \mathrm{O}$ & 1.62 & --- & --- & --- & --- & --- & $54.97 \%$ & $41.00 \%$ & --- \\
\hline $\mathrm{Na}_{3} \mathrm{PO}_{4} \cdot 8 \mathrm{H}_{2} \mathrm{O}$ & 1.8 & --- & --- & --- & --- & --- & $\begin{array}{ll}-- \\
-1\end{array}$ & -- & $\begin{array}{ll}-- \\
-1\end{array}$ \\
\hline $\mathrm{Na}_{4} \mathrm{P}_{2} \mathrm{O}_{7} \cdot 10 \mathrm{H}_{2} \mathrm{O}$ & 1.83 & --- & --- & --- & --- & --- & --- & -- & --- \\
\hline $\mathrm{Na}_{6}\left(\mathrm{SO}_{4}\right)_{2} \mathrm{CO}_{3}$ & 2.64 & --- & --- & --- & --- & --- & $--\cdot$ & --- & -- \\
\hline $\mathrm{NaF}$ & 2.78 & --- & --- & --- & --- & --- & --- & --- & $0.87 \%$ \\
\hline $\mathrm{Na}_{7} \mathrm{~F}\left(\mathrm{PO}_{4}\right)_{2} \bullet 19 \mathrm{H}_{2} \mathrm{O}$ & 1.75 & --- & $9.58 \%$ & --- & $7.35 \%$ & $1.63 \%$ & $4.14 \%$ & $4.10 \%$ & $64.35 \%$ \\
\hline $\mathrm{NaIICO}_{3}$ & 2.159 & -- & $\cdots$ & $-\cdot$ & $--\cdot$ & -- & $\cdots$ & -- & $\cdots$ \\
\hline $\mathrm{NaNO}_{2}$ & 2.168 & --- & --- & --- & --- & --- & --- & --- & --- \\
\hline $\mathrm{NaNO}_{3}$ & 2.26 & -- & --- & --- & --- & --- & --- & --- & --- \\
\hline
\end{tabular}


Table C.1. (contd)

\begin{tabular}{|c|c|c|c|c|c|c|c|c|c|}
\hline & $\begin{array}{l}\text { Density } \\
(\mathrm{g} / \mathrm{mL})\end{array}$ & 241-BX-108 & 241-BX-109 & $241-B X-110$ & 241-BX-111 & 241-BX-112 & 241-BY-101 & 241-BY-102 & 241-BY-103 \\
\hline Total kg solids for tank & \multirow{9}{*}{$11 / a$} & $1.11 \mathrm{E}+05$ & $2.22 \mathrm{E}+05$ & $1.03 \mathrm{E}+06$ & $8.25 \mathrm{E}+05$ & $2.59 \mathrm{E}+05$ & $1.92 \mathrm{E}+06$ & $5.80 \mathrm{E}+05$ & $1.77 \mathbf{E}+06$ \\
\hline Total kL solids for tank & & $5.27 \mathrm{E}+01$ & $1.07 \mathrm{E}+02$ & $4.74 \mathrm{E}+02$ & $3.76 \mathrm{E}+02$ & $1.25 \mathrm{E}+02$ & $9.37 \mathrm{E}+02$ & $2.64 \mathrm{E}+02$ & $8.02 \mathrm{E}+02$ \\
\hline Vol. frac. solid phase in tank & & 0.443 & 0.147 & 0.611 & 0.526 & 0.201 & 0.670 & 0.252 & 0.509 \\
\hline Avg g/mL, all solid & & 2.111 & 2.075 & 2.173 & 2.196 & 2.075 & 2.051 & 2.197 & 2.214 \\
\hline Total kg non-salt solids for tank & & $2.33 \mathrm{E}+04$ & $8.43 \mathrm{E}+04$ & $1.39 \mathrm{E}+05$ & $6.50 \mathrm{E}+04$ & $8.22 \mathrm{E}+04$ & $1.90 \mathrm{E}+05$ & $7.07 \mathrm{E}+04$ & $2.63 \mathrm{E}+05$ \\
\hline Total kL non-salt solids for tank & & $7.12 \mathrm{E}+00$ & $2.32 \mathrm{E}+01$ & $4.88 \mathrm{E}+0 \mathrm{I}$ & $2.36 \mathrm{E}+01$ & $2.63 \mathrm{E}+01$ & $7.13 \mathrm{E}+01$ & $2.72 \mathrm{E}+01$ & $1.05 \mathrm{E}+02$ \\
\hline Wt. frac. non-salts in solid & & 0.209 & 0.379 & 0.135 & 0.079 & 0.317 & 0.099 & 0.122 & 0.148 \\
\hline Avg g/mL, non-salt solid & & 3.264 & 3.632 & 2.846 & 2.760 & 3.130 & 2.668 & 2.600 & 2.513 \\
\hline Vol. frac. non-salts in solid & & 0.135 & 0.216 & 0.103 & $0.06 \hat{3}$ & 0.210 & 0.076 & 0.103 & 0.131 \\
\hline \multicolumn{10}{|l|}{ non-salts: mass fracs in total solid } \\
\hline Ag & 10.5 & --- & --- & --- & --- & --- & --- & --- & --- \\
\hline $\mathrm{Ag}_{2} \mathrm{O}$ & 7.143 & --- & $0.00 \%$ & --- & -- & $0.01 \%$ & --- & --- & -- \\
\hline $\mathrm{Bi}_{2} \mathrm{O}_{3}$ & 8.9 & $1.80 \%$ & $0.20 \%$ & $1.32 \%$ & $0.54 \%$ & --- & $0.01 \%$ & --- & $0.02 \%$ \\
\hline $\mathrm{BiFeO}_{3}$ & 7.9 & $\ldots$ & $\ldots$ & -.- & $\ldots$ & $8.34 \%$ & $\ldots$ & $\ldots$ & $\ldots$ \\
\hline $\mathrm{Ca}(\mathrm{OH})_{2}$ & 2.24 & $\cdots$ & $\cdots$ & $\cdots$ & $\cdots$ & 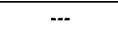 & $\cdots$ & $\cdots$ & $\cdots$ \\
\hline $\mathrm{Ca}_{5} \mathrm{OH}\left(\mathrm{PO}_{4}\right)_{3}$ & 3.14 & $1.27 \%$ & $3.41 \%$ & $0.23 \%$ & $0.07 \%$ & -- & $0.66 \%$ & $\cdots$ & $0.22 \%$ \\
\hline $\mathrm{CaC}_{2} \mathrm{O}_{4} \cdot \mathrm{H}_{2} \mathrm{O}$ & 2.2 & -- & --- & --- & --- & --- & --- & --- & -- \\
\hline $\mathrm{CaCO}_{3}$ & 2.71 & --- & --- & -- & --- & --- & --- & --- & -- \\
\hline $\mathrm{CaF}_{2}$ & 3.18 & --- & --- & --- & --- & $0.71 \%$ & --- & $0.28 \%$ & --- \\
\hline $\mathrm{CrOOH}$ & 4.11 & $\cdots$ & $\cdots$ & $0.82 \%$ & $0.47 \%$ & $0.63 \%$ & $0.27 \%$ & $0.58 \%$ & $0.42 \%$ \\
\hline $\mathrm{FePO}_{4} \cdot 2 \mathrm{H}_{2} \mathrm{O}$ & 3.15 & -- & --- & $2.27 \%$ & $1.33 \%$ & $3.82 \%$ & $1.06 \%$ & $0.87 \%$ & $0.80 \%$ \\
\hline $\mathrm{FeOOH}$ & 4.26 & $6.44 \%$ & $16.97 \%$ & --- & --- & --- & --- & --- & -- \\
\hline Gibbsite & 2.42 & $0.70 \%$ & $2.16 \%$ & $6.32 \%$ & $4.19 \%$ & $3.40 \%$ & $4.36 \%$ & $7.92 \%$ & $12.14 \%$ \\
\hline Boehmite & 3.01 & --- & --- & --- & --- & --- & --- & --- & -- \\
\hline $\mathrm{HgO}$ (co-precip. with $\mathrm{Ag}_{2} \mathrm{O}$ ) & 7.143 & $0.02 \%$ & $0.06 \%$ & --- & --- & --- & --- & --- & --- \\
\hline $\mathrm{KAlSiO}_{4}$ & 2.61 & --- & --- & $0.17 \%$ & -- & --- & $0.51 \%$ & $2.20 \%$ & $0.28 \%$ \\
\hline $\mathrm{La}(\mathrm{OH})_{3}$ & 2.3 & -- & --- & --- & $0.00 \%$ & --- & $0.00 \%$ & --- & -- \\
\hline $\mathrm{LaPO}_{4} \cdot 2 \mathrm{H}_{2} \mathrm{O}$ & 6.51 & $0.00 \%$ & --- & $0.00 \%$ & -- & $0.00 \%$ & --- & $0.00 \%$ & $0.00 \%$ \\
\hline $\mathrm{Mn}_{3}\left(\mathrm{PO}_{4}\right)_{2}$ & 3.102 & $0.06 \%$ & $0.15 \%$ & --- & --- & -- & --- & -- & -- \\
\hline $\mathrm{MnO}_{2}$ & 5.026 & -- & --- & --- & --. & $0.16 \%$ & $\ldots$ & --- & $\ldots$ \\
\hline $\mathrm{Na}_{2}\left(\mathrm{UO}_{2}\right)_{2}\left(\mathrm{PO}_{4}\right)_{2} \cdot 2 \mathrm{H}_{2} \mathrm{O}$ & 3.5 & $3.01 \%$ & $12.66 \%$ & --- & --- & --- & --- & $\begin{array}{ll}-- \\
\end{array}$ & --- \\
\hline $\mathrm{Na}_{2} \mathrm{U}_{2} \mathrm{O}_{7}$ & 5.617 & -- & --- & $0.23 \%$ & $0.12 \%$ & $0.44 \%$ & $0.50 \%$ & $0.18 \%$ & $0.11 \%$ \\
\hline $\mathrm{NaAICO}_{3}(\mathrm{OH})_{2}$ & 2.42 & $3.99 \%$ & --- & -- & --- & --- & --- & --- & -- \\
\hline $\mathrm{NaAlSiO}_{4}$ & 2.365 & $3.29 \%$ & $1.32 \%$ & $2.03 \%$ & $1.12 \%$ & $14.03 \%$ & $2.21 \%$ & --- & $0.72 \%$ \\
\hline $\mathrm{Ni}(\mathrm{OH})_{2}$ & 4.15 & $0.02 \%$ & $0.06 \%$ & $0.02 \%$ & $0.00 \%$ & $0.00 \%$ & $0.24 \%$ & $0.08 \%$ & $0.05 \%$ \\
\hline $\mathrm{Ni}_{3}\left(\mathrm{PO}_{4}\right)$ & 3.93 & -. & $-\cdots$ & $-\cdots$ & -- & -.. & $-\cdot$ & -. & -. \\
\hline $\mathrm{NiC}_{2} \mathrm{O}_{4} \cdot 2 \mathrm{H}_{2} \mathrm{O}$ (on Fe phase) & 4.26 & --- & --- & --- & --- & --- & --- & --- & --- \\
\hline $\mathrm{Pb}(\mathrm{OH})_{2}$ & 7.1 & $0.11 \%$ & $0.39 \%$ & $0.02 \%$ & $0.02 \%$ & $0.06 \%$ & $0.04 \%$ & $0.03 \%$ & $0.03 \%$ \\
\hline $\mathrm{Pb}_{3}\left(\mathrm{PO}_{4}\right)_{2}$ & 7.1 & --- & --- & -- & $\begin{array}{c}-- \\
-\end{array}$ & --- & 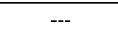 & --- & $\begin{array}{ll}-- \\
-1\end{array}$ \\
\hline $\mathrm{PbCO}_{3}$ & 6.6 & -- & --- & -- & --- & --- & --- & --- & -- \\
\hline $\mathrm{Pu}(\mathrm{OH})_{4}$ (co-precip. on Fe phase) & 4.26 & $0.00 \%$ & $0.00 \%$ & $0.00 \%$ & $0.00 \%$ & $0.00 \%$ & $0.00 \%$ & $0.00 \%$ & $0.00 \%$ \\
\hline $\mathrm{PuO}_{2}$ & 11.43 & -- & --- & -- & --- & --- & --- & --- & -- \\
\hline $\mathrm{SiO}_{2}$ & 2.6 & $\begin{array}{l}-- \\
\end{array}$ & --- & --- & $\begin{array}{l}-- \\
\end{array}$ & --- & --- & --- & --- \\
\hline $\mathrm{Sr}_{3}\left(\mathrm{PO}_{4}\right)_{2}$ & 3.5 & --- & $0.51 \%$ & --- & --- & --- & --- & --- & --- \\
\hline $\mathrm{SrCO}_{3}$ & 3.5 & $0.17 \%$ & --- & $0.03 \%$ & $0.01 \%$ & $0.07 \%$ & $0.03 \%$ & $0.06 \%$ & $0.03 \%$ \\
\hline $\mathrm{ZrO}_{2}$ & 5.68 & $0.01 \%$ & 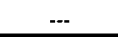 & $0.01 \%$ & $0.00 \%$ & $0.02 \%$ & $0.00 \%$ & $0.00 \%$ & $0.01 \%$ \\
\hline \multicolumn{10}{|l|}{ salts: mass fracs in total solid } \\
\hline $\mathrm{KNO}_{3}$ & 2.109 & $+\cdots$ & $\cdots$ & $-\cdots$ & $+\cdots$ & $-\cdots$ & $\cdots$ & $\begin{array}{ll}-\cdot \\
\end{array}$ & $-\cdots$ \\
\hline $\mathrm{Na}_{2} \mathrm{C}_{2} \mathrm{O}_{4}$ & 2.34 & $0.37 \%$ & $0.79 \%$ & $0.55 \%$ & $0.50 \%$ & $0.77 \%$ & $1.15 \%$ & $5.37 \%$ & $10.60 \%$ \\
\hline $\mathrm{Na}_{2} \mathrm{CO}_{3} \cdot \mathrm{H}_{2} \mathrm{O}$ & 2.25 & -- & $0.00 \%$ & -- & $0.09 \%$ & $0.01 \%$ & $2.19 \%$ & $17.11 \%$ & $16.27 \%$ \\
\hline $\mathrm{Na}_{2} \mathrm{SO}_{4}$ & 2.68 & -- & --- & --- & --- & --- & --- & --- & -- \\
\hline $\mathrm{Na}_{2} \mathrm{SO}_{4} \cdot 10 \mathrm{H}_{2} \mathrm{O}$ & 1.464 & -- & --- & -- & --- & --- & --- & --- & -- \\
\hline $\mathrm{Na}_{3} \mathrm{FSO}_{4}$ & 2.65 & -- & --- & $2.86 \%$ & --- & --- & $0.47 \%$ & --- & $4.78 \%$ \\
\hline $\mathrm{Na}_{3} \mathrm{NO}_{3} \mathrm{SO}_{4} \cdot \mathrm{H}_{2} \mathrm{O}$ & 2.3 & -- & $0.19 \%$ & -- & --- & --- & --- & --- & -- \\
\hline $\mathrm{Na}_{3} \mathrm{PO}_{4} \bullet 0.25 \mathrm{NaOH} \bullet 12 \mathrm{H}_{2} \mathrm{O}$ & 1.62 & --- & $55.67 \%$ & --- & --- & --- & $12.00 \%$ & --- & --- \\
\hline $\mathrm{Na}_{3} \mathrm{PO}_{4} \bullet 8 \mathrm{H}_{2} \mathrm{O}$ & 1.8 & -- & --- & -- & $\begin{array}{c}-- \\
-1\end{array}$ & --- & --- & --- & -- \\
\hline $\mathrm{Na}_{4} \mathrm{P}_{2} \mathrm{O}_{7} \cdot 10 \mathrm{H}_{2} \mathrm{O}$ & 1.83 & $20.71 \%$ & $5.47 \%$ & --- & --- & --- & --- & --- & --- \\
\hline $\mathrm{Na}_{6}\left(\mathrm{SO}_{4}\right)_{2} \mathrm{CO}_{3}$ & 2.64 & --- & --- &.-- & -- & --. & --- & -- & --- \\
\hline $\mathrm{NaF}$ & 2.78 & -- & --- & $0.07 \%$ & $0.48 \%$ & $3.99 \%$ & --- & $9.71 \%$ & $2.03 \%$ \\
\hline $\mathrm{Na}_{7} \mathrm{~F}\left(\mathrm{PO}_{4}\right)_{2} \cdot 19 \mathrm{H}_{2} \mathrm{O}$ & 1.75 & $29.54 \%$ & $\begin{array}{l}-- \\
\end{array}$ & $24.74 \%$ & $15.21 \%$ & $63.54 \%$ & $24.23 \%$ & $21.97 \%$ & $16.96 \%$ \\
\hline $\mathrm{NaIICO}_{3}$ & 2.159 &.-- &.-- & -- & -- & $\cdots$ & $\cdots$ & $\cdots$ & -- \\
\hline $\mathrm{NaNO}_{2}$ & 2.168 & -- & --- & --- & --- & --- & --- & -- & -- \\
\hline $\mathrm{NaNO}_{3}$ & 2.26 & $28.50 \%$ & --- & $58.31 \%$ & $75.84 \%$ & --- & $50.08 \%$ & $33.64 \%$ & $34.52 \%$ \\
\hline
\end{tabular}


Table C.1. (contd)

\begin{tabular}{|c|c|c|c|c|c|c|c|c|c|}
\hline & $\begin{array}{l}\text { Density } \\
(\mathrm{g} / \mathrm{mL})\end{array}$ & 241-BY-104 & 241-BY-105 & 241-BY-106 & 241-BY-107 & 241-BY-108 & 241-BY-109 & 241-BY-110 & 241-BY-111 \\
\hline Total kg solids for tank & \multirow{9}{*}{ 11/a } & $1.08 \mathrm{E}+06$ & $2.26 \mathrm{E}+06$ & $1.12 \mathrm{E}+06$ & $6.13 \mathrm{E}+05$ & $8.35 \mathrm{E}+05$ & $7.31 E+05$ & $9.71 \mathrm{E}+05$ & $1.32 \mathrm{E}+06$ \\
\hline Total kL solids for tank & & $4.54 \mathrm{E}+02$ & $9.68 \mathrm{E}+02$ & $4.80 \mathrm{E}+02$ & $2.64 \mathrm{E}+02$ & $3.49 \mathrm{E}+02$ & $3.33 \mathrm{E}+02$ & $4.06 \mathrm{E}+02$ & $5.82 \mathrm{E}+02$ \\
\hline Vol. frac. solid phase in tank & & 0.334 & 0.523 & 0.236 & 0.257 & 0.415 & 0.318 & 0.294 & 0.509 \\
\hline Avg g/mL, all solid & & 2.383 & 2.336 & 2.344 & 2.324 & 2.391 & 2.193 & 2.389 & 2.272 \\
\hline Total kg non-salt solids for tank & & $2.65 \mathrm{E}+05$ & $4.33 \mathrm{E}+05$ & $1.86 \mathrm{E}+05$ & $1.51 \mathrm{E}+05$ & $2.98 \mathrm{E}+05$ & $1.97 \mathrm{E}+05$ & $1.96 \mathrm{E}+05$ & $2.97 \mathbf{E}+05$ \\
\hline Total kL non-salt solids for tank & & $9.76 \mathrm{E}+01$ & $1.62 \mathrm{E}+02$ & $6.85 \mathrm{E}+01$ & $5.86 \mathrm{E}+01$ & $1.12 \mathrm{E}+02$ & $8.04 \mathrm{E}+01$ & $6.82 \mathrm{E}+01$ & $1.21 \mathbf{E}+02$ \\
\hline Wt. frac. non-salts in solid & & 0.245 & 0.192 & 0.165 & 0.246 & 0.357 & 0.270 & 0.202 & 0.225 \\
\hline Avg g/mL, non-salt solid & & 2.711 & 2.678 & 2.709 & 2.573 & 2.664 & 2.454 & 2.875 & 2.456 \\
\hline Vol. frac. non-salts in solid & & 0.215 & 0.167 & 0.143 & 0.222 & 0.320 & 0.241 & 0.168 & 0.208 \\
\hline \multicolumn{10}{|l|}{ non-salts: mass fracs in total solid } \\
\hline $\mathrm{Ag}$ & 10.5 & -- & --- & --- & --- & --- & --- & --- & -- \\
\hline $\mathrm{Ag}_{2} \mathrm{O}$ & 7.143 & -- & $0.00 \%$ & --- & -- & -- & $0.00 \%$ & -- & -- \\
\hline $\mathrm{Bi}_{2} \mathrm{O}_{3}$ & 8.9 & $0.07 \%$ & $0.05 \%$ & $0.05 \%$ & $0.05 \%$ & $0.05 \%$ & $0.01 \%$ & $0.07 \%$ & $0.01 \%$ \\
\hline $\mathrm{BiFeO}_{3}$ & 7.9 & $\cdots$ & $\ldots$ & -- & $\cdots$ & $--\cdot$ & $-\cdots$ & $\cdots$ & $\cdots$ \\
\hline $\mathrm{Ca}(\mathrm{OH})_{2}$ & 2.24 & $\cdots$ & $\cdots$ & $\cdots$ & $\cdots$ & $\cdots$ & $\cdots$ & $\cdots$ & $\cdots$ \\
\hline $\mathrm{Ca}_{5} \mathrm{OH}\left(\mathrm{PO}_{4}\right)_{3}$ & 3.14 & $0.74 \%$ & $3.57 \%$ & $0.57 \%$ & $0.61 \%$ & $1.51 \%$ & $0.14 \%$ & $1.92 \%$ & $0.20 \%$ \\
\hline $\mathrm{CaC}_{2} \mathrm{O}_{4} * \mathrm{H}_{2} \mathrm{O}$ & 2.2 & -- & --- & --- & -- & --- & --- & -- & -- \\
\hline $\mathrm{CaCO}_{3}$ & 2.71 & -- & $0.76 \%$ & --- & --- & --- & --- & --- & -- \\
\hline $\mathrm{CaF}_{2}$ & 3.18 & --- & $0.26 \%$ & --- & --- & --- & --- & --- & -- \\
\hline $\mathrm{CrOOH}$ & 4.11 & $0.70 \%$ & $0.09 \%$ & $0.33 \%$ & $0.01 \%$ & $0.06 \%$ & $0.44 \%$ & $0.64 \%$ & $0.12 \%$ \\
\hline $\mathrm{FePO}_{4} \cdot 2 \mathrm{H}_{2} \mathrm{O}$ & 3.15 & $3.84 \%$ & --- & $2.21 \%$ & $2.49 \%$ & $4.17 \%$ & $0.59 \%$ & $4.29 \%$ & $2.53 \%$ \\
\hline $\mathrm{FeOOH}$ & 4.26 & --- & $0.82 \%$ & --- & --- & --- & --- & --- & --- \\
\hline Gibbsite & 2.42 & $15.78 \%$ & $6.82 \%$ & $10.98 \%$ & $18.70 \%$ & $25.31 \%$ & $21.10 \%$ & $9.56 \%$ & --- \\
\hline Boehmite & 3.01 & --- & --- & --- & --- & --- & --- & --- & -- \\
\hline $\mathrm{HgO}$ (co-precip. with $\mathrm{Ag}_{2} \mathrm{O}$ ) & 7.143 & $0.00 \%$ & $0.00 \%$ & --- & --- & --- & --- & --- & --- \\
\hline $\mathrm{KAlSiO}_{4}$ & 2.61 & --- & $\cdots$ & --- & -- & --- & --- & --- & $\ldots$ \\
\hline $\mathrm{La}(\mathrm{OH})_{3}$ & 2.3 & $\begin{array}{ll}-- \\
\end{array}$ & $\begin{array}{l}-- \\
\end{array}$ & --- & $\begin{array}{ll}-- \\
\end{array}$ & $0.00 \%$ & $\begin{array}{ll}-- \\
\end{array}$ & --- & $0.00 \%$ \\
\hline $\mathrm{LaPO}_{4} \cdot 2 \mathrm{H}_{2} \mathrm{O}$ & 6.51 & $0.01 \%$ & $0.00 \%$ & $0.00 \%$ & $0.00 \%$ & --- & $0.00 \%$ & $0.00 \%$ & -- \\
\hline $\mathrm{Mn}_{3}\left(\mathrm{PO}_{4}\right)_{2}$ & 3.102 & -- & --- & --- & -- & -- & --- & --- & -- \\
\hline $\mathrm{MnO}_{2}$ & 5.026 & $0.02 \%$ & $0.01 \%$ & -.- & --. & -.. & $0.02 \%$ & --- & -- \\
\hline $\mathrm{Na}_{2}\left(\mathrm{UO}_{2}\right)_{2}\left(\mathrm{PO}_{4}\right)_{2} \cdot 2 \mathrm{H}_{2} \mathrm{O}$ & 3.5 & $\begin{array}{ll}-- \\
\end{array}$ & $\begin{array}{ll}-- \\
\end{array}$ & --- & --- & --- & $\begin{array}{ll}-- \\
\end{array}$ & --- & -- \\
\hline $\mathrm{Na}_{2} \mathrm{U}_{2} \mathrm{O}_{7}$ & 5.617 & $1.25 \%$ & $0.60 \%$ & $0.76 \%$ & $0.93 \%$ & $2.23 \%$ & $0.11 \%$ & $1.53 \%$ & $0.10 \%$ \\
\hline $\mathrm{NaAICO}_{3}(\mathrm{OH})_{2}$ & 2.42 & --- & --- & -- & --- & --- & --- & --- & -- \\
\hline $\mathrm{NaAlSiO}_{4}$ & 2.365 & $0.64 \%$ & $5.54 \%$ & $0.29 \%$ & $1.23 \%$ & $0.57 \%$ & $4.46 \%$ & $0.69 \%$ & $19.45 \%$ \\
\hline $\mathrm{Ni}(\mathrm{OH})_{2}$ & 4.15 & $0.46 \%$ & $0.22 \%$ & $0.88 \%$ & $0.21 \%$ & $0.60 \%$ & $0.05 \%$ & $0.73 \%$ & $0.04 \%$ \\
\hline $\mathrm{Ni}_{3}\left(\mathrm{PO}_{4}\right)$ & 3.93 & -- & -- & -- & -- & -- & -- & $\cdots$ & -- \\
\hline $\mathrm{NiC}_{2} \mathrm{O}_{4} \cdot 2 \mathrm{H}_{2} \mathrm{O}$ (on $\mathrm{Fe}$ phase) & 4.26 & --- & --- & --- & --- & --- & --- & -- & -- \\
\hline $\mathrm{Pb}(\mathrm{OH})_{2}$ & 7.1 & $0.05 \%$ & $0.02 \%$ & $0.05 \%$ & $0.05 \%$ & $0.08 \%$ & $0.02 \%$ & $0.14 \%$ & $0.02 \%$ \\
\hline $\mathrm{Pb}_{3}\left(\mathrm{PO}_{4}\right)_{2}$ & 7.1 & $\begin{array}{ll}-- \\
-1\end{array}$ & $\begin{array}{ll}-- \\
-\end{array}$ & --- & $\begin{array}{ll}-- \\
\end{array}$ & $\begin{array}{ll}-- \\
-1\end{array}$ & --- & $\begin{array}{ll}-- \\
\end{array}$ & --- \\
\hline $\mathrm{PbCO}_{3}$ & 6.6 & -- & --- & -- & --- & --- & --- & --- & -- \\
\hline $\mathrm{Pu}(\mathrm{OH})_{4}$ (co-precip. on Fe phase) & 4.26 & $0.00 \%$ & $0.00 \%$ & $0.00 \%$ & $0.00 \%$ & $0.00 \%$ & $0.00 \%$ & $0.00 \%$ & $0.00 \%$ \\
\hline $\mathrm{PuO}_{2}$ & 11.43 & -- & --- & -- & -- & -- & --- & --- & -- \\
\hline $\mathrm{SiO}_{2}$ & 2.6 & --- & $\begin{array}{l}-- \\
\end{array}$ & --- & $\begin{array}{l}-- \\
\end{array}$ & --- & --- & --- & --- \\
\hline $\mathrm{Sr}_{3}\left(\mathrm{PO}_{4}\right)_{2}$ & 3.5 & --- & --- & --- & --- & --- & --- & --- & -- \\
\hline $\mathrm{SrCO}_{3}$ & 3.5 & $0.92 \%$ & $0.40 \%$ & $0.39 \%$ & $0.28 \%$ & $1.10 \%$ & $0.03 \%$ & $0.61 \%$ & $0.03 \%$ \\
\hline $\mathrm{ZrO}_{2}$ & 5.68 & $0.00 \%$ & $0.00 \%$ & $0.00 \%$ & --. & $0.00 \%$ & $0.01 \%$ & $0.00 \%$ & $0.00 \%$ \\
\hline \multicolumn{10}{|l|}{ salts: mass fracs in total solid } \\
\hline $\mathrm{KNO}_{3}$ & 2.109 & $-\cdots$ & $\cdots$ & $-\cdots$ & $\cdots$ & $-\cdots$ & $\cdots$ & $\cdots$ & $-\cdots$ \\
\hline $\mathrm{Na}_{2} \mathrm{C}_{2} \mathrm{O}_{4}$ & 2.34 & $4.70 \%$ & $0.99 \%$ & $3.18 \%$ & $5.12 \%$ & $2.09 \%$ & $5.17 \%$ & $3.49 \%$ & $4.39 \%$ \\
\hline $\mathrm{Na}_{2} \mathrm{CO}_{3} \cdot \mathrm{H}_{2} \mathrm{O}$ & 2.25 & $26.63 \%$ & $4.85 \%$ & $5.80 \%$ & $13,20 \%$ & $7.36 \%$ & $0.00 \%$ & $56.69 \%$ & $28.86 \%$ \\
\hline $\mathrm{Na}_{2} \mathrm{SO}_{4}$ & 2.68 & -- & --- & --- & --- & --- & --- & --- & -- \\
\hline $\mathrm{Na}_{2} \mathrm{SO}_{4} \cdot 10 \mathrm{H}_{2} \mathrm{O}$ & 1.464 & -- & $\begin{array}{ll}-- \\
\end{array}$ & --- & --- & $\begin{array}{ll}-- \\
\end{array}$ & $\begin{array}{ll}-- \\
\end{array}$ & --- & -- \\
\hline $\mathrm{Na}_{3} \mathrm{FSO}_{4}$ & 2.65 & $7.70 \%$ & $1.73 \%$ & $5.08 \%$ & $7.13 \%$ & $7.29 \%$ & $24.36 \%$ & $9.46 \%$ & $10.26 \%$ \\
\hline $\mathrm{Na}_{3} \mathrm{NO}_{3} \mathrm{SO}_{4} \cdot \mathrm{H}_{2} \mathrm{O}$ & 2.3 & -- & --- & -- & -- & --- & --- & --- & -- \\
\hline $\mathrm{Na}_{3} \mathrm{PO}_{4} \cdot 0.25 \mathrm{NaOH} \cdot 12 \mathrm{H}_{2} \mathrm{O}$ & 1.62 & --- & --- & --- & --- & --- & --- & --- & --- \\
\hline $\mathrm{Na}_{3} \mathrm{PO}_{4} \cdot 8 \mathrm{H}_{2} \mathrm{O}$ & 1.8 & -- & --- & -- & --- & --- & --- & --- & -- \\
\hline $\mathrm{Na}_{4} \mathrm{P}_{2} \mathrm{O}_{7} \cdot 10 \mathrm{H}_{2} \mathrm{O}$ & 1.83 & --- & --- & --- & --- & --- & --- & --- & -- \\
\hline $\mathrm{Na}_{6}\left(\mathrm{SO}_{4}\right)_{2} \mathrm{CO}_{3}$ & 2.64 & --- & --- & -- & -- & -- & --- & --- & --- \\
\hline $\mathrm{NaF}$ & 2.78 & $0.43 \%$ & --- & $1.43 \%$ & $0.28 \%$ & $1.11 \%$ & $7.80 \%$ & $0.48 \%$ & $0.28 \%$ \\
\hline $\mathrm{Na}_{7} \mathrm{~F}\left(\mathrm{PO}_{4}\right)_{2} \bullet 19 \mathrm{H}_{2} \mathrm{O}$ & 1.75 & $0.63 \%$ & --- & $0.84 \%$ & $5.00 \%$ & $4.11 \%$ & $35.68 \%$ & $0.89 \%$ & $9.79 \%$ \\
\hline $\mathrm{NaIICO}_{3}$ & 2.159 & -- & $\cdots$ & -- & $\cdots$ & --. & $\cdots$ & $\cdots$ & -- \\
\hline $\mathrm{NaNO}_{2}$ & 2.168 & --- & --- & --- & --- & $1.45 \%$ & --- & --- & -- \\
\hline $\mathrm{NaNO}_{3}$ & 2.26 & $35.42 \%$ & $73.27 \%$ & $67.16 \%$ & $44.70 \%$ & $40.93 \%$ & --- & $8.80 \%$ & $23.93 \%$ \\
\hline
\end{tabular}


Table C.1. (contd)

\begin{tabular}{|c|c|c|c|c|c|c|c|c|c|c|}
\hline & $\begin{array}{l}\text { Density } \\
(\mathrm{g} / \mathrm{mL})\end{array}$ & 241-BY-112 & $241-C-101$ & $241-\mathrm{C}-102$ & $241-\mathrm{C}-103$ & 241-C-104 & 241-C-105 & 241-C-106 & $241-\mathrm{C}-107$ & $241-C-108$ \\
\hline Total kg solids for tank & \multirow{9}{*}{ 11/a } & $1.22 \mathrm{E}+06$ & $4.12 \mathrm{E}+05$ & $3.66 E+05$ & $5.98 \mathrm{E}+05$ & $5.26 \mathrm{E}+05$ & $6.71 \mathrm{E}+05$ & $1.62 \mathrm{E}+04$ & $4.88 \mathrm{E}+05$ & $1.45 \mathrm{E}+05$ \\
\hline Total kL solids for tank & & $5.25 \mathrm{E}+02$ & $1.70 \mathrm{E}+02$ & $1.41 \mathrm{E}+02$ & $2.37 \mathrm{E}+02$ & $1.74 \mathrm{E}+02$ & $2.73 \mathrm{E}+02$ & $5.38 \mathrm{E}+00$ & $1.84 \mathrm{E}+02$ & $6.46 \mathrm{E}+01$ \\
\hline Vol. frac. solid phase in tank & & 0.485 & 0.511 & 0.118 & 0.313 & 0.178 & 0.546 & 0.039 & 0.195 & 0.258 \\
\hline Avg g $/ \mathrm{mL}$, all solid & & 2.327 & 2.420 & 2.594 & 2.525 & 3.015 & 2.457 & 3.018 & 2.655 & 2.239 \\
\hline Total kg non-salt solids for tank & & 1. $42 \mathrm{E}+05$ & $3.19 \mathrm{E}+05$ & $3.65 \mathrm{E}+05$ & $5.96 \mathrm{E}+05$ & $4.26 \mathrm{E}+05$ & $6.45 \mathrm{E}+05$ & $1.52 \mathrm{E}+04$ & $3.62 \mathrm{E}+05$ & $8.68 \mathrm{E}+04$ \\
\hline Total kL non-salt solids for tank & & $4.76 \mathrm{E}+01$ & $1.21 \mathrm{E}+02$ & $1.41 E+02$ & $2.36 \mathrm{E}+02$ & $1.36 \mathrm{E}+02$ & $2.61 \mathrm{E}+02$ & $4.95 \mathrm{E}+00$ & $1.12 \mathrm{E}+02$ & $3.17 \mathrm{E}+0 \mathrm{I}$ \\
\hline Wt. frac. non-salts in solid & & 0.116 & 0.774 & 1.000 & 0.997 & 0.809 & 0.962 & 0.938 & 0.743 & 0.601 \\
\hline Avg g/mL, non-salt solid & & 2.984 & 2.632 & 2.594 & 2.526 & 3.132 & 2.471 & 3.077 & 3.234 & 2.742 \\
\hline Vol. frac non-salts in solid & & 0.091 & 0.712 & 1.000 & 0.996 & 0.779 & 0.957 & 0.920 & 0.610 & 0.490 \\
\hline \multicolumn{11}{|l|}{ non-salts: mass fracs in total solid } \\
\hline $\mathrm{Ag}$ & 10.5 & -- & --- & $\overline{---}$ & --- & --- & --- & $0.17 \%$ & --- & --- \\
\hline $\mathrm{Ag}_{2} \mathrm{O}$ & 7.143 & -- & --- & $0.04 \%$ & --- & $0.20 \%$ & $0,01 \%$ & -- & $0.06 \%$ & --- \\
\hline $\mathrm{Bi}_{2} \mathrm{O}_{3}$ & 8.9 & -- & $0.20 \%$ & $0.61 \%$ & $0.08 \%$ & $0.01 \%$ & $0.07 \%$ & $0.01 \%$ & $2.17 \%$ & $3.37 \%$ \\
\hline $\mathrm{BiFeO}_{3}$ & 7.9 & $\ldots$ & $\ldots$ & -.. & -.. & ..- & ... & -.. & -- & -.- \\
\hline $\mathrm{Ca}(\mathrm{OH})_{2}$ & 2.24 & $\cdots$ & $\cdots$ & $\cdots$ & $\cdots$ & $\cdots$ & $\cdots$ & $\cdots$ & $\ldots$ & $\cdots$ \\
\hline $\mathrm{Ca}_{5} \mathrm{OH}\left(\mathrm{PO}_{4}\right)_{3}$ & 3.14 & $0.22 \%$ & $2.65 \%$ & $2.53 \%$ & $1.13 \%$ & $0.99 \%$ & $0.83 \%$ & $1.32 \%$ & $0.58 \%$ & $7.89 \%$ \\
\hline $\mathrm{CaC}_{2} \mathrm{O}_{4} * \mathrm{H}_{2} \mathrm{O}$ & 2.2 & -- & --- & --- & -- & -- & -- & -- & --- & --- \\
\hline $\mathrm{CaCO}_{3}$ & 2.71 & -- & --- & --- & -- & $0.32 \%$ & --- & -- & --- & --- \\
\hline $\mathrm{CaF}_{2}$ & 3.18 & -- & --- & $0.82 \%$ & $0.11 \%$ & -- & --- & -- & --- & --- \\
\hline $\mathrm{CrOOH}$ & 4.11 & $4.02 \%$ & ... & -.. & $0.21 \%$ & -.- & -.. & $0.59 \%$ & -.. & ... \\
\hline $\mathrm{FePO}_{4} \cdot 2 \mathrm{H}_{2} \mathrm{O}$ & 3.15 & $1.54 \%$ & --- & --- & --- & -- & -- & --- & --- & --- \\
\hline $\mathrm{FeOOH}$ & 4.26 & --- & $7.25 \%$ & $6.73 \%$ & $3.42 \%$ & $7.67 \%$ & $0.75 \%$ & $31.08 \%$ & $30.74 \%$ & $2.84 \%$ \\
\hline Gibbsite & 2.42 & $3.68 \%$ & $43.24 \%$ & $11.17 \%$ & $60.03 \%$ & $36.68 \%$ & $71.84 \%$ & $23.91 \%$ & $30.21 \%$ & $31.99 \%$ \\
\hline Boehmite & 3.01 & --- & --- & -- & --- & -- & --- & --- & -- & --- \\
\hline $\mathrm{HgO}$ (co-precip. with $\mathrm{Ag}_{2} \mathrm{O}$ ) & 7.143 & --- & $0.01 \%$ & --- & $0.03 \%$ & $0.01 \%$ & $0.00 \%$ & $0.00 \%$ & $0.01 \%$ & $0.01 \%$ \\
\hline $\mathrm{KAlSiO}_{4}$ & 2.61 & -- & $-\cdots$ & -- & -- & --- & $0.25 \%$ & $\ldots$ & --- & --- \\
\hline $\mathrm{La}(\mathrm{OH})_{3}$ & 2.3 & $0.00 \%$ & --- & --- & --- & $0.01 \%$ & --- & --- & --- & $\begin{array}{l}-- \\
\end{array}$ \\
\hline $\mathrm{LaPO}_{4} \cdot 2 \mathrm{H}_{2} \mathrm{O}$ & 6.51 & -- & $0.11 \%$ & $0.08 \%$ & $0.05 \%$ & --- & $0.12 \%$ & $0.15 \%$ & $0.10 \%$ & $0.01 \%$ \\
\hline $\mathrm{Mn}_{3}\left(\mathrm{PO}_{4}\right)_{2}$ & 3.102 & --- & $0.28 \%$ & $0.73 \%$ & -- & --- & --- & -- & $2.03 \%$ & $0.08 \%$ \\
\hline $\mathrm{MnO}_{2}$ & 5.026 & -- & $--\cdot$ & $-\cdots$ & $0.10 \%$ & $1.92 \%$ & $0.50 \%$ & $7.67 \%$ & --- & -- \\
\hline $\mathrm{Na}_{2}\left(\mathrm{UO}_{2}\right)_{2}\left(\mathrm{PO}_{4}\right)_{2} \cdot 2 \mathrm{H}_{2} \mathrm{O}$ & 3.5 & -- & $\begin{array}{ll}-- \\
\end{array}$ & --- & --- & --- & $\begin{array}{ll}-- \\
\end{array}$ & --- & --- & $\begin{array}{l}-- \\
\end{array}$ \\
\hline $\mathrm{Na}_{2} \mathrm{U}_{2} \mathrm{O}_{7}$ & 5.617 & $0.23 \%$ & $3.64 \%$ & $2.22 \%$ & $1.53 \%$ & $8.21 \%$ & $2.19 \%$ & $1.02 \%$ & $2.31 \%$ & $0.14 \%$ \\
\hline $\mathrm{NaAICO}_{3}(\mathrm{OH})_{2}$ & 2.42 & -- & $7.69 \%$ & $32.97 \%$ & $9.76 \%$ & -- & --- & $25.36 \%$ & $1.22 \%$ & $3.21 \%$ \\
\hline $\mathrm{NaAlSiO}_{4}$ & 2.365 & $1.82 \%$ & $11.51 \%$ & $38.07 \%$ & $19.87 \%$ & $8.67 \%$ & $19.08 \%$ & $0.17 \%$ & $1.62 \%$ & $6.77 \%$ \\
\hline $\mathrm{Ni}(\mathrm{OH})_{2}$ & 4.15 & $0.05 \%$ & $0.38 \%$ & $2.23 \%$ & $1.05 \%$ & $0.73 \%$ & $0.44 \%$ & $1.07 \%$ & $0.92 \%$ & $3,30 \%$ \\
\hline $\mathrm{Ni}_{3}\left(\mathrm{PO}_{4}\right)$ & 3.93 & $\cdots$ & -- & -- & -- & -- & -- & $\cdots$ & -- & -- \\
\hline $\mathrm{NiC}_{2} \mathrm{O}_{4} \cdot 2 \mathrm{H}_{2} \mathrm{O}$ (on $\mathrm{Fe}$ phase) & 4.26 & -- & --- & --- & -- & -- & -- & -- & -- & -- \\
\hline $\mathrm{Pb}(\mathrm{OH})_{2}$ & 7.1 & $0.02 \%$ & $0.26 \%$ & $0.27 \%$ & $0.10 \%$ & $0.17 \%$ & $0.07 \%$ & $1.20 \%$ & $2.21 \%$ & $0.25 \%$ \\
\hline $\mathrm{Pb}_{3}\left(\mathrm{PO}_{4}\right)_{2}$ & 7.1 & -- & --- & -- & --- & -- & --- & --- & --- & -- \\
\hline $\mathrm{PbCO}_{3}$ & 6.6 & -- & --- & --- & -- & --- & --- & --- & --- & --- \\
\hline $\mathrm{Pu}(\mathrm{OH})_{4}$ (co-precip. on Fe phase) & 4.26 & $0.00 \%$ & $0.00 \%$ & $0.02 \%$ & $0.02 \%$ & $0.02 \%$ & $0.01 \%$ & $0.01 \%$ & $0.01 \%$ & $0.00 \%$ \\
\hline $\mathrm{PuO}_{2}$ & 11.43 & -- & -- & -- & -- & --- & --- & -- & -- & -- \\
\hline $\mathrm{SiO}_{2}$ & 2.6 & --- & --- & --- & --- & --- & --- & --- & --- & --- \\
\hline $\mathrm{Sr}_{3}\left(\mathrm{PO}_{4}\right)_{2}$ & 3.5 & --- & $0.13 \%$ & --- & --- & --- & --- & --- & --- & --- \\
\hline $\mathrm{SrCO}_{3}$ & 3.5 & $0.04 \%$ & --- & $0.06 \%$ & $0.01 \%$ & $0.03 \%$ & $0.04 \%$ & $0.07 \%$ & $0.08 \%$ & $0.17 \%$ \\
\hline $\mathrm{ZrO}_{2}$ & 5.68 & $0.00 \%$ & $0.08 \%$ & $1.46 \%$ & $2.15 \%$ & $15.31 \%$ & $0.04 \%$ & $0.02 \%$ & $0.02 \%$ & $0.03 \%$ \\
\hline \multicolumn{11}{|l|}{ salts: mass fracs in total solid } \\
\hline $\mathrm{KNO}_{3}$ & 2.109 & $\cdots$ & $\cdots$ & $-\cdots$ & $\cdots$ & $\cdots$ & $\cdots$ & $\cdots$ & $-\cdots$ & $\cdots$ \\
\hline $\mathrm{Na}_{2} \mathrm{C}_{2} \mathrm{O}_{4}$ & 2.34 & $7.13 \%$ & $0.92 \%$ & -- & $0.26 \%$ & $6.79 \%$ & $2.16 \%$ & $6.10 \%$ & --- & $0.40 \%$ \\
\hline $\mathrm{Na}_{2} \mathrm{CO}_{3} \cdot \mathrm{H}_{2} \mathrm{O}$ & 2.25 & $55.43 \%$ & $\cdots$ & $0.02 \%$ & $0.08 \%$ & $0.11 \%$ & $0.00 \%$ & $0.10 \%$ & $0.03 \%$ & -- \\
\hline $\mathrm{Na}_{2} \mathrm{SO}_{4}$ & 2.68 & -- & -- & -- & -- & --- & -- & -- & -- & -- \\
\hline $\mathrm{Na}_{2} \mathrm{SO}_{4} \cdot 10 \mathrm{H}_{2} \mathrm{O}$ & 1.464 & $\begin{array}{ll}-- \\
-1\end{array}$ & --- & --- & --- & --- & --- & --- & --- & $\begin{array}{ll}-- \\
\end{array}$ \\
\hline $\mathrm{Na}_{3} \mathrm{FSO}_{4}$ & 2.65 & $8.65 \%$ & --- & --- & --- & -- & $0.39 \%$ & --- & --- & -- \\
\hline $\mathrm{Na}_{3} \mathrm{NO}_{3} \mathrm{SO}_{4} \cdot \mathrm{H}_{2} \mathrm{O}$ & 2.3 & -- & $2.27 \%$ & -- & -- & --- & -- & -- & --- & -- \\
\hline $\mathrm{Na}_{3} \mathrm{PO}_{4} \cdot 0.25 \mathrm{NaOH} \cdot 12 \mathrm{H}_{2} \mathrm{O}$ & 1.62 & --- & --- & --- & -- & --- & --- & --- & --- & --- \\
\hline $\mathrm{Na}_{3} \mathrm{PO}_{4} \cdot 8 \mathrm{H}_{2} \mathrm{O}$ & 1.8 & -- & --- & --- & -- & $\begin{array}{c}-- \\
\end{array}$ & --- & --- & --- & --- \\
\hline $\mathrm{Na}_{4} \mathrm{P}_{2} \mathrm{O}_{7} \cdot 10 \mathrm{H}_{2} \mathrm{O}$ & 1.83 & --- & $12.61 \%$ & --- & --- & --- & --- & --- & --- & $0.15 \%$ \\
\hline $\mathrm{Na}_{6}\left(\mathrm{SO}_{4}\right)_{2} \mathrm{CO}_{3}$ & 2.64 & -- & --- & -- & --- & -- & $\cdots$ & $\cdots$ & --- & --- \\
\hline $\mathrm{NaF}$ & 2.78 & $0.73 \%$ & -- & -- & --- & $12.18 \%$ & -- & --- & -- & -- \\
\hline $\mathrm{Na}_{7} \mathrm{~F}\left(\mathrm{PO}_{4}\right)_{2} \cdot 19 \mathrm{H}_{2} \mathrm{O}$ & 1.75 & $4.60 \%$ & $4.90 \%$ & --- & --- & --- & $1.21 \%$ & --- & $25.69 \%$ & $39.39 \%$ \\
\hline $\mathrm{NaI} \mathrm{ICO}_{3}$ & 2.159 & -- & $\cdots$ &.-- & -- & -.- & $\cdots$ & -- & $-\cdot$ & $\cdots$ \\
\hline $\mathrm{NaNO}_{2}$ & 2.168 & -- & --- & --- & -- & --- & --- & --- & --- & -- \\
\hline $\mathrm{NaNO}_{3}$ & 2.26 & $11.84 \%$ & $1.87 \%$ & --- & --- & --- & --- & --- & --- & --- \\
\hline
\end{tabular}


Table C.1. (contd)

\begin{tabular}{|c|c|c|c|c|c|c|c|c|c|c|}
\hline & $\begin{array}{l}\text { Density } \\
(\mathrm{g} / \mathrm{mL})\end{array}$ & 241-C-109 & $241-C-110$ & $241-C-111$ & 241-C-112 & 241-C-201 & 241-C-202 & 241-C-203 & 241-C-204 & 241-S-101 \\
\hline Total kg solids for tank & \multirow{9}{*}{ 11/a } & $1.92 \mathrm{E}+05$ & $2.66 \mathrm{E}+05$ & $2.00 \mathrm{E}+05$ & $1.86 \mathrm{E}+05$ & $7.81 \mathrm{IE}+03$ & $6.15 \mathrm{E}+03$ & $1.50 \mathrm{E}+04$ & $5.59 \mathrm{E}+03$ & $6.84 \mathrm{E}+05$ \\
\hline Total kL solids for tank & & 8. $19 \mathrm{E}+01$ & $1.26 \mathrm{E}+02$ & $7.66 \mathrm{E}+01$ & $6.59 \mathrm{E}+01$ & $2.19 \mathrm{E}+00$ & $1.68 \mathrm{E}+00$ & $3.96 \mathrm{E}+00$ & $1.38 \mathrm{E}+00$ & $2.58 \mathrm{E}+02$ \\
\hline Vol. frac. solid phase in tank & & 0.341 & 0.187 & 0.353 & 0.168 & 0.547 & 0.421 & 0.396 & 0.138 & 0.161 \\
\hline Avg g/mL, all solid & & 2.345 & 2.109 & 2.609 & 2.826 & 3.567 & 3.654 & 3.789 & 4.041 & 2.654 \\
\hline Total $\mathrm{kg}$ non-salt solids for tank & & $1.36 \mathrm{E}+05$ & $1.03 \mathrm{E}+05$ & $1.68 \mathrm{E}+05$ & $1.43 \mathrm{E}+05$ & $5.77 \mathrm{E}+03$ & $4.70 \mathrm{E}+03$ & $1.24 \mathrm{E}+04$ & $4.98 \mathrm{E}+03$ & $3.65 \mathrm{E}+05$ \\
\hline Total kL non-salt solids for tank & & $4.92 \mathrm{E}+01$ & $3.36 \mathrm{E}+01$ & $5.96 \mathrm{E}+01$ & $4.29 \mathrm{E}+01$ & $1.29 \mathrm{E}+00$ & $1.05 \mathrm{E}+00$ & $2.83 \mathrm{E}+00$ & $1.12 \mathrm{E}+00$ & $1.17 \mathrm{E}+02$ \\
\hline Wt. frac. non-salts in solid & & 0.710 & 0.388 & 0.842 & 0.768 & 0.739 & 0.764 & 0.830 & 0.891 & 0.533 \\
\hline Avg g/mL, non-salt solid & & 2.771 & 3.074 & 2.823 & 3.331 & 4.463 & 4.465 & 4.404 & 4.435 & 3.116 \\
\hline Vol. frac. non-salts in solid & & 0.601 & 0.266 & 0.778 & 0.651 & 0.591 & 0.625 & 0.714 & 0.812 & 0.454 \\
\hline \multicolumn{11}{|l|}{ non-salts: mass fracs in total solid } \\
\hline $\mathrm{Ag}$ & 10.5 & --- & --- & --- & --- & --- & --- & --- & --- & --- \\
\hline $\mathrm{Ag}_{2} \mathrm{O}$ & 7.143 & --- & $0.00 \%$ & --- & $0.00 \%$ & --- & --- & --- & --- & --- \\
\hline $\mathrm{Bi}_{2} \mathrm{O}_{3}$ & 8.9 & $0.75 \%$ & -- & $1.05 \%$ & $1.12 \%$ & --- & --- & --- & --- & $0.01 \%$ \\
\hline $\mathrm{BiFeO}_{3}$ & 7.9 & $\ldots$ & $8.38 \%$ & $\ldots$ &.-- & $\ldots$ & $\ldots$ & $\ldots$ & -- & -- \\
\hline $\mathrm{Ca}(\mathrm{OH})_{2}$ & 2.24 & $\cdots$ & $\cdots$ & $\cdots$ & 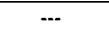 & $\cdots$ & $\cdots$ & $\cdots$ & $\cdots$ & $\cdots$ \\
\hline $\mathrm{Ca}_{5} \mathrm{OH}\left(\mathrm{PO}_{4}\right)_{3}$ & 3.14 & $8.33 \%$ & $0.08 \%$ & $5.92 \%$ & $14.50 \%$ & $0.07 \%$ & -- & -.. & -- & $0.27 \%$ \\
\hline $\mathrm{CaC}_{2} \mathrm{O}_{4} * \mathrm{H}_{2} \mathrm{O}$ & 2.2 & --- & --- & --- & -- & -- & --- & $2.06 \%$ & --- & --- \\
\hline $\mathrm{CaCO}_{3}$ & 2.71 & --- & --- & --- & --- & $5.74 \%$ & $5.44 \%$ & $4.72 \%$ & $6.50 \%$ & --- \\
\hline $\mathrm{CaF}_{2}$ & 3.18 & --- & $0.71 \%$ & --- & --- & $0.49 \%$ & $0.88 \%$ & $0.72 \%$ & $0.76 \%$ & --- \\
\hline $\mathrm{CrOOH}$ & 4.11 & $0.10 \%$ & $0.22 \%$ & $\cdots$ & $0.08 \%$ & -.- & ..- & ... & ..- & $3.46 \%$ \\
\hline $\mathrm{FePO}_{4} \cdot 2 \mathrm{H}_{2} \mathrm{O}$ & 3.15 & --- & $7.45 \%$ & --- & --- & --- & -- & --- & -- & -- \\
\hline $\mathrm{FeOOH}$ & 4.26 & $6.11 \%$ & --- & $12.50 \%$ & $10.78 \%$ & $35.07 \%$ & $35.77 \%$ & $38.37 \%$ & $40.76 \%$ & $0.50 \%$ \\
\hline Gibbsite & 2.42 & $30.46 \%$ & $6.63 \%$ & $40.96 \%$ & $6.57 \%$ & --- & --- & --- & --- & --- \\
\hline Boehmite & 3.01 & -- & --- & --- & -- & --- & --- & --- & --- & $45,02 \%$ \\
\hline $\mathrm{HgO}$ (co-precip. with $\mathrm{Ag}_{2} \mathrm{O}$ ) & 7.143 & --- & --- & --- & --- & --- & --- & --- & --- & --- \\
\hline $\mathrm{KAISiO}_{4}$ & 2.61 & -- & --- & $-\cdots$ & --- & --- & --- & --- & $-\cdots$ & --- \\
\hline $\mathrm{La}(\mathrm{OH})_{3}$ & 2.3 & $-\cdots$ & --- & --- & --- & --- & --- & $\begin{array}{ll}-- \\
\end{array}$ & --- & --- \\
\hline $\mathrm{LaPO}_{4} \cdot 2 \mathrm{H}_{2} \mathrm{O}$ & 6.51 & $0.00 \%$ & $0.00 \%$ & $0.12 \%$ & $0.04 \%$ & $0.48 \%$ & $0.49 \%$ & $0.53 \%$ & $0.56 \%$ & $0.01 \%$ \\
\hline $\mathrm{Mn}_{3}\left(\mathrm{PO}_{4}\right)_{2}$ & 3.102 & $0.09 \%$ & $0.04 \%$ & $0.10 \%$ & $0.13 \%$ & -- & -- & --- & -- & -- \\
\hline $\mathrm{MnO}_{2}$ & 5.026 & -- & $\ldots$ & --- &.-- & $0.01 \%$ & $0.01 \%$ & $\ldots$ & $0.01 \%$ & $0.54 \%$ \\
\hline $\mathrm{Na}_{2}\left(\mathrm{UO}_{2}\right)_{2}\left(\mathrm{PO}_{4}\right)_{2} \cdot 2 \mathrm{H}_{2} \mathrm{O}$ & 3.5 & --- & --- & --- & --- & --- & --- & --- & --- & --- \\
\hline $\mathrm{Na}_{2} \mathrm{U}_{2} \mathrm{O}_{7}$ & 5.617 & $3.18 \%$ & $0.99 \%$ & $3.34 \%$ & $15.87 \%$ & $0.03 \%$ & --- & --- & --- & $1.84 \%$ \\
\hline $\mathrm{NaAICO}_{3}(\mathrm{OH})_{2}$ & 2.42 & $10.89 \%$ & $2.45 \%$ & $9.54 \%$ & $17.02 \%$ & -- & $3.09 \%$ & $3.31 \%$ & $3.52 \%$ & --- \\
\hline $\mathrm{NaAlSiO}_{4}$ & 2.365 & $6.20 \%$ & $11.58 \%$ & $4.94 \%$ & $3.52 \%$ & $3.00 \%$ & --- & --- & -- & $1.45 \%$ \\
\hline $\mathrm{Ni}(\mathrm{OH})_{2}$ & 4.15 & $4.25 \%$ & $0.01 \%$ & $4.56 \%$ & $6.08 \%$ & $4.50 \%$ & $4.58 \%$ & $3.08 \%$ & $5.20 \%$ & $0.05 \%$ \\
\hline $\mathrm{Ni}_{3}\left(\mathrm{PO}_{4}\right)$ & 3.93 & -- & $--\cdot$ & $\cdots$ & -- & --- & -- & -- & $\cdots$ & -- \\
\hline $\mathrm{NiC}_{2} \mathrm{O}_{4} \cdot 2 \mathrm{H}_{2} \mathrm{O}$ (on Fe phase) & 4.26 & --- & --- & --- & --- & --- & --- & $3.61 \%$ & --- & -- \\
\hline $\mathrm{Pb}(\mathrm{OH})_{2}$ & 7.1 & $0.57 \%$ & $0.10 \%$ & $0.43 \%$ & $0.85 \%$ & $23.50 \%$ & $11.86 \%$ & $25.56 \%$ & --- & $0.02 \%$ \\
\hline $\mathrm{Pb}_{3}\left(\mathrm{PO}_{4}\right)_{2}$ & 7.1 & -- & --- & $0.60 \%$ & --- & -- & $\begin{array}{ll}-- \\
-1\end{array}$ & --- & --- & --- \\
\hline $\mathrm{PbCO}_{3}$ & 6.6 & -- & --- & --- & --- & -- & $13.25 \%$ & --- & $30.14 \%$ & --- \\
\hline $\mathrm{Pu}(\mathrm{OH})_{4}$ (co-precip. on Fe phase) & 4.26 & $0.00 \%$ & $0.00 \%$ & $0.00 \%$ & $0.00 \%$ & $0.03 \%$ & $0.01 \%$ & $0.00 \%$ & $0.00 \%$ & $0.00 \%$ \\
\hline $\mathrm{PuO}_{2}$ & 11.43 & -- & -- & --- & -- & -- & -- & -- & -- & -- \\
\hline $\mathrm{SiO}_{2}$ & 2.6 & --- & $\begin{array}{l}-- \\
\end{array}$ & --- & --- & --- & --- & --- & $0.53 \%$ & --- \\
\hline $\mathrm{Sr}_{3}\left(\mathrm{PO}_{4}\right)_{2}$ & 3.5 & --- & --- & $0.06 \%$ & $0.16 \%$ & --- & --- & --- & -- & --- \\
\hline $\mathrm{SrCO}_{3}$ & 3.5 & $0.12 \%$ & $0.07 \%$ & --- & --- & $0.98 \%$ & $0.99 \%$ & $1.07 \%$ & $1.13 \%$ & $0.11 \%$ \\
\hline $\mathrm{ZrO}_{2}$ & 5.68 & $0.01 \%$ & $0.08 \%$ & $0.04 \%$ & $0.01 \%$ & --- & --- & --- & -- & $0.01 \%$ \\
\hline \multicolumn{11}{|l|}{ salts: mass fracs in total solid } \\
\hline $\mathrm{KNO}_{3}$ & 2.109 & $-\cdots$ & $\begin{array}{ll}-\cdot \\
\end{array}$ & $\cdots$ & $-\cdots$ & $\begin{array}{ll}-- \\
\end{array}$ & $-\cdots$ & $\cdots$ & $-\cdots$ & 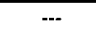 \\
\hline $\mathrm{Na}_{2} \mathrm{C}_{2} \mathrm{O}_{4}$ & 2.34 & $1.16 \%$ & $0.07 \%$ & $0.26 \%$ & $3.01 \%$ & $7.06 \%$ & $12.85 \%$ & --- & $10.88 \%$ & $3.04 \%$ \\
\hline $\mathrm{Na}_{2} \mathrm{CO}_{3} \cdot \mathrm{H}_{2} \mathrm{O}$ & 2.25 & -- & $0.00 \%$ & $\cdots$ & $0.00 \%$ & $16.47 \%$ & $3.50 \%$ & $11.96 \%$ & $0.01 \%$ & $1.43 \%$ \\
\hline $\mathrm{Na}_{2} \mathrm{SO}_{4}$ & 2.68 & --- & --- & --- & -- & -- & --- & --- & -- & -- \\
\hline $\mathrm{Na}_{2} \mathrm{SO}_{4} \cdot 10 \mathrm{H}_{2} \mathrm{O}$ & 1.464 & --- & --- & --- & --- & --- & --- & --- & --- & --- \\
\hline $\mathrm{Na}_{3} \mathrm{FSO}_{4}$ & 2.65 & --- & --- & --- & --- & --- & --- & --- & --- & --- \\
\hline $\mathrm{Na}_{3} \mathrm{NO}_{3} \mathrm{SO}_{4} \cdot \mathrm{H}_{2} \mathrm{O}$ & 2.3 & --- & --- & --- & --- & -- & -- & --- & -- & --- \\
\hline $\mathrm{Na}_{3} \mathrm{PO}_{4} \cdot 0.25 \mathrm{NaOH} \cdot 12 \mathrm{H}_{2} \mathrm{O}$ & 1.62 & $13.65 \%$ & --- & --- & --- & --- & --- & --- & --- & --- \\
\hline $\mathrm{Na}_{3} \mathrm{PO}_{4} \cdot 8 \mathrm{H}_{2} \mathrm{O}$ & 1.8 & --- & --- & --- & --- & --- & --- & --- & --- & --- \\
\hline $\mathrm{Na}_{4} \mathrm{P}_{2} \mathrm{O}_{7} \cdot 10 \mathrm{H}_{2} \mathrm{O}$ & 1.83 & --- & --- & $14.99 \%$ & $20.24 \%$ & -- & --- & --- & --- & --- \\
\hline $\mathrm{Na}_{6}\left(\mathrm{SO}_{4}\right)_{2} \mathrm{CO}_{3}$ & 2.64 & --- & -- & $\cdots$ & -- & -- & --- & --- & -- & $3.16 \%$ \\
\hline $\mathrm{NaF}$ & 2.78 & -- & $0.84 \%$ & $0.57 \%$ & --- & --- & --- & --- & --- & -- \\
\hline $\mathrm{Na}_{7} \mathrm{~F}\left(\mathrm{PO}_{4}\right)_{2} \cdot 19 \mathrm{H}_{2} \mathrm{O}$ & 1.75 & $14.17 \%$ & $60.31 \%$ & --- & --- & --- & --- & --- & --- & $1.22 \%$ \\
\hline $\mathrm{NaIICO}$ & 2.159 & -- & -.- & $\cdots$ & -.. & --- & ..- & $\cdots$ & --. & ..- \\
\hline $\mathrm{NaNO}_{2}$ & 2.168 & --- & --- & --- & --- & --- & -- & --- & --- & -- \\
\hline $\mathrm{NaNO}_{3}$ & 2.26 & --- & --- & --- & --- & $2.57 \%$ & $7.27 \%$ & $5.03 \%$ & --- & $37.84 \%$ \\
\hline
\end{tabular}


Table C.1. (contd)

\begin{tabular}{|c|c|c|c|c|c|c|c|c|c|c|}
\hline & $\begin{array}{l}\text { Density } \\
(\mathrm{g} / \mathrm{mL})\end{array}$ & $241-\mathrm{S}-102$ & $241-S-103$ & 241-S-104 & 241-S-105 & 241-S-106 & $241-S-107$ & 241-S-108 & 241-S-109 & $241-S-110$ \\
\hline Total kg solids for tank & \multirow{9}{*}{ 11/a } & $7.28 \mathrm{E}+05$ & $5.38 \mathrm{E}+05$ & $7.08 \mathrm{E}+05$ & $1.22 \mathrm{E}+06$ & $1.54 \mathrm{E}+06$ & $4.03 \mathrm{E}+05$ & $1.71 \mathrm{E}+06$ & $2.22 \mathrm{E}+06$ & $1.77 \mathrm{E}+06$ \\
\hline Total kL solids for tank & & $3.44 \mathrm{E}+02$ & $2.40 \mathrm{E}+02$ & $2.62 \mathrm{E}+02$ & $5.71 \mathrm{E}+02$ & $7.05 \mathrm{E}+02$ & $1.41 \mathrm{E}+02$ & $7.79 \mathrm{E}+02$ & $9.98 \mathrm{E}+02$ & $7.38 \mathrm{E}+02$ \\
\hline Vol. frac. solid phase in tank & & 0.276 & 0.268 & 0.240 & 0.372 & 0.409 & 0.099 & 0.374 & 0.495 & 0.501 \\
\hline Avg g/mL, all solid & & 2.113 & 2,236 & 2.704 & 2.144 & 2.190 & 2.851 & 2.191 & 2.224 & 2.391 \\
\hline Total $\mathrm{kg}$ non-salt solids for tank & & $1.08 \mathrm{E}+05$ & $1.00 \mathrm{E}+05$ & $4.53 \mathrm{E}+05$ & $1.42 \mathrm{E}+05$ & $1.39 \mathrm{E}+05$ & $2.80 \mathrm{E}+05$ & $2.03 \mathrm{E}+05$ & $9.29 \mathrm{E}+03$ & $3.51 \mathrm{E}+05$ \\
\hline Total kL non-salt solids for tank & & $4.06 \mathrm{E}+01$ & $3.87 \mathrm{E}+01$ & $1.49 \mathrm{E}+02$ & $5.43 \mathrm{E}+01$ & $5.43 \mathrm{E}+01$ & $8.47 \mathrm{E}+01$ & $7.71 \mathrm{E}+01$ & $2.20 \mathrm{E}+00$ & $1.13 \mathrm{E}+02$ \\
\hline Wt. frac. non-salts in solid & & 0.148 & 0.186 & 0.640 & 0.116 & 0.090 & 0.694 & 0.119 & 0.004 & 0.199 \\
\hline Avg g/mL, non-salt solid & & 2.648 & 2.582 & 3.037 & 2.619 & 2.558 & 3.301 & 2.635 & 4.226 & 3.112 \\
\hline Vol. frac. non-salts in solid & & 0.118 & 0.161 & 0.570 & 0.095 & 0.077 & 0.599 & 0.099 & 0.002 & 0.153 \\
\hline \multicolumn{11}{|l|}{ non-salts: mass fracs in total solid } \\
\hline $\mathrm{Ag}$ & 10.5 & --- & --- & --- & --- & --- & --- & --- & --- & --- \\
\hline $\mathrm{Ag}_{2} \mathrm{O}$ & 7.143 & --- & --- & --- & --- & --- & $0.02 \%$ & --- & $0.00 \%$ & --- \\
\hline $\mathrm{Bi}_{2} \mathrm{O}_{3}$ & 8.9 & $0.02 \%$ & $0.02 \%$ & $0.00 \%$ & $0.01 \%$ & $0.02 \%$ & $0.02 \%$ & $0.01 \%$ & $0.01 \%$ & $0.04 \%$ \\
\hline $\mathrm{BiFeO}_{3}$ & 7.9 & $\ldots$ & $\ldots$ & $\ldots$ &.- & -.. & $\ldots$ & $\ldots$ & -- & -- \\
\hline $\mathrm{Ca}(\mathrm{OH})_{2}$ & 2.24 & $\cdots$ & $\cdots$ & $\cdots$ & $\cdots$ & $\cdots$ & $\cdots$ & 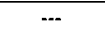 & $\cdots$ & $\cdots$ \\
\hline $\mathrm{Ca}_{5} \mathrm{OH}\left(\mathrm{PO}_{4}\right)_{3}$ & 3.14 & $0.37 \%$ & $0.15 \%$ & $0.03 \%$ & $0.11 \%$ & $0.06 \%$ & -- & $0.12 \%$ & $0.04 \%$ & $0.12 \%$ \\
\hline $\mathrm{CaC}_{2} \mathrm{O}_{4} * \mathrm{H}_{2} \mathrm{O}$ & 2.2 & --- & --- & --- & -- & --- & --- & --- & --- & --- \\
\hline $\mathrm{CaCO}_{3}$ & 2.71 & --- & --- & $0.09 \%$ & --- & --- & --- & --- & --- & --- \\
\hline $\mathrm{CaF}_{2}$ & 3.18 & --- & --- & $0.02 \%$ & --- & --- & $0.38 \%$ & --- & --- & --- \\
\hline $\mathrm{CrOOH}$ & 4.11 & $1.06 \%$ & $1.76 \%$ & $0.18 \%$ & $1.19 \%$ & $0.57 \%$ & $1.48 \%$ & $1.27 \%$ & $0.19 \%$ & $1.10 \%$ \\
\hline $\mathrm{FePO}_{4} \cdot 2 \mathrm{H}_{2} \mathrm{O}$ & 3.15 & -- & --- & --- & --- & --- & --- & --- & --- & -- \\
\hline $\mathrm{FeOOH}$ & 4.26 & $0.31 \%$ & $0.46 \%$ & $0.45 \%$ & $0.42 \%$ & $0.40 \%$ & $1.53 \%$ & $0.45 \%$ & $0.13 \%$ & $0.64 \%$ \\
\hline Gibbsite & 2.42 & $11.23 \%$ & $15.08 \%$ & -- & $9.04 \%$ & $7.64 \%$ & --- & $9.00 \%$ & --- & --- \\
\hline Boehmite & 3.01 & -- & --- & $59.83 \%$ & --- & --- & $46.32 \%$ & --- & --- & $16.38 \%$ \\
\hline $\mathrm{HgO}$ (co-precip. with $\mathrm{Ag}_{2} \mathrm{O}$ ) & 7.143 & --- & --- & --- & --- & --- & --- & --- & --- & --- \\
\hline $\mathrm{KAISiO}_{4}$ & 2.61 & -- & --- & $-\cdots$ & --- & -- & --- & --- & $-\cdots$ & --- \\
\hline $\mathrm{La}(\mathrm{OH})_{3}$ & 2.3 & --- & --- & $0.00 \%$ & --- & --- & --- & --- & --- & $\begin{array}{ll}-- \\
-1\end{array}$ \\
\hline $\mathrm{LaPO}_{4} \cdot 2 \mathrm{H}_{2} \mathrm{O}$ & 6.51 & $0.01 \%$ & $0.01 \%$ & --- & $0.01 \%$ & $0.01 \%$ & $0.02 \%$ & $0.01 \%$ & $0.01 \%$ & $0.01 \%$ \\
\hline $\mathrm{Mn}_{3}\left(\mathrm{PO}_{4}\right)_{2}$ & 3.102 & -- & --- & --- & -- & -- & -- & --- & -- & -- \\
\hline $\mathrm{MnO}_{2}$ & 5.026 & $0.13 \%$ & $0.07 \%$ & $0.40 \%$ & $0.07 \%$ & -.- & $0.34 \%$ & $0.12 \%$ & --- & $0.17 \%$ \\
\hline $\mathrm{Na}_{2}\left(\mathrm{UO}_{2}\right)_{2}\left(\mathrm{PO}_{4}\right)_{2} \cdot 2 \mathrm{H}_{2} \mathrm{O}$ & 3.5 & --- & --- & --- & --- & --- & --- & --- & --- & --- \\
\hline $\mathrm{Na}_{2} \mathrm{U}_{2} \mathrm{O}_{7}$ & 5.617 & $0.92 \%$ & $0.22 \%$ & $1.35 \%$ & $0.19 \%$ & $0.06 \%$ & $4.41 \%$ & $0.21 \%$ & $0.03 \%$ & $0.56 \%$ \\
\hline $\mathrm{NaAICO}_{3}(\mathrm{OH})_{2}$ & 2.42 & -- & --- & --- & --- & -- & -- & -- & -- & -- \\
\hline $\mathrm{NaAlSiO}_{4}$ & 2.365 & $0.70 \%$ & $0.73 \%$ & $1.51 \%$ & $0.51 \%$ & $0.21 \%$ & $5.02 \%$ & $0.63 \%$ & -- & $0.78 \%$ \\
\hline $\mathrm{Ni}(\mathrm{OH})_{2}$ & 4.15 & $0.01 \%$ & $0.06 \%$ & $0.02 \%$ & $0.03 \%$ & $0.01 \%$ & $0.88 \%$ & $0.03 \%$ & $0.01 \%$ & $0.02 \%$ \\
\hline $\mathrm{Ni}_{3}\left(\mathrm{PO}_{4}\right)$ & 3.93 & -- & $--\cdot$ & $\cdots$ & -- & --- & -- & $--\cdot$ & $\cdots$ & -- \\
\hline $\mathrm{NiC}_{2} \mathrm{O}_{4} \cdot 2 \mathrm{H}_{2} \mathrm{O}$ (on Fe phase) & 4.26 & --- & --- & --- & --- & --- & --- & --- & --- & --- \\
\hline $\mathrm{Pb}(\mathrm{OH})_{2}$ & 7.1 & $0.03 \%$ & $0.03 \%$ & $0.01 \%$ & $0.03 \%$ & $0.01 \%$ & $0.06 \%$ & $0.03 \%$ & $0.01 \%$ & $0.01 \%$ \\
\hline $\mathrm{Pb}_{3}\left(\mathrm{PO}_{4}\right)_{2}$ & 7.1 & -- & --- & --- & --- & -- & $\begin{array}{ll}-- \\
-1\end{array}$ & --- & -- & --- \\
\hline $\mathrm{PbCO}_{3}$ & 6.6 & -- & --- & --- & --- & -- & --- & --- & --- & --- \\
\hline $\mathrm{Pu}(\mathrm{OH})_{4}$ (co-precip. on Fe phase) & 4.26 & $0.00 \%$ & $0.00 \%$ & $0.00 \%$ & $0.00 \%$ & $0.00 \%$ & $0.01 \%$ & $0.00 \%$ & $0.00 \%$ & $0.00 \%$ \\
\hline $\mathrm{PuO}_{2}$ & 11.43 & -- & --- & --- & --- & -- & -- & -- & -- & -- \\
\hline $\mathrm{SiO}_{2}$ & 2.6 & --- & $\begin{array}{l}-- \\
\end{array}$ & --- & --- & --- & --- & --- & --- & --- \\
\hline $\mathrm{Sr}_{3}\left(\mathrm{PO}_{4}\right)_{2}$ & 3.5 & --- & --- & --- & --- & --- & -- & --- & --- & -- \\
\hline $\mathrm{SrCO}_{3}$ & 3.5 & $0.00 \%$ & $0.01 \%$ & $0.12 \%$ & $0.01 \%$ & $0.00 \%$ & $0.19 \%$ & $0.01 \%$ & $0.00 \%$ & $0.05 \%$ \\
\hline $\mathrm{ZrO}_{2}$ & 5.68 & $0.01 \%$ & $0.01 \%$ & $0.01 \%$ & $0.01 \%$ & --- & $8.71 \%$ & $0.01 \%$ & -- & $0.00 \%$ \\
\hline \multicolumn{11}{|l|}{ salts: mass fracs in total solid } \\
\hline $\mathrm{KNO}_{3}$ & 2.109 & $-\cdots$ & $\begin{array}{ll}-\cdot \\
\end{array}$ & $\cdots$ & 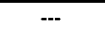 & $\begin{array}{ll}-- \\
\end{array}$ & $-\cdots$ & $\cdots$ & -- & 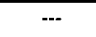 \\
\hline $\mathrm{Na}_{2} \mathrm{C}_{2} \mathrm{O}_{4}$ & 2.34 & $3.14 \%$ & $3.43 \%$ & $1.11 \%$ & --- & $1.36 \%$ & $1.97 \%$ & --- & -- & $1.67 \%$ \\
\hline $\mathrm{Na}_{2} \mathrm{CO}_{3} \cdot \mathrm{H}_{2} \mathrm{O}$ & 2.25 & -- & $5.07 \%$ & $\cdots$ & $9.60 \%$ & $3.91 \%$ & $0.01 \%$ & $8.78 \%$ & $1.40 \%$ & $6.17 \%$ \\
\hline $\mathrm{Na}_{2} \mathrm{SO}_{4}$ & 2.68 & --- & --- & --- & --- & -- & --- & --- & -- & -- \\
\hline $\mathrm{Na}_{2} \mathrm{SO}_{4} \cdot 10 \mathrm{H}_{2} \mathrm{O}$ & 1.464 & --- & --- & --- & --- & --- & --- & --- & --- & --- \\
\hline $\mathrm{Na}_{3} \mathrm{FSO}_{4}$ & 2.65 & $0.34 \%$ & --- & --- & $4.57 \%$ & $1.70 \%$ & --- & $0.09 \%$ & --- & $0.58 \%$ \\
\hline $\mathrm{Na}_{3} \mathrm{NO}_{3} \mathrm{SO}_{4} \cdot \mathrm{H}_{2} \mathrm{O}$ & 2.3 & -- & --- & --- & -- & -- & -- & --- & -- & -- \\
\hline $\mathrm{Na}_{3} \mathrm{PO}_{4} \cdot 0.25 \mathrm{NaOH} \cdot 12 \mathrm{H}_{2} \mathrm{O}$ & 1.62 & --- & $0.53 \%$ & --- & $18.75 \%$ & $5.43 \%$ & --- & --- & $4.32 \%$ & --- \\
\hline $\mathrm{Na}_{3} \mathrm{PO}_{4} \cdot 8 \mathrm{H}_{2} \mathrm{O}$ & 1.8 & --- & --- & --- & --- & --- & --- & --- & --- & --- \\
\hline $\mathrm{Na}_{4} \mathrm{P}_{2} \mathrm{O}_{7} \cdot 10 \mathrm{H}_{2} \mathrm{O}$ & 1.83 & --- & --- & --- & --- & -- & --- & --- & --- & --- \\
\hline $\mathrm{Na}_{6}\left(\mathrm{SO}_{4}\right)_{2} \mathrm{CO}_{3}$ & 2.64 & --- & $3.93 \%$ & -- & --- & -- & --- & $4.65 \%$ & $1.24 \%$ & $1.91 \%$ \\
\hline $\mathrm{NaF}$ & 2.78 & -- & --- & --- & --- & --- & $14.86 \%$ & --- & -- & -- \\
\hline $\mathrm{Na}_{7} \mathrm{~F}\left(\mathrm{PO}_{4}\right)_{2} \cdot 19 \mathrm{H}_{2} \mathrm{O}$ & 1.75 & $31.93 \%$ & $13.14 \%$ & --- & $0.85 \%$ & $8.17 \%$ & $13.77 \%$ & $18.79 \%$ & $0.91 \%$ & $1.17 \%$ \\
\hline $\mathrm{NaIICO}_{3}$ & 2.159 & -- & -.- & $\cdots$ & -.- & --- & -.. & -.. &.-- & -.- \\
\hline $\mathrm{NaNO}_{2}$ & 2.168 & -- & --- & --- & -- & --- & --- & -- & -- & --- \\
\hline $\mathrm{NaNO}_{3}$ & 2.26 & $49.80 \%$ & $55.30 \%$ & $34.88 \%$ & $54.61 \%$ & $70.43 \%$ & -- & $55.78 \%$ & $91.71 \%$ & $68.61 \%$ \\
\hline
\end{tabular}


Table C.1. (contd)

\begin{tabular}{|c|c|c|c|c|c|c|c|c|c|c|}
\hline & $\begin{array}{l}\text { Density } \\
(\mathrm{g} / \mathrm{mL})\end{array}$ & $241-\mathrm{S}-111$ & 241-S-112 & 241-SX-101 & 241-SX-102 & 241-SX-103 & 241-SX-104 & 241-SX-105 & 241-SX-106 & 241-SX-107 \\
\hline Total kg solids for tank & \multirow{9}{*}{ 11/a } & $1.21 \mathrm{E}+06$ & $3.23 \mathrm{E}+06$ & $1.09 \mathrm{E}+06$ & $6.00 \mathrm{E}+05$ & $1.20 \mathrm{E}+06$ & $1.11 \mathrm{E}+06$ & $5.22 \mathrm{E}+05$ & $7.33 \mathrm{E}+05$ & $1.87 \mathrm{E}+05$ \\
\hline Total kL solids for tank & & $5.33 \mathrm{E}+02$ & $1.42 \mathrm{E}+03$ & $4.71 \mathrm{E}+02$ & $2.52 \mathrm{E}+02$ & $5.03 \mathrm{E}+02$ & $4.57 \mathrm{E}+02$ & $2.21 \mathrm{E}+02$ & $3.25 \mathrm{E}+02$ & $6.30 \mathrm{E}+01$ \\
\hline Vol. frac. solid phase in tank & & 0.305 & 0.603 & 0.301 & 0.132 & 0.266 & 0.270 & 0.176 & 0.216 & 0.176 \\
\hline Avg g/mL, all solid & & 2.272 & 2.278 & 2.320 & 2.379 & 2.395 & 2.436 & 2.358 & 2.256 & 2.970 \\
\hline Total kg non-salt solids for tank & & $3.99 \mathrm{E}+05$ & 4. $17 \mathrm{E}+04$ & $1.66 \mathrm{E}+05$ & $1.70 \mathrm{E}+05$ & $2.12 \mathrm{E}+05$ & $3.96 \mathrm{E}+05$ & $1.42 \mathrm{E}+05$ & $1.04 \mathrm{E}+05$ & $1.70 \mathrm{E}+05$ \\
\hline Total kL non-salt solids for tank & & $1.64 \mathrm{E}+02$ & $1.5 ! \mathrm{E}+01$ & $6.15 \mathrm{E}+01$ & $6.44 \mathrm{E}+01$ & $6.76 \mathrm{E}+0 \mathrm{I}$ & $1.28 \mathrm{E}+02$ & $5.51 \mathrm{IE}+0 \mathrm{l}$ & $3.89 \mathrm{E}+01$ & $5.54 \mathrm{E}+0 \mathrm{l}$ \\
\hline Wt. frac. non-salts in solid & & 0.329 & 0.013 & 0.152 & 0.283 & 0.176 & 0.356 & 0.272 & 0.141 & 0.907 \\
\hline Avg g/mL, non-salt solid & & 2.438 & 2.755 & 2.701 & 2.635 & 3.136 & 3.090 & 2.582 & 2.666 & 3.062 \\
\hline Vol. frac. non-salts in solid & & 0.307 & 0.011 & 0.131 & 0.256 & 0.135 & 0.281 & 0.249 & 0.120 & 0.880 \\
\hline \multicolumn{11}{|l|}{ non-salts: mass fracs in total solid } \\
\hline $\mathrm{Ag}$ & 10.5 & --- & --- & --- & --- & --- & --- & --- & --- & --- \\
\hline $\mathrm{Ag}_{2} \mathrm{O}$ & 7.143 & --- & -- & --- & --- & --- & --- & --- & -- & -- \\
\hline $\mathrm{Bi}_{2} \mathrm{O}_{3}$ & 8.9 & $0.01 \%$ & --- & $0.00 \%$ & $0.02 \%$ & $0.01 \%$ & $0.02 \%$ & $0.03 \%$ & $0.02 \%$ & $0.02 \%$ \\
\hline $\mathrm{BiFeO}_{3}$ & 7.9 & $\cdots$ & $\cdots$ & $\ldots$ & -- & $\cdots$ & -.- & $\cdots$ & $\cdots$ & $--\cdot$ \\
\hline $\mathrm{Ca}(\mathrm{OH})_{2}$ & 2.24 & $\cdots$ & $\cdots$ & $\cdots$ & $\cdots$ & $\cdots$ & $\cdots$ & $\cdots$ & $\cdots$ & $\cdots$ \\
\hline $\mathrm{Ca}_{5} \mathrm{OH}\left(\mathrm{PO}_{4}\right)_{3}$ & 3.14 & $0.08 \%$ & $0.04 \%$ & $0.08 \%$ & $0.25 \%$ & $0.14 \%$ & $0.21 \%$ & $0.31 \%$ & $0.19 \%$ & $0.40 \%$ \\
\hline $\mathrm{CaC}_{2} \mathrm{O}_{4} * \mathrm{H}_{2} \mathrm{O}$ & 2.2 & -- & -- & --- & --- & --- & --- & --- & --- & --- \\
\hline $\mathrm{CaCO}_{3}$ & 2.71 & --- & --- & --- & --- & --- & --- & --- & --- & --- \\
\hline $\mathrm{CaF}_{2}$ & 3.18 & -- & --- & --- & --- & -- & --- & --- & --- & --- \\
\hline $\mathrm{CrOOH}$ & 4.11 & $0.37 \%$ & $0.20 \%$ & $2.44 \%$ & $3.92 \%$ & $1.87 \%$ & $1.62 \%$ & $1.97 \%$ & $2.20 \%$ & $0.96 \%$ \\
\hline $\mathrm{FePO}_{4} \cdot 2 \mathrm{H}_{2} \mathrm{O}$ & 3.15 & --- & --- & --- & --- & --- & --- & --- & --- & --- \\
\hline $\mathrm{FeOOH}$ & 4.26 & $0.04 \%$ & $0.07 \%$ & $0.58 \%$ & $0.65 \%$ & $0.47 \%$ & $0.77 \%$ & $0.91 \%$ & $0.49 \%$ & $1.53 \%$ \\
\hline Gibbsite & 2.42 & $32.36 \%$ & $0.04 \%$ & $11.21 \%$ & $21.91 \%$ & --- & --- & $22.21 \%$ & $9.74 \%$ & -- \\
\hline Boehmite & 3.01 & -- & --- & --- & --- & $13.97 \%$ & $30.40 \%$ & --- & --- & $81.96 \%$ \\
\hline $\mathrm{HgO}$ (co-precip. with $\mathrm{Ag}_{2} \mathrm{O}$ ) & 7.143 & --- & --- & --- & --- & --- & --- & --- & --- & $0.00 \%$ \\
\hline $\mathrm{KAlSiO}_{4}$ & 2.61 & --- & --- & --- & --- & $--\cdot$ & --- & --- & -- & -- \\
\hline $\mathrm{La}(\mathrm{OH})_{3}$ & 2.3 & --- & $0.01 \%$ & --- & $\begin{array}{ll}-- \\
\end{array}$ & $\begin{array}{ll}-- \\
\end{array}$ & --- & $\begin{array}{ll}-- \\
\end{array}$ & --- & --- \\
\hline $\mathrm{LaPO}_{+} \cdot 2 \mathrm{H}_{2} \mathrm{O}$ & 6.51 & $0.01 \%$ & --- & $0.01 \%$ & $0.02 \%$ & $0.01 \%$ & $0.01 \%$ & $0.02 \%$ & $0.02 \%$ & $0.01 \%$ \\
\hline $\mathrm{Mn}_{3}\left(\mathrm{PO}_{4}\right)_{2}$ & 3.102 & -- & --- & --- & -- & -- & -- & --- & -- & --- \\
\hline $\mathrm{MnO}_{2}$ & 5.026 & -- & $\ldots$ & --- & $0.20 \%$ & $0.28 \%$ & $0.37 \%$ & $0.35 \%$ & $0.03 \%$ & $0.87 \%$ \\
\hline $\mathrm{Na}_{2}\left(\mathrm{UO}_{2}\right)_{2}\left(\mathrm{PO}_{4}\right)_{2} \cdot 2 \mathrm{H}_{2} \mathrm{O}$ & 3.5 & --- & $\begin{array}{ll}-- \\
\end{array}$ & --- & --- & $\begin{array}{ll}-- \\
\end{array}$ & +-- & $\begin{array}{ll}-- \\
\end{array}$ & --- & --- \\
\hline $\mathrm{Na}_{2} \mathrm{U}_{2} \mathrm{O}_{7}$ & 5.617 & $0.06 \%$ & $0.07 \%$ & $0.47 \%$ & $0.32 \%$ & $0.16 \%$ & $0.70 \%$ & $0.31 \%$ & $0.16 \%$ & $1.81 \%$ \\
\hline $\mathrm{NaAICO}_{3}(\mathrm{OH})_{2}$ & 2.42 & -- & -- & --- & --- & -- & --- & --- & --- & --- \\
\hline $\mathrm{NaAlSiO}_{4}$ & 2.365 & -- & $0.84 \%$ & $0.32 \%$ & $0.87 \%$ & $0.61 \%$ & $1.22 \%$ & $1.00 \%$ & $1.21 \%$ & $2.27 \%$ \\
\hline $\mathrm{Ni}(\mathrm{OH})_{2}$ & 4.15 & $0.01 \%$ & $0.01 \%$ & $0.02 \%$ & $0.06 \%$ & $0.04 \%$ & $0.22 \%$ & $0.07 \%$ & $0.04 \%$ & $0.68 \%$ \\
\hline $\mathrm{Ni}_{3}\left(\mathrm{PO}_{4}\right)$ & 3.93 & -- & -- & $\cdots$ & -- & -- & -- & -- & $\cdots$ & $\cdots$ \\
\hline $\mathrm{NiC}_{2} \mathrm{O}_{4} \cdot 2 \mathrm{H}_{2} \mathrm{O}$ (on Fe phase) & 4.26 & -- & --- & --- & --- & -- & -- & --- & -- & --- \\
\hline $\mathrm{Pb}(\mathrm{OH})_{2}$ & 7.1 & $0.01 \%$ & $0.01 \%$ & $0.03 \%$ & $0.04 \%$ & $0.03 \%$ & $0.03 \%$ & $0.04 \%$ & $0.03 \%$ & $0.03 \%$ \\
\hline $\mathrm{Pb}_{3}\left(\mathrm{PO}_{4}\right)_{2}$ & 7.1 & -- & $\begin{array}{ll}-- \\
\end{array}$ & --- & $\begin{array}{ll}-- \\
\end{array}$ & --- & --- & $\begin{array}{ll}-- \\
\end{array}$ & -- & --- \\
\hline $\mathrm{PbCO}_{3}$ & 6.6 & -- & --- & --- & --- & -- & --- & --- & --- & --- \\
\hline $\mathrm{Pu}(\mathrm{OH})_{4}$ (co-precip. on Fe phase) & 4.26 & $0.00 \%$ & $0.00 \%$ & $0.00 \%$ & $0.00 \%$ & $0.00 \%$ & $0.00 \%$ & $0.00 \%$ & $0.00 \%$ & $0.00 \%$ \\
\hline $\mathrm{PuO}_{2}$ & 11.43 & -- & --- & --- & --- & --- & -- & --- & --- & -- \\
\hline $\mathrm{SiO}_{2}$ & 2.6 & --- & --- & --- & --- & --- & --- & --- & --- & --- \\
\hline $\mathrm{Sr}_{3}\left(\mathrm{PO}_{4}\right)_{2}$ & 3.5 & -- & --- & $0.04 \%$ & --- & --- & --- & $0.01 \%$ & --- & --- \\
\hline $\mathrm{SrCO}_{3}$ & 3.5 & $0.00 \%$ & $0.00 \%$ & --- & $0.01 \%$ & $0.01 \%$ & $0.05 \%$ & --- & $0.00 \%$ & $0.14 \%$ \\
\hline $\mathrm{ZrO}_{2}$ & 5.68 & -- & $0.00 \%$ & $0.01 \%$ & $0.02 \%$ & $0.01 \%$ & $0.01 \%$ & $0.02 \%$ & $0.01 \%$ & $0.03 \%$ \\
\hline \multicolumn{11}{|l|}{ salts: mass fracs in total solid } \\
\hline $\mathrm{KNO}_{3}$ & 2.109 & $-\cdots$ & $\begin{array}{ll}-\cdot \\
\end{array}$ & $\cdots$ & $-\cdots$ & +-- & $-\cdots$ & $-\cdots$ & $-\cdots$ & 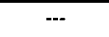 \\
\hline $\mathrm{Na}_{2} \mathrm{C}_{2} \mathrm{O}_{4}$ & 2.34 & $1.54 \%$ & $1.01 \%$ & --- & $5.46 \%$ & $3.74 \%$ & $2.99 \%$ & $3.61 \%$ & $3.01 \%$ & $2.78 \%$ \\
\hline $\mathrm{Na}_{2} \mathrm{CO}_{3} \cdot \mathrm{H}_{2} \mathrm{O}$ & 2.25 & $4.36 \%$ & $18.74 \%$ & $0.42 \%$ & $18.12 \%$ & $10.93 \%$ & $8.16 \%$ & $12.49 \%$ & $4.12 \%$ & $0.18 \%$ \\
\hline $\mathrm{Na}_{2} \mathrm{SO}_{4}$ & 2.68 & -- & -- & --- & --- & --- & --- & --- & --- & --- \\
\hline $\mathrm{Na}_{2} \mathrm{SO}_{4} \cdot 10 \mathrm{H}_{2} \mathrm{O}$ & 1.464 & --- & --- & $\begin{array}{ll}-- \\
\end{array}$ & $\begin{array}{ll}-- \\
\end{array}$ & --- & $\begin{array}{ll}-- \\
\end{array}$ & --- & --- & $\begin{array}{ll}-- \\
\end{array}$ \\
\hline $\mathrm{Na}_{3} \mathrm{FSO}_{4}$ & 2.65 & $0.05 \%$ & --- & $0.14 \%$ & $0.03 \%$ & $0.07 \%$ & $1.58 \%$ & --- & --- & $0.35 \%$ \\
\hline $\mathrm{Na}_{3} \mathrm{NO}_{3} \mathrm{SO}_{4} \cdot \mathrm{H}_{2} \mathrm{O}$ & 2.3 & -- & -- & --- & --- & -- & --- & --- & -- & -- \\
\hline $\mathrm{Na}_{3} \mathrm{PO}_{4} \cdot 0.25 \mathrm{NaOH} \cdot 12 \mathrm{H}_{2} \mathrm{O}$ & 1.62 & --- & $1.55 \%$ & --- & --- & --- & --- & --- & $1.92 \%$ & --- \\
\hline $\mathrm{Na}_{3} \mathrm{PO}_{4} \cdot 8 \mathrm{H}_{2} \mathrm{O}$ & 1.8 & -- & --- & --- & --- & --- & --- & --- & --- & --- \\
\hline $\mathrm{Na}_{4} \mathrm{P}_{2} \mathrm{O}_{7} \cdot 10 \mathrm{H}_{2} \mathrm{O}$ & 1.83 & -- & --- & --- & --- & --- & --- & --- & -- & --- \\
\hline $\mathrm{Na}_{6}\left(\mathrm{SO}_{4}\right)_{2} \mathrm{CO}_{3}$ & 2.64 & $4.67 \%$ & $9.92 \%$ & $0.63 \%$ & $6.10 \%$ & $4.49 \%$ & $1.32 \%$ & $4.74 \%$ & $1.66 \%$ & --- \\
\hline $\mathrm{NaF}$ & 2.78 & -- & --- & --- & --- & --- & --- & --- & --- & --- \\
\hline $\mathrm{Na}_{7} \mathrm{~F}\left(\mathrm{PO}_{4}\right)_{2} \cdot 19 \mathrm{H}_{2} \mathrm{O}$ & 1.75 & $8.96 \%$ & $0.74 \%$ & --- & --- & --- & $9.74 \%$ & --- & $6.43 \%$ & --- \\
\hline $\mathrm{NaIICO}_{3}$ & 2.159 & -- & -.- & $\cdots$ & $-\cdot$ & -- & $\cdots$ & $\cdots$ & $--\cdot$ & $\cdots$ \\
\hline $\mathrm{NaNO}_{2}$ & 2.168 & --- & --- & --- & --- & --- & --- & --- & --- & -- \\
\hline $\mathrm{NaNO}_{3}$ & 2.26 & $47.48 \%$ & $66.74 \%$ & $83.61 \%$ & $41.99 \%$ & $63.16 \%$ & $40.58 \%$ & $51.91 \%$ & $68.72 \%$ & $5.98 \%$ \\
\hline
\end{tabular}


Table C.1. (contd)

\begin{tabular}{|c|c|c|c|c|c|c|c|c|c|c|}
\hline & $\begin{array}{l}\text { Density } \\
(\mathrm{g} / \mathrm{mL})\end{array}$ & 241-SX-108 & 241-SX-109 & $241-S X-110$ & 241-SX-111 & 241-SX-112 & 241-SX-113 & $241-\mathrm{SX}-114$ & 241-SX-115 & 241-SY-101 \\
\hline Total kg solids for tank & \multirow{9}{*}{ 11/a } & $2.36 \mathrm{E}+05$ & $7.55 \mathrm{E}+05$ & $1.40 \mathrm{E}+05$ & $2.03 E+05$ & $1.67 \mathrm{E}+05$ & $4.24 \mathrm{E}+04$ & $3.06 \mathrm{E}+05$ & $7.67 \mathrm{E}+03$ & $4.99 \mathrm{E}+05$ \\
\hline Total kL solids for tank & & $9.49 \mathrm{E}+01$ & $3.02 \mathrm{E}+02$ & $5.25 \mathrm{E}+01$ & $7.00 \mathrm{E}+01$ & $5.88 \mathrm{E}+01$ & $1.63 \mathrm{E}+01$ & $1.19 \mathrm{E}+02$ & $2.18 \mathrm{E}+00$ & $2.13 \mathrm{E}+02$ \\
\hline Vol. frac. solid phase in tank & & 0.344 & 0.331 & 0.248 & 0.161 & 0.208 & 0.230 & 0.200 & 0.156 & 0.059 \\
\hline Avg g/mL, all solid & & 2.488 & 2,502 & 2.658 & 2.905 & 2.837 & 2.598 & 2.575 & 3.512 & 2.341 \\
\hline Total $\mathrm{kg}$ non-salt solids for tank & & $6.11 \mathrm{E}+04$ & $2.81 \mathrm{E}+05$ & $8.04 \mathrm{E}+04$ & $1.72 \mathrm{E}+05$ & $1.28 \mathrm{E}+05$ & $4.24 \mathrm{E}+04$ & $1.42 \mathrm{E}+05$ & $7.67 \mathrm{E}+03$ & $2.95 \mathrm{E}+05$ \\
\hline Total kL non-salt solids for tank & & $1.75 \mathrm{E}+01$ & $9.25 \mathrm{E}+01$ & $2.64 \mathrm{E}+01$ & $5.61 \mathrm{E}+01$ & $4.19 \mathrm{E}+01$ & $1.63 \mathrm{E}+01$ & $4.63 \mathrm{E}+0 \mathrm{l}$ & $2.18 \mathrm{E}+00$ & $1.16 \mathrm{E}+02$ \\
\hline Wt. frac. non-salts in solid & & 0.259 & 0.373 & 0.576 & 0.844 & 0.770 & 1.000 & 0.464 & 1.000 & 0.591 \\
\hline Avg g/mL, non-salt solid & & 3.499 & 3.043 & 3.051 & 3.062 & 3.062 & 2.598 & 3.066 & 3.512 & 2.542 \\
\hline Vol. frac. non-salts in solid & & 0.184 & 0.306 & 0.502 & 0.801 & 0.714 & 1.000 & 0.590 & 1.000 & 0.544 \\
\hline \multicolumn{11}{|l|}{ non-salts: mass fracs in total solid } \\
\hline $\mathrm{Ag}$ & 10.5 & --- & --- & --- & --- & --- & --- & --- & --- & --- \\
\hline $\mathrm{Ag}_{2} \mathrm{O}$ & 7.143 & --- & --- & --- & --- & --- & $0.00 \%$ & --- & --- & --- \\
\hline $\mathrm{Bi}_{2} \mathrm{O}_{3}$ & 8.9 & $0.01 \%$ & $0.00 \%$ & $0.01 \%$ & $0.02 \%$ & $0.01 \%$ & $0.00 \%$ & $0.00 \%$ & $0.02 \%$ & $0.02 \%$ \\
\hline $\mathrm{BiFeO}_{3}$ & 7.9 & $\ldots$ & $\ldots$ & $\ldots$ &.-- & -.. & $\ldots$ & $\ldots$ & -- & -- \\
\hline $\mathrm{Ca}(\mathrm{OH})_{2}$ & 2.24 & $\cdots$ & $\cdots$ & $\cdots$ & $\cdots$ & $\cdots$ & 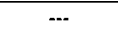 & 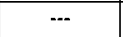 & $\cdots$ & $\cdots$ \\
\hline $\mathrm{Ca}_{5} \mathrm{OH}\left(\mathrm{PO}_{4}\right)_{3}$ & 3.14 & $0.12 \%$ & $0.16 \%$ & $0.26 \%$ & $0.39 \%$ & $0.33 \%$ & $0.19 \%$ & $0.23 \%$ & $4.81 \%$ & $0.35 \%$ \\
\hline $\mathrm{CaC}_{2} \mathrm{O}_{4} * \mathrm{H}_{2} \mathrm{O}$ & 2.2 & -- & --- & --- & -- & -- & $0.09 \%$ & --- & --- & --- \\
\hline $\mathrm{CaCO}_{3}$ & 2.71 & $0.81 \%$ & --- & --- & --- & --- & $0.48 \%$ & --- & --- & --- \\
\hline $\mathrm{CaF}_{2}$ & 3.18 & --- & --- & --- & --- & -- & $0.04 \%$ & --- & $0.59 \%$ & --- \\
\hline $\mathrm{CrOOH}$ & 4.11 & $1.77 \%$ & $\cdots$ & $\cdots$ & $0.82 \%$ & $0.87 \%$ & ..- & $0.85 \%$ & ..- & $5.76 \%$ \\
\hline $\mathrm{FePO}_{4} \cdot 2 \mathrm{H}_{2} \mathrm{O}$ & 3.15 & --- & --- & --- & --- & --- & -- & --- & -- & --- \\
\hline $\mathrm{FeOOH}$ & 4.26 & $6.09 \%$ & $0.49 \%$ & $0.90 \%$ & $1.43 \%$ & $1.27 \%$ & $7.87 \%$ & $0.72 \%$ & $47.43 \%$ & $0.42 \%$ \\
\hline Gibbsite & 2.42 & --- & --- & -- & --- & --- & --- & --- & --- & $52.17 \%$ \\
\hline Boehmite & 3.01 & $11.57 \%$ & $33.89 \%$ & $52.35 \%$ & $75.94 \%$ & $69.59 \%$ & --- & $40.78 \%$ & $25.37 \%$ & -- \\
\hline $\mathrm{HgO}$ (co-precip. with $\mathrm{Ag}_{2} \mathrm{O}$ ) & 7.143 & $0.00 \%$ & --- & --- & --- & --- & --- & --- & $0.01 \%$ & --- \\
\hline $\mathrm{KAISiO}_{4}$ & 2.61 & --- & --- & $-\cdots$ & --- & --- & $1.85 \%$ & --- & -- & --- \\
\hline $\mathrm{La}(\mathrm{OH})_{3}$ & 2.3 & --- & --- & --- & --- & --- & $\begin{array}{l}-- \\
--\end{array}$ & --- & $\begin{array}{ll}-- \\
-1\end{array}$ & $\begin{array}{ll}-- \\
-1\end{array}$ \\
\hline $\mathrm{LaPO}_{4} \cdot 2 \mathrm{H}_{2} \mathrm{O}$ & 6.51 & $0.05 \%$ & $0.00 \%$ & $0.01 \%$ & $0.01 \%$ & $0.01 \%$ & $0.00 \%$ & $0.00 \%$ & $0.02 \%$ & $0.02 \%$ \\
\hline $\mathrm{Mn}_{3}\left(\mathrm{PO}_{4}\right)_{2}$ & 3.102 & -- & --- & -- & -- & -- & -- & --- & -- & -- \\
\hline $\mathrm{MnO}_{2}$ & 5.026 & $2.02 \%$ & $0.33 \%$ & $0.60 \%$ & $0.88 \%$ & $0.72 \%$ & $0.26 \%$ & $0.54 \%$ & $5.68 \%$ & $--\cdot$ \\
\hline $\mathrm{Na}_{2}\left(\mathrm{UO}_{2}\right)_{2}\left(\mathrm{PO}_{4}\right)_{2} \cdot 2 \mathrm{H}_{2} \mathrm{O}$ & 3.5 & --- & --- & --- & --- & --- & --- & --- & --- & --- \\
\hline $\mathrm{Na}_{2} \mathrm{U}_{2} \mathrm{O}_{7}$ & 5.617 & $1.47 \%$ & $0.87 \%$ & $1.27 \%$ & $1.80 \%$ & $1.54 \%$ & $0.18 \%$ & $1.17 \%$ & $2.02 \%$ & $0.08 \%$ \\
\hline $\mathrm{NaAICO}_{3}(\mathrm{OH})_{2}$ & 2.42 & --- & -- & --- & --- & --- & --- & -- & $4.03 \%$ & --- \\
\hline $\mathrm{NaAlSiO}_{4}$ & 2.365 & $1.17 \%$ & $1.29 \%$ & $1.74 \%$ & $2.35 \%$ & $1.95 \%$ & $34.55 \%$ & $1.72 \%$ & $8.89 \%$ & --- \\
\hline $\mathrm{Ni}(\mathrm{OH})_{2}$ & 4.15 & $0.40 \%$ & $0.14 \%$ & $0.35 \%$ & $0.61 \%$ & $0.55 \%$ & $0.05 \%$ & $0.24 \%$ & $0.82 \%$ & $0.17 \%$ \\
\hline $\mathrm{Ni}_{3}\left(\mathrm{PO}_{4}\right)$ & 3.93 & -- & $--\cdot$ & $\cdots$ & -- & --- & -- & $--\cdot$ & $\cdots$ & -- \\
\hline $\mathrm{NiC}_{2} \mathrm{O}_{4} \cdot 2 \mathrm{H}_{2} \mathrm{O}$ (on Fe phase) & 4.26 & --- & --- & --- & --- & --- & --- & --- & --- & --- \\
\hline $\mathrm{Pb}(\mathrm{OH})_{2}$ & 7.1 & $0.06 \%$ & $0.02 \%$ & $0.02 \%$ & $0.03 \%$ & $0.02 \%$ & $0.00 \%$ & $0.02 \%$ & $0.03 \%$ & $0.10 \%$ \\
\hline $\mathrm{Pb}_{3}\left(\mathrm{PO}_{4}\right)_{2}$ & 7.1 & -- & --- & --- & --- & -- & $\begin{array}{ll}-- \\
-1\end{array}$ & --- & -- & --- \\
\hline $\mathrm{PbCO}_{3}$ & 6.6 & -- & --- & --- & --- & -- & --- & --- & --- & --- \\
\hline $\mathrm{Pu}(\mathrm{OH})_{4}$ (co-precip. on Fe phase) & 4.26 & $0.01 \%$ & $0.00 \%$ & $0.00 \%$ & $0.00 \%$ & $0.00 \%$ & $0.00 \%$ & $0.00 \%$ & $0.10 \%$ & $0.00 \%$ \\
\hline $\mathrm{PuO}_{2}$ & 11.43 & -- & --- & --- & --- & -- & -- & -- & -- & -- \\
\hline $\mathrm{SiO}_{2}$ & 2.6 & $\begin{array}{l}-- \\
\end{array}$ & $\begin{array}{l}-- \\
\end{array}$ & --- & --- & --- & $54.41 \%$ & --- & --- & --- \\
\hline $\mathrm{Sr}_{3}\left(\mathrm{PO}_{4}\right)_{2}$ & 3.5 & --- & --- & --- & --- & --- & --- & $0.09 \%$ & $0.16 \%$ & --- \\
\hline $\mathrm{SrCO}_{3}$ & 3.5 & $0.20 \%$ & $0.06 \%$ & $0.09 \%$ & $0.14 \%$ & $0.12 \%$ & --- & --- & --- & $0.01 \%$ \\
\hline $\mathrm{ZrO}_{2}$ & 5.68 & $0.12 \%$ & $0.01 \%$ & $0.02 \%$ & $0.03 \%$ & $0.02 \%$ & $0.01 \%$ & $0.01 \%$ & $0.03 \%$ & $--\cdot$ \\
\hline \multicolumn{11}{|l|}{ salts: mass fracs in total solid } \\
\hline $\mathrm{KNO}_{3}$ & 2.109 & $\begin{array}{ll}-- \\
\end{array}$ & $\begin{array}{ll}-\cdot \\
\end{array}$ & $\cdots$ & $-\cdots$ & $\begin{array}{ll}-- \\
\end{array}$ & $\overline{-\cdots}$ & 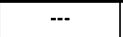 & -- & 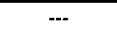 \\
\hline $\mathrm{Na}_{2} \mathrm{C}_{2} \mathrm{O}_{4}$ & 2.34 & $0.36 \%$ & $0.64 \%$ & $0.96 \%$ & $2.69 \%$ & $2.35 \%$ & --- & $1.67 \%$ & -- & $29.91 \%$ \\
\hline $\mathrm{Na}_{2} \mathrm{CO}_{3} \cdot \mathrm{H}_{2} \mathrm{O}$ & 2.25 & $1.87 \%$ & $0.14 \%$ & $\cdots$ & $--\cdot$ & $0.50 \%$ & $0.00 \%$ & $\cdots$ & -- & -- \\
\hline $\mathrm{Na}_{2} \mathrm{SO}_{4}$ & 2.68 & -- & --- & --- & --- & -- & --- & --- & -- & --- \\
\hline $\mathrm{Na}_{2} \mathrm{SO}_{4} \cdot 10 \mathrm{H}_{2} \mathrm{O}$ & 1.464 & --- & $\begin{array}{ll}-- \\
\end{array}$ & --- & --- & --- & --- & --- & --- & --- \\
\hline $\mathrm{Na}_{3} \mathrm{FSO}_{4}$ & 2.65 & --- & $0.36 \%$ & $0.15 \%$ & --- & $0.65 \%$ & --- & --- & --- & --- \\
\hline $\mathrm{Na}_{3} \mathrm{NO}_{3} \mathrm{SO}_{4} \cdot \mathrm{H}_{2} \mathrm{O}$ & 2.3 & --- & -- & --- & --- & --- & --- & --- & -- & --- \\
\hline $\mathrm{Na}_{3} \mathrm{PO}_{4} \cdot 0.25 \mathrm{NaOH} \cdot 12 \mathrm{H}_{2} \mathrm{O}$ & 1.62 & --- & --- & --- & --- & --- & --- & --- & --- & $9.12 \%$ \\
\hline $\mathrm{Na}_{3} \mathrm{PO}_{4} \cdot 8 \mathrm{H}_{2} \mathrm{O}$ & 1.8 & --- & --- & --- & --- & --- & --- & --- & --- & --- \\
\hline $\mathrm{Na}_{4} \mathrm{P}_{2} \mathrm{O}_{7} \cdot 10 \mathrm{H}_{2} \mathrm{O}$ & 1.83 & --- & --- & --- & --- & -- & --- & --- & --- & --- \\
\hline $\mathrm{Na}_{6}\left(\mathrm{SO}_{4}\right)_{2} \mathrm{CO}_{3}$ & 2.64 & --- & --- & -- & --- & -- & --- & $\cdots$ & -- & --- \\
\hline $\mathrm{NaF}$ & 2.78 & -- & --- & --- & --- & --- & --- & --- & --- & -- \\
\hline $\mathrm{Na}_{7} \mathrm{~F}\left(\mathrm{PO}_{4}\right)_{2} \cdot 19 \mathrm{H}_{2} \mathrm{O}$ & 1.75 & --- & --- & --- & --- & --- & --- & --- & --- & $1.86 \%$ \\
\hline $\mathrm{NaIICO}_{3}$ & 2.159 & --- & .-. & $\cdots$ & -.. & --- & ..- & $\cdots$ & -- & -- \\
\hline $\mathrm{NaNO}_{2}$ & 2.168 & -- & -- & --- & -- & --- & --- & --- & --- & --- \\
\hline $\mathrm{NaNO}_{3}$ & 2.26 & $71.88 \%$ & $61.59 \%$ & $41.28 \%$ & $12.86 \%$ & $19.49 \%$ & -- & $51.95 \%$ & --- & --- \\
\hline
\end{tabular}


Table C.1. (contd)

\begin{tabular}{|c|c|c|c|c|c|c|c|c|c|c|}
\hline & $\begin{array}{l}\text { Density } \\
(\mathrm{g} / \mathrm{mL})\end{array}$ & 241-SY-102 & 241-SY-103 & $241-\mathrm{T}-101$ & $241-T-102$ & 241-T-103 & 241-T-104 & 241-T-105 & $241-T-106$ & 241-T-107 \\
\hline Total kg solids for tank & \multirow{9}{*}{ 11/a } & $3.28 \mathrm{E}+05$ & $6.49 \mathrm{E}+05$ & $1.87 \mathrm{E}+05$ & $1.16 \mathrm{E}+05$ & $6.04 \mathrm{E}+04$ & $4.32 \mathrm{E}+05$ & $1.31 \mathrm{IE}+05$ & $7.54 \mathrm{E}+04$ & $2.98 \mathrm{E}+05$ \\
\hline Total kL solids for tank & & 1. $34 \mathrm{E}+02$ & $2.82 \mathrm{E}+02$ & $8.12 \mathrm{E}+01$ & $4.69 \mathrm{E}+01$ & $2.37 \mathrm{E}+01$ & $2.04 \mathrm{E}+02$ & $4.31 \mathrm{E}+01$ & $3.43 \mathrm{E}+01$ & $1.07 \mathrm{E}+02$ \\
\hline Vol. frac. solid phase in tank & & 0.034 & 0.102 & 0.216 & 0.388 & 0.233 & 0.170 & 0.116 & 0.419 & 0.164 \\
\hline Avg g/mL, all solid & & 2.446 & 2,300 & 2.298 & 2.479 & 2.544 & 2.115 & 3.040 & 2.195 & 2.779 \\
\hline Total $\mathrm{kg}$ non-salt solids for tank & & $2.35 \mathrm{E}+05$ & $2.45 \mathrm{E}+05$ & $1.06 \mathrm{E}+05$ & $1.16 \mathrm{E}+05$ & $6.01 \mathrm{E}+04$ & $1.62 \mathrm{E}+05$ & $1.28 \mathrm{E}+05$ & $4.49 \mathrm{E}+04$ & $2.57 \mathrm{E}+05$ \\
\hline Total kL non-salt solids for tank & & $8.83 \mathrm{E}+01$ & $9.55 \mathrm{E}+01$ & $4.29 \mathrm{E}+01$ & $4.68 \mathrm{E}+01$ & $2.36 \mathrm{E}+0 \mathrm{I}$ & $5.23 \mathrm{E}+01$ & 4. $18 \mathrm{E}+01$ & $1.71 \mathrm{E}+01$ & $8.71 \mathrm{E}+01$ \\
\hline Wt. frac. non-salts in solid & & 0.716 & 0.377 & 0.569 & 0.997 & 0.995 & 0.376 & 0.976 & 0.596 & 0.861 \\
\hline Avg g/mL, non-salt solid & & 2.662 & 2.564 & 2.477 & 2.479 & 2.545 & 3.104 & 3.063 & 2.618 & 2.949 \\
\hline Vol. frac. non-salts in solid & & 0.658 & 0.338 & 0.528 & 0.997 & 0.994 & 0.256 & 0.968 & 0.500 & 0.811 \\
\hline \multicolumn{11}{|l|}{ non-salts: mass fracs in total solid } \\
\hline $\mathrm{Ag}$ & 10.5 & --- & --- & --- & --- & --- & --- & --- & --- & --- \\
\hline $\mathrm{Ag}_{2} \mathrm{O}$ & 7.143 & --- & --- & --- & $0.04 \%$ & --- & $0.00 \%$ & $0.01 \%$ & --- & $0.00 \%$ \\
\hline $\mathrm{Bi}_{2} \mathrm{O}_{3}$ & 8.9 & $1.05 \%$ & $0.02 \%$ & $0.08 \%$ & $0.22 \%$ & $0.48 \%$ & --- & --- & $3.24 \%$ & --- \\
\hline $\mathrm{BiFeO}_{3}$ & 7.9 & $\ldots$ & $\ldots$ & $\ldots$ &.- & -.. & $9.97 \%$ & $15.32 \%$ & -- & $4.72 \%$ \\
\hline $\mathrm{Ca}(\mathrm{OH})_{2}$ & 2.24 & $\cdots$ & $\cdots$ & $\cdots$ & $\cdots$ & $\cdots$ & $\cdots$ & 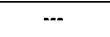 & $\cdots$ & $\cdots$ \\
\hline $\mathrm{Ca}_{5} \mathrm{OH}\left(\mathrm{PO}_{4}\right)_{3}$ & 3.14 & $1.10 \%$ & $0.42 \%$ & $0.23 \%$ & $0.24 \%$ & $0.35 \%$ & -- & $1.29 \%$ & -- & -- \\
\hline $\mathrm{CaC}_{2} \mathrm{O}_{4} * \mathrm{H}_{2} \mathrm{O}$ & 2.2 & -- & --- & --- & -- & --- & --- & -- & --- & --- \\
\hline $\mathrm{CaCO}_{3}$ & 2.71 & --- & --- & --- & --- & --- & --- & --- & --- & --- \\
\hline $\mathrm{CaF}_{2}$ & 3.18 & --- & --- & --- & --- & $0.19 \%$ & $1.00 \%$ & --- & $0.32 \%$ & $1.23 \%$ \\
\hline $\mathrm{CrOOH}$ & 4.11 & $7.21 \%$ & $3.13 \%$ & -.. & $0.00 \%$ & $0,07 \%$ & ... & $1.00 \%$ & $\ldots$ & $0.20 \%$ \\
\hline $\mathrm{FePO}_{4} \cdot 2 \mathrm{H}_{2} \mathrm{O}$ & 3.15 & --- & --- & --- & --- & --- & $4.65 \%$ & $0.21 \%$ & --- & $27.99 \%$ \\
\hline $\mathrm{FeOOH}$ & 4.26 & $4.06 \%$ & $1.11 \%$ & $0.63 \%$ & $2.82 \%$ & $6.67 \%$ & --- & $16.87 \%$ & $2.90 \%$ & --- \\
\hline Gibbsite & 2.42 & $56.78 \%$ & $30.85 \%$ & $52.89 \%$ & $86.00 \%$ & $72.80 \%$ & $10.63 \%$ & $37.72 \%$ & $41.28 \%$ & $25.80 \%$ \\
\hline Boehmite & 3.01 & -- & --- & -- & --- & --- & --- & --- & -- & -- \\
\hline $\mathrm{HgO}$ (co-precip. with $\mathrm{Ag}_{2} \mathrm{O}$ ) & 7.143 & --- & --- & --- & --- & --- & --- & --- & --- & --- \\
\hline $\mathrm{KAISiO}_{4}$ & 2.61 & -- & --- & $-\cdots$ & --- & --- & --- & --- & $-\cdots$ & --- \\
\hline $\mathrm{La}(\mathrm{OH})_{3}$ & 2.3 & --- & --- & --- & $\begin{array}{ll}-- \\
\end{array}$ & --- & --- & $\begin{array}{ll}-- \\
\end{array}$ & --- & --- \\
\hline $\mathrm{LaPO}_{4} \cdot 2 \mathrm{H}_{2} \mathrm{O}$ & 6.51 & $0.08 \%$ & $0.02 \%$ & $0.02 \%$ & $0.02 \%$ & $0.04 \%$ & $0.00 \%$ & $0.03 \%$ & $0.01 \%$ & $0.00 \%$ \\
\hline $\mathrm{Mn}_{3}\left(\mathrm{PO}_{4}\right)_{2}$ & 3.102 & -- & -- & --- & $0.24 \%$ & $0.45 \%$ & $0.05 \%$ & --- & $0.05 \%$ & $0.30 \%$ \\
\hline $\mathrm{MnO}_{2}$ & 5.026 & $0.51 \%$ & $\ldots$ & $0.11 \%$ &.-- & -- & $--\cdot$ & $2.48 \%$ & $\ldots$ & -.- \\
\hline $\mathrm{Na}_{2}\left(\mathrm{UO}_{2}\right)_{2}\left(\mathrm{PO}_{4}\right)_{2} \cdot 2 \mathrm{H}_{2} \mathrm{O}$ & 3.5 & --- & $\begin{array}{ll}-- \\
\end{array}$ & --- & --- & --- & --- & --- & --- & --- \\
\hline $\mathrm{Na}_{2} \mathrm{U}_{2} \mathrm{O}_{7}$ & 5.617 & $0.31 \%$ & $0.29 \%$ & $0.98 \%$ & $1.44 \%$ & $1.50 \%$ & $0.42 \%$ & $1.16 \%$ & $1.12 \%$ & $8.85 \%$ \\
\hline $\mathrm{NaAICO}_{3}(\mathrm{OH})_{2}$ & 2.42 & -- & --- & --- & $6.97 \%$ & $8.26 \%$ & --- & --- & $3.76 \%$ & $8.15 \%$ \\
\hline $\mathrm{NaAlSiO}_{4}$ & 2.365 & --- & $1.71 \%$ & $1.38 \%$ & $1.61 \%$ & $7.39 \%$ & $10.78 \%$ & $20.90 \%$ & $6.37 \%$ & $7.92 \%$ \\
\hline $\mathrm{Ni}(\mathrm{OH})_{2}$ & 4.15 & $0.18 \%$ & $0.06 \%$ & $0.09 \%$ & $0.02 \%$ & $0.45 \%$ & $0.01 \%$ & $0.04 \%$ & $0.07 \%$ & $0.07 \%$ \\
\hline $\mathrm{Ni}_{3}\left(\mathrm{PO}_{4}\right)$ & 3.93 & -- & $--\cdot$ & $\cdots$ & -- & --- & -- & $--\cdot$ & $\cdots$ & -- \\
\hline $\mathrm{NiC}_{2} \mathrm{O}_{4} \cdot 2 \mathrm{H}_{2} \mathrm{O}$ (on Fe phase) & 4.26 & -- & --- & --- & --- & -- & -- & --- & -- & --- \\
\hline $\mathrm{Pb}(\mathrm{OH})_{2}$ & 7.1 & $0.26 \%$ & $0.09 \%$ & $0.40 \%$ & $0.08 \%$ & $0.47 \%$ & $0.02 \%$ & $0.36 \%$ & $0.36 \%$ & $0.28 \%$ \\
\hline $\mathrm{Pb}_{3}\left(\mathrm{PO}_{4}\right)_{2}$ & 7.1 & --- & --- & --- & --- & -- & $\begin{array}{ll}-- \\
-1\end{array}$ & --- & -- & --- \\
\hline $\mathrm{PbCO}_{3}$ & 6.6 & -- & --- & --- & --- & -- & --- & --- & --- & --- \\
\hline $\mathrm{Pu}(\mathrm{OH})_{4}$ (co-precip. on Fe phase) & 4.26 & --- & $0.00 \%$ & $0.00 \%$ & $0.00 \%$ & $0.00 \%$ & $0.00 \%$ & $0.00 \%$ & $0.00 \%$ & $0.00 \%$ \\
\hline $\mathrm{PuO}_{2}$ & 11.43 & $0.02 \%$ & --- & --- & --- & --- & --- & --- & --- & --- \\
\hline $\mathrm{SiO}_{2}$ & 2.6 & --- & $\begin{array}{l}-- \\
\end{array}$ & --- & --- & --- & --- & --- & --- & --- \\
\hline $\mathrm{Sr}_{3}\left(\mathrm{PO}_{4}\right)_{2}$ & 3.5 & --- & --- & --- & --- & --- & $0.06 \%$ & --- & $0.05 \%$ & --- \\
\hline $\mathrm{SrCO}_{3}$ & 3.5 & $0.03 \%$ & $0.00 \%$ & $0.01 \%$ & $0.01 \%$ & $0.02 \%$ & --- & $0.15 \%$ & --- & $0.51 \%$ \\
\hline $\mathrm{ZrO}_{2}$ & 5.68 & $0.01 \%$ & $0.02 \%$ & $0.03 \%$ & $0.01 \%$ & $0.35 \%$ & $0.03 \%$ & $0.03 \%$ & $0.05 \%$ & $0.02 \%$ \\
\hline \multicolumn{11}{|l|}{ salts: mass fracs in total solid } \\
\hline $\mathrm{KNO}_{3}$ & 2.109 & $-\cdots$ & $\begin{array}{ll}-\cdot \\
\end{array}$ & $\cdots$ & $-\cdots$ & $\begin{array}{ll}-- \\
\end{array}$ & $\overline{-\cdots}$ & $-\cdots$ & $-\cdots$ & 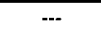 \\
\hline $\mathrm{Na}_{2} \mathrm{C}_{2} \mathrm{O}_{4}$ & 2.34 & $17.47 \%$ & $9.71 \%$ & --- & $0.26 \%$ & $0.51 \%$ & --- & $2.41 \%$ & $0.62 \%$ & $0.31 \%$ \\
\hline $\mathrm{Na}_{2} \mathrm{CO}_{3} \cdot \mathrm{H}_{2} \mathrm{O}$ & 2.25 & -- & $3,56 \%$ & $\cdots$ & $0.02 \%$ & --- & $0.00 \%$ & $0.00 \%$ & -- & $0.00 \%$ \\
\hline $\mathrm{Na}_{2} \mathrm{SO}_{4}$ & 2.68 & --- & --- & --- & -- & --- & --- & --- & -- & -- \\
\hline $\mathrm{Na}_{2} \mathrm{SO}_{4} \cdot 10 \mathrm{H}_{2} \mathrm{O}$ & 1.464 & --- & --- & --- & --- & --- & --- & --- & --- & --- \\
\hline $\mathrm{Na}_{3} \mathrm{FSO}_{4}$ & 2.65 & --- & --- & --- & --- & --- & --- & --- & --- & --- \\
\hline $\mathrm{Na}_{3} \mathrm{NO}_{3} \mathrm{SO}_{4} \cdot \mathrm{H}_{2} \mathrm{O}$ & 2.3 & --- & --- & --- & --- & --- & -- & --- & -- & --- \\
\hline $\mathrm{Na}_{3} \mathrm{PO}_{4} \cdot 0.25 \mathrm{NaOH} \cdot 12 \mathrm{H}_{2} \mathrm{O}$ & 1.62 & $5.91 \%$ & --- & --- & --- & --- & --- & --- & --- & --- \\
\hline $\mathrm{Na}_{3} \mathrm{PO}_{4} \cdot 8 \mathrm{H}_{2} \mathrm{O}$ & 1.8 & --- & --- & --- & --- & --- & --- & --- & -- & --- \\
\hline $\mathrm{Na}_{4} \mathrm{P}_{2} \mathrm{O}_{7} \cdot 10 \mathrm{H}_{2} \mathrm{O}$ & 1.83 & --- & --- & --- & --- & --- & $17.72 \%$ & --- & $2.19 \%$ & $5.10 \%$ \\
\hline $\mathrm{Na}_{6}\left(\mathrm{SO}_{4}\right)_{2} \mathrm{CO}_{3}$ & 2.64 & --- & $3.13 \%$ & -- & --- & -- & --- & -- & --- & -- \\
\hline $\mathrm{NaF}$ & 2.78 & -- & --- & --- & --- & --- & $0.21 \%$ & $0.02 \%$ & $0.77 \%$ & $4.66 \%$ \\
\hline $\mathrm{Na}_{7} \mathrm{~F}\left(\mathrm{PO}_{4}\right)_{2} \cdot 19 \mathrm{H}_{2} \mathrm{O}$ & 1.75 & $5.01 \%$ & $12.05 \%$ & $11.36 \%$ & --- & --- & $44.46 \%$ & --- & $36.84 \%$ & $3.87 \%$ \\
\hline $\mathrm{NaIICO}_{3}$ & 2.159 & -- & -.- & -.. & -.. & --- & ..- & $\ldots$ &.-- & -- \\
\hline $\mathrm{NaNO}_{2}$ & 2.168 & --- & --- & --- & --- & --- & --- & --- & --- & --- \\
\hline $\mathrm{NaNO}_{3}$ & 2.26 & --- & $33.82 \%$ & $31.78 \%$ & --- & --- & -- & --- & --- & --- \\
\hline
\end{tabular}


Table C.1. (contd)

\begin{tabular}{|c|c|c|c|c|c|c|c|c|c|c|}
\hline & $\begin{array}{l}\text { Density } \\
(\mathrm{g} / \mathrm{mL})\end{array}$ & 241-T-108 & 241-T-109 & $241-\mathrm{T}-110$ & 241-T-111 & 241-T-112 & 241-T-201 & 241-T-202 & $241-\mathrm{T}-203$ & 241-T-204 \\
\hline Total kg solids for tank & \multirow{9}{*}{ 11/a } & $6.16 \mathrm{E}+04$ & $1.89 \mathrm{E}+05$ & $8.32 \mathrm{E}+04$ & $2.32 \mathrm{E}+05$ & $3.78 \mathrm{E}+04$ & $2.68 \mathrm{E}+04$ & $8.77 \mathrm{E}+03$ & $1.57 \mathrm{E}+04$ & $1.91 E+04$ \\
\hline Total kL solids for tank & & $3.06 \mathrm{E}+01$ & $1.03 \mathrm{E}+02$ & $2.05 \mathrm{E}+01$ & $6.76 \mathrm{E}+01$ & $1.12 \mathrm{E}+01$ & $4.37 \mathrm{E}+00$ & $1.41 \mathrm{E}+00$ & $2.71 \mathrm{E}+00$ & $2.85 \mathrm{E}+00$ \\
\hline Vol. frac. solid phase in tank & & 0.511 & 0.439 & 0.015 & 0.040 & 0.045 & 0.037 & 0.017 & 0.019 & 0.020 \\
\hline Avg g/mL, all solid & & 2.010 & 1.832 & 4.054 & 3.429 & 3.361 & 6.132 & 6.221 & 5.776 & 6.703 \\
\hline Total $\mathrm{kg}$ non-salt solids for tank & & $4.32 \mathrm{E}+03$ & $7.67 \mathrm{E}+03$ & $8.24 \mathrm{E}+04$ & $1.77 \mathrm{E}+05$ & $3.11 \mathrm{E}+04$ & $2.68 \mathrm{E}+04$ & $8.73 E+03$ & $1.45 \mathrm{E}+04$ & $1.91 \mathrm{E}+04$ \\
\hline Total kL non-salt solids for tank & & $1.34 \mathrm{E}+00$ & $2.53 \mathrm{E}+00$ & $2.02 \mathrm{E}+01$ & $3.87 \mathrm{E}+01$ & $7.14 \mathrm{E}+00$ & $4.37 \mathrm{E}+00$ & $1.39 \mathrm{E}+00$ & $2.21 \mathrm{E}+00$ & $2.85 \mathrm{E}+00$ \\
\hline Wt. frac. non-salts in solid & & 0.070 & 0.041 & 0.991 & 0.763 & 0.823 & 1.000 & 0.994 & 0.927 & 1.000 \\
\hline Avg g/mL, non-salt solid & & 3.235 & 3.030 & 4.082 & 4.562 & 4.356 & 6.132 & 6.265 & 6.587 & 6.704 \\
\hline Vol. frac. non-salts in solid & & 0.044 & 0.025 & 0.984 & 0.573 & 0.636 & 1.000 & 0.987 & 0.813 & 1.000 \\
\hline \multicolumn{11}{|l|}{ non-salts: mass fracs in total solid } \\
\hline $\mathrm{Ag}$ & 10.5 & --- & --- & --- & --- & --- & --- & --- & --- & --- \\
\hline $\mathrm{Ag}_{2} \mathrm{O}$ & 7.143 & --- & --- & --- & $0.09 \%$ & --- & $0.00 \%$ & $0.00 \%$ & $0.00 \%$ & $0.00 \%$ \\
\hline $\mathrm{Bi}_{2} \mathrm{O}_{3}$ & 8.9 & $1.12 \%$ & $0.03 \%$ & --- & $23.68 \%$ & $24.45 \%$ & $53.34 \%$ & $44.73 \%$ & $45.24 \%$ & $51.39 \%$ \\
\hline $\mathrm{BiFeO}_{3}$ & 7.9 & $\ldots$ & $\ldots$ & $39.00 \%$ &.- & -.. & $\ldots$ & $\ldots$ & -- & $\ldots$ \\
\hline $\mathrm{Ca}(\mathrm{OH})_{2}$ & 2.24 & $\cdots$ & $\cdots$ & $\cdots$ & $\cdots$ & $\cdots$ & $\cdots$ & $\cdots$ & $\cdots$ & $\cdots$ \\
\hline $\mathrm{Ca}_{5} \mathrm{OH}\left(\mathrm{PO}_{4}\right)_{3}$ & 3.14 & $0.14 \%$ & -- & $0.06 \%$ & -- & $1.82 \%$ & -- & -.. & -- & --. \\
\hline $\mathrm{CaC}_{2} \mathrm{O}_{4} * \mathrm{H}_{2} \mathrm{O}$ & 2.2 & -- & --- & --- & --- & --- & --- & --- & --- & --- \\
\hline $\mathrm{CaCO}_{3}$ & 2.71 & --- & --- & --- & --- & --- & --- & --- & --- & --- \\
\hline $\mathrm{CaF}_{2}$ & 3.18 & -- & $0.09 \%$ & $2.96 \%$ & $3.11 \%$ & --- & $0.73 \%$ & $0.55 \%$ & $0.72 \%$ & $0.36 \%$ \\
\hline $\mathrm{CrOOH}$ & 4.11 & $0.05 \%$ & $0.01 \%$ & $2.65 \%$ & -.- & $2,00 \%$ & $3.62 \%$ & ... & $\cdots$ & $0.72 \%$ \\
\hline $\mathrm{FePO}_{4} \cdot 2 \mathrm{H}_{2} \mathrm{O}$ & 3.15 & --- & $2.74 \%$ & $33.26 \%$ & --- & --- & --- & --- & --- & --- \\
\hline $\mathrm{FeOOH}$ & 4.26 & $1.70 \%$ & --- & $3.70 \%$ & $22.69 \%$ & $19.89 \%$ & $5.87 \%$ & $9.83 \%$ & $6.85 \%$ & $5.75 \%$ \\
\hline Gibbsite & 2.42 & $1.30 \%$ & --- & --- & --- & --- & --- & --- & --- & --- \\
\hline Boehmite & 3.01 & -- & --- & --- & --- & --- & --- & --- & --- & --- \\
\hline $\mathrm{HgO}$ (co-precip. with $\mathrm{Ag}_{2} \mathrm{O}$ ) & 7.143 & --- & --- & --- & --- & --- & --- & --- & --- & --- \\
\hline $\mathrm{KAISiO}_{4}$ & 2.61 & -- & --- & $-\cdots$ & --- & $4.93 \%$ & $0.23 \%$ & $0.43 \%$ & $0.35 \%$ & $0.24 \%$ \\
\hline $\mathrm{La}(\mathrm{OH})_{3}$ & 2.3 & $-\cdots$ & --- & --- & --- & -- & $4.34 \%$ & $1.18 \%$ & --- & --- \\
\hline $\mathrm{LaPO}_{4} \cdot 2 \mathrm{H}_{2} \mathrm{O}$ & 6.51 & $0.01 \%$ & $0.01 \%$ & $1.19 \%$ & $6.49 \%$ & $7.11 \%$ & $12.35 \%$ & $21.08 \%$ & $20.49 \%$ & $20.03 \%$ \\
\hline $\mathrm{Mn}_{3}\left(\mathrm{PO}_{4}\right)_{2}$ & 3.102 & -- & -- & $1.71 \%$ & $14.64 \%$ & -- & -- & --- & -- & -- \\
\hline $\mathrm{MnO}_{2}$ & 5.026 & $0.03 \%$ & $0.19 \%$ & --- &.-- & $7.19 \%$ & $18.21 \%$ & $19.99 \%$ & $17.76 \%$ & $19.91 \%$ \\
\hline $\mathrm{Na}_{2}\left(\mathrm{UO}_{2}\right)_{2}\left(\mathrm{PO}_{4}\right)_{2} \cdot 2 \mathrm{H}_{2} \mathrm{O}$ & 3.5 & --- & --- & --- & --- & --- & --- & --- & --- & --- \\
\hline $\mathrm{Na}_{2} \mathrm{U}_{2} \mathrm{O}_{7}$ & 5.617 & $0.26 \%$ & --- & --- & --- & $0.72 \%$ & $0.09 \%$ & $0.16 \%$ & $0.13 \%$ & $0.17 \%$ \\
\hline $\mathrm{NaAICO}_{3}(\mathrm{OH})_{2}$ & 2.42 & --- & $0.94 \%$ & $12.12 \%$ & $1.68 \%$ & -- & --- & --- & -- & --- \\
\hline $\mathrm{NaAlSiO}_{4}$ & 2.365 & $2.32 \%$ & --- & $0.46 \%$ & --- & $13.70 \%$ & --- & --- & -- & -- \\
\hline $\mathrm{Ni}(\mathrm{OH})_{2}$ & 4.15 & $0.01 \%$ & $0.00 \%$ & $0.12 \%$ & --- & $0.13 \%$ & $0.29 \%$ & $0.21 \%$ & $0.25 \%$ & $0.34 \%$ \\
\hline $\mathrm{Ni}_{3}\left(\mathrm{PO}_{4}\right)$ & 3.93 & -- & $--\cdot$ & $\cdots$ & -- & --- & -- & $--\cdot$ & $\cdots$ & -- \\
\hline $\mathrm{NiC}_{2} \mathrm{O}_{4} \cdot 2 \mathrm{H}_{2} \mathrm{O}$ (on Fe phase) & 4.26 & --- & --- & --- & $0.30 \%$ & --- & --- & --- & -- & --- \\
\hline $\mathrm{Pb}(\mathrm{OH})_{2}$ & 7.1 & $0.06 \%$ & $0.05 \%$ & $1.26 \%$ & --- & $0.40 \%$ & $0.14 \%$ & $0.12 \%$ & $0.03 \%$ & $0.32 \%$ \\
\hline $\mathrm{Pb}_{3}\left(\mathrm{PO}_{4}\right)_{2}$ & 7.1 & -- & --- & --- & $0.31 \%$ & -- & $\begin{array}{ll}-- \\
-1\end{array}$ & --- & -- & --- \\
\hline $\mathrm{PbCO}_{3}$ & 6.6 & -- & --- & --- & --- & -- & --- & --- & --- & --- \\
\hline $\mathrm{Pu}(\mathrm{OH})_{4}$ (co-precip. on Fe phase) & 4.26 & $0.00 \%$ & $0.00 \%$ & $0.00 \%$ & $0.00 \%$ & $0.00 \%$ & $0.01 \%$ & $0.00 \%$ & $0.00 \%$ & $0.00 \%$ \\
\hline $\mathrm{PuO}_{2}$ & 11.43 & -- & -- & --- & --- & -- & -- & --- & -- & -- \\
\hline $\mathrm{SiO}_{2}$ & 2.6 & $\begin{array}{l}-- \\
\end{array}$ & $\begin{array}{l}-- \\
\end{array}$ & --- & $2.83 \%$ & --- & --- & --- & --- & --- \\
\hline $\mathrm{Sr}_{3}\left(\mathrm{PO}_{4}\right)_{2}$ & 3.5 & --- & --- & --- & --- & --- & --- & --- & --- & --- \\
\hline $\mathrm{SrCO}_{3}$ & 3.5 & $0.02 \%$ & $0.00 \%$ & $0.55 \%$ & $0.45 \%$ & $0.00 \%$ & $0.78 \%$ & $0.85 \%$ & $0.89 \%$ & $0.75 \%$ \\
\hline $\mathrm{ZrO}_{2}$ & 5.68 & $0.01 \%$ & $0.00 \%$ & $0.01 \%$ & $0.00 \%$ & --- & $0.00 \%$ & --- & $0.00 \%$ & $0.00 \%$ \\
\hline \multicolumn{11}{|l|}{ salts: mass fracs in total solid } \\
\hline $\mathrm{KNO}_{3}$ & 2.109 & $-\cdots$ & $\bar{c}$ & $\cdots$ & $-\cdots$ & $\begin{array}{ll}-- \\
\end{array}$ & $\overline{-\cdots}$ & $\cdots$ & -- & 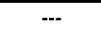 \\
\hline $\mathrm{Na}_{2} \mathrm{C}_{2} \mathrm{O}_{4}$ & 2.34 & $0.16 \%$ & --- & $0.94 \%$ & $4.33 \%$ & $0.28 \%$ & --- & --- & -- & --- \\
\hline $\mathrm{Na}_{2} \mathrm{CO}_{3} \cdot \mathrm{H}_{2} \mathrm{O}$ & 2.25 & -- & $0.27 \%$ & $\cdots$ & $0.05 \%$ & --- & $0.00 \%$ & $0.00 \%$ & $7.29 \%$ & $0.00 \%$ \\
\hline $\mathrm{Na}_{2} \mathrm{SO}_{4}$ & 2.68 & --- & --- & --- & -- & --- & --- & --- & -- & -- \\
\hline $\mathrm{Na}_{2} \mathrm{SO}_{4} \cdot 10 \mathrm{H}_{2} \mathrm{O}$ & 1.464 & --- & --- & --- & --- & --- & --- & --- & --- & --- \\
\hline $\mathrm{Na}_{3} \mathrm{FSO}_{4}$ & 2.65 & --- & --- & --- & --- & --- & --- & --- & --- & --- \\
\hline $\mathrm{Na}_{3} \mathrm{NO}_{3} \mathrm{SO}_{4} \cdot \mathrm{H}_{2} \mathrm{O}$ & 2.3 & --- & --- & --- & --- & --- & -- & --- & -- & --- \\
\hline $\mathrm{Na}_{3} \mathrm{PO}_{4} \cdot 0.25 \mathrm{NaOH} \cdot 12 \mathrm{H}_{2} \mathrm{O}$ & 1.62 & --- & --- & --- & --- & $17.37 \%$ & --- & --- & --- & --- \\
\hline $\mathrm{Na}_{3} \mathrm{PO}_{4} \cdot 8 \mathrm{H}_{2} \mathrm{O}$ & 1.8 & --- & --- & --- & --- & --- & --- & --- & --- & --- \\
\hline $\mathrm{Na}_{4} \mathrm{P}_{2} \mathrm{O}_{7} \cdot 10 \mathrm{H}_{2} \mathrm{O}$ & 1.83 & --- & $45.14 \%$ & --- & $19.35 \%$ & --- & --- & --- & --- & --- \\
\hline $\mathrm{Na}_{6}\left(\mathrm{SO}_{4}\right)_{2} \mathrm{CO}_{3}$ & 2.64 & -- &.- & -- & -- & -- & --- & -- & -- & --- \\
\hline $\mathrm{NaF}$ & 2.78 & $0.01 \%$ & $1.98 \%$ & --- & --- & --- & --- & $0.57 \%$ & --- & --- \\
\hline $\mathrm{Na}_{7} \mathrm{~F}\left(\mathrm{PO}_{4}\right)_{2} \cdot 19 \mathrm{H}_{2} \mathrm{O}$ & 1.75 & $49.89 \%$ & $48.56 \%$ & --- & --- & --- & --- & --- & --- & --- \\
\hline $\mathrm{NaIICO}_{3}$ & 2.159 & -- & -.- & $\cdots$ & -.. & --- & ..- & $\cdots$ & --. & ..- \\
\hline $\mathrm{NaNO}_{2}$ & 2.168 & --- & --- & --- & --- & --- & --- & --- & --- & --- \\
\hline $\mathrm{NaNO}_{3}$ & 2.26 & $42.91 \%$ & --- & --- & --- & --- & -- & --- & --- & -- \\
\hline
\end{tabular}


Table C.1. (contd)

\begin{tabular}{|c|c|c|c|c|c|c|c|c|c|}
\hline & $\begin{array}{l}\text { Density } \\
(\mathrm{g} / \mathrm{mL})\end{array}$ & 241-TX-101 & 241-TX-102 & 241-TX-103 & 241-TX-104 & 241-TX-105 & 241-TX-106 & 241-TX-107 & 241-TX-108 \\
\hline Total kg solids for tank & \multirow{9}{*}{$11 / a$} & $2.91 \mathbf{E}+05$ & $6.64 \mathrm{E}+05$ & $4.87 \mathrm{E}+05$ & $2.35 \mathrm{E}+05$ & $2.21 \mathrm{E}+06$ & $1.12 \mathrm{E}+06$ & $1.21 \mathrm{E}+05$ & $4.12 \mathrm{E}+05$ \\
\hline Total kL solids for tank & & $1.21 \mathrm{E}+02$ & $2.92 \mathrm{E}+02$ & $2.17 \mathrm{E}+02$ & $1.02 \mathrm{E}+02$ & $9.74 \mathrm{E}+02$ & $4.92 \mathrm{E}+02$ & $5.32 \mathrm{E}+01$ & $1.83 \mathrm{E}+02$ \\
\hline Vol. frac. solid phase in tank & & 0.351 & 0.356 & 0.396 & 0.389 & 0.446 & 0.373 & 0.475 & 0.374 \\
\hline Avg $\mathrm{g} / \mathrm{mL}$, all solid & & 2.411 & 2.274 & 2.246 & 2.306 & 2.266 & 2.274 & 2.279 & 2.253 \\
\hline Total kg non-salt solids for tank & & 2.12E+05 & $9.12 \mathrm{E}+04$ & $6.17 \mathrm{E}+04$ & $1.27 \mathrm{E}+05$ & $2.13 \mathrm{E}+05$ & $1.60 \mathrm{E}+05$ & $2.68 \mathrm{E}+04$ & $6.24 \mathrm{E}+04$ \\
\hline Total kL non-salt solids for tank & & $8.49 \mathrm{E}+01$ & $3.57 \mathrm{E}+01$ & $2.44 \mathrm{E}+01$ & $5.19 \mathrm{E}+01$ & $8.17 \mathrm{E}+0 \mathrm{l}$ & $6.34 \mathrm{E}+01$ & $1.08 \mathrm{E}+01$ & $2.43 \mathrm{E}+01$ \\
\hline Wt. frac. non-salts in solid & & 0.728 & 0.137 & 0.127 & 0.542 & 0.096 & 0.143 & 0.221 & 0.152 \\
\hline Avg g/mL, non-salt solid & & 2.496 & 2.552 & 2.531 & 2.449 & 2.602 & 2.531 & 2.484 & 2.573 \\
\hline Vol. frac. non-salts in solid & & 0.704 & 0.122 & 0.112 & 0.510 & 0.084 & 0.129 & 0.203 & 0.133 \\
\hline \multicolumn{10}{|l|}{ non-salts: mass fracs in total solid } \\
\hline Ag & 10.5 & --- & --- & --- & --- & --- & --- & --- & --- \\
\hline $\mathrm{Ag}_{2} \mathrm{O}$ & 7.143 & --- & --- & --- & --- & --- & --- & --- & -- \\
\hline $\mathrm{Bi}_{2} \mathrm{O}_{3}$ & 8.9 & $0.02 \%$ & $0.01 \%$ & $0.01 \%$ & $0.02 \%$ & $0.01 \%$ & $0.01 \%$ & $0.01 \%$ & $0.02 \%$ \\
\hline $\mathrm{BiFeO}_{3}$ & 7.9 & ..- & $\ldots$ & -.- & $\ldots$ & -.. & $\ldots$ & $\ldots$ & $\ldots$ \\
\hline $\mathrm{Ca}(\mathrm{OH})_{2}$ & 2.24 & $\cdots$ & $\cdots$ & $\cdots$ & $\cdots$ & 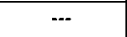 & $\cdots$ & 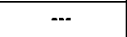 & $\cdots$ \\
\hline $\mathrm{Ca}_{5} \mathrm{OH}\left(\mathrm{PO}_{4}\right)_{3}$ & 3.14 & $0.31 \%$ & $0.12 \%$ & $0.11 \%$ & $0.16 \%$ & $0.11 \%$ & $0.12 \%$ & $0.10 \%$ & $0.17 \%$ \\
\hline $\mathrm{CaC}_{2} \mathrm{O}_{4} \cdot \mathrm{H}_{2} \mathrm{O}$ & 2.2 & -- & --- & --- & --- & --- & --- & --- & -- \\
\hline $\mathrm{CaCO}_{3}$ & 2.71 & -- & --- & -- & --- & --- & --- & --- & --- \\
\hline $\mathrm{CaF}_{2}$ & 3.18 & -- & --- & --- & --- & --- & --- & --- & -- \\
\hline $\mathrm{CrOOH}$ & 4.11 & $0.58 \%$ & $0.24 \%$ & $0.22 \%$ & $0.34 \%$ & $0.22 \%$ & $0.24 \%$ & $0.29 \%$ & $0.23 \%$ \\
\hline $\mathrm{FePO}_{4} \cdot 2 \mathrm{H}_{2} \mathrm{O}$ & 3.15 & -- & $0.89 \%$ & $0.87 \%$ & --- & $0.82 \%$ & $0.88 \%$ & $0.65 \%$ & $1.41 \%$ \\
\hline $\mathrm{FeOOH}$ & 4.26 & $1.20 \%$ & --- & --- & $0.58 \%$ & --- & --- & --- & --- \\
\hline Gibbsite & 2.42 & $66.90 \%$ & $10.96 \%$ & $10.24 \%$ & $52.58 \%$ & $7.05 \%$ & $11.69 \%$ & $15.05 \%$ & $8.04 \%$ \\
\hline Boehmite & 3.01 & --- & --- & -- & --- & --- & --- & --- & -- \\
\hline $\mathrm{HgO}$ (co-precip. with $\mathrm{Ag}_{2} \mathrm{O}$ ) & 7.143 & $0.00 \%$ & --- & --- & $0.00 \%$ & --- & --- & --- & --- \\
\hline $\mathrm{KAlSiO}_{4}$ & 2.61 & --- & --- & $-\cdots$ & -- & --- & --- & --- & $\ldots$ \\
\hline $\mathrm{La}(\mathrm{OH})_{3}$ & 2.3 & -- & -- & --- & --- & --- & --- & --- & --- \\
\hline $\mathrm{LaPO}_{4} \cdot 2 \mathrm{H}_{2} \mathrm{O}$ & 6.51 & $0.01 \%$ & $0.01 \%$ & $0.01 \%$ & $0.01 \%$ & $0.01 \%$ & $0.01 \%$ & $0.01 \%$ & $0.01 \%$ \\
\hline $\mathrm{Mn}_{3}\left(\mathrm{PO}_{4}\right)_{2}$ & 3.102 & -- & --- & -- & -- & -- & --- & --- & -- \\
\hline $\mathrm{MnO}_{2}$ & 5.026 & $0.38 \%$ & $0.15 \%$ & $0.14 \%$ & $0.13 \%$ & $0.12 \%$ & $0.15 \%$ & $0.16 \%$ & $0.15 \%$ \\
\hline $\mathrm{Na}_{2}\left(\mathrm{UO}_{2}\right)_{2}\left(\mathrm{PO}_{4}\right)_{2} \cdot 2 \mathrm{H}_{2} \mathrm{O}$ & 3.5 & --- & --- & --- & --- & --- & --- & --- & --- \\
\hline $\mathrm{Na}_{2} \mathrm{U}_{2} \mathrm{O}_{7}$ & 5.617 & $1.71 \%$ & $0.47 \%$ & $0.22 \%$ & $0.21 \%$ & $0.49 \%$ & $0.32 \%$ & $0.28 \%$ & $0.57 \%$ \\
\hline $\mathrm{NaAICO}_{3}(\mathrm{OH})_{2}$ & 2.42 & -- & --- & -- & --- & --- & --- & $4.75 \%$ & $3.68 \%$ \\
\hline $\mathrm{NaAlSiO}_{4}$ & 2.365 & $1.17 \%$ & $0.82 \%$ & $0.77 \%$ & $0.09 \%$ & $0.76 \%$ & $0.83 \%$ & $0.75 \%$ & $0.81 \%$ \\
\hline $\mathrm{Ni}(\mathrm{OH})_{2}$ & 4.15 & $0.41 \%$ & $0.02 \%$ & $0.02 \%$ & $0.00 \%$ & $0.01 \%$ & $0.02 \%$ & $0.02 \%$ & $0.02 \%$ \\
\hline $\mathrm{Ni}_{3}\left(\mathrm{PO}_{4}\right)$ & 3.93 & -. & -.. & $-\cdots$ & -- & -.. & $\cdots$ & -. & $\ldots$ \\
\hline $\mathrm{NiC}_{2} \mathrm{O}_{4} \cdot 2 \mathrm{H}_{2} \mathrm{O}$ (on Fe phase) & 4.26 & --- & --- & --- & --- & --- & --- & --- & --- \\
\hline $\mathrm{Pb}(\mathrm{OH})_{2}$ & 7.1 & $0.03 \%$ & $0.04 \%$ & $0.04 \%$ & $0.02 \%$ & $0.03 \%$ & $0.04 \%$ & $0.03 \%$ & $0.04 \%$ \\
\hline $\mathrm{Pb}_{3}\left(\mathrm{PO}_{4}\right)_{2}$ & 7.1 & --- & --- & -- & --- & --- & 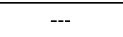 & --- & $\begin{array}{ll}-- \\
-1\end{array}$ \\
\hline $\mathrm{PbCO}_{3}$ & 6.6 & -- & --- & -- & --- & --- & --- & --- & -- \\
\hline $\mathrm{Pu}(\mathrm{OH})_{4}$ (co-precip. on Fe phase) & 4.26 & -- & --- & -- & --- & -- & --- & --- & -- \\
\hline $\mathrm{PuO}_{2}$ & 11.43 & $0.00 \%$ & $0.00 \%$ & $0.00 \%$ & $0.00 \%$ & $0.00 \%$ & $0.00 \%$ & $0.00 \%$ & $0.00 \%$ \\
\hline $\mathrm{SiO}_{2}$ & 2.6 & --- & $\begin{array}{l}-- \\
\end{array}$ & --- & $\begin{array}{l}-- \\
\end{array}$ & --- & --- & --- & --- \\
\hline $\mathrm{Sr}_{3}\left(\mathrm{PO}_{4}\right)_{2}$ & 3.5 & --- & --- & --- & --- & --- & --- & --- & --- \\
\hline $\mathrm{SrCO}_{3}$ & 3.5 & $0.08 \%$ & $0.00 \%$ & $0.00 \%$ & $0.00 \%$ & $0.00 \%$ & $0.00 \%$ & $0.01 \%$ & $0.01 \%$ \\
\hline $\mathrm{ZrO}_{2}$ & 5.68 & $0.02 \%$ & $0.01 \%$ & $0.01 \%$ & $0.00 \%$ & $0.01 \%$ & $0.01 \%$ & $0.01 \%$ & $0.01 \%$ \\
\hline \multicolumn{10}{|l|}{ salts: mass fracs in total solid } \\
\hline $\mathrm{KNO}_{3}$ & 2.109 & $\overline{c--}$ & $\cdots$ & $-\cdots$ & $+\cdots$ & $-\cdots$ & $\cdots$ & $\begin{array}{ll}-\cdot \\
\end{array}$ & $-\cdots$ \\
\hline $\mathrm{Na}_{2} \mathrm{C}_{2} \mathrm{O}_{4}$ & 2.34 & --- & $0.54 \%$ & $0.56 \%$ & $0.79 \%$ & $0.52 \%$ & $0.52 \%$ & $0.64 \%$ & $0.49 \%$ \\
\hline $\mathrm{Na}_{2} \mathrm{CO}_{3} \cdot \mathrm{H}_{2} \mathrm{O}$ & 2.25 & $1.34 \%$ & $2.11 \%$ & $3.61 \%$ & $-\cdot$ & $8.46 \%$ & $2.10 \%$ & $0.32 \%$ & -- \\
\hline $\mathrm{Na}_{2} \mathrm{SO}_{4}$ & 2.68 & -- & --- & -- & --- & --- & --- & --- & $4.12 \%$ \\
\hline $\mathrm{Na}_{2} \mathrm{SO}_{4} \cdot 10 \mathrm{H}_{2} \mathrm{O}$ & 1.464 & -- & --- & -- & --- & --- & --- & --- & -- \\
\hline $\mathrm{Na}_{3} \mathrm{FSO}_{4}$ & 2.65 & $1.69 \%$ & $2.18 \%$ & $2.13 \%$ & --- & $1.69 \%$ & $2.15 \%$ & $1.58 \%$ & -- \\
\hline $\mathrm{Na}_{3} \mathrm{NO}_{3} \mathrm{SO}_{4} \cdot \mathrm{H}_{2} \mathrm{O}$ & 2.3 & -- & --- & $7.02 \%$ & --- & --- & --- & $5.72 \%$ & -- \\
\hline $\mathrm{Na}_{3} \mathrm{PO}_{4} \bullet 0.25 \mathrm{NaOH} \bullet 12 \mathrm{H}_{2} \mathrm{O}$ & 1.62 & --- & --- & --- & --- & --- & --- & --- & --- \\
\hline $\mathrm{Na}_{3} \mathrm{PO}_{4} \bullet 8 \mathrm{H}_{2} \mathrm{O}$ & 1.8 & -- & --- & -- & $\begin{array}{c}-- \\
-1\end{array}$ & --- & --- & --- & -- \\
\hline $\mathrm{Na}_{4} \mathrm{P}_{2} \mathrm{O}_{7} \cdot 10 \mathrm{H}_{2} \mathrm{O}$ & 1.83 & --- & --- & --- & --- & --- & --- & --- & --- \\
\hline $\mathrm{Na}_{6}\left(\mathrm{SO}_{4}\right)_{2} \mathrm{CO}_{3}$ & 2.64 & --- & $6.70 \%$ &.-- & --- & $6.35 \%$ & $6.44 \%$ & -- & --- \\
\hline $\mathrm{NaF}$ & 2.78 & -- & $0.10 \%$ & $0.10 \%$ & --- & $0.09 \%$ & $0.10 \%$ & $0.07 \%$ & $0.16 \%$ \\
\hline $\mathrm{Na}_{7} \mathrm{~F}\left(\mathrm{PO}_{4}\right)_{2} \cdot 19 \mathrm{H}_{2} \mathrm{O}$ & 1.75 & $2.90 \%$ & $7.85 \%$ & $8.32 \%$ & $7.57 \%$ & $7.37 \%$ & $7.56 \%$ & $5.22 \%$ & $9.81 \%$ \\
\hline $\mathrm{NaIICO}_{3}$ & 2.159 & --- &.-- & -- & -.- & $\cdots$ &.-- & -.. & -- \\
\hline $\mathrm{NaNO}_{2}$ & 2.168 & -- & -- & --- & --- & -- & --- & -- & -- \\
\hline $\mathrm{NaNO}_{\mathrm{s}}$ & 2.26 & $21.25 \%$ & $66.77 \%$ & $65.60 \%$ & $37.47 \%$ & $65.88 \%$ & $66.78 \%$ & $64.33 \%$ & $70.27 \%$ \\
\hline
\end{tabular}


Table C.1. (contd)

\begin{tabular}{|c|c|c|c|c|c|c|c|c|c|}
\hline & $\begin{array}{l}\text { Density } \\
(\mathrm{g} / \mathrm{mL})\end{array}$ & 241-TX-109 & 241-TX-110 & 241-TX-111 & $241-\mathrm{TX}-112$ & 241-TX-113 & 241-TX-114 & 241-TX-115 & 241-TX-116 \\
\hline Total kg solids for tank & \multirow{9}{*}{$11 / a$} & $6.67 \mathrm{E}+05$ & $1.50 \mathrm{E}+06$ & $1.15 \mathrm{E}+06$ & $2.30 \mathrm{E}+06$ & $3.32 \mathrm{E}+06$ & $1.87 E+06$ & $1.97 \mathrm{E}+06$ & $2.49 \mathrm{E}+06$ \\
\hline Total kL solids for tank & & $3.21 \mathbf{E}+02$ & $6.59 \mathrm{E}+02$ & $5.10 \mathrm{E}+02$ & $1.03 \mathrm{E}+03$ & $1.44 \mathrm{E}+03$ & $8.38 \mathrm{E}+02$ & $8.71 \mathrm{E}+02$ & $1.07 \mathrm{E}+03$ \\
\hline Vol. frac. solid phase in tank & & 0.233 & 0.373 & 0.369 & 0.428 & 0.596 & 0.416 & 0.416 & 0.474 \\
\hline Avg g/mL, all solid & & 2.080 & 2.269 & 2.256 & 2.243 & 2,303 & 2.229 & 2.258 & 2.326 \\
\hline Total kg non-salt solids for tank & & 2.12E+05 & $2.65 \mathrm{E}+05$ & $2.23 \mathrm{E}+05$ & $3.24 \mathrm{E}+05$ & $1.50 \mathrm{E}+05$ & $2.69 \mathrm{E}+05$ & $2.87 \mathrm{E}+05$ & $5.00 \mathrm{E}+05$ \\
\hline Total kL non-salt solids for tank & & $6.75 \mathrm{E}+01$ & $1.04 \mathrm{E}+02$ & $8.69 \mathrm{E}+01$ & $1.28 \mathrm{E}+02$ & $5.46 \mathrm{E}+01$ & $1.06 \mathrm{E}+02$ & $1.13 \mathrm{E}+02$ & $2.00 \mathrm{E}+02$ \\
\hline Wt. frac. non-salts in solid & & 0.318 & 0.177 & 0.194 & 0.141 & 0.045 & 0.144 & 0.146 & 0.201 \\
\hline Avg g/mL, non-salt solid & & 3.136 & 2.553 & 2.566 & 2.521 & 2.745 & 2.532 & 2.528 & 2.505 \\
\hline Vol. frac. non-salts in solid & & 0.211 & 0.158 & 0.170 & 0.125 & 0.038 & 0.127 & 0.130 & 0.187 \\
\hline \multicolumn{10}{|l|}{ non-salts: mass fracs in total solid } \\
\hline Ag & 10.5 & --- & --- & --- & --- & --- & --- & --- & -- \\
\hline $\mathrm{Ag}_{2} \mathrm{O}$ & 7.143 & --- & --- & --- & -- & -- & --- & --- & -- \\
\hline $\mathrm{Bi}_{2} \mathrm{O}_{3}$ & 8.9 & --- & $0.31 \%$ & $0.46 \%$ & $0.01 \%$ & $0.41 \%$ & $0.04 \%$ & $0.01 \%$ & $0.05 \%$ \\
\hline $\mathrm{BiFeO}_{3}$ & 7.9 & $7.95 \%$ & $\ldots$ & -.- & $\ldots$ & -.. & $\ldots$ & $\ldots$ & $\ldots$ \\
\hline $\mathrm{Ca}(\mathrm{OH})_{2}$ & 2.24 & $\cdots$ & $\cdots$ & $\cdots$ & 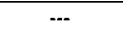 & 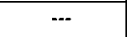 & $\cdots$ & 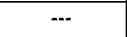 & $\cdots$ \\
\hline $\mathrm{Ca}_{5} \mathrm{OH}\left(\mathrm{PO}_{4}\right)_{3}$ & 3.14 & $0.54 \%$ & $0.14 \%$ & $0.15 \%$ & $0.11 \%$ & $0.09 \%$ & $0.11 \%$ & $0.13 \%$ & $0.18 \%$ \\
\hline $\mathrm{CaC}_{2} \mathrm{O}_{4} \cdot \mathrm{H}_{2} \mathrm{O}$ & 2.2 & --- & --- & -- & --- & --- & --- & --- & -- \\
\hline $\mathrm{CaCO}_{3}$ & 2.71 & -- & --- & -- & --- & --- & --- & --- & --- \\
\hline $\mathrm{CaF}_{2}$ & 3.18 & $0.01 \%$ & --- & --- & --- & --- & --- & --- & --- \\
\hline $\mathrm{CrOOH}$ & 4.11 & -.- & $0.25 \%$ & $0.25 \%$ & $0.22 \%$ & $0.03 \%$ & $0.23 \%$ & $0.22 \%$ & $0.06 \%$ \\
\hline $\mathrm{FePO}_{4} \cdot 2 \mathrm{H}_{2} \mathrm{O}$ & 3.15 & $5.58 \%$ & $1.37 \%$ & $1.63 \%$ & $0.91 \%$ & --- & $1.11 \%$ & $1.02 \%$ & $2.26 \%$ \\
\hline $\mathrm{FeOOH}$ & 4.26 & --- & --- & --- & --- & $0.40 \%$ & --- & --- & $0.04 \%$ \\
\hline Gibbsite & 2.42 & $6.93 \%$ & $5.27 \%$ & $4.00 \%$ & $6.38 \%$ & --- & $4.87 \%$ & $7.02 \%$ & --- \\
\hline Boehmite & 3.01 & --- & --- & --- & --- & --- & --- & --- & --- \\
\hline $\mathrm{HgO}$ (co-precip. with $\mathrm{Ag}_{2} \mathrm{O}$ ) & 7.143 & --- & --- & --- & --- & --- & --- & --- & --- \\
\hline $\mathrm{KAlSiO}_{4}$ & 2.61 & -- & -- & $-\cdots$ & -- & --- & --- & --- & $\ldots$ \\
\hline $\mathrm{La}(\mathrm{OH})_{3}$ & 2.3 & -- & --- & --- & --- & --- & --- & --- & --- \\
\hline $\mathrm{LaPO}_{4} \cdot 2 \mathrm{H}_{2} \mathrm{O}$ & 6.51 & $0.00 \%$ & $0.01 \%$ & $0.01 \%$ & $0.01 \%$ & $0.01 \%$ & $0.01 \%$ & $0.01 \%$ & $0.01 \%$ \\
\hline $\mathrm{Mn}_{3}\left(\mathrm{PO}_{4}\right)_{2}$ & 3.102 & -- & --- & --- & -- & -- & --- & --- & -- \\
\hline $\mathrm{MnO}_{2}$ & 5.026 & $0.04 \%$ & $0.15 \%$ & $0.15 \%$ & $0.14 \%$ & $0.00 \%$ & $0.15 \%$ & $0.14 \%$ & $0.14 \%$ \\
\hline $\mathrm{Na}_{2}\left(\mathrm{UO}_{2}\right)_{2}\left(\mathrm{PO}_{4}\right)_{2} \cdot 2 \mathrm{H}_{2} \mathrm{O}$ & 3.5 & --- & --- & --- & --- & --- & --- & --- & --- \\
\hline $\mathrm{Na}_{2} \mathrm{U}_{2} \mathrm{O}_{7}$ & 5.617 & $0.75 \%$ & $0.27 \%$ & $0.29 \%$ & $0.22 \%$ & --- & $0.22 \%$ & $0.27 \%$ & -- \\
\hline $\mathrm{NaAICO}_{3}(\mathrm{OH})_{2}$ & 2.42 & -- & $8.57 \%$ & $10.81 \%$ & $5.18 \%$ & $3.53 \%$ & $6.72 \%$ & $4.88 \%$ & $13.35 \%$ \\
\hline $\mathrm{NaAISiO}_{4}$ & 2.365 & $9.77 \%$ & $1.32 \%$ & $1.56 \%$ & $0.79 \%$ & --- & $0.87 \%$ & $0.79 \%$ & $3.94 \%$ \\
\hline $\mathrm{Ni}(\mathrm{OH})_{2}$ & 4.15 & $0.01 \%$ & $0.02 \%$ & $0.02 \%$ & $0.02 \%$ & $0.00 \%$ & $0.01 \%$ & $0.02 \%$ & $0.01 \%$ \\
\hline $\mathrm{Ni}_{3}\left(\mathrm{PO}_{4}\right)$ & 3.93 &.- & -.. & $-\cdots$ & -- & $-\cdot$ & $-\cdot$ & -. & -. \\
\hline $\mathrm{NiC}_{2} \mathrm{O}_{4} \cdot 2 \mathrm{H}_{2} \mathrm{O}$ (on Fe phase) & 4.26 & --- & --- & --- & --- & --- & --- & --- & --- \\
\hline $\mathrm{Pb}(\mathrm{OH})_{2}$ & 7.1 & $0.06 \%$ & $0.04 \%$ & $0.04 \%$ & $0.04 \%$ & --- & $0.04 \%$ & $0.04 \%$ & $0.06 \%$ \\
\hline $\mathrm{Pb}_{3}\left(\mathrm{PO}_{4}\right)_{2}$ & 7.1 & --- & --- & --- & $\begin{array}{c}-- \\
-\end{array}$ & $0.02 \%$ & 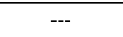 & --- & -- \\
\hline $\mathrm{PbCO}_{3}$ & 6.6 & -- & --- & -- & --- & -- & --- & --- & -- \\
\hline $\mathrm{Pu}(\mathrm{OH})_{4}$ (co-precip. on Fe phase) & 4.26 & -- & --- & -- & --- & -- & --- & --- & -- \\
\hline $\mathrm{PuO}_{2}$ & 11.43 & $0.00 \%$ & $0.00 \%$ & $0.00 \%$ & $0.00 \%$ & $0.00 \%$ & $0.00 \%$ & $0.00 \%$ & $0.00 \%$ \\
\hline $\mathrm{SiO}_{2}$ & 2.6 & $\begin{array}{l}-- \\
\end{array}$ & $\begin{array}{l}-- \\
\end{array}$ & --- & $\begin{array}{l}-- \\
\end{array}$ & --- & --- & --- & --- \\
\hline $\mathrm{Sr}_{3}\left(\mathrm{PO}_{4}\right)_{2}$ & 3.5 & --- & --- & --- & --- & --- & --- & --- & --- \\
\hline $\mathrm{SrCO}_{3}$ & 3.5 & $0.07 \%$ & $0.01 \%$ & $0.01 \%$ & $0.00 \%$ & $0.01 \%$ & $0.00 \%$ & $0.00 \%$ & $0.00 \%$ \\
\hline $\mathrm{ZrO}_{2}$ & 5.68 & $0.05 \%$ & $0.01 \%$ & $0.01 \%$ & $0.01 \%$ & $0.01 \%$ & $0.01 \%$ & $0.01 \%$ & $0.00 \%$ \\
\hline \multicolumn{10}{|l|}{ salts: mass fracs in total solid } \\
\hline $\mathrm{KNO}_{3}$ & 2.109 & -- & $\cdots$ & $\begin{array}{ll}-\cdot \\
\end{array}$ & $+\cdots$ & $-\cdots$ & $-\cdots$ & $+\cdots$ & $-\cdots$ \\
\hline $\mathrm{Na}_{2} \mathrm{C}_{2} \mathrm{O}_{4}$ & 2.34 & $0.28 \%$ & $0.55 \%$ & $0.53 \%$ & $0.65 \%$ & --- & $1.14 \%$ & $0.56 \%$ & $1.99 \%$ \\
\hline $\mathrm{Na}_{2} \mathrm{CO}_{3} \cdot \mathrm{H}_{2} \mathrm{O}$ & 2.25 & -- & $\cdots$ & -- & $0.16 \%$ & $0.01 \%$ & $\cdots$ & $\cdots$ & $0.21 \%$ \\
\hline $\mathrm{Na}_{2} \mathrm{SO}_{4}$ & 2.68 & -- & --- & --- & --- & -- & --- & --- & -- \\
\hline $\mathrm{Na}_{2} \mathrm{SO}_{4} \cdot 10 \mathrm{H}_{2} \mathrm{O}$ & 1.464 & -- & --- & -- & --- & --- & --- & --- & -- \\
\hline $\mathrm{Na}_{3} \mathrm{FSO}_{4}$ & 2.65 & $4.51 \%$ & $2.65 \%$ & $2.82 \%$ & $1.92 \%$ & $7.73 \%$ & $1.33 \%$ & $2.03 \%$ & $5.20 \%$ \\
\hline $\mathrm{Na}_{3} \mathrm{NO}_{3} \mathrm{SO}_{4} \cdot \mathrm{H}_{2} \mathrm{O}$ & 2.3 & -- & --- & $5.27 \%$ & $6.93 \%$ & $9.82 \%$ & --- & $7.06 \%$ & $1.00 \%$ \\
\hline $\mathrm{Na}_{3} \mathrm{PO}_{4} \bullet 0.25 \mathrm{NaOH} \bullet 12 \mathrm{H}_{2} \mathrm{O}$ & 1.62 & --- & --- & --- & --- & --- & --- & --- & --- \\
\hline $\mathrm{Na}_{3} \mathrm{PO}_{4} \bullet 8 \mathrm{H}_{2} \mathrm{O}$ & 1.8 & -- & --- & -- & $\begin{array}{c}-- \\
-1\end{array}$ & --- & --- & --- & -- \\
\hline $\mathrm{Na}_{4} \mathrm{P}_{2} \mathrm{O}_{7} \cdot 10 \mathrm{H}_{2} \mathrm{O}$ & 1.83 & --- & --- & --- & --- & --- & --- & --- & --- \\
\hline $\mathrm{Na}_{6}\left(\mathrm{SO}_{4}\right)_{2} \mathrm{CO}_{3}$ & 2.64 & -- & $4.65 \%$ &.-- & --- & --- & $5.97 \%$ & -- & -- \\
\hline $\mathrm{NaF}$ & 2.78 & $0.63 \%$ & $0.15 \%$ & $0.18 \%$ & $0.10 \%$ & --- & $0.12 \%$ & $0.11 \%$ & --- \\
\hline $\mathrm{Na}_{7} \mathrm{~F}\left(\mathrm{PO}_{4}\right)_{2} \cdot 19 \mathrm{H}_{2} \mathrm{O}$ & 1.75 & $62.82 \%$ & $9.41 \%$ & $10.52 \%$ & $9.13 \%$ & --- & $13.95 \%$ & $7.18 \%$ & --- \\
\hline $\mathrm{NaIICO}_{3}$ & 2.159 & --- &.-- & -- &.-- & $4.70 \%$ & -- & -.. & -- \\
\hline $\mathrm{NaNO}_{2}$ & 2.168 & --- & -- & --- & --- & -- & --- & -- & -- \\
\hline $\mathrm{NaNO}_{\mathrm{s}}$ & 2.26 & --- & $64.85 \%$ & $61.30 \%$ & $67.05 \%$ & $73.24 \%$ & $63.09 \%$ & $68.49 \%$ & $71.48 \%$ \\
\hline
\end{tabular}


Table C.1. (contd)

\begin{tabular}{|c|c|c|c|c|c|c|c|c|c|}
\hline & $\begin{array}{l}\text { Density } \\
(\mathrm{g} / \mathrm{mL})\end{array}$ & $241-\mathrm{TX}-117$ & 241-TX-118 & 241-TY-101 & 241-TY-102 & 241-TY-103 & 241-TY-104 & 241-TY-105 & 241-TY-106 \\
\hline Total kg solids for tank & \multirow{9}{*}{$11 / a$} & $1.71 \mathbf{E}+06$ & $8.62 \mathrm{E}+05$ & $3.84 \mathrm{E}+05$ & $2.37 \mathrm{E}+05$ & $2.61 E+05$ & $9.15 \mathrm{E}+04$ & $7.59 \mathrm{E}+05$ & $6.63 \mathrm{E}+04$ \\
\hline Total kL solids for tank & & $7.95 \mathrm{E}+02$ & $4.64 \mathrm{E}+02$ & $1.53 \mathrm{E}+02$ & $1.07 \mathrm{E}+02$ & $1.07 \mathrm{E}+02$ & $3.94 \mathrm{E}+01$ & $3.99 \mathrm{E}+02$ & $2.72 \mathrm{E}+01$ \\
\hline Vol. frac. solid phase in tank & & 0.437 & 0.479 & 0.342 & 0.409 & 0.182 & 0.236 & 0.456 & 0.439 \\
\hline Avg g/mL, all solid & & 2.156 & 1.858 & 2.511 & 2.224 & 2.432 & 2.322 & 1.904 & 2.435 \\
\hline Total kg non-salt solids for tank & & $2.42 \mathrm{E}+05$ & $9.19 \mathrm{E}+04$ & $2.64 \mathrm{E}+05$ & $9.85 \mathrm{E}+03$ & $1.17 \mathrm{E}+05$ & $3.83 \mathrm{E}+04$ & $9.40 \mathrm{E}+04$ & $3.48 \mathrm{E}+04$ \\
\hline Total kL non-salt solids for tank & & $9.55 \mathrm{E}+01$ & $3.36 \mathrm{E}+01$ & $8.74 \mathrm{E}+01$ & $3.92 \mathrm{E}+00$ & $2.77 \mathrm{E}+01$ & $1.07 \mathrm{E}+01$ & $2.65 \mathrm{E}+01$ & 1. $17 \mathrm{E}+01$ \\
\hline Wt. frac. non-salts in solid & & 0.141 & 0.107 & 0.688 & 0.041 & 0.448 & 0.419 & 0.124 & 0.524 \\
\hline Avg g/mL, non-salt solid & & 2.533 & 2.740 & 3.022 & 2.514 & 4.216 & 3.590 & 3.552 & 2.963 \\
\hline Vol. frac. non-salts in solid & & 0.120 & 0.072 & 0.571 & 0.037 & 0.258 & 0.271 & 0.066 & 0.431 \\
\hline \multicolumn{10}{|l|}{ non-salts: mass fracs in total solid } \\
\hline Ag & 10.5 & --- & --- & --- & --- & --- & --- & --- & -- \\
\hline $\mathrm{Ag}_{2} \mathrm{O}$ & 7.143 & --- & --- & $0.00 \%$ & --- & $0.00 \%$ & $0.00 \%$ & --- & -- \\
\hline $\mathrm{Bi}_{2} \mathrm{O}_{3}$ & 8.9 & $0.04 \%$ & $0.07 \%$ & $6.17 \%$ & $0.01 \%$ & $10.04 \%$ & $5.15 \%$ & $0.08 \%$ & $0.11 \%$ \\
\hline $\mathrm{BiFeO}_{3}$ & 7.9 & ..- & $\ldots$ & -.- & $\ldots$ & $\ldots$ & $\ldots$ & $\ldots$ & $\ldots$ \\
\hline $\mathrm{Ca}(\mathrm{OH})_{2}$ & 2.24 & $\cdots$ & $\cdots$ & $\cdots$ & $\cdots$ & 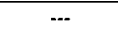 & $\cdots$ & 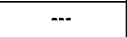 & $\cdots$ \\
\hline $\mathrm{Ca}_{5} \mathrm{OH}\left(\mathrm{PO}_{4}\right)_{3}$ & 3.14 & $0.15 \%$ & $\cdots$ & $2.16 \%$ & -- & $3.87 \%$ & $4.14 \%$ & $1.64 \%$ & $1.43 \%$ \\
\hline $\mathrm{CaC}_{2} \mathrm{O}_{4} \cdot \mathrm{H}_{2} \mathrm{O}$ & 2.2 & -- & --- & --- & --- & --- & --- & --- & -- \\
\hline $\mathrm{CaCO}_{3}$ & 2.71 & --- & --- & -- & --- & --- & --- & --- & --- \\
\hline $\mathrm{CaF}_{2}$ & 3.18 & --- & $0.10 \%$ & $1.40 \%$ & $0.07 \%$ & --- & $0.93 \%$ & --- & --- \\
\hline $\mathrm{CrOOH}$ & 4.11 & $0.28 \%$ & $0.80 \%$ & $2.76 \%$ & $0.01 \%$ & $0.44 \%$ & $0.34 \%$ & $\cdots$ & $\cdots$ \\
\hline $\mathrm{FePO}_{4} \cdot 2 \mathrm{H}_{2} \mathrm{O}$ & 3.15 & $1.89 \%$ & $2.08 \%$ & -- & $0.45 \%$ & --- & --- & --- & -- \\
\hline $\mathrm{FeOOH}$ & 4.26 & -- & --- & $12.97 \%$ & --- & $12.62 \%$ & $13.46 \%$ & $6.47 \%$ & $14.04 \%$ \\
\hline Gibbsite & 2.42 & --- & $1.59 \%$ & --- & --- & --- & --- & --- & --- \\
\hline Boehmite & 3.01 & --- & --- & -- & --- & --- & --- & --- & --- \\
\hline $\mathrm{HgO}$ (co-precip. with $\mathrm{Ag}_{2} \mathrm{O}$ ) & 7.143 & --- & --- & --- & --- & $0.01 \%$ & $0.01 \%$ & $0.03 \%$ & $0.01 \%$ \\
\hline $\mathrm{KAlSiO}_{4}$ & 2.61 & --- & --- & $-\cdots$ & -- & --- & -- & -- & -- \\
\hline $\mathrm{La}(\mathrm{OH})_{3}$ & 2.3 & -- & -- & --- & --- & --- & --- & --- & -- \\
\hline $\mathrm{LaPO}_{4} \cdot 2 \mathrm{H}_{2} \mathrm{O}$ & 6.51 & $0.01 \%$ & $0.01 \%$ & $0.00 \%$ & $0.01 \%$ & $0.00 \%$ & --- & --- & -- \\
\hline $\mathrm{Mn}_{3}\left(\mathrm{PO}_{4}\right)_{2}$ & 3.102 & -- & $0.74 \%$ & $0.24 \%$ & -- & $0.12 \%$ & --- & $0.06 \%$ & $0.23 \%$ \\
\hline $\mathrm{MnO}_{2}$ & 5.026 & $0.15 \%$ & --- &.-- & $0.01 \%$ & $\ldots$ & $0.71 \%$ & $\ldots$ & -- \\
\hline $\mathrm{Na}_{2}\left(\mathrm{UO}_{2}\right)_{2}\left(\mathrm{PO}_{4}\right)_{2} \cdot 2 \mathrm{H}_{2} \mathrm{O}$ & 3.5 & --- & --- & --- & --- & --- & --- & $1.79 \%$ & --- \\
\hline $\mathrm{Na}_{2} \mathrm{U}_{2} \mathrm{O}_{7}$ & 5.617 & -- & $0.27 \%$ & $0.63 \%$ & --- & $8.18 \%$ & $3.71 \%$ & --- & $2.07 \%$ \\
\hline $\mathrm{NaAICO}_{3}(\mathrm{OH})_{2}$ & 2.42 & $6.66 \%$ & $3.81 \%$ & $12.41 \%$ & $3.57 \%$ & --- & $5.86 \%$ & $1.79 \%$ & --- \\
\hline $\mathrm{NaAlSiO}_{4}$ & 2.365 & $4.87 \%$ & $1.10 \%$ & $18.82 \%$ & --- & $7.85 \%$ & $6.62 \%$ & $0.19 \%$ & $6.99 \%$ \\
\hline $\mathrm{Ni}(\mathrm{OH})_{2}$ & 4.15 & $0.01 \%$ & $0.02 \%$ & $0.59 \%$ & $0.01 \%$ & $1.23 \%$ & $0.61 \%$ & $0.03 \%$ & $0.02 \%$ \\
\hline $\mathrm{Ni}_{3}\left(\mathrm{PO}_{4}\right)$ & 3.93 &.- & $-\cdots$ & $0.69 \%$ & -- & -- &.- & -. & $\ldots$ \\
\hline $\mathrm{NiC}_{2} \mathrm{O}_{4} \cdot 2 \mathrm{H}_{2} \mathrm{O}$ (on Fe phase) & 4.26 & --- & --- & $1.02 \%$ & --- & --- & --- & --- & --- \\
\hline $\mathrm{Pb}(\mathrm{OH})_{2}$ & 7.1 & $0.05 \%$ & $0.05 \%$ & --- & --- & $0.20 \%$ & $0.17 \%$ & $0.09 \%$ & $0.09 \%$ \\
\hline $\mathrm{Pb}_{3}\left(\mathrm{PO}_{4}\right)_{2}$ & 7.1 & --- & --- & $0.06 \%$ & --- & --- & 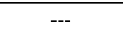 & --- & $\begin{array}{ll}-- \\
-1\end{array}$ \\
\hline $\mathrm{PbCO}_{3}$ & 6.6 & -- & --- & -- & $0.02 \%$ & --- & --- & --- & -- \\
\hline $\mathrm{Pu}(\mathrm{OH})_{4}$ (co-precip. on Fe phase) & 4.26 & -- & --- & $0.00 \%$ & $0.00 \%$ & $0.00 \%$ & $0.00 \%$ & $0.00 \%$ & $0.00 \%$ \\
\hline $\mathrm{PuO}_{2}$ & 11.43 & $0.00 \%$ & $0.02 \%$ & -- & -- & --- & --- & --- & -- \\
\hline $\mathrm{SiO}_{2}$ & 2.6 & --- & --- & $8.72 \%$ & $\begin{array}{l}-- \\
\end{array}$ & --- & --- & --- & $27.24 \%$ \\
\hline $\mathrm{Sr}_{3}\left(\mathrm{PO}_{4}\right)_{2}$ & 3.5 & --- & $0.00 \%$ & -- & --- & --- & --- & --- & -- \\
\hline $\mathrm{SrCO}_{3}$ & 3.5 & $0.00 \%$ & --- & --- & $0.00 \%$ & $0.12 \%$ & $0.07 \%$ & $0.21 \%$ & $0.06 \%$ \\
\hline $\mathrm{ZrO}_{2}$ & 5.68 & $0.00 \%$ & $0.01 \%$ & $0.11 \%$ & $0.00 \%$ & $0.11 \%$ & $0.06 \%$ & $-\cdots$ & $0.17 \%$ \\
\hline \multicolumn{10}{|l|}{ salts: mass fracs in total solid } \\
\hline $\mathrm{KNO}_{3}$ & 2.109 & $-\cdots$ & $\cdots$ & $\cdots$ & $\begin{array}{ll}-\cdot \\
\end{array}$ & $-\cdots$ & $\cdots$ & $\begin{array}{l}-\cdot \\
\end{array}$ & $-\cdots$ \\
\hline $\mathrm{Na}_{2} \mathrm{C}_{2} \mathrm{O}_{4}$ & 2.34 & $2.28 \%$ & $5.21 \%$ & -- & $0.17 \%$ & $1.03 \%$ & $7.86 \%$ & $0.41 \%$ & $1.21 \%$ \\
\hline $\mathrm{Na}_{2} \mathrm{CO}_{3} \cdot \mathrm{H}_{2} \mathrm{O}$ & 2.25 & $0.22 \%$ & $\cdots$ & $0.00 \%$ & $0.01 \%$ & $0.00 \%$ & $1.02 \%$ & $\cdots$ & -- \\
\hline $\mathrm{Na}_{2} \mathrm{SO}_{4}$ & 2.68 & -- & --- & -- & --- & --- & --- & --- & -- \\
\hline $\mathrm{Na}_{2} \mathrm{SO}_{4} \cdot 10 \mathrm{H}_{2} \mathrm{O}$ & 1.464 & -- & --- & --- & --- & --- & --- & --- & -- \\
\hline $\mathrm{Na}_{3} \mathrm{FSO}_{4}$ & 2.65 & $3.44 \%$ & $1.68 \%$ & --- & --- & --- & --- & --- & -- \\
\hline $\mathrm{Na}_{3} \mathrm{NO}_{3} \mathrm{SO}_{4} \cdot \mathrm{H}_{2} \mathrm{O}$ & 2.3 & -- & --- & -- & --- & --- & --- & $6.15 \%$ & -- \\
\hline $\mathrm{Na}_{3} \mathrm{PO}_{4} \bullet 0.25 \mathrm{NaOH} \bullet 12 \mathrm{H}_{2} \mathrm{O}$ & 1.62 & --- & --- & --- & --- & --- & --- & $50.54 \%$ & --- \\
\hline $\mathrm{Na}_{3} \mathrm{PO}_{4} \bullet 8 \mathrm{H}_{2} \mathrm{O}$ & 1.8 & -- & --- & -- & -- & --- & --- & --- & -- \\
\hline $\mathrm{Na}_{4} \mathrm{P}_{2} \mathrm{O}_{7} \cdot 10 \mathrm{H}_{2} \mathrm{O}$ & 1.83 & $7.66 \%$ & --- & $31.24 \%$ & $9.74 \%$ & $36.11 \%$ & $22.36 \%$ & $14.41 \%$ & $22.55 \%$ \\
\hline $\mathrm{Na}_{6}\left(\mathrm{SO}_{4}\right)_{2} \mathrm{CO}_{3}$ & 2.64 & $2.02 \%$ & --- & --- & --- & --- & --- & --- & -- \\
\hline $\mathrm{NaF}$ & 2.78 & -- & $0.22 \%$ & -- & $1.23 \%$ & --- & --- & --- & --- \\
\hline $\mathrm{Na}_{7} \mathrm{~F}\left(\mathrm{PO}_{4}\right)_{2} \cdot 19 \mathrm{H}_{2} \mathrm{O}$ & 1.75 & $18.67 \%$ & $82.24 \%$ & --- & --- & $18.05 \%$ & $26.90 \%$ & --- & --- \\
\hline $\mathrm{NaIICO}_{3}$ & 2.159 & -- & $--\cdot$ & -- & $--\cdot$ & --- & -- & $--\cdot$ & -- \\
\hline $\mathrm{NaNO}_{2}$ & 2.168 & -- & --- & --- & --- & --- & --- & -- & -- \\
\hline $\mathrm{NaNO}_{3}$ & 2.26 & $51.60 \%$ & --- & --- & $84.70 \%$ & --- & --- & $16.11 \%$ & $23.79 \%$ \\
\hline
\end{tabular}


Table C.1. (contd)

\begin{tabular}{|c|c|c|c|c|c|c|c|c|c|c|}
\hline & $\begin{array}{l}\text { Density } \\
(\mathrm{g} / \mathrm{mL})\end{array}$ & 241-U-101 & 241-U-102 & $241-\mathrm{U}-103$ & 241-U-104 & $241-U-105$ & 241-U-106 & 241-U-107 & 241-U-108 & 241-U-109 \\
\hline Total kg solids for tank & \multirow{9}{*}{ 11/a } & $6.85 \mathrm{E}+04$ & $6.16 \mathrm{E}+05$ & $8.48 \mathrm{E}+05$ & $1.56 \mathrm{E}+05$ & $1.35 \mathrm{E}+06$ & $1.46 \mathrm{E}+05$ & $1.25 \mathrm{E}+06$ & $1.18 \mathrm{E}+06$ & $8.80 \mathrm{E}+05$ \\
\hline Total kL solids for tank & & $2.82 \mathrm{E}+01$ & $2.64 \mathrm{E}+02$ & $3.84 \mathrm{E}+02$ & $6.46 \mathrm{E}+01$ & $5.85 \mathrm{E}+02$ & $6.14 \mathrm{E}+01$ & $5.97 \mathrm{E}+02$ & $5.12 \mathrm{E}+02$ & $4.08 \mathrm{E}+02$ \\
\hline Vol. frac. solid phase in tank & & 0.317 & 0.239 & 0.272 & 0.140 & 0.438 & 0.095 & 0.394 & 0.289 & 0.378 \\
\hline Avg g/mL, all solid & & 2.430 & 2.331 & 2.212 & 2.419 & 2.299 & 2.380 & 2.088 & 2.302 & 2.157 \\
\hline Total kg non-salt solids for tank & & $6.09 \mathrm{E}+04$ & $2.23 \mathrm{E}+05$ & $8.26 \mathrm{E}+04$ & $1.56 \mathrm{E}+05$ & $2.32 \mathrm{E}+05$ & $4.68 \mathrm{E}+04$ & $1.49 \mathrm{E}+05$ & $1.34 \mathrm{E}+05$ & $1.67 \mathrm{E}+05$ \\
\hline Total kL non-salt solids for tank & & $2.47 \mathrm{E}+01$ & $8.93 \mathrm{E}+01$ & $3.05 \mathrm{E}+01$ & $6.45 \mathrm{E}+01$ & $8.65 \mathrm{E}+0 \mathrm{I}$ & $1.73 \mathrm{E}+01$ & $5.76 \mathrm{E}+01$ & $4.48 \mathrm{E}+01$ & $6.71 \mathrm{E}+01$ \\
\hline Wt. frac. non-salts in solid & & 0.889 & 0.363 & 0.097 & 0.998 & 0.172 & 0.320 & 0.120 & 0.113 & 0.190 \\
\hline Avg g/mL, non-salt solid & & 2.469 & 2.502 & 2.706 & 2.420 & 2.677 & 2.707 & 2.590 & 2.986 & 2.494 \\
\hline Vol. frac. non-salts in solid & & 0.875 & 0.338 & 0.080 & 0.998 & 0.148 & 0.281 & 0.097 & 0.087 & 0.164 \\
\hline \multicolumn{11}{|l|}{ non-salts: mass fracs in total solid } \\
\hline $\mathrm{Ag}$ & 10.5 & --- & --- & --- & --- & --- & --- & --- & --- & --- \\
\hline $\mathrm{Ag}_{2} \mathrm{O}$ & 7.143 & --- & -- & --- & --- & --- & --- & --- & -- & -- \\
\hline $\mathrm{Bi}_{2} \mathrm{O}_{3}$ & 8.9 & $0.02 \%$ & $0.02 \%$ & $0.01 \%$ & $0.01 \%$ & $0.01 \%$ & $0.04 \%$ & $0.02 \%$ & $0.01 \%$ & $0.01 \%$ \\
\hline $\mathrm{BiFeO}_{3}$ & 7.9 & $\cdots$ & $\cdots$ & $\ldots$ & -- & $\cdots$ & -.- & $\cdots$ & $\cdots$ & $--\cdot$ \\
\hline $\mathrm{Ca}(\mathrm{OH})_{2}$ & 2.24 & $\cdots$ & $\cdots$ & $\cdots$ & $\cdots$ & $\cdots$ & $\cdots$ & $\cdots$ & $\cdots$ & $\cdots$ \\
\hline $\mathrm{Ca}_{5} \mathrm{OH}\left(\mathrm{PO}_{4}\right)_{3}$ & 3.14 & $0.28 \%$ & $0.25 \%$ & $0.39 \%$ & $0.76 \%$ & $0.17 \%$ & $0.55 \%$ & $0.22 \%$ & $0.39 \%$ & $0.11 \%$ \\
\hline $\mathrm{CaC}_{2} \mathrm{O}_{4} * \mathrm{H}_{2} \mathrm{O}$ & 2.2 & -- & -- & --- & --- & --- & --- & --- & --- & --- \\
\hline $\mathrm{CaCO}_{3}$ & 2.71 & --- & --- & --- & --- & --- & --- & --- & --- & --- \\
\hline $\mathrm{CaF}_{2}$ & 3.18 & --- & --- & --- & --- & -- & --- & --- & --- & --- \\
\hline $\mathrm{CrOOH}$ & 4.11 & $\cdots$ & $1.26 \%$ & $1.22 \%$ & $\cdots$ & $0.70 \%$ & $2.14 \%$ & $0.62 \%$ & $1.07 \%$ & $1.06 \%$ \\
\hline $\mathrm{FePO}_{4} \cdot 2 \mathrm{H}_{2} \mathrm{O}$ & 3.15 & -- & --- & --- & --- & --- & --- & --- & -- & --- \\
\hline $\mathrm{FeOOH}$ & 4.26 & $1.11 \%$ & $0.41 \%$ & $0.65 \%$ & $1.55 \%$ & $0.84 \%$ & $2.78 \%$ & $0.62 \%$ & $0.45 \%$ & $0.08 \%$ \\
\hline Gibbsite & 2.42 & $84.05 \%$ & $28.40 \%$ & $5.62 \%$ & $18.46 \%$ & $13.23 \%$ & $20.62 \%$ & $9.81 \%$ & $7.04 \%$ & $17.37 \%$ \\
\hline Boehmite & 3.01 & --- & --- & --- & --- & --- & --- & --- & --- & --- \\
\hline $\mathrm{HgO}$ (co-precip. with $\mathrm{Ag}_{2} \mathrm{O}$ ) & 7.143 & $0.00 \%$ & --- & --- & --- & --- & --- & --- & --- & --- \\
\hline $\mathrm{KAlSiO}_{4}$ & 2.61 & -- & -- & --- & --- & $--\cdot$ & --- & --- & -- & --- \\
\hline $\mathrm{La}(\mathrm{OH})_{3}$ & 2.3 & --- & $\begin{array}{ll}-- \\
\end{array}$ & --- & $\begin{array}{ll}-- \\
\end{array}$ & --- & --- & --- & $0.01 \%$ & --- \\
\hline $\mathrm{LaPO}_{+} \cdot 2 \mathrm{H}_{2} \mathrm{O}$ & 6.51 & $0.01 \%$ & $0.02 \%$ & $0.01 \%$ & $0.01 \%$ & $0.01 \%$ & $0.04 \%$ & $0.01 \%$ & --- & $0.01 \%$ \\
\hline $\mathrm{Mn}_{3}\left(\mathrm{PO}_{4}\right)_{2}$ & 3.102 & -- & --- & --- & -- & -- & -- & -- & --- & --- \\
\hline $\mathrm{MnO}_{2}$ & 5.026 & $0.46 \%$ & $0.19 \%$ & --- & $0.43 \%$ & $0.27 \%$ & $0.97 \%$ & $0.20 \%$ & --- & $0.02 \%$ \\
\hline $\mathrm{Na}_{2}\left(\mathrm{UO}_{2}\right)_{2}\left(\mathrm{PO}_{4}\right)_{2} \cdot 2 \mathrm{H}_{2} \mathrm{O}$ & 3.5 & +-- & --- & --- & --- & $\begin{array}{ll}-- \\
\end{array}$ & --- & $\begin{array}{ll}-- \\
\end{array}$ & --- & --- \\
\hline $\mathrm{Na}_{2} \mathrm{U}_{2} \mathrm{O}_{7}$ & 5.617 & $1.26 \%$ & $0.40 \%$ & $0.22 \%$ & $0.89 \%$ & $1.34 \%$ & $0.52 \%$ & $0.11 \%$ & $1.57 \%$ & $0.05 \%$ \\
\hline $\mathrm{NaAICO}_{3}(\mathrm{OH})_{2}$ & 2.42 & -- & $4.72 \%$ & --- & --- & -- & $3.28 \%$ & --- & -- & --- \\
\hline $\mathrm{NaAlSiO}_{4}$ & 2.365 & $1.10 \%$ & $0.42 \%$ & $1.52 \%$ & $77.26 \%$ & $0.54 \%$ & $0.40 \%$ & $0.31 \%$ & --- & $0.25 \%$ \\
\hline $\mathrm{Ni}(\mathrm{OH})_{2}$ & 4.15 & $0.51 \%$ & $0.15 \%$ & $0.05 \%$ & $0.36 \%$ & $0.04 \%$ & $0.33 \%$ & $0.01 \%$ & $0.02 \%$ & $0.01 \%$ \\
\hline $\mathrm{Ni}_{3}\left(\mathrm{PO}_{4}\right)$ & 3.93 & -- & -- & $\cdots$ & -- & -- & -- & -- & $\cdots$ & $\cdots$ \\
\hline $\mathrm{NiC}_{2} \mathrm{O}_{4} \cdot 2 \mathrm{H}_{2} \mathrm{O}$ (on Fe phase) & 4.26 & -- & --- & --- & --- & --- & -- & --- & -- & --- \\
\hline $\mathrm{Pb}(\mathrm{OH})_{2}$ & 7.1 & $0.02 \%$ & $0.03 \%$ & $0.03 \%$ & $0.01 \%$ & $0.04 \%$ & $0.23 \%$ & $0.04 \%$ & $0.79 \%$ & $0.01 \%$ \\
\hline $\mathrm{Pb}_{3}\left(\mathrm{PO}_{4}\right)_{2}$ & 7.1 & -- & $\begin{array}{ll}-- \\
\end{array}$ & --- & $\begin{array}{ll}-- \\
\end{array}$ & --- & --- & $\begin{array}{ll}-- \\
\end{array}$ & -- & --- \\
\hline $\mathrm{PbCO}_{3}$ & 6.6 & -- & --- & --- & --- & -- & --- & --- & --- & --- \\
\hline $\mathrm{Pu}(\mathrm{OH})_{4}$ (co-precip. on Fe phase) & 4.26 & $0.00 \%$ & $0.00 \%$ & $0.00 \%$ & $0.00 \%$ & $0.00 \%$ & $0.01 \%$ & $0.00 \%$ & $0.00 \%$ & $0.00 \%$ \\
\hline $\mathrm{PuO}_{2}$ & 11.43 & -- & --- & --- & --- & --- & -- & --- & --- & -- \\
\hline $\mathrm{SiO}_{2}$ & 2.6 & --- & --- & --- & --- & --- & --- & --- & --- & --- \\
\hline $\mathrm{Sr}_{3}\left(\mathrm{PO}_{4}\right)_{2}$ & 3.5 & -- & --- & --- & --- & --- & --- & --- & --- & --- \\
\hline $\mathrm{SrCO}_{3}$ & 3.5 & $0.10 \%$ & $0.02 \%$ & $0.01 \%$ & $0.07 \%$ & $0.00 \%$ & $0.01 \%$ & $0.00 \%$ & $0.00 \%$ & $0.00 \%$ \\
\hline $\mathrm{ZrO}_{2}$ & 5.68 & $0.02 \%$ & $0.01 \%$ & $0.01 \%$ & $0.01 \%$ & $0.01 \%$ & $0.09 \%$ & $0.00 \%$ & -.- & $0.00 \%$ \\
\hline \multicolumn{11}{|l|}{ salts: mass fracs in total solid } \\
\hline $\mathrm{KNO}_{3}$ & 2.109 & $-\cdots$ & $\begin{array}{ll}-\cdot \\
\end{array}$ & $\cdots$ & $-\cdots$ & +-- & $-\cdots$ & $\cdots$ & $-\cdots$ & 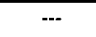 \\
\hline $\mathrm{Na}_{2} \mathrm{C}_{2} \mathrm{O}_{4}$ & 2.34 & $0.99 \%$ & $4.96 \%$ & $1.94 \%$ & $0.17 \%$ & $2.58 \%$ & $7.61 \%$ & $0.74 \%$ & $1.19 \%$ & $1.49 \%$ \\
\hline $\mathrm{Na}_{2} \mathrm{CO}_{3} \cdot \mathrm{H}_{2} \mathrm{O}$ & 2.25 & -- & $\cdots$ & $10.28 \%$ & -- & $9.87 \%$ & --. & $\cdots$ & $10.40 \%$ & $2.93 \%$ \\
\hline $\mathrm{Na}_{2} \mathrm{SO}_{4}$ & 2.68 & --- & --- & --- & --- & --- & --- & --- & --- & --- \\
\hline $\mathrm{Na}_{2} \mathrm{SO}_{4} \cdot 10 \mathrm{H}_{2} \mathrm{O}$ & 1.464 & --- & --- & --- & --- & --- & $\begin{array}{ll}-- \\
\end{array}$ & --- & --- & --- \\
\hline $\mathrm{Na}_{3} \mathrm{FSO}_{4}$ & 2.65 & --- & --- & --- & --- & $0.44 \%$ & $3.83 \%$ & --- & --- & --- \\
\hline $\mathrm{Na}_{3} \mathrm{NO}_{3} \mathrm{SO}_{4} \cdot \mathrm{H}_{2} \mathrm{O}$ & 2.3 & -- & --- & -- & --- & -- & --- & -- & -- & -- \\
\hline $\mathrm{Na}_{3} \mathrm{PO}_{4} \cdot 0.25 \mathrm{NaOH} \cdot 12 \mathrm{H}_{2} \mathrm{O}$ & 1.62 & --- & --- & $6.87 \%$ & --- & --- & --- & $22.80 \%$ & --- & $6.46 \%$ \\
\hline $\mathrm{Na}_{3} \mathrm{PO}_{4} \cdot 8 \mathrm{H}_{2} \mathrm{O}$ & 1.8 & -- & --- & --- & --- & --- & --- & --- & --- & --- \\
\hline $\mathrm{Na}_{4} \mathrm{P}_{2} \mathrm{O}_{7} \cdot 10 \mathrm{H}_{2} \mathrm{O}$ & 1.83 & --- & --- & --- & --- & --- & --- & --- & -- & --- \\
\hline $\mathrm{Na}_{6}\left(\mathrm{SO}_{4}\right)_{2} \mathrm{CO}_{3}$ & 2.64 & --- & $3.49 \%$ & $6.02 \%$ & -- & $5.13 \%$ & --- & $--\cdot$ & $5.34 \%$ & $4.07 \%$ \\
\hline $\mathrm{NaF}$ & 2.78 & --- & --- & --- & --- & -- & --- & --- & --- & -- \\
\hline $\mathrm{Na}_{7} \mathrm{~F}\left(\mathrm{PO}_{4}\right)_{2} \cdot 19 \mathrm{H}_{2} \mathrm{O}$ & 1.75 & $1.89 \%$ & $3.91 \%$ & $6.71 \%$ & --- & $6.31 \%$ & $3.73 \%$ & $2.75 \%$ & $5.79 \%$ & $15.88 \%$ \\
\hline $\mathrm{NaIICO}_{3}$ & 2.159 & -- & -.- & $\cdots$ & $-\cdot$ & -- & $\cdots$ & $\cdots$ & $--\cdot$ & $\cdots$ \\
\hline $\mathrm{NaNO}_{2}$ & 2.168 & --- & --- & --- & --- & --- & --- & --- & --- & --- \\
\hline $\mathrm{NaNO}_{3}$ & 2.26 & $8.19 \%$ & $51.35 \%$ & $58.44 \%$ & --- & $58.46 \%$ & $52.83 \%$ & $61.74 \%$ & $65.95 \%$ & $50.17 \%$ \\
\hline
\end{tabular}


Table C.1. (contd)

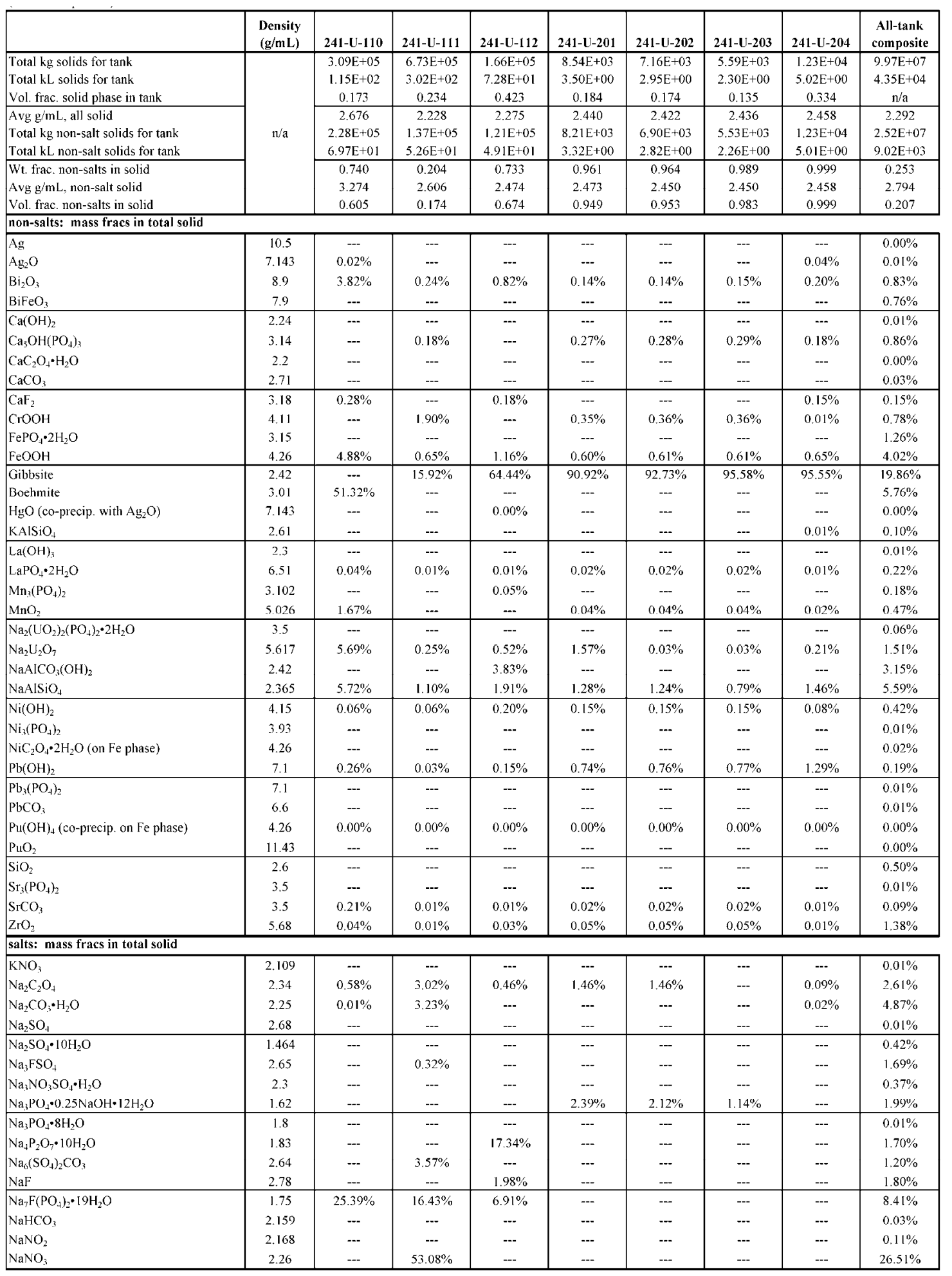


Table C.1. (contd)

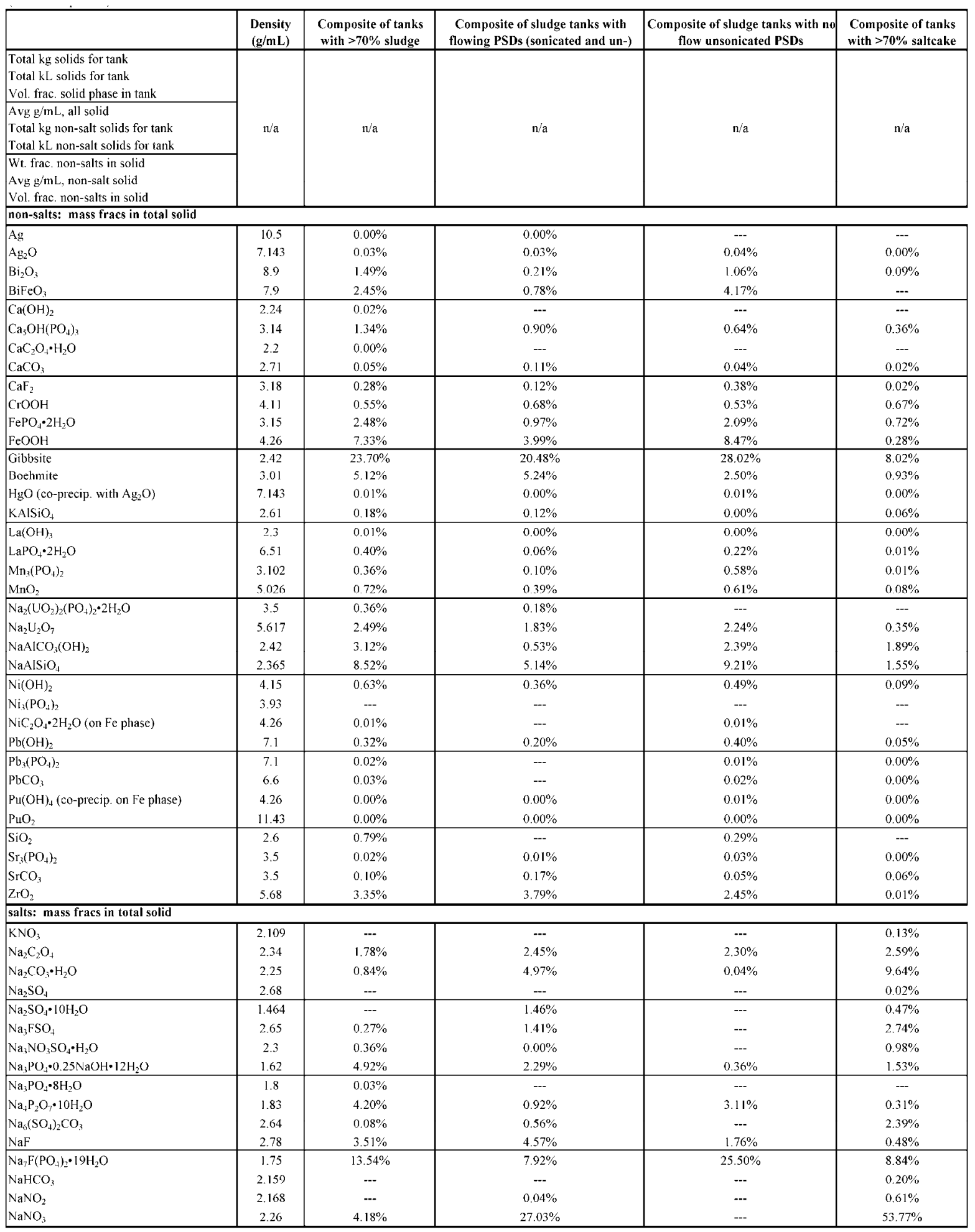


Table C.1. (contd)

\begin{tabular}{|c|c|c|c|c|}
\hline & $\begin{array}{l}\text { Density } \\
(\mathrm{g} / \mathrm{mL})\end{array}$ & $\begin{array}{c}\text { Composite of saltcake tanks with } \\
\text { flowing sonicated PSDs }\end{array}$ & $\begin{array}{l}\text { Composite of saltcake tanks with } \\
\text { flowing unsonicated PSDs }\end{array}$ & $\begin{array}{c}\text { Composite of saltcake tanks with } \\
\text { no-flow unsonicated PSDs }\end{array}$ \\
\hline $\begin{array}{l}\text { Total kg solids for tank } \\
\text { Total kL solids for tank } \\
\text { Vol. frac. solid phase in tank }\end{array}$ & \multirow{3}{*}{ 11/a } & \multirow{3}{*}{$\mathrm{n} / \mathrm{a}$} & \multirow{3}{*}{ n/a } & \multirow{3}{*}{ n/a } \\
\hline $\begin{array}{l}\text { Avg g/mL, all solid } \\
\text { Total kg non-salt solids for tank } \\
\text { Total } \mathrm{kL} \text { non-salt solids for tank }\end{array}$ & & & & \\
\hline $\begin{array}{l}\text { Wt. frac. non-salts in solid } \\
\text { Avg g/mL, non-salt solid } \\
\text { Vol. frac. non-salts in solid }\end{array}$ & & & & \\
\hline \multicolumn{5}{|l|}{ non-salts: mass fracs in total solid } \\
\hline$A g$ & 10.5 & --- & --- & --- \\
\hline $\mathrm{Ag}_{2} \mathrm{O}$ & 7.143 & $0.00 \%$ & $0.00 \%$ & --- \\
\hline $\mathrm{Bi}_{2} \mathrm{O}_{3}$ & 8.9 & $0.18 \%$ & $0.18 \%$ & $0.02 \%$ \\
\hline $\mathrm{BiFeO}_{3}$ & 7.9 & $--\cdot$ & -.- & --. \\
\hline $\mathrm{Ca}(\mathrm{OH})_{2}$ & 2.24 & $\ldots$ & $\cdots$ & $\cdots$ \\
\hline $\mathrm{Ca}_{5} \mathrm{OH}\left(\mathrm{PO}_{4}\right)_{3}$ & 3.14 & $0.83 \%$ & $0.83 \%$ & $0.39 \%$ \\
\hline $\mathrm{CaC}_{2} \mathrm{O}_{4} \cdot \mathrm{H}_{2} \mathrm{O}$ & 2.2 & --- & --- & --- \\
\hline $\mathrm{CaCO}_{3}$ & 2.71 & $0.11 \%$ & $0.10 \%$ & $0.01 \%$ \\
\hline $\mathrm{CaF}_{2}$ & 3.18 & $0.04 \%$ & $0.04 \%$ & $0.00 \%$ \\
\hline $\mathrm{CrOOH}$ & 4.11 & $0.61 \%$ & $0.64 \%$ & $1.11 \%$ \\
\hline $\mathrm{FePO}_{4} \cdot 2 \mathrm{H}_{2} \mathrm{O}$ & 3.15 & $0.82 \%$ & $0.81 \%$ & $0.90 \%$ \\
\hline $\mathrm{FeOOH}$ & 4.26 & $0.37 \%$ & $0.35 \%$ & $0.21 \%$ \\
\hline Gibbsite & 2.42 & $7.96 \%$ & $7.88 \%$ & $9.98 \%$ \\
\hline Boehmite & 3.01 & $0.79 \%$ & $1.35 \%$ & $5.30 \%$ \\
\hline $\mathrm{HgO}$ (co-precip. with $\mathrm{Ag}_{2} \mathrm{O}$ ) & 7.143 & $0.00 \%$ & $0.00 \%$ & $0.00 \%$ \\
\hline $\mathrm{KAlSiO}_{4}$ & 2.61 & $0.00 \%$ & $0.00 \%$ & $0.10 \%$ \\
\hline $\mathrm{La}(\mathrm{OH})_{3}$ & 2.3 & $0.00 \%$ & $0.00 \%$ & $0.00 \%$ \\
\hline $\mathrm{LaPO}_{4} \cdot 2 \mathrm{H}_{2} \mathrm{O}$ & 6.51 & $0.01 \%$ & $0.01 \%$ & $0.01 \%$ \\
\hline $\mathrm{Mn}_{3}\left(\mathrm{PO}_{4}\right)_{2}$ & 3.102 & -- & --- & --- \\
\hline $\mathrm{MnO}_{2}$ & 5.026 & $0.02 \%$ & $0.02 \%$ & $0.04 \%$ \\
\hline $\mathrm{Na}_{2}\left(\mathrm{UO}_{2}\right)_{2}\left(\mathrm{PO}_{4}\right)_{2} \cdot 2 \mathrm{H}_{2} \mathrm{O}$ & 3.5 & --- & $\begin{array}{ll}-- \\
\end{array}$ & --- \\
\hline $\mathrm{Na}_{2} \mathrm{U}_{2} \mathrm{O}_{7}$ & 5.617 & $0.47 \%$ & $0.49 \%$ & $0.54 \%$ \\
\hline $\mathrm{NaAICO}_{3}(\mathrm{OH})_{2}$ & 2.42 & $1.20 \%$ & $1.02 \%$ & --- \\
\hline $\mathrm{NaAISiO}_{4}$ & 2.365 & $1.25 \%$ & $1.25 \%$ & $0.57 \%$ \\
\hline $\mathrm{Ni}(\mathrm{OH})_{2}$ & 4.15 & $0.15 \%$ & $0.14 \%$ & $0.13 \%$ \\
\hline $\mathrm{Ni}_{3}\left(\mathrm{PO}_{4}\right)$ & 3.93 & --- & -- & -- \\
\hline $\mathrm{NiC}_{2} \mathrm{O}_{4} \cdot 2 \mathrm{H}_{2} \mathrm{O}$ (on Fe phase) & 4.26 & --- & --- & --- \\
\hline $\mathrm{Pb}(\mathrm{OH})_{2}$ & 7.1 & $0.06 \%$ & $0.06 \%$ & $0.05 \%$ \\
\hline $\mathrm{Pb}_{3}\left(\mathrm{PO}_{4}\right)_{2}$ & 7.1 & $0.01 \%$ & $0.01 \%$ & --- \\
\hline $\mathrm{PbCO}_{3}$ & 6.6 & --- & -- & --- \\
\hline $\mathrm{Pu}(\mathrm{OH})_{4}$ (co-precip. on Fe phase) & 4.26 & $0.00 \%$ & $0.00 \%$ & $0.00 \%$ \\
\hline $\mathrm{PuO}_{2}$ & 11.43 & $0.00 \%$ & $0.00 \%$ & --- \\
\hline $\mathrm{SiO}_{2}$ & 2.6 & --- & --- & --- \\
\hline $\mathrm{Sr}_{3}\left(\mathrm{PO}_{4}\right)_{2}$ & 3.5 & $0.00 \%$ & $0.00 \%$ & --- \\
\hline $\mathrm{SrCO}_{3}$ & 3.5 & $0.20 \%$ & $0.20 \%$ & $0.23 \%$ \\
\hline $\mathrm{ZrO}_{2}$ & 5.68 & $0.01 \%$ & $0.01 \%$ & $0.01 \%$ \\
\hline \multicolumn{5}{|l|}{ salts: mass fracs in total solid } \\
\hline $\mathrm{KNO}_{3}$ & 2.109 & $\cdots$ & -- & $2.10 \%$ \\
\hline $\mathrm{Na}_{2} \mathrm{C}_{2} \mathrm{O}_{4}$ & 2.34 & $2.08 \%$ & $2.10 \%$ & $6.07 \%$ \\
\hline $\mathrm{Na}_{2} \mathrm{CO}_{3} \cdot \mathrm{H}_{2} \mathrm{O}$ & 2.25 & $11.37 \%$ & $11.28 \%$ & $20.11 \%$ \\
\hline $\mathrm{Na}_{2} \mathrm{SO}_{4}$ & 2.68 & --- & --- & --- \\
\hline $\mathrm{Na}_{2} \mathrm{SO}_{4} \cdot 10 \mathrm{H}_{2} \mathrm{O}$ & 1.464 & --- & --- & --- \\
\hline $\mathrm{Na}_{3} \mathrm{FSO}_{4}$ & 2.65 & $5.09 \%$ & $4.98 \%$ & $2.88 \%$ \\
\hline $\mathrm{Na}_{3} \mathrm{NO}_{3} \mathrm{SO}_{4} \cdot \mathrm{H}_{2} \mathrm{O}$ & 2.3 & $2.86 \%$ & $2.83 \%$ & --- \\
\hline $\mathrm{Na}_{3} \mathrm{PO}_{4} \cdot 0.25 \mathrm{NaOH} \cdot 12 \mathrm{H}_{2} \mathrm{O}$ & 1.62 & $0.61 \%$ & $0.60 \%$ & $0.46 \%$ \\
\hline $\mathrm{Na}_{3} \mathrm{PO}_{4} \bullet 8 \mathrm{H}_{2} \mathrm{O}$ & 1.8 & --- & -- & --- \\
\hline $\mathrm{Na}_{4} \mathrm{P}_{2} \mathrm{O}_{7} \cdot 10 \mathrm{H}_{2} \mathrm{O}$ & 1.83 & $0.06 \%$ & $0.06 \%$ & --- \\
\hline $\mathrm{Na}_{6}\left(\mathrm{SO}_{4}\right)_{2} \mathrm{CO}_{3}$ & 2.64 & $1.00 \%$ & $1.03 \%$ & $1.67 \%$ \\
\hline $\mathrm{NaF}$ & 2.78 & $0.29 \%$ & $0.26 \%$ & $0.10 \%$ \\
\hline $\mathrm{Na}_{7} \mathrm{~F}\left(\mathrm{PO}_{4}\right)_{2} \cdot 19 \mathrm{H}_{2} \mathrm{O}$ & 1.75 & $3.64 \%$ & $3.62 \%$ & $3.11 \%$ \\
\hline $\mathrm{NaIICO}_{3}$ & 2.159 & $1.37 \%$ & $1.36 \%$ & -- \\
\hline $\mathrm{NaNO}_{2}$ & 2.168 & $0.08 \%$ & $0.08 \%$ & $1.47 \%$ \\
\hline $\mathrm{NaNO}_{3}$ & 2.26 & $56.47 \%$ & $56.40 \%$ & $42.43 \%$ \\
\hline
\end{tabular}


Table C.2. As-Is Solid-Phase Compositions of Waste Types Based on 2002 BBI ("---" = not present, "n/a" = insufficient data)

\begin{tabular}{|c|c|c|c|c|c|c|c|c|c|c|}
\hline & $\begin{array}{l}\text { density } \\
(\mathrm{g} / \mathrm{mL}) \\
\end{array}$ & $\begin{array}{l}\text { Mol. wt. } \\
\text { (g/mol) }\end{array}$ & $\begin{array}{c}\text { Sludge } \\
\text { IC }\end{array}$ & $\begin{array}{c}\text { Sludge } \\
\text { R1 (boiling) } \\
\end{array}$ & $\begin{array}{c}\text { Sludge } \\
2 \mathrm{C}\end{array}$ & $\begin{array}{c}\text { Sludge } \\
\text { TBP }\end{array}$ & $\begin{array}{l}\text { Sludge } \\
\text { CWP2 } \\
\end{array}$ & $\begin{array}{c}\text { Sludge } \\
\text { 224 Post-1949 } \\
\end{array}$ & $\begin{array}{l}\text { Sludge } \\
\text { CWP1 } \\
\end{array}$ & $\begin{array}{r}\text { Sludge } \\
\text { CWZr2 } \\
\end{array}$ \\
\hline Avg g/mL, non-salt solid & & & 3.179 & 3.499 & 3.966 & 3.632 & 2.479 & 6.414 & 2.471 & 4.327 \\
\hline Mass frac non-salts in solid & & & 0.364 & 0.259 & 0.643 & 0.379 & 0.997 & 0.962 & 0.962 & 0.587 \\
\hline Vol. frac. non-salts in solid & $\mathrm{n} / \mathrm{a}$ & $\mathrm{n} / \mathrm{a}$ & 0.241 & 0.184 & 0.442 & 0.216 & 0.997 & 0.898 & 0.957 & 0.476 \\
\hline Avg $g / m L$, salt solid & & & 1.765 & 2.260 & 1.741 & 1.645 & 2.333 & 2.250 & 2.134 & 2.764 \\
\hline \multicolumn{11}{|l|}{ mass fracs in total solid } \\
\hline$\overline{A g}$ & 10.5 & 107.87 & $\overline{---}$ & $\overline{---}$ & $\overline{---}$ & $\overline{---}$ & $\overline{---}$ & $\overline{---}$ & $\overline{---}$ & $\overline{---}$ \\
\hline $\mathrm{Ag}_{2} \mathrm{O}$ & 7.143 & 231.74 & $0.0 \%$ & --- & $0.0 \%$ & $0.0 \%$ & $0.0 \%$ & $0.0 \%$ & $0.0 \%$ & $0.1 \%$ \\
\hline $\mathrm{Bi}_{2} \mathrm{O}_{3}$ & 8.9 & 465.96 & -- & $0.0 \%$ & -- & $0.2 \%$ & $0.2 \%$ & $45.1 \%$ & $0.1 \%$ & $0.1 \%$ \\
\hline $\mathrm{BiFeO}_{3}$ & 7.9 & 312.83 & $10.1 \%$ & --- & $23.2 \%$ & --- & --- & -- & --- & --- \\
\hline$\overline{\mathrm{Ca}(\mathrm{OH})_{2}}$ & 2.24 & 74.09 & 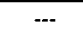 & --- & $\overline{---}$ & $\overline{c--}$ & $\overline{c--}$ & $-\cdots$ & $\overline{---}$ & $-\cdots$ \\
\hline $\mathrm{Ca}_{5} \mathrm{OH}\left(\mathrm{PO}_{4}\right)_{3}$ & 3.14 & 502.31 & $0.0 \%$ & $0.1 \%$ & $0.9 \%$ & $3.4 \%$ & $0.2 \%$ & --- & $0.8 \%$ & --- \\
\hline $\mathrm{CaC}_{2} \mathrm{O}_{4} \cdot \mathrm{H}_{2} \mathrm{O}$ & 2.2 & 146.11 & -- & --- & --- & --- & --- & -- & -- & -- \\
\hline $\mathrm{CaCO}_{3}$ & 2.71 & 100.09 & -- & $0.8 \%$ & -- & --- & --- & -- & --- & -- \\
\hline $\mathrm{CaF}_{2}$ & 3.18 & 78.07 & $0.8 \%$ & -- & $0.8 \%$ & -- & $--\cdot$ & $0.5 \%$ & -- & $0.3 \%$ \\
\hline $\mathrm{CrOOH}$ & 4.11 & 85 & $0.0 \%$ & $1.8 \%$ & $1.3 \%$ & --- & $0.0 \%$ & $2.9 \%$ & --- & $0.7 \%$ \\
\hline $\mathrm{FePO}_{4} \cdot 2 \mathrm{H}_{2} \mathrm{O}$ & 3.15 & 186.852 & $5.2 \%$ & --- & $29.0 \%$ & --- & --- & --- & --- & --- \\
\hline $\mathrm{FeOOH}$ & 4.26 & 88.85 & --- & $6.1 \%$ & $1.0 \%$ & $17.0 \%$ & $2.8 \%$ & $5.8 \%$ & $0.7 \%$ & $0.7 \%$ \\
\hline Gibbsite & 2.42 & 78 & $8.1 \%$ & $\begin{array}{c}-- \\
\end{array}$ & $\overline{---}$ & $2.2 \%$ & $86.0 \%$ & $\overline{---}$ & $71.8 \%$ & $11.1 \%$ \\
\hline Boehmite & 3.01 & 59.99 & --- & $11.6 \%$ & -- & -- & --- & -- & --- & -- \\
\hline $\mathrm{HgO}$ (co-precip. with $\mathrm{Ag}_{2} \mathrm{O}$ ) & 7.143 & 216.59 & --- & $0.0 \%$ & --- & $0.1 \%$ & --- & --- & $0.0 \%$ & --- \\
\hline $\mathrm{KAISiO}_{4}$ & 2.61 & 158.16 & -- & --- & --- & --- & --- & $0.3 \%$ & $0.2 \%$ & $2.2 \%$ \\
\hline $\mathrm{La}(\mathrm{OH})_{3}$ & 2.3 & 189.93 & -- & --- & -- & --- & -- & $0.5 \%$ & -- & --- \\
\hline $\mathrm{LaPO}_{4} \cdot 2 \mathrm{H}_{2} \mathrm{O}$ & 6.51 & 269.91 & $0.0 \%$ & $0.1 \%$ & $0.4 \%$ & --- & $0.0 \%$ & $19.3 \%$ & $0.1 \%$ & $0.2 \%$ \\
\hline $\mathrm{Mn}_{3}\left(\mathrm{PO}_{4}\right)_{2}$ & 3.102 & 354.76 & $0.0 \%$ & --- & $0.5 \%$ & $0.2 \%$ & $0.2 \%$ & --- & --- & -- \\
\hline $\mathrm{MnO}_{2}$ & 5.026 & 86.94 & -- & $2.0 \%$ & $0.0 \%$ & -- & -- & $20.2 \%$ & $0.5 \%$ & $0.2 \%$ \\
\hline $\mathrm{Na}_{2}\left(\mathrm{UO}_{2}\right)_{2}\left(\mathrm{PO}_{4}\right)_{2} \cdot 2 \mathrm{H}_{2} \mathrm{O}$ & 3.5 & 811.952 & --- & --- & $\begin{array}{c}-- \\
-\end{array}$ & $12.7 \%$ & --- & --- & --- & $\begin{array}{c}-- \\
-1\end{array}$ \\
\hline $\mathrm{Na}_{2} \mathrm{U}_{2} \mathrm{O}_{7}$ & 5.617 & 634.03 & $0.7 \%$ & $1.5 \%$ & $0.1 \%$ & --- & $1.4 \%$ & $0.2 \%$ & $2.2 \%$ & $3.6 \%$ \\
\hline $\mathrm{NaAlCO}_{3}(\mathrm{OH})_{2}$ & 2.42 & 144 & $0.7 \%$ & --- & $3.3 \%$ & --- & $7.0 \%$ & --- & --- & --- \\
\hline $\mathrm{NaAlSiO}_{4}$ & 2.365 & 142.05 & $10.6 \%$ & $1.2 \%$ & $2.8 \%$ & $1.3 \%$ & $1.6 \%$ & $\cdots$ & $19.1 \%$ & $\cdots$ \\
\hline $\mathrm{Ni}(\mathrm{OH})_{2}$ & 4.15 & 92.71 & $0.0 \%$ & $0.4 \%$ & $0.0 \%$ & $0.1 \%$ & $0.0 \%$ & $0.3 \%$ & $0.4 \%$ & $0.1 \%$ \\
\hline $\mathrm{Ni}_{3}\left(\mathrm{PO}_{4}\right)_{2}$ & 3.93 & 366.02 & -- & --- & --- & --- & -- & --- & --- & -- \\
\hline $\mathrm{NiC}_{2} \mathrm{O}_{4} \cdot 2 \mathrm{H}_{2} \mathrm{O}$ (on $\mathrm{Fe}$ phase) & 4.26 & 182.74 & -- & -- & -- & -- & -- & -- & -- & -- \\
\hline $\mathrm{Pb}(\mathrm{OH})_{2}$ & 7.1 & 241.21 & $0.0 \%$ & $0.1 \%$ & $0.6 \%$ & $0.4 \%$ & $0.1 \%$ & $0.2 \%$ & $0.1 \%$ & $0.0 \%$ \\
\hline $\mathrm{Pb}_{3}\left(\mathrm{PO}_{4}\right)_{2}$ & 7.1 & 811.54 & --- & --- & --- & --- & --- & -- & --- & --- \\
\hline $\mathrm{PbCO}_{3}$ & 6.6 & 267.21 & -- & --- & -- & -- & --- & -- & -- & -- \\
\hline Pu(OH $)_{4}$ (co-precip. on Fe phase) & 4.26 & 307.03 & $0.0 \%$ & $0.0 \%$ & $0.0 \%$ & $0.0 \%$ & $0.0 \%$ & $0.0 \%$ & $0.0 \%$ & $0.0 \%$ \\
\hline $\mathrm{PuO}_{2}$ & 11.43 & 271 & --- & --- & -- & --- & --- & -- & --- & --- \\
\hline $\mathrm{SiO}_{2}$ & 2.6 & 60.08 & -- & $\cdots$ & -- & -- & -- & -- & -- & -- \\
\hline $\mathrm{Sr}_{3}\left(\mathrm{PO}_{4}\right)_{2}$ & 3.5 & 452.8 & $0.1 \%$ & --- & --- & $0.5 \%$ & --- & --- & --- & --- \\
\hline $\mathrm{SrCO}_{3}$ & 3.5 & 147.63 & $0.0 \%$ & $0.2 \%$ & $0.3 \%$ & -- & $0.0 \%$ & $0.8 \%$ & $0.0 \%$ & $0.0 \%$ \\
\hline $\mathrm{ZrO}_{2}$ & 5.68 & 123.22 & $0.1 \%$ & $0.1 \%$ & $0.0 \%$ & -- & $0.0 \%$ & $0.0 \%$ & $0.0 \%$ & $39.2 \%$ \\
\hline \multicolumn{11}{|l|}{ salts: mass fracs in total solid } \\
\hline $\mathrm{KNO}_{3}$ & 2.109 & 101.1 & -- & --- & --- & -- & -- & -- & -- & -- \\
\hline $\mathrm{Na}_{2} \mathrm{C}_{2} \mathrm{O}_{4}$ & 2.34 & 134 & $0.1 \%$ & $0.4 \%$ & $1.0 \%$ & $0.8 \%$ & $0.3 \%$ & -- & $2.2 \%$ & $1.2 \%$ \\
\hline $\mathrm{Na}_{2} \mathrm{CO}_{3} \cdot \mathrm{H}_{2} \mathrm{O}$ & 2.25 & 124 & $0.0 \%$ & $1.9 \%$ & $0.0 \%$ & $0.0 \%$ & $0.0 \%$ & $3.8 \%$ & $0.0 \%$ & $0.1 \%$ \\
\hline $\mathrm{Na}_{2} \mathrm{SO}_{4}$ & 2.68 & 142.04 & --- & -- & --- & --- & --- & --- & -- & --- \\
\hline $\mathrm{Na}_{2} \mathrm{SO}_{4} \cdot 10 \mathrm{H}_{2} \mathrm{O}$ & 1.464 & 322.2 & -- & +-- & +-- & $\begin{array}{c}-- \\
\end{array}$ & --- & $\overline{-\cdots}$ & --- & $\overline{---}$ \\
\hline $\mathrm{Na}_{3} \mathrm{FSO}_{4}$ & 2.65 & 184.03 & --- & --- & -- & -- & --- & --- & $0.4 \%$ & -- \\
\hline $\mathrm{Na}_{3} \mathrm{NO}_{3} \mathrm{SO}_{4} \cdot \mathrm{H}_{2} \mathrm{O}$ & 2.3 & 245.05 & -- & --- & -- & $0.2 \%$ & --- & -- & -- & --- \\
\hline $\mathrm{Na}_{3} \mathrm{PO}_{4} \cdot 0.25 \mathrm{NaOH} \cdot 12 \mathrm{H}_{2} \mathrm{O}$ & 1.62 & 390.13 & -- & -- & $5.4 \%$ & $55.7 \%$ &.- & $--\cdot$ & -- & -- \\
\hline $\mathrm{Na}_{3} \mathrm{PO}_{4} \cdot 8 \mathrm{H}_{2} \mathrm{O}$ & 1.8 & 308.06 & -- & $\cdots$ & $-\cdots$ & $-\cdots$ & $-\cdots$ & $-\cdots$ & $-\cdots$ & -- \\
\hline $\mathrm{Na}_{4} \mathrm{P}_{2} \mathrm{O}_{7} \cdot 10 \mathrm{H}_{2} \mathrm{O}$ & 1.83 & 446.06 & $5.9 \%$ & --- & --- & $5.5 \%$ & --- & --- & --- & --- \\
\hline $\mathrm{Na}_{6}\left(\mathrm{SO}_{4}\right)_{2} \mathrm{CO}_{3}$ & 2.64 & 390.08 & --- & $\cdots$ & -- & -- & $--\cdot$ & -- & -- & -- \\
\hline $\mathrm{NaF}$ & 2.78 & 41.99 & $0.6 \%$ & --. & $--\cdot$ & -- & $--\cdot$ & --- & --. & $40.1 \%$ \\
\hline $\mathrm{Na}_{7} \mathrm{~F}\left(\mathrm{PO}_{4}\right)_{2} \cdot 19 \mathrm{H}_{2} \mathrm{O}$ & 1.75 & 712.16 & $56.9 \%$ & --- & $29.3 \%$ & --- & --- & --- & $1.2 \%$ & --- \\
\hline $\mathrm{NaHCO}_{3}$ & 2.159 & 84.01 & --- & -- & -- & -- & --- & -- & -- & --- \\
\hline $\mathrm{NaNO}_{2}$ & 2,168 & 69 & --- & $\cdots$ & $\cdots$ & -- & -- & -- & -- & -- \\
\hline $\mathrm{NaNO}_{3}$ & 2.26 & 84.99 & -- & $71.9 \%$ & --- & -- & --- & -- & -- & -- \\
\hline
\end{tabular}


Table C.2. (contd)

\begin{tabular}{|c|c|c|c|c|c|c|c|c|c|c|c|}
\hline & $\begin{array}{l}\text { density } \\
(\mathrm{g} / \mathrm{mL})\end{array}$ & $\begin{array}{l}\text { Sludge } \\
\text { CWR1 } \\
\end{array}$ & $\begin{array}{c}\text { Sludge } \\
\text { DE }\end{array}$ & $\begin{array}{c}\text { Sludge } \\
\text { RI (non-boiling) } \\
\end{array}$ & $\begin{array}{l}\text { Sludge } \\
\text { PFeCN }\end{array}$ & $\begin{array}{c}\text { Sludge } \\
\text { BL }\end{array}$ & $\begin{array}{l}\text { Sludge } \\
\text { TFeCN }\end{array}$ & $\begin{array}{c}\begin{array}{c}\text { Sludge } \\
\text { P3 }\end{array} \\
\end{array}$ & \begin{tabular}{|c|} 
Sludge \\
ICFeCN
\end{tabular} & $\begin{array}{l}\text { Sludge } \\
\text { CWR2 }\end{array}$ & $\begin{array}{c}\text { Sludge } \\
\text { SRR }\end{array}$ \\
\hline Avg g/mL, non-salt solid & \multirow{4}{*}{$\mathrm{n} / \mathrm{a}$} & 2.488 & 2.505 & 2.586 & 2.686 & 3.433 & 2.917 & 2.984 & 3.022 & 2.737 & \multirow{4}{*}{$n / a$} \\
\hline Mass frac non-salts in solid & & 0.219 & 0.201 & 0.221 & 0.294 & 0.972 & 0.158 & 0.999 & 0.688 & 0.176 & \\
\hline vol. frac non-salts in solid & & 0.191 & 0.187 & 0.198 & 0.261 & 0.960 & 0.114 & 0.999 & 0.571 & 0.148 & \\
\hline Avg $\mathrm{g} / \mathrm{mL}$, salt solid & & 2.090 & 2.284 & 2.251 & 2.280 & 2.340 & 1.997 & 2.250 & 1.830 & 2.226 & \\
\hline \multicolumn{12}{|l|}{ mass fracs in total solid } \\
\hline$\overline{A g}$ & 10.5 & -- & -- & --- & $-\cdots$ & 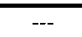 & --- & $-\cdots$ & -- & -- & \multirow{36}{*}{$\mathrm{n} / \mathrm{a}$} \\
\hline $\mathrm{Ag}_{2} \mathrm{O}$ & 7.143 & $0.0 \%$ & -- & --- & -- & $0.0 \%$ & $0.0 \%$ & $0.1 \%$ & $0.0 \%$ & -- & \\
\hline $\mathrm{Bi}_{2} \mathrm{O}_{3}$ & 8.9 & $0.0 \%$ & $0.0 \%$ & $0.0 \%$ & $0.1 \%$ & $0.1 \%$ & $0.1 \%$ & $0.0 \%$ & $6.2 \%$ & $0.0 \%$ & \\
\hline $\mathrm{BiFeO}_{3}$ & 7.9 & -- & -- & --- & -- & --- & -- & -- & -- & --- & \\
\hline $\mathrm{Ca}(\mathrm{OH})_{2}$ & 2.24 & $-\cdots$ & -- & --- & --- & --- & -- & $-\cdots$ & --- & -- & \\
\hline $\mathrm{Ca}_{5} \mathrm{OH}\left(\mathrm{PO}_{4}\right)_{3}$ & 3.14 & $0.1 \%$ & $0.2 \%$ & $0.1 \%$ & $1.1 \%$ & $4.1 \%$ & $1.9 \%$ & $1.3 \%$ & $2.2 \%$ & $0.4 \%$ & \\
\hline $\mathrm{CaC}_{2} \mathrm{O}_{4} \cdot \mathrm{H}_{2} \mathrm{O}$ & 2.2 & -- & -- & --- & --- & --- & --- & --- & --- & --- & \\
\hline $\mathrm{CaCO}_{3}$ & 2.71 & -- & -- & -- & -- &.-- &.-- & -- & -- & -- & \\
\hline $\mathrm{CaF}_{2}$ & 3.18 & $0.0 \%$ & -.. & $-\cdots$ & -.. & 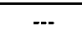 & -.. & $\cdots$ & $1.4 \%$ & -- & \\
\hline $\mathrm{CrOOH}$ & 4.11 & $1.0 \%$ & $0.1 \%$ & $2.1 \%$ & $0.4 \%$ & $0.9 \%$ & $0.2 \%$ & $0.2 \%$ & $2.8 \%$ & $0.9 \%$ & \\
\hline $\mathrm{FePO}_{4} \cdot 2 \mathrm{H}_{2} \mathrm{O}$ & 3.15 & --- & $2.3 \%$ & --- & $4.0 \%$ & --- & $1.0 \%$ & --- & -- & --- & \\
\hline $\mathrm{FeOOH}$ & 4.26 & $0.1 \%$ & $0.0 \%$ & $0.6 \%$ & --- & $49.1 \%$ & $1.0 \%$ & $28.2 \%$ & $13.0 \%$ & $0.5 \%$ & \\
\hline Gibbsite & 2.42 & $20.2 \%$ & -- & $18.5 \%$ & $19.9 \%$ & $31.6 \%$ & $4.6 \%$ & $42.4 \%$ & --- & $13.3 \%$ & \\
\hline Boelhmite & 3.01 & --- & --- & -- & -- & --- & --- & -- & -- & -- & \\
\hline $\mathrm{HgO}$ (co-precip. with $\mathrm{Ag}_{2} \mathrm{O}$ ) & 7.143 & -- & --- & $0.0 \%$ & $0.0 \%$ & $0.0 \%$ & --- & --- & --- & -- & \\
\hline $\mathrm{KAISiO}_{4}$ & 2.61 & $0.0 \%$ & --- & --- & --- & --- & $0.5 \%$ & --- & --- & --- & \\
\hline $\mathrm{La}(\mathrm{OH})_{3}$ & 2.3 & -- & -- & -- & $0.0 \%$ & -- & $0.0 \%$ & -- & -- & $0.0 \%$ & \\
\hline $\mathrm{LaPO}_{4} \cdot 2 \mathrm{H}_{2} \mathrm{O}$ & 6.51 & $0.0 \%$ & $0.0 \%$ & $0.0 \%$ & $0.0 \%$ & $0.8 \%$ & $0.0 \%$ & $1.3 \%$ & $0.0 \%$ & $0.0 \%$ & \\
\hline $\mathrm{Mn}_{3}\left(\mathrm{PO}_{4}\right)_{2}$ & 3.102 & --- & -- & --- & --- & --- & $0.0 \%$ & --- & $0.2 \%$ & -- & \\
\hline $\mathrm{MnO}_{2}$ & 5.026 & $0.0 \%$ & $0.1 \%$ & $0.0 \%$ & $0.0 \%$ & $6.5 \%$ & --- & $0.5 \%$ & --- & $0.0 \%$ & \\
\hline $\mathrm{Na}_{2}\left(\mathrm{UO}_{2}\right)_{2}\left(\mathrm{PO}_{4}\right)_{2} \cdot 2 \mathrm{H}_{2} \mathrm{O}$ & 3.5 & -- & -- & --- & --- & -- & -- & -- & -- & -- & \\
\hline $\mathrm{Na}_{2} \mathrm{U}_{2} \mathrm{O}_{7}$ & 5.617 & $0.1 \%$ & -- & $0.4 \%$ & $1.7 \%$ & $1.4 \%$ & $1.9 \%$ & $1.9 \%$ & $0.6 \%$ & $1.5 \%$ & \\
\hline $\mathrm{NaAlCO}_{3}(\mathrm{OH})_{2}$ & 2.42 & -- & $13.3 \%$ & --- & --- & -- & $1.5 \%$ & $11.2 \%$ & $12.4 \%$ & --- & \\
\hline $\mathrm{NaAlSiO}_{4}$ & 2.365 & $0.3 \%$ & $3.9 \%$ & $0.3 \%$ & $0.6 \%$ & -- & $2.3 \%$ & $5.0 \%$ & $18.8 \%$ & $0.2 \%$ & \\
\hline $\mathrm{Ni}(\mathrm{OH})_{2}$ & 4.15 & $0.0 \%$ & $0.0 \%$ & $0.0 \%$ & $0.5 \%$ & $1.2 \%$ & $0.8 \%$ & $1.7 \%$ & $0.6 \%$ & $0.0 \%$ & \\
\hline $\mathrm{Ni}_{3}\left(\mathrm{PO}_{4}\right)_{2}$ & 3.93 & -- & -- & --- & -- & -- & -- & -- & $0.7 \%$ & -- & \\
\hline $\mathrm{NiC}_{2} \mathrm{O}_{4} \cdot 2 \mathrm{H}_{2} \mathrm{O}$ (on Fe phase) & 4.26 & $\cdots$ & -- & -- & -.- & $\ldots$ & -.. & $\ldots$ & $1.0 \%$ & -.. & \\
\hline $\mathrm{Pb}(\mathrm{OH})_{2}$ & 7.1 & $0.0 \%$ & $0.1 \%$ & $0.0 \%$ & $0.1 \%$ & $1.5 \%$ & $0.1 \%$ & $0.2 \%$ & --- & $0.7 \%$ & \\
\hline $\mathrm{Pb}_{3}\left(\mathrm{PO}_{4}\right)_{2}$ & 7.1 & --- & -- & --- & --- & -- & --- & --- & $0.1 \%$ & --- & \\
\hline $\mathrm{PbCO}_{3}$ & 6.6 & --- & -- & -- & -- & -- & --- & -- & -- & -- & \\
\hline $\mathrm{Pu}(\mathrm{OH})_{4}$ (co-precip. on Fe phase) & 4.26 & $0.0 \%$ & -- & $0.0 \%$ & $0.0 \%$ & $0.0 \%$ & $0.0 \%$ & $0.0 \%$ & $0.0 \%$ & $0.0 \%$ & \\
\hline $\mathrm{PuO}_{2}$ & 11.43 & --- & $0.0 \%$ & $0.0 \%$ & --- & -- & --- & --- & -- & -- & \\
\hline $\mathrm{SiO}_{2}$ & 2.6 & -- & -- & --- & $-\cdots$ & $-\cdots$ & $-\cdots$ & $\cdots$ & $8.7 \%$ & $-\cdots$ & \\
\hline $\mathrm{Sr}_{3}\left(\mathrm{PO}_{4}\right)_{2}$ & 3.5 & -- & -- & $0.0 \%$ & --- & --- & $00 \%$ & --- & --- & --- & \\
\hline $\mathrm{SrCO}_{3}$ & 3.5 & $0.0 \%$ & $0.0 \%$ & $0.0 \%$ & $1.0 \%$ & $0.1 \%$ & $0.0 \%$ & $0.1 \%$ & -- & $0.0 \%$ & \\
\hline $\mathrm{ZrO}_{2}$ & 5.68 & $0.0 \%$ & $0.0 \%$ & $0.0 \%$ & $0.0 \%$ & $0.0 \%$ & $0.0 \%$ & $5.8 \%$ & $0.1 \%$ & $0.0 \%$ & \\
\hline \multicolumn{12}{|l|}{ salts: mass fracs in total solid } \\
\hline $\mathrm{KNO}_{3}$ & 2.109 & -- & -- & +-- & --- & -- & -- & -- & -- & -- & \\
\hline $\mathrm{Na}_{2} \mathrm{C}_{2} \mathrm{O}_{4}$ & 2.34 & $1.5 \%$ & $2.0 \%$ & $0.1 \%$ & $3.6 \%$ & $2.8 \%$ & $1.3 \%$ & $\cdots$ & $\cdots$ & $1.0 \%$ & \\
\hline $\mathrm{Na}_{2} \mathrm{CO}_{3} \cdot \mathrm{H}_{2} \mathrm{O}$ & 2.25 & $2.8 \%$ & $0.2 \%$ & $0.3 \%$ & $18.2 \%$ & $0.0 \%$ & $2.0 \%$ & $0.1 \%$ & $0.0 \%$ & $9.0 \%$ & \\
\hline $\mathrm{Na}_{2} \mathrm{SO}_{4}$ & 2.68 & --- & -- & --- & -- & -- & -- & --- & -- & --- & \\
\hline $\mathrm{Na}_{2} \mathrm{SO}_{4} \cdot 10 \mathrm{H}_{2} \mathrm{O}$ & 1.464 & -- & -- & --- & --- & $-\cdots$ & $-\cdots$ & $-\cdots$ & $-\cdots$ & --- & \\
\hline $\mathrm{Na}_{3} \mathrm{FSO}_{4}$ & 2.65 & --- & $5.2 \%$ & $0.1 \%$ & $7.5 \%$ & --- & $0.4 \%$ & --- & --- & --- & \\
\hline $\mathrm{Na}_{3} \mathrm{NO}_{3} \mathrm{SO}_{4} \cdot \mathrm{H}_{2} \mathrm{O}$ & 2.3 & -- & $1.0 \%$ & -- & --- & --- & --- & -- & -- & -- & \\
\hline $\mathrm{Na}_{3} \mathrm{PO}_{4} \cdot 0,25 \mathrm{NaOH} \cdot 12 \mathrm{H}_{2} \mathrm{O}$ & 1.62 & $6.3 \%$ &.- & --- & -- & 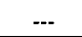 & $10.9 \%$ & $-\cdots$ & $-\cdots$ & $--\cdot$ & \\
\hline $\mathrm{Na}_{3} \mathrm{PO}_{4} \cdot 8 \mathrm{H}_{2} \mathrm{O}$ & 1.8 & -- & -- & --- & -- & -- & --- & -- & --- & --- & \\
\hline $\mathrm{Na}_{4} \mathrm{P}_{2} \mathrm{O}_{7} \cdot 10 \mathrm{H}_{2} \mathrm{O}$ & 1.83 & -- & -- & --- & --- & --- & $1.8 \%$ & --- & $31.2 \%$ & --- & \\
\hline $\mathrm{Na}_{6}\left(\mathrm{SO}_{4}\right)_{2} \mathrm{CO}_{3}$ & 2.64 & $3.9 \%$ & $\ldots$ & $0.5 \%$ & -.- & -.- & -.. & -.. & -.. & $4.6 \%$ & \\
\hline $\mathrm{NaF}$ & 2.78 & -- & -- & -- & $0.7 \%$ & -- & --- & --- & --- & -- & \\
\hline $\mathrm{Na}_{7} \mathrm{~F}\left(\mathrm{PO}_{4}\right)_{2} \cdot 19 \mathrm{H}_{2} \mathrm{O}$ & 1.75 & $15.3 \%$ & -- & $1.3 \%$ & $2.1 \%$ & --- & $22.1 \%$ & --- & --- & $6.6 \%$ & \\
\hline $\mathrm{NaHCO}_{3}$ & 2.159 & -- & -- & --- & --- & --- & --- & --- & -- & --- & \\
\hline $\mathrm{NaNO}_{2}$ & 2.168 & -- & -.. & -.- & $0.6 \%$ & -- & -.. & -- & --- & -- & \\
\hline $\mathrm{NaNO}_{3}$ & 2.26 & $48.3 \%$ & $71.5 \%$ & $75.5 \%$ & $37.8 \%$ & --- & $45.7 \%$ & --- & --- & $61.3 \%$ & \\
\hline
\end{tabular}


Table C.2. (contd)

\begin{tabular}{|c|c|c|c|c|c|c|c|c|c|c|c|}
\hline & $\begin{array}{l}\text { density } \\
(\mathrm{g} / \mathrm{mL})\end{array}$ & $\begin{array}{c}\text { Sludge } \\
\text { AR }\end{array}$ & $\begin{array}{c}\text { Sludge } \\
\text { P2 }\end{array}$ & $\begin{array}{l}\text { Sludge } \\
\text { MW }\end{array}$ & $\begin{array}{c}\text { Sludge } \\
\text { PL2 }\end{array}$ & $\begin{array}{c}\text { Sludge } \\
224 \text { Pre-1949 } \\
\end{array}$ & \begin{tabular}{|c|} 
Sludge \\
CWZrI \\
\end{tabular} & \begin{tabular}{|c|} 
Sludge \\
$Z$
\end{tabular} & \begin{tabular}{|l|} 
Sludge \\
OWw3 \\
\end{tabular} & \begin{tabular}{|c|}
$\begin{array}{c}\text { Sludge } \\
\text { THI }\end{array}$ \\
\end{tabular} & $\begin{array}{c}\text { Sludge } \\
\text { HS }\end{array}$ \\
\hline $\begin{array}{l}\text { Avg g/mL, non-salt solid } \\
\text { Mass frac non-salts in solid } \\
\text { vol. frac. non-salts in solid } \\
\text { Avg } g / \mathrm{mL} \text {, salt solid }\end{array}$ & $\mathrm{n} / \mathrm{a}$ & $\begin{array}{l}3.077 \\
0.938 \\
0.920 \\
2.339\end{array}$ & $\begin{array}{l}3.452 \\
0.986 \\
0.978 \\
2.250\end{array}$ & $\begin{array}{l}2.724 \\
0.950 \\
0.937 \\
2.107\end{array}$ & $\begin{array}{l}3.047 \\
0.366 \\
0.334 \\
2.652\end{array}$ & $\begin{array}{l}5.847 \\
0.902 \\
0.780 \\
2.259\end{array}$ & $\mathrm{n} / \mathrm{a}$ & $n / a$ & $\mathrm{n} / \mathrm{a}$ & $\mathrm{n} / \mathrm{a}$ & $\begin{array}{l}4.432 \\
0.808 \\
0.683 \\
2.278\end{array}$ \\
\hline mass fracs in total solid & & & & & & & & & & & \\
\hline $\begin{array}{l}\mathrm{Ag} \\
\mathrm{Ag}_{2} \mathrm{O} \\
\mathrm{Bi}_{2} \mathrm{O}_{3} \\
\mathrm{BiFeO}_{3} \\
\end{array}$ & $\begin{array}{c}10.5 \\
7.143 \\
8.9 \\
7.9 \\
\end{array}$ & $\begin{array}{c}0.2 \% \\
--- \\
0.0 \% \\
--- \\
\end{array}$ & $\begin{array}{l}-- \\
-- \\
-- \\
--\end{array}$ & $\begin{array}{c}-- \\
0.0 \% \\
0.1 \% \\
--- \\
\end{array}$ & $\begin{array}{c}--- \\
0.0 \% \\
0.0 \% \\
--- \\
\end{array}$ & $\begin{array}{c}--- \\
0.0 \% \\
44.1 \% \\
--- \\
\end{array}$ & \multirow{27}{*}{$\mathrm{n} / \mathrm{a}$} & \multirow{27}{*}{$\mathrm{n} / \mathrm{a}$} & \multirow{27}{*}{$\mathrm{n} / \mathrm{a}$} & \multirow{27}{*}{$\mathrm{n} / \mathrm{a}$} & $\begin{array}{l}-- \\
--- \\
-- \\
--\end{array}$ \\
\hline $\begin{array}{l}\mathrm{Ca}(\mathrm{OH})_{2} \\
\mathrm{Ca}_{5} \mathrm{OH}\left(\mathrm{PO}_{4}\right)_{3} \\
\mathrm{CaC}_{2} \mathrm{O}_{4} \cdot \mathrm{H}_{2} \mathrm{O} \\
\mathrm{CaCO}_{3}\end{array}$ & $\begin{array}{c}2.24 \\
3.14 \\
2.2 \\
2.71 \\
\end{array}$ & $\begin{array}{c}--- \\
1.3 \% \\
--- \\
--- \\
\end{array}$ & $\begin{array}{c}--- \\
0.4 \% \\
--- \\
3.7 \% \\
\end{array}$ & $\begin{array}{c}--- \\
0.9 \% \\
-- \\
--- \\
\end{array}$ & $\begin{array}{c}--- \\
1.8 \% \\
--- \\
--- \\
\end{array}$ & $\begin{array}{c}--- \\
0.6 \% \\
--- \\
--- \\
\end{array}$ & & & & & $\begin{array}{l}--- \\
0.0 \% \\
0.9 \% \\
5.4 \% \\
\end{array}$ \\
\hline $\begin{array}{l}\mathrm{CaF}_{2} \\
\mathrm{CrOOH} \\
\mathrm{FePO}_{4} \cdot 2 \mathrm{H}_{2} \mathrm{O} \\
\mathrm{FeOOH}\end{array}$ & $\begin{array}{l}3.18 \\
4.11 \\
3.15 \\
4.26\end{array}$ & $\begin{array}{c}-- \\
0.6 \% \\
--- \\
31.1 \%\end{array}$ & $\begin{array}{c}0.0 \% \\
-- \\
-- \\
58.2 \%\end{array}$ & $\begin{array}{c}-- \\
1.1 \% \\
--- \\
1.5 \%\end{array}$ & $\begin{array}{c}-- \\
2.5 \% \\
--- \\
2.4 \%\end{array}$ & $\begin{array}{c}4.6 \% \\
1.9 \% \\
--- \\
7.8 \% \\
\end{array}$ & & & & & $\begin{array}{c}0.7 \% \\
--- \\
--- \\
37.5 \%\end{array}$ \\
\hline Gibbsite & 2.42 & $23.9 \%$ & $11.0 \%$ & $73.2 \%$ & $18.5 \%$ & --- & & & & & --- \\
\hline Boehmite & 3.01 & --- & --- & --- & --- & --- & & & & & --- \\
\hline $\mathrm{HgO}$ (co-precip. with $\mathrm{Ag}_{2} \mathrm{O}$ ) & 7.143 & $0.0 \%$ & $0.0 \%$ & --- & --- & --- & & & & & --- \\
\hline $\mathrm{KAISiO}_{4}$ & 2.61 & --- & --- & --- & $2.9 \%$ & $2.7 \%$ & & & & & --- \\
\hline $\mathrm{La}(\mathrm{OH})_{3}$ & 2.3 & -- & -- & -- & -- & -- & & & & & -- \\
\hline $\mathrm{LaPO}_{4} \cdot 2 \mathrm{H}_{2} \mathrm{O}$ & 6.51 & $0.2 \%$ & $0.4 \%$ & $0.0 \%$ & $0.0 \%$ & $11.0 \%$ & & & & & $0.5 \%$ \\
\hline $\mathrm{Mn}_{3}\left(\mathrm{PO}_{4}\right)_{2}$ & 3.102 & --- & -- & -- & -- & $2.2 \%$ & & & & & --- \\
\hline $\mathrm{MnO}_{2}$ & 5.026 & $7.7 \%$ & $1.0 \%$ & $0.4 \%$ & $0.7 \%$ & $14.1 \%$ & & & & & $0.0 \%$ \\
\hline $\mathrm{Na}_{2}\left(\mathrm{UO}_{2}\right)_{2}\left(\mathrm{PO}_{4}\right)_{2} \cdot 2 \mathrm{H}_{2} \mathrm{O}$ & 3.5 & --- & --- & --- & --- & --- & & & & & --- \\
\hline $\mathrm{Na}_{2} \mathrm{U}_{2} \mathrm{O}_{7}$ & 5.617 & $1.0 \%$ & $0.6 \%$ & $15.4 \%$ & $5.7 \%$ & $0.1 \%$ & & & & & $0.0 \%$ \\
\hline $\mathrm{NaAlCO}_{3}(\mathrm{OH})_{2}$ & 2.42 & $25.4 \%$ & $17.3 \%$ & --- & --- & --- & & & & & $2.6 \%$ \\
\hline $\mathrm{NaAlSiO}_{4}$ & 2.365 & $0.2 \%$ & $0.5 \%$ & $1.9 \%$ & --- & --- & & & & & $0.7 \%$ \\
\hline $\mathrm{Ni}(\mathrm{OH})_{2}$ & 4.15 & $1.1 \%$ & $3.1 \%$ & $0.1 \%$ & $0.1 \%$ & $0.3 \%$ & & & & & $4.0 \%$ \\
\hline $\mathrm{Ni}_{3}\left(\mathrm{PO}_{4}\right)_{2}$ & 3.93 & -- & -- & --- & -- & -- & & & & & -- \\
\hline $\mathrm{NiC}_{2} \mathrm{O}_{4} \cdot 2 \mathrm{H}_{2} \mathrm{O}$ (on Fe phase) & 4.26 & -- & $--\cdot$ & $--\cdot$ & -- & $--\cdot$ & & & & & $1.6 \%$ \\
\hline $\mathrm{Pb}(\mathrm{OH})_{2}$ & 7.1 & $1.2 \%$ & $1.5 \%$ & $0.2 \%$ & $0.1 \%$ & $0.1 \%$ & & & & & $18.5 \%$ \\
\hline $\mathrm{Pb}_{3}\left(\mathrm{PO}_{4}\right)_{2}$ & 7.1 & --- & -- & --- & --- & --- & & & & & --- \\
\hline $\mathrm{PbCO}_{3}$ & 6.6 & -- & --- & --- & -- & --- & & & & & $7.2 \%$ \\
\hline $\mathrm{Pu}(\mathrm{OH})_{4}$ (co-precip. on Fe phase) & 4.26 & $0.0 \%$ & $0.0 \%$ & $0.0 \%$ & $0.0 \%$ & $0.0 \%$ & & & & & $0.0 \%$ \\
\hline $\mathrm{PuO}_{2}$ & 11.43 & -- & -- & --- & --- & -- & & & & & --- \\
\hline $\mathrm{SiO}_{2}$ & 2.6 & $-\cdots$ & $-\cdots$ & $-\cdots$ & $-\cdots$ & $-\cdots$ & & & & & $0.1 \%$ \\
\hline $\mathrm{Sr}_{3}\left(\mathrm{PO}_{4}\right)_{2}$ & 3.5 & --- & --- & --- & --- & --- & & & & & --- \\
\hline $\mathrm{SrCO}_{3}$ & 3.5 & $0.1 \%$ & $0.2 \%$ & $0.0 \%$ & $0.0 \%$ & $0.7 \%$ & & & & & $1.0 \%$ \\
\hline $\mathrm{ZrO}_{2}$ & 5.68 & $0.0 \%$ & $0.7 \%$ & $0.2 \%$ & $1.9 \%$ & $0.0 \%$ & & & & & -- \\
\hline \multicolumn{12}{|l|}{ salts: mass fracs in total solid } \\
\hline$\overline{\mathrm{KNO}_{3}}$ & 2.109 & $-\overline{--}$ & $-\overline{--}$ & $\overline{---}$ & $\overline{---}$ & $\overline{---}$ & \multirow{16}{*}{$n / a$} & \multirow{16}{*}{$\mathrm{n} / \mathrm{a}$} & \multirow{16}{*}{$\mathrm{n} / \mathrm{a}$} & \multirow{16}{*}{$n / a$} & $\overline{---}$ \\
\hline $\mathrm{Na}_{2} \mathrm{C}_{2} \mathrm{O}_{4}$ & 2.34 & $6.1 \%$ & -- & $3.3 \%$ & $7.9 \%$ & $1.0 \%$ & & & & & $5.6 \%$ \\
\hline $\mathrm{Na}_{2} \mathrm{CO}_{3} \cdot \mathrm{H}_{2} \mathrm{O}$ & 2.25 & $0.1 \%$ & $1.4 \%$ & $0.0 \%$ & $0.0 \%$ & $8.8 \%$ & & & & & $9.5 \%$ \\
\hline $\mathrm{Na}_{2} \mathrm{SO}_{4}$ & 2.68 & --- & -- & -- & -- & -- & & & & & --- \\
\hline $\mathrm{Na}_{2} \mathrm{SO}_{4} \cdot 10 \mathrm{H}_{2} \mathrm{O}$ & 1.464 & 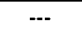 & $--\cdot$ & $-\cdots$ & --- & --- & & & & & --- \\
\hline $\mathrm{Na}_{3} \mathrm{FSO}_{4}$ & 2.65 & --- & --- & --- & $18.9 \%$ & --- & & & & & --- \\
\hline $\mathrm{Na}_{3} \mathrm{NO}_{3} \mathrm{SO}_{4} \cdot \mathrm{H}_{2} \mathrm{O}$ & 2.3 & --- & -- & --- & --- & --- & & & & & --- \\
\hline $\mathrm{Na}_{3} \mathrm{PO}_{4} \cdot 0,25 \mathrm{NaOH} \cdot 12 \mathrm{H}_{2} \mathrm{O}$ & 1.62 &.-- & $\ldots$ & $\cdots$ &.- &.- & & & & & -- \\
\hline $\mathrm{Na}_{3} \mathrm{PO}_{4} \cdot 8 \mathrm{H}_{2} \mathrm{O}$ & 1.8 & --- & --- & --- & $\begin{array}{c}-- \\
-1\end{array}$ & $\begin{array}{c}-- \\
\end{array}$ & & & & & $-\cdots$ \\
\hline $\mathrm{Na}_{4} \mathrm{P}_{2} \mathrm{O}_{7} \bullet 10 \mathrm{H}_{2} \mathrm{O}$ & 1.83 & --- & -- & --- & -- & --- & & & & & --- \\
\hline $\mathrm{Na}_{6}\left(\mathrm{SO}_{4}\right)_{2} \mathrm{CO}_{3}$ & 2.64 & $\cdots$ & -- & $\cdots$ & -- & -- & & & & & -- \\
\hline $\mathrm{NaF}$ & 2.78 & --- & --- & --- & $35.5 \%$ & --- & & & & & --- \\
\hline $\mathrm{Na}_{7} \mathrm{~F}\left(\mathrm{PO}_{4}\right)_{2} \cdot 19 \mathrm{H}_{2} \mathrm{O}$ & 1.75 & --- & -- & $1.6 \%$ & $1.1 \%$ & --- & & & & & --- \\
\hline $\mathrm{NaHCO}_{3}$ & 2.159 & --- & -- & --- & -- & --- & & & & & --- \\
\hline $\mathrm{NaNO}_{2}$ & 2.168 & $--\cdot$ & $--\cdot$ & $\cdots$ & -- & -- & & & & & --- \\
\hline $\mathrm{NaNO}_{3}$ & 2.26 & --- & --- & --- & -- & --- & & & & & $4.1 \%$ \\
\hline
\end{tabular}


Table C.2. (contd)

\begin{tabular}{|c|c|c|c|c|c|c|c|c|c|c|c|}
\hline & $\begin{array}{l}\text { density } \\
(\mathrm{g} / \mathrm{mL})\end{array}$ & $\begin{array}{c}\text { Sludge } \\
\text { Portland Cement } \\
\end{array}$ & $\begin{array}{c}\text { Sludge } \\
\text { B }\end{array}$ & \begin{tabular}{|c|} 
Sludge \\
P1
\end{tabular} & \begin{tabular}{|c|} 
Saltcake \\
SI salt \\
\end{tabular} & $\begin{array}{c}\text { Saltcake } \\
\text { T2 salt } \\
\end{array}$ & \begin{tabular}{|c|} 
Saltcake \\
BY salt \\
\end{tabular} & $\begin{array}{c}\text { Saltcake } \\
\text { A2 salt } \\
\end{array}$ & \begin{tabular}{|l|} 
Saltcake \\
S2 salt \\
\end{tabular} & $\begin{array}{c}\text { Saltcake } \\
\text { Al salt } \\
\end{array}$ & $\begin{array}{c}\text { Saltcake } \\
\text { R salt } \\
\end{array}$ \\
\hline $\begin{array}{l}\text { Avg g/mL, non-salt solid } \\
\text { Mass frac non-salts in solid } \\
\text { vol. frac. non-salts in solid } \\
\text { Avg } g / \mathrm{mL} \text {, salt solid }\end{array}$ & $\mathrm{n} / \mathrm{a}$ & $1 / \mathbf{a}$ & $\mathrm{n} / \mathrm{a}$ & $\mathrm{n} / \mathrm{a}$ & $\begin{array}{l}2.605 \\
0.113 \\
0.094 \\
2.135\end{array}$ & $\begin{array}{l}2.745 \\
0.045 \\
0.038 \\
2.286\end{array}$ & $\begin{array}{l}2.604 \\
0.163 \\
0.143 \\
2.224\end{array}$ & $\begin{array}{l}2.780 \\
0.035 \\
0.028 \\
2.241\end{array}$ & $\begin{array}{l}2.564 \\
0.377 \\
0.338 \\
2.165\end{array}$ & $\begin{array}{l}2.667 \\
0.090 \\
0.079 \\
2.297\end{array}$ & $\begin{array}{l}3.043 \\
0.373 \\
0.306 \\
2.263\end{array}$ \\
\hline \multicolumn{12}{|l|}{ mass fracs in total solid } \\
\hline $\begin{array}{l}\mathrm{Ag} \\
\mathrm{Ag}_{2} \mathrm{O} \\
\mathrm{Bi}_{2} \mathrm{O}_{3} \\
\mathrm{BiFeO}_{3} \\
\end{array}$ & $\begin{array}{c}10.5 \\
7.143 \\
8.9 \\
7.9 \\
\end{array}$ & \multirow{9}{*}{$\mathrm{n} / \mathrm{a}$} & \multirow{9}{*}{$n / a$} & \multirow{9}{*}{$\mathrm{n} / \mathrm{a}$} & $\begin{array}{c}-- \\
--- \\
0.0 \% \\
--- \\
\end{array}$ & $\begin{array}{c}--- \\
--- \\
0.4 \% \\
--- \\
\end{array}$ & $\begin{array}{c}--- \\
--- \\
0.0 \% \\
--- \\
\end{array}$ & $\begin{array}{c}--- \\
--- \\
0.0 \% \\
--- \\
\end{array}$ & $\begin{array}{c}--- \\
--- \\
0.0 \% \\
--- \\
\end{array}$ & $\begin{array}{c}--- \\
--- \\
0.0 \% \\
--- \\
\end{array}$ & $\begin{array}{c}--- \\
--- \\
0.0 \% \\
--- \\
\end{array}$ \\
\hline $\begin{array}{l}\mathrm{Ca}(\mathrm{OH})_{2} \\
\mathrm{Ca}_{5} \mathrm{OH}\left(\mathrm{PO}_{4}\right)_{3} \\
\mathrm{CaC}_{2} \mathrm{O}_{4} \cdot \mathrm{H}_{2} \mathrm{O} \\
\mathrm{CaCO}_{3}\end{array}$ & $\begin{array}{c}2.24 \\
3.14 \\
2.2 \\
2.71 \\
\end{array}$ & & & & $\begin{array}{c}--- \\
0.1 \% \\
--- \\
--- \\
\end{array}$ & $\begin{array}{c}--- \\
0.1 \% \\
--- \\
--- \\
\end{array}$ & $\begin{array}{c}--- \\
0.2 \% \\
--- \\
--- \\
\end{array}$ & $\begin{array}{c}--- \\
0.2 \% \\
--- \\
--- \\
\end{array}$ & $\begin{array}{c}--- \\
0.4 \% \\
--- \\
--- \\
\end{array}$ & $\begin{array}{c}--- \\
0.2 \% \\
--- \\
--- \\
\end{array}$ & $\begin{array}{c}--- \\
0.2 \% \\
--- \\
--- \\
\end{array}$ \\
\hline $\begin{array}{l}\mathrm{CaF}_{2} \\
\mathrm{CrOOH} \\
\mathrm{FePO} \cdot 2 \mathrm{H}_{2} \mathrm{O} \\
\mathrm{FeOOH}\end{array}$ & $\begin{array}{l}3.18 \\
4.11 \\
3.15 \\
4.26 \\
\end{array}$ & & & & $\begin{array}{c}--- \\
0.9 \% \\
--- \\
0.5 \%\end{array}$ & $\begin{array}{c}--\cdot \\
0.0 \% \\
--- \\
0.4 \%\end{array}$ & $\begin{array}{c}0.1 \% \\
1.7 \% \\
1.8 \% \\
--- \\
\end{array}$ & $\begin{array}{c}--- \\
0.6 \% \\
--- \\
0.1 \%\end{array}$ & $\begin{array}{c}--- \\
3.1 \% \\
--- \\
1.1 \% \\
\end{array}$ & $\begin{array}{c}--- \\
1.1 \% \\
--- \\
0.2 \% \\
\end{array}$ & $\begin{array}{c}-\cdots \\
--- \\
--- \\
0.5 \%\end{array}$ \\
\hline $\begin{array}{l}\text { Gibbsite } \\
\text { Boehmite } \\
\mathrm{HgO} \text { (co-precip. with } \mathrm{Ag}_{2} \mathrm{O} \text { ) } \\
\mathrm{KAISiO}_{+}\end{array}$ & $\begin{array}{l}2.42 \\
3.01 \\
7.143 \\
2.61 \\
\end{array}$ & & & & $\begin{array}{c}8.9 \% \\
--- \\
--- \\
--- \\
\end{array}$ & $\begin{array}{l}-- \\
--- \\
--- \\
--- \\
\end{array}$ & $\begin{array}{c}2.9 \% \\
--- \\
--- \\
0.4 \% \\
\end{array}$ & $\begin{array}{c}1.9 \% \\
--- \\
--- \\
0.4 \%\end{array}$ & $\begin{array}{c}30.8 \% \\
--- \\
--- \\
--- \\
\end{array}$ & $\begin{array}{c}6.1 \% \\
--- \\
--- \\
--- \\
\end{array}$ & $\begin{array}{c}-- \\
33.9 \% \\
--- \\
--- \\
\end{array}$ \\
\hline $\begin{array}{l}\mathrm{La}(\mathrm{OH})_{3} \\
\mathrm{LaPO} \cdot 2 \mathrm{H}_{2} \mathrm{O} \\
\mathrm{Mn}_{3}\left(\mathrm{PO}_{4}\right)_{2} \\
\mathrm{MnO}_{2}\end{array}$ & $\begin{array}{c}2.3 \\
6.51 \\
3.102 \\
5.026 \\
\end{array}$ & & & & $\begin{array}{c}-- \\
0.0 \% \\
--- \\
0.1 \% \\
\end{array}$ & $\begin{array}{c}-- \\
0.0 \% \\
--- \\
0.0 \% \\
\end{array}$ & $\begin{array}{c}0.0 \% \\
0.0 \% \\
--- \\
--- \\
\end{array}$ & $\begin{array}{c}0.0 \% \\
0.0 \% \\
--- \\
--- \\
\end{array}$ & $\begin{array}{c}-- \\
0.0 \% \\
--- \\
--- \\
\end{array}$ & $\begin{array}{c}-- \\
0.0 \% \\
--- \\
--- \\
\end{array}$ & $\begin{array}{c}-- \\
0.0 \% \\
--- \\
0.3 \% \\
\end{array}$ \\
\hline $\begin{array}{l}\mathrm{Na}_{2}\left(\mathrm{UO}_{2}\right)_{2}\left(\mathrm{PO}_{4}\right)_{2} \cdot 2 \mathrm{H}_{2} \mathrm{O} \\
\mathrm{Na}_{2} \mathrm{U}_{2} \mathrm{O}_{7} \\
\mathrm{NaAlCO}_{3}(\mathrm{OH})_{2} \\
\mathrm{NaAlSiO}_{4}\end{array}$ & $\begin{array}{c}3.5 \\
5.617 \\
2.42 \\
2.365 \\
\end{array}$ & & & & $\begin{array}{c}-- \\
0.1 \% \\
0.2 \% \\
0.3 \% \\
\end{array}$ & $\begin{array}{c}-- \\
--- \\
3.5 \% \\
--- \\
\end{array}$ & $\begin{array}{c}-- \\
0.2 \% \\
--- \\
8.9 \% \\
\end{array}$ & $\begin{array}{c}-- \\
0.1 \% \\
--- \\
0.2 \% \\
\end{array}$ & $\begin{array}{c}-- \\
0.3 \% \\
--- \\
1.7 \% \\
\end{array}$ & $\begin{array}{c}-- \\
0.3 \% \\
--- \\
0.9 \% \\
\end{array}$ & $\begin{array}{c}-- \\
0.9 \% \\
--- \\
1.3 \% \\
\end{array}$ \\
\hline $\begin{array}{l}\mathrm{Ni}(\mathrm{OH})_{2} \\
\mathrm{Ni}_{3}\left(\mathrm{PO}_{4}\right)_{2} \\
\mathrm{NiC} \mathrm{O}_{4} \cdot 2 \mathrm{H}_{2} \mathrm{O} \text { (on Fe phase) } \\
\mathrm{Pb}(\mathrm{OH})_{2}\end{array}$ & $\begin{array}{c}4.15 \\
3.93 \\
4.26 \\
7.1 \\
\end{array}$ & & & & $\begin{array}{c}0.0 \% \\
--- \\
\cdots- \\
0.0 \%\end{array}$ & $\begin{array}{c}0.0 \% \\
--- \\
--- \\
---\end{array}$ & $\begin{array}{c}0.0 \% \\
--- \\
--- \\
0.0 \% \\
\end{array}$ & $\begin{array}{c}0.0 \% \\
--- \\
\cdots \\
0.0 \% \\
\end{array}$ & $\begin{array}{c}0.1 \% \\
--- \\
--- \\
0.1 \% \\
\end{array}$ & $\begin{array}{c}0.0 \% \\
--- \\
\cdots \\
0.1 \% \\
\end{array}$ & $\begin{array}{c}0.1 \% \\
--- \\
--- \\
0.0 \%\end{array}$ \\
\hline $\begin{array}{l}\mathrm{Pb}_{3}\left(\mathrm{PO}_{4}\right)_{2} \\
\mathrm{PbCO}_{3} \\
\mathrm{Pu}(\mathrm{OH})_{4} \text { (co-precip. on Fe phase) } \\
\mathrm{PuO}_{2}\end{array}$ & $\begin{array}{r}7.1 \\
6.6 \\
4.26 \\
11.43\end{array}$ & & & & $\begin{array}{c}--- \\
--- \\
0.0 \% \\
---\end{array}$ & $\begin{array}{c}0.0 \% \\
--- \\
--- \\
0.0 \%\end{array}$ & $\begin{array}{c}--- \\
--- \\
0.0 \% \\
---\end{array}$ & $\begin{array}{c}--- \\
--- \\
0.0 \% \\
---\end{array}$ & $\begin{array}{c}--- \\
--- \\
0.0 \% \\
---\end{array}$ & $\begin{array}{c}--- \\
--- \\
0.0 \% \\
---\end{array}$ & $\begin{array}{c}--- \\
--- \\
0.0 \% \\
---\end{array}$ \\
\hline $\begin{array}{l}\mathrm{SiO}_{2} \\
\mathrm{Sr}_{3}\left(\mathrm{PO}_{4}\right)_{2} \\
\mathrm{SrCO}_{3} \\
\mathrm{ZrO}_{2}\end{array}$ & $\begin{array}{l}2.6 \\
3.5 \\
3.5 \\
5.68 \\
\end{array}$ & & & & \begin{tabular}{c|}
--- \\
--- \\
$0.0 \%$ \\
$0.0 \%$ \\
\end{tabular} & $\begin{array}{c}-- \\
--- \\
0.0 \% \\
0.0 \%\end{array}$ & $\begin{array}{c}-- \\
--- \\
0.0 \% \\
0.0 \%\end{array}$ & $\begin{array}{c}-- \\
--- \\
0.0 \% \\
0.0 \% \\
\end{array}$ & $\begin{array}{c}-- \\
--- \\
0.0 \% \\
0.0 \%\end{array}$ & $\begin{array}{c}--- \\
--- \\
0.0 \% \\
0.0 \% \\
\end{array}$ & $\begin{array}{c}-- \\
--- \\
0.1 \% \\
0.0 \%\end{array}$ \\
\hline \multicolumn{12}{|l|}{ salts: mass fracs in total solid } \\
\hline $\begin{array}{l}\mathrm{KNO}_{3} \\
\mathrm{Na}_{2} \mathrm{C}_{2} \mathrm{O}_{4} \\
\mathrm{Na}_{2} \mathrm{CO}_{3} \cdot \mathrm{H}_{2} \mathrm{O} \\
\mathrm{Na}_{2} \mathrm{SO}_{4}\end{array}$ & $\begin{array}{l}2.109 \\
2.34 \\
2.25 \\
2.68 \\
\end{array}$ & \multirow{4}{*}{$1 / \mathrm{a}$} & \multirow{4}{*}{$\mathrm{n} / \mathrm{a}$} & \multirow{4}{*}{$17 / \mathrm{a}$} & $\begin{array}{l}-- \\
1.1 \% \\
6.1 \% \\
--- \\
\end{array}$ & $\begin{array}{c}-- \\
--- \\
0.0 \% \\
--- \\
\end{array}$ & $\begin{array}{c}-- \\
5.6 \% \\
37.1 \% \\
--- \\
\end{array}$ & $\begin{array}{c}2.4 \% \\
3.7 \% \\
20.4 \% \\
--- \\
\end{array}$ & $\begin{array}{c}-- \\
9.7 \% \\
3.6 \% \\
--- \\
\end{array}$ & $\begin{array}{c}--- \\
4.9 \% \\
34.4 \% \\
--- \\
\end{array}$ & $\begin{array}{c}-- \\
0.6 \% \\
0.1 \% \\
--- \\
\end{array}$ \\
\hline $\begin{array}{l}\mathrm{Na}_{2} \mathrm{SO}_{4} \cdot 10 \mathrm{H}_{2} \mathrm{O} \\
\mathrm{Na}_{3} \mathrm{FSO}_{4} \\
\mathrm{Na}_{3} \mathrm{NO}_{3} \mathrm{SO}_{4} \cdot \mathrm{H}_{2} \mathrm{O} \\
\mathrm{Na}_{3} \mathrm{PO}_{4} \cdot 0.25 \mathrm{NaOH} \cdot 12 \mathrm{H}_{2} \mathrm{O}\end{array}$ & $\begin{array}{c}1.464 \\
2.65 \\
2.3 \\
1.62 \\
\end{array}$ & & & & $\begin{array}{c}-- \\
3.0 \% \\
--- \\
10.7 \% \\
\end{array}$ & $\begin{array}{c}-- \\
7.7 \% \\
9.8 \% \\
--- \\
\end{array}$ & $\begin{array}{c}-- \\
7.7 \% \\
--- \\
-- \\
\end{array}$ & $\begin{array}{c}-- \\
1.8 \% \\
--- \\
--- \\
\end{array}$ & $\begin{array}{l}-- \\
-- \\
-- \\
---\end{array}$ & $\begin{array}{c}--- \\
0.3 \% \\
--- \\
--- \\
\end{array}$ & $\begin{array}{c}-- \\
0.4 \% \\
--- \\
-- \\
\end{array}$ \\
\hline $\begin{array}{l}\mathrm{Na}_{3} \mathrm{PO}_{4} \cdot 8 \mathrm{H}_{2} \mathrm{O} \\
\mathrm{Na}_{4} \mathrm{P}_{2} \mathrm{O}_{7} \cdot 10 \mathrm{H}_{2} \mathrm{O} \\
\mathrm{Na}_{6}\left(\mathrm{SO}_{4}\right)_{2} \mathrm{CO}_{3} \\
\mathrm{NaF}\end{array}$ & $\begin{array}{l}1.8 \\
1.83 \\
2.64 \\
2.78\end{array}$ & & & & $\begin{array}{l}-- \\
-- \\
-- \\
---\end{array}$ & $\begin{array}{l}--- \\
-- \\
-- \\
---\end{array}$ & $\begin{array}{c}--- \\
--- \\
--- \\
2.2 \%\end{array}$ & $\begin{array}{c}--- \\
--- \\
1.6 \% \\
---\end{array}$ & $\begin{array}{c}-- \\
--- \\
3.1 \% \\
---\end{array}$ & $\begin{array}{c}--- \\
--- \\
9.8 \% \\
---\end{array}$ & $\begin{array}{l}--- \\
--- \\
-- \\
---\end{array}$ \\
\hline $\begin{array}{l}\mathrm{Na}_{7} \mathrm{~F}\left(\mathrm{PO}_{4}\right)_{2} \cdot 19 \mathrm{H}_{2} \mathrm{O} \\
\mathrm{NaHCO}_{3} \\
\mathrm{NaNO}_{2} \\
\mathrm{NaNO}_{3}\end{array}$ & $\begin{array}{c}1.75 \\
2.159 \\
2.168 \\
2.26\end{array}$ & & & & $\begin{array}{c}4.9 \% \\
--- \\
--- \\
62.9 \%\end{array}$ & $\begin{array}{c}--- \\
4.7 \% \\
--- \\
73.2 \%\end{array}$ & $\begin{array}{c}10.0 \% \\
--- \\
\ldots \\
21.0 \%\end{array}$ & $\begin{array}{c}2.5 \% \\
--- \\
11.2 \% \\
52.8 \%\end{array}$ & $\begin{array}{c}12.1 \% \\
--- \\
--- \\
33.8 \%\end{array}$ & $\begin{array}{c}--- \\
--- \\
--- \\
41.6 \%\end{array}$ & $\begin{array}{c}--- \\
--- \\
--- \\
61.6 \%\end{array}$ \\
\hline
\end{tabular}


Table C.2. (contd)

\begin{tabular}{|c|c|c|c|c|}
\hline & $\begin{array}{l}\text { density } \\
\text { (g/mL) }\end{array}$ & \begin{tabular}{|c|} 
Saltcake \\
T1 salt
\end{tabular} & \begin{tabular}{|c|} 
Saltcake \\
B salt
\end{tabular} & $\begin{array}{c}\text { Saltcake } \\
\text { R1 salt }\end{array}$ \\
\hline $\begin{array}{l}\text { Avg } g / m L, \text { non-salt solid } \\
\text { Mass frac non-salts in solid } \\
\text { Vol. frac. non-salts in solid } \\
\text { Avg g/mL, salt solid }\end{array}$ & $\mathrm{n} / \mathrm{a}$ & $\begin{array}{l}3.030 \\
0.041 \\
0.025 \\
1.802\end{array}$ & $\begin{array}{l}2.493 \\
0.213 \\
0.157 \\
1.718\end{array}$ & $\begin{array}{l}3.116 \\
0.533 \\
0.454 \\
2.270\end{array}$ \\
\hline \multicolumn{5}{|l|}{ mass fracs in total solid } \\
\hline $\begin{array}{l}\mathrm{Ag} \\
\mathrm{Ag}_{2} \mathrm{O} \\
\mathrm{Bi}_{2} \mathrm{O}_{3} \\
\mathrm{BiFeO}_{3} \\
\end{array}$ & $\begin{array}{c}10.5 \\
7.143 \\
8.9 \\
7.9 \\
\end{array}$ & $\begin{array}{c}--- \\
-- \\
0.0 \% \\
--- \\
\end{array}$ & $\begin{array}{c}--- \\
-- \\
0.2 \% \\
--- \\
\end{array}$ & $\begin{array}{c}--- \\
--- \\
0.0 \% \\
--- \\
\end{array}$ \\
\hline $\begin{array}{l}\mathrm{Ca}(\mathrm{OH})_{2} \\
\mathrm{Ca}_{3} \mathrm{OH}\left(\mathrm{PO}_{4}\right)_{3} \\
\mathrm{CaC}_{2} \mathrm{O}_{4} \cdot \mathrm{H}_{2} \mathrm{O} \\
\mathrm{CaCO}_{3} \\
\end{array}$ & $\begin{array}{c}2.24 \\
3.14 \\
2.2 \\
2.71 \\
\end{array}$ & $\begin{array}{l}-- \\
--- \\
--- \\
--- \\
\end{array}$ & $\begin{array}{c}-- \\
0.4 \% \\
-- \\
-- \\
\end{array}$ & $\begin{array}{c}--- \\
0.3 \% \\
--- \\
--- \\
\end{array}$ \\
\hline \begin{tabular}{|l|}
$\mathrm{CaF}_{2}$ \\
$\mathrm{CrOOH}$ \\
$\mathrm{FePO} \cdot 2 \mathrm{H}_{2} \mathrm{O}$ \\
$\mathrm{FeOOH}$ \\
\end{tabular} & $\begin{array}{l}3.18 \\
4.11 \\
3.15 \\
4.26\end{array}$ & $\begin{array}{c}0.1 \% \\
0.0 \% \\
2.7 \% \\
---\end{array}$ & $\begin{array}{c}-- \\
0.0 \% \\
--- \\
0.2 \%\end{array}$ & $\begin{array}{c}-- \\
3.5 \% \\
--- \\
0.5 \%\end{array}$ \\
\hline $\begin{array}{l}\text { Gibbsite } \\
\text { Boehmite } \\
\mathrm{HgO} \text { (co-precip. with } \mathrm{Ag}_{2} \mathrm{O} \text { ) } \\
\mathrm{KAlSiO}_{4}\end{array}$ & $\begin{array}{c}2.42 \\
3.01 \\
7.143 \\
2.61 \\
\end{array}$ & $\begin{array}{l}--- \\
--- \\
--\end{array}$ & $\begin{array}{c}19.4 \% \\
-- \\
-- \\
-- \\
\end{array}$ & $\begin{array}{c}-\cdots \\
45.0 \% \\
--- \\
--- \\
\end{array}$ \\
\hline $\begin{array}{l}\mathrm{La}(\mathrm{OH})_{3} \\
\mathrm{LaPO} \cdot 2 \mathrm{H}_{2} \mathrm{O} \\
\mathrm{Mn}_{3}\left(\mathrm{PO}_{4}\right)_{2} \\
\mathrm{MnO}_{2} \\
\end{array}$ & $\begin{array}{c}2.3 \\
6.51 \\
3.102 \\
5.026 \\
\end{array}$ & $\begin{array}{c}--- \\
0.0 \% \\
-- \\
0.2 \% \\
\end{array}$ & $\begin{array}{c}\cdots \\
0.0 \% \\
\ldots .- \\
0.0 \% \\
\end{array}$ & $\begin{array}{c}-- \\
0.0 \% \\
\cdots \\
0.5 \% \\
\end{array}$ \\
\hline $\begin{array}{l}\mathrm{Na}_{2}\left(\mathrm{UO}_{2}\right)_{2}\left(\mathrm{PO}_{4}\right)_{2} \cdot 2 \mathrm{H}_{2} \mathrm{O} \\
\mathrm{Na}_{2} \mathrm{U}_{2} \mathrm{O}_{7} \\
\mathrm{NaAlCO}_{3}(\mathrm{OH})_{2} \\
\mathrm{NaAlSiO}_{4}\end{array}$ & $\begin{array}{c}3.5 \\
5.617 \\
2.42 \\
2.365 \\
\end{array}$ & $\begin{array}{c}-- \\
--- \\
0.9 \% \\
-\cdots \\
\end{array}$ & $\begin{array}{c}-- \\
0.3 \% \\
--- \\
0.5 \% \\
\end{array}$ & $\begin{array}{c}-- \\
1.8 \% \\
--- \\
1.5 \% \\
\end{array}$ \\
\hline $\begin{array}{l}\mathrm{Ni}(\mathrm{OH})_{2} \\
\mathrm{Ni}_{3}\left(\mathrm{PO}_{4}\right)_{2} \\
\mathrm{NiC}_{2} \mathrm{O}_{4} \cdot 2 \mathrm{H}_{2} \mathrm{O} \text { (on Fe phase) } \\
\mathrm{Pb}(\mathrm{OH})_{2}\end{array}$ & $\begin{array}{c}4.15 \\
3.93 \\
4.26 \\
7.1 \\
\end{array}$ & $\begin{array}{c}0.0 \% \\
-- \\
-- \\
0.0 \% \\
\end{array}$ & $\begin{array}{c}0.1 \% \\
-- \\
-- \\
0.1 \% \\
\end{array}$ & $\begin{array}{c}0.0 \% \\
--- \\
--- \\
0.0 \% \\
\end{array}$ \\
\hline $\begin{array}{l}\mathrm{Pb}_{3}\left(\mathrm{PO}_{4}\right)_{2} \\
\mathrm{PbCO}_{3} \\
\mathrm{Pu}(\mathrm{OH})_{4} \text { (co-precip. on Fe phase) } \\
\mathrm{PuO}_{2}\end{array}$ & $\begin{array}{c}7.1 \\
6.6 \\
4.26 \\
11.43 \\
\end{array}$ & $\begin{array}{c}-- \\
--- \\
0.0 \% \\
--- \\
\end{array}$ & $\begin{array}{c}-- \\
-- \\
0.0 \% \\
-- \\
\end{array}$ & $\begin{array}{c}-- \\
--- \\
0.0 \% \\
--- \\
\end{array}$ \\
\hline $\begin{array}{l}\mathrm{SiO}_{2} \\
\mathrm{Sr}_{3}\left(\mathrm{PO}_{4}\right)_{2} \\
\mathrm{SrCO}_{3} \\
\mathrm{ZrO}_{2}\end{array}$ & $\begin{array}{l}2.6 \\
3.5 \\
3.5 \\
5.68\end{array}$ & $\begin{array}{c}--- \\
--- \\
0.0 \% \\
0.0 \%\end{array}$ & $\begin{array}{c}--- \\
-- \\
0.0 \% \\
0.0 \%\end{array}$ & $\begin{array}{c}--- \\
--- \\
0.1 \% \\
0.0 \%\end{array}$ \\
\hline \multicolumn{5}{|l|}{ salts: mass fracs in total solid } \\
\hline $\begin{array}{l}\mathrm{KNO}_{3} \\
\mathrm{Na}_{2} \mathrm{C}_{2} \mathrm{O}_{4} \\
\mathrm{Na}_{2} \mathrm{CO}_{3} \cdot \mathrm{H}_{2} \mathrm{O} \\
\mathrm{Na}_{2} \mathrm{SO}_{4} \\
\end{array}$ & $\begin{array}{l}2.109 \\
2.34 \\
2.25 \\
2.68 \\
\end{array}$ & $\begin{array}{c}-- \\
--- \\
0.3 \% \\
--- \\
\end{array}$ & $\begin{array}{c}-- \\
0.5 \% \\
-- \\
-- \\
\end{array}$ & $\begin{array}{c}-- \\
3.0 \% \\
1.4 \% \\
--- \\
\end{array}$ \\
\hline $\begin{array}{l}\mathrm{Na}_{2} \mathrm{SO}_{4} \cdot 10 \mathrm{H}_{2} \mathrm{O} \\
\mathrm{Na}_{3} \mathrm{FSO}_{4} \\
\mathrm{Na}_{3} \mathrm{NO}_{3} \mathrm{SO}_{4} \cdot \mathrm{H}_{2} \mathrm{O} \\
\mathrm{Na}_{3} \mathrm{PO}_{4} \cdot 0.25 \mathrm{NaOH} \cdot 12 \mathrm{H}_{2} \mathrm{O}\end{array}$ & $\begin{array}{l}1.464 \\
2.65 \\
2.3 \\
1.62\end{array}$ & $\begin{array}{l}--- \\
-- \\
-- \\
--\end{array}$ & $\begin{array}{c}26.9 \% \\
-- \\
-- \\
--\end{array}$ & $\begin{array}{l}--- \\
--- \\
---\end{array}$ \\
\hline $\begin{array}{l}\mathrm{Na}_{3} \mathrm{PO}_{4} \cdot 8 \mathrm{H}_{2} \mathrm{O} \\
\mathrm{Na}_{4} \mathrm{P}_{2} \mathrm{O}_{7} \cdot 10 \mathrm{H}_{2} \mathrm{O} \\
\mathrm{Na}_{6}\left(\mathrm{SO}_{4}\right)_{2} \mathrm{CO}_{3} \\
\mathrm{NaF}\end{array}$ & $\begin{array}{l}1.8 \\
1.83 \\
2.64 \\
2.78\end{array}$ & $\begin{array}{c}-- \\
45.1 \% \\
--- \\
2.0 \%\end{array}$ & $\begin{array}{c}-- \\
--- \\
-- \\
5.6 \%\end{array}$ & $\begin{array}{c}-- \\
--- \\
3.2 \% \\
--- \\
\end{array}$ \\
\hline $\begin{array}{l}\mathrm{Na}_{7} \mathrm{~F}\left(\mathrm{PO}_{4}\right)_{2} \cdot 19 \mathrm{H}_{2} \mathrm{O} \\
\mathrm{NaHCO}_{3} \\
\mathrm{NaNO}_{2} \\
\mathrm{NaNO}_{3}\end{array}$ & $\begin{array}{c}1.75 \\
2.159 \\
2.168 \\
2.26\end{array}$ & $\begin{array}{c}48.6 \% \\
-- \\
-- \\
---\end{array}$ & $\begin{array}{c}38.8 \% \\
-- \\
\cdots \\
7.0 \%\end{array}$ & $\begin{array}{c}1.2 \% \\
--- \\
--- \\
37.8 \%\end{array}$ \\
\hline
\end{tabular}


Table C.3. Fully-Washed ${ }^{(a)}$ Solid-Phase Compositions of Individual Tanks Based on 2002 BBI ("---" = not present)

\begin{tabular}{|c|c|c|c|c|c|c|c|c|c|}
\hline & Density $(\mathrm{g} / \mathrm{mL})$ & $\begin{array}{l}\text { Mol. wt. } \\
\text { (g/mol) }\end{array}$ & 241-A-101 & 241-A-102 & 241-A-103 & 241-A-104 & 241-A-105 & 241-A-106 & 241-AN-101 \\
\hline $\begin{array}{l}\text { Total } \mathrm{kg} \text { washed solids for tank } \\
\text { Total } \mathrm{kL} \text { washed solids for tank } \\
\text { Avg g/mL, washed solid }\end{array}$ & $\mathrm{n} / \mathrm{a}$ & $\mathrm{n} / \mathrm{a}$ & $\begin{array}{c}5.84 \mathrm{E}+04 \\
1.99 \mathrm{E}+01 \\
2.933\end{array}$ & $\begin{array}{c}3.45 \mathrm{E}+04 \\
1.09 \mathrm{E}+01 \\
3.173\end{array}$ & $\begin{array}{c}1.14 \mathrm{E}+05 \\
4.60 \mathrm{E}+01 \\
2.477\end{array}$ & $\begin{array}{c}7.94 \mathrm{E}+04 \\
2.21 \mathrm{E}+01 \\
3.586\end{array}$ & $\begin{array}{c}6.46 \mathrm{E}+04 \\
2.00 \mathrm{E}+01 \\
3.223\end{array}$ & $\begin{array}{c}1.63 \mathrm{E}+05 \\
5.99 \mathrm{E}+01 \\
2.722\end{array}$ & $\begin{array}{c}2.28 \mathrm{E}+04 \\
9.40 \mathrm{E}+00 \\
2.427\end{array}$ \\
\hline \multicolumn{10}{|l|}{ mass fracs of solids in washed solid } \\
\hline $\begin{array}{l}\mathrm{Ag}_{2} \mathrm{O} \\
\mathrm{Bi}_{2} \mathrm{O}_{3} \\
\mathrm{BiFeO}_{3}\end{array}$ & $\begin{array}{c}7.143 \\
8.9 \\
7.9\end{array}$ & $\begin{array}{l}231.74 \\
465.96 \\
312.83\end{array}$ & $\begin{array}{c}--- \\
0.60 \% \\
---\end{array}$ & $\begin{array}{c}0.02 \% \\
0.18 \% \\
---\end{array}$ & $\begin{array}{c}--- \\
0.13 \% \\
---\end{array}$ & $\begin{array}{l}--- \\
--- \\
---\end{array}$ & $\begin{array}{l}-- \\
-- \\
--\end{array}$ & $\begin{array}{c}--- \\
0.11 \% \\
---\end{array}$ & $\begin{array}{c}0.03 \% \\
0.12 \% \\
---\end{array}$ \\
\hline $\begin{array}{l}\mathrm{Ca}(\mathrm{OH})_{2} \\
\mathrm{CrOOH} \\
\mathrm{FeOOH}\end{array}$ & $\begin{array}{l}2.24 \\
4.11 \\
4.26\end{array}$ & $\begin{array}{c}74.09 \\
85 \\
88.85\end{array}$ & $\begin{array}{c}3.72 \% \\
24.81 \% \\
4.62 \%\end{array}$ & $\begin{array}{c}0.60 \% \\
6.78 \% \\
14.68 \%\end{array}$ & $\begin{array}{l}.79 \% \\
3.33 \% \\
2.32 \%\end{array}$ & $\begin{array}{c}4.38 \% \\
-- \\
63.89 \%\end{array}$ & $\begin{array}{c}5.32 \% \\
-- \\
45.11 \%\end{array}$ & $\begin{array}{l}5.30 \% \\
2.25 \% \\
22.87 \%\end{array}$ & $\begin{array}{c}0.11 \% \\
--- \\
0.05 \%\end{array}$ \\
\hline $\begin{array}{l}\text { Gibbsite } \\
\text { Boehmite } \\
\mathrm{HgO} \text { (co-precip. with } \mathrm{Ag}_{2} \mathrm{O} \text { ) }\end{array}$ & $\begin{array}{c}2.42 \\
3.01 \\
7.143\end{array}$ & $\begin{array}{c}78 \\
59.99 \\
216.59\end{array}$ & $\begin{array}{c}32.83 \% \\
--- \\
---\end{array}$ & $\begin{array}{c}42.69 \% \\
--- \\
---\end{array}$ & $\begin{array}{c}3.71 \% \\
--- \\
---\end{array}$ & $\begin{array}{c}3.44 \% \\
--- \\
0.06 \%\end{array}$ & $\begin{array}{c}\cdots \\
\cdots-- \\
0.08 \%\end{array}$ & $\begin{array}{l}-- \\
--- \\
0.03 \%\end{array}$ & $\begin{array}{c}99.29 \% \\
--- \\
---\end{array}$ \\
\hline $\begin{array}{l}\mathrm{KAlSiO}_{4} \\
\mathrm{La}(\mathrm{OH})_{3} \\
\mathrm{MnO}_{2}\end{array}$ & $\begin{array}{c}2.61 \\
2.3 \\
5.026 \\
\end{array}$ & $\begin{array}{c}158.16 \\
189.93 \\
86.94 \\
\end{array}$ & $\begin{array}{c}-- \\
0.37 \% \\
--- \\
\end{array}$ & $\begin{array}{c}-- \\
0.07 \% \\
2.49 \% \\
\end{array}$ & $\begin{array}{c}-- \\
0.13 \% \\
--- \\
\end{array}$ & $\begin{array}{c}-- \\
0.02 \% \\
8.88 \% \\
\end{array}$ & $\begin{array}{c}-- \\
0.74 \% \\
1.18 \% \\
\end{array}$ & $\begin{array}{c}-- \\
0.06 \% \\
1.22 \% \\
\end{array}$ & $\begin{array}{c}--- \\
0.07 \% \\
--- \\
\end{array}$ \\
\hline $\begin{array}{l}\mathrm{Na}_{2} \mathrm{U}_{2} \mathrm{O}_{7} \\
\mathrm{NaAlSiO}_{4} \\
\mathrm{Ni}(\mathrm{OH})_{2}\end{array}$ & $\begin{array}{c}5.617 \\
2.365 \\
4.15 \\
\end{array}$ & $\begin{array}{c}634.03 \\
142.05 \\
92.71 \\
\end{array}$ & $\begin{array}{c}6.88 \% \\
22.87 \% \\
1.25 \% \\
\end{array}$ & $\begin{array}{c}22.10 \% \\
8.91 \% \\
0.31 \% \\
\end{array}$ & $\begin{array}{c}2.80 \% \\
81.78 \% \\
0.37 \% \\
\end{array}$ & $\begin{array}{c}0.11 \% \\
16.98 \% \\
1.93 \% \\
\end{array}$ & $\begin{array}{c}0.01 \% \\
36.04 \% \\
5.41 \% \\
\end{array}$ & $\begin{array}{c}0.58 \% \\
65.44 \% \\
0.54 \% \\
\end{array}$ & $\begin{array}{c}0.17 \% \\
--- \\
0.01 \% \\
\end{array}$ \\
\hline $\begin{array}{l}\mathrm{Pb}(\mathrm{OH})_{2} \\
\mathrm{Pu}(\mathrm{OH})_{4} \text { (co-precip. on Fe phase) } \\
\mathrm{PuO}_{2}\end{array}$ & $\begin{array}{c}7.1 \\
4.26 \\
11.43\end{array}$ & $\begin{array}{c}241.21 \\
307.03 \\
271\end{array}$ & $\begin{array}{c}1.45 \% \\
0.01 \% \\
---\end{array}$ & $\begin{array}{c}0.82 \% \\
0.02 \% \\
\ldots--\end{array}$ & $\begin{array}{c}0.24 \% \\
0.00 \% \\
---\end{array}$ & $\begin{array}{c}0.11 \% \\
0.02 \% \\
---\end{array}$ & $\begin{array}{c}3.97 \% \\
0.02 \% \\
\ldots\end{array}$ & $\begin{array}{c}0.66 \% \\
0.02 \% \\
\cdots\end{array}$ & $\begin{array}{c}0.13 \% \\
0.00 \% \\
---\end{array}$ \\
\hline $\begin{array}{l}\mathrm{SiO}_{2} \\
\mathrm{Sr}(\mathrm{OH})_{2} \\
\mathrm{ZrO}_{2}\end{array}$ & $\begin{array}{c}2.6 \\
3.625 \\
5.68\end{array}$ & $\begin{array}{c}60.08 \\
119.62 \\
123.22\end{array}$ & $\begin{array}{c}-- \\
0.15 \% \\
0.43 \%\end{array}$ & $\begin{array}{c}-- \\
0.07 \% \\
0.31 \%\end{array}$ & $\begin{array}{c}-- \\
0.0 \% \% \\
0.38 \%\end{array}$ & $\begin{array}{l}-- \\
0.13 \% \\
0.05 \%\end{array}$ & $\begin{array}{l}-- \\
0.15 \% \\
1.96 \%\end{array}$ & $\begin{array}{c}-- \\
0.04 \% \\
0.89 \%\end{array}$ & $\begin{array}{c}-- \\
0.01 \% \\
---\end{array}$ \\
\hline
\end{tabular}


Table C.3. (contd)

\begin{tabular}{|c|c|c|c|c|c|c|c|c|c|}
\hline & Density $(\mathrm{g} / \mathrm{mL})$ & 241-AN-102 & 241-AN-103 & 241-AN-104 & 241-AN-105 & 241-AN-106 & 241-AN-107 & 241-AP-101 & 241-AP-102 \\
\hline $\begin{array}{l}\text { Total kg washed solids for tank } \\
\text { Total kL washed solids for tank } \\
\text { Avg g/mL, washed solid }\end{array}$ & $\mathrm{n} / \mathrm{a}$ & $\begin{array}{c}1.80 \mathrm{E}+05 \\
7.25 \mathrm{E}+01 \\
2.484 \\
\end{array}$ & $\begin{array}{c}3.48 \mathrm{E}+04 \\
1.33 \mathrm{E}+01 \\
2.619 \\
\end{array}$ & $\begin{array}{c}1.79 \mathrm{E}+04 \\
5.81 \mathrm{E}+00 \\
3.089 \\
\end{array}$ & $\begin{array}{c}7.33 \mathrm{E}+04 \\
2.82 \mathrm{E}+01 \\
2.597 \\
\end{array}$ & $\begin{array}{c}9.09 \mathrm{E}+03 \\
3.57 \mathrm{E}+00 \\
2.546 \\
\end{array}$ & $\begin{array}{c}1.43 \mathrm{E}+05 \\
5.28 \mathrm{E}+01 \\
2.716\end{array}$ & $\begin{array}{c}8.79 \mathrm{E}+03 \\
3.54 \mathrm{E}+00 \\
2.486 \\
\end{array}$ & $\begin{array}{c}1.26 \mathrm{E}+04 \\
5.21 \mathrm{E}+00 \\
2.424 \\
\end{array}$ \\
\hline \multicolumn{10}{|l|}{ mass fracs of solids in washed solid } \\
\hline $\begin{array}{l}\mathrm{Ag}_{2} \mathrm{O} \\
\mathrm{Bi}_{2} \mathrm{O}_{3} \\
\mathrm{BiFeO}_{3}\end{array}$ & $\begin{array}{c}7.143 \\
8.9 \\
7.9 \\
\end{array}$ & $\begin{array}{c}\cdots \\
0.14 \% \\
--- \\
\end{array}$ & $\begin{array}{c}--- \\
0.53 \% \\
--- \\
\end{array}$ & $\begin{array}{c}--- \\
0.60 \% \\
--- \\
\end{array}$ & $\begin{array}{c}--- \\
0.16 \% \\
--- \\
\end{array}$ & $\begin{array}{c}--- \\
0.25 \% \\
--- \\
\end{array}$ & $\begin{array}{c}0.01 \% \\
0.03 \% \\
--- \\
\end{array}$ & $\begin{array}{l}\cdots- \\
--- \\
--- \\
\end{array}$ & $\begin{array}{c}0.01 \% \\
0.05 \% \\
--- \\
\end{array}$ \\
\hline $\begin{array}{l}\mathrm{Ca}(\mathrm{OH})_{2} \\
\mathrm{CrOOH} \\
\mathrm{FeOOH}\end{array}$ & $\begin{array}{l}2.24 \\
4.11 \\
4.26 \\
\end{array}$ & $\begin{array}{l}3.51 \% \\
1.02 \% \\
1.34 \% \\
\end{array}$ & $\begin{array}{l}2.78 \% \\
8.96 \% \\
0.97 \% \\
\end{array}$ & $\begin{array}{c}7.65 \% \\
42.41 \% \\
3.50 \% \\
\end{array}$ & $\begin{array}{c}4.76 \% \\
14.82 \% \\
0.85 \% \\
\end{array}$ & $\begin{array}{l}0.95 \% \\
8.27 \% \\
0.61 \% \\
\end{array}$ & $\begin{array}{l}3.60 \% \\
0.88 \% \\
15.61 \% \\
\end{array}$ & $\begin{array}{c}0.67 \% \\
--- \\
0.19 \% \\
\end{array}$ & $\begin{array}{c}0.38 \% \\
--- \\
0.04 \% \\
\end{array}$ \\
\hline $\begin{array}{l}\text { Gibbsite } \\
\text { Boehmite } \\
\mathrm{HgO} \text { (co-precip. with } \mathrm{Ag}_{2} \mathrm{O} \text { ) }\end{array}$ & $\begin{array}{c}2.42 \\
3.01 \\
7.143 \\
\end{array}$ & $\begin{array}{c}90.43 \% \\
--- \\
-- \\
\end{array}$ & $\begin{array}{c}56.55 \% \\
--- \\
--- \\
\end{array}$ & $\begin{array}{l}-- \\
--- \\
---\end{array}$ & $\begin{array}{c}78.15 \% \\
\cdots \\
--- \\
\end{array}$ & $\begin{array}{c}87.08 \% \\
--- \\
---\end{array}$ & $\begin{array}{c}71.84 \% \\
--- \\
--- \\
\end{array}$ & $\begin{array}{c}94.47 \% \\
--- \\
--- \\
\end{array}$ & $\begin{array}{c}99.22 \% \\
--- \\
---\end{array}$ \\
\hline $\begin{array}{l}\mathrm{KAlSiO}_{4} \\
\mathrm{La}(\mathrm{OH})_{3} \\
\mathrm{MnO}_{2} \\
\end{array}$ & $\begin{array}{c}2.61 \\
2.3 \\
5.026 \\
\end{array}$ & $\begin{array}{c}-- \\
0.02 \% \\
0.04 \% \\
\end{array}$ & $\begin{array}{c}27.69 \% \\
0.34 \% \\
--- \\
\end{array}$ & $\begin{array}{c}-- \\
0.93 \% \\
--- \\
\end{array}$ & $\begin{array}{c}-- \\
0.11 \% \\
--- \\
\end{array}$ & $\begin{array}{l}0.57 \% \\
0.10 \% \\
0.08 \% \\
\end{array}$ & $\begin{array}{c}-- \\
0.16 \% \\
3.19 \% \\
\end{array}$ & $\begin{array}{c}-- \\
0.08 \% \\
--- \\
\end{array}$ & $\begin{array}{c}-- \\
0.01 \% \\
0.09 \% \\
\end{array}$ \\
\hline $\begin{array}{l}\mathrm{Na}_{2} \mathrm{U}_{2} \mathrm{O}_{7} \\
\mathrm{NaAlSiO}_{4} \\
\mathrm{Ni}(\mathrm{OH})_{2} \\
\end{array}$ & $\begin{array}{r}5.617 \\
2.365 \\
4.15 \\
\end{array}$ & $\begin{array}{c}0.81 \% \\
--- \\
1.85 \% \\
\end{array}$ & $\begin{array}{c}0.53 \% \\
--- \\
0.11 \% \\
\end{array}$ & $\begin{array}{c}3.85 \% \\
37.96 \% \\
1.46 \% \\
\end{array}$ & $\begin{array}{l}0.46 \% \\
0.02 \% \\
0.24 \% \\
\end{array}$ & $\begin{array}{c}1.29 \% \\
--- \\
0.48 \% \\
\end{array}$ & $\begin{array}{c}--- \\
--- \\
2.69 \% \\
\end{array}$ & $\begin{array}{c}3.48 \% \\
--- \\
0.34 \% \\
\end{array}$ & $\begin{array}{c}0.04 \% \\
--- \\
0.10 \% \\
\end{array}$ \\
\hline $\begin{array}{l}\mathrm{Pb}(\mathrm{OH})_{2} \\
\mathrm{Pu}(\mathrm{OH})_{4} \text { (co-precip. on Fe phase) } \\
\mathrm{PuO}_{2}\end{array}$ & $\begin{array}{c}7.1 \\
4.26 \\
11.43 \\
\end{array}$ & $\begin{array}{c}0.54 \% \\
0.00 \% \\
\cdots \\
\end{array}$ & $\begin{array}{c}1.42 \% \\
0.00 \% \\
--- \\
\end{array}$ & $\begin{array}{c}1.44 \% \\
0.00 \% \\
--- \\
\end{array}$ & $\begin{array}{c}0.39 \% \\
0.00 \% \\
\cdots \\
\end{array}$ & $\begin{array}{c}0.14 \% \\
0.01 \% \\
--- \\
\end{array}$ & $\begin{array}{c}1.56 \% \\
0.00 \% \\
--- \\
\end{array}$ & $\begin{array}{c}0.76 \% \\
0.00 \% \\
--. \\
\end{array}$ & $\begin{array}{c}0.07 \% \\
0.00 \% \\
--. \\
\end{array}$ \\
\hline $\begin{array}{l}\mathrm{SiO}_{2} \\
\mathrm{Sr}(\mathrm{OH})_{2} \\
\mathrm{ZrO}_{2}\end{array}$ & $\begin{array}{c}2.6 \\
3.625 \\
5.68 \\
\end{array}$ & $\begin{array}{c}-- \\
0.03 \% \\
0.25 \%\end{array}$ & $\begin{array}{c}-- \\
0.10 \% \\
--- \\
\end{array}$ & $\begin{array}{c}--- \\
0.16 \% \\
0.04 \%\end{array}$ & $\begin{array}{c}--- \\
0.04 \% \\
--- \\
\end{array}$ & $\begin{array}{c}--- \\
0.04 \% \\
0.11 \%\end{array}$ & $\begin{array}{c}--- \\
0.02 \% \\
0.43 \%\end{array}$ & $\begin{array}{c}--- \\
0.02 \% \\
--- \\
\end{array}$ & $\begin{array}{l}--- \\
--- \\
---\end{array}$ \\
\hline
\end{tabular}


Table C.3. (contd)

\begin{tabular}{|c|c|c|c|c|c|c|c|c|c|}
\hline & Density $(\mathrm{g} / \mathrm{mL})$ & 241-AP-103 & 241-AP-104 & 241-AP-105 & 241-AP-106 & 241-AP-107 & 241-AP-108 & 241-AW-101 & 241-AW-102 \\
\hline $\begin{array}{l}\text { Total kg washed solids for tank } \\
\text { Total kL washed solids for tank } \\
\text { Avg g/mL, washed solid }\end{array}$ & $\mathrm{n} / \mathrm{a}$ & $\begin{array}{c}1.91 \mathrm{E}+04 \\
7.79 \mathrm{E}+00 \\
2.452 \\
\end{array}$ & $\begin{array}{c}1.24 \mathrm{E}+05 \\
5.09 \mathrm{E}+01 \\
2.426 \\
\end{array}$ & $\begin{array}{c}2.74 \mathrm{E}+05 \\
1.13 \mathrm{E}+02 \\
2.430 \\
\end{array}$ & $\begin{array}{c}1.19 \mathrm{E}+05 \\
4.90 \mathrm{E}+01 \\
2.425 \\
\end{array}$ & $\begin{array}{c}5.14 \mathrm{E}+03 \\
2.12 \mathrm{E}+00 \\
2.425 \\
\end{array}$ & $\begin{array}{c}1.13 \mathrm{E}+05 \\
4.66 \mathrm{E}+01 \\
2.424 \\
\end{array}$ & $\begin{array}{c}1.34 \mathrm{E}+04 \\
3.90 \mathrm{E}+00 \\
3.436\end{array}$ & $\begin{array}{c}1.93 \mathrm{E}+05 \\
7.74 \mathrm{E}+01 \\
2.492 \\
\end{array}$ \\
\hline \multicolumn{10}{|l|}{ mass fracs of solids in washed solid } \\
\hline $\begin{array}{l}\mathrm{Ag}_{2} \mathrm{O} \\
\mathrm{Bi}_{2} \mathrm{O}_{3} \\
\mathrm{BiFeO}_{3} \\
\end{array}$ & $\begin{array}{c}7.143 \\
8.9 \\
7.9 \\
\end{array}$ & $\begin{array}{c}0.07 \% \\
0.36 \% \\
--- \\
\end{array}$ & $\begin{array}{c}--- \\
0.08 \% \\
--- \\
\end{array}$ & $\begin{array}{c}--- \\
0.05 \% \\
--- \\
\end{array}$ & $\begin{array}{l}--- \\
--- \\
--- \\
\end{array}$ & $\begin{array}{c}--- \\
0.06 \% \\
--- \\
\end{array}$ & $\begin{array}{c}0.01 \% \\
0.06 \% \\
--- \\
\end{array}$ & $\begin{array}{l}--- \\
--- \\
--- \\
\end{array}$ & $\begin{array}{c}--- \\
0.15 \% \\
-- \\
\end{array}$ \\
\hline $\begin{array}{l}\mathrm{Ca}(\mathrm{OH})_{2} \\
\mathrm{CrOOH} \\
\mathrm{FeOOH}\end{array}$ & $\begin{array}{l}2.24 \\
4.11 \\
4.26 \\
\end{array}$ & $\begin{array}{c}0.64 \% \\
--- \\
0.27 \% \\
\end{array}$ & $\begin{array}{c}0.35 \% \\
--- \\
0.07 \% \\
\end{array}$ & $\begin{array}{c}0.39 \% \\
--- \\
0.21 \% \\
\end{array}$ & $\begin{array}{c}0.52 \% \\
--- \\
0.03 \% \\
\end{array}$ & $\begin{array}{c}0.32 \% \\
--- \\
0.07 \% \\
\end{array}$ & $\begin{array}{c}0.19 \% \\
--- \\
0.05 \% \\
\end{array}$ & $\begin{array}{l}10.67 \% \\
9.41 \% \\
9.62 \% \\
\end{array}$ & $\begin{array}{l}1.19 \% \\
0.07 \% \\
2.97 \% \\
\end{array}$ \\
\hline $\begin{array}{l}\text { Gibbsite } \\
\text { Boehmite } \\
\mathrm{HgO} \text { (co-precip. with } \mathrm{Ag}_{2} \mathrm{O} \text { ) }\end{array}$ & $\begin{array}{c}2.42 \\
3.01 \\
7.143 \\
\end{array}$ & $\begin{array}{c}96.77 \% \\
--- \\
--- \\
\end{array}$ & $\begin{array}{c}99.14 \% \\
--- \\
---\end{array}$ & $\begin{array}{c}98.76 \% \\
--- \\
--- \\
\end{array}$ & $\begin{array}{c}98.99 \% \\
--- \\
---\end{array}$ & $\begin{array}{c}99.22 \% \\
--- \\
---\end{array}$ & $\begin{array}{c}99.47 \% \\
--- \\
-- \\
\end{array}$ & $\begin{array}{l}-- \\
\cdots \\
---\end{array}$ & $\begin{array}{c}92.76 \% \\
--- \\
--- \\
\end{array}$ \\
\hline $\begin{array}{l}\mathrm{KAlSiO}_{4} \\
\mathrm{La}(\mathrm{OH})_{3} \\
\mathrm{MnO}_{2} \\
\end{array}$ & $\begin{array}{c}2.61 \\
2.3 \\
5.026 \\
\end{array}$ & $\begin{array}{c}-- \\
0.01 \% \\
--- \\
\end{array}$ & $\begin{array}{c}-- \\
0.00 \% \\
--- \\
\end{array}$ & $\begin{array}{c}-- \\
0.02 \% \\
--- \\
\end{array}$ & $\begin{array}{c}-- \\
0.01 \% \\
0.20 \% \\
\end{array}$ & $\begin{array}{c}--- \\
0.01 \% \\
--- \\
\end{array}$ & $\begin{array}{c}-- \\
0.01 \% \\
--- \\
\end{array}$ & $\begin{array}{c}36.04 \% \\
0.24 \% \\
--- \\
\end{array}$ & $\begin{array}{c}-- \\
0.06 \% \\
-- \\
\end{array}$ \\
\hline $\begin{array}{l}\mathrm{Na}_{2} \mathrm{U}_{2} \mathrm{O}_{7} \\
\mathrm{NaAlSiO} \\
\mathrm{Ni}(\mathrm{OH})_{2} \\
\end{array}$ & $\begin{array}{c}5.617 \\
2.365 \\
4.15 \\
\end{array}$ & $\begin{array}{c}0.25 \% \\
--- \\
1.18 \% \\
\end{array}$ & $\begin{array}{c}0.05 \% \\
--- \\
0.21 \% \\
\end{array}$ & $\begin{array}{c}0.29 \% \\
--- \\
0.14 \% \\
\end{array}$ & $\begin{array}{c}0.02 \% \\
--- \\
0.16 \% \\
\end{array}$ & $\begin{array}{c}0.01 \% \\
--- \\
0.21 \% \\
\end{array}$ & $\begin{array}{c}0.01 \% \\
--- \\
0.13 \% \\
\end{array}$ & $\begin{array}{c}26.31 \% \\
--- \\
0.63 \% \\
\end{array}$ & $\begin{array}{c}2.21 \% \\
--- \\
0.38 \% \\
\end{array}$ \\
\hline $\begin{array}{l}\mathrm{Pb}(\mathrm{OH})_{2} \\
\mathrm{Pu}(\mathrm{OH})_{4} \text { (co-precip. on Fe phase) } \\
\mathrm{PuO}_{2}\end{array}$ & $\begin{array}{c}7.1 \\
4.26 \\
11.43 \\
\end{array}$ & $\begin{array}{c}0.40 \% \\
0.00 \% \\
\ldots \\
\end{array}$ & $\begin{array}{c}0.10 \% \\
0.00 \% \\
--- \\
\end{array}$ & $\begin{array}{c}0.12 \% \\
0.00 \% \\
--- \\
\end{array}$ & $\begin{array}{c}0.02 \% \\
0.00 \% \\
\ldots \\
\end{array}$ & $\begin{array}{c}0.09 \% \\
0.00 \% \\
--- \\
\end{array}$ & $\begin{array}{c}0.06 \% \\
0.00 \% \\
--- \\
\end{array}$ & $\begin{array}{c}5.96 \% \\
0.02 \% \\
--- \\
\end{array}$ & $\begin{array}{c}0.19 \% \\
0.00 \% \\
-.- \\
\end{array}$ \\
\hline $\begin{array}{l}\mathrm{SiO}_{2} \\
\mathrm{Sr}(\mathrm{OH})_{2} \\
\mathrm{ZrO}_{2}\end{array}$ & $\begin{array}{c}2.6 \\
3.625 \\
5.68\end{array}$ & $\begin{array}{c}-- \\
0.05 \% \\
--- \\
\end{array}$ & $\begin{array}{c}-- \\
0.01 \% \\
---\end{array}$ & $\begin{array}{c}--- \\
0.01 \% \\
--- \\
\end{array}$ & $\begin{array}{c}--- \\
0.00 \% \\
0.05 \%\end{array}$ & $\begin{array}{c}--- \\
0.01 \% \\
--- \\
\end{array}$ & $\begin{array}{c}--- \\
0.01 \% \\
--- \\
\end{array}$ & $\begin{array}{c}--- \\
0.06 \% \\
1.05 \%\end{array}$ & $\begin{array}{c}--- \\
0.02 \% \\
--- \\
\end{array}$ \\
\hline
\end{tabular}


Table C.3. (contd)

\begin{tabular}{|c|c|c|c|c|c|c|c|c|c|}
\hline & Density $(\mathrm{g} / \mathrm{mL})$ & 241-AW-103 & 241-AW-104 & 241-AW-105 & 241-AW-106 & 241-AX-101 & 241-AX-102 & 241-AX-103 & 241-AX-104 \\
\hline $\begin{array}{l}\text { Total kg washed solids for tank } \\
\text { Total kL washed solids for tank } \\
\text { Avg g/mL, washed solid }\end{array}$ & $\mathrm{n} / \mathrm{a}$ & $\begin{array}{c}3.75 \mathrm{E}+05 \\
9.29 \mathrm{E}+01 \\
4.041 \\
\end{array}$ & $\begin{array}{c}6.19 \mathrm{E}+04 \\
2.06 \mathrm{E}+01 \\
2.999 \\
\end{array}$ & $\begin{array}{c}1.71 \mathrm{E}+05 \\
3.43 \mathrm{E}+01 \\
4.999 \\
\end{array}$ & $\begin{array}{c}1.09 \mathrm{E}+05 \\
4.27 \mathrm{E}+01 \\
2.554 \\
\end{array}$ & $\begin{array}{c}1.31 \mathrm{E}+05 \\
5.19 \mathrm{E}+01 \\
2.528 \\
\end{array}$ & $\begin{array}{c}3.07 \mathrm{E}+04 \\
1.08 \mathrm{E}+01 \\
2.842 \\
\end{array}$ & $\begin{array}{c}3.69 \mathrm{E}+04 \\
1.32 \mathrm{E}+01 \\
2.794 \\
\end{array}$ & $\begin{array}{c}3.45 \mathrm{E}+04 \\
9.70 \mathrm{E}+00 \\
3.551 \\
\end{array}$ \\
\hline \multicolumn{10}{|l|}{ mass fracs of solids in washed solid } \\
\hline $\begin{array}{l}\mathrm{Ag}_{2} \mathrm{O} \\
\mathrm{Bi}_{2} \mathrm{O}_{3} \\
\mathrm{BiFeO}_{3}\end{array}$ & $\begin{array}{c}7.143 \\
8.9 \\
7.9 \\
\end{array}$ & $\begin{array}{c}0.20 \% \\
0.16 \% \\
--- \\
\end{array}$ & $\begin{array}{c}0.04 \% \\
0.06 \% \\
--- \\
\end{array}$ & $\begin{array}{c}0.35 \% \\
0.10 \% \\
--- \\
\end{array}$ & $\begin{array}{c}--- \\
0.28 \% \\
--- \\
\end{array}$ & $\begin{array}{c}--- \\
0.12 \% \\
--- \\
\end{array}$ & $\begin{array}{c}--- \\
0.03 \% \\
--- \\
\end{array}$ & $\begin{array}{c}--- \\
0.06 \% \\
--- \\
\end{array}$ & $\begin{array}{l}--- \\
--- \\
---\end{array}$ \\
\hline $\begin{array}{l}\mathrm{Ca}(\mathrm{OH})_{2} \\
\mathrm{CrOOH} \\
\mathrm{FeOOH} \\
\end{array}$ & $\begin{array}{l}2.24 \\
4.11 \\
4.26 \\
\end{array}$ & $\begin{array}{l}0.26 \% \\
1.43 \% \\
0.69 \% \\
\end{array}$ & $\begin{array}{l}3.74 \% \\
6.82 \% \\
6.76 \% \\
\end{array}$ & $\begin{array}{l}0.86 \% \\
0.85 \% \\
2.36 \% \\
\end{array}$ & $\begin{array}{l}1.13 \% \\
8.65 \% \\
0.75 \% \\
\end{array}$ & $\begin{array}{l}1.30 \% \\
6.78 \% \\
1.56 \% \\
\end{array}$ & $\begin{array}{c}1.65 \% \\
--- \\
22.93 \% \\
\end{array}$ & $\begin{array}{r}1.65 \% \\
5.23 \% \\
18.59 \% \\
\end{array}$ & $\begin{array}{c}3.37 \% \\
--- \\
65.10 \% \\
\end{array}$ \\
\hline $\begin{array}{l}\text { Gibbsite } \\
\text { Boehmite } \\
\mathrm{HgO} \text { (co-precip. with } \mathrm{Ag}_{2} \mathrm{O} \text { ) }\end{array}$ & $\begin{array}{c}2.42 \\
3.01 \\
7.143 \\
\end{array}$ & $\begin{array}{c}26.64 \% \\
--- \\
--- \\
\end{array}$ & $\begin{array}{c}51.11 \% \\
--- \\
--- \\
\end{array}$ & $\begin{array}{c}2.19 \% \\
--- \\
--- \\
\end{array}$ & $\begin{array}{c}85.25 \% \\
--- \\
-- \\
\end{array}$ & $\begin{array}{c}84.44 \% \\
--- \\
--- \\
\end{array}$ & $\begin{array}{c}48.06 \% \\
--- \\
0.05 \% \\
\end{array}$ & $\begin{array}{c}53.38 \% \\
--- \\
--- \\
\end{array}$ & $\begin{array}{c}22.75 \% \\
--- \\
0.04 \% \\
\end{array}$ \\
\hline $\begin{array}{l}\mathrm{KAlSiO}_{4} \\
\mathrm{La}(\mathrm{OH})_{3} \\
\mathrm{MnO}_{2} \\
\end{array}$ & $\begin{array}{c}2.61 \\
2.3 \\
5.026 \\
\end{array}$ & $\begin{array}{c}2.79 \% \\
0.17 \% \\
--- \\
\end{array}$ & $\begin{array}{l}.96 \% \\
0.09 \% \\
1.81 \% \\
\end{array}$ & $\begin{array}{l}6.11 \% \\
0.56 \% \\
1.15 \% \\
\end{array}$ & $\begin{array}{c}-- \\
0.12 \% \\
--- \\
\end{array}$ & $\begin{array}{c}-- \\
0.11 \% \\
--- \\
\end{array}$ & $\begin{array}{c}-- \\
0.02 \% \\
1.76 \% \\
\end{array}$ & $\begin{array}{c}-- \\
0.32 \% \\
2.12 \% \\
\end{array}$ & $\begin{array}{c}--- \\
0.30 \% \\
1.12 \% \\
\end{array}$ \\
\hline $\begin{array}{l}\mathrm{Na}_{2} \mathrm{U}_{2} \mathrm{O}_{7} \\
\mathrm{NaAlSiO}_{4} \\
\mathrm{Ni}(\mathrm{OH})_{2} \\
\end{array}$ & $\begin{array}{c}5.617 \\
2.365 \\
4.15 \\
\end{array}$ & $\begin{array}{c}4.37 \% \\
--- \\
0.05 \% \\
\end{array}$ & $\begin{array}{c}15.76 \% \\
--- \\
0.22 \% \\
\end{array}$ & $\begin{array}{c}.96 \% \\
--- \\
0.19 \% \\
\end{array}$ & $\begin{array}{l}1.48 \% \\
1.43 \% \\
0.56 \% \\
\end{array}$ & $\begin{array}{l}.05 \% \\
4.01 \% \\
0.23 \% \\
\end{array}$ & $\begin{array}{c}0.34 \% \\
15.05 \% \\
9.94 \% \\
\end{array}$ & $\begin{array}{c}0.85 \% \\
14.07 \% \\
1.10 \% \\
\end{array}$ & $\begin{array}{l}0.65 \% \\
0.55 \% \\
3.47 \% \\
\end{array}$ \\
\hline $\begin{array}{l}\mathrm{Pb}(\mathrm{OH})_{2} \\
\mathrm{Pu}(\mathrm{OH})_{4} \text { (co-precip. on Fe phase) } \\
\mathrm{PuO}_{2}\end{array}$ & $\begin{array}{c}7.1 \\
4.26 \\
11.43 \\
\end{array}$ & $\begin{array}{c}0.05 \% \\
0.00 \% \\
--- \\
\end{array}$ & $\begin{array}{c}0.26 \% \\
0.02 \% \\
\cdots \\
\end{array}$ & $\begin{array}{c}0.03 \% \\
0.01 \% \\
--- \\
\end{array}$ & $\begin{array}{c}0.15 \% \\
0.02 \% \\
-- \\
\end{array}$ & $\begin{array}{c}0.31 \% \\
0.00 \% \\
--- \\
\end{array}$ & $\begin{array}{c}0.12 \% \\
0.02 \% \\
\cdots \\
\end{array}$ & $\begin{array}{c}.70 \% \\
0.01 \% \\
--- \\
\end{array}$ & $\begin{array}{c}1.62 \% \\
0.02 \% \\
-.- \\
\end{array}$ \\
\hline $\begin{array}{l}\mathrm{SiO}_{2} \\
\mathrm{Sr}(\mathrm{OH})_{2} \\
\mathrm{ZrO}_{2}\end{array}$ & $\begin{array}{c}2.6 \\
3.625 \\
5.68\end{array}$ & $\begin{array}{c}-- \\
0.01 \% \\
63.17 \%\end{array}$ & $\begin{array}{c}-- \\
0.02 \% \\
5.31 \%\end{array}$ & $\begin{array}{c}--- \\
0.00 \% \\
75.28 \%\end{array}$ & $\begin{array}{c}--- \\
0.05 \% \\
0.13 \%\end{array}$ & $\begin{array}{c}--- \\
0.02 \% \\
0.06 \%\end{array}$ & $\begin{array}{c}--- \\
0.00 \% \\
0.03 \%\end{array}$ & $\begin{array}{c}--- \\
0.19 \% \\
0.75 \%\end{array}$ & $\begin{array}{c}--- \\
0.20 \% \\
0.80 \%\end{array}$ \\
\hline
\end{tabular}


Table C.3. (contd)

\begin{tabular}{|c|c|c|c|c|c|c|c|c|c|}
\hline & Density $(\mathrm{g} / \mathrm{mL})$ & 241-AY-101 & 241-AY-102 & 241-AZ-101 & 241-AZ-102 & 241-B-101 & 241-B-102 & 241-B-103 & 241-B-104 \\
\hline $\begin{array}{l}\text { Total kg washed solids for tank } \\
\text { Total kL washed solids for tank } \\
\text { Avg g/mL, washed solid }\end{array}$ & $\mathrm{n} / \mathrm{a}$ & $\begin{array}{c}1.67 \mathrm{E}+05 \\
5.38 \mathrm{E}+01 \\
3.109 \\
\end{array}$ & $\begin{array}{c}2.79 \mathrm{E}+05 \\
8.30 \mathrm{E}+01 \\
3.368 \\
\end{array}$ & $\begin{array}{c}1.20 \mathrm{E}+05 \\
4.05 \mathrm{E}+01 \\
2.971 \\
\end{array}$ & $\begin{array}{c}1.17 \mathrm{E}+05 \\
3.91 \mathrm{E}+01 \\
2.986 \\
\end{array}$ & $\begin{array}{c}1.60 \mathrm{E}+05 \\
5.77 \mathrm{E}+01 \\
2.771 \\
\end{array}$ & $\begin{array}{c}1.75 \mathrm{E}+04 \\
6.90 \mathrm{E}+00 \\
2.530 \\
\end{array}$ & $\begin{array}{c}3.29 \mathrm{E}+04 \\
1.29 \mathrm{E}+01 \\
2.562 \\
\end{array}$ & $\begin{array}{c}8.84 \mathrm{E}+04 \\
2.09 \mathrm{E}+01 \\
4.236 \\
\end{array}$ \\
\hline \multicolumn{10}{|l|}{ mass fracs of solids in washed solid } \\
\hline $\begin{array}{l}\mathrm{Ag}_{2} \mathrm{O} \\
\mathrm{Bi}_{2} \mathrm{O}_{3} \\
\mathrm{BiFeO}_{3}\end{array}$ & $\begin{array}{c}7.143 \\
8.9 \\
7.9 \\
\end{array}$ & $\begin{array}{c}0.09 \% \\
--- \\
--- \\
\end{array}$ & $\begin{array}{c}0.01 \% \\
0.06 \% \\
--- \\
\end{array}$ & $\begin{array}{c}0.06 \% \\
--- \\
--- \\
\end{array}$ & $\begin{array}{c}0.22 \% \\
0.02 \% \\
--- \\
\end{array}$ & $\begin{array}{l}-- \\
--- \\
--- \\
\end{array}$ & $\begin{array}{c}--- \\
2.17 \% \\
--- \\
\end{array}$ & $\begin{array}{c}-\cdots \\
2.13 \% \\
--- \\
\end{array}$ & $\begin{array}{c}--- \\
--- \\
41.12 \% \\
\end{array}$ \\
\hline $\begin{array}{l}\mathrm{Ca}(\mathrm{OH})_{2} \\
\mathrm{CrOOH} \\
\mathrm{FeOOH}\end{array}$ & $\begin{array}{l}2.24 \\
4.11 \\
4.26 \\
\end{array}$ & $\begin{array}{r}1.47 \% \\
1.22 \% \\
44.19 \% \\
\end{array}$ & $\begin{array}{r}3.17 \% \\
0.90 \% \\
51.20 \% \\
\end{array}$ & $\begin{array}{c}0.99 \% \\
0.06 \% \\
27.63 \% \\
\end{array}$ & $\begin{array}{c}1.10 \% \\
0.33 \% \\
32.37 \% \\
\end{array}$ & $\begin{array}{r}2.77 \% \\
0.43 \% \\
19.98 \% \\
\end{array}$ & $\begin{array}{l}1.93 \% \\
1.28 \% \\
2.43 \% \\
\end{array}$ & $\begin{array}{l}1.95 \% \\
1.36 \% \\
2.56 \% \\
\end{array}$ & $\begin{array}{c}1.47 \% \\
1.22 \% \\
28.77 \% \\
\end{array}$ \\
\hline $\begin{array}{l}\text { Gibbsite } \\
\text { Boehmite } \\
\mathrm{HgO} \text { (co-precip. with } \mathrm{Ag}_{2} \mathrm{O} \text { ) }\end{array}$ & $\begin{array}{c}2.42 \\
3.01 \\
7.143 \\
\end{array}$ & $\begin{array}{c}46.12 \% \\
--- \\
--- \\
\end{array}$ & $\begin{array}{c}33.00 \% \\
--- \\
0.00 \% \\
\end{array}$ & $\begin{array}{c}56.20 \% \\
--- \\
--- \\
\end{array}$ & $\begin{array}{c}46.76 \% \\
--- \\
--- \\
\end{array}$ & $\begin{array}{c}28.80 \% \\
--- \\
0.07 \% \\
\end{array}$ & $\begin{array}{c}82.03 \% \\
--- \\
--- \\
\end{array}$ & $\begin{array}{c}79.66 \% \\
--- \\
--- \\
\end{array}$ & $\begin{array}{l}-- \\
\cdots- \\
---\end{array}$ \\
\hline $\begin{array}{l}\mathrm{KAlSiO}_{4} \\
\mathrm{La}(\mathrm{OH})_{3} \\
\mathrm{MnO}_{2} \\
\end{array}$ & $\begin{array}{c}2.61 \\
2.3 \\
5.026 \\
\end{array}$ & $\begin{array}{l}-- \\
1.11 \% \\
1.99 \% \\
\end{array}$ & $\begin{array}{c}-- \\
0.57 \% \\
6.73 \% \\
\end{array}$ & $\begin{array}{c}-- \\
1.05 \% \\
0.35 \% \\
\end{array}$ & $\begin{array}{c}-- \\
0.89 \% \\
0.66 \% \\
\end{array}$ & $\begin{array}{c}-- \\
0.00 \% \\
0.03 \% \\
\end{array}$ & $\begin{array}{c}--- \\
--- \\
0.27 \% \\
\end{array}$ & $\begin{array}{c}-- \\
0.00 \% \\
0.29 \% \\
\end{array}$ & $\begin{array}{l}-- \\
0.11 \% \\
0.48 \% \\
\end{array}$ \\
\hline $\begin{array}{l}\mathrm{Na}_{2} \mathrm{U}_{2} \mathrm{O}_{7} \\
\mathrm{NaAlSiO}_{4} \\
\mathrm{Ni}(\mathrm{OH})_{2} \\
\end{array}$ & $\begin{array}{r}5.617 \\
2.365 \\
4.15 \\
\end{array}$ & $\begin{array}{l}0.50 \% \\
0.57 \% \\
1.40 \% \\
\end{array}$ & $\begin{array}{c}1.47 \% \\
--- \\
1.23 \% \\
\end{array}$ & $\begin{array}{l}1.71 \% \\
1.74 \% \\
1.69 \% \\
\end{array}$ & $\begin{array}{l}.43 \% \\
8.98 \% \\
1.90 \% \\
\end{array}$ & $\begin{array}{c}2.09 \% \\
37.07 \% \\
8.56 \% \\
\end{array}$ & $\begin{array}{l}1.95 \% \\
7.46 \% \\
0.07 \% \\
\end{array}$ & $\begin{array}{l}3.94 \% \\
7.64 \% \\
0.07 \% \\
\end{array}$ & $\begin{array}{c}-- \\
23.52 \% \\
0.20 \% \\
\end{array}$ \\
\hline $\begin{array}{l}\mathrm{Pb}(\mathrm{OH})_{2} \\
\mathrm{Pu}(\mathrm{OH})_{4} \text { (co-precip. on Fe phase) } \\
\mathrm{PuO}_{2}\end{array}$ & $\begin{array}{c}7.1 \\
4.26 \\
11.43 \\
\end{array}$ & $\begin{array}{c}0.74 \% \\
0.01 \% \\
\cdots \\
\end{array}$ & $\begin{array}{c}1.52 \% \\
0.01 \% \\
--- \\
\end{array}$ & $\begin{array}{c}0.08 \% \\
0.02 \% \\
--- \\
\end{array}$ & $\begin{array}{c}0.29 \% \\
0.03 \% \\
\cdots \\
\end{array}$ & $\begin{array}{c}0.13 \% \\
0.01 \% \\
--- \\
\end{array}$ & $\begin{array}{c}0.28 \% \\
0.00 \% \\
--- \\
\end{array}$ & $\begin{array}{c}0.29 \% \\
0.00 \% \\
--. \\
\end{array}$ & $\begin{array}{c}2.06 \% \\
0.00 \% \\
--. \\
\end{array}$ \\
\hline $\begin{array}{l}\mathrm{SiO}_{2} \\
\mathrm{Sr}(\mathrm{OH})_{2} \\
\mathrm{ZrO}_{2}\end{array}$ & $\begin{array}{c}2.6 \\
3.625 \\
5.68 \\
\end{array}$ & $\begin{array}{c}-- \\
0.06 \% \\
0.53 \%\end{array}$ & $\begin{array}{c}--- \\
0.12 \% \\
0.01 \%\end{array}$ & $\begin{array}{c}--- \\
0.11 \% \\
8.31 \%\end{array}$ & $\begin{array}{c}--- \\
0.06 \% \\
3.95 \%\end{array}$ & $\begin{array}{c}--- \\
0.03 \% \\
0.03 \%\end{array}$ & $\begin{array}{c}--- \\
0.07 \% \\
0.06 \%\end{array}$ & $\begin{array}{c}--- \\
0.07 \% \\
0.06 \%\end{array}$ & $\begin{array}{c}--- \\
0.81 \% \\
0.22 \%\end{array}$ \\
\hline
\end{tabular}


Table C.3. (contd)

\begin{tabular}{|c|c|c|c|c|c|c|c|c|c|}
\hline & Density $(\mathrm{g} / \mathrm{mL})$ & 241-B-105 & 241-B-106 & 241-B-107 & 241-B-108 & 241-B-109 & 241-B-110 & 241-B-111 & 241-B-112 \\
\hline $\begin{array}{l}\text { Total kg washed solids for tank } \\
\text { Total kL washed solids for tank } \\
\text { Avg g/mL, washed solid }\end{array}$ & $\mathrm{n} / \mathrm{a}$ & $\begin{array}{c}1.97 \mathrm{E}+05 \\
7.68 \mathrm{E}+01 \\
2.571\end{array}$ & $\begin{array}{c}4.40 \mathrm{E}+04 \\
1.17 \mathrm{E}+01 \\
3.769\end{array}$ & $\begin{array}{c}7.82 \mathrm{E}+04 \\
2.60 \mathrm{E}+01 \\
3.006 \\
\end{array}$ & $\begin{array}{c}8.31 \mathrm{E}+04 \\
3.35 \mathrm{E}+01 \\
2.480\end{array}$ & $\begin{array}{c}1.57 \mathrm{E}+05 \\
5.92 \mathrm{E}+01 \\
2.645\end{array}$ & $\begin{array}{c}8.10 \mathrm{E}+04 \\
1.68 \mathrm{E}+01 \\
4.831 \\
\end{array}$ & $\begin{array}{c}9.07 \mathrm{E}+04 \\
2.18 \mathrm{E}+01 \\
4.149 \\
\end{array}$ & $\begin{array}{c}1.79 \mathrm{E}+04 \\
5.74 \mathrm{E}+00 \\
3.118\end{array}$ \\
\hline \multicolumn{10}{|l|}{ mass fracs of solids in washed solid } \\
\hline $\begin{array}{l}\mathrm{Ag}_{2} \mathrm{O} \\
\mathrm{Bi}_{2} \mathrm{O}_{3} \\
\mathrm{BiFeO}_{3} \\
\end{array}$ & $\begin{array}{c}7.143 \\
8.9 \\
7.9 \\
\end{array}$ & $\begin{array}{c}--- \\
3.53 \% \\
--- \\
\end{array}$ & $\begin{array}{c}0.06 \% \\
12.39 \% \\
--- \\
\end{array}$ & $\begin{array}{c}0.01 \% \\
11.00 \% \\
--- \\
\end{array}$ & $\begin{array}{c}--- \\
1.16 \% \\
--- \\
\end{array}$ & $\begin{array}{c}--- \\
2.21 \% \\
--- \\
\end{array}$ & $\begin{array}{c}0.08 \% \\
--- \\
47.22 \% \\
\end{array}$ & $\begin{array}{c}0.01 \% \\
--- \\
38.71 \% \\
\end{array}$ & $\begin{array}{c}--- \\
17.63 \% \\
--- \\
\end{array}$ \\
\hline $\begin{array}{l}\mathrm{Ca}(\mathrm{OH})_{2} \\
\mathrm{CrOOH} \\
\mathrm{FeOOH}\end{array}$ & $\begin{array}{l}2.24 \\
4.11 \\
4.26\end{array}$ & $\begin{array}{l}1.97 \% \\
1.36 \% \\
3.79 \%\end{array}$ & $\begin{array}{c}4.72 \% \\
0.53 \% \\
33.50 \%\end{array}$ & $\begin{array}{c}0.95 \% \\
0.39 \% \\
22.88 \% \\
\end{array}$ & $\begin{array}{l}1.32 \% \\
0.08 \% \\
0.86 \%\end{array}$ & $\begin{array}{l}1.11 \% \\
1.35 \% \\
4.20 \%\end{array}$ & $\begin{array}{c}2.55 \% \\
2.15 \% \\
35.70 \% \\
\end{array}$ & $\begin{array}{c}1.70 \% \\
2.08 \% \\
24.05 \%\end{array}$ & $\begin{array}{c}2.23 \% \\
1.46 \% \\
20.43 \%\end{array}$ \\
\hline $\begin{array}{l}\text { Gibbsite } \\
\text { Boehmite } \\
\mathrm{HgO} \text { (co-precip. with } \mathrm{Ag}_{2} \mathrm{O} \text { ) }\end{array}$ & $\begin{array}{c}2.42 \\
3.01 \\
7.143 \\
\end{array}$ & $\begin{array}{c}76.93 \% \\
--- \\
--- \\
\end{array}$ & $\begin{array}{c}24.54 \% \\
--- \\
0.08 \% \\
\end{array}$ & $\begin{array}{c}34.69 \% \\
--- \\
--- \\
\end{array}$ & $\begin{array}{c}91.82 \% \\
--- \\
--\end{array}$ & $\begin{array}{c}69.24 \% \\
--- \\
---\end{array}$ & $\begin{array}{l}--- \\
--- \\
--- \\
\end{array}$ & $\begin{array}{c}3.48 \% \\
--- \\
--- \\
\end{array}$ & $\begin{array}{c}24.55 \% \\
--- \\
---\end{array}$ \\
\hline $\begin{array}{l}\mathrm{KAISiO}_{4} \\
\mathrm{La}(\mathrm{OH})_{3} \\
\mathrm{MnO}_{2}\end{array}$ & $\begin{array}{c}2.61 \\
2.3 \\
5.026\end{array}$ & $\begin{array}{c}-- \\
0.00 \% \\
0.11 \%\end{array}$ & $\begin{array}{c}-- \\
0.07 \% \\
0.32 \%\end{array}$ & $\begin{array}{c}-- \\
0.01 \% \\
0.15 \%\end{array}$ & $\begin{array}{l}-- \\
0.03 \% \\
0.00 \%\end{array}$ & $\begin{array}{l}0.26 \% \\
0.03 \% \\
0.36 \%\end{array}$ & $\begin{array}{c}-- \\
0.07 \% \\
0.18 \%\end{array}$ & $\begin{array}{l}-- \\
0.01 \% \\
0.17 \%\end{array}$ & $\begin{array}{c}-- \\
0.05 \% \\
0.14 \%\end{array}$ \\
\hline $\begin{array}{l}\mathrm{Na}_{2} \mathrm{U}_{2} \mathrm{O}_{7} \\
\mathrm{NaAlSiO}_{4} \\
\mathrm{Ni}(\mathrm{OH})_{2} \\
\end{array}$ & $\begin{array}{r}5.617 \\
2.365 \\
4.15 \\
\end{array}$ & $\begin{array}{l}2.03 \% \\
9.78 \% \\
0.06 \% \\
\end{array}$ & $\begin{array}{c}20.23 \% \\
2.03 \% \\
0.11 \% \\
\end{array}$ & $\begin{array}{c}2.67 \% \\
26.32 \% \\
0.06 \% \\
\end{array}$ & $\begin{array}{l}1.26 \% \\
2.33 \% \\
0.52 \% \\
\end{array}$ & $\begin{array}{c}7.23 \% \\
12.87 \% \\
0.53 \% \\
\end{array}$ & $\begin{array}{l}0.47 \% \\
9.99 \% \\
0.05 \% \\
\end{array}$ & $\begin{array}{l}--- \\
--- \\
0.03 \% \\
\end{array}$ & $\begin{array}{c}0.33 \% \\
31.73 \% \\
0.19 \% \\
\end{array}$ \\
\hline $\begin{array}{l}\mathrm{Pb}(\mathrm{OH})_{2} \\
\mathrm{Pu}(\mathrm{OH})_{4} \text { (co-precip. on Fe phase) } \\
\mathrm{PuO}_{2}\end{array}$ & $\begin{array}{c}7.1 \\
4.26 \\
11.43 \\
\end{array}$ & $\begin{array}{c}0.31 \% \\
0.00 \% \\
\quad-. \\
\end{array}$ & $\begin{array}{c}0.56 \% \\
0.00 \% \\
--- \\
\end{array}$ & $\begin{array}{c}0.55 \% \\
0.00 \% \\
--- \\
\end{array}$ & $\begin{array}{c}0.51 \% \\
0.00 \% \\
\ldots \\
\end{array}$ & $\begin{array}{c}0.49 \% \\
0.00 \% \\
--- \\
\end{array}$ & $\begin{array}{c}1.05 \% \\
0.00 \% \\
--- \\
\end{array}$ & $\begin{array}{c}2.43 \% \\
0.00 \% \\
\ldots \\
-. \\
\end{array}$ & $\begin{array}{c}0.90 \% \\
0.00 \% \\
-- \\
\end{array}$ \\
\hline $\begin{array}{l}\mathrm{SiO}_{2} \\
\mathrm{Sr}(\mathrm{OH})_{2} \\
\mathrm{ZrO}_{2}\end{array}$ & $\begin{array}{c}2.6 \\
3.625 \\
5.68 \\
\end{array}$ & $\begin{array}{c}-- \\
0.09 \% \\
0.05 \%\end{array}$ & $\begin{array}{c}--- \\
0.71 \% \\
0.14 \%\end{array}$ & $\begin{array}{c}--- \\
0.15 \% \\
0.17 \%\end{array}$ & $\begin{array}{c}--- \\
0.05 \% \\
0.07 \%\end{array}$ & $\begin{array}{c}--- \\
0.04 \% \\
0.08 \%\end{array}$ & $\begin{array}{c}--- \\
0.49 \% \\
0.01 \%\end{array}$ & $\begin{array}{c}26.90 \% \\
0.40 \% \\
0.03 \%\end{array}$ & $\begin{array}{c}--- \\
0.34 \% \\
0.01 \%\end{array}$ \\
\hline
\end{tabular}


Table C.3. (contd)

\begin{tabular}{|c|c|c|c|c|c|c|c|c|c|}
\hline & Density $(\mathrm{g} / \mathrm{mL})$ & 241-B-201 & 241-B-202 & 241-B-203 & 241-B-204 & 241-BX-101 & 241-BX-102 & 241-BX-103 & 241-BX-104 \\
\hline $\begin{array}{l}\text { Total kg washed solids for tank } \\
\text { Total kL washed solids for tank } \\
\text { Avg g/mL, washed solid }\end{array}$ & $\mathrm{n} / \mathrm{a}$ & $\begin{array}{c}3.12 \mathrm{E}+04 \\
6.24 \mathrm{E}+00 \\
4.996 \\
\end{array}$ & $\begin{array}{c}1.58 \mathrm{E}+04 \\
3.88 \mathrm{E}+00 \\
4.081 \\
\end{array}$ & $\begin{array}{c}2.18 \mathrm{E}+04 \\
4.32 \mathrm{E}+00 \\
5.038\end{array}$ & $\begin{array}{c}2.38 \mathrm{E}+04 \\
4.59 \mathrm{E}+00 \\
5.190\end{array}$ & $\begin{array}{c}1.03 \mathrm{E}+05 \\
3.98 \mathrm{E}+01 \\
2.582\end{array}$ & $\begin{array}{c}2.15 \mathrm{E}+05 \\
8.46 \mathrm{E}+01 \\
2.540\end{array}$ & $\begin{array}{c}8.63 \mathrm{E}+04 \\
3.30 \mathrm{E}+01 \\
2.613\end{array}$ & $\begin{array}{c}3.20 \mathrm{E}+05 \\
1.18 \mathrm{E}+02 \\
2.716\end{array}$ \\
\hline \multicolumn{10}{|l|}{ mass fracs of solids in washed solid } \\
\hline $\begin{array}{l}\mathrm{Ag}_{2} \mathrm{O} \\
\mathrm{Bi}_{2} \mathrm{O}_{3} \\
\mathrm{BiFeO}_{3} \\
\end{array}$ & $\begin{array}{c}7.143 \\
8.9 \\
7.9 \\
\end{array}$ & $\begin{array}{c}0.00 \% \\
51.35 \% \\
-- \\
\end{array}$ & $\begin{array}{c}0.01 \% \\
32.97 \% \\
--- \\
\end{array}$ & $\begin{array}{c}0.00 \% \\
48.54 \% \\
--- \\
\end{array}$ & $\begin{array}{c}0.01 \% \\
51.31 \% \\
--- \\
\end{array}$ & $\begin{array}{c}--- \\
0.60 \% \\
--- \\
\end{array}$ & $\begin{array}{c}--- \\
0.38 \% \\
--- \\
\end{array}$ & $\begin{array}{c}--- \\
0.56 \% \\
--- \\
\end{array}$ & $\begin{array}{c}0.01 \% \\
0.13 \% \\
--- \\
\end{array}$ \\
\hline $\begin{array}{l}\mathrm{Ca}(\mathrm{OH})_{2} \\
\mathrm{CrOOH} \\
\mathrm{FeOOH}\end{array}$ & $\begin{array}{l}2.24 \\
4.11 \\
4.26\end{array}$ & $\begin{array}{l}5.60 \% \\
2.20 \% \\
9.08 \%\end{array}$ & $\begin{array}{l}10.18 \% \\
2.93 \% \\
16.40 \%\end{array}$ & $\begin{array}{l}0.43 \% \\
5.20 \% \\
5.52 \%\end{array}$ & $\begin{array}{l}0.47 \% \\
5.00 \% \\
5.62 \%\end{array}$ & $\begin{array}{c}0.84 \% \\
--- \\
10.40 \%\end{array}$ & $\begin{array}{c}0.90 \% \\
--- \\
6.58 \%\end{array}$ & $\begin{array}{c}1.15 \% \\
0.06 \% \\
11.87 \%\end{array}$ & $\begin{array}{l}0.66 \% \\
1.20 \% \\
1.61 \%\end{array}$ \\
\hline $\begin{array}{l}\text { Gibbsite } \\
\text { Boehmite } \\
\mathrm{HgO} \text { (co-precip. with } \mathrm{Ag}_{2} \mathrm{O} \text { ) }\end{array}$ & $\begin{array}{c}2.42 \\
3.01 \\
7.143 \\
\end{array}$ & $\begin{array}{l}--- \\
--- \\
-- \\
\end{array}$ & $\begin{array}{c}2.73 \% \\
--- \\
--- \\
\end{array}$ & $\begin{array}{l}-- \\
--- \\
---\end{array}$ & $\begin{array}{l}-- \\
--- \\
--- \\
\end{array}$ & $\begin{array}{c}75.98 \% \\
--- \\
0.01 \% \\
\end{array}$ & $\begin{array}{c}40.14 \% \\
--- \\
--- \\
\end{array}$ & $\begin{array}{c}74.26 \% \\
\cdots \\
0.01 \% \\
\end{array}$ & $\begin{array}{c}77.19 \% \\
--- \\
--- \\
\end{array}$ \\
\hline $\begin{array}{l}\mathrm{KAISiO}_{4} \\
\mathrm{La}(\mathrm{OH})_{3} \\
\mathrm{MnO}_{2}\end{array}$ & $\begin{array}{c}2.61 \\
2.3 \\
5.026\end{array}$ & $\begin{array}{l}3.16 \% \\
9.02 \% \\
18.33 \%\end{array}$ & $\begin{array}{l}1.58 \% \\
14.18 \% \\
17.12 \% \\
\end{array}$ & $\begin{array}{l}0.32 \% \\
14.92 \% \\
23.50 \%\end{array}$ & $\begin{array}{l}0.31 \% \\
13.51 \% \\
22.45 \%\end{array}$ & $\begin{array}{l}-- \\
0.03 \% \\
0.41 \%\end{array}$ & $\begin{array}{l}0.22 \% \\
0.02 \% \\
0.26 \%\end{array}$ & $\begin{array}{l}-- \\
0.03 \% \\
0.39 \%\end{array}$ & $\begin{array}{l}-- \\
0.04 \% \\
0.37 \%\end{array}$ \\
\hline $\begin{array}{l}\mathrm{Na}_{2} \mathrm{U}_{2} \mathrm{O}_{7} \\
\mathrm{NaAlSiO}_{4} \\
\mathrm{Ni}(\mathrm{OH})_{2} \\
\end{array}$ & $\begin{array}{r}5.617 \\
2.365 \\
4.15 \\
\end{array}$ & $\begin{array}{c}0.10 \% \\
--- \\
0.35 \% \\
\end{array}$ & $\begin{array}{c}0.37 \% \\
--- \\
0.28 \% \\
\end{array}$ & $\begin{array}{c}0.25 \% \\
--- \\
0.30 \% \\
\end{array}$ & $\begin{array}{c}0.21 \% \\
--- \\
0.35 \% \\
\end{array}$ & $\begin{array}{l}1.12 \% \\
9.14 \% \\
0.54 \% \\
\end{array}$ & $\begin{array}{c}0.05 \% \\
27.05 \% \\
0.36 \% \\
\end{array}$ & $\begin{array}{l}1.96 \% \\
8.19 \% \\
0.51 \% \\
\end{array}$ & $\begin{array}{l}16.24 \% \\
2.05 \% \\
0.05 \% \\
\end{array}$ \\
\hline $\begin{array}{l}\mathrm{Pb}(\mathrm{OH})_{2} \\
\mathrm{Pu}(\mathrm{OH})_{4} \text { (co-precip. on Fe phase) } \\
\mathrm{PuO}_{2}\end{array}$ & $\begin{array}{c}7.1 \\
4.26 \\
11.43 \\
\end{array}$ & $\begin{array}{c}0.17 \% \\
0.01 \% \\
\ldots \\
\end{array}$ & $\begin{array}{c}0.66 \% \\
0.00 \% \\
--- \\
\end{array}$ & $\begin{array}{c}0.30 \% \\
0.00 \% \\
--- \\
\end{array}$ & $\begin{array}{c}0.25 \% \\
0.00 \% \\
\ldots \\
\end{array}$ & $\begin{array}{c}0.45 \% \\
0.00 \% \\
--- \\
\end{array}$ & $\begin{array}{c}0.28 \% \\
0.00 \% \\
--- \\
\end{array}$ & $\begin{array}{c}0.48 \% \\
0.02 \% \\
--- \\
\end{array}$ & $\begin{array}{c}0.19 \% \\
0.00 \% \\
--- \\
\end{array}$ \\
\hline $\begin{array}{l}\mathrm{SiO}_{2} \\
\mathrm{Sr}(\mathrm{OH})_{2} \\
\mathrm{ZrO}_{2}\end{array}$ & $\begin{array}{c}2.6 \\
3.625 \\
5.68\end{array}$ & $\begin{array}{c}-- \\
0.61 \% \\
0.01 \%\end{array}$ & $\begin{array}{c}-- \\
0.58 \% \\
---\end{array}$ & $\begin{array}{c}--- \\
0.71 \% \\
0.00 \%\end{array}$ & $\begin{array}{c}--- \\
0.50 \% \\
0.00 \%\end{array}$ & $\begin{array}{c}--- \\
0.07 \% \\
0.42 \%\end{array}$ & $\begin{array}{c}23.46 \% \\
0.01 \% \\
0.28 \%\end{array}$ & $\begin{array}{c}--- \\
0.11 \% \\
0.39 \%\end{array}$ & $\begin{array}{c}--- \\
0.02 \% \\
0.23 \%\end{array}$ \\
\hline
\end{tabular}


Table C.3. (contd)

\begin{tabular}{|c|c|c|c|c|c|c|c|c|c|}
\hline & Density $(\mathrm{g} / \mathrm{mL})$ & 241-BX-105 & 241-BX-106 & 241-BX-107 & 241-BX-108 & 241-BX-109 & 241-BX-110 & 241-BX-111 & 241-BX-112 \\
\hline $\begin{array}{l}\text { Total kg washed solids for tank } \\
\text { Total kL washed solids for tank } \\
\text { Avg g/mL, washed solid }\end{array}$ & $\mathrm{n} / \mathrm{a}$ & $\begin{array}{c}6.28 \mathrm{E}+04 \\
2.21 \mathrm{E}+01 \\
2.838 \\
\end{array}$ & $\begin{array}{c}7.73 \mathrm{E}+04 \\
3.06 \mathrm{E}+01 \\
2.530 \\
\end{array}$ & $\begin{array}{c}1.93 \mathrm{E}+05 \\
5.76 \mathrm{E}+01 \\
3.352\end{array}$ & $\begin{array}{c}2.01 \mathrm{E}+04 \\
5.78 \mathrm{E}+00 \\
3.468\end{array}$ & $\begin{array}{c}7.58 \mathrm{E}+04 \\
1.90 \mathrm{E}+01 \\
3.985 \\
\end{array}$ & $\begin{array}{c}1.26 \mathrm{E}+05 \\
4.40 \mathrm{E}+01 \\
2.862\end{array}$ & $\begin{array}{c}5.89 \mathrm{E}+04 \\
2.12 \mathrm{E}+01 \\
2.785\end{array}$ & $\begin{array}{c}7.68 \mathrm{E}+04 \\
2.44 \mathrm{E}+01 \\
3.148\end{array}$ \\
\hline \multicolumn{10}{|l|}{ mass fracs of solids in washed solid } \\
\hline $\begin{array}{l}\mathrm{Ag}_{2} \mathrm{O} \\
\mathrm{Bi}_{2} \mathrm{O}_{3} \\
\mathrm{BiFeO}_{3} \\
\end{array}$ & $\begin{array}{c}7.143 \\
8.9 \\
7.9 \\
\end{array}$ & $\begin{array}{c}--- \\
0.64 \% \\
--- \\
\end{array}$ & $\begin{array}{c}0.01 \% \\
0.38 \% \\
--- \\
\end{array}$ & $\begin{array}{c}--- \\
--- \\
34.50 \% \\
\end{array}$ & $\begin{array}{c}--- \\
9.98 \% \\
--- \\
\end{array}$ & $\begin{array}{c}0.00 \% \\
0.57 \% \\
--- \\
\end{array}$ & $\begin{array}{c}--- \\
10.82 \% \\
--- \\
\end{array}$ & $\begin{array}{c}--- \\
--- \\
10.12 \% \\
\end{array}$ & $\begin{array}{c}0.04 \% \\
--- \\
28.14 \% \\
\end{array}$ \\
\hline $\begin{array}{l}\mathrm{Ca}(\mathrm{OH})_{2} \\
\mathrm{CrOOH} \\
\mathrm{FeOOH}\end{array}$ & $\begin{array}{l}2.24 \\
4.11 \\
4.26\end{array}$ & $\begin{array}{c}5.26 \% \\
10.54 \% \\
7.49 \%\end{array}$ & $\begin{array}{l}5.22 \% \\
4.79 \% \\
4.30 \%\end{array}$ & $\begin{array}{c}1.83 \% \\
--- \\
6.89 \% \\
\end{array}$ & $\begin{array}{c}5.22 \% \\
--- \\
35.74 \%\end{array}$ & $\begin{array}{c}7.37 \% \\
--- \\
49.81 \%\end{array}$ & $\begin{array}{l}1.39 \% \\
6.69 \% \\
8.81 \%\end{array}$ & $\begin{array}{l}0.73 \% \\
6.64 \% \\
6.01 \%\end{array}$ & $\begin{array}{l}2.29 \% \\
2.11 \% \\
6.13 \%\end{array}$ \\
\hline $\begin{array}{l}\text { Gibbsite } \\
\text { Boehmite } \\
\mathrm{HgO} \text { (co-precip. with } \mathrm{Ag}_{2} \mathrm{O} \text { ) }\end{array}$ & $\begin{array}{c}2.42 \\
3.01 \\
7.143 \\
\end{array}$ & $\begin{array}{c}-- \\
--- \\
0.03 \%\end{array}$ & $\begin{array}{l}--- \\
--- \\
0.02 \%\end{array}$ & $\begin{array}{c}22.08 \% \\
--- \\
--- \\
\end{array}$ & $\begin{array}{c}15.85 \% \\
--- \\
0.11 \% \\
\end{array}$ & $\begin{array}{c}6.33 \% \\
--- \\
0.17 \% \\
\end{array}$ & $\begin{array}{c}51.69 \% \\
--- \\
--- \\
\end{array}$ & $\begin{array}{c}58.68 \% \\
--- \\
--- \\
\end{array}$ & $\begin{array}{c}11.47 \% \\
--- \\
--- \\
\end{array}$ \\
\hline $\begin{array}{l}\mathrm{KAISiO}_{4} \\
\mathrm{La}(\mathrm{OH})_{3} \\
\mathrm{MnO}_{2}\end{array}$ & $\begin{array}{c}2.61 \\
2.3 \\
5.026\end{array}$ & $\begin{array}{l}0.26 \% \\
0.29 \% \\
1.83 \%\end{array}$ & $\begin{array}{l}1.04 \% \\
0.05 \% \\
1.05 \%\end{array}$ & $\begin{array}{c}-- \\
0.00 \% \\
0.10 \%\end{array}$ & $\begin{array}{c}-- \\
0.00 \% \\
0.26 \%\end{array}$ & $\begin{array}{c}--- \\
--- \\
0.33 \%\end{array}$ & $\begin{array}{c}1.40 \% \\
0.00 \% \\
---\end{array}$ & $\begin{array}{c}-- \\
0.00 \% \\
---\end{array}$ & $\begin{array}{c}-- \\
0.00 \% \\
0.53 \%\end{array}$ \\
\hline $\begin{array}{l}\mathrm{Na}_{2} \mathrm{U}_{2} \mathrm{O}_{7} \\
\mathrm{NaAlSiO}_{4} \\
\mathrm{Ni}(\mathrm{OH})_{2} \\
\end{array}$ & $\begin{array}{r}5.617 \\
2.365 \\
4.15 \\
\end{array}$ & $\begin{array}{c}12.16 \% \\
60.43 \% \\
0.18 \% \\
\end{array}$ & $\begin{array}{c}2.62 \% \\
79.87 \% \\
0.11 \% \\
\end{array}$ & $\begin{array}{c}2.65 \% \\
31.47 \% \\
0.02 \% \\
\end{array}$ & $\begin{array}{l}13.04 \% \\
18.25 \% \\
0.12 \% \\
\end{array}$ & $\begin{array}{c}29.03 \% \\
3.87 \% \\
0.18 \% \\
\end{array}$ & $\begin{array}{c}1.90 \% \\
16.60 \% \\
0.19 \% \\
\end{array}$ & $\begin{array}{c}1.67 \% \\
15.73 \% \\
0.04 \% \\
\end{array}$ & $\begin{array}{c}1.50 \% \\
47.33 \% \\
0.00 \% \\
\end{array}$ \\
\hline $\begin{array}{l}\mathrm{Pb}(\mathrm{OH})_{2} \\
\mathrm{Pu}(\mathrm{OH})_{4} \text { (co-precip. on Fe phase) } \\
\mathrm{PuO}_{2}\end{array}$ & $\begin{array}{c}7.1 \\
4.26 \\
11.43 \\
\end{array}$ & $\begin{array}{c}0.49 \% \\
0.00 \% \\
\quad-. \\
\end{array}$ & $\begin{array}{c}0.30 \% \\
0.00 \% \\
---\end{array}$ & $\begin{array}{c}0.07 \% \\
0.00 \% \\
--- \\
\end{array}$ & $\begin{array}{c}0.59 \% \\
0.00 \% \\
\ldots \\
\end{array}$ & $\begin{array}{c}1.14 \% \\
0.00 \% \\
--- \\
\end{array}$ & $\begin{array}{c}0.19 \% \\
0.00 \% \\
--- \\
\end{array}$ & $\begin{array}{c}0.26 \% \\
0.00 \% \\
\ldots \\
-. \\
\end{array}$ & $\begin{array}{c}0.19 \% \\
0.00 \% \\
--- \\
\end{array}$ \\
\hline $\begin{array}{l}\mathrm{SiO}_{2} \\
\mathrm{Sr}(\mathrm{OH})_{2} \\
\mathrm{ZrO}_{2}\end{array}$ & $\begin{array}{c}2.6 \\
3.625 \\
5.68\end{array}$ & $\begin{array}{c}-- \\
0.05 \% \\
0.35 \%\end{array}$ & $\begin{array}{c}-- \\
0.03 \% \\
0.20 \%\end{array}$ & $\begin{array}{c}--- \\
0.22 \% \\
0.17 \%\end{array}$ & $\begin{array}{c}--- \\
0.75 \% \\
0.08 \%\end{array}$ & $\begin{array}{c}-- \\
1.19 \% \\
---\end{array}$ & $\begin{array}{c}--- \\
0.21 \% \\
0.10 \%\end{array}$ & $\begin{array}{c}--- \\
0.09 \% \\
0.04 \%\end{array}$ & $\begin{array}{c}--- \\
0.18 \% \\
0.08 \%\end{array}$ \\
\hline
\end{tabular}


Table C.3. (contd)

\begin{tabular}{|c|c|c|c|c|c|c|c|c|c|}
\hline & Density $(\mathrm{g} / \mathrm{mL})$ & 241-BY-101 & 241-BY-102 & 241-BY-103 & 241-BY-104 & 241-BY-105 & 241-BY-106 & 241-BY-107 & 241-BY-108 \\
\hline $\begin{array}{l}\text { Total kg washed solids for tank } \\
\text { Total kL washed solids for tank } \\
\text { Avg g/mL, washed solid }\end{array}$ & $\mathrm{n} / \mathrm{a}$ & $\begin{array}{c}1.76 \mathrm{E}+05 \\
6.72 \mathrm{E}+01 \\
2.620\end{array}$ & $\begin{array}{c}6.79 \mathrm{E}+04 \\
2.63 \mathrm{E}+01 \\
2.581 \\
\end{array}$ & $\begin{array}{c}2.55 \mathrm{E}+05 \\
1.02 \mathrm{E}+02 \\
2.500\end{array}$ & $\begin{array}{c}2.39 \mathrm{E}+05 \\
8.86 \mathrm{E}+01 \\
2.697\end{array}$ & $\begin{array}{c}4.06 \mathrm{E}+05 \\
1.62 \mathrm{E}+02 \\
2.503 \\
\end{array}$ & $\begin{array}{c}1.70 \mathrm{E}+05 \\
6.32 \mathrm{E}+01 \\
2.691\end{array}$ & $\begin{array}{c}1.41 \mathrm{E}+05 \\
5.53 \mathrm{E}+01 \\
2.554\end{array}$ & $\begin{array}{c}2.75 \mathrm{E}+05 \\
1.04 \mathrm{E}+02 \\
2.635\end{array}$ \\
\hline \multicolumn{10}{|l|}{ mass fracs of solids in washed solid } \\
\hline $\begin{array}{l}\mathrm{Ag}_{2} \mathrm{O} \\
\mathrm{Bi}_{2} \mathrm{O}_{3} \\
\mathrm{BiFeO}_{3} \\
\end{array}$ & $\begin{array}{c}7.143 \\
8.9 \\
7.9 \\
\end{array}$ & $\begin{array}{c}--- \\
0.10 \% \\
--- \\
\end{array}$ & $\begin{array}{l}--- \\
--- \\
--- \\
\end{array}$ & $\begin{array}{c}--- \\
0.15 \% \\
--- \\
\end{array}$ & $\begin{array}{c}--- \\
0.33 \% \\
--- \\
\end{array}$ & $\begin{array}{c}0.01 \% \\
0.28 \% \\
--- \\
\end{array}$ & $\begin{array}{c}--- \\
0.32 \% \\
--- \\
\end{array}$ & $\begin{array}{c}--- \\
0.20 \% \\
--- \\
\end{array}$ & $\begin{array}{c}--- \\
0.16 \% \\
--- \\
\end{array}$ \\
\hline $\begin{array}{l}\mathrm{Ca}(\mathrm{OH})_{2} \\
\mathrm{CrOOH} \\
\mathrm{FeOOH}\end{array}$ & $\begin{array}{l}2.24 \\
4.11 \\
4.26\end{array}$ & $\begin{array}{l}5.35 \% \\
2.90 \% \\
5.49 \%\end{array}$ & $\begin{array}{l}2.27 \% \\
4.99 \% \\
3.54 \%\end{array}$ & $\begin{array}{l}1.14 \% \\
2.92 \% \\
2.65 \%\end{array}$ & $\begin{array}{l}2.46 \% \\
3.16 \% \\
8.27 \%\end{array}$ & $\begin{array}{l}19.13 \% \\
0.49 \% \\
4.59 \%\end{array}$ & $\begin{array}{l}2.79 \% \\
2.15 \% \\
6.96 \%\end{array}$ & $\begin{array}{l}1.94 \% \\
0.06 \% \\
5.15 \%\end{array}$ & $\begin{array}{l}3.39 \% \\
0.17 \% \\
6.03 \%\end{array}$ \\
\hline $\begin{array}{l}\text { Gibbsite } \\
\text { Boehmite } \\
\mathrm{HgO} \text { (co-precip. with } \mathrm{Ag}_{2} \mathrm{O} \text { ) }\end{array}$ & $\begin{array}{c}2.42 \\
3.01 \\
7.143 \\
\end{array}$ & $\begin{array}{c}47.58 \% \\
--- \\
---\end{array}$ & $\begin{array}{c}67.53 \% \\
--- \\
--- \\
\end{array}$ & $\begin{array}{c}84.54 \% \\
--- \\
--- \\
\end{array}$ & $\begin{array}{c}71.38 \% \\
--- \\
0.00 \% \\
\end{array}$ & $\begin{array}{c}37.99 \% \\
--- \\
0.01 \% \\
\end{array}$ & $\begin{array}{c}72.58 \% \\
--- \\
--- \\
\end{array}$ & $\begin{array}{c}81.15 \% \\
--- \\
--- \\
\end{array}$ & $\begin{array}{c}76.96 \% \\
--- \\
--- \\
\end{array}$ \\
\hline $\begin{array}{l}\mathrm{KAISiO}_{4} \\
\mathrm{La}(\mathrm{OH})_{3} \\
\mathrm{MnO}_{2}\end{array}$ & $\begin{array}{c}2.61 \\
2.3 \\
5.026\end{array}$ & $\begin{array}{c}5.59 \% \\
0.01 \% \\
---\end{array}$ & $\begin{array}{c}18.76 \% \\
0.00 \% \\
---\end{array}$ & $\begin{array}{c}1.96 \% \\
0.00 \% \\
---\end{array}$ & $\begin{array}{c}-- \\
0.04 \% \\
0.11 \%\end{array}$ & $\begin{array}{l}-- \\
0.00 \% \\
0.07 \%\end{array}$ & $\begin{array}{c}-- \\
0.01 \% \\
---\end{array}$ & $\begin{array}{c}-- \\
0.00 \% \\
---\end{array}$ & $\begin{array}{c}-- \\
0.01 \% \\
---\end{array}$ \\
\hline $\begin{array}{l}\mathrm{Na}_{2} \mathrm{U}_{2} \mathrm{O}_{7} \\
\mathrm{NaAlSiO}_{4} \\
\mathrm{Ni}(\mathrm{OH})_{2} \\
\end{array}$ & $\begin{array}{r}5.617 \\
2.365 \\
4.15 \\
\end{array}$ & $\begin{array}{c}5.43 \% \\
24.19 \% \\
2.59 \% \\
\end{array}$ & $\begin{array}{c}1.56 \% \\
--- \\
0.65 \% \\
\end{array}$ & $\begin{array}{l}0.75 \% \\
5.05 \% \\
0.38 \% \\
\end{array}$ & $\begin{array}{l}5.67 \% \\
2.87 \% \\
2.06 \% \\
\end{array}$ & $\begin{array}{c}3.35 \% \\
30.87 \% \\
1.23 \% \\
\end{array}$ & $\begin{array}{l}5.03 \% \\
1.92 \% \\
5.80 \% \\
\end{array}$ & $\begin{array}{l}4.04 \% \\
5.34 \% \\
0.93 \% \\
\end{array}$ & $\begin{array}{l}6.78 \% \\
1.74 \% \\
1.82 \% \\
\end{array}$ \\
\hline $\begin{array}{l}\mathrm{Pb}(\mathrm{OH})_{2} \\
\mathrm{Pu}(\mathrm{OH})_{4} \text { (co-precip. on Fe phase) } \\
\mathrm{PuO}_{2}\end{array}$ & $\begin{array}{c}7.1 \\
4.26 \\
11.43 \\
\end{array}$ & $\begin{array}{c}0.48 \% \\
0.00 \% \\
\quad-. \\
\end{array}$ & $\begin{array}{c}0.28 \% \\
0.00 \% \\
--- \\
\end{array}$ & $\begin{array}{c}0.19 \% \\
0.00 \% \\
--- \\
\end{array}$ & $\begin{array}{c}0.25 \% \\
0.00 \% \\
\ldots \\
\end{array}$ & $\begin{array}{c}0.13 \% \\
0.00 \% \\
--- \\
\end{array}$ & $\begin{array}{c}0.36 \% \\
0.00 \% \\
--- \\
\end{array}$ & $\begin{array}{c}0.20 \% \\
0.00 \% \\
\ldots- \\
\end{array}$ & $\begin{array}{c}0.23 \% \\
0.00 \% \\
--- \\
\end{array}$ \\
\hline $\begin{array}{l}\mathrm{SiO}_{2} \\
\mathrm{Sr}(\mathrm{OH})_{2} \\
\mathrm{ZrO}_{2}\end{array}$ & $\begin{array}{c}2.6 \\
3.625 \\
5.68\end{array}$ & $\begin{array}{c}-- \\
0.28 \% \\
0.02 \%\end{array}$ & $\begin{array}{c}--- \\
0.40 \% \\
0.01 \%\end{array}$ & $\begin{array}{c}--- \\
0.19 \% \\
0.06 \%\end{array}$ & $\begin{array}{c}--- \\
3.36 \% \\
0.01 \%\end{array}$ & $\begin{array}{c}--- \\
1.83 \% \\
0.01 \%\end{array}$ & $\begin{array}{c}--- \\
2.07 \% \\
0.01 \%\end{array}$ & $\begin{array}{c}-- \\
1.00 \% \\
---\end{array}$ & $\begin{array}{c}--- \\
2.71 \% \\
0.01 \%\end{array}$ \\
\hline
\end{tabular}


Table C.3. (contd)

\begin{tabular}{|c|c|c|c|c|c|c|c|c|c|}
\hline & Density $(\mathrm{g} / \mathrm{mL})$ & 241-BY-109 & 241-BY-110 & 241-BY-111 & 241-BY-112 & 241-C-101 & 241-C-102 & 241-C-103 & 241-C-104 \\
\hline $\begin{array}{l}\text { Total kg washed solids for tank } \\
\text { Total } \mathrm{kL} \text { washed solids for tank } \\
\text { Avg g/mL, washed solid }\end{array}$ & $\mathrm{n} / \mathrm{a}$ & $\begin{array}{c}1.95 \mathrm{E}+05 \\
7.95 \mathrm{E}+01 \\
2.450\end{array}$ & $\begin{array}{c}1.68 \mathrm{E}+05 \\
5.95 \mathrm{E}+01 \\
2.828\end{array}$ & $\begin{array}{c}2.79 \mathrm{E}+05 \\
1.14 \mathrm{E}+02 \\
2.444\end{array}$ & $\begin{array}{c}1.31 \mathrm{E}+05 \\
4.37 \mathrm{E}+01 \\
3.004\end{array}$ & $\begin{array}{c}3.01 \mathrm{E}+05 \\
1.15 \mathrm{E}+02 \\
2.615\end{array}$ & $\begin{array}{c}3.07 \mathrm{E}+05 \\
1.18 \mathrm{E}+02 \\
2.599\end{array}$ & $\begin{array}{c}5.68 \mathrm{E}+05 \\
2.25 \mathrm{E}+02 \\
2.520\end{array}$ & $\begin{array}{c}4.24 \mathrm{E}+05 \\
1.36 \mathrm{E}+02 \\
3.119\end{array}$ \\
\hline \multicolumn{10}{|l|}{ mass fracs of solids in washed solid } \\
\hline $\begin{array}{l}\mathrm{Ag}_{2} \mathrm{O} \\
\mathrm{Bi}_{2} \mathrm{O}_{3} \\
\mathrm{BiFeO}_{3}\end{array}$ & $\begin{array}{c}7.143 \\
8.9 \\
7.9 \\
\end{array}$ & $\begin{array}{c}0.01 \% \\
0.05 \% \\
--- \\
\end{array}$ & $\begin{array}{c}-- \\
0.42 \% \\
--- \\
\end{array}$ & $\begin{array}{c}-- \\
0.05 \% \\
--- \\
\end{array}$ & $\begin{array}{l}-\cdot \\
\cdots- \\
--- \\
\end{array}$ & $\begin{array}{c}-- \\
0.27 \% \\
--- \\
\end{array}$ & $\begin{array}{c}0.04 \% \\
0.73 \% \\
--- \\
\end{array}$ & $\begin{array}{c}-- \\
0.08 \% \\
--- \\
\end{array}$ & $\begin{array}{c}0.25 \% \\
0.01 \% \\
--- \\
\end{array}$ \\
\hline $\begin{array}{l}\mathrm{Ca}(\mathrm{OH})_{2} \\
\mathrm{CrOOH} \\
\mathrm{FeOOH}\end{array}$ & $\begin{array}{l}2.24 \\
4.11 \\
4.26\end{array}$ & $\begin{array}{l}0.38 \% \\
1.64 \% \\
1.06 \%\end{array}$ & $\begin{array}{l}8.20 \% \\
3.69 \% \\
11.77 \%\end{array}$ & $\begin{array}{l}0.69 \% \\
0.58 \% \\
5.70 \%\end{array}$ & $\begin{array}{c}1.53 \% \\
37.41 \% \\
6.81 \%\end{array}$ & $\begin{array}{c}2.67 \% \\
--- \\
9.91 \%\end{array}$ & $\begin{array}{c}3.14 \% \\
--- \\
8.02 \%\end{array}$ & $\begin{array}{l}0.98 \% \\
0.23 \% \\
3.61 \%\end{array}$ & $\begin{array}{l}1.20 \% \\
--- \\
9.52 \%\end{array}$ \\
\hline $\begin{array}{l}\text { Gibbsite } \\
\text { Boehmite } \\
\mathrm{HgO} \text { (co-precip. with } \mathrm{Ag}_{2} \mathrm{O} \text { ) }\end{array}$ & $\begin{array}{c}2.42 \\
3.01 \\
7.143 \\
\end{array}$ & $\begin{array}{c}79.25 \% \\
--- \\
---\end{array}$ & $\begin{array}{c}55.18 \% \\
--- \\
--- \\
\end{array}$ & $\begin{array}{l}-- \\
--- \\
---\end{array}$ & $\begin{array}{c}34.27 \% \\
\cdots- \\
\cdots- \\
\end{array}$ & $\begin{array}{c}64.87 \% \\
--- \\
0.02 \%\end{array}$ & $\begin{array}{c}34.58 \% \\
--- \\
--- \\
\end{array}$ & $\begin{array}{c}68.85 \% \\
\cdots- \\
0.03 \% \\
\end{array}$ & $\begin{array}{c}45.52 \% \\
--- \\
0.01 \% \\
\end{array}$ \\
\hline $\begin{array}{l}\mathrm{KAlSiO}_{4} \\
\mathrm{La}(\mathrm{OH})_{3} \\
\mathrm{MnO}_{2}\end{array}$ & $\begin{array}{c}2.61 \\
2.3 \\
5.026\end{array}$ & $\begin{array}{c}-- \\
0.00 \% \\
0.08 \%\end{array}$ & $\begin{array}{c}--- \\
0.01 \% \\
---\end{array}$ & $\begin{array}{c}-- \\
0.00 \% \\
---\end{array}$ & $\begin{array}{c}--- \\
0.00 \% \\
---\end{array}$ & $\begin{array}{l}-- \\
0.11 \% \\
0.28 \%\end{array}$ & $\begin{array}{l}-- \\
0.07 \% \\
0.64 \%\end{array}$ & $\begin{array}{l}-- \\
0.04 \% \\
0.11 \%\end{array}$ & $\begin{array}{l}-- \\
0.01 \% \\
2.39 \%\end{array}$ \\
\hline $\begin{array}{l}\mathrm{Na}_{2} \mathrm{U}_{2} \mathrm{O}_{7} \\
\mathrm{NaAlSiO}_{4} \\
\mathrm{Ni}(\mathrm{OH})_{2} \\
\end{array}$ & $\begin{array}{r}5.617 \\
2.365 \\
4.15 \\
\end{array}$ & $\begin{array}{c}0.42 \% \\
16.74 \% \\
0.17 \% \\
\end{array}$ & $\begin{array}{l}8.82 \% \\
4.01 \% \\
4.21 \% \\
\end{array}$ & $\begin{array}{c}0.47 \% \\
92.10 \% \\
0.20 \% \\
\end{array}$ & $\begin{array}{c}2.18 \% \\
16.90 \% \\
0.43 \% \\
\end{array}$ & $\begin{array}{c}4.98 \% \\
15.76 \% \\
0.52 \% \\
\end{array}$ & $\begin{array}{c}2.64 \% \\
45.35 \% \\
2.65 \% \\
\end{array}$ & $\begin{array}{c}1.61 \% \\
20.95 \% \\
1.10 \% \\
\end{array}$ & $\begin{array}{l}10.19 \% \\
10.76 \% \\
0.90 \% \\
\end{array}$ \\
\hline $\begin{array}{l}\mathrm{Pb}(\mathrm{OH})_{2} \\
\mathrm{Pu}(\mathrm{OH})_{4} \text { (co-precip. on Fe phase) } \\
\mathrm{PuO}_{2}\end{array}$ & $\begin{array}{c}7.1 \\
4.26 \\
11.43 \\
\end{array}$ & $\begin{array}{c}0.08 \% \\
0.00 \% \\
\quad-. \\
\end{array}$ & $\begin{array}{c}0.81 \% \\
0.00 \% \\
---\end{array}$ & $\begin{array}{c}0.08 \% \\
0.00 \% \\
--- \\
\end{array}$ & $\begin{array}{c}0.18 \% \\
0.00 \% \\
\ldots \\
\end{array}$ & $\begin{array}{c}0.35 \% \\
0.00 \% \\
--- \\
\end{array}$ & $\begin{array}{c}0.32 \% \\
0.02 \% \\
--- \\
\end{array}$ & $\begin{array}{c}0.11 \% \\
0.02 \% \\
\ldots \\
-. \\
\end{array}$ & $\begin{array}{c}0.21 \% \\
0.03 \% \\
--- \\
\end{array}$ \\
\hline $\begin{array}{l}\mathrm{SiO}_{2} \\
\mathrm{Sr}(\mathrm{OH})_{2} \\
\mathrm{ZrO}_{2}\end{array}$ & $\begin{array}{c}2.6 \\
3.625 \\
5.68\end{array}$ & $\begin{array}{c}-- \\
0.09 \% \\
0.04 \%\end{array}$ & $\begin{array}{c}-- \\
2.85 \% \\
0.02 \%\end{array}$ & $\begin{array}{c}--- \\
0.12 \% \\
0.00 \%\end{array}$ & $\begin{array}{c}--- \\
0.27 \% \\
0.02 \%\end{array}$ & $\begin{array}{c}--- \\
0.15 \% \\
0.10 \%\end{array}$ & $\begin{array}{c}--- \\
0.05 \% \\
1.74 \%\end{array}$ & $\begin{array}{c}--- \\
0.01 \% \\
2.26 \%\end{array}$ & $\begin{array}{c}--- \\
0.03 \% \\
19.00 \%\end{array}$ \\
\hline
\end{tabular}


Table C.3. (contd)

\begin{tabular}{|c|c|c|c|c|c|c|c|c|c|}
\hline & Density $(\mathrm{g} / \mathrm{mL})$ & 241-C-105 & 241-C-106 & 241-C-107 & 241-C-108 & 241-C-109 & $241-C-110$ & 241-C-111 & 241-C-112 \\
\hline $\begin{array}{l}\text { Total kg washed solids for tank } \\
\text { Total kL washed solids for tank } \\
\text { Avg g/mL, washed solid }\end{array}$ & $\mathrm{n} / \mathrm{a}$ & $\begin{array}{c}6.44 \mathrm{E}+05 \\
2.61 \mathrm{E}+02 \\
2.463\end{array}$ & $\begin{array}{c}1.33 \mathrm{E}+04 \\
4.18 \mathrm{E}+00 \\
3.179\end{array}$ & $\begin{array}{c}3.56 \mathrm{E}+05 \\
1.09 \mathrm{E}+02 \\
3.265\end{array}$ & $\begin{array}{c}8.16 \mathrm{E}+04 \\
3.09 \mathrm{E}+01 \\
2.644\end{array}$ & $\begin{array}{c}1.23 \mathrm{E}+05 \\
4.54 \mathrm{E}+01 \\
2.700\end{array}$ & $\begin{array}{c}8.96 \mathrm{E}+04 \\
2.84 \mathrm{E}+01 \\
3.151\end{array}$ & $\begin{array}{c}1.56 \mathrm{E}+05 \\
5.61 \mathrm{E}+01 \\
2.784\end{array}$ & $\begin{array}{c}1.21 \mathrm{E}+05 \\
3.72 \mathrm{E}+01 \\
3.262\end{array}$ \\
\hline \multicolumn{10}{|l|}{ mass fracs of solids in washed solid } \\
\hline $\begin{array}{l}\mathrm{Ag}_{2} \mathrm{O} \\
\mathrm{Bi}_{2} \mathrm{O}_{3} \\
\mathrm{BiFeO}_{3}\end{array}$ & $\begin{array}{c}7.143 \\
8.9 \\
7.9 \\
\end{array}$ & $\begin{array}{c}0.01 \% \\
0.07 \% \\
--- \\
\end{array}$ & $\begin{array}{c}0.23 \% \\
0.01 \% \\
--- \\
\end{array}$ & $\begin{array}{c}0.08 \% \\
--- \\
4.01 \% \\
\end{array}$ & $\begin{array}{c}--- \\
5.97 \% \\
--- \\
\end{array}$ & $\begin{array}{c}--- \\
1.17 \% \\
--- \\
\end{array}$ & $\begin{array}{c}0.00 \% \\
--- \\
24.88 \% \\
\end{array}$ & $\begin{array}{c}--- \\
1.34 \% \\
--- \\
\end{array}$ & $\begin{array}{c}0.00 \% \\
1.73 \% \\
--- \\
\end{array}$ \\
\hline $\begin{array}{l}\mathrm{Ca}(\mathrm{OH})_{2} \\
\mathrm{CrOOH} \\
\mathrm{FeOOH}\end{array}$ & $\begin{array}{l}2.24 \\
4.11 \\
4.26\end{array}$ & $\begin{array}{c}0.63 \% \\
--- \\
0.78 \%\end{array}$ & $\begin{array}{c}1.19 \% \\
0.72 \% \\
38.00 \%\end{array}$ & $\begin{array}{c}0.59 \% \\
--- \\
41.03 \%\end{array}$ & $\begin{array}{c}10.30 \% \\
--- \\
5.02 \%\end{array}$ & $\begin{array}{l}9.62 \% \\
0.15 \% \\
9.57 \%\end{array}$ & $\begin{array}{c}2.18 \% \\
0.65 \% \\
10.52 \%\end{array}$ & $\begin{array}{c}5.59 \% \\
--- \\
16.00 \%\end{array}$ & $\begin{array}{c}16.44 \% \\
0.12 \% \\
16.57 \%\end{array}$ \\
\hline $\begin{array}{l}\text { Gibbsite } \\
\text { Boehmite } \\
\mathrm{HgO} \text { (co-precip. with } \mathrm{Ag}_{2} \mathrm{O} \text { ) }\end{array}$ & $\begin{array}{c}2.42 \\
3.01 \\
7.143 \\
\end{array}$ & $\begin{array}{c}74.85 \% \\
--- \\
0.00 \% \\
\end{array}$ & $\begin{array}{c}46.03 \% \\
--- \\
0.00 \% \\
\end{array}$ & $\begin{array}{c}42.35 \% \\
--- \\
0.01 \% \\
\end{array}$ & $\begin{array}{c}59.75 \% \\
--- \\
0.02 \% \\
\end{array}$ & $\begin{array}{c}56.98 \% \\
--- \\
--- \\
\end{array}$ & $\begin{array}{c}23.63 \% \\
--- \\
--- \\
\end{array}$ & $\begin{array}{c}59.06 \% \\
--- \\
--- \\
\end{array}$ & $\begin{array}{c}24.28 \% \\
--- \\
---\end{array}$ \\
\hline $\begin{array}{l}\mathrm{KAlSiO}_{4} \\
\mathrm{La}(\mathrm{OH})_{3} \\
\mathrm{MnO}_{2}\end{array}$ & $\begin{array}{c}2.61 \\
2.3 \\
5.026\end{array}$ & $\begin{array}{l}0.26 \% \\
0.09 \% \\
0.52 \%\end{array}$ & $\begin{array}{l}-- \\
0.13 \% \\
9.37 \%\end{array}$ & $\begin{array}{c}-- \\
0.10 \% \\
2.04 \%\end{array}$ & $\begin{array}{c}-- \\
0.01 \% \\
0.11 \%\end{array}$ & $\begin{array}{l}-- \\
0.00 \% \\
0.11 \%\end{array}$ & $\begin{array}{c}-- \\
0.00 \% \\
0.08 \%\end{array}$ & $\begin{array}{l}-- \\
0.11 \% \\
0.10 \%\end{array}$ & $\begin{array}{l}-- \\
0.05 \% \\
0.15 \%\end{array}$ \\
\hline $\begin{array}{l}\mathrm{Na}_{2} \mathrm{U}_{2} \mathrm{O}_{7} \\
\mathrm{NaAlSiO}_{4} \\
\mathrm{Ni}(\mathrm{OH})_{2} \\
\end{array}$ & $\begin{array}{r}5.617 \\
2.365 \\
4.15 \\
\end{array}$ & $\begin{array}{c}2.28 \% \\
19.88 \% \\
0.46 \% \\
\end{array}$ & $\begin{array}{l}1.24 \% \\
0.21 \% \\
1.31 \% \\
\end{array}$ & $\begin{array}{l}3.16 \% \\
2.22 \% \\
1.26 \% \\
\end{array}$ & $\begin{array}{c}0.24 \% \\
11.99 \% \\
5.85 \% \\
\end{array}$ & $\begin{array}{l}4.98 \% \\
9.71 \% \\
6.65 \% \\
\end{array}$ & $\begin{array}{c}2.93 \% \\
34.39 \% \\
0.04 \% \\
\end{array}$ & $\begin{array}{l}4.28 \% \\
6.32 \% \\
5.84 \% \\
\end{array}$ & $\begin{array}{c}24.39 \% \\
5.41 \% \\
9.35 \% \\
\end{array}$ \\
\hline $\begin{array}{l}\mathrm{Pb}(\mathrm{OH})_{2} \\
\mathrm{Pu}(\mathrm{OH})_{4} \text { (co-precip. on Fe phase) } \\
\mathrm{PuO}_{2}\end{array}$ & $\begin{array}{c}7.1 \\
4.26 \\
11.43 \\
\end{array}$ & $\begin{array}{c}0.07 \% \\
0.01 \% \\
\quad-. \\
\end{array}$ & $\begin{array}{c}.46 \% \\
0.01 \% \\
--- \\
\end{array}$ & $\begin{array}{c}3.03 \% \\
0.01 \% \\
--- \\
\end{array}$ & $\begin{array}{c}0.44 \% \\
0.00 \% \\
\ldots \\
\end{array}$ & $\begin{array}{c}0.89 \% \\
0.00 \% \\
--- \\
\end{array}$ & $\begin{array}{c}0.29 \% \\
0.00 \% \\
--- \\
\end{array}$ & $\begin{array}{c}1.23 \% \\
0.00 \% \\
\ldots \\
-.\end{array}$ & $\begin{array}{c}1.31 \% \\
0.00 \% \\
\cdots \\
--\end{array}$ \\
\hline $\begin{array}{l}\mathrm{SiO}_{2} \\
\mathrm{Sr}(\mathrm{OH})_{2} \\
\mathrm{ZrO}_{2}\end{array}$ & $\begin{array}{c}2.6 \\
3.625 \\
5.68\end{array}$ & $\begin{array}{c}-- \\
0.03 \% \\
0.04 \%\end{array}$ & $\begin{array}{c}--- \\
0.07 \% \\
0.03 \%\end{array}$ & $\begin{array}{c}--- \\
0.09 \% \\
0.03 \%\end{array}$ & $\begin{array}{c}--- \\
0.25 \% \\
0.05 \%\end{array}$ & $\begin{array}{c}--- \\
0.16 \% \\
0.01 \%\end{array}$ & $\begin{array}{c}--- \\
0.17 \% \\
0.23 \%\end{array}$ & $\begin{array}{c}--- \\
0.06 \% \\
0.05 \%\end{array}$ & $\begin{array}{c}--- \\
0.20 \% \\
0.01 \%\end{array}$ \\
\hline
\end{tabular}


Table C.3. (contd)

\begin{tabular}{|c|c|c|c|c|c|c|c|c|c|}
\hline & Density $(\mathrm{g} / \mathrm{mL})$ & 241-C-201 & 241-C-202 & 241-C-203 & 241-C-204 & 241-S-101 & 241-S-102 & $241-S-103$ & 241-S-104 \\
\hline $\begin{array}{l}\text { Total kg washed solids for tank } \\
\text { Total kL washed solids for tank } \\
\text { Avg g/mL, washed solid }\end{array}$ & $\mathrm{n} / \mathrm{a}$ & $\begin{array}{c}5.63 \mathrm{E}+03 \\
1.28 \mathrm{E}+00 \\
4.392 \\
\end{array}$ & $\begin{array}{c}4.42 \mathrm{E}+03 \\
9.90 \mathrm{E}-01 \\
4.465\end{array}$ & $\begin{array}{c}1.16 \mathrm{E}+04 \\
2.59 \mathrm{E}+00 \\
4.466\end{array}$ & $\begin{array}{c}4.61 \mathrm{E}+03 \\
1.04 \mathrm{E}+00 \\
4.448\end{array}$ & $\begin{array}{c}3.64 \mathrm{E}+05 \\
1.17 \mathrm{E}+02 \\
3.111\end{array}$ & $\begin{array}{c}1.07 \mathrm{E}+05 \\
4.07 \mathrm{E}+01 \\
2.627\end{array}$ & $\begin{array}{c}9.98 \mathrm{E}+04 \\
3.87 \mathrm{E}+01 \\
2.575 \\
\end{array}$ & $\begin{array}{c}4.53 \mathrm{E}+05 \\
1.49 \mathrm{E}+02 \\
3.036\end{array}$ \\
\hline \multicolumn{10}{|l|}{ mass fracs of solids in washed solid } \\
\hline $\begin{array}{l}\mathrm{Ag}_{2} \mathrm{O} \\
\mathrm{Bi}_{2} \mathrm{O}_{3} \\
\mathrm{BiFeO}_{3}\end{array}$ & $\begin{array}{c}7.143 \\
8.9 \\
7.9 \\
\end{array}$ & $\begin{array}{l}--- \\
--- \\
--- \\
\end{array}$ & $\begin{array}{l}--- \\
--- \\
--- \\
\end{array}$ & $\begin{array}{l}-- \\
--- \\
--- \\
\end{array}$ & $\begin{array}{l}-\cdots \\
--- \\
--- \\
\end{array}$ & $\begin{array}{c}--- \\
0.02 \% \\
--- \\
\end{array}$ & $\begin{array}{c}--- \\
0.12 \% \\
--- \\
\end{array}$ & $\begin{array}{c}--- \\
0.09 \% \\
--- \\
\end{array}$ & $\begin{array}{c}--- \\
0.00 \% \\
--- \\
\end{array}$ \\
\hline $\begin{array}{l}\mathrm{Ca}(\mathrm{OH})_{2} \\
\mathrm{CrOOH} \\
\mathrm{FeOOH}\end{array}$ & $\begin{array}{l}2.24 \\
4.11 \\
4.26\end{array}$ & $\begin{array}{c}6.62 \% \\
--- \\
48.68 \%\end{array}$ & $\begin{array}{c}6.77 \% \\
--- \\
49.77 \%\end{array}$ & $\begin{array}{c}6.77 \% \\
--- \\
49.77 \%\end{array}$ & $\begin{array}{c}6.71 \% \\
--- \\
49.43 \%\end{array}$ & $\begin{array}{l}0.38 \% \\
6.51 \% \\
0.94 \%\end{array}$ & $\begin{array}{l}1.84 \% \\
7.20 \% \\
2.14 \%\end{array}$ & $\begin{array}{l}0.60 \% \\
9.49 \% \\
2.46 \%\end{array}$ & $\begin{array}{l}0.17 \% \\
0.28 \% \\
0.70 \%\end{array}$ \\
\hline $\begin{array}{l}\text { Gibbsite } \\
\text { Boehmite } \\
\mathrm{HgO} \text { (co-precip. with } \mathrm{Ag}_{2} \mathrm{O} \text { ) }\end{array}$ & $\begin{array}{c}2.42 \\
3.01 \\
7.143 \\
\end{array}$ & $\begin{array}{l}-- \\
--- \\
--- \\
\end{array}$ & $\begin{array}{c}2.33 \% \\
--- \\
--- \\
\end{array}$ & $\begin{array}{c}2.33 \% \\
--- \\
--- \\
\end{array}$ & $\begin{array}{c}2.31 \% \\
--- \\
--- \\
\end{array}$ & $\begin{array}{c}--- \\
84.61 \% \\
---\end{array}$ & $\begin{array}{c}76.48 \% \\
--- \\
--- \\
\end{array}$ & $\begin{array}{c}81.29 \% \\
--- \\
--- \\
\end{array}$ & $\begin{array}{c}-- \\
93.55 \% \\
--- \\
\end{array}$ \\
\hline $\begin{array}{l}\mathrm{KAlSiO}_{4} \\
\mathrm{La}(\mathrm{OH})_{3} \\
\mathrm{MnO}_{2}\end{array}$ & $\begin{array}{c}2.61 \\
2.3 \\
5.026\end{array}$ & $\begin{array}{c}-- \\
0.47 \% \\
0.01 \%\end{array}$ & $\begin{array}{c}-- \\
0.48 \% \\
0.01 \%\end{array}$ & $\begin{array}{c}-- \\
0.48 \% \\
---\end{array}$ & $\begin{array}{l}-- \\
0.48 \% \\
0.01 \%\end{array}$ & $\begin{array}{l}-- \\
0.01 \% \\
1.02 \%\end{array}$ & $\begin{array}{c}-- \\
0.06 \% \\
0.86 \%\end{array}$ & $\begin{array}{l}-- \\
0.05 \% \\
0.35 \%\end{array}$ & $\begin{array}{c}-- \\
0.00 \% \\
0.62 \%\end{array}$ \\
\hline $\begin{array}{l}\mathrm{Na}_{2} \mathrm{U}_{2} \mathrm{O}_{7} \\
\mathrm{NaAlSiO}_{4} \\
\mathrm{Ni}(\mathrm{OH})_{2} \\
\end{array}$ & $\begin{array}{r}5.617 \\
2.365 \\
4.15 \\
\end{array}$ & $\begin{array}{l}0.04 \% \\
4.16 \% \\
6.25 \% \\
\end{array}$ & $\begin{array}{l}--- \\
--- \\
6.37 \% \\
\end{array}$ & $\begin{array}{l}--- \\
--- \\
6.36 \% \\
\end{array}$ & $\begin{array}{l}--- \\
--- \\
6.31 \% \\
\end{array}$ & $\begin{array}{l}3.46 \% \\
2.73 \% \\
0.09 \% \\
\end{array}$ & $\begin{array}{l}6.24 \% \\
4.74 \% \\
0.08 \% \\
\end{array}$ & $\begin{array}{l}1.19 \% \\
3.96 \% \\
0.30 \% \\
\end{array}$ & $\begin{array}{l}2.11 \% \\
2.35 \% \\
0.03 \% \\
\end{array}$ \\
\hline $\begin{array}{l}\mathrm{Pb}(\mathrm{OH})_{2} \\
\mathrm{Pu}(\mathrm{OH})_{4} \text { (co-precip. on Fe phase) } \\
\mathrm{PuO}_{2}\end{array}$ & $\begin{array}{c}7.1 \\
4.26 \\
11.43 \\
\end{array}$ & $\begin{array}{c}32.63 \% \\
0.04 \% \\
\ldots \\
-. \\
\end{array}$ & $\begin{array}{c}33.13 \% \\
0.02 \% \\
--- \\
\end{array}$ & $\begin{array}{c}33.16 \% \\
0.01 \% \\
--- \\
\end{array}$ & $\begin{array}{c}33.00 \% \\
0.00 \% \\
\ldots \\
-. \\
\end{array}$ & $\begin{array}{c}0.04 \% \\
0.00 \% \\
--- \\
\end{array}$ & $\begin{array}{c}0.18 \% \\
0.00 \% \\
--- \\
\end{array}$ & $\begin{array}{c}0.15 \% \\
0.00 \% \\
\quad-. \\
\end{array}$ & $\begin{array}{c}0.01 \% \\
0.00 \% \\
-.- \\
\end{array}$ \\
\hline $\begin{array}{l}\mathrm{SiO}_{2} \\
\mathrm{Sr}(\mathrm{OH})_{2} \\
\mathrm{ZrO}_{2}\end{array}$ & $\begin{array}{c}2.6 \\
3.625 \\
5.68 \\
\end{array}$ & $\begin{array}{l}-- \\
1.10 \% \\
---\end{array}$ & $\begin{array}{c}--- \\
1.12 \% \\
---\end{array}$ & $\begin{array}{c}--- \\
1.12 \% \\
---\end{array}$ & $\begin{array}{c}0.64 \% \\
1.11 \% \\
---\end{array}$ & $\begin{array}{c}--- \\
0.17 \% \\
0.01 \%\end{array}$ & $\begin{array}{c}--- \\
0.02 \% \\
0.04 \%\end{array}$ & $\begin{array}{c}--- \\
0.04 \% \\
0.03 \%\end{array}$ & $\begin{array}{c}--- \\
0.15 \% \\
0.02 \%\end{array}$ \\
\hline
\end{tabular}


Table C.3. (contd)

\begin{tabular}{|c|c|c|c|c|c|c|c|c|c|}
\hline & Density $(\mathrm{g} / \mathrm{mL})$ & 241-S-105 & 241-S-106 & 241-S-107 & 241-S-108 & 241-S-109 & 241-S-110 & 241-S-111 & $241-S-112$ \\
\hline $\begin{array}{l}\text { Total kg washed solids for tank } \\
\text { Total kL washed solids for tank } \\
\text { Avg g/mL, washed solid }\end{array}$ & $\mathrm{n} / \mathrm{a}$ & $\begin{array}{c}1.42 \mathrm{E}+05 \\
5.43 \mathrm{E}+01 \\
2.609\end{array}$ & $\begin{array}{c}1.39 \mathrm{E}+05 \\
5.43 \mathrm{E}+01 \\
2.552 \\
\end{array}$ & $\begin{array}{c}2.79 \mathrm{E}+05 \\
8.48 \mathrm{E}+01 \\
3.293 \\
\end{array}$ & $\begin{array}{c}2.03 \mathrm{E}+05 \\
7.72 \mathrm{E}+01 \\
2.625 \\
\end{array}$ & $\begin{array}{c}9.02 \mathrm{E}+03 \\
2.23 \mathrm{E}+00 \\
4.053 \\
\end{array}$ & $\begin{array}{c}3.50 \mathrm{E}+05 \\
1.13 \mathrm{E}+02 \\
3.105\end{array}$ & $\begin{array}{c}3.98 \mathrm{E}+05 \\
1.64 \mathrm{E}+02 \\
2.436 \\
\end{array}$ & $\begin{array}{c}4.14 \mathrm{E}+04 \\
1.52 \mathrm{E}+01 \\
2.729\end{array}$ \\
\hline \multicolumn{10}{|l|}{ mass fracs of solids in washed solid } \\
\hline $\begin{array}{l}\mathrm{Ag}_{2} \mathrm{O} \\
\mathrm{Bi}_{2} \mathrm{O}_{3} \\
\mathrm{BiFeO}_{3}\end{array}$ & $\begin{array}{c}7.143 \\
8.9 \\
7.9 \\
\end{array}$ & $\begin{array}{l}--- \\
0.11 \% \\
--- \\
\end{array}$ & $\begin{array}{c}--- \\
0.25 \% \\
--- \\
\end{array}$ & $\begin{array}{c}0.04 \% \\
0.03 \% \\
--- \\
\end{array}$ & $\begin{array}{c}--- \\
0.12 \% \\
--- \\
\end{array}$ & $\begin{array}{c}0.28 \% \\
1.78 \% \\
--- \\
\end{array}$ & $\begin{array}{l}--- \\
0.18 \% \\
--- \\
\end{array}$ & $\begin{array}{c}--- \\
0.02 \% \\
--- \\
\end{array}$ & $\begin{array}{l}--- \\
--- \\
--- \\
\end{array}$ \\
\hline $\begin{array}{l}\mathrm{Ca}(\mathrm{OH})_{2} \\
\mathrm{CrOOH} \\
\mathrm{FeOOH}\end{array}$ & $\begin{array}{l}2.24 \\
4.11 \\
4.26\end{array}$ & $\begin{array}{c}0.73 \% \\
10.25 \% \\
3.60 \%\end{array}$ & $\begin{array}{l}0.47 \% \\
6.40 \% \\
4.43 \%\end{array}$ & $\begin{array}{l}0.52 \% \\
2.13 \% \\
2.21 \%\end{array}$ & $\begin{array}{c}0.75 \% \\
10.72 \% \\
3.80 \%\end{array}$ & $\begin{array}{c}6.57 \% \\
46.18 \% \\
31.51 \% \\
\end{array}$ & $\begin{array}{l}0.46 \% \\
5.57 \% \\
3.23 \%\end{array}$ & $\begin{array}{l}0.19 \% \\
1.12 \% \\
0.12 \%\end{array}$ & $\begin{array}{c}2.37 \% \\
15.94 \% \\
5.78 \%\end{array}$ \\
\hline $\begin{array}{l}\text { Gibbsite } \\
\text { Boehmite } \\
\mathrm{HgO} \text { (co-precip. with } \mathrm{Ag}_{2} \mathrm{O} \text { ) }\end{array}$ & $\begin{array}{c}2.42 \\
3.01 \\
7.143 \\
\end{array}$ & $\begin{array}{c}77.99 \% \\
--- \\
---\end{array}$ & $\begin{array}{c}85.13 \% \\
--- \\
--- \\
\end{array}$ & $\begin{array}{c}-- \\
66.81 \% \\
--- \\
\end{array}$ & $\begin{array}{c}75.81 \% \\
--- \\
--\end{array}$ & $\begin{array}{l}-- \\
--- \\
--- \\
\end{array}$ & $\begin{array}{c}-- \\
82.54 \% \\
--- \\
\end{array}$ & $\begin{array}{c}98.28 \% \\
--- \\
--- \\
\end{array}$ & $\begin{array}{c}2.91 \% \\
--- \\
--- \\
\end{array}$ \\
\hline $\begin{array}{l}\mathrm{KAlSiO}_{4} \\
\mathrm{La}(\mathrm{OH})_{3} \\
\mathrm{MnO}_{2}\end{array}$ & $\begin{array}{c}2.61 \\
2.3 \\
5.026\end{array}$ & $\begin{array}{c}-- \\
0.06 \% \\
0.61 \%\end{array}$ & $\begin{array}{c}-- \\
0.08 \% \\
---\end{array}$ & $\begin{array}{c}-- \\
0.02 \% \\
0.49 \%\end{array}$ & $\begin{array}{c}-- \\
0.07 \% \\
1.05 \%\end{array}$ & $\begin{array}{c}-- \\
1.16 \% \\
---\end{array}$ & $\begin{array}{l}-- \\
0.03 \% \\
0.86 \%\end{array}$ & $\begin{array}{c}-- \\
0.02 \% \\
---\end{array}$ & $\begin{array}{c}-- \\
0.49 \% \\
---\end{array}$ \\
\hline $\begin{array}{l}\mathrm{Na}_{2} \mathrm{U}_{2} \mathrm{O}_{7} \\
\mathrm{NaAlSiO}_{4} \\
\mathrm{Ni}(\mathrm{OH})_{2} \\
\end{array}$ & $\begin{array}{r}5.617 \\
2.365 \\
4.15 \\
\end{array}$ & $\begin{array}{l}1.63 \% \\
4.39 \% \\
0.27 \% \\
\end{array}$ & $\begin{array}{l}0.66 \% \\
2.31 \% \\
0.09 \% \\
\end{array}$ & $\begin{array}{l}6.36 \% \\
7.23 \% \\
1.27 \% \\
\end{array}$ & $\begin{array}{l}1.73 \% \\
5.30 \% \\
0.29 \% \\
\end{array}$ & $\begin{array}{c}7.31 \% \\
--- \\
2.47 \% \\
\end{array}$ & $\begin{array}{l}2.81 \% \\
3.96 \% \\
0.09 \% \\
\end{array}$ & $\begin{array}{c}0.19 \% \\
--- \\
0.02 \% \\
\end{array}$ & $\begin{array}{c}5.31 \% \\
65.36 \% \\
0.70 \% \\
\end{array}$ \\
\hline $\begin{array}{l}\mathrm{Pb}(\mathrm{OH})_{2} \\
\mathrm{Pu}(\mathrm{OH})_{4} \text { (co-precip. on Fe phase) } \\
\mathrm{PuO}_{2}\end{array}$ & $\begin{array}{c}7.1 \\
4.26 \\
11.43 \\
\end{array}$ & $\begin{array}{c}0.26 \% \\
0.01 \% \\
\ldots \\
\end{array}$ & $\begin{array}{c}0.16 \% \\
0.00 \% \\
--- \\
\end{array}$ & $\begin{array}{c}0.09 \% \\
0.01 \% \\
--- \\
\end{array}$ & $\begin{array}{c}0.24 \% \\
0.01 \% \\
\ldots \\
\end{array}$ & $\begin{array}{c}2.16 \% \\
0.02 \% \\
--- \\
\end{array}$ & $\begin{array}{c}0.04 \% \\
0.00 \% \\
--- \\
\end{array}$ & $\begin{array}{c}0.03 \% \\
0.00 \% \\
\ldots \\
-. \\
\end{array}$ & $\begin{array}{c}0.97 \% \\
0.00 \% \\
-.- \\
\end{array}$ \\
\hline $\begin{array}{l}\mathrm{SiO}_{2} \\
\mathrm{Sr}(\mathrm{OH})_{2} \\
\mathrm{ZrO}_{2}\end{array}$ & $\begin{array}{c}2.6 \\
3.625 \\
5.68 \\
\end{array}$ & $\begin{array}{c}-- \\
0.04 \% \\
0.06 \%\end{array}$ & $\begin{array}{c}--- \\
0.02 \% \\
---\end{array}$ & $\begin{array}{c}--- \\
0.22 \% \\
12.56 \%\end{array}$ & $\begin{array}{c}--- \\
0.04 \% \\
0.07 \%\end{array}$ & $\begin{array}{c}--- \\
0.56 \% \\
---\end{array}$ & $\begin{array}{c}--- \\
0.21 \% \\
0.02 \%\end{array}$ & $\begin{array}{c}--- \\
0.00 \% \\
---\end{array}$ & $\begin{array}{c}--- \\
0.15 \% \\
0.02 \%\end{array}$ \\
\hline
\end{tabular}


Table C.3. (contd)

\begin{tabular}{|c|c|c|c|c|c|c|c|c|c|}
\hline & Density $(\mathrm{g} / \mathrm{mL})$ & 241-SX-101 & $241-S X-102$ & 241-SX-103 & 241-SX-104 & 241-SX-105 & 241-SX-106 & 241-SX-107 & 241-SX-108 \\
\hline $\begin{array}{l}\text { Total kg washed solids for tank } \\
\text { Total kL washed solids for tank } \\
\text { Avg g/mL, washed solid }\end{array}$ & $\mathrm{n} / \mathrm{a}$ & $\begin{array}{c}1.66 \mathrm{E}+05 \\
6.15 \mathrm{E}+01 \\
2.695 \\
\end{array}$ & $\begin{array}{c}1.69 \mathrm{E}+05 \\
6.44 \mathrm{E}+01 \\
2.627 \\
\end{array}$ & $\begin{array}{c}2.11 \mathrm{E}+05 \\
6.76 \mathrm{E}+01 \\
3.127\end{array}$ & $\begin{array}{c}3.96 \mathrm{E}+05 \\
1.28 \mathrm{E}+02 \\
3.084\end{array}$ & $\begin{array}{c}1.42 \mathrm{E}+05 \\
5.51 \mathrm{E}+01 \\
2.572\end{array}$ & $\begin{array}{c}1.03 \mathrm{E}+05 \\
3.89 \mathrm{E}+01 \\
2.653\end{array}$ & $\begin{array}{c}1.69 \mathrm{E}+05 \\
5.54 \mathrm{E}+01 \\
3.058\end{array}$ & $\begin{array}{c}6.05 \mathrm{E}+04 \\
1.74 \mathrm{E}+01 \\
3.476 \\
\end{array}$ \\
\hline \multicolumn{10}{|l|}{ mass fracs of solids in washed solid } \\
\hline $\begin{array}{l}\mathrm{Ag}_{2} \mathrm{O} \\
\mathrm{Bi}_{2} \mathrm{O}_{3} \\
\mathrm{BiFeO}_{3} \\
\end{array}$ & $\begin{array}{c}7.143 \\
8.9 \\
7.9 \\
\end{array}$ & $\begin{array}{c}--- \\
0.03 \% \\
--- \\
\end{array}$ & $\begin{array}{c}--- \\
0.09 \% \\
--- \\
\end{array}$ & $\begin{array}{c}--- \\
0.08 \% \\
--- \\
\end{array}$ & $\begin{array}{c}--- \\
0.04 \% \\
--- \\
\end{array}$ & $\begin{array}{c}--- \\
0.10 \% \\
--- \\
\end{array}$ & $\begin{array}{c}--- \\
0.13 \% \\
--- \\
\end{array}$ & $\begin{array}{c}--- \\
0.02 \% \\
--- \\
\end{array}$ & $\begin{array}{c}--- \\
0.06 \% \\
--- \\
\end{array}$ \\
\hline $\begin{array}{l}\mathrm{Ca}(\mathrm{OH})_{2} \\
\mathrm{CrOOH} \\
\mathrm{FeOOH}\end{array}$ & $\begin{array}{l}2.24 \\
4.11 \\
4.26\end{array}$ & $\begin{array}{c}0.40 \% \\
16.07 \% \\
3.79 \%\end{array}$ & $\begin{array}{c}0.66 \% \\
13.87 \% \\
2.30 \%\end{array}$ & $\begin{array}{c}0.61 \% \\
10.63 \% \\
2.67 \%\end{array}$ & $\begin{array}{l}0.43 \% \\
4.56 \% \\
2.17 \%\end{array}$ & $\begin{array}{l}0.85 \% \\
7.27 \% \\
3.37 \%\end{array}$ & $\begin{array}{c}1.01 \% \\
15.62 \% \\
3.49 \%\end{array}$ & $\begin{array}{l}0.32 \% \\
1.06 \% \\
1.69 \%\end{array}$ & $\begin{array}{c}2.69 \% \\
6.93 \% \\
23.79 \%\end{array}$ \\
\hline $\begin{array}{l}\text { Gibbsite } \\
\text { Boehmite } \\
\mathrm{HgO} \text { (co-precip. with } \mathrm{Ag}_{2} \mathrm{O} \text { ) }\end{array}$ & $\begin{array}{c}2.42 \\
3.01 \\
7.143 \\
\end{array}$ & $\begin{array}{c}73.90 \% \\
--- \\
--- \\
\end{array}$ & $\begin{array}{c}77.62 \% \\
--- \\
--- \\
\end{array}$ & $\begin{array}{c}-- \\
79.55 \% \\
--- \\
\end{array}$ & $\begin{array}{c}--- \\
85.47 \% \\
---\end{array}$ & $\begin{array}{c}81.76 \% \\
--- \\
---\end{array}$ & $\begin{array}{c}69.17 \% \\
--- \\
--- \\
\end{array}$ & $\begin{array}{c}-- \\
90.48 \% \\
0.00 \%\end{array}$ & $\begin{array}{c}-- \\
45.20 \% \\
0.02 \%\end{array}$ \\
\hline $\begin{array}{l}\mathrm{KAISiO}_{4} \\
\mathrm{La}(\mathrm{OH})_{3} \\
\mathrm{MnO}_{2}\end{array}$ & $\begin{array}{c}2.61 \\
2.3 \\
5.026\end{array}$ & $\begin{array}{c}-- \\
0.04 \% \\
---\end{array}$ & $\begin{array}{c}-- \\
0.05 \% \\
0.69 \%\end{array}$ & $\begin{array}{l}-- \\
0.05 \% \\
1.57 \%\end{array}$ & $\begin{array}{l}-- \\
0.02 \% \\
1.03 \%\end{array}$ & $\begin{array}{c}-- \\
0.05 \% \\
1.28 \%\end{array}$ & $\begin{array}{c}-- \\
0.08 \% \\
0.18 \%\end{array}$ & $\begin{array}{l}-- \\
0.01 \% \\
0.96 \%\end{array}$ & $\begin{array}{l}-- \\
0.14 \% \\
7.89 \%\end{array}$ \\
\hline $\begin{array}{l}\mathrm{Na}_{2} \mathrm{U}_{2} \mathrm{O}_{7} \\
\mathrm{NaAlSiO}_{4} \\
\mathrm{Ni}(\mathrm{OH})_{2} \\
\end{array}$ & $\begin{array}{r}5.617 \\
2.365 \\
4.15 \\
\end{array}$ & $\begin{array}{l}.09 \% \\
2.11 \% \\
0.10 \% \\
\end{array}$ & $\begin{array}{l}.15 \% \\
3.10 \% \\
0.23 \% \\
\end{array}$ & $\begin{array}{l}0.91 \% \\
3.49 \% \\
0.21 \% \\
\end{array}$ & $\begin{array}{l}1.98 \% \\
3.42 \% \\
0.63 \% \\
\end{array}$ & $\begin{array}{l}1.15 \% \\
3.69 \% \\
0.26 \% \\
\end{array}$ & $\begin{array}{l}1.16 \% \\
8.62 \% \\
0.26 \% \\
\end{array}$ & $\begin{array}{l}2.00 \% \\
2.50 \% \\
0.75 \% \\
\end{array}$ & $\begin{array}{l}5.73 \% \\
4.59 \% \\
1.57 \% \\
\end{array}$ \\
\hline $\begin{array}{l}\mathrm{Pb}(\mathrm{OH})_{2} \\
\mathrm{Pu}(\mathrm{OH})_{4} \text { (co-precip. on Fe phase) } \\
\mathrm{PuO}_{2}\end{array}$ & $\begin{array}{c}7.1 \\
4.26 \\
11.43 \\
\end{array}$ & $\begin{array}{c}0.17 \% \\
0.01 \% \\
\quad-. \\
\end{array}$ & $\begin{array}{c}0.13 \% \\
0.00 \% \\
--- \\
\end{array}$ & $\begin{array}{c}0.15 \% \\
0.00 \% \\
-- \\
\end{array}$ & $\begin{array}{c}0.08 \% \\
0.01 \% \\
\ldots \\
\end{array}$ & $\begin{array}{c}0.13 \% \\
0.01 \% \\
--- \\
\end{array}$ & $\begin{array}{c}0.22 \% \\
0.01 \% \\
--- \\
\end{array}$ & $\begin{array}{c}0.03 \% \\
0.01 \% \\
-.- \\
\end{array}$ & $\begin{array}{c}0.23 \% \\
0.03 \% \\
--- \\
\end{array}$ \\
\hline $\begin{array}{l}\mathrm{SiO}_{2} \\
\mathrm{Sr}(\mathrm{OH})_{2} \\
\mathrm{ZrO}_{2}\end{array}$ & $\begin{array}{c}2.6 \\
3.625 \\
5.68\end{array}$ & $\begin{array}{c}-- \\
0.23 \% \\
0.05 \%\end{array}$ & $\begin{array}{c}-- \\
0.03 \% \\
0.08 \%\end{array}$ & $\begin{array}{c}--- \\
0.05 \% \\
0.03 \%\end{array}$ & $\begin{array}{c}--- \\
0.11 \% \\
0.04 \%\end{array}$ & $\begin{array}{c}--- \\
0.03 \% \\
0.06 \%\end{array}$ & $\begin{array}{c}--- \\
0.02 \% \\
0.04 \%\end{array}$ & $\begin{array}{c}--- \\
0.13 \% \\
0.03 \%\end{array}$ & $\begin{array}{c}--- \\
0.64 \% \\
0.49 \%\end{array}$ \\
\hline
\end{tabular}


Table C.3. (contd)

\begin{tabular}{|c|c|c|c|c|c|c|c|c|c|}
\hline & Density $(\mathrm{g} / \mathrm{mL})$ & 241-SX-109 & 241-SX-110 & 241-SX-111 & 241-SX-112 & 241-SX-113 & 241-SX-114 & 241-SX-115 & 241-SY-101 \\
\hline $\begin{array}{l}\text { Total kg washed solids for tank } \\
\text { Total kL washed solids for tank } \\
\text { Avg g/mL, washed solid }\end{array}$ & $\mathrm{n} / \mathrm{a}$ & $\begin{array}{c}2.81 \mathrm{E}+05 \\
9.25 \mathrm{E}+01 \\
3.039 \\
\end{array}$ & $\begin{array}{c}8.03 \mathrm{E}+04 \\
2.64 \mathrm{E}+01 \\
3.046 \\
\end{array}$ & $\begin{array}{c}1.72 \mathrm{E}+05 \\
5.61 \mathrm{E}+01 \\
3.058\end{array}$ & $\begin{array}{c}1.28 \mathrm{E}+05 \\
4.19 \mathrm{E}+01 \\
3.057\end{array}$ & $\begin{array}{c}4.23 \mathrm{E}+04 \\
1.63 \mathrm{E}+01 \\
2.595 \\
\end{array}$ & $\begin{array}{c}1.42 \mathrm{E}+05 \\
4.63 \mathrm{E}+01 \\
3.061\end{array}$ & $\begin{array}{c}7.43 \mathrm{E}+03 \\
2.13 \mathrm{E}+00 \\
3.480\end{array}$ & $\begin{array}{c}2.95 \mathrm{E}+05 \\
1.16 \mathrm{E}+02 \\
2.538 \\
\end{array}$ \\
\hline \multicolumn{10}{|l|}{ mass fracs of solids in washed solid } \\
\hline $\begin{array}{l}\mathrm{Ag}_{2} \mathrm{O} \\
\mathrm{Bi}_{2} \mathrm{O}_{3} \\
\mathrm{BiFeO}_{3}\end{array}$ & $\begin{array}{c}7.143 \\
8.9 \\
7.9 \\
\end{array}$ & $\begin{array}{c}--- \\
0.00 \% \\
--- \\
\end{array}$ & $\begin{array}{c}--- \\
0.01 \% \\
--- \\
\end{array}$ & $\begin{array}{c}--- \\
0.02 \% \\
--- \\
\end{array}$ & $\begin{array}{c}--- \\
0.02 \% \\
--- \\
\end{array}$ & $\begin{array}{c}0.00 \% \\
0.00 \% \\
--- \\
\end{array}$ & $\begin{array}{c}--- \\
0.01 \% \\
--- \\
\end{array}$ & $\begin{array}{c}-\cdots \\
0.02 \% \\
--- \\
\end{array}$ & $\begin{array}{c}--- \\
0.04 \% \\
--- \\
\end{array}$ \\
\hline $\begin{array}{l}\mathrm{Ca}(\mathrm{OH})_{2} \\
\mathrm{CrOOH} \\
\mathrm{FeOOH}\end{array}$ & $\begin{array}{l}2.24 \\
4.11 \\
4.26\end{array}$ & $\begin{array}{c}0.32 \% \\
--- \\
1.32 \%\end{array}$ & $\begin{array}{c}0.33 \% \\
--- \\
1.57 \%\end{array}$ & $\begin{array}{l}0.34 \% \\
0.97 \% \\
1.70 \%\end{array}$ & $\begin{array}{l}0.32 \% \\
1.13 \% \\
1.65 \%\end{array}$ & $\begin{array}{c}0.59 \% \\
--- \\
7.89 \%\end{array}$ & $\begin{array}{l}0.37 \% \\
1.84 \% \\
1.55 \%\end{array}$ & $\begin{array}{c}4.25 \% \\
--- \\
48.98 \%\end{array}$ & $\begin{array}{l}0.44 \% \\
9.76 \% \\
0.72 \%\end{array}$ \\
\hline $\begin{array}{l}\text { Gibbsite } \\
\text { Boehmite } \\
\mathrm{HgO} \text { (co-precip. with } \mathrm{Ag}_{2} \mathrm{O} \text { ) }\end{array}$ & $\begin{array}{c}2.42 \\
3.01 \\
7.143 \\
\end{array}$ & $\begin{array}{c}-- \\
91.07 \% \\
--- \\
\end{array}$ & $\begin{array}{c}-- \\
91.00 \% \\
--- \\
\end{array}$ & $\begin{array}{c}-- \\
90.07 \% \\
--- \\
\end{array}$ & $\begin{array}{c}--- \\
90.50 \% \\
---\end{array}$ & $\begin{array}{l}-- \\
--- \\
---\end{array}$ & $\begin{array}{c}-- \\
88.08 \% \\
--- \\
\end{array}$ & $\begin{array}{c}2.25 \% \\
26.20 \% \\
0.01 \% \\
\end{array}$ & $\begin{array}{c}88.42 \% \\
--- \\
---\end{array}$ \\
\hline $\begin{array}{l}\mathrm{KAlSiO}_{4} \\
\mathrm{La}(\mathrm{OH})_{3} \\
\mathrm{MnO}_{2}\end{array}$ & $\begin{array}{c}2.61 \\
2.3 \\
5.026\end{array}$ & $\begin{array}{l}-- \\
0.00 \% \\
0.89 \%\end{array}$ & $\begin{array}{l}-- \\
0.01 \% \\
1.05 \%\end{array}$ & $\begin{array}{l}-- \\
0.01 \% \\
1.04 \%\end{array}$ & $\begin{array}{l}-- \\
0.01 \% \\
0.94 \%\end{array}$ & $\begin{array}{l}.85 \% \\
0.00 \% \\
0.26 \%\end{array}$ & $\begin{array}{l}-- \\
0.01 \% \\
1.17 \%\end{array}$ & $\begin{array}{l}-- \\
0.01 \% \\
5.86 \%\end{array}$ & $\begin{array}{c}-- \\
0.02 \% \\
---\end{array}$ \\
\hline $\begin{array}{l}\mathrm{Na}_{2} \mathrm{U}_{2} \mathrm{O}_{7} \\
\mathrm{NaAlSiO}_{4} \\
\mathrm{Ni}(\mathrm{OH})_{2} \\
\end{array}$ & $\begin{array}{r}5.617 \\
2.365 \\
4.15 \\
\end{array}$ & $\begin{array}{l}.34 \% \\
3.46 \% \\
0.38 \% \\
\end{array}$ & $\begin{array}{l}.20 \% \\
3.02 \% \\
0.60 \% \\
\end{array}$ & $\begin{array}{l}2.14 \% \\
2.78 \% \\
0.72 \% \\
\end{array}$ & $\begin{array}{l}2.00 \% \\
2.53 \% \\
0.72 \% \\
\end{array}$ & $\begin{array}{c}0.18 \% \\
34.63 \% \\
0.05 \% \\
\end{array}$ & $\begin{array}{l}2.52 \% \\
3.71 \% \\
0.51 \% \\
\end{array}$ & $\begin{array}{l}2.09 \% \\
9.18 \% \\
0.84 \% \\
\end{array}$ & $\begin{array}{c}0.14 \% \\
--- \\
0.29 \% \\
\end{array}$ \\
\hline $\begin{array}{l}\mathrm{Pb}(\mathrm{OH})_{2} \\
\mathrm{Pu}(\mathrm{OH})_{4} \text { (co-precip. on Fe phase) } \\
\mathrm{PuO}_{2}\end{array}$ & $\begin{array}{c}7.1 \\
4.26 \\
11.43 \\
\end{array}$ & $\begin{array}{c}0.04 \% \\
0.00 \% \\
\quad-. \\
\end{array}$ & $\begin{array}{c}0.04 \% \\
0.00 \% \\
--- \\
\end{array}$ & $\begin{array}{c}0.04 \% \\
0.01 \% \\
--- \\
\end{array}$ & $\begin{array}{c}0.03 \% \\
0.01 \% \\
\ldots \\
\end{array}$ & $\begin{array}{c}0.00 \% \\
0.00 \% \\
--- \\
\end{array}$ & $\begin{array}{c}0.05 \% \\
0.00 \% \\
--- \\
\end{array}$ & $\begin{array}{c}0.03 \% \\
0.11 \% \\
\ldots- \\
\end{array}$ & $\begin{array}{c}0.17 \% \\
0.00 \% \\
--- \\
\end{array}$ \\
\hline $\begin{array}{l}\mathrm{SiO}_{2} \\
\mathrm{Sr}(\mathrm{OH})_{2} \\
\mathrm{ZrO}_{2}\end{array}$ & $\begin{array}{c}2.6 \\
3.625 \\
5.68\end{array}$ & $\begin{array}{c}-- \\
0.14 \% \\
0.02 \%\end{array}$ & $\begin{array}{c}--- \\
0.13 \% \\
0.03 \%\end{array}$ & $\begin{array}{c}--- \\
0.13 \% \\
0.03 \%\end{array}$ & $\begin{array}{c}--- \\
0.12 \% \\
0.03 \%\end{array}$ & $\begin{array}{c}54.53 \% \\
--- \\
0.01 \%\end{array}$ & $\begin{array}{c}--- \\
0.15 \% \\
0.03 \%\end{array}$ & $\begin{array}{c}--- \\
0.13 \% \\
0.03 \%\end{array}$ & $\begin{array}{c}--- \\
0.01 \% \\
---\end{array}$ \\
\hline
\end{tabular}


Table C.3. (contd)

\begin{tabular}{|c|c|c|c|c|c|c|c|c|c|}
\hline & Density $(\mathrm{g} / \mathrm{mL})$ & 241-SY-102 & 241-SY-103 & 241-T-101 & 241-T-102 & 241-T-103 & 241-T-104 & 241-T-105 & 241-T-106 \\
\hline $\begin{array}{l}\text { Total kg washed solids for tank } \\
\text { Total kL washed solids for tank } \\
\text { Avg g/mL, washed solid }\end{array}$ & $\mathrm{n} / \mathrm{a}$ & $\begin{array}{c}2.34 \mathrm{E}+05 \\
8.84 \mathrm{E}+01 \\
2.648 \\
\end{array}$ & $\begin{array}{c}2.44 \mathrm{E}+05 \\
9.55 \mathrm{E}+01 \\
2.554 \\
\end{array}$ & $\begin{array}{c}1.06 \mathrm{E}+05 \\
4.29 \mathrm{E}+01 \\
2.473 \\
\end{array}$ & $\begin{array}{c}1.12 \mathrm{E}+05 \\
4.52 \mathrm{E}+01 \\
2.480\end{array}$ & $\begin{array}{c}5.77 \mathrm{E}+04 \\
2.26 \mathrm{E}+01 \\
2.548 \\
\end{array}$ & $\begin{array}{c}1.52 \mathrm{E}+05 \\
4.86 \mathrm{E}+01 \\
3.117\end{array}$ & $\begin{array}{c}1.27 \mathrm{E}+05 \\
4.17 \mathrm{E}+01 \\
3.051\end{array}$ & $\begin{array}{c}4.36 \mathrm{E}+04 \\
1.66 \mathrm{E}+01 \\
2.620\end{array}$ \\
\hline \multicolumn{10}{|l|}{ mass fracs of solids in washed solid } \\
\hline $\begin{array}{l}\mathrm{Ag}_{2} \mathrm{O} \\
\mathrm{Bi}_{2} \mathrm{O}_{3} \\
\mathrm{BiFeO}_{3}\end{array}$ & $\begin{array}{c}7.143 \\
8.9 \\
7.9 \\
\end{array}$ & $\begin{array}{c}--- \\
1.48 \% \\
--- \\
\end{array}$ & $\begin{array}{c}--- \\
0.07 \% \\
--- \\
\end{array}$ & $\begin{array}{c}--- \\
0.15 \% \\
--- \\
\end{array}$ & $\begin{array}{c}0.04 \% \\
0.23 \% \\
--- \\
\end{array}$ & $\begin{array}{c}--- \\
0.51 \% \\
--- \\
\end{array}$ & $\begin{array}{c}0.00 \% \\
--- \\
28.40 \% \\
\end{array}$ & $\begin{array}{c}0.01 \% \\
--- \\
15.78 \% \\
\end{array}$ & $\begin{array}{c}--- \\
5.60 \% \\
--- \\
\end{array}$ \\
\hline $\begin{array}{l}\mathrm{Ca}(\mathrm{OH})_{2} \\
\mathrm{CrOOH} \\
\mathrm{FeOOH}\end{array}$ & $\begin{array}{l}2.24 \\
4.11 \\
4.26\end{array}$ & $\begin{array}{c}1.14 \% \\
10.11 \% \\
5.70 \%\end{array}$ & $\begin{array}{l}0.83 \% \\
8.33 \% \\
2.95 \%\end{array}$ & $\begin{array}{c}0.30 \% \\
--- \\
1.12 \%\end{array}$ & $\begin{array}{l}0.19 \% \\
0.01 \% \\
2.92 \%\end{array}$ & $\begin{array}{l}0.46 \% \\
0.07 \% \\
6.98 \%\end{array}$ & $\begin{array}{c}2.69 \% \\
--- \\
6.30 \%\end{array}$ & $\begin{array}{l}0.98 \% \\
1.03 \% \\
17.48 \%\end{array}$ & $\begin{array}{c}0.53 \% \\
--- \\
5.01 \%\end{array}$ \\
\hline $\begin{array}{l}\text { Gibbsite } \\
\text { Boehmite } \\
\mathrm{HgO} \text { (co-precip. with } \mathrm{Ag}_{2} \mathrm{O} \text { ) }\end{array}$ & $\begin{array}{c}2.42 \\
3.01 \\
7.143 \\
\end{array}$ & $\begin{array}{c}79.65 \% \\
--- \\
--- \\
\end{array}$ & $\begin{array}{c}82.03 \% \\
--- \\
--- \\
\end{array}$ & $\begin{array}{c}93.13 \% \\
--- \\
--- \\
\end{array}$ & $\begin{array}{c}93.14 \% \\
--- \\
---\end{array}$ & $\begin{array}{c}80.94 \% \\
--- \\
---\end{array}$ & $\begin{array}{c}30.28 \% \\
--- \\
--- \\
\end{array}$ & $\begin{array}{c}38.86 \% \\
--- \\
--- \\
\end{array}$ & $\begin{array}{c}74.93 \% \\
--- \\
---\end{array}$ \\
\hline $\begin{array}{l}\mathrm{KAlSiO}_{4} \\
\mathrm{La}(\mathrm{OH})_{3} \\
\mathrm{MnO}_{2}\end{array}$ & $\begin{array}{c}2.61 \\
2.3 \\
5.026\end{array}$ & $\begin{array}{l}-- \\
0.08 \% \\
0.72 \%\end{array}$ & $\begin{array}{c}--- \\
0.04 \% \\
---\end{array}$ & $\begin{array}{c}-- \\
0.03 \% \\
0.20 \%\end{array}$ & $\begin{array}{l}-- \\
0.02 \% \\
0.18 \%\end{array}$ & $\begin{array}{l}-- \\
0.03 \% \\
0.35 \%\end{array}$ & $\begin{array}{c}-- \\
0.00 \% \\
0.10 \%\end{array}$ & $\begin{array}{c}-- \\
0.02 \% \\
2.55 \%\end{array}$ & $\begin{array}{l}-- \\
0.01 \% \\
0.07 \%\end{array}$ \\
\hline $\begin{array}{l}\mathrm{Na}_{2} \mathrm{U}_{2} \mathrm{O}_{7} \\
\mathrm{NaAlSiO}_{4} \\
\mathrm{Ni}(\mathrm{OH})_{2} \\
\end{array}$ & $\begin{array}{r}5.617 \\
2.365 \\
4.15 \\
\end{array}$ & $\begin{array}{c}0.44 \% \\
--- \\
0.25 \% \\
\end{array}$ & $\begin{array}{l}0.76 \% \\
4.53 \% \\
0.16 \% \\
\end{array}$ & $\begin{array}{l}1.73 \% \\
2.43 \% \\
0.15 \% \\
\end{array}$ & $\begin{array}{l}1.49 \% \\
1.67 \% \\
0.02 \% \\
\end{array}$ & $\begin{array}{l}1.57 \% \\
7.74 \% \\
0.47 \% \\
\end{array}$ & $\begin{array}{c}1.20 \% \\
30.72 \% \\
0.02 \% \\
\end{array}$ & $\begin{array}{c}1.19 \% \\
21.52 \% \\
0.04 \% \\
\end{array}$ & $\begin{array}{l}1.93 \% \\
11.01 \% \\
0.12 \% \\
\end{array}$ \\
\hline $\begin{array}{l}\mathrm{Pb}(\mathrm{OH})_{2} \\
\mathrm{Pu}(\mathrm{OH})_{4} \text { (co-precip. on Fe phase) } \\
\mathrm{PuO}_{2}\end{array}$ & $\begin{array}{c}7.1 \\
4.26 \\
11.43 \\
\end{array}$ & $\begin{array}{c}0.36 \% \\
--- \\
0.03 \% \\
\end{array}$ & $\begin{array}{c}0.24 \% \\
0.00 \% \\
---\end{array}$ & $\begin{array}{c}0.70 \% \\
0.00 \% \\
--- \\
\end{array}$ & $\begin{array}{c}0.08 \% \\
0.00 \% \\
\ldots \\
\end{array}$ & $\begin{array}{c}0.49 \% \\
0.00 \% \\
--- \\
\end{array}$ & $\begin{array}{c}0.06 \% \\
0.00 \% \\
--- \\
\end{array}$ & $\begin{array}{c}0.37 \% \\
0.00 \% \\
\ldots \\
-. \\
\end{array}$ & $\begin{array}{c}0.62 \% \\
0.00 \% \\
--. \\
\end{array}$ \\
\hline $\begin{array}{l}\mathrm{SiO}_{2} \\
\mathrm{Sr}(\mathrm{OH})_{2} \\
\mathrm{ZrO}_{2}\end{array}$ & $\begin{array}{c}2.6 \\
3.625 \\
5.68 \\
\end{array}$ & $\begin{array}{c}-- \\
0.03 \% \\
0.01 \%\end{array}$ & $\begin{array}{c}-- \\
0.01 \% \\
0.04 \%\end{array}$ & $\begin{array}{c}--- \\
0.02 \% \\
0.05 \%\end{array}$ & $\begin{array}{c}--- \\
0.00 \% \\
0.01 \%\end{array}$ & $\begin{array}{c}--- \\
0.02 \% \\
0.36 \%\end{array}$ & $\begin{array}{c}--- \\
0.14 \% \\
0.09 \%\end{array}$ & $\begin{array}{c}--- \\
0.12 \% \\
0.03 \%\end{array}$ & $\begin{array}{c}--- \\
0.07 \% \\
0.09 \%\end{array}$ \\
\hline
\end{tabular}


Table C.3. (contd)

\begin{tabular}{|c|c|c|c|c|c|c|c|c|c|}
\hline & Density $(\mathrm{g} / \mathrm{mL})$ & 241-T-107 & 241-T-108 & 241-T-109 & 241-T-110 & 241-T-111 & $241-T-112$ & 241-T-201 & 241-T-202 \\
\hline $\begin{array}{l}\text { Total kg washed solids for tank } \\
\text { Total kL washed solids for tank } \\
\text { Avg g/mL, washed solid }\end{array}$ & $\mathrm{n} / \mathrm{a}$ & $\begin{array}{c}2.01 \mathrm{E}+05 \\
6.54 \mathrm{E}+01 \\
3.075 \\
\end{array}$ & $\begin{array}{c}4.27 \mathrm{E}+03 \\
1.32 \mathrm{E}+00 \\
3.248 \\
\end{array}$ & $\begin{array}{c}4.12 \mathrm{E}+03 \\
1.15 \mathrm{E}+00 \\
3.591 \\
\end{array}$ & $\begin{array}{c}6.24 \mathrm{E}+04 \\
1.27 \mathrm{E}+01 \\
4.904 \\
\end{array}$ & $\begin{array}{c}1.58 \mathrm{E}+05 \\
3.32 \mathrm{E}+01 \\
4.771 \\
\end{array}$ & $\begin{array}{c}2.98 \mathrm{E}+04 \\
7.26 \mathrm{E}+00 \\
4.099 \\
\end{array}$ & $\begin{array}{c}2.58 \mathrm{E}+04 \\
4.89 \mathrm{E}+00 \\
5.278 \\
\end{array}$ & $\begin{array}{c}8.16 \mathrm{E}+03 \\
1.67 \mathrm{E}+00 \\
4.872 \\
\end{array}$ \\
\hline \multicolumn{10}{|l|}{ mass fracs of solids in washed solid } \\
\hline $\begin{array}{l}\mathrm{Ag}_{2} \mathrm{O} \\
\mathrm{Bi}_{2} \mathrm{O}_{3} \\
\mathrm{BiFeO}_{3}\end{array}$ & $\begin{array}{c}7.143 \\
8.9 \\
7.9 \\
\end{array}$ & $\begin{array}{c}0.00 \% \\
--- \\
7.00 \% \\
\end{array}$ & $\begin{array}{c}--- \\
--- \\
21.63 \% \\
\end{array}$ & $\begin{array}{l}--- \\
1.25 \% \\
--- \\
\end{array}$ & $\begin{array}{c}--- \\
--- \\
52.02 \% \\
\end{array}$ & $\begin{array}{c}0.13 \% \\
--- \\
46.52 \% \\
\end{array}$ & $\begin{array}{c}--- \\
--- \\
41.65 \% \\
\end{array}$ & $\begin{array}{c}0.00 \% \\
55.47 \% \\
--- \\
\end{array}$ & $\begin{array}{c}0.00 \% \\
48.10 \% \\
--- \\
\end{array}$ \\
\hline $\begin{array}{l}\mathrm{Ca}(\mathrm{OH})_{2} \\
\mathrm{CrOOH} \\
\mathrm{FeOOH}\end{array}$ & $\begin{array}{l}2.24 \\
4.11 \\
4.26\end{array}$ & $\begin{array}{c}1.73 \% \\
0.30 \% \\
19.75 \%\end{array}$ & $\begin{array}{l}1.47 \% \\
0.79 \% \\
18.33 \%\end{array}$ & $\begin{array}{c}3.92 \% \\
0.29 \% \\
59.80 \% \\
\end{array}$ & $\begin{array}{c}3.80 \% \\
3.53 \% \\
26.04 \% \\
\end{array}$ & $\begin{array}{c}4.32 \% \\
--- \\
19.98 \%\end{array}$ & $\begin{array}{l}1.70 \% \\
2.54 \% \\
13.41 \% \\
\end{array}$ & $\begin{array}{l}0.72 \% \\
3.77 \% \\
6.10 \% \\
\end{array}$ & $\begin{array}{c}0.56 \% \\
--- \\
10.57 \%\end{array}$ \\
\hline $\begin{array}{l}\text { Gibbsite } \\
\text { Boehmite } \\
\mathrm{HgO} \text { (co-precip. with } \mathrm{Ag}_{2} \mathrm{O} \text { ) }\end{array}$ & $\begin{array}{c}2.42 \\
3.01 \\
7.143 \\
\end{array}$ & $\begin{array}{c}4.83 \% \\
--- \\
--- \\
\end{array}$ & $\begin{array}{c}18.78 \% \\
--- \\
---\end{array}$ & $\begin{array}{c}23.31 \% \\
--- \\
---\end{array}$ & $\begin{array}{c}8.76 \% \\
--- \\
--- \\
\end{array}$ & $\begin{array}{c}1.33 \% \\
--- \\
--- \\
\end{array}$ & $\begin{array}{l}-- \\
-- \\
---\end{array}$ & $\begin{array}{l}\cdots \\
\cdots \\
\cdots \\
---\end{array}$ & $\begin{array}{l}-- \\
\cdots- \\
---\end{array}$ \\
\hline $\begin{array}{l}\mathrm{KAlSiO}_{4} \\
\mathrm{La}(\mathrm{OH})_{3} \\
\mathrm{MnO}_{2} \\
\end{array}$ & $\begin{array}{c}2.61 \\
2.3 \\
5.026 \\
\end{array}$ & $\begin{array}{c}-- \\
0.00 \% \\
0.33 \% \\
\end{array}$ & $\begin{array}{c}-- \\
0.07 \% \\
0.39 \% \\
\end{array}$ & $\begin{array}{c}-- \\
0.23 \% \\
8.58 \% \\
\end{array}$ & $\begin{array}{l}-- \\
1.11 \% \\
1.68 \% \\
\end{array}$ & $\begin{array}{c}-- \\
6.68 \% \\
15.75 \% \\
\end{array}$ & $\begin{array}{l}6.26 \% \\
6.35 \% \\
9.12 \% \\
\end{array}$ & $\begin{array}{l}0.24 \% \\
13.56 \% \\
18.93 \% \\
\end{array}$ & $\begin{array}{c}0.46 \% \\
17.54 \% \\
21.50 \% \\
\end{array}$ \\
\hline $\begin{array}{l}\mathrm{Na}_{2} \mathrm{U}_{2} \mathrm{O}_{7} \\
\mathrm{NaAlSiO}_{4} \\
\mathrm{Ni}(\mathrm{OH})_{2} \\
\end{array}$ & $\begin{array}{r}5.617 \\
2.365 \\
4.15 \\
\end{array}$ & $\begin{array}{l}13.13 \% \\
11.75 \% \\
0.10 \% \\
\end{array}$ & $\begin{array}{c}3.81 \% \\
33.43 \% \\
0.07 \% \\
\end{array}$ & $\begin{array}{l}--- \\
--- \\
0.21 \% \\
\end{array}$ & \begin{tabular}{c|}
--- \\
$0.62 \%$ \\
$0.16 \%$ \\
\end{tabular} & $\begin{array}{c}--- \\
--- \\
0.22 \% \\
\end{array}$ & $\begin{array}{c}0.91 \% \\
17.38 \% \\
0.16 \% \\
\end{array}$ & $\begin{array}{c}0.10 \% \\
--- \\
0.30 \% \\
\end{array}$ & $\begin{array}{c}0.17 \% \\
--- \\
0.22 \% \\
\end{array}$ \\
\hline $\begin{array}{l}\mathrm{Pb}(\mathrm{OH})_{2} \\
\mathrm{Pu}(\mathrm{OH})_{4} \text { (co-precip. on Fe phase) } \\
\mathrm{PuO}_{2}\end{array}$ & $\begin{array}{c}7.1 \\
4.26 \\
11.43 \\
\end{array}$ & $\begin{array}{c}0.42 \% \\
0.00 \% \\
-.- \\
\end{array}$ & $\begin{array}{c}0.90 \% \\
0.00 \% \\
--- \\
\end{array}$ & $\begin{array}{c}2.19 \% \\
0.00 \% \\
--- \\
\end{array}$ & $\begin{array}{c}1.68 \% \\
0.00 \% \\
\cdots \\
\end{array}$ & $\begin{array}{c}0.40 \% \\
0.00 \% \\
--- \\
\end{array}$ & $\begin{array}{c}0.51 \% \\
0.00 \% \\
--- \\
\end{array}$ & $\begin{array}{c}0.15 \% \\
0.01 \% \\
--. \\
\end{array}$ & $\begin{array}{c}0.13 \% \\
0.01 \% \\
--. \\
\end{array}$ \\
\hline $\begin{array}{l}\mathrm{SiO}_{2} \\
\mathrm{Sr}(\mathrm{OH})_{2} \\
\mathrm{ZrO}_{2}\end{array}$ & $\begin{array}{c}2.6 \\
3.625 \\
5.68 \\
\end{array}$ & $\begin{array}{c}-- \\
0.62 \% \\
0.03 \%\end{array}$ & $\begin{array}{c}--- \\
0.18 \% \\
0.14 \%\end{array}$ & $\begin{array}{c}--- \\
0.08 \% \\
0.14 \%\end{array}$ & $\begin{array}{c}--- \\
0.60 \% \\
0.02 \%\end{array}$ & $\begin{array}{l}.13 \% \\
0.53 \% \\
0.00 \%\end{array}$ & $\begin{array}{c}--- \\
0.00 \% \\
--- \\
\end{array}$ & $\begin{array}{c}--- \\
0.66 \% \\
0.00 \%\end{array}$ & $\begin{array}{c}--- \\
0.74 \% \\
--- \\
\end{array}$ \\
\hline
\end{tabular}


Table C.3. (contd)

\begin{tabular}{|c|c|c|c|c|c|c|c|c|c|}
\hline & Density $(\mathrm{g} / \mathrm{mL})$ & 241-T-203 & 241-T-204 & 241-TX-101 & 241-TX-102 & 241-TX-103 & 241-TX-104 & 241-TX-105 & 241-TX-106 \\
\hline $\begin{array}{l}\text { Total kg washed solids for tank } \\
\text { Total } \mathrm{kL} \text { washed solids for tank } \\
\text { Avg g/mL, washed solid }\end{array}$ & $\mathrm{n} / \mathrm{a}$ & $\begin{array}{c}1.36 \mathrm{E}+04 \\
2.70 \mathrm{E}+00 \\
5.020\end{array}$ & $\begin{array}{c}1.79 \mathrm{E}+04 \\
3.43 \mathrm{E}+00 \\
5.228 \\
\end{array}$ & $\begin{array}{c}2.12 \mathrm{E}+05 \\
8.49 \mathrm{E}+01 \\
2.493\end{array}$ & $\begin{array}{c}8.79 \mathrm{E}+04 \\
3.45 \mathrm{E}+01 \\
2.544\end{array}$ & $\begin{array}{c}5.93 \mathrm{E}+04 \\
2.35 \mathrm{E}+01 \\
2.522 \\
\end{array}$ & $\begin{array}{c}1.27 \mathrm{E}+05 \\
5.19 \mathrm{E}+01 \\
2.447\end{array}$ & $\begin{array}{c}2.02 \mathrm{E}+05 \\
7.80 \mathrm{E}+01 \\
2.593\end{array}$ & $\begin{array}{c}1.55 \mathrm{E}+05 \\
6.14 \mathrm{E}+01 \\
2.523\end{array}$ \\
\hline \multicolumn{10}{|l|}{ mass fracs of solids in washed solid } \\
\hline $\begin{array}{l}\mathrm{Ag}_{2} \mathrm{O} \\
\mathrm{Bi}_{2} \mathrm{O}_{3} \\
\mathrm{BiFeO}_{3}\end{array}$ & $\begin{array}{c}7.143 \\
8.9 \\
7.9 \\
\end{array}$ & $\begin{array}{c}0.00 \% \\
52.34 \% \\
--- \\
\end{array}$ & $\begin{array}{c}0.00 \% \\
54.73 \% \\
--- \\
\end{array}$ & $\begin{array}{c}--- \\
0.02 \% \\
--- \\
\end{array}$ & $\begin{array}{c}--- \\
0.11 \% \\
--- \\
\end{array}$ & $\begin{array}{c}--- \\
0.12 \% \\
--- \\
\end{array}$ & $\begin{array}{c}--- \\
0.04 \% \\
--- \\
\end{array}$ & $\begin{array}{c}--- \\
0.13 \% \\
--- \\
\end{array}$ & $\begin{array}{c}--- \\
0.10 \% \\
--- \\
\end{array}$ \\
\hline $\begin{array}{l}\mathrm{Ca}(\mathrm{OH})_{2} \\
\mathrm{CrOOH} \\
\mathrm{FeOOH}\end{array}$ & $\begin{array}{l}2.24 \\
4.11 \\
4.26\end{array}$ & $\begin{array}{c}0.79 \% \\
--- \\
7.92 \%\end{array}$ & $\begin{array}{l}0.36 \% \\
0.77 \% \\
6.13 \%\end{array}$ & $\begin{array}{l}0.32 \% \\
0.80 \% \\
1.65 \%\end{array}$ & $\begin{array}{l}0.67 \% \\
1.79 \% \\
3.20 \%\end{array}$ & $\begin{array}{l}0.66 \% \\
1.81 \% \\
3.38 \%\end{array}$ & $\begin{array}{l}0.22 \% \\
0.64 \% \\
1.08 \%\end{array}$ & $\begin{array}{l}0.86 \% \\
2.36 \% \\
4.23 \%\end{array}$ & $\begin{array}{l}0.63 \% \\
1.73 \% \\
3.03 \%\end{array}$ \\
\hline $\begin{array}{l}\text { Gibbsite } \\
\text { Boehmite } \\
\mathrm{HgO} \text { (co-precip. with } \mathrm{Ag}_{2} \mathrm{O} \text { ) }\end{array}$ & $\begin{array}{c}2.42 \\
3.01 \\
7.143 \\
\end{array}$ & $\begin{array}{l}-- \\
--- \\
-- \\
\end{array}$ & $\begin{array}{l}-- \\
--- \\
---\end{array}$ & $\begin{array}{c}92.00 \% \\
--- \\
0.00 \% \\
\end{array}$ & $\begin{array}{c}82.80 \% \\
--- \\
---\end{array}$ & $\begin{array}{c}84.14 \% \\
--- \\
---\end{array}$ & $\begin{array}{c}97.17 \% \\
--- \\
0.00 \% \\
\end{array}$ & $\begin{array}{c}76.83 \% \\
--- \\
--- \\
\end{array}$ & $\begin{array}{c}84.53 \% \\
--- \\
--- \\
\end{array}$ \\
\hline $\begin{array}{l}\mathrm{KAlSiO}_{4} \\
\mathrm{La}(\mathrm{OH})_{3} \\
\mathrm{MnO}_{2}\end{array}$ & $\begin{array}{c}2.61 \\
2.3 \\
5.026\end{array}$ & $\begin{array}{l}0.40 \% \\
16.68 \% \\
20.55 \% \\
\end{array}$ & $\begin{array}{l}0.25 \% \\
15.01 \% \\
21.20 \% \\
\end{array}$ & $\begin{array}{l}-- \\
0.01 \% \\
0.53 \%\end{array}$ & $\begin{array}{c}-- \\
0.06 \% \\
1.13 \%\end{array}$ & $\begin{array}{c}-- \\
0.07 \% \\
1.17 \%\end{array}$ & $\begin{array}{l}-- \\
0.01 \% \\
0.23 \%\end{array}$ & $\begin{array}{l}-- \\
0.09 \% \\
1.32 \%\end{array}$ & $\begin{array}{l}-- \\
0.06 \% \\
1.12 \%\end{array}$ \\
\hline $\begin{array}{l}\mathrm{Na}_{2} \mathrm{U}_{2} \mathrm{O}_{7} \\
\mathrm{NaAlSiO}_{4} \\
\mathrm{Ni}(\mathrm{OH})_{2} \\
\end{array}$ & $\begin{array}{r}5.617 \\
2.365 \\
4.15 \\
\end{array}$ & $\begin{array}{c}0.15 \% \\
--- \\
0.29 \% \\
\end{array}$ & $\begin{array}{c}0.18 \% \\
--- \\
0.36 \% \\
\end{array}$ & $\begin{array}{l}2.35 \% \\
1.61 \% \\
0.57 \% \\
\end{array}$ & $\begin{array}{l}3.52 \% \\
6.20 \% \\
0.13 \% \\
\end{array}$ & $\begin{array}{l}.83 \% \\
6.31 \% \\
0.13 \% \\
\end{array}$ & $\begin{array}{l}0.39 \% \\
0.17 \% \\
0.01 \% \\
\end{array}$ & $\begin{array}{l}5.29 \% \\
8.27 \% \\
0.16 \% \\
\end{array}$ & $\begin{array}{l}2.28 \% \\
6.00 \% \\
0.17 \% \\
\end{array}$ \\
\hline $\begin{array}{l}\mathrm{Pb}(\mathrm{OH})_{2} \\
\mathrm{Pu}(\mathrm{OH})_{4} \text { (co-precip. on Fe phase) } \\
\mathrm{PuO}_{2}\end{array}$ & $\begin{array}{c}7.1 \\
4.26 \\
11.43 \\
\end{array}$ & $\begin{array}{c}0.03 \% \\
0.00 \% \\
\quad-. \\
\end{array}$ & $\begin{array}{c}0.34 \% \\
0.00 \% \\
--- \\
\end{array}$ & $\begin{array}{c}0.04 \% \\
--- \\
0.00 \% \\
\end{array}$ & $\begin{array}{c}0.31 \% \\
--- \\
0.01 \% \\
\end{array}$ & $\begin{array}{c}0.32 \% \\
--- \\
0.01 \% \\
\end{array}$ & $\begin{array}{c}0.03 \% \\
--- \\
0.00 \% \\
\end{array}$ & $\begin{array}{c}0.38 \% \\
--- \\
0.01 \% \\
\end{array}$ & $\begin{array}{c}0.28 \% \\
--- \\
0.01 \% \\
\end{array}$ \\
\hline $\begin{array}{l}\mathrm{SiO}_{2} \\
\mathrm{Sr}(\mathrm{OH})_{2} \\
\mathrm{ZrO}_{2}\end{array}$ & $\begin{array}{c}2.6 \\
3.625 \\
5.68\end{array}$ & $\begin{array}{c}-- \\
0.84 \% \\
0.00 \%\end{array}$ & $\begin{array}{c}--- \\
0.65 \% \\
0.00 \%\end{array}$ & $\begin{array}{c}--- \\
0.09 \% \\
0.02 \%\end{array}$ & $\begin{array}{c}--- \\
0.01 \% \\
0.05 \%\end{array}$ & $\begin{array}{c}--- \\
0.01 \% \\
0.05 \%\end{array}$ & $\begin{array}{c}--- \\
0.00 \% \\
0.01 \%\end{array}$ & $\begin{array}{c}--- \\
0.02 \% \\
0.06 \%\end{array}$ & $\begin{array}{c}--- \\
0.02 \% \\
0.05 \%\end{array}$ \\
\hline
\end{tabular}


Table C.3. (contd)

\begin{tabular}{|c|c|c|c|c|c|c|c|c|c|}
\hline & Density $(\mathrm{g} / \mathrm{mL})$ & 241-TX-107 & 241-TX-108 & 241-TX-109 & 241-TX-110 & 241-TX-111 & 241-TX-112 & 241-TX-113 & 241-TX-114 \\
\hline $\begin{array}{l}\text { Total kg washed solids for tank } \\
\text { Total kL washed solids for tank } \\
\text { Avg g/mL, washed solid }\end{array}$ & $\mathrm{n} / \mathrm{a}$ & $\begin{array}{c}2.37 \mathrm{E}+04 \\
9.55 \mathrm{E}+00 \\
2.485 \\
\end{array}$ & $\begin{array}{c}5.22 \mathrm{E}+04 \\
2.02 \mathrm{E}+01 \\
2.585 \\
\end{array}$ & $\begin{array}{c}1.91 \mathrm{E}+05 \\
5.99 \mathrm{E}+01 \\
3.193 \\
\end{array}$ & $\begin{array}{c}1.95 \mathrm{E}+05 \\
7.53 \mathrm{E}+01 \\
2.590 \\
\end{array}$ & $\begin{array}{c}1.55 \mathrm{E}+05 \\
5.94 \mathrm{E}+01 \\
2.619 \\
\end{array}$ & $\begin{array}{c}2.57 \mathrm{E}+05 \\
1.02 \mathrm{E}+02 \\
2.534 \\
\end{array}$ & $\begin{array}{c}9.46 \mathrm{E}+04 \\
3.20 \mathrm{E}+01 \\
2.954 \\
\end{array}$ & $\begin{array}{c}2.00 \mathrm{E}+05 \\
7.82 \mathrm{E}+01 \\
2.556 \\
\end{array}$ \\
\hline \multicolumn{10}{|l|}{ mass fracs of solids in washed solid } \\
\hline $\begin{array}{l}\mathrm{Ag}_{2} \mathrm{O} \\
\mathrm{Bi}_{2} \mathrm{O}_{3} \\
\mathrm{BiFeO}_{3}\end{array}$ & $\begin{array}{c}7.143 \\
8.9 \\
7.9 \\
\end{array}$ & $\begin{array}{c}--- \\
0.05 \% \\
--- \\
\end{array}$ & $\begin{array}{c}--- \\
0.13 \% \\
--- \\
\end{array}$ & $\begin{array}{c}--- \\
--- \\
27.74 \% \\
\end{array}$ & $\begin{array}{c}--- \\
--- \\
3.19 \% \\
\end{array}$ & $\begin{array}{c}--- \\
--- \\
4.57 \% \\
\end{array}$ & $\begin{array}{l}--- \\
0.13 \% \\
--- \\
\end{array}$ & $\begin{array}{c}--- \\
--- \\
19.43 \% \\
\end{array}$ & $\begin{array}{c}--- \\
--- \\
0.55 \% \\
\end{array}$ \\
\hline $\begin{array}{l}\mathrm{Ca}(\mathrm{OH})_{2} \\
\mathrm{CrOOH} \\
\mathrm{FeOOH}\end{array}$ & $\begin{array}{l}2.24 \\
4.11 \\
4.26\end{array}$ & $\begin{array}{l}0.37 \% \\
1.46 \% \\
1.59 \%\end{array}$ & $\begin{array}{l}1.00 \% \\
1.80 \% \\
5.27 \%\end{array}$ & $\begin{array}{c}1.41 \% \\
--- \\
9.26 \%\end{array}$ & $\begin{array}{l}0.79 \% \\
1.88 \% \\
4.11 \% \\
\end{array}$ & $\begin{array}{l}0.83 \% \\
1.84 \% \\
4.42 \% \\
\end{array}$ & $\begin{array}{l}0.72 \% \\
1.99 \% \\
3.87 \%\end{array}$ & $\begin{array}{l}2.42 \% \\
1.07 \% \\
8.47 \%\end{array}$ & $\begin{array}{l}0.78 \% \\
2.15 \% \\
4.77 \%\end{array}$ \\
\hline $\begin{array}{l}\text { Gibbsite } \\
\text { Boehmite } \\
\mathrm{HgO} \text { (co-precip. with } \mathrm{Ag}_{2} \mathrm{O} \text { ) }\end{array}$ & $\begin{array}{c}2.42 \\
3.01 \\
7.143 \\
\end{array}$ & $\begin{array}{c}90.09 \% \\
--- \\
-- \\
\end{array}$ & $\begin{array}{c}79.08 \% \\
--- \\
--- \\
\end{array}$ & $\begin{array}{c}24.17 \% \\
--- \\
--- \\
\end{array}$ & $\begin{array}{c}76.06 \% \\
\cdots \\
--- \\
\end{array}$ & $\begin{array}{c}2.96 \% \\
--- \\
--- \\
\end{array}$ & $\begin{array}{c}82.26 \% \\
--- \\
--- \\
\end{array}$ & $\begin{array}{c}67.08 \% \\
\cdots \\
--- \\
\end{array}$ & $\begin{array}{c}79.51 \% \\
--- \\
--- \\
\end{array}$ \\
\hline $\begin{array}{l}\mathrm{KAlSiO}_{4} \\
\mathrm{La}(\mathrm{OH})_{3} \\
\mathrm{MnO}_{2} \\
\end{array}$ & $\begin{array}{c}2.61 \\
2.3 \\
5.026 \\
\end{array}$ & $\begin{array}{c}-- \\
0.03 \% \\
0.83 \% \\
\end{array}$ & \begin{tabular}{c|}
--- \\
$0.07 \%$ \\
$1.18 \%$ \\
\end{tabular} & $\begin{array}{c}-- \\
0.01 \% \\
0.13 \% \\
\end{array}$ & \begin{tabular}{c|}
--- \\
$0.07 \%$ \\
$1.16 \%$ \\
\end{tabular} & $\begin{array}{c}-- \\
0.06 \% \\
1.10 \% \\
\end{array}$ & $\begin{array}{c}-- \\
0.07 \% \\
1.29 \% \\
\end{array}$ & $\begin{array}{c}-- \\
0.26 \% \\
0.17 \% \\
\end{array}$ & $\begin{array}{c}-- \\
0.08 \% \\
1.37 \% \\
\end{array}$ \\
\hline $\begin{array}{l}\mathrm{Na}_{2} \mathrm{U}_{2} \mathrm{O}_{7} \\
\mathrm{NaAlSiO}_{4} \\
\mathrm{Ni}(\mathrm{OH})_{2} \\
\end{array}$ & $\begin{array}{r}5.617 \\
2.365 \\
4.15 \\
\end{array}$ & $\begin{array}{l}.43 \% \\
3.82 \% \\
0.08 \% \\
\end{array}$ & $\begin{array}{l}4.47 \% \\
6.40 \% \\
0.14 \% \\
\end{array}$ & $\begin{array}{c}2.62 \% \\
34.07 \% \\
0.03 \% \\
\end{array}$ & $\begin{array}{c}2.09 \% \\
10.13 \% \\
0.13 \% \\
\end{array}$ & $\begin{array}{c}2.13 \% \\
11.54 \% \\
0.12 \% \\
\end{array}$ & $\begin{array}{l}2.00 \% \\
7.12 \% \\
0.14 \% \\
\end{array}$ & $\begin{array}{c}-- \\
--- \\
0.16 \% \\
\end{array}$ & $\begin{array}{l}2.09 \% \\
8.10 \% \\
0.14 \% \\
\end{array}$ \\
\hline $\begin{array}{l}\mathrm{Pb}(\mathrm{OH})_{2} \\
\mathrm{Pu}(\mathrm{OH})_{4} \text { (co-precip. on Fe phase) } \\
\mathrm{PuO}_{2}\end{array}$ & $\begin{array}{c}7.1 \\
4.26 \\
11.43 \\
\end{array}$ & $\begin{array}{c}0.16 \% \\
\cdots \\
0.02 \% \\
\end{array}$ & $\begin{array}{c}0.34 \% \\
--- \\
0.01 \% \\
\end{array}$ & $\begin{array}{c}0.20 \% \\
--- \\
0.00 \% \\
\end{array}$ & $\begin{array}{c}0.30 \% \\
\cdots-- \\
0.01 \% \\
\end{array}$ & $\begin{array}{c}0.29 \% \\
--- \\
0.01 \% \\
\end{array}$ & $\begin{array}{c}0.33 \% \\
--- \\
0.01 \% \\
\end{array}$ & $\begin{array}{c}0.57 \% \\
--- \\
0.00 \% \\
\end{array}$ & $\begin{array}{c}0.37 \% \\
--- \\
0.01 \% \\
\end{array}$ \\
\hline $\begin{array}{l}\mathrm{SiO}_{2} \\
\mathrm{Sr}(\mathrm{OH})_{2} \\
\mathrm{ZrO}_{2}\end{array}$ & $\begin{array}{c}2.6 \\
3.625 \\
5.68\end{array}$ & $\begin{array}{c}-- \\
0.04 \% \\
0.03 \%\end{array}$ & $\begin{array}{c}-- \\
0.06 \% \\
0.05 \%\end{array}$ & $\begin{array}{c}--- \\
0.20 \% \\
0.17 \%\end{array}$ & $\begin{array}{c}--- \\
0.03 \% \\
0.07 \%\end{array}$ & $\begin{array}{c}--- \\
0.04 \% \\
0.08 \%\end{array}$ & $\begin{array}{c}--- \\
0.01 \% \\
0.06 \%\end{array}$ & $\begin{array}{c}--- \\
0.18 \% \\
0.20 \%\end{array}$ & $\begin{array}{c}--- \\
0.02 \% \\
0.06 \%\end{array}$ \\
\hline
\end{tabular}


Table C.3. (contd)

\begin{tabular}{|c|c|c|c|c|c|c|c|c|c|}
\hline & Density $(\mathrm{g} / \mathrm{mL})$ & 241-TX-115 & 241-TX-116 & 241-TX-117 & 241-TX-118 & 241-TY-101 & 241-TY-102 & 241-TY-103 & 241-TY-104 \\
\hline $\begin{array}{l}\text { Total kg washed solids for tank } \\
\text { Total kL washed solids for tank } \\
\text { Avg g/mL, washed solid }\end{array}$ & $\mathrm{n} / \mathrm{a}$ & $\begin{array}{c}2.31 \mathrm{E}+05 \\
9.11 \mathrm{E}+01 \\
2.538 \\
\end{array}$ & $\begin{array}{c}3.17 \mathrm{E}+05 \\
1.25 \mathrm{E}+02 \\
2.532 \\
\end{array}$ & $\begin{array}{c}1.72 \mathrm{E}+05 \\
6.73 \mathrm{E}+01 \\
2.554 \\
\end{array}$ & $\begin{array}{c}6.57 \mathrm{E}+04 \\
2.26 \mathrm{E}+01 \\
2.905 \\
\end{array}$ & $\begin{array}{c}2.37 \mathrm{E}+05 \\
7.82 \mathrm{E}+01 \\
3.028 \\
\end{array}$ & $\begin{array}{c}5.38 \mathrm{E}+03 \\
2.11 \mathrm{E}+00 \\
2.550 \\
\end{array}$ & $\begin{array}{c}1.14 \mathrm{E}+05 \\
2.77 \mathrm{E}+01 \\
4.110 \\
\end{array}$ & $\begin{array}{c}3.48 \mathrm{E}+04 \\
9.78 \mathrm{E}+00 \\
3.556 \\
\end{array}$ \\
\hline \multicolumn{10}{|l|}{ mass fracs of solids in washed solid } \\
\hline $\begin{array}{l}\mathrm{Ag}_{2} \mathrm{O} \\
\mathrm{Bi}_{2} \mathrm{O}_{3} \\
\mathrm{BiFeO}_{3}\end{array}$ & $\begin{array}{c}7.143 \\
8.9 \\
7.9 \\
\end{array}$ & $\begin{array}{c}\cdots \\
0.12 \% \\
--- \\
\end{array}$ & $\begin{array}{c}--- \\
0.36 \% \\
--- \\
\end{array}$ & $\begin{array}{c}--- \\
0.36 \% \\
--- \\
\end{array}$ & $\begin{array}{l}--- \\
0.91 \% \\
--- \\
\end{array}$ & $\begin{array}{c}0.00 \% \\
10.01 \% \\
--- \\
\end{array}$ & $\begin{array}{c}--- \\
0.37 \% \\
--- \\
\end{array}$ & $\begin{array}{c}0.00 \% \\
22.95 \% \\
--- \\
\end{array}$ & $\begin{array}{c}0.01 \% \\
13.54 \% \\
--- \\
\end{array}$ \\
\hline $\begin{array}{l}\mathrm{Ca}(\mathrm{OH})_{2} \\
\mathrm{CrOOH} \\
\mathrm{FeOOH}\end{array}$ & $\begin{array}{l}2.24 \\
4.11 \\
4.26\end{array}$ & $\begin{array}{l}0.81 \% \\
1.91 \% \\
4.12 \%\end{array}$ & $\begin{array}{l}.03 \% \\
0.49 \% \\
8.76 \%\end{array}$ & $\begin{array}{l}1.13 \% \\
2.76 \% \\
8.96 \%\end{array}$ & $\begin{array}{c}1.21 \% \\
10.51 \% \\
12.94 \% \\
\end{array}$ & $\begin{array}{l}4.73 \% \\
4.47 \% \\
21.03 \% \\
\end{array}$ & $\begin{array}{l}2.76 \% \\
0.25 \% \\
9.48 \%\end{array}$ & $\begin{array}{c}6.53 \% \\
1.00 \% \\
28.87 \% \\
\end{array}$ & $\begin{array}{c}10.35 \% \\
0.91 \% \\
35.40 \% \\
\end{array}$ \\
\hline $\begin{array}{l}\text { Gibbsite } \\
\text { Boehmite } \\
\mathrm{HgO} \text { (co-precip. with } \mathrm{Ag}_{2} \mathrm{O} \text { ) }\end{array}$ & $\begin{array}{c}2.42 \\
3.01 \\
7.143 \\
\end{array}$ & $\begin{array}{c}82.20 \% \\
--- \\
--\end{array}$ & $\begin{array}{c}56.65 \% \\
--- \\
--- \\
\end{array}$ & $\begin{array}{c}35.93 \% \\
--- \\
--- \\
\end{array}$ & $\begin{array}{c}47.90 \% \\
--- \\
--- \\
\end{array}$ & $\begin{array}{c}10.90 \% \\
--- \\
--- \\
\end{array}$ & $\begin{array}{c}85.30 \% \\
--- \\
--- \\
\end{array}$ & $\begin{array}{c}-- \\
--- \\
0.03 \% \\
\end{array}$ & $\begin{array}{c}8.35 \% \\
--- \\
0.02 \% \\
\end{array}$ \\
\hline $\begin{array}{l}\mathrm{KAlSiO}_{4} \\
\mathrm{La}(\mathrm{OH})_{3} \\
\mathrm{MnO}_{2} \\
\end{array}$ & $\begin{array}{c}2.61 \\
2.3 \\
5.026 \\
\end{array}$ & $\begin{array}{c}-- \\
0.07 \% \\
1.23 \% \\
\end{array}$ & \begin{tabular}{c|}
--- \\
$0.06 \%$ \\
$1.13 \%$ \\
\end{tabular} & $\begin{array}{c}-- \\
0.09 \% \\
1.51 \% \\
\end{array}$ & $\begin{array}{c}-- \\
0.10 \% \\
7.10 \% \\
\end{array}$ & $\begin{array}{c}-- \\
0.00 \% \\
0.29 \% \\
\end{array}$ & $\begin{array}{c}-- \\
0.28 \% \\
0.41 \% \\
\end{array}$ & $\begin{array}{l}-- \\
0.01 \% \\
0.20 \% \\
\end{array}$ & $\begin{array}{c}-- \\
--- \\
1.87 \% \\
\end{array}$ \\
\hline $\begin{array}{l}\mathrm{Na}_{2} \mathrm{U}_{2} \mathrm{O}_{7} \\
\mathrm{NaAlSiO}_{4} \\
\mathrm{Ni}(\mathrm{OH})_{2} \\
\end{array}$ & $\begin{array}{r}5.617 \\
2.365 \\
4.15 \\
\end{array}$ & $\begin{array}{l}2.26 \% \\
6.73 \% \\
0.14 \% \\
\end{array}$ & $\begin{array}{c}-- \\
30.89 \% \\
0.08 \% \\
\end{array}$ & $\begin{array}{c}-- \\
48.55 \% \\
0.11 \% \\
\end{array}$ & $\begin{array}{c}3.57 \% \\
14.40 \% \\
0.28 \% \\
\end{array}$ & $\begin{array}{c}1.02 \% \\
30.50 \% \\
2.65 \% \\
\end{array}$ & $\begin{array}{c}--- \\
--- \\
0.27 \% \\
\end{array}$ & $\begin{array}{l}18.71 \% \\
17.95 \% \\
2.80 \% \\
\end{array}$ & $\begin{array}{c}9.77 \% \\
17.40 \% \\
1.61 \% \\
\end{array}$ \\
\hline $\begin{array}{l}\mathrm{Pb}(\mathrm{OH})_{2} \\
\mathrm{Pu}(\mathrm{OH})_{4} \text { (co-precip. on Fe phase) } \\
\mathrm{PuO}_{2}\end{array}$ & $\begin{array}{c}7.1 \\
4.26 \\
11.43 \\
\end{array}$ & $\begin{array}{c}0.32 \% \\
\cdots \\
0.01 \% \\
\end{array}$ & $\begin{array}{c}0.48 \% \\
--- \\
0.00 \% \\
\end{array}$ & $\begin{array}{c}0.52 \% \\
--- \\
0.00 \% \\
\end{array}$ & $\begin{array}{c}0.72 \% \\
\cdots \\
0.22 \% \\
\end{array}$ & $\begin{array}{c}0.08 \% \\
0.00 \% \\
--- \\
\end{array}$ & $\begin{array}{c}0.67 \% \\
0.00 \% \\
--- \\
\end{array}$ & $\begin{array}{c}0.47 \% \\
0.00 \% \\
--. \\
\end{array}$ & $\begin{array}{c}0.46 \% \\
0.00 \% \\
--. \\
\end{array}$ \\
\hline $\begin{array}{l}\mathrm{SiO}_{2} \\
\mathrm{Sr}(\mathrm{OH})_{2} \\
\mathrm{ZrO}_{2}\end{array}$ & $\begin{array}{c}2.6 \\
3.625 \\
5.68 \\
\end{array}$ & $\begin{array}{c}--- \\
0.03 \% \\
0.05 \%\end{array}$ & $\begin{array}{c}-- \\
0.03 \% \\
0.03 \%\end{array}$ & $\begin{array}{c}--- \\
0.03 \% \\
0.05 \%\end{array}$ & $\begin{array}{c}--- \\
0.04 \% \\
0.10 \%\end{array}$ & $\begin{array}{c}14.13 \% \\
--- \\
0.17 \%\end{array}$ & $\begin{array}{c}--- \\
0.10 \% \\
0.11 \%\end{array}$ & $\begin{array}{c}--- \\
0.23 \% \\
0.25 \%\end{array}$ & $\begin{array}{c}--- \\
0.15 \% \\
0.17 \%\end{array}$ \\
\hline
\end{tabular}


Table C.3. (contd)

\begin{tabular}{|c|c|c|c|c|c|c|c|c|c|}
\hline & Density $(\mathrm{g} / \mathrm{mL})$ & 241-TY-105 & 241-TY-106 & 241-U-101 & 241-U-102 & $241-\mathrm{U}-103$ & 241-U-104 & 241-U-105 & $241-\mathrm{U}-106$ \\
\hline $\begin{array}{l}\text { Total kg washed solids for tank } \\
\text { Total kL washed solids for tank } \\
\text { Avg g/mL, washed solid }\end{array}$ & $\mathrm{n} / \mathrm{a}$ & $\begin{array}{c}8.11 \mathrm{E}+04 \\
2.18 \mathrm{E}+01 \\
3.714\end{array}$ & $\begin{array}{c}3.45 \mathrm{E}+04 \\
1.17 \mathrm{E}+01 \\
2.943 \\
\end{array}$ & $\begin{array}{c}6.09 \mathrm{E}+04 \\
2.47 \mathrm{E}+01 \\
2.466 \\
\end{array}$ & $\begin{array}{c}2.10 \mathrm{E}+05 \\
8.38 \mathrm{E}+01 \\
2.501\end{array}$ & $\begin{array}{c}8.17 \mathrm{E}+04 \\
3.06 \mathrm{E}+01 \\
2.671\end{array}$ & $\begin{array}{c}1.56 \mathrm{E}+05 \\
6.45 \mathrm{E}+01 \\
2.414 \\
\end{array}$ & $\begin{array}{c}2.31 \mathrm{E}+05 \\
8.66 \mathrm{E}+01 \\
2.668\end{array}$ & $\begin{array}{c}.43 \mathrm{E}+04 \\
1.64 \mathrm{E}+01 \\
2.706 \\
\end{array}$ \\
\hline \multicolumn{10}{|l|}{ mass fracs of solids in washed solid } \\
\hline $\begin{array}{l}\mathrm{Ag}_{2} \mathrm{O} \\
\mathrm{Bi}_{2} \mathrm{O}_{3} \\
\mathrm{BiFeO}_{3} \\
\end{array}$ & $\begin{array}{c}7.143 \\
8.9 \\
7.9 \\
\end{array}$ & $\begin{array}{c}--- \\
0.78 \% \\
--- \\
\end{array}$ & $\begin{array}{c}--- \\
0.22 \% \\
--- \\
\end{array}$ & $\begin{array}{c}--- \\
0.02 \% \\
--- \\
\end{array}$ & $\begin{array}{c}--- \\
0.06 \% \\
--- \\
\end{array}$ & $\begin{array}{c}--- \\
0.14 \% \\
--- \\
\end{array}$ & $\begin{array}{c}--- \\
0.01 \% \\
--- \\
\end{array}$ & $\begin{array}{c}--- \\
0.08 \% \\
--- \\
\end{array}$ & $\begin{array}{c}--- \\
0.13 \% \\
--- \\
\end{array}$ \\
\hline $\begin{array}{l}\mathrm{Ca}(\mathrm{OH})_{2} \\
\mathrm{CrOOH} \\
\mathrm{FeOOH}\end{array}$ & $\begin{array}{l}2.24 \\
4.11 \\
4.26\end{array}$ & $\begin{array}{c}11.30 \% \\
--- \\
60.58 \%\end{array}$ & $\begin{array}{c}2.03 \% \\
--- \\
27.00 \%\end{array}$ & $\begin{array}{c}0.23 \% \\
--- \\
1.25 \%\end{array}$ & $\begin{array}{l}0.54 \% \\
3.70 \% \\
1.21 \%\end{array}$ & $\begin{array}{c}2.99 \% \\
12.66 \% \\
6.76 \%\end{array}$ & $\begin{array}{c}0.56 \% \\
--- \\
1.56 \%\end{array}$ & $\begin{array}{l}0.72 \% \\
4.05 \% \\
4.90 \%\end{array}$ & $\begin{array}{l}1.34 \% \\
7.04 \% \\
9.17 \%\end{array}$ \\
\hline $\begin{array}{l}\text { Gibbsite } \\
\text { Boehmite } \\
\mathrm{HgO} \text { (co-precip. with } \mathrm{Ag}_{2} \mathrm{O} \text { ) }\end{array}$ & $\begin{array}{c}2.42 \\
3.01 \\
7.143 \\
\end{array}$ & $\begin{array}{c}9.10 \% \\
--- \\
0.28 \% \\
\end{array}$ & $\begin{array}{l}-- \\
--- \\
0.01 \% \\
\end{array}$ & $\begin{array}{c}94.61 \% \\
--- \\
0.00 \% \\
\end{array}$ & $\begin{array}{c}90.90 \% \\
--- \\
---\end{array}$ & $\begin{array}{c}58.34 \% \\
--- \\
--- \\
\end{array}$ & $\begin{array}{c}18.53 \% \\
--- \\
--- \\
\end{array}$ & $\begin{array}{c}77.08 \% \\
\cdots- \\
--- \\
\end{array}$ & $\begin{array}{c}73.81 \% \\
--- \\
---\end{array}$ \\
\hline $\begin{array}{l}\mathrm{KAISiO}_{4} \\
\mathrm{La}(\mathrm{OH})_{3} \\
\mathrm{MnO}_{2}\end{array}$ & $\begin{array}{c}2.61 \\
2.3 \\
5.026\end{array}$ & $\begin{array}{c}-- \\
--- \\
0.43 \%\end{array}$ & $\begin{array}{c}--- \\
--- \\
0.33 \%\end{array}$ & $\begin{array}{l}-- \\
0.01 \% \\
0.52 \%\end{array}$ & $\begin{array}{l}-- \\
0.03 \% \\
0.55 \%\end{array}$ & $\begin{array}{c}--- \\
0.09 \% \\
---\end{array}$ & $\begin{array}{c}-- \\
0.00 \% \\
0.43 \%\end{array}$ & $\begin{array}{l}-- \\
0.05 \% \\
1.60 \%\end{array}$ & $\begin{array}{l}-- \\
0.10 \% \\
3.20 \%\end{array}$ \\
\hline $\begin{array}{l}\mathrm{Na}_{2} \mathrm{U}_{2} \mathrm{O}_{7} \\
\mathrm{NaAlSiO}_{4} \\
\mathrm{Ni}(\mathrm{OH})_{2} \\
\end{array}$ & $\begin{array}{r}5.617 \\
2.365 \\
4.15 \\
\end{array}$ & $\begin{array}{c}13.11 \% \\
1.73 \% \\
0.24 \% \\
\end{array}$ & $\begin{array}{c}3.98 \% \\
13.43 \% \\
0.04 \% \\
\end{array}$ & $\begin{array}{l}1.42 \% \\
1.23 \% \\
0.57 \% \\
\end{array}$ & $\begin{array}{l}1.16 \% \\
1.24 \% \\
0.43 \% \\
\end{array}$ & $\begin{array}{c}2.29 \% \\
15.76 \% \\
0.51 \% \\
\end{array}$ & $\begin{array}{c}0.89 \% \\
77.56 \% \\
0.37 \% \\
\end{array}$ & $\begin{array}{l}7.81 \% \\
3.14 \% \\
0.26 \% \\
\end{array}$ & $\begin{array}{l}1.70 \% \\
1.33 \% \\
1.08 \% \\
\end{array}$ \\
\hline $\begin{array}{l}\mathrm{Pb}(\mathrm{OH})_{2} \\
\mathrm{Pu}(\mathrm{OH})_{4} \text { (co-precip. on Fe phase) } \\
\mathrm{PuO}_{2}\end{array}$ & $\begin{array}{c}7.1 \\
4.26 \\
11.43 \\
\end{array}$ & $\begin{array}{c}0.81 \% \\
0.00 \% \\
\quad-. \\
\end{array}$ & $\begin{array}{c}0.17 \% \\
0.00 \% \\
--- \\
\end{array}$ & $\begin{array}{c}0.02 \% \\
0.00 \% \\
--- \\
\end{array}$ & $\begin{array}{c}0.09 \% \\
0.00 \% \\
\ldots \\
\end{array}$ & $\begin{array}{c}0.34 \% \\
0.00 \% \\
--- \\
\end{array}$ & $\begin{array}{c}0.01 \% \\
0.00 \% \\
--- \\
\end{array}$ & $\begin{array}{c}0.26 \% \\
0.01 \% \\
\ldots \\
-.\end{array}$ & $\begin{array}{c}0.77 \% \\
0.02 \% \\
-- \\
\end{array}$ \\
\hline $\begin{array}{l}\mathrm{SiO}_{2} \\
\mathrm{Sr}(\mathrm{OH})_{2} \\
\mathrm{ZrO}_{2}\end{array}$ & $\begin{array}{c}2.6 \\
3.625 \\
5.68 \\
\end{array}$ & $\begin{array}{c}-- \\
1.63 \% \\
---\end{array}$ & $\begin{array}{l}52.38 \% \\
0.09 \% \\
0.33 \%\end{array}$ & $\begin{array}{c}--- \\
0.09 \% \\
0.02 \%\end{array}$ & $\begin{array}{c}--- \\
0.06 \% \\
0.02 \%\end{array}$ & $\begin{array}{c}--- \\
0.07 \% \\
0.06 \%\end{array}$ & $\begin{array}{c}--- \\
0.06 \% \\
0.01 \%\end{array}$ & $\begin{array}{c}--- \\
0.01 \% \\
0.04 \%\end{array}$ & $\begin{array}{c}--- \\
0.02 \% \\
0.30 \%\end{array}$ \\
\hline
\end{tabular}


Table C.3. (contd)

\begin{tabular}{|c|c|c|c|c|c|c|c|c|c|}
\hline & Density $(\mathrm{g} / \mathrm{mL})$ & 241-U-107 & 241-( /-108 & $241-\mathrm{U}-109$ & 241-U-110 & 241-U -111 & $241-\mathrm{U}-112$ & 241-U-201 & 241- I-202 \\
\hline $\begin{array}{l}\text { Total kg washed solids for tank } \\
\text { Total kL washed solids for tank } \\
\text { Avg g/mL, washed solid }\end{array}$ & $\mathrm{n} / \mathrm{a}$ & $\begin{array}{c}1.48 \mathrm{E}+05 \\
5.76 \mathrm{E}+01 \\
2.574 \\
\end{array}$ & $\begin{array}{c}1.32 \mathrm{E}+05 \\
4.48 \mathrm{E}+01 \\
2.956 \\
\end{array}$ & $\begin{array}{c}1.67 \mathrm{E}+05 \\
6.71 \mathrm{E}+01 \\
2.489 \\
\end{array}$ & $\begin{array}{c}2.28 \mathrm{E}+05 \\
6.98 \mathrm{E}+01 \\
3.268 \\
\end{array}$ & $\begin{array}{c}1.37 \mathrm{E}+05 \\
5.26 \mathrm{E}+01 \\
2.598 \\
\end{array}$ & $\begin{array}{c}1.19 \mathrm{E}+05 \\
4.79 \mathrm{E}+01 \\
2.474 \\
\end{array}$ & $\begin{array}{c}8.21 \mathrm{E}+03 \\
3.32 \mathrm{E}+00 \\
2.470 \\
\end{array}$ & $\begin{array}{c}6.90 \mathrm{E}+03 \\
2.82 \mathrm{E}+00 \\
2.448 \\
\end{array}$ \\
\hline \multicolumn{10}{|l|}{ mass fracs of solids in washed solid } \\
\hline $\begin{array}{l}\mathrm{Ag}_{2} \mathrm{O} \\
\mathrm{Bi}_{2} \mathrm{O}_{3} \\
\mathrm{BiFeO}_{3}\end{array}$ & $\begin{array}{c}7.143 \\
8.9 \\
7.9 \\
\end{array}$ & $\begin{array}{c}\cdots \\
0.14 \% \\
--- \\
\end{array}$ & $\begin{array}{c}--- \\
0.10 \% \\
--- \\
\end{array}$ & $\begin{array}{c}--- \\
0.07 \% \\
--- \\
\end{array}$ & $\begin{array}{c}0.03 \% \\
5.16 \% \\
-- \\
\end{array}$ & $\begin{array}{c}--- \\
1.18 \% \\
--- \\
\end{array}$ & $\begin{array}{l}--- \\
1.15 \% \\
--- \\
\end{array}$ & $\begin{array}{c}--- \\
0.15 \% \\
--- \\
\end{array}$ & $\begin{array}{c}--- \\
0.15 \% \\
--- \\
\end{array}$ \\
\hline $\begin{array}{l}\mathrm{Ca}(\mathrm{OH})_{2} \\
\mathrm{CrOOH} \\
\mathrm{FeOOH}\end{array}$ & $\begin{array}{l}2.24 \\
4.11 \\
4.26 \\
\end{array}$ & $\begin{array}{l}1.37 \% \\
5.17 \% \\
5.23 \% \\
\end{array}$ & $\begin{array}{l}2.56 \% \\
9.55 \% \\
3.98 \% \\
\end{array}$ & $\begin{array}{l}0.44 \% \\
5.60 \% \\
0.44 \% \\
\end{array}$ & $\begin{array}{c}0.36 \% \\
--- \\
6.60 \% \\
\end{array}$ & $\begin{array}{l}0.66 \% \\
9.33 \% \\
3.20 \% \\
\end{array}$ & $\begin{array}{c}0.23 \% \\
--- \\
1.62 \% \\
\end{array}$ & $\begin{array}{l}0.21 \% \\
0.36 \% \\
0.62 \% \\
\end{array}$ & $\begin{array}{l}0.21 \% \\
0.37 \% \\
0.63 \% \\
\end{array}$ \\
\hline $\begin{array}{l}\text { Gibbsite } \\
\text { Boehmite } \\
\mathrm{HgO} \text { (co-precip. with } \mathrm{Ag}_{2} \mathrm{O} \text { ) }\end{array}$ & $\begin{array}{c}2.42 \\
3.01 \\
7.143 \\
\end{array}$ & $\begin{array}{c}2.34 \% \\
--- \\
-- \\
\end{array}$ & $\begin{array}{c}62.63 \% \\
--- \\
--- \\
\end{array}$ & $\begin{array}{c}1.58 \% \\
--- \\
---\end{array}$ & $\begin{array}{c}--- \\
69.40 \% \\
--\end{array}$ & $\begin{array}{c}78.40 \% \\
--- \\
--- \\
\end{array}$ & $\begin{array}{c}93.00 \% \\
--- \\
0.00 \% \\
\end{array}$ & $\begin{array}{c}94.64 \% \\
--- \\
--- \\
\end{array}$ & $\begin{array}{c}96.26 \% \\
--- \\
-- \\
\end{array}$ \\
\hline $\begin{array}{l}\mathrm{KAlSiO}_{4} \\
\mathrm{La}(\mathrm{OH})_{3} \\
\mathrm{MnO}_{2} \\
\end{array}$ & $\begin{array}{c}2.61 \\
2.3 \\
5.026 \\
\end{array}$ & $\begin{array}{c}-- \\
0.06 \% \\
1.70 \% \\
\end{array}$ & $\begin{array}{c}-- \\
0.06 \% \\
--- \\
\end{array}$ & $\begin{array}{c}-- \\
0.04 \% \\
0.11 \% \\
\end{array}$ & $\begin{array}{c}-- \\
0.03 \% \\
2.26 \% \\
\end{array}$ & $\begin{array}{c}-- \\
0.05 \% \\
--- \\
\end{array}$ & $\begin{array}{c}-- \\
0.01 \% \\
0.05 \% \\
\end{array}$ & $\begin{array}{c}-- \\
0.02 \% \\
0.04 \% \\
\end{array}$ & $\begin{array}{c}-- \\
0.02 \% \\
0.04 \% \\
\end{array}$ \\
\hline $\begin{array}{l}\mathrm{Na}_{2} \mathrm{U}_{2} \mathrm{O}_{7} \\
\mathrm{NaAlSiO}_{4} \\
\mathrm{Ni}(\mathrm{OH})_{2} \\
\end{array}$ & $\begin{array}{r}5.617 \\
2.365 \\
4.15 \\
\end{array}$ & $\begin{array}{l}0.88 \% \\
2.60 \% \\
0.12 \% \\
\end{array}$ & $\begin{array}{c}13.95 \% \\
--- \\
0.14 \% \\
\end{array}$ & $\begin{array}{l}0.25 \% \\
1.31 \% \\
0.07 \% \\
\end{array}$ & $\begin{array}{l}7.70 \% \\
7.73 \% \\
0.08 \% \\
\end{array}$ & $\begin{array}{l}1.22 \% \\
5.42 \% \\
0.31 \% \\
\end{array}$ & $\begin{array}{l}0.73 \% \\
2.67 \% \\
0.28 \% \\
\end{array}$ & $\begin{array}{l}1.63 \% \\
1.34 \% \\
0.15 \% \\
\end{array}$ & $\begin{array}{l}0.03 \% \\
1.28 \% \\
0.15 \% \\
\end{array}$ \\
\hline $\begin{array}{l}\mathrm{Pb}(\mathrm{OH})_{2} \\
\mathrm{Pu}(\mathrm{OH})_{4} \text { (co-precip. on Fe phase) } \\
\mathrm{PuO}_{2}\end{array}$ & $\begin{array}{c}7.1 \\
4.26 \\
11.43 \\
\end{array}$ & $\begin{array}{c}0.34 \% \\
0.01 \% \\
\cdots \\
\end{array}$ & $\begin{array}{c}6.99 \% \\
0.01 \% \\
--- \\
\end{array}$ & $\begin{array}{c}0.07 \% \\
0.00 \% \\
--- \\
\end{array}$ & $\begin{array}{c}0.35 \% \\
0.00 \% \\
\cdots \\
\end{array}$ & $\begin{array}{c}0.15 \% \\
0.00 \% \\
--- \\
\end{array}$ & $\begin{array}{c}0.21 \% \\
0.00 \% \\
--- \\
\end{array}$ & $\begin{array}{c}0.77 \% \\
0.00 \% \\
--. \\
\end{array}$ & $\begin{array}{c}0.78 \% \\
0.00 \% \\
--. \\
\end{array}$ \\
\hline $\begin{array}{l}\mathrm{SiO}_{2} \\
\mathrm{Sr}(\mathrm{OH})_{2} \\
\mathrm{ZrO}_{2}\end{array}$ & $\begin{array}{c}2.6 \\
3.625 \\
5.68 \\
\end{array}$ & $\begin{array}{c}-- \\
0.02 \% \\
0.02 \%\end{array}$ & $\begin{array}{c}-- \\
0.02 \% \\
--- \\
\end{array}$ & $\begin{array}{c}--- \\
0.01 \% \\
0.01 \%\end{array}$ & $\begin{array}{c}--- \\
0.23 \% \\
0.06 \%\end{array}$ & $\begin{array}{c}--- \\
0.05 \% \\
0.03 \%\end{array}$ & $\begin{array}{c}--- \\
0.01 \% \\
0.04 \%\end{array}$ & $\begin{array}{c}--- \\
0.02 \% \\
0.05 \%\end{array}$ & $\begin{array}{c}--- \\
0.02 \% \\
0.05 \%\end{array}$ \\
\hline
\end{tabular}


Table C.3. (contd)

\begin{tabular}{|c|c|c|c|c|}
\hline & Density $(\mathrm{g} / \mathrm{mL})$ & 241-U-203 & 241-U J-204 & $\begin{array}{c}\text { All-tank } \\
\text { composite }\end{array}$ \\
\hline $\begin{array}{l}\text { Total } \mathrm{kg} \text { washed solids for tank } \\
\text { Total kL washed solids for tank } \\
\text { Avg g/mL, washed solid }\end{array}$ & $\mathrm{n} / \mathrm{a}$ & $\begin{array}{c}5.52 \mathrm{E}+03 \\
2.26 \mathrm{E}+00 \\
2.448\end{array}$ & $\begin{array}{c}1.23 \mathrm{E}+04 \\
5.01 \mathrm{E}+00 \\
2.455\end{array}$ & $\begin{array}{c}2.36 \mathrm{E}+07 \\
8.44 \mathrm{E}+03 \\
2.793\end{array}$ \\
\hline \multicolumn{5}{|l|}{ mass fracs of solids in washed solid } \\
\hline $\begin{array}{l}\mathrm{Ag}_{2} \mathrm{O} \\
\mathrm{Bi}_{2} \mathrm{O}_{3} \\
\mathrm{BiFeO}_{3}\end{array}$ & $\begin{array}{c}7.143 \\
8.9 \\
7.9 \\
\end{array}$ & $\begin{array}{c}--- \\
0.15 \% \\
--- \\
\end{array}$ & $\begin{array}{c}0.04 \% \\
0.20 \% \\
--- \\
\end{array}$ & $\begin{array}{l}0.02 \% \\
1.00 \% \\
2.22 \% \\
\end{array}$ \\
\hline $\begin{array}{l}\mathrm{Ca}(\mathrm{OH})_{2} \\
\mathrm{CrOOH} \\
\mathrm{FeOOH}\end{array}$ & $\begin{array}{l}2.24 \\
4.11 \\
4.26\end{array}$ & $\begin{array}{l}0.21 \% \\
0.36 \% \\
0.62 \%\end{array}$ & $\begin{array}{l}0.28 \% \\
0.01 \% \\
0.65 \%\end{array}$ & $\begin{array}{l}1.97 \% \\
2.94 \% \\
8.22 \%\end{array}$ \\
\hline $\begin{array}{l}\text { Gibbsite } \\
\text { Boehmite } \\
\mathrm{HgO} \text { (co-precip. with } \mathrm{Ag}_{2} \mathrm{O} \text { ) }\end{array}$ & $\begin{array}{c}2.42 \\
3.01 \\
7.143 \\
\end{array}$ & $\begin{array}{c}96.76 \% \\
-- \\
--\end{array}$ & $\begin{array}{c}95.71 \% \\
--- \\
--- \\
\end{array}$ & $\begin{array}{l}50.53 \% \\
11.79 \% \\
0.01 \% \\
\end{array}$ \\
\hline $\begin{array}{l}\mathrm{KAlSiO}_{4} \\
\mathrm{La}(\mathrm{OH})_{3} \\
\mathrm{MnO}_{2} \\
\end{array}$ & $\begin{array}{c}2.61 \\
2.3 \\
5.026 \\
\end{array}$ & $\begin{array}{c}-- \\
0.02 \% \\
0.04 \% \\
\end{array}$ & $\begin{array}{l}0.01 \% \\
0.01 \% \\
0.02 \% \\
\end{array}$ & $\begin{array}{l}0.33 \% \\
0.22 \% \\
1.00 \% \\
\end{array}$ \\
\hline $\begin{array}{l}\mathrm{Na}_{2} \mathrm{U}_{2} \mathrm{O}_{7} \\
\mathrm{NaAlSiO}_{4} \\
\mathrm{Ni}(\mathrm{OH})_{2} \\
\end{array}$ & $\begin{array}{c}5.617 \\
2.365 \\
4.15 \\
\end{array}$ & $\begin{array}{l}0.03 \% \\
0.80 \% \\
0.15 \% \\
\end{array}$ & $\begin{array}{l}0.21 \% \\
1.46 \% \\
0.08 \% \\
\end{array}$ & $\begin{array}{c}3.26 \% \\
12.07 \% \\
0.82 \% \\
\end{array}$ \\
\hline $\begin{array}{l}\mathrm{Pb}(\mathrm{OH})_{2} \\
\mathrm{Pu}(\mathrm{OH})_{4} \text { (co-precip. on Fe phase) } \\
\mathrm{PuO}_{2}\end{array}$ & $\begin{array}{c}7.1 \\
4.26 \\
11.43 \\
\end{array}$ & $\begin{array}{c}0.78 \% \\
0.00 \% \\
\ldots\end{array}$ & $\begin{array}{c}.29 \% \\
0.00 \% \\
---\end{array}$ & $\begin{array}{l}0.43 \% \\
0.00 \% \\
0.00 \% \\
\end{array}$ \\
\hline $\begin{array}{l}\mathrm{SiO}_{2} \\
\mathrm{Sr}(\mathrm{OH})_{2} \\
\mathrm{ZrO}_{2}\end{array}$ & $\begin{array}{c}2.6 \\
3.625 \\
5.68\end{array}$ & $\begin{array}{c}-- \\
0.02 \% \\
0.05 \%\end{array}$ & $\begin{array}{c}-- \\
0.01 \% \\
0.01 \%\end{array}$ & $\begin{array}{l}0.66 \% \\
0.24 \% \\
2.27 \%\end{array}$ \\
\hline
\end{tabular}

(a) This "fully-washed" composition is not the same as that which results from applying the BBI wash factors, which is also referred to as "fully washed" in other contexts. 
Table C.4. Fully-Washed Solid-Phase Compositions of Waste Types Based on 2002 BBI ("---" = not present, "n/a" = insufficient data)

\begin{tabular}{|c|c|c|c|c|c|c|c|c|c|c|c|}
\hline & $\begin{array}{l}\text { density } \\
(\mathrm{g} / \mathrm{mL})\end{array}$ & $\begin{array}{c}\text { Mol. wt. } \\
(\mathrm{g} / \mathrm{mol})\end{array}$ & $\begin{array}{c}\text { Sludge } \\
\text { IC }\end{array}$ & $\begin{array}{c}\text { Sludge } \\
\text { R1 (boiling) }\end{array}$ & $\begin{array}{c}\text { Sludge } \\
2 \mathrm{C}\end{array}$ & $\begin{array}{c}\text { Sludge } \\
\text { TBP }\end{array}$ & $\begin{array}{l}\text { Sludge } \\
\text { CWP2 }\end{array}$ & $\begin{array}{c}\text { Sludge } \\
\text { 224 Post-1949 }\end{array}$ & $\begin{array}{l}\text { Sludge } \\
\text { CWP1 }\end{array}$ & $\begin{array}{l}\text { Sludge } \\
\text { CWZr2 }\end{array}$ & $\begin{array}{l}\text { Sludge } \\
\text { CWR1 }\end{array}$ \\
\hline Avg g/mL, washed solid & $n / a$ & $\mathrm{n} / \mathrm{a}$ & 3.225 & 3.476 & 4.862 & 3.985 & 2.480 & 5.031 & 2.463 & 4.299 & 2.483 \\
\hline \multicolumn{12}{|l|}{ mass fracs of solids in washed solid } \\
\hline $\mathrm{Ag}_{2} \mathrm{O}$ & 7.143 & 231.74 & $0.0 \%$ & --- & $0.0 \%$ & $0.0 \%$ & $0.0 \%$ & $0.0 \%$ & $0.0 \%$ & $0.2 \%$ & $0.0 \%$ \\
\hline $\mathrm{Bi}_{2} \mathrm{O}_{3}$ & 8.9 & 465.96 & --- & $0.1 \%$ & --- & $0.6 \%$ & $0.2 \%$ & $50.0 \%$ & $0.1 \%$ & $0.1 \%$ & $0.1 \%$ \\
\hline $\mathrm{BiFeO}_{3}$ & 7.9 & 312.83 & $30.4 \%$ & --- & $49.3 \%$ & --- & -- & -- & --- & -- & -- \\
\hline $\mathrm{Ca}(\mathrm{OH})_{2}$ & 2.24 & 74.09 & $2.2 \%$ & $2.7 \%$ & $3.1 \%$ & $7.4 \%$ & $0.2 \%$ & $0.6 \%$ & $0.6 \%$ & $0.5 \%$ & $0.4 \%$ \\
\hline $\mathrm{CrOOH}$ & 4.11 & 85 & $0.1 \%$ & $6.9 \%$ & $2.7 \%$ & --- & $0.0 \%$ & $3.2 \%$ & --- & $1.3 \%$ & $4.7 \%$ \\
\hline $\mathrm{FeOOH}$ & 4.26 & 88.85 & $7.4 \%$ & $23.8 \%$ & $31.5 \%$ & $49.8 \%$ & $2.9 \%$ & $6.4 \%$ & $0.8 \%$ & $1.2 \%$ & $0.5 \%$ \\
\hline Gibbsite & 2.42 & 78 & $25.3 \%$ & --- & $3.8 \%$ & $6.3 \%$ & $93.1 \%$ & --- & $74.9 \%$ & $19.0 \%$ & $92.3 \%$ \\
\hline Boehmite & 3.01 & 59.99 & --- & $45.2 \%$ & -.. & --. & --. & --. & ..- & -.- & --. \\
\hline $\mathrm{HgO}$ (co-precip. with $\mathrm{Ag}_{2} \mathrm{O}$ ) & 7.143 & 216.59 & --- & $0.0 \%$ & --- & $0.2 \%$ & --- & --- & $0.0 \%$ & --- & --- \\
\hline $\mathrm{KAISiO}_{4}$ & 2.61 & 158.16 & -- & -- & --- & -- & -- & $0.3 \%$ & $0.3 \%$ & $3.8 \%$ & $0.0 \%$ \\
\hline $\mathrm{La}(\mathrm{OH})_{3}$ & 2.3 & 189.93 & $0.0 \%$ & $0.1 \%$ & $0.5 \%$ & -- & $0.0 \%$ & $15.6 \%$ & $0.1 \%$ & $0.3 \%$ & $0.0 \%$ \\
\hline $\mathrm{MnO}_{2}$ & 5.026 & 86.94 & $0.1 \%$ & $7.9 \%$ & $0.8 \%$ & $0.3 \%$ & $0.2 \%$ & $22.4 \%$ & $0.5 \%$ & $0.4 \%$ & $0.1 \%$ \\
\hline $\mathrm{Na}_{2} \mathrm{U}_{2} \mathrm{O}_{7}$ & 5.617 & 634.03 & $2.2 \%$ & $5.7 \%$ & $0.3 \%$ & $29.0 \%$ & $1.5 \%$ & $0.2 \%$ & $2.3 \%$ & $6.1 \%$ & $0.3 \%$ \\
\hline $\mathrm{Ni}(\mathrm{OH})_{2}$ & 4.15 & 92.71 & $0.0 \%$ & $1.6 \%$ & $0.1 \%$ & $0.2 \%$ & $0.0 \%$ & $0.3 \%$ & $0.5 \%$ & $0.1 \%$ & $0.1 \%$ \\
\hline $\mathrm{Pb}(\mathrm{OH})_{2}$ & 7.1 & 241.21 & $0.1 \%$ & $0.2 \%$ & $1.3 \%$ & $1.1 \%$ & $0.1 \%$ & $0.2 \%$ & $0.1 \%$ & $0.0 \%$ & $0.2 \%$ \\
\hline $\mathrm{Pu}(\mathrm{OH})_{4}$ (co-precip. on Fe phase) & 4.26 & 307.03 & $0.0 \%$ & $0.0 \%$ & $0.0 \%$ & $0.0 \%$ & $0.0 \%$ & $0.0 \%$ & $0.0 \%$ & $0.0 \%$ & $0.0 \%$ \\
\hline $\mathrm{PuO}_{2}$ & 11.43 & 271 & --- & --- & --- & --- & --- & -- & -- & -- & --- \\
\hline $\mathrm{SiO}_{2}$ & 2.6 & 60.08 & --- & --- & --- & $\begin{array}{c}-- \\
\end{array}$ & --- & $\begin{array}{c}-- \\
\end{array}$ & $\begin{array}{c}-- \\
\end{array}$ & --- & --- \\
\hline $\mathrm{Sr}(\mathrm{OH})_{2}$ & 3.625 & 119.62 & $0.2 \%$ & $0.6 \%$ & $0.5 \%$ & $1.2 \%$ & $0.0 \%$ & $0.8 \%$ & $0.0 \%$ & $0.0 \%$ & $0.0 \%$ \\
\hline $\mathrm{ZrO}_{2}$ & 5.68 & 123.22 & $0.2 \%$ & $0.5 \%$ & $0.0 \%$ & -- & $0.0 \%$ & $0.0 \%$ & $0.0 \%$ & $67.0 \%$ & $0.0 \%$ \\
\hline
\end{tabular}


Table C.4. (contd)

\begin{tabular}{|c|c|c|c|c|c|c|c|c|c|c|c|c|}
\hline & $\begin{array}{l}\text { density } \\
(\mathrm{g} / \mathrm{mL})\end{array}$ & $\begin{array}{c}\text { Sludge } \\
\text { DE }\end{array}$ & $\begin{array}{c}\text { Sludge } \\
\text { R1 (non-boiling) }\end{array}$ & $\begin{array}{l}\text { Sludge } \\
\text { PFeCN }\end{array}$ & $\begin{array}{c}\text { Sludge } \\
\text { BL }\end{array}$ & $\begin{array}{l}\text { Sludge } \\
\text { TFeCN }\end{array}$ & $\begin{array}{c}\text { Sludge } \\
\text { P3 }\end{array}$ & $\begin{array}{c}\text { Sludge } \\
\text { 1CFeCN }\end{array}$ & $\begin{array}{l}\text { Sludge } \\
\text { CWR2 }\end{array}$ & $\begin{array}{l}\text { Sludge } \\
\text { SRR }\end{array}$ & $\begin{array}{c}\text { Sludge } \\
\text { AR }\end{array}$ & $\begin{array}{c}\text { Sludge } \\
\text { P2 }\end{array}$ \\
\hline Avg g/mL, washed solid & $\mathrm{n} / \mathrm{a}$ & 2.532 & 2.582 & 2.664 & 3.368 & 2.849 & 2.978 & 3.028 & 2.720 & $\mathrm{n} / \mathrm{a}$ & 3.179 & 3.551 \\
\hline \multicolumn{13}{|l|}{ mass fracs of solids in washed solid } \\
\hline $\mathrm{Ag}_{2} \mathrm{O}$ & 7.143 & --- & $--\cdot$ & $--\cdot$ & $0.0 \%$ & $0.0 \%$ & $0.1 \%$ & $0.0 \%$ & $--\cdot$ & \multirow{21}{*}{$\mathrm{n} / \mathrm{a}$} & $0.2 \%$ & --- \\
\hline $\mathrm{Bi}_{2} \mathrm{O}_{3}$ & 8.9 & $0.4 \%$ & $0.0 \%$ & $0.2 \%$ & $0.1 \%$ & $0.8 \%$ & $0.0 \%$ & $10.0 \%$ & $0.1 \%$ & & $0.0 \%$ & --- \\
\hline $\mathrm{BiFeO}_{3}$ & 7.9 & --- & --- & --- & --- & -- & --- & --- & --- & & --- & --- \\
\hline $\mathrm{Ca}(\mathrm{OH})_{2}$ & 2.24 & $1.0 \%$ & $0.3 \%$ & $3.0 \%$ & $3.2 \%$ & $9.9 \%$ & $1.0 \%$ & $4.7 \%$ & $1.6 \%$ & & $1.2 \%$ & $3.4 \%$ \\
\hline $\mathrm{CrOOH}$ & 4.11 & $0.5 \%$ & $9.4 \%$ & $1.6 \%$ & $0.9 \%$ & $1.8 \%$ & $0.2 \%$ & $4.5 \%$ & $5.3 \%$ & & $0.7 \%$ & --- \\
\hline $\mathrm{FeOOH}$ & 4.26 & $8.8 \%$ & $2.6 \%$ & $7.1 \%$ & $51.2 \%$ & $10.0 \%$ & $30.0 \%$ & $21.0 \%$ & $2.7 \%$ & & $38.0 \%$ & $65.1 \%$ \\
\hline Gibbsite & 2.42 & $56.6 \%$ & $84.0 \%$ & $74.4 \%$ & $33.0 \%$ & $38.1 \%$ & $51.6 \%$ & $10.9 \%$ & $76.2 \%$ & & $46.0 \%$ & $22.8 \%$ \\
\hline Boehmite & 3.01 & -- & --- & --- & --- &.-- &.-- & --- & --- & & $\cdots$ & --- \\
\hline $\mathrm{HgO}$ (co-precip. with $\mathrm{Ag}_{2} \mathrm{O}$ ) & 7.143 & --- & $0.0 \%$ & $0.0 \%$ & $0.0 \%$ & --- & --- & --- & --- & & $0.0 \%$ & $0.0 \%$ \\
\hline $\mathrm{KAlSiO}_{4}$ & 2.61 & $-\cdots$ & $-\cdots$ & --- & --- & $3.3 \%$ & $-\cdots$ & --- & --- & & $-\cdots$ & --- \\
\hline $\mathrm{La}(\mathrm{OH})_{3}$ & 2.3 & $0.1 \%$ & $0.0 \%$ & $0.0 \%$ & $0.6 \%$ & $0.0 \%$ & $1.0 \%$ & $0.0 \%$ & $0.0 \%$ & & $0.1 \%$ & $0.3 \%$ \\
\hline $\mathrm{MnO}_{2}$ & 5.026 & $1.1 \%$ & $0.1 \%$ & $0.1 \%$ & $6.7 \%$ & $0.1 \%$ & $0.5 \%$ & $0.3 \%$ & $0.1 \%$ & & $9.4 \%$ & $1.1 \%$ \\
\hline $\mathrm{Na}_{2} \mathrm{U}_{2} \mathrm{O}_{7}$ & 5.617 & --- & $1.9 \%$ & $6.3 \%$ & $1.5 \%$ & $13.2 \%$ & $2.1 \%$ & $1.0 \%$ & $8.5 \%$ & & $1.2 \%$ & $0.7 \%$ \\
\hline $\mathrm{NaAlSiO}_{4}$ & 2.365 & $30.9 \%$ & $1.3 \%$ & $2.3 \%$ & --- & $16.5 \%$ & $5.3 \%$ & $30.5 \%$ & $1.1 \%$ & & $0.2 \%$ & $0.6 \%$ \\
\hline $\mathrm{Ni}(\mathrm{OH})_{2}$ & 4.15 & $0.1 \%$ & $0.1 \%$ & $1.9 \%$ & $1.2 \%$ & $5.3 \%$ & $1.8 \%$ & $2.7 \%$ & $0.1 \%$ & & $1.3 \%$ & $3.5 \%$ \\
\hline $\mathrm{Pb}(\mathrm{OH})_{2}$ & 7.1 & $0.5 \%$ & $0.1 \%$ & $0.2 \%$ & $1.5 \%$ & $0.8 \%$ & $0.2 \%$ & $0.1 \%$ & $4.2 \%$ & & $1.5 \%$ & $1.6 \%$ \\
\hline $\mathrm{Pu}(\mathrm{OH})_{4}$ (co-precip. on Fe phase) & 4.26 & --- & $0.0 \%$ & $0.0 \%$ & $0.0 \%$ & $0.0 \%$ & $0.0 \%$ & $0.0 \%$ & $0.0 \%$ & & $0.0 \%$ & $0.0 \%$ \\
\hline $\mathrm{PuO}_{2}$ & 11.43 & $0.0 \%$ & $0.0 \%$ & --- & --- & --- & --- & --- & --- & & --- & --- \\
\hline $\mathrm{SiO}_{2}$ & 2.6 & --- & --- & --- & --- & --- & --- & $14.1 \%$ & --- & & --- & --- \\
\hline $\mathrm{Sr}(\mathrm{OH})_{2}$ & 3.625 & $0.0 \%$ & $0.1 \%$ & $3.0 \%$ & $0.1 \%$ & $0.2 \%$ & $0.1 \%$ & -- & $0.0 \%$ & & $0.1 \%$ & $0.2 \%$ \\
\hline $\mathrm{ZrO}_{2}$ & 5.68 & $0.0 \%$ & $0.0 \%$ & $0.0 \%$ & $0.0 \%$ & $0.0 \%$ & $6.2 \%$ & $0.2 \%$ & $0.0 \%$ & & $0.0 \%$ & $0.8 \%$ \\
\hline
\end{tabular}


Table C.4. (contd)

\begin{tabular}{|c|c|c|c|c|c|c|c|c|c|c|c|}
\hline & $\begin{array}{l}\text { density } \\
(\mathrm{g} / \mathrm{mL})\end{array}$ & $\begin{array}{l}\text { Sludge } \\
\text { NW }\end{array}$ & $\begin{array}{c}\text { Sludge } \\
\text { PL2 }\end{array}$ & $\begin{array}{c}\text { Sludge } \\
\text { 224 Pre-1949 }\end{array}$ & $\begin{array}{l}\text { Sludge } \\
\text { CWZr1 }\end{array}$ & $\begin{array}{c}\text { Sludge } \\
\mathrm{z}\end{array}$ & $\begin{array}{l}\text { Sludge } \\
\text { OWw3 }\end{array}$ & $\begin{array}{c}\text { Sludge } \\
\text { TH1 }\end{array}$ & $\begin{array}{c}\text { Sludge } \\
\text { HS }\end{array}$ & $\begin{array}{c}\text { Sludge } \\
\text { Portland Cement }\end{array}$ & $\begin{array}{c}\text { Sludge } \\
\text { B }\end{array}$ \\
\hline Avg g/mL, washed solid & $n / a$ & 2.716 & 2.999 & 4.996 & $\mathrm{n} / \mathrm{a}$ & $\mathrm{n} / \mathrm{a}$ & $\mathrm{n} / \mathrm{a}$ & $\mathrm{n} / \mathrm{a}$ & 4.447 & $\mathrm{n} / \mathrm{a}$ & $\mathrm{n} / \mathrm{a}$ \\
\hline \multicolumn{12}{|l|}{ mass fracs of solids in washed solid } \\
\hline $\mathrm{Ag}_{2} \mathrm{O}$ & 7.143 & $0.0 \%$ & $0.0 \%$ & $0.0 \%$ & \multirow{21}{*}{$\mathrm{n} / \mathrm{a}$} & \multirow{21}{*}{$\mathrm{n} / \mathrm{a}$} & \multirow{21}{*}{ n/a } & \multirow{21}{*}{$\mathrm{n} / \mathrm{a}$} & -- & \multirow{21}{*}{$\mathrm{n} / \mathrm{a}$} & \multirow{21}{*}{$\mathrm{n} / \mathrm{a}$} \\
\hline $\mathrm{Bi}_{2} \mathrm{O}_{3}$ & 8.9 & $0.1 \%$ & $0.1 \%$ & $51.3 \%$ & & & & & -- & & \\
\hline $\mathrm{BiFeO}_{3}$ & 7.9 & --- & --- & --- & & & & & -- & & \\
\hline $\mathrm{Ca}(\mathrm{OH})_{2}$ & 2.24 & $0.7 \%$ & $3.7 \%$ & $5.6 \%$ & & & & & $6.7 \%$ & & \\
\hline $\mathrm{CrOOH}$ & 4.11 & $1.2 \%$ & $6.8 \%$ & $2.2 \%$ & & & & & -- & & \\
\hline $\mathrm{FeOOH}$ & 4.26 & $1.6 \%$ & $6.8 \%$ & $9.1 \%$ & & & & & $49.5 \%$ & & \\
\hline Gibbsite & 2.42 & $77.2 \%$ & $51.1 \%$ & $-\ldots$ & & & & & $1.8 \%$ & & \\
\hline Boehmite & 3.01 & --. & --- & -- & & & & & -- & & \\
\hline $\mathrm{HgO}$ (co-precip. with $\mathrm{Ag}_{2} \mathrm{O}$ ) & 7.143 & --- & --- & -- & & & & & -- & & \\
\hline $\mathrm{KAISiO}_{4}$ & 2.61 & 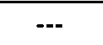 & $8.0 \%$ & $3.2 \%$ & & & & & --.- & & \\
\hline $\mathrm{La}(\mathrm{OH})_{3}$ & 2.3 & $0.0 \%$ & $0.1 \%$ & $9.0 \%$ & & & & & $0.5 \%$ & & \\
\hline $\mathrm{MnO}_{2}$ & 5.026 & $0.4 \%$ & $1.8 \%$ & $18.3 \%$ & & & & & $0.0 \%$ & & \\
\hline $\mathrm{Na}_{2} \mathrm{U}_{2} \mathrm{O}_{7}$ & 5.617 & $16.2 \%$ & $15.8 \%$ & $0.1 \%$ & & & & & $0.0 \%$ & & \\
\hline $\mathrm{NaAlSiO}_{4}$ & 2.365 & $2.1 \%$ & --- & --- & & & & & $0.9 \%$ & & \\
\hline $\mathrm{Ni}(\mathrm{OH})_{2}$ & 4.15 & $0.1 \%$ & $0.2 \%$ & $0.4 \%$ & & & & & $6.3 \%$ & & \\
\hline $\mathrm{Pb}(\mathrm{OH})_{2}$ & 7.1 & $0.2 \%$ & $0.3 \%$ & $0.2 \%$ & & & & & $33.0 \%$ & & \\
\hline $\mathrm{Pu}(\mathrm{OH})_{4}$ (co-precip. on Fe phase) & 4.26 & $0.0 \%$ & $0.0 \%$ & $0.0 \%$ & & & & & $0.0 \%$ & & \\
\hline $\mathrm{PuO}_{2}$ & 11.43 & $-\cdots$ & --- & - & & & & & -- & & \\
\hline $\mathrm{SiO}_{2}$ & 2.6 & $-\cdots$ & --- & --- & & & & & $0.1 \%$ & & \\
\hline $\mathrm{Sr}(\mathrm{OH})_{2}$ & 3.625 & $0.0 \%$ & $0.0 \%$ & $0.6 \%$ & & & & & $1.1 \%$ & & \\
\hline $\mathrm{ZrO}_{2}$ & 5.68 & $0.2 \%$ & $5.3 \%$ & $0.0 \%$ & & & & & --- & & \\
\hline
\end{tabular}


Table C.4. (contd)

\begin{tabular}{|c|c|c|c|c|c|c|c|c|c|c|c|c|}
\hline & $\begin{array}{l}\text { density } \\
(\mathrm{g} / \mathrm{mL})\end{array}$ & $\begin{array}{c}\text { Sludge } \\
\text { P1 }\end{array}$ & $\begin{array}{l}\text { Salt } \\
\text { S1 }\end{array}$ & $\begin{array}{l}\text { Salt } \\
\text { T2 }\end{array}$ & $\begin{array}{l}\text { Salt } \\
\text { BY }\end{array}$ & $\begin{array}{l}\text { Salt } \\
\text { A2 }\end{array}$ & $\begin{array}{c}\text { Salt } \\
\text { S2 }\end{array}$ & $\begin{array}{l}\text { Salt } \\
\text { A1 }\end{array}$ & $\begin{array}{c}\text { Salt } \\
\mathbf{R}\end{array}$ & $\begin{array}{c}\text { Salt } \\
\text { T1 }\end{array}$ & $\begin{array}{c}\text { Salt } \\
\text { B }\end{array}$ & $\begin{array}{l}\text { Salt } \\
\text { R1 }\end{array}$ \\
\hline Avg g/mL, washed solid & $n / a$ & $\mathrm{n} / \mathrm{a}$ & 2.597 & 2.954 & 2.596 & 2.722 & 2.554 & 2.641 & 3.039 & 3.591 & 2.480 & 3.111 \\
\hline \multicolumn{13}{|l|}{ mass fracs of solids in washed solid } \\
\hline $\mathrm{Ag}_{2} \mathrm{O}$ & 7.143 & \multirow{20}{*}{$\mathrm{n} / \mathrm{a}$} & --- & --- & $\cdots$ & --- & --- & --- & --- & --- & --- & --- \\
\hline $\mathrm{Bi}_{2} \mathrm{O}_{3}$ & 8.9 & & $0.2 \%$ & --- & $0.0 \%$ & $0.3 \%$ & $0.1 \%$ & $0.3 \%$ & $0.0 \%$ & $1.2 \%$ & $1.2 \%$ & $0.0 \%$ \\
\hline $\mathrm{BiFeO}_{3}$ & 7.9 & & --- & $19.4 \%$ & --- & --- & -- & --- & --- & --- & --- & --- \\
\hline $\mathrm{Ca}(\mathrm{OH})_{2}$ & 2.24 & & $0.7 \%$ & $2.4 \%$ & $1.1 \%$ & $5.2 \%$ & $0.8 \%$ & $2.0 \%$ & $0.3 \%$ & $3.9 \%$ & $1.3 \%$ & $0.4 \%$ \\
\hline $\mathrm{CrOOH}$ & 4.11 & & $8.2 \%$ & $1.1 \%$ & $11.3 \%$ & $16.4 \%$ & $8.3 \%$ & $12.3 \%$ & --- & $0.3 \%$ & $0.1 \%$ & $6.5 \%$ \\
\hline $\mathrm{FeOOH}$ & 4.26 & & $4.7 \%$ & $8.5 \%$ & $5.7 \%$ & $2.1 \%$ & $3.0 \%$ & $2.5 \%$ & $1.3 \%$ & $59.8 \%$ & $0.9 \%$ & $0.9 \%$ \\
\hline Gibbsite & 2.42 & & $80.5 \%$ & $67.1 \%$ & $19.0 \%$ & $55.2 \%$ & $82.0 \%$ & $68.5 \%$ & --- & $23.3 \%$ & $91.8 \%$ & --- \\
\hline Boehmite & 3.01 & & -- & -- & --- & --- & -- & --. & $91.1 \%$ & -- & $\cdots$ & $84.6 \%$ \\
\hline $\mathrm{HgO}$ (co-precip. with $\mathrm{Ag}_{2} \mathrm{O}$ ) & 7.143 & & --- & --- & --- & --- & --- & --- & --- & --- & --- & --- \\
\hline $\mathrm{KAlSiO}_{4}$ & 2.61 & & --- & --- & $2.7 \%$ & $10.4 \%$ & --- & --- & --- & --- & --- & --- \\
\hline $\mathrm{La}(\mathrm{OH})_{3}$ & 2.3 & & $0.1 \%$ & $0.3 \%$ & $0.0 \%$ & $0.3 \%$ & $0.0 \%$ & $0.2 \%$ & $0.0 \%$ & $0.2 \%$ & $0.0 \%$ & $0.0 \%$ \\
\hline $\mathrm{MnO}_{2}$ & 5.026 & & $0.7 \%$ & $0.2 \%$ & -- & -- & --- & --- & $0.9 \%$ & $8.6 \%$ & $0.0 \%$ & $1.0 \%$ \\
\hline $\mathrm{Na}_{2} \mathrm{U}_{2} \mathrm{O}_{7}$ & 5.617 & & $1.2 \%$ & --- & $1.1 \%$ & $3.4 \%$ & $0.8 \%$ & $2.8 \%$ & $2.3 \%$ & --- & $1.3 \%$ & $3.5 \%$ \\
\hline $\mathrm{Ni}(\mathrm{OH})_{2}$ & 4.15 & & $0.3 \%$ & $0.2 \%$ & $0.3 \%$ & $0.4 \%$ & $0.2 \%$ & $0.5 \%$ & $0.4 \%$ & $0.2 \%$ & $0.5 \%$ & $0.1 \%$ \\
\hline $\mathrm{Pb}(\mathrm{OH})_{2}$ & 7.1 & & $0.3 \%$ & $0.6 \%$ & $0.1 \%$ & $1.3 \%$ & $0.2 \%$ & $0.7 \%$ & $0.0 \%$ & $2.2 \%$ & $0.5 \%$ & $0.0 \%$ \\
\hline $\mathrm{Pu}(\mathrm{OH})_{4}$ (co-precip. on Fe phase) & 4.26 & & $0.0 \%$ & --- & $0.0 \%$ & $0.0 \%$ & $0.0 \%$ & $0.0 \%$ & $0.0 \%$ & $0.0 \%$ & $0.0 \%$ & $0.0 \%$ \\
\hline $\mathrm{PuO}_{2}$ & 11.43 & & --- & $0.0 \%$ & --- & --- & --- & --- & --- & --- & --- & --- \\
\hline $\mathrm{SiO}_{2}$ & 2.6 & & --- & --- & --- & --- & --- & --- & --- & --- & --- & --- \\
\hline $\mathrm{Sr}(\mathrm{OH})_{2}$ & 3.625 & & $0.0 \%$ & $0.2 \%$ & $0.2 \%$ & $0.1 \%$ & $0.0 \%$ & $0.1 \%$ & $0.1 \%$ & $0.1 \%$ & $0.0 \%$ & $0.2 \%$ \\
\hline $\mathrm{ZrO}_{2}$ & 5.68 & & $0.1 \%$ & $0.2 \%$ & $0.0 \%$ & $0.1 \%$ & $0.0 \%$ & $0.2 \%$ & $0.0 \%$ & $0.1 \%$ & $0.1 \%$ & $0.0 \%$ \\
\hline
\end{tabular}





\section{Appendix D}

\section{UDS Primary Particle Size and Shape}





\section{Appendix D: UDS Primary Particle Size and Shape}

This appendix lists the expert panel convened to review and revise the particle size estimates and determine consensus options for those compounds without information (Section D.1), expert panel meeting notes (Section D.2), a summary of the analyzed/consensus particle size information, including information from Wells et al. (2007) (Section D.3), and the analyzed images (Section D.4).

\section{D.1 Expert Panel}

$\begin{array}{ll}\text { Edgar Buck } & \text { PNNL } \\ \text { Bill Callaway } & \text { WRPS } \\ \text { Gary Cooke } & \text { WRPS } \\ \text { Dan Greer } & \text { WRPS } \\ \text { Dan Herting } & \text { WRPS/WTP } \\ \text { Jim Huckaby } & \text { PNNL } \\ \text { Dean Kurath } & \text { PNNL } \\ \text { Gregg Lumetta } & \text { PNNL } \\ \text { Lenna Mahoney } & \text { PNNL } \\ \text { Brian Rapko } & \text { PNNL } \\ \text { Juergen Rasmussen } & \text { WRPS } \\ \text { Jacob Reynolds } & \text { WRPS } \\ \text { Joel Tingey } & \text { PNNL } \\ \text { Beric Wells } & \text { PNNL }\end{array}$

\section{D.2 Expert Panel Meeting Notes}

From 11/4/2010 Meeting:

\section{$\underline{\text { Ag compounds }}$}

Gary: He has seen Ag-O, but couldn't say that it's $\mathrm{Ag}_{2} \mathrm{O}$ specifically. Because of carbon mounting of the samples, can’t distinguish Ag oxide from carbonate.

Resolution: The newly determined particle size for Ag was accepted. Using the RPT-153 " $\mathrm{Ag}_{2} \mathrm{CO}_{3}$ " sizes for current report's $\mathrm{Ag}_{2} \mathrm{O}$ was also accepted.

\section{$\underline{\text { Gibbsite }}$}

Dan: The RPT-153 maximum size of $20 \mu \mathrm{m}$ needs to be increased in view of the $\sim 200-\mu \mathrm{m}$ primary particles seen in S-102 and S-112 heels.

\section{Ca compounds}

Dan: Are so many different Ca compounds necessary for the purpose? 
Gary: Has often seen Ca-P particles up to $1 \mu \mathrm{m}$, apparently primary particles. Recommended changing max size from $0.1 \mu \mathrm{m}$ to $1 \mu \mathrm{m}$ for $\mathrm{Ca}_{5} \mathrm{OH}\left(\mathrm{PO}_{4}\right)_{3}$. He hasn't explicitly seen $\mathrm{Ca}(\mathrm{OH})_{2}$ or $\mathrm{CaC}_{2} \mathrm{O}_{4}$, but others have mentioned seeing the latter.

Resolution: The max size for $\mathrm{Ca}_{5} \mathrm{OH}\left(\mathrm{PO}_{4}\right)_{3}$ will be increased to $1 \mu \mathrm{m}$. The same size distribution will be assigned to $\mathrm{Ca}(\mathrm{OH})_{2}$ and $\mathrm{CaC}_{2} \mathrm{O}_{4} \cdot \mathrm{H}_{2} \mathrm{O}$ that is used for $\mathrm{Ca}_{5} \mathrm{OH}\left(\mathrm{PO}_{4}\right)_{3}$. No change in chemical breakdown of Ca compounds - it may be more detail than needed, but to some extent reflects density uncertainty and the fix would be hard to fit into review schedule.

\section{$\underline{\text { Hg compounds }}$}

Gary: The only time he has seen Hg has been in association with Ag; there was more than one example.

Resolution: Assign $\mathrm{HgO}$ the same sizes as $\mathrm{Ag}_{2} \mathrm{O}$, and assign it the same density as $\operatorname{Ag}_{2} \mathrm{O}$, even in tanks where no $\mathrm{Ag}$ is supposed to be in the inventory. The latter is a minor spreadsheet change.

$\underline{\mathrm{KAlSiO}}_{4}$

Resolution: Assign same size info as for sodium aluminosilicate.

\section{$\underline{\text { La compounds }}$}

Gary: He hasn't seen La-rich particles. Lanthanum is often found in association with $\mathrm{Ca}, \mathrm{Fe}, \mathrm{Al}, \mathrm{Sr}$, rare earths (such as $\mathrm{Nd}$ ), and $\mathrm{P}$. The La used in the process was not pure, but combined to some extent with other rare earths. La-containing particles are always small.

Actions: Joel will look into La-related info. Edgar will look for sizes of rare-earth particles.

Resolution: $\mathrm{La}(\mathrm{OH})_{3}$ will be given the same size data as $\mathrm{LaPO}_{4} \cdot 2 \mathrm{H}_{2} \mathrm{O}$. The size data for the latter may be changed from the RPT-153 values, depending on what Edgar and Joel and Gary come up with.

\section{$\underline{\text { Ni compounds }}$}

Gregg: Ni oxalate doesn't exist, it can be zeroed out. If not, it can be assigned the max $~ 2 \mu \mathrm{m}$ size data of $\mathrm{Fe}_{2} \mathrm{O}_{3}$.

Gary: Ni often found with Mn, Fe, P.

Resolution: Treat $\mathrm{Ni}_{3}\left(\mathrm{PO}_{4}\right)_{2}$ as having the same size data as $\mathrm{Mn}_{3}\left(\mathrm{PO}_{4}\right)_{2}$, since the compounds are analogous. Treat $\mathrm{NiC}_{2} \mathrm{O}_{4} \cdot 2 \mathrm{H}_{2} \mathrm{O}$ as having the size data and density of $\mathrm{Fe}_{2} \mathrm{O}_{3}$ from RTP-153.

\section{$\underline{\text { Pb compounds }}$}

Gary: Pb particles observed before 2004 probably came from the Pb-shielded sample carriers, not the waste, except in $\mathrm{C}$ tanks. When a switch was made from Pb-shielded carriers to W-shielded carriers, small $\mathrm{Pb}$ particles stopped appearing and were replaced by small $\mathrm{W}$ particles. The true waste $\mathrm{Pb}$ particles were also small. 
Jake: The heels left behind in the $\mathrm{C}-200$ series of tanks did not contain $\mathrm{Pb}$, in spite of high $\mathrm{Pb}$ content in inventories before retrieval. This suggests that $\mathrm{Pb}$ particles in waste were small and easily carried over.

Edgar: Would make sense to assign Fe-compound density and size data to $\mathrm{Pb}$ compounds, except in $\mathrm{C}-200$ series where $\mathrm{Pb}$ compounds are large fraction of solids. This would follow from frequent observations of small amounts of $\mathrm{Pb}$ in Fe-rich mixed phases.

Resolution: Use the size data for $\mathrm{Pb}_{5} \mathrm{OH}\left(\mathrm{PO}_{4}\right)_{3}$ for all $\mathrm{Pb}$ compounds, since those data show small sizes consistent with observations of Pb-containing particles. Will mention the approach that Edgar suggested as part of the mixed-phase data-gap discussion, but leave the composition calculations as-is in view of the schedule and review constraints.

\section{$\underline{\text { Pu compounds }}$}

Dan: If the RPT-153 max size of $40 \mu \mathrm{m}$ is going to be continued into the new report, it needs to be made clear that it is not an equivalent spherical diameter but a length of an elongated shape.

Jake: There's an Andy Felmy paper that identified the $\mathrm{Pu}$ in certain trenches as $\mathrm{Pu}(\mathrm{OH})_{4}$. The significance is that this particular trench waste came from the same source that fed SY-102, TX-118, and possibly other TX tanks. However, Jake wasn't sure how definite Andy's paper was about the phase identification. Apparently PFP (Plutonium Finishing Plant), the waste source, was in this period trying to dissolve high-fired $\mathrm{PuO}_{2}$ material from Rocky Flats and possibly didn't dissolve all of it, and didn't filter the process material to remove solids before sending it out as waste.

Dan and Joel: Both agree that high-fired $\mathrm{PuO}_{2}$ wouldn’t change to $\mathrm{Pu}(\mathrm{OH})_{4}$ in the trenches.

Gary: Saw an individual (i.e., probably not co-precipitate) Pu-rich particle on a mat of other particles in a sample from AY-102. It was about $3 \mu \mathrm{m}$ in size (no statement about shape). Some $\mathrm{P}$ and $\mathrm{O}$ were also present but may have been from the mat. These Pu-rich particles do not have large a ratio of length to width as the $40-\mu \mathrm{m}$-long particle Dan noted.

Action: Jake to check the Felmy report to see how definitely $\mathrm{Pu}(\mathrm{OH})_{4}$ was identified.

Resolution: Make sure new report makes it clear that Pu particles have not been spherical or near-spherical in shape. Continue with approach of assigning $\mathrm{PuO}_{2}$ properties to SY-102 and TX farm $\mathrm{Pu}$, and assigning $\mathrm{Pu}(\mathrm{OH})_{4}$ identity and $\mathrm{FeOOH}$ properties to $\mathrm{Pu}$ in all other tanks (treating it as co-precipitate).

\section{$\underline{\text { Sr compounds }}$}

Gary: $\mathrm{Sr}$ is usually found in hydro-uvarovite. He believes there can be $\mathrm{SrCO}_{3}$ in some wastes; a dissolution test seemed to indicate its presence in a saltcake waste sample. A relatively large particle of nastrophite was found in C-104, about $20 \mu \mathrm{m}$ (?) maximum.

Jake: There was a nastrophite particle of about $200 \mu \mathrm{m}$ size in C-108. $\mathrm{SrCO}_{3}$ hasn't been observed, which may mean its small particles.

Action: Gary will look for information (images etc.) on large nastrophite particles and send it to Edgar.

\section{D.3}


Resolution: Change the $\mathrm{Sr}_{3}\left(\mathrm{PO}_{4}\right)_{2}$ size range to a larger one that reflects the nastrophite observations. Use the RPT-153 $\mathrm{Sr}_{3}\left(\mathrm{PO}_{4}\right)_{2}$ size range (small particles) to describe $\mathrm{SrCO}_{3}$.

Amorphous phases

Some general discussion.

Jake: The amorphous particles are typically the result of rapid precipitation and as a result have small primary particle size ( 0.1 um).

Gary: On the other hand, can’t rule out cemented aggregates.

Resolution: Add these general remarks to discussion of amorphous phases in report.

$\underline{\text { Salts }}$

Gary: He can't systematically assemble a set of images of salt at this time-maybe around Thanksgiving or so. Regarding individual salts.

He hasn’t seen $\mathrm{KNO}_{3}$.

Has seen $\mathrm{Na}_{2} \mathrm{C}_{2} \mathrm{O}_{4}$, frequently. It has needle shape. Typically has an upper limit on size of about $20 \mu \mathrm{m}$ long, $5 \mu \mathrm{m}$ wide.

Has seen $\mathrm{Na}_{2} \mathrm{CO}_{3} \cdot \mathrm{H}_{2} \mathrm{O}$. It too has an upper limit on size, he doesn’t recall what.

He has seen both $\mathrm{Na}_{2} \mathrm{SO}_{4}$ and $\mathrm{Na}_{2} \mathrm{SO}_{4} \cdot 10 \mathrm{H}_{2} \mathrm{O}$, but the latter has been so infrequent he just saw it for the first time. Usually if there's any F present, the sulfates prefer to precipitate as the fluoride sulfate.

He has seen $\mathrm{Na}_{3} \mathrm{FSO}_{4}$ and thinks the top size was (?) $200 \mathrm{um}$.

He hasn't seen $\mathrm{Na}_{3} \mathrm{NO}_{3} \mathrm{SO}_{4} \cdot \mathrm{H}_{2} \mathrm{O}$, but recalls that Dan Herting thought he had seen it in a sample from a TX tank. (Dan wasn't around at this point in the meeting to speak to the subject.)

He has seen plenty of $\mathrm{Na}_{3} \mathrm{PO}_{4} \cdot 0.25 \mathrm{NaOH} \bullet 12 \mathrm{H}_{2} \mathrm{O}$. There is no obvious max length; they keep right on growing. They have a needle shape, and he has images that show hollow needles in some cases. This has implications for the effective density.

He has not seen $\mathrm{Na}_{3} \mathrm{PO}_{4} \bullet 8 \mathrm{H}_{2} \mathrm{O}$.

Jake: That phosphate form should be around. The evaporator runs targeted conditions that would produce it, in preference to the dodecahydrate with its difficult flow behavior.

Gary: Not too sure about the pyrophosphates $\left(\mathrm{Na}_{4} \mathrm{P}_{2} \mathrm{O}_{7} \bullet 10 \mathrm{H}_{2} \mathrm{O}\right)$.

He has seen $\mathrm{Na}_{6}\left(\mathrm{SO}_{4}\right)_{2} \mathrm{CO}_{3}$.

He has definitely seen $\mathrm{NaF}$, which is always small particles. 
He has seen plenty of $\mathrm{Na}_{2} \mathrm{~F}\left(\mathrm{PO}_{4}\right)_{2} \cdot 19 \mathrm{H}_{2} \mathrm{O}$ in all sizes. Showed a picture of a 3-inch chunk. There is no apparent upper limit on size.

$\mathrm{NaHCO}_{3}$. Its presence is $\mathrm{pH}$-related.

He has seen $\mathrm{NaNO}_{2}$, but it may have been an artifact of drying interstitial liquid.

His observations of $\mathrm{NaNO}_{3}$ suggest no upper limit on particle size. It's another source of very large particles.

Gary: In general, although salts are hard they are easy to break up, owing to their cleavage planes.

Jake: Recommended Dave Schuford (?) as having process knowledge pertinent to salts.

Action: Gary to supply Edgar with sets of images, but not for at least 2 weeks. Edgar to look into reports to find them in the meantime.

Resolution: Don’t have recommended size ranges for salts at this time.

Table D.1. 11/4/2010 Meeting Notes Summary Table

\begin{tabular}{|c|c|c|c|c|}
\hline & Max & Mode & Min & \\
\hline & $(\mu \mathrm{m})$ & $(\mu \mathrm{m})$ & $(\mu \mathrm{m})$ & Data Source and Comments \\
\hline Ag & 2 & -- & -- & $\begin{array}{l}\text { Data review in present study; } \\
\text { only one particle }\end{array}$ \\
\hline $\mathrm{Ag}_{2} \mathrm{O}$ & 4 & -- & -- & $\begin{array}{l}\text { As for } \mathrm{Ag}_{2} \mathrm{CO}_{3} \text { in } \\
\text { WTP-RPT-153, Table 3.2.10 }\end{array}$ \\
\hline $\mathrm{Al}(\mathrm{OH})_{3}$, gibbsite & 200 & ? & 0.8 & $\begin{array}{l}\text { Updated max from Dan } \\
\text { Herting (need reference); will } \\
\text { need to generate new mode; } \\
\text { min from WTP-RPT-153, } \\
\text { Table 3.2.10. }\end{array}$ \\
\hline AlOOH, boehmite & 0.052 & 0.048 & 0.015 & $\begin{array}{l}\text { Non-spherical, so equiv. } \\
\text { diameter is given; } \\
\text { WTP-RPT-153, p. } 3.48\end{array}$ \\
\hline $\mathrm{BiFeO}_{3}$ & 0.1 & 0.06 & 0.03 & Data review in present study \\
\hline $\mathrm{Bi}_{2} \mathrm{O}_{3}$ & 10 & 2 & 1 & $\begin{array}{l}\text { As for } \mathrm{BiPO}_{4} \text { in } \\
\text { WTP-RPT-153, Table 3.2.10 }\end{array}$ \\
\hline $\mathrm{Ca}(\mathrm{OH})_{2}{ }^{[1]}$ & \multirow{2}{*}{\multicolumn{3}{|c|}{ As for $\mathrm{Ca}_{5} \mathrm{OH}\left(\mathrm{PO}_{4}\right)_{3}$}} & Agreed in meeting \\
\hline $\mathrm{CaC}_{2} \mathrm{O}_{4} \cdot \mathrm{H}_{2} \mathrm{O}^{[1]}$ & & & & Agreed in meeting \\
\hline $\mathrm{CaCO}_{3}$ & 14 & -- & -- & WTP-RPT-153, Table 3.2.10 \\
\hline $\mathrm{CaF}_{2}$ & 15 & -- & -- & WTP-RPT-153, Table 3.2.10 \\
\hline
\end{tabular}


Table D.1. (contd)

\begin{tabular}{|c|c|c|c|c|}
\hline \multirow{3}{*}{$\mathrm{Ca}_{5} \mathrm{OH}\left(\mathrm{PO}_{4}\right)_{3}$} & \multirow{2}{*}{$\begin{array}{l}\text { Max } \\
(\mu \mathrm{m})\end{array}$} & \multirow{2}{*}{$\frac{\text { Mode }}{(\mu \mathrm{m})}$} & \multirow{2}{*}{$\frac{\text { Min }}{(\mu \mathrm{m})}$} & \multirow[b]{2}{*}{ Data Source and Comments } \\
\hline & & & & \\
\hline & 1 & ? & 0.05 & $\begin{array}{l}\text { Updated max from Gary } \\
\text { Cooke (need reference); will } \\
\text { need to generate new mode; } \\
\text { min from WTP-RPT-153, } \\
\text { Table 3.2.10. }\end{array}$ \\
\hline $\mathrm{CrOOH}$ & 0.43 & 0.105 & 0.022 & Data review in present study \\
\hline $\mathrm{FeOOH}$ & 0.015 & 0.008 & 0.004 & Data review in present study \\
\hline $\mathrm{FePO}_{4} \cdot 2 \mathrm{H}_{2} \mathrm{O}$ & 0.02 & 0.015 & 0.005 & Data review in present study \\
\hline $\mathrm{HgO}^{[2]}$ & \multicolumn{3}{|c|}{ as for $\mathrm{Ag}_{2} \mathrm{O}$} & $\begin{array}{l}\text { Agreed in meeting; density } \\
\text { will also be set to that of } \\
\mathrm{Ag}_{2} \mathrm{O} \text {, even in tanks where no } \\
\mathrm{Ag} \text { is present. }\end{array}$ \\
\hline $\mathrm{KAlSiO}_{4}$ & \multicolumn{3}{|c|}{$\begin{array}{c}\text { as for } \\
\left(\mathrm{NaSiO}_{4}\right)_{6} \cdot 2\left(\mathrm{NaNO}_{3}\right)_{1.6} \cdot 2 \mathrm{H}_{2} \mathrm{O}\end{array}$} & Agreed in meeting \\
\hline $\mathrm{La}(\mathrm{OH})_{3}$ & \multicolumn{3}{|c|}{ as for $\mathrm{LaPO}_{4} \cdot 2 \mathrm{H}_{2} \mathrm{O}$} & Agreed in meeting \\
\hline $\mathrm{LaPO}_{4} \cdot 2 \mathrm{H}_{2} \mathrm{O}$ & 3 ? & $0.2 ?$ & $0.1 ?$ & $\begin{array}{l}\text { Values are from } \\
\text { WTP-RPT-153, Table 3.2.10; } \\
\text { they may change after new } \\
\text { information has been } \\
\text { reviewed. }\end{array}$ \\
\hline $\mathrm{MnO}_{2}$ & 10 & 1.3 & 0.3 & WTP-RPT-153, Table 3.2.10 \\
\hline $\mathrm{Mn}_{3}\left(\mathrm{PO}_{4}\right)_{2}$ & 8 & -- & 8 & WTP-RPT-153, Table 3.2 .10 \\
\hline $\mathrm{NaAlCO}_{3}(\mathrm{OH})_{2}$ & 4.2 & 0.94 & 0.14 & $\begin{array}{l}\text { Non-spherical, so equiv. } \\
\text { diameter is given; } \\
\text { WTP-RPT-153, p. 3.48. }\end{array}$ \\
\hline$\left(\mathrm{NaSiO}_{4}\right)_{6} \cdot 2\left(\mathrm{NaNO}_{3}\right)_{1.6} \cdot 2 \mathrm{H}_{2} \mathrm{O}$ & 8 & 2.5 & 1 & WTP-RPT-153, Table 3.2.10 \\
\hline $\mathrm{Na}_{2}\left(\mathrm{UO}_{2}\right)_{2}\left(\mathrm{PO}_{4}\right)_{2} \cdot 2 \mathrm{H}_{2} \mathrm{O}$ & 10 & 5 & 2 & Data review in present study \\
\hline $\mathrm{Na}_{2} \mathrm{U}_{2} \mathrm{O}_{7}$ & 15 & 0.1 & 0.05 & WTP-RPT-153, Table 3.2.10 \\
\hline $\mathrm{Ni}(\mathrm{OH})_{2}$ & 0.5 & 0.2 & 0.005 & WTP-RPT-153, Table 3.2.10 \\
\hline $\mathrm{Ni}_{3}\left(\mathrm{PO}_{4}\right)_{2}$ & \multicolumn{3}{|c|}{ as for $\mathrm{Mn}_{3}\left(\mathrm{PO}_{4}\right)_{2}$} & Agreed in meeting \\
\hline $\mathrm{NiC}_{2} \mathrm{O}_{4} \cdot 2 \mathrm{H}_{2} \mathrm{O}$ & 1.6 & 0.88 & 0.037 & $\begin{array}{l}\text { Agreed in meeting; as for } \\
\mathrm{Fe}_{2} \mathrm{O}_{3} \text { in WTP-RPT-153, } \\
\text { p. 3.48; non-spherical, so } \\
\text { equiv. diameter is given; } \\
\text { density is also that of } \mathrm{Fe}_{2} \mathrm{O}_{3} \text {, } \\
\text { assumes co-precipitation. }\end{array}$ \\
\hline $\mathrm{Pb}(\mathrm{OH})_{2}$ & \multicolumn{3}{|c|}{ as for $\mathrm{Pb}_{3}\left(\mathrm{PO}_{4}\right)_{2}$} & Agreed in meeting \\
\hline $\mathrm{Pb}_{3}\left(\mathrm{PO}_{4}\right)_{2}$ & 0.4 & -- & 0.05 & $\begin{array}{l}\text { As for } \mathrm{Pb}_{5} \mathrm{OH}\left(\mathrm{PO}_{4}\right)_{3} \text { in } \\
\text { WTP-RPT-153, Table 3.2.10 }\end{array}$ \\
\hline $\mathrm{PbCO}_{3}$ & \multicolumn{3}{|c|}{ as for $\mathrm{Pb}_{3}\left(\mathrm{PO}_{4}\right)_{2}$} & Agreed in meeting \\
\hline
\end{tabular}


Table D.1. (contd)

\begin{tabular}{|c|c|c|c|c|}
\hline & Max & Mode & Min & \\
\hline & $(\mu \mathrm{m})$ & $(\mu \mathrm{m})$ & $(\mu \mathrm{m})$ & Data Source and Comments \\
\hline $\mathrm{PuO}_{2}$ & 40 & 8 & 1 & $\begin{array}{l}\text { Non-spherical; values given } \\
\text { are lengths and need to be } \\
\text { converted to equivalent } \\
\text { diameters. }\end{array}$ \\
\hline $\mathrm{Pu}(\mathrm{OH})_{4}[4]$ & \multicolumn{3}{|c|}{ as for $\mathrm{FeOOH}$} & $\begin{array}{l}\text { Agreed in meeting; density is } \\
\text { also that of FeOOH, assumes } \\
\text { mixed phase. }\end{array}$ \\
\hline $\mathrm{SiO}_{2}$ & 100 & -- & -- & WTP-RPT-153, Table 3.2.10 \\
\hline $\mathrm{Sr}_{3}\left(\mathrm{PO}_{4}\right)_{2}$ & $200 ?$ & ? & ? & $\begin{array}{l}\text { Will be based on images of } \\
\text { large nastrophite particles; } \\
\text { need references. }\end{array}$ \\
\hline $\mathrm{SrCO}_{3}$ & 0.3 & 0.1 & 0.03 & $\begin{array}{l}\text { Data review for } \mathrm{Sr}_{3}\left(\mathrm{PO}_{4}\right)_{2} \text { in } \\
\text { present study, but did not } \\
\text { include nastrophite; agreed in } \\
\text { meeting. }\end{array}$ \\
\hline $\mathrm{Sr}(\mathrm{OH})_{2}$ & \multicolumn{3}{|c|}{ as for $\mathrm{SrCO}_{3}$} & $\begin{array}{l}\text { Not discussed in meeting - } \\
\text { need concurrence }\end{array}$ \\
\hline $\mathrm{ZrO}_{2}$ & 50 & 0.2 & 0.05 & WTP-RPT-153, Table 3.2.10 \\
\hline
\end{tabular}

Note: The compounds $\mathrm{Ag}_{2} \mathrm{CO}_{3}$ and $\mathrm{Fe}_{2} \mathrm{O}_{3}$ (hematite), which were used in WTP-RPT-153, are not used in the present study and so are omitted from the above table. 


\section{D.3 Analysis Summary}

A summary of analyzed/consensus particle size information, including information from Wells et al. (2007), is provided in Table D.2.

Table D.2. Summary of Particle Sizes (including information from Wells et al. 2007)

\begin{tabular}{|c|c|c|c|c|c|}
\hline Mol. Formula & Mineral Name & \multicolumn{3}{|c|}{$\begin{array}{l}\text { Triangular Distribution of } \\
\text { Primary Particles }(\mu \mathrm{m})\end{array}$} & Source Reference and Comments[h] \\
\hline $\mathrm{Ag}$ & metallic silver (alloy with $\mathrm{Hg}$ ) & 2 & & & Cantrell et al. 2008b, PNNL-16738 \\
\hline $\mathrm{Bi}_{2} \mathrm{O}_{3}$ & bismite & 3 & 1 & 1 & $\begin{array}{l}\text { Data collected by EC Buck for Adam Poloski } 2006 \\
\text { (Bismuth Phosphate processing waste) }\end{array}$ \\
\hline $\mathrm{BiFeO}_{3}$ & $\begin{array}{l}\text { bismuth iron silicate- } \\
\text { phosphate (bismutoferrite } \\
\text { related) }\end{array}$ & 0.1 & 0.06 & 0.035 & Lumetta et al. 2009, PNNL-17992, pg. 3.34, Figure 3.22 \\
\hline $\mathrm{Ca}(\mathrm{OH})_{2}[\mathbf{a}]$ & & & & & $\begin{array}{l}\text { Use same distribution as calcium phosphate per Committee } \\
\text { Agreement (11/4/10 meeting notes) }\end{array}$ \\
\hline $\mathrm{Ca}_{5} \mathrm{OH}\left(\mathrm{PO}_{4}\right)_{3}$ & apatite-related phase & 9 & & 0.05 & $\begin{array}{l}\text { Cantrell et al. 2008b, PNNL-16738 } \\
\text { Apatite: see } \\
\text { WRPS-1000365 JM Frye and GA Cooke to DE Place, } \\
\text { April 9, 2010, Investigation of the Cause of Discrepancies in } \\
\text { the Ion Chromatography and Inductively Coupled Plasma } \\
\text { Arc Spectrometry Data for Sulfate and Phosphate in Tank } \\
\text { 241-AP-103 CORE 332, 241-SY-103 CORE 326, AND } \\
\text { 241-AP-102 2007 Supernate Grab Samples, Washington } \\
\text { River Protection Solutions. } \\
\text { LAB-RPT-10-00001 Revision 0, WS Callaway and HJ } \\
\text { Huber, June 2010, Results of Physicochemical } \\
\text { Characterization and Caustic Dissolution Tests on Tank 241- } \\
\text { C-108 Heel Solids, Washington River Protection Solutions. }\end{array}$ \\
\hline $\mathrm{CaC}_{2} \mathrm{O}_{4} \cdot \mathrm{H}_{2} \mathrm{O}$ & & & & & $\begin{array}{l}\text { Use same distribution as calcium phosphate per Committee } \\
\text { Agreement (11/4/10 meeting notes) }\end{array}$ \\
\hline $\mathrm{CaCO}_{3}$ & calcite & 55 & & & Cantrell et al. 2008b, PNNL-16738 \\
\hline
\end{tabular}


Table D.2. (contd)

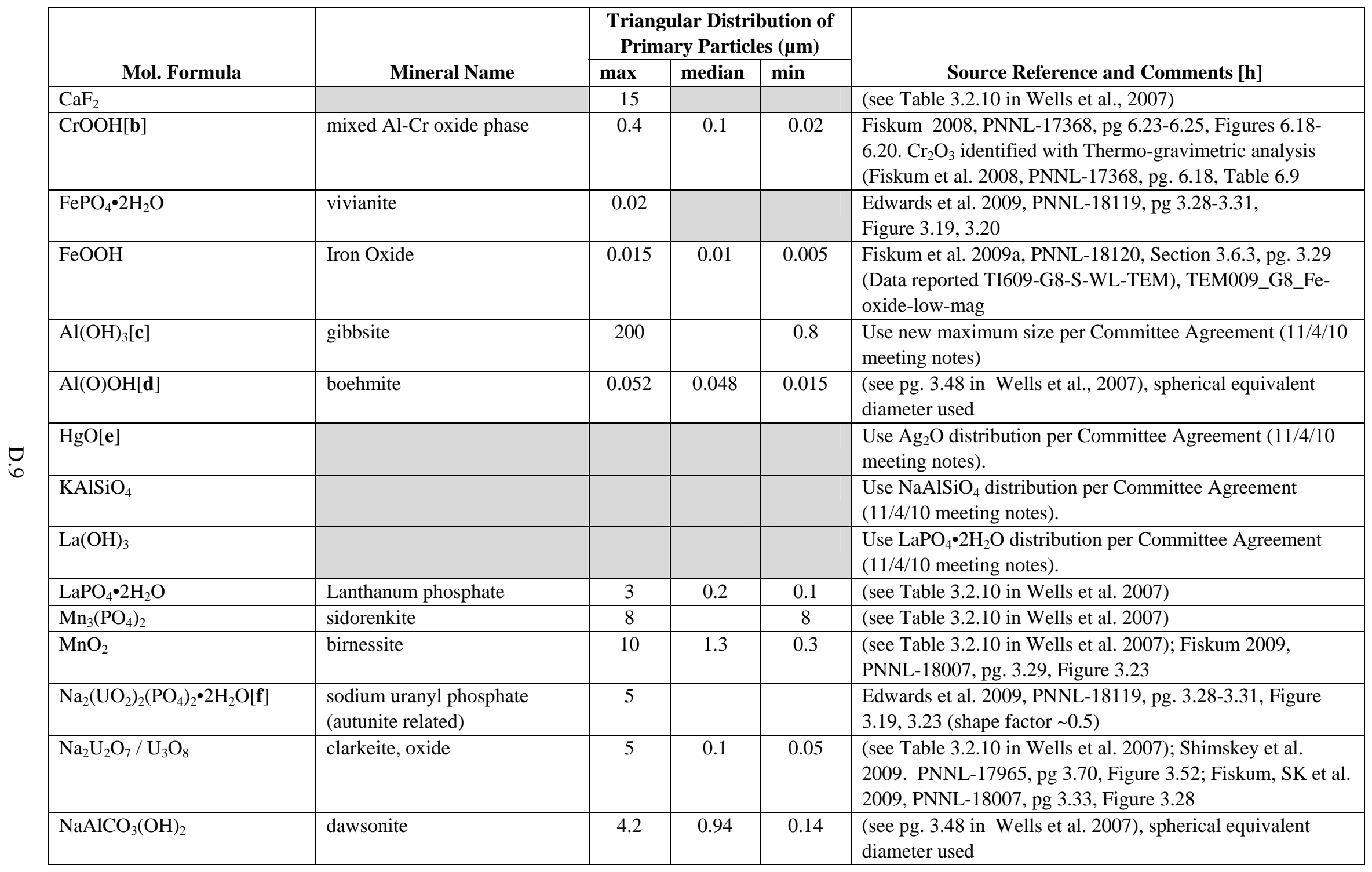


Table D.2. (contd)

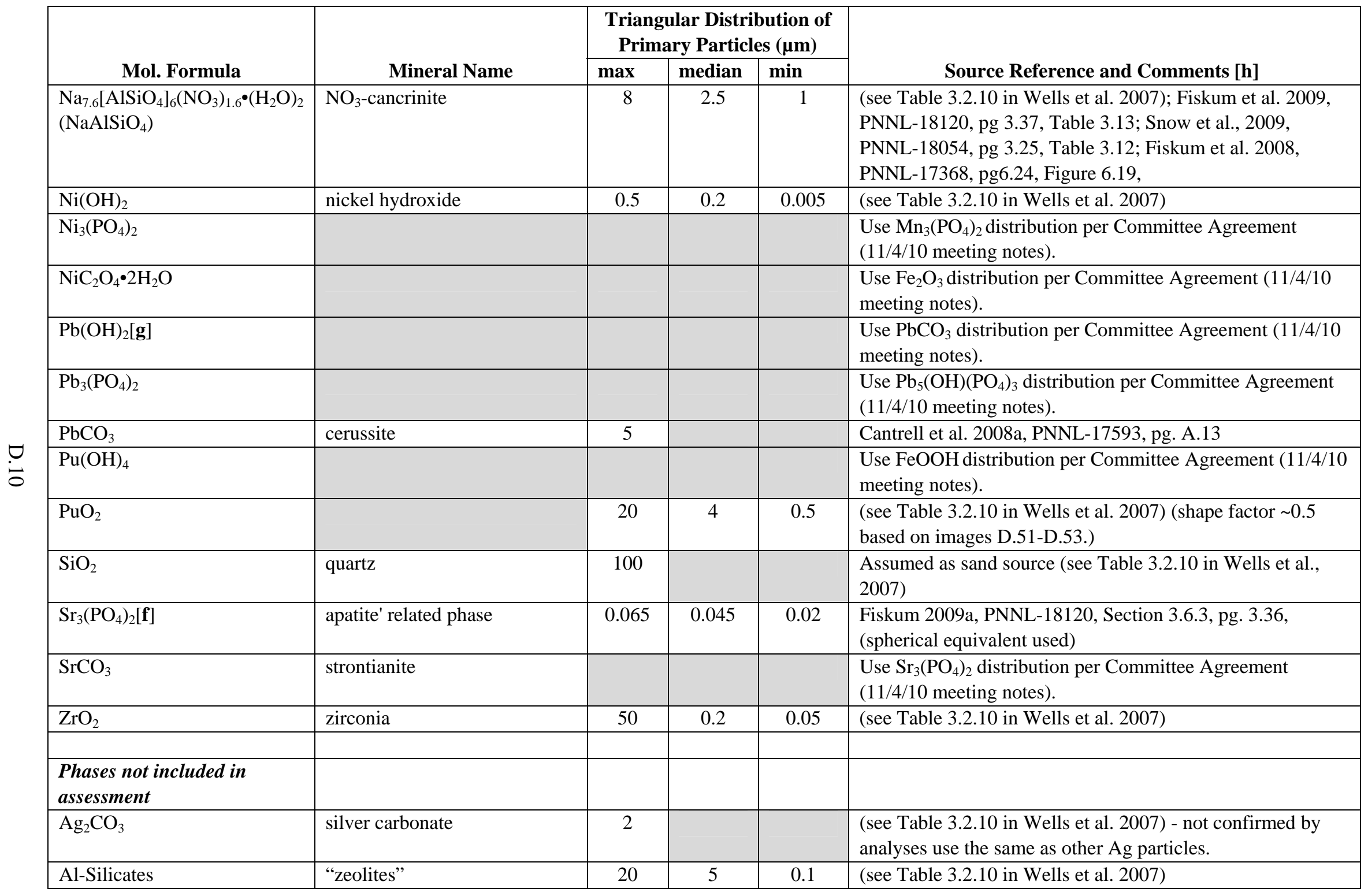


Table D.2. (contd)

\begin{tabular}{|c|c|c|c|c|c|}
\hline \multirow[b]{2}{*}{ Mol. Formula } & \multirow[b]{2}{*}{ Mineral Name } & \multicolumn{3}{|c|}{$\begin{array}{l}\text { Triangular Distribution of } \\
\text { Primary Particles }(\mu \mathrm{m})\end{array}$} & \multirow[b]{2}{*}{ Source Reference and Comments [h] } \\
\hline & & $\max$ & median & $\min$ & \\
\hline Al-Silicates & "clays" & 0.6 & 0.3 & 0.1 & (see Table 3.2.10 in Wells et al. 2007) \\
\hline $\mathrm{BiPO}_{4}$ & bismuth phosphate & 10 & 2 & 1 & $\begin{array}{l}\text { (see Table } 3.2 .10 \text { in Wells et al. 2007), also identified by } \\
\text { others }\end{array}$ \\
\hline $\mathrm{Fe}_{2} \mathrm{O}_{3}$ & hematite & 1.6 & 0.88 & 0.037 & $\begin{array}{l}\text { (see pg. 3-48 in Wells et al. 2007), spherical equivalent } \\
\text { diameter used. }\end{array}$ \\
\hline $\mathrm{Na}_{4}\left(\mathrm{UO}_{2}\right)\left(\mathrm{CO}_{3}\right)_{3}$ & cejkaite & 50 & 7.5 & 2 & (see Table 3.2.10 in Wells et al. 2007) \\
\hline $\mathrm{Pb}_{5}(\mathrm{OH})\left(\mathrm{PO}_{4}\right)_{3}$ & pyromorphite & 0.4 & & 0.05 & (see Table 3.2.10 in Wells et al. 2007) \\
\hline $\mathrm{ZrOOH}$ & zirconium hydroxide & 10 & & & $\begin{array}{l}\text { Cantrell et al. 2008b, PNNL-16738 - particle agglomerate- } \\
\text { actual size is considerably smaller based on data collected } \\
\text { by others. Distribution based on data collected by E. Buck } \\
\text { for Cantrell (2011). }\end{array}$ \\
\hline
\end{tabular}

[a] With the techniques used, it was not possible to distinguish $\mathrm{CaC}_{2} \mathrm{O}_{4} \cdot \mathrm{H}_{2} \mathrm{O}$ from $\mathrm{Ca}(\mathrm{OH})_{2}$.

[b] The $\mathrm{CrOOH}$ phase is always present as a mixed Al-Cr phase; further analysis has suggested that this phase is more closely

related to $\mathrm{Cr}_{2} \mathrm{O}_{3}$ rather than $\mathrm{CrOOH}$.

[c] Additional Al-phases include diaspore (25 mm long $\times 1 \mathrm{~mm}$ thick), Al-phosphate, $\mathrm{Ca}_{3} \mathrm{Al}_{2} \mathrm{O}_{6}$.

[d] Values were calculated from XRD broadening.

[e] Ag-Hg phase observed.

[f] Many of the phosphate particles are acicular and have been corrected by a shape factor.

[g] Lead is often associated with iron oxides in tank sludge. This suggests that the iron oxide density and size distribution

should be used.

[h] (11/4/10 meeting notes)—see Section D.2. 
Table D.3. Summary of Maximum Salt Particle Sizes

\begin{tabular}{|c|c|c|c|c|c|c|c|}
\hline Mineral Name & Formula & $\operatorname{Max}(\mu \mathrm{m})$ & Shape & $\begin{array}{c}\text { Density } \\
\left(\mathrm{g} / \mathrm{cm}^{3}\right)[1]\end{array}$ & $\begin{array}{l}\text { Shape } \\
\text { Factor }\end{array}$ & $\begin{array}{l}\text { Spherical } \\
\text { Equivalent }\end{array}$ & Comment \\
\hline Natroxalate & $\mathrm{Na}_{2} \mathrm{C}_{2} \mathrm{O}_{4}$ & 20 & acicular & 2.35 & 0.4 & 8 & CCP-EMSP-0010 \\
\hline Thermonatrite & $\mathrm{Na}_{2} \mathrm{CO}_{3} \cdot\left(\mathrm{H}_{2} \mathrm{O}\right)$ & 100 & platy & 2.26 & 0.8 & 80 & CCP-EMSP-0010 \\
\hline Na Sulfate & $\mathrm{Na}_{2} \mathrm{SO}_{4} \cdot 10 \mathrm{H}_{2} \mathrm{O}$ & & & & & & Use $\mathrm{Na}_{2} \mathrm{SO}_{4}$ size \\
\hline Schairerite & $\mathrm{Na}_{3} \mathrm{FSO}_{4}$ & 220 & $\begin{array}{l}\text { hexagonal } \\
\text { plates }\end{array}$ & 2.63 & 0.8 & 176 & CCP-EMSP-0010 \\
\hline Darapskite & $\mathrm{Na}_{3} \mathrm{NO}_{3} \mathrm{SO}_{4} \cdot \mathrm{H}_{2} \mathrm{O}$ & 100 & $\begin{array}{l}\text { rectangular } \\
\text { plates }\end{array}$ & 2.2 & 0.8 & 80 & HNF-11585, pg. 4-28 \\
\hline $\begin{array}{l}\text { Na Phosphate } \\
\text { Dodecahydrate }\end{array}$ & $\mathrm{Na}_{3} \mathrm{PO}_{4} \cdot 12 \mathrm{H}_{2} \mathrm{O}$ & 1100 & acicular & 1.62 & 0.4 & 440 & CCP-EMSP-0010 \\
\hline Na Phosphate & $\mathrm{Na}_{3} \mathrm{PO}_{4} \cdot 8 \mathrm{H}_{2} \mathrm{O}$ & & & & & & Use PSD maximum size \\
\hline Na Phosphate & 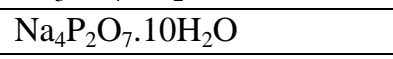 & & & & & & Use PSD maximum size \\
\hline Burkeite & $\mathrm{Na}_{6} \mathrm{CO}_{3}\left(\mathrm{SO}_{4}\right)_{2}$ & 40 & platy & 2.58 & 0.8 & 32 & CCP-EMSP-0010 \\
\hline Apophyllite & $\mathrm{NaF}$ & 12 & rhombohedral & 2.38 & 1 & 12 & CCP-EMSP-0010 \\
\hline Natrophosphate & $\mathrm{Na}_{7} \mathrm{~F}\left(\mathrm{PO}_{4}\right)_{2} \cdot 19 \mathrm{H}_{2} \mathrm{O}$ & 2100 & round particles & 1.76 & 1 & 2100 & $\begin{array}{l}\text { no upper limit, } \\
\text { CCP-EMSP-0010 }\end{array}$ \\
\hline Trona (NaHCO3) & $\mathrm{Na}_{3}\left(\mathrm{CO}_{3}\right)\left(\mathrm{HCO}_{3}\right) \cdot 2\left(\mathrm{H}_{2} \mathrm{O}\right)$ & 410 & platy & 2.13 & 0.8 & 328 & CCP-EMSP-0010 \\
\hline Sodium nitrite & $\mathrm{NaNO}_{2}$ & & & & & & Use PSD maximum size \\
\hline Nitratine & $\mathrm{NaNO}_{3}$ & 650 & cubical & 2.26 & 1 & 650 & $\begin{array}{l}\text { no upper limit (HNF- } \\
\text { 11858, Figure 4.1.2-4, pg. } \\
4-6)\end{array}$ \\
\hline \multicolumn{8}{|c|}{$\begin{array}{l}\text { Phases not included in } \\
\text { assessment }\end{array}$} \\
\hline Cryolite & $\mathrm{Na}_{3} \mathrm{AlF}_{6}$ & 3.2 & spherical & 2.96 & 1 & 3.2 & CCP-EMSP-0010 \\
\hline Nastrophite & $\mathrm{NaSrPO}_{4} \cdot 9 \mathrm{H}_{2} \mathrm{O}$ & 260 & elongated & 2.14 & 0.4 & 104 & CCP-EMSP-0010 \\
\hline Hydrouvarovite* & $\left((\mathrm{Ca}, \mathrm{Sr})_{3}(\mathrm{Cr}, \mathrm{Al})_{2}(\mathrm{OH})_{12}\right)$ & 12 & cubical & -- & 1 & 12 & CCP-EMSP-0010 \\
\hline
\end{tabular}


Table D.3. (contd)

[1] Densities obtained from Web-Mineral (http://webmineral.com/).

* Mineral name used in analysis. This is likely to be incorrect as uvarovite is a garnet and would be unlikely to form in the

Hanford tanks. Phase may be a carbonate.

Suggest using a density of 2.5 for this phase in agreement with the other salts.

Round, square, cubical

Assumed Shape Factor

platy, hexagonal plates

1

platy, hexagonal plates

acicular, elongated

Conservative shape correction factors were used because the particles in the images were irregular, and the values varied. 


\section{D.4 Particle Images}

\section{Gibbsite}

\begin{tabular}{|l|l|}
\hline & \\
\hline
\end{tabular}




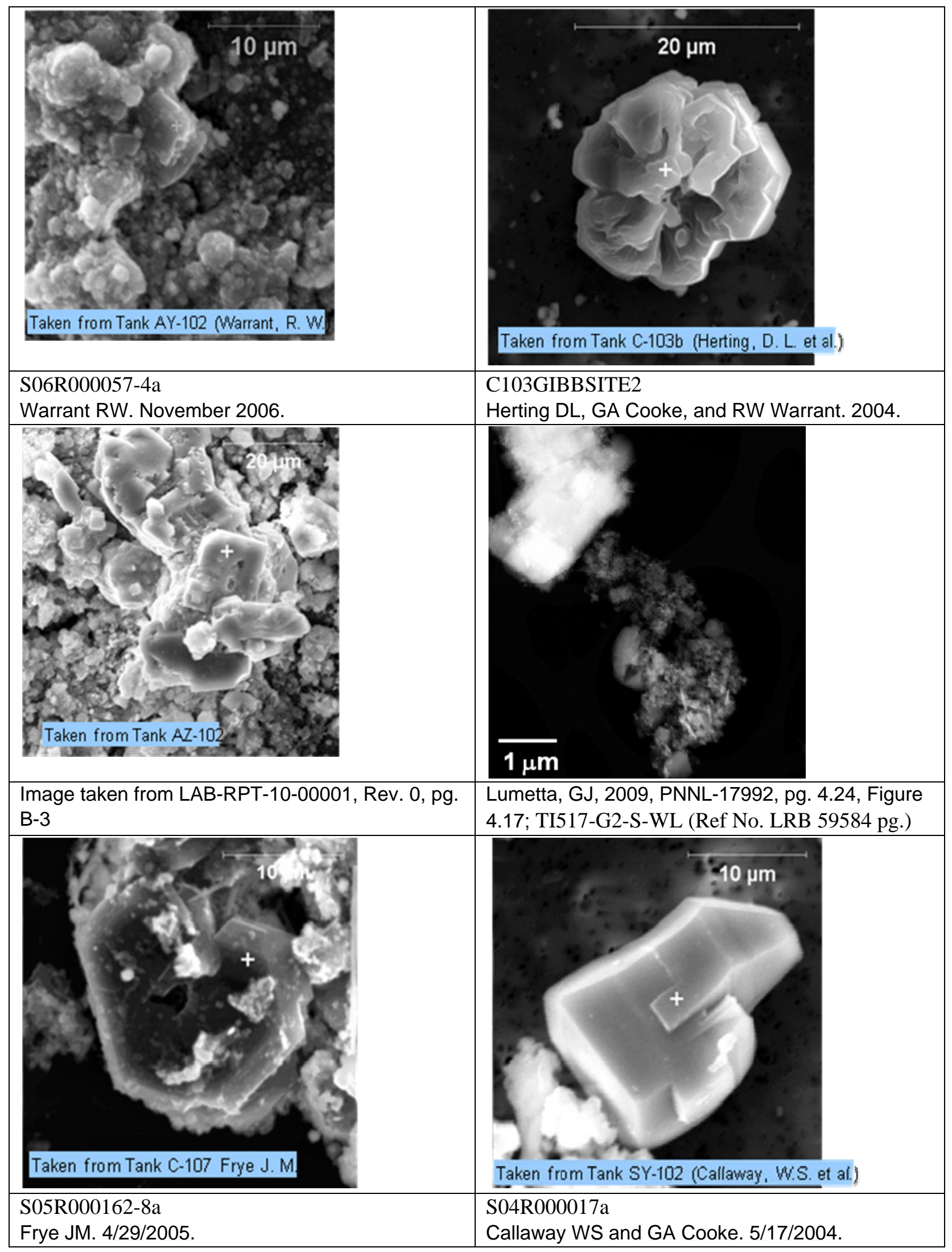




\section{Boehmite Particles}

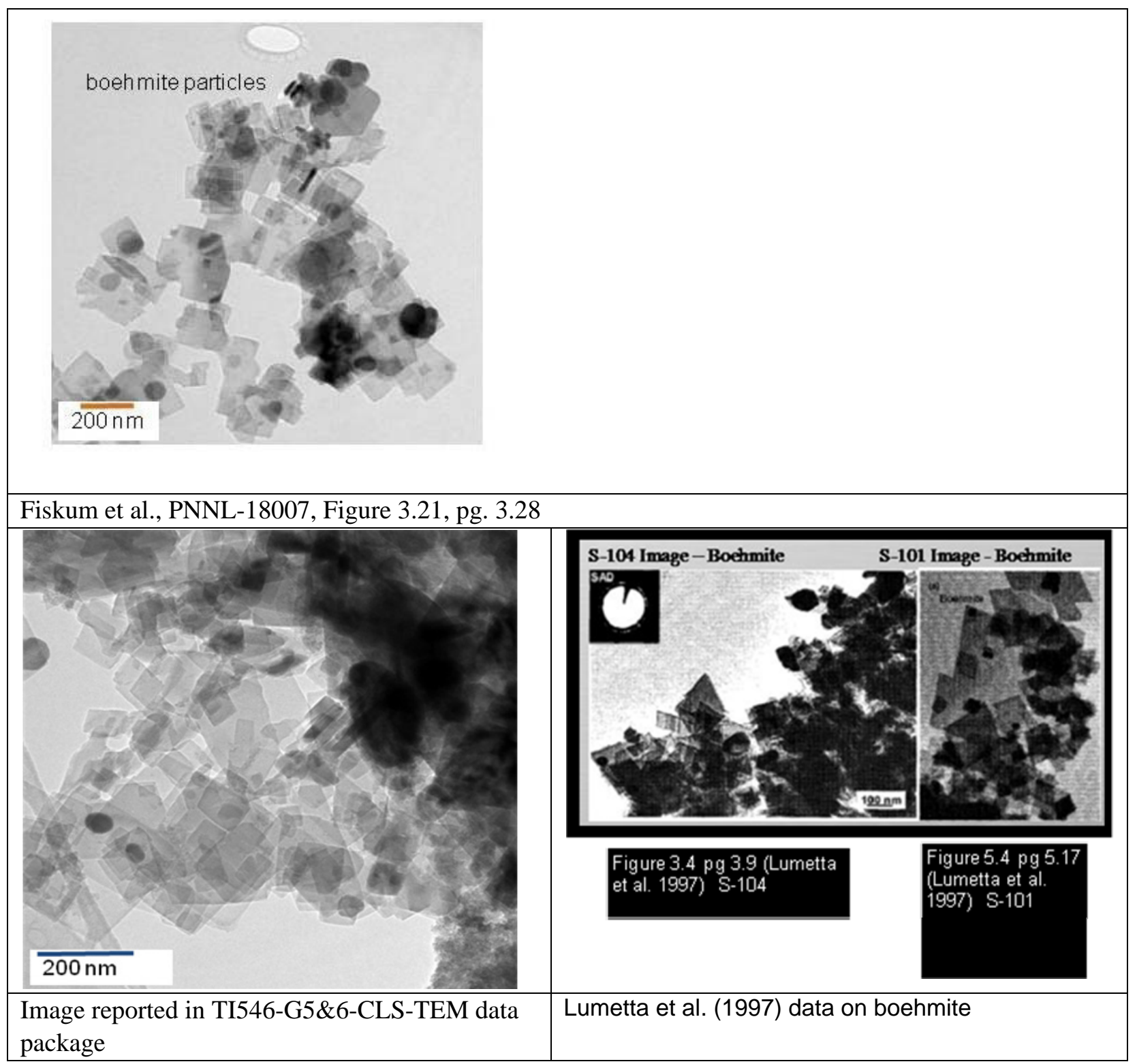




\section{Dawsonite Particles}

\begin{tabular}{|l|l|}
\hline & \\
\hline
\end{tabular}




\section{Iron Oxides}

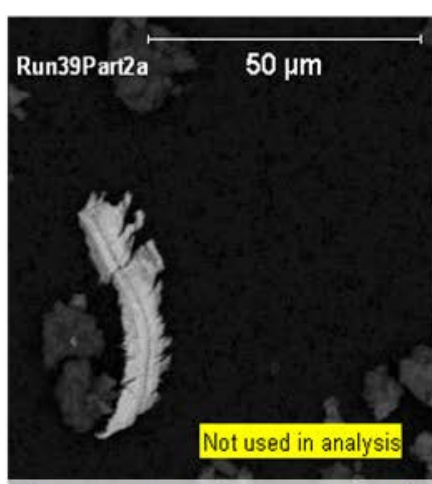

Tank SY-102 (Callaway, W. S. et al.)

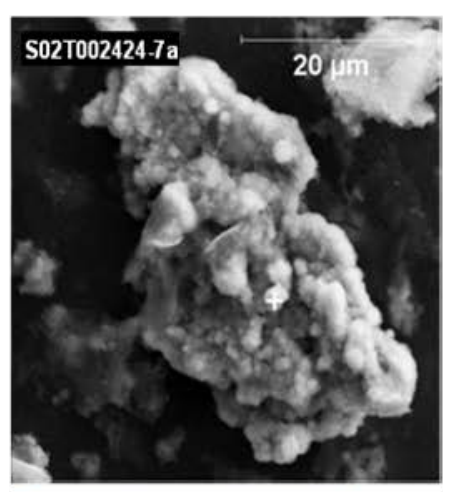

Tank AN-107 (Herting D. L. et al.)

Images obtained from Callaway and Cooke 2004, Warrant 2002

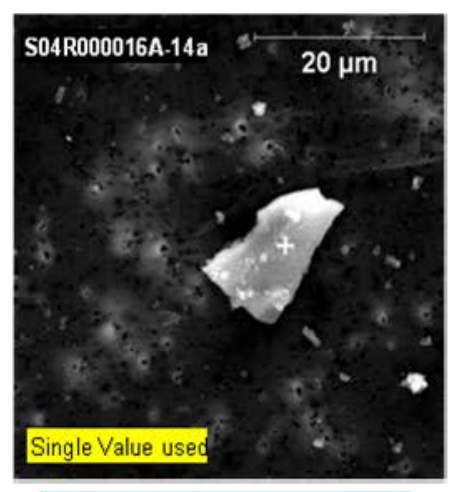

Tank SY-102 (Callaway, W. S., et al.)

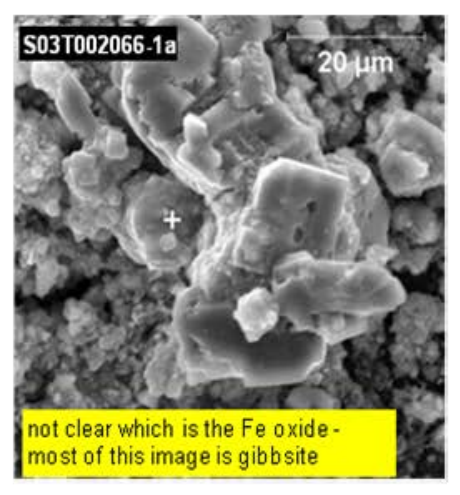

Tank AZ-102

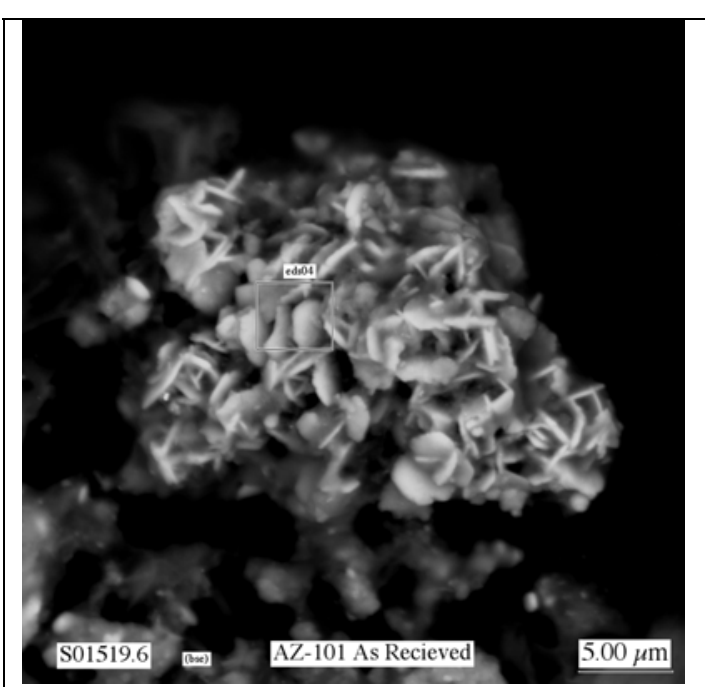

Figure 4.11 pg. 4.14 (AZ-101) Buck et al. 2003.

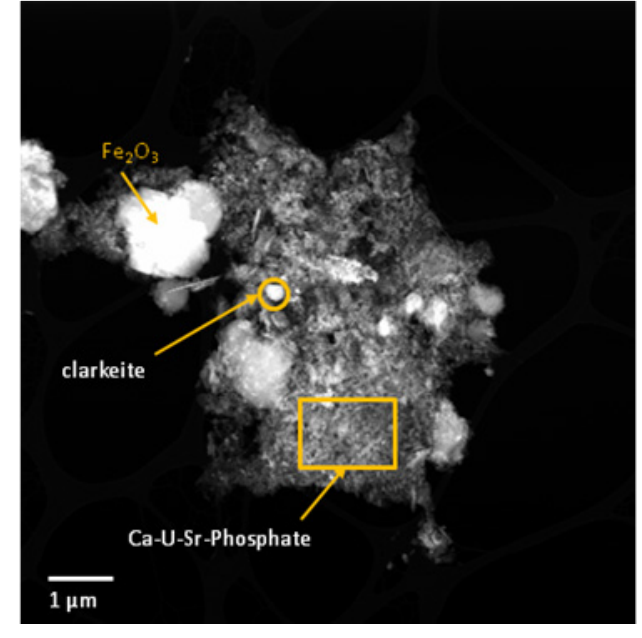

Fiskum, SK., 2009, PNNL-18120, pg. 3.29, Figure 3.16; TI609-G8-S-WL-TEM, STEM004-190K-EDS 


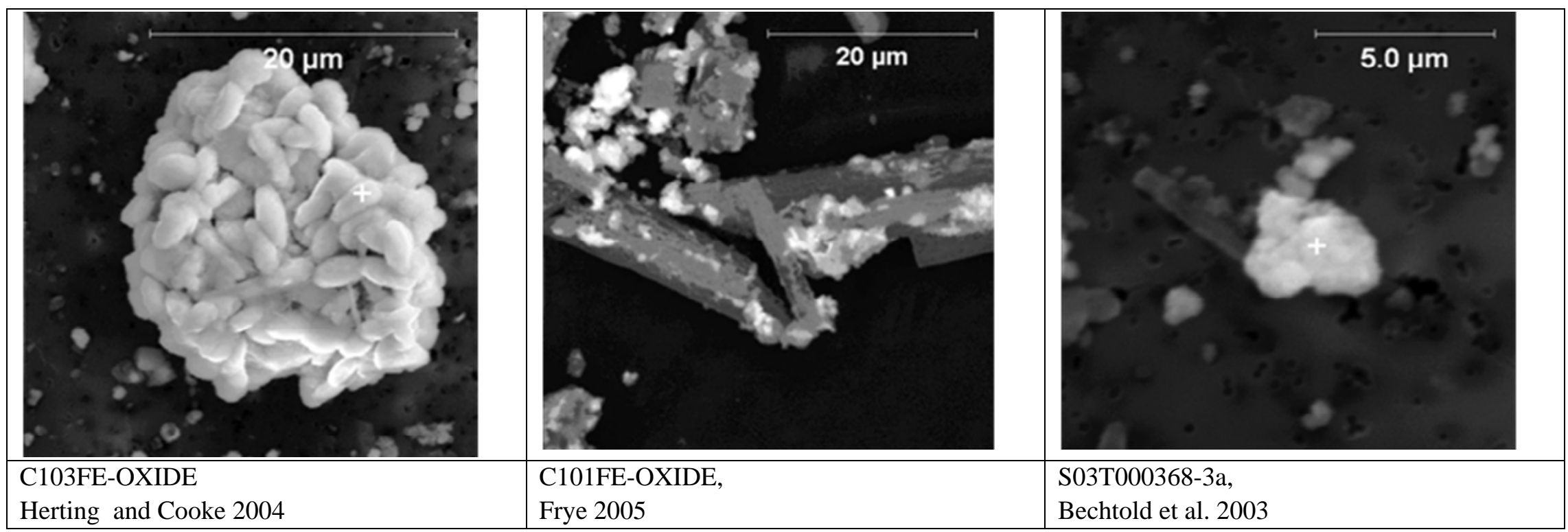

$\underset{v}{0}$ 


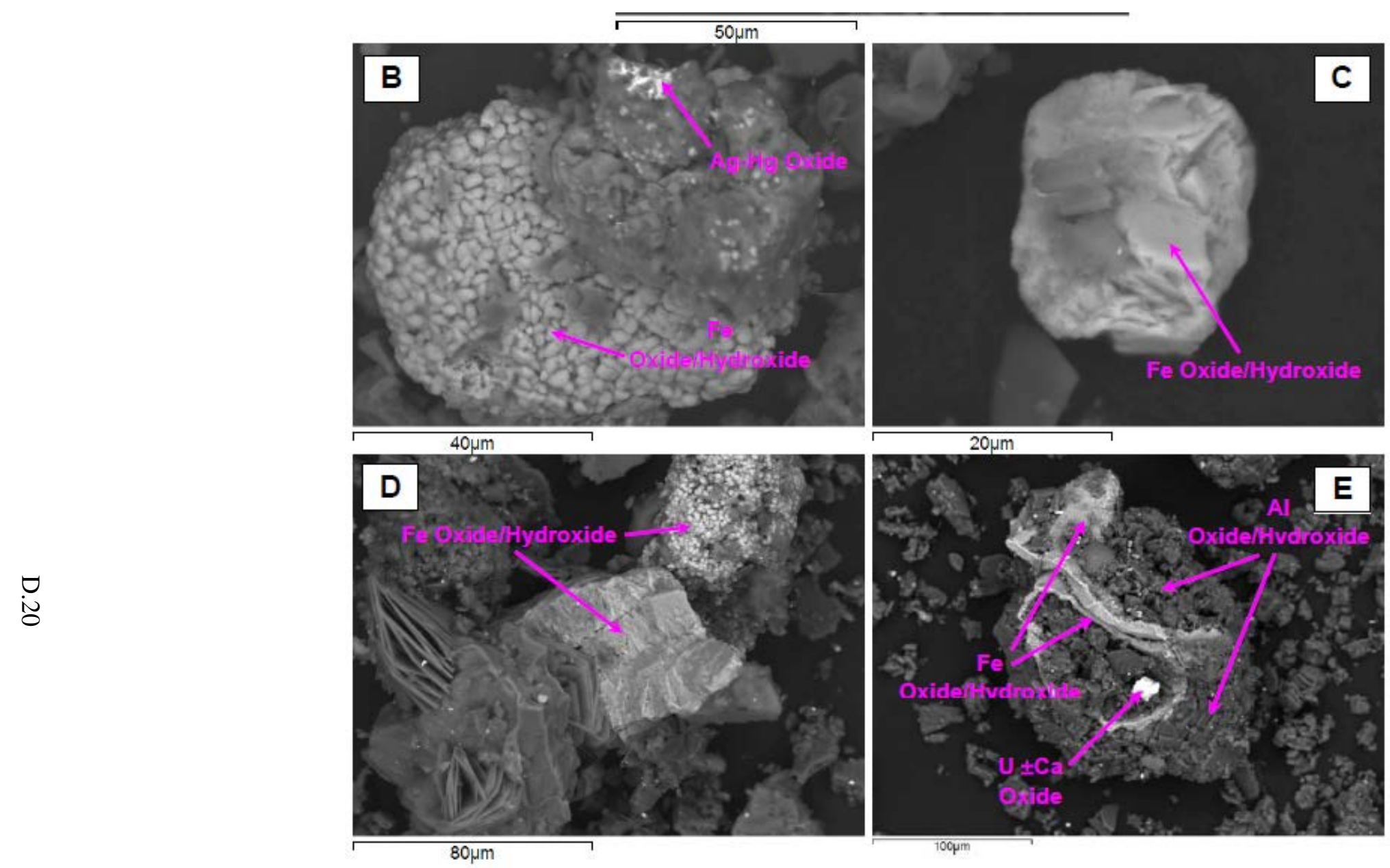

KJ Cantrell et al. 2008. Hanford Tank 241-C-103 Residual Waste Contaminant Release Models and Supporting Data. PNNL-16738, pg. 3.70, Figure 3.7. 


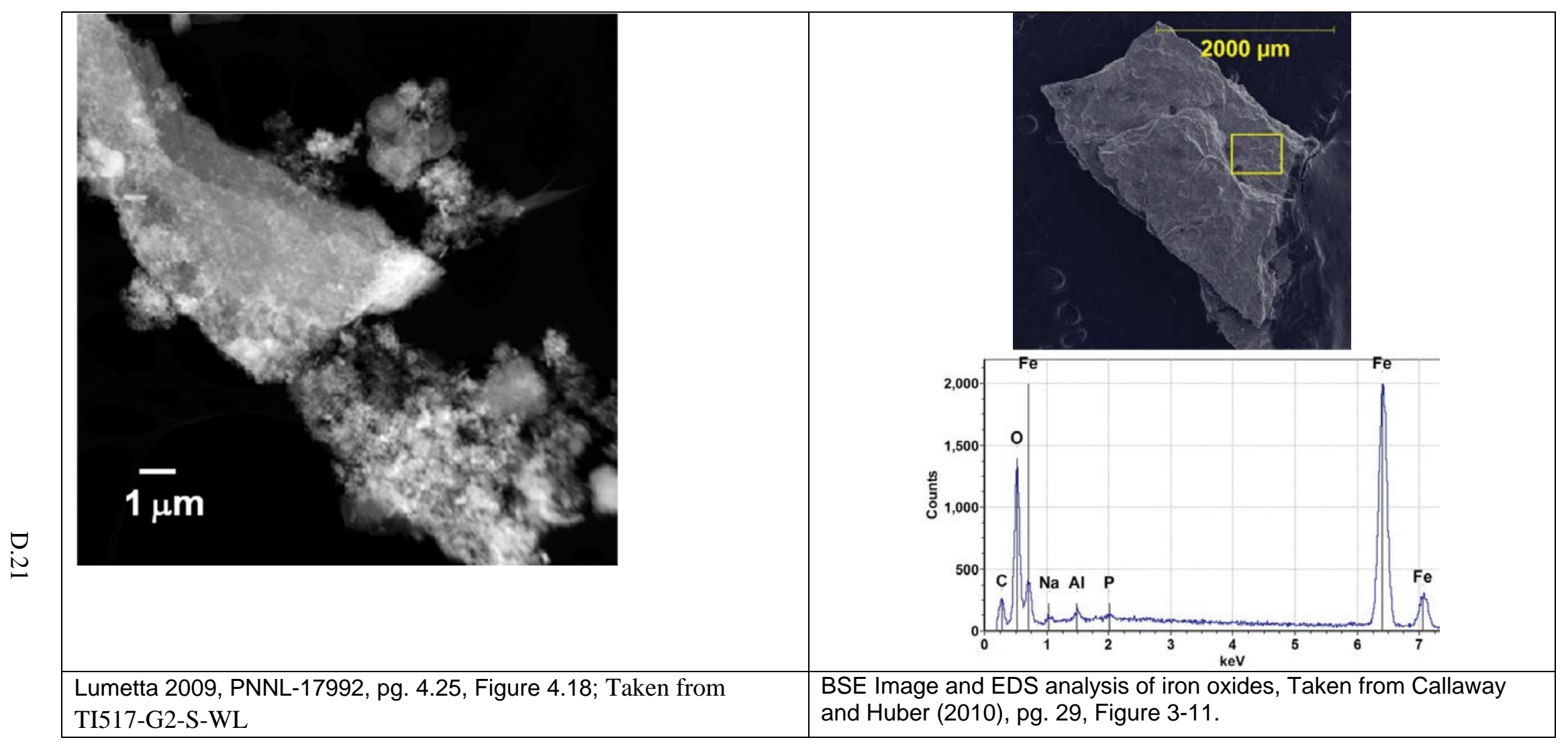




\section{Uranium and Actinide Phases}

\begin{tabular}{|l|l|}
\hline & \\
\hline &
\end{tabular} \mid




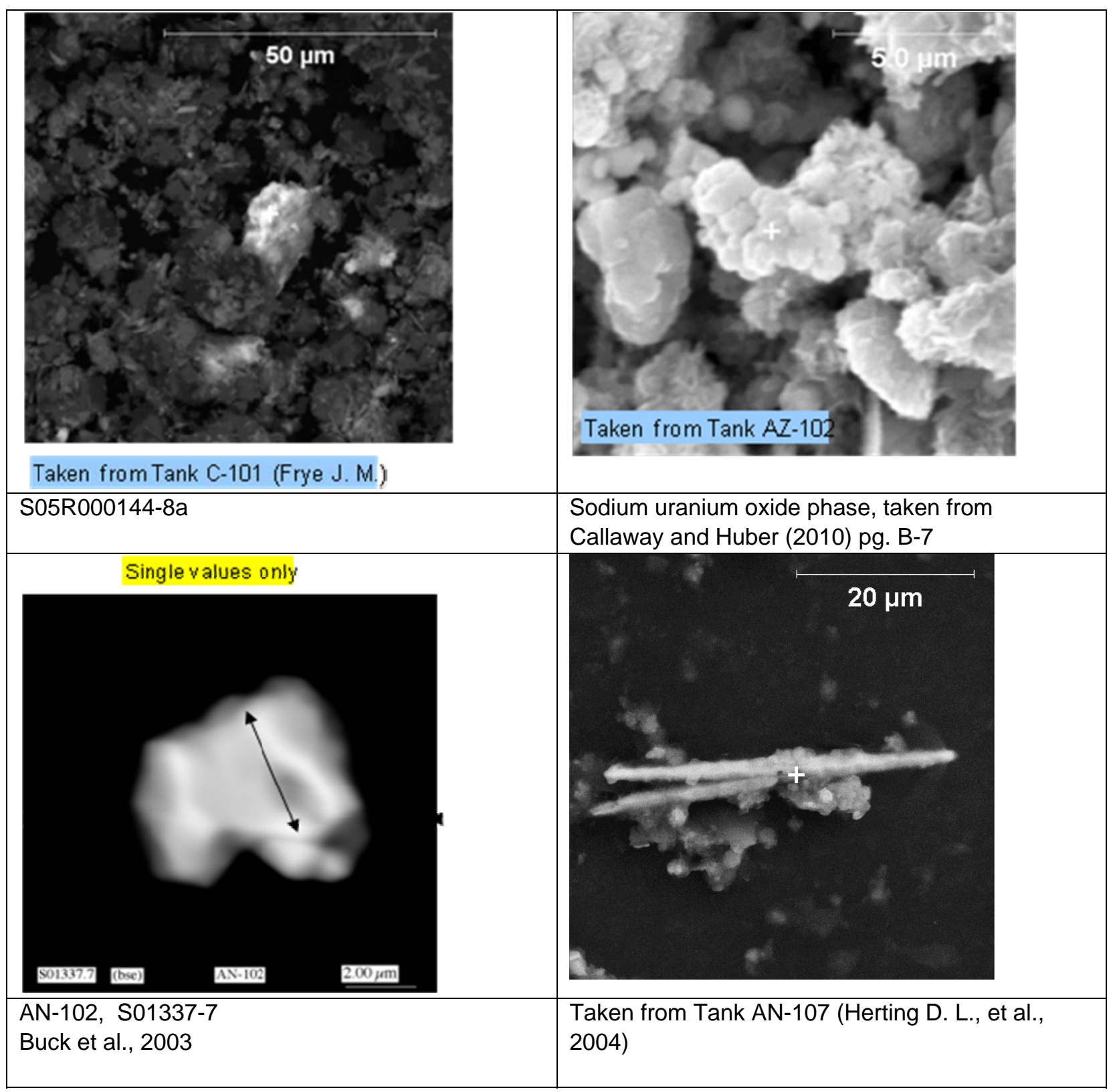




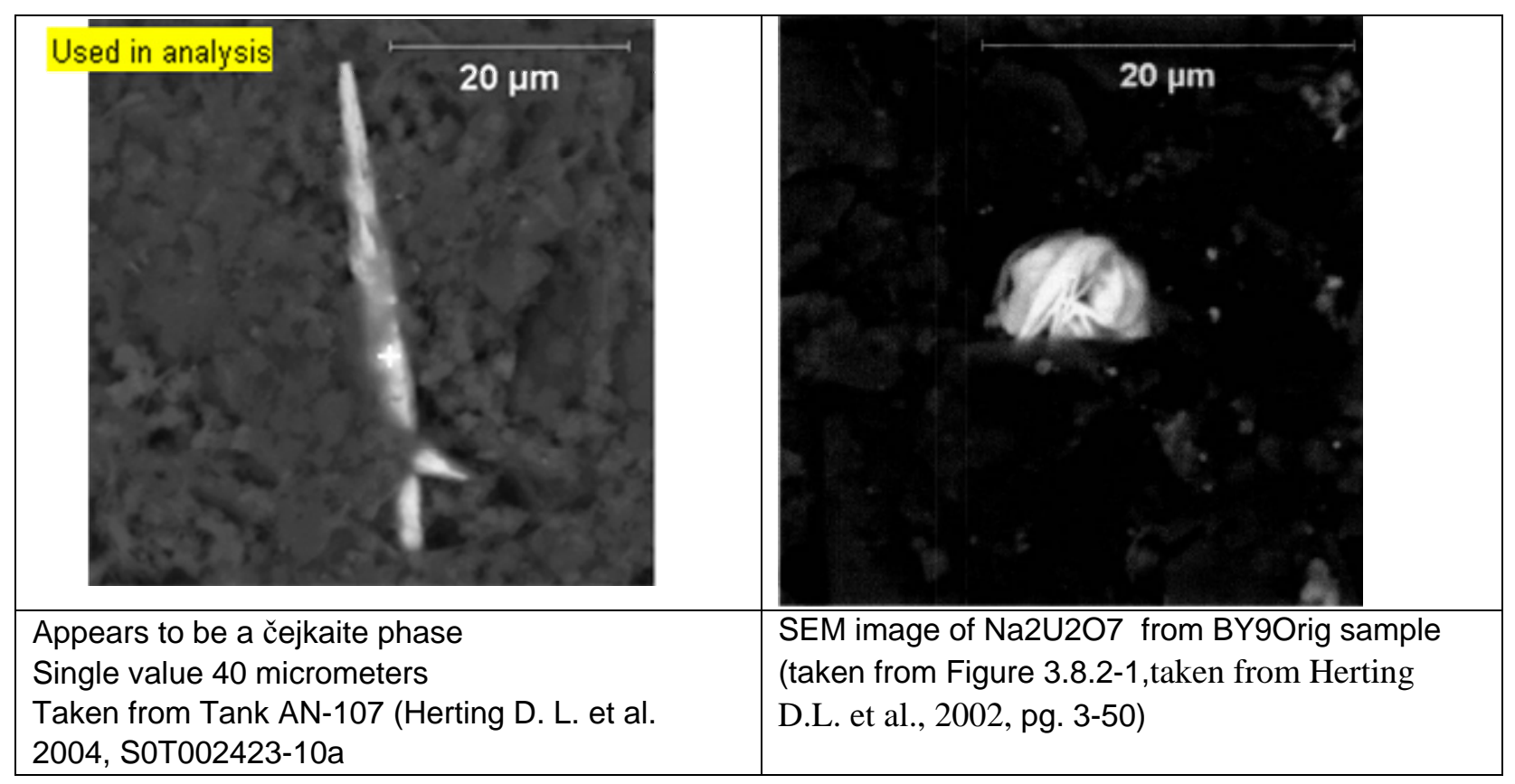




\section{Bismuth Phases}

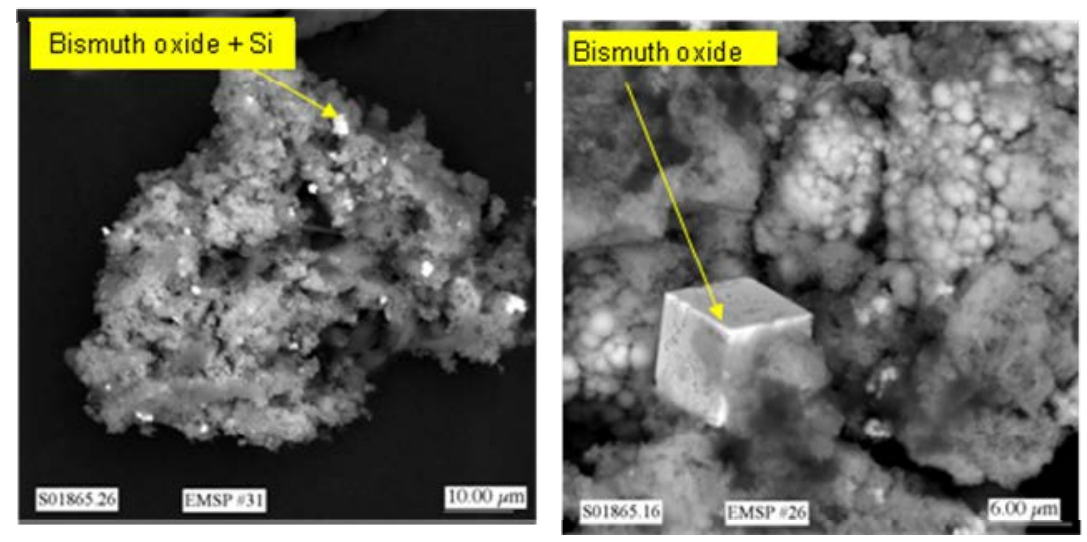

Images taken from 'Bismuth Phosphate Tank Waste.pptx” submitted.

\section{Manganese Phases}

\begin{tabular}{|l|l|l|}
\hline & \\
& &
\end{tabular}




\section{Cancrinite}

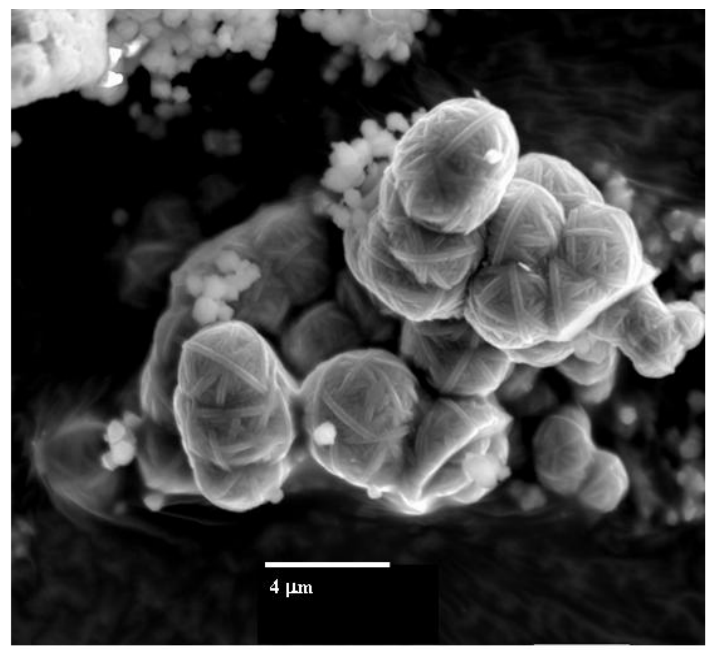

Taken from EC Buck and BK McNamara, Environ. Sci. Technol., 38 (2004) 4432-4438
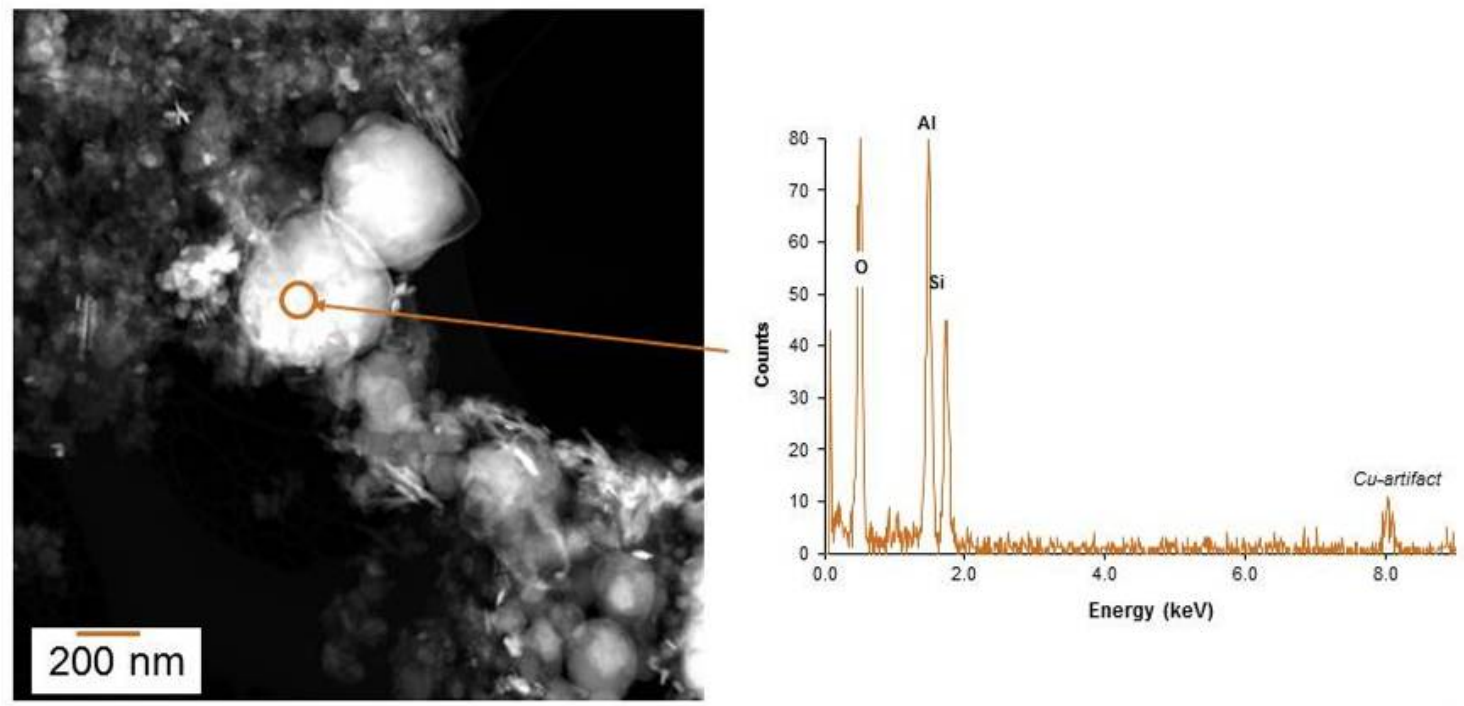

Image taken from TI546-G5\&6-CLS-TEM data package. 


\section{Zeolite Phase}

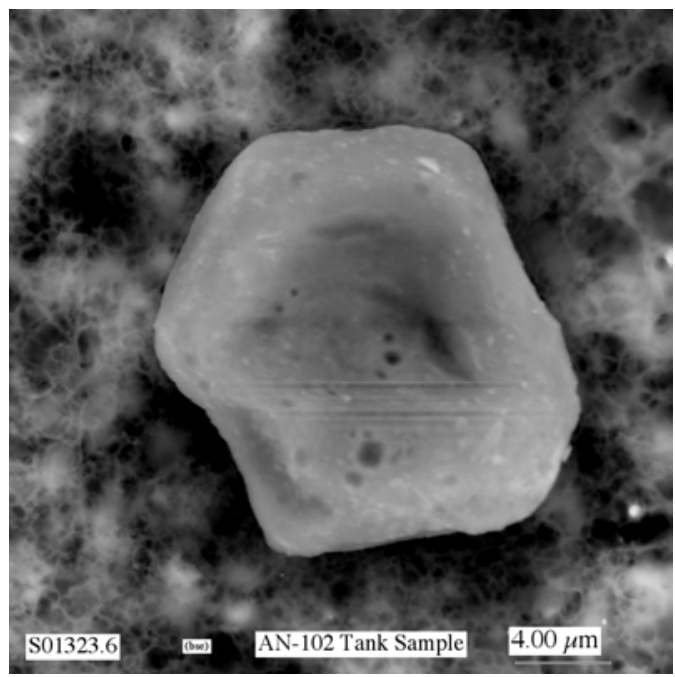

Zeolite like phase (Buck et al., 2003) 


\section{Calcium Phases}

\section{Calcium Phosphate}

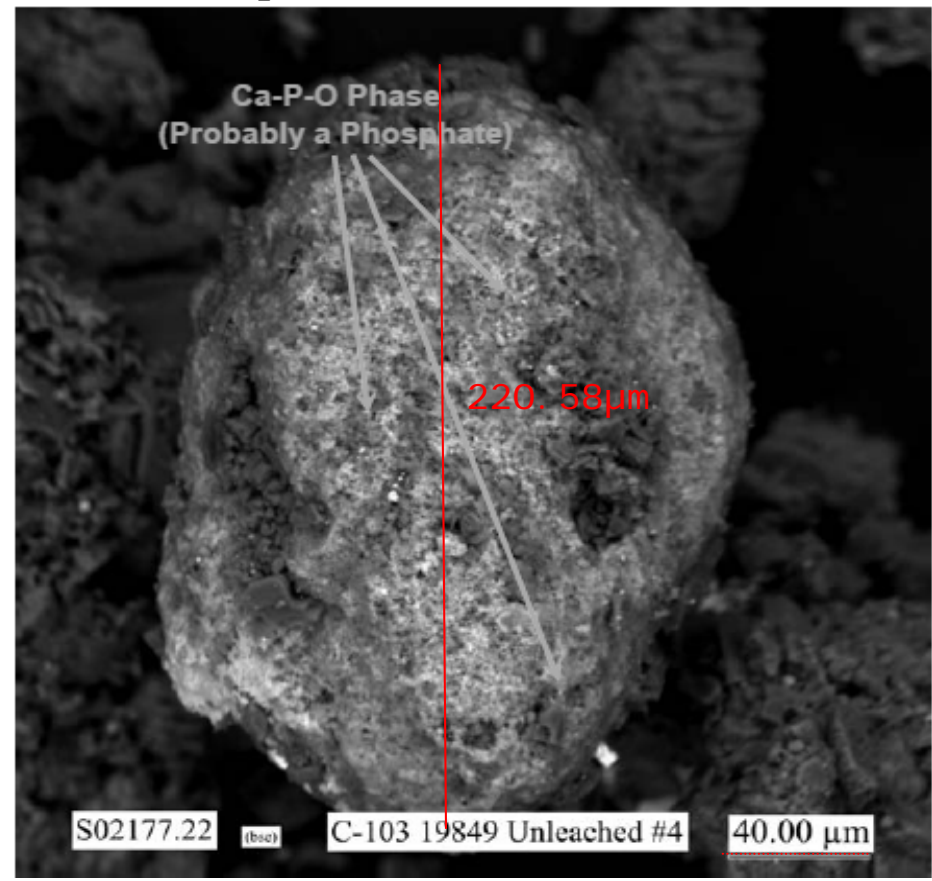

KJ Cantrell et al., 2008. Hanford Tank 241-C-103 Residual Waste Contaminant Release Models and Supporting Data. PNNL-16738, pg. 3.75, Figure 3.12.

\section{Calcium Carbonate}

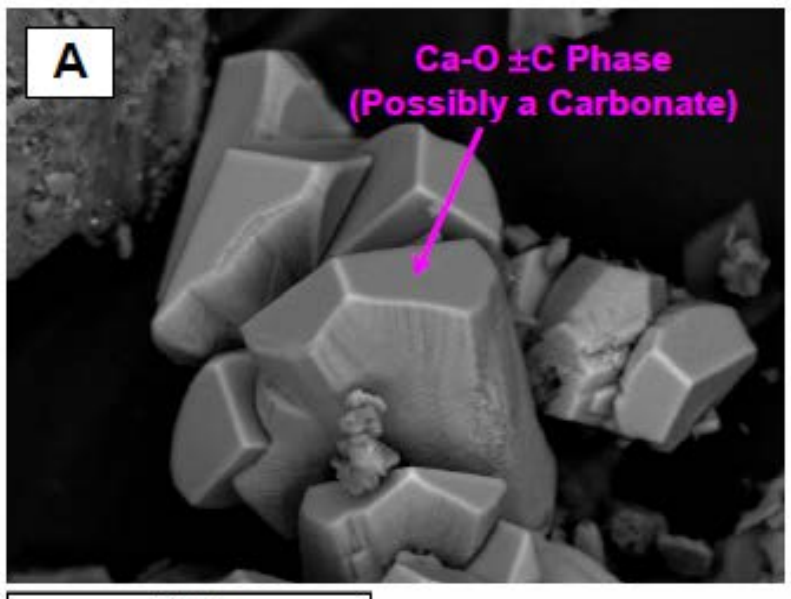

$60 \mu \mathrm{m}$

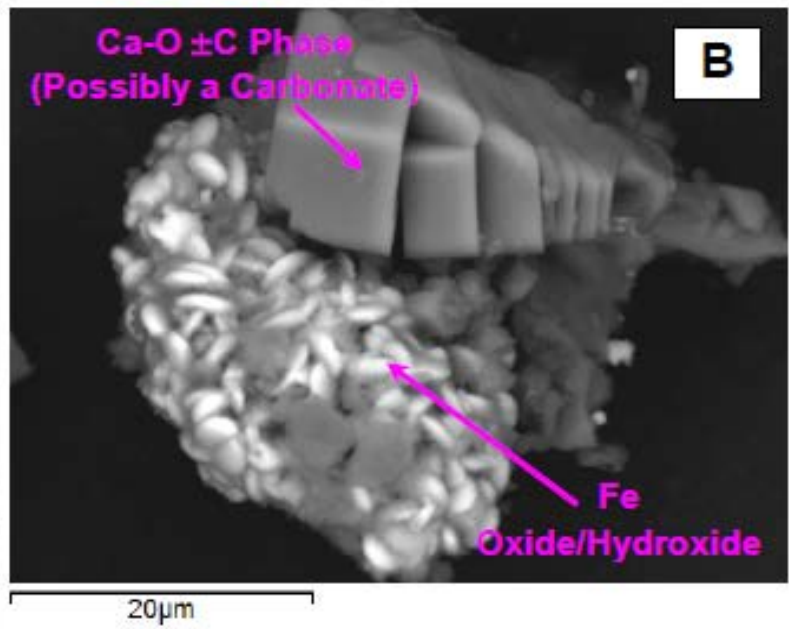

KJ Cantrell et al. 2008. Hanford Tank 241-C-103 Residual Waste Contaminant Release Models and Supporting Data. PNNL-16738, pg. 3.76, Figure 3.13. 


\section{Lead Carbonate}

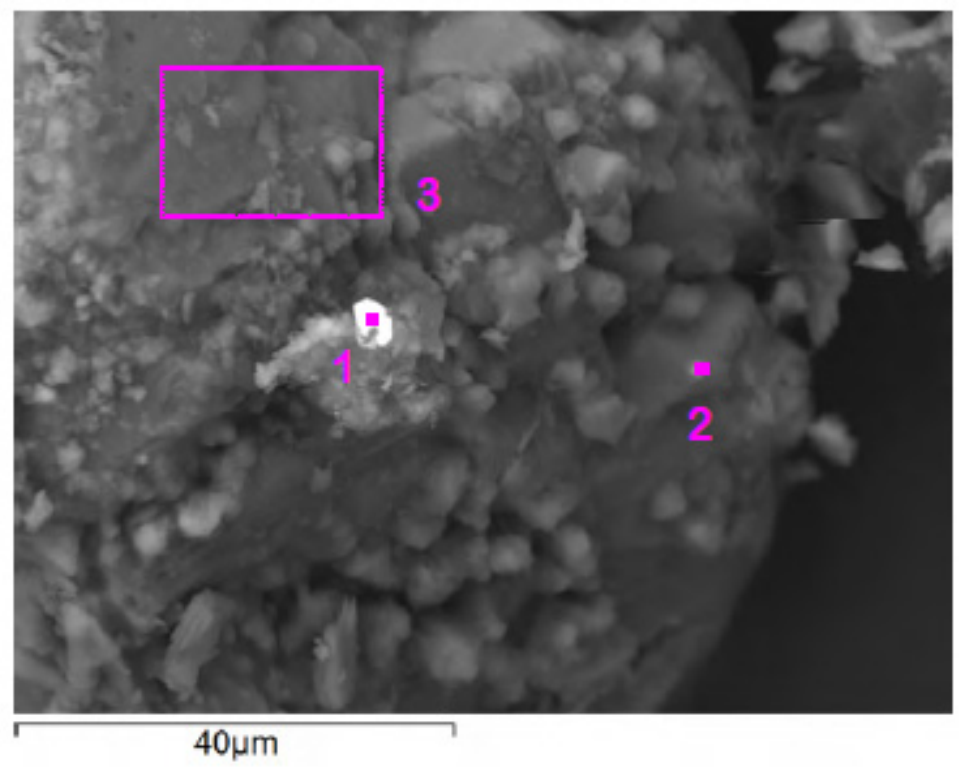

KJ Cantrell et al. 2008. Hanford Tank 241-S-112 Residual Waste Composition and Leach Test Data. PNNL-17593, pg. A.13. 


\section{Salts}

\section{$\mathrm{NaNO}_{3}$}

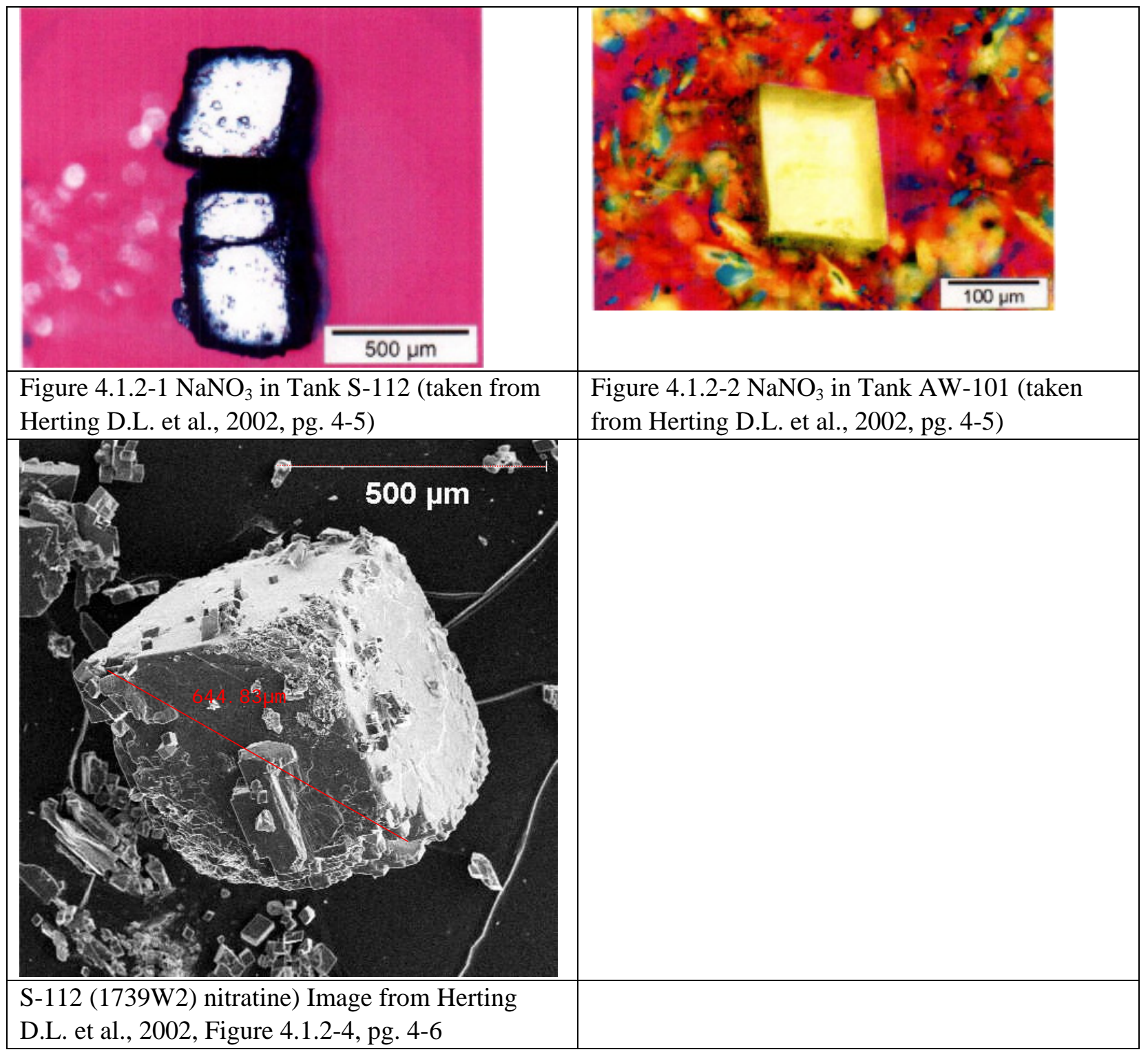




\section{$\mathrm{Na}_{4} \mathrm{P}_{2} \mathrm{O}_{7} \cdot 10 \mathrm{H}_{2} \mathrm{O}$}

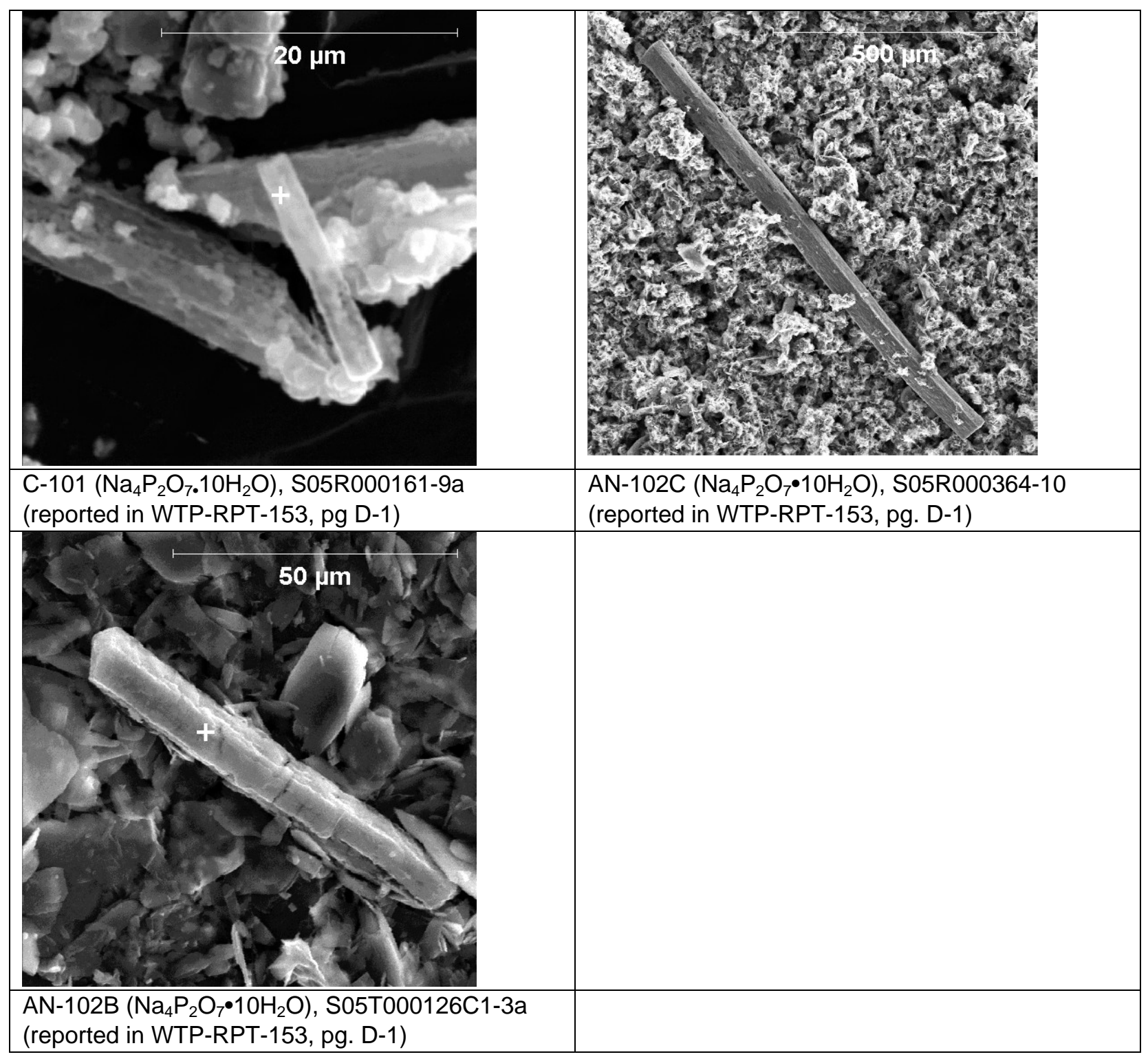


$\mathrm{Na}_{7} \mathrm{~F}\left(\mathrm{PO}_{4}\right)_{2} \cdot 19 \mathrm{H}_{2} \mathrm{O}$

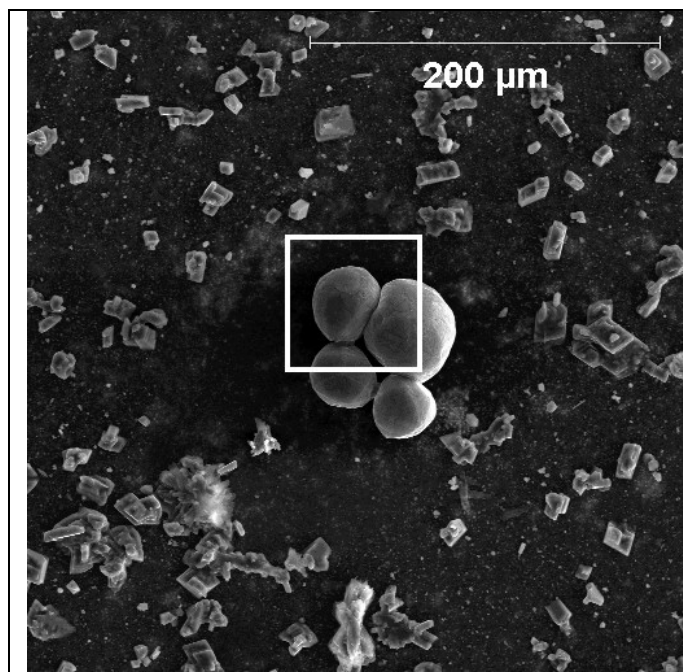

AN-102B $\left(\mathrm{Na}_{7} \mathrm{~F}\left(\mathrm{PO}_{4}\right)_{2} \cdot 19 \mathrm{H}_{2} \mathrm{O}\right)$, S05T000139C3-1 provided by $\mathrm{G}$ Cooke by e-mail

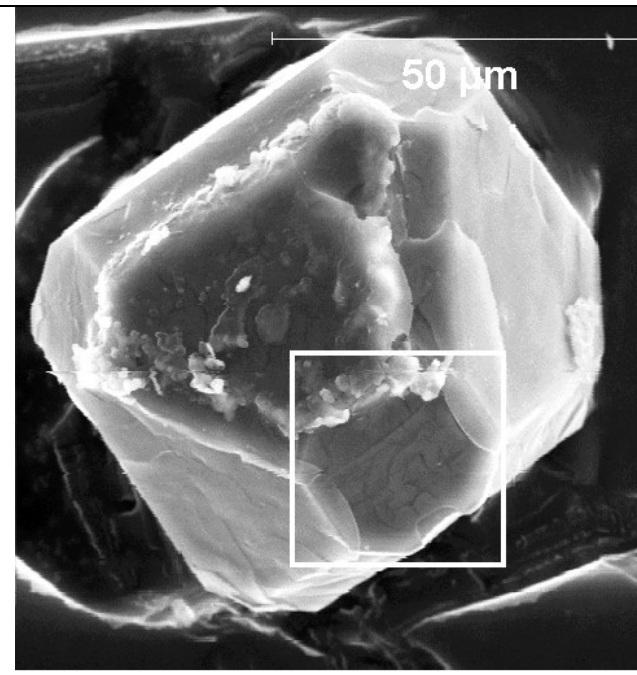

AN-107 $\left(\mathrm{Na}_{7} \mathrm{~F}\left(\mathrm{PO}_{4}\right)_{2} \cdot 19 \mathrm{H}_{2} \mathrm{O}\right)$, S02T002450-1 provided by $\mathrm{G}$ Cooke by e-mail
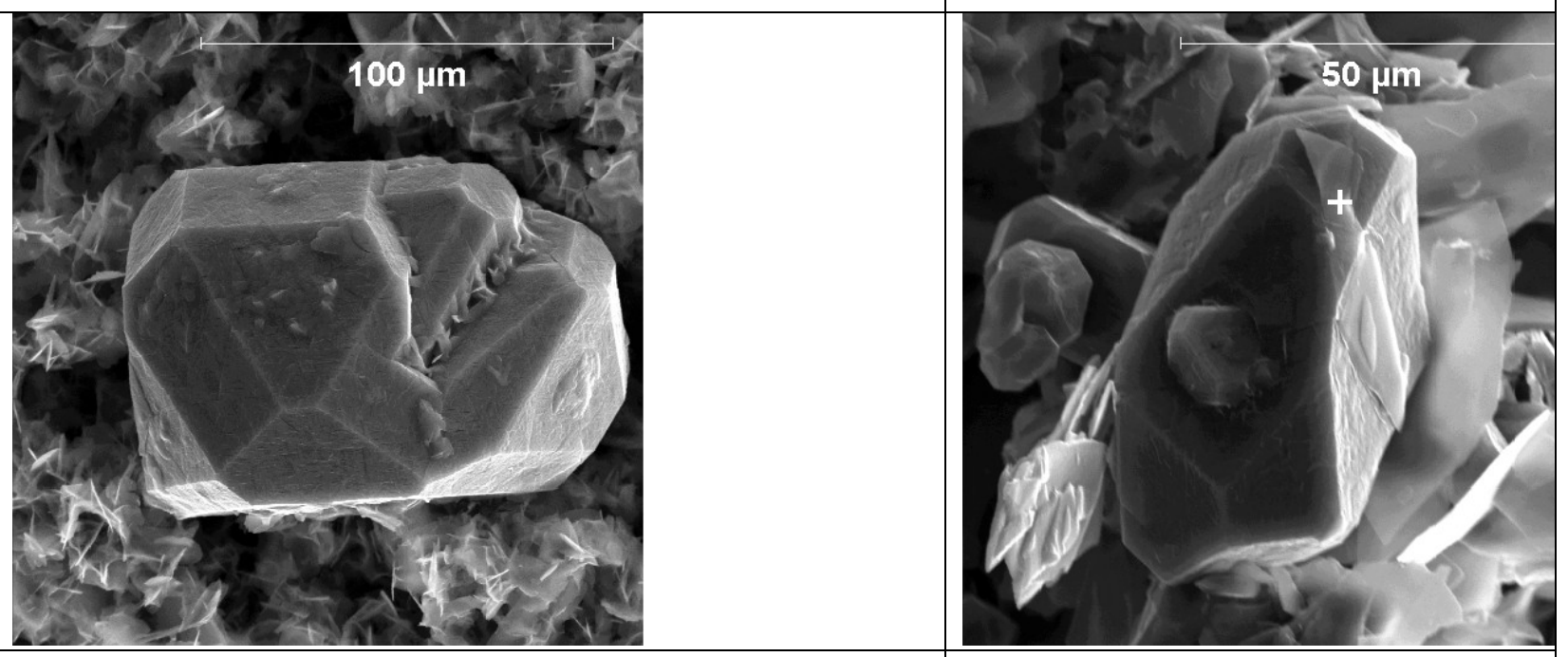

AN-102C $\left(\mathrm{Na}_{7} \mathrm{~F}\left(\mathrm{PO}_{4}\right)_{2} \cdot 19 \mathrm{H}_{2} \mathrm{O}\right)$, S05R000364-8a provided by $G$ Cooke by e-mail

AN-102C $\left(\mathrm{Na}_{7} \mathrm{~F}\left(\mathrm{PO}_{4}\right)_{2} \cdot 19 \mathrm{H}_{2} \mathrm{O}\right)$, S05R000321-3a provided by $\mathrm{G}$ Cooke by e-mail 


\begin{tabular}{|l|l|l|}
\hline & $500 \mu m$ \\
\hline &
\end{tabular}


Thermonatrite $\left[\mathrm{Na}_{2} \mathrm{CO}_{3} \cdot\left(\mathrm{H}_{2} \mathrm{O}\right)\right]$

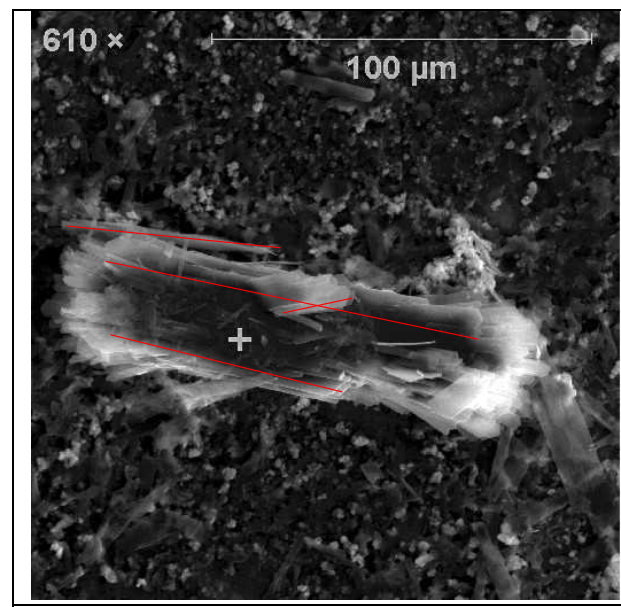

Image provided by Gary Cooke (222s) via email

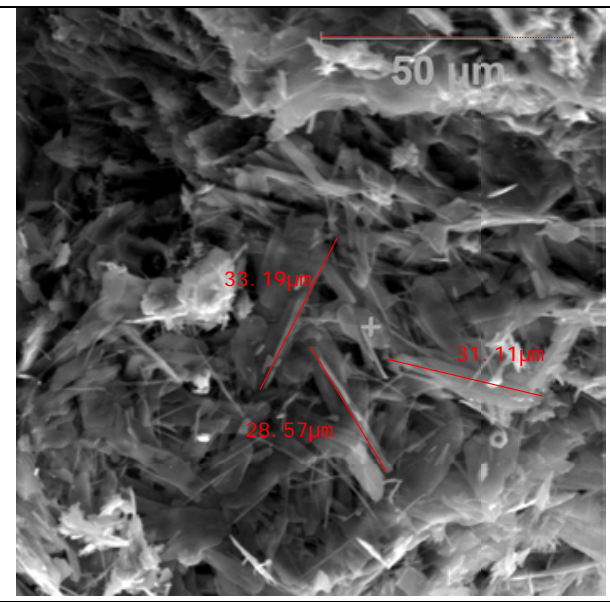

Image provided by Gary Cooke (222s) via email

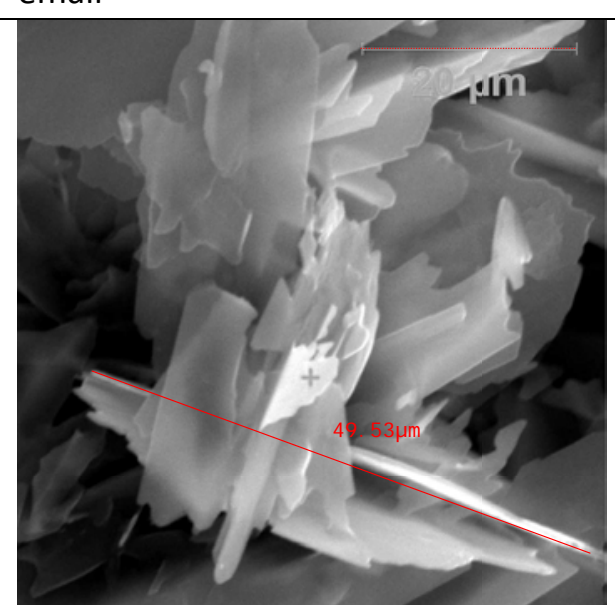

Image provided by Gary Cooke (222s) via email 
Sodium Sulfate-Thenardite $\left[\mathrm{Na}_{2} \mathrm{SO}_{4}\right]$

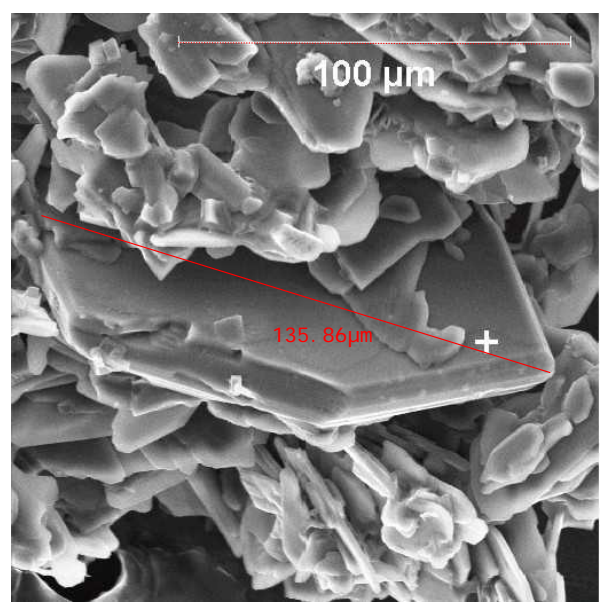

Image provided by Gary Cooke (222s) via email

\section{Sodium Phosphate Dodecahydrate $\left[\mathrm{Na}_{3} \mathrm{PO}_{4} \cdot 12 \mathrm{H}_{2} \mathrm{O} \cdot 0.25 \mathrm{NaOH}\right]$}

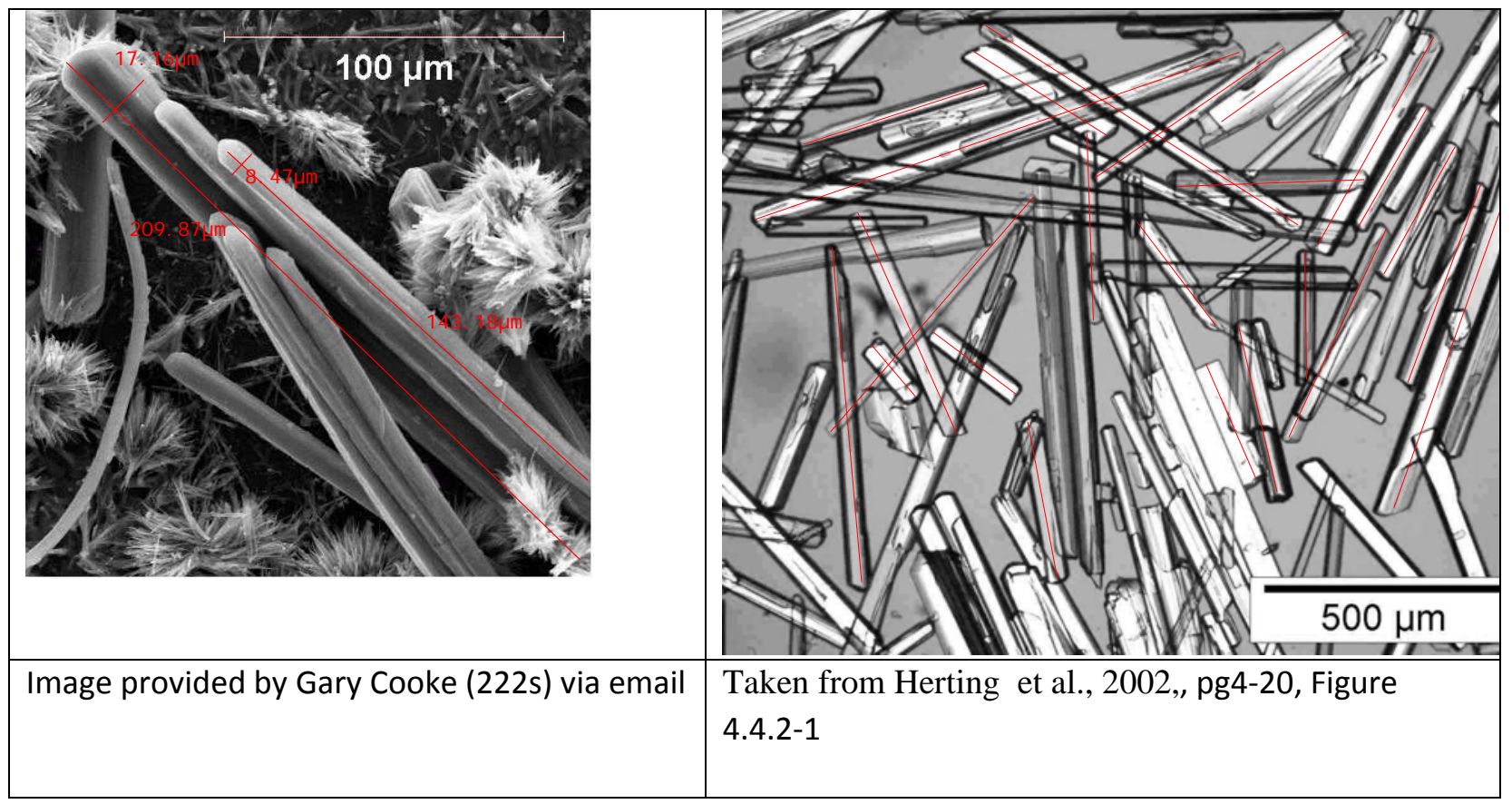




\section{Hydrouvarovite $\left[(\mathrm{Ca}, \mathrm{Sr})_{3}(\mathrm{Cr}, \mathrm{Al})_{2}(\mathrm{OH})_{12}\right]$}

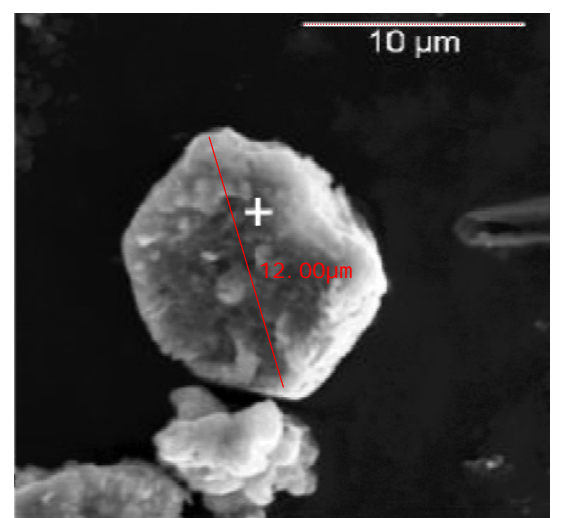

SEM image of particle in the BY-109 residue sample thought to be a hydrouvarovite, Image taken from Herting et al., 2002, pg3-53, Figure 3.9.2-3

\section{Sodium Sulfate-Fluoride Double Salt -Schairerite $\left[\mathrm{Na}_{3} \mathrm{FSO}_{4}\right]$}

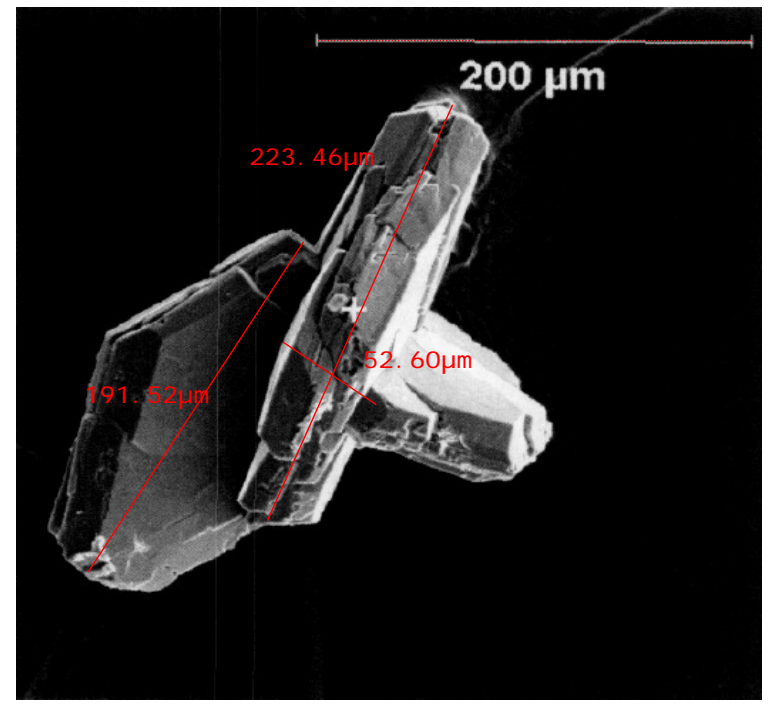

Taken from Herting et al. 2002. pg3-17, Figure 3.2.2-4. 
Trona $\left[\mathrm{Na}_{3}\left(\mathrm{CO}_{3}\right)\left(\mathrm{HCO}_{3}\right) \cdot 2\left(\mathrm{H}_{2} \mathrm{O}\right)\right]$

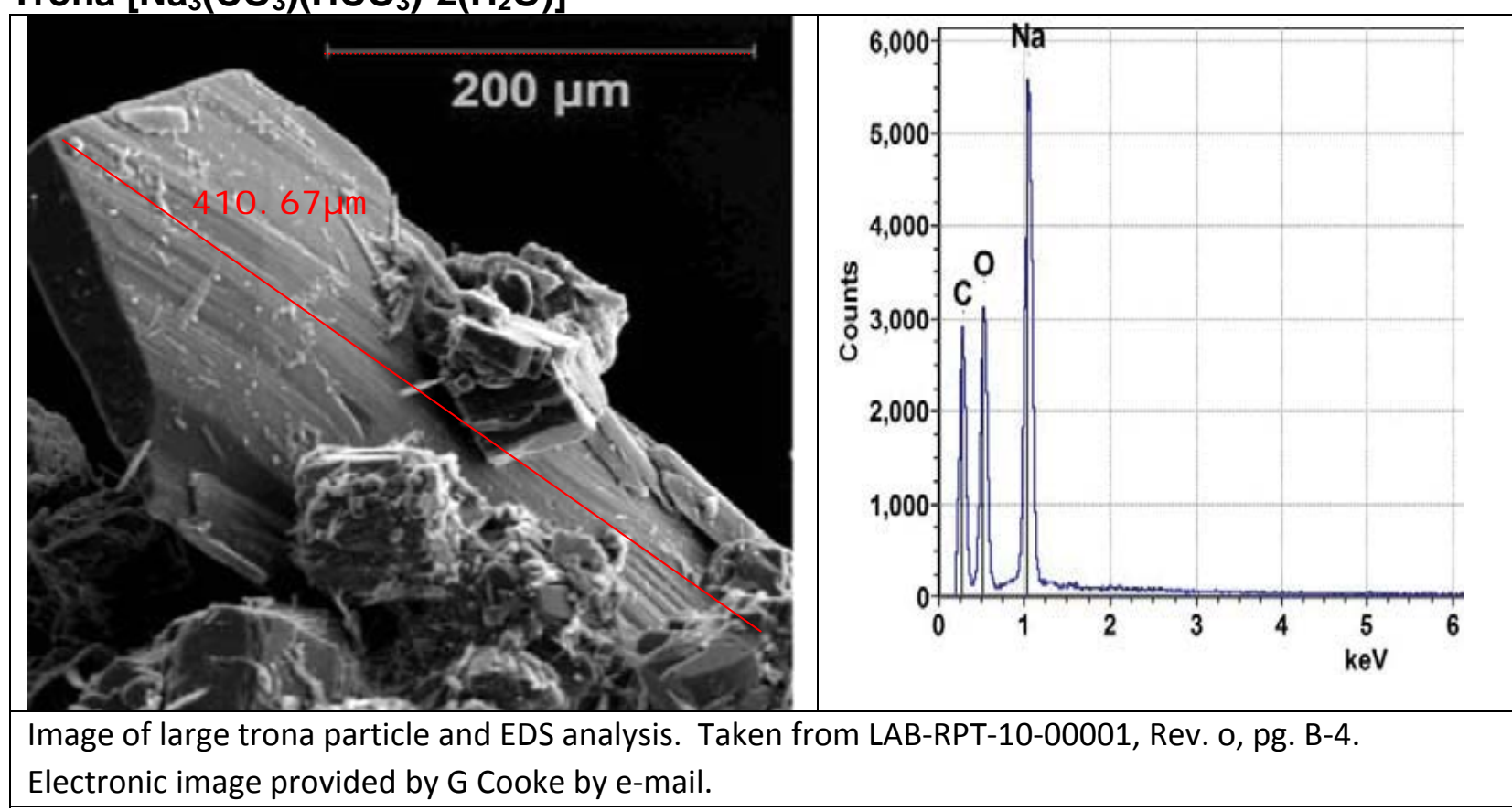

\section{Burkeite $\left[\mathrm{Na}_{6} \mathrm{CO}_{3}\left(\mathrm{SO}_{4}\right)_{2}\right]$}

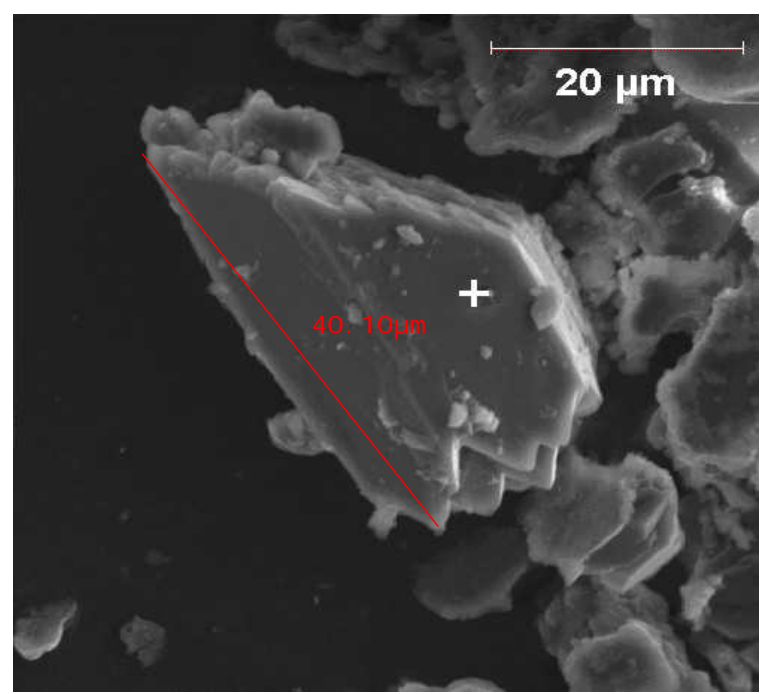

Image provided by G Cooke by e-mail. 
Darapskite $\mathrm{Na}_{3} \mathrm{NO}_{3} \mathrm{SO}_{4} \cdot \mathrm{H}_{2} \mathrm{O}$

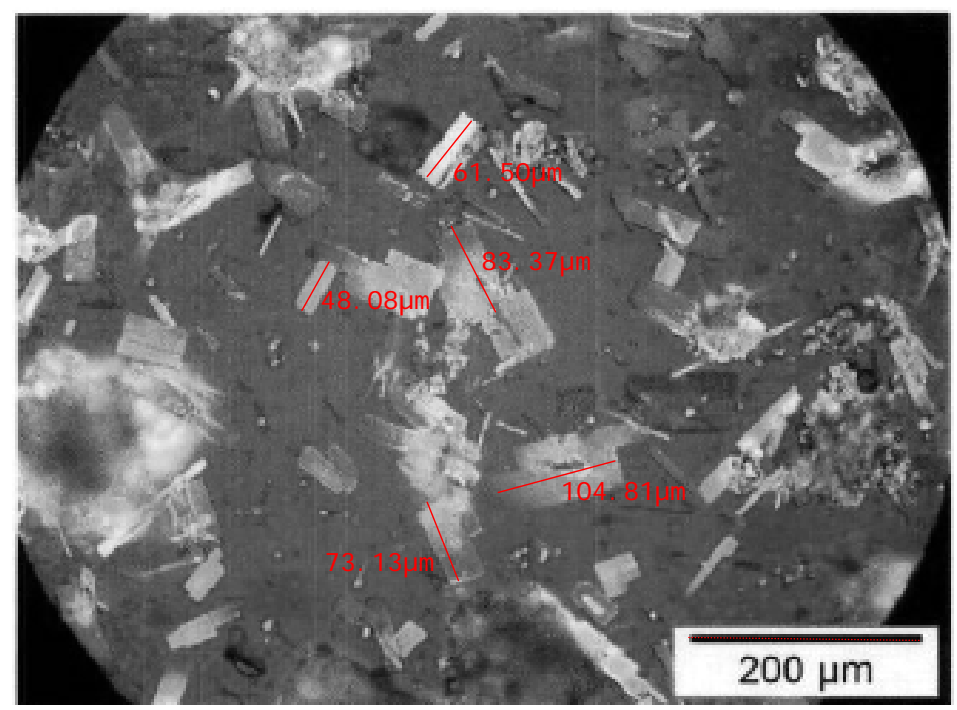

Image taken from Herting et al. 2002. pg 4-28. 


\section{Apophyllite [NaF]}

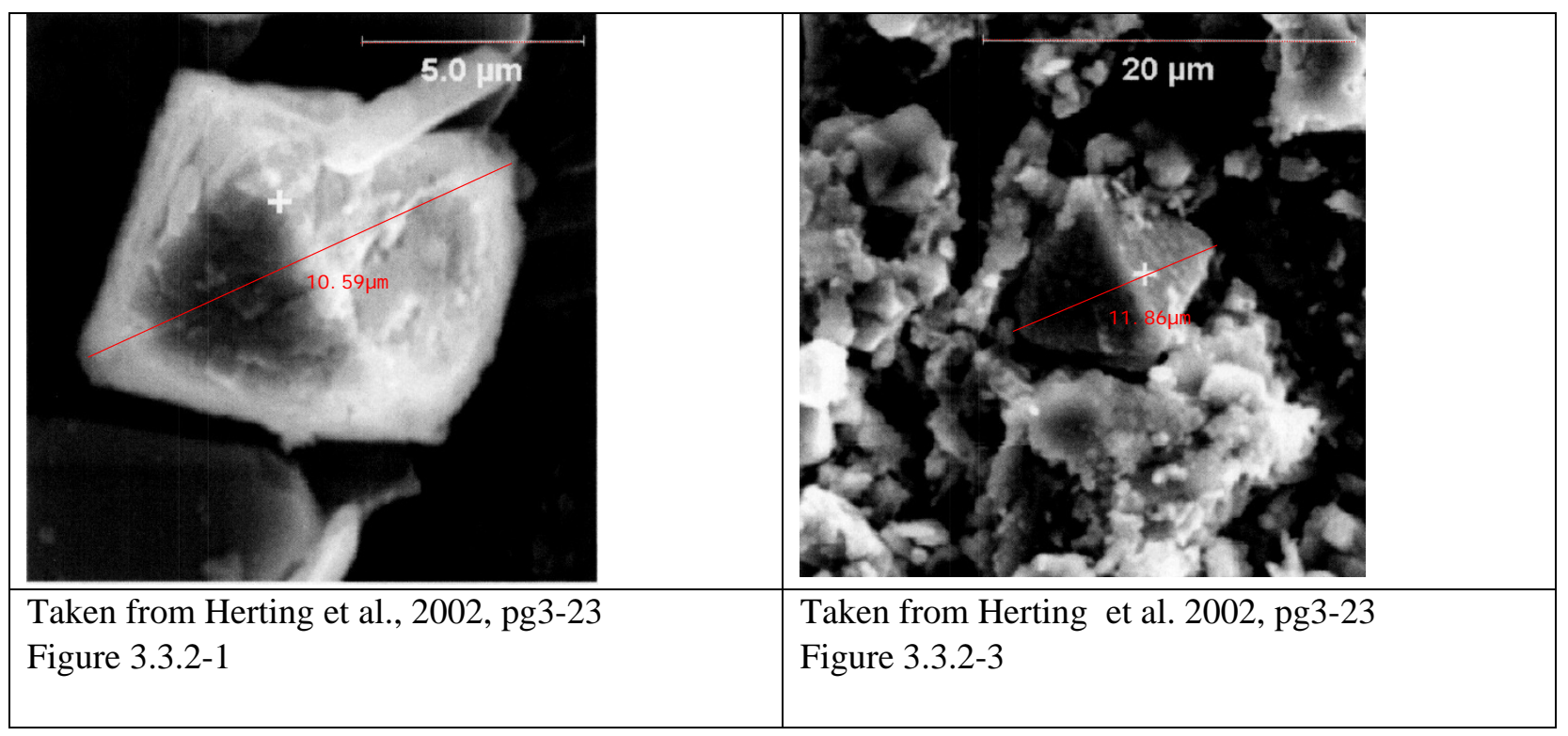




\section{Cryolite $\left[\mathrm{Na}_{3} \mathrm{AIF}_{6}\right]$}

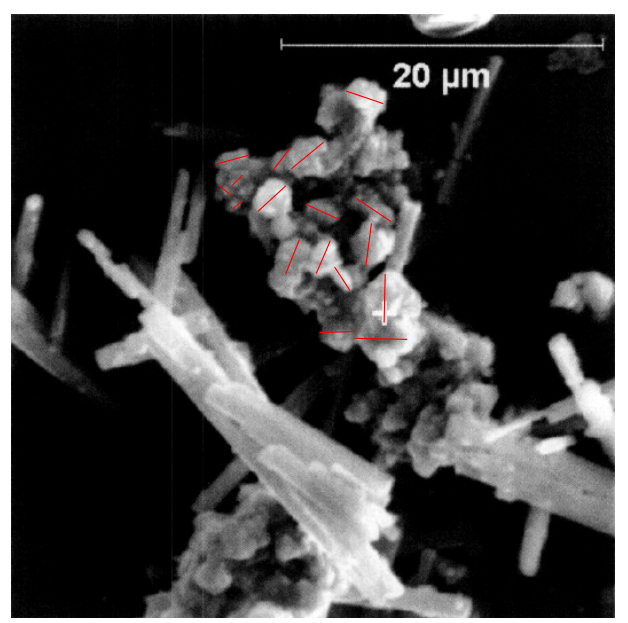

Taken from Herting et al. 2002. pg3-48, Figure 3.7.2-1. 


\section{Sodium Oxalate-Natroxalate $\left[\mathrm{Na}_{2} \mathrm{C}_{2} \mathrm{O}_{4}\right]$}

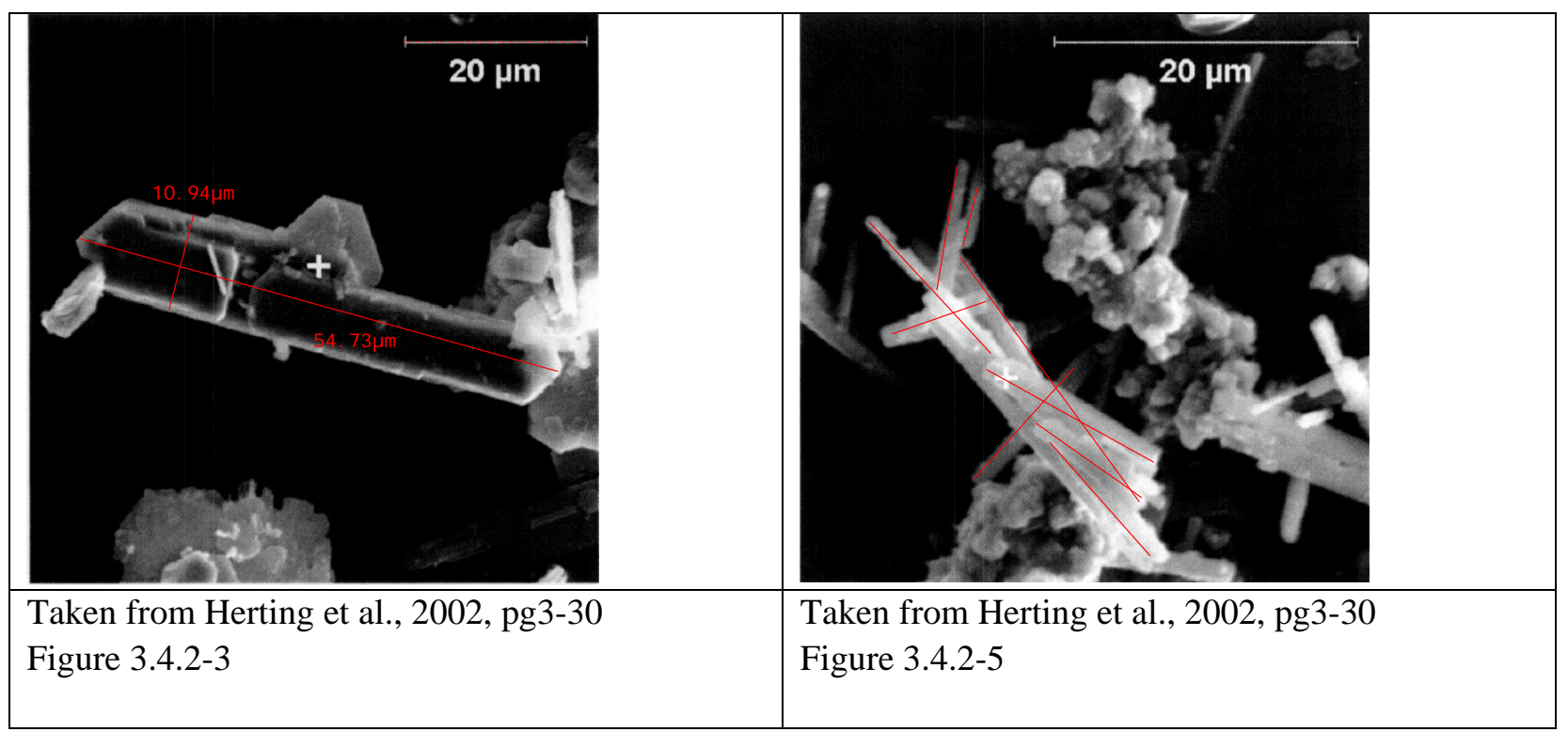




\section{Nastrophite $\left[\mathrm{NaSrPO}_{4} \cdot 9 \mathrm{H}_{2} \mathrm{O}\right]$}
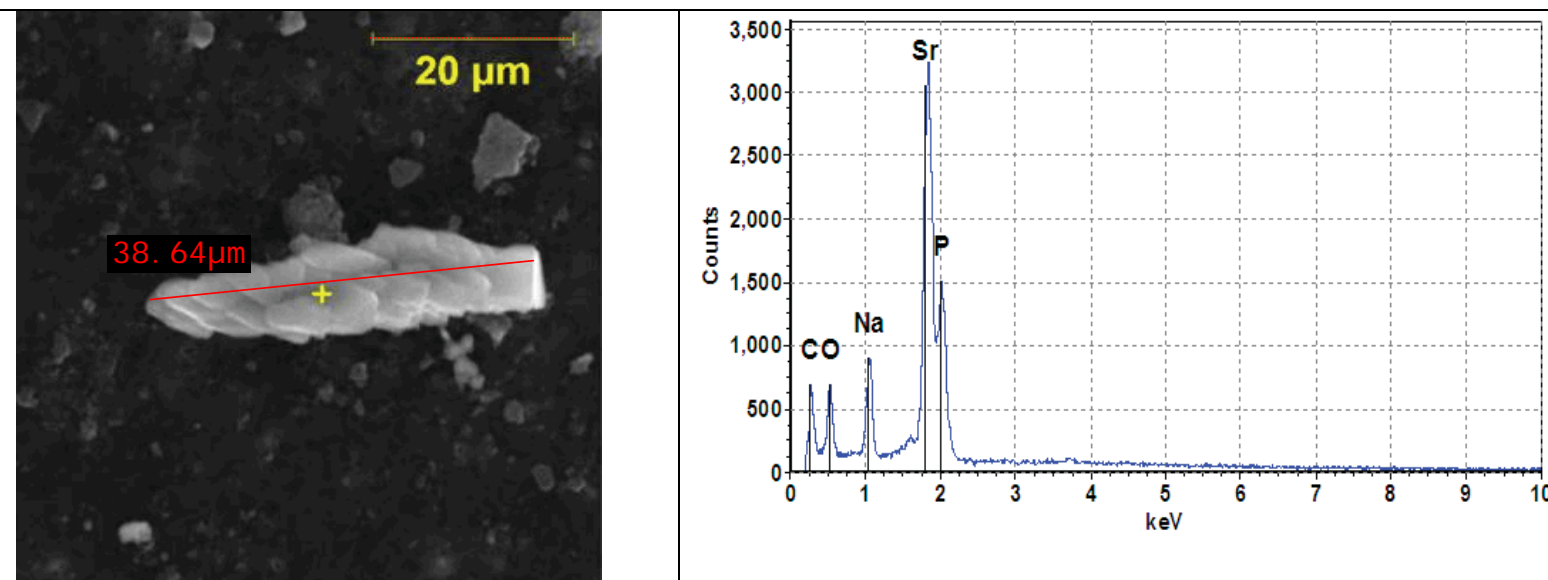

C108 Heel showing nastrophite particle and EDS analysis, taken from Callaway and Huber (2010), Figure 3-14, pg. 31.
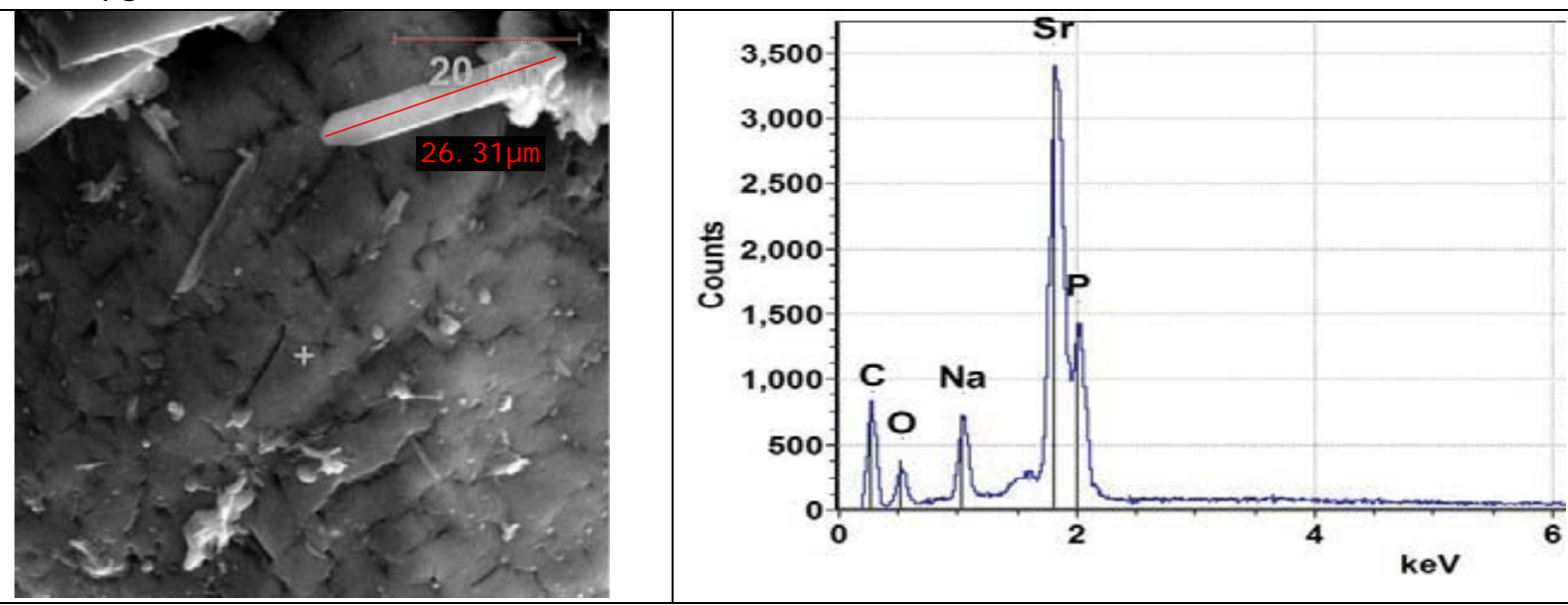

BSE image of nastrophite particle and EDS analysis taken from Callaway and Huber (2010), pg. B-4

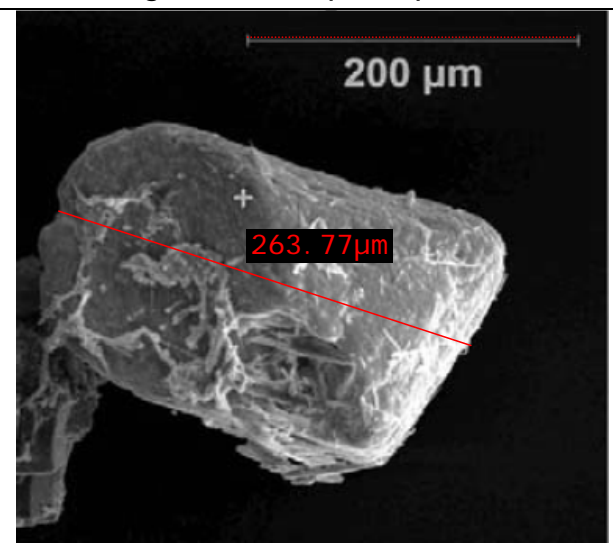

Callaway and Huber (2010)

pg. B-7 
Appendix E PSD Sampling and Analysis 



\section{Appendix E: PSD Sampling and Analysis}

A general discussion of the difficulties with PSD sampling and analysis is provided. The bulk of the discussion is taken directly from Wells et al. (2007).

The goal of any PSD analysis is to obtain a particle size measurement that is representative of the tested sample under specified conditions. Material collection must be controlled to eliminate bias in the sampling of the tank waste. However, the tank configuration and the chemistry and physical properties of the sludge contained therein makes representative sampling difficult, if not impossible.

To begin, tanks containing particulate matter generally contain a large volume of sludge, typically 10 to 1000 kiloliters, that can be sampled, whereas analytical testing (and the safety requirements tests) dictates tank sample volumes on the order of liters. Particle size analysis must, as a result, derive a representative size distribution from only a fraction of the tank material. Significant sampling difficulties derive from the fact that the sludge chemistry and physical properties within a given tank may not be uniform. Tank heterogeneity derives from a number of processes, including (but not limited to) the addition of different process wastes into the same tank over a number of years (resulting in layered waste types), uncontrolled precipitation of multiple chemical species, and continued chemical changes within the tank (which may also vary within the tank because of temperature differentials). The result is possible variation of solid species concentrations and particle sizes, among other things, with location in the tank.

Spatial variations make the definition of a representative material for a given tank relatively ambiguous, especially when the analysis is aimed at characterizing all the solid material in that tank. Under such circumstances, it would be ideal to homogenize the entire tank before sampling or to pull multiple samples and homogenize them before analysis. Because of their size, homogenization of entire tanks is not practical. In addition, tanks are configured such that there is limited control over where waste samples can be taken, so multiple sampling and subsequent homogenization also may not provide a means of obtaining a representative sample. With the acknowledgement of these factors, the PSDs for a waste sample may not be representative of the PSD of the waste in the tank as a whole.

Even if a representative sample is obtained through rigorous sampling, the testing protocol used in PSD analysis can influence the results dramatically. For example, the choice of suspending fluid can influence PSD results. If the suspending fluid viscosity is low, large or dense particles may settle out of suspension before analysis in static cells or may not be suspended in flow systems. In this case, a bias toward fine particles would be introduced to the PSD results. In addition, the ionic strength and $\mathrm{pH}$ of the suspending medium may also alter the measured PSD through dissolution or precipitation or through changes in the interparticle forces that govern the formation and stability of flocs and/or aggregates.

Shear forces applied to the sample during PSD measurement can also influence the measured distribution size. For example, weak flocs that exist under quiescent conditions may be disrupted under shear or by an applied ultrasonic field. Indeed, ultrasonic fields are usually used to break down weak flocs in samples where the distribution of primary particles or hard agglomerates is of interest. Shear and ultrasonics typically shift the PSD to lower particle sizes. Ultrasonics can cause particle agglomeration if particle-particle repulsion forces (e.g., electrostatic repulsion) are overcome by the energy provided from ultrasonics. The force required to disrupt the weak flocs depends strongly on the nature of forces holding 
the particles together. In some cases, the degree of agitation required to suspend the samples' particulate matter may also be sufficient to break weak flocs.

PSD analyses were performed with a number of instruments that employ different measurement principles and flow geometries. The latter is especially significant because some instruments measure the dispersion under static/quiescent conditions, whereas others circulate and stir the dispersion during the analysis. Both correspond to dramatically different shear environments. Even where measurements have employed flow systems, differences in the circulation rate and line diameter as well as the presence of sample reservoir agitation (e.g., stirring or sonication) result in variations in the effective shear applied to the sample. These differences mean that the PSD measurements contained in the referenced reports are not always directly comparable.

Further, the PSD data for Hanford waste were prepared over a number of years and by different organizations, and, as a result, the PSD measurements were not governed by a single universal procedure. Tank sampling methods differ from report to report in the location and method of extraction (e.g., rotary core, push-mode core, slurry grab samples, etc.). Some reports considered waste samples extracted from a single height in the tank, while others retrieved samples from multiple heights and homogenized them into a single sample. Differences in treatment of tank waste samples after extraction but before PSD analysis arise from different storage protocols after extraction and different experimental objectives.

For example, some tank waste samples were reported to have dried during storage because of evaporation. To counteract evaporative losses, the original sample mass was restored by adding deionized water to the sample. In such cases, it would be difficult to evaluate what influence this process had on the size distribution of particles in the affected samples because the process of precipitation on drying may not be immediately reversible.

The vast majority if not all of the samples from the M12 actual waste evaluation had been evaporated to dryness and were re-hydrated before analysis (Fiskum et al. 2008, Fiskum et al. 2009, Lumetta et al. 2009, Snow et al. 2009, Edwards et al. 2009). A qualitative comparison of PSD data for the waste types of the M12 analyses and related as-received samples from Wells et al. (2007) is made in Figure E.1 through E.11. These comparisons have not been reviewed and are thus for information only. In the figures, the data labeled as tank or waste type are from Wells et al. (2007); all other data are from the previously specified M12 reports. Both Flowing Sonicated and Flowing Unsonicated data are presented where possible. Unless otherwise specified, the pump rate for the M12 data is $3000 \mathrm{rpm}$. There is no conclusive evidence that the evaporation and rehydration of the samples impacted the PSD results. 


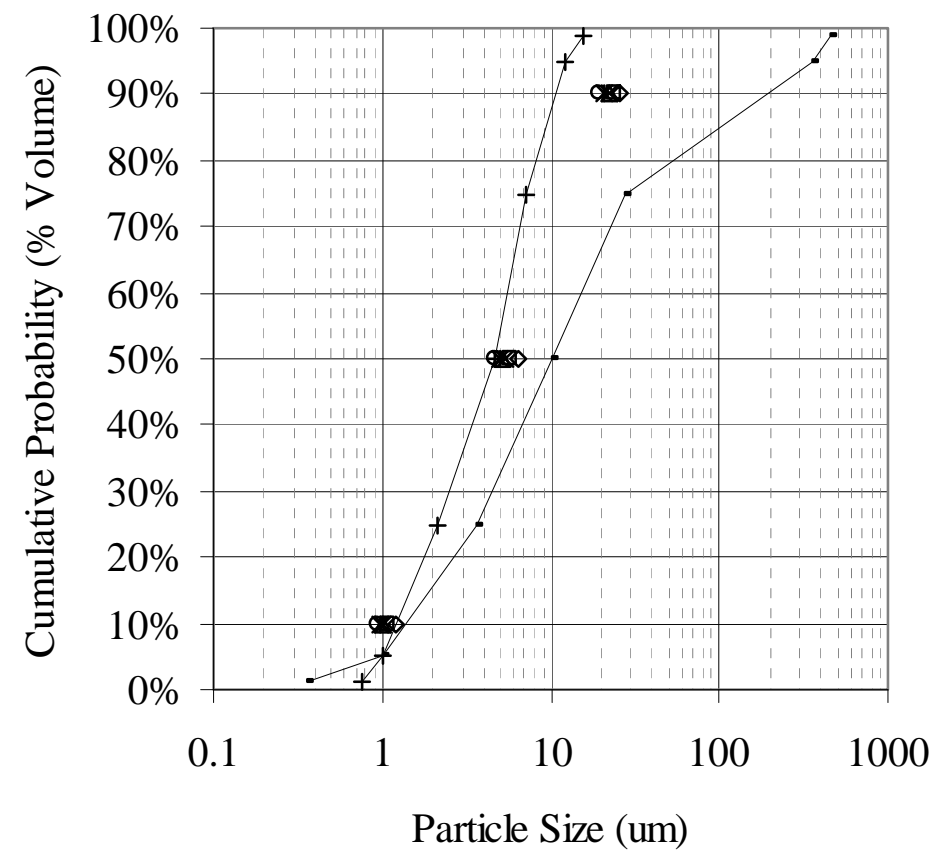
$\diamond$ TI483-G1-S-WL-PSD-1 0.25
口 TI483-G1-S-WL-PSD-1 0.5
$\Delta$ TI483-G1-S-WL-PSD-1 0.75
$\times \quad$ TI483-G1-S-WL-PSD-2 0.25
* TI483-G1-S-WL-PSD-2 0.5
○ TI483-G1-S-WL-PSD-2 0.75
+ 1C, sonicated, WTP-RPT-153
_. 2C, sonicated, WTP-RPT-153

Figure E.1. 1C and 2C Sludge Waste Type PSD Comparison, Flowing Sonicated. For Information Only.

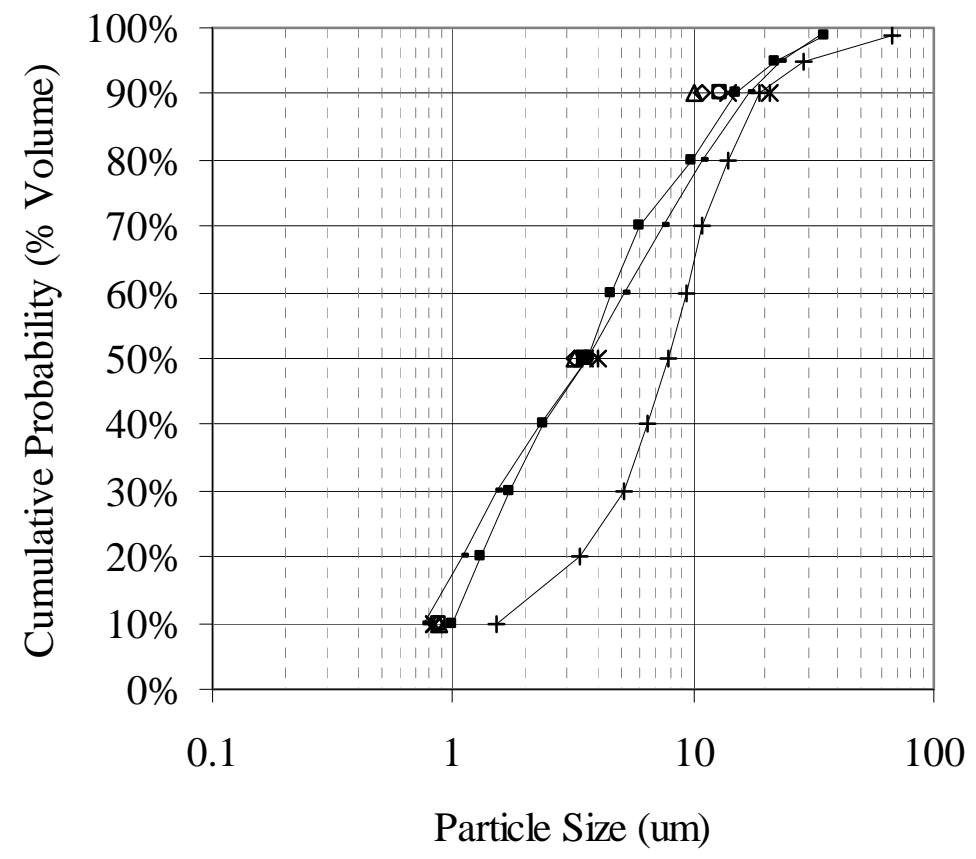

Figure E.2. BY and T Saltcake Waste Type PSD Comparison, Flowing Unsonicated. For Information Only. 


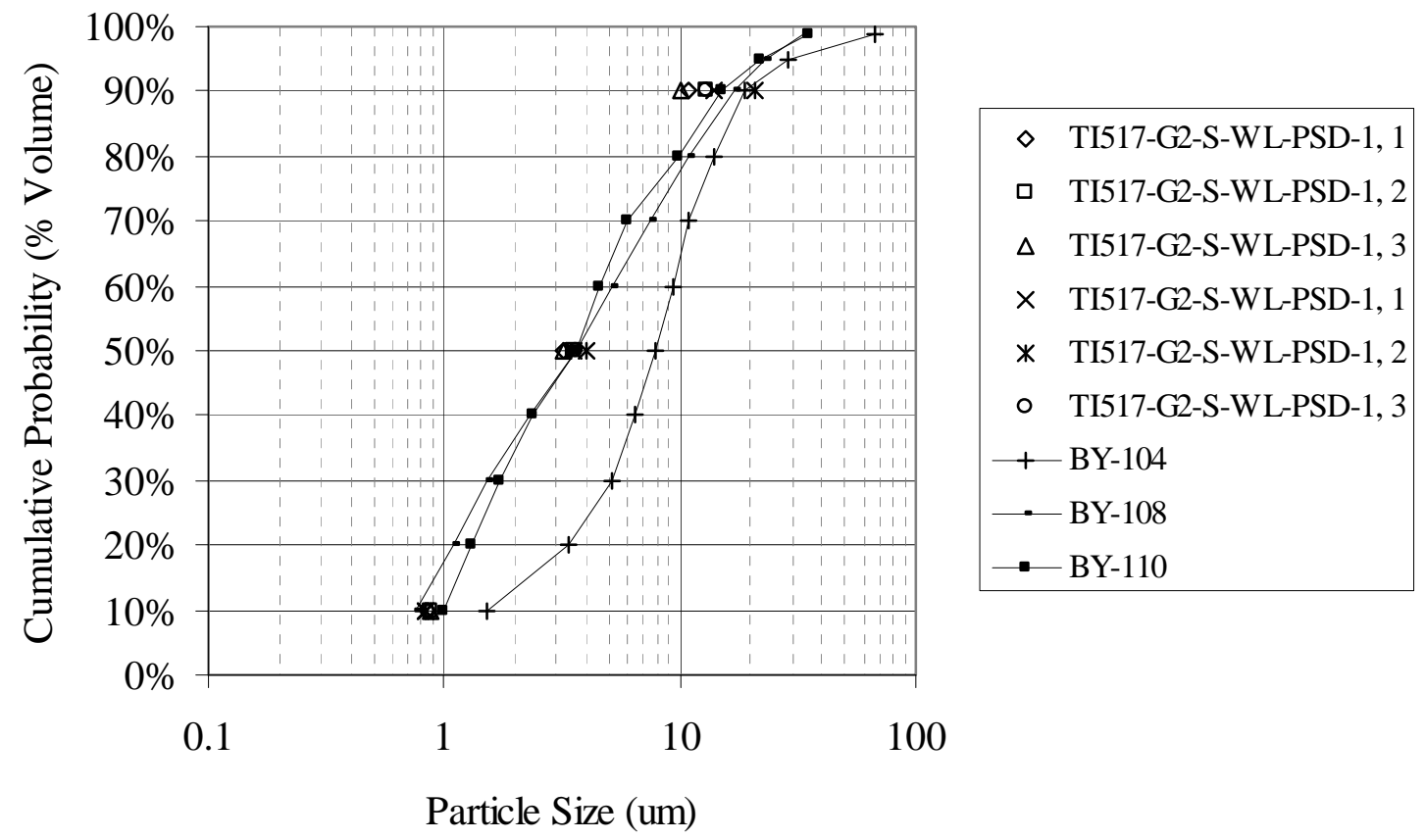

Figure E.3. BY and T Saltcake Waste Type PSD Comparison, Flowing Sonicated. For Information Only.
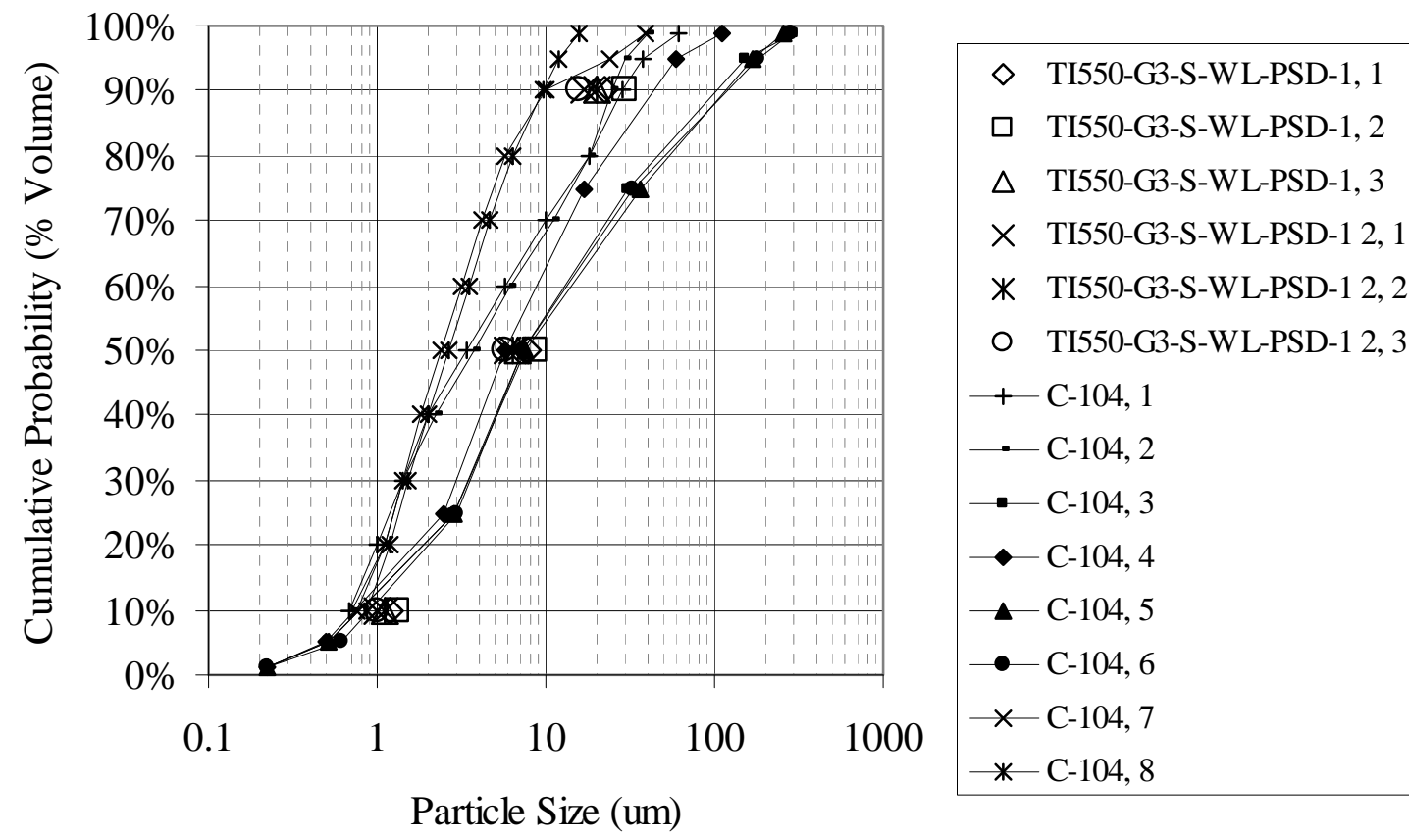

Figure E.4. CWP Sludge Waste Type PSD Comparison, Flowing Unsonicated. For Information Only. 


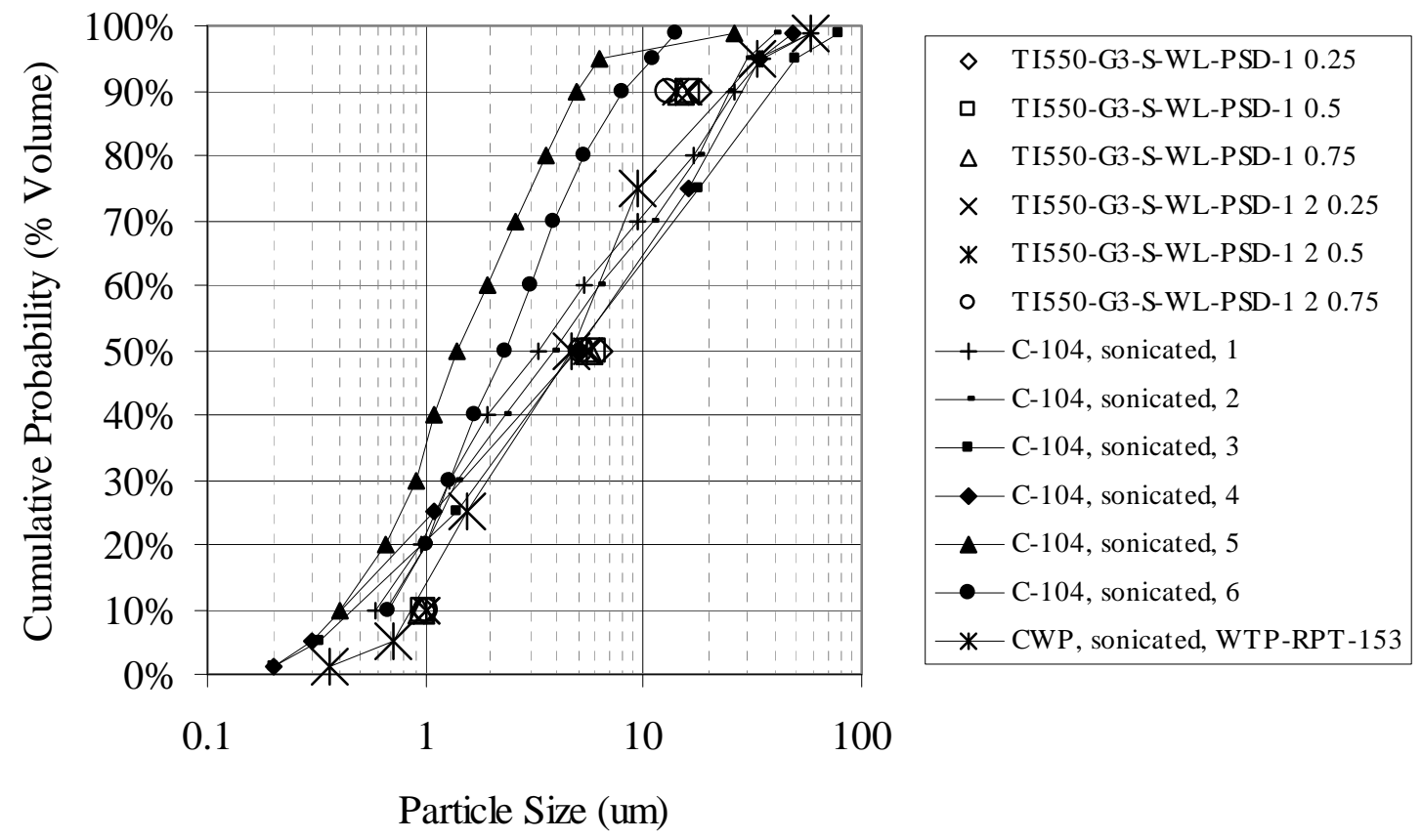

Figure E.5. CWP Sludge Waste Type PSD Comparison, Flowing Sonicated. For Information Only.

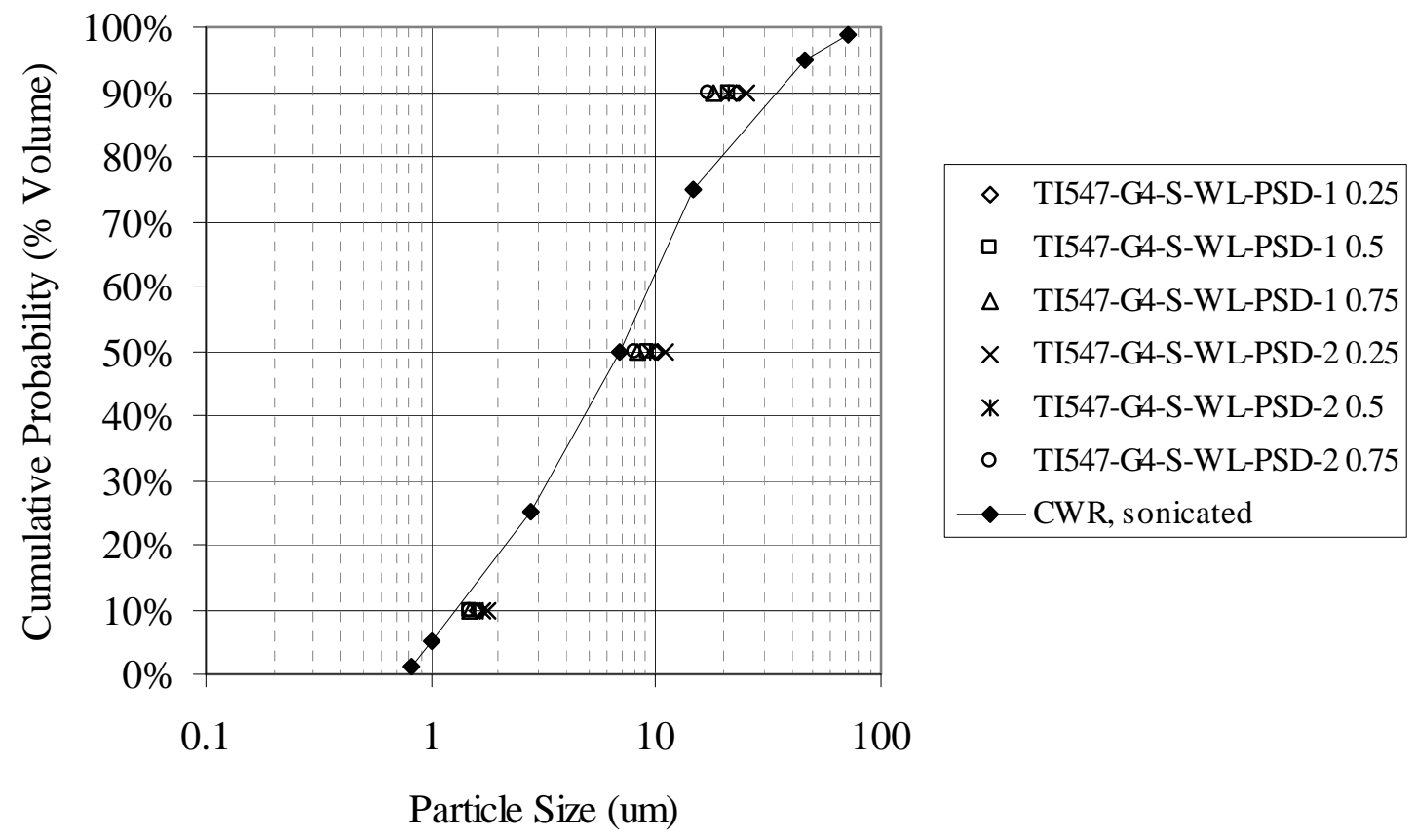

Figure E.6. CWR Sludge Waste Type PSD Comparison, Flowing Sonicated. For Information Only. 


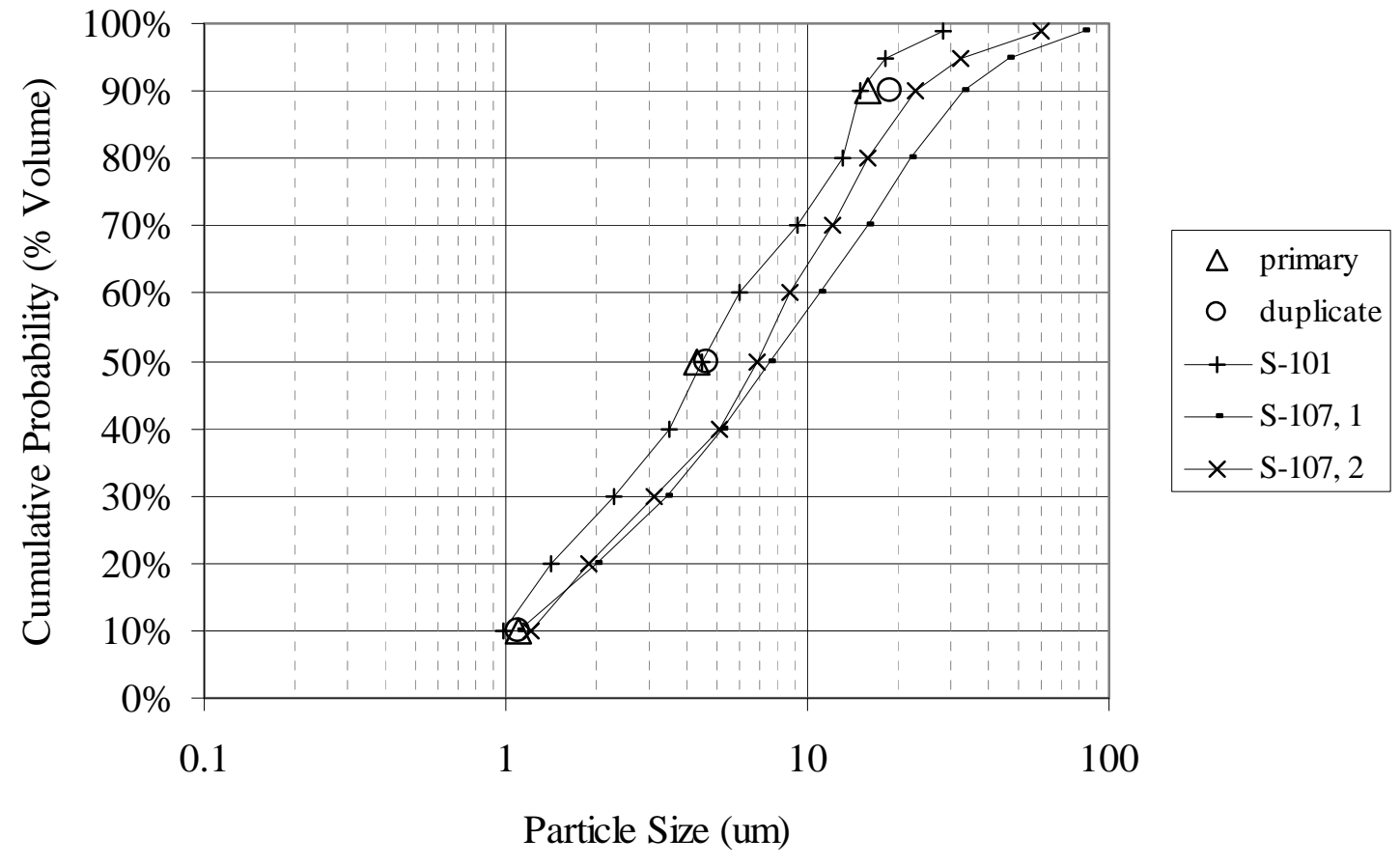

Figure E.7. R (boiling) Sludge Waste Type PSD Comparison, Flowing Unsonicated. For Information Only.

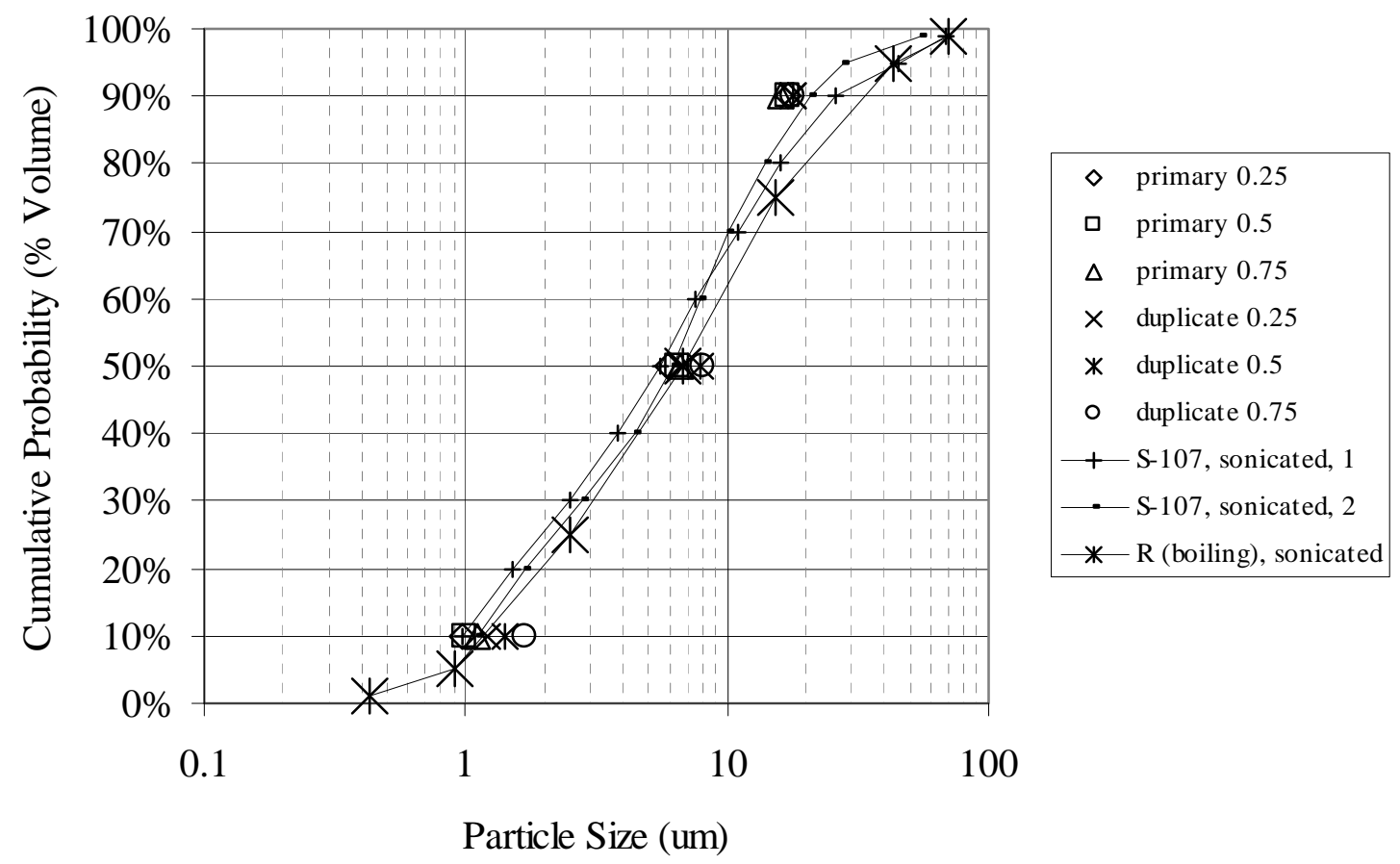

Figure E.8. R (boiling) Sludge Waste Type PSD Comparison, Flowing Sonicated. For Information Only. 


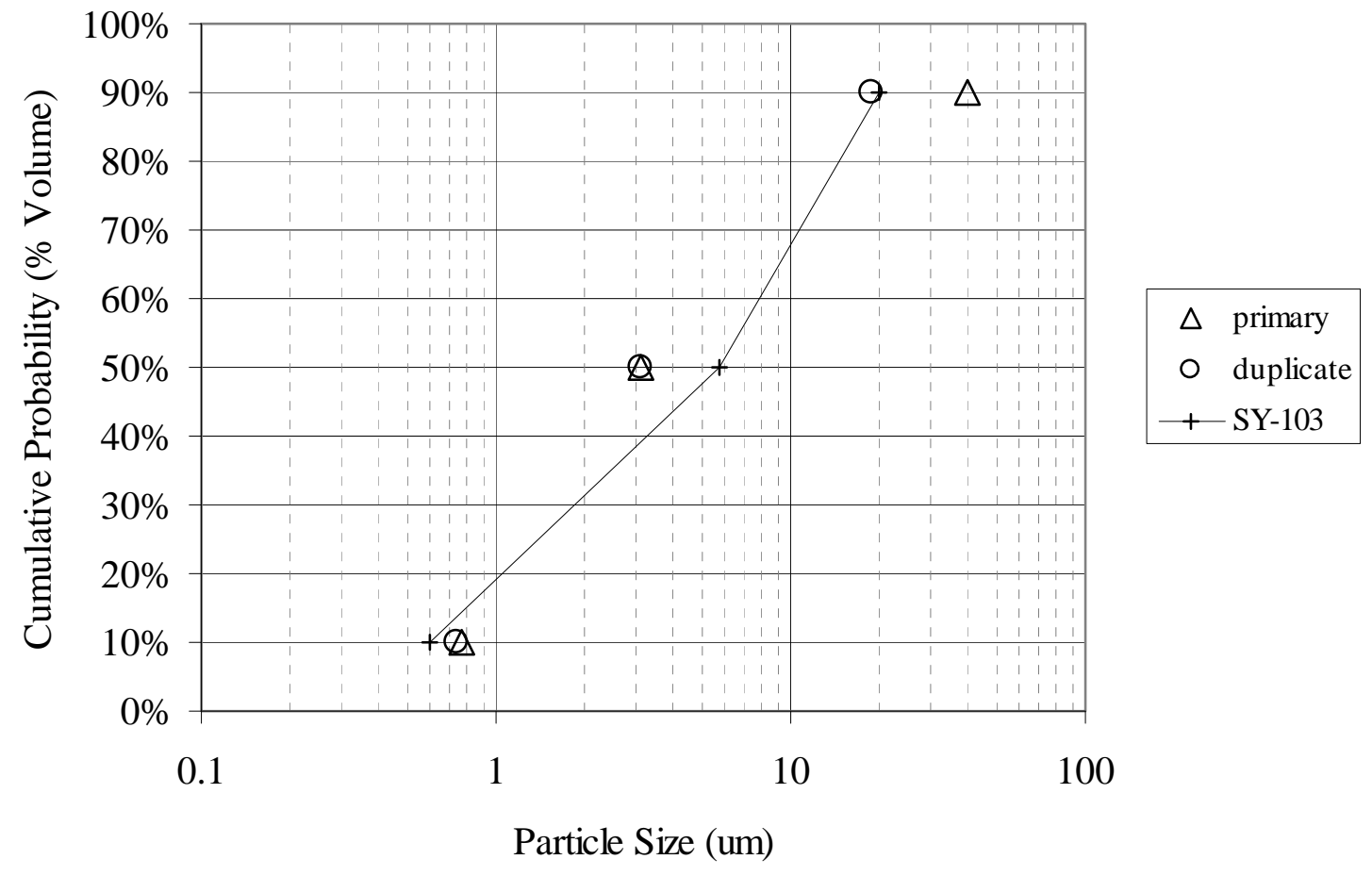

Figure E.9. S Saltcake Waste Type PSD Comparison, Flowing Unsonicated. For Information Only.

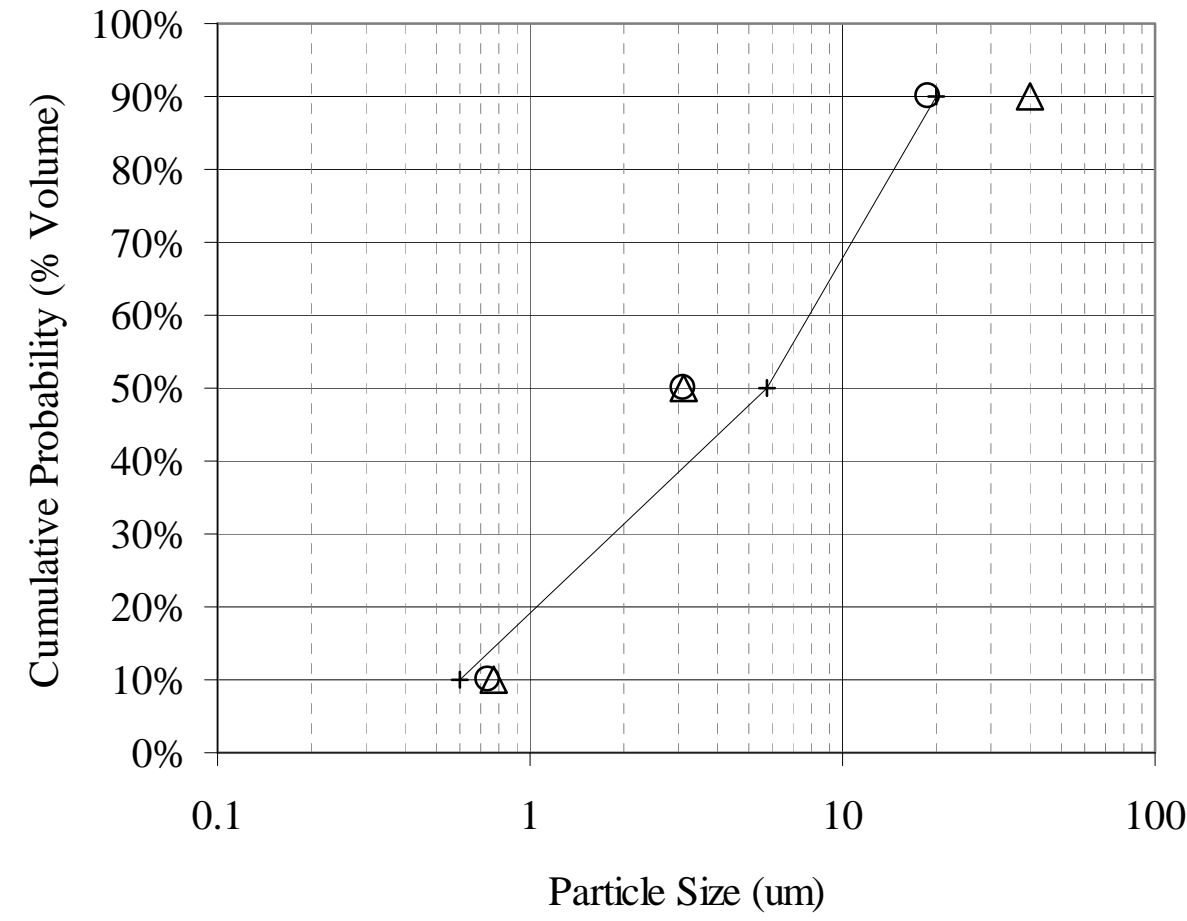

Figure E.10. S Saltcake Waste Type PSD Comparison, Flowing Unsonicated. For Information Only. 


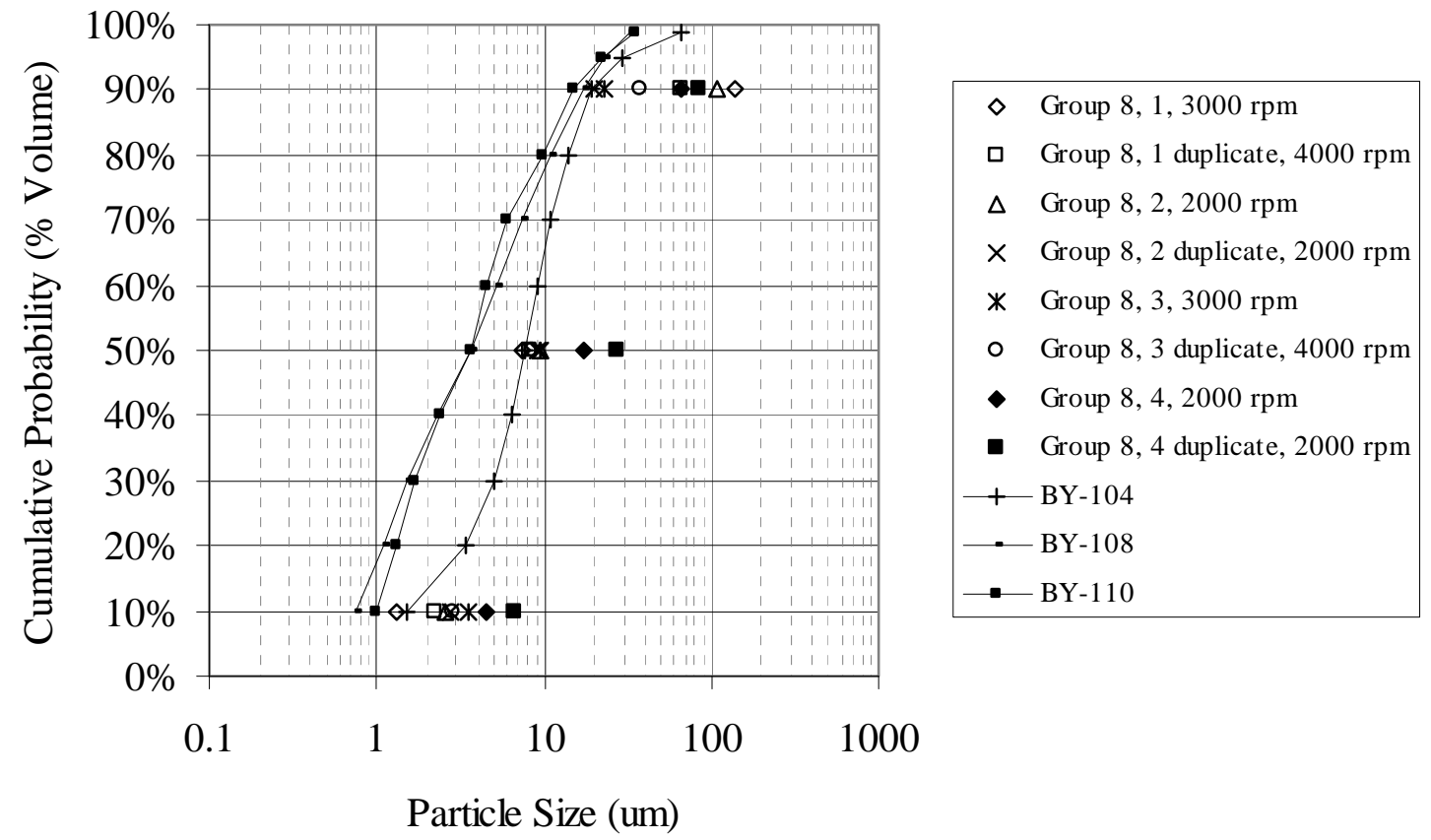

Figure E.11. 1CFeCN, PFeCN, and TFeCN Sludge Waste Type PSD Comparison, Flowing Unsonicated. For Information Only.

Additional comparison of the effect of evaporation to dryness and rehydration on the PSD of a sample is made for AZ-101 data. In Figure E.12, PSD data for AZ-101 waste samples analyzed by Bell (2001) and Callaway (2000) are provided. The data from Bell (2001) are particle sizes of the UDS suspended during the time period immediately following final mixer pump operation in the tank (the "settling test," Carlson et al. 2001) are reported in Bell (2001). Grab samples were taken at various elevations and times after the cessation of mixing. It is noted in Bell (2001) that at the time of the particle size analysis, the samples had evaporated to dryness and were stored in that condition for an extended period of time. It is further noted that the particle size "...results should be treated as suspect as related to their representation of the PSDs of the originally sampled materials.” The data from Callaway (2000) are for waste composite samples kept in a hydrated condition. The same instrumentation and configuration thereof was used for both studies. The comparison shows that, as with the M12 data, there is no conclusive evidence that the evaporation and rehydration of the samples impacted the PSD results. 


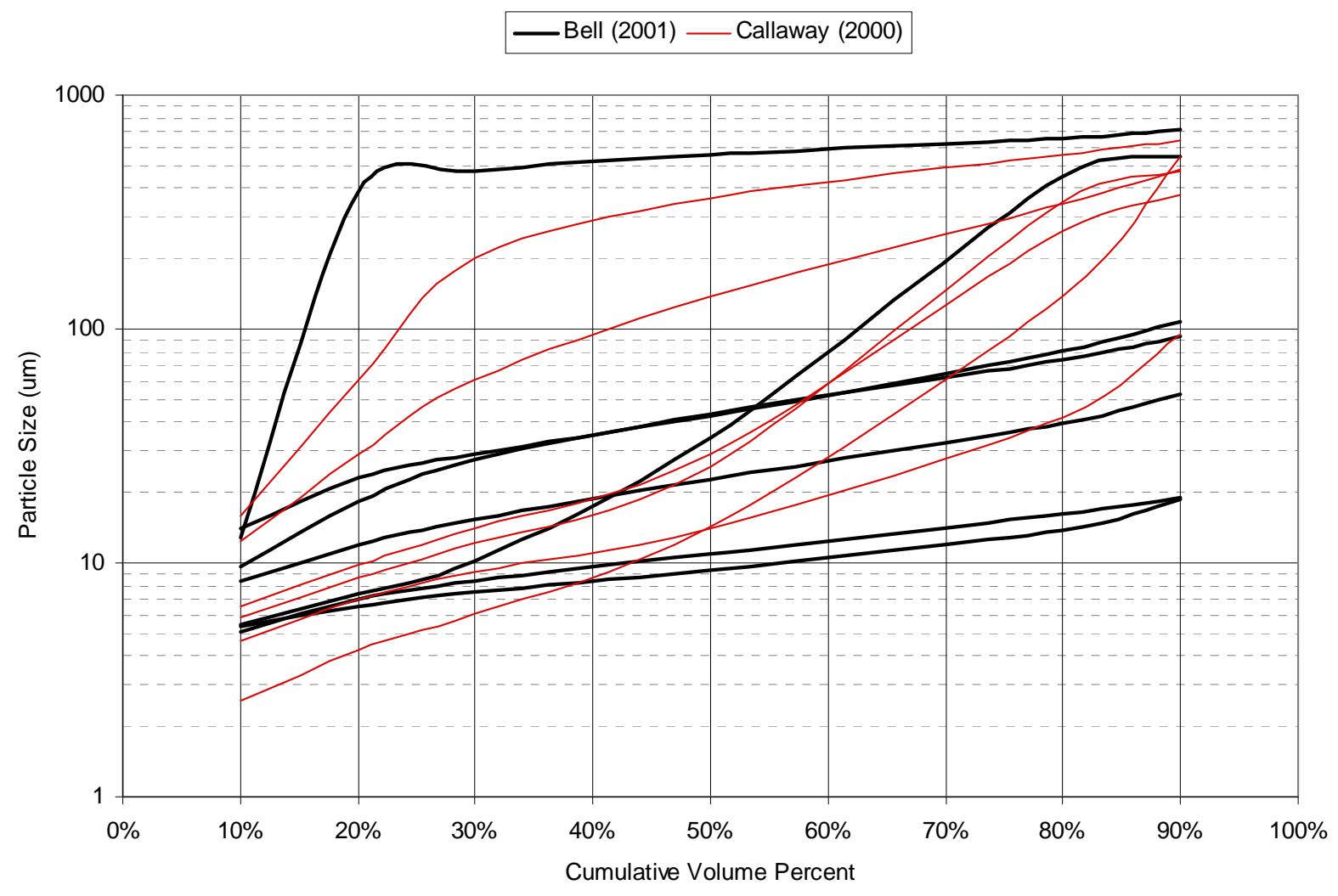

Figure E.12. AZ-101 PSD Comparison, No-Flow Unsonicated. For Information Only.

\section{REFERENCES}

Bell KE. 2001. Tank 241-AZ-101 Grab Samples From Mixer Pump Test Events 5, 7, 8, and 9 Analytical Results for the Final Results for the Report. HNF-6062, Rev 0, Fluor Hanford, Richland, Washington.

Callaway WS. 2000. Results of Retrieval Testing of Sludge from Tank 241-AZ-101. HNF-7078, Rev. 0, Fluor Hanford, Richland, Washington.

Carlson AB, PJ Certa, TM Hohl, JR Bellomy III, TW Crawford, DC Hedengren, AM Templeton, HS Fisher, SJ Greenwood, DG Douglas, and WJ Ulbright Jr. 2001. Test Report, 241-AZ-101 Mixer Pump Test. RPP-6548, Rev. 1, Numatec Hanford Corporation, Richland, Washington.

Edwards MK, JM Billing, DL Blanchard, EC Buck, AJ Casella, AM Casella, JV Crum, RC Daniel, KE Draper, SL Fiskum, LK Jagoda, ED Jenson, AE Kozelisky, PJ MacFarlan, RA Peterson, RW Shimskey, LA Snow and RG Swoboda. 2009. Characterization, Leaching, and Filtration Testing for Tributyl Phosphate (TBP, Group 7) Actual Waste Sample Composites. PNNL-18119 (WTP-RPT-169 Rev. 0), Pacific Northwest National Laboratory, Richland, Washington.

Fiskum SK, AE Kozelisky, EC Buck, GJ Lumetta, RC Daniel, PJ MacFarlan, K Draper, BK McNamara, MK Edwards, RA Peterson, TL Hubler, SI Sinkov, LK Jagoda, LA Snow, ED Jenson, and RG Swoboda. 
2008. Characterization and Leach Testing for REDOX Sludge and S-Saltcake Actual Waste Sample Composites. PNNL-17368, WTP-RPT-157, Pacific Northwest National Laboratory, Richland, Washington.

Fiskum SK, JM Billing, JV Crum, RC Daniel, MK Edwards, RW Shimskey, RA Peterson, PJ MacFarlan, EC Buck, KE Draper and AE Kozelisky. 2009. Characterization, Leaching, and Filtration Testing of Ferrocyanide Tank Sludge (Group 8) Actual Waste Composite. PNNL-18120 (WTP-RPT-170 Rev. 0), Pacific Northwest National Laboratory, Richland, Washington.

Lumetta GJ, EC Buck, RC Daniel, K Draper, MK Edwards, SK Fiskum, RT Hallen, LK Jagoda, ED Jenson, AE Kozelisky, PJ MacFarlan, RA Peterson, RW Shimskey, SI Sinkov and LA Snow. 2009. Characterization, Leaching, and Filtration Testing for Bismuth Phosphate Sludge (Group 1) and Bismuth Phosphate Saltcake (Group 2) Actual Waste Sample Composites. PNNL-17992 (WTP-RPT-166 Rev. 0), Pacific Northwest National Laboratory, Richland, Washington.

Snow LA, EC Buck, AJ Casella, JV Crum, RC Daniel, KE Draper, MK Edwards, SK Fiskum, LK Jagoda, ED Jenson, AE Kozelisky, PJ MacFarlan, RA Peterson, and RG Swoboda. 2009.

Characterization and Leach Testing for PUREX Cladding Waste Sludge (Group 3) and REDOX Cladding Waste Sludge (Group 4) Actual Waste Sample Composites. PNNL-18054, WTP-RPT-167. Pacific Northwest National Laboratory, Richland, Washington.

Wells BE, MA Knight, EC Buck, SK Cooley, RC Daniel, LA Mahoney, PA Meyer, AP Poloski, JM Tingey, WS Callaway III, GA Cooke, ME Johnson, MG Thien, DJ Washenfelder, JJ Davis, MN Hall, GL Smith, SL Thomson, and Y Onishi. 2007. Estimate of Hanford Waste Insoluble Solid Particle Size and Density Distribution. PNWD-3824 (WTP-RPT-153, Rev. 0), Battelle-Pacific Northwest Division, Richland, Washington. 
Appendix F

PSD Method Examples 



\section{Appendix F: PSD Method Examples}

Examples illustrating the calculations conducted to determine the probabilities associated with sizes included in a combined distribution are provided.

Consider the sonicated sludge data for four samples from waste Tank AY-101. The working data matrix (rounded to 3 decimal places) for this example is given in Table F.1 below.

Table F.1. Working Data Matrix for AY-101 (values are particle sizes in microns)

\begin{tabular}{|c|c|c|c|c|c|c|c|c|c|c|c|c|c|c|c|}
\hline Percentile & 0.01 & 0.05 & 0.10 & 0.20 & 0.25 & 0.30 & 0.40 & 0.50 & 0.60 & 0.70 & 0.75 & 0.80 & 0.90 & 0.95 & 0.99 \\
\hline Sample 1 & 0.300 & 0.610 & 0.859 & 1.704 & 2.400 & 2.833 & 3.947 & 5.500 & 6.607 & 7.938 & 8.700 & 9.902 & 12.828 & 14.600 & 9.900 \\
\hline Sample 2 & 0.260 & 0.560 & 0.750 & 1.344 & 1.800 & 2.122 & 2.950 & 4.100 & 5.020 & 46 & 6.800 & 7.771 & & 00 & 100 \\
\hline Sample 3 & 0.213 & 0.346 & \begin{tabular}{|l|l|}
0.548 \\
\end{tabular} & 0.979 & 1.207 & 1.505 & 3.047 & 5.461 & 6.784 & 7.914 & 8.533 & $\mid$ & 20 & 10 & 18.951 \\
\hline Sample 4 & 0.175 & 0.279 & 0.415 & 0.784 & 0.970 & 1.168 & 1.809 & 3.649 & 5.724 & 7.097 & 7.752 & 8.469 & 10.460 & 12.410 & 17.231 \\
\hline
\end{tabular}

Assuming a combined distribution is to be formed based only on these four samples, the combined distribution would represent a single waste tank. Thus, the volume weight associated with this tank would be 1.0. The probability associated with a listed size for this example is calculated by (incremental probability)*(volume weight)/(number of samples). For Sample 2, the first size listed is P01 $=0.260 \mu \mathrm{m}$. The associated probability for $0.26 \mu \mathrm{m}$ is $(0.01) *(1.0) / 4=0.0025$. The size listed as P75 for Sample 4 is $7.752 \mu \mathrm{m}$. The associated probability for this size value is $(0.05) *(1.0) / 4=0.0125$. These values can be found in the Probability column of Table F.2 corresponding to these two size values. The other Probability values listed in Table F.2 are calculated in the same way.

Once the combined distribution was formed for a given waste tank, waste type, or composite, linear interpolation was used to determine the particle sizes that correspond to the non-P100 percentiles of interest. This interpolation process is illustrated in Table F.2 for the sonicated sludge data for four samples from waste Tank AY-101. Note that for this waste tank, P100 values were not available for the analyzed samples. Thus, the working data matrix given above in Table F.1 and Table F.2 only describe percentiles up to P99.

Table F.2. Interpolations for Forming Combined Distribution, AY-101 Example (Sizes are in microns)

\begin{tabular}{|c|c|c|c|c|}
\hline Size & Probability & Cumulative Probability & Percentile & Percentile \\
\hline 0.174567 & 0.0025 & 0.0025 & & \\
\hline 0.21329 & 0.0025 & 0.005 & & \\
\hline 0.26 & 0.0025 & 0.0075 & & \\
\hline 0.264737 & & & & \\
\hline 0.278949 & 0.01 & 0.0175 & & \\
\hline 0.3 & 0.0025 & 0.02 & & \\
\hline 0.346036 & 0.01 & 0.03 & & \\
\hline 0.415 & 0.0125 & 0.0425 & 0.05 & P 05 \\
\hline 0.4948 & & & & \\
\hline 0.548 & 0.0125 & 0.055 & & \\
\hline
\end{tabular}


Table F.2. (contd)

\begin{tabular}{|c|c|c|c|c|}
\hline Size & Probability & Cumulative Probability & Percentile & Percentile \\
\hline 0.56 & 0.01 & 0.065 & & \\
\hline 0.61 & 0.01 & 0.075 & & \\
\hline 0.749824 & 0.0125 & 0.0875 & & \\
\hline 0.766912 & & & 0.10 & P10 \\
\hline 0.784 & 0.025 & 0.1125 & & \\
\hline 0.859113 & 0.0125 & 0.125 & & \\
\hline 0.969987 & 0.0125 & 0.1375 & & \\
\hline 0.979 & 0.025 & 0.1625 & & \\
\hline 1.168 & 0.0125 & 0.175 & & \\
\hline 1.207113 & 0.0125 & 0.1875 & & \\
\hline 1.275715 & & & 0.20 & P20 \\
\hline 1.344316 & 0.025 & 0.2125 & & \\
\hline 1.505 & 0.0125 & 0.225 & & \\
\hline 1.704084 & 0.025 & 0.25 & 0.25 & P25 \\
\hline 1.8 & 0.0125 & 0.2625 & & \\
\hline 1.809 & 0.025 & 0.2875 & & \\
\hline 2.122144 & 0.0125 & 0.3 & 0.30 & P30 \\
\hline 2.4 & 0.0125 & 0.3125 & & \\
\hline 2.832967 & 0.0125 & 0.325 & & \\
\hline 2.94971 & 0.025 & 0.35 & & \\
\hline 3.047 & 0.025 & 0.375 & & \\
\hline 3.649 & 0.025 & 0.4 & 0.40 & P40 \\
\hline 3.947318 & 0.025 & 0.425 & & \\
\hline 4.1 & 0.025 & 0.45 & & \\
\hline 5.019655 & 0.025 & 0.475 & & \\
\hline 5.461 & 0.025 & 0.5 & 0.50 & $\mathrm{P} 50$ \\
\hline 5.5 & 0.025 & 0.525 & & \\
\hline 5.724 & 0.025 & 0.55 & & \\
\hline 6.145595 & 0.025 & 0.575 & & \\
\hline 6.60732 & 0.025 & 0.6 & 0.60 & P60 \\
\hline 6.784 & 0.025 & 0.625 & & \\
\hline 6.8 & 0.0125 & 0.6375 & & \\
\hline 7.097 & 0.025 & 0.6625 & & \\
\hline 7.752408 & 0.0125 & 0.675 & & \\
\hline 7.771345 & 0.0125 & 0.6875 & & \\
\hline 7.842672 & & & 0.70 & P70 \\
\hline 7.914 & 0.025 & 0.7125 & & \\
\hline 7.937577 & 0.025 & 0.7375 & & \\
\hline 8.469 & 0.0125 & 0.75 & 0.75 & P75 \\
\hline 8.532556 & 0.0125 & 0.7625 & & \\
\hline 8.7 & 0.0125 & 0.775 & & \\
\hline 9.196 & 0.0125 & 0.7875 & & \\
\hline 9.902108 & 0.0125 & 0.8 & 0.80 & P80 \\
\hline 10.15011 & 0.025 & 0.825 & & \\
\hline 10.46 & 0.025 & 0.85 & & \\
\hline 11.22 & 0.025 & 0.875 & & \\
\hline 11.6 & 0.0125 & 0.8875 & & \\
\hline 12.41 & 0.0125 & 0.9 & 0.90 & P90 \\
\hline 12.82757 & 0.025 & 0.925 & & \\
\hline 13.31 & 0.0125 & 0.9375 & & \\
\hline 14.6 & 0.0125 & 0.95 & 0.95 & P95 \\
\hline
\end{tabular}


Table F.2. (contd)

\begin{tabular}{|c|c|c|c|c|}
\hline Size & Probability & Cumulative Probability & Percentile & Percentile \\
\hline 16.1 & 0.01 & 0.96 & & \\
\hline 17.23065 & 0.01 & 0.97 & & \\
\hline 18.95061 & 0.01 & 0.98 & & \\
\hline 19.9 & 0.01 & 0.99 & 0.99 & P99 \\
\hline
\end{tabular}

In Table F.2, particle sizes shown in red were obtained by linear interpolation. The Probability column shows the probability associated with each of the listed sizes. The Cumulative Probability column shows the cumulative probability associated with each of the listed sizes, obtained by summing on the associated probabilities, after ordering the sizes. The final two columns show the cumulative percentiles and corresponding percentiles of interest. Thus, taking just the specified percentiles of interest, the PSD summary for this waste tank includes the following estimates of "typical” particle sizes based on use of the combined distribution. The PSD summary is provided in Table F.3.

Table F.3. Typical Particle Size Estimates from Combined Distribution, AY-101 Example (sizes are in microns)

\begin{tabular}{|c|c|}
\hline Size & Percentile \\
\hline 0.264737 & 0.01 \\
\hline 0.4948 & 0.05 \\
\hline 0.766912 & 0.10 \\
\hline 1.275715 & 0.20 \\
\hline 1.704084 & 0.25 \\
\hline 2.122144 & 0.30 \\
\hline 3.649 & 0.40 \\
\hline 5.461 & 0.50 \\
\hline 6.60732 & 0.60 \\
\hline 7.842672 & 0.70 \\
\hline 8.469 & 0.75 \\
\hline 9.902108 & 0.80 \\
\hline 12.41 & 0.90 \\
\hline 14.6 & 0.95 \\
\hline 19.9 & 0.99 \\
\hline
\end{tabular}

Table F.4 provides an example that illustrates how the working data matrix for the sonicated sludge data for waste tank AY-101 was used to obtain median sizes at each percentile of interest. These median values were also reported in the PSD summary for AY-101 to estimate "typical" particle sizes for that waste tank.

Table F.4. Working Data Matrix and Percentile Medians for AY-101 Samples

(values are particle sizes in microns)

\begin{tabular}{|c|c|c|c|c|c|c|c|c|c|c|c|c|c|c|c|}
\hline Per & 01 & $\overline{05}$ & 10 & 20 & 0.25 & 0.30 & 0.4 & 50 & 0.60 & 0.70 & 0.75 & 0.80 & 0.90 & 0.95 & 0.99 \\
\hline $\mathrm{Sa}$ & 0 & & & & & & & & & & & & & & \\
\hline & & & & & & & & & & & & & & & \\
\hline & & & & & & & & & & & & & & & \\
\hline & & & & & & & & & & & 2 & & & & 231 \\
\hline (I) & 231 & .453 & 0.649 & 1.162 & 1.504 & 1.814 & 2.998 & 4.781 & 6.166 & 7.506 & 8.142 & 8.833 & 10.840 & 12.860 & 18.091 \\
\hline
\end{tabular}


Entries in the above table have been rounded to 3 decimal places. The PSD summary for this waste tank includes the estimates of "typical” particle sizes (shown to 6 decimal places) based on medians over the sample data.

Table F.5. Typical Particle Size Estimates from Percentile Medians, AY-101 Example (sizes are in microns)

\begin{tabular}{|c|c|}
\hline Size & Percentile \\
\hline 0.236645 & 0.01 \\
\hline 0.453018 & 0.05 \\
\hline 0.648912 & 0.10 \\
\hline 1.161658 & 0.20 \\
\hline 1.503557 & 0.25 \\
\hline 1.813572 & 0.30 \\
\hline 2.998355 & 0.40 \\
\hline 4.7805 & 0.50 \\
\hline 6.165660 & 0.60 \\
\hline 7.5055 & 0.70 \\
\hline 8.142482 & 0.75 \\
\hline 8.8325 & 0.80 \\
\hline 10.84 & 0.90 \\
\hline 12.86 & 0.95 \\
\hline 18.090629 & 0.99 \\
\hline
\end{tabular}

Note that the estimates of "typical" particle sizes obtained using the combined distribution approach are similar to those obtained by taking medians over the sample data.

The AY-101 example is continued to illustrate the two methods used to obtain "typical" P100 estimates. Table F.6 shows the 8 highest percentiles represented by the summary version of the combined distribution along with the transformed values for the corresponding cumulative percentiles. The least-squares regression would be conducted using the sizes as the response variable $Y$ and the transformed percentiles as the predictor variable $X$.

Table F.6. Least-Squares Regression Inputs to Estimate Typical P100 Value for AY-101 Using Gumbel Distribution (sizes are in microns)

\begin{tabular}{|c|c|c|}
\hline$Y=$ Size & Percentile & $\boldsymbol{X}=-\ln (-\ln ($ Prop.) $)$ \\
\hline 5.461 & 0.50 & 0.36651292 \\
\hline 6.60732 & 0.60 & 0.67172699 \\
\hline 7.842672 & 0.70 & 1.03093043 \\
\hline 8.469 & 0.75 & 1.2458993 \\
\hline 9.902108 & 0.80 & 1.49993999 \\
\hline 12.41 & 0.90 & 2.25036733 \\
\hline 14.6 & 0.95 & 2.97019525 \\
\hline 19.9 & 0.99 & 4.60014923 \\
\hline
\end{tabular}

The least-squares regression for this example yields estimated slope and intercept values of 3.41833467 and 4.39528823, respectively. Approximating a cumulative percentile of 1.0 with 0.999 , the estimated slope and intercept values are then used to calculate an estimated P100 size as follows.

$$
\hat{P} 100=4.39528823+3.41833467\{-\ln [-\ln (0.999)]\}=28.00659770
$$


Thus, the estimated "typical" particle size for P100 determined using the combined distribution and reported in the PSD summary for sonicated sludge samples from AY-101 was 28.00659770 (rounded to 8 decimal places).

To illustrate the use of medians to determine a similar estimate for this AY-101 data, the Gumbel distribution and least-squares regression must be used to determine P100 estimates for each of the four available samples from AY-101. The process applied to the first sample is illustrated here. For the first sample, the regression would be conducted using the 8 highest percentiles from the analyzed sample data. These values are given in Table F.7. Again, the sizes serve as the response variable $Y$, and the transformed percentiles serve as the predictor variable $X$.

Table F.7. Least-Squares Regression Inputs to Estimate P100 for Sample 1 from AY-101 Using Gumbel Distribution (sizes are in microns)

\begin{tabular}{|c|c|c|}
\hline $\boldsymbol{Y}=$ Size & Percentile & $\boldsymbol{X}=-\ln (-\ln$ (Prop.)) \\
\hline 5.5 & 0.50 & 0.36651292 \\
\hline 6.60731961 & 0.60 & 0.67172699 \\
\hline 7.93757679 & 0.70 & 1.03093043 \\
\hline 8.7 & 0.75 & 1.2458993 \\
\hline 9.90210788 & 0.80 & 1.49993999 \\
\hline 12.82757182 & 0.90 & 2.25036733 \\
\hline 14.6 & 0.95 & 2.97019525 \\
\hline 19.9 & 0.99 & 4.60014923 \\
\hline
\end{tabular}

For this sample, least-squares regression yields estimated slope and intercept values of 3.41163863 and 4.50534793, respectively. Again, approximating a cumulative percentile of 1.0 with 0.999 , the estimated slope and intercept values are used to calculate an estimated P100 size.

$$
\hat{P} 100=4.50534793+3.41163863\{-\ln [-\ln (0.999)]\}=28.07040613
$$

The same process is followed to estimate P100 for the other three samples from AY-101. Table F.8 summarizes the P100 estimates for the four samples in this example.

Table F.8. Estimates of P100 (in microns) for Four Samples from AY-101

\begin{tabular}{|c|c|}
\hline Sample & P100 Estimate \\
\hline 1 & 28.07040613 \\
\hline 2 & 22.84278816 \\
\hline 3 & 25.80099724 \\
\hline 4 & 24.40086705 \\
\hline
\end{tabular}

The median of these four P100 estimates is 25.10093214 (rounded to 8 decimal places). This was the estimated "typical” particle size for P100 determined using medians and reported in the PSD summary for sonicated sludge samples from AY-101.

Sample data for sonicated sludge samples from AY-101 is again used to illustrate how minimum and maximum values are used as lower and upper limits for the non-P100 percentiles of interest. The working data matrix is again given in Table F.9 (rounded to 3 decimal places) along with the minimum and maximum values for each of the percentiles. These minimum and maximum values are reported in the PSD summary for sonicated sludge samples from AY-101. 
Table F.9. Working Data Matrix with Minimum and Maximum Percentile Values for AY-101 (values are particle sizes in microns)

\begin{tabular}{|c|c|c|c|c|c|c|c|c|c|c|c|c|c|c|c|}
\hline ntile & 01 & 05 & .10 & .20 & 0.25 & 0.30 & 0.40 & 0.50 & 0.60 & 0.70 & 0.75 & $\begin{array}{l}0.80 \\
\end{array}$ & 0.90 & 0.95 & 0.99 \\
\hline Sample 1 & 300 & 10 & 850 & 704 & 00 & 833 & 3947 & 500 & 607 & 070 & 8.700 & & 838 & 0 & \\
\hline & & & & & & & & & & & & & & & \\
\hline & & & & & & & & & & & & & & & \\
\hline & & 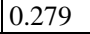 & & & & & & 3.6 & & 7.0 & & & & & \\
\hline & & 279 & & 07 & & & & 3.6 & 5.020 & 6 & 6.800 & 7. & & & \\
\hline $\mathrm{Im}$ & 300 & 0.610 & 0.859 & 1.704 & 2.400 & 2.833 & 3.947 & 5.500 & 6.784 & 7.938 & 8.700 & 9.902 & 12.828 & 14.600 & 19.900 \\
\hline
\end{tabular}

The lower and upper limit estimates on P100 were determined by taking the minimum and maximum of the analyzed P100 values, respectively. For the example data from AY-101, the P100 estimates, listed to 8 decimal places, for the four samples (all non-M12 samples) are 28.07040613, 22.84278816, 25.80099724, and 24.40086705. Thus, the lower and upper limits on P100 listed in the PSD summary for this example were 22.84278816 and 28.07040613, respectively. 
Appendix G

Particle Size Distribution Measurement Uncertainty - Additional Discussion 



\section{Appendix G: Particle Size Distribution Measurement Uncertainty - Additional Discussion}

\section{G.1 Particle Size Measurement Methodology}

One of the key measurements in the physical behavior of tank wastes is particle size. An understanding of the size of the solid particles in a tank waste sample is crucial in determining sedimentation rates, the ease with which the solids can be filtered, the flow behavior of the solids when pumped through a pipe, and the force required to suspend solids and keep the solids suspended in a pipe or tank. Solid particles in tank waste slurries and sludges are generally polydisperse with irregular shapes; therefore, a mean particle diameter does not provide sufficient information to design systems for handling slurries and sludges of Hanford tank waste. Data on the distribution of particle sizes is necessary to characterize the solids and their resulting slurries and sludges.

Multiple methods have been developed to determine the size of particles in polydisperse systems. These methods include microscopy, wet and dry sieving, sedimentation, electrical pulse counting, hydrodynamic, electroacoustics, and light scattering. Multiple methods have been used in characterizing the particle size of tank wastes with the primary methods being microscopy, sieving, sedimentation, and light scattering. Different sizing methods can be expected to give different results because each method is measuring a different aspect of the particle size distribution (PSD). Since each of these methods interacts with the particle in different ways, comparison of the results obtained from each of these methods provides insight into the deviation of the particles in the sample from monodisperse spherical particles.

Microscopy methods, including optical microscopy, transmission electron microscopy (TEM), scanning electron microscopy (SEM), and atomic force microscopy, have been used to determine the size and shape of individual particles in a tank waste sample. These methods create an image of a small sample of the particles, and the image can be analyzed to determine the size and shape of the particles. Microscopy is generally the method used to determine the shape of the particles in tank wastes, but sampling errors can be very large when using microscopy methods to determine the PSD. Direct observation of PSD by microscopy has the limitation that the sample is generally mounted in an environment different than the slurry or sludge where the sample is taken (often the sample dried), and only a very small fraction of the sample can be analyzed. Changing the solution chemistry around the particle may significantly alter the aggregation of the particles, thus modifying the size and shape of the measured particles.

Sieving a sample provides a simple, inexpensive, and straightforward measurement of the PSD of the

sample. For slurries and sludges, dry-sieving the sample will likely modify the agglomeration behavior of the sample and include dissolved solids that remained with the UDS after drying the sample; therefore, wet-sieving is required for obtaining the PSD of tank waste samples. The liquid used in wet-sieving needs to be carefully selected to maintain the proper solvation environment for the particles. The particles in each sieve cut must also be carefully measured to get an accurate measurement of the mass of the UDS, not including dissolved solids or interstitial liquid. A proper sieving technique will provide the smallest radius of the particles, but this requires substantial effort to avoid clumping of materials, blinding 
of the sieves, and suspending the material sufficient that the smallest diameter of the particle can align with the sieve mesh. Sieve methods are also limited in the number of discrete size ranges that are measured. This method requires a substantial amount of sample, which requires performing any PSD measurement of tank wastes in a hot cell. The range of sizes that can be measured by sieving is limited to coarser particles. Standard sieves have openings that range from $37 \mu \mathrm{m}$ to $8 \mathrm{~mm}$, which can be expanded down to about $2 \mu \mathrm{m}$ with micro-mesh sieves.

Sedimentation methods have been used for many years to determine the PSD in a slurry. Gravity settling and centrifugation have both been used in these sedimentation methods. Stokes Law is the basis for determining the PSD of the particles in a sample by measuring the concentration of the particles at different heights in a sample that is settling. In this method, spherical particles are assumed; therefore, the measured size is the equivalent settling radius, which is the radius of a sphere of the same density, which settles at the same rate. The settling rate may be affected by thermal diffusive motion of the particles and by particle-particle interactions; therefore, this method is sensitive to differences in temperatures and solids concentration in the sample. Small, lower density particles also settle at slow rates requiring long measurement times for samples containing small particles at low densities. Gravity settling measurements are generally only applicable to particles above $1 \mu \mathrm{m}$ in size. Smaller sizes can be measured using centrifugal force methods (see Figure G.1).

Electrical pulse counting is capable of counting the number of particles in a sample and determining the volume of each particle. The most common system of electrical pulse counting is the Coulter counter. This system works by drawing a suspension through a small hole with an electrode on each side of the hole. Particles passing through the hole disrupt the flow of current between the electrodes, resulting in pulses in the resistance. Each disruption is a particle, and the height of the pulse is proportional to the volume of the particle. Dilute solutions must be used in this system to avoid multiple particles passing through the hole simultaneously. These systems are capable of measuring particles with diameters between $1 \mu \mathrm{m}$ and $1 \mathrm{~mm}$.

Hydrodynamic methods measure the PSD by forcing the sample through a long packed column or a fine capillary tube and collecting fractions of the sample exiting the column as a function of time. Particles of different sizes travel through the column or capillary at different speeds; therefore, particles are fractionated by size as they move through the column. Increased resolution of the PSD can be achieved by subjecting the sample to a steady field (magnetic, thermal, electrical, or centrifugal) at right angles to the flow. Longer times are required to make these measurements, but the particles of varying sizes are physically separated and can be further characterized by other physical or chemical means.

Light-scattering methods are currently the dominant methods for characterizing the PSD of solid particles suspended in air or liquid. Light-scattering methods for determining PSD include laser diffraction and dynamic light scattering. Laser diffraction systems determine particle size by analyzing the scattering pattern (intensity of the scattered light as a function of angle from the incident light) created when light is scattered from the particle. This method is described in much more detail in the later sections of this report. Dynamic light scattering expands the size range of particles that can be accurately measured by laser diffraction. In dynamic light scattering, also called quasi-elastic scattering (QELS) or photon correlation spectroscopy (PCS), the time-dependent fluctuations in the intensity of scattered light that occurs because the particles are undergoing random, Brownian motion is measured; therefore, the diameter of smaller particles can be measured. 
Ultrasound can be used in place of light for collecting the PSD of particles suspended in an appropriate media. Dispersed particles absorb and scatter ultrasound in a manner similar to light. With acoustic measurements, the attenuation of the transmitted energy from the incident energy is measured as a function of frequency. Dilute samples are not necessary for these measurements; therefore, samples with high solids content and high ionic strengths can be measured directly using acoustic methods.

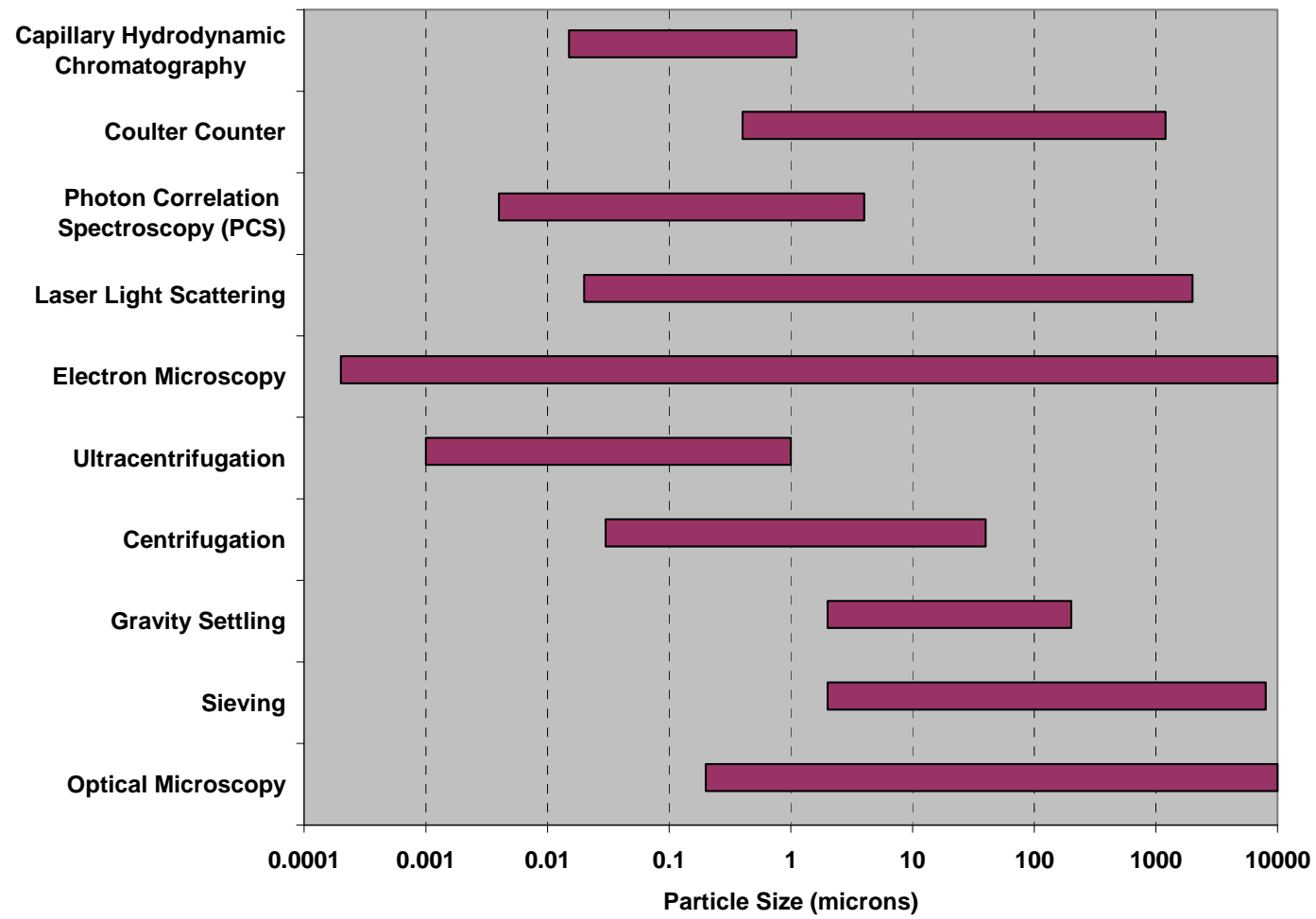

Figure G.1. Approximate Measuring Range of PSD Methods

At Hanford, the majority of PSD measurements have been made by light scattering. Previously, PSDs measured using two different light scattering systems were compared to each other and with sieve measurements, settling tests, and microscopic observations (Jewett 2003 and Bechtold et al. 2002). These tests showed that the light-scattering methods respond reliably to suspensions that have a wide distribution of dense particles and that the results are sufficiently reliable and accurate. However, it is noted in Bechtold et al. (2002) that, in comparison to sieving analysis of particle size, the light-scattering particle size analyzer was poor at finding the largest size classes above $500 \mu \mathrm{m}$ in size.

\section{G.2 Laser Diffraction Particle Size Distribution System}

The PSD of the tank waste simulants was determined by laser light-scattering techniques.

Measurements were made using a Malvern Mastersizer PSD system. The system consists of a dispersion unit, an optical bench, and a computer for control and data analysis. A schematic of the system is shown in Figure G.2. 


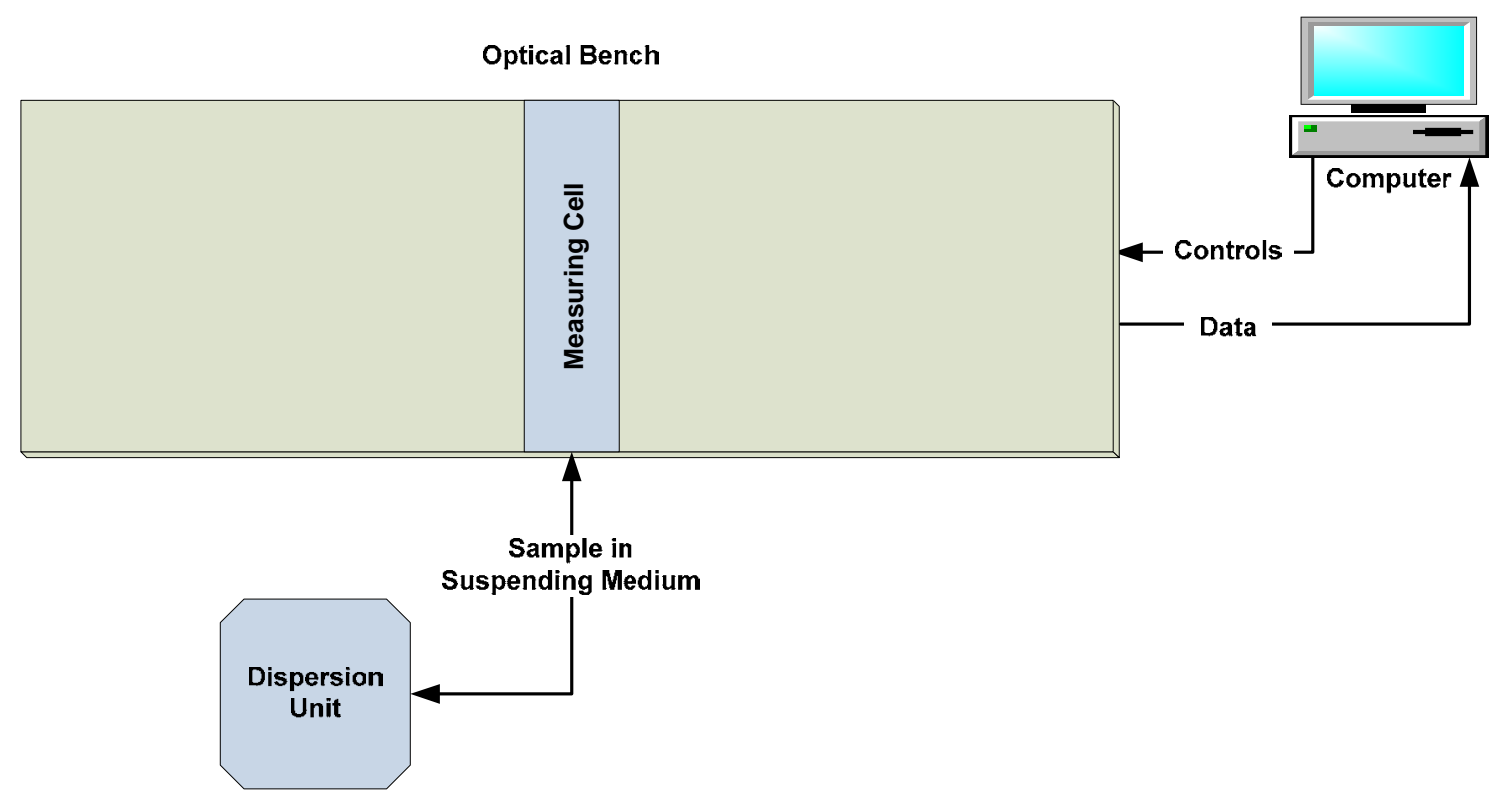

Figure G.2. Schematic of the Mastersizer PSD System

The dispersion unit presents the sample to the measurement cell where a focused laser beam is scattered by the particles. The laser, optics, and detectors for measuring light intensity at specified angles are located in the optical bench. In the Mastersizer PSD system, a dual wavelength detection system is employed to obtain better resolution of sub-micron particles. A short wavelength light source (blue light from a solid state light source at a wavelength of $466 \mathrm{~nm}$ ) is used in conjunction with a helium-neon laser (red light at a wavelength of $632.8 \mathrm{~nm}$ ) to accurately obtain a wide range of particle sizes (Malvern 2007). The intensity and angle of the scattered light from the blue light source is measured by backscatter as well as the wide angle forward scatter detectors. Forward scattering, side scattering, and back scattering detectors are used to measure the angle and intensity of the scattered light from the helium-neon laser. A schematic of the typical optical bench is shown in Figure G.3.

The size range accessible during the measurement is directly related to the angular range of the scattering measurement. Detectors for most modern systems are set over the scattering range from 0.02 to 135 degrees. The exact scattering range of the Malvern Mastersizer is not known, but the system does have backscattering detectors as well as large- and wide-angle detectors. The system consists of 61 detectors that have been positioned to optimize the resolution across the entire range of available particle sizes $(0.02$ to $2000 \mu \mathrm{m})$. A logarithmic detector sequence, where the detectors are grouped closely together at small angles and more widely spaced at wide angles, yields the optimum sensitivity. The detector sequence is designed such that equal volumes of particles of different sizes produce a similar measured signal. This requires the size of the detectors to be increased as the measured scattering angle increases (Malvern 2007). 


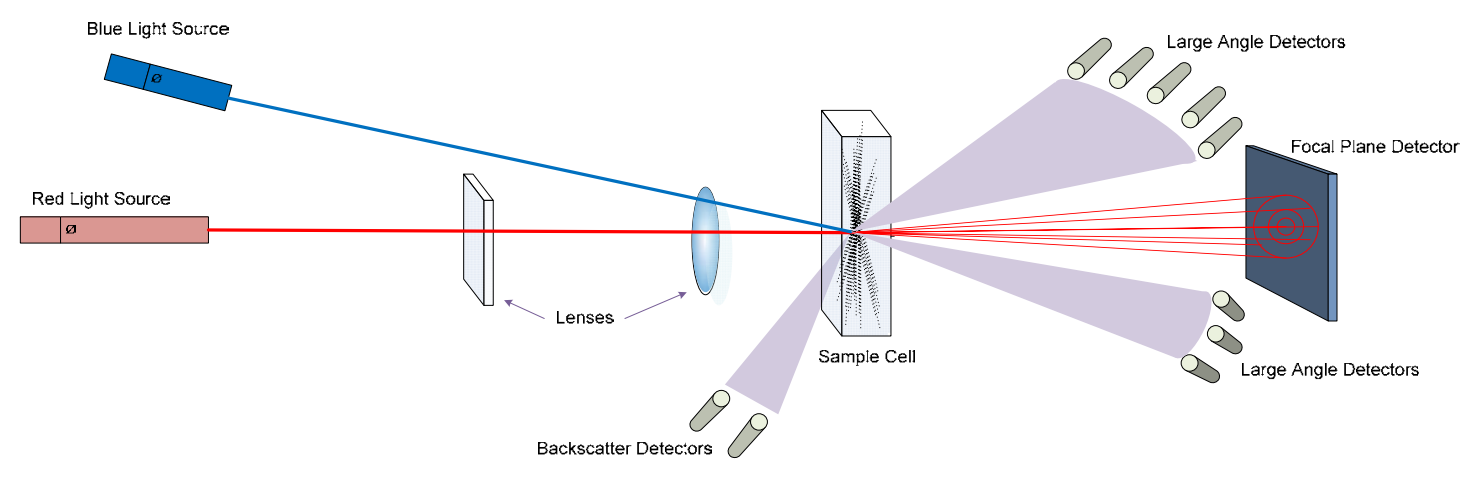

Figure G.3. Typical Optical Bench Schematic for a Light Scattering (Laser Diffraction) PSD System

In light-scattering techniques, PSDs are calculated by comparing a sample's scattering pattern with an appropriate optical model using a mathematical inversion process. Traditionally, two different models are used: the Fraunhofer Approximation and Mie Theory. The Mastersizer system uses the Mie Theory model to calculate the PSD from the scattering pattern. More details about Mie Theory will be provided in the next section.

Several particle dispersion units can be used with this light-scattering system, and the appropriate dispersion unit must be selected to perform accurate PSD measurements. This determination is based upon the sample size, suspending medium, and size and density of the solid particles. The dispersion unit should confirm that the sample passes through the laser beam as a homogeneous stream of particles in a known, reproducible state of dispersion at a concentration applicable to the light scattering technique. The suspension must be dilute, such that the scattered light is measured before it is re-scattered by other particles; therefore, the dispersion unit is also used to dilute the sample with the suspending medium. The suspension is sonicated and circulated in the dispersion unit to obtain a homogeneous stream of representative particles.

\section{G.3 Light Scattering (Laser Diffraction) Particle Size Distribution Theory}

Light scattering is based upon the principle that particles passing through coherent light will scatter that light at an angle that depends on the size of the particles. The observed scattering intensity is also dependent on particle size and diminishes, to a good approximation, in relation to the particle's cross-sectional area. Large particles, therefore, scatter light at narrow angles with high intensity, whereas small particles scatter at wider angles but with low intensity.

To determine the PSD of the sample measured by light scattering, the scattering pattern must be deconvoluted into the scattering pattern for each individual particle followed by a mathematical inversion of that individual pattern. Mie theory is used by Malvern to manipulate the data to obtain a PSD.

Mie theory provides a rigorous solution to calculate PSDs from light scattering data and is based on Maxwell's electromagnetic field equations. A detailed description of Maxwell's electromagnetic field equations and the derivation of the Mie theory can be found in the NIST-recommended practice guide for 
particle size analysis (Jillavenkatesa 2001). Mie theory can be applied to a suspension of particles, small or large, transparent or opaque, provided the following assumptions are valid:

- The particles being measured are spherical.

- The suspension is dilute, such that light is scattered by only one particle.

- The particles are homogeneous.

Some additional modeling and some measurement procedures provide adjustments for these deviations. These adjustments provide more accurate results, but error associated with these theory adjustments is also observed.

Only a small number of the particles observed in tank waste and very few used in preparing simulants for tank waste are spherical; therefore, the particle size of irregular particles is expressed in terms of a spherical equivalent diameter. Equivalent spherical diameters are the diameters of spheres that would give the same behavior as that recorded from the true sample by the light scattering method. In the case of light scattering, the diameter of the sphere that yields an equivalent light-scattering pattern to the particle being measured corresponds approximately to the sphere of equivalent average cross-sectional area. A more detailed discussion of equivalent spherical diameters is provided in an article in the Proceedings of the Royal Society of London (Jennings 1988).

Malvern incorporates mathematical models to calculate the spherical equivalent diameter based upon the irregularity of the particle as defined by the circularity, convexity, and elongation of the particle. An estimate of the degree of irregularity of the particles must be input into the model to calculate accurate PSDs of non-spherical particles. Most modern light scattering systems provide the user with the ability to select an irregular-particle analysis mode; accurate results can be verified for products such as crystallites (Rawle). Even in mixtures of different shapes, selecting an analysis mode that accounts for irregular shapes is more accurate that analyzing the data as if the particles were spherical.

Figure G.4 provides one example of the differences in light-scattering intensity as a function of angle observed for spherical and irregular particles having similar, volume-based, size distributions. The differences observed, particularly at large angles (high detector numbers), are compensated for within the analysis software to yield more accurate results.

PSD measurements in light scattering systems are performed on very dilute samples to minimize errors in the analysis associated with scattering of light from multiple particles, light scattered from one particle to another. The system measures the obscuration of the sample, and valid measurements are made within the specified obscuration range (Malvern 2007).

Solid particles are not homogeneous in Hanford tank waste samples, and errors are associated with the assumption that these particles are homogeneous. Average or primary particle optical properties are included in the analysis to reduce errors associated with inhomogeneity of the solid particles. 


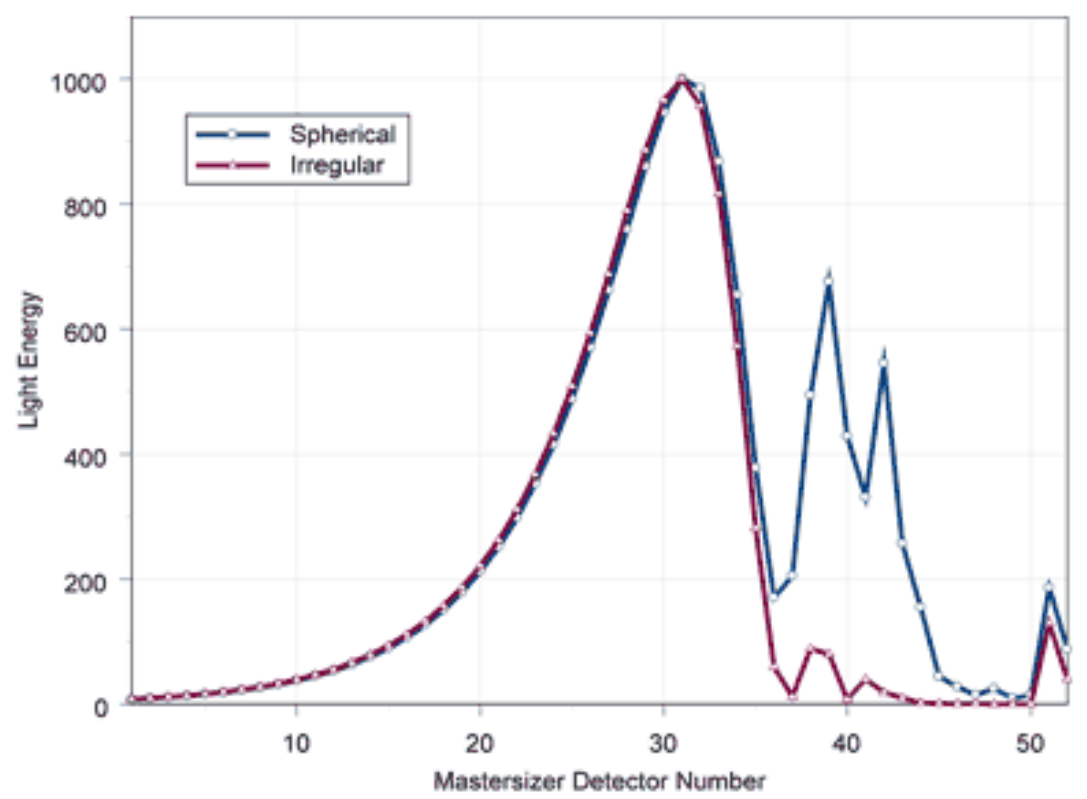

Figure G.4. Comparison of the PSD of Irregular and Spherical Particles (higher detector numbers correspond to higher angle)

Mie Theory predicts the primary scattering response observed from the surface of the particle, with the intensity predicted by the refractive index difference between the particle and the dispersion medium. It also predicts how the particle's absorption and net effect of reflection from the exterior and internal surfaces of the particle affects the secondary scattering signal caused by light refraction within the particle. The refractive index of the particle and the suspending medium as well as the particle absorption index must be input into the model; therefore, the accuracy of the model is dependent upon the accuracy of these optical properties. The particle absorption index is especially important for particles below $50 \mu \mathrm{m}$ in diameter and is extremely important when the particle is transparent, as stated in the international standard for laser diffraction measurements (ISO13320-1 1999). Figure G.5, provides a schematic description of the impact of these variables.

Refractive Index of Dispersing Medium $\neq$ Refractive Index of the Particle

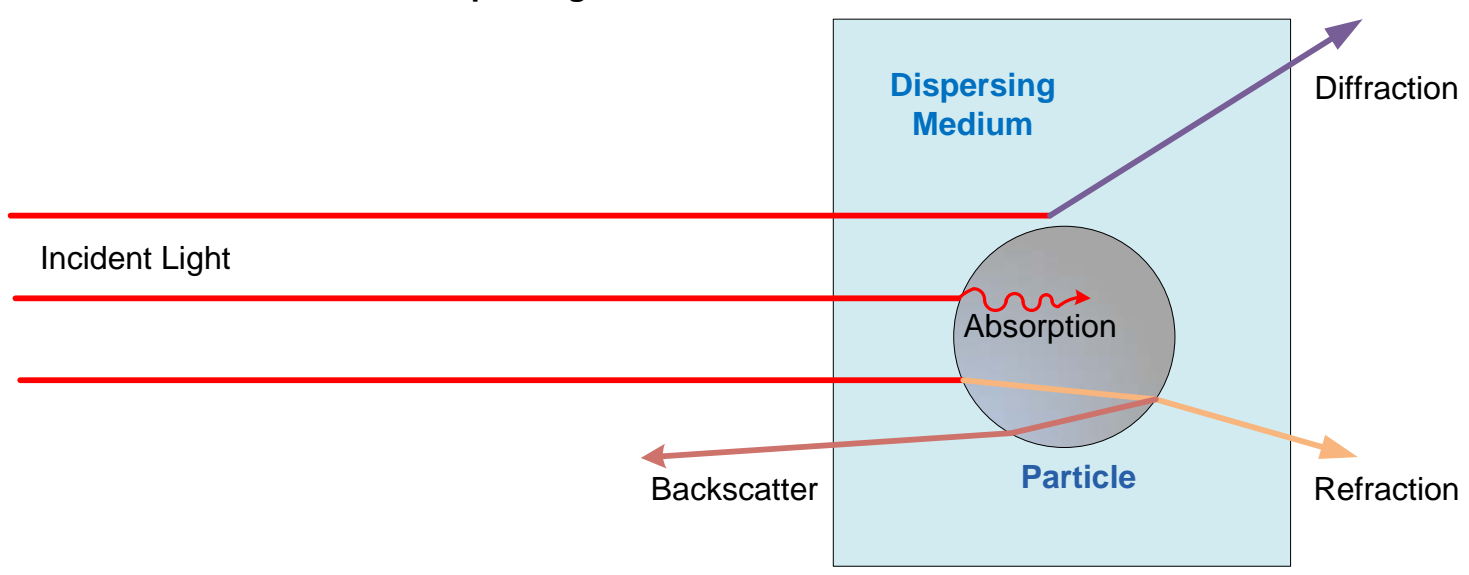

Figure G.5. Schematic of the Optical Properties Required in Mie Theory 
The refractive index of the suspending media and the solid particle is a critical input when determining the PSD. This refractive index has both a real component and an imaginary component. In general, the real component of the refractive index is well known, but it is often difficult to obtain the value of the imaginary component. The imaginary component is an indication of the amount of absorption of the light by the particle. Figure 6.2 in the NIST-recommended practice guide for particle size characterization shows the impact of the imaginary component on the PSD. This figure is copied in this section as Figure G.6. The PSD of particles with a real component refractive index of 1.43 and 1.44 are plotted as a function of the imaginary component of the refractive index. The PSD is represented as the $\mathrm{d} 90$ ( $90 \%$ of the particles are smaller than the reported value), $\mathrm{d} 50$ (the mean particle diameter), and d10 (10\% of the particles are smaller than the reported value). A logarithmic scale is used for the imaginary component of the refractive index where an imaginary component equal to zero represents a non-absorbing material. At an imaginary component of 1, the PSD is much tighter than at an imaginary component of 0.01 . The mean particle diameter is also slightly higher at an imaginary refractive index component of 1 compared to 0.01 . The error in particle size from a deviation in the imaginary component of the refractive index is much greater at d90 and d10 than is observed at d50.

Most PSD measurement systems currently do not include the ability to use several refractive indices in the analysis of the scattering data; therefore, a single refractive index for the particles must be selected. Selecting an appropriate refractive index for systems containing several materials is difficult and introduces error in the PSD measurement. 


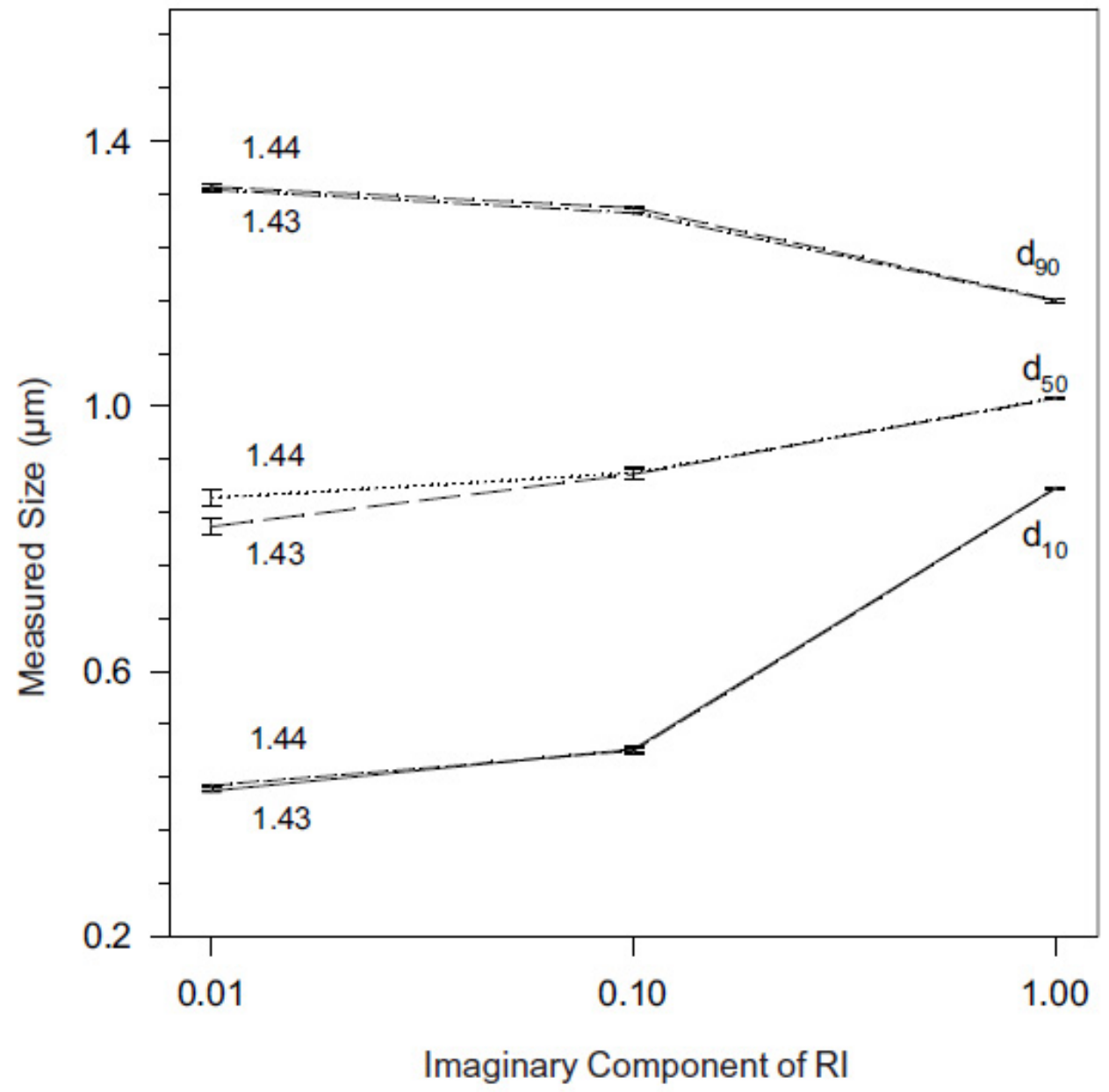

Figure G.6. Impact of the Imaginary Component of Refractive Index on PSD

Light scattering methods provide a rapid method for determining the PSD of particles in the submicron to millimeter size range, but error in these measurements can result from poor sampling, lack of detailed information on optical properties of the particles, deviations from spherical configurations of the particles, concentrated suspensions, and the presence of agglomerates. Variations in the calculation algorithm, input parameters, and sample preparation may cause significant differences in the PSD of the same sample. Care must be exercised in measuring the PSD, analyzing the data, and reporting the data to verify that accurate information on PSD is presented.

The impact of the various issues identified above on measurement uncertainty is discussed in more detail in Section G.4, which provides an overview of the measurement and analysis parameters that users of typical particle size analyzer systems must select. G.4 expands upon the discussion of uncertainty in PSD with respect to the model, the refractive index, and the dispersion concentration already introduced in G.2 and G.3, and describes additional measurement parameters, such as frequency of measurement and duration of analysis, which impact the PSD uncertainty. 


\section{G.4 Measurement and Analysis Approach}

Measuring a PSD often requires selecting the dispersing phase, and using surfactants, numerous operational parameters (such as circulator flow rate), and numerous analysis parameters, such as those required by Mie theory. The following sections provide a summary of the impact of run parameters, dispersing phase chemistry, and analysis parameters. The discussion is based on operational experience and observations when measuring the PSD of a wet dispersion with the Malvern Mastersizer 2000 (various dispersion units were used, such as the Hydro G, with the Mastersizer 2000 optical bench). An effort has been made to make the discussion as general as possible so that the discussion can be applied to wet dispersion systems manufactured by other companies.

\section{G4.1 Impact of Run Parameters on Measurement Uncertainty}

PSDs measured by light scattering can depend on the conditions under which the dispersion or powder is presented to the laser beam and the duration and frequency of the interactions between the particles in the dispersion or powder and the laser beam. Modern PSD analyzers typically allow control of operational parameters that impact the state of dispersion and the interaction time of particles with the laser beam, which impacts measurement statistics. These operational parameters define how a given sample dispersion (or powder) is "run," and once executed, these run parameters cannot be changed without re-running the sample. Typical run parameters include, but are not limited to:

- recirculation rate between the dispersion reservoir and observation/flow cell (if the two exist as separate entities)

- stirrer/agitator rate in either the dispersion reservoir and/or observation/flow cell

- dispersion sonication (either before or during measurement)

- frequency of scattering signal observation (i.e., the data collection rate)

- integration time (i.e., the total duration of observation or the number of observations that make up a single PSD measurement)

- choice of dispersing phase and use of surfactants/dispersants.

It should be noted that the limitations outlined above apply only to wet dispersion systems. Size analyzers that work with dry powders are available and have their own unique run parameters that affect measurement uncertainty. In the following sections, the impact of each run parameter listed above on the measured PSD data is discussed briefly.

\section{G4.1.1 Impact of Flow Conditions}

When dispersions are prepared using liquid suspending phases, the particle size analyzer typically employs either a static or flow configuration to present the dispersion to the laser beam. In static configurations, the dispersion is placed into a cuvette (or similar container), which is inserted into the laser beam. When no mechanism for maintaining particle dispersion is present, the stability of the dispersion in static cells is governed by how quickly the particles settle under gravity or clarify through aggregation. Some static cells, such as the Horiba LA-910, may have in-cell agitators (such as micro magnetic stir bars) that prevent the dispersion from settling during the measurement. In flow cell 
configurations, the dispersion is continuously circulated between a dispersion reservoir and an observation cell (through which the laser beam interacts with the particles). Depending on the rate selected, recirculation of the dispersion may provide sufficient energy to either partially or completely suspend particles in the dispersion. In addition, dispersion reservoirs are often equipped with mixing impellers to maintain suspension of the dispersion outside of the recirculation path. When considered overall, dispersion mixing in flow configurations may be affected by both the recirculation rate and the rate of agitation in the dispersion reservoir.

Uncertainty in PSD measurements with respect to flow and mixing conditions derives from the inability to fully suspend all or a fraction of the particles that comprise the dispersion and by shear-induced disruption or agglomeration of the particulate matter. Selected flow conditions may not fully suspend all particles in the dispersion. More energy is required to disperse large particles or particles with high density than is required to disperse small or low density particles. Particle size systems with flow configurations typically list an upper recommended particle size (with the caveat that the actual size limit is density dependent). Example limits for various Malvern Mastersizer 2000 dispersion units are listed in Table G.1.

Table G.1. Example Dispersion Units Used with the Malvern Mastersizer 2000 and Their Operating Size Limit

\begin{tabular}{|c|c|c|}
\hline Dispersion Unit & Dispersion Mechanisms & Upper Size Limit, $\boldsymbol{\mu m}$ \\
\hline $\begin{array}{c}\text { Hydro } \mathrm{G}-800 \mathrm{~mL} \text { cell for use } \\
\text { with non-corrosive dispersions }\end{array}$ & Independent stirrer and circulator \\
\hline $\begin{array}{c}\text { Hydro S }-150 \mathrm{~mL} \text { cell for use } \\
\text { with chemically aggressive } \\
\text { dispersions }\end{array}$ & Independent stirrer and circulator & 600 \\
\hline Hydro $\mu \mathrm{P}$ & & 150 \\
\hline
\end{tabular}

Beyond this upper size limit, the dispersion unit may not be able to reliably suspend particles or introduce them into the observation cell. The consequence is that any particle fraction and/or species that cannot be reliably suspended may not be presented to the laser beam and will not contribute to the integrated diffraction pattern. The uncertainty introduced in the measured PSD (by volume) will be on the order of the relative volume contribution and size range of the "difficult-to-suspend" species/particles.

Flow conditions may also cause disruption of agglomerates. This is generally desirable as the distribution of primary particle sizes in the dispersion is often sought; however, unless the primary size is known by direct imaging techniques, there will be uncertainty as to whether or not the flow condition used in the measurement yields the primary particle size or yields only partial breakdown of agglomerates. In general, higher flow and mixing rates yield increasing disruption of agglomerates. Shear disruption of agglomerates is revealed by a reduction in measured particle size with time (under flowing conditions) and/or increasing flow and/or mixing rates.

Increased flow rates can also yield an increase in the frequency of particle-particle interactions resulting in increased agglomeration instead of the expected disruption of agglomerates. If the particles have a propensity to stick to one another, then increasing flow and/or mixing rates can cause particle aggregation. Aggregation is manifested by an increase in particle size (or the appearance of a peak with a characteristic diameter much larger than that of the sizes associated with the unsheared dispersion) with 
time and/or flow and mixing rates. Unfortunately, increases in particle size with increasing shear rate can also be associated with improved suspension of "difficult-to-suspend" particles. Depending on the size range of particles in the primary dispersion, it may be possible to evaluate shear-induced agglomeration by examining the post-measurement dispersion for evidence of large particles not observed in the primary dispersion (prior to the measurement).

\section{G4.1.2 Impact of Sonication}

Modern particle size analyzers typically come equipped with a liquid dispersion sonicator. Even when the particle size analyzer does not include a sonicator, a separate sonic bath or horn is often used to disperse the particles before introduction to the analyzer. Sonicators are employed to disrupt particle agglomerates, and disperse particles to the primary particle size; however, as with shear-induced disruption of agglomerates, uncertainty with regards to the primary particle size exists without information from direct visual observation of the dispersion. Although the general effect of sonication is to reduce particle size, the energy it deposits into the dispersion may cause particle agglomeration. The latter often manifests itself as an increase in measured particle size or the appearance of a peak diameter at sizes above that characteristic of the unsonicated dispersion.

\section{G4.1.3 Frequency of Observation and Measurement Integration Time}

When particles interact with the analyzer's laser beams, a light scattering pattern is generated and measured by ring detectors that capture the scattered light intensity as a function of detector position. While beam broadening techniques can increase the number of particles interacting with the laser at any given time, the ability of a given measurement to capture a representative sampling of the dispersion's diffraction pattern depends on both the frequency of observation and the number of observations used to generate an "averaged" diffraction pattern. Each observation (or snapshot) of the diffraction pattern should be considered as a point reading of the current light intensity levels at the detectors relative to that of the "clean" background intensities. In addition, each observation only evaluates the diffraction pattern generated by particles that reside in the path of the broadened laser beam. The number of particles captured by each observation is in turn dependent on the observation cell geometry (such as cell thickness or beam length), the concentration of suspended particles that are carried to the observation cell, and the PSD. A PSD generated by light scattering is only considered representative when a sufficient number of observations have been made to yield high confidence that the entire speciation of particle size, shape, and orientation have been observed in the observation cell. For optical microscopy, representative size sampling requires on the order of 10,000 images. A similar number of snapshots are expected to obtain representative sampling of laser diffraction patterns. Representative measurements of PSD by laser diffraction are obtained by integrating (or averaging) individual snapshots over long periods of time. Snapshots are typically taken at high frequency to minimize the time required to achieve the 10,000 snapshots required for representativeness. For example, a typical measurement frequency and duration for Malvern Mastersizer 2000 measurements is $1000 \mathrm{~Hz}$ and 10 seconds, respectively, which yield “single” PSD measurements composed of 10,000 individual scattering pattern snapshots.

As discussed above, a large number of snapshots are taken to reduce uncertainty with respect to the representativeness of the PSD. The larger the number of snapshots taken, the higher the degree of certainty that all particle sizes, shapes, and orientations have been observed. Collection of additional snapshots requires longer measuring times, which can impact the physical and chemical state of the 
dispersion. For example, if the particles exist as agglomerates, longer measuring time can see shear-induced disruption of those agglomerates and a change in the particle size with time. Time dependent processes can include agglomerate disruption and formation, particle dissolution or growth if equilibrium does not exist between particle and suspending phase, and changes in the chemistry of the suspending phase (such as absorption of $\mathrm{CO}_{2}$ from the atmosphere). Thus, to reduce (or better characterize) the uncertainty in the measured PSD with respect to time-dependent processes, measurement times should be optimized so that they occur on time-scales much faster than time-dependent processes or done after process equilibrium is reached. Determination of equilibrium is often difficult without extensive testing of the dispersion.

\section{G4.2 Dispersion Considerations}

There are several considerations that must be made with respect to the state of the dispersion when measuring PSD. These include

- selection of the dispersing phase used to suspend the particles

- use of surfactants or dispersants

- concentration of the particles in the dispersion.

While not controlled through the particle size analyzer, these parameters are still selected and implemented by the user. Selection of the dispersing medium and surfactants can affect the state of particle aggregation in the dispersion. Proper selection of suspending phase and use of surfactants is perhaps the most important means of achieving dispersion of primary particles.

\section{G4.2.1 Impact of Dispersing Phase and Use of Surfactants/Dispersants}

The dispersing phase chemistry can directly impact the state of particle agglomeration. As stated previously, proper selection of the dispersing phase and/or dispersants can yield particles dispersed to their primary size. As with dispersion accomplished by shear-induced disruption and sonication, uncertainty with respect to the true "primary" particle size exists without direct imaging of the particles. One application in which the importance of the dispersing phase chemistry is demonstrated is with Malvern's verification standard for the Hydro $\mu \mathrm{P}$ dispersion unit. This standard is hydrophobic, is not wet well by water, and as such, will not disperse well in water. If water is used as the dispersing phase, then the measured median particle size is frequently higher than listed on the verification standard's certificate. However, if isopropanol is used, then the standard disperses properly and measurement of the standard returns the expected median particle size.

\section{G4.2.2 Dispersion Concentration}

Control of the concentration of particles presented to the laser beam during diffraction experiments is important to optimizing PSD measurements. Concentration of the particles in the dispersion is typically monitored by the instrument software through obscuration of the laser beam. For measurements with the Malvern Mastersizer 2000, optimal obscuration limits are typically 15-20\% for large particles dispersion ( $100 \mu \mathrm{m}$ or above), $10 \%$ for mid-range particle dispersions ( 1 to $100 \mu \mathrm{m}$ ), and less than $5 \%$ for submicron particle dispersions. In general, higher obscurations are needed for large particles to increase the number of particles counted per snapshot observation so that representative sampling is achieved. If 
higher than optimal obscurations are used, the laser beam may encounter multiple scattering centers. In contrast, if less than optimal obscurations are used, then electronic and measurement noise is increased relative to the measurement signal. Both multiple scattering and measurement noise introduce uncertainty in the PSD. Multiple scattering typically yields increased scattering at high angles such that the contribution of fine particles is overestimated. Increased noise-to-signal ratios as a result of low particle concentrations can result in artificial peaks associated with random spikes in the diffraction signal, particularly for detectors at angles where the dispersion does not scatter strongly. In addition to these effects, dispersion concentration can impact the PSD with respect to particle aggregation. Higher particle concentrations yield more frequent particle interactions, which can result in particle agglomeration and an increase in the particle size with time. Agglomeration lends to uncertainty in the measurement with respect to the "true" steady-state PSD.

Source dispersions prepared for particle size analysis are usually prepared outside the particle size analyzer at concentrations much higher than those required for testing. These dispersions are then added drop-wise to the dispersion reservoir (while the dispersion is circulating) until the test obscuration is within the desired range. The system to be characterized may initially be in powder form. This powder is sometimes added directly to dispersant circulating through the size analyzer. This process of dilution can have a number of impacts on the measured size distribution. First, the particles in the concentrated dispersion or powder are likely agglomerated. Dilution and dispersion in the suspending phase typically disrupts these agglomerates; however, the dispersion may not fully disrupt these agglomerates, especially if particle-particle contact has caused sintering of the materials. Secondly, the particles may not be at chemical equilibrium with the suspending phase. Efforts should be made to match the dispersing phase with the dispersant in the source material. While matching is straight-forward for most systems, matching suspending phase chemistry may not be possible for certain complex or chemically difficult (hazardous) materials. For example, PSDs of radioactive tank waste at PNNL often requires that the complex tank waste supernate be matched with non-radioactive simulants that mimic (but do not entirely match) the waste supernate chemistry. Mismatch may result in dynamic changes in interactions between solids and supernate, and particle dissolution may occur upon dilution. Alternate approaches that attempt to avoid the need for a complex suspending phase wash the solids with water to remove any soluble solids before size characterization. Depending on the wash efficiency and the soluble solids dissolution kinetics, dilution of the washed particle solids may result in additional dissolution of soluble material during the particle size measurement. Dissolution of particles can both reduce particle size (if large particle species shrink) or increase the particle size (if small particles are consumed). Regardless of the impact, dissolution introduces uncertainty with respect to the true size distribution of soluble and insoluble solid particles. Dissolution not only impacts the particle size of the dispersion, but can also interfere with the diffraction pattern. Rapid dissolution can create localized increases in the refractive index of the suspending phase which cause diffraction of the laser. If the effect is sufficiently severe, these can be falsely interpreted as large particles by the analyzer.

\section{G 4.3 Impact of Analysis Parameters}

Section G.4.1 discussed run parameters that for a given measurement are fixed and cannot be changed without re-running the sample. Analysis parameters can be altered after the measurement is completed. As the name implies, the analysis parameters determine how averaged diffraction data is interpreted when calculating the PSD of the dispersion. Analysis parameters include (but are not limited to): 
- interpretive model for the diffraction pattern (e.g., single narrow distribution, multiple narrow distribution, and broad general distribution),

- refractive index of both the particle and suspending phase, and

- absorption index of the particle.

\section{G4.3.1 Impact of Interpretive Model}

As discussed in Section G.3, interpretation of the averaged diffraction pattern is accomplished through application of Mie scattering theory. The diffraction pattern is translated to a size distribution by calculating the size distribution of spherical particles that yield approximately the same diffraction pattern as measured. Conversion of the diffraction pattern to size distribution is typically done by the software supplied by the instrument vendor. The Malvern Mastersizer 2000 software provides several models to allow for optimization of calculated distribution depending on the assumed particle morphology. For example, if the distribution is known to be composed of relatively monodisperse particles (with respect to size), then calculation of the distribution may be optimized using a single narrow PSD model. Further optimizations can be made by specifying if the particles are spherical or have an irregular shape. With respect to model selections in the Malvern Mastersizer 2000, the most general model for systems with unknown PSDs is a general purpose broad distribution with irregular particle shape.

A degree of uncertainty in the PSD result is associated with model selection. Direct visual observation of the particles through a microscope may help guide model selection. However, unless the particles are clearly spherical and monodisperse with respect to size or a mixture of monodisperse spherical particles of different sizes, the general purpose broad PSD model should be selected. Based on operational experience with the Malvern Mastersizer 2000 software, application of the broad PSD model for interpretation has the following results:

- When used to interpret single narrow distributions of particles, the resulting PSD is broader than the actual distribution. This is observed in analysis of samples created by sieve cuts. Using the broad size distribution model tends to produce PSD measurements that are broadened far beyond the cut point including beyond the size range caused by irregular particle shape.

- $\quad$ Non-spherical particles are cast in terms of spherical particles.

- PSD is smoothed and contains no slope discontinuities in either differential or cumulative form.

The overall impact of translation by the general purpose model is that the calculated PSDs will generally have a broader size range than the actual distribution. Size discontinuities will be smoothed, such that any size category where particles are actually missing may be interpreted as a non-zero minimum in the differential distribution.

\section{G4.3.2 Impact of Refractive and Absorption Indices}

To translate the diffraction pattern to a PSD, the refractive indices for both particle and suspending phase at the wavelength of light used in the measurement must be input to the Mie theory. The refractive index for common liquid suspending phases is available in reference documentation or peer-reviewed literature or can be determined using a standard bench-top refractometer. Small errors may be observed when using a refractive index reported for a solvent at a different wavelength that is used in the 
measurement. For example the refractive index of water at ambient temperature and pressure measured at wavelengths in the visible light spectrum (404 to $707 \mathrm{~nm}$ ) varies between 1.343 and 1.331 (Lide 1990 \& Schiebener 1990). Likewise for common powders, selection of refractive index is relatively straightforward as refractive indices for common materials are frequently reported in the literature. However, for less common materials, the particle refractive index may not be immediately available but can be assessed by optical microscopy techniques such as Becke line method or by estimating refractive index from materials of similar composition and crystal phase.

Difficulty in selection of particle refractive index results when the dispersion being characterized contains multiple particle species of differing refractive index. Currently, no mixing rule exists that allows calculation of refractive index for multi-component particle dispersions. In addition, the chemical composition and/or morphology and crystal phase of the particles may not be known at the time of analysis. Under these circumstances, the user is forced to assume a single particle refractive index based on a defensible argument such as physical appearance, known process history of the dispersion, and/or analytical data such as chemical speciation. Because the assumed refractive index impacts calculation of PSD from measured diffraction data, any error in the assumed refractive index can be corrected through post-measurement reanalysis of the data. As such, selection of an appropriate refractive index is not crucial to successfully conducting the measurement; however, it is important when presenting or using data for comparative analysis.

Another parameter important in proper calculation of dispersion PSD is the particle absorption index. This index describes the degree of particle transparency: opaque particles have an absorption index of 1 whereas completely transparent particles have an absorption index of 0 . The absorption index determines the importance of internal reflections of the laser beam within the particle on the resulting diffraction pattern. Particles can be semi-transparent, such that the absorption index falls between 0 and 1 . Like refractive index, the absorption index of common materials may be available in the reference documents. For less common materials, estimates of transparency can be made through microscopy examination of individual particles or by analysis of the laser obscuration of a dispersion as a function of particle concentration. According to the Malvern Mastersizer 2000 reference documentation on size analyses, order of magnitude estimates of the particle absorption index (i.e., 0.01, 0.1, or 1) are sufficient for correct presentation of PSD.

The impact of particle and suspending phase refractive indices and particle absorption index can be easily assessed by calculating the PSD using different refractive and absorption index parameters. What is generally observed is that changes in refractive index do not dramatically alter the calculated PSD when the relative refractive index (which is the different between particle and suspending phase refractive index) is high (>1). As the relative refractive index approaches zero, changes in refractive index yield progressively larger changes in the calculated PSD. It should also be noted that the degree to which the PSD changes with variations in refractive index depends on the size range of particles contained in the dispersion and on the optimal value for refractive index (i.e., that which gives the best measure of refractive index for mixed particle systems). In general, PSDs measured for large particles (>10 $\mu \mathrm{m}$ in diameter) are relatively insensitive to changes in refractive index. Indeed, for sufficiently large particles, analysis of the diffraction pattern does not require knowledge of refractive index (such as in Fraunhofer diffraction). For fine particles (i.e., those below $10 \mu \mathrm{m}$ ), lowering the relative refractive index typically causes a shift in the lower PSD boundary to smaller diameters and may increase the relative volume contribution of fines. 
With respect to absorption index, changes in this parameter typically alter the relative contribution of particle fines below $1 \mu \mathrm{m}$. Incorrect assignment of particle absorption index can yield improper interpretation of high-angle diffraction readings. For example, if a transparent particle dispersion is analyzed using an absorption index of 1 (i.e., as an opaque dispersion), the high-angle light-intensity readings will be attributed (improperly) to the existence of a significant relative volume fraction of fines when the correct interpretation of the high-angle readings is internal reflection of the laser beam in the particle interior.

Without experimental knowledge of the actual particle and suspending phase refractive index and the particle absorption index, it is difficult to assess the appropriateness of the optical properties selected for analysis. A rough evaluation can be performed by comparing the measured diffraction pattern to the diffraction pattern fit by the analysis software. For example, on the Malvern Mastersizer 2000 analyzer, significant difference between fit and measured light intensity on high-angle detectors can indicate that an inappropriate absorption index has been assigned to the particle. Likewise, a persistent deviation between fit and measured diffraction patterns over low and mid-range angle detectors can suggest incorrect selection of either particle or dispersing medium refractive index. Evaluation of the diffraction pattern fit should be used for indication only, as poor fits may not always result from improper selection of optical properties. Indeed, other issues such as a poor background (i.e., clean reference cell) measurement, unclean observation cell windows, and particle adhesion to the cell windows during measurement may alter the diffraction pattern measured in ways that cannot be accommodated by the diffraction model.

The uncertainty in the PSD associated with the particle and suspending medium optical properties can be assessed by observing the variation in calculated PSDs resulting from parametric variations in selected optical properties. If the particle refractive index is unknown and cannot be easily determined, it is recommended that the refractive index of similar crystal phases or compounds be employed to define a range of refractive indices. Then, variation of the calculated PSD over this index range can be determined and used to assess uncertainty. A similar approach can be applied to determine uncertainty with respect to the remaining optical properties. 


\section{G.5 General Difficulties with Light Scattering}

The previous section discussed uncertainty with respect to parameters that the analyst can control, including run and analysis parameters and dispersing phase considerations. In this section, difficulties with the light scattering methodology beyond the analysts control are discussed. The most significant of these difficulties is the accuracy of light scattering applied to sizing of particle dispersions with particles whose diameters range over several orders of magnitude and contain several different materials. This difficulty derives from the fact that the broader the distribution of particles, the greater the disparity between the number of large and small particles that are subjected to the measurement. Poor sampling statistics are obtained for the large particles in the presence of much smaller particles unless the sampling time is long and the flow rate of the system is high. Moreover, control of the process may only require knowledge of a material's apparent PSD (as characterized by light scattering) rather than knowledge of the true size distribution of particles.

Hanford tank waste sludges and slurries are complex mixtures of crystalline and amorphous precipitated metal hydroxides. Typical speciation includes aluminum and iron bearing minerals of broad (several orders of magnitudes) size distributions. Knowledge of particle size in Hanford tank wastes is key to evaluating processing, transport, and mixing of these wastes at the Waste Treatment Plant. To date, most characterizations have employed light scattering. When applied to multicomponent systems with a broad size distribution, light scattering models generally cannot resolve sharp peaks associated with individual species, especially when those peaks overlap. Even when the individual peaks are well separated, the analyzer may merge two peaks into a single peak if the peak intensities are on the same order of magnitude, especially when the broad PSD model is used. When peaks are well separated in size range, the analyzer may be able to distinguish the peaks separately but may not characterize the range of particle sizes in each peak correctly. Even though peaks may be well-separated, there can be overlap of the diffraction patterns of large and small particles. In these cases, small particle scattering may be confused with the stronger scattering from large particles, especially if the small particle species is present in limited amounts (e.g., 5 vol\%).

To illustrate these points, case studies for measurement of mixed component (both with respect to chemical species and size) PSDs are presented and discussed. In these studies, a mixture of known composition was created from components whose size distribution had been determined by light scattering. The PSD of this mixture was measured and compared to the distribution calculated from the individual components. In this way, the difference between single component and mixed systems can be assessed and the uncertainty associated with mixed system PSD measurements evaluated.

\section{G5.1 Case 1: Particle Size Distribution of a Mixture of $X$ and $Y$ Narrow Size Distribution Glass Beads}

Case 1 consists of a Bi-component system made up of two narrow distributions, highly spherical glass beads mixed in different ratios. Both particles are soda lime glass and hence have the same optical properties for analysis purposes. The PSDs of this system at two different ratios are given in Figures G.7 and G.8. The normalized component distribution for both particles has been plotted with the measured PSDs for the mix. Two different models have been used to obtain the measured PSDs shown. First, a general purpose-irregular model used for irregular shaped particles typical of milled and naturally 
occurring samples has been used to illustrate the PSD generated using this model. Even though the distributions of these two particle modes are well separated, overlap of the diffraction patterns of the large and small particles using this model results in one broad distribution skewed toward the particle size of the dominant particle. Changing the model to multiple narrow-spherical produces a PSD with two distinct modes indicative of the two different distributions present. This model is only appropriate for samples consisting of 1 or more very narrow distributions and as discussed previously should not be used unless microscopic evidence distinguishes multiple narrow size bands. The impact of particle shape has been discussed previously and spherical particles should only be used in the model if there is microscopic evidence of highly spherical, smooth particles that are typical of latices or emulsions.

Figures G.7 and G.8 clearly illustrate that as the concentration of larger glass beads increases the ability of the multiple narrow-spherical model to accurately characterize the mixed distribution decreases due to the stronger diffraction pattern of the larger beads. This phenomenon results in an overestimation of the larger bead contribution to the overall PSD and consequently an underestimation of the contribution from the smaller beads. The choice of model, based on knowledge of the material being analyzed, can significantly impact the observed PSD. A very different PSD is obtained if a general purpose model is assumed, which is usually the default model on light scattering instruments. If a narrow mode distribution model is used for a broad continuous material anomalous discrete particle sizes can appear that may not be indicative of the true PSD of the material.

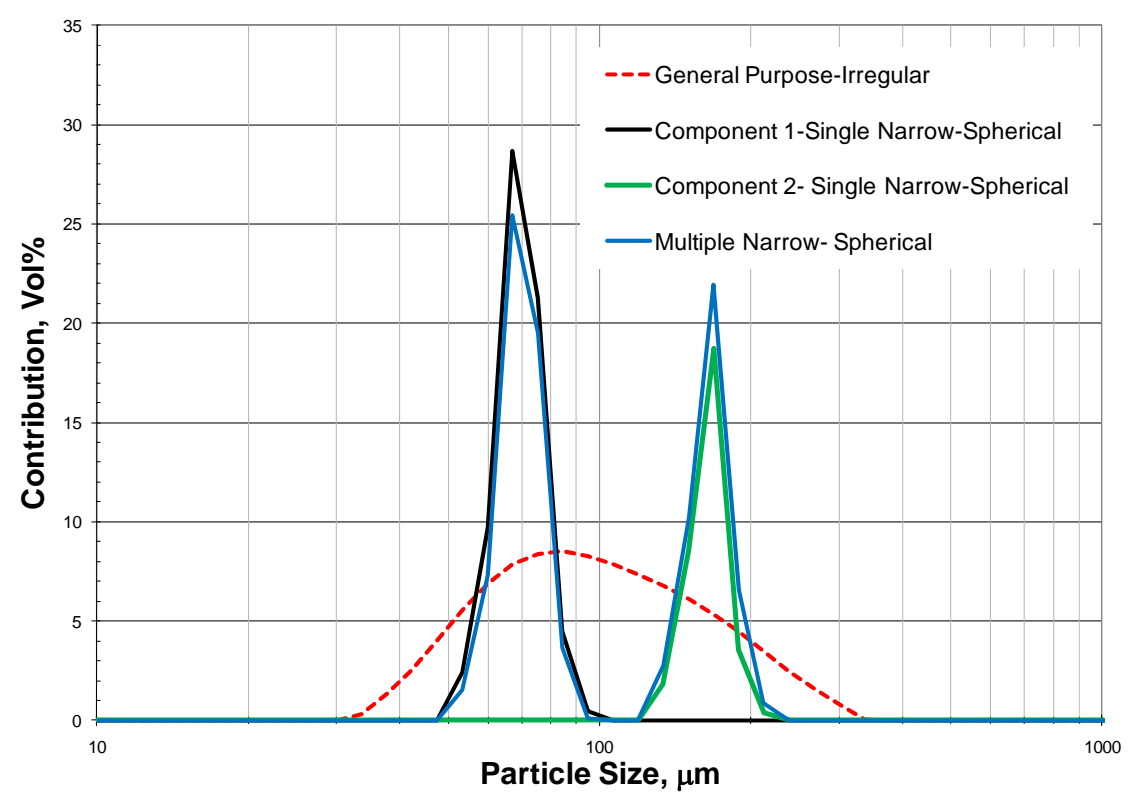

Figure G.7. Bi-Component, Narrow Distribution Spherical Glass Beads. XLSciTech, 63-75 $\mu \mathrm{m}$ soda lime glass (67 wt \%) mixed with XLSciTech, 150-180 $\mu \mathrm{m}$ soda lime glass (33 wt\%). 


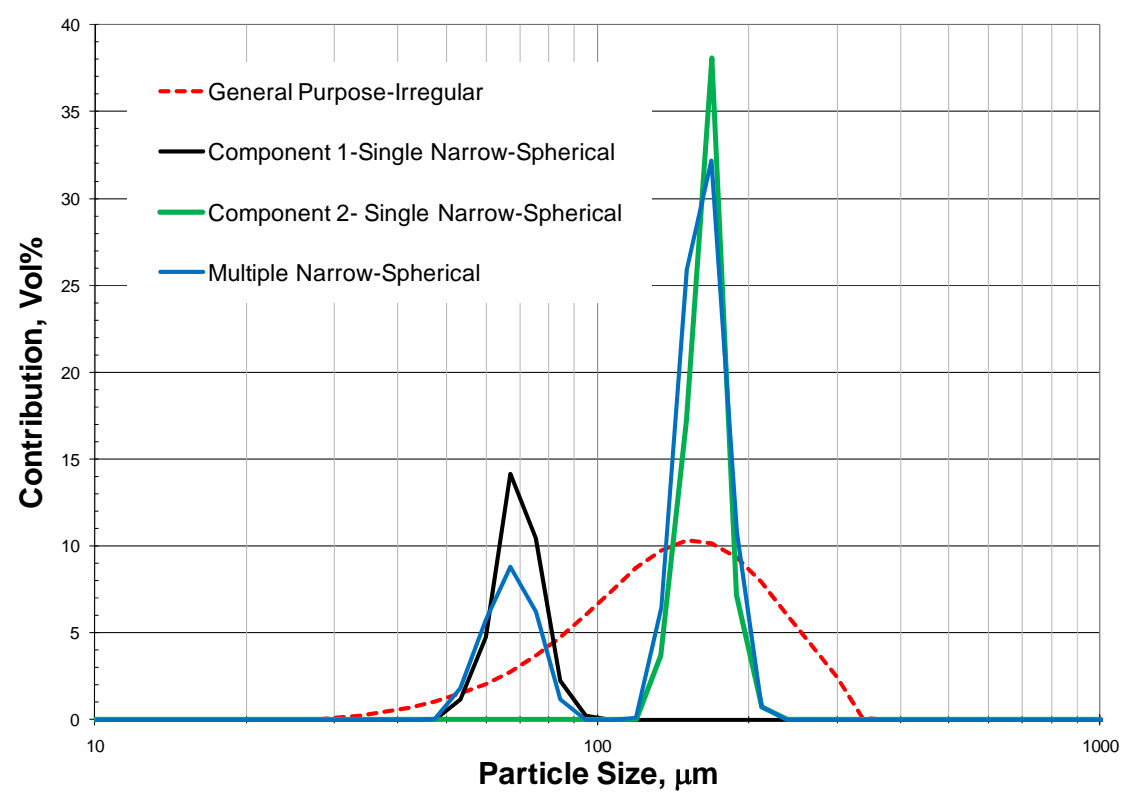

Figure G.8. Bi-Component, Narrow Distribution Spherical Glass Beads. XLSciTech, 63-75 $\mu \mathrm{m}$ soda lime glass (33 wt \%) mixed with XLSciTech, 150-180 $\mu$ m soda lime glass (67 wt\%).

\section{G5.2 Case 2: Multi-component System with Discrete Broad Particle Size Distributions}

Case 2 consists of a multi-component system with discrete broad PSDs. The individual normalized PSDs of the components and the measured combined PSD are given in Figure G.9 below. A general purpose-irregular model has been applied to all of the data as well as a single set of particle optical properties. This multiple component composite was constructed with different particle types (irregular, spherical), materials with different optical properties, and different distribution types. Component 3 is a very narrow distribution spherical glass bead that would normally be analyzed individually with a single narrow spherical model, while both components 1 and 2 are broad distribution irregular shaped particles. The optical properties of the composite range from transparent to opaque with particle refractive indices ranging from 1.5 to 2.4. As with the bi-component mix discussed in Case 1, the measured PSD of the composite exhibits the same tendencies of overestimating the distributions of the larger particles. It is surprising that in the measured PSD of the complex mix, the discrete distributions of the individual components are well aligned. Multiple aliquots of the complex mix also yield consistent results. The precision of the light scattering technique is discussed later. 


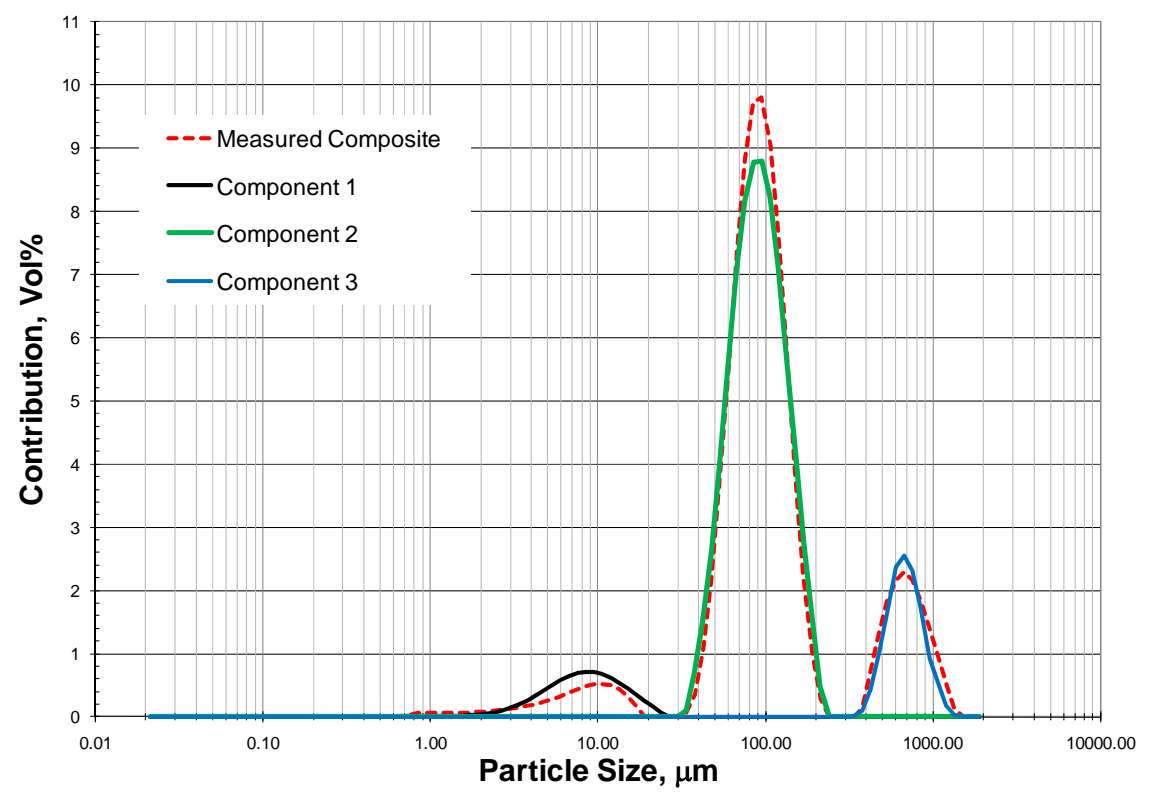

Figure G.9. Multi-Component Composite Sample with Discrete Broad PSDs and the Individual Component PSDs

The multi component composite given in Figure G.9 was sieved and the fractions collected, reanalyzed, and compared with the individual component PSDs. These comparisons are given in Figures G.10 through G.12. It can be seen that the recovered fractions overlap perfectly with the individual components of the composite. The fact that we can recover the individual component PSDs demonstrates that the components were not altered physically or chemically during compositing and can be recovered; therefore, the deviation in the resulting measured composite PSD compared to the PSD of the individual components in Figure G.9 is indicative of the true measurement error and not due to changes in the components from handling and mixing. 


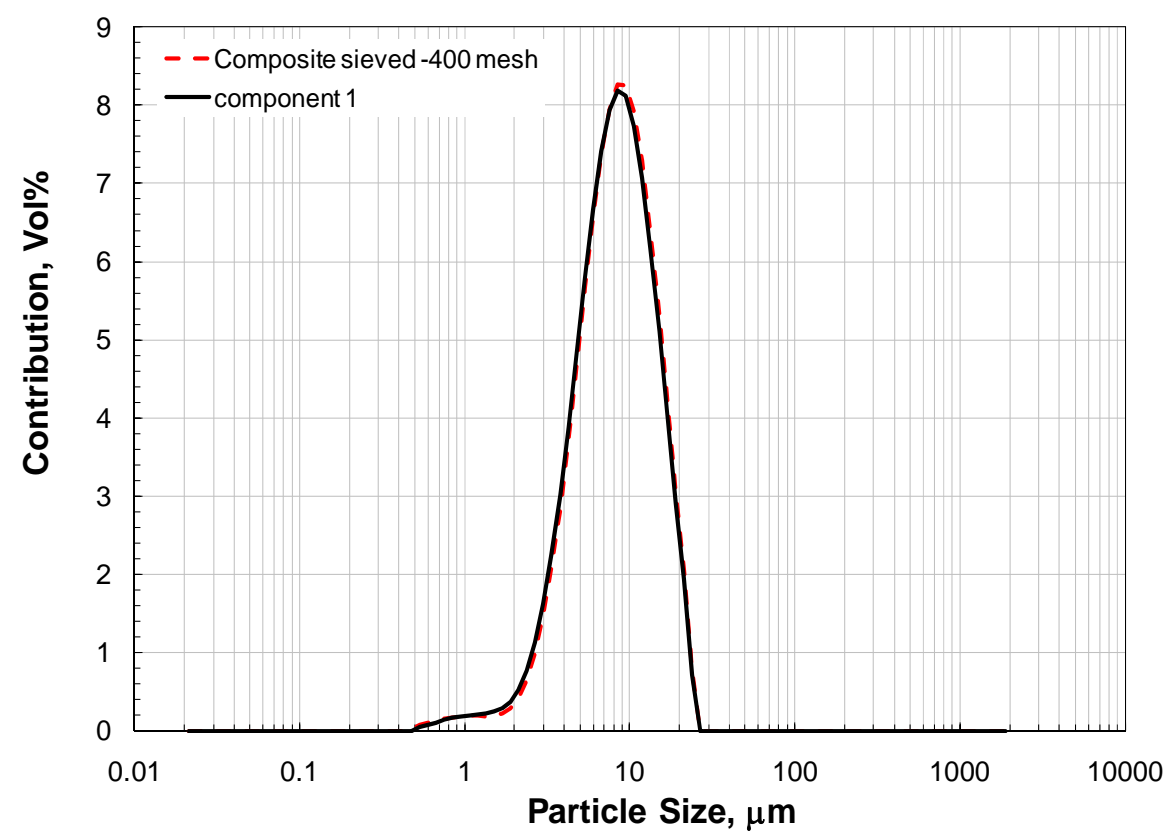

Figure G.10. PSDs of Component 1 and the -400 Mesh Sieved Fraction from the Multi-Component Composite

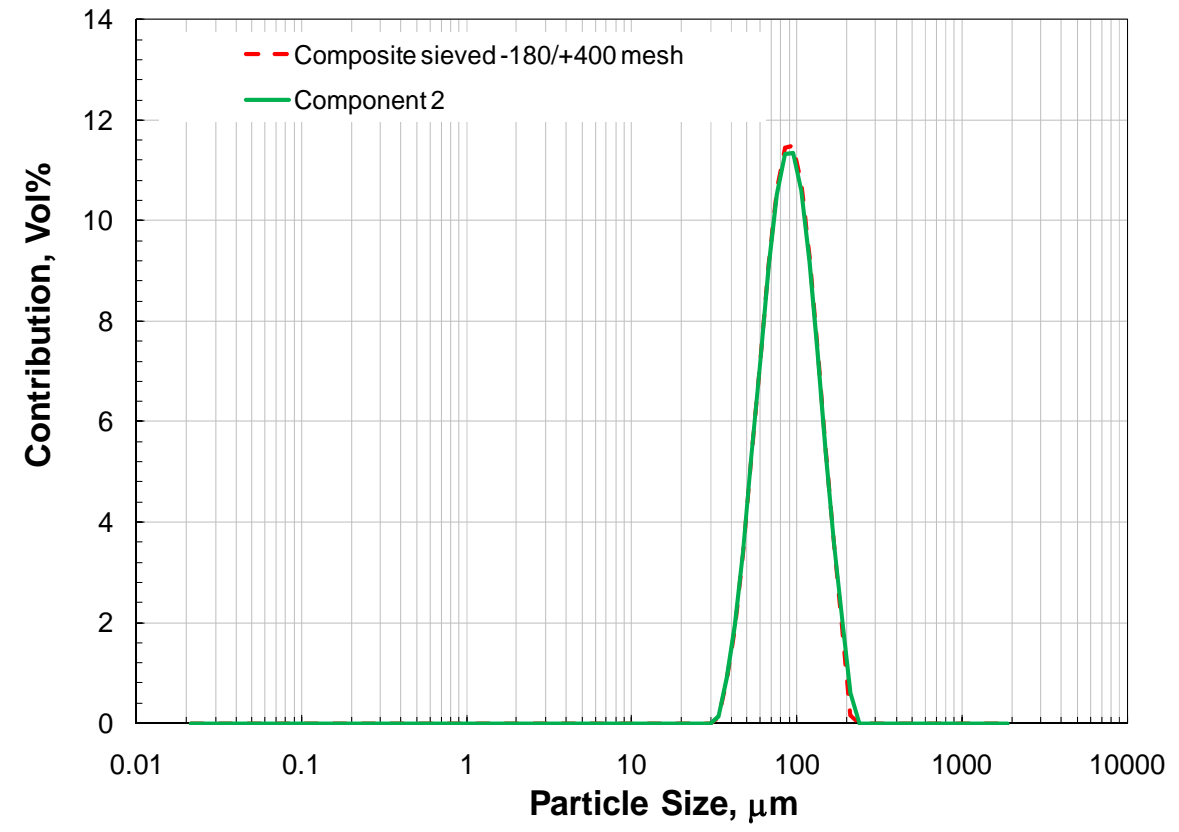

Figure G.11. PSDs of Component 2 and the $-180 /+400$ Mesh Sieved Fraction from Multi-Component Composite 


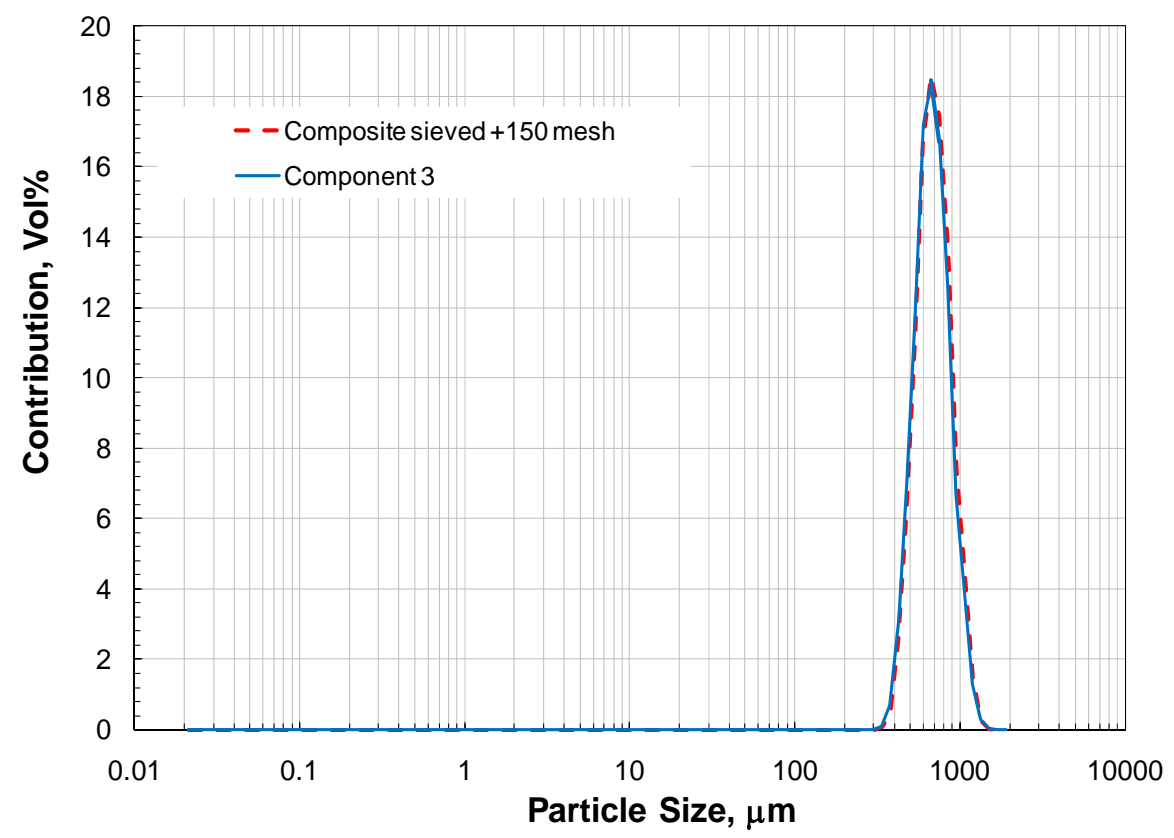

Figure G.12. PSDs of Component 3 and the +150 Mesh Sieved Fraction from Multi-Component Composite

\section{G5.3 Case 3: Characterization of a Two-Component System with Overlapping Particle Size Distributions}

A two-component system was created by mixing aluminum oxide (component 1 ) with sand (component 2). The PSDs for the individual components are shown in Figure G.13. The aluminum oxide (component 1) has a median diameter of $136 \mu \mathrm{m}$, while the sand (component 2) has a median diameter of $310 \mu \mathrm{m}$. As indicated by the PSD results, the peaks for aluminum oxide and sand are clearly distinguishable when plotted side-by-side, but overlap to a significant extent over the 100 to $300 \mu \mathrm{m}$ range. 


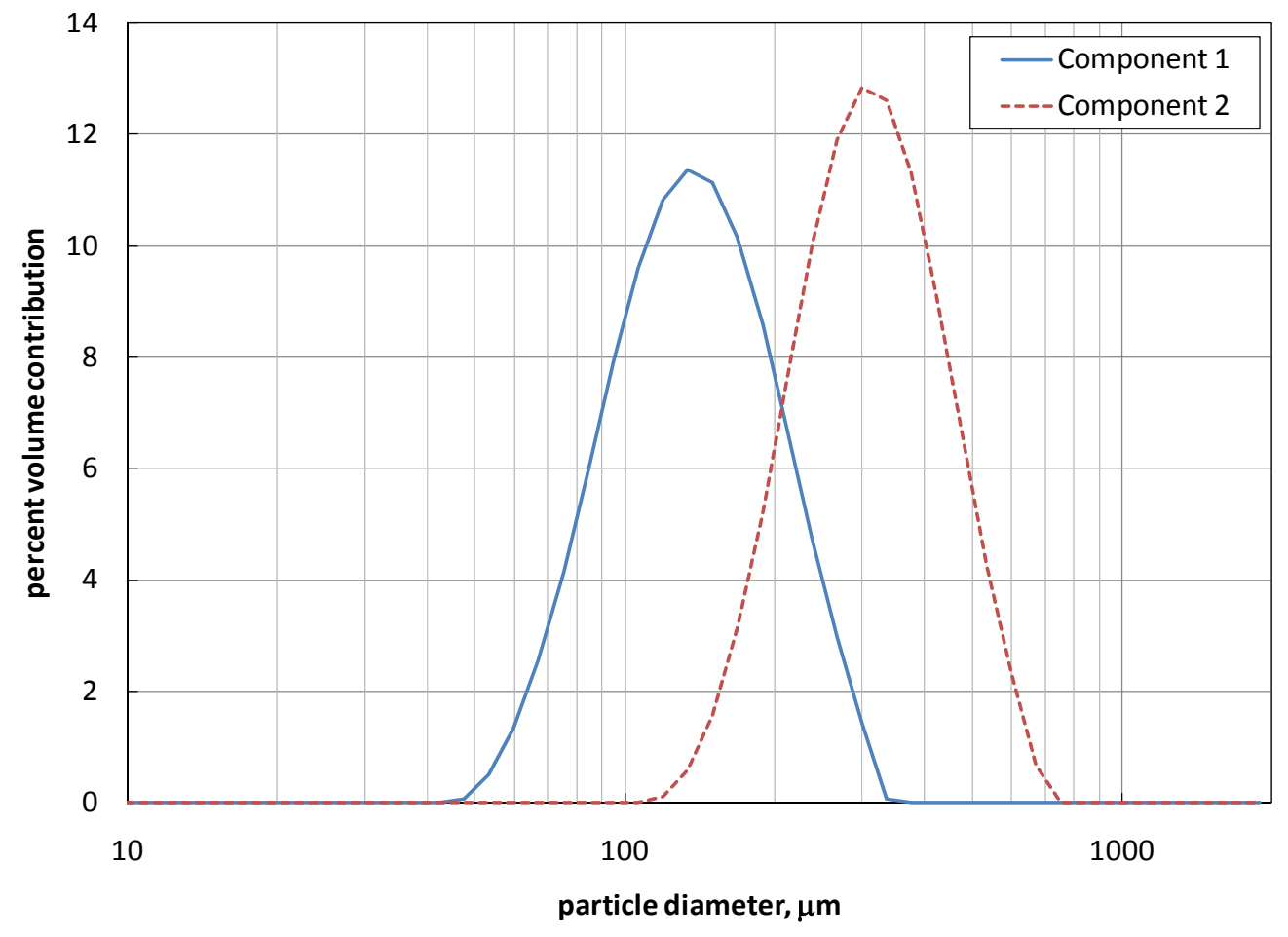

Figure G.13. PSDs for Case 3 Components (1: aluminum oxide, 2: sand)

Figure G.14 compares the measured and calculated PSDs for a mixture of 96 vol\% aluminum oxide and 4 vol\% sand. Given that the majority of the particle volume corresponds to aluminum oxide, it is not surprising that the PSD resembles the component 1 peak in Figure G.13. However, as shown by the calculated PSD, the contribution of sand is underestimated in the measured PSD. The calculation suggests that the PSD should show a shoulder that begins between 300-400 $\mu \mathrm{m}$ and continues out to $700 \mu \mathrm{m}$. The measured PSD for the mixture does not show this expected shoulder, and relative to the aluminum oxide PSD shown in Figure G.13, only shows a minor increase in the contribution of particles in the 300 to $400 \mu \mathrm{m}$ range. Based on the measured PSD in Figure G.14, it appears that the size analyzer is unable to recognize the contribution of the $4 \mathrm{vol} \%$ of sand to the diffraction pattern. Indeed, the light-scattering system appears to miss entirely the addition of the large sand particles to the smaller aluminum oxide powder. 


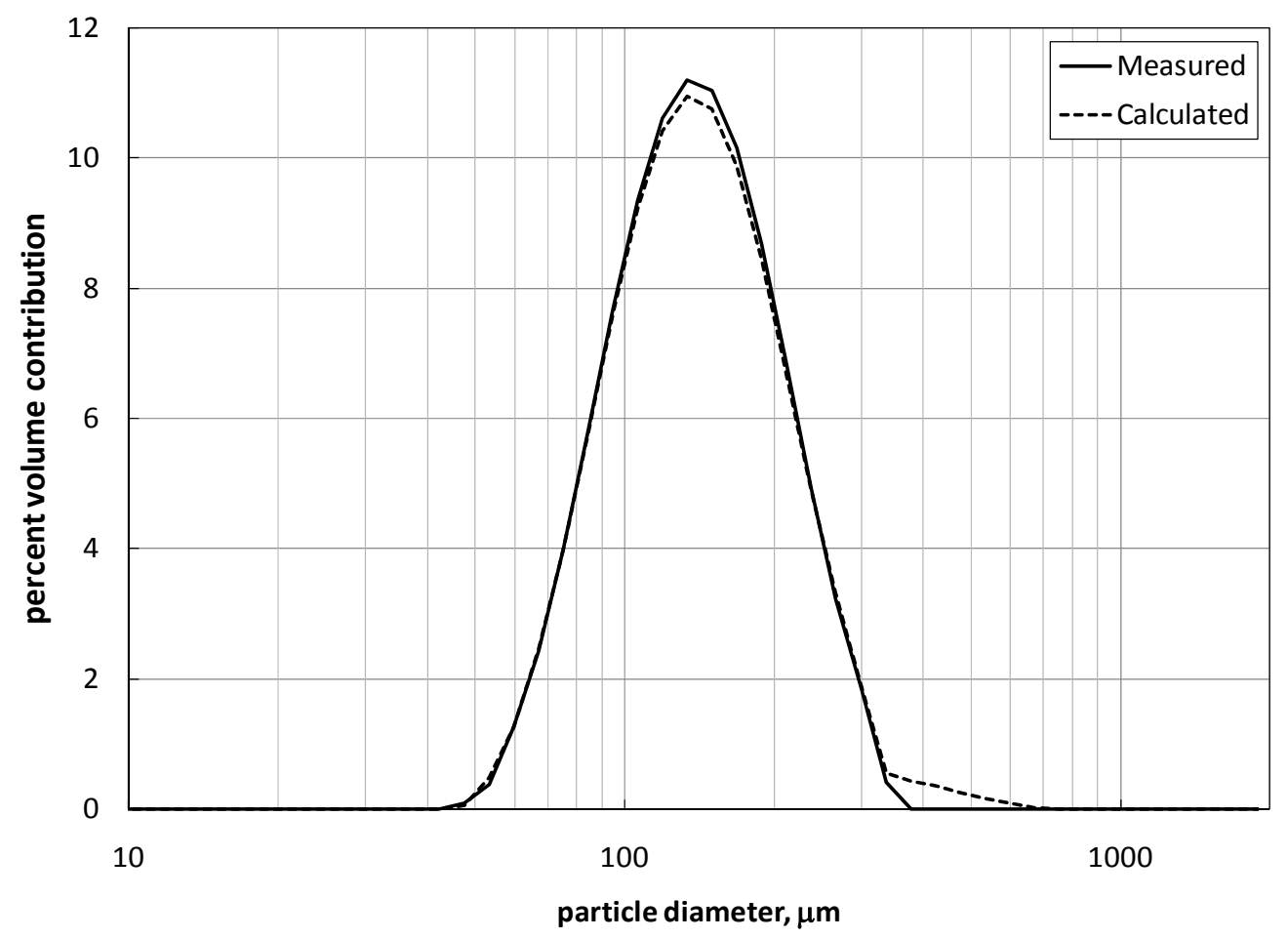

Figure G.14. Measured and Calculated PSDs for a Mixture of 96 vol\% Aluminum Oxide and 4 vol\% Sand. Calculated PSD is based upon component PSDs shown in Figure G.13.

Figure G.15 compares the measured and calculated PSDs for a mixture of 85 vol\% aluminum oxide and 15 vol\% sand. Here, the higher concentration of sand particles was added to determine if increased concentration would improve component detection. While the higher concentration of sand clearly improves detection of particles in the 300 to $700 \mu \mathrm{m}$ range, the measured concentration still falls below the actual value as determined by calculation from the component peaks. It is interesting to note that the measured PSD shows a population shoulder of large particles that begins at $\sim 600 \mu \mathrm{m}$, peaks at $\sim 1000 \mu \mathrm{m}$, and ends at $\sim 2000 \mu \mathrm{m}$. The range of sizes exhibited in this peak is greater than that characterized in either individual component PSDs. Since the component particles for both sand and aluminum oxide are well beyond the colloidal size range, it is unlikely that this shoulder peak results from particle agglomeration. Indeed, the large particle peak appears to result from misinterpretation of the overlapping diffraction patterns for aluminum and sand. Unlike the previous system, the presence of 15 vol\% sand in the aluminum oxide yields an apparent PSD that overestimates the size of the largest particles by nearly a factor of 3 . 


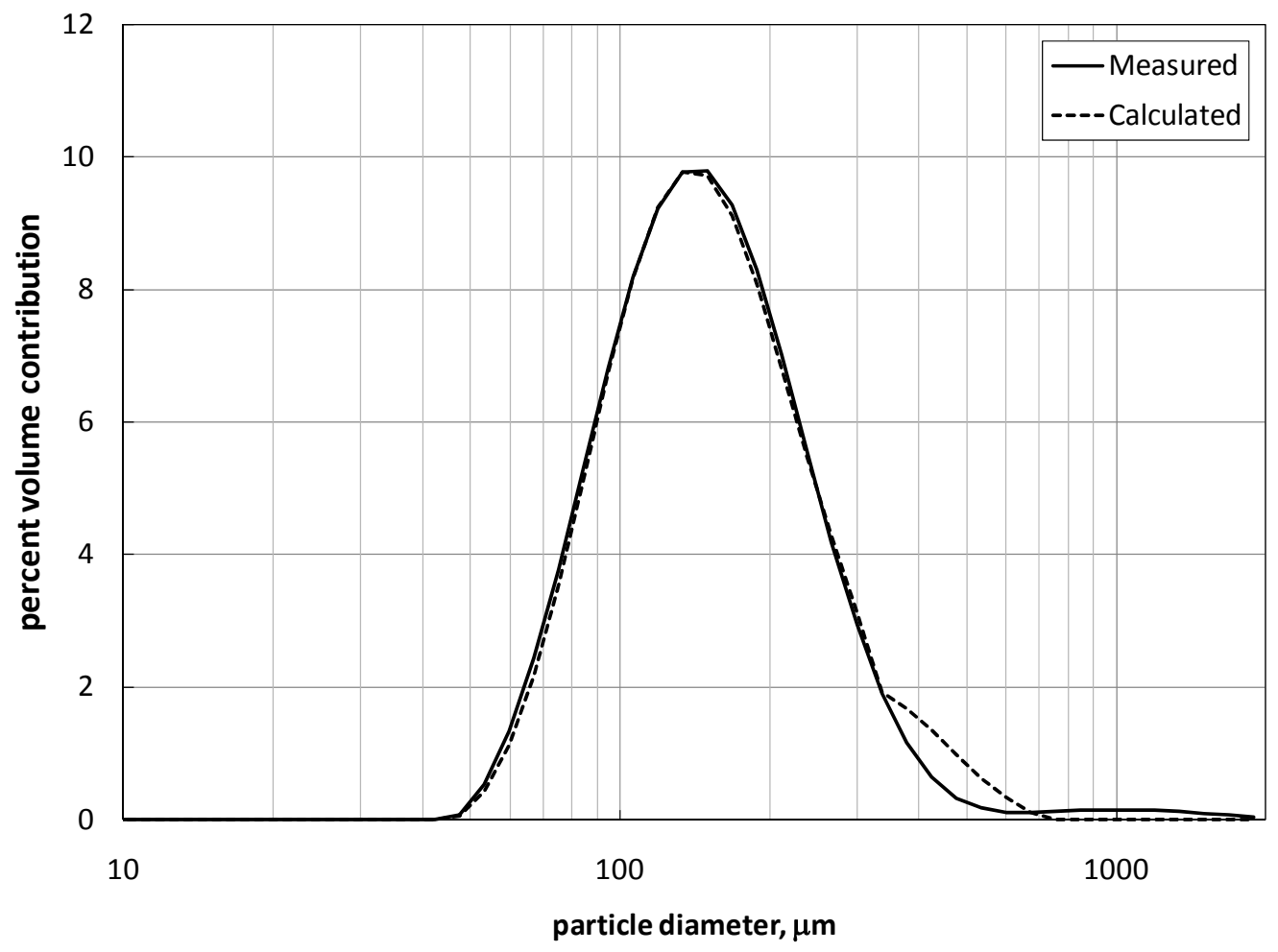

Figure G.15. Measured and Calculated PSDs for a Mixture of 85 vol\% Aluminum Oxide and 15 vol\% Sand. Calculated PSD is based upon component PSDs shown in Figure G.13.

As discussed above, Case 3 compares measured and calculated PSDs for a two-component system where the component size distributions overlap moderately. The measurements presented show that, for the specific cases where sand (which has the larger diameter of the two components) is present in low concentrations, analysis of the mixture by light scattering can both fail to detect the larger sand component and overestimate the size of the sand component while the major component (i.e., aluminum oxide) PSD is comparable to its single component measurement. The contribution of large particles (which derives from sand additions to the mixture) is not well captured at small concentrations in a matrix with primarily smaller diameter particles.

\section{G5.4 Case4 : Multi-component System with Overlapping Particle Size Distributions}

Case 4 is a multi-component composite sample constructed from 3 components with broad overlapping PSDs. The composite sample consisted of gibbsite (17.6 vol\% with diameters ranging from 1 to $45 \mu \mathrm{m}$ ), boehmite ( $82.2 \mathrm{vol} \%$ with diameters ranging from 0.15 to $17 \mu \mathrm{m}$ ), and tungsten carbide ( 0.1 vol\% with diameters ranging from 0.3 to $25 \mu \mathrm{m}$ ). These materials differ greatly in optical properties but are all irregular shaped particles. The normalized individual component PSDs are shown in Figure G.16 along with the measured composite PSD and the calculated composite PSD. An overestimation of the large particle contribution is observed in the measured composite PSD, unlike the previous examples. This multi-component system has a submicron fraction which was not present in any of the other cases. A large portion of the submicron fraction is not resolved and is lost in the composite PSD. To facilitate data interpretation, the normalized calculated composite PSD has been included in 
Figure G.16. Figure G.16 illustrates the difficulties in effectively and accurately measuring PSDs of complex systems with diameters ranging over multiple orders of magnitude.

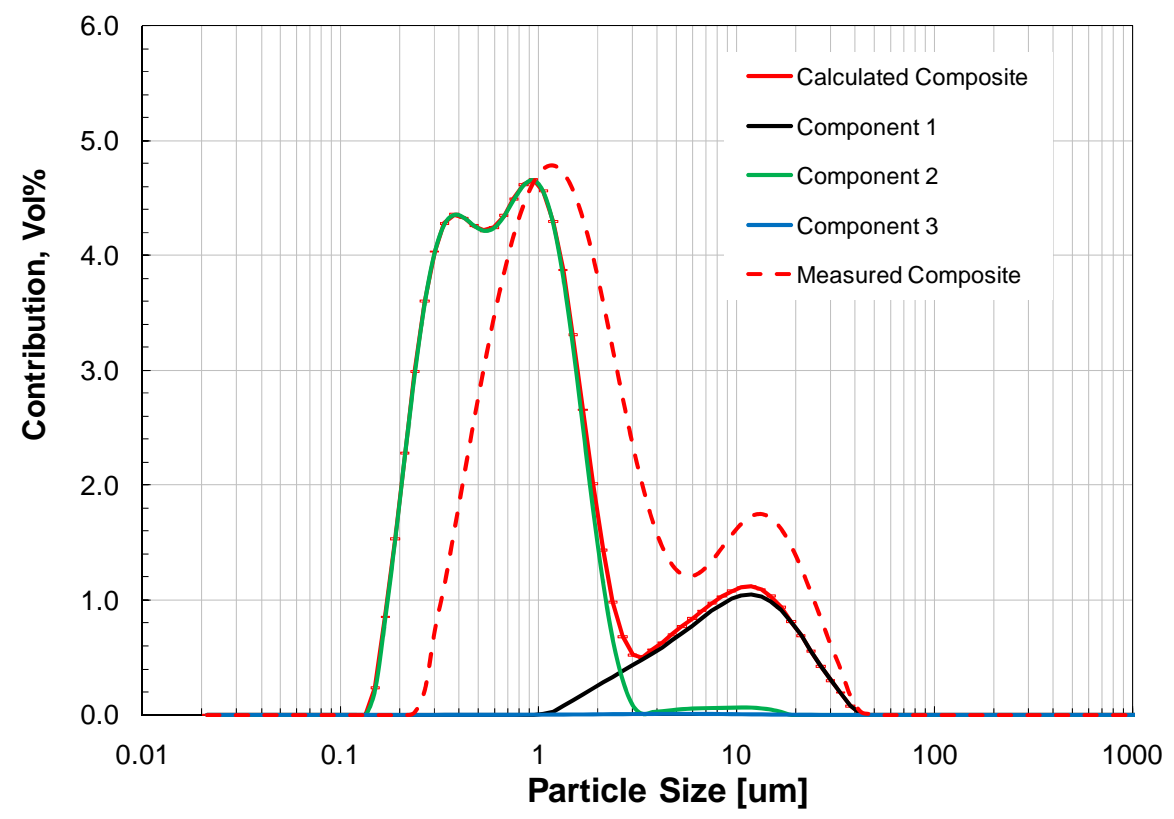

Figure G.16. Multi-Component Composite with Overlapping PSDs, Individual Component PSD and the Calculated Composite PSD

\section{G5.5. Case 5: Precision of Multi-component Broad Particle Size Distribution Measurements}

As seen in the previous four cases, significant difficulty can be encountered when attempting to "accurately" characterize multi-component system PSDs. However, it should be recognized that some of the error associated with these measurements can also be associated with random measurement errors, sampling errors, or other sources of uncertainty described in Section G.4. Careful control of sampling and reproduction of measurement conditions can yield relatively reproducible PSDs, even across multiple subsamples and subaliquots. Figure G.17 shows an example of the precision obtained for well-controlled particle size measurements of a broad multi-component system. The average and maximum and minimum PSDs based on the average and standard deviation of multiple measurements of the same system is shown for a broad range ( 0.3 to $200 \mu \mathrm{m})$ multi-component Hanford tank waste simulant. The PSD shown is the average of 7 measurements of separate sample aliquots. The standard deviation bars are calculated as the sample standard deviation of each measurement bin, with the PSD being represented in 100 bins scaled logarithmically over 0.02 to $2000 \mu \mathrm{m}$. Over most of the PSD size range, the variation in measured PSD is small, such that when graphed as in Figure G.17, the standard deviation curves are nearly indistinguishable from the average. Some variation is seen in the shoulder of the PSD from 70 to $200 \mu \mathrm{m}$. Such variation is typically observed and is usually attributed to difficulties in representatively sampling large particles. Sample difficulties for large particle derive from the fact that, given equal volume contribution of large and small particles, the large particles are present in much fewer numbers. For example, the volume contribution of one $100 \mu \mathrm{m}$ particle is equaled by the volume contribution of one-thousand $10 \mu \mathrm{m}$ particles. The consequence for PSD measurements is that in a given sample aliquot, fewer shape configurations are observed for large particles relative to that of small particles. 


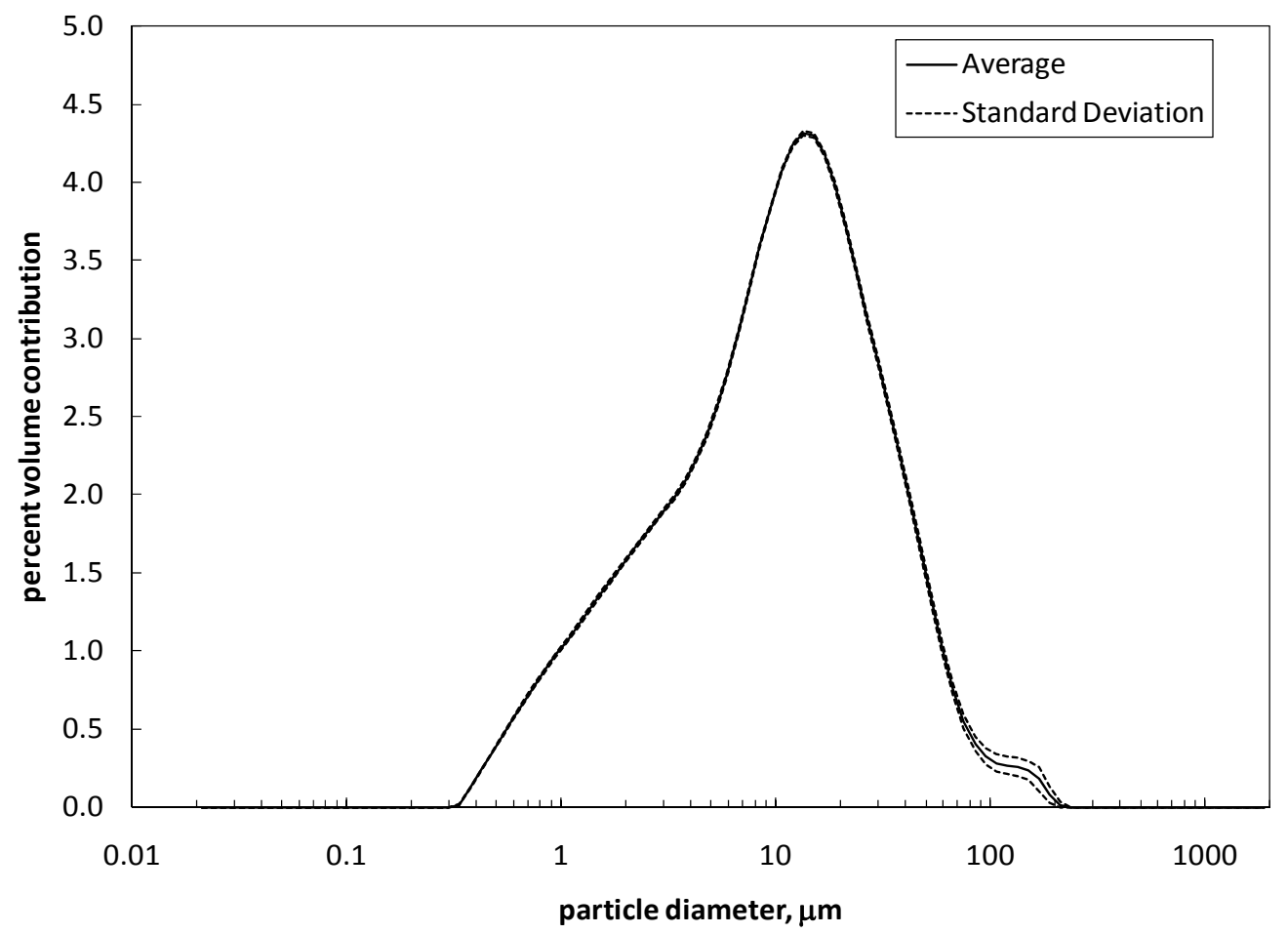

Figure G.17. Average, Maximum, and Minimum PSDs Based on the Average and Standard Deviation Calculated from PSD Measurements of Seven Subsamples of a Hanford Tank Waste Simulant

Table G.2 presents select diameter percentiles for cumulative percent volume undersize for the distribution shown in Figure G.17. The results show that for up to the $80^{\text {th }}$ percentile, the percentile diameters show a relative standard deviation of $1.5 \%$ or lower. The variation in the measured percentiles increases rapidly toward the upper end of the distribution. The $99^{\text {th }}$ percentile shows a variation of $14 \%$. Again, it is expected that this variation is caused by the difficulty with respect to having a representative sampling set of large particles. Based on these results, the precision of PSD measurements is found to be approximately 1 to $3 \%$ for most common percentiles. It should be noted that this precision may only be applicable to the dispersion tested and the instrument on which it was tested (in this example, the dispersion was measured on the Malvern Mastersizer 2000 with the Hydro G dispersion unit). It is promising to note that the magnitude of relative percent variation is comparable to that recommended when verifying the performance of the Malvern Mastersizer 2000 with one of Malvern Instrument's Verification powders on the Hydro G. Malvern's performance specification for their verification powders is $2 \%$ for the $50^{\text {th }}$ percentile and $3 \%$ for the $10^{\text {th }}$ and $90^{\text {th }}$ percentiles. It should be noted that other instrument configurations, such as use of different dispersion units, may alter the typical range of variation. 
Table G.2. Average, Standard Deviation, and Relative Percent Standard Deviation for Select PSD Percentiles for the Data Plotted in Figure G.17

\begin{tabular}{|c|c|c|c|}
\hline Percentile & Average Diameter, $\boldsymbol{\mu} \mathbf{m}$ & $\begin{array}{c}\text { Standard Deviation, } \\
\boldsymbol{\mu \mathbf { m }}\end{array}$ & $\begin{array}{c}\text { Relative Percent } \\
\text { Standard Deviation }\end{array}$ \\
\hline 1 & 0.5214 & 0.0032 & 0.61 \\
\hline 5 & 1.088 & 0.011 & 0.97 \\
\hline 10 & 1.925 & 0.024 & 1.3 \\
\hline 20 & 4.392 & 0.060 & 1.4 \\
\hline 25 & 6.134 & 0.085 & 1.4 \\
\hline 30 & 8.20 & 0.11 & 1.4 \\
\hline 40 & 13.03 & 0.16 & 1.2 \\
\hline 50 & 18.77 & 0.21 & 1.1 \\
\hline 60 & 25.99 & 0.28 & 1.1 \\
\hline 70 & 36.05 & 0.42 & 1.2 \\
\hline 75 & 43.09 & 0.55 & 1.3 \\
\hline 80 & 52.50 & 0.77 & 1.5 \\
\hline 90 & 86.9 & 2.0 & 2.3 \\
\hline 95 & 128.6 & 5.0 & 3.9 \\
\hline 99 & 322 & 45 & 14 \\
\hline
\end{tabular}

\section{G 6 Conclusions}

The preceding sections have discussed particle sizing by light scattering, with an emphasis on the Malvern Mastersizer 2000 particle size system, which has been used for both actual waste and waste simulant particle size analyses conducted at PNNL over the past several years. The theory underlying light scattering was presented, and uncertainty in the light scattering PSD results was discussed extensively with respect to instrument operating and run parameters, result analysis parameters, dispersing phase chemistry, particle morphology and chemistry, and weaknesses in the light scattering method. Because measurement uncertainty derives from many sources, such as the state of particle agglomeration and how it responds to flow/mixing conditions and dispersing phase chemistry as well as difficulties in deconvoluting overlapping diffraction patterns in multicomponent systems, assigning an estimate to PSD measurement uncertainty is difficult.

The discussions of uncertainty in the preceding sections, along with the examples given in Section G.5, show that the PSD measured by light scattering may not accurately capture the true distribution of particle sizes in a given dispersion or powder with respect to either the diameter or volume contribution of particles. Certainly, the accuracy of light scattering is improved for systems composed of highly monodisperse, spherical particles with uniform well-known optical properties. However, even the inclusion of a second monodisperse spherical particle of different size into this ideal system can yield unexpected results, especially if the model used to interpret the diffraction pattern is not optimized for that system. For more complex systems, such as broad (several order of magnitudes) mixtures of particle size and multicomponent systems, PSD analysis by light scattering can provide a general guide as to the range, size, and relative volumes of particles; however, light scattering may not be capable of detecting all particle sizes and may incorrectly interpret the relative volume contributions of each species. The potential for misinterpretation of even simple systems creates significant uncertainty with respect to what is the "true" PSD of the dispersion or powder. Indeed, the PSD characterized by light scattering is best 
characterized as an apparent PSD although the technique is arguably adequate to characterize the general sizes of particles present in the waste.

The current state-of-art for light scattering does not inform on the absolute uncertainty with respect to PSD measurement. While it may be possible to develop a better understanding of uncertainty, it would require development and study of model systems, such as well-characterized mixtures of particles that span the entire measuring range of the instrument $(0.02$ to $2000 \mu \mathrm{m})$ and encompass the range of particle optical properties, chemistries, and morphologies encountered in Hanford tank wastes. A thorough exploration of uncertainty would require a significant investment of resources. A smaller effort involving a relatively simple set of measurements with individual broad based particles and combinations of particles could probably provide reasonable estimates of the uncertainty.

Despite the uncertainty with regard to PSD measurement accuracy, study of multi-component broad PSD dispersions at PNNL has demonstrated that when measuring conditions are well-controlled, PSD measurements of complex systems are highly reproducible. A case study in Section G.5 showed typical variations of 1 to $3 \%$ in select percentiles. It is observed that precision decreases for larger particles (greater than $70 \mu \mathrm{m}$ in the case presented) up to $14 \%$ at the $99^{\text {th }}$ percentile (320 $\mu \mathrm{m}$ ). This difficulty derives from difficulty associated with obtaining a representative sampling of large particle morphologies in mixtures with broad PSDs. While PSD measurements may not always be accurate, they can be precise under controlled conditions. 


\section{G.7 References}

Bechtold DB, WS Callaway, GA Cooke, JB Duncan, DL Herting, JR Jewett, JC Person, and JM Tingey. 2002. Particle Property Analyses of High-Level Waste Tank Sludges. HNF-8862 Rev. 0, Fluor Hanford, Inc., Richland, Washington.

ISO13320-1. 1999. "Particle Size Analysis_Laser Diffraction Methods. Part 1: General Principles”, International Organization for Standardization, Geneva, Switzerland.

Jennings BR and K Parslow. 1988. "Particle Size Measurement: The Equivalent Spherical Diameter”, Proc. R. Soc. Lond. A, 419, 137-149.

Jewett JR, TJ Conrads, LJ Julyk, DA Reynolds, L Jensen, NW Kirch, SD Estey, DB Bechtold, WS Callaway III, GA Cooke, DL Herting, JC Person, JB Duncan, Y Onishi, and JM Tingey. 2003.

"Waste Slurry Particle Properties for Use in Slurry Flow Modeling." Proceedings of the 2003 Symposium on Waste Management, February 2003, Tucson, Arizona.

Jillavenkatesa A, SJ Dapkunas, and L-S H Lum. 2001. "Particle Size Characterization, NIST Recommended Practice Guide.” Special Publication 960-1, National Institute of Standards and Technology, Washington, D.C.

Lide DR, Ed. 1990. CRC Handbook of Chemistry and Physics, $71^{\text {st }}$ Addition, CRC Press, Inc., Boca Raton, Florida.

Malvern Instruments Limited. 2007. “Mastersizer 2000 User Manual.” MAN0384, Issue 1.0, Malvern, Worcestershire, UK.

Rawle A. "Basic Principles of Particle Size Analysis.” MRK034, Malvern Instruments Limited, Malvern, Worcestershire, UK.

Schiebener P, J Straub, JMH Leveit Sengers, and JS Gallagher. 1990. "Refractive Index of Water and Steam as Function of Wavelength, Temperature and Density.” J. Phys. Chem. Ref. Data 19:3. 

Appendix $\mathrm{H}$

\section{PSD Results}





\section{Appendix H: PSD Results}

The PSDs are provided as the "typical” particle sizes for the different percentiles based on the combined distribution, estimates of "typical" particle sizes based on the percentile medians, estimates of the lower limits on particle sizes for the different percentiles based on the percentile minimums, and estimates of the upper limits on particle sizes for the different percentiles based on the percentile maximums. Tank, waste type, and composite PSDs for the percentiles of interest are provided in Table H.1, Table H.2, and Table H.3, respectively.

The differential PSDs presented in Section 4.0 as examples of the PSD changes due to the pretreatment processes observed for the M12 waste groups are provided in Table H.4. All of the PSDs in Table H.4 were measured with the Malvern Mastersizer (see Section 3.2.5.1) circulation pump running at $2000 \mathrm{rpm}$ and its ultrasonic agitator turned on. 
Table H.1. Tank PSDs

\begin{tabular}{|c|c|c|c|c|c|c|c|c|c|c|c|c|c|c|c|c|c|c|}
\hline PSD Type & Tank & PSD & 0.01 & 0.05 & 0.1 & 0.2 & 0.25 & 0.3 & 0.4 & 0.5 & 0.6 & 0.7 & 0.75 & 0.8 & 0.9 & 0.95 & 0.99 & 1 \\
\hline \multirow{31}{*}{\begin{tabular}{|l} 
Sludge, \\
Flowing \\
Sonicated
\end{tabular}} & AW-103 & Min & 0.18 & 0.27 & 0.38 & 0.74 & 1.04 & 1.20 & 1.50 & 1.99 & 2.75 & 3.76 & 4.47 & 5.43 & 9.24 & 11.10 & 15.30 & 22.28 \\
\hline & AW-103 & Comb. & 0.27 & 0.66 & 0.95 & 1.36 & 1.68 & 2.10 & 2.75 & 3.80 & 5.31 & 7.53 & 9.27 & 11.29 & 21.35 & 83.14 & 258.00 & 342.96 \\
\hline & \begin{tabular}{|l|}
$A W-103$ \\
\end{tabular} & Max & 0.70 & 1.08 & 1.36 & 2.17 & 2.74 & 3.50 & 5.70 & 9.30 & 21.63 & 50.30 & 76.70 & 92.82 & 135.93 & 164.50 & 258.00 & 399.23 \\
\hline & AW-103 & Median & 0.31 & 0.54 & 0.77 & 1.07 & 1.21 & 1.49 & 2.40 & 3.59 & 4.88 & 6.27 & 7.10 & 8.29 & 15.53 & 18.80 & 26.70 & 40.09 \\
\hline & AY-101 & Min & 0.17 & 0.28 & 0.42 & 0.78 & 0.97 & 1.17 & 1.81 & 3.65 & 5.02 & 6.15 & 6.80 & 7.77 & 10.15 & 11.60 & 16.10 & 22.84 \\
\hline & AY-101 & Comb. & 0.26 & 0.49 & 0.77 & 1.28 & 1.70 & 2.12 & 3.65 & 5.46 & 6.61 & 7.84 & 8.47 & 9.90 & 12.41 & 14.60 & 19.90 & 28.01 \\
\hline & AY-101 & Max & 0.30 & 0.61 & 0.86 & 1.70 & 2.40 & 2.83 & 3.95 & 5.50 & 6.78 & 7.94 & 8.70 & 9.90 & 12.83 & 14.60 & 19.90 & 28.07 \\
\hline & AY-101 & Median & 0.24 & 0.45 & 0.65 & 1.16 & 1.50 & 1.81 & 3.00 & 4.78 & 6.17 & 7.51 & 8.14 & 8.83 & 10.84 & 12.86 & 18.09 & 25.10 \\
\hline & \begin{tabular}{|l|} 
AY-102 \\
\end{tabular} & Min & 0.31 & 0.41 & 0.48 & 0.59 & 0.66 & 0.75 & 0.93 & 1.14 & 1.71 & 3.04 & 3.60 & 4.25 & 5.93 & 7.00 & 9.40 & 14.17 \\
\hline & AY-102 & Comb. & 0.41 & 0.56 & 0.74 & 0.98 & 1.08 & 1.28 & 2.17 & 3.32 & 4.98 & 7.00 & 8.66 & 10.41 & 15.41 & 21.06 & 39.59 & 56.54 \\
\hline & \begin{tabular}{|l|} 
AY-102 \\
\end{tabular} & Max & 0.60 & 0.75 & 0.83 & 1.03 & 1.28 & 1.67 & 2.52 & 4.17 & 6.88 & 10.19 & 12.21 & 14.58 & 21.24 & 27.22 & 39.59 & 59.77 \\
\hline & AY-102 & Median & 0.35 & 0.45 & 0.58 & 0.91 & 1.07 & 1.26 & 1.99 & 3.11 & 4.51 & 6.53 & 7.84 & 9.27 & 13.23 & 16.45 & 23.85 & 35.97 \\
\hline & \begin{tabular}{|l|} 
AZ-101 \\
\end{tabular} & Min & 0.47 & 0.56 & 0.70 & 1.10 & 1.33 & 1.60 & 2.30 & 3.15 & 4.30 & 6.00 & 6.93 & 8.00 & 15.00 & 20.00 & 27.00 & 41.97 \\
\hline & \begin{tabular}{|l|} 
AZ-101 \\
\end{tabular} & Comb. & 0.47 & 0.56 & 0.70 & 1.10 & 1.33 & 1.60 & 2.30 & 3.15 & 4.30 & 6.00 & 6.93 & 8.00 & 15.00 & 20.00 & 27.00 & 41.97 \\
\hline & AZ-101 & Max & 0.47 & 0.56 & 0.70 & 1.10 & 1.33 & 1.60 & 2.30 & 3.15 & 4.30 & 6.00 & 6.93 & 8.00 & 15.00 & 20.00 & 27.00 & 41.97 \\
\hline & \begin{tabular}{|l|} 
AZ-101 \\
\end{tabular} & Median & 0.47 & 0.56 & 0.70 & 1.10 & 1.33 & 1.60 & 2.30 & 3.15 & 4.30 & 6.00 & 6.93 & 8.00 & 15.00 & 20.00 & 27.00 & 41.97 \\
\hline & \begin{tabular}{|l|} 
AZ-102 \\
\end{tabular} & Min & 0.55 & 1.10 & 1.60 & 2.13 & 2.40 & 2.65 & 3.24 & 3.90 & 4.84 & 6.03 & 6.80 & 7.82 & 11.00 & 14.01 & 20.79 & 29.96 \\
\hline & AZ-102 & Comb. & 1.10 & 1.79 & 2.50 & 3.69 & 4.40 & 5.15 & 7.08 & 10.07 & 13.28 & 18.37 & 25.05 & 35.77 & 124.62 & 176.09 & 671.65 & 890.37 \\
\hline & AZ-102 & Max & 1.39 & 1.98 & 3.13 & 5.77 & 7.43 & 9.43 & 14.77 & 23.48 & 107.42 & 128.83 & 138.14 & 147.48 & 176.39 & 458.17 & 671.65 & 1026.23 \\
\hline & \begin{tabular}{|l|} 
AZ-102 \\
\end{tabular} & Median & 0.99 & 1.55 & 2.00 & 3.30 & 4.25 & 4.87 & 6.52 & 9.04 & 12.09 & 15.93 & 18.04 & 21.66 & 35.13 & 58.06 & 225.09 & 291.88 \\
\hline & B-203 & Min & 0.47 & 1.01 & 1.57 & 2.58 & 3.11 & 3.68 & 4.97 & 6.48 & 8.28 & 10.55 & 12.01 & 13.90 & 24.02 & 375.68 & 517.93 & 799.97 \\
\hline & B-203 & Comb. & 0.47 & 1.01 & 1.57 & 2.58 & 3.11 & 3.68 & 4.97 & 6.48 & 8.28 & 10.55 & 12.01 & 13.90 & 24.02 & 375.68 & 517.93 & 799.97 \\
\hline & B-203 & Max & 0.47 & 1.01 & 1.57 & 2.58 & 3.11 & 3.68 & 4.97 & 6.48 & 8.28 & 10.55 & 12.01 & 13.90 & 24.02 & 375.68 & 517.93 & 799.97 \\
\hline & B-203 & Median & 0.47 & 1.01 & 1.57 & 2.58 & 3.11 & 3.68 & 4.97 & 6.48 & 8.28 & 10.55 & 12.01 & 13.90 & 24.02 & 375.68 & 517.93 & 799.97 \\
\hline & C-104 & Min & 0.20 & 0.30 & 0.41 & 0.66 & 0.79 & 0.91 & 1.14 & 1.42 & 1.88 & 2.65 & 3.08 & 3.60 & 4.88 & 6.18 & 14.44 & 21.77 \\
\hline & C-104 & Comb. & 0.26 & 0.41 & 0.66 & 1.00 & 1.14 & 1.38 & 2.27 & 3.18 & 4.89 & 8.15 & 10.79 & 14.33 & 26.47 & 37.66 & 79.00 & 113.77 \\
\hline & C-104 & Max & 0.26 & 0.45 & 0.68 & 1.03 & 1.35 & 1.74 & 2.89 & 4.90 & 8.18 & 13.94 & 18.20 & 23.45 & 38.95 & 50.20 & 79.00 & 120.83 \\
\hline & C-104 & Median & 0.21 & 0.35 & 0.53 & 0.95 & 1.14 & 1.37 & 2.10 & 3.57 & 5.87 & 10.12 & 13.60 & 17.33 & 25.38 & 32.07 & 44.47 & 68.87 \\
\hline & C-106 & Min & 0.18 & 0.27 & 0.45 & 1.09 & 1.44 & 1.90 & 2.92 & 3.93 & 5.17 & 6.95 & 8.16 & 9.63 & 13.53 & 16.60 & 22.82 & 34.03 \\
\hline & C-106 & Comb. & 0.18 & 0.27 & 0.45 & 1.09 & 1.44 & 1.90 & 2.92 & 3.93 & 5.17 & 6.95 & 8.16 & 9.63 & 13.53 & 16.60 & 22.82 & 34.03 \\
\hline & C-106 & Max & 0.18 & 0.27 & 0.45 & 1.09 & 1.44 & 1.90 & 2.92 & 3.93 & 5.17 & 6.95 & 8.16 & 9.63 & 13.53 & 16.60 & 22.82 & 34.03 \\
\hline
\end{tabular}


Table H.1. (contd)

\begin{tabular}{|c|c|c|c|c|c|c|c|c|c|c|c|c|c|c|c|c|c|c|}
\hline PSD Type & Tank & PSD & 0.01 & 0.05 & 0.1 & 0.2 & 0.25 & 0.3 & 0.4 & 0.5 & 0.6 & 0.7 & 0.75 & 0.8 & 0.9 & 0.95 & 0.99 & 1 \\
\hline & C-106 & Median & 0.18 & 0.27 & 0.45 & 1.09 & 1.44 & 1.90 & 2.92 & 3.93 & 5.17 & 6.95 & 8.16 & 9.63 & 13.53 & 16.60 & 22.82 & 34.03 \\
\hline & C-107 & Min & 0.62 & 0.78 & 0.87 & 1.10 & 1.23 & 1.42 & 1.91 & 2.56 & 3.27 & 4.18 & 4.73 & 5.34 & 6.81 & 7.69 & 9.85 & 14.26 \\
\hline & C-107 & Comb. & 0.65 & 0.84 & 0.97 & 1.28 & 1.42 & 1.81 & 2.56 & 3.31 & 4.39 & 5.76 & 6.50 & 6.88 & 9.98 & 11.59 & 15.79 & 23.05 \\
\hline & C-107 & Max & 0.65 & 0.84 & 0.97 & 1.31 & 1.52 & 1.81 & 2.56 & 3.62 & 4.68 & 6.05 & 6.88 & 7.88 & 10.34 & 11.85 & 15.79 & 22.99 \\
\hline & C-107 & Median & 0.64 & 0.81 & 0.92 & 1.20 & 1.37 & 1.63 & 2.29 & 3.23 & 4.19 & 5.44 & 6.20 & 7.08 & 9.24 & 10.55 & 13.77 & 20.11 \\
\hline & S-107 & Min & 0.32 & 0.66 & 0.97 & 1.54 & 1.96 & 2.50 & 3.84 & 5.47 & 7.59 & 10.36 & 12.19 & 14.41 & 20.79 & 28.25 & 54.92 & 76.65 \\
\hline & S-107 & Comb. & 0.43 & 0.84 & 1.12 & 1.75 & 2.21 & 2.81 & 4.55 & 6.22 & 7.91 & 10.79 & 13.06 & 15.91 & 25.91 & 44.63 & 68.54 & 102.56 \\
\hline & S-107 & Max & 0.43 & 0.84 & 1.12 & 1.75 & 2.21 & 2.81 & 4.55 & 6.22 & 7.91 & 10.79 & 13.06 & 15.91 & 25.91 & 44.63 & 68.54 & 102.97 \\
\hline & S-107 & Median & 0.38 & 0.75 & 1.05 & 1.64 & 2.08 & 2.65 & 4.19 & 5.85 & 7.75 & 10.58 & 12.63 & 15.16 & 23.35 & 36.44 & 61.73 & 89.81 \\
\hline & SX-108 & Min & 0.18 & 0.26 & 0.40 & 0.82 & 1.00 & 1.22 & 2.19 & 4.27 & 7.50 & 13.70 & 17.93 & 21.35 & 27.25 & 30.98 & 37.06 & 59.28 \\
\hline & \begin{tabular}{|l|} 
SX-108 \\
\end{tabular} & Comb. & 0.18 & 0.26 & 0.40 & 0.82 & 1.00 & 1.22 & 2.19 & 4.27 & 7.50 & 13.70 & 17.93 & 21.35 & 27.25 & 30.98 & 37.06 & 59.28 \\
\hline & \begin{tabular}{|l|} 
SX-108 \\
\end{tabular} & Max & 0.18 & 0.26 & 0.40 & 0.82 & 1.00 & 1.22 & 2.19 & 4.27 & 7.50 & 13.70 & 17.93 & 21.35 & 27.25 & 30.98 & 37.06 & 59.28 \\
\hline & SX-108 & Median & 0.18 & 0.26 & 0.40 & 0.82 & 1.00 & 1.22 & 2.19 & 4.27 & 7.50 & 13.70 & 17.93 & 21.35 & 27.25 & 30.98 & 37.06 & 59.28 \\
\hline & \begin{tabular}{|l|} 
SY-102 \\
\end{tabular} & Min & 0.23 & 0.45 & 0.60 & 1.03 & 1.14 & 1.28 & 1.71 & 2.38 & 3.01 & 3.74 & 4.26 & 4.95 & 7.37 & 9.79 & 14.72 & 21.45 \\
\hline & \begin{tabular}{|l|} 
SY-102 \\
\end{tabular} & Comb. & 0.44 & 0.57 & 0.80 & 1.11 & 1.40 & 1.68 & 2.36 & 3.20 & 4.11 & 5.29 & 6.00 & 7.21 & 10.23 & 12.80 & 18.90 & 27.61 \\
\hline & \begin{tabular}{|l|} 
SY-102 \\
\end{tabular} & Max & 0.44 & 0.65 & 0.80 & 1.11 & 1.50 & 1.75 & 2.36 & 3.20 & 4.17 & 5.43 & 6.20 & 7.43 & 10.68 & 12.80 & 18.90 & 27.68 \\
\hline & \begin{tabular}{|l|} 
SY-102 \\
\end{tabular} & Median & 0.23 & 0.45 & 0.61 & 1.05 & 1.40 & 1.65 & 2.30 & 3.20 & 4.11 & 5.29 & 6.00 & 7.06 & 9.77 & 11.50 & 16.30 & 23.78 \\
\hline & T-110 & Min & 0.34 & 0.61 & 1.07 & 2.37 & 3.12 & 3.94 & 5.88 & 8.46 & 12.44 & 20.02 & 25.75 & 32.41 & 50.82 & 70.27 & 427.45 & 531.43 \\
\hline & T-110 & Comb. & 0.34 & 0.61 & 1.07 & 2.37 & 3.12 & 3.94 & 5.88 & 8.46 & 12.44 & 20.02 & 25.75 & 32.41 & 50.82 & 70.27 & 427.45 & 531.43 \\
\hline & T-110 & Max & 0.34 & 0.61 & 1.07 & 2.37 & 3.12 & 3.94 & 5.88 & 8.46 & 12.44 & 20.02 & 25.75 & 32.41 & 50.82 & 70.27 & 427.45 & 531.43 \\
\hline & T-110 & Median & 0.34 & 0.61 & 1.07 & 2.37 & 3.12 & 3.94 & 5.88 & 8.46 & 12.44 & 20.02 & 25.75 & 32.41 & 50.82 & 70.27 & 427.45 & 531.43 \\
\hline & T-203 & Min & 0.44 & 0.87 & 1.37 & 2.30 & 2.80 & 3.34 & 4.57 & 6.01 & 7.70 & 9.78 & 11.09 & 12.72 & 20.11 & 401.33 & 505.83 & 799.58 \\
\hline & T-203 & Comb. & 0.44 & 0.87 & 1.37 & 2.30 & 2.80 & 3.34 & 4.57 & 6.01 & 7.70 & 9.78 & 11.09 & 12.72 & 20.11 & 401.33 & 505.83 & 799.58 \\
\hline & T-203 & Max & 0.44 & 0.87 & 1.37 & 2.30 & 2.80 & 3.34 & 4.57 & 6.01 & 7.70 & 9.78 & 11.09 & 12.72 & 20.11 & 401.33 & 505.83 & 799.58 \\
\hline & T-203 & Median & 0.44 & 0.87 & 1.37 & 2.30 & 2.80 & 3.34 & 4.57 & 6.01 & 7.70 & 9.78 & 11.09 & 12.72 & 20.11 & 401.33 & 505.83 & 799.58 \\
\hline & \begin{tabular}{|l|} 
T-204 \\
\end{tabular} & Min & 0.45 & 0.87 & 1.42 & 2.49 & 3.03 & 3.60 & 4.85 & 6.28 & 8.00 & 10.20 & 11.63 & 13.46 & 21.40 & 303.76 & 505.17 & 745.48 \\
\hline & T-204 & Comb. & 0.45 & 0.87 & 1.42 & 2.49 & 3.03 & 3.60 & 4.85 & 6.28 & 8.00 & 10.20 & 11.63 & 13.46 & 21.40 & 303.76 & 505.17 & 745.48 \\
\hline & T-204 & Max & 0.45 & 0.87 & 1.42 & 2.49 & 3.03 & 3.60 & 4.85 & 6.28 & 8.00 & 10.20 & 11.63 & 13.46 & 21.40 & 303.76 & 505.17 & 745.48 \\
\hline & T-204 & Median & 0.45 & 0.87 & 1.42 & 2.49 & 3.03 & 3.60 & 4.85 & 6.28 & 8.00 & 10.20 & 11.63 & 13.46 & 21.40 & 303.76 & 505.17 & 745.48 \\
\hline \multirow{4}{*}{\begin{tabular}{|l|} 
Sludge, \\
Flowing \\
Unsonicated
\end{tabular}} & AW-103 & Min & 0.18 & 0.30 & 0.51 & 1.20 & 1.31 & 1.45 & 1.78 & 2.31 & 3.07 & 4.06 & 4.67 & 5.39 & 7.49 & 9.63 & 14.90 & 20.65 \\
\hline & AW-103 & Comb. & 0.31 & 0.84 & 1.36 & 2.14 & 2.73 & 3.16 & 4.12 & 5.41 & 7.29 & 9.71 & 10.96 & 13.26 & 37.26 & 150.90 & 447.50 & 600.68 \\
\hline & AW-103 & Max & 1.30 & 1.90 & 2.30 & 3.38 & 4.10 & 4.99 & 7.41 & 11.00 & 31.36 & 89.38 & 150.90 & 185.30 & 279.41 & 343.10 & 447.50 & 738.41 \\
\hline & AW-103 & Median & 0.38 & 0.74 & 0.96 & 1.91 & 2.95 & 3.31 & 4.16 & 5.35 & 6.62 & 8.25 & 9.23 & 10.85 & 15.98 & 21.00 & 37.97 & 54.15 \\
\hline
\end{tabular}


Table H.1. (contd)

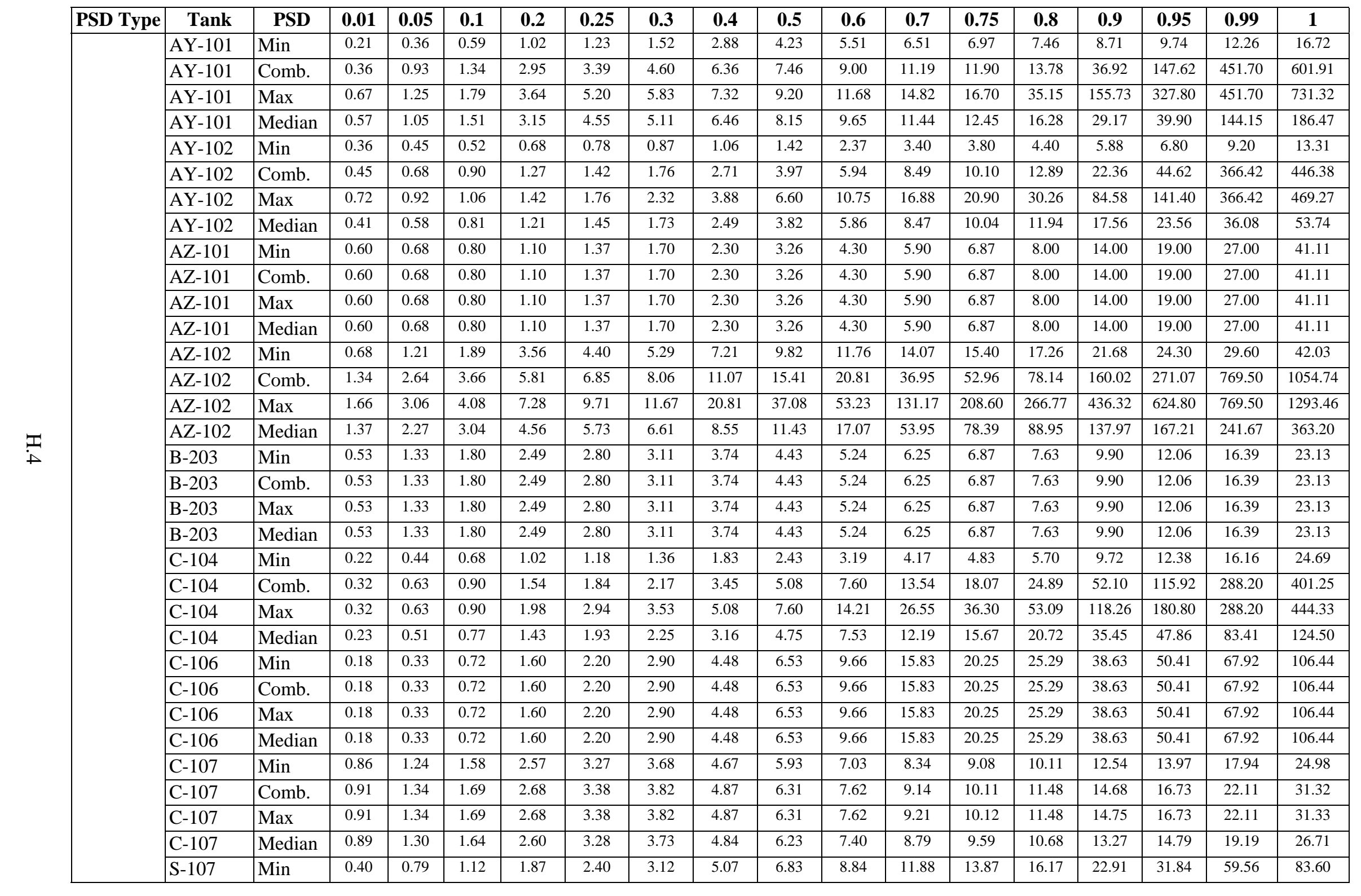


Table H.1. (contd)

\begin{tabular}{|c|c|c|c|c|c|c|c|c|c|c|c|c|c|c|c|c|c|c|}
\hline PSD Type & Tank & PSD & 0.01 & 0.05 & 0.1 & 0.2 & 0.25 & 0.3 & 0.4 & 0.5 & 0.6 & 0.7 & 0.75 & 0.8 & 0.9 & 0.95 & 0.99 & 1 \\
\hline & S-107 & Comb. & 0.43 & 0.86 & 1.18 & 1.97 & 2.64 & 3.41 & 5.24 & 7.52 & 10.79 & 14.75 & 16.17 & 21.90 & 32.27 & 45.72 & 81.70 & 117.73 \\
\hline & \begin{tabular}{|l|} 
S-107 \\
\end{tabular} & Max & 0.43 & 0.86 & 1.18 & 1.97 & 2.64 & 3.41 & 5.24 & 7.52 & 10.79 & 15.62 & 18.51 & 21.90 & 32.69 & 45.72 & 81.70 & 117.48 \\
\hline & S-107 & Median & 0.42 & 0.83 & 1.15 & 1.92 & 2.52 & 3.27 & 5.16 & 7.17 & 9.81 & 13.75 & 16.19 & 19.04 & 27.80 & 38.78 & 70.63 & 100.54 \\
\hline & SX-108 & Min & 0.41 & 0.67 & 0.90 & 1.37 & 1.79 & 2.55 & 5.27 & 8.51 & 13.92 & 20.74 & 23.20 & 25.67 & 30.44 & 33.77 & 40.31 & 60.06 \\
\hline & \begin{tabular}{|l|} 
SX-108 \\
\end{tabular} & Comb. & 0.41 & 0.67 & 0.90 & 1.37 & 1.79 & 2.55 & 5.27 & 8.51 & 13.92 & 20.74 & 23.20 & 25.67 & 30.44 & 33.77 & 40.31 & 60.06 \\
\hline & \begin{tabular}{|l|} 
SX-108 \\
\end{tabular} & Max & 0.41 & 0.67 & 0.90 & 1.37 & 1.79 & 2.55 & 5.27 & 8.51 & 13.92 & 20.74 & 23.20 & 25.67 & 30.44 & 33.77 & 40.31 & 60.06 \\
\hline & SX-108 & Median & 0.41 & 0.67 & 0.90 & 1.37 & 1.79 & 2.55 & 5.27 & 8.51 & 13.92 & 20.74 & 23.20 & 25.67 & 30.44 & 33.77 & 40.31 & 60.06 \\
\hline & \begin{tabular}{|l|} 
SY-102 \\
\end{tabular} & Min & 0.29 & 0.80 & 1.11 & 1.43 & 1.71 & 2.05 & 2.90 & 3.92 & 5.18 & 6.39 & 7.10 & 8.41 & 11.81 & 14.00 & 20.90 & 29.99 \\
\hline & \begin{tabular}{|l|} 
SY-102 \\
\end{tabular} & Comb. & 0.53 & 0.96 & 1.22 & 1.95 & 2.50 & 2.77 & 3.41 & 4.20 & 5.24 & 7.06 & 8.29 & 9.84 & 15.10 & 23.21 & 36.03 & 53.11 \\
\hline & \begin{tabular}{|l|} 
SY-102 \\
\end{tabular} & Max & 0.53 & 0.96 & 1.22 & 1.97 & 2.50 & 2.77 & 3.41 & 4.20 & 5.24 & 7.06 & 8.29 & 9.84 & 19.12 & 32.44 & 36.03 & 59.41 \\
\hline & \begin{tabular}{|l|} 
SY-102 \\
\end{tabular} & Median & 0.32 & 0.89 & 1.15 & 1.93 & 2.50 & 2.77 & 3.41 & 4.20 & 5.24 & 6.54 & 7.30 & 8.75 & 12.59 & 15.10 & 34.90 & 46.85 \\
\hline & T-110 & Min & 0.45 & 1.16 & 2.24 & 4.43 & 5.69 & 7.12 & 10.85 & 16.92 & 29.68 & 59.60 & 82.76 & 110.58 & 188.74 & 260.29 & 414.64 & 638.94 \\
\hline & T-110 & Comb. & 0.45 & 1.16 & 2.24 & 4.43 & 5.69 & 7.12 & 10.85 & 16.92 & 29.68 & 59.60 & 82.76 & 110.58 & 188.74 & 260.29 & 414.64 & 638.94 \\
\hline & T-110 & Max & 0.45 & 1.16 & 2.24 & 4.43 & 5.69 & 7.12 & 10.85 & 16.92 & 29.68 & 59.60 & 82.76 & 110.58 & 188.74 & 260.29 & 414.64 & 638.94 \\
\hline & T-110 & Median & 0.45 & 1.16 & 2.24 & 4.43 & 5.69 & 7.12 & 10.85 & 16.92 & 29.68 & 59.60 & 82.76 & 110.58 & 188.74 & 260.29 & 414.64 & 638.94 \\
\hline & T-203 & Min & 0.73 & 1.55 & 2.15 & 3.17 & 3.68 & 4.21 & 5.40 & 6.86 & 8.80 & 11.63 & 13.67 & 16.48 & 27.90 & 42.56 & 85.64 & 120.92 \\
\hline & T-203 & Comb. & 0.73 & 1.55 & 2.15 & 3.17 & 3.68 & 4.21 & 5.40 & 6.86 & 8.80 & 11.63 & 13.67 & 16.48 & 27.90 & 42.56 & 85.64 & 120.92 \\
\hline & T-203 & Max & 0.73 & 1.55 & 2.15 & 3.17 & 3.68 & 4.21 & 5.40 & 6.86 & 8.80 & 11.63 & 13.67 & 16.48 & 27.90 & 42.56 & 85.64 & 120.92 \\
\hline & T-203 & Median & 0.73 & 1.55 & 2.15 & 3.17 & 3.68 & 4.21 & 5.40 & 6.86 & 8.80 & 11.63 & 13.67 & 16.48 & 27.90 & 42.56 & 85.64 & 120.92 \\
\hline & T-204 & Min & 0.74 & 1.62 & 2.35 & 3.65 & 4.33 & 5.07 & 6.81 & 9.15 & 12.70 & 19.00 & 24.22 & 31.64 & 57.06 & 83.70 & 149.78 & 221.09 \\
\hline & T-204 & Comb. & 0.74 & 1.62 & 2.35 & 3.65 & 4.33 & 5.07 & 6.81 & 9.15 & 12.70 & 19.00 & 24.22 & 31.64 & 57.06 & 83.70 & 149.78 & 221.09 \\
\hline & T-204 & Max & 0.74 & 1.62 & 2.35 & 3.65 & 4.33 & 5.07 & 6.81 & 9.15 & 12.70 & 19.00 & 24.22 & 31.64 & 57.06 & 83.70 & 149.78 & 221.09 \\
\hline & T-204 & Median & 0.74 & 1.62 & 2.35 & 3.65 & 4.33 & 5.07 & 6.81 & 9.15 & 12.70 & 19.00 & 24.22 & 31.64 & 57.06 & 83.70 & 149.78 & 221.09 \\
\hline \multirow{10}{*}{$\begin{array}{l}\text { Sludge, No- } \\
\text { Flow } \\
\text { Unsonicated }\end{array}$} & C-107 & Min & 0.38 & 0.51 & 0.59 & 0.71 & 0.78 & 0.85 & 1.13 & 2.10 & 3.77 & 7.05 & 8.60 & 10.02 & 13.70 & 17.00 & 21.89 & 32.22 \\
\hline & C-107 & Comb. & 0.58 & 1.00 & 2.30 & 4.80 & 6.35 & 7.92 & 10.53 & 15.02 & 22.33 & 37.29 & 46.88 & 62.47 & 128.73 & 253.80 & 741.37 & 1004.29 \\
\hline & \begin{tabular}{|l|}
$\mathrm{C}-107$ \\
\end{tabular} & Max & 4.60 & 10.20 & 17.45 & 31.57 & 38.00 & 43.27 & 54.74 & 68.00 & 84.07 & 127.01 & 164.00 & 209.99 & 337.29 & 464.00 & 741.37 & 1106.73 \\
\hline & \begin{tabular}{|l|}
$\mathrm{C}-107$ \\
\end{tabular} & Median & 1.27 & 2.10 & 2.96 & 4.73 & 5.65 & 6.60 & 8.75 & 11.50 & 15.44 & 25.02 & 34.00 & 46.35 & 82.00 & 124.50 & 224.01 & 325.13 \\
\hline & AY-102 & Min & 0.41 & 0.71 & 1.03 & 1.74 & 2.06 & 2.38 & 3.12 & 4.11 & 5.46 & 7.03 & 7.92 & 8.98 & 12.39 & 15.00 & 21.85 & 30.71 \\
\hline & AY-102 & Comb. & 0.69 & 1.11 & 1.77 & 2.61 & 3.12 & 3.81 & 5.53 & 7.67 & 10.79 & 16.21 & 23.92 & 49.10 & 208.10 & 388.02 & 829.94 & 1214.09 \\
\hline & AY-102 & Max & 1.12 & 1.70 & 2.20 & 5.69 & 8.61 & 12.37 & 29.81 & 152.55 & 365.11 & 546.28 & 591.09 & 621.11 & 689.19 & 742.48 & 829.94 & 1268.34 \\
\hline & AY-102 & Median & 0.48 & 0.94 & 1.41 & 2.27 & 2.68 & 3.24 & 4.63 & 6.50 & 8.85 & 11.88 & 15.01 & 20.83 & 88.30 & 192.44 & 290.10 & 447.25 \\
\hline & C-104 & Min & 4.65 & 11.72 & 18.25 & 29.78 & 34.51 & 39.83 & 51.37 & 64.98 & 79.25 & 93.48 & 101.01 & 108.19 & 126.24 & 140.27 & 162.53 & 223.25 \\
\hline & C-104 & Comb. & 4.65 & 11.72 & 18.25 & 29.78 & 34.51 & 39.83 & 51.37 & 64.98 & 79.25 & 93.48 & 101.01 & 108.19 & 126.24 & 140.27 & 162.53 & 223.25 \\
\hline
\end{tabular}


Table H.1. (contd)

\begin{tabular}{|c|c|c|c|c|c|c|c|c|c|c|c|c|c|c|c|c|c|c|}
\hline PSD Type & Tank & PSD & 0.01 & 0.05 & 0.1 & 0.2 & 0.25 & 0.3 & 0.4 & 0.5 & 0.6 & 0.7 & 0.75 & 0.8 & 0.9 & 0.95 & 0.99 & 1 \\
\hline & C-104 & Max & 4.65 & 11.72 & 18.25 & 29.78 & 34.51 & 39.83 & 51.37 & 64.98 & 79.25 & 93.48 & 101.01 & 108.19 & 126.24 & 140.27 & 162.53 & 223.25 \\
\hline & C-104 & Median & 4.65 & 11.72 & 18.25 & 29.78 & 34.51 & 39.83 & 51.37 & 64.98 & 79.25 & 93.48 & 101.01 & 108.19 & 126.24 & 140.27 & 162.53 & 223.25 \\
\hline & \begin{tabular}{|l|} 
AZ-101 \\
\end{tabular} & Min & 0.30 & 1.10 & 1.41 & 2.04 & 2.56 & 3.04 & 3.62 & 4.28 & 4.98 & 5.74 & 6.21 & 6.89 & 9.84 & 10.69 & 12.73 & 18.14 \\
\hline & AZ-101 & Comb. & 1.25 & 2.57 & 4.01 & 6.06 & 7.28 & 8.66 & 12.29 & 18.72 & 32.44 & 60.98 & 105.15 & 235.18 & 520.24 & 629.65 & 858.48 & 1441.13 \\
\hline & AZ-101 & Max & 4.58 & 9.08 & 16.04 & 386.80 & 444.92 & 472.69 & 521.15 & 552.93 & 586.57 & 620.51 & 638.02 & 656.06 & 711.92 & 756.52 & 858.48 & 1347.58 \\
\hline & AZ-101 & Median & 2.49 & 4.35 & 5.40 & 7.44 & 8.67 & 10.19 & 14.88 & 22.74 & 29.03 & 57.72 & 66.59 & 73.45 & 95.54 & 130.16 & 171.83 & 247.98 \\
\hline & SY-102 & Min & 1.11 & 1.70 & 2.24 & 3.42 & 4.00 & 4.58 & 5.75 & 7.10 & 8.88 & 12.13 & 16.00 & 80.92 & 196.84 & 258.00 & 369.11 & 584.52 \\
\hline & SY-102 & Comb. & 1.84 & 3.25 & 4.51 & 7.21 & 8.53 & 10.78 & 20.32 & 55.00 & 90.68 & 138.87 & 196.84 & 233.51 & 346.00 & 463.50 & 816.12 & 1198.19 \\
\hline & \begin{tabular}{|l|} 
SY-102 \\
\end{tabular} & Max & 2.86 & 7.40 & 16.17 & 48.25 & 65.00 & 81.52 & 117.52 & 160.00 & 207.61 & 296.38 & 346.00 & 381.61 & 466.64 & 590.00 & 816.12 & 1191.93 \\
\hline & \begin{tabular}{|l|} 
SY-102 \\
\end{tabular} & Median & 2.03 & 3.60 & 4.66 & 6.62 & 7.80 & 9.47 & 14.68 & 29.00 & 60.60 & 94.82 & 119.50 & 152.44 & 254.88 & 316.00 & 424.19 & 701.93 \\
\hline & U-110 & Min & 0.82 & 1.26 & 1.80 & 2.90 & 3.43 & 3.96 & 4.84 & 5.47 & 7.25 & 9.76 & 12.57 & 15.39 & 26.29 & 45.20 & 60.32 & 84.77 \\
\hline & U-110 & Comb. & 0.93 & 1.75 & 2.79 & 4.75 & 5.18 & 6.36 & 9.76 & 15.39 & 26.29 & 39.37 & 44.55 & 46.47 & 54.30 & 60.90 & 66.48 & 100.03 \\
\hline & U-110 & Max & 1.54 & 4.88 & 7.59 & 14.96 & 19.11 & 23.03 & 31.43 & 39.37 & 44.55 & 47.74 & 49.43 & 51.21 & 57.38 & 63.20 & 66.48 & 94.26 \\
\hline & U-110 & Median & 1.18 & 3.07 & 4.70 & 8.93 & 11.27 & 13.50 & 18.13 & 22.42 & 25.90 & 28.75 & 31.00 & 33.30 & 41.84 & 54.20 & 63.40 & 89.51 \\
\hline & \begin{tabular}{|l|} 
AZ-102 \\
\end{tabular} & Min & 0.53 & 1.50 & 2.44 & 4.23 & 4.99 & 5.95 & 12.50 & 54.74 & 59.30 & 79.97 & 83.36 & 86.74 & 98.17 & 121.72 & 144.34 & 198.45 \\
\hline & AZ-102 & Comb. & 0.53 & 1.50 & 2.44 & 4.23 & 4.99 & 5.95 & 12.50 & 54.74 & 59.30 & 79.97 & 83.36 & 86.74 & 98.17 & 121.72 & 144.34 & 198.45 \\
\hline & AZ-102 & Max & 0.53 & 1.50 & 2.44 & 4.23 & 4.99 & 5.95 & 12.50 & 54.74 & 59.30 & 79.97 & 83.36 & 86.74 & 98.17 & 121.72 & 144.34 & 198.45 \\
\hline & AZ-102 & Median & 0.53 & 1.50 & 2.44 & 4.23 & 4.99 & 5.95 & 12.50 & 54.74 & 59.30 & 79.97 & 83.36 & 86.74 & 98.17 & 121.72 & 144.34 & 198.45 \\
\hline & B-201 & Min & 0.97 & 1.61 & 2.98 & 4.60 & 5.61 & 6.59 & 9.87 & 15.40 & 19.31 & 23.10 & 26.34 & 28.00 & 30.60 & 33.59 & 36.84 & 50.83 \\
\hline & B-201 & Comb. & 1.13 & 2.98 & 4.44 & 8.63 & 12.64 & 18.40 & 23.10 & 30.60 & 40.49 & 64.71 & 75.34 & 82.12 & 104.44 & 121.94 & 136.85 & 210.78 \\
\hline & B-201 & Max & 3.03 & 7.39 & 18.40 & 38.72 & 47.58 & 55.02 & 63.60 & 74.70 & 81.06 & 91.65 & 97.44 & 102.64 & 120.62 & 127.20 & 136.85 & 181.00 \\
\hline & B-201 & Median & 2.00 & 4.50 & 10.69 & 21.66 & 26.59 & 30.81 & 36.74 & 45.05 & 50.19 & 57.37 & 61.89 & 65.32 & 75.61 & 80.40 & 86.84 & 115.91 \\
\hline & B-111 & Min & 0.72 & 0.96 & 1.19 & 1.76 & 2.08 & 2.45 & 3.20 & 3.70 & 4.06 & 4.51 & 4.73 & 5.02 & 6.07 & 7.46 & 8.61 & 11.73 \\
\hline & B-111 & Comb. & 0.72 & 0.96 & 1.19 & 1.76 & 2.08 & 2.45 & 3.20 & 3.70 & 4.06 & 4.51 & 4.73 & 5.02 & 6.07 & 7.46 & 8.61 & 11.73 \\
\hline & B-111 & Max & 0.72 & 0.96 & 1.19 & 1.76 & 2.08 & 2.45 & 3.20 & 3.70 & 4.06 & 4.51 & 4.73 & 5.02 & 6.07 & 7.46 & 8.61 & 11.73 \\
\hline & B-111 & Median & 0.72 & 0.96 & 1.19 & 1.76 & 2.08 & 2.45 & 3.20 & 3.70 & 4.06 & 4.51 & 4.73 & 5.02 & 6.07 & 7.46 & 8.61 & 11.73 \\
\hline & BX-107 & Min & 0.65 & 0.94 & 1.25 & 2.00 & 2.66 & 3.11 & 3.89 & 4.71 & 5.47 & 6.53 & 7.24 & 9.08 & 12.08 & 13.90 & 14.96 & 22.84 \\
\hline & BX-107 & Comb. & 0.65 & 0.94 & 1.25 & 2.00 & 2.66 & 3.11 & 3.89 & 4.71 & 5.47 & 6.53 & 7.24 & 9.08 & 12.08 & 13.90 & 14.96 & 22.84 \\
\hline & BX-107 & Max & 0.65 & 0.94 & 1.25 & 2.00 & 2.66 & 3.11 & 3.89 & 4.71 & 5.47 & 6.53 & 7.24 & 9.08 & 12.08 & 13.90 & 14.96 & 22.84 \\
\hline & BX-107 & Median & 0.65 & 0.94 & 1.25 & 2.00 & 2.66 & 3.11 & 3.89 & 4.71 & 5.47 & 6.53 & 7.24 & 9.08 & 12.08 & 13.90 & 14.96 & 22.84 \\
\hline & C-103 & Min & 0.48 & 0.62 & 0.69 & 0.81 & 0.86 & 0.90 & 0.97 & 1.07 & 1.11 & 1.18 & 1.21 & 1.26 & 1.35 & 1.45 & 1.62 & 1.95 \\
\hline & \begin{tabular}{|l|}
$\mathrm{C}-103$ \\
\end{tabular} & Comb. & 0.48 & 0.62 & 0.69 & 0.81 & 0.86 & 0.90 & 0.97 & 1.07 & 1.11 & 1.18 & 1.21 & 1.26 & 1.35 & 1.45 & 1.62 & 1.95 \\
\hline & C-103 & Max & 0.48 & 0.62 & 0.69 & 0.81 & 0.86 & 0.90 & 0.97 & 1.07 & 1.11 & 1.18 & 1.21 & 1.26 & 1.35 & 1.45 & 1.62 & 1.95 \\
\hline
\end{tabular}


Table H.1. (contd)

\begin{tabular}{|c|c|c|c|c|c|c|c|c|c|c|c|c|c|c|c|c|c|c|}
\hline PSD Type & Tank & PSD & 0.01 & 0.05 & 0.1 & 0.2 & 0.25 & 0.3 & 0.4 & 0.5 & 0.6 & 0.7 & 0.75 & 0.8 & 0.9 & 0.95 & 0.99 & 1 \\
\hline & C-103 & Median & 0.48 & 0.62 & 0.69 & 0.81 & 0.86 & 0.90 & 0.97 & 1.07 & 1.11 & 1.18 & 1.21 & 1.26 & 1.35 & 1.45 & 1.62 & 1.95 \\
\hline & T-104 & Min & 0.64 & 0.90 & 1.27 & 2.39 & 2.92 & 3.35 & 3.90 & 4.48 & 4.99 & 5.82 & 6.22 & 6.59 & 8.32 & 10.43 & 11.67 & 16.55 \\
\hline & T-104 & Comb. & 0.64 & 0.90 & 1.27 & 2.39 & 2.92 & 3.35 & 3.90 & 4.48 & 4.99 & 5.82 & 6.22 & 6.59 & 8.32 & 10.43 & 11.67 & 16.55 \\
\hline & T-104 & Max & 0.64 & 0.90 & 1.27 & 2.39 & 2.92 & 3.35 & 3.90 & 4.48 & 4.99 & 5.82 & 6.22 & 6.59 & 8.32 & 10.43 & 11.67 & 16.55 \\
\hline & T-104 & Median & 0.64 & 0.90 & 1.27 & 2.39 & 2.92 & 3.35 & 3.90 & 4.48 & 4.99 & 5.82 & 6.22 & 6.59 & 8.32 & 10.43 & 11.67 & 16.55 \\
\hline & T-111 & Min & 1.04 & 1.70 & 2.34 & 3.08 & 3.39 & 3.60 & 4.06 & 4.44 & 4.88 & 5.39 & 5.71 & 6.07 & 7.65 & 9.56 & 11.67 & 16.06 \\
\hline & T-111 & Comb. & 1.04 & 1.70 & 2.34 & 3.08 & 3.39 & 3.60 & 4.06 & 4.44 & 4.88 & 5.39 & 5.71 & 6.07 & 7.65 & 9.56 & 11.67 & 16.06 \\
\hline & T-111 & Max & 1.04 & 1.70 & 2.34 & 3.08 & 3.39 & 3.60 & 4.06 & 4.44 & 4.88 & 5.39 & 5.71 & 6.07 & 7.65 & 9.56 & 11.67 & 16.06 \\
\hline & T-111 & Median & 1.04 & 1.70 & 2.34 & 3.08 & 3.39 & 3.60 & 4.06 & 4.44 & 4.88 & 5.39 & 5.71 & 6.07 & 7.65 & 9.56 & 11.67 & 16.06 \\
\hline \multirow{24}{*}{\begin{tabular}{|l|} 
Saltcake, \\
Flowing \\
Sonicated
\end{tabular}} & AN-104 & Min & 0.49 & 0.52 & 0.57 & 0.66 & 0.71 & 0.77 & 0.91 & 1.05 & 1.19 & 1.34 & 1.43 & 1.53 & 1.83 & 2.19 & 4.06 & 5.23 \\
\hline & \begin{tabular}{|l|} 
AN-104 \\
\end{tabular} & Comb. & 0.50 & 0.57 & 0.66 & 0.89 & 1.03 & 1.15 & 1.35 & 1.70 & 2.17 & 4.10 & 4.64 & 6.09 & 10.24 & 12.44 & 16.02 & 25.63 \\
\hline & \begin{tabular}{|l} 
AN-104 \\
\end{tabular} & Max & 0.72 & 1.10 & 1.35 & 1.70 & 1.92 & 2.17 & 3.15 & 4.50 & 5.90 & 7.83 & 8.86 & 10.03 & 12.16 & 13.56 & 16.02 & 23.48 \\
\hline & \begin{tabular}{|l} 
AN-104 \\
\end{tabular} & Median & 0.61 & 0.81 & 0.96 & 1.18 & 1.32 & 1.47 & 2.03 & 2.77 & 3.54 & 4.59 & 5.15 & 5.78 & 7.00 & 7.88 & 10.04 & 14.36 \\
\hline & \begin{tabular}{|l|} 
AN-102 \\
\end{tabular} & Min & 0.19 & 0.27 & 0.32 & 0.40 & 0.44 & 0.47 & 0.54 & 0.61 & 0.68 & 0.70 & 0.71 & 0.73 & 0.75 & 0.77 & 4.85 & 5.64 \\
\hline & \begin{tabular}{|l|} 
AN-102 \\
\end{tabular} & Comb. & 0.26 & 0.37 & 0.45 & 0.63 & 0.70 & 0.78 & 1.03 & 1.52 & 2.74 & 5.24 & 7.92 & 10.40 & 18.73 & 40.74 & 803.73 & 948.99 \\
\hline & \begin{tabular}{|l|} 
AN-102 \\
\end{tabular} & Max & 0.31 & 0.96 & 5.25 & 8.59 & 10.04 & 11.67 & 16.47 & 42.68 & 317.07 & 439.00 & 481.03 & 523.09 & 616.84 & 678.09 & 803.73 & 1237.70 \\
\hline & \begin{tabular}{|l|} 
AN-102 \\
\end{tabular} & Median & 0.22 & 0.34 & 0.46 & 0.63 & 0.70 & 0.77 & 0.92 & 1.15 & 1.58 & 4.76 & 6.55 & 8.27 & 12.86 & 17.12 & 24.34 & 39.25 \\
\hline & \begin{tabular}{|l|} 
AN-107 \\
\end{tabular} & Min & 0.16 & 0.20 & 0.24 & 0.29 & 0.32 & 0.34 & 0.40 & 0.46 & 0.50 & 0.80 & 0.94 & 1.13 & 1.74 & 2.42 & 4.15 & 5.96 \\
\hline & AN-107 & Comb. & 0.17 & 0.23 & 0.29 & 0.33 & 0.37 & 0.41 & 0.50 & 0.63 & 0.86 & 1.15 & 1.36 & 1.91 & 4.08 & 6.79 & 19.75 & 26.74 \\
\hline & \begin{tabular}{|l|} 
AN-107 \\
\end{tabular} & Max & 0.17 & 0.23 & 0.29 & 0.38 & 0.43 & 0.49 & 0.66 & 0.91 & 1.35 & 2.28 & 3.13 & 4.46 & 7.75 & 11.13 & 19.75 & 29.59 \\
\hline & \begin{tabular}{|l} 
AN-107 \\
\end{tabular} & Median & 0.16 & 0.21 & 0.25 & 0.31 & 0.34 & 0.37 & 0.45 & 0.54 & 0.68 & 0.90 & 1.07 & 1.32 & 2.35 & 3.81 & 7.60 & 10.96 \\
\hline & BY-104 & Min & 0.32 & 0.57 & 0.81 & 1.29 & 1.59 & 1.97 & 2.98 & 4.26 & 5.41 & 6.61 & 7.49 & 8.47 & 14.97 & 30.72 & 48.03 & 71.05 \\
\hline & \begin{tabular}{|l|} 
BY-104 \\
\end{tabular} & Comb. & 0.32 & 0.57 & 0.81 & 1.29 & 1.59 & 1.97 & 2.98 & 4.26 & 5.41 & 6.61 & 7.49 & 8.47 & 14.97 & 30.72 & 48.03 & 71.05 \\
\hline & BY-104 & Max & 0.32 & 0.57 & 0.81 & 1.29 & 1.59 & 1.97 & 2.98 & 4.26 & 5.41 & 6.61 & 7.49 & 8.47 & 14.97 & 30.72 & 48.03 & 71.05 \\
\hline & BY-104 & Median & 0.32 & 0.57 & 0.81 & 1.29 & 1.59 & 1.97 & 2.98 & 4.26 & 5.41 & 6.61 & 7.49 & 8.47 & 14.97 & 30.72 & 48.03 & 71.05 \\
\hline & \begin{tabular}{|l|} 
BY-108 \\
\end{tabular} & Min & 0.20 & 0.28 & 0.39 & 0.72 & 0.87 & 1.05 & 1.40 & 2.01 & 2.99 & 4.29 & 5.25 & 6.42 & 11.06 & 15.87 & 28.64 & 41.80 \\
\hline & \begin{tabular}{|l|} 
BY-108 \\
\end{tabular} & Comb. & 0.20 & 0.28 & 0.39 & 0.72 & 0.87 & 1.05 & 1.40 & 2.01 & 2.99 & 4.29 & 5.25 & 6.42 & 11.06 & 15.87 & 28.64 & 41.80 \\
\hline & BY-108 & Max & 0.20 & 0.28 & 0.39 & 0.72 & 0.87 & 1.05 & 1.40 & 2.01 & 2.99 & 4.29 & 5.25 & 6.42 & 11.06 & 15.87 & 28.64 & 41.80 \\
\hline & \begin{tabular}{|l|} 
BY-108 \\
\end{tabular} & Median & 0.20 & 0.28 & 0.39 & 0.72 & 0.87 & 1.05 & 1.40 & 2.01 & 2.99 & 4.29 & 5.25 & 6.42 & 11.06 & 15.87 & 28.64 & 41.80 \\
\hline & BY-110 & Min & 0.24 & 0.38 & 0.51 & 0.78 & 0.96 & 1.17 & 1.46 & 1.88 & 3.38 & 4.35 & 5.07 & 5.91 & 12.58 & 21.24 & 34.58 & 51.93 \\
\hline & BY-110 & Comb. & 0.24 & 0.38 & 0.51 & 0.78 & 0.96 & 1.17 & 1.46 & 1.88 & 3.38 & 4.35 & 5.07 & 5.91 & 12.58 & 21.24 & 34.58 & 51.93 \\
\hline & BY-110 & Max & 0.24 & 0.38 & 0.51 & 0.78 & 0.96 & 1.17 & 1.46 & 1.88 & 3.38 & 4.35 & 5.07 & 5.91 & 12.58 & 21.24 & 34.58 & 51.93 \\
\hline & BY-110 & Median & 0.24 & 0.38 & 0.51 & 0.78 & 0.96 & 1.17 & 1.46 & 1.88 & 3.38 & 4.35 & 5.07 & 5.91 & 12.58 & 21.24 & 34.58 & 51.93 \\
\hline
\end{tabular}


Table H.1. (contd)

\begin{tabular}{|c|c|c|c|c|c|c|c|c|c|c|c|c|c|c|c|c|c|c|}
\hline PSD Type & Tank & PSD & 0.01 & 0.05 & 0.1 & 0.2 & 0.25 & 0.3 & 0.4 & 0.5 & 0.6 & 0.7 & 0.75 & 0.8 & 0.9 & 0.95 & 0.99 & 1 \\
\hline & S-104 & Min & 0.13 & 0.17 & 0.22 & 0.30 & 0.36 & 0.44 & 0.71 & 1.19 & 2.19 & 3.69 & 4.66 & 5.89 & 9.61 & 14.28 & 25.71 & 37.88 \\
\hline & S-104 & Comb. & 0.13 & 0.17 & 0.22 & 0.30 & 0.36 & 0.44 & 0.71 & 1.19 & 2.19 & 3.69 & 4.66 & 5.89 & 9.61 & 14.28 & 25.71 & 37.88 \\
\hline & S-104 & Max & 0.13 & 0.17 & 0.22 & 0.30 & 0.36 & 0.44 & 0.71 & 1.19 & 2.19 & 3.69 & 4.66 & 5.89 & 9.61 & 14.28 & 25.71 & 37.88 \\
\hline & S-104 & Median & 0.13 & 0.17 & 0.22 & 0.30 & 0.36 & 0.44 & 0.71 & 1.19 & 2.19 & 3.69 & 4.66 & 5.89 & 9.61 & 14.28 & 25.71 & 37.88 \\
\hline \multirow{28}{*}{$\begin{array}{l}\text { Saltcake, } \\
\text { Flowing } \\
\text { Unsonicated }\end{array}$} & AN-104 & Min & 0.71 & 0.78 & 0.88 & 1.04 & 1.10 & 1.16 & 1.26 & 1.36 & 1.45 & 1.56 & 1.62 & 1.70 & 1.94 & 2.34 & 4.75 & 5.88 \\
\hline & AN-104 & Comb. & 0.72 & 0.88 & 1.02 & 1.23 & 1.31 & 1.36 & 1.56 & 1.68 & 1.94 & 2.88 & 3.88 & 4.78 & 5.88 & 8.21 & 15.23 & 21.54 \\
\hline & AN-104 & Max & 0.91 & 1.19 & 1.36 & 1.57 & 1.68 & 1.80 & 2.19 & 3.41 & 4.36 & 5.00 & 5.35 & 5.72 & 7.35 & 11.66 & 15.23 & 21.90 \\
\hline & AN-104 & Median & 0.81 & 0.99 & 1.12 & 1.30 & 1.39 & 1.48 & 1.73 & 2.38 & 2.90 & 3.28 & 3.49 & 3.71 & 4.65 & 7.00 & 9.99 & 13.89 \\
\hline & AN-102 & Min & 0.21 & 0.33 & 0.46 & 0.83 & 1.06 & 1.36 & 1.87 & 2.45 & 3.28 & 5.09 & 7.54 & 13.36 & 23.62 & 30.72 & 46.06 & 68.94 \\
\hline & AN-102 & Comb. & 0.29 & 0.57 & 0.89 & 1.72 & 2.45 & 3.17 & 6.97 & 13.36 & 21.74 & 40.91 & 54.34 & 100.42 & 366.52 & 513.18 & 839.79 & 1325.86 \\
\hline & AN-102 & Max & 0.45 & 0.89 & 2.05 & 13.82 & 29.25 & 70.33 & 191.20 & 278.39 & 353.21 & 423.26 & 459.62 & 497.97 & 596.13 & 676.92 & 839.79 & 1168.18 \\
\hline & AN-102 & Median & 0.25 & 0.47 & 0.81 & 1.57 & 2.19 & 2.97 & 5.51 & 9.23 & 14.35 & 21.74 & 26.72 & 33.05 & 53.34 & 76.81 & 214.65 & 296.88 \\
\hline & BY-104 & Min & 0.49 & 0.92 & 1.49 & 3.42 & 4.18 & 5.10 & 6.45 & 7.78 & 9.30 & 11.23 & 12.53 & 13.99 & 19.38 & 28.81 & 66.54 & 88.67 \\
\hline & BY-104 & Comb. & 0.49 & 0.92 & 1.49 & 3.42 & 4.18 & 5.10 & 6.45 & 7.78 & 9.30 & 11.23 & 12.53 & 13.99 & 19.38 & 28.81 & 66.54 & 88.67 \\
\hline & BY-104 & Max & 0.49 & 0.92 & 1.49 & 3.42 & 4.18 & 5.10 & 6.45 & 7.78 & 9.30 & 11.23 & 12.53 & 13.99 & 19.38 & 28.81 & 66.54 & 88.67 \\
\hline & BY-104 & Median & 0.49 & 0.92 & 1.49 & 3.42 & 4.18 & 5.10 & 6.45 & 7.78 & 9.30 & 11.23 & 12.53 & 13.99 & 19.38 & 28.81 & 66.54 & 88.67 \\
\hline & BY-108 & Min & 0.21 & 0.42 & 0.76 & 1.14 & 1.33 & 1.54 & 2.31 & 3.56 & 5.17 & 7.40 & 9.00 & 10.95 & 17.20 & 23.23 & 33.37 & 50.83 \\
\hline & \begin{tabular}{|l|} 
BY -108 \\
\end{tabular} & Comb. & 0.21 & 0.42 & 0.76 & 1.14 & 1.33 & 1.54 & 2.31 & 3.56 & 5.17 & 7.40 & 9.00 & 10.95 & 17.20 & 23.23 & 33.37 & 50.83 \\
\hline & BY-108 & Max & 0.21 & 0.42 & 0.76 & 1.14 & 1.33 & 1.54 & 2.31 & 3.56 & 5.17 & 7.40 & 9.00 & 10.95 & 17.20 & 23.23 & 33.37 & 50.83 \\
\hline & BY-108 & Median & 0.21 & 0.42 & 0.76 & 1.14 & 1.33 & 1.54 & 2.31 & 3.56 & 5.17 & 7.40 & 9.00 & 10.95 & 17.20 & 23.23 & 33.37 & 50.83 \\
\hline & BY-110 & Min & 0.33 & 0.69 & 1.01 & 1.35 & 1.50 & 1.68 & 2.43 & 3.57 & 4.51 & 6.01 & 7.71 & 9.88 & 14.75 & 22.42 & 34.66 & 51.72 \\
\hline & BY-110 & Comb. & 0.33 & 0.69 & 1.01 & 1.35 & 1.50 & 1.68 & 2.43 & 3.57 & 4.51 & 6.01 & 7.71 & 9.88 & 14.75 & 22.42 & 34.66 & 51.72 \\
\hline & BY-110 & Max & 0.33 & 0.69 & 1.01 & 1.35 & 1.50 & 1.68 & 2.43 & 3.57 & 4.51 & 6.01 & 7.71 & 9.88 & 14.75 & 22.42 & 34.66 & 51.72 \\
\hline & BY-110 & Median & 0.33 & 0.69 & 1.01 & 1.35 & 1.50 & 1.68 & 2.43 & 3.57 & 4.51 & 6.01 & 7.71 & 9.88 & 14.75 & 22.42 & 34.66 & 51.72 \\
\hline & S-104 & Min & 0.20 & 0.28 & 0.44 & 0.91 & 1.04 & 1.20 & 1.51 & 2.12 & 3.60 & 6.47 & 8.35 & 10.77 & 20.40 & 28.64 & 46.59 & 71.18 \\
\hline & S-104 & Comb. & 0.20 & 0.28 & 0.44 & 0.91 & 1.04 & 1.20 & 1.51 & 2.12 & 3.60 & 6.47 & 8.35 & 10.77 & 20.40 & 28.64 & 46.59 & 71.18 \\
\hline & S-104 & Max & 0.20 & 0.28 & 0.44 & 0.91 & 1.04 & 1.20 & 1.51 & 2.12 & 3.60 & 6.47 & 8.35 & 10.77 & 20.40 & 28.64 & 46.59 & 71.18 \\
\hline & S-104 & Median & 0.20 & 0.28 & 0.44 & 0.91 & 1.04 & 1.20 & 1.51 & 2.12 & 3.60 & 6.47 & 8.35 & 10.77 & 20.40 & 28.64 & 46.59 & 71.18 \\
\hline & S-101 & Min & 0.71 & 0.82 & 0.98 & 1.42 & 1.81 & 2.30 & 3.49 & 4.53 & 5.88 & 9.30 & 10.87 & 12.71 & 15.47 & 17.91 & 27.93 & 40.06 \\
\hline & S-101 & Comb. & 0.71 & 0.82 & 0.98 & 1.42 & 1.81 & 2.30 & 3.49 & 4.53 & 5.88 & 9.30 & 10.87 & 12.71 & 15.47 & 17.91 & 27.93 & 40.06 \\
\hline & S-101 & Max & 0.71 & 0.82 & 0.98 & 1.42 & 1.81 & 2.30 & 3.49 & 4.53 & 5.88 & 9.30 & 10.87 & 12.71 & 15.47 & 17.91 & 27.93 & 40.06 \\
\hline & S-101 & Median & 0.71 & 0.82 & 0.98 & 1.42 & 1.81 & 2.30 & 3.49 & 4.53 & 5.88 & 9.30 & 10.87 & 12.71 & 15.47 & 17.91 & 27.93 & 40.06 \\
\hline Saltcake, No- & SY-101 & Min & 0.21 & 0.64 & 0.75 & 0.97 & 1.08 & 1.20 & 1.35 & 1.50 & 1.89 & 2.29 & 2.74 & 3.19 & 4.09 & 4.54 & 4.90 & 7.44 \\
\hline
\end{tabular}


Table H.1. (contd)

\begin{tabular}{|c|c|c|c|c|c|c|c|c|c|c|c|c|c|c|c|c|c|c|}
\hline PSD Type & Tank & PSD & 0.01 & 0.05 & 0.1 & 0.2 & 0.25 & 0.3 & 0.4 & 0.5 & 0.6 & 0.7 & 0.75 & 0.8 & 0.9 & 0.95 & 0.99 & 1 \\
\hline \multirow{27}{*}{\begin{tabular}{|l|} 
Flow \\
Unsonicated
\end{tabular}} & SY-101 & Comb. & 0.62 & 0.94 & 1.26 & 1.88 & 2.27 & 2.78 & 4.03 & 5.67 & 10.46 & 32.70 & 51.81 & 90.72 & 176.60 & 252.12 & 1006.49 & 1329.69 \\
\hline & SY-101 & Max & 6.26 & 14.21 & 39.58 & 81.61 & 95.46 & 111.67 & 139.29 & 183.65 & 209.78 & 228.01 & 235.25 & 242.71 & 896.70 & 956.10 & 1006.49 & 1795.52 \\
\hline & SY-101 & Median & 0.55 & 0.80 & 1.12 & 1.77 & 2.11 & 2.45 & 3.50 & 4.31 & 6.19 & 8.08 & 17.27 & 22.41 & 32.70 & 37.84 & 41.96 & 69.79 \\
\hline & S-104 & Min & 0.62 & 0.90 & 1.37 & 2.61 & 3.05 & 3.49 & 4.20 & 5.30 & 6.27 & 8.91 & 10.36 & 13.33 & 15.08 & 18.30 & 19.43 & 30.07 \\
\hline & S-104 & Comb. & 0.62 & 0.90 & 1.37 & 2.61 & 3.05 & 3.49 & 4.20 & 5.30 & 6.27 & 8.91 & 10.36 & 13.33 & 15.08 & 18.30 & 19.43 & 30.07 \\
\hline & S-104 & Max & 0.62 & 0.90 & 1.37 & 2.61 & 3.05 & 3.49 & 4.20 & 5.30 & 6.27 & 8.91 & 10.36 & 13.33 & 15.08 & 18.30 & 19.43 & 30.07 \\
\hline & S-104 & Median & 0.62 & 0.90 & 1.37 & 2.61 & 3.05 & 3.49 & 4.20 & 5.30 & 6.27 & 8.91 & 10.36 & 13.33 & 15.08 & 18.30 & 19.43 & 30.07 \\
\hline & SY-103 & Min & 0.73 & 1.04 & 1.35 & 2.54 & 3.14 & 3.66 & 4.53 & 5.73 & 7.12 & 12.56 & 15.55 & 18.06 & 22.47 & 24.95 & 29.30 & 45.62 \\
\hline & SY-103 & Comb. & 0.73 & 1.04 & 1.35 & 2.54 & 3.14 & 3.66 & 4.53 & 5.73 & 7.12 & 12.56 & 15.55 & 18.06 & 22.47 & 24.95 & 29.30 & 45.62 \\
\hline & SY-103 & Max & 0.73 & 1.04 & 1.35 & 2.54 & 3.14 & 3.66 & 4.53 & 5.73 & 7.12 & 12.56 & 15.55 & 18.06 & 22.47 & 24.95 & 29.30 & 45.62 \\
\hline & SY-103 & Median & 0.73 & 1.04 & 1.35 & 2.54 & 3.14 & 3.66 & 4.53 & 5.73 & 7.12 & 12.56 & 15.55 & 18.06 & 22.47 & 24.95 & 29.30 & 45.62 \\
\hline & AW-101 & Min & 11.84 & 44.77 & 82.30 & 144.46 & 190.49 & 217.58 & 258.49 & 314.06 & 371.74 & 411.64 & 427.67 & 444.27 & 488.84 & 514.13 & 839.17 & 1032.53 \\
\hline & AW-101 & Comb. & 11.84 & 44.77 & 82.30 & 144.46 & 190.49 & 217.58 & 258.49 & 314.06 & 371.74 & 411.64 & 427.67 & 444.27 & 488.84 & 514.13 & 839.17 & 1032.53 \\
\hline & AW-101 & Max & 11.84 & 44.77 & 82.30 & 144.46 & 190.49 & 217.58 & 258.49 & 314.06 & 371.74 & 411.64 & 427.67 & 444.27 & 488.84 & 514.13 & 839.17 & 1032.53 \\
\hline & AW-101 & Median & 11.84 & 44.77 & 82.30 & 144.46 & 190.49 & 217.58 & 258.49 & 314.06 & 371.74 & 411.64 & 427.67 & 444.27 & 488.84 & 514.13 & 839.17 & 1032.53 \\
\hline & BY-104 & Min & 0.00 & 1.18 & 2.62 & 3.65 & 4.16 & 4.68 & 7.84 & 11.00 & 20.45 & 29.90 & 36.41 & 42.91 & 55.91 & 62.42 & 67.62 & 108.54 \\
\hline & BY-104 & Comb. & 1.13 & 2.10 & 3.65 & 7.82 & 11.00 & 17.12 & 24.98 & 35.05 & 42.42 & 52.16 & 56.34 & 67.69 & 79.03 & 97.22 & 130.82 & 184.58 \\
\hline & BY-104 & Max & 1.69 & 2.10 & 6.54 & 17.25 & 22.61 & 27.97 & 35.36 & 42.76 & 56.34 & 69.92 & 80.42 & 90.92 & 111.92 & 122.42 & 130.82 & 195.02 \\
\hline & BY-104 & Median & 0.56 & 1.67 & 4.21 & 9.29 & 11.83 & 14.37 & 23.37 & 32.37 & 38.56 & 44.76 & 51.46 & 58.17 & 71.58 & 78.28 & 83.65 & 121.84 \\
\hline & AN-105 & Min & 0.20 & 1.00 & 1.37 & 2.13 & 2.54 & 2.95 & 3.58 & 4.23 & 4.93 & 6.57 & 8.80 & 17.83 & 38.81 & 44.63 & 48.93 & 86.47 \\
\hline & AN-105 & Comb. & 0.22 & 1.03 & 1.39 & 2.16 & 2.73 & 3.25 & 4.23 & 4.93 & 6.57 & 17.83 & 20.20 & 38.81 & 50.34 & 63.72 & 69.30 & 119.90 \\
\hline & AN-105 & Max & 0.22 & 1.03 & 1.39 & 2.16 & 2.73 & 3.25 & 4.27 & 5.76 & 8.84 & 20.20 & 40.02 & 47.66 & 63.02 & 66.51 & 69.30 & 121.78 \\
\hline & AN-105 & Median & 0.21 & 1.02 & 1.38 & 2.14 & 2.64 & 3.10 & 3.93 & 4.99 & 6.89 & 13.39 & 24.41 & 32.74 & 50.91 & 55.57 & 59.11 & 104.12 \\
\hline & AN-104 & Min & 0.53 & 0.63 & 0.77 & 1.09 & 1.43 & 1.78 & 2.89 & 4.85 & 20.74 & 24.19 & 25.91 & 27.64 & 33.34 & 36.20 & 39.24 & 59.59 \\
\hline & AN-104 & Comb. & 0.53 & 0.63 & 0.77 & 1.09 & 1.43 & 1.78 & 2.89 & 4.85 & 20.74 & 24.19 & 25.91 & 27.64 & 33.34 & 36.20 & 39.24 & 59.59 \\
\hline & AN-104 & Max & 0.53 & 0.63 & 0.77 & 1.09 & 1.43 & 1.78 & 2.89 & 4.85 & 20.74 & 24.19 & 25.91 & 27.64 & 33.34 & 36.20 & 39.24 & 59.59 \\
\hline & $\mathrm{AN}-104$ & Median & 0.53 & 0.63 & 0.77 & 1.09 & 1.43 & 1.78 & 2.89 & 4.85 & 20.74 & 24.19 & 25.91 & 27.64 & 33.34 & 36.20 & 39.24 & 59.59 \\
\hline \multirow{4}{*}{$\begin{array}{l}\text { Saltcake, } \\
\text { Flowing } \\
\text { Sonicated, } \\
\text { Washed }\end{array}$} & AN-102 & Min & 0.76 & 1.07 & 1.32 & 1.93 & 2.29 & 2.62 & 3.25 & 3.86 & 4.54 & 5.39 & 5.98 & 6.72 & 9.96 & 15.81 & 34.29 & 46.47 \\
\hline & AN-102 & Comb. & 0.76 & 1.08 & 1.34 & 1.99 & 2.36 & 2.72 & 3.37 & 4.02 & 4.76 & 5.72 & 6.37 & 7.30 & 11.54 & 19.31 & 43.43 & 59.11 \\
\hline & AN-102 & Max & 0.76 & 1.08 & 1.34 & 1.99 & 2.36 & 2.72 & 3.37 & 4.02 & 4.76 & 5.72 & 6.37 & 7.30 & 11.54 & 19.31 & 43.43 & 59.11 \\
\hline & AN-102 & Median & 0.76 & 1.07 & 1.33 & 1.96 & 2.32 & 2.67 & 3.31 & 3.94 & 4.65 & 5.56 & 6.18 & 7.01 & 10.75 & 17.56 & 38.86 & 52.79 \\
\hline \multirow{2}{*}{$\begin{array}{l}\text { Saltcake, } \\
\text { Flowing }\end{array}$} & AN-102 & Min & 0.76 & 1.09 & 1.35 & 2.12 & 2.51 & 2.88 & 3.59 & 4.33 & 5.21 & 6.44 & 7.41 & 8.84 & 17.18 & 31.14 & 79.66 & 108.37 \\
\hline & AN-102 & Comb. & 0.82 & 1.12 & 1.43 & 2.23 & 2.67 & 3.04 & 3.80 & 4.65 & 5.76 & 7.48 & 8.84 & 12.76 & 36.06 & 86.06 & 310.93 & 409.13 \\
\hline
\end{tabular}


Table H.1. (contd)

\begin{tabular}{|c|c|c|c|c|c|c|c|c|c|c|c|c|c|c|c|c|c|c|}
\hline PSD Type & Tank & PSD & 0.01 & 0.05 & 0.1 & 0.2 & 0.25 & 0.3 & 0.4 & 0.5 & 0.6 & 0.7 & 0.75 & 0.8 & 0.9 & 0.95 & 0.99 & 1 \\
\hline \multirow{2}{*}{$\begin{array}{l}\text { Unsonicated, } \\
\text { Washed }\end{array}$} & AN-102 & Max & 0.82 & 1.12 & 1.43 & 2.23 & 2.67 & 3.04 & 3.80 & 4.65 & 5.76 & 7.55 & 9.22 & 12.76 & 40.98 & 111.65 & 310.93 & 424.47 \\
\hline & AN-102 & Median & 0.79 & 1.10 & 1.39 & 2.17 & 2.59 & 2.96 & 3.69 & 4.49 & 5.48 & 6.99 & 8.31 & 10.80 & 29.08 & 71.39 & 195.30 & 266.42 \\
\hline
\end{tabular}


Table H.2. Waste Type PSDs

\begin{tabular}{|c|c|c|c|c|c|c|c|c|c|c|c|c|c|c|c|c|c|c|c|}
\hline $\begin{array}{l}\text { PSD } \\
\text { Type }\end{array}$ & Waste Type & $\begin{array}{c}\text { PSD Data } \\
\text { Set(s) }\end{array}$ & PSD & 0.01 & 0.05 & 0.1 & 0.2 & 0.25 & 0.3 & 0.4 & 0.5 & 0.6 & 0.7 & 0.75 & 0.8 & 0.9 & 0.95 & 0.99 & 1 \\
\hline \multirow{32}{*}{$\begin{array}{l}\text { Sludge, } \\
\text { Flowing } \\
\text { Sonicated }\end{array}$} & 1C and $2 \mathrm{C}$ sludge & $\begin{array}{l}\text { M12 } \\
\text { Group 1 }\end{array}$ & Min & 0.41 & 0.65 & 0.92 & 1.49 & 1.81 & 2.17 & 3.14 & 4.54 & 6.42 & 8.97 & 10.69 & 12.91 & 21.34 & 33.55 & 67.72 & 141.59 \\
\hline & 1C and 2C sludge & & Comb. & 0.43 & 0.70 & 1.00 & 1.62 & 1.98 & 2.40 & 3.49 & 5.04 & 7.07 & 9.83 & 11.67 & 14.05 & 22.90 & 35.42 & 71.46 & 158.87 \\
\hline & 1C and 2C sludge & & Max & 0.43 & 0.70 & 1.00 & 1.62 & 1.98 & 2.40 & 3.49 & 5.04 & 7.07 & 9.83 & 11.67 & 14.05 & 22.90 & 35.42 & 71.46 & 158.87 \\
\hline & 1C and 2C sludge & & Median & 0.42 & 0.67 & 0.96 & 1.55 & 1.89 & 2.28 & 3.32 & 4.79 & 6.75 & 9.40 & 11.18 & 13.48 & 22.12 & 34.49 & 69.59 & 150.23 \\
\hline & 224 Post-1949 sludge & $\begin{array}{l}\text { B-203, T-203, } \\
\text { T-204 }\end{array}$ & Min & 0.44 & 0.87 & 1.37 & 2.30 & 2.80 & 3.34 & 4.57 & 6.01 & 7.70 & 9.78 & 11.09 & 12.72 & 20.11 & 303.76 & 505.17 & 745.48 \\
\hline & 224 Post-1949 sludge & & Comb. & 0.47 & 1.01 & 1.57 & 2.58 & 3.11 & 3.68 & 4.97 & 6.48 & 8.28 & 10.55 & 12.01 & 13.90 & 24.02 & 401.33 & 517.93 & 814.00 \\
\hline & 224 Post-1949 sludge & & Max & 0.47 & 1.01 & 1.57 & 2.58 & 3.11 & 3.68 & 4.97 & 6.48 & 8.28 & 10.55 & 12.01 & 13.90 & 24.02 & 401.33 & 517.93 & 799.97 \\
\hline & 224 Post-1949 sludge & & Median & 0.45 & 0.87 & 1.42 & 2.49 & 3.03 & 3.60 & 4.85 & 6.28 & 8.00 & 10.20 & 11.63 & 13.46 & 21.40 & 375.68 & 505.83 & 799.58 \\
\hline & 2C sludge & $\mathrm{T}-110$ & Min & 0.34 & 0.61 & 1.07 & 2.37 & 3.12 & 3.94 & 5.88 & 8.46 & 12.44 & 20.02 & 25.75 & 32.41 & 50.82 & \begin{tabular}{|l|}
70.27 \\
\end{tabular} & 427.45 & 531.43 \\
\hline & 2C sludge & & Comb. & 0.34 & 0.61 & 1.07 & 2.37 & 3.12 & 3.94 & 5.88 & 8.46 & 12.44 & 20.02 & 25.75 & 32.41 & 50.82 & 70.27 & 427.45 & 531.43 \\
\hline & 2C sludge & & Max & 0.34 & 0.61 & 1.07 & 2.37 & 3.12 & 3.94 & 5.88 & 8.46 & 12.44 & 20.02 & 25.75 & 32.41 & 50.82 & 70.27 & 427.45 & 531.43 \\
\hline & 2C sludge & & Median & 0.34 & 0.61 & 1.07 & 2.37 & 3.12 & 3.94 & 5.88 & 8.46 & 12.44 & 20.02 & 25.75 & 32.41 & 50.82 & 70.27 & 427.45 & 531.43 \\
\hline & AR sludge & C-106 & Min & 0.18 & 0.27 & 0.45 & 1.09 & 1.44 & 1.90 & 2.92 & 3.93 & 5.17 & 6.95 & 8.16 & 9.63 & 13.53 & 16.60 & 22.82 & 34.03 \\
\hline & AR sludge & & Comb. & 0.18 & 0.27 & 0.45 & 1.09 & 1.44 & 1.90 & 2.92 & 3.93 & 5.17 & 6.95 & 8.16 & 9.63 & 13.53 & 16.60 & 22.82 & 34.03 \\
\hline & AR sludge & & Max & 0.18 & 0.27 & 0.45 & 1.09 & 1.44 & 1.90 & 2.92 & 3.93 & 5.17 & 6.95 & 8.16 & 9.63 & 13.53 & 16.60 & 22.82 & 34.03 \\
\hline & AR sludge & & Median & 0.18 & 0.27 & 0.45 & 1.09 & 1.44 & 1.90 & 2.92 & 3.93 & 5.17 & 6.95 & 8.16 & 9.63 & 13.53 & 16.60 & 22.82 & 34.03 \\
\hline & BL sludge & AY-102 & Min & 0.31 & 0.41 & 0.48 & 0.59 & 0.66 & 0.75 & 0.93 & 1.14 & 1.71 & 3.04 & 3.60 & 4.25 & 5.93 & 7.00 & 9.40 & 14.17 \\
\hline & BL sludge & & Comb. & 0.41 & 0.56 & 0.74 & 0.98 & 1.08 & 1.28 & 2.17 & 3.32 & 4.98 & 7.00 & 8.66 & 10.41 & 15.41 & 21.06 & 39.59 & 56.54 \\
\hline & BL sludge & & Max & 0.60 & 0.75 & 0.83 & 1.03 & 1.28 & 1.67 & 2.52 & 4.17 & 6.88 & 10.19 & 12.21 & 14.58 & 21.24 & 27.22 & 39.59 & 59.77 \\
\hline & \begin{tabular}{|l|} 
BL sludge \\
\end{tabular} & & Median & 0.35 & 0.45 & 0.58 & 0.91 & 1.07 & 1.26 & 1.99 & 3.11 & 4.51 & 6.53 & 7.84 & 9.27 & 13.23 & \begin{tabular}{|l|}
16.45 \\
\end{tabular} & 23.85 & 35.97 \\
\hline & $\begin{array}{l}\text { CWP1 and CWP2 } \\
\text { sludge }\end{array}$ & M12 Group 3 & Min & 0.35 & 0.63 & 1.00 & 1.88 & 2.36 & 2.87 & 3.95 & 5.09 & 6.36 & 7.85 & 8.73 & 9.75 & 12.60 & 14.99 & 18.75 & 22.44 \\
\hline & $\begin{array}{l}\text { CWP1 and CWP2 } \\
\text { sludge }\end{array}$ & & Comb. & 0.35 & 0.67 & 1.10 & 2.11 & 2.69 & 3.31 & 4.51 & 5.71 & 7.07 & 8.73 & 9.75 & 10.99 & 14.61 & 17.31 & 21.70 & 25.18 \\
\hline & $\begin{array}{l}\text { CWP1 and CWP2 } \\
\text { sludge }\end{array}$ & & Max & 0.35 & 0.67 & 1.10 & 2.11 & 2.69 & 3.31 & 4.63 & 6.01 & 7.50 & 9.22 & 10.23 & 11.40 & 14.61 & 17.31 & 21.70 & 25.18 \\
\hline & $\begin{array}{l}\text { CWP1 and CWP2 } \\
\text { sludge }\end{array}$ & & Median & 0.35 & 0.65 & 1.05 & 2.00 & 2.53 & 3.09 & 4.29 & 5.55 & 6.93 & 8.54 & 9.48 & 10.57 & 13.61 & 16.15 & 20.23 & 23.81 \\
\hline & \begin{tabular}{|l|} 
CWR1 sludge \\
\end{tabular} & M12 Group 4 & Min & 0.36 & 0.79 & 1.55 & 3.42 & 4.28 & 5.06 & 6.53 & 8.01 & 9.65 & 11.61 & 12.81 & 14.25 & 18.77 & 23.87 & 60.11 & 112.47 \\
\hline & CWR1 sludge & & Comb. & 0.37 & 0.85 & 1.79 & 4.05 & 5.03 & 5.80 & 7.26 & 8.80 & 10.78 & 13.57 & 15.51 & 18.28 & 34.50 & 62.12 & 101.96 & 141.59 \\
\hline & \begin{tabular}{|l} 
CWR1 sludge \\
\end{tabular} & & Max & 0.37 & 0.85 & 1.79 & 4.05 & 5.03 & 5.94 & 7.73 & 9.67 & 12.05 & 15.51 & 18.28 & 23.14 & 50.55 & \begin{tabular}{|l|}
70.17 \\
\end{tabular} & 101.96 & 141.59 \\
\hline & CWR1 sludge & & Median & 0.36 & 0.82 & 1.67 & 3.74 & 4.65 & 5.50 & 7.13 & 8.84 & 10.85 & 13.56 & 15.54 & 18.70 & 34.66 & 47.02 & 81.03 & 127.03 \\
\hline & CWZr2 sludge & AW-103 & Min & 0.18 & 0.27 & 0.38 & 0.74 & 1.04 & 1.20 & 1.50 & 1.99 & 2.75 & 3.76 & 4.47 & 5.43 & 9.24 & 11.10 & 15.30 & 22.28 \\
\hline & CWZr2 sludge & & Comb. & 0.27 & 0.66 & 0.95 & 1.36 & 1.68 & 2.10 & 2.75 & 3.80 & 5.31 & 7.53 & 9.27 & 11.29 & 21.35 & 83.14 & 258.00 & 342.96 \\
\hline & \begin{tabular}{|l} 
CWZr2 sludge \\
\end{tabular} & & Max & 0.70 & 1.08 & 1.36 & 2.17 & 2.74 & 3.50 & 5.70 & 9.30 & 21.63 & 50.30 & 76.70 & 92.82 & 135.93 & 164.50 & 258.00 & 399.23 \\
\hline & CWZr2 sludge & & Median & 0.31 & 0.54 & 0.77 & 1.07 & 1.21 & 1.49 & 2.40 & 3.59 & 4.88 & 6.27 & 7.10 & 8.29 & 15.53 & 18.80 & 26.70 & 40.09 \\
\hline
\end{tabular}


Table H.2. (contd)

\begin{tabular}{|c|c|c|c|c|c|c|c|c|c|c|c|c|c|c|c|c|c|c|c|}
\hline $\begin{array}{l}\text { PSD } \\
\text { Type }\end{array}$ & Waste Type & $\begin{array}{c}\text { PSD Data } \\
\text { Set(s) }\end{array}$ & PSD & 0.01 & 0.05 & 0.1 & 0.2 & 0.25 & 0.3 & 0.4 & 0.5 & 0.6 & 0.7 & 0.75 & 0.8 & 0.9 & 0.95 & 0.99 & 1 \\
\hline & P3 sludge & AZ-101, AZ-102 & Min & 0.47 & 0.56 & 0.70 & 1.10 & 1.33 & 1.60 & 2.30 & 3.15 & 4.30 & 6.00 & 6.80 & 7.82 & 11.00 & \begin{tabular}{|l|}
14.01 \\
\end{tabular} & 20.79 & 29.96 \\
\hline & P3 sludge & & Comb. & 0.55 & 0.97 & 1.10 & 2.22 & 2.71 & 3.15 & 4.30 & 5.97 & 8.00 & 13.59 & 14.95 & 19.71 & 39.52 & 128.76 & 671.65 & 845.58 \\
\hline & P3 sludge & & Max & 1.39 & 1.98 & 3.13 & 5.77 & 7.43 & 9.43 & 14.77 & 23.48 & \begin{tabular}{|l|}
107.42 \\
\end{tabular} & 128.83 & 138.14 & 147.48 & 176.39 & 458.17 & 671.65 & 1026.23 \\
\hline & P3 sludge & & Median & 0.99 & 1.53 & 1.98 & 3.26 & 4.19 & 4.86 & 6.45 & 8.90 & \begin{tabular}{|l|}
11.58 \\
\end{tabular} & 14.60 & 16.40 & 20.97 & 34.26 & 49.23 & 222.90 & 273.07 \\
\hline & R1 (boiling) sludge & $\begin{array}{l}\text { SX-108, M12 } \\
\text { Group } 5\end{array}$ & Min & 0.18 & 0.26 & 0.40 & 0.82 & 1.00 & 1.22 & 2.19 & 4.27 & 7.50 & 10.02 & 11.14 & 12.49 & 16.58 & 20.82 & 31.99 & 50.24 \\
\hline & R1 (boiling) sludge & & Comb. & 0.24 & 0.66 & 1.00 & 2.20 & 3.02 & 4.12 & 5.72 & 7.46 & 9.03 & 11.86 & 13.44 & 14.68 & 21.75 & 30.98 & 61.74 & 112.47 \\
\hline & R1 (boiling) sludge & & Max & 0.44 & 0.96 & 1.88 & 3.97 & 4.76 & 5.47 & 6.82 & 8.20 & 9.73 & 13.70 & 17.93 & 21.35 & 27.25 & \begin{tabular}{|l|}
30.98 \\
\end{tabular} & 63.50 & 112.47 \\
\hline & R1 (boiling) sludge & & Median & 0.36 & 0.64 & 1.04 & 2.22 & 2.95 & 3.69 & 5.13 & 6.57 & 8.15 & 11.55 & 12.66 & 13.98 & 18.05 & 22.56 & 37.06 & 59.28 \\
\hline & Unidentified sludge & AY-101 & Min & 0.17 & 0.28 & 0.42 & 0.78 & 0.97 & 1.17 & 1.81 & 3.65 & 5.02 & 6.15 & 6.80 & 7.77 & 10.15 & 11.60 & 16.10 & 22.84 \\
\hline & Unidentified sludge & & Comb. & 0.26 & 0.49 & 0.77 & 1.28 & 1.70 & 2.12 & 3.65 & 5.46 & 6.61 & 7.84 & 8.47 & 9.90 & 12.41 & 14.60 & 19.90 & 28.01 \\
\hline & Unidentified sludge & & Max & 0.30 & 0.61 & 0.86 & 1.70 & 2.40 & 2.83 & 3.95 & 5.50 & 6.78 & 7.94 & 8.70 & 9.90 & 12.83 & 14.60 & 19.90 & 28.07 \\
\hline & Unidentified sludge & & Median & 0.24 & 0.45 & 0.65 & 1.16 & 1.50 & 1.81 & 3.00 & 4.78 & 6.17 & 7.51 & 8.14 & 8.83 & 10.84 & 12.86 & 18.09 & 25.10 \\
\hline \multirow{24}{*}{\begin{tabular}{|l|} 
Sludge, \\
Flowing \\
Unsonicated
\end{tabular}} & 224 Post-1949 sludge & $\begin{array}{l}\text { B-203, T-203, } \\
\text { T-204 }\end{array}$ & Min & 0.53 & 1.33 & 1.80 & 2.49 & 2.80 & 3.11 & 3.74 & 4.43 & 5.24 & 6.25 & 6.87 & 7.63 & 9.90 & 12.06 & 16.39 & 23.13 \\
\hline & 224 Post-1949 sludge & & Comb. & 0.74 & 1.62 & 2.35 & 3.15 & 3.67 & 3.87 & 5.15 & 6.40 & 7.96 & 10.40 & 12.25 & 16.04 & 30.10 & 56.54 & 149.78 & 203.75 \\
\hline & 224 Post-1949 sludge & & Max & 0.74 & 1.62 & 2.35 & 3.65 & 4.33 & 5.07 & 6.81 & 9.15 & 12.70 & 19.00 & 24.22 & 31.64 & 57.06 & 83.70 & 149.78 & 221.09 \\
\hline & 224 Post-1949 sludge & & Median & 0.73 & 1.55 & 2.15 & 3.17 & 3.68 & 4.21 & 5.40 & 6.86 & 8.80 & 11.63 & 13.67 & 16.48 & 27.90 & 42.56 & 85.64 & 120.92 \\
\hline & 2C sludge & $\mathrm{T}-110$ & Min & 0.45 & 1.16 & 2.24 & 4.43 & 5.69 & 7.12 & 10.85 & 16.92 & 29.68 & 59.60 & 82.76 & 110.58 & 188.74 & 260.29 & 414.64 & 638.94 \\
\hline & 2C sludge & & Comb. & 0.45 & 1.16 & 2.24 & 4.43 & 5.69 & 7.12 & 10.85 & 16.92 & 29.68 & 59.60 & 82.76 & 110.58 & 188.74 & 260.29 & 414.64 & 638.94 \\
\hline & 2C sludge & & Max & 0.45 & 1.16 & 2.24 & 4.43 & 5.69 & 7.12 & 10.85 & 16.92 & 29.68 & 59.60 & 82.76 & 110.58 & 188.74 & 260.29 & 414.64 & 638.94 \\
\hline & 2C sludge & & Median & 0.45 & 1.16 & 2.24 & 4.43 & 5.69 & 7.12 & 10.85 & 16.92 & 29.68 & 59.60 & 82.76 & 110.58 & 188.74 & 260.29 & 414.64 & 638.94 \\
\hline & AR sludge & C-106 & Min & 0.18 & 0.33 & 0.72 & 1.60 & 2.20 & 2.90 & 4.48 & 6.53 & 9.66 & 15.83 & 20.25 & 25.29 & 38.63 & 50.41 & 67.92 & 106.44 \\
\hline & AR sludge & & Comb. & 0.18 & 0.33 & 0.72 & 1.60 & 2.20 & 2.90 & 4.48 & 6.53 & 9.66 & 15.83 & 20.25 & 25.29 & 38.63 & 50.41 & 67.92 & 106.44 \\
\hline & AR sludge & & Max & 0.18 & 0.33 & 0.72 & 1.60 & 2.20 & 2.90 & 4.48 & 6.53 & 9.66 & 15.83 & 20.25 & 25.29 & 38.63 & 50.41 & 67.92 & 106.44 \\
\hline & AR sludge & & Median & 0.18 & 0.33 & 0.72 & 1.60 & 2.20 & 2.90 & 4.48 & 6.53 & 9.66 & 15.83 & 20.25 & 25.29 & 38.63 & 50.41 & 67.92 & 106.44 \\
\hline & BL sludge & AY-102 & Min & 0.36 & 0.45 & 0.52 & 0.68 & 0.78 & 0.87 & 1.06 & 1.42 & 2.37 & 3.40 & 3.80 & 4.40 & 5.88 & 6.80 & 9.20 & 13.31 \\
\hline & BL sludge & & Comb. & 0.45 & 0.68 & 0.90 & 1.27 & 1.42 & 1.76 & 2.71 & 3.97 & 5.94 & 8.49 & 10.10 & 12.89 & 22.36 & 44.62 & 366.42 & 446.38 \\
\hline & BL sludge & & Max & 0.72 & 0.92 & 1.06 & 1.42 & 1.76 & 2.32 & 3.88 & 6.60 & 10.75 & 16.88 & 20.90 & 30.26 & 84.58 & 141.40 & 366.42 & 469.27 \\
\hline & \begin{tabular}{|l|} 
BL sludge \\
\end{tabular} & & Median & 0.41 & 0.58 & 0.81 & 1.21 & 1.45 & 1.73 & 2.49 & 3.82 & 5.86 & 8.47 & 10.04 & 11.94 & 17.56 & 23.56 & 36.08 & 53.74 \\
\hline & CWZr2 sludge & AW-103 & Min & 0.18 & 0.30 & 0.51 & 1.20 & 1.31 & 1.45 & 1.78 & 2.31 & 3.07 & 4.06 & 4.67 & 5.39 & 7.49 & 9.63 & 14.90 & 20.65 \\
\hline & CWZr2 sludge & & Comb. & 0.31 & 0.84 & 1.36 & 2.14 & 2.73 & 3.16 & 4.12 & 5.41 & 7.29 & 9.71 & 10.96 & 13.26 & 37.26 & 150.90 & 447.50 & 600.68 \\
\hline & CWZr2 sludge & & Max & 1.30 & 1.90 & 2.30 & 3.38 & 4.10 & 4.99 & 7.41 & 11.00 & 31.36 & 89.38 & 150.90 & 185.30 & 279.41 & 343.10 & 447.50 & 738.41 \\
\hline & \begin{tabular}{|l|} 
CWZr2 sludge \\
\end{tabular} & & Median & 0.38 & 0.74 & 0.96 & 1.91 & 2.95 & 3.31 & 4.16 & 5.35 & 6.62 & 8.25 & 9.23 & 10.85 & 15.98 & 21.00 & 37.97 & 54.15 \\
\hline & P3 sludge & AZ-101, AZ-102 & Min & 0.60 & 0.68 & 0.80 & 1.10 & 1.37 & 1.70 & 2.30 & 3.26 & 4.30 & 5.90 & 6.87 & 8.00 & 14.00 & 19.00 & 27.00 & 41.11 \\
\hline & P3 sludge & & Comb. & 0.62 & 0.81 & 1.33 & 2.29 & 3.21 & 4.13 & 5.85 & 7.27 & 11.70 & 16.08 & 19.94 & 26.18 & 88.21 & 163.41 & 769.50 & 988.23 \\
\hline & \begin{tabular}{|l|} 
P3 sludge \\
\end{tabular} & & Max & 1.66 & 3.06 & 4.08 & 7.28 & 9.71 & 11.67 & 20.81 & 37.08 & 53.23 & 131.17 & 208.60 & 266.77 & 436.32 & 624.80 & 769.50 & 1293.46 \\
\hline & P3 sludge & & Median & 1.36 & 2.26 & 3.04 & 4.55 & 5.59 & 6.45 & 8.49 & 11.25 & 16.54 & 52.29 & 70.89 & 87.48 & 128.96 & 159.24 & 234.19 & 345.06 \\
\hline
\end{tabular}


Table H.2. (contd)

\begin{tabular}{|c|c|c|c|c|c|c|c|c|c|c|c|c|c|c|c|c|c|c|c|}
\hline $\begin{array}{l}\text { PSD } \\
\text { Type }\end{array}$ & Waste Type & $\begin{array}{l}\text { PSD Data } \\
\text { Set(s) }\end{array}$ & PSD & 0.01 & 0.05 & 0.1 & 0.2 & 0.25 & 0.3 & 0.4 & 0.5 & 0.6 & 0.7 & 0.75 & 0.8 & 0.9 & 0.95 & 0.99 & 1 \\
\hline & Unidentified sludge & AY-101 & Min & 0.21 & 0.36 & 0.59 & 1.02 & 1.23 & 1.52 & 2.88 & 4.23 & 5.51 & 6.51 & 6.97 & 7.46 & 8.71 & 9.74 & 12.26 & 16.72 \\
\hline & Unidentified sludge & & Comb. & 0.36 & 0.93 & 1.34 & 2.95 & 3.39 & 4.60 & 6.36 & 7.46 & 9.00 & 11.19 & 11.90 & 13.78 & 36.92 & 147.62 & 451.70 & 601.91 \\
\hline & Unidentified sludge & & Max & 0.67 & 1.25 & 1.79 & 3.64 & 5.20 & 5.83 & 7.32 & 9.20 & 11.68 & 14.82 & 16.70 & 35.15 & 155.73 & 327.80 & 451.70 & 731.32 \\
\hline & Unidentified sludge & & Median & 0.57 & 1.05 & 1.51 & 3.15 & 4.55 & 5.11 & 6.46 & 8.15 & 9.65 & 11.44 & 12.45 & 16.28 & 29.17 & 39.90 & 144.15 & 186.47 \\
\hline & R1 (boiling) sludge & $\begin{array}{l}\text { SX-108, M12 } \\
\text { Group } 5\end{array}$ & Min & 0.41 & 0.67 & 0.90 & 1.37 & 1.79 & 2.23 & 3.05 & 4.25 & 5.90 & 8.03 & 9.35 & 10.94 & 15.99 & 21.40 & 33.78 & 60.06 \\
\hline & R1 (boiling) sludge & & Comb. & 0.44 & 0.76 & 1.08 & 1.71 & 2.04 & 2.55 & 3.69 & 5.30 & 7.81 & 10.70 & 13.23 & 15.65 & 26.36 & 33.77 & 46.85 & 79.62 \\
\hline & R1 (boiling) sludge & & Max & 0.44 & 0.76 & 1.08 & 1.71 & 2.04 & 2.55 & 5.27 & 8.51 & 13.92 & 20.74 & 23.20 & 25.67 & 30.44 & 33.77 & 46.85 & 79.62 \\
\hline & R1 (boiling) sludge & & Median & 0.41 & 0.74 & 1.06 & 1.62 & 1.91 & 2.41 & 3.32 & 4.57 & 6.28 & 8.65 & 10.23 & 12.24 & 19.25 & 27.77 & 40.31 & 70.96 \\
\hline & 1C and 2C sludge & M12 Group 1 & Min & 0.42 & 0.73 & 1.09 & 1.83 & 2.26 & 2.76 & 4.03 & 5.72 & 8.00 & 11.33 & 13.66 & 16.74 & 27.65 & 39.73 & 67.19 & 112.47 \\
\hline & $1 \mathrm{C}$ and $2 \mathrm{C}$ sludge & & Comb. & 0.44 & 0.80 & 1.23 & 2.16 & 2.71 & 3.34 & 4.81 & 6.77 & 9.49 & 13.53 & 16.25 & 19.79 & 31.47 & 43.13 & 68.73 & 141.59 \\
\hline & 1C and 2C sludge & & Max & 0.44 & 0.80 & 1.23 & 2.16 & 2.71 & 3.36 & 4.93 & 6.93 & 9.62 & 13.53 & 16.25 & 19.79 & 31.47 & 43.13 & 68.73 & 141.59 \\
\hline & 1C and 2C sludge & & Median & 0.43 & 0.77 & 1.16 & 1.99 & 2.49 & 3.06 & 4.48 & 6.33 & 8.81 & 12.43 & 14.95 & 18.27 & 29.56 & 41.43 & 67.96 & 127.03 \\
\hline & \begin{tabular}{|l|} 
CWP1 and CWP2 \\
sludge \\
\end{tabular} & M12 Group 3 & Min & 0.35 & 0.65 & 1.04 & 1.96 & 2.48 & 3.07 & 4.43 & 6.02 & 7.85 & 10.05 & 11.36 & 12.89 & 17.20 & 20.90 & 27.07 & 35.57 \\
\hline & $\begin{array}{l}\text { CWP1 and CWP2 } \\
\text { sludge }\end{array}$ & & Comb. & 0.36 & 0.70 & 1.17 & 2.27 & 2.93 & 3.70 & 5.19 & 6.90 & 8.89 & 11.36 & 12.89 & 14.76 & 20.90 & 25.95 & 33.31 & 39.91 \\
\hline & $\begin{array}{l}\text { CWP1 and CWP2 } \\
\text { sludge }\end{array}$ & & Max & 0.36 & 0.70 & 1.17 & 2.27 & 2.93 & 3.70 & 5.53 & 7.66 & 10.03 & 12.79 & 14.40 & 16.28 & 21.50 & 25.95 & 33.31 & 39.91 \\
\hline & $\begin{array}{l}\text { CWP1 and CWP2 } \\
\text { sludge }\end{array}$ & & Median & 0.35 & 0.67 & 1.11 & 2.11 & 2.71 & 3.39 & 4.98 & 6.84 & 8.94 & 11.42 & 12.88 & 14.58 & 19.35 & 23.42 & 30.19 & 37.74 \\
\hline & \begin{tabular}{|l|} 
CWR1 sludge \\
\end{tabular} & M12 Group 4 & Min & 0.38 & 0.95 & 2.09 & 4.88 & 6.16 & 7.38 & 9.79 & 12.38 & 15.38 & 19.18 & 21.59 & 24.56 & 34.12 & 44.35 & 72.46 & 158.87 \\
\hline & CWR1 sludge & & Comb. & 0.41 & 1.32 & 3.18 & 6.16 & 7.38 & 8.58 & 12.11 & 15.38 & 20.16 & 28.46 & 34.12 & 44.35 & 70.36 & \begin{tabular}{|l|}
86.19 \\
\end{tabular} & 130.38 & 282.51 \\
\hline & CWR1 sludge & & Max & 0.41 & 1.32 & 3.18 & 6.90 & 8.55 & 10.25 & 14.24 & 20.16 & 30.52 & 44.69 & 52.35 & 60.70 & 82.57 & 100.64 & 130.38 & 282.51 \\
\hline & CWR1 sludge & & Median & 0.39 & 1.13 & 2.64 & 5.89 & 7.36 & 8.82 & 12.01 & 16.27 & 22.95 & 31.93 & 36.97 & 42.63 & 58.35 & 72.49 & 101.42 & 220.69 \\
\hline & TBP sludge & M12 Group 7 & Min & 0.34 & 0.59 & 1.05 & 2.41 & 2.96 & 3.47 & 4.46 & 5.53 & 6.82 & 8.53 & 9.68 & 11.17 & 16.62 & 24.57 & 68.28 & 141.59 \\
\hline & TBP sludge & & Comb. & 0.40 & 0.70 & 1.14 & 2.96 & 3.96 & 4.58 & 6.13 & 8.17 & 11.17 & 19.06 & 38.96 & 57.44 & 92.45 & 141.59 & 241.25 & 632.46 \\
\hline & TBP sludge & & Max & 0.40 & 0.70 & 1.14 & 4.58 & 5.80 & 6.94 & 9.68 & 19.06 & 48.53 & 66.75 & 77.13 & 89.37 & 126.34 & 163.80 & 267.07 & 632.46 \\
\hline & TBP sludge & & Median & 0.37 & 0.64 & 1.10 & 3.50 & 4.38 & 5.20 & 7.07 & 12.29 & 27.67 & 37.64 & 43.41 & 50.27 & 71.48 & 94.18 & 167.68 & 387.03 \\
\hline & PFeCN sludge & M12 Group 8 & Min & 0.37 & 0.73 & 1.26 & 2.42 & 3.04 & 3.71 & 5.28 & 7.47 & 11.08 & 13.22 & 14.61 & 16.39 & 23.04 & 34.91 & 86.19 & 158.87 \\
\hline & PFeCN sludge & & Comb. & 0.49 & 1.15 & 1.83 & 3.71 & 4.55 & 5.32 & 7.32 & 9.01 & 11.17 & 14.71 & 18.88 & 34.91 & 101.11 & 158.87 & 379.09 & 709.63 \\
\hline & PFeCN sludge & & Max & 0.49 & 1.43 & 3.54 & 5.32 & 6.01 & 6.67 & 7.98 & 9.41 & 11.17 & 53.38 & 78.26 & 97.09 & 142.63 & 184.37 & 444.00 & 709.63 \\
\hline & PFeCN sludge & & Median & 0.43 & 1.08 & 2.40 & 3.87 & 4.53 & 5.19 & 6.63 & 8.44 & 11.12 & 33.30 & 46.44 & 56.74 & 82.83 & 109.64 & 265.09 & 434.25 \\
\hline \multirow{6}{*}{$\begin{array}{l}\text { Sludge, No- } \\
\text { Flow } \\
\text { Unsonicated }\end{array}$} & 1C sludge & BX-107, T-104 & Min & 0.64 & 0.90 & 1.25 & 2.00 & 2.66 & 3.11 & 3.89 & 4.48 & 4.99 & 5.82 & 6.22 & 6.59 & 8.32 & 10.43 & 11.67 & 16.55 \\
\hline & 1C sludge & & Comb. & 0.65 & 0.94 & 1.27 & 2.39 & 2.92 & 3.35 & 3.90 & 4.71 & 5.47 & 6.42 & 6.78 & 8.00 & 11.82 & 12.87 & 14.96 & 22.26 \\
\hline & 1C sludge & & Max & 0.65 & 0.94 & 1.27 & 2.39 & 2.92 & 3.35 & 3.90 & 4.71 & 5.47 & 6.53 & 7.24 & 9.08 & 12.08 & 13.90 & 14.96 & 22.84 \\
\hline & 1C sludge & & Median & 0.65 & 0.92 & 1.26 & 2.19 & 2.79 & 3.23 & 3.89 & 4.60 & 5.23 & 6.18 & 6.73 & 7.84 & 10.20 & 12.16 & 13.32 & 19.69 \\
\hline & 224 Pre-1949 sludge & B-201 & Min & 0.97 & 1.61 & 2.98 & 4.60 & 5.61 & 6.59 & 9.87 & 15.40 & 19.31 & 23.10 & 26.34 & 28.00 & 30.60 & 33.59 & 36.84 & 50.83 \\
\hline & 224 Pre-1949 sludge & & Comb. & 1.13 & 2.98 & 4.44 & 8.63 & 12.64 & 18.40 & 23.10 & 30.60 & 40.49 & 64.71 & 75.34 & 82.12 & 104.44 & 121.94 & 136.85 & 210.78 \\
\hline
\end{tabular}


Table H.2. (contd)

\begin{tabular}{|c|c|c|c|c|c|c|c|c|c|c|c|c|c|c|c|c|c|c|c|}
\hline $\begin{array}{l}\text { PSD } \\
\text { Type }\end{array}$ & Waste Type & $\begin{array}{c}\text { PSD Data } \\
\text { Set(s) }\end{array}$ & PSD & 0.01 & 0.05 & 0.1 & 0.2 & 0.25 & 0.3 & 0.4 & 0.5 & 0.6 & 0.7 & 0.75 & 0.8 & 0.9 & 0.95 & 0.99 & 1 \\
\hline & 224 Pre-1949 sludge & & Max & 3.03 & 7.39 & 18.40 & 38.72 & 47.58 & 55.02 & 63.60 & 74.70 & 81.06 & 91.65 & 97.44 & 102.64 & 120.62 & 127.20 & 136.85 & 181.00 \\
\hline & 224 Pre-1949 sludge & & Median & 2.00 & 4.50 & 10.69 & 21.66 & 26.59 & 30.81 & 36.74 & 45.05 & 50.19 & 57.37 & 61.89 & 65.32 & 75.61 & 80.40 & 86.84 & 115.91 \\
\hline & 2C sludge & B-111 & Min & 0.72 & 0.96 & 1.19 & 1.76 & 2.08 & 2.45 & 3.20 & 3.70 & 4.06 & 4.51 & 4.73 & 5.02 & 6.07 & 7.46 & 8.61 & 11.73 \\
\hline & 2C sludge & & Comb. & 0.72 & 0.96 & 1.19 & 1.76 & 2.08 & 2.45 & 3.20 & 3.70 & 4.06 & 4.51 & 4.73 & 5.02 & 6.07 & 7.46 & 8.61 & 11.73 \\
\hline & 2C sludge & & Max & 0.72 & 0.96 & 1.19 & 1.76 & 2.08 & 2.45 & 3.20 & 3.70 & 4.06 & 4.51 & 4.73 & 5.02 & 6.07 & 7.46 & 8.61 & 11.73 \\
\hline & 2C sludge & & Median & 0.72 & 0.96 & 1.19 & 1.76 & 2.08 & 2.45 & 3.20 & 3.70 & 4.06 & 4.51 & 4.73 & 5.02 & 6.07 & 7.46 & 8.61 & 11.73 \\
\hline & BL sludge & AY-102 & Min & 0.41 & 0.71 & 1.03 & 1.74 & 2.06 & 2.38 & 3.12 & 4.11 & 5.46 & 7.03 & 7.92 & 8.98 & 12.39 & 15.00 & 21.85 & 30.71 \\
\hline & BL sludge & & Comb. & 0.69 & 1.11 & 1.77 & 2.61 & 3.12 & 3.81 & 5.53 & 7.67 & 10.79 & 16.21 & 23.92 & 49.10 & 208.10 & 388.02 & 829.94 & 1214.09 \\
\hline & BL sludge & & Max & 1.12 & 1.70 & 2.20 & 5.69 & 8.61 & 12.37 & 29.81 & 152.55 & 365.11 & 546.28 & 591.09 & 621.11 & 689.19 & 742.48 & 829.94 & 1268.34 \\
\hline & BL sludge & & Median & 0.48 & 0.94 & 1.41 & 2.27 & 2.68 & 3.24 & 4.63 & 6.50 & 8.85 & 11.88 & 15.01 & 20.83 & 88.30 & 192.44 & 290.10 & 447.25 \\
\hline & P3 sludge & AZ-101, AZ-102 & Min & 0.30 & 1.10 & 1.41 & 2.04 & 2.56 & 3.04 & 3.62 & 4.28 & 4.98 & 5.74 & 6.21 & 6.89 & 9.84 & 10.69 & 12.73 & 18.14 \\
\hline & P3 sludge & & Comb. & 1.25 & 2.16 & 4.01 & 4.99 & 5.95 & 8.66 & 12.50 & 34.06 & 58.76 & 78.78 & 84.84 & 96.90 & 240.90 & 521.41 & \begin{tabular}{|l|}
858.48 \\
\end{tabular} & 1287.51 \\
\hline & \begin{tabular}{|l|} 
P3 sludge \\
\end{tabular} & & Max & 4.58 & 9.08 & 16.04 & 386.80 & 444.92 & 472.69 & 521.15 & 552.93 & 586.57 & 620.51 & 638.02 & \begin{tabular}{|l|}
656.06 \\
\end{tabular} & 711.92 & 756.52 & \begin{tabular}{|l|}
858.48 \\
\end{tabular} & 1347.58 \\
\hline & P3 sludge & & Median & 2.48 & 4.34 & 5.36 & 7.25 & 8.38 & 9.65 & 13.69 & 24.14 & 38.76 & 59.31 & 69.51 & 77.35 & 96.85 & 125.94 & \begin{tabular}{|l|}
163.36 \\
\end{tabular} & 232.37 \\
\hline \multirow{16}{*}{$\begin{array}{l}\text { Saltcake, } \\
\text { Flowing } \\
\text { Sonicated }\end{array}$} & A2 saltcake & $\begin{array}{l}\text { AN-104, AN-102, } \\
\text { AN-107 }\end{array}$ & Min & 0.16 & 0.20 & 0.24 & 0.29 & 0.32 & 0.34 & 0.40 & 0.46 & 0.50 & 0.70 & 0.71 & 0.73 & 0.75 & 0.77 & 4.06 & 5.23 \\
\hline & A2 saltcake & & Comb. & 0.24 & 0.36 & 0.48 & 0.66 & 0.77 & 0.89 & 1.16 & 1.52 & 2.15 & 4.46 & 5.27 & 7.77 & 12.57 & 18.73 & 803.73 & 935.76 \\
\hline & A2 saltcake & & Max & 0.72 & 1.10 & 5.25 & 8.59 & 10.04 & 11.67 & $\mid 16.47$ & 42.68 & 317.07 & 439.00 & 481.03 & 523.09 & 616.84 & 678.09 & 803.73 & 1237.70 \\
\hline & A2 saltcake & & Median & 0.20 & 0.27 & 0.33 & 0.45 & 0.51 & 0.57 & 0.72 & 0.93 & 1.20 & 1.83 & 2.24 & 2.51 & 3.86 & 5.56 & 9.72 & 13.89 \\
\hline & BY saltcake & $\begin{array}{l}\text { BY-104, BY-108, } \\
\text { BY-110 }\end{array}$ & Min & 0.20 & 0.28 & 0.39 & 0.72 & 0.87 & 1.05 & 1.40 & 1.88 & 2.99 & 4.29 & 5.07 & 5.91 & 11.06 & 15.87 & 28.64 & 41.80 \\
\hline & BY saltcake & & Comb. & 0.26 & 0.40 & 0.68 & 1.00 & 1.25 & 1.42 & 1.98 & 3.11 & 4.31 & 5.61 & 6.55 & 8.47 & 14.97 & 29.53 & 48.03 & 71.47 \\
\hline & BY saltcake & & Max & 0.32 & 0.57 & 0.81 & 1.29 & 1.59 & 1.97 & 2.98 & 4.26 & 5.41 & 6.61 & 7.49 & 8.47 & 14.97 & 30.72 & 48.03 & 71.05 \\
\hline & BY saltcake & & Median & 0.24 & 0.38 & 0.51 & 0.78 & 0.96 & 1.17 & 1.46 & 2.01 & 3.38 & 4.35 & 5.25 & 6.42 & 12.58 & 21.24 & 34.58 & 51.93 \\
\hline & $\begin{array}{l}\text { BY, T1, and T2 } \\
\text { saltcake }\end{array}$ & M12 Group 2 & Min & 0.36 & 0.55 & 0.77 & 1.23 & 1.49 & 1.75 & 2.35 & 3.07 & 3.96 & 5.13 & 5.88 & 6.81 & 9.62 & 12.26 & 17.21 & 25.18 \\
\hline & \begin{tabular}{|l|}
$\begin{array}{l}\text { BY, T1, and T2 } \\
\text { saltcake }\end{array}$ \\
\end{tabular} & & Comb. & 0.37 & 0.59 & 0.84 & 1.37 & 1.66 & 1.96 & 2.65 & 3.48 & 4.50 & 5.88 & 6.81 & 7.89 & 11.08 & 14.03 & 19.48 & 25.18 \\
\hline & \begin{tabular}{|l|} 
BY, T1, and T2 \\
saltcake \\
\end{tabular} & & Max & 0.37 & 0.59 & 0.84 & 1.37 & 1.66 & 1.96 & 2.65 & 3.50 & 4.56 & 5.95 & 6.83 & 7.89 & 11.08 & 14.03 & 19.48 & 25.18 \\
\hline & \begin{tabular}{|l|} 
BY, T1, and T2 \\
saltcake
\end{tabular} & & Median & 0.37 & 0.57 & 0.80 & 1.30 & 1.57 & 1.86 & 2.50 & 3.28 & 4.26 & 5.54 & 6.35 & 7.35 & 10.35 & 13.14 & 18.35 & 25.18 \\
\hline & S1 and S2 saltcake & M12 Group 6 & Min & 0.36 & 0.57 & 0.76 & 1.13 & 1.34 & 1.55 & 2.05 & 2.69 & 3.56 & 4.81 & 5.67 & 6.74 & 9.96 & 12.69 & 17.40 & 25.18 \\
\hline & S1 and S2 saltcake & & Comb. & 0.39 & 0.57 & 0.82 & 1.34 & 1.55 & 1.79 & 2.55 & 3.56 & 4.81 & 6.87 & 8.33 & 10.19 & 25.18 & 59.18 & 94.87 & 141.59 \\
\hline & S1 and S2 saltcake & & Max & 0.39 & 0.57 & 0.82 & 1.37 & 1.70 & 2.09 & 3.12 & 4.65 & 6.87 & 10.19 & 12.82 & 18.09 & 59.18 & 75.90 & 101.20 & 141.59 \\
\hline & S1 and S2 saltcake & & Median & 0.37 & 0.57 & 0.79 & 1.25 & 1.52 & 1.82 & 2.59 & 3.67 & 5.21 & 7.50 & 9.24 & 12.42 & 34.57 & 44.29 & 59.30 & 83.39 \\
\hline \multirow{4}{*}{\begin{tabular}{|l} 
Saltcake, \\
Flowing \\
Unsonicated
\end{tabular}} & A2 saltcake & AN-104, AN-102 & Min & 0.21 & 0.33 & 0.46 & 0.83 & 1.06 & 1.16 & 1.26 & 1.36 & 1.45 & 1.56 & 1.62 & 1.70 & 1.94 & 2.34 & 4.75 & 5.88 \\
\hline & A2 saltcake & & Comb. & 0.38 & 0.82 & 1.02 & 1.31 & 1.43 & 1.57 & 1.93 & 3.30 & 5.04 & 10.18 & 13.90 & 22.93 & 176.49 & 391.10 & 839.79 & 1223.66 \\
\hline & \begin{tabular}{|l|} 
A2 saltcake \\
\end{tabular} & & Max & 0.91 & 1.19 & 2.05 & 13.82 & 29.25 & 70.33 & 191.20 & 278.39 & 353.21 & 423.26 & 459.62 & \begin{tabular}{|l|}
497.97 \\
\end{tabular} & 596.13 & 676.92 & \begin{tabular}{|l|}
839.79 \\
\end{tabular} & 1168.18 \\
\hline & A2 saltcake & & Median & 0.34 & 0.77 & 0.88 & 1.57 & 1.68 & 1.80 & 2.53 & 4.68 & 7.85 & 11.47 & 13.64 & 16.18 & 40.91 & 76.49 & 171.24 & 239.56 \\
\hline
\end{tabular}


Table H.2. (contd)

\begin{tabular}{|c|c|c|c|c|c|c|c|c|c|c|c|c|c|c|c|c|c|c|c|}
\hline $\begin{array}{l}\text { PSD } \\
\text { Type }\end{array}$ & Waste Type & $\begin{array}{c}\text { PSD Data } \\
\text { Set(s) }\end{array}$ & PSD & 0.01 & 0.05 & 0.1 & 0.2 & 0.25 & 0.3 & 0.4 & 0.5 & 0.6 & 0.7 & 0.75 & 0.8 & 0.9 & 0.95 & 0.99 & 1 \\
\hline & BY saltcake & $\begin{array}{l}\text { BY-104, BY-108, } \\
\text { BY-110 }\end{array}$ & Min & 0.21 & 0.42 & 0.76 & 1.14 & 1.33 & 1.54 & 2.31 & 3.56 & 4.51 & 6.01 & 7.71 & 9.88 & 14.75 & 22.42 & 33.37 & 50.83 \\
\hline & BY saltcake & & Comb. & 0.36 & 0.78 & 1.10 & 1.52 & 2.28 & 2.79 & 4.01 & 5.51 & 7.64 & 9.53 & 11.14 & 13.99 & 19.38 & 28.81 & 66.54 & 90.53 \\
\hline & BY saltcake & & Max & 0.49 & 0.92 & 1.49 & 3.42 & 4.18 & 5.10 & 6.45 & 7.78 & 9.30 & 11.23 & 12.53 & 13.99 & 19.38 & 28.81 & 66.54 & 88.67 \\
\hline & BY saltcake & & Median & 0.33 & 0.69 & 1.01 & 1.35 & 1.50 & 1.68 & 2.43 & 3.57 & 5.17 & 7.40 & 9.00 & 10.95 & 17.20 & 23.23 & 34.66 & 51.72 \\
\hline & $\begin{array}{l}\text { BY, T1, and T2 } \\
\text { saltcake }\end{array}$ & M12 Group 2 & Min & 0.39 & 0.60 & 0.83 & 1.28 & 1.53 & 1.79 & 2.38 & 3.17 & 4.23 & 5.69 & 6.61 & 7.74 & 11.10 & 14.15 & 19.76 & 28.25 \\
\hline & \begin{tabular}{|l|} 
BY, T1, and T2 \\
saltcake
\end{tabular} & & Comb. & 0.42 & 0.64 & 0.86 & 1.30 & 1.54 & 1.82 & 2.52 & 3.48 & 4.82 & 6.61 & 7.74 & 9.16 & 14.15 & 18.95 & 29.26 & 56.37 \\
\hline & \begin{tabular}{|l|} 
BY, T1, and T2 \\
saltcake \\
\end{tabular} & & Max & 0.42 & 0.64 & 0.86 & 1.30 & 1.54 & 1.82 & 2.52 & 3.48 & 4.82 & 6.69 & 7.91 & 9.42 & 14.18 & 18.95 & 29.59 & 56.37 \\
\hline & $\begin{array}{l}\text { BY, T1, and T2 } \\
\text { saltcake }\end{array}$ & & Median & 0.40 & 0.62 & 0.85 & 1.29 & 1.54 & 1.81 & 2.45 & 3.32 & 4.53 & 6.19 & 7.26 & 8.58 & 12.64 & 16.55 & 24.68 & 42.31 \\
\hline & \begin{tabular}{|l} 
S1 and S2 saltcake \\
\end{tabular} & M12 Group 6 & Min & 0.37 & 0.55 & 0.74 & 1.11 & 1.32 & 1.57 & 2.18 & 3.07 & 4.44 & 6.63 & 8.26 & \begin{tabular}{|l|}
10.46 \\
\end{tabular} & \begin{tabular}{|l|}
18.64 \\
\end{tabular} & \begin{tabular}{|l|}
57.77 \\
\end{tabular} & 79.35 & 112.47 \\
\hline & S1 and S2 saltcake & & Comb. & 0.39 & 0.58 & 0.77 & 1.16 & 1.37 & 1.61 & 2.21 & 3.14 & 4.77 & 7.62 & 9.77 & 12.87 & 39.95 & 74.52 & 121.20 & 158.87 \\
\hline & S1 and S2 saltcake & & Max & 0.39 & 0.58 & 0.77 & 1.16 & 1.37 & 1.61 & 2.21 & 3.14 & 4.77 & 7.62 & 9.77 & 12.87 & 39.95 & 74.52 & 124.10 & 158.87 \\
\hline & S1 and S2 saltcake & & Median & 0.38 & 0.57 & 0.75 & 1.13 & 1.35 & 1.59 & 2.20 & 3.11 & 4.60 & 7.13 & 9.01 & 11.66 & 29.30 & 66.14 & 101.73 & 135.67 \\
\hline \multirow{12}{*}{\begin{tabular}{|l|} 
Saltcake, \\
No-Flow \\
Unsonicated
\end{tabular}} & A2 saltcake & $\begin{array}{l}\text { AW-101, AN-105, } \\
\text { AN-104 }\end{array}$ & Min & 0.20 & 0.63 & 0.77 & 1.09 & 1.43 & 1.78 & 2.89 & 4.23 & 4.93 & 6.57 & 8.80 & 17.83 & 33.34 & 36.20 & 39.24 & 59.59 \\
\hline & \begin{tabular}{|l|} 
A2 saltcake \\
\end{tabular} & & Comb. & 0.55 & 1.03 & 1.39 & 2.83 & 3.98 & 4.76 & 20.30 & 27.58 & 44.67 & 136.40 & 228.00 & \begin{tabular}{|l|}
294.01 \\
\end{tabular} & 424.72 & 479.59 & 839.17 & 1278.76 \\
\hline & \begin{tabular}{|l|} 
A2 saltcake \\
\end{tabular} & & Max & 11.84 & 44.77 & 82.30 & 144.46 & 190.49 & 217.58 & 258.49 & 314.06 & 371.74 & 411.64 & 427.67 & 4444.27 & 488.84 & 514.13 & 839.17 & 1032.53 \\
\hline & A2 saltcake & & Median & 0.37 & 1.02 & 1.38 & 2.14 & 2.64 & 3.10 & 3.93 & 5.31 & 14.79 & 22.20 & 32.97 & 37.65 & 50.91 & 55.57 & 59.11 & 104.12 \\
\hline & BY saltcake & BY-104 & Min & 0.00 & 1.18 & 2.62 & 3.65 & 4.16 & 4.68 & 7.84 & 11.00 & 20.45 & 29.90 & 36.41 & 42.91 & 55.91 & 62.42 & 67.62 & 108.54 \\
\hline & BY saltcake & & Comb. & 1.13 & 2.10 & 3.65 & 7.82 & 11.00 & 17.12 & 24.98 & 35.05 & 42.42 & 52.16 & 56.34 & 67.69 & 79.03 & 97.22 & 130.82 & 184.58 \\
\hline & BY saltcake & & Max & 1.69 & 2.10 & 6.54 & 17.25 & 22.61 & 27.97 & 35.36 & 42.76 & 56.34 & 69.92 & 80.42 & 90.92 & 111.92 & 122.42 & 130.82 & 195.02 \\
\hline & BY saltcake & & Median & 0.56 & 1.67 & 4.21 & 9.29 & 11.83 & 14.37 & 23.37 & 32.37 & 38.56 & 44.76 & 51.46 & 58.17 & 71.58 & 78.28 & 83.65 & 121.84 \\
\hline & S2 saltcake & SY-101, SY-103 & Min & 0.21 & 0.64 & 0.75 & 0.97 & 1.08 & 1.20 & 1.35 & 1.50 & 1.89 & 2.29 & 2.74 & 3.19 & 4.09 & 4.54 & 4.90 & 7.44 \\
\hline & S2 saltcake & & Comb. & 0.72 & 1.02 & 1.35 & 2.52 & 2.90 & 3.44 & 4.49 & 5.77 & 10.46 & 15.42 & 21.21 & 23.09 & 77.32 & 172.50 & 1006.49 & 1263.74 \\
\hline & S2 saltcake & & Max & 6.26 & 14.21 & 39.58 & 81.61 & 95.46 & 111.67 & 139.29 & 183.65 & 209.78 & 228.01 & 235.25 & 242.71 & 896.70 & 956.10 & 1006.49 & 1795.52 \\
\hline & S2 saltcake & & Median & 0.55 & 0.80 & 1.12 & 1.79 & 2.14 & 2.50 & 3.78 & 4.56 & 6.78 & 9.00 & 15.55 & 19.93 & 32.70 & 36.60 & 39.72 & 63.95 \\
\hline \multirow{4}{*}{$\begin{array}{l}\text { Saltcake, } \\
\text { Flowing } \\
\text { Sonicated, } \\
\text { Washed }\end{array}$} & A2 saltcake & AN-102 & Min & 0.76 & 1.07 & 1.32 & 1.93 & 2.29 & 2.62 & 3.25 & 3.86 & 4.54 & 5.39 & 5.98 & 6.72 & 9.96 & 15.81 & 34.29 & 46.47 \\
\hline & A2 saltcake & & Comb. & 0.76 & 1.08 & 1.34 & 1.99 & 2.36 & 2.72 & 3.37 & 4.02 & 4.76 & 5.72 & 6.37 & 7.30 & \begin{tabular}{|l|}
11.54 \\
\end{tabular} & \begin{tabular}{|l|}
19.31 \\
\end{tabular} & 43.43 & 59.11 \\
\hline & A2 saltcake & & Max & 0.76 & 1.08 & 1.34 & 1.99 & 2.36 & 2.72 & 3.37 & 4.02 & 4.76 & 5.72 & 6.37 & 7.30 & 11.54 & 19.31 & 43.43 & 59.11 \\
\hline & A2 saltcake & & Median & 0.76 & 1.07 & 1.33 & 1.96 & 2.32 & 2.67 & 3.31 & 3.94 & 4.65 & 5.56 & 6.18 & 7.01 & \begin{tabular}{|l|}
10.75 \\
\end{tabular} & \begin{tabular}{|l|}
17.56 \\
\end{tabular} & 38.86 & 52.79 \\
\hline \multirow{4}{*}{\begin{tabular}{|l|} 
Saltcake, \\
Flowing \\
Unsonicated, \\
Washed \\
\end{tabular}} & A2 saltcake & AN-102 & Min & 0.76 & 1.09 & 1.35 & 2.12 & 2.51 & 2.88 & 3.59 & 4.33 & 5.21 & 6.44 & 7.41 & 8.84 & \begin{tabular}{|l|}
17.18 \\
\end{tabular} & \begin{tabular}{|l|}
31.14 \\
\end{tabular} & 79.66 & \begin{tabular}{|l|}
108.37 \\
\end{tabular} \\
\hline & A2 saltcake & & Comb. & 0.82 & 1.12 & 1.43 & 2.23 & 2.67 & 3.04 & 3.80 & 4.65 & 5.76 & 7.48 & 8.84 & 12.76 & 36.06 & 86.06 & 310.93 & 409.13 \\
\hline & A2 saltcake & & Max & 0.82 & 1.12 & 1.43 & 2.23 & 2.67 & 3.04 & 3.80 & 4.65 & 5.76 & 7.55 & 9.22 & 12.76 & 40.98 & 111.65 & 310.93 & 424.47 \\
\hline & A2 saltcake & & Median & 0.79 & 1.10 & 1.39 & 2.17 & 2.59 & 2.96 & 3.69 & 4.49 & 5.48 & 6.99 & 8.31 & 10.80 & 29.08 & \begin{tabular}{|l|}
71.39 \\
\end{tabular} & 195.30 & 266.42 \\
\hline
\end{tabular}


Table H.3. Composite PSDs

\begin{tabular}{|c|c|c|c|c|c|c|c|c|c|c|c|c|c|c|c|c|c|}
\hline PSD Type & PSD & 0.01 & 0.05 & 0.1 & 0.2 & 0.25 & 0.3 & 0.4 & 0.5 & 0.6 & 0.7 & 0.75 & 0.8 & 0.9 & 0.95 & 0.99 & 1 \\
\hline \multirow{4}{*}{$\begin{array}{l}\text { Sludge, } \\
\text { Flowing } \\
\text { Sonicated }\end{array}$} & Min & 0.17 & 0.26 & 0.38 & 0.59 & 0.66 & 0.75 & 0.93 & 1.14 & 1.71 & 2.65 & 3.08 & 3.60 & 4.88 & 6.18 & 9.40 & 14.17 \\
\hline & Comb. & 0.36 & 0.69 & 1.00 & 1.71 & 2.19 & 2.68 & 3.95 & 5.37 & 7.07 & 9.22 & 10.68 & 12.59 & 18.74 & 29.19 & 135.27 & 791.67 \\
\hline & Max & 1.39 & 1.98 & 3.13 & 5.77 & 7.43 & 9.43 & 14.77 & 23.48 & 107.42 & 128.83 & 138.14 & 147.48 & 176.39 & 458.17 & 671.65 & 1026.23 \\
\hline & Median & 0.44 & 0.73 & 0.97 & 1.60 & 1.96 & 2.36 & 3.14 & 4.90 & 6.86 & 9.44 & 11.09 & 12.69 & 17.38 & 22.56 & 32.93 & 50.24 \\
\hline \multirow{4}{*}{$\begin{array}{l}\text { Sludge, } \\
\text { Flowing } \\
\text { Unsonicated }\end{array}$} & Min & 0.18 & 0.30 & 0.51 & 0.68 & 0.78 & 0.87 & 1.06 & 1.42 & 2.37 & 3.40 & 3.80 & 4.40 & 5.88 & 6.80 & 9.20 & 13.31 \\
\hline & Comb. & 0.48 & 0.94 & 1.34 & 2.31 & 2.93 & 3.63 & 5.19 & 6.90 & 9.22 & 12.73 & 14.69 & 18.04 & 33.18 & 68.68 & 275.12 & 930.79 \\
\hline & Max & 1.66 & 3.06 & 4.08 & 7.28 & 9.71 & 11.67 & 20.81 & 37.08 & 53.23 & 131.17 & 208.60 & 266.77 & 436.32 & 624.80 & 769.50 & 1293.46 \\
\hline & Median & 0.44 & 0.88 & 1.15 & 2.16 & 2.81 & 3.41 & 4.85 & 6.53 & 8.84 & 11.88 & 14.19 & 17.06 & 25.07 & 32.44 & 59.56 & 83.60 \\
\hline \multirow{4}{*}{$\begin{array}{l}\text { Sludge, No- } \\
\text { Flow } \\
\text { Unsonicated }\end{array}$} & Min & 0.30 & 0.51 & 0.59 & 0.71 & 0.78 & 0.85 & 0.97 & 1.07 & 1.11 & 1.18 & 1.21 & 1.26 & 1.35 & 1.45 & 1.62 & 1.95 \\
\hline & Comb. & 0.62 & 0.88 & 1.11 & 1.76 & 2.58 & 3.34 & 4.70 & 6.22 & 9.08 & 15.20 & 29.77 & 47.67 & 98.74 & 162.41 & 858.48 & 1093.55 \\
\hline & Max & 4.65 & 11.72 & 18.40 & 386.80 & 444.92 & 472.69 & 521.15 & 552.93 & 586.57 & 620.51 & 638.02 & 656.06 & 711.92 & 756.52 & 858.48 & 1347.58 \\
\hline & Median & 1.05 & 1.70 & 2.36 & 4.23 & 4.99 & 5.95 & 8.12 & 10.93 & 14.52 & 23.10 & 32.00 & 42.02 & 89.36 & 140.27 & 245.59 & 356.36 \\
\hline \multirow{4}{*}{$\begin{array}{l}\text { Saltcake, } \\
\text { Flowing } \\
\text { Sonicated }\end{array}$} & Min & 0.13 & 0.17 & 0.22 & 0.29 & 0.32 & 0.34 & 0.40 & 0.46 & 0.50 & 0.70 & 0.71 & 0.73 & 0.75 & 0.77 & 4.06 & 5.23 \\
\hline & Comb. & 0.31 & 0.57 & 0.77 & 1.19 & 1.39 & 1.70 & 2.35 & 3.30 & 4.50 & 5.92 & 6.85 & 8.77 & 13.88 & 20.56 & 80.37 & 939.22 \\
\hline & Max & 0.72 & 1.10 & 5.25 & 8.59 & 10.04 & 11.67 & 16.47 & 42.68 & 317.07 & 439.00 & 481.03 & 523.09 & 616.84 & 678.09 & 803.73 & 1237.70 \\
\hline & Median & 0.20 & 0.30 & 0.39 & 0.58 & 0.64 & 0.73 & 0.89 & 1.05 & 1.34 & 3.18 & 3.98 & 5.17 & 8.68 & 11.69 & 17.31 & 25.18 \\
\hline \multirow{4}{*}{$\begin{array}{l}\text { Saltcake, } \\
\text { Flowing } \\
\text { Unsonicated }\end{array}$} & Min & 0.20 & 0.28 & 0.44 & 0.83 & 1.04 & 1.16 & 1.26 & 1.36 & 1.45 & 1.56 & 1.62 & 1.70 & 1.94 & 2.34 & 4.75 & 5.88 \\
\hline & Comb. & 0.41 & 0.72 & 0.92 & 1.34 & 1.55 & 1.83 & 2.58 & 3.66 & 5.36 & 7.72 & 9.30 & 11.16 & 19.15 & 39.02 & 175.63 & 1003.44 \\
\hline & Max & 0.91 & 1.19 & 2.05 & 13.82 & 29.25 & 70.33 & 191.20 & 278.39 & 353.21 & 423.26 & 459.62 & $\begin{array}{l}497.97 \\
\end{array}$ & 596.13 & $\begin{array}{l}676.92 \\
\end{array}$ & 839.79 & 1168.18 \\
\hline & Median & 0.38 & 0.62 & 0.85 & 1.29 & 1.52 & 1.73 & 2.41 & 3.52 & 4.79 & 7.05 & 8.68 & 11.83 & 19.01 & 28.73 & 46.32 & 70.06 \\
\hline \multirow{4}{*}{$\begin{array}{l}\text { Saltcake, } \\
\text { No-Flow } \\
\text { Unsonicated }\end{array}$} & Min & 0.00 & 0.63 & 0.75 & 0.97 & 1.08 & 1.20 & 1.35 & 1.50 & 1.89 & 2.29 & 2.74 & 3.19 & 4.09 & 4.54 & 4.90 & 7.44 \\
\hline & Comb. & 0.63 & 1.09 & 1.70 & 3.04 & 4.18 & 4.79 & 7.94 & 18.03 & 27.48 & 42.66 & 62.58 & 87.73 & 316.50 & 437.80 & 1006.49 & 1457.54 \\
\hline & Max & 11.84 & 44.77 & 82.30 & 144.46 & 190.49 & 217.58 & 258.49 & 314.06 & 371.74 & 411.64 & $\begin{array}{l}427.67 \\
\end{array}$ & 444.27 & 896.70 & 956.10 & 1006.49 & 1795.52 \\
\hline & Median & 0.55 & 1.02 & 1.37 & 2.35 & 2.89 & 3.37 & 4.35 & 5.75 & 10.28 & 18.65 & 24.90 & 33.20 & 47.36 & 53.53 & 58.27 & 97.50 \\
\hline
\end{tabular}


Table H.4. PSDs for M12 Waste Groups During Pretreatment Processes

\begin{tabular}{|c|c|c|c|c|c|c|c|c|c|c|c|c|c|}
\hline & \multicolumn{13}{|c|}{ \% Below Size } \\
\hline & \multicolumn{4}{|c|}{ Group 1/2 Mixture } & \multicolumn{3}{|c|}{ Group 3/4 Mixture } & \multicolumn{4}{|c|}{ Group 5/6 Mixture } & \multicolumn{2}{|c|}{ Group 8} \\
\hline $\begin{array}{l}\text { Particle } \\
\text { Size } \\
(\mu \mathrm{m})\end{array}$ & $\begin{array}{c}\text { High- } \\
\text { Solids, } \\
\text { Pre- } \\
\text { Leach }\end{array}$ & $\begin{array}{c}\text { Post- } \\
\text { Caustic } \\
\text { Leach }\end{array}$ & $\begin{array}{c}\text { Post- } \\
\text { Caustic } \\
\text { Leach, } \\
\text { Washed }\end{array}$ & $\begin{array}{c}\text { Post- } \\
\text { Oxidative } \\
\text { Leach, } \\
\text { Washed }\end{array}$ & $\begin{array}{c}\text { High- } \\
\text { Solids, } \\
\text { Pre- } \\
\text { Leach }\end{array}$ & $\begin{array}{c}\text { Post- } \\
\text { Caustic } \\
\text { Leach }\end{array}$ & $\begin{array}{c}\text { Post- } \\
\text { Caustic } \\
\text { Leach, } \\
\text { Washed }\end{array}$ & $\begin{array}{c}\text { High- } \\
\text { Solids, } \\
\text { Pre- } \\
\text { Leach }\end{array}$ & $\begin{array}{c}\text { Post- } \\
\text { Caustic } \\
\text { Leach }\end{array}$ & $\begin{array}{c}\text { Post- } \\
\text { Caustic } \\
\text { Leach, } \\
\text { Washed }\end{array}$ & $\begin{array}{c}\text { Post- } \\
\text { Oxidative } \\
\text { Leach, } \\
\text { Washed }\end{array}$ & $\begin{array}{l}\text { High- } \\
\text { Solids, } \\
\text { Pre- } \\
\text { Leach }\end{array}$ & $\begin{array}{c}\text { Post- } \\
\text { Caustic } \\
\text { Leach, } \\
\text { Washed }\end{array}$ \\
\hline 0.11 & - & - & 0.04 & 0.15 & - & - & - & - & - & - & - & - & - \\
\hline 0.13 & - & - & 0.77 & 1.35 & - & - & - & - & - & - & - & - & - \\
\hline 0.14 & - & - & 2.30 & 2.97 & - & - & - & - & - & - & - & - & - \\
\hline 0.16 & - & - & 3.65 & 4.46 & - & - & - & - & - & - & - & - & - \\
\hline 0.18 & - & - & 4.84 & 5.72 & - & - & - & - & - & - & - & - & - \\
\hline 0.20 & 0.02 & - & 5.81 & 6.67 & - & 0.01 & 0.02 & - & - & - & - & - & - \\
\hline 0.22 & 0.15 & - & 6.49 & 7.27 & - & 0.05 & 0.41 & 0.02 & - & - & - & - & - \\
\hline 0.25 & 0.35 & - & 6.82 & 7.48 & 0.10 & 0.20 & 0.71 & 0.16 & 0.08 & 0.05 & 0.22 & - & - \\
\hline 0.28 & 0.63 & - & 6.77 & 7.32 & 0.19 & 0.40 & 1.00 & 0.41 & 0.29 & 0.18 & 0.75 & 0.03 & - \\
\hline 0.32 & 0.89 & 0.05 & 6.36 & 6.83 & 0.36 & 0.55 & 1.31 & 0.66 & 0.76 & 0.54 & 1.37 & 0.09 & 0.04 \\
\hline 0.36 & $\begin{array}{l}1.17 \\
\end{array}$ & 0.28 & 5.65 & 6.09 & 0.48 & 0.71 & 1.59 & 0.96 & 1.12 & 0.94 & 2.11 & 0.12 & 0.08 \\
\hline 0.40 & 1.44 & 0.57 & 4.74 & 5.18 & 0.59 & 0.85 & 1.82 & 1.28 & 1.53 & 1.42 & 2.86 & 0.16 & 0.10 \\
\hline 0.45 & 1.68 & 0.93 & 3.77 & 4.23 & 0.70 & 0.97 & 2.00 & 1.58 & 1.92 & 1.91 & 3.56 & 0.18 & 0.12 \\
\hline 0.50 & $\begin{array}{ll}1.88 \\
\end{array}$ & 1.37 & 2.87 & 3.32 & 0.79 & $\begin{array}{l}1.07 \\
\end{array}$ & 2.12 & 1.86 & 2.28 & 2.40 & 4.15 & 0.21 & 0.13 \\
\hline 0.56 & 2.05 & 1.84 & 2.16 & 2.57 & 0.87 & 1.13 & 2.19 & 2.11 & 2.59 & 2.83 & 4.57 & 0.22 & 0.15 \\
\hline 0.63 & 2.18 & 2.36 & 1.68 & 2.02 & 0.95 & 1.18 & 2.20 & 2.32 & 2.88 & 3.20 & 4.83 & 0.23 & 0.15 \\
\hline 0.71 & 2.30 & 2.90 & 1.48 & 1.71 & 1.02 & 1.20 & 2.19 & 2.49 & 3.12 & 3.49 & 4.91 & 0.23 & 0.15 \\
\hline 0.80 & 2.40 & 3.46 & 1.49 & 1.59 & 1.10 & 1.22 & 2.15 & 2.62 & 3.33 & 3.72 & 4.86 & 0.23 & 0.14 \\
\hline 0.89 & 2.52 & 4.01 & 1.66 & 1.61 & 1.19 & 1.24 & 2.13 & 2.72 & 3.53 & 3.90 & 4.71 & 0.22 & 0.13 \\
\hline 1.00 & 2.67 & 4.54 & 1.89 & 1.71 & 1.30 & 1.27 & 2.12 & 2.80 & 3.72 & 4.05 & 4.53 & 0.21 & 0.12 \\
\hline 1.12 & 2.85 & 5.04 & 2.12 & 1.81 & 1.44 & 1.31 & 2.16 & 2.88 & 3.91 & 4.20 & 4.35 & 0.20 & 0.11 \\
\hline 1.26 & 3.08 & 5.46 & 2.29 & 1.87 & 1.61 & 1.39 & 2.24 & 2.95 & 4.10 & 4.36 & 4.21 & 0.20 & 0.11 \\
\hline 1.42 & 3.34 & 5.79 & 2.36 & 1.86 & 1.81 & 1.49 & 2.36 & 3.03 & 4.28 & 4.52 & 4.12 & 0.21 & 0.12 \\
\hline 1.59 & 3.63 & 5.97 & 2.34 & 1.76 & 2.04 & 1.62 & 2.50 & 3.11 & 4.44 & 4.68 & 4.08 & 0.25 & 0.14 \\
\hline $\begin{array}{l}1.78 \\
\end{array}$ & 3.91 & 5.99 & 2.23 & $\begin{array}{l}1.59 \\
\end{array}$ & 2.29 & 1.77 & 2.64 & 3.18 & 4.56 & 4.81 & 4.06 & $\begin{array}{l}0.33 \\
\end{array}$ & $\begin{array}{l}0.19 \\
\end{array}$ \\
\hline 2.00 & 4.15 & 5.84 & 2.07 & 1.39 & 2.56 & 1.94 & 2.76 & 3.25 & 4.63 & 4.90 & 4.03 & 0.44 & 0.28 \\
\hline
\end{tabular}


Table H.4. (contd)

\begin{tabular}{|c|c|c|c|c|c|c|c|c|c|c|c|c|c|}
\hline & \multicolumn{13}{|c|}{ \% Below Size } \\
\hline & \multicolumn{4}{|c|}{ Group 1/2 Mixture } & \multicolumn{3}{|c|}{ Group 3/4 Mixture } & \multicolumn{4}{|c|}{ Group 5/6 Mixture } & \multicolumn{2}{|c|}{ Group 8} \\
\hline $\begin{array}{c}\text { Particle } \\
\text { Size } \\
(\mu \mathrm{m})\end{array}$ & $\begin{array}{l}\text { High- } \\
\text { Solids, } \\
\text { Pre- } \\
\text { Leach }\end{array}$ & $\begin{array}{l}\text { Post- } \\
\text { Caustic } \\
\text { Leach }\end{array}$ & $\begin{array}{c}\text { Post- } \\
\text { Caustic } \\
\text { Leach, } \\
\text { Washed }\end{array}$ & $\begin{array}{c}\text { Post- } \\
\text { Oxidative } \\
\text { Leach, } \\
\text { Washed }\end{array}$ & $\begin{array}{l}\text { High- } \\
\text { Solids, } \\
\text { Pre- } \\
\text { Leach }\end{array}$ & $\begin{array}{c}\text { Post- } \\
\text { Caustic } \\
\text { Leach }\end{array}$ & $\begin{array}{c}\text { Post- } \\
\text { Caustic } \\
\text { Leach, } \\
\text { Washed }\end{array}$ & $\begin{array}{c}\text { High- } \\
\text { Solids, } \\
\text { Pre- } \\
\text { Leach }\end{array}$ & $\begin{array}{l}\text { Post- } \\
\text { Caustic } \\
\text { Leach }\end{array}$ & $\begin{array}{c}\text { Post- } \\
\text { Caustic } \\
\text { Leach, } \\
\text { Washed }\end{array}$ & $\begin{array}{c}\text { Post- } \\
\text { Oxidative } \\
\text { Leach, } \\
\text { Washed }\end{array}$ & $\begin{array}{c}\text { High- } \\
\text { Solids, } \\
\text { Pre- } \\
\text { Leach }\end{array}$ & $\begin{array}{c}\text { Post- } \\
\text { Caustic } \\
\text { Leach, } \\
\text { Washed }\end{array}$ \\
\hline 2.24 & 4.33 & 5.53 & 1.90 & 1.19 & 2.84 & 2.13 & 2.85 & 3.32 & 4.62 & 4.90 & 3.95 & 0.60 & 0.40 \\
\hline 2.52 & 4.44 & 5.09 & 1.76 & 1.02 & 3.12 & 2.31 & 2.90 & 3.39 & 4.54 & 4.82 & 3.81 & 0.82 & 0.56 \\
\hline 2.83 & 4.46 & 4.59 & 1.67 & 0.90 & 3.39 & 2.50 & 2.92 & 3.46 & 4.39 & 4.64 & 3.60 & 1.07 & 0.75 \\
\hline 3.17 & 4.40 & 4.06 & 1.62 & 0.84 & 3.64 & 2.69 & 2.91 & 3.54 & 4.18 & 4.37 & 3.31 & 1.38 & 0.97 \\
\hline 3.56 & 4.27 & 3.56 & 1.60 & 0.84 & 3.87 & 2.87 & 2.89 & 3.62 & 3.93 & 4.04 & 2.98 & 1.70 & 1.20 \\
\hline 3.99 & 4.08 & 3.10 & 1.58 & 0.86 & 4.09 & 3.05 & 2.88 & 3.72 & 3.65 & 3.64 & 2.61 & 2.03 & 1.42 \\
\hline 4.48 & 3.84 & 2.72 & 1.54 & 0.89 & 4.27 & 3.23 & 2.89 & 3.80 & 3.36 & 3.22 & 2.24 & 2.33 & 1.63 \\
\hline 5.02 & 3.58 & 2.41 & 1.40 & 0.89 & 4.43 & 3.42 & 2.93 & 3.87 & 3.05 & 2.79 & 1.88 & 2.60 & 1.79 \\
\hline 5.64 & 3.31 & 2.15 & 1.18 & 0.82 & 4.55 & 3.61 & 3.00 & 3.90 & 2.76 & 2.37 & 1.56 & 2.80 & 1.90 \\
\hline 6.32 & 3.03 & 1.92 & 0.80 & 0.69 & 4.64 & 3.81 & 3.10 & 3.88 & 2.47 & 1.97 & 1.29 & 2.92 & 1.95 \\
\hline 7.10 & 2.76 & 1.72 & 0.29 & 0.49 & 4.67 & 4.03 & 3.22 & 3.78 & 2.19 & 1.61 & 1.06 & 2.96 & 1.95 \\
\hline 7.96 & 2.51 & 1.52 & 0.02 & 0.06 & 4.65 & 4.24 & 3.33 & 3.59 & 1.91 & 1.30 & 0.87 & 2.93 & 1.91 \\
\hline 8.93 & 2.25 & 1.33 & - & - & 4.57 & 4.45 & 3.42 & 3.33 & 1.64 & 1.04 & 0.72 & 2.82 & 1.85 \\
\hline 10.02 & 2.02 & 1.15 & - & - & 4.41 & 4.63 & 3.47 & 2.99 & 1.37 & 0.83 & 0.59 & 2.68 & 1.80 \\
\hline 11.25 & 1.78 & 0.97 & - & - & 4.17 & 4.74 & 3.44 & 2.59 & 1.10 & 0.66 & 0.48 & 2.50 & 1.79 \\
\hline 12.62 & 1.55 & 0.76 & - & - & 3.85 & 4.76 & 3.32 & 2.15 & 0.83 & 0.53 & 0.37 & 2.34 & 1.85 \\
\hline 14.16 & 1.31 & 0.57 & - & - & 3.45 & 4.64 & 3.09 & 1.71 & 0.58 & 0.42 & 0.26 & 2.20 & 2.00 \\
\hline 15.89 & 1.06 & 0.32 & - & - & 2.98 & 4.37 & 2.76 & 1.28 & 0.28 & 0.33 & 0.13 & 2.13 & 2.26 \\
\hline 17.83 & 0.80 & 0.12 & - & - & 2.47 & 3.93 & 2.32 & 0.89 & 0.05 & 0.24 & 0.03 & 2.14 & 2.62 \\
\hline 20.00 & 0.56 & 0.02 & - & - & 1.93 & 3.34 & 1.79 & 0.56 & 0.01 & 0.12 & - & 2.23 & 3.06 \\
\hline 22.44 & 0.27 & - & - & - & 1.40 & 2.61 & 1.29 & 0.20 & - & 0.05 & - & 2.41 & 3.56 \\
\hline 25.18 & 0.12 & - & - & - & 0.86 & 1.88 & 0.59 & 0.06 & - & - & - & 2.67 & 4.06 \\
\hline 28.25 & - & - & - & - & 0.33 & 1.11 & 0.08 & - & - & - & - & 2.96 & 4.53 \\
\hline 31.70 & - & - & - & - & 0.01 & 0.09 & - & - & - & - & - & 3.27 & 4.92 \\
\hline 35.57 & - & - & - & - & - & - & - & - & - & - & - & 3.56 & 5.18 \\
\hline 39.91 & - & - & - & - & - & - & - & - & - & - & - & 3.80 & 5.32 \\
\hline 44.77 & - & - & - & - & - & - & - & - & - & - & - & 3.97 & 5.32 \\
\hline
\end{tabular}


Table H.4. (contd)

\begin{tabular}{|c|c|c|c|c|c|c|c|c|c|c|c|c|c|}
\hline & \multicolumn{13}{|c|}{ \% Below Size } \\
\hline & \multicolumn{4}{|c|}{ Group 1/2 Mixture } & \multicolumn{3}{|c|}{ Group 3/4 Mixture } & \multicolumn{4}{|c|}{ Group 5/6 Mixture } & \multicolumn{2}{|c|}{ Group 8} \\
\hline $\begin{array}{c}\text { Particle } \\
\text { Size } \\
(\mu \mathrm{m})\end{array}$ & $\begin{array}{l}\text { High- } \\
\text { Solids, } \\
\text { Pre- } \\
\text { Leach }\end{array}$ & $\begin{array}{c}\text { Post- } \\
\text { Caustic } \\
\text { Leach }\end{array}$ & $\begin{array}{c}\text { Post- } \\
\text { Caustic } \\
\text { Leach, } \\
\text { Washed }\end{array}$ & $\begin{array}{c}\text { Post- } \\
\text { Oxidative } \\
\text { Leach, } \\
\text { Washed }\end{array}$ & $\begin{array}{l}\text { High- } \\
\text { Solids, } \\
\text { Pre- } \\
\text { Leach }\end{array}$ & $\begin{array}{c}\text { Post- } \\
\text { Caustic } \\
\text { Leach }\end{array}$ & $\begin{array}{c}\text { Post- } \\
\text { Caustic } \\
\text { Leach, } \\
\text { Washed }\end{array}$ & $\begin{array}{c}\text { High- } \\
\text { Solids, } \\
\text { Pre- } \\
\text { Leach }\end{array}$ & $\begin{array}{l}\text { Post- } \\
\text { Caustic } \\
\text { Leach }\end{array}$ & $\begin{array}{c}\text { Post- } \\
\text { Caustic } \\
\text { Leach, } \\
\text { Washed }\end{array}$ & $\begin{array}{c}\text { Post- } \\
\text { Oxidative } \\
\text { Leach, } \\
\text { Washed }\end{array}$ & $\begin{array}{c}\text { High- } \\
\text { Solids, } \\
\text { Pre- } \\
\text { Leach }\end{array}$ & $\begin{array}{c}\text { Post- } \\
\text { Caustic } \\
\text { Leach, } \\
\text { Washed }\end{array}$ \\
\hline 50.24 & - & - & - & - & - & - & - & - & - & - & - & 4.05 & 5.19 \\
\hline 56.37 & - & - & - & - & - & - & - & - & - & - & - & 4.04 & 4.94 \\
\hline 63.25 & - & - & - & - & - & - & - & - & - & - & - & 3.95 & 4.61 \\
\hline 70.96 & - & - & - & - & - & - & - & - & - & - & - & 3.78 & 4.19 \\
\hline 79.62 & - & - & - & - & - & - & - & - & - & - & - & 3.53 & 3.69 \\
\hline 89.34 & - & - & - & - & - & - & - & - & - & - & - & 3.18 & 3.12 \\
\hline 100.24 & - & - & - & - & - & - & - & - & - & - & - & 2.75 & 2.46 \\
\hline 112.47 & - & - & - & - & - & - & - & - & - & - & - & 2.22 & 1.77 \\
\hline 126.19 & - & - & - & - & - & - & - & - & - & - & - & 1.59 & 1.07 \\
\hline 141.59 & - & - & - & - & - & - & - & - & - & - & - & 0.99 & 0.17 \\
\hline 158.87 & - & - & - & - & - & - & - & - & - & - & - & 0.30 & - \\
\hline 178.25 & - & - & - & - & - & - & - & - & - & - & - & 0.03 & - \\
\hline
\end{tabular}





\section{Appendix I}

Rheology Data 



\section{Appendix I: Rheology Data}

Data for Hanford waste liquid rheology is tabulated in Table I.1, shear strength in Table I.2, and Bingham rheology in Table I.3. 
Table I.1. Liquid Rheology

\begin{tabular}{|c|c|c|c|c|c|}
\hline Tank & Primary Waste Type & $\begin{array}{c}\text { Primary } \\
\text { Waste Type } \\
\text { Fraction } \\
\end{array}$ & $\begin{array}{l}\text { Sample } \\
\text { Density } \\
(\mathrm{g} / \mathrm{mL})\end{array}$ & $\begin{array}{c}\text { Sample } \\
\text { Temperature } \\
\left({ }^{\circ} \mathrm{C}\right) \\
\end{array}$ & $\begin{array}{c}\text { Viscosity } \\
\text { (cP) }\end{array}$ \\
\hline $\mathrm{AN}-103$ & A2 saltcake & 1.00 & 1.48 & 29 & 27.4 \\
\hline AN-103 & - & - & 1.48 & 45 & 13 \\
\hline $\mathrm{AN}-103$ & - & - & 1.48 & 65 & 7.6 \\
\hline $\mathrm{AN}-103$ & - & - & 1.43 & 29 & 8.8 \\
\hline AN-103 & - & - & 1.43 & 45 & 5.9 \\
\hline $\mathrm{AN}-103$ & - & - & 1.43 & 65 & 4 \\
\hline AN-103 & - & - & 1.38 & 29 & 7.8 \\
\hline $\mathrm{AN}-103$ & - & - & 1.38 & 45 & 5.4 \\
\hline $\mathrm{AN}-103$ & - & - & 1.38 & 65 & 3.7 \\
\hline $\mathrm{AN}-103$ & - & - & 1.35 & 29 & 11.6 \\
\hline AN-103 & - & - & 1.35 & 45 & 6.7 \\
\hline $\mathrm{AN}-103$ & - & - & 1.35 & 65 & 4.6 \\
\hline AN-104 & A2 saltcake & 1.00 & 1.25 & 25 & 3.5 \\
\hline AN-104 & - & - & 1.4 & 25 & 16.9 \\
\hline AN-104 & - & - & 1.4 & 45 & 8.1 \\
\hline AN-104 & - & - & 1.4 & 65 & 4.6 \\
\hline AN-104 & - & - & 1.3 & 25 & 17.5 \\
\hline AN-104 & - & - & 1.3 & 45 & 8.1 \\
\hline $\mathrm{AN}-104$ & - & - & 1.3 & 65 & 5.4 \\
\hline AN-105 & A2 saltcake & 1.00 & 1.42 & 25 & 12.3 \\
\hline AN-105 & - & - & 1.42 & 45 & 6.3 \\
\hline AN-105 & - & - & 1.42 & 65 & 3.5 \\
\hline AN-105 & - & - & 1.35 & 25 & 9.4 \\
\hline $\mathrm{AN}-105$ & - & - & 1.35 & 45 & 4.2 \\
\hline $\mathrm{AN}-105$ & - & - & 1.35 & 65 & 2.4 \\
\hline AW-101 & A2 salteake & 1.00 & 1.47 & 25 & 22 \\
\hline $\mathrm{AW}-101$ & - & - & 1.47 & 45 & 8 \\
\hline AW-101 & - & - & 1.47 & 65 & 5.4 \\
\hline AW-101 & - & - & 1.38 & 25 & 5.9 \\
\hline AW-101 & - & - & 1.38 & 45 & 3 \\
\hline AW-101 & - & - & 1.38 & 65 & 1.8 \\
\hline AW-101 & - & - & 1.34 & 25 & 11.5 \\
\hline AW-101 & - & - & 1.34 & 45 & 5.4 \\
\hline AW-101 & - & - & 1.34 & 65 & 3.3 \\
\hline S-112 & S1 saltcake & 0.99 & 1.27 & 20 & 5.7 \\
\hline $\mathrm{S}-112$ & - & - & 1.27 & 35 & 4 \\
\hline $\mathrm{S}-112$ & - & - & 1.27 & 50 & 2.9 \\
\hline $\mathrm{S}-112$ & - & - & 1.12 & 20 & 2 \\
\hline S-112 & - & - & 1.12 & 35 & 1.8 \\
\hline $\mathrm{S}-112$ & - & - & 1.12 & 50 & 1.4 \\
\hline$S Y-\mid 01$ & S2 saltcake & 1.00 & 1.5 & 26 & 25.5 \\
\hline SY-101 & - & - & 1.5 & 47 & 10.5 \\
\hline SY-101 & - & - & 1.49 & 30 & 20.5 \\
\hline SY-101 & - & - & 1.49 & 47 & 10.8 \\
\hline SY-101 & - & - & 1.3 & 27 & 4.7 \\
\hline SY-101 & - & - & 1.3 & 47 & 3.2 \\
\hline SY-10I & - & - & 1.32 & 24 & 6 \\
\hline SY-101 & - & - & 1.32 & 47 & 3.7 \\
\hline $\mathrm{AY}-102$ & BL sludge & 1.00 & 1.15 & 27 & 1.8 \\
\hline $\mathrm{AY}-102$ & - & - & 1.15 & 45 & 1.1 \\
\hline $\mathrm{AZ}-101$ & P3 sludge & 1.00 & 1.24 & 28 & 2.3 \\
\hline AZ-101 & - & - & 1.24 & 45 & 1.8 \\
\hline $\mathrm{AZ}-101$ & - & - & 1.24 & 65 & 1.5 \\
\hline C-104 & CWP1 sludge & 0.34 & 1.17 & 25 & 1.2 \\
\hline C-104 & - & - & 1.17 & 45 & 1.2 \\
\hline C-104 & - & - & 1.17 & 65 & 1.3 \\
\hline C- 107 & 1C sludge & 0.54 & 1.02 & 45 & 2.4 \\
\hline C-107 & - & - & 1.02 & 65 & 1.1 \\
\hline C-107 & - & - & 1.03 & 25 & 1.5 \\
\hline $\mathrm{C}-107$ & - & - & 1.03 & 45 & 1.3 \\
\hline C-107 & - & - & 1.03 & 25 & 1.7 \\
\hline AP-104 & $\mathrm{n} / \mathrm{a}$ & 0.00 & 1.41 & 25 & 3.6 \\
\hline AP-104 & - & - & 1.41 & 40 & 2.3 \\
\hline
\end{tabular}


Table I.2. Shear Strength

\begin{tabular}{|c|c|c|c|c|c|c|c|c|}
\hline \multirow[b]{2}{*}{ Tank } & \multirow[b]{2}{*}{ Primary Waste Type } & \multirow[b]{2}{*}{$\begin{array}{l}\text { Primary } \\
\text { Waste Type } \\
\text { Fraction }\end{array}$} & \multirow[b]{2}{*}{$\begin{array}{l}\text { Elevation in } \\
\text { Tank (in.) }\end{array}$} & \multirow[b]{2}{*}{$\begin{array}{l}\text { Average } \\
\text { Mass } \\
\text { Fraction } \\
\text { UDS }\end{array}$} & \multirow[b]{2}{*}{$\begin{array}{l}\text { Specific Mass } \\
\text { Fraction UDS }\end{array}$} & \multicolumn{3}{|c|}{ Shear Strength (Pa) } \\
\hline & & & & & & Shear Vane & Core Extrusion & Ball Rheometer \\
\hline$A-101$ & Al saltcake & 0.99 & 175 & 0.34 & - & - & 110 & - \\
\hline$A-101$ & - & - & 195 & - & - & - & 700 & - \\
\hline A-101 & - & - & 232 & - & - & - & 480 & - \\
\hline$A-101$ & - & - & 255 & - & - & - & 220 & - \\
\hline A-101 & - & - & 295 & - & - & - & 270 & - \\
\hline A-101 & - & - & 312 & - & - & - & 440 & - \\
\hline A-101 & - & - & 325 & - & - & - & 110 & - \\
\hline A-101 & - & - & 335 & - & - & - & 400 & - \\
\hline$A-101$ & - & - & 180 & - & - & - & 60 & - \\
\hline A-10I & - & - & 218 & - & - & - & 360 & - \\
\hline A-101 & - & - & 237 & - & - & - & 600 & - \\
\hline A-101 & - & - & 255 & - & - & - & 700 & - \\
\hline $\mathrm{A}-101$ & - & - & 270 & - & - & - & 400 & - \\
\hline$A-101$ & - & - & 280 & - & - & - & 400 & - \\
\hline A-101 & - & - & 295 & - & - & - & 670 & - \\
\hline A-101 & - & - & 312 & - & - & - & 630 & - \\
\hline$\Lambda-101$ & - & - & 345 & - & - & - & 25 & - \\
\hline A-101 & - & - & 313 & - & - & - & 443 & - \\
\hline A-101 & - & - & 313 & - & - & - & 443 & - \\
\hline AN- 102 & A2 saltcake & 1,00 & $\mathrm{n} / \mathrm{a}$ & 0.20 & - & 1450 & - & - \\
\hline $\mathrm{AN}-102$ & - & - & $n / a$ & - & - & 560 & - & - \\
\hline AN- 102 & - & - & $\mathrm{n} / \mathrm{a}$ & - & - & 955 & - & - \\
\hline AN-102 & - & - & $\mathrm{n} / \mathrm{a}$ & - & - & 874 & - & - \\
\hline $\mathrm{AN}-102$ & - & - & $n / a$ & - & - & 337 & - & - \\
\hline $\mathrm{AN}-102$ & - & - & $n / a$ & - & - & 187 & - & - \\
\hline $\mathrm{AN}-103$ & A2 saltcake & 1.00 & 63 & 0.07 & - & - & - & 900 \\
\hline$A N-103$ & - & - & 64 & - & - & - & - & 196 \\
\hline $\mathrm{AN}-103$ & - & - & 75 & - & - & - & - & 188 \\
\hline $\mathrm{AN}-103$ & - & - & 79 & - & - & - & - & 186 \\
\hline $\mathrm{AN}-103$ & - & - & 83 & - & - & - & - & 183 \\
\hline$A N-103$ & - & - & 87 & - & - & - & - & 179 \\
\hline AN-103 & - & - & 91 & - & - & - & - & 174 \\
\hline $\mathrm{AN}-103$ & - & - & 94 & - & - & - & - & 169 \\
\hline AN-103 & - & - & 98 & - & - & - & - & 163 \\
\hline AN-103 & - & - & 102 & - & - & - & - & 157 \\
\hline AN-103 & - & - & 106 & - & - & - & - & 149 \\
\hline $\mathrm{AN}-103$ & - & - & 110 & - & - & - & - & 141 \\
\hline $\mathrm{AN}-103$ & - & - & 114 & - & - & - & - & 132 \\
\hline AN-103 & - & - & 118 & - & - & - & - & 121 \\
\hline AN- 103 & - & - & 122 & - & - & - & - & 110 \\
\hline $\mathrm{AN}-103$ & - & - & 126 & - & - & - & - & 97 \\
\hline $\mathrm{AN}-103$ & - & - & 130 & - & - & - & - & 83 \\
\hline AN-103 & - & - & 134 & - & - & - & - & 68 \\
\hline AN-103 & - & - & 138 & - & - & - & - & 51 \\
\hline AN-103 & - & - & 91 & - & - & - & - & 900 \\
\hline $\mathrm{AN}-103$ & - & - & 91 & - & - & - & - & 240 \\
\hline $\mathrm{AN}-103$ & - & - & 98 & - & - & - & - & 218 \\
\hline AN-103 & - & - & 102 & - & - & - & - & 206 \\
\hline AN-103 & - & - & 106 & - & - & - & - & 193 \\
\hline AN-103 & - & - & 110 & - & - & - & - & 179 \\
\hline AN-103 & - & - & 114 & - & - & - & - & 165 \\
\hline AN-103 & - & - & 118 & - & - & - & - & 150 \\
\hline AN-103 & - & - & 122 & - & - & - & - & 134 \\
\hline $\mathrm{AN}-103$ & - & - & 126 & - & - & - & - & 117 \\
\hline $\mathrm{AN}-103$ & - & - & 130 & - & - & - & - & 99 \\
\hline $\mathrm{AN}-103$ & - & - & 134 & - & - & - & - & 80 \\
\hline $\mathrm{AN}-103$ & - & - & 138 & - & - & - & - & 61 \\
\hline $\mathrm{AN}-103$ & - & - & 152 & - & - & - & 100 & - \\
\hline AN-103 & - & - & 133 & - & - & - & 150 & - \\
\hline AN-103 & - & - & 95 & - & - & - & 1050 & - \\
\hline AN-103 & - & - & 76 & - & - & - & 750 & - \\
\hline AN-103 & - & - & 38 & - & - & - & 1050 & - \\
\hline AN-103 & - & - & 114 & - & - & - & 779 & - \\
\hline
\end{tabular}


Table I.2. (contd)

\begin{tabular}{|c|c|c|c|c|c|c|c|c|}
\hline \multirow[b]{2}{*}{ Tank } & \multirow[b]{2}{*}{ Primary Waste Type } & \multirow[b]{2}{*}{$\begin{array}{l}\text { Primary } \\
\text { Waste Type } \\
\text { Fraction }\end{array}$} & \multirow[b]{2}{*}{$\begin{array}{l}\text { Elevation in } \\
\text { Tank (in.) }\end{array}$} & \multirow[b]{2}{*}{$\begin{array}{l}\text { Average } \\
\text { Mass } \\
\text { Fraction } \\
\text { UDS } \\
\end{array}$} & \multirow[b]{2}{*}{$\begin{array}{l}\text { Specific Mass } \\
\text { Fraction UDS }\end{array}$} & \multicolumn{3}{|c|}{ Shear Strength (Pa) } \\
\hline & & & & & & Shear Vane & Core Extrusion & Ball Rheometer \\
\hline $\mathrm{AN}-103$ & - & - & 76 & - & - & - & 1255 & - \\
\hline $\mathrm{AN}-\mathrm{l03}$ & - & - & 57 & - & - & - & 1428 & - \\
\hline AN-103 & - & - & 76 & - & - & - & 1763 & - \\
\hline $\mathrm{AN}-103$ & - & - & 76 & - & - & - & 991 & - \\
\hline $\mathrm{AN}-103$ & - & - & 38 & - & - & - & 991 & - \\
\hline $\mathrm{AN}-103$ & - & - & 57 & - & - & - & 991 & - \\
\hline AN- 103 & - & - & 19 & - & - & - & 803 & - \\
\hline $\mathrm{AN}-103$ & - & - & 133 & - & - & - & 357 & - \\
\hline$A N-103$ & - & - & 76 & - & - & - & 558 & - \\
\hline AN-103 & - & - & 57 & - & - & - & 728 & - \\
\hline$A N-103$ & - & - & 76 & - & - & - & 1200 & - \\
\hline $\mathrm{AN}-103$ & - & - & 19 & - & - & - & 2618 & - \\
\hline $\mathrm{AN}-103$ & - & - & 126 & - & - & 5000 & - & - \\
\hline $\mathrm{AN}-103$ & - & - & 126 & - & - & 6000 & - & - \\
\hline AN-103 & - & - & 126 & - & - & 8000 & - & - \\
\hline AN-104 & A2 saltcake & 1.00 & 25 & 0.14 & - & - & - & 900 \\
\hline$\Lambda N-104$ & - & - & 26 & - & - & - & - & 146 \\
\hline $\mathrm{AN}-104$ & - & - & 31 & - & - & - & - & 141 \\
\hline AN-104 & - & - & 35 & - & - & - & - & 137 \\
\hline AN-104 & - & - & 39 & - & - & - & - & 133 \\
\hline $\mathrm{AN}-104$ & - & - & 43 & - & - & - & - & 129 \\
\hline AN-104 & - & - & 47 & - & - & - & - & 125 \\
\hline AN-104 & - & - & 51 & - & - & - & - & 122 \\
\hline $\mathrm{AN}-104$ & - & - & 55 & - & - & - & - & 118 \\
\hline $\mathrm{AN}-104$ & - & - & 59 & - & - & - & - & 115 \\
\hline $\mathrm{AN}-104$ & - & - & 63 & - & - & - & - & 112 \\
\hline AN-104 & - & - & 67 & - & - & - & - & 109 \\
\hline AN-I04 & - & - & 71 & - & - & - & - & 106 \\
\hline $\mathrm{AN}-104$ & - & - & 75 & - & - & - & - & 103 \\
\hline $\mathrm{AN}-104$ & - & - & 79 & - & - & - & - & 101 \\
\hline AN-104 & - & - & 83 & - & - & - & - & 98 \\
\hline AN-104 & - & - & 87 & - & - & - & - & 98 \\
\hline AN-104 & - & - & 91 & - & - & - & - & 96 \\
\hline AN-104 & - & - & 94 & - & - & - & - & 93 \\
\hline AN-104 & - & - & 98 & - & - & - & - & 90 \\
\hline AN-104 & - & - & 102 & - & - & - & - & 87 \\
\hline $\mathrm{AN}-104$ & - & - & 106 & - & - & - & - & 84 \\
\hline $\mathrm{AN}-104$ & - & - & 110 & - & - & - & - & 80 \\
\hline AN-104 & - & - & 114 & - & - & - & - & 76 \\
\hline AN-104 & - & - & 118 & - & - & - & - & 72 \\
\hline $\mathrm{AN}-104$ & - & - & 122 & - & - & - & - & 67 \\
\hline $\mathrm{AN}-104$ & - & - & 126 & - & - & - & - & 62 \\
\hline AN-104 & - & - & 130 & - & - & - & - & 56 \\
\hline $\mathrm{AN}-104$ & - & - & 134 & - & - & - & - & 50 \\
\hline AN-104 & - & - & 138 & - & - & - & - & 43 \\
\hline $\mathrm{AN}-104$ & - & - & 24 & - & - & - & - & 900 \\
\hline $\mathrm{AN}-104$ & - & - & 24 & - & - & - & - & 256 \\
\hline AN-104 & - & - & 39 & - & - & - & - & 216 \\
\hline AN-104 & - & - & 43 & - & - & - & - & 206 \\
\hline AN-104 & - & - & 47 & - & - & - & - & 195 \\
\hline AN-104 & - & - & 51 & - & - & - & - & 185 \\
\hline AN-104 & - & - & 55 & - & - & - & - & 175 \\
\hline AN-104 & - & - & 59 & - & - & - & - & 165 \\
\hline $\mathrm{AN}-104$ & - & - & 63 & - & - & - & - & 155 \\
\hline $\mathrm{AN}-104$ & - & - & 67 & - & - & - & - & 146 \\
\hline $\mathrm{AN}-104$ & - & - & 71 & - & - & - & - & 136 \\
\hline AN-104 & - & - & 75 & - & - & - & - & 126 \\
\hline AN-104 & - & - & 79 & - & - & - & - & 117 \\
\hline AN-104 & - & - & 83 & - & - & - & - & 108 \\
\hline AN-104 & - & - & 87 & - & - & - & - & 99 \\
\hline AN-104 & - & - & 91 & - & - & - & - & 90 \\
\hline AN-104 & - & - & 94 & - & - & - & - & 81 \\
\hline AN-104 & - & - & 98 & - & - & - & - & 72 \\
\hline
\end{tabular}


Table I.2. (contd)

\begin{tabular}{|c|c|c|c|c|c|c|c|c|}
\hline \multirow[b]{2}{*}{ Tank } & \multirow[b]{2}{*}{ Primary Waste Type } & \multirow[b]{2}{*}{$\begin{array}{l}\text { Primary } \\
\text { Waste Type } \\
\text { Fraction }\end{array}$} & \multirow[b]{2}{*}{$\begin{array}{l}\text { Elevation in } \\
\text { Tank (in.) }\end{array}$} & \multirow{2}{*}{$\begin{array}{l}\text { Average } \\
\text { Mass } \\
\text { Fraction } \\
\text { UDS }\end{array}$} & \multirow[b]{2}{*}{$\begin{array}{l}\text { Specific Mass } \\
\text { Fraction UDS }\end{array}$} & \multicolumn{3}{|c|}{ Shear Strength (Pa) } \\
\hline & & & & & & Shear Vane & Core Extrusion & Ball Rheometer \\
\hline AN-104 & - & - & 102 & - & - & - & - & 64 \\
\hline $\mathrm{AN}-\mathrm{I04}$ & - & - & 106 & - & - & - & - & 55 \\
\hline AN-104 & - & - & 110 & - & - & - & - & 47 \\
\hline $\mathrm{AN}-104$ & - & - & 114 & - & - & - & - & 39 \\
\hline $\mathrm{AN}-104$ & - & - & 118 & - & - & - & - & 32 \\
\hline $\mathrm{AN}-104$ & - & - & 122 & - & - & - & - & 24 \\
\hline AN-104 & - & - & 126 & - & - & - & - & 17 \\
\hline AN-104 & - & - & 130 & - & - & - & - & 10 \\
\hline$A N-104$ & - & - & 114 & - & - & - & 127 & - \\
\hline AN-104 & - & - & 95 & - & - & - & 286 & - \\
\hline AN-104 & - & - & 38 & - & - & - & 606 & - \\
\hline AN-104 & - & - & 19 & - & - & - & 795 & - \\
\hline $\mathrm{AN}-104$ & - & - & 95 & - & - & - & 226 & - \\
\hline $\mathrm{AN}-105$ & A2 saltcake & 1.00 & 47 & 0.12 & - & - & - & 900 \\
\hline AN-105 & - & - & 47 & - & - & - & - & 216 \\
\hline AN-105 & - & - & 58 & - & - & - & - & 206 \\
\hline$\Lambda N-105$ & - & - & 77 & - & - & - & - & 189 \\
\hline AN-105 & - & - & 97 & - & - & - & - & 165 \\
\hline$A N-105$ & - & - & 117 & - & - & - & - & 134 \\
\hline AN-105 & - & - & 137 & - & - & - & - & 96 \\
\hline $\mathrm{AN}-105$ & - & - & 156 & - & - & - & - & 52 \\
\hline AN-105 & - & - & 5 & - & - & - & - & 900 \\
\hline AN-105 & - & - & 6 & - & - & - & - & 180 \\
\hline $\mathrm{AN}-105$ & - & - & 18 & - & - & - & - & 173 \\
\hline $\mathrm{AN}-105$ & - & - & 38 & - & - & - & - & 162 \\
\hline $\mathrm{AN}-105$ & - & - & 58 & - & - & - & - & 146 \\
\hline$A N-105$ & - & - & 77 & - & - & - & - & 126 \\
\hline $\mathrm{AN}-105$ & - & - & 97 & - & - & - & - & 102 \\
\hline $\mathrm{AN}-105$ & - & - & 117 & - & - & - & - & 72 \\
\hline $\mathrm{AN}-105$ & - & - & 137 & - & - & - & - & 38 \\
\hline$A N-107$ & A2 saltcake & 1.00 & $n / a$ & 0.07 & - & 3 & - & - \\
\hline AN-107 & - & - & $\mathrm{n} / \mathrm{a}$ & - & - & 33 & - & - \\
\hline AN-107 & - & - & $\mathrm{n} / \mathrm{a}$ & - & - & 46 & - & - \\
\hline AN-107 & - & - & $\mathrm{n} / \mathrm{a}$ & - & - & 42 & - & - \\
\hline AN-107 & - & - & $\mathrm{n} / \mathrm{a}$ & - & - & 42 & - & - \\
\hline AN-107 & - & - & $\mathrm{n} / \mathrm{a}$ & - & - & 206 & - & - \\
\hline $\mathrm{AN}-107$ & - & - & $\mathrm{n} / \mathrm{a}$ & - & - & 258 & - & - \\
\hline $\mathrm{AN}-107$ & - & - & $n / a$ & - & - & 323 & - & - \\
\hline AN-107 & - & - & $11 / \mathrm{a}$ & - & - & 106 & - & - \\
\hline AN- 107 & - & - & $\mathrm{n} / \mathrm{a}$ & - & - & 411 & - & - \\
\hline $\mathrm{AN}-107$ & - & - & $\mathrm{n} / \mathrm{a}$ & - & - & 610 & - & - \\
\hline $\mathrm{AN}-107$ & - & - & $\mathrm{n} / \mathrm{a}$ & - & - & 60 & - & - \\
\hline AN-107 & - & - & $\mathrm{n} / \mathrm{a}$ & - & - & 56 & - & - \\
\hline $\mathrm{AW}-101$ & A2 saltcake & 1.00 & 39 & 0.04 & - & - & - & 900 \\
\hline $\mathrm{AW}-101$ & - & - & 39 & - & - & - & - & 300 \\
\hline AW-101 & - & - & 46 & - & - & - & - & 276 \\
\hline AW-101 & - & - & 56 & - & - & - & - & 241 \\
\hline $\mathrm{AW}-101$ & - & - & 66 & - & - & - & - & 201 \\
\hline AW-101 & - & - & 76 & - & - & - & - & 156 \\
\hline $\mathrm{AW}-101$ & - & - & 85 & - & - & - & - & 104 \\
\hline AW-101 & - & - & 95 & - & - & - & - & 44 \\
\hline AW-101 & - & - & 5 & - & - & - & - & 900 \\
\hline AW-101 & - & - & 5 & - & - & - & - & 217 \\
\hline AW-101 & - & - & 18 & - & - & - & - & 201 \\
\hline AW-101 & - & - & 37 & - & - & - & - & 174 \\
\hline AW-101 & - & - & 57 & - & - & - & - & 142 \\
\hline AW-101 & - & - & 77 & - & - & - & - & 102 \\
\hline AW-101 & - & - & 96 & - & - & - & - & 50 \\
\hline $\mathrm{AW}-101$ & - & - & 102 & - & - & - & - & 34 \\
\hline $\mathrm{AW}-101$ & - & - & 108 & - & - & - & - & 11 \\
\hline AW-101 & - & - & 109 & - & - & - & - & 4 \\
\hline AW-101 & - & - & 95 & - & - & - & 100 & - \\
\hline AW-101 & - & - & 57 & - & - & - & 100 & - \\
\hline
\end{tabular}


Table I.2. (contd)

\begin{tabular}{|c|c|c|c|c|c|c|c|c|}
\hline \multirow[b]{2}{*}{ Tank } & \multirow[b]{2}{*}{ Primary Waste Type } & \multirow[b]{2}{*}{$\begin{array}{l}\text { Primary } \\
\text { Waste Type } \\
\text { Fraction }\end{array}$} & \multirow[b]{2}{*}{$\begin{array}{l}\text { Elevation in } \\
\text { Tank (in.) }\end{array}$} & \multirow[b]{2}{*}{$\begin{array}{l}\text { Average } \\
\text { Mass } \\
\text { Fraction } \\
\text { UDS } \\
\end{array}$} & \multirow[b]{2}{*}{$\begin{array}{l}\text { Specific Mass } \\
\text { Fraction UDS }\end{array}$} & \multicolumn{3}{|c|}{ Shear Strength (Pa) } \\
\hline & & & & & & Shear Vane & Core Extrusion & Ball Rheometer \\
\hline $\mathrm{AW}-101$ & - & - & 19 & - & - & - & \begin{tabular}{|c|}
100 \\
\end{tabular} & - \\
\hline $\mathrm{AW}-10 \mathrm{I}$ & - & - & 38 & - & - & - & 351 & - \\
\hline$A W-101$ & - & - & 57 & - & - & - & 372 & - \\
\hline AW-101 & - & - & 57 & - & - & - & 165 & - \\
\hline $\mathrm{AW}-101$ & - & - & 10 & - & - & 1600 & - & - \\
\hline AW-101 & - & - & 30 & - & - & 350 & - & - \\
\hline AW-101 & - & - & 69 & - & - & 550 & - & - \\
\hline $\mathrm{AW}-103$ & CWZr2 sludge & 0.87 & 96 & 0.26 & - & 456 & - & - \\
\hline AW-103 & - & - & 77 & - & - & 913 & - & - \\
\hline $\mathrm{AW}-\mathrm{I03}$ & - & - & 58 & - & - & 931 & - & - \\
\hline AW-103 & - & - & 39 & - & - & 521 & - & - \\
\hline $\mathrm{AW}-103$ & - & - & 20 & - & - & 1769 & - & - \\
\hline $\mathrm{AW}-103$ & - & - & 5 & - & - & 3538 & - & - \\
\hline AW-103 & - & - & 95 & - & - & 279 & - & - \\
\hline $\mathrm{AW}-103$ & - & - & 76 & - & - & 521 & - & - \\
\hline AW-103 & - & - & 57 & - & - & 1583 & - & - \\
\hline$\Lambda \mathrm{W}-103$ & - & - & 38 & - & - & 326 & - & - \\
\hline $\mathrm{AW}-103$ & - & - & 19 & - & - & 587 & - & - \\
\hline $\mathrm{AY}-101$ & Unidentified sludge & 1.00 & 13 & 0.63 & - & 2720 & - & - \\
\hline AY -101 & - & - & 4 & - & - & 6745 & - & - \\
\hline $\mathrm{AY}-101$ & - & - & 9 & - & - & 1193 & - & - \\
\hline AY-10I & - & - & 23 & - & - & 402 & - & - \\
\hline $\mathrm{AY}-101$ & - & - & 14 & - & - & 18288 & - & - \\
\hline $\mathrm{AY}-101$ & - & - & 12 & - & - & 1442 & - & - \\
\hline AY-101 & - & - & 21 & - & - & 420 & - & - \\
\hline AY-101 & - & - & 22 & - & - & 302 & - & - \\
\hline$A Y-101$ & - & - & 9 & - & - & 1050 & - & - \\
\hline$A Y-101$ & - & - & 32 & - & - & 9 & - & - \\
\hline$A Y-101$ & - & - & 22 & - & - & 3695 & - & - \\
\hline $\mathrm{AY}-10 \mathrm{I}$ & - & - & 5 & - & - & 2598 & - & - \\
\hline$A Y-101$ & - & - & 32 & - & - & 45 & - & - \\
\hline AY-102 & BL sludge & 1.00 & 27 & 0.30 & - & - & 1460 & - \\
\hline AY -102 & - & - & 9 & - & - & - & 1030 & - \\
\hline $\mathrm{AY}-102$ & - & - & 9 & - & - & - & 1300 & - \\
\hline $\mathrm{AY}-102$ & - & - & 9 & - & - & - & 1760 & - \\
\hline AY -102 & - & - & 58 & - & - & - & 1440 & - \\
\hline $\mathrm{AY}-102$ & - & - & 25 & - & - & - & 1830 & - \\
\hline $\mathrm{AY}-102$ & - & - & 59 & - & - & - & 1550 & - \\
\hline$A Y-102$ & - & - & 26 & - & - & - & 1550 & - \\
\hline $\mathrm{AY}-102$ & - & - & 26 & - & - & - & 1340 & - \\
\hline $\mathrm{AY}-102$ & - & - & 8 & - & - & - & 620 & - \\
\hline $\mathrm{AY}-102$ & - & - & 45 & - & - & - & 860 & - \\
\hline$A Y-102$ & - & - & 8 & - & - & - & 790 & - \\
\hline$A Y-102$ & - & - & 8 & - & - & - & 960 & - \\
\hline $\mathrm{AY}-102$ & - & - & 8 & - & - & - & 1340 & - \\
\hline $\mathrm{AY}-102$ & - & - & $\mathrm{n} / \mathrm{a}$ & - & - & - & 1370 & - \\
\hline $\mathrm{AY}-102$ & - & - & $\mathrm{n} / \mathrm{a}$ & - & - & - & 1550 & - \\
\hline AY -102 & - & - & $n / a$ & - & - & - & 760 & - \\
\hline $\mathrm{AY}-102$ & - & - & $n / a$ & - & - & - & 690 & - \\
\hline$A Y-102$ & - & - & $\mathrm{n} / \mathrm{a}$ & - & - & - & 1020 & - \\
\hline $\mathrm{AY}-102$ & - & - & $\mathrm{n} / \mathrm{a}$ & - & - & - & 1790 & - \\
\hline $\mathrm{AY}-102$ & - & - & $\mathrm{n} / \mathrm{a}$ & - & - & - & 850 & - \\
\hline AY -102 & - & - & $n / a$ & - & - & - & 1410 & - \\
\hline $\mathrm{AY}-102$ & - & - & $\mathrm{n} / \mathrm{a}$ & - & - & - & 670 & - \\
\hline AY -102 & - & - & $\mathrm{n} / \mathrm{a}$ & - & - & - & 480 & - \\
\hline $\mathrm{AY}-102$ & - & - & $\mathrm{n} / \mathrm{a}$ & - & - & - & 390 & - \\
\hline $\mathrm{AY}-102$ & - & - & $\mathrm{n} / \mathrm{a}$ & - & - & 43 & - & - \\
\hline $\mathrm{AY}-102$ & - & - & 50 & - & - & 6189 & - & - \\
\hline$A Y-102$ & - & - & 41 & - & - & 177 & - & - \\
\hline $\mathrm{AY}-102$ & - & - & 31 & - & - & 70 & - & - \\
\hline$A Y-102$ & - & - & 22 & - & - & 3318 & - & - \\
\hline $\mathrm{AY}-102$ & - & - & 13 & - & - & 890 & - & - \\
\hline AY -102 & - & - & 4 & - & - & 839 & - & - \\
\hline
\end{tabular}


Table I.2. (contd)

\begin{tabular}{|c|c|c|c|c|c|c|c|c|}
\hline \multirow[b]{2}{*}{ Tank } & \multirow[b]{2}{*}{ Primary Waste Type } & \multirow[b]{2}{*}{$\begin{array}{l}\text { Primary } \\
\text { Waste Type } \\
\text { Fraction }\end{array}$} & \multirow[b]{2}{*}{$\begin{array}{l}\text { Elevation in } \\
\text { Tank (in.) }\end{array}$} & \multirow[b]{2}{*}{$\begin{array}{l}\text { Average } \\
\text { Mass } \\
\text { Fraction } \\
\text { UDS }\end{array}$} & \multirow[b]{2}{*}{$\begin{array}{l}\text { Specific Mass } \\
\text { Fraction UDS }\end{array}$} & \multicolumn{3}{|c|}{ Shear Strength (Pa) } \\
\hline & & & & & & Shear Vane & Core Extrusion & Ball Rheometer \\
\hline $\mathrm{AY}-102$ & - & - & 59 & - & - & \begin{tabular}{|l|}
1251 \\
\end{tabular} & - & - \\
\hline $\mathrm{AY}-102$ & - & - & 50 & - & - & 507 & - & - \\
\hline $\mathrm{AY}-102$ & - & - & 40 & - & - & 250 & - & - \\
\hline $\mathrm{AY}-102$ & - & - & 12 & - & - & 474 & - & - \\
\hline $\mathrm{AY}-102$ & - & - & 4 & - & - & 8096 & - & - \\
\hline $\mathrm{AY}-102$ & - & - & 58 & - & - & 953 & - & - \\
\hline $\mathrm{AY}-102$ & - & - & 48 & - & - & 414 & - & - \\
\hline $\mathrm{AY}-102$ & - & - & 39 & - & - & 940 & - & - \\
\hline $\mathrm{AY}-102$ & - & - & 29 & - & - & 164 & - & - \\
\hline $\mathrm{AY}-102$ & - & - & 20 & - & - & 392 & - & - \\
\hline$A Y-102$ & - & - & 31 & - & - & 93 & - & - \\
\hline $\mathrm{AY}-102$ & - & - & 21 & - & - & 57 & - & - \\
\hline $\mathrm{AY}-102$ & - & - & 59 & - & - & 1104 & - & - \\
\hline$A Y-102$ & - & - & 11 & - & - & 720 & - & - \\
\hline$A Y-102$ & - & - & 4 & - & - & 479 & - & - \\
\hline $\mathrm{AY}-102$ & - & - & 50 & - & - & 435 & - & - \\
\hline$\Lambda Y-102$ & - & - & 40 & - & - & 378 & - & - \\
\hline $\mathrm{AY}-102$ & - & - & 31 & - & - & 470 & - & - \\
\hline $\mathrm{AY}-102$ & - & - & 21 & - & - & 751 & - & - \\
\hline $\mathrm{AY}-102$ & - & - & 12 & - & - & 835 & - & - \\
\hline $\mathrm{AY}-102$ & - & - & 4 & - & - & 533 & - & - \\
\hline$A Z-101$ & P3 sludge & 1.00 & $\mathrm{n} / \mathrm{a}$ & & 0.400586941 & 1500 & - & - \\
\hline$A Z-101$ & - & - & 7 & - & 0.48 & 4190 & - & - \\
\hline $\mathrm{AZ}-101$ & - & - & 8 & - & 0.48 & 1769 & - & - \\
\hline AZ-102 & P3 sludge & 1.00 & 10 & 0.31 & - & 866 & - & - \\
\hline $\mathrm{AZ}-102$ & - & - & 8 & - & - & 345 & - & - \\
\hline AZ- 102 & - & - & 44 & - & - & 736 & - & - \\
\hline $\mathrm{AZ}-102$ & - & - & 27 & - & - & 8194 & - & - \\
\hline AZ-102 & - & - & 27 & - & - & 12105 & - & - \\
\hline $\mathrm{AZ}-102$ & - & - & 13 & - & - & 1117 & - & - \\
\hline $\mathrm{AZ}-102$ & - & - & 4 & - & - & 549 & - & - \\
\hline B-111 & 2C sludge & 0.89 & $\mathrm{n} / \mathrm{a}$ & 0.11 & - & 90 & - & - \\
\hline B-111 & - & - & $\mathrm{n} / \mathrm{a}$ & - & - & 30 & - & - \\
\hline B-201 & 224 Pre-1949 sludge & 1.00 & $\mathrm{n} / \mathrm{a}$ & 0.16 & - & 1410 & - & - \\
\hline B-201 & - & - & $\mathrm{n} / \mathrm{a}$ & - & - & 1310 & - & - \\
\hline B-201 & - & - & $\mathrm{n} / \mathrm{a}$ & - & - & 1220 & - & - \\
\hline B-202 & 224 Post-1949 sludge & 1.00 & $\mathrm{n} / \mathrm{a}$ & 0.05 & - & 200 & - & - \\
\hline B-202 & - & - & $n / a$ & - & - & 270 & - & - \\
\hline B-202 & - & - & $11 / \mathrm{a}$ & - & - & 750 & - & - \\
\hline B-202 & - & - & $\mathrm{n} / \mathrm{a}$ & - & - & 470 & - & - \\
\hline B-202 & - & - & $\mathrm{n} / \mathrm{a}$ & - & - & 750 & - & - \\
\hline B-202 & - & - & $\mathrm{n} / \mathrm{a}$ & - & - & 270 & - & - \\
\hline B-203 & 224 Post- 1949 sludge & 1.00 & 258 & 0.14 & - & - & 62 & - \\
\hline B-203 & - & - & 210 & - & - & - & 50 & - \\
\hline B-203 & - & - & 210 & - & - & - & 923 & - \\
\hline B-203 & - & - & 182 & - & - & - & 1445 & - \\
\hline B-203 & - & - & 191 & - & - & - & 865 & - \\
\hline B-203 & - & - & 163 & - & - & - & 1615 & - \\
\hline B-203 & - & - & 172 & - & - & - & 765 & - \\
\hline B-203 & - & - & 144 & - & - & - & 1179 & - \\
\hline B-203 & - & - & 153 & - & - & - & 789 & - \\
\hline B-203 & - & - & 125 & - & - & - & 891 & - \\
\hline B-203 & - & - & 134 & - & - & - & 1354 & - \\
\hline B-203 & - & - & 106 & - & - & - & 1095 & - \\
\hline B-203 & - & - & 87 & - & - & - & 1316 & - \\
\hline B-203 & - & - & 96 & - & - & - & 1968 & - \\
\hline B-203 & - & - & $\mathrm{n} / \mathrm{a}$ & - & 0.313 & 2280 & - & - \\
\hline B-203 & - & - & $\mathrm{n} / \mathrm{a}$ & - & 0.214 & 60 & - & - \\
\hline B-204 & 224 Post-1949 sludge & 1.00 & 248 & 0.14 & - & - & 26 & - \\
\hline B-204 & - & - & 229 & - & - & - & 104 & - \\
\hline B-204 & - & - & 220 & - & - & - & 450 & - \\
\hline B-204 & - & - & 191 & - & - & - & 730 & - \\
\hline B-204 & - & - & 163 & - & - & - & 889 & - \\
\hline
\end{tabular}


Table I.2. (contd)

\begin{tabular}{|c|c|c|c|c|c|c|c|c|}
\hline \multirow[b]{2}{*}{ Tank } & \multirow[b]{2}{*}{ Primary Waste Type } & \multirow[b]{2}{*}{$\begin{array}{l}\text { Primary } \\
\text { Waste Type } \\
\text { Fraction }\end{array}$} & \multirow[b]{2}{*}{$\begin{array}{l}\text { Elevation in } \\
\text { Tank (in.) }\end{array}$} & \multirow{2}{*}{$\begin{array}{l}\text { Average } \\
\text { Mass } \\
\text { Fraction } \\
\text { UDS }\end{array}$} & \multirow[b]{2}{*}{$\begin{array}{l}\text { Specific Mass } \\
\text { Fraction UDS }\end{array}$} & \multicolumn{3}{|c|}{ Shear Strength (Pa) } \\
\hline & & & & & & Shear Vane & Core Extrusion & Ball Rheometer \\
\hline B-204 & - & - & 172 & - & - & - & 298 & - \\
\hline B-204 & - & - & 163 & - & - & - & 1586 & - \\
\hline B-204 & - & - & 144 & - & - & - & 988 & - \\
\hline B-204 & - & - & 125 & - & - & - & 1213 & - \\
\hline B-204 & - & - & 135 & - & - & - & 1152 & - \\
\hline B-204 & - & - & 106 & - & - & - & 1000 & - \\
\hline B-204 & - & - & 115 & - & - & - & 1534 & - \\
\hline B-204 & - & - & 87 & - & - & - & 935 & - \\
\hline B-204 & - & - & 96 & - & - & - & 860 & - \\
\hline B-204 & - & - & 77 & - & - & - & 554 & - \\
\hline B-204 & - & - & 49 & - & - & - & 1153 & - \\
\hline B-204 & - & - & 30 & - & - & - & 1009 & - \\
\hline B-204 & - & - & 39 & - & - & - & 921 & - \\
\hline B-204 & - & - & 11 & - & - & - & 500 & - \\
\hline B-204 & - & - & 20 & - & - & - & 1066 & - \\
\hline B-204 & - & - & 248 & - & - & - & 20 & - \\
\hline B-204 & - & - & 239 & - & - & - & 46 & - \\
\hline B-204 & - & - & 239 & - & - & - & 216 & - \\
\hline B-204 & - & - & 220 & - & - & - & 102 & - \\
\hline B-204 & - & - & 201 & - & - & - & 497 & - \\
\hline B-204 & - & - & 182 & - & - & - & 253 & - \\
\hline B-204 & - & - & 163 & - & - & - & 347 & - \\
\hline B-204 & - & - & 125 & - & - & - & 753 & - \\
\hline B-204 & - & - & 134 & - & - & - & 821 & - \\
\hline B-204 & - & - & 106 & - & - & - & 1201 & - \\
\hline B-204 & - & - & 115 & - & - & - & 700 & - \\
\hline B-204 & - & - & 87 & - & - & - & 875 & - \\
\hline B-204 & - & - & 96 & - & - & - & 629 & - \\
\hline B-204 & - & - & 68 & - & - & - & 1207 & - \\
\hline B-204 & - & - & 68 & - & - & - & 1217 & - \\
\hline B-204 & - & - & 49 & - & - & - & 826 & - \\
\hline B-204 & - & - & 49 & - & - & - & 1178 & - \\
\hline B-204 & - & - & 30 & - & - & - & 1410 & - \\
\hline B-204 & - & - & 39 & - & - & - & 594 & - \\
\hline B-204 & - & - & 11 & - & - & - & 1091 & - \\
\hline B-204 & - & - & 20 & - & - & - & 996 & - \\
\hline BX-107 & 1C sludge & 1.00 & $\mathrm{n} / \mathrm{a}$ & 0.50 & - & 300 & - & - \\
\hline BX-107 & - & - & $n / a$ & - & - & 880 & - & - \\
\hline BX-107 & - & - & $11 / \mathrm{a}$ & - & - & 480 & - & - \\
\hline C- 104 & CWP1 sludge & 0.34 & 80 & 0.54 & - & 345 & - & - \\
\hline C-104 & - & - & 75 & - & - & 289 & - & - \\
\hline C-104 & - & - & 68 & - & - & 521 & - & - \\
\hline C-104 & - & - & 58 & - & - & 2421 & - & - \\
\hline C-104 & - & - & 54 & - & - & 885 & - & - \\
\hline C-104 & - & - & 49 & - & - & 1304 & - & - \\
\hline C-104 & - & - & 44 & - & - & 791 & - & - \\
\hline C-104 & - & - & 39 & - & - & 2607 & - & - \\
\hline C-104 & - & - & 30 & - & - & 810 & - & - \\
\hline C-104 & - & - & 20 & - & - & 7077 & - & - \\
\hline C- 107 & 1C sludge & 0.54 & $\mathrm{n} / \mathrm{a}$ & 0.50 & - & 1183 & - & - \\
\hline C-107 & - & - & $n / a$ & - & - & {$[149$} & - & - \\
\hline C-107 & - & - & $\mathrm{n} / \mathrm{a}$ & - & - & 7826 & - & - \\
\hline C- 107 & - & - & $\mathrm{n} / \mathrm{a}$ & - & - & 228 & - & - \\
\hline C-107 & - & - & $\mathrm{n} / \mathrm{a}$ & - & - & 1087 & - & - \\
\hline $\mathrm{C}-107$ & - & - & $\mathrm{n} / \mathrm{a}$ & - & - & 350 & - & - \\
\hline C-107 & - & - & $\mathrm{n} / \mathrm{a}$ & - & - & 432 & - & - \\
\hline C-107 & - & - & $\mathrm{n} / \mathrm{a}$ & - & - & 1050 & - & - \\
\hline C-107 & - & - & $\mathrm{n} / \mathrm{a}$ & - & - & 1230 & - & - \\
\hline C-107 & - & - & $\mathrm{n} / \mathrm{a}$ & - & - & 1119 & - & - \\
\hline C-107 & - & - & $\mathrm{n} / \mathrm{a}$ & - & - & 1014 & - & - \\
\hline C-107 & - & - & $\mathrm{n} / \mathrm{a}$ & - & - & 75 & - & - \\
\hline C-107 & - & - & $\mathrm{n} / \mathrm{a}$ & - & - & 650 & - & - \\
\hline C-109 & TFeCN sludge & 0.38 & $\mathrm{n} / \mathrm{a}$ & 0.43 & - & 1730 & - & - \\
\hline
\end{tabular}


Table I.2. (contd)

\begin{tabular}{|c|c|c|c|c|c|c|c|c|}
\hline \multirow[b]{2}{*}{ Tank } & \multirow[b]{2}{*}{ Primary Waste Type } & \multirow[b]{2}{*}{$\begin{array}{l}\text { Primary } \\
\text { Waste Type } \\
\text { Fraction }\end{array}$} & \multirow[b]{2}{*}{$\begin{array}{l}\text { Elevation in } \\
\text { Tank (in.) }\end{array}$} & \multirow[b]{2}{*}{$\begin{array}{l}\text { Average } \\
\text { Mass } \\
\text { Fraction } \\
\text { UDS }\end{array}$} & \multirow[b]{2}{*}{$\begin{array}{l}\text { Specific Mass } \\
\text { Fraction UDS }\end{array}$} & \multicolumn{3}{|c|}{ Shear Strength (Pa) } \\
\hline & & & & & & Shear Vane & Core Extrusion & Ball Rheometer \\
\hline $\mathrm{C}-110$ & 1C sludge & 1.00 & $\mathrm{n} / \mathrm{a}$ & 0.49 & - & 500 & - & \begin{tabular}{|c|}
- \\
\end{tabular} \\
\hline $\mathrm{C}-110$ & - & - & $\mathrm{n} / \mathrm{a}$ & - & - & 500 & - & - \\
\hline C-112 & TFeCN sludge & 0.69 & $\mathrm{n} / \mathrm{a}$ & 0.44 & - & 1600 & - & - \\
\hline Group 1 & $1 \mathrm{C}$ and $2 \mathrm{C}$ sludge & $\mathrm{n} / \mathrm{a}$ & $\mathrm{n} / \mathrm{a}$ & & 0.09 & 15 & - & - \\
\hline Group 2 & $\mathrm{BY}, \mathrm{T} 1$, and $\mathrm{T} 2$ saltcake & $\mathrm{n} / \mathrm{a}$ & $\mathrm{n} / \mathrm{a}$ & & 0.374 & 21 & - & - \\
\hline Group 3 & CWP1 and CWP2 sludge & $n / a$ & $n / a$ & & 0.288 & 700 & - & - \\
\hline Group 4 & CWR1 sludge & $n / a$ & $\mathrm{n} / \mathrm{a}$ & & 0.297 & 100 & - & - \\
\hline Group 4 & - & - & $\mathrm{n} / \mathrm{a}$ & - & 0.297 & 340 & - & - \\
\hline Group 4 & - & - & $\mathrm{n} / \mathrm{a}$ & - & 0.297 & 290 & - & - \\
\hline Group 5 & RI (boiling) sludge & $\mathrm{n} / \mathrm{a}$ & $n / a$ & & 0.185 & 290 & - & - \\
\hline Group 5 & - & - & $11 / \mathrm{a}$ & - & 0.185 & 430 & - & - \\
\hline Group 5 & - & - & $\mathrm{n} / \mathrm{a}$ & - & 0.185 & 72 & - & - \\
\hline Group 5 & - & - & $\mathrm{n} / \mathrm{a}$ & - & 0.185 & 350 & - & - \\
\hline Group 5 & - & - & $\mathrm{n} / \mathrm{a}$ & - & 0.185 & 360 & - & - \\
\hline Group 6 & S1 and S2 Saltcake & $\mathrm{n} / \mathrm{a}$ & $\mathrm{n} / \mathrm{a}$ & & 0.147 & 20 & - & - \\
\hline Group 7 & TBP sludge & $\mathrm{n} / \mathrm{a}$ & $\mathrm{n} / \mathrm{a}$ & & 0.1 & 23 & - & - \\
\hline Group 8 & PFeCN sludge & $\mathrm{n} / \mathrm{a}$ & $\mathrm{n} / \mathrm{a}$ & & 0.114 & 11 & - & - \\
\hline Group 8 & - & - & $\mathrm{n} / \mathrm{a}$ & - & 0.114 & 13 & - & - \\
\hline Group 8 & - & - & $\mathrm{n} / \mathrm{a}$ & - & 0.114 & 13 & - & - \\
\hline S-102 & S2 saltcake & 0.59 & 22 & 0.14 & - & - & 800 & - \\
\hline S-102 & - & - & 35 & - & - & - & 1000 & - \\
\hline S-102 & - & - & 55 & - & - & - & 800 & - \\
\hline S-102 & - & - & 73 & - & - & - & 1000 & - \\
\hline S-102 & - & - & 113 & - & - & - & 800 & - \\
\hline S-102 & - & - & 130 & - & - & - & 500 & - \\
\hline S-102 & - & - & 150 & - & - & - & 190 & - \\
\hline S-102 & - & - & 168 & - & - & - & 170 & - \\
\hline S-102 & - & - & 190 & - & - & - & 500 & - \\
\hline S-102 & - & - & 12 & - & - & - & 600 & - \\
\hline S-102 & - & - & 38 & - & - & - & 800 & - \\
\hline S-102 & - & - & 70 & - & - & - & 800 & - \\
\hline S-102 & - & - & 80 & - & - & - & 450 & - \\
\hline S-102 & - & - & 95 & - & - & - & 70 & - \\
\hline S-102 & - & - & 108 & - & - & - & 300 & - \\
\hline S-102 & - & - & 113 & - & - & - & 50 & - \\
\hline $\mathrm{S}-102$ & - & - & 120 & - & - & - & 50 & - \\
\hline S-102 & - & - & 132 & - & - & - & 500 & - \\
\hline S-102 & - & - & 152 & - & - & - & 130 & - \\
\hline S-102 & - & - & 168 & - & - & - & 130 & - \\
\hline S-102 & - & - & 190 & - & - & - & 100 & - \\
\hline S-102 & - & - & 190 & - & - & - & 70 & - \\
\hline S-102 & - & - & 171 & - & - & - & 225 & - \\
\hline S-102 & - & - & 152 & - & - & - & 375 & - \\
\hline S-102 & - & - & 133 & - & - & - & 950 & - \\
\hline S-102 & - & - & 114 & - & - & - & 150 & - \\
\hline S-102 & - & - & 114 & - & - & - & 375 & - \\
\hline S-102 & - & - & 95 & - & - & - & 263 & - \\
\hline S-102 & - & - & 76 & - & - & - & 800 & - \\
\hline S-102 & - & - & 76 & - & - & - & 1050 & - \\
\hline S-102 & - & - & 57 & - & - & - & 1350 & - \\
\hline S-102 & - & - & 38 & - & - & - & 1500 & - \\
\hline S-102 & - & - & 19 & - & - & - & 600 & - \\
\hline S- 104 & R saltcake & 0.47 & $\mathrm{n} / \mathrm{a}$ & 0.36 & - & 1040 & - & - \\
\hline S-104 & - & - & $\mathrm{n} / \mathrm{a}$ & - & - & 6380 & - & - \\
\hline SY-101 & S2 saltcake & 1.00 & $\mathrm{n} / \mathrm{a}$ & 0.34 & - & 13036 & - & - \\
\hline SY-101 & - & - & 143 & - & - & 4750 & - & - \\
\hline SY-101 & - & - & 143 & - & - & 730 & - & - \\
\hline SY-101 & - & - & 143 & - & - & 2100 & - & - \\
\hline SY-101 & - & - & 143 & - & - & 530 & - & - \\
\hline SY-101 & - & - & 67 & - & - & 1500 & - & - \\
\hline SY-101 & - & - & 67 & - & - & 640 & - & - \\
\hline SY-101 & - & - & 67 & - & - & 300 & - & - \\
\hline SY-101 & - & - & 67 & - & - & 510 & - & - \\
\hline
\end{tabular}


Table I.2. (contd)

\begin{tabular}{|c|c|c|c|c|c|c|c|c|}
\hline \multirow[b]{2}{*}{ Tank } & \multirow[b]{2}{*}{ Primary Waste Type } & \multirow[b]{2}{*}{$\begin{array}{l}\text { Primary } \\
\text { Waste Type } \\
\text { Fraction }\end{array}$} & \multirow[b]{2}{*}{$\begin{array}{l}\text { Elevation in } \\
\text { Tank (in.) }\end{array}$} & \multirow[b]{2}{*}{$\begin{array}{l}\text { Average } \\
\text { Mass } \\
\text { Fraction } \\
\text { UDS }\end{array}$} & \multirow[b]{2}{*}{$\begin{array}{l}\text { Specific Mass } \\
\text { Fraction UDS }\end{array}$} & \multicolumn{3}{|c|}{ Shear Strength (Pa) } \\
\hline & & & & & & Shear Vane & Core Extrusion & Ball Rheometer \\
\hline SY-101 & - & - & 10 & - & - & 11600 & - & - \\
\hline SY-I0I & - & - & 10 & - & - & 2640 & - & - \\
\hline SY-101 & - & - & 10 & - & - & 400 & - & - \\
\hline SY-101 & - & - & 10 & - & - & 1400 & - & - \\
\hline SY-101 & - & - & 143 & - & - & 1720 & - & - \\
\hline SY-101 & - & - & 143 & - & - & 450 & - & - \\
\hline SY-101 & - & - & 143 & - & - & 790 & - & - \\
\hline SY-101 & - & - & 143 & - & - & 230 & - & - \\
\hline SY-101 & - & - & 67 & - & - & 1850 & - & - \\
\hline SY-I0I & - & - & 67 & - & - & 370 & - & - \\
\hline SY-101 & - & - & 67 & - & - & 730 & - & - \\
\hline SY-101 & - & - & 67 & - & - & 670 & - & - \\
\hline SY-101 & - & - & 124 & - & - & 240 & - & - \\
\hline SY-101 & - & - & 124 & - & - & 330 & - & - \\
\hline SY-101 & - & - & 86 & - & - & 190 & - & - \\
\hline SY-101 & - & - & $\mathrm{n} / \mathrm{a}$ & - & - & 60 & - & - \\
\hline SY-101 & - & - & $\mathrm{n} / \mathrm{a}$ & - & - & 110 & - & - \\
\hline SY-101 & - & - & 124 & - & - & 220 & - & - \\
\hline SY-101 & - & - & 124 & - & - & 170 & - & - \\
\hline SY-101 & - & - & 124 & - & - & 80 & - & - \\
\hline SY-101 & - & - & 124 & - & - & 200 & - & - \\
\hline SY-I0I & - & - & 124 & - & - & 430 & - & - \\
\hline SY-101 & - & - & 124 & - & - & 380 & - & - \\
\hline SY-101 & - & - & 124 & - & - & 260 & - & - \\
\hline SY-101 & - & - & 124 & - & - & 370 & - & - \\
\hline SY-101 & - & - & 124 & - & - & 360 & - & - \\
\hline SY-101 & - & - & 124 & - & - & 420 & - & - \\
\hline SY-10I & - & - & 124 & - & - & 400 & - & - \\
\hline SY-101 & - & - & 124 & - & - & 190 & - & - \\
\hline SY-10I & - & - & 86 & - & - & 170 & - & - \\
\hline SY-101 & - & - & 86 & - & - & 190 & - & - \\
\hline SY-101 & - & - & 86 & - & - & 170 & - & - \\
\hline SY-101 & - & - & 86 & - & - & 440 & - & - \\
\hline SY-101 & - & - & 86 & - & - & 40 & - & - \\
\hline SY-101 & - & - & 86 & - & - & 130 & - & - \\
\hline SY-101 & - & - & $\mathrm{n} / \mathrm{a}$ & - & - & 60 & - & - \\
\hline SY-101 & - & - & $\mathrm{n} / \mathrm{a}$ & - & - & 110 & - & - \\
\hline SY-101 & - & - & 10 & - & - & 2400 & - & - \\
\hline SY-102 & Unidentified sludge & 0.51 & $11 / \mathrm{a}$ & 0.51 & - & 3900 & - & - \\
\hline SY-102 & - & - & $\mathrm{n} / \mathrm{a}$ & - & - & 279 & - & - \\
\hline SY-102 & - & - & $\mathrm{n} / \mathrm{a}$ & - & - & 304 & - & - \\
\hline SY-102 & - & - & $\mathrm{n} / \mathrm{a}$ & - & - & 101 & - & - \\
\hline SY-103 & S2 saltcake & 1.00 & 40 & 0.11 & - & - & - & 900 \\
\hline SY-103 & - & - & 41 & - & - & - & - & 225 \\
\hline SY-103 & - & - & 47 & - & - & - & - & 200 \\
\hline SY-103 & - & - & 51 & - & - & - & - & 185 \\
\hline SY-103 & - & - & 55 & - & - & - & - & 170 \\
\hline SY-103 & - & - & 59 & - & - & - & - & 155 \\
\hline SY-103 & - & - & 63 & - & - & - & - & 142 \\
\hline SY-103 & - & - & 67 & - & - & - & - & 128 \\
\hline SY-103 & - & - & 71 & - & - & - & - & 116 \\
\hline SY-103 & - & - & 75 & - & - & - & - & 104 \\
\hline SY-103 & - & - & 79 & - & - & - & - & 92 \\
\hline SY-103 & - & - & 83 & - & - & - & - & 81 \\
\hline SY-103 & - & - & 87 & - & - & - & - & 71 \\
\hline SY-103 & - & - & 91 & - & - & - & - & 61 \\
\hline SY-103 & - & - & 94 & - & - & - & - & 52 \\
\hline SY-103 & - & - & 98 & - & - & - & - & 43 \\
\hline SY-103 & - & - & 102 & - & - & - & - & 35 \\
\hline SY-103 & - & - & 106 & - & - & - & - & 28 \\
\hline SY-103 & - & - & 110 & - & - & - & - & 21 \\
\hline SY-103 & - & - & 114 & - & - & - & - & 16 \\
\hline SY-103 & - & - & 118 & - & - & - & - & 10 \\
\hline
\end{tabular}


Table I.2. (contd)

\begin{tabular}{|c|c|c|c|c|c|c|c|c|}
\hline \multirow[b]{2}{*}{ Tank } & \multirow[b]{2}{*}{ Primary Waste Type } & \multirow[b]{2}{*}{$\begin{array}{l}\text { Primary } \\
\text { Waste Type } \\
\text { Fraction }\end{array}$} & \multirow[b]{2}{*}{$\begin{array}{l}\text { Elevation in } \\
\text { Tank (in.) }\end{array}$} & \multirow{2}{*}{$\begin{array}{l}\text { Average } \\
\text { Mass } \\
\text { Fraction } \\
\text { UDS }\end{array}$} & \multirow[b]{2}{*}{$\begin{array}{l}\text { Specific Mass } \\
\text { Fraction UDS }\end{array}$} & \multicolumn{3}{|c|}{ Shear Strength (Pa) } \\
\hline & & & & & & Shear Vane & Core Extrusion & Ball Rheometer \\
\hline SY-103 & - & - & 122 & - & - & - & - & 6 \\
\hline SY-I03 & - & - & 124 & - & - & - & - & 2 \\
\hline SY-103 & - & - & 41 & - & - & - & - & 900 \\
\hline SY-103 & - & - & 41 & - & - & - & - & 293 \\
\hline SY-103 & - & - & 47 & - & - & - & - & 275 \\
\hline SY-103 & - & - & 51 & - & - & - & - & 263 \\
\hline SY-103 & - & - & 55 & - & - & - & - & 252 \\
\hline SY-103 & - & - & 59 & - & - & - & - & 239 \\
\hline SY-103 & - & - & 63 & - & - & - & - & 227 \\
\hline SY-I03 & - & - & 67 & - & - & - & - & 215 \\
\hline SY-103 & - & - & 71 & - & - & - & - & 203 \\
\hline SY-103 & - & - & 75 & - & - & - & - & 190 \\
\hline SY-103 & - & - & 79 & - & - & - & - & 178 \\
\hline SY-103 & - & - & 83 & - & - & - & - & 165 \\
\hline SY-103 & - & - & 87 & - & - & - & - & 153 \\
\hline SY-103 & - & - & 91 & - & - & - & - & 140 \\
\hline SY-103 & - & - & 94 & - & - & - & - & 127 \\
\hline SY-103 & - & - & 98 & - & - & - & - & 114 \\
\hline SY-103 & - & - & 102 & - & - & - & - & 100 \\
\hline SY-103 & - & - & 106 & - & - & - & - & 87 \\
\hline SY-103 & - & - & 110 & - & - & - & - & 73 \\
\hline SY-I03 & - & - & 114 & - & - & - & - & 59 \\
\hline SY-103 & - & - & 118 & - & - & - & - & 45 \\
\hline SY-103 & - & - & 122 & - & - & - & - & 31 \\
\hline SY-103 & - & - & 126 & - & - & - & - & 16 \\
\hline SY-103 & - & - & 130 & - & - & - & - & 1 \\
\hline SY-103 & - & - & 114 & - & - & - & 50 & - \\
\hline SY-103 & - & - & 94 & - & - & - & 150 & - \\
\hline SY-103 & - & - & 76 & - & - & - & 200 & - \\
\hline SY-103 & - & - & 57 & - & - & - & 375 & - \\
\hline SY-103 & - & - & 38 & - & - & - & 200 & - \\
\hline SY-103 & - & - & 57 & - & - & - & 206 & - \\
\hline SY-103 & - & - & 38 & - & - & - & 312 & - \\
\hline SY-103 & - & - & 105 & - & - & 980 & - & - \\
\hline SY-103 & - & - & 86 & - & - & 1100 & - & - \\
\hline SY-103 & - & - & 67 & - & - & 1900 & - & - \\
\hline SY-103 & - & - & 48 & - & - & 1300 & - & - \\
\hline SY-103 & - & - & 29 & - & - & 1900 & - & - \\
\hline SY-103 & - & - & 28 & - & - & 2000 & - & - \\
\hline SY-103 & - & - & 47 & - & - & $\mathrm{I} 400$ & - & - \\
\hline SY-103 & - & - & 63 & - & - & 2000 & - & - \\
\hline SY-103 & - & - & 87 & - & - & 1300 & - & - \\
\hline SY-103 & - & - & 106 & - & - & 1000 & - & - \\
\hline $\mathrm{T}-107$ & 1C sludge & 0.85 & $n / a$ & 0.49 & - & 720 & - & - \\
\hline $\mathrm{T}-110$ & 2C sludge & 0.97 & 131 & 0.22 & - & - & 194 & - \\
\hline $\mathrm{T}-110$ & - & - & 93 & - & - & - & 1000 & - \\
\hline $\mathrm{T}-110$ & - & - & 36 & - & - & - & 971 & - \\
\hline $\mathrm{T}-110$ & - & - & 36 & - & - & - & 1586 & - \\
\hline $\mathrm{T}-110$ & - & - & 17 & - & - & - & 1990 & - \\
\hline $\mathrm{T}-110$ & - & - & 27 & - & - & - & 1296 & - \\
\hline $\mathrm{T}-110$ & - & - & $n / a$ & - & 0.507 & 70 & - & - \\
\hline $\mathrm{T}-111$ & 2C sludge & 0.55 & $\mathrm{n} / \mathrm{a}$ & 0.17 & - & 180 & - & - \\
\hline $\mathrm{T}-111$ & - & - & $\mathrm{n} / \mathrm{a}$ & - & - & 450 & - & - \\
\hline $\mathrm{T}-201$ & 224 Pre-1949 sludge & 1.00 & 52 & 0.23 & - & - & 1469 & - \\
\hline $\mathrm{T}-201$ & - & - & 61 & - & - & - & 864 & - \\
\hline T-201 & - & - & 33 & - & - & - & 2065 & - \\
\hline $\mathrm{T}-201$ & - & - & 42 & - & - & - & 2738 & - \\
\hline $\mathrm{T}-202$ & 224 Post-1949 sludge & 1.00 & 90 & 0.08 & - & - & 84 & - \\
\hline $\mathrm{T}-202$ & - & - & 99 & - & - & - & 68 & - \\
\hline $\mathrm{T}-202$ & - & - & 52 & - & - & - & 572 & - \\
\hline $\mathrm{T}-202$ & - & - & 33 & - & - & - & 996 & - \\
\hline $\mathrm{T}-202$ & - & - & 42 & - & - & - & 901 & - \\
\hline $\mathrm{T}-202$ & - & - & 14 & - & - & - & 794 & - \\
\hline
\end{tabular}


Table I.2. (contd)

\begin{tabular}{|c|c|c|c|c|c|c|c|c|}
\hline \multirow[b]{2}{*}{ Tank } & \multirow[b]{2}{*}{ Primary Waste Type } & \multirow[b]{2}{*}{$\begin{array}{l}\text { Primary } \\
\text { Waste Type } \\
\text { Fraction }\end{array}$} & \multirow[b]{2}{*}{$\begin{array}{l}\text { Elevation in } \\
\text { Tank (in.) }\end{array}$} & \multirow{2}{*}{$\begin{array}{l}\text { Average } \\
\text { Mass } \\
\text { Fraction } \\
\text { UDS }\end{array}$} & \multirow[b]{2}{*}{$\begin{array}{l}\text { Specific Mass } \\
\text { Fraction UDS }\end{array}$} & \multicolumn{3}{|c|}{ Shear Strength (Pa) } \\
\hline & & & & & & Shear Vane & Core Extrusion & Ball Rheometer \\
\hline $\mathrm{T}-202$ & - & - & 14 & - & - & -2 & 1112 & - \\
\hline $\mathrm{T}-202$ & - & - & 23 & - & - & - & 1027 & - \\
\hline $\mathrm{T}-203$ & 224 Post-1949 sludge & 1.00 & 175 & 0.12 & - & - & 79 & - \\
\hline T-203 & - & - & 185 & - & - & - & 70 & - \\
\hline $\mathrm{T}-203$ & - & - & 185 & - & - & - & 62 & - \\
\hline $\mathrm{T}-203$ & - & - & 156 & - & - & - & 387 & - \\
\hline T-203 & - & - & 118 & - & - & - & 903 & - \\
\hline T-203 & - & - & 99 & - & - & - & 1315 & - \\
\hline $\mathrm{T}-203$ & - & - & 109 & - & - & - & 926 & - \\
\hline $\mathrm{T}-203$ & - & - & 80 & - & - & - & 1196 & - \\
\hline $\mathrm{T}-203$ & - & - & 61 & - & - & - & 835 & - \\
\hline $\mathrm{T}-203$ & - & - & 42 & - & - & - & 1130 & - \\
\hline $\mathrm{T}-203$ & - & - & 23 & - & - & - & 1605 & - \\
\hline $\mathrm{T}-203$ & - & - & 33 & - & - & - & 1337 & - \\
\hline T-203 & - & - & 175 & - & - & - & 863 & - \\
\hline $\mathrm{T}-203$ & - & - & $\mathrm{n} / \mathrm{a}$ & - & 0.243 & 3770 & - & - \\
\hline $\mathrm{T}-203$ & - & - & $\mathrm{n} / \mathrm{a}$ & - & 0.22 & 310 & - & - \\
\hline $\mathrm{T}-204$ & 224 Post-1949 sludge & 1.00 & 187 & 0.08 & - & - & 95 & - \\
\hline T-204 & - & - & 196 & - & - & - & 47 & - \\
\hline T-204 & - & - & 177 & - & - & - & 63 & - \\
\hline T-204 & - & - & 158 & - & - & - & 58 & - \\
\hline T-204 & - & - & 130 & - & - & - & 581 & - \\
\hline T-204 & - & - & 111 & - & - & - & 752 & - \\
\hline $\mathrm{T}-204$ & - & - & 92 & - & - & - & 1476 & - \\
\hline T-204 & - & - & 101 & - & - & - & 1086 & - \\
\hline $\mathrm{T}-204$ & - & - & 82 & - & - & - & 1017 & - \\
\hline $\mathrm{T}-204$ & - & - & 54 & - & - & - & 1637 & - \\
\hline T-204 & - & - & 63 & - & - & - & 1165 & - \\
\hline T-204 & - & - & 35 & - & - & - & 1990 & - \\
\hline T-204 & - & - & 44 & - & - & - & 747 & - \\
\hline $\mathrm{T}-204$ & - & - & $\mathrm{n} / \mathrm{a}$ & - & 0.174 & 1520 & - & - \\
\hline T-204 & - & - & $\mathrm{n} / \mathrm{a}$ & - & 0.159 & 842 & - & - \\
\hline T-204 & - & - & $\mathrm{n} / \mathrm{a}$ & - & 0.103825137 & 20 & - & - \\
\hline U-103 & S1 saltcake & 0.69 & 160 & 0.15 & - & - & 100 & - \\
\hline U-103 & - & - & 140 & - & - & - & 200 & - \\
\hline U-103 & - & - & 110 & - & - & - & 1750 & - \\
\hline U-103 & - & - & 100 & - & - & - & 1300 & - \\
\hline U-103 & - & - & 85 & - & - & - & 500 & - \\
\hline $\mathrm{U}-103$ & - & - & 70 & - & - & - & 1200 & - \\
\hline U-103 & - & - & 60 & - & - & - & 800 & - \\
\hline U-103 & - & - & 50 & - & - & - & 20 & - \\
\hline U-103 & - & - & 25 & - & - & - & 700 & - \\
\hline U-103 & - & - & 18 & - & - & - & 150 & - \\
\hline U-103 & - & - & 10 & - & - & - & 1000 & - \\
\hline U-103 & - & - & 170 & - & - & - & 50 & - \\
\hline U-103 & - & - & 135 & - & - & - & 70 & - \\
\hline U-103 & - & - & 120 & - & - & - & 150 & - \\
\hline U-103 & - & - & 110 & - & - & - & 200 & - \\
\hline U-103 & - & - & 78 & - & - & - & 1000 & - \\
\hline U-103 & - & - & 167 & - & - & - & 60 & - \\
\hline U-103 & - & - & 157 & - & - & - & 250 & - \\
\hline U-103 & - & - & 172 & - & - & - & 25 & - \\
\hline U-103 & - & - & 142 & - & - & - & 175 & - \\
\hline U-103 & - & - & 170 & - & - & - & 35 & - \\
\hline U-103 & - & - & 133 & - & - & - & 60 & - \\
\hline U-103 & - & - & 110 & - & - & - & 225 & - \\
\hline U-103 & - & - & 48 & - & - & - & 25 & - \\
\hline U-107 & S2 saltcake & 0.91 & 137 & 0.60 & - & - & 15 & - \\
\hline U-107 & - & - & 128 & - & - & - & 20 & - \\
\hline U-107 & - & - & 118 & - & - & - & 25 & - \\
\hline U-107 & - & - & 112 & - & - & - & 50 & - \\
\hline U-107 & - & - & 108 & - & - & - & 70 & - \\
\hline U-107 & - & - & 131 & - & - & - & 50 & - \\
\hline
\end{tabular}


Table I.2. (contd)

\begin{tabular}{|c|c|c|c|c|c|c|c|c|}
\hline \multirow[b]{2}{*}{ Tank } & \multirow[b]{2}{*}{ Primary Waste Type } & \multirow[b]{2}{*}{$\begin{array}{l}\text { Primary } \\
\text { Waste Type } \\
\text { Fraction }\end{array}$} & \multirow[b]{2}{*}{$\begin{array}{l}\text { Elevation in } \\
\text { Tank (in.) }\end{array}$} & \multirow[b]{2}{*}{$\begin{array}{l}\text { Average } \\
\text { Mass } \\
\text { Fraction } \\
\text { UDS }\end{array}$} & \multirow[b]{2}{*}{$\begin{array}{l}\text { Specific Mass } \\
\text { Fraction UDS }\end{array}$} & \multicolumn{3}{|c|}{ Shear Strength (Pa) } \\
\hline & & & & & & Shear Vane & Core Extrusion & Ball Rheometer \\
\hline U-107 & - & - & 112 & - & - & - & 150 & - \\
\hline$U-107$ & - & - & 102 & - & - & - & 50 & - \\
\hline U-107 & - & - & 93 & - & - & - & 200 & - \\
\hline U-107 & - & - & 82 & - & - & - & 550 & - \\
\hline U-107 & - & - & 79 & - & - & - & 130 & - \\
\hline U-107 & - & - & 73 & - & - & - & 380 & - \\
\hline U-107 & - & - & 70 & - & - & - & 380 & - \\
\hline U- 107 & - & - & 58 & - & - & - & 50 & - \\
\hline U-107 & - & - & 54 & - & - & - & 400 & - \\
\hline$U-107$ & - & - & 141 & - & - & - & 30 & - \\
\hline U-107 & - & - & 137 & - & - & - & 50 & - \\
\hline U-107 & - & - & 77 & - & - & - & 375 & - \\
\hline U-107 & - & - & 62 & - & - & - & 300 & - \\
\hline U-107 & - & - & 58 & - & - & - & 450 & - \\
\hline U-107 & - & - & 55 & - & - & - & 230 & - \\
\hline U-107 & - & - & 50 & - & - & - & 100 & - \\
\hline U-107 & - & - & 40 & - & - & - & 225 & - \\
\hline U-107 & - & - & 12 & - & - & - & 50 & - \\
\hline U-107 & - & - & 140 & - & - & - & 38 & - \\
\hline U-107 & - & - & 39 & - & - & - & 150 & - \\
\hline U-107 & - & - & 39 & - & - & - & 300 & - \\
\hline U- 107 & - & - & 135 & - & - & - & 25 & - \\
\hline U-107 & - & - & 130 & - & - & - & 50 & - \\
\hline U-107 & - & - & 118 & - & - & - & 40 & - \\
\hline U-107 & - & - & 108 & - & - & - & 100 & - \\
\hline U-107 & - & - & 92 & - & - & - & 225 & - \\
\hline U- 107 & - & - & 94 & - & - & - & 200 & - \\
\hline U-107 & - & - & 127 & - & - & - & 25 & - \\
\hline U-107 & - & - & 127 & - & - & - & 25 & - \\
\hline U-107 & - & - & 90 & - & - & 51 & - & - \\
\hline
\end{tabular}


Table I.3. Bingham Rheology

\begin{tabular}{|c|c|c|c|c|c|c|}
\hline \multirow[b]{2}{*}{ Tank } & \multirow[b]{2}{*}{ Primary Waste Type } & \multirow[b]{2}{*}{$\begin{array}{c}\text { Primary } \\
\text { Waste Type } \\
\text { Fraction }\end{array}$} & \multirow[b]{2}{*}{\begin{tabular}{|l|} 
Sample Mass \\
Fraction UDS
\end{tabular}} & \multirow[b]{2}{*}{$\begin{array}{c}\text { Sample } \\
\text { Temperature }\left({ }^{\circ} \mathrm{C}\right)\end{array}$} & \multicolumn{2}{|c|}{ Bingham Parameters } \\
\hline & & & & & $\begin{array}{c}\text { Yield Stress } \\
(\mathrm{Pa})\end{array}$ & $\begin{array}{c}\text { Viscosity } \\
\text { (cP) }\end{array}$ \\
\hline Group 1 & $1 \mathrm{C}$ and $2 \mathrm{C}$ sludge & $\mathrm{n} / \mathrm{a}$ & 0.09 & 25 & 0 & 6.4 \\
\hline Group 1 & - & - & 0.09 & 25 & 0.3 & 6.7 \\
\hline Group 1 & - & - & 0.09 & 40 & 0.5 & 6.3 \\
\hline Group 1 & - & - & 0.09 & 60 & 0.5 & 5.6 \\
\hline BX-107 & lC sludge & 1.00 & 0.01 & 25 & 0.1 & 1.8 \\
\hline BX-107 & - & - & 0.01 & 25 & 0.3 & 1.9 \\
\hline BX-107 & - & - & 0.02 & 25 & 0.1 & 2.3 \\
\hline BX-107 & - & - & 0.06 & 25 & 0 & 3.3 \\
\hline BX-107 & - & - & 0.08 & 25 & 0.2 & 4.5 \\
\hline BX-107 & - & - & 0.08 & 25 & 0.4 & 4.3 \\
\hline BX-107 & - & - & 0.01 & 95 & 0 & 1.4 \\
\hline BX-107 & - & - & 0.01 & 95 & 0 & 1.4 \\
\hline BX-107 & - & - & 0.02 & 95 & 0 & 1.5 \\
\hline BX-107 & - & - & 0.06 & 95 & 0.2 & 2 \\
\hline BX-107 & - & - & 0.08 & 95 & 0.9 & 1.2 \\
\hline BX-I07 & - & - & 0.08 & 95 & 1.4 & 4.6 \\
\hline C- 107 & 1C sludge & 0.54 & 0.10 & 20 & 0 & 1.5 \\
\hline C-107 & - & - & 0.10 & 20 & 0 & 1.6 \\
\hline C- 107 & - & - & 0.11 & 25 & 0 & 1.1 \\
\hline C-107 & - & - & 0.17 & 25 & 0 & 1.3 \\
\hline C-107 & - & - & 0.21 & 25 & 0 & 2.5 \\
\hline C-107 & 1C sludge & 0.54 & 0.16 & 25 & 0 & 2.6 \\
\hline C-107 & - & - & 0.16 & 25 & 0 & 1.5 \\
\hline C-107 & - & - & 0.21 & 25 & 0 & 1.8 \\
\hline C-107 & - & - & 0.21 & 25 & 0 & 1 \\
\hline C-107 & - & - & 0.11 & 25 & 0 & 1.7 \\
\hline C-107 & - & - & 0.17 & 25 & 0 & 1.4 \\
\hline C-107 & - & - & 0.21 & 25 & 0.3 & 2.1 \\
\hline C-107 & lC sludge & 0.54 & 0.16 & 45 & 0 & 1.4 \\
\hline C-107 & - & - & 0.21 & 45 & 0 & 1 \\
\hline C-107 & - & - & 0.11 & 45 & 0 & 1.6 \\
\hline C-107 & 1C sludge & 0.54 & 0.10 & 45 & 0 & 1.2 \\
\hline C-107 & - & - & 0.17 & 45 & 0 & 1.1 \\
\hline C-107 & - & - & 0.21 & 15 & 0 & 2 \\
\hline C-107 & - & - & 0.11 & 65 & 0 & 1.6 \\
\hline C-107 & 1C sludge & 0.54 & 0.10 & 65 & 0 & 0.9 \\
\hline C-107 & - & - & 0.16 & 65 & 0 & 1.3 \\
\hline C-107 & - & - & 0.21 & 65 & 0 & 0.9 \\
\hline C-107 & - & - & 0.17 & 65 & 0 & 1.2 \\
\hline C- 107 & - & - & 0.21 & 65 & 0 & 1.5 \\
\hline C-110 & 1C sludge & 1.00 & 0.01 & 30 & 0 & 2.1 \\
\hline C-110 & - & - & 0.01 & 30 & 0 & 2.2 \\
\hline C-110 & - & - & 0.04 & 30 & 0.2 & 3 \\
\hline C- 110 & - & - & 0.07 & 30 & 0.7 & 2.6 \\
\hline C-110 & - & - & 0.01 & 95 & 0.2 & 1.3 \\
\hline C-110 & - & - & 0.01 & 95 & 0 & 1.4 \\
\hline C-110 & - & - & 0.04 & 95 & 0.4 & 1.9 \\
\hline C-110 & - & - & 0.07 & 95 & 0.2 & 1.8 \\
\hline T-107 & 1C sludge & 0.85 & 0.10 & 25 & 0 & 4.5 \\
\hline T-107 & - & - & 0.19 & 25 & 0.9 & 6.7 \\
\hline T-107 & - & - & 0.10 & 90 & 0 & 2.6 \\
\hline T-107 & - & - & 0.19 & 90 & 0.6 & 6.6 \\
\hline B-202 & 224 Post- 1949 sludge & 1.00 & 0.04 & 29 & 0 & 2 \\
\hline B-202 & - & - & 0.04 & 29 & 0 & 2 \\
\hline B-202 & - & - & 0.09 & 29 & 1.1 & 3.7 \\
\hline B-202 & - & - & 0.10 & 29 & 1.1 & 3.4 \\
\hline B-202 & - & - & 0.04 & 30 & 0 & 2.2 \\
\hline B-202 & - & - & 0.04 & 30 & 0 & 2 \\
\hline B-202 & - & - & 0.04 & 30 & 0 & 2 \\
\hline B-202 & - & - & 0.09 & 30 & 0.6 & 2.7 \\
\hline B-202 & - & - & 0.09 & 30 & 0.5 & 2.7 \\
\hline B-202 & - & - & 0.09 & 30 & 0.9 & 3.5 \\
\hline B-202 & - & - & 0.10 & 30 & $\mathrm{I}$ & 30.8 \\
\hline
\end{tabular}


Table I.3. (contd)

\begin{tabular}{|c|c|c|c|c|c|c|}
\hline \multirow[b]{2}{*}{ Tank } & \multirow[b]{2}{*}{ Primary Waste Type } & \multirow[b]{2}{*}{$\begin{array}{c}\text { Primary } \\
\text { Waste Type } \\
\text { Fraction } \\
\end{array}$} & \multirow[b]{2}{*}{$\begin{array}{l}\text { Sample Mass } \\
\text { Fraction UDS }\end{array}$} & \multirow[b]{2}{*}{$\begin{array}{c}\text { Sample } \\
\text { Temperature }\left({ }^{\circ} \mathrm{C}\right)\end{array}$} & \multicolumn{2}{|c|}{ Bingham Parameters } \\
\hline & & & & & $\begin{array}{c}\text { Yield Stress } \\
\text { (Pa) }\end{array}$ & $\begin{array}{c}\text { Viscosity } \\
\text { (cP) }\end{array}$ \\
\hline $\mathrm{B}-202$ & - & - & 0.04 & 95 & 0 & 1.5 \\
\hline B-202 & - & - & 0.04 & 95 & 0 & 1 \\
\hline B-202 & - & - & 0.04 & 95 & 0 & 2 \\
\hline $\mathrm{B}-202$ & - & - & 0.04 & 95 & 0 & 2 \\
\hline $\mathrm{B}-202$ & - & - & 0.04 & 95 & 0 & 1 \\
\hline B-202 & - & - & 0.05 & 95 & 0 & 1.2 \\
\hline B-202 & - & - & 0.09 & 95 & 1.4 & 2.6 \\
\hline B-202 & - & - & 0.09 & 95 & 0.7 & 2.1 \\
\hline B-202 & - & - & 0.09 & 95 & 0.9 & 2.2 \\
\hline B-202 & - & - & 0.09 & 95 & 0.9 & 1.7 \\
\hline B-202 & - & - & 0.10 & 95 & 1.1 & 3.4 \\
\hline B-202 & - & - & 0.10 & 95 & 1.1 & 2.2 \\
\hline B-203 & 224 Post- 1949 sludge & 1.00 & 0.06 & 25 & 0.1 & 1.9 \\
\hline B-203 & - & - & 0.14 & 25 & 3.1 & 4.7 \\
\hline B-203 & - & - & 0.14 & 25 & 3.2 & 4.7 \\
\hline B-203 & - & - & 0.21 & 25 & 10.9 & 7.9 \\
\hline $\mathrm{T}-203$ & 224 Post- 1949 sludge & 1.00 & 0.04 & 25 & 0.1 & 1.5 \\
\hline $\mathrm{T}-203$ & - & - & 0.10 & 25 & 3.2 & 3.1 \\
\hline $\mathrm{T}-203$ & - & - & 0.22 & 25 & 35.5 & 14.8 \\
\hline $\mathrm{T}-204$ & 224 Post- 1949 sludge & 1.00 & 0.03 & 25 & 0.2 & 1.9 \\
\hline $\mathrm{T}-204$ & - & - & 0.07 & 25 & 3.1 & 3.8 \\
\hline B-201 & 224 Pre-1949 sludge & 1.00 & 0.05 & 30 & 0.3 & 1.9 \\
\hline $\mathrm{B}-201$ & - & - & 0.05 & 30 & 0.4 & 1.7 \\
\hline B-201 & - & - & 0.06 & 30 & 0.2 & 1.6 \\
\hline B-20I & - & - & 0.06 & 30 & 0.4 & 1.7 \\
\hline B-201 & - & - & 0.10 & 30 & 0.4 & 2 \\
\hline B-201 & - & - & 0.11 & 30 & 0.3 & 1.9 \\
\hline B-201 & - & - & 0.13 & 30 & 9.7 & 11.7 \\
\hline B-201 & - & - & 0.13 & 30 & 7.3 & 11.7 \\
\hline B-201 & - & - & 0.18 & 30 & 4.4 & 10.3 \\
\hline B-201 & - & - & 0.18 & 30 & 6.6 & 9.2 \\
\hline B-201 & - & - & 0.30 & 30 & 1.2 & 4 \\
\hline B-20I & - & - & 0.30 & 30 & 2.4 & 5 \\
\hline$T-110$ & 2C sludge & 0.97 & 0.01 & 25 & 0 & 1.3 \\
\hline $\mathrm{T}-110$ & - & - & 0.09 & 25 & 0.1 & 2.3 \\
\hline $\mathrm{T}-110$ & - & - & 0.20 & 25 & 1.5 & 5.9 \\
\hline $\mathrm{T}-111$ & 2C sludge & 0.55 & 0.00 & 27 & 0 & 3.8 \\
\hline$T-111$ & - & - & 0.08 & 27 & 0.6 & 5.4 \\
\hline $\mathrm{T}-111$ & - & - & 0.00 & 28 & 0 & 1.8 \\
\hline $\mathrm{T}-111$ & - & - & 0.00 & 95 & 0 & 0.8 \\
\hline $\mathrm{T}-111$ & - & - & 0.00 & 95 & 0 & 1.6 \\
\hline $\mathrm{T}-111$ & - & - & 0.03 & 95 & 0.2 & 1.6 \\
\hline $\mathrm{T}-111$ & - & - & 0.08 & 95 & 0.8 & 2.6 \\
\hline $\mathrm{AY}-102$ & BL sludge & 1.00 & 0.06 & 25 & 1.5 & 4.3 \\
\hline AY-102 & BL sludge & 1.00 & 0.08 & 27 & 0 & 2.3 \\
\hline $\mathrm{AY}-102$ & - & - & 0.15 & 27 & 0 & 3 \\
\hline AY-102 & - & - & 0.17 & 27 & 0 & 2.1 \\
\hline AY-102 & - & - & 0.25 & 27 & 0 & 3.6 \\
\hline $\mathrm{AY}-102$ & - & - & 0.06 & 40 & 1.4 & 3.1 \\
\hline $\mathrm{AY}-102$ & BL sludge & 1.00 & 0.08 & 45 & 0 & 0.9 \\
\hline AY-102 & - & - & 0.15 & 45 & 0 & 1.7 \\
\hline $\mathrm{AY}-102$ & - & - & 0.17 & 45 & 0 & 1.2 \\
\hline $\mathrm{AY}-102$ & - & - & 0.25 & 45 & 0 & 2.7 \\
\hline AY-102 & - & - & 0.00 & 65 & 0 & 0.3 \\
\hline $\mathrm{AY}-102$ & - & - & 0.08 & 65 & 0 & 1.1 \\
\hline $\mathrm{AY}-102$ & - & - & 0.15 & 65 & 0 & 0.9 \\
\hline $\mathrm{AY}-102$ & - & - & 0.17 & 65 & 0 & 1.3 \\
\hline $\mathrm{AY}-102$ & - & - & 0.25 & 65 & 0 & 1.7 \\
\hline Group 2 & $\mathrm{BY}, \mathrm{T} 1$, and $\mathrm{T} 2$ saltcake & $\mathrm{n} / \mathrm{a}$ & 0.37 & 25 & 1.7 & 14 \\
\hline Group 2 & - & - & 0.37 & 25 & 1.1 & 13 \\
\hline Group 2 & - & - & 0.37 & 40 & 1.3 & 10 \\
\hline Group 2 & - & - & 0.37 & 60 & 1.1 & 8.6 \\
\hline Group 3 & CWP1 and CWP2 sludge & $\mathrm{n} / \mathrm{a}$ & 0.29 & 25 & 0 & 3.2 \\
\hline
\end{tabular}


Table I.3. (contd)

\begin{tabular}{|c|c|c|c|c|c|c|}
\hline \multirow[b]{2}{*}{ Tank } & \multirow[b]{2}{*}{ Primary Waste Type } & \multirow[b]{2}{*}{$\begin{array}{c}\text { Primary } \\
\text { Waste Type } \\
\text { Fraction } \\
\end{array}$} & \multirow[b]{2}{*}{$\begin{array}{l}\text { Sample Mass } \\
\text { Fraction UDS }\end{array}$} & \multirow[b]{2}{*}{$\begin{array}{c}\text { Sample } \\
\text { Temperature }\left({ }^{\circ} \mathrm{C}\right)\end{array}$} & \multicolumn{2}{|c|}{ Bingham Parameters } \\
\hline & & & & & $\begin{array}{c}\text { Yield Stress } \\
\text { (Pa) }\end{array}$ & $\begin{array}{c}\text { Viscosity } \\
\text { (cP) }\end{array}$ \\
\hline Group 3 & - & - & 0.29 & 25 & 0 & 3.4 \\
\hline Group 3 & - & - & 0.29 & 40 & 0 & 2.3 \\
\hline Group 3 & - & - & 0.29 & 60 & 0 & 1.7 \\
\hline C-104 & CWP1 sludge & 0.34 & 0.07 & 25 & 0.2 & 1.4 \\
\hline C-104 & - & - & 0.07 & 25 & 0 & 1.7 \\
\hline C-104 & - & - & 0.11 & 25 & 0.2 & 1.7 \\
\hline C-104 & - & - & 0.14 & 25 & 0.4 & 3.1 \\
\hline C-104 & - & - & 0.21 & 25 & 2.5 & 5.1 \\
\hline C-104 & - & - & 0.21 & 25 & 0.7 & 3.5 \\
\hline C-104 & - & - & 0.22 & 25 & 1.8 & 7.7 \\
\hline $\mathrm{C}-104$ & - & - & 0.24 & 25 & 20.4 & 1.1 \\
\hline C-104 & - & - & 0.27 & 25 & 2.8 & 10.8 \\
\hline C- 104 & - & - & 0.56 & 25 & 4.3 & 10.2 \\
\hline C-104 & CWP1 sludge & 0.34 & 0.07 & 45 & 0 & 1.1 \\
\hline C- 104 & - & - & 0.11 & 45 & 0.1 & 1.6 \\
\hline C-104 & - & - & 0.14 & 45 & 0.3 & 2.4 \\
\hline C-104 & - & - & 0.21 & 45 & 0.8 & 2.9 \\
\hline C-104 & - & - & 0.27 & 45 & 3.3 & 9.3 \\
\hline $\mathrm{C}-104$ & - & - & 0.56 & 45 & 2.1 & 5.9 \\
\hline$C-104$ & CWP1 sludge & 0.34 & 0.07 & 65 & 0 & 1.1 \\
\hline C-104 & - & - & 0.11 & 65 & 0.1 & 1.2 \\
\hline C-104 & - & - & 0.14 & 65 & 0.3 & 1.5 \\
\hline C-104 & - & - & 0.21 & 65 & 0.7 & 2.6 \\
\hline C-104 & - & - & 0.27 & 65 & 3.8 & 7.5 \\
\hline C-104 & - & - & 0.56 & 65 & 1.9 & 4.2 \\
\hline T-102 & CWP2 sludge & 0.89 & 0.05 & 30 & 0 & 1 \\
\hline $\mathrm{T}-102$ & - & - & 0.10 & 30 & 0 & 1.2 \\
\hline $\mathrm{T}-102$ & - & - & 0.05 & 90 & 0 & 0.2 \\
\hline $\mathrm{T}-102$ & - & - & 0.10 & 90 & 0 & 0.9 \\
\hline Group 4 & CWR1 sludge & $\mathrm{n} / \mathrm{a}$ & 0.30 & 25 & 0 & 2.3 \\
\hline Group 4 & - & - & 0.30 & 25 & 0 & 2.4 \\
\hline Group 4 & - & - & 0.30 & 40 & 0 & 1.4 \\
\hline Group 4 & - & - & 0.30 & 60 & 0 & $\mathrm{I} .1$ \\
\hline$\Lambda Z-101$ & P3 sludge & 1.00 & 0.11 & 25 & 1.1 & 2.9 \\
\hline$A Z-101$ & - & - & 0.14 & 25 & 0.3 & 2.2 \\
\hline AZ-I0I & - & - & 0.14 & 25 & 0.2 & 2.8 \\
\hline AZ-101 & - & - & 0.14 & 25 & 0.3 & 3 \\
\hline$A Z-101$ & - & - & 0.14 & 25 & 0.2 & 3 \\
\hline$A Z-101$ & - & - & 0.14 & 25 & 0.2 & 3.5 \\
\hline AZ-101 & - & - & 0.14 & 25 & 0.2 & 3 \\
\hline AZ-10I & - & - & 0.45 & 25 & 4 & 11 \\
\hline AZ-101 & - & - & 0.45 & 25 & 4 & 11 \\
\hline AZ-101 & - & - & 0.45 & 25 & 2.4 & 11 \\
\hline $\mathrm{AZ}-101$ & - & - & 0.45 & 25 & 4 & 10 \\
\hline AZ-101 & - & - & 0.45 & 25 & 2.9 & 11 \\
\hline AZ-101 & - & - & 0.45 & 25 & 4.7 & 17 \\
\hline$A Z-101$ & - & - & 0.45 & 25 & 3 & 18 \\
\hline AZ-101 & - & - & 0.45 & 25 & 4.4 & 16 \\
\hline$A Z-101$ & - & - & 0.03 & 27 & 0 & 1.8 \\
\hline$A Z-101$ & - & - & 0.25 & 27 & 0.9 & 7.9 \\
\hline AZ-101 & - & - & 0.12 & 27 & 0 & 2.7 \\
\hline$A Z-101$ & - & - & 0.25 & 27 & 0.8 & 7.1 \\
\hline$A Z-101$ & - & - & 0.01 & 28 & 0 & 2.3 \\
\hline AZ-101 & P3 sludge & 1.00 & 0.14 & 40 & 0.1 & 2.5 \\
\hline AZ-I0I & - & - & 0.14 & 40 & 0.2 & 2.6 \\
\hline AZ-101 & - & - & 0.14 & 40 & 0.2 & 2.6 \\
\hline AZ-101 & - & - & 0.14 & 40 & 0.2 & 2.6 \\
\hline $\mathrm{AZ}-101$ & - & - & 0.14 & 40 & 0.2 & 2.5 \\
\hline AZ-101 & - & - & 0.14 & 40 & 0.2 & 2.4 \\
\hline AZ-101 & P3 sludge & 1.00 & 0.01 & 45 & 0 & 1.9 \\
\hline AZ-101 & - & - & 0.25 & 45 & 0.7 & 4.7 \\
\hline $\mathrm{AZ}-101$ & - & - & 0.25 & 45 & 0.8 & 5.7 \\
\hline AZ-101 & - & - & 0.03 & 45 & 0 & 1.6 \\
\hline
\end{tabular}


Table I.3. (contd)

\begin{tabular}{|c|c|c|c|c|c|c|}
\hline \multirow[b]{2}{*}{ Tank } & \multirow[b]{2}{*}{ Primary Waste Type } & \multirow[b]{2}{*}{$\begin{array}{c}\text { Primary } \\
\text { Waste Type } \\
\text { Fraction }\end{array}$} & \multirow[b]{2}{*}{\begin{tabular}{|l|} 
Sample Mass \\
Fraction UDS \\
\end{tabular}} & \multirow[b]{2}{*}{$\begin{array}{c}\text { Sample } \\
\text { Temperature }\left({ }^{\circ} \mathrm{C}\right)\end{array}$} & \multicolumn{2}{|c|}{ Bingham Parameters } \\
\hline & & & & & $\begin{array}{c}\text { Yield Stress } \\
(\mathrm{Pa})\end{array}$ & $\begin{array}{c}\text { Viscosity } \\
\text { (cP) }\end{array}$ \\
\hline AZ-101 & - & - & 0.12 & 45 & 0 & 1.8 \\
\hline AZ-101 & - & - & 0.03 & 65 & 0 & 1.5 \\
\hline AZ-101 & - & - & 0.25 & 65 & 0.4 & 3 \\
\hline AZ-101 & P3 sludge & 1.00 & 0.01 & 65 & 0 & 1.6 \\
\hline AZ-101 & - & - & 0.12 & 65 & 0 & 1.4 \\
\hline AZ-102 & P3 sludge & 1.00 & 0.05 & 25 & 0.1 & 2.2 \\
\hline AZ-102 & - & - & 0.09 & 25 & 6.5 & 7.9 \\
\hline AZ-102 & - & - & 0.14 & 25 & 1.4 & 5.6 \\
\hline AZ-102 & - & - & 0.02 & 27 & 0 & 2.1 \\
\hline AZ-102 & - & - & 0.03 & 27 & 0 & 2.1 \\
\hline AZ-102 & - & - & 0.32 & 27 & 0.9 & 4.8 \\
\hline $\mathrm{AZ}-102$ & - & - & 0.17 & 27 & 0 & 2.4 \\
\hline AZ-102 & - & - & 0.17 & 45 & 0 & 1.5 \\
\hline AZ-102 & - & - & 0.02 & 45 & 0 & 1.8 \\
\hline $\mathrm{AZ}-102$ & - & - & 0.03 & 15 & 0 & 1.5 \\
\hline AZ- 102 & - & - & 0.32 & 45 & 0.6 & 3.2 \\
\hline $\mathrm{AZ}-102$ & - & - & 0.32 & 65 & 0.5 & 2.3 \\
\hline$A Z-102$ & - & - & 0.03 & 65 & 0 & 1 \\
\hline $\mathrm{AZ}-102$ & - & - & 0.17 & 65 & 0 & 1.2 \\
\hline Group 8 & PFeCN sludge & $\mathrm{n} / \mathrm{a}$ & 0.11 & 25 & 0 & 3.3 \\
\hline Group 8 & - & - & 0.11 & 25 & 0 & 2.6 \\
\hline Group 8 & - & - & 0.11 & 40 & 0 & 2.2 \\
\hline Group 8 & - & - & 0.11 & 60 & 0 & 1.4 \\
\hline Group 5 & R1 (boiling) sludge & $\mathrm{n} / \mathrm{a}$ & 0.19 & 25 & 57 & 13 \\
\hline Group 5 & - & - & 0.19 & 40 & 66 & 13 \\
\hline Group 5 & - & - & 0.19 & 60 & 74 & 17 \\
\hline Group 7 & TBP sludge & $\mathrm{n} / \mathrm{a}$ & 0.10 & 25 & 4.1 & 11 \\
\hline Group 7 & - & - & 0.10 & 25 & 3.3 & 12 \\
\hline Group 7 & - & - & 0.10 & 40 & 2.8 & 11 \\
\hline Group 7 & - & - & 0.10 & 60 & 3.5 & 8.9 \\
\hline C-109 & TFeCN sludge & 0.38 & 0.05 & 30 & 0.9 & 2.6 \\
\hline C-109 & - & - & 0.08 & 30 & 37.2 & 56.5 \\
\hline $\mathrm{C}-112$ & TFeCN sludge & 0.69 & 0.07 & 25 & 0.7 & 3.9 \\
\hline C-112 & - & - & 0.07 & 95 & 0.1 & 1.8 \\
\hline SY-102 & Unidentified sludge & 0.51 & 0.23 & 25 & 0 & 0.8 \\
\hline SY -102 & - & - & 0.59 & 25 & 2.1 & 6.2 \\
\hline SY-102 & - & - & 0.60 & 25 & 2.6 & 4.7 \\
\hline $\mathrm{AN}-102$ & A2 saltcake & 1.00 & 0.02 & 25 & 1.6 & 14.7 \\
\hline $\mathrm{AN}-102$ & - & - & 0.02 & 35 & 1.4 & 9.9 \\
\hline AN-102 & - & - & 0.02 & 50 & 0.7 & 9.4 \\
\hline AN-103 & A2 saltcake & 1.00 & 0.01 & 29 & 0 & 19.6 \\
\hline $\mathrm{AN}-103$ & - & - & 0.02 & 29 & 0 & 9.5 \\
\hline $\mathrm{AN}-103$ & - & - & 0.25 & 29 & 0 & 35.3 \\
\hline $\mathrm{AN}-103$ & A2 saltcake & 1.00 & 0.01 & 45 & 0 & 10.8 \\
\hline AN-103 & - & - & 0.02 & 45 & 0 & 5.8 \\
\hline $\mathrm{AN}-103$ & - & - & 0.25 & 45 & 0 & 20.3 \\
\hline $\mathrm{AN}-103$ & A2 saltcake & 1.00 & 0.01 & 65 & 0 & 6.1 \\
\hline AN-103 & - & - & 0.02 & 65 & 0 & 4 \\
\hline $\mathrm{AN}-103$ & - & - & 0.25 & 65 & 0 & 11.9 \\
\hline $\mathrm{AN}-104$ & A2 saltcake & 1.00 & 0.01 & 25 & 0 & 3.9 \\
\hline $\mathrm{AN}-104$ & - & - & 0.01 & 25 & 0.1 & 27 \\
\hline AN-104 & - & - & 0.02 & 25 & 1.5 & 1.9 \\
\hline $\mathrm{AN}-104$ & - & - & 0.02 & 25 & 0 & 8.7 \\
\hline $\mathrm{AN}-104$ & - & - & 0.05 & 25 & 0 & 2.9 \\
\hline $\mathrm{AN}-104$ & - & - & 0.11 & 25 & 0.1 & 55.9 \\
\hline $\mathrm{AN}-104$ & A2 saltcake & 1.00 & 0.01 & 45 & 0.1 & 12.5 \\
\hline $\mathrm{AN}-104$ & - & - & 0.02 & 45 & 0.2 & 4.7 \\
\hline AN-104 & - & - & 0.05 & 45 & 0.2 & 13.1 \\
\hline AN-104 & - & - & 0.11 & 45 & 0.1 & 24.7 \\
\hline $\mathrm{AN}-104$ & A2 saltcake & 1.00 & 0.01 & 65 & 0.1 & 7.2 \\
\hline AN-104 & - & - & 0.02 & 65 & 0.3 & 3.4 \\
\hline AN-104 & - & - & 0.05 & 65 & 0.2 & 6.7 \\
\hline $\mathrm{AN}-104$ & - & - & 0.11 & 65 & 0.4 & 14.1 \\
\hline
\end{tabular}


Table I.3. (contd)

\begin{tabular}{|c|c|c|c|c|c|c|}
\hline \multirow[b]{2}{*}{ Tauk } & \multirow[b]{2}{*}{ Primary Waste Type } & \multirow[b]{2}{*}{$\begin{array}{c}\text { Primary } \\
\text { Waste Type } \\
\text { Fraclion }\end{array}$} & \multirow[b]{2}{*}{\begin{tabular}{|l|} 
Sample Mass \\
Fraction UDS
\end{tabular}} & \multirow[b]{2}{*}{$\begin{array}{c}\text { Sample } \\
\text { Temperature }\left({ }^{\circ} \mathrm{C}\right)\end{array}$} & \multicolumn{2}{|c|}{ Bingham Parameters } \\
\hline & & & & & $\begin{array}{c}\text { Yield Stress } \\
(\mathrm{Pa}) \\
\end{array}$ & $\begin{array}{c}\text { Viscosity } \\
\text { (cP) }\end{array}$ \\
\hline$A N-105$ & A2 saltcake & 1.00 & 0.01 & 25 & 0 & 6 \\
\hline $\mathrm{AN}-105$ & - & - & 0.09 & 25 & 0 & 7.1 \\
\hline $\mathrm{AN}-105$ & - & - & 0.12 & 25 & 0 & 19.4 \\
\hline $\mathrm{AN}-105$ & - & - & 0.16 & 25 & 0 & 50.9 \\
\hline $\mathrm{AN}-105$ & A2 saltcake & 1.00 & 0.01 & 45 & 0 & 3.6 \\
\hline $\mathrm{AN}-105$ & - & - & 0.09 & 45 & 0 & 3.9 \\
\hline $\mathrm{AN}-105$ & - & - & 0.12 & 45 & 0 & 10 \\
\hline $\mathrm{AN}-105$ & - & - & 0.16 & 45 & 0.5 & 21.5 \\
\hline $\mathrm{AN}-105$ & A2 saltcake & 1.00 & 0.01 & 65 & 0 & 2.5 \\
\hline $\mathrm{AN}-105$ & - & - & 0.09 & 65 & 0 & 2.7 \\
\hline $\mathrm{AN}-105$ & - & - & 0.12 & 65 & 0 & 6.4 \\
\hline $\mathrm{AN}-105$ & - & - & 0.16 & 65 & 0.6 & 13.3 \\
\hline $\mathrm{AN}-107$ & A2 saltcake & 1.00 & 0.00 & 25 & 1.5 & 9.4 \\
\hline $\mathrm{AN}-107$ & - & - & 0.00 & 40 & 1.1 & 5.9 \\
\hline AW-101 & A2 saltcake & 1.00 & 0.01 & 25 & 0 & 8.2 \\
\hline AW-101 & - & - & 0.02 & 25 & 0 & 5.4 \\
\hline AW-101 & - & - & 0.03 & 25 & 0.1 & 46.5 \\
\hline AW-101 & A2 saltcake & 1.00 & 0.01 & 45 & 0 & 4.2 \\
\hline AW-101 & - & - & 0.02 & 45 & 0 & 2.8 \\
\hline AW-101 & - & - & 0.03 & 45 & 0.1 & 16.9 \\
\hline AW-101 & A2 saltcake & 1.00 & 0.01 & 65 & 0 & 2.6 \\
\hline AW-101 & - & - & 0.02 & 65 & 0 & 1.8 \\
\hline AW-101 & - & - & 0.03 & 65 & 0.2 & 10.5 \\
\hline S-104 & R saltcake & 0.47 & 0.15 & 28 & 17.8 & 20.7 \\
\hline S-104 & - & - & 0.06 & 30 & 4.6 & 9.7 \\
\hline S-104 & - & - & 0.08 & 30 & 1.4 & 4.5 \\
\hline S-104 & - & - & 0.17 & 30 & 15.3 & 112.5 \\
\hline S-104 & - & - & 0.20 & 30 & 3.9 & 8.1 \\
\hline S-104 & - & - & 0.06 & 95 & 4.8 & 7.2 \\
\hline S-104 & - & - & 0.08 & 95 & 1.6 & 3.7 \\
\hline S-104 & - & - & 0.15 & 95 & 10.5 & 10.9 \\
\hline S-104 & - & - & 0.20 & 95 & 2.5 & 6.4 \\
\hline SY-101 & S2 saltcake & 1.00 & 0.02 & 32 & 0.3 & 44.7 \\
\hline SY-101 & - & - & 0.12 & 32 & 0.4 & 60.4 \\
\hline SY-101 & - & - & 0.12 & 32 & 0.3 & 54.3 \\
\hline SY-10I & S2 saltcake & 1.00 & 0.02 & 50 & 0.7 & 15.5 \\
\hline SY-101 & - & - & 0.05 & 50 & 0.3 & 20.2 \\
\hline SY-101 & - & - & 0.07 & 50 & 0.6 & 5.8 \\
\hline SY-101 & - & - & 0.13 & 50 & 0.3 & 24.7 \\
\hline SY-101 & - & - & 0.15 & 50 & 0.3 & 29.2 \\
\hline SY-101 & - & - & 0.16 & 50 & 1.3 & 26.9 \\
\hline SY-101 & - & - & 0.29 & 50 & 3.4 & 79.4 \\
\hline SY-101 & - & - & 0.36 & 50 & 5.1 & 81.9 \\
\hline SY-101 & - & - & 0.07 & 65 & 0.2 & 13.5 \\
\hline SY-101 & - & - & 0.12 & 65 & 0 & 7.7 \\
\hline$S Y-101$ & - & - & 0.15 & 65 & 0.3 & 15.4 \\
\hline SY-101 & - & - & 0.04 & 70 & 2.2 & 25.3 \\
\hline SY-101 & - & - & 0.12 & 70 & 5.2 & 46.4 \\
\hline SY-101 & - & - & 0.20 & 70 & 5.1 & 59.2 \\
\hline$S Y-101$ & - & - & 0.09 & 80 & 0 & 5.5 \\
\hline SY-101 & - & - & 0.10 & 80 & 0.2 & 11.8 \\
\hline SY-101 & - & - & 0.12 & 80 & 0.3 & 11.6 \\
\hline SY-101 & - & - & 0.02 & 90 & 2.7 & 19.2 \\
\hline$S Y-101$ & - & - & 0.02 & 90 & 2.6 & 18.5 \\
\hline SY-101 & - & - & 0.12 & 90 & 8.1 & 48 \\
\hline Group 6 & S-Saltcake (S1 and S2) & $\mathrm{n} / \mathrm{a}$ & 0.15 & 25 & 0 & 6.5 \\
\hline Group 6 & - & - & 0.15 & 25 & 0 & 8 \\
\hline Group 6 & - & - & 0.15 & 40 & 0 & 6.9 \\
\hline Group 6 & - & - & 0.15 & 60 & 0 & 4.6 \\
\hline
\end{tabular}


PNNL-20646

EMSP-RPT-006

\section{Distribution}

No. of

Copies

7 Department of Energy

Office of Environmental Management

D Govans

HE Johnson

NP Machara*

SP Schneider*

J Shultz*

GL Smith*

HG Sutter (consultant)

17 Department of Energy

Office of River Protection

W Abdul

H6-60

DH Alexander

H6-60

GE Brunson

VL Callahan

K Chen

TW Fletcher*

RA Gilbert

BJ Harp

PG Harrington

TA Hu

AA Kruger

C-K Liu

BM Mauss

SH Pfaff*

JJ Rambo

JA Reddick

JH Wicks
H6-60

H6-60

H6-60

H6-60

H6-60

H6-60

H6-60

H6-60

H6-60

H6-60

H6-60

H6-60

H6-60

H6-60

H6-60
No. of

Copies

1 Oak Ridge National Laboratory

EM Pierce*

2 CRESP/Vanderbilt

DS Kosson

SL Krahn

13 Washington River Protection Services

WB Barton

S7-90

KD Boomer

E6-47

RA Burk

R3-26

WS Callaway

T6-07

GA Cooke

T6-07

DA Greer

B1-55

SJ Harrington

B1-55

DL Herting*

T6-07

JE Meacham

R2-58

RE Mendoza

B1-55

JH Rasmussen

B1-55

JG Reynolds*

B1-55

MG Thien*

B1-55

$1 \quad$ Washington State Department of Ecology JJ Lyon

$1 \quad$ Columbia Energy and Environmental $\underline{\text { Services }}$

SF Agnew

3 Defense Nuclear Facility Safety Board

PA Meyer

AP Poloski*

RG Quirk

$3 \quad$ Savannah River National Laboratory

E Hansen

M Poirier

W Wilmarth 
PNNL-20646

EMSP-RPT-006

\section{Distribution (con't)}

No. of

\section{Copies}

8

Bechtel National, Inc.

AV Arakali*

SM Barnes

FW Damerow

G Duncan*

RL Hanson*

PJ Keuhlen

ED Lee

PS Sundar

18 Pacific Northwest National Laboratory

KK Anderson

EC Buck

K7-20

CA Burns

SK Cooley

RC Daniel

AR Felmy

LK Holton

JL Huckaby*

DE Knutson

DE Kurath*

LA Mahoney

TE Michener

MJ Minette

Y Onishi

RA Peterson

MW Rinker

JM Tingey*

BE Wells*
P7-27

P7-25

K7-20

P7-22

K8-96

H6-61

K7-15

K3-52

K7-15

K7-15

P7-25

K7-15

P7-22

K5-22

K8-41

K7-15
No. of

Copies

External

D Dickey

A Etchells

*Will be receiving hard copy distribution. 



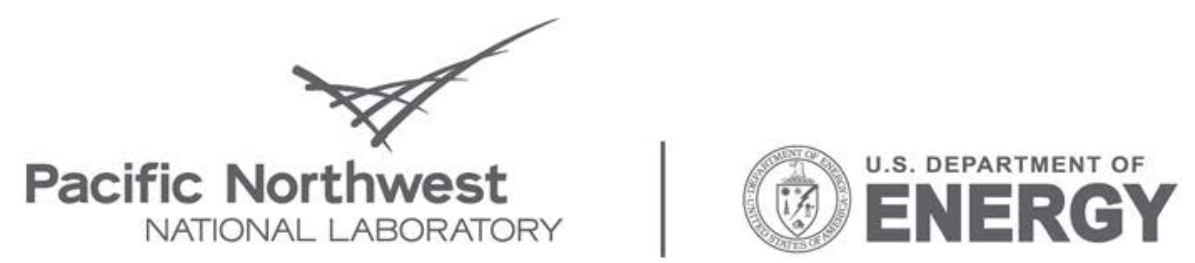

Proudly Operated by Battelle Since 1965

902 Battelle Boulevard

P.O. Box 999

Richland, WA 99352

1-888-375-PNNL (7665)

www.pnl.gov 